258

$11 / 12$

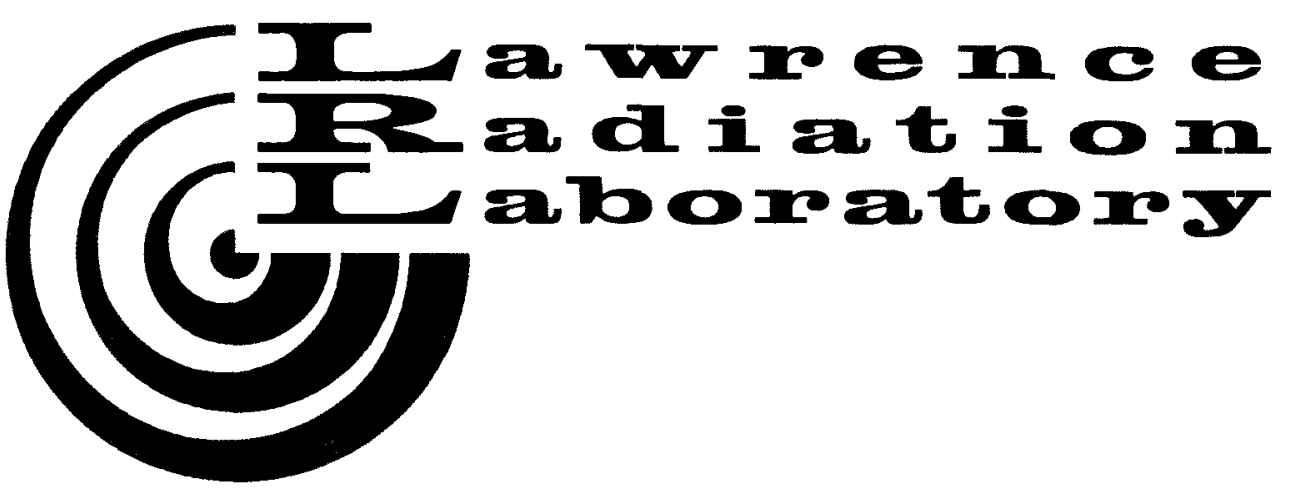

UCRL-50163 Part IV

PREDICTION OF THE MAXIMUM DOSAGE TO MAN

FROM THE FALLOUT OF NUCLEAR DEVICES

IV. HANDBOOK FOR ESTIMATING THE

MAXIMUM INTERNAL DOSE FROM RADIONUCLIDES

RELEASED TO THE BIOSPHERE

Bio-Medical Division

Yook C. Ng, C. Ann Burton,

Stanley E. Thompson, Robert K. Tandy,

Helen K. Kretner, and Michael W. Pratt

May 14,1968

UNIVERSITY Of CALIFORNIA L I V E R M O RE 


\section{DISCLAIMER}

This report was prepared as an account of work sponsored by an agency of the United States Government. Neither the United States Government nor any agency Thereof, nor any of their employees, makes any warranty, express or implied, or assumes any legal liability or responsibility for the accuracy, completeness, or usefulness of any information, apparatus, product, or process disclosed, or represents that its use would not infringe privately owned rights. Reference herein to any specific commercial product, process, or service by trade name, trademark, manufacturer, or otherwise does not necessarily constitute or imply its endorsement, recommendation, or favoring by the United States Government or any agency thereof. The views and opinions of authors expressed herein do not necessarily state or reflect those of the United States Government or any agency thereof. 


\section{DISCLAIMER}

Portions of this document may be illegible in electronic image products. Images are produced from the best available original document. 
TID $-4500, \quad$ UC -48

Biology and Medicine

\author{
Inawremoe Radiation Iaboratory \\ UNIVERSITY OF CALIFORNIA \\ LIVERMORE
}

UCRL-50163 Part IV

\title{
PREDICTION OF THE MAXIMUM DOSAGE TO MAN \\ FROM THE FALLOUT OF NUCLEAR DEVICES \\ IV. HANDBOOK FOR ESTIMATING THE \\ MAXIMUM INTERNAL DOSE FROM RADIONUCLIDES \\ RELEASED TO THE BIOSPHERE \\ Bio-Medical Division \\ Yook C. Ng, C. Ann Burton, \\ Stanley E. Thompson, Robert K. Tandy, \\ Helen K. Kretner, and Michael W. Pratt
}

May 14, 1968

LEGAL NOTICE

This report was prepared as an account of Government sponsored work. Neither the United States, nor the Commisston, nor any person acting on behalf of the Commisalon:

A. Makes any warranty or representation, expressed or implied, with respect to the accuracy, completeness, or usefulness of the information contained in this report, or that the use of any information, apparatus, method, or process disclosed in this report may not infringe privately owned rights; or

B. Assumes any liabilities with respect to the use of, or for damages resulting from the se of any information, apparatus, method, or process disclosed in thls report.

As used in the above, "person acting on behalf of the Commission" includes any employee or contractor of the Commission, or employee of such contractor, to the extent that

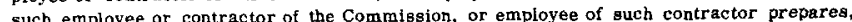
dis with the Commission, or his employment with such contractor. 
8

•

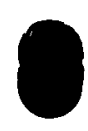




\section{Contents}

PREFACE . . . . . . . . . . . . . . . . .

ACKNOWLEDGMENTS . . . . . . . . . . . . . . . viii

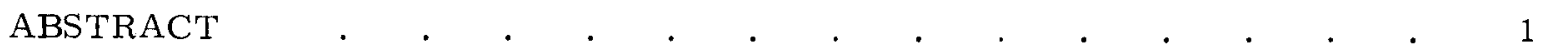

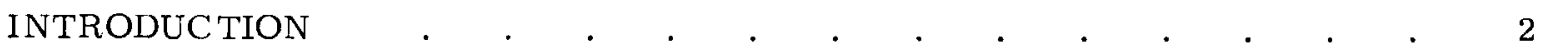

List of Symbols $\quad . \quad$. . . . . . . . . . . . . . . 3

HANDBOOK TABLES: ESTIMATES OF INPUT PARAMETERS AND

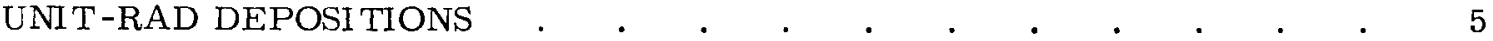

1. Mass of Organs in Standard Man and the One-Year Child . . 6.1

2. Q: Maximum Available Energy from the Decay of

3. $\mathrm{f}_{\mathrm{M}}$ : Fraction of the Daily Intake Secreted in Milk . . . . . 13

4. $\mathrm{C}_{\mathrm{S}}$ : Average Concentration in Soil $\quad . \quad$. . . . . . . 15

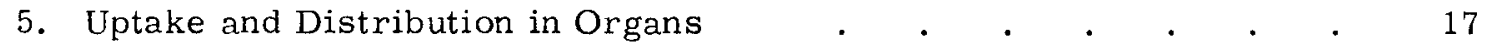

$\mathrm{f}_{\mathrm{B}}$ : Fractional Uptake . . . . . . . . . . 17

$\mathrm{T}_{\mathrm{B}}$ : Biological Half-Life.$\quad \cdot \quad \cdot \quad \cdot \quad \cdot \quad \cdot \quad \cdot \quad \cdot 25$

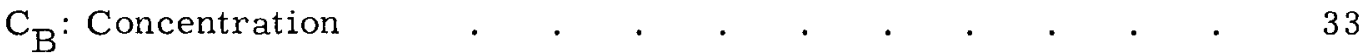

6. $\mathrm{F}_{1}$ : Unit Rad Deposition . . . . . . . . . . . 41

Forage-to-Cow-to-Milk Pathway . . . . . . . . 41

Soil-Root Pathway . . . . . . . . . . 77

7. $\mathrm{C}_{\mathrm{A}}$ : Concentration in Water . . . . . . . . . . 103

Seawater • • . . . . . . . . . . . 103

Freshwater . . . . . . . . . . . . 105

8. C* : Ratio of Aquatic Diet to Terrestrial Diet . . . . . 107

Seawater . . . . . . . . . . . . . . . 108

Freshwater . . . . . . . . . . . . 109

9. $\mathrm{F}_{\mathrm{A}}$ : Unit-Rad Contamination . . . . . . . . . 111

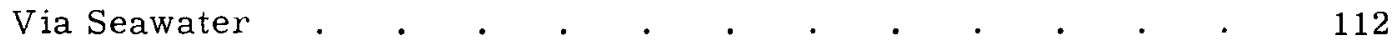

Via Freshwater . . . . . . . . . . . . . 153

10. Concentration in Terrestrial Foodstuffs $\quad . \quad$. . . . . . . . 193

Plants . . . . . . . . . . . . . 193

Meats . . . . . . . . . . . . . . . 195

11. Concentration in Seawater Foodstuffs . . . . . . . . . . . 197

Plants . . . . . . . . . . . . . . 198

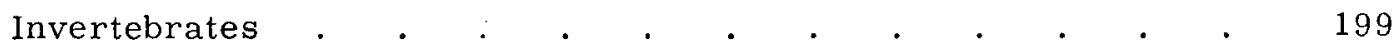

Fish . . . . . . . . . . . . . . . 200

12. Concentration in Freshwater Foodstuffs . . . . . . . 201

Plants . . . . . . . . . . . 202

Invertebrates . . . . . . . . . . . . 203

Fish . . . . . . . . . . . . . 204 
APPENDIX: ESTIMATES OF INPUT PARAMETERS USING

COLLATERAL DATA

Introduction

1. General Equations

2. Estimation of the Daily Intake . . . . . . . . . A.3

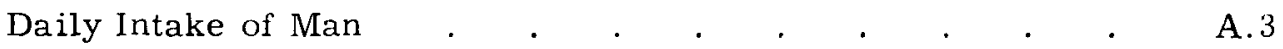

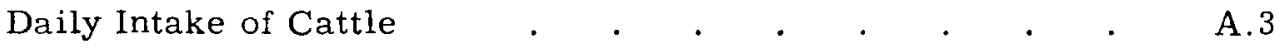

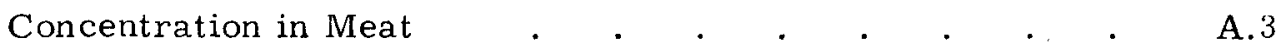

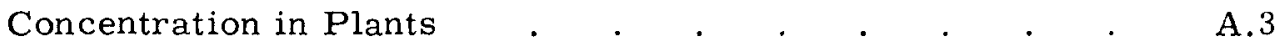

3. Estimation of $\mathrm{f}_{\mathrm{B}}$ : Fractional Uptake by Ingestion . . . . A.4

4. Estimation of $\mathrm{T}_{\mathrm{B}}$ : Biological Half-Life . . . . . A.5

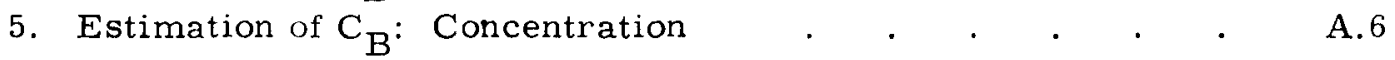

6. Special Considerations in Estimation of $f_{B}, T_{B}$, and $C_{B} \cdot \quad \cdot \quad A .7$

Functional Dependence of Unit-rad Deposition on

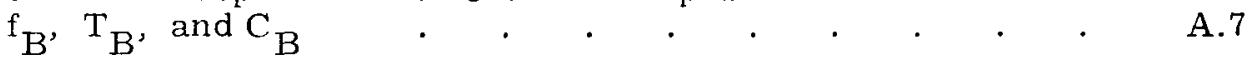

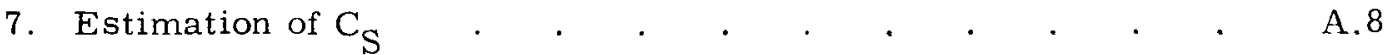

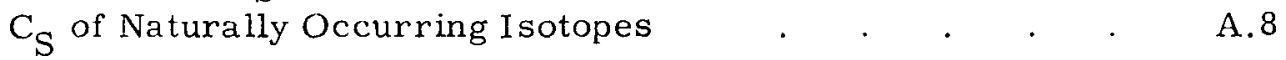

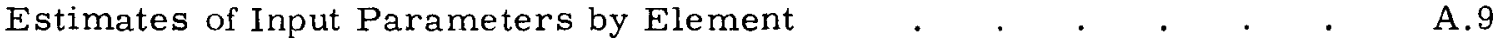

BIBLIOGRA PHIC CITATIONS FOR SELECTED ACCESSION NUMBERS . . $\quad 205$

HANDBOOK REQUEST FORMS 


\section{Preface}

The extensive coverage given to $\mathrm{I}^{131}$ and infant thyroid dosages subsequent to nuclear device testing at the Nevada Test Site is sufficient evidence to indicate that both preshot prediction and postshot documentation of the dosage to humans from internal emitters were inadequate. So far as $\mathrm{I}^{131}$ is concerned, the postshot documentation was partially corrected in 1957. However, this documentation capability was not integrated with preshot prediction and as a result "surprises" occurred following the July 1962 tests.

Whether or not such "surprises" could have been anticipated in July 1962 is at this time academic. It is important to point out, however, that there is every reason to believe that these "surprises" need not occur in future events if an adequate program of preshot prediction is integrated with an adequate program of postshot documentation. Off-site radiological safety programs should and can be conducted with the same degree of planning and precision as laboratory experiments. Thus preshot prediction should not serve the sole purpose of preshot rad-safe analysis. Rather, it should also have the function of guiding the postshot documentation by suggesting what to measure, where to measure it, and the precision required in the measurement. Furthermore, the preshot prediction program should feed on the postshot documentation results in order to improve subsequent predictions.

There are two other points that appear to be obvious conclusions from previous test results: (1) no radionuclide that is produced should be ignored until a critical analysis demonstrates that it is insignificant, and (2) preshot predictions and postshot documentation must encompass distances extending 2000 to 3000 miles from the site of detonation.

The Information Integration Group of the Bio-Medical Division of this laboratory has accepted the responsibility for developing this preshot predictive capability. UCRL-50163 (Parts I, II, III and IV) presents our approach to predicting the dosage from each and every radionuclide that is released to the atmosphere and deposited on agricultural lands remote from the site of detonation. Our group is also investigating the aquatic transport of nuclear debris by surface and ground water into fresh water and marine ecosystems. This predictive approach will be presented in subsequent reports in this series.

Part I of this report presents the approach we use to estimate the fallout levels as a function of cloud travel time for periods up to $50 \mathrm{hr}$ post-detonation. Part II shows how these fallout estimates can be combined with radionuclide production estimates and biological uptake relationships to arrive at estimates of burden and dosage for man. Part III shows how this predictive approach can supply guidelines for the design of nuclear devices for peaceful purposes. Part IV is a handbook which lists the input parameters required for the estimation of dosage. 
There are three questions that could be asked about the outcome of the detonation of a nuclear device. It is essential that the reader recognize which of the three we are trying to answer and why we feel it to be the most appropriate. The three possible questions are:

1. What is the worst situation that could develop?

2. What is the most likely situation that will develop?

3. What would be the situation if everything went off perfectly?

We choose to answer the first question and to direct our efforts to predicting the worst case. However, in the process of answering the first question we can generally answer the second. Quite obviously, the answer to the third question has no meaning with respect to public health and safety.

We choose to answer the first question because we feel that only the worst case should be compared with prescribed tolerances in a preshot rad-safe analysis. Furthermore, only when we know the worst case can we establish an adequate system of postshot monitoring to document the actual case and to insure that appropriate countermeasures are instituted when and if needed. In other words, it is only by this approach that uncertainties concerning dosimetry, such as presently exist for $\mathrm{I}^{131}$, can be eliminated.

Furthermore, we are attempting a thorough analysis and are considering each and every radionuclide on the chart of the nuclides. In this respect, our estimates may indicate that a particular radionuclide is a hazard for one of two reasons: either (1) it will be a hazard because of what we know about it, or (2) it will be a hazard because of what we don't know about it. If a pertinent relationship is not known for a particular radionuclide, we make worst-case estimates of the relationship and hence maximize our estimates of hazard. Nevertheless, despite its conservative nature, this approach still allows us to eliminate most of the radionuclides from consideration and to indicate those that are potentially the most hazardous. Obviously, it also allows us to estimate the upper limit of the potential burden and dosage; but due to the perversity of nature, the precise dosage can only be determined by postshot documentation in the affected areas.

It is obvious that had $\mathrm{I}^{131}$ been measured in milk during the early period of testing, its dosimetry would not now be a problem. Thus, we wish to be able through our predictive approach to indicate what should be measured, where it should be measured, and with what precision it should be measured. There appears to be no other way to insure unambiguous dosimetry for future events and to assure that the need for countermeasures is recognized in time so that they can be planned for and instituted when and if needed. The most appropriate countermeasures lie in device design, and Part III of this report shows how this predictive approach can supply guidelines for the design of nuclear devices that might be used in the construction of a sealevel canal. 
Thus, this predictive approach is meant to serve three purposes:

1. In preshot rad-safe analysis, to determine whether or not a particular event can be conducted without exceeding existing tolerances.

2. In guidance for postshot documentation, to indicate what should be measured, where it should be measured, and with what precision it should be measured.

3 . In guidance for device design, to indicate the maximum amount of a radionuclide that can be produced and subsequently released to the environment without exceeding prescribed tolerances. 


\section{Acknowledgments}

Many individuals contributed to the preparation of this Handbook. The authors are grateful to Mary Tamplin, Thelma Smith and Yvonne Ricker for documentation, and to Marguerite Longmate for data handling; and they are grateful to June Ellingson and Judith Fudala, who prepared the manuscript. 


\title{
PREDICTION OF THE MAXIMUM DOSAGE TO MAN FROM THE FALLOUT OF NUCLEAR DEVICES IV. HANDBOOK FOR ESTIMATING THE MAXIMUM INTERNAL DOSE FROM RADIONUCLIDES RELEASED TO THE BIOSPHERE
}

\begin{abstract}
Part IV of this series is a specialhours. The first issue and first addition purpose Handbook for estimating the maximum internal dose to man from radionuclides released to the biosphere. are devoted to estimation of the dosage resulting from contamination of agricultural lands as described in UCRLThis Handbook, which is to be continu50163, Part II, and the dosage resulting ously updated and is to be expandable, lists the pertinent data for all isotopes whose half-lives are greater than 12 from contamination of aquatic environments as described in UCRL-50163, Part V.
\end{abstract}




\section{Introduction}

This document is a Handbook for estimating the maximum internal dose from the deposition of radionuclides released to the biosphere. The Handbook, which is to be continuously updated, lists the pertinent data for all isotopes whose half-lives are greater than 12 hours. At this time the Handbook contains only the unit-rad depositions for the radionuclides and the input parameters used for their calculation. The unit-rad deposition $F_{1}$ is required for estimation of the maximum internal dose from the contamination of agricultural land as described in the other parts of the UCRL-50163 series $(5573,5574)$. * In subsequent updatings the pertinent data for estimating the dosage from contamination of aquatic environments will be included.

This document serves a very special purpose, that of estimating the maximum dosage from radionuclides released to the biosphere. A value is assigned to the parameters of each element. The values include conservative worst-case estimates and maximum or near maximum values. The Handbook, therefore, is not to be considered as representative of the average or mean for the biosphere. A Handbook of the Biological Exchangeable Pool will subsequently be issued which will list means and ranges of welldocumented values and values derivable with confidence from collateral data.

Input parameters were estimated following general procedures designated in the tables by code numbers. When data were

*The numbers 5573 and 5574 are accession numbers by which we designate specific documents in our Information File and in the bibliography of this report. not available, conservative worst-case estimates were made. When this procedure led to the identification of potentially hazardous nuclides, that is, nuclides whose $\mathrm{m}^{2}$-rad values were within the first three orders of magnitude, more reasonable values based on collateral data were obtained where possible. The use of collateral data for the re-evaluation of parameters used in UCRL-50163, Part II (5574), is described in detail in the Appendix under the heading of the appropriate element.

It is intended that this Handbook be updated as more data become available. Furthermore we intend to reevaluate the parameters for additional elements beyond those represented in the first three orders of $\mathrm{m}^{2}$-rad values. We are publishing the Handbook at this time for two important reasons:

(1) To solicit any additional data that may be pertinent to the various aspects to the problem.

(2) To solicit useful criticism that will permit the analysis to be more precise.

In this respect it should be pointed out that much of the information in the Handbook is necessarily based on animal data. We would be grateful for the receipt of any pertinent human data.

At this time, we are not certain of the frequency with which this Handbook will be updated. The revisions will be in the form of new tables or pages for replacement or addition to this existing document. The final two pages of this report contain address and change-of-address forms that the reader may use to keep us informed of his address so that revisions can be sent to him. 


\section{LIST OF SYMBOLS}

Listed below are the symbols which appear throughout this Handbook, together with the quantities they represent:

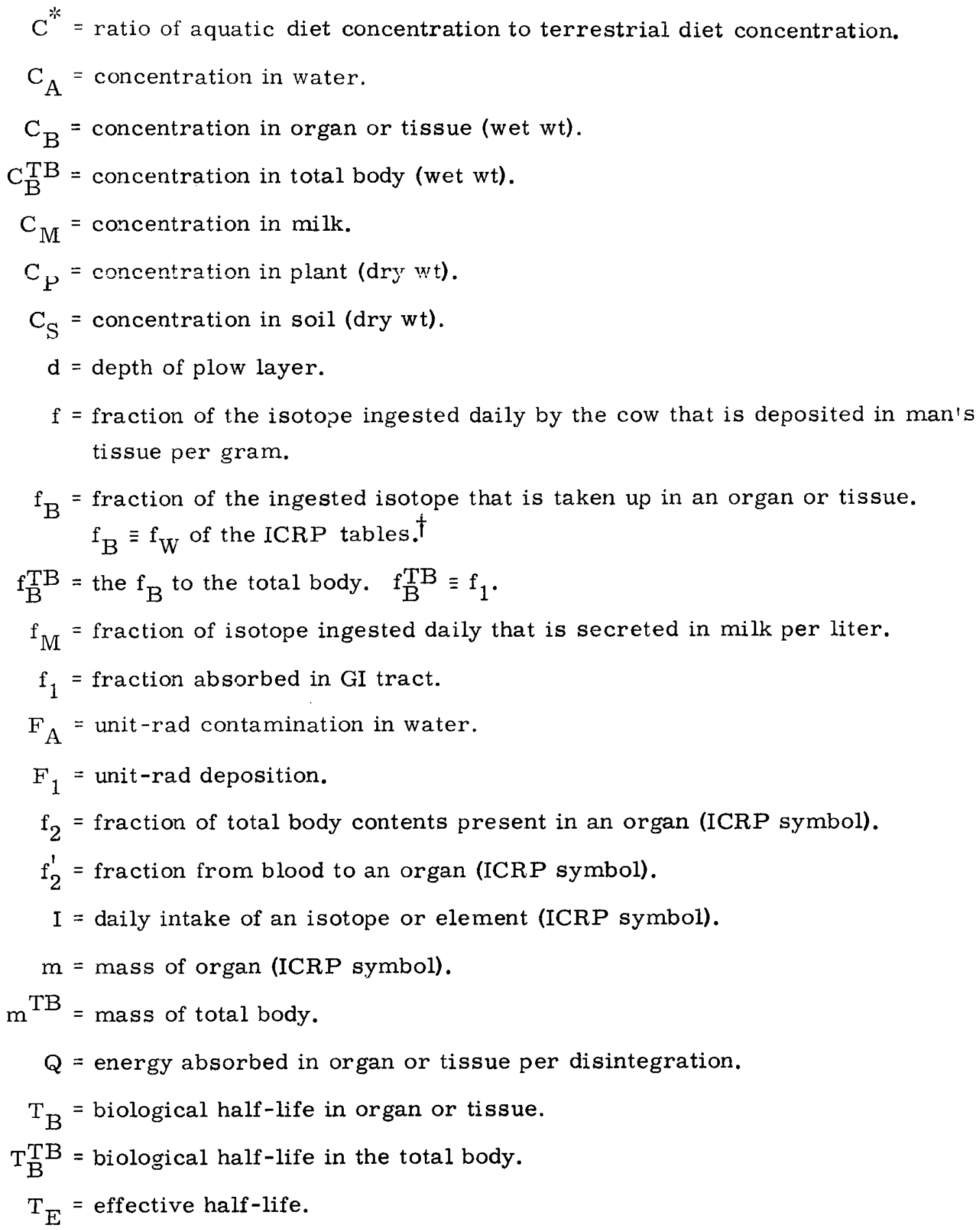

\footnotetext{
$t_{\text {Reference }}$ is made throughout this document to the tables of Report of ICRP Committee II on Permissible Dose for Internal Radiation (5592, 5593).
} 
$T_{P}=$ effective half-life on forage.

$T_{R}=$ half-life for radioactive decay.

(UAF) = "utilized area factor," the effective area of pasture grazed daily by the cow. $\rho=$ density of the medium. 


\section{Handbook Tables: Estimates of Input Parameters and Unit-Rad Depositions}

These tables list the required input parameters for calculation of unit-rad depositions, together with their reference sources, and the calculated unit-rad depositions of the elements in selected human organs. The organs listed are limited to those that have so far been considered in our predictive model for estimation of the maximum internal dose from fallout (5573, 5574).

The tables are in twelve sections:

1. Mass of organs in standard man and the one-year child.

2. Maximum available energy from the decay of radionuclides.

3. Fraction of the daily intake secreted in milk.

4. Average concentration in soil.

5. Uptake and distribution in organs.
A. Fraction of ingested isotope de- posited.
B. Biological half-life.
C. Concentration.

6. Unit-rad deposition.
A. Via milk.
B. Via typical agricultural soil.

7. Concentration in water
A. Seawater
B. Freshwater

8. Ratio of aquatic diet to terrestrial diet
A. Seawater
B. Freshwater

9. Unit-rad contamination
A. Via seawater
B. Via freshwater

10. Concentration in terrestrial foodstuffs
A. Plants
B. Meat

11. Concentration in seawater foodstuffs
A. Plants
B. Invertebrates
C. Fish

12. Concentration in freshwater foodstuffs
A. Plants
B. Invertebrates
C. Fish

The numerical values in the tables are expressed in E-format. Thus

2.40-03 signifies $2.40 \times 10^{-3}$. 

1. MASS OF ORGANS IN STANDARD MAN AND THE ONE-YEAR CHILD

The mass of organs in standard man and the one-year child are listed in Table 1 together with reference sources. The masses for the adult are for the most part the revised standard man values of Snyder et al. (5307). The masses for the one-year child are based on handbook values from a number of sources.

The mass listed for the gastrointestinal (GI) tract is the mass of the GI tract contents, which is used to estimate the unitrad deposition to the GI tract via milk. The value listed for the child assumes that the mass ratios of GI tract to total body are equal in the child and the adult. 
TABLE 1. MASS OF ORGANS IN STANDARO MAN ANO THE ONE-YEAR CHILD.

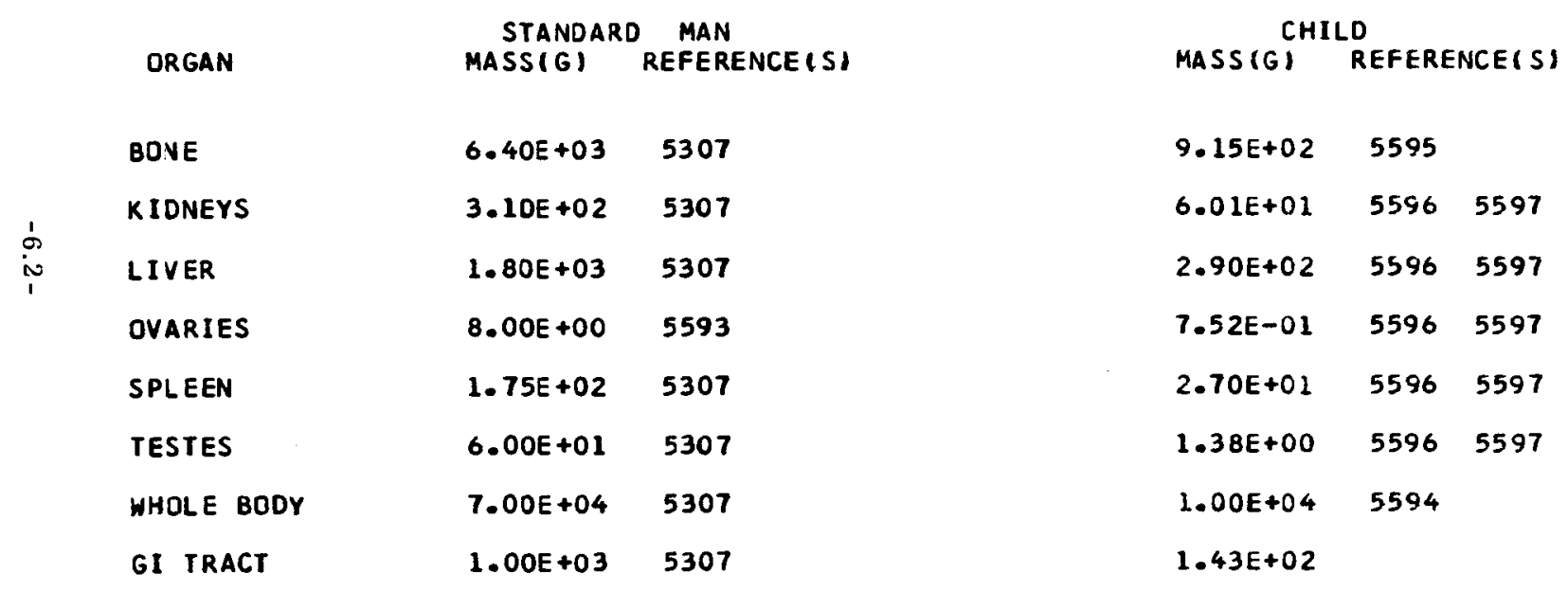

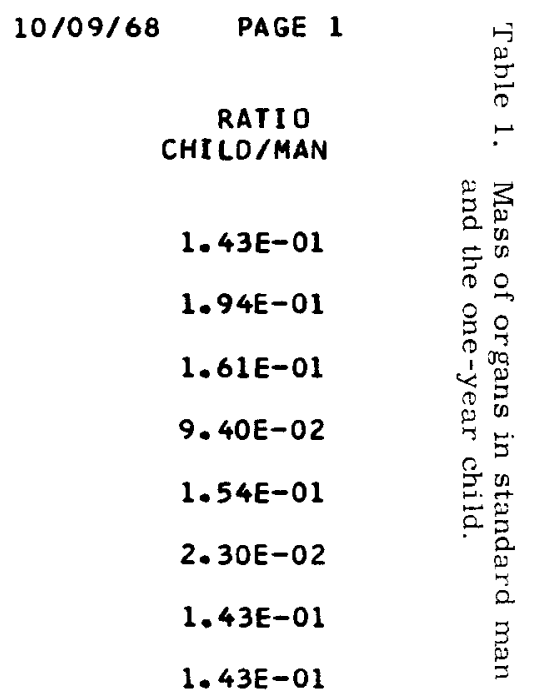


2. Q: MAXIMUM AVAILABLE ENERGY FROM THE DECAY OF RADIONUCLIDES

The values listed in Table 2 for $Q$ are the estimates of Burton and Maxwell of the total available disintegration energy in $\mathrm{MeV}$ for every nuclide $(Z=1$ to $Z=100)$ whose half-life is greater than 12 hours (5572). Each nuclide is identified by its atomic number $Z$, its mass $A$, and its radiological half-life $T_{R}$ in days. 


\begin{tabular}{|c|c|c|c|c|}
\hline $\begin{array}{l}\text { ELEMENT } \\
\text { SYMBOL }\end{array}$ & $\begin{array}{l}\text { ATOMIC } \\
\text { NO. (Z) }\end{array}$ & $\begin{array}{l}\text { ISOTOPE } \\
\text { MASS(A) }\end{array}$ & $\begin{array}{l}\text { HALF-LIFE } \\
T_{R} \text { (DAY) }\end{array}$ & $\begin{array}{l}\text { MAX. AVAI LABLE } \\
\text { ENERGY } Q \text { (MEV) }\end{array}$ \\
\hline$H$ & 1 & 3 & $4.47 E+03$ & $6.29 E-03$ \\
\hline $\mathrm{BE}$ & 4 & 7 & $5.36 E+01$ & $4.93 E-02$ \\
\hline $\mathrm{BE}$ & 4 & 10 & $9.85 E+08$ & $2.90 E-J 1$ \\
\hline$C$ & 6 & 14 & $2.09 E+06$ & $6.09 E-02$ \\
\hline NA & 11 & 22 & $9.42 E+02$ & $2.37 E+00$ \\
\hline NA & 11 & 24 & $6.25 \mathrm{E}-01$ & 4. $98 E+00$ \\
\hline MG & 12 & 28 & $8.88 E-01$ & $5.55 E+00$ \\
\hline $\mathrm{AL}$ & 13 & 26 & $2.70 E+08$ & $3.16 E+00$ \\
\hline SI & 14 & 32 & $2.55 E+05$ & $1.11 E+00$ \\
\hline $\mathbf{P}$ & 15 & 32 & $1.43 E+01$ & $1.08 E+00$ \\
\hline$p$ & 15 & 33 & $2.50 E+01$ & $9.52 \mathrm{E}-02$ \\
\hline 5 & 16 & 35 & $8.67 E+01$ & $5.92 \mathrm{E}-02$ \\
\hline $\mathrm{CL}$ & 17 & 36 & $1.10 E+08$ & $3.33 E-01$ \\
\hline$A R$ & 18 & 37 & $3.51 \mathrm{E}+01$ & $2.82 E-03$ \\
\hline$A R$ & 18 & 39 & $9.85 E+04$ & $2.55 \mathrm{E}-01$ \\
\hline$A R$ & 18 & 42 & $1.20 E+04$ & $3.06 E+00$ \\
\hline$k$ & 19 & 40 & $4.74 E+11$ & $8.24 E-01$ \\
\hline K & 19 & 42 & $5.17 E-01$ & $2.79 E+00$ \\
\hline$k$ & 19 & 43 & $9.33 E-01$ & $1.44 E+00$ \\
\hline CA & 20 & 41 & $2.81 E+07$ & $3.61 E-03$ \\
\hline CA & 20 & 45 & $1.65 E+02$ & $9.07 \mathrm{E}-02$ \\
\hline CA & 20 & 47 & $4.70 E+00$ & $1.90 E+00$ \\
\hline CA & 20 & 48 & $7 \cdot 30 E+18$ & $3.67 E+00$ \\
\hline SC & 21 & $44 M$ & $2.40 E+00$ & $2.97 E+00$ \\
\hline SC & 21 & 46 & $8.40 E+01$ & $2.15 E+00$ \\
\hline SC & 21 & 47 & $3.40 E+00$ & $3.19 \mathrm{E}-01$ \\
\hline SC & 21 & 48 & $1.83 E+00$ & $3.63 E+00$ \\
\hline TI & 22 & 44 & $1.72 \mathrm{E}+04$ & $2.85 E+00$ \\
\hline$v$ & 23 & 48 & $1.61 E+01$ & $3.05 E+00$ \\
\hline v & 23 & 49 & $3.30 E+02$ & $5.00 E-03$ \\
\hline$v$ & 23 & 50 & $2.19 E+18$ & $1.25 E+00$ \\
\hline$C R$ & 24 & 48 & $9.58 \mathrm{E}-01$ & $3.47 E+00$ \\
\hline $\mathrm{CR}$ & 24 & 51 & $2.78 E+01$ & $3.45 E-02$ \\
\hline$M N$ & 25 & 52 & $5.70 E+00$ & $3.57 E+00$ \\
\hline MN & 25 & 53 & $7.30 E+08$ & $6.00 E-03$ \\
\hline$M N$ & 25 & 54 & $3.03 E+02$ & $8.41 E-01$ \\
\hline $\mathrm{FE}$ & 26 & 55 & $9.85 E+02$ & $6.54 \mathrm{E}-03$ \\
\hline FE & 26 & 59 & $4.50 E+01$ & $1.32 E+00$ \\
\hline $\mathrm{FE}$ & 26 & 60 & $3.65 E+07$ & $2.70 E+00$ \\
\hline CO & 27 & 55 & $7.50 \mathrm{E}-01$ & $2.45 E+00$ \\
\hline $\mathrm{CO}$ & 27 & 56 & $7.73 E+01$ & $2.09 E+00$ \\
\hline CO & 27 & 57 & $2.67 E+02$ & $1.43 \mathrm{E}-01$ \\
\hline CO & 27 & 58 & $7.10 E+01$ & $1.01 E+00$ \\
\hline $\mathrm{CO}$ & 27 & 60 & $1.92 \mathrm{E}+03$ & $2.62 E+00$ \\
\hline NI & 28 & 56 & $6.10 E+00$ & $3.85 E+00$ \\
\hline NI & 28 & 57 & $1.50 E+00$ & $2.01 E+00$ \\
\hline N I & 28 & 59 & $2.92 E+07$ & 7. $70 \mathrm{E}-03$ \\
\hline N I & 28 & 63 & $3.36 E+04$ & $1.78 E-02$ \\
\hline N I & 28 & 66 & $2.29 E+00$ & $1.84 E+00$ \\
\hline $\mathrm{CU}$ & 29 & 64 & $5.38 E-01$ & $3.50 E-01$ \\
\hline
\end{tabular}

ELEMENT ATOMIC

I SOTJPE

HALF-LIFE $T_{R}$ (DAY)

$2.54 E+00$
$2.45 E+02$
$5.83 E-01$
$1.94 E+00$
$3.25 E+00$
$5.88 E-01$
$2.80 E+02$
$1.67 E+00$
$1.10 E+01$
$2.58 E+00$
$1.08 E+00$
$7.60 E+01$
$1.80 E+01$
$1.10 E+00$
$1.63 E+00$
$8.40 E+00$
$1.20 E+02$
$2.55 E+07$
$6.88 E-01$
$2.42 E+00$
$1.47 E+00$
$6 \cdot 17 E-01$
$1.44 E+00$
$7.30 E+07$
$3.93 E+03$
$8.30 E+01$
$3.30 E+01$
$1.88 E+01$
$1.72 E+13$
$2.55 E+01$
$1.38 E+00$
$6.40 E+01$
$5.04 E+01$
$1.02 E+04$
$6.08 E-01$
$3.33 E+00$
$5.83 E-01$
$1.08 E+02$
$2.67 E+00$
$5.90 E+01$
$7.08 E-01$
$8.50 E+01$
$3.27 E+00$
$3.47 E+08$
$6.50 E+01$
$7.08 E-01$
$6.08 E-01$
$7.30 E+11$
$6.20 E+01$
$1.01 E+01$
1.80

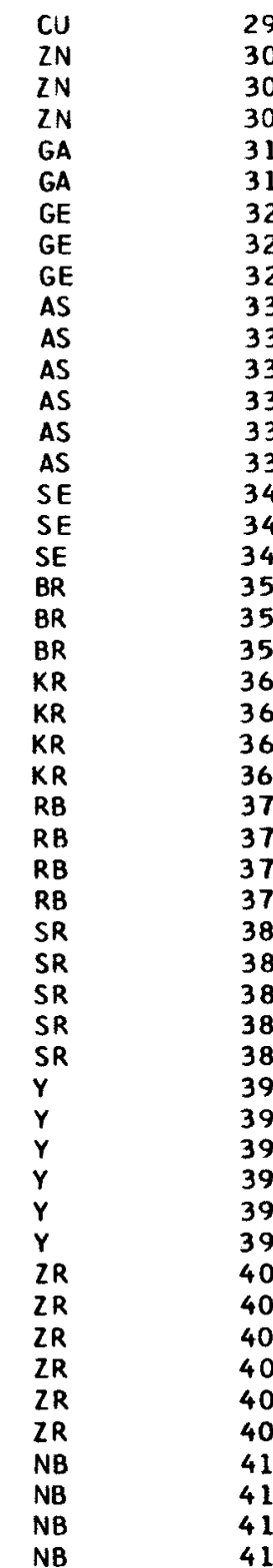

67
65
$69 M$
72
67
72
68
69
71
71
72
73
74
76
77
72
75
79
76
77
82
76
79
81
85
83
84
86
87
82
83
85
89
90
86
87
$87 M$
88
90
91
86
88
89
93
95
97
90
91
$91 M$
92
9

MAX. AVAILABLE ENERGY Q (MEV)

\begin{tabular}{l}
$2.97 E-01$ \\
$5.22 E-01$ \\
$8.66 E-01$ \\
$4.52 E+00$ \\
$2.04 E-01$ \\
$3.14 E+00$ \\
$1.64 E+00$ \\
$1.87 E+00$ \\
$1.04 E-02$ \\
$6.67 E-01$ \\
$3.11 E+00$ \\
$1.10 E-02$ \\
$1.82 E+00$ \\
$2.23 E+00$ \\
$2.94 E-01$ \\
$3.17 E+00$ \\
$5.92 E-01$ \\
$4.67 E-02$ \\
$2.14 E+00$ \\
$1.02 E+00$ \\
$2.81 E+00$ \\
$2.56 E+00$ \\
$8.65 E-01$ \\
$2.50 E-02$ \\
$2.88 E-01$ \\
$5.80 E-01$ \\
$1.88 E+00$ \\
$1.04 E+00$ \\
$8.64 E-02$ \\
$3.45 E+00$ \\
$1.12 E+00$ \\
$5.28 E-01$ \\
$7.69 E-01$ \\
$1.57 E+00$ \\
$4.31 E+00$ \\
$8.87 E-01$ \\
$1.27 E+00$ \\
$2.75 E+00$ \\
$1.36 E+00$ \\
$8.18 E-01$ \\
$4.57 E+00$ \\
$3.16 E+00$ \\
$1.86 E+00$ \\
$2.01 E-02$ \\
$1.67 E+00$ \\
$3.11 E+00$ \\
$5.27 E+00$ \\
$1.80 E-02$ \\
$2.34 E-01$ \\
$9.75 E-01$ \\
\hline
\end{tabular}


NO. (2) ISOTOPE

HALF-LIFE

$T_{R}$ (DAY)

MAX. AVA I LABLE

ENERGY Q (MEV)

$1.78 E+00$

8. $10 E-01$

. $04 E+00$

$2.69 E+00$

1. $90 \mathrm{E}-02$

8. $72 E-01$

8. $11 E-01$

. $07 E+00$

$2.50 E+00$

2. $00 E-02$

$1.16 \mathrm{E}-01$

$1.49 E+00$

$9.02 E-02$

$6.08 E-01$

5. $95 E-01$

$2.49 E+00$

$4.84 E-01$

$4.84 E-01$

$1.56 E+00$

$3.34 \mathrm{E}-01$

3. $39 \mathrm{E}-01$

1. $71 E+00$

$2.43 E-01$

$1.66 E+00$

$6.85 E-02$

$1.23 E-03$

5. $48 E-01$

$3.15 E+00$

$1.12 E+00$

2. $65 \mathrm{E}+00$

$2.56 E+00$

$2.80 E+00$

$4.69 E-01$

$1.13 E-01$

2. $11 E-01$

$1.27 E+00$

$1.06 \mathrm{E}+00$

4. $47 \mathrm{E}-01$

$E+00$

2.19E-01

4. 20E-01

3. $20 E-01$

8. $90 \mathrm{E}-02$

1.21E-01

1. 37E-01

6.96E-01

1. $90 E+00$

$3.31 E+00$

5. $30 E-02$

$2.54 E+00$
SYMBOL

NO. (Z) ISOTOPE

HALF-LIFE

$T_{R}$ (DAY)

2. $80 E+00$

$6.02 E+01$

$9.85 E+02$

$1.25 E+01$

3. $90 E+00$

$6.00 E+00$

$6.67 \mathrm{E}-01$

4. $70 E+00$

$1.70 E+01$

$1.50 E+02$

4. $38 E+15$

$1.04 E+02$

5. $80 E+01$

$1.05 E+02$

$3.30 E+01$

1. $20 \mathrm{E}+00$

$3.25 E+00$

$5.42 E-01$

4. $20 \mathrm{E}+00$

$6.02 E+01$

$1.32 E+01$

$5.84 E+09$

$5.21 E-01$

$8.05 E+00$

8. $75 E-01$

7. $92 E-01$

7.50E-01

$3.64 E+01$

8. $00 E+00$

$1.20 E+01$

$5.27 E+00$

$2.30 E+00$

1. $29 E+00$

$9.70 E+00$

$6.58 E+00$

$7.67 E+02$

$7.30 E+08$

$1-30 E+01$

$1.10 E+04$

2. $40 E+00$

$1.16 E+01$

$2.63 E+03$

$1.63 E+00$

$1.21 E+00$

$1.28 E+01$

$8.08 E-01$

$3.19 E+07$

$4.02 E+13$

$1.67 E+00$

$3.00 E+00$
MAX. AVAILABLE

ENERGY O (MEV) $2.40 E+00$

$5.37 E-01$

$1.26 E+00$

$1.92 \mathrm{E}+00$

$2.18 E+00$

5. $96 E-01$

8. $91 E-01$

3. $05 \mathrm{E}-02$

$2.78 \mathrm{E}-01$

$3.47 E-01$

$9.41 E-01$

$2.51 E+00$

$3.12 E+00$

$2.22 \mathrm{E}-01$

$2.25 E+00$

$6.70 E-02$

$7.69 E-02$

$2.48 E+00$

$6.01 E-01$
$1.25 E+00$

$2.78 E+00$

$5.60 E-01$

2. $36 E-01$

2.36E-01

$1.64 E-01$

4. $19 E-01$

6. $20 E-01$

$3.50 E-02$

$6.91 \mathrm{E}-01$

$1.93 E+00$

5. $50 E-02$

$2.33 E+00$

8. $10 E-01$

2. $56 E+00$

$1.11 E+00$

$6.82 E-01$

$2.68 \mathrm{E}-01$

3. $72 E+00$

8. $99 \mathrm{E}-01$

$3.74 \mathrm{E}-02$

$3.15 E+00$

$1.39 E+00$ 

SYMBOL

ATOMIC ISOTOPE
NO. ( 2 ) MASS(A)

HALF-LIFE

$T_{R}$ (DAY)

MAX. AVAILABLE

NERGY Q (MEV)

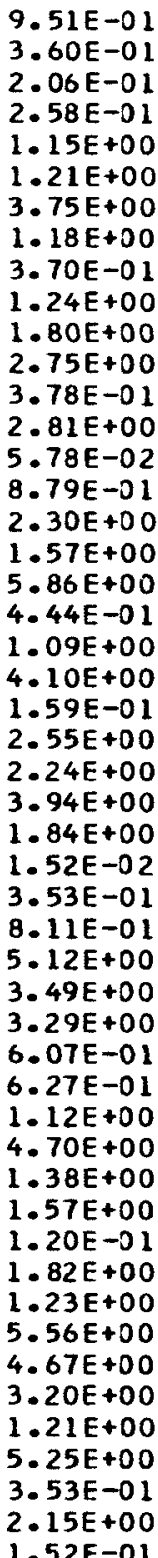

7. 50E-01

$1.44 E+00$

$1.40 E+02$
$3.25 E+01$

1. $83 E+18$

1. $38 E+00$

$2.85 E+02$

$8.00 E-01$

1. $37 E+01$

3. $30 E+00$

8. $76 E+17$

$1.11 E+01$

$2.65 E+02$

$3.65 E+02$

$6.57 E+03$

$1.60 E+03$

$9.85 E+02$

$5.39 E+00$

4. $06 E+01$

$2.21 E+00$

$1.18 E+00$

2. $00 E+01$

$3.40 E+02$

4. $38 E+10$

$4.02 E+13$

$4.38 E+15$

$1.46 E+17$

$3.28 E+04$

$1.96 E+00$

$5.90 E+00$

$4.60 \mathrm{E}+00$

$1.58 E+00$

$2.40 E+01$

$5.40 E+01$

$1.06 E+02$

1. $83 E+03$

$5.33 E-01$
$4.53 E+03$

$4.53 E+03$

$5.84 E+03$

. $52 \mathrm{E}+01$

$6.33 E-01$

4. $80 E+01$

$1.04 E+00$

$3.10 E+04$

9. $00 E+00$

4. $38 E+07$

1. $20 E+02$

4. $02 E+16$
ELEMENT SYMBOL

ATOMIC

I SOTOPE

$\begin{array}{lll}\text { GD } & 64 & 159 \\ \text { GD } & 64 & 162 \\ \text { TB } & 65 & 151 \\ \text { TB } & 65 & 152 \\ \text { TB } & 65 & 153 \\ \text { TB } & 65 & 154 \\ \text { TB } & 65 & 155 \\ \text { TB } & 65 & 156 \\ \text { TB } & 65 & 157 \\ \text { TB } & 65 & 158 \\ \text { TB } & 65 & 160 \\ \text { TB } & 65 & 161 \\ \text { TB } & 65 & 164 \\ \text { DY } & 66 & 154 \\ \text { DY } & 66 & 154 M \\ \text { DY } & 66 & 156 \\ \text { DY } & 66 & 159 \\ \text { DY } & 66 & 166 \\ \text { HO } & 67 & 163 \\ \text { HO } & 67 & 166 \\ \text { HO } & 67 & 166 M \\ \text { ER } & 68 & 160 \\ \text { ER } & 68 & 169 \\ \text { ER } & 68 & 172 \\ \text { IM } & 69 & 165 \\ \text { TM } & 69 & 167 \\ \text { TM } & 69 & 168 \\ \text { TM } & 69 & 170 \\ \text { TM } & 69 & 171 \\ \text { TM } & 69 & 172 \\ \text { YB } & 70 & 166 \\ \text { YB } & 70 & 169 \\ \text { YB } & 70 & 175 \\ \text { LU } & 71 & 169 \\ \text { LU } & 71 & 170 \\ \text { LU } & 71 & 171 \\ \text { LU } & 71 & 172 \\ \text { LU } & 71 & 173 \\ \text { LU } & 71 & 174 \\ \text { LU } & 71 & 174 M \\ \text { LU } & 71 & 176 \\ \text { LU } & 71 & 177 \\ \text { LU } & 71 & 177 M \\ \text { HF } & 72 & 170 \\ \text { HF } & 72 & 171 \\ \text { HF } & 72 & 172 \\ \text { HF } & 72 & 173 \\ \text { HF } & 72 & 174 \\ \text { HF } & 72 & 175 \\ \text { HF } & 72 & 181 \\ & & \end{array}$

HALF-LIFE

$T_{R}$ (DAY)

7. $50 \mathrm{OE}-01$

$3.65 E+02$

7. $50 E-01$

$7.50 E-01$
$2.5 B E+00$

$8.75 E-01$

$5.60 E+00$

$5.40 E+00$

$5.47 E+04$

$5.47 E+04$

$7.20 E+01$

6. $90 E+00$

$1.00 E+00$

$3.65 E+08$

5. $42 E-01$

$7.30 E+16$

$1.44 E+02$

3. $42 E+00$

3. $65 E+05$

$1.13 E+00$

4. $38 E+05$

$1.21 E+00$

$9.40 E+00$

$2.04 E+00$

$1.21 E+00$

$9.60 E+00$

8. $60 E+01$

$1.25 E+02$

6. $94 E+02$

$2.67 E+00$

$2.42 E+00$

$3.20 E+01$

$4.20 E+00$

$1.50 E+00$

$2.00 E+00$

8. $20 E+00$

$6.70 E+00$

$5.11 E+02$

1. $30 E+03$

$1.65 E+02$

$8.03 E+12$

$6.80 \mathrm{E}+00$

1. $55 E+02$

$5.00 E-01$

5.83E- 01

1. $83 E+03$

1. $00 E+00$

$1.57 E+18$

7. $00 E+01$

4. $46 E+01$
MAX. AVAILABLE

ENERGY Q (MEV)

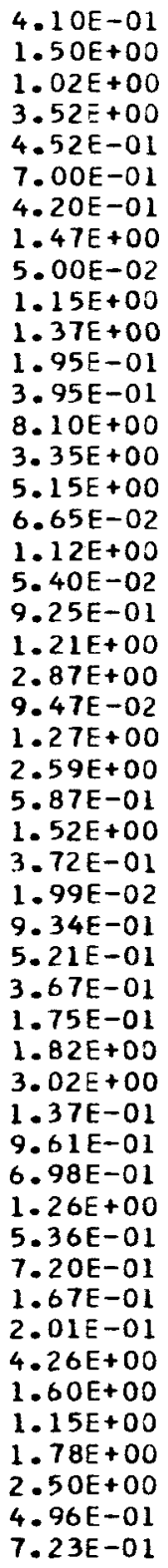



SYMBOL

$\begin{array}{ll}\text { ATOMIC } & \text { I SOTOPE } \\ \text { NO. }(Z) & \text { MASS(A) }\end{array}$

HALF-LIFE $T_{R}$ (DAY)

MAX. AVAILABLE ENERGY Q (MEV)

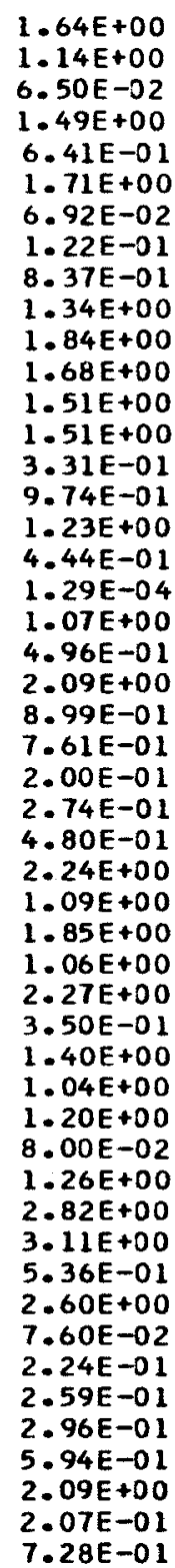

ELEMENT

ATOMIC ISOTOPE NO. (Z) MASS(A) SYMBOL

\begin{tabular}{ll} 
AU & 79 \\
AU & 79 \\
HG & 80 \\
HG & 80 \\
HG & 80 \\
HG & 80 \\
HG & 80 \\
TL & 81 \\
TL & 81 \\
TL & 81 \\
TL & 81 \\
PB & 82 \\
PB & 82 \\
PB & 82 \\
PB & 82 \\
PB & 82 \\
PB & 82 \\
BI & 83 \\
BI & 83 \\
BI & 83 \\
BI & 83 \\
BI & 83 \\
BI & 83 \\
PO & 84 \\
PO & 84 \\
PO & 84 \\
PO & 84 \\
RN & 86 \\
RN & 86 \\
RA & 88 \\
RA & 88 \\
RA & 88 \\
RA & 88 \\
RA & 88 \\
AC & 89 \\
AC & 89 \\
TH & 90 \\
TH & 90 \\
TH & 90 \\
TH & 90 \\
TH & 90 \\
TH & 90 \\
TH & 90 \\
PA & 91 \\
PA & 91 \\
PA & 91 \\
PA & 91 \\
PA & 91 \\
PA & 91 \\
U & 92 \\
\hline
\end{tabular}

HALF-LIFE $T_{R}$ (DAY)

198
199
194
$195 M$
197
$197 M$
203
200
201
202
204
200
202
203
204
205
210
205
206
207
208
210
$210 M$
206
208
209
210
211
222
223
224
225
226
228
225
227
227
228
229
230
231
232
234
228
229
230
231
232
233
230
29
29
29
29
29

MAX. AVAILABLE ENERGY O (MEV)

$$
\begin{aligned}
& 7.50 E-01 \\
& 2.33 E-01 \\
& 2.17 E+00 \\
& 1.66 E+00 \\
& 3.50 E-01 \\
& 6.41 E-01 \\
& 3.25 E-01 \\
& 1.43 E+00 \\
& 2.50 E-01 \\
& 5.05 E-01 \\
& 3.05 E-01 \\
& 1.79 E+00 \\
& 5.91 E-01 \\
& 7.64 E-01 \\
& 2.60 E+00 \\
& 8.60 E-02 \\
& 5.78 E+00 \\
& 1.95 E+00 \\
& 1.81 E+00 \\
& 1.85 E+00 \\
& 2.70 E+00 \\
& 5.74 E+00 \\
& 5.57 E+00 \\
& 3.08 E+00 \\
& 7.71 E+00 \\
& 4.97 E+00 \\
& 5.30 E+00 \\
& 9.57 E+00 \\
& 2.63 E+01 \\
& 2.79 E+01 \\
& 2.90 E+01 \\
& 2.83 E+01 \\
& 3.11 E+01 \\
& 3.58 E+01 \\
& 2.83 E+01 \\
& 3.39 E+01 \\
& 3.40 E+01 \\
& 3.45 E+01 \\
& 3.34 E+01 \\
& 3.57 E+01 \\
& 3.91 E+01 \\
& 3.98 E+01 \\
& 4.20 E+01 \\
& 3.64 E+01 \\
& 3.35 E+01 \\
& 3.73 E+01 \\
& 3.90 E+01 \\
& 4.03 E+01 \\
& 3.86 E+01 \\
& 3.94 E+01 \\
& 3.9
\end{aligned}
$$




\begin{tabular}{|c|c|c|c|c|c|c|c|c|c|}
\hline $\begin{array}{l}\text { ELEMENT } \\
\text { SYMBOL }\end{array}$ & $\begin{array}{l}\text { ATOMIC } \\
\text { NO. }(Z)\end{array}$ & $\begin{array}{l}\text { ISOTOPE } \\
\text { MASS(A) }\end{array}$ & $\begin{array}{l}\text { HALF-LIFE } \\
T_{R}(D A Y)\end{array}$ & $\begin{array}{l}\text { MAX. AVAI LABLE } \\
\text { ENERGY } Q \text { (MEV) }\end{array}$ & $\begin{array}{l}\text { EL EMENT } \\
\text { SYMBOL }\end{array}$ & $\begin{array}{l}\text { ATOMIC } \\
\text { NO. }(Z)\end{array}$ & $\begin{array}{l}\text { I SO TOPE } \\
\text { MASS(A) }\end{array}$ & $\begin{array}{l}\text { HALF-LIFE } \\
T_{R}(D A Y)\end{array}$ & $\begin{array}{l}\text { MAX. AVAILABLE } \\
\text { ENERGY Q (MEV) }\end{array}$ \\
\hline $\begin{array}{l}U \\
U \\
U \\
U \\
U \\
U \\
U \\
U \\
U \\
N P \\
N P \\
N P \\
N P \\
N P \\
N P \\
N P \\
P U \\
P U \\
P U \\
P U \\
P U \\
P U \\
P U \\
P U \\
P U \\
A M \\
A M \\
A M \\
A M \\
A M \\
A M\end{array}$ & $\begin{array}{l}92 \\
92 \\
92 \\
92 \\
92 \\
92 \\
92 \\
92 \\
92 \\
93 \\
93 \\
93 \\
93 \\
93 \\
93 \\
93 \\
94 \\
94 \\
94 \\
94 \\
94 \\
94 \\
94 \\
94 \\
94 \\
95 \\
95 \\
95 \\
95 \\
95 \\
95\end{array}$ & $\begin{array}{l}231 \\
232 \\
233 \\
234 \\
235 \\
236 \\
237 \\
238 \\
240 \\
234 \\
235 \\
236 \\
236 M \\
237 \\
238 \\
239 \\
236 \\
237 \\
238 \\
239 \\
240 \\
241 \\
242 \\
244 \\
246 \\
239 \\
240 \\
241 \\
242 \\
242 M \\
243\end{array}$ & $\begin{array}{l}4.20 E+00 \\
2.63 E+04 \\
5.91 E+07 \\
9.05 E+07 \\
2.60 E+11 \\
8.72 E+09 \\
6.75 E+00 \\
1.65 E+12 \\
5.88 E-01 \\
4.40 E+00 \\
4.10 E+02 \\
1.83 E+06 \\
9.17 E-01 \\
7.81 E+08 \\
2.10 E+00 \\
2.35 E+00 \\
1.04 E+03 \\
4.56 E+01 \\
3.25 E+04 \\
8.89 E+06 \\
2.47 E+06 \\
4.74 E+03 \\
1.38 E+08 \\
2.77 E+10 \\
1.08 E+01 \\
5.00 E-01 \\
2.13 E+00 \\
1.67 E+05 \\
6.67 E-01 \\
5.55 E+04 \\
2.79 E+06\end{array}$ & $\begin{array}{l}3 \cdot 92 E+01 \\
3 \cdot 98 E+01 \\
3 \cdot 82 E+01 \\
4.05 E+01 \\
4 \cdot 37 E+01 \\
4.43 E+01 \\
4 \cdot 37 E+01 \\
4.62 E+01 \\
5 \cdot 12 E+01 \\
4 \cdot 22 E+01 \\
4 \cdot 39 E+01 \\
4 \cdot 59 E+01 \\
4 \cdot 52 E+01 \\
4 \cdot 34 E+01 \\
4 \cdot 68 E+01 \\
4 \cdot 91 E+01 \\
4 \cdot 55 E+01 \\
4 \cdot 36 E+01 \\
4.60 E+01 \\
4.88 E+01 \\
4 \cdot 95 E+01 \\
4 \cdot 89 E+01 \\
5.11 E+01 \\
5.72 E+01 \\
5.82 E+01 \\
4.92 E+01 \\
5.10 E+01 \\
4 \cdot 89 E+01 \\
5.22 E+01 \\
5.22 E+01 \\
5.43 E+01\end{array}$ & $\begin{array}{l}C M \\
C M \\
C M \\
C M \\
C M \\
C M \\
C M \\
C M \\
C M \\
B K \\
B K \\
B K \\
B K \\
B K \\
C F \\
C F \\
C F \\
C F \\
C F \\
C F \\
C F \\
E S \\
E S \\
E S \\
E S \\
E S \\
E S \\
F M \\
F M \\
F M \\
F M\end{array}$ & $\begin{array}{l}96 \\
96 \\
96 \\
96 \\
96 \\
96 \\
96 \\
96 \\
96 \\
97 \\
97 \\
97 \\
97 \\
97 \\
98 \\
98 \\
98 \\
98 \\
98 \\
98 \\
98 \\
99 \\
99 \\
99 \\
99 \\
99 \\
99 \\
100 \\
100 \\
100 \\
100\end{array}$ & $\begin{array}{l}240 \\
241 \\
242 \\
243 \\
244 \\
245 \\
246 \\
247 \\
248 \\
245 \\
246 \\
247 \\
248 \\
249 \\
246 \\
248 \\
249 \\
250 \\
251 \\
252 \\
253 \\
251 \\
252 \\
253 \\
254 \\
254 M \\
255 \\
252 \\
253 \\
255 \\
257\end{array}$ & $\begin{array}{l}2.68 E+01 \\
3.50 E+01 \\
1.63 E+02 \\
1.17 E+04 \\
6.61 E+03 \\
3.40 E+06 \\
2.00 E+06 \\
6.10 E+09 \\
1.72 E+08 \\
4.95 E+00 \\
1.80 E+00 \\
3.65 E+06 \\
9.58 E-01 \\
3.14 E+02 \\
1.49 E+00 \\
3.50 E+02 \\
1.31 E+05 \\
3.65 E+03 \\
2.92 E+05 \\
9.31 E+02 \\
1.90 E+01 \\
1.50 E+00 \\
1.40 E+02 \\
2.00 E+01 \\
4.80 E+02 \\
1.63 E+00 \\
2.40 E+01 \\
9.58 E-01 \\
4.50 E+00 \\
8.33 E-01 \\
7.90 E+01\end{array}$ & $\begin{array}{l}5.18 E+01 \\
4.98 E+01 \\
5.21 E+01 \\
5.49 E+01 \\
5.53 E+01 \\
5.44 E+01 \\
5.65 E+01 \\
6.05 E+01 \\
6.22 E+01 \\
5.50 E+01 \\
5.77 E+01 \\
6.00 E+01 \\
6.19 E+01 \\
6.06 E+01 \\
5.89 E+01 \\
6.15 E+01 \\
6.06 E+01 \\
6.25 E+01 \\
6.63 E+01 \\
6.83 E+01 \\
6.73 E+01 \\
6.65 E+01 \\
6.85 E+01 \\
6.72 E+01 \\
7.03 E+01 \\
7.01 E+01 \\
7.36 E+01 \\
6.86 E+01 \\
6.74 E+01 \\
7.35 E+01 \\
7.38 E+01\end{array}$ \\
\hline
\end{tabular}




\section{3. $f_{M}$ : FRACTION OF THE DAILY} INTAKE SECRETED IN MILK

The values adopted for $f_{M}$, the fraction of the isotope ingested daily by the cow that is secreted in milk per liter, are shown in Table 3 . The $\mathrm{f}_{\mathrm{M}}$ values listed in Table 3 purposely include greater than average values with maximum and near maximum values. Thus, the $\mathrm{f}_{M}$ listed for $I$ is $2 \times 10^{-2} /$ liter rather than the average value of $1 \times 10^{-2} /$ liter (1794). The values were assigned according to the procedures and code numbers shown below.

Rules for estimating $\mathrm{f}_{\mathrm{M}}$

Code \#

1 The $f_{M}$ was estimated from tracer data. Maximum or near maximum values were assumed.

2 The $f_{M I}$ was estimated from concentrations in milk and in the diet from which the milk was derived.
3 The $f_{M}$ was estimated from concentrations in milk and forage as summarized below. Maximum or near maximum concentrations in milk, and average concentrations in forage were assumed.

4 The $f_{M}$ of $H$ and $O$ was estimated assuming daily consumption of 50 liters of water and daily secretion of 10 liters of milk, i. e., $f_{M}=0.02 /$ liter. The $f_{M}$ of the inert gases arbitrarily was also assumed to be $0.02 /$ liter to facilitate programming.

5 The $f_{\mathrm{M}}$ was assumed to be 0.05 times the fraction absorbed per liter. The $f_{B}$ to the whole body $\left(f_{B}^{T B}\right.$ or $\left.f_{1}\right)$ was assumed as the fraction absorbed.

6 The $\mathrm{f}_{\mathrm{M}}$ was conservatively estimated from unpublished tracer data of G. D. Potter, Bio-Medical Division, Lawrence Radiation Laboratory, Livermore, California.

Estimation of $\mathrm{f}_{\mathrm{M}}$ from concentrations in milk and forage by Rule 3 . Estimation of $f_{M}$ from concentrations in milk and forage by Rule 3 .

\begin{tabular}{|c|c|c|c|c|c|}
\hline Element & $\begin{array}{c}\mathrm{C}_{\mathrm{M}} \\
\text { (mg/liter) }\end{array}$ & Ref. & $\begin{array}{c}\mathrm{C}_{\mathrm{P}} \\
(\mathrm{mg} / \mathrm{kg})\end{array}$ & Ref. & $\underset{\text { (per liter) }}{\mathrm{f}_{\mathrm{M}}}$ \\
\hline $5^{\mathrm{B}}$ & $8 \times 10^{-1}$ & 2539 & $3 \times 10^{1}$ & 2579 & $2.7 \times 10^{-3}$ \\
\hline${ }_{6}^{\mathrm{C}}$ & $6.5 \times 10^{4}$ & 5387 & $4.5 \times 10^{5}$ & 5306 & $1.5 \times 10^{-2}$ \\
\hline $7^{\mathrm{N}}$ & $5.4 \times 10^{3}$ & 2554 & $2.5 \times 10^{4}$ & 5306 & $2.2 \times 10^{-2}$ \\
\hline${ }_{9}^{\mathrm{F}}$ & $2.8 \times 10^{-1}$ & 2596 & 2 & 5306 & $1.4 \times 10^{-2}$ \\
\hline $13^{\mathrm{Al}}$ & $9.7 \times 10^{-1}$ & 2549 & $\times 10^{2}$ & 2899 & $\times 10^{-4}$ \\
\hline $14^{\mathrm{Si}}$ & 7.2 & 2597 & $7 \times 10^{3}$ & 5373 & $10^{-4}$ \\
\hline $16^{\mathrm{S}}$ & $4.34 \times 10^{2}$ & 2751 & $2.5 \times 10^{3}$ & 4162 & $1.75 \times 10^{-2}$ \\
\hline${ }_{17} \mathrm{Cl}$ & $2.25 \times 10^{3}$ & 2543 & $4.5 \times 10^{3}$ & 4162 & $\times 10^{-2}$ \\
\hline $19^{\mathrm{K}}$ & $2 \times 10^{3}$ & 2544 & $2 \times 10^{4}$ & 4162 & $10^{-2}$ \\
\hline $24^{\mathrm{Cr}}$ & $1.5 \times 10^{-2}$ & 5387 & $7 \times 10^{-1}$ & 2218 & $2.15 \times 10^{-3}$ \\
\hline $25^{\mathrm{Mn}}$ & $1.1 \times 10^{-1}$ & 2543 & $4.4 \times 10^{1}$ & 4162 & $2.5 \times 10^{-4}$ \\
\hline${ }_{26} \mathrm{Fe}$ & 1.85 & 2536 & $1.6 \times 10^{2}$ & 4162 & $1.2 \times 10^{-3}$ \\
\hline $28^{\mathrm{Ni}}$ & $10^{-1}$ & 2597 & 1.5 & 2620 & $6.7 \times 10^{-3}$ \\
\hline $29^{\mathrm{Cu}}$ & 1.26 & 2793 & 9.0 & 4162 & $1.4 \times 10^{-2}$ \\
\hline $30^{\mathrm{Zn}}$ & 6.6 & 2544 & $1.7 \times 10^{1}$ & 4162 & $3.9 \times 10^{-2}$ \\
\hline $33^{\mathrm{As}}$ & $\times 10^{-2}$ & 2596 & 1.0 & 2877 & $\times 10^{-3}$ \\
\hline $37^{\mathrm{Rb}}$ & 3.0 & 2597 & $10^{1}$ & 5306 & $\times 10^{-2}$ \\
\hline $82^{\mathrm{Pb}}$ & $4.3 \times 10^{-2}$ & 2543 & 7 & 2877 & $6.2 \times 10^{-4}$ \\
\hline
\end{tabular}




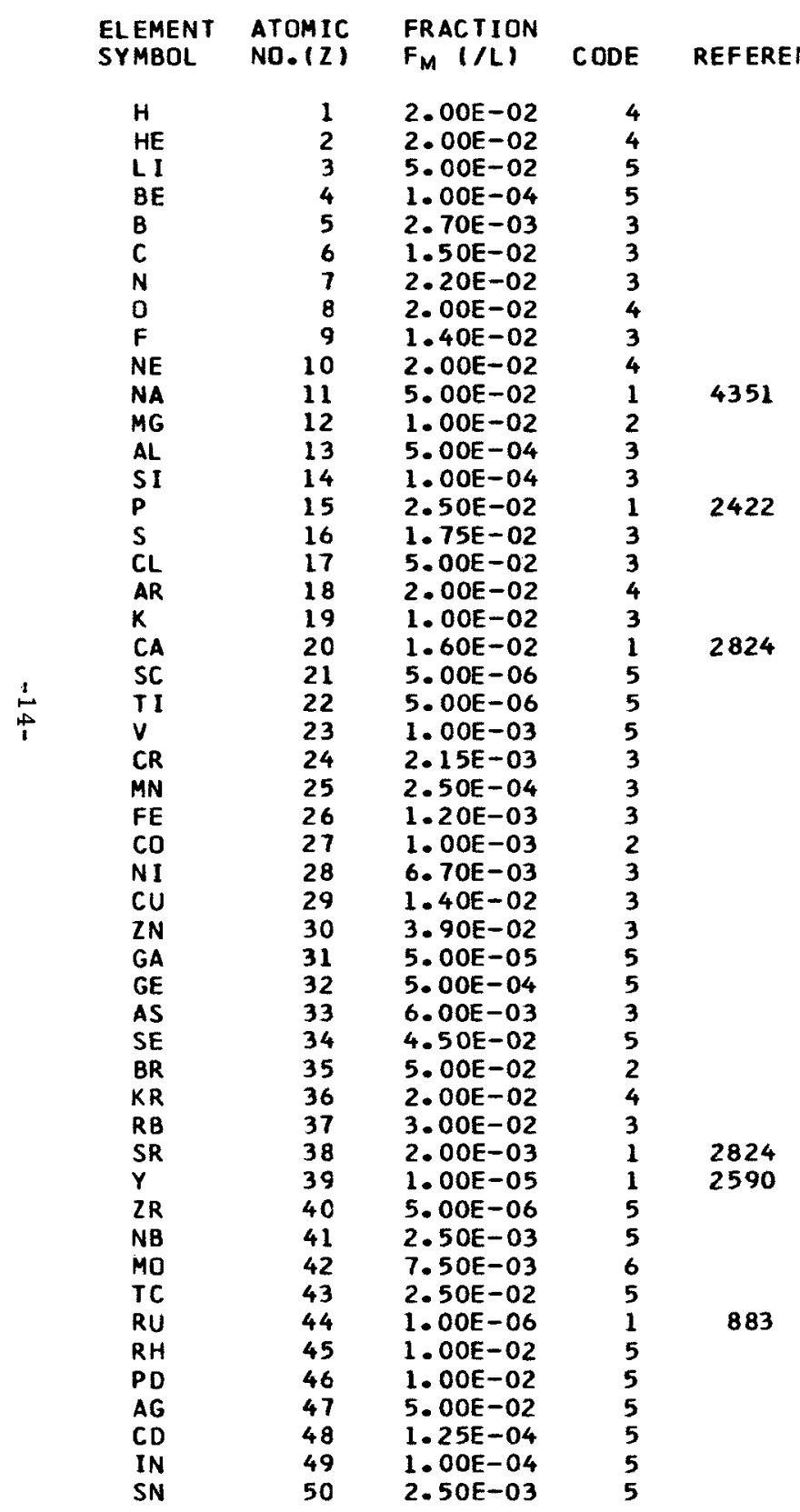

ELEMENT ATOMIC SYMBOL

NO. 121

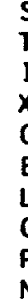

TE

$X E$

CS

BA

CE

PR

PM

EM

GU

GD

oY

HO

TM

$Y B$

LU

HF

TA

RE
FRACTION

$F_{M}(/ L) \quad$ CODE

1. $50 \mathrm{E}-03$ $1.00 \mathrm{E}-03$ 2. OOE- 02 $2.00 E-02$ $1.40 \mathrm{E}-02$ 1. $40 \mathrm{E}-02$ 6.00E-04 5.00E-06 2. $00 E-05$ 5.00E-06 5. 00 E- 06 5. 00 E-06 5. 00 E- 06 5. OOE-06 5. 0 OE-06 5. $00 \mathrm{E}-06$ $5.00 E-06$ 5.00E-06 5. 0 OE-06 5.0OE-06 5. $00 \mathrm{E}-06$ 5. $00 \mathrm{E}-06$ 5. $00 \mathrm{E}-06$ 5. 00 E- 06 2. 50E-02 5. 00 EE- 04 $2.50 \mathrm{OE}-02$ 2.5 OE-02 5. OOE-03 5.00E-03 $5.00 \mathrm{E}-03$

5. $00 \mathrm{E}-03$

3.75E- 02 $2.25 E-02$ 6.2 OE- 04 $5.00 E-04$ 3. OOE-O3 $5.00 \mathrm{E}-02$ $5.00 E-02$
$2.00 E-02$ 5.00E-02 1.50E-02

5.00E-06

$5.00 E-06$

$5.00 E-06$ $5.00 E-04$ 5. 00 E-06 5. $1.50 E-06$ $1.50 E-06$ 5.00E-06 5. 00 E-06 1. 50E-06 $1.50 E-06$ $1.50 E-06$ 1.5OE-06
REFERENCE (S)

268
1794
4761
2568
2569


4. $\mathrm{C}_{\mathrm{S}}$ :AVERAGE CONCENTRATION IN SOIL

The values adopted for $\mathrm{C}_{\mathrm{S}}$ were the average concentrations in typical agricultural soil and are shown in Table 4. The $\mathrm{C}_{\mathrm{S}}$ values were assigned according to the procedures and code numbers shown below.

Rules for estimating $\mathrm{C}_{\mathrm{S}}$

Code \#

1 The $\mathrm{C}_{\mathrm{S}}$ was assumed to be the average concentration in soil reported by Vinogradov (2532).

2 The $\mathrm{C}_{\mathrm{S}}$ was assumed to be the minimum value found in the literature.
3 The $\mathrm{C}_{\mathrm{S}}$ was assigned to give $\mathrm{C}_{\mathrm{B}} / \mathrm{C}_{\mathrm{S}}=100$ when $\mathrm{C}_{\mathrm{B}}$ was known and $\mathrm{C}_{\mathrm{S}}$ was not. $\left(\mathrm{A} \mathrm{C}_{\mathrm{B}} / \mathrm{C}_{\mathrm{S}}\right.$ of 100 appears to be a maximum or near maximum ratio based on the observed $\mathrm{C}_{\mathrm{B}} / \mathrm{C}_{\mathrm{S}}$ for $\mathrm{I}$ in the thyroid and $\mathrm{Cd}$ in the kidneys.)

5 The $\mathrm{C}_{\mathrm{S}}$ (of inert gases) was assumed to be that attributable to the soil atmosphere. The calculations are summarized under $2 \mathrm{He}$ in the Appendix.

6 The $C_{S}$ was assumed to be $10^{-30} \mu \mathrm{g} / \mathrm{g}$ for all transplutonium elements.

99 The $\mathrm{C}_{\mathrm{S}}$ was estimated from collateral data as described in the Appendix. 
$\begin{array}{ll}\text { ELEMENT } & \text { ATOMIC } \\ \text { SYMBOL } & \text { NO. }(Z)\end{array}$

CONC

$C_{S}$ (PPM) CODE REFERENCE(S)

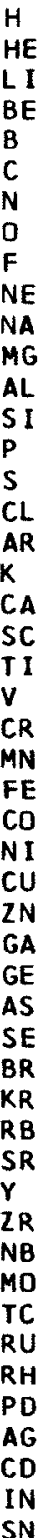

2. $00 E+04 \quad 99$

1. 0 OOE -04

3. $00 E+01$

6. $00 E+00$

2. $00 E+01$

2. $00 E+04$

$1.00 E+03$

4. $90 \mathrm{O}+05$

2.0OE +02

$1.00 E-03$

6. $30 E+03$

7. $14 E+04$

3. $30 E+05$

$8.00 E+02$

8. $50 E+02$

1. $00 E+02$

1. $00 E+00$

1. $36 E+04$

$1.37 E+04$

7. $00 E+00$

4. $60 \mathrm{E}+03$

$1.00 E+02$

2. $00 E+02$
8. $50 E+02$

8. $50 E+02$

3. $80 E+04$

$8.00 E+00$

2. $00 E+01$

5. $00 E+01$

3. $00 E+01$

$1.00 E+00$

5. $0 O E+00$

1. $00 E-01$

5. $00 E+00$

1. $00 \mathrm{E}-04$

1. $00 E+02$

3. $00 E+02$

$5.00 E+01$

3. $00 E+02$

5. $30 E+00$

2. $00 E+00$

1. $00 E-13$

1. OOE-03

1. $00 E-03$

1. $00 E-03$

5. 00E-01

1. OOE-O1

1. $00 E+01$
2532

2532

2532

2532

2532

2532
2532

2532

2532

2532

2532

2532

2532
2532

2532

2532

2532

2532

2532

2532

2532
2532

2532

2532

2532

2532

2532

2532

2532

2532

2531
2532

2532

2532

2532

2219

2532

2532

2532

2532
ELEMENT SYMBOL

ATOMIC NO. (Z)

CONC.

$C_{S}$ (PPM) CODE REFERENCE(S)

SE

I

$X E$
$C S$
$B A$

BA

LA

PR

ND

SM

EU

GD

TB

HO

ER

$Y B$

LU

HF

TA

RE

OS

PT

AU

HG

PB

BI

PO

RN

FR

AC
TH
PA

PA

UP

PU

AM

CM

CF

ES

$\begin{array}{rr}2.30 E+00 & 1 \\ 1.00 E-03 & 99 \\ 5.00 E+00 & 1 \\ 1.00 E-05 & 5 \\ 5.00 E+00 & 1 \\ 5.00 E+02 & 1 \\ 4.00 E+01 & 1 \\ 5.30 E+01 & 1 \\ 6.70 E+00 & 1 \\ 2.50 E+01 & 1 \\ 1.00 E-18 & 99 \\ 6.40 E+00 & 1 \\ 1.00 E+00 & 1 \\ 4.20 E+00 & 1 \\ 7.00 E-01 & 1 \\ 3.00 E+00 & 1 \\ 7.00 E-01 & 1 \\ 8.00 E-01 & 1 \\ 7.00 E-01 & 1 \\ 8.00 E-01 & 1 \\ 7.00 E-01 & 1 \\ 6.00 E+00 & 1 \\ 1.00 E+00 & 99 \\ 1.00 E+00 & 2 \\ 1.00 E-03 & 99 \\ 1.00 E-03 & 99 \\ 1.00 E-05 & 2 \\ 1.00 E-02 & 2 \\ 1.00 E-01 & 2 \\ 1.00 E-02 & 1 \\ 1.00 E-01 & 99 \\ 1.00 E+01 & 1 \\ 1.00 E-01 & 99 \\ 1.00 E-11 & 99 \\ 1.00 E-19 & 99 \\ 1.00 E-13 & 5 \\ 1.00 E-18 & 99 \\ 8.00 E-07 & 1 \\ 1.00 E-10 & 99 \\ 6.00 E+00 & 1 \\ 1.00 E-07 & 99 \\ 1.00 E+00 & 1 \\ 1.00 E-12 & 99 \\ 1.00 E-13 & 99 \\ 1.00 E-30 & 6 \\ 1.00 E-30 & 6 \\ 1.00 E-30 & 6 \\ 1.00 E-30 & 6 \\ 1.00 E-30 & 6 \\ 1.00 E-30 & 6 \\ & \end{array}$

2532

2532

\section{2}

2532

2532
2532

2532

2532

2532

2532

2532

2532

2532

2532

2532

2532

2532

2532

2532

5213

$5786 \quad 5799$

$\begin{array}{lll}5782 & 5799 & 5803\end{array}$

2531

2532

2532

2532

2532

2532 


\section{UPTAKE AND DISTRIBUTION IN ORGANS}

\section{$\mathrm{f}_{\mathrm{B}}$ : Fractional Uptake}

The values adopted for $f_{B}$, the fraction that is taken up by an organ following ingestion, are shown in Table 5A. The values were assigned according to the procedures and code numbers shown below.

\section{Rules for estimating $\mathrm{f}_{B}$}

\section{Code \#}

1 The $f_{B}$ was assumed to be the ICRP estimate of " $f$, fraction reaching the organ by ingestion."

2 The $\mathrm{f}_{\mathrm{B}}$ was assumed to be the fraction absorbed times the fraction from blood to the tissue. The fraction absorbed was assumed to be the ICRP value of " $f_{1}$, fraction from GI tract to blood." The fraction from blood to the tissue was based on the values of " $f_{2}^{\prime}$ fraction from blood to tissue" listed in the ICRP tables. The tissue was assumed to take up from blood all the isotope that is not taken up by the other tissues. The $\mathrm{f}_{2}^{\prime}$ for the tissue was assumed to be 0.1 if the fraction not taken up by the other tissues is less than 0.1 ; the $f_{2}^{\prime}$ was assumed to be 1 if the fraction not taken up by the other tissues is equal to or greater than 0.1 .

3 The $f_{B}$ was obtained from data on uptake in humans.

5 The $\mathrm{f}_{\mathrm{B}}$ (of $\mathrm{H}, \mathrm{C}, \mathrm{N}, \mathrm{O}, \mathrm{Na}, \mathrm{Cl}, \mathrm{K}$, $\mathrm{Br}, \mathrm{Rb}, \mathrm{Cs}$ and the inert gases) was assumed to be the ratio of organ mass to total body mass. The organ and total body masses were those shown in Table 1.

6 The $f_{B}$ in the GI tract (contents) was assumed to be $1-\mathrm{f}_{\mathrm{B}}^{\mathrm{TB}}$ or 0.1 , whichever is greater.

99 The $f_{B}$ was estimated from collateral data as described in the Appendix. 


$\begin{array}{cccr}\text { ELEMENT } & \text { ATOMIC } & \text { FRACTION } & \\ \text { SYMBOL } & \text { NO.121 } & \text { F } & \text { CODE } \\ & & & \\ \text { H } & 1 & 9.10 E-02 & 5 \\ \text { HE } & 2 & 9.10 E-02 & 5 \\ \text { LI } & 3 & 1.00 E+00 & 2 \\ \text { BE } & 4 & 6.40 E-04 & 1 \\ \text { B } & 5 & 9.00 E-01 & 2 \\ \text { C } & 6 & 2.50 E-02 & 1 \\ \text { N } & 7 & 9.10 E-02 & 5 \\ \text { O } & 8 & 9.10 E-02 & 5 \\ \text { F } & 9 & 5.30 E-01 & 1 \\ \text { NE } & 1 C & 9.10 E-02 & 5 \\ \text { NA } & 11 & 9.10 E-02 & 5 \\ \text { MG } & 12 & 5.00 E-02 & 1 \\ \text { AL } & 13 & 1.00 E-01 & 2 \\ \text { SI } & 14 & 8.50 E-01 & 2 \\ \text { P } & 15 & 3.75 E-01 & 1 \\ \text { S } & 16 & 3.00 E-02 & 1 \\ \text { CL } & 17 & 9.10 E-02 & 5 \\ \text { AR } & 18 & 9.10 E-02 & 5 \\ \text { K } & 19 & 9.10 E-02 & 5 \\ \text { CA } & 20 & 5.40 E-01 & 1 \\ \text { SC } & 21 & 2.00 E-05 & 1 \\ \text { T I } & 22 & 1.00 E-04 & 2 \\ \text { V } & 23 & 2.80 E-03 & 1 \\ \text { CR } & 24 & 5.00 E-03 & 2 \\ \text { MN } & 25 & 8.50 E-03 & 99 \\ \text { FE } & 26 & 1.00 E-02 & 1 \\ \text { CO } & 27 & 2.00 E-02 & 99 \\ \text { NI } & 28 & 1.50 E-01 & 1 \\ \text { CU } & 29 & 2.40 E-01 & 99 \\ \text { IN } & 30 & 1.50 E-02 & 1 \\ \text { GA } & 31 & 3.00 E-04 & 1 \\ \text { GE } & 32 & 1.00 E-02 & 2 \\ \text { AS } & 33 & 3.00 E-02 & 2 \\ \text { SE } & 34 & 9.00 E-01 & 2 \\ \text { BR } & 35 & 9.10 E-02 & 5 \\ \text { KR } & 36 & 9.10 E-02 & 5 \\ \text { RB } & 37 & 9.10 E-02 & 5 \\ \text { SR } & 38 & 2.10 E-01 & 1 \\ \text { Y } & 39 & 7.50 E-05 & 1 \\ \text { IR } & 40 & 3.00 E-05 & 1 \\ \text { NB } & 41 & 1.90 E-02 & 99 \\ \text { MO } & 42 & 1.00 E-01 & 99 \\ \text { IC } & 43 & 2.00 E-03 & 99 \\ \text { RU } & 44 & 3.30 E-02 & 99 \\ \text { RH } & 45 & 1.00 E-02 & 1 \\ \text { PO } & 46 & 4.00 E-03 & 99 \\ \text { AG } & 47 & 5.00 E-02 & 99 \\ \text { CD } & 48 & 9.00 E-05 & 99 \\ \text { IN } & 49 & 3.40 E-04 & 1 \\ \text { SN } & 50 & 2.00 E-02 & 1\end{array}$

REFERENCE ( $S$ )
ELEMENT ATOMIC SYMBOL

NO. $(Z)$

FRACTION

$F_{B}$

COOE

REFERENEE (S)

SB $\quad 5$

$\begin{array}{ll}T E & 52 \\ I & 53\end{array}$

I E

$X E$
$C S$

BA

LA

CE

ND

ND

$5 M$

EU

T B

DY

HO

ER

TM

$Y B$

LU

TA

WE

OS

IR

PT

AU

HG

PB

51
52
53
54

3. $00 E-03$

$2.30 E-02$

7. 0 OE-02

$9.10 E-02$

$9.10 E-02$

3. $50 E-02$

4. $00 E-05$

3. OOE -05

4. ODE- 05

3. $50 \mathrm{E}-05$

$3.50 E-05$

3.5 OE-05

3. $60 E-05$

4. $50 \mathrm{E}-05$

$6.00 E-05$

$6.00 E-05$

6. $40 E-05$

6. OOE- 05

$6.50 E-05$

$5.80 E-05$

6. $80 E-05$

$1.50 E-05$

1. OOE-02

$7.00 \mathrm{E}-03$

$5.00 E-03$

1. $0 O E-01$

l. $.00 E-01$

$1.00 E-01$

$1.00 E-01$

7.5 OE- 01

2. $50 \mathrm{OE}-02$

2. 0 OE- 02

$3.00 \mathrm{E}-04$

6. 0 OE- 03

1. $O O E+O O$

1. $00 E-01$

$1.00 E+00$

l. $50 \mathrm{OF}-01$

3. $00 \mathrm{E}-05$

$7.00 E-05$

4. $50 \mathrm{OE}-05$

$1.10 E-03$

5. $40 E-05$

$2.40 \mathrm{E}-05$

$2.50 E-05$

$3.00 \mathrm{E}-05$

$2.40 E-05$

$2.40 E-05$

$2.40 E-05$ 


$\begin{array}{lrrr}\text { ELEMENT } & \text { ATOMIC } & \text { FRACIION } & \\ \text { SYMBOL } & \text { NO. }(2) & \text { F } & \text { CODE } \\ \text { H } & & & \\ \text { HE } & 1 & 4.40 E-03 & 5 \\ \text { LI } & 2 & 4.40 E-03 & 5 \\ \text { BE } & 3 & 1.00 E+00 & 2 \\ \text { B } & 4 & 6.00 E-05 & 1 \\ \text { C } & 5 & 9.00 E-01 & 2 \\ \text { N } & 6 & 4.40 E-03 & 5 \\ \text { O } & 7 & 4.40 E-03 & 5 \\ \text { F } & 8 & 4.40 E-03 & 5 \\ \text { NE } & 9 & 1.00 E+00 & 2 \\ \text { NA } & 10 & 4.40 E-03 & 5 \\ \text { MG } & 11 & 4.40 E-03 & 5 \\ \text { AL } & 12 & 1.00 E-01 & 2 \\ \text { SI } & 13 & 1.00 E-01 & 2 \\ \text { P } & 14 & 8.50 E-01 & 2 \\ \text { S } & 15 & 2.00 E-02 & 99 \\ \text { CL } & 16 & 2.50 E-03 & 99 \\ \text { AR } & 17 & 4.40 E-03 & 5 \\ \text { K } & 18 & 4.40 E-03 & 5 \\ \text { CA } & 19 & 4.40 E-03 & 5 \\ \text { SC } & 20 & 3.00 E-03 & 99 \\ \text { TI } & 21 & 2.00 E-06 & 1 \\ \text { V } & 22 & 1.00 E-04 & 2 \\ \text { CR } & 23 & 8.00 E-04 & 1 \\ \text { MN } & 24 & 1.30 E-05 & 1 \\ \text { FE } & 25 & 5.00 E-03 & 1 \\ \text { CO } & 26 & 3.00 E-03 & 99 \\ \text { NI } & 27 & 3.00 E-01 & 2 \\ \text { CU } & 28 & 3.00 E-01 & 2 \\ \text { ZN } & 29 & 1.00 E-02 & 1 \\ \text { GA } & 30 & 4.00 E-03 & 1 \\ \text { GE } & 31 & 2.00 E-05 & 1 \\ \text { AS } & 32 & 3.00 E-04 & 1 \\ \text { SE } & 33 & 3.00 E-04 & 1 \\ \text { BR } & 34 & 4.00 E-02 & 1 \\ \text { KR } & 35 & 4.40 E-03 & 5 \\ \text { RB } & 36 & 4.40 E-03 & 5 \\ \text { SR } & 37 & 4.40 E-03 & 5 \\ \text { Y } & 38 & 1.30 E-03 & 99 \\ \text { ZR } & 39 & 3.00 E-06 & 99 \\ \text { NB } & 40 & 2.00 E-06 & 1 \\ \text { MD } & 41 & 1.00 E-03 & 99 \\ \text { TC } & 42 & 4.80 E-02 & 99 \\ \text { RU } & 43 & 1.00 E-02 & 99 \\ \text { RH } & 44 & 1.80 E-02 & 99 \\ \text { PD } & 45 & 6.00 E-03 & 1 \\ \text { AG } & 46 & 2.00 E-02 & 1 \\ \text { CD } & 48 & 2.00 E-02 & 99 \\ \text { IN } & 49 & 8.50 E-04 & 1 \\ \text { SN } & 50 & 1.50 E-05 & 1 \\ & & & 99\end{array}$

$\begin{array}{crrr}\text { ELEMENT } & \text { ATOMIC } & \text { FRACTION } & \\ \text { SYMBOL } & \text { NO.1Z1 } & \text { FB } & \text { CODE } \\ & & & \\ \text { SB } & 51 & 5.00 E-05 & 99 \\ \text { TE } & 52 & 2.00 E-02 & 1 \\ \text { I } & 53 & 4.00 E-02 & 1 \\ \text { XE } & 54 & 4.40 E-03 & 5 \\ \text { CS } & 55 & 4.40 E-03 & 5 \\ \text { BA } & 56 & 5.00 E-06 & 1 \\ \text { LA } & 57 & 1.00 E-04 & 2 \\ \text { CE } & 58 & 2.00 E-06 & 1 \\ \text { PR } & 59 & 2.00 E-06 & 1 \\ \text { ND } & 60 & 5.00 E-06 & 1 \\ \text { PM } & 61 & 2.00 E-06 & 1 \\ \text { SM } & 62 & 2.00 E-06 & 1 \\ \text { EU } & 63 & 3.00 E-06 & 1 \\ \text { GD } & 64 & 2.00 E-06 & 99 \\ \text { TB } & 65 & 3.00 E-06 & 1 \\ \text { DY } & 66 & 1.00 E-04 & 2 \\ \text { HO } & 67 & 2.00 E-06 & 1 \\ \text { ER } & 68 & 2.00 E-06 & 1 \\ \text { TM } & 69 & 2.00 E-06 & 1 \\ \text { YB } & 70 & 5.00 E-06 & 1 \\ \text { LU } & 71 & 1.00 E-06 & 1 \\ \text { HF } & 72 & 2.00 E-06 & 1 \\ \text { TA } & 73 & 1.50 E-03 & 99 \\ \text { W } & 74 & 1.00 E-01 & 2 \\ \text { RE } & 75 & 5.00 E-01 & 2 \\ \text { OS } & 76 & 5.00 E-03 & 1 \\ \text { IR } & 77 & 4.50 E-03 & 1 \\ \text { PT } & 78 & 1.00 E-02 & 1 \\ \text { AU } & 79 & 3.00 E-03 & 1 \\ \text { HG } & 80 & 2.60 E-01 & 1 \\ \text { TL } & 81 & 2.30 E-02 & 1 \\ \text { PB } & 82 & 1.00 E-02 & 1 \\ \text { BI } & 83 & 3.00 E-03 & 1 \\ \text { PO } & 84 & 4.00 E-03 & 1 \\ \text { AT } & 85 & 1.00 E+00 & 2 \\ \text { RN } & 86 & 4.40 E-03 & 5 \\ \text { FR } & 87 & 1.00 E+00 & 5 \\ \text { RA } & 88 & 6.00 E-04 & 1 \\ \text { AC } & 89 & 1.00 E-06 & 1 \\ \text { TH } & 90 & 5.00 E-06 & 1 \\ \text { PA } & 91 & 4.00 E-06 & 1 \\ \text { U } & 92 & 1.10 E-03 & 1 \\ \text { NP } & 93 & 3.00 E-06 & 1 \\ \text { PU } & 94 & 6.00 E-07 & 1 \\ \text { AM } & 95 & 3.00 E-06 & 1 \\ \text { CM } & 96 & 2.00 E-06 & 1 \\ \text { BK } & 97 & 3.00 E-05 & 2 \\ \text { CF } & 98 & 3.00 E-05 & 2 \\ \text { ES } & 99 & 3.00 E-05 & 2 \\ \text { FM } & 100 & 3.00 E-05 & 2 \\ & & & \end{array}$

REFERENCE (S)

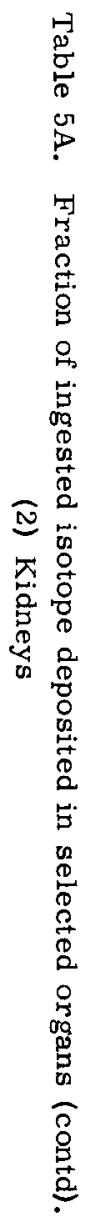




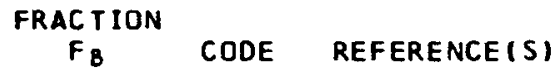

2. $60 \mathrm{E}-02 \quad 5$

2. $60 \mathrm{E}-02$

1. $00 E+00$

2. 00 E- 04

1. 20E-01

2. $60 \mathrm{E}-02$

2. $60 \mathrm{E}-02$

$2.60 \mathrm{E}-02$

$1.00 E+00$

2. $60 \mathrm{E}-02$

2. $60 \mathrm{E}-02$

1. $00 \mathrm{E}-01$

1. $00 E-01$

8. 50E-01

1. $00 E-01$

5. $00 \mathrm{E}-03$

2. $60 \mathrm{E}-02$

$2.60 E-02$

2. $60 \mathrm{E}-02$

2. $00 E-02$

$1.50 E-05$

$1.00 E-04$

1.2OE-03

5. $00 \mathrm{E}-03$

2. $00 \mathrm{E}-02$

1. $30 E-02$

7. $00 E-03$

2. $00 E-02$

2. $00 E-02$

3. 50E-02

2. $50 E-04$

2. $00 E-04$

9. $00 \mathrm{E}-04$

6. $00 E-02$

2. $60 \mathrm{E}-02$

$2.60 \mathrm{E}-02$

$2.60 E-02$

7. $80 \mathrm{E}-03$

1. $70 E-05$

7. $00 \mathrm{E}-06$

4. 50E-03

2. $40 E-01$

3. $00 E-03$

$6.30 E-02$

. 00E-03

. $00 E-02$

$.00 E-02$

1. $90 \mathrm{E}-03$

2. $80 \mathrm{E}-04$

5. OOE-04

\section{ELEMENT}

SYMBOL

ATOMIC

NO. (Z)

FRACTION

$F_{B}$ CODE

REFERENCE (S)

\begin{tabular}{ll} 
SB & 51 \\
TE & 52 \\
I & 53 \\
XE & 54 \\
CS & 55 \\
BA & 56 \\
LA & 57 \\
CE & 58 \\
PR & 59 \\
ND & 60 \\
PM & 61 \\
SM & 62 \\
EU & 63 \\
GD & 64 \\
TB & 65 \\
DY & 66 \\
HO & 67 \\
ER & 68 \\
TM & 69 \\
YB & 70 \\
LU & 71 \\
HF & 72 \\
TA & 73 \\
W & 74 \\
RE & 75 \\
OS & 76 \\
IR & 77 \\
PT & 78 \\
AU & 79 \\
HG & 80 \\
TL & 81 \\
PB & 82 \\
BI & 83 \\
PO & 84 \\
AT & 85 \\
RN & 86 \\
FR & 87 \\
RA & 88 \\
AC & 89 \\
TH & 90 \\
PA & 91 \\
U & 92 \\
NP & 93 \\
PU & 94 \\
AM & 95 \\
CM & 96 \\
BK & 97 \\
CF & 98 \\
ES & 99 \\
FM & 100 \\
\hline
\end{tabular}

$6.00 E-05$

1. $00 \mathrm{OE}-02$

$1.20 \mathrm{E}-01$

$2.60 E-02$

$2.60 E-02$

3. $00 E-05$
$1.50 E-05$

$1.50 E-05$
$2.50 E-05$

2. $50 E-05$

5.0OE-05

$6.00 E-06$

3.5 OE-O5

2. $50 \mathrm{E}-05$

1. $20 \mathrm{E}-05$

2.00E- 05

$6.00 E-06$

$6.00 E-06$

$3.00 \mathrm{E}-06$

9.00 E- 06

$6.00 \mathrm{E}-06$

$1.00 E-04$

4. 50E-05

$1.50 \mathrm{E}-02$

$6.00 E-03$

5.00E-03

4. 0 OE -03

2. 3OE-02

8.00E-04

4.00E-03

$1.10 E-01$

$2.00 \mathrm{E}-02$

$6.40 E-03$

1. $50 E-03$

$1.00 \mathrm{E}-02$

$1.00 E+00$

$2.60 E-02$

$1.00 E+00$

1. 20E- 04

5. 00 E- 05

5. $00 E-06$

5. $00 E-06$

$1.00 E-02$

5. 00 E- 06

4. 5 OE-06

3. $50 E-05$

4. $00 \mathrm{OE}-05$

3.00E-05

3. OOE-0 5

3. 0 OE- 05

3. OOE-O5 


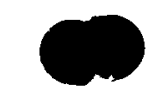

TABLE 5A. FRACTION OF INGESTED ISOTOPE DEPOSITED IN OVARIES

$\begin{array}{lrrr}\text { ELEMENT } & \text { ATOMIC } & \text { FRACTION } & \\ \text { SYMBOL } & \text { NO. } 121 & \text { F } & \text { CODE } \\ \text { H } & & & \\ \text { HE } & 1 & 1.10 E-04 & 5 \\ \text { LI } & 2 & 1.10 E-04 & 5 \\ \text { BE } & 3 & 1.00 E-04 & 1 \\ \text { B } & 4 & 2.00 E-03 & 2 \\ \text { C } & 5 & 9.00 E-01 & 2 \\ \text { N } & 6 & 1.10 E-04 & 5 \\ \text { O } & 7 & 1.10 E-04 & 5 \\ \text { F } & 8 & 1.10 E-04 & 5 \\ \text { NE } & 9 & 1.00 E+00 & 2 \\ \text { NA } & 10 & 1.10 E-04 & 5 \\ \text { MG } & 11 & 1.10 E-04 & 5 \\ \text { AL } & 12 & 1.00 E-01 & 2 \\ \text { SI } & 13 & 1.00 E-01 & 2 \\ \text { P } & 14 & 5.00 E-05 & 1 \\ \text { S } & 15 & 5.00 E-04 & 99 \\ \text { CL } & 16 & 2.00 E-04 & 99 \\ \text { AR } & 17 & 1.10 E-04 & 5 \\ \text { K } & 18 & 1.10 E-04 & 5 \\ \text { CA } & 19 & 1.10 E-04 & 5 \\ \text { SC } & 20 & 7.00 E-05 & 99 \\ \text { II } & 21 & 2.70 E-06 & 99 \\ \text { V } & 22 & 1.00 E-04 & 2 \\ \text { CR } & 23 & 2.00 E-02 & 2 \\ \text { MN } & 24 & 5.00 E-03 & 2 \\ \text { FE } & 25 & 1.00 E-03 & 99 \\ \text { CD } & 26 & 1.00 E-04 & 99 \\ \text { NI } & 27 & 3.00 E-05 & 99 \\ \text { CU } & 28 & 3.00 E-01 & 2 \\ \text { ZN } & 29 & 9.00 E-04 & 99 \\ \text { GA } & 30 & 4.00 E-05 & 1 \\ \text { GE } & 31 & 1.00 E-03 & 2 \\ \text { AS } & 32 & 1.00 E-02 & 2 \\ \text { SE } & 33 & 3.00 E-02 & 2 \\ \text { BR } & 34 & 9.00 E-01 & 2 \\ \text { KR } & 35 & 1.10 E-04 & 5 \\ \text { RB } & 37 & 1.10 E-04 & 5 \\ \text { SR } & 38 & 1.10 E-04 & 5 \\ \text { Y } & 39 & 1.00 E-05 & 99 \\ \text { ZR } & 40 & 1.00 E-04 & 99 \\ \text { NB } & 41 & 1.00 E-04 & 2 \\ \text { MO } & 42 & 1.30 E-04 & 99 \\ \text { TC } & 43 & 5.00 E-01 & 2 \\ \text { RU } & 44 & 9.00 E-05 & 99 \\ \text { RH } & 45 & 6.00 E-03 & 99 \\ \text { PD } & 46 & 6.00 E-04 & 99 \\ \text { AG } & 47 & 6.00 E-04 & 99 \\ \text { CD } & 48 & 1.90 E-06 & 99 \\ \text { IN } & 49 & 2.00 E-03 & 2 \\ \text { SN } & 50 & 2.00 E-04 & 99\end{array}$

$\begin{array}{lrrr}\text { ELEMENT } & \text { ATOMIC } & \text { FRACTION } \\ \text { SYMBOL } & \text { NO.12 } & \text { FB } & \text { CODE } \\ \text { SB } & 51 & 8.00 E-06 & 99 \\ \text { TE } & 52 & 2.00 E-04 & 99 \\ \text { I } & 53 & 1.00 E+00 & 2 \\ \text { XE } & 54 & 1.10 E-04 & 5 \\ \text { CS } & 55 & 1.10 E-04 & 5 \\ \text { BA } & 56 & 2.00 E-05 & 1 \\ \text { LA } & 57 & 1.00 E-04 & 2 \\ \text { CE } & 58 & 2.00 E-07 & 99 \\ \text { PR } & 59 & 1.00 E-04 & 2 \\ \text { ND } & 60 & 1.00 E-05 & 2 \\ \text { PM } & 61 & 9.00 E-07 & 99 \\ \text { SM } & 6 & 1.00 E-07 & 99 \\ \text { EU } & 63 & 5.00 E-07 & 99 \\ \text { GD } & 64 & 1.00 E-06 & 99 \\ \text { IB } & 65 & 1.00 E-06 & 99 \\ \text { DY } & 66 & 1.00 E-04 & 2 \\ \text { HO } & 67 & 1.00 E-04 & 2 \\ \text { ER } & 68 & 1.00 E-04 & 2 \\ \text { IM } & 69 & 7.50 E-07 & 99 \\ \text { YB } & 70 & 1.50 E-06 & 99 \\ \text { LU } & 71 & 1.00 E-04 & 2 \\ \text { HF } & 72 & 1.00 E-05 & 99 \\ \text { TA } & 73 & 2.50 E-03 & 99 \\ \text { W } & 74 & 1.00 E-01 & 2 \\ \text { RE } & 75 & 5.00 E-01 & 2 \\ \text { OS } & 76 & 1.00 E-01 & 2 \\ \text { IR } & 77 & 1.00 E-01 & 2 \\ \text { PI } & 78 & 1.00 E-01 & 2 \\ \text { AU } & 79 & 1.00 E-01 & 2 \\ \text { HG } & 80 & 7.50 E-01 & 2 \\ \text { TL } & 81 & 1.40 E-03 & 99 \\ \text { PB } & 82 & 8.00 E-02 & 2 \\ \text { BI } & 83 & 1.00 E-02 & 2 \\ \text { PO } & 84 & 6.00 E-02 & 2 \\ \text { AT } & 85 & 2.00 E-03 & 1 \\ \text { RN } & 86 & 1.10 E-04 & 5 \\ \text { FR } & 87 & 1.00 E+00 & 2 \\ \text { RA } & 88 & 3.00 E-01 & 2 \\ \text { AC } & 89 & 1.00 E-04 & 2 \\ \text { TH } & 90 & 1.00 E-04 & 2 \\ \text { PA } & 91 & 1.00 E-02 & 2 \\ \text { U } & 92 & 1.00 E-04 & 2 \\ \text { NP } & 93 & 1.00 E-04 & 2 \\ \text { PU } & 94 & 3.00 E-06 & 2 \\ \text { AM } & 95 & 1.00 E-04 & 2 \\ \text { CM } & 96 & 1.00 E-04 & 2 \\ \text { BK } & 97 & 3.00 E-05 & 2 \\ \text { CF } & 98 & 3.00 E-05 & 2 \\ \text { ES } & 99 & 3.00 E-05 & 2 \\ \text { FM } & 100 & 3.00 E-05 & 2 \\ \text { FM } & & & \end{array}$

REFERENCE (S) 


$\begin{array}{ll}\text { ELEMENT } & \text { ATOM } \\ \text { SYMBOL } & \text { NO } \\ & \\ \text { H } & \\ \text { HE } & \\ \text { LI } & \\ \text { BE } & \\ \text { B } & \\ \text { C } & \\ \text { N } & \\ \text { D } & \\ \text { F } & \\ \text { NE } & \\ \text { NA } & \\ \text { MG } & 1 \\ \text { AL } & 12 \\ \text { SI } & 14 \\ \text { P } & 15 \\ \text { S } & 16 \\ \text { CL } & 1 \\ \text { AR } & 18 \\ \text { K } & \\ \text { CA } & 20 \\ \text { SC } & 21 \\ \text { TI } & 22 \\ \text { V } & 23 \\ \text { CR } & 24 \\ \text { MN } & 25 \\ \text { FE } & 26 \\ \text { CO } & 27 \\ \text { NI } & 28 \\ \text { CU } & 29 \\ \text { ZN } & 30 \\ \text { GA } & 31 \\ \text { GE } & 32 \\ \text { AS } & 33 \\ \text { SE } & 34 \\ \text { BR } & 35 \\ \text { KR } & 36 \\ \text { RB } & 3 \\ \text { SR } & 38 \\ \text { Y } & \\ \text { ZR } & 40 \\ \text { NB } & 41 \\ \text { MO } & 42 \\ \text { TC } & 43 \\ \text { RU } & 44 \\ \text { RH } & 45 \\ \text { PD } & 46 \\ \text { AG } & 47 \\ \text { CD } & 48 \\ \text { IN } & 49 \\ \text { SN } & 5 \\ & \end{array}$

FRACTION

$$
F_{B} \text { CODE }
$$

REFERENCE (S)

2. $50 E-03$ $2.50 E-03$

1. $00 \mathrm{OOE}+00$

4. $00 E-06$ 9. $00 E-01$ $2.50 E-03$ 2.5OE-03 $2.50 E-03$ 1. $00 E+00$ 2. $50 \mathrm{E}-03$ $2.50 E-03$
$2.50 E-03$ 2. $50 \mathrm{E}-03$ $1.00 \mathrm{E}-01$ 2. $00 E-04$ 8. 50E-01

1. $00 E-02$ 3. $00 E-04$ 2. $50 E-03$ $2.50 E-03$ $2.50 E-03$ 2. $00 E-03$ $5.30 E-06$ 1. $00 \mathrm{E}-06$ 2. $00 E-04$ 5. $00 E-03$ 6. $00 E-04$ 2. $00 E-03$ 2.00E-03 4. $20 \mathrm{E}-04$ 3. $00 \mathrm{E}-01$ 2. $00 \mathrm{E}-02$

1. $00 E-02$

1. $00 E-05$

1. $00 E-02$ 3. OOE -02 $4.50 E-03$ 4. $50 \mathrm{E}-03$ 2.50E-03 2. $50 \mathrm{E}-03$ $2.50 E-03$ 7. $50 \mathrm{OE}-04$

3. 50E-07

6. $00 E-07$ 4. $00 E-04$ 8. $00 E-03$ 5. $00 E-01$ 3. $90 \mathrm{E}-03$ 2. $00 \mathrm{E}-03$ 2. $00 \mathrm{E}-03$ 1. $10 \mathrm{E}-03$ 9. $30 E-06$ . OOE-O 4. $50 E-05$
2
ELEMENT ATOMIC
SYMBOL

FRACT ION

$F_{B}$

CODE

REFERENCE (S)

\begin{tabular}{ll} 
SB & 51 \\
TE & 52 \\
I & 53 \\
XE & 54 \\
CS & 55 \\
BA & 56 \\
LA & 57 \\
CE & 58 \\
PR & 59 \\
ND & 60 \\
PM & 61 \\
SM & 62 \\
EU & 63 \\
GD & 64 \\
TB & 65 \\
OY & 66 \\
HO & 67 \\
ER & 68 \\
TM & 69 \\
YB & 70 \\
LU & 71 \\
HF & 72 \\
TA & 73 \\
W & 74 \\
RE & 75 \\
OS & 76 \\
IR & 77 \\
PI & 78 \\
AU & 79 \\
HG & 80 \\
TL & 81 \\
PB & 82 \\
BI & 83 \\
PO & 84 \\
AT & 85 \\
RN & 86 \\
FR & 87 \\
RA & 88 \\
AC & 89 \\
TH & 90 \\
PA & 91 \\
U & 92 \\
NP & 93 \\
PU & 94 \\
AM & 95 \\
CM & 96 \\
BK & 97 \\
CF & 98 \\
ES & 99 \\
FM & 100 \\
& \\
\hline & 69
\end{tabular}

8.50E-06

2. $50 E-03$

5. $00 \mathrm{E}-03$

$2.50 E-03$

$2.50 E-03$

2. 50E-06

$1.00 E-04$

3. $60 \mathrm{E}-06$

1. $00 E-04$

$1.00 E-05$

$1.00 E-07$

3.00E- 07

$3.00 E-07$

$1.50 E-07$

3. $00 \mathrm{E}-07$

1. OOE- 04

$1.00 E-04$

$1.00 E-04$

$9.00 E-07$

$6.00 E-07$

$1.00 E-04$

1. $30 E-05$

5. OOE-04

$1.00 E-01$

5. $00 \mathrm{E}-01$

$1.00 \mathrm{E}-01$

2. $00 \mathrm{OE}-03$

$8.00 \mathrm{E}-04$

5.0OE-04

$2.00 E-02$

2.0OE- 03

$8.00 E-02$

$1.00 E-04$

$2.00 \mathrm{E}-03$

3.00E-02

$2.50 \mathrm{E}-03$

$1.00 E+00$

3. $00 \mathrm{E}-01$

$1.00 E-04$

$1.00 E-04$

$1.00 \mathrm{E}-04$

$1.00 E-02$

$1.00 E-04$

$3.00 E-06$

$1.00 E-04$

1. 00 E -04

3.0OE-O5

3. $00 E-05$

3. $00 E-05$

3.00E-05

\section{9}

1 
FRACTION

F $B$ CODE REFERENCE $(S)$

$8.60 \mathrm{E}-04$ 8. $60 E-04$ 4. $20 \mathrm{OE}-04$ 2. $00 E-03$ 9. $00 E-01$

8. $60 \mathrm{E}-04$

$8.60 E-04$ $8.60 E-04$ $1.00 E+00$ 8. $60 E-04$ . $60 \mathrm{E}-04$ . $00 E-01$ . $00 \mathrm{E}-01$ 4. $30 E-04$

4. $00 E-03$

1. $30 \mathrm{E}-03$ 8.60E-04 8. $60 \mathrm{E}-04$ 8. $60 \mathrm{E}-04$ 5. $00 E-04$ 2. $70 E-06$ 1. $00 \mathrm{E}-04$ 2. $00 E-02$

5. $00 \mathrm{E}-03$ 1. $00 E-03$ 7. $50 E-04$ 2. $00 E-04$ 3. $00 \mathrm{E}-01$ 7. $00 \mathrm{E}-03$ $9.00 E-05$

1. $00 \mathrm{E}-03$

1. $0 O E-02$

3. $0 O E-02$

9. $00 E-01$

8.60E-04

$8.60 E-04$

8. $60 \mathrm{E}-04$ 2. $60 \mathrm{E}-04$ $1.40 E-07$

2. $00 E-06$

1. $00 E-04$

1. $00 \mathrm{E}-03$

5. $00 \mathrm{E}-01$

2. $60 E-03$

$6.00 E-03$

$6.00 E-04$

1. $00 E-03$

6. $30 E-05$

2. OOE-04
ELEMENT ATOMIC

FRACTION

$F_{B} \quad$ CODE

REFERENCE (S)

SB

I

XE

CS

CE

PR

PM

SM

$G D$

TB

DY

HO

ER

$Y B$

LU

TA

RE

RE

IR

PT

HG

PB

BI

8.00E-06 7. $50 E-04$ 5. 0 OE-03 $8.60 E-04$ 8. 60E-04 5.00E-05

1. $00 \mathrm{E}-04$ 2.00E-07 1. $00 \mathrm{E}-04$ $1.00 E-05$ 9. 0 OE- 07 $1.00 \mathrm{E}-07$ 5. OOE-07 $1.00 E-06$ $1.00 \mathrm{E}-06$ $1.00 E-04$ 1.00E-0 4 1. $00 E-04$ 7. 5 OE- 07 $1.50 E-06$ $1.00 E-04$ 2. $70 E-08$ $2.50 E-03$

$1.00 E-01$ 5.00E-01 $1.00 E-01$

$1.00 E-01$

1. $00 E-01$

$1.00 E-01$

$7.50 E-01$

$1.40 \mathrm{E}-03$

$8.00 E-02$

$1.00 \mathrm{E}-02$

$6.00 \mathrm{E}-02$

$1.00 E+00$

$8.60 E-04$

$1.00 E+00$

3. $00 E-01$

1. $00 E-04$

$1.00 E-04$

$1.00 E-04$

$1.00 E-04$

I. 00 EE- 04

3. $00 \mathrm{E}-06$

$1.00 \mathrm{E}-04$

$1.00 E-04$

3. $00 E-05$

3.00E-05

3. 00 OE-05

3. OOE- 05 


ELEMENT ATOMIC
SYMBOL NO.

FRACTION

${ }_{B}$

CODE REFERENCE (S)

1. $00 E+00$

$1.00 E+00$

1. $00 E+00$

2. $00 E-03$

9. $00 E-01$

1. $00 E+00$

1. $00 E+00$

1. $00 E+00$

$1.0 O E+00$

$1.00 E+00$

1. $O O E+O O$

1. $00 E-01$

1. $00 E-01$

8. $50 \mathrm{OE}-01$

7. $50 \mathrm{E}-01$

$1.00 E+00$

1. $00 E+00$

1. $0 O E+00$

1. $O O E+O O$

6. $00 E-01$

1. $00 E-04$

$1.00 E-04$

2. OOE-02

5. $00 E-03$

1. OOE-01

$1.00 E-01$

3. 00E-01

3. $00 E-01$

2. $80 E-01$

1. OOE-01

1. 0 OE -03

1. OOE-02

3. $00 E-02$

$9.00 E-01$

1. $00 E+00$

1. $00 E+00$

1. $00 E+00$

3. $00 E-01$

1. $00 E-04$

1. $00 E-04$

5. $00 E-02$

8. OOE-01

1. $00 E+00$

3. $00 E-01$

2. $00 E-01$

2. $00 E-01$

1. $00 E+00$

2. 50E-03

2. $00 E-03$

5. OOE-02
ELEMENT

ATOMIC

FRACTION

$F_{B}$

CODE

REFERENCE (S)

I

$X E$

CS

BA

CE

PR
ND

PM

SM

GD

$T B$

DY

ER

TM

LU

HF

TA

RE

IR

PT

AU

HG

P L

$B$

PO

RN

FR

RA

AC

PA

UP

NP

AM

CM

BK

CF

FM

3.00E-02

2. $50 E-01$

$1.00 E+00$

$1.00 E+00$

5.00E-02

1. $00 \mathrm{E}-04$

$1.00 \mathrm{E}-04$

$1.00 E-04$
$1.00 E-04$

$1.00 E-04$

$1.00 E-04$

$1.00 E-04$

1. $00 \mathrm{E}-04$

$1.00 E-04$

$1.00 E-0$

1. 00E-04

1. OOE-04

1. 00 OE-04

$1.00 E-04$

$1.00 E-04$

5. $00 \mathrm{E}-02$

$1.00 E-01$

5. OOE- 0

$1.00 E-01$

$1.00 E-01$

$1.00 E-01$

$1.00 E-01$

7.50E-01

4.5OE-01

8. OOE-02

$1.00 E-02$

6. $00 E-02$

$1.00 E+00$

$1.00 E+00$

$1.00 E+00$

3. OOE-01

1. $00 \mathrm{OE}-04$

1. 0 OE- 04

1. $00 E-04$

1. 00 E- 04

1. $00 \mathrm{E}-04$

$3.00 E-05$

$1.00 E-04$

$1.00 E-04$

3. 0 OE-05

3.00E-05

3. $00 E-05$
3. DOE- 05 
TABLE 5A. FRACTION OF IVGESIEU ISUTOPE DEPOSITED IN GI TRACT

$10 / 09 / 68$

PAGE 8

$\begin{array}{cccc}\text { ELEMENT } & \text { ATOMIC } & \text { FRACTION } & \\ \text { SYMBOL } & \text { NO. }(2) & \text { F B } & \text { CODE } \\ \text { H } & & 1.00 E-01 & 6 \\ \text { HE } & 1 & 1.00 E-01 & 6 \\ \text { LI } & 3 & 1.00 E-01 & 6 \\ \text { BE } & 3 & 1.00 E-01 & 6 \\ \text { B } & 5 & 1.00 E+00 & 6 \\ \text { C } & 6 & 1.00 E-01 & 6 \\ \text { N } & 7 & 1.00 E-01 & 6 \\ \text { D } & 8 & 1.00 E-01 & 6 \\ \text { F } & 9 & 1.00 E-01 & 6 \\ \text { NE } & 10 & 1.00 E-01 & 6 \\ \text { NA } & 11 & 1.00 E-01 & 6 \\ \text { MG } & 12 & 9.00 E-01 & 6 \\ \text { AL } & 13 & 9.00 E-01 & 6 \\ \text { SI } & 14 & 1.50 E-01 & 6 \\ \text { P } & 15 & 2.50 E-01 & 6 \\ \text { S } & 16 & 1.00 E-01 & 6 \\ \text { CL } & 17 & 1.00 E-01 & 6 \\ \text { AR } & 18 & 1.00 E-01 & 6 \\ \text { K } & 19 & 1.00 E-01 & 6 \\ \text { CA } & 20 & 4.00 E-01 & 6 \\ \text { SC } & 21 & 1.00 E+00 & 6 \\ \text { TI } & 22 & 1.00 E+00 & 6 \\ \text { V } & 23 & 1.00 E+00 & 6 \\ \text { CR } & 24 & 1.00 E+00 & 6 \\ \text { MN } & 25 & 9.00 E-01 & 6 \\ \text { FE } & 26 & 9.00 E-01 & 6 \\ \text { CD } & 27 & 7.00 E-01 & 6 \\ \text { NI } & 28 & 7.00 E-01 & 6 \\ \text { CU } & 29 & 7.20 E-01 & 6 \\ \text { ZN } & 30 & 9.00 E-01 & 6 \\ \text { GA } & 31 & 1.00 E+00 & 6 \\ \text { GE } & 32 & 1.00 E+00 & 6 \\ \text { AS } & 33 & 1.00 E+00 & 6 \\ \text { SE } & 34 & 1.00 E-01 & 6 \\ \text { BR } & 35 & 1.00 E-01 & 6 \\ \text { KR } & 36 & 1.00 E-01 & 6 \\ \text { RB } & 37 & 1.00 E-01 & 6 \\ \text { SR } & 38 & 7.00 E-01 & 6 \\ \text { Y } & 39 & 1.00 E+00 & 6 \\ \text { ZR } & 40 & 1.00 E+00 & 6 \\ \text { NB } & 41 & 1.00 E+00 & 6 \\ \text { MO } & 42 & 2.00 E-01 & 6 \\ \text { TC } & 43 & 1.00 E-01 & 6 \\ \text { RU } & 44 & 7.00 E-01 & 6 \\ \text { RH } & 45 & 8.00 E-01 & 6 \\ \text { PD } & 46 & 8.00 E-01 & 6 \\ \text { AG } & 47 & 1.00 E-01 & 6 \\ \text { CD } & 48 & 1.00 E+00 & 6 \\ \text { IV } & 49 & 1.00 E+00 & 6 \\ \text { SN } & 50 & 1.00 E+00 & 6\end{array}$

$\begin{array}{cccc}\text { ELEMENT } & \text { ATOMIC } & \text { FRACTIOV } & \\ \text { SYMBOL } & \text { NO. }(21 & \text { F B } & \text { CODE } \\ & & & \\ \text { SB } & 51 & 1.00 E+00 & 6 \\ \text { TE } & 52 & 7.50 E-01 & 6 \\ \text { I } & 53 & 1.00 E-01 & 6 \\ \text { XE } & 54 & 1.00 E-01 & 6 \\ \text { CS } & 55 & 1.00 E-01 & 6 \\ \text { BA } & 56 & 1.00 E+00 & 6 \\ \text { LA } & 57 & 1.00 E+00 & 6 \\ \text { CE } & 58 & 1.00 E+00 & 6 \\ \text { PR } & 59 & 1.00 E+00 & 6 \\ \text { ND } & 60 & 1.00 E+00 & 6 \\ \text { PM } & 61 & 1.00 E+00 & 6 \\ \text { SM } & 62 & 1.00 E+00 & 6 \\ \text { EU } & 63 & 1.00 E+00 & 6 \\ \text { GD } & 64 & 1.00 E+00 & 6 \\ \text { TB } & 65 & 1.00 E+00 & 6 \\ \text { DY } & 66 & 1.00 E+00 & 6 \\ \text { HO } & 67 & 1.00 E+00 & 6 \\ \text { ER } & 68 & 1.00 E+00 & 6 \\ \text { TM } & 69 & 1.00 E+00 & 6 \\ \text { YB } & 70 & 1.00 E+00 & 6 \\ \text { LU } & 71 & 1.00 E+00 & 6 \\ \text { HF } & 72 & 1.00 E+00 & 6 \\ \text { TA } & 73 & 1.00 E+00 & 6 \\ \text { W } & 74 & 2.00 E-01 & 6 \\ \text { RE } & 75 & 5.00 E-01 & 6 \\ \text { OS } & 76 & 9.00 E-01 & 6 \\ \text { IR } & 77 & 9.00 E-01 & 6 \\ \text { PT } & 78 & 9.00 E-01 & 6 \\ \text { AU } & 79 & 9.00 E-01 & 6 \\ \text { HG } & 80 & 2.50 E-01 & 6 \\ \text { TL } & 81 & 5.50 E-01 & 6 \\ \text { FB } & 82 & 9.20 E-01 & 6 \\ \text { BI } & 83 & 1.00 E+00 & 6 \\ \text { PO } & 84 & 9.40 E-01 & 6 \\ \text { AT } & 85 & 1.00 E-01 & 6 \\ \text { RN } & 86 & 1.00 E-01 & 6 \\ \text { FR } & 87 & 1.00 E-01 & 6 \\ \text { RA } & 88 & 7.00 E-01 & 6 \\ \text { AC } & 89 & 1.00 E+00 & 6 \\ \text { TH } & 90 & 1.00 E+00 & 6 \\ \text { PA } & 91 & 1.00 E+00 & 6 \\ \text { U } & 92 & 1.00 E+00 & 6 \\ \text { NP } & 93 & 1.00 E+00 & 6 \\ \text { PU } & 94 & 1.00 E+00 & 6 \\ \text { AM } & 95 & 1.00 E+00 & 6 \\ \text { CM } & 96 & 1.00 E+00 & 6 \\ \text { BK } & 97 & 1.00 E+00 & 6 \\ \text { CF } & 98 & 1.00 E+00 & 6 \\ \text { ES } & 99 & 1.00 E+00 & 6 \\ \text { FM } & 100 & 1.00 E+00 & 6\end{array}$

REFERENCE (S) 
$\bullet$

-

- 
5. UPTAKE AND DISTRIBUTION IN ORGANS (Continued)

$I_{B}$ : Biological Half-Life

The biological half-life $T_{B}$ is required in the estimation of the effective half-life $\mathrm{T}_{\mathrm{E}}$. The effective half-life is equal to the product of the biological and radiological half-lives divided by the sum, i. e., $T_{E}=T_{B} T_{R} /\left(T_{B}+T_{R}\right)$. The values adopted for $\mathrm{T}_{\mathrm{B}}$ are shown in Table $5 \mathrm{~B}$. These values were assigned according to the procedures and code numbers shown below.

\section{Rules for estimating $\mathrm{T}_{\mathrm{B}}$}

\section{Code \#}

1 The $T_{B}$ was assumed to be the ICRP estimate of $T_{B}$.

3 The $T_{B}$ was assumed to be $3.65 \times 10^{5}$ days via milk and zero via soil (so that $F_{1}$ is minimized by both routes).

4 The $\mathrm{T}_{\mathrm{B}}$ (of $\mathrm{H}, \mathrm{C}, \mathrm{N}, \mathrm{O}, \mathrm{Na}, \mathrm{Cl}, \mathrm{K}$, $\mathrm{Br}, \mathrm{Rb}, \mathrm{Cs}$ and the inert gases) was assumed to be the $T_{B}$ for total body.

5 The $\mathrm{T}_{\mathrm{B}}$ was obtained from human retention data.

6 The $T_{B}$ in the GI tract is the same for all elements. This value, 1.5 days, corresponds to a mean life of about 2 days, which approximates the transit time of material in the GI tract following ingestion (2839).

99 The $T_{B}$ was estimated from collateral data as described in the Appendix. 


$\begin{array}{llll}\text { ELEMENT } & \text { ATOMIC } & \text { HALF-LIFE } \\ \text { SYMBOL } & \text { NO. }(2) & T_{B}(D A Y) & \text { CODE }\end{array}$

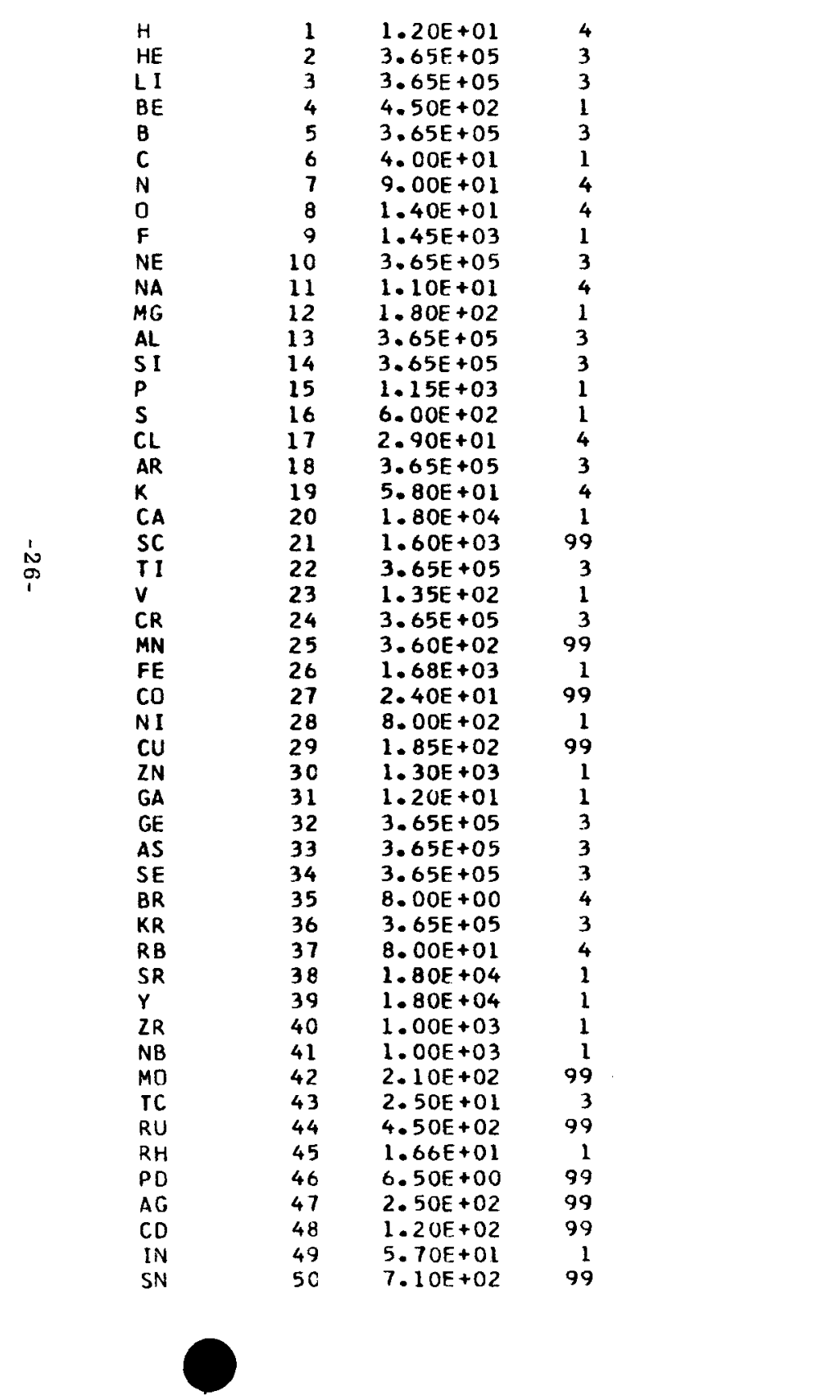

$\begin{array}{llll}\text { ELEMENT } & \text { ATOMIC } & \text { HALF-LIFE } \\ \text { SYMBOL } & \text { NO. }(Z) & T_{B}(D A Y) & \\ & & \end{array}$

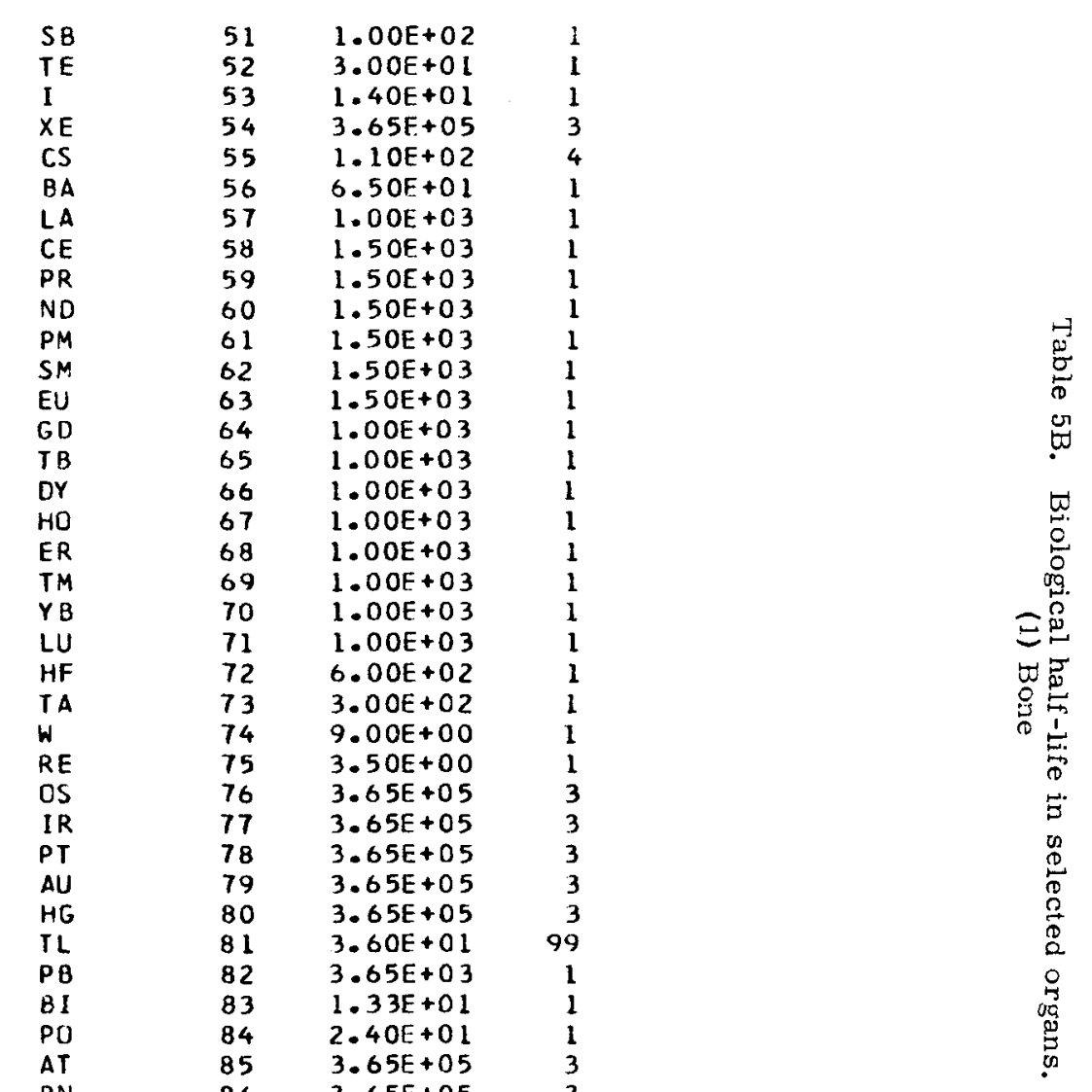




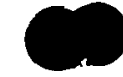

TABLE 5B. BIOLOGICAL HALF-LIFE IN KIDNEYS

$\begin{array}{lrrr}\text { ELEMENT } & \text { ATOMIC } & \text { HALF-LIFE } \\ \text { SYMBOL } & \text { NO. (2) } & \text { T } B(D A Y) & \text { CODE } \\ \text { H } & & & \\ \text { HE } & 1 & 1.20 E+01 & 4 \\ \text { LI } & 2 & 3.65 E+05 & 3 \\ \text { BE } & 3 & 3.65 E+05 & 3 \\ \text { B } & 4 & 1.20 E+02 & 1 \\ \text { C } & 5 & 3.65 E+05 & 3 \\ \text { N } & 6 & 1.00 E+01 & 4 \\ \text { O } & 7 & 9.00 E+01 & 4 \\ \text { F } & 8 & 1.40 E+01 & 4 \\ \text { NE } & 9 & 3.65 E+05 & 3 \\ \text { NA } & 10 & 3.65 E+05 & 3 \\ \text { MG } & 11 & 1.10 E+01 & 4 \\ \text { AL } & 12 & 3.65 E+05 & 3 \\ \text { SI } & 13 & 3.65 E+05 & 3 \\ \text { P } & 14 & 3.65 E+05 & 3 \\ \text { S } & 15 & 1.80 E+01 & 99 \\ \text { CL } & 16 & 3.65 E+05 & 3 \\ \text { AR } & 17 & 2.90 E+01 & 4 \\ \text { K } & 18 & 3.65 E+05 & 3 \\ \text { CA } & 19 & 5.80 E+01 & 4 \\ \text { SC } & 20 & 1.70 E+01 & 99 \\ \text { TI } & 21 & 1.60 E+03 & 99 \\ \text { V } & 22 & 3.65 E+05 & 3 \\ \text { CR } & 23 & 7.40 E+01 & 1 \\ \text { MN } & 24 & 6.16 E+02 & 1 \\ \text { FE } & 25 & 2.50 E+01 & 99 \\ \text { CO } & 26 & 7.20 E+02 & 99 \\ \text { NI } & 27 & 2.40 E+01 & 99 \\ \text { CU } & 28 & 3.65 E+05 & 3 \\ \text { ZN } & 29 & 9.00 E+01 & 99 \\ \text { GA } & 30 & 1.49 E+02 & 1 \\ \text { GE } & 31 & 9.00 E+00 & 1 \\ \text { AS } & 32 & 1.20 E+01 & 1 \\ \text { SE } & 34 & 5.50 E+02 & 1 \\ \text { BR } & 35 & 1.10 E+01 & 1 \\ \text { KR } & 36 & 3.00 E+00 & 4 \\ \text { RB } & 37 & 8.05 E+05 & 3 \\ \text { SR } & 38 & 1.60 E+01 & 4 \\ \text { Y } & 39 & 4.20 E+01 & 99 \\ \text { ZR } & 40 & 9.00 E+02 & 99 \\ \text { NB } & 41 & 7.60 E+02 & 1 \\ \text { MO } & 42 & 2.40 E+01 & 99 \\ \text { TC } & 43 & 2.00 E+01 & 1 \\ \text { RU } & 44 & 1.80 E+02 & 99 \\ \text { RH } & 45 & 2.80 E+01 & 1 \\ \text { PD } & 46 & 3.00 E+01 & 1 \\ \text { AG } & 47 & 4.00 E+00 & 99 \\ \text { CD } & 48 & 3.00 E+02 & 1 \\ \text { IN } & 49 & 6.00 E+01 & 1 \\ \text { SN } & 50 & 6.60 E+02 & 99\end{array}$

\begin{tabular}{|c|c|c|c|c|}
\hline $\begin{array}{l}\text { ELEMENT } \\
\text { SYMBOL }\end{array}$ & $\begin{array}{l}\text { ATOMIC } \\
\text { NO. (Z) }\end{array}$ & $\begin{array}{l}\text { HALF-LIFE } \\
T_{B} \text { (DAY) }\end{array}$ & CODE & REF EREN $=E(S)$ \\
\hline SB & 51 & $3.65 E+05$ & 3 & \\
\hline TE & 52 & $3.00 E+01$ & 1 & \\
\hline$I$ & 53 & $7.00 E+00$ & 1 & \\
\hline$X E$ & 54 & $3.65 E+05$ & 3 & \\
\hline CS & 55 & $1.10 E+02$ & 4 & \\
\hline BA & 56 & $8.50 E+00$ & 1 & \\
\hline LA & 57 & $3.65 E+05$ & 3 & \\
\hline$C E$ & 58 & $5.63 E+02$ & 1 & \\
\hline PR & 59 & $7.50 E+02$ & 1 & \\
\hline ND & 60 & $6.56 E+02$ & $i$ & \\
\hline PM & 61 & $6.56 E+02$ & 1 & \\
\hline SM & 62 & $6.56 E+02$ & 1 & \\
\hline EU & 63 & $1.48 E+03$ & $i$ & \\
\hline GD & 64 & $1.30 E+03$ & 99 & \\
\hline$T B$ & 65 & $7.00 E+02$ & 1 & \\
\hline DY & 66 & $3.65 E+05$ & 3 & \\
\hline $\mathrm{HO}$ & 67 & $8.00 E+02$ & 1 & \\
\hline ER & 68 & $6.50 E+02$ & 1 & \\
\hline TM & 69 & $3.35 E+02$ & 1 & \\
\hline YB & 70 & $6.85 E+02$ & 1 & \\
\hline LU & 71 & $7.50 E+02$ & 1 & \\
\hline HF & 72 & $5.63 E+02$ & 1 & \\
\hline TA & 73 & $4.00 E+02$ & 1 & \\
\hline$W$ & 74 & $3.65 E+05$ & 3 & \\
\hline RE & 75 & $3.65 E+05$ & 3 & \\
\hline OS & 76 & $5.00 E+00$ & 1 & \\
\hline IR & 77 & $5.00 E+01$ & 1 & \\
\hline PI & 78 & $6.00 E+01$ & 1 & \\
\hline$A U$ & 79 & $2.80 E+02$ & 1 & : \\
\hline HG & 80 & $1.45 E+01$ & 1 & \\
\hline$T L$ & 81 & $3.50 E+01$ & 99 & \\
\hline PB & 82 & $5.31 E+02$ & 1 & \\
\hline B I & 83 & $6.00 E+00$ & 1 & \\
\hline PO & 84 & $7.00 E+01$ & 1 & \\
\hline AT & 85 & $3.65 E+05$ & 3 & \\
\hline RN & 86 & $3.65 E+05$ & 3 & \\
\hline$F R$ & 87 & $3.65 E+05$ & 3 & \\
\hline RA & 88 & $1.00 E+01$ & 1 & \\
\hline AC & 89 & $2.40 E+04$ & 1 & \\
\hline TH & 90 & $2.20 E+04$ & 1 & \\
\hline PA & 91 & $5.10 E+04$ & 1 & \\
\hline$u$ & 92 & $1.50 E+01$ & 1 & \\
\hline NP & 93 & $6.40 E+04$ & 1 & \\
\hline PU & 94 & $3.20 E+04$ & 1 & \\
\hline$A M$ & 95 & $2.70 E+04$ & 1 & \\
\hline CM & 96 & $2.40 E+04$ & 1 & \\
\hline BK & 97 & $3.65 E+05$ & 3 & \\
\hline CF & 98 & $3.65 E+05$ & 3 & \\
\hline ES & 99 & $3.65 E+05$ & 3 & \\
\hline FM & 100 & $3.65 E+05$ & 3 & \\
\hline
\end{tabular}




\begin{tabular}{|c|c|c|c|}
\hline $\begin{array}{l}\text { ELEMENT } \\
\text { SYMBOL }\end{array}$ & $\begin{array}{l}\text { ATOMIC } \\
\text { NO. }(Z)\end{array}$ & $\begin{array}{l}\text { HALF-LIFE } \\
T_{B} \text { (DAY) }\end{array}$ & CODE \\
\hline H & 1 & $1.20 E+01$ & 4 \\
\hline HE & 2 & $3.65 E+05$ & 3 \\
\hline LI & 3 & $3.65 E+05$ & 3 \\
\hline BE & 4 & $2.70 E+02$ & 1 \\
\hline B & 5 & $5.00 E-01$ & 1 \\
\hline$c$ & 6 & $1.00 E+01$ & 4 \\
\hline $\mathbf{N}$ & 7 & $9.00 E+01$ & 4 \\
\hline 0 & 8 & $1.40 E+01$ & 4 \\
\hline$F$ & 9 & $3.65 E+05$ & 3 \\
\hline NE & 10 & $3.65 E+05$ & 3 \\
\hline NA & 11 & $1.10 E+01$ & 4 \\
\hline MG & 12 & $3.65 E+05$ & 3 \\
\hline AL & 13 & $3.65 E+05$ & 3 \\
\hline SI & 14 & $3.65 E+05$ & 3 \\
\hline $\mathbf{P}$ & 15 & $3.40 E+01$ & 99 \\
\hline $\mathbf{S}$ & 16 & $3.65 E+05$ & 3 \\
\hline $\mathrm{CL}$ & 17 & $2.90 E+01$ & 4 \\
\hline AR & 18 & $3.65 E+05$ & 3 \\
\hline$k$ & 19 & 5. $80 E+01$ & 1 \\
\hline CA & 20 & 8. OOE +00 & 99 \\
\hline SC & 21 & $1.70 E+03$ & 99 \\
\hline TI & 22 & $3.65 E+05$ & 3 \\
\hline v & 23 & $7.00 E+01$ & 1 \\
\hline CR & 24 & $3.65 E+05$ & 3 \\
\hline MN & 25 & $7.10 E+01$ & 99 \\
\hline FE & 26 & $5.54 E+02$ & 1 \\
\hline CO & 27 & $2.40 E+01$ & 99 \\
\hline NI & 28 & $5.00 E+02$ & 1 \\
\hline $\mathrm{CU}$ & 29 & $1.20 E+03$ & 99 \\
\hline $\mathrm{ZN}$ & 30 & $9.10 E+01$ & 1 \\
\hline GA & 31 & $4.80 E+00$ & 1 \\
\hline GE & 32 & $7.50 E+00$ & 1 \\
\hline AS & 33 & $5.50 E+02$ & 1 \\
\hline SE & 34 & $2.40 E+01$ & 1 \\
\hline BR & 35 & $8.00 E+00$ & 4 \\
\hline$K R$ & 36 & $3.65 E+05$ & 3 \\
\hline RB & 37 & $8.00 E+01$ & 4 \\
\hline SR & 38 & $6.00 E+00$ & 99 \\
\hline $\mathbf{Y}$ & 39 & $1.40 E+03$ & 99 \\
\hline$Z R$ & 40 & $3.20 E+02$ & 1 \\
\hline NB & 41 & $8.45 E+02$ & 1 \\
\hline MO & 42 & $9.50 E+01$ & 99 \\
\hline TC & 43 & $3.00 E+01$ & 1 \\
\hline RU & 44 & $7.00 E+01$ & 99 \\
\hline RH & 45 & $1.82 \mathrm{E}+01$ & 1 \\
\hline PD & 46 & $1.90 E+01$ & 1 \\
\hline $\mathbf{A G}$ & 47 & $5.20 E+01$ & 5 \\
\hline CD & 48 & $2.00 E+02$ & 1 \\
\hline IN & 49 & $5.80 E+01$ & 1 \\
\hline SN & 50 & $1.60 E+03$ & 99 \\
\hline
\end{tabular}

REFERENCE (S)

2932

$\begin{array}{lrrr}\text { ELEMENT } & \text { ATOMIC } & \text { HALF-LI IFE } & \\ \text { SYMBOL } & \text { NO. } 121 & T_{B}(D A Y) & \text { CODE } \\ & & & \\ \text { SB } & 51 & 3.80 E+01 & 1 \\ \text { TE } & 52 & 3.00 E+01 & 1 \\ \text { I } & 53 & 7.00 E+00 & 1 \\ \text { XE } & 54 & 3.65 E+05 & 3 \\ \text { CS } & 55 & 1.10 E+02 & 4 \\ \text { BA } & 56 & 9.75 E+02 & 1 \\ \text { LA } & 57 & 4.00 E+02 & 1 \\ \text { CE } & 58 & 2.93 E+02 & 1 \\ \text { PR } & 59 & 3.75 E+02 & 1 \\ \text { ND } & 60 & 1.31 E+02 & 1 \\ \text { PM } & 61 & 6.56 E+02 & 1 \\ \text { SM } & 62 & 1.87 E+02 & 1 \\ \text { EU } & 63 & 1.27 E+02 & 1 \\ \text { GD } & 64 & 4.60 E+02 & 1 \\ \text { TB } & 65 & 6.70 E+01 & 99 \\ \text { DY } & 66 & 5.00 E+02 & 1 \\ \text { HO } & 67 & 8.75 E+02 & 1 \\ \text { ER } & 68 & 4.33 E+02 & 1 \\ \text { IM } & 69 & 7.00 E+01 & 99 \\ \text { YB } & 70 & 7.00 E+01 & 99 \\ \text { LU } & 71 & 3.65 E+05 & 3 \\ \text { HF } & 72 & 6.25 E+02 & 1 \\ \text { TA } & 73 & 4.00 E+02 & 1 \\ \text { W } & 74 & 4.00 E+00 & 1 \\ \text { RE } & 75 & 1.40 E+01 & 1 \\ \text { OS } & 76 & 5.50 E+00 & 1 \\ \text { IR } & 77 & 2.70 E+01 & 1 \\ \text { PT } & 78 & 2.00 E+01 & 1 \\ \text { AU } & 79 & 3.00 E+02 & 1 \\ \text { HG } & 80 & 1.35 E+01 & 1 \\ \text { TL } & 81 & 2.50 E+01 & 99 \\ \text { PB } & 82 & 1.95 E+03 & 1 \\ \text { BI } & 83 & 1.50 E+01 & 1 \\ \text { PO } & 84 & 4.10 E+01 & 1 \\ \text { AT } & 85 & 3.65 E+05 & 3 \\ \text { RN } & 86 & 3.65 E+05 & 3 \\ \text { FR } & 87 & 3.65 E+05 & 3 \\ \text { RA } & 88 & 1.00 E+01 & 1 \\ \text { AC } & 89 & 2.40 E+03 & 1 \\ \text { TH } & 90 & 5.70 E+04 & 1 \\ \text { PA } & 91 & 5.80 E+04 & 1 \\ \text { U } & 92 & 3.65 E+05 & 3 \\ \text { NP } & 93 & 5.40 E+04 & 1 \\ \text { PU } & 94 & 3.00 E+04 & 1 \\ \text { AM } & 95 & 3.48 E+03 & 1 \\ \text { CM } & 96 & 3.00 E+03 & 1 \\ \text { BK } & 97 & 3.65 E+05 & 3 \\ \text { CF } & 98 & 3.65 E+05 & 3 \\ \text { ES } & 99 & 3.65 E+05 & 3 \\ \text { FM } & 100 & 3.65 E+05 & 3 \\ & & & \end{array}$

REFERENCE (S)

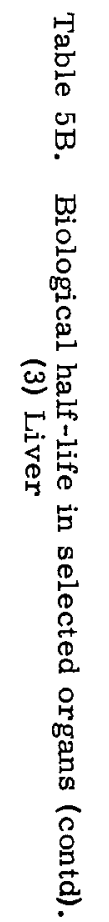




$\begin{array}{lrrr}\text { ELEMENT } & \text { ATOMIC } & \text { HALF-LIFE } \\ \text { SYMBDL } & \text { NO. }(2) & \text { T } B(D A Y) & \text { CODE } \\ \text { H } & & & \\ \text { HE } & 1 & 1.20 E+01 & 4 \\ \text { LI } & 2 & 3.65 E+05 & 3 \\ \text { BE } & 3 & 2.00 E+00 & 1 \\ \text { B } & 4 & 3.65 E+05 & 3 \\ \text { C } & 5 & 3.65 E+05 & 3 \\ \text { N } & 6 & 1.00 E+01 & 4 \\ \text { O } & 7 & 9.00 E+01 & 4 \\ \text { F } & 8 & 1.40 E+01 & 4 \\ \text { NE } & 9 & 3.65 E+05 & 3 \\ \text { NA } & 10 & 3.65 E+05 & 3 \\ \text { MG } & 11 & 1.10 E+01 & 4 \\ \text { AL } & 12 & 3.65 E+05 & 3 \\ \text { SI } & 13 & 3.65 E+05 & 3 \\ \text { P } & 14 & 6.00 E+01 & 1 \\ \text { S } & 15 & 1.20 E+01 & 99 \\ \text { CL } & 16 & 3.65 E+05 & 3 \\ \text { AR } & 17 & 2.90 E+01 & 4 \\ \text { K } & 18 & 3.65 E+05 & 3 \\ \text { CA } & 19 & 5.80 E+01 & 4 \\ \text { SC } & 20 & 3.70 E+01 & 99 \\ \text { TI } & 21 & 3.65 E+05 & 3 \\ \text { V } & 22 & 3.65 E+05 & 3 \\ \text { CR } & 23 & 3.65 E+05 & 3 \\ \text { MN } & 24 & 3.65 E+05 & 3 \\ \text { FE } & 25 & 1.00 E+00 & 99 \\ \text { CO } & 26 & 4.50 E+02 & 99 \\ \text { NI } & 27 & 2.40 E+01 & 99 \\ \text { CU } & 28 & 3.65 E+05 & 3 \\ \text { ZN } & 30 & 1.50 E+01 & 99 \\ \text { GA } & 31 & 3.07 E+02 & 1 \\ \text { GE } & 32 & 3.65 E+05 & 3 \\ \text { AS } & 33 & 3.65 E+05 & 3 \\ \text { SE } & 34 & 3.65 E+05 & 3 \\ \text { BR } & 35 & 8.00 E+00 & 4 \\ \text { KR } & 36 & 3.65 E+05 & 3 \\ \text { RB } & 37 & 8.00 E+01 & 4 \\ \text { SR } & 38 & 4.50 E+01 & 99 \\ \text { Y } & 39 & 3.65 E+05 & 3 \\ \text { ZR } & 40 & 3.65 E+05 & 3 \\ \text { NB } & 41 & 3.65 E+05 & 3 \\ \text { MD } & 42 & 1.70 E+01 & 99 \\ \text { TC } & 43 & 3.65 E+05 & 3 \\ \text { RU } & 44 & 7.00 E+01 & 99 \\ \text { RH } & 45 & 3.65 E+05 & 3 \\ \text { PD } & 46 & 3.65 E+05 & 3 \\ \text { AG } & 47 & 3.00 E+00 & 99 \\ \text { CD } & 48 & 6.50 E+01 & 99 \\ \text { IN } & 49 & 3.65 E+05 & 3 \\ \text { SN } & 50 & 4.50 E+01 & 99 \\ & & & \end{array}$

$\begin{array}{lrrr}\text { ELEMENT } & \text { ATOMIC } & \text { HALF-LIFE } \\ \text { SYMBOL } & \text { NO.12I } & \text { T } B(D A Y) & \text { CODE } \\ & & & \\ \text { SB } & 51 & 3.65 E+05 & 3 \\ \text { TE } & 52 & 3.00 E+01 & 99 \\ \text { I } & 53 & 3.65 E+05 & 3 \\ \text { XE } & 54 & 3.65 E+05 & 3 \\ \text { CS } & 55 & 1.10 E+02 & 4 \\ \text { BA } & 56 & 4.90 E+00 & 1 \\ \text { LA } & 57 & 3.65 E+05 & 3 \\ \text { CE } & 58 & 3.65 E+05 & 3 \\ \text { PR } & 59 & 3.65 E+05 & 3 \\ \text { ND } & 60 & 3.65 E+05 & 3 \\ \text { PM } & 61 & 3.65 E+05 & 3 \\ \text { SM } & 62 & 3.65 E+05 & 3 \\ \text { EU } & 63 & 3.65 E+05 & 3 \\ \text { GD } & 64 & 3.65 E+05 & 3 \\ \text { TB } & 65 & 3.65 E+05 & 3 \\ \text { DY } & 66 & 3.65 E+05 & 3 \\ \text { HO } & 67 & 3.65 E+05 & 3 \\ \text { ER } & 68 & 3.65 E+05 & 3 \\ \text { TM } & 69 & 3.65 E+05 & 3 \\ \text { YB } & 70 & 3.65 E+05 & 3 \\ \text { LU } & 71 & 3.65 E+05 & 3 \\ \text { HF } & 72 & 5.63 E+02 & 99 \\ \text { TA } & 73 & 4.60 E+02 & 99 \\ \text { W } & 74 & 3.65 E+05 & 3 \\ \text { RE } & 75 & 3.65 E+05 & 3 \\ \text { OS } & 76 & 3.65 E+05 & 3 \\ \text { IR } & 77 & 3.65 E+05 & 3 \\ \text { PT } & 78 & 3.65 E+05 & 3 \\ \text { AU } & 79 & 3.65 E+05 & 3 \\ \text { HG } & 80 & 3.65 E+05 & 3 \\ \text { TL } & 81 & 3.65 E+05 & 3 \\ \text { PB } & 82 & 3.65 E+05 & 3 \\ \text { BI } & 83 & 3.65 E+05 & 3 \\ \text { PD } & 84 & 3.65 E+05 & 3 \\ \text { AT } & 85 & 1.35 E+01 & 1 \\ \text { RN } & 86 & 3.65 E+05 & 3 \\ \text { FR } & 87 & 3.65 E+05 & 3 \\ \text { RA } & 88 & 3.65 E+05 & 3 \\ \text { AC } & 89 & 3.65 E+05 & 3 \\ \text { TH } & 90 & 3.65 E+05 & 3 \\ \text { PA } & 91 & 3.65 E+05 & 3 \\ \text { U } & 92 & 3.65 E+05 & 3 \\ \text { NP } & 93 & 3.65 E+05 & 3 \\ \text { PU } & 94 & 3.65 E+05 & 3 \\ \text { AM } & 95 & 3.65 E+05 & 3 \\ \text { CM } & 96 & 3.65 E+05 & 3 \\ \text { BK } & 97 & 3.65 E+05 & 3 \\ \text { CF } & 98 & 3.65 E+05 & 3 \\ \text { ES } & 99 & 3.65 E+05 & 3 \\ \text { FM } & 100 & 3.65 E+05 & 3 \\ & & & \end{array}$




$\begin{array}{lrrr}\text { ELEMENT } & \text { ATOMIC } & \text { HALF-LIFE } & \\ \text { SYMBOL } & \text { NO. (Z) } & \text { T } B \text { (DAY) } & \text { CODE } \\ \text { H } & & & \\ \text { HE } & 1 & 1.20 E+01 & 4 \\ \text { LI } & 2 & 3.65 E+05 & 3 \\ \text { BE } & 3 & 3.65 E+05 & 3 \\ \text { B } & 4 & 5.40 E+02 & 1 \\ \text { C } & 5 & 3.65 E+05 & 3 \\ \text { N } & 6 & 1.00 E+01 & 4 \\ \text { O } & 7 & 9.00 E+01 & 4 \\ \text { F } & 8 & 1.40 E+01 & 4 \\ \text { NE } & 9 & 3.65 E+05 & 3 \\ \text { NA } & 10 & 3.65 E+05 & 3 \\ \text { MG } & 11 & 1.10 E+01 & 4 \\ \text { AL } & 12 & 3.65 E+05 & 3 \\ \text { SI } & 13 & 5.00 E+02 & 1 \\ \text { P } & 14 & 3.65 E+05 & 3 \\ \text { S } & 15 & 2.50 E+01 & 99 \\ \text { CL } & 16 & 3.65 E+05 & 3 \\ \text { AR } & 17 & 2.90 E+01 & 4 \\ \text { K } & 18 & 3.65 E+05 & 3 \\ \text { CA } & 19 & 5.80 E+01 & 1 \\ \text { SC } & 20 & 9.00 E+00 & 99 \\ \text { TI } & 21 & 1.43 E+03 & 99 \\ \text { V } & 22 & 3.20 E+02 & 1 \\ \text { CR } & 23 & 9.00 E+01 & 1 \\ \text { MN } & 24 & 3.65 E+05 & 3 \\ \text { FE } & 25 & 1.60 E+01 & 99 \\ \text { CO } & 26 & 6.00 E+02 & 1 \\ \text { NI } & 27 & 2.40 E+01 & 99 \\ \text { CU } & 28 & 3.65 E+05 & 3 \\ \text { ZN } & 29 & 1.20 E+01 & 99 \\ \text { GA } & 30 & 3.65 E+05 & 3 \\ \text { GE } & 31 & 6.00 E+00 & 1 \\ \text { AS } & 33 & 3.65 E+05 & 3 \\ \text { SE } & 34 & 3.65 E+05 & 3 \\ \text { BR } & 35 & 8.00 E+01 & 1 \\ \text { KR } & 36 & 3.65 E+00 & 4 \\ \text { RB } & 37 & 8.00 E+01 & 3 \\ \text { SR } & 38 & 1.20 E+01 & 99 \\ \text { Y } & 39 & 1.00 E+04 & 99 \\ \text { ZR } & 40 & 9.00 E+02 & 1 \\ \text { NB } & 41 & 9.50 E+02 & 1 \\ \text { MO } & 42 & 8.00 E+00 & 99 \\ \text { TC } & 43 & 3.65 E+05 & 3 \\ \text { RU } & 44 & 1.60 E+02 & 99 \\ \text { RH } & 45 & 2.08 E+01 & 1 \\ \text { PD } & 46 & 1.50 E+01 & 1 \\ \text { AG } & 47 & 2.20 E+01 & 99 \\ \text { CD } & 48 & 2.20 E+02 & 99 \\ \text { IN } & 49 & 4.80 E+01 & 1 \\ \text { SN } & 50 & 8.80 E+02 & 99\end{array}$

$\begin{array}{ll}\text { ELEMENT } & \text { ATOMIC } \\ \text { SYMBOL } & \text { NO. }(Z)\end{array}$

HALF-LI FE

B (DAY) CODE

REFERENEE(S)

3. $65 E+05$

3. 0 OE +01

7. $00 E+00$

$3.65 E+05$

$1.10 E+02$

$1.30 E+01$

$3.65 E+05$

$8.45 E+02$

$3.65 E+05$

$3.65 E+05$

7. $30 E+02$

$7.20 E+02$

4. $45 E+02$

$3.85 E+02$

$3.40 E+02$

$3.65 E+05$

$3.65 E+05$

$3.65 E+05$

4. $10 E+02$

$3.65 E+05$

$3.65 E+05$

$3.50 E+02$

2. $40 E+02$

$3.65 E+05$

$3.65 E+05$

$3.65 E+0.5$

$5.00 E+01$

$6.00 E+01$

$2.40 E+02$

$.00 E+01$

$2.50 E+01$

$3.65 E+05$

1. $00 E+01$

6.00E+01

$2.70 E+01$

$3.65 E+05$

$3.65 E+05$

$3.65 E+05$

$3.65 E+05$

$3.65 E+05$

$3.65 E+05$

$3.65 E+05$

$3.65 E+05$

$3.65 E+05$

$3.65 E+05$

$3.65 E+05$

$3.65 \mathrm{E}+05$

$3.65 E+05$
$3.65 E+0.5$

$3.65 E+05$

$3.65 E+05$ 
HALF-LIFE

$T_{B}$ (DAY) CODE REFERENCE (S)

$\begin{array}{rr}1.20 E+01 & 4 \\ 3.65 E+05 & 3 \\ 2.00 E+00 & 1 \\ 3.65 E+05 & 3 \\ 3.65 E+05 & 3 \\ 1.00 E+01 & 4 \\ 9.00 E+01 & 4 \\ 1.40 E+01 & 4 \\ 3.65 E+05 & 3 \\ 3.65 E+05 & 3 \\ 1.10 E+01 & 4 \\ 3.65 E+05 & 3 \\ 3.65 E+05 & 3 \\ 6.00 E+01 & 1 \\ 1.80 E+01 & 99 \\ 6.23 E+02 & 1 \\ 2.90 E+01 & 4 \\ 3.65 E+05 & 3 \\ 5.80 E+01 & 4 \\ 1.70 E+01 & 99 \\ 3.65 E+05 & 3 \\ 3.65 E+05 & 3 \\ 3.65 E+05 & 3 \\ 3.65 E+05 & 3 \\ 2.60 E+01 & 99 \\ 2.40 E+02 & 99 \\ 2.40 E+01 & 99 \\ 3.65 E+05 & 3 \\ 1.00 E+01 & 99 \\ 2.70 E+02 & 1 \\ 3.65 E+05 & 3 \\ 3.65 E+05 & 3 \\ 3.65 E+05 & 3 \\ 3.65 E+05 & 3 \\ 8.00 E+00 & 4 \\ 3.65 E+05 & 3 \\ 8.00 E+01 & 4 \\ 2.00 E+01 & 99 \\ 8.40 E+03 & 99 \\ 8.00 E+02 & 99 \\ 3.65 E+05 & 3 \\ 1.80 E+01 & 99 \\ 3.65 E+05 & 3 \\ 1.80 E+02 & 99 \\ 3.65 E+05 & 3 \\ 3.65 E+05 & 3 \\ 7.00 E+00 & 99 \\ 3.65 E+05 & 3 \\ 3.65 E+05 & 3 \\ 6.00 E+01 & 99 \\ & \end{array}$

$\begin{array}{lrrr}\text { ELEMENT } & \text { ATOMIC } & \text { HALF-LI FE } & \\ \text { SYMBOL } & \text { ND. }(2) & \text { TB }(D A Y) & \text { CODE } \\ \text { SB } & & & \\ \text { TE } & 51 & 3.65 E+05 & 3 \\ \text { I } & 52 & 3.00 E+01 & 1 \\ \text { XE } & 53 & 7.00 E+00 & 1 \\ \text { CS } & 54 & 3.65 E+05 & 3 \\ \text { BA } & 55 & 1.10 E+02 & 4 \\ \text { LA } & 56 & 6.20 E+00 & 1 \\ \text { CE } & 57 & 3.65 E+05 & 3 \\ \text { PR } & 58 & 3.40 E+02 & 99 \\ \text { ND } & 59 & 3.65 E+05 & 3 \\ \text { PM } & 60 & 3.65 E+05 & 3 \\ \text { SM } & 61 & 3.95 E+02 & 99 \\ \text { EU } & 62 & 3.95 E+02 & 99 \\ \text { GD } & 63 & 3.80 E+02 & 99 \\ \text { TB } & 64 & 3.30 E+02 & 99 \\ \text { DY } & 65 & 4.00 E+02 & 99 \\ \text { HO } & 66 & 3.65 E+05 & 3 \\ \text { ER } & 67 & 3.65 E+05 & 3 \\ \text { TM } & 68 & 3.65 E+05 & 3 \\ \text { YB } & 69 & 4.00 E+02 & 99 \\ \text { LU } & 70 & 4.10 E+02 & 99 \\ \text { HF } & 71 & 3.65 E+05 & 3 \\ \text { TA } & 72 & 5.63 E+02 & 99 \\ \text { W } & 73 & 4.60 E+02 & 99 \\ \text { RE } & 74 & 3.65 E+05 & 3 \\ \text { OS } & 75 & 3.65 E+05 & 3 \\ \text { IR } & 76 & 3.65 E+05 & 3 \\ \text { PT } & 77 & 3.65 E+05 & 3 \\ \text { AU } & 78 & 3.65 E+05 & 3 \\ \text { HG } & 89 & 3.65 E+05 & 3 \\ \text { TL } & 81 & 3.65 E+05 & 3 \\ \text { PB } & 82 & 3.65 E+05 & 3 \\ \text { BI } & 83 & 3.65 E+05 & 3 \\ \text { PO } & 84 & 3.65 E+05 & 3 \\ \text { AT } & 85 & 3.65 E+05 & 3 \\ \text { RN } & 86 & 3.65 E+05 & 3 \\ \text { FR } & 87 & 3.65 E+05 & 3 \\ \text { RA } & 88 & 3.65 E+05 & 3 \\ \text { AC } & 89 & 3.65 E+05 & 3 \\ \text { TH } & 90 & 3.65 E+05 & 3 \\ \text { PA } & 91 & 3.65 E+05 & 3 \\ \text { U } & 92 & 3.65 E+05 & 3 \\ \text { NP } & 93 & 3.65 E+05 & 3 \\ \text { PU } & 94 & 3.65 E+05 & 3 \\ \text { AM } & 95 & 3.65 E+05 & 3 \\ \text { CM } & 96 & 3.65 E+05 & 3 \\ \text { BK } & 97 & 3.65 E+05 & 3 \\ \text { CF } & 98 & 3.65 E+05 & 3 \\ \text { ES } & 99 & 3.65 E+05 & 3 \\ \text { FM } & 100 & 3.65 E+05 & 3 \\ & & & \end{array}$

REFERENCE (5)

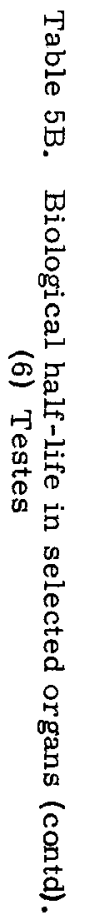


ELEMENT ATOMIC HALF-LIFE

SYMBOL NO.IZ) TB (DAY) CODE REFERENCE(S)

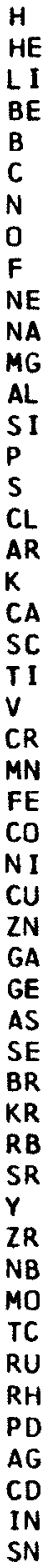

1. $20 E+01$ 3. $65 E+05$ 2. $00 E+00$ 1. $80 E+02$

5. OOE-O1

1. $00 E+01$

$9.00 E+01$

1. $40 E+01$

B. $08 E+02$

3. $65 E+05$

$1.10 E+01$

1. $80 E+02$

$5.50 E+02$

$6.00 E+01$

$2.57 E+02$

9. $00 E+01$

2. $90 \mathrm{E}+01$

$3.65 E+05$

$5.80 E+01$

$1.64 E+04$

$1.43 E+03$

$3.20 \mathrm{E}+02$

4. $20 E+01$

$6.16 E+02$

3. $60 E+01$

$8.00 E+02$

$2.40 E+01$

$6.67 E+02$

2. $50 E+02$

9. $33 E+02$

$6.00 E+00$

1. $00 E+00$

2. $80 E+02$

1. $10 E+01$

8. $00 E+00$

$3.65 E+05$

$8.00 E+01$

I. $30 E+04$

$1.40 E+04$

4. $50 E+02$

7. $60 E+02$

1. $00 E+02$

3. $0 O E+00$

1. $30 E+02$

1. $04 E+01$

5. $00 E+00$

5. $20 E+01$

2. $00 E+02$

4. $80 E+01$
$2.0 O E+02$

2. $00 E+02$
ELEMENT

SYMBOL NO. (Z)

$S$

TE

XE

CS

BA

CE

PR

$4419 \quad 5417$

2669

5427

4419

2932
HAL F-L IFE

$T_{B}$ (DAY) CODE REFERENCE(S)

$3.80 E+01$

1. $50 E+01$

1. $38 E+02$

$3.65 E+05$

1. $10 E+02$

6. $50 E+01$

$5.00 E+02$

$5.63 E+02$

$7.50 E+02$

6. $6 E+02$

.

(2)

$6.35 E+02$

5. $50 \mathrm{E}+02$

$6.70 E+02$

$7.00 E+02$

$7.50 E+02$

$6.50 E+02$

$6.75 E+02$

7.50E+02

$5.63 E+02$

$2.4 O E+02$

$1.00 \mathrm{E}+00$

$7.00 E+00$

$2.00 E+00$

2. $00 E+01$

$2.40 \mathrm{E}+01$

1. $20 E+02$

1. $00 E+01$

$1.00 E+01$
$2.50 E+01$

$2.50 E+01$

$1.46 E+03$

$5.00 \mathrm{E}+00$

$3.00 E+01$

2.7OE+01

$3.65 E+05$

$7.00 E+01$

$8.10 E+03$

$2.40 E+04$

$5.70 E+04$

4. $10 E+04$

$1.0 O E+02$

3. $90 E+04$

$6.50 E+04$

. 00 E 04

$2.00+04$

$2.40 E+04$

$6.50 E+04$

$6.50 E+04$

6. $50 E+04$

6. $50 E+04$

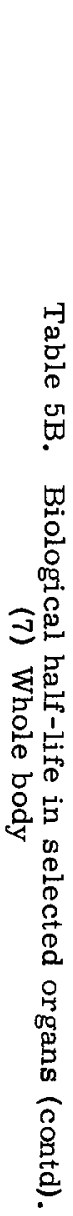




\begin{tabular}{|c|c|c|c|}
\hline $\begin{array}{l}\text { ELEMENT } \\
\text { SYMBOL }\end{array}$ & $\begin{array}{l}\text { ATOMIC } \\
\text { ND. }(Z)\end{array}$ & $\begin{array}{l}\text { HALF-LIFE } \\
T_{B}(D A Y)\end{array}$ & CODE \\
\hline H & 1 & $1.50 E+00$ & 6 \\
\hline HE & 2 & $1.50 E+00$ & 6 \\
\hline LI & 3 & $1.50 E+00$ & 6 \\
\hline $\mathrm{BE}$ & 4 & $1.50 E+00$ & 6 \\
\hline B & 5 & $1.50 E+00$ & 6 \\
\hline c & 6 & 1. $50 E+00$ & 6 \\
\hline$N$ & 7 & $1.50 \mathrm{E}+00$ & 6 \\
\hline 0 & 8 & $1.50 E+00$ & 6 \\
\hline $\mathbf{F}$ & 9 & $1.50 E+00$ & 6 \\
\hline VE & 10 & $1.50 E+00$ & 6 \\
\hline NA & 11 & $1.50 E+00$ & 6 \\
\hline MG & 12 & $1.50 E+00$ & 6 \\
\hline AL & 13 & $1.50 E+00$ & 6 \\
\hline SI & 14 & 1. $50 E+00$ & 6 \\
\hline $\mathbf{P}$ & 15 & $1.50 E+00$ & 6 \\
\hline$S$ & 16 & $1.50 E+00$ & 6 \\
\hline $\mathrm{CL}$ & 17 & $1.50 E+00$ & 6 \\
\hline$A R$ & 18 & 1. $50 E+00$ & 6 \\
\hline$k$ & 19 & $1.50 E+00$ & 6 \\
\hline CA & 20 & $1.50 E+00$ & 6 \\
\hline SC & 21 & $1.50 E+00$ & 6 \\
\hline TI & 22 & $1.50 E+00$ & 6 \\
\hline$v$ & 23 & $1.50 E+00$ & 6 \\
\hline CR & 24 & $1.50 E+00$ & 6 \\
\hline MN & 25 & $1.50 E+00$ & 6 \\
\hline $\begin{array}{l}\text { FE } \\
\text { CO }\end{array}$ & $\begin{array}{l}26 \\
27\end{array}$ & 1. $50 E+00$ & 6 \\
\hline $\begin{array}{l}\text { CO } \\
\text { NI }\end{array}$ & $\begin{array}{l}27 \\
28\end{array}$ & $\begin{array}{l}1.50 E+00 \\
1.50 E+00\end{array}$ & $\begin{array}{l}6 \\
6\end{array}$ \\
\hline $\mathrm{CU}$ & 29 & $1.50 E+00$ & 6 \\
\hline$Z N$ & 30 & $1.50 E+00$ & 6 \\
\hline GA & 31 & $1.50 E+00$ & 6 \\
\hline GE & 32 & $1.50 E+00$ & 6 \\
\hline AS & 33 & $1.50 E+00$ & 6 \\
\hline SE & 34 & $1.50 E+00$ & 6 \\
\hline BR & 35 & $1.50 E+00$ & 6 \\
\hline KR & 36 & $1.50 E+00$ & 6 \\
\hline RB & 37 & 1. $50 E+00$ & 6 \\
\hline$S R$ & 38 & $1.50 E+00$ & 6 \\
\hline$Y$ & 39 & $1.50 E+00$ & 6 \\
\hline $2 R$ & 40 & $1.50 E+00$ & 6 \\
\hline NB & 41 & 1. $50 E+00$ & 6 \\
\hline MO & 42 & $1.50 E+00$ & 6 \\
\hline TC & 43 & $1.50 E+00$ & 6 \\
\hline RU & 44 & $1.50 E+00$ & 6 \\
\hline RH & 45 & $1.50 E+00$ & 6 \\
\hline PD & 46 & $1.50 E+00$ & 6 \\
\hline AG & 47 & $1.50 E+00$ & 6 \\
\hline CD & 48 & $1.50 E+00$ & 6 \\
\hline IN & 49 & $50 E+00$ & 6 \\
\hline SN & 50 & $5 O E+O O$ & 6 \\
\hline
\end{tabular}

REFERENCE(S)

6

6

6

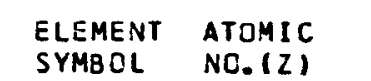

LLF-LIFE

SYMBOL NO.(Z)

B (DAY)

CODE

REFERENCE (S)

$\begin{array}{ll}\text { SB } & 51 \\ \text { TE } & 52 \\ \text { I } & 53 \\ \text { XE } & 54 \\ \text { CS } & 55 \\ \text { BA } & 56 \\ \text { LA } & 57 \\ \text { CE } & 58 \\ \text { PR } & 59 \\ \text { ND } & 60 \\ \text { PM } & 61 \\ \text { SM } & 62 \\ \text { EU } & 63 \\ \text { GD } & 64 \\ \text { TB } & 65 \\ \text { DY } & 66 \\ \text { HO } & 67 \\ \text { ER } & 68 \\ \text { TM } & 69 \\ \text { YB } & 70 \\ \text { LJ } & 71 \\ \text { HF } & 72 \\ \text { TA } & 73 \\ \text { W } & 74 \\ \text { RE } & 75 \\ \text { OS } & 76 \\ \text { IR } & 77 \\ \text { PT } & 78 \\ \text { AU } & 79 \\ \text { HG } & 80 \\ \text { IL } & 81 \\ \text { PB } & 82 \\ \text { BI } & 93 \\ \text { PO } & 94 \\ \text { AT } & 85 \\ \text { RN } & 86 \\ \text { FR } & 87 \\ \text { RA } & 88 \\ \text { AC } & 89 \\ \text { TH } & 90 \\ \text { PA } & 91 \\ \text { U } & 92 \\ \text { NP } & 93 \\ \text { PU } & 94 \\ \text { AM } & 95 \\ \text { CM } & 96 \\ \text { BK } & 97 \\ \text { CF } & 98 \\ \text { ES } & 99 \\ \text { FM } & 100 \\ & \end{array}$

$1.50 E+00$

1. $50 E+00$

$1.50 E+00$

$1.50 E+00$

1. $50 E+00$

$1.50 E+00$

$1.50 E+00$

$1.50 E+00$

$1.50 E+00$

$1.50 E+00$

$1.50 E+00$

$1.50 E+00$

$1.50 E+00$

1. $50 E+00$

$1.50 E+00$

$1.50 E+00$

$1.50 E+00$

1. $50 E+00$

$1.50 E+00$

$1.50 E+00$

$1.50 E+00$

$1.50 E+00$

$1.50 E+00$

$1.50 E+00$

$1.50 E+00$

1. $50 E+00$

$1.50 E+00$

$1.50 E+00$

$1.50=+00$

$1.50 E+00$

$1.50 E+00$

$1.50 E+00$

$1.50 E+00$

$1.50 E+00$

$1.50 E+D 0$

$1.50 E+00$

$1.50 E+00$

$1.50 E+00$

$1.50 \bar{E}+00$

$1.50 E+00$

$1.50 E+00$

$1.50 E+00$

$1.50 \mathrm{E}+00$

$1.50 E+00$

1. $50 E+00$

$1.50 E+00$

$1.50 E+00$

1. $50 E+00$

$1.50 E+00$

$1.50 E+00$

$$
\begin{aligned}
& 6 \\
& 6 \\
& 6 \\
& 6 \\
& 6 \\
& 6 \\
& 6 \\
& 6 \\
& 6 \\
& 6 \\
& 6 \\
& 6 \\
& 6 \\
& 6 \\
& 6 \\
& 6 \\
& 6 \\
& 6 \\
& 6 \\
& 6 \\
& 6 \\
& 6 \\
& 6 \\
& 6 \\
& 6 \\
& 6 \\
& 6 \\
& 6 \\
& 6 \\
& 6 \\
& 6 \\
& 6 \\
& 6 \\
& 6 \\
& 6 \\
& 6 \\
& 6 \\
& 6 \\
& 6 \\
& 6 \\
& 6 \\
& 6 \\
& 6 \\
& 6 \\
& 6 \\
& 6 \\
& 6 \\
& 6 \\
& 6 \\
& 6 \\
&
\end{aligned}
$$


-

○

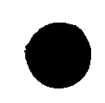




\section{UPTAKE AND DISTRIBUTION IN ORGANS (Continued)}

\section{$\mathrm{C}_{\mathrm{B}}$ : Concentration}

The values adopted for $C_{B}$, the concentration in an organ or tissue, are shown in Table $5 \mathrm{C}$. The $\mathrm{C}_{\mathrm{B}}$ in the GI tract was estimated as the concentration in a predominantly terrestrial diet. This value is meant to approximate the concentration in the GI tract contents and is used to estimate the unit-rad deposition to the GI tract via soil. It is also used to estimate the unit-rad contamination via aquatic environments as outlined in Section 9 (6354). The values were assigned according to the procedures and code numbers shown below.

\section{Rules for estimating $\mathrm{C}_{\mathrm{B}}$}

Code \#

1 The $\mathrm{C}_{B}$ was assumed to be the 90th percentile value of $U$. S. subjects (1798).

2 The $C_{B}$ was assumed to be greatest of the maxima in the literature.

3 The $C_{B}$ was assumed to be the maximum of the mean or median values in the literature.

4 The $C_{B}$ was assumed to be the ICRP value.

5 The $\mathrm{C}_{\mathrm{B}}$ was assumed to be 100 times the average soil concentration $\mathrm{C}_{\mathrm{S}}$.

6 The $\mathrm{C}_{\mathrm{B}}$ of $\mathrm{C}, \mathrm{N}, \mathrm{H}$ and $\mathrm{O}$ was assumed to be the $C_{B}^{T B}$.

7 The $C_{B}$ was assumed to be $C_{B}^{T B}$ times the ratio of total body to organ mass. (The total body con- tent was assumed to be concentrated in the organ.)

8 The $C_{B}^{T B}$ was estimated from data on "Elemental Composition of Total Body of Standard Man" (5280).

9 The $\mathrm{C}_{\mathrm{B}}$ in the daily diet was estimated from the daily intake I assuming an intake of $1.3 \mathrm{~kg} /$ day. The daily intake I was calculated by Eq. (12) in the Appendix using the $\mathrm{C}_{\mathrm{P}}$ from Table $10 \mathrm{~A}$ and the $\mathrm{C}_{\text {meat }}$ from Table 10B.

10 The $\mathrm{C}_{B}$ in the daily diet was estimated from the daily intake I assuming a total intake of $1.3 \mathrm{~kg} /$ day. The daily intake I was assumed to be the ICRP estimate.

11 The $C_{B}$ in the daily diet was estimated from the daily intake $I$ assuming a total intake of $1.3 \mathrm{~kg} /$ day. The daily intake I was estimated from values reported in the literature.

12 The $\mathrm{C}_{\mathrm{B}}$ in the daily diet was estimated from the daily intake I assuming a total intake of $1.3 \mathrm{~kg} /$ day. The daily intake I was based on values measured or estimated by H. A. Schroeder as referenced in the table.

13 The $\mathrm{C}_{\mathrm{B}}$ (of inert gases) was assumed to be $\mathrm{C}_{\mathrm{A}}$ for freshwater (Table $7 \mathrm{~B}$ ).

99 The $C_{B}$ was estimated from collateral data as described in the Appendix.

It may be noted that the $\mathrm{C}_{B}$ values adopted for Table $5 \mathrm{C}$ include maximum and near-maximum values, in accordance with our stated objectives. 
CONC NO. (Z) $C_{B}$ (PPM) CODE REFERENCE (S)

$1.42 E+03$

$2 \cdot 60 \mathrm{E}+01$

$3.30 E+02$

8. $00 E+04$

$2.40 E+03$
$1.70 E+03$

1. $00 E+02$

$6.24 E+0$

9. $36 \mathrm{E}+04$

5. $00 E-02$

1. $00 E+00$

$1.50 E+00$

4. $80 E+00$

$2.40 E+00$

6. $00 E+02$

5. $00 \mathrm{E}+00$

$1.66 E+01$

$8.40 E+00$

$1.08 E+02$

1. $00 E+00$

1. $00 E+02$

1. $00 E-02$

1. $00 E+01$

3. OOE-O

1. $00 E-02$

$1.00 E+01$

8. $60 E+01$

5. $00 E+00$

1. $00 E+00$

$5.30 E+02$

4. $80 \mathrm{E}-01$

$1.00 E-11$

5. $00 E-03$

$1.00 E-01$

$1.00 E-01$

1. $00 \mathrm{E}-01$

5. $00 \mathrm{E}-0$

$1.00 E+0$

$4.80 F+00$
3868

3868

3868

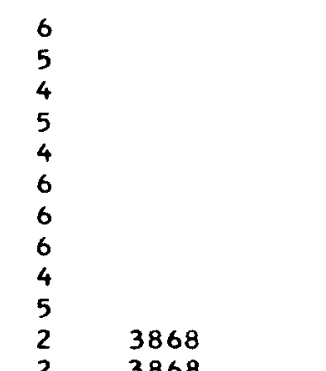

3868

3868

3868

3868

3868

3868

3868

3868

3868

3868

3868

$\begin{array}{ll}\text { ELEMENT } & \text { ATOMIC } \\ \text { SYMBOL } & \text { NO. }(Z)\end{array}$

CONC.

SYMBOL NO. (Z) $C_{B}$ (PPM) CODE REFERENLE(S)

$\begin{array}{lrrr}\text { SB } & 51 & 2.00 E-01 & 4 \\ \text { TE } & 52 & 5.00 E-03 & 99 \\ \text { I } & 53 & 3.00 E-01 & 4 \\ \text { XE } & 54 & 1.00 E-03 & 5 \\ \text { CS } & 55 & 6.00 E+00 & 4 \\ \text { BA } & 56 & 1.60 E+00 & 4 \\ \text { LA } & 57 & 4.00 E+03 & 5 \\ \text { CE } & 58 & 5.00 E-01 & 99 \\ \text { PR } & 59 & 6.70 E+02 & 5 \\ \text { ND } & 60 & 2.50 E+03 & 5 \\ \text { PM } & 61 & 5.00 E-21 & 99 \\ \text { SM } & 62 & 5.00 E-02 & 99 \\ \text { EU } & 63 & 5.00 E-03 & 99 \\ \text { GD } & 64 & 5.00 E-02 & 99 \\ \text { TB } & 65 & 5.00 E-03 & 99 \\ \text { OY } & 66 & 3.00 E+02 & 5 \\ \text { HD } & 67 & 7.00 E+01 & 5 \\ \text { ER } & 68 & 8.00 E+01 & 5 \\ \text { TM } & 69 & 5.00 E-03 & 99 \\ \text { YB } & 70 & 5.00 E-03 & 99 \\ \text { LU } & 71 & 7.00 E+01 & 5 \\ \text { HF } & 72 & 5.00 E-01 & 99 \\ \text { TA } & 73 & 1.00 E+00 & 99 \\ \text { W } & 74 & 1.00 E+02 & 5 \\ \text { RE } & 75 & 1.00 E-01 & 5 \\ \text { OS } & 76 & 1.00 E-01 & 5 \\ \text { IR } & 77 & 1.00 E-03 & 5 \\ \text { PT } & 78 & 1.00 \mathrm{~F}+00 & 5 \\ \text { AU } & 79 & 2.50 E+00 & 4 \\ \text { HG } & 80 & 1.00 E+00 & 5 \\ \text { TL } & 81 & 1.00 E-01 & 99 \\ \text { PB } & 82 & 3.36 F+01 & 2 \\ \text { BI } & 83 & 1.00 E+01 & 5 \\ \text { PU } & 84 & 1.00 E-09 & 5 \\ \text { AT } & 85 & 1.00 E-17 & 5 \\ \text { RN } & 86 & 1.00 E-11 & 5 \\ \text { FR } & 87 & 1.00 E-16 & 5 \\ \text { RA } & 88 & 1.70 E-08 & 4 \\ \text { AC } & 89 & 1.00 E-08 & 5 \\ \text { TH } & 90 & 6.00 E+02 & 5 \\ \text { PA } & 91 & 1.00 E-05 & 5 \\ \text { U } & 92 & 1.00 E-03 & 4 \\ \text { NP } & 93 & 1.00 E-10 & 5 \\ \text { PU } & 94 & 1.00 F-11 & 5 \\ \text { AM } & 95 & 1.00 E-28 & 5 \\ \text { CM } & 96 & 1.00 E-2.8 & 5 \\ \text { BK } & 97 & 1.00 E-28 & 5 \\ \text { CF } & 98 & 1.00 E-28 & 5 \\ \text { ES } & 99 & 1.00 F-28 & 5 \\ \text { FM } & 100 & 1.00 E-28 & 5\end{array}$




$\begin{array}{lrrrr}\text { ELEMENT } & \text { ATOMIC } & \text { CONC } & & \\ \text { SYMBOL } & \text { NO. }(2) & \text { C B (PPM) } & \text { CODE } & \text { REFEREND } \\ \text { H } & & & & \\ \text { HE } & 1 & 1.00 E+05 & 6 & \\ \text { LI } & 2 & 1.00 E-02 & 5 & \\ \text { BE } & 3 & 8.00 E-03 & 4 & \\ \text { B } & 4 & 3.00 E-02 & 4 & \\ \text { C } & 5 & 1.10 E-01 & 1 & 1798 \\ \text { N } & 6 & 2.40 E+05 & 6 & \\ \text { O } & 7 & 2.90 E+04 & 6 & \\ \text { F } & 8 & 6.00 E+05 & 6 & \\ \text { NE } & 9 & 8.00 E-01 & 4 & \\ \text { NA } & 10 & 1.00 E-01 & 5 & \\ \text { MG } & 11 & 2.20 E+03 & 4 & \\ \text { AL } & 12 & 1.90 E+02 & 1 & 1798 \\ \text { SI } & 13 & 9.00 E-01 & 1 & 1798 \\ \text { P } & 14 & 2.10 E+01 & 4 & \\ \text { S } & 15 & 2.00 E+03 & 1 & 1798 \\ \text { CL } & 16 & 1.00 E+04 & 99 & \\ \text { AR } & 17 & 2.10 E+03 & 4 & \\ \text { K } & 18 & 1.00 E+02 & 5 & \\ \text { CA } & 19 & 2.30 E+03 & 1 & 1798 \\ \text { SC } & 20 & 1.80 E+02 & 1 & 1798 \\ \text { TI } & 21 & 1.00 E-01 & 99 & \\ \text { V } & 22 & 5.50 E-02 & 1 & 1798 \\ \text { CR } & 23 & 8.00 E-02 & 2 & 1774 \\ \text { MN } & 24 & 5.00 E-02 & 1 & 1798 \\ \text { FE } & 25 & 1.70 E+00 & 1 & 1798 \\ \text { CO } & 26 & 1.20 E+02 & 1 & 1798 \\ \text { NI } & 27 & 2.20 E-02 & 1 & 1798 \\ \text { CU } & 28 & 1.20 E-01 & 1 & 1798 \\ \text { ZN } & 29 & 3.74 E+00 & 1 & 1798 \\ \text { GA } & 30 & 8.10 E+01 & 1 & 1798 \\ \text { GE } & 31 & 1.00 E-02 & 4 & \\ \text { AS } & 32 & 1.00 E+02 & 5 & \\ \text { SE } & 33 & 1.00 E-02 & 3 & 893 \\ \text { BR } & 34 & 1.80 E-01 & 3 & 893 \\ \text { KR } & 35 & 3.00 E+00 & 3 & 893 \\ \text { RB } & 36 & 1.00 E-02 & 5 & \\ \text { SR } & 37 & 1.40 E+00 & 3 & 893 \\ \text { Y } & 38 & 1.20 E-01 & 1 & 1798 \\ \text { ZR } & 39 & 5.00 E+00 & 99 & \\ \text { NB } & 40 & 1.00 E+00 & 4 & \\ \text { MO } & 41 & 1.00 E+00 & 4 & \\ \text { TC } & 42 & 5.30 E-01 & 1 & 1798 \\ \text { RU } & 43 & 1.00 E-11 & 5 & \\ \text { RH } & 44 & 5.00 E-02 & 99 & \\ \text { PD } & 45 & 1.00 E-01 & 5 & \\ \text { AG } & 46 & 1.00 E-01 & 5 & \\ \text { CD } & 48 & 1.10 E-02 & 1 & 1798 \\ \text { IN } & 49 & 1.00 E+01 & 1 & 1798 \\ \text { SN } & 50 & 5.70 E-01 & 5 & \\ & & & 1 & 1798\end{array}$

$\begin{array}{lrrr}\text { ELEMENT } & \text { ATOMIC } & \text { CONC. } \\ \text { SYMBOL } & \text { NO. (2) } & \text { C } \text { B (PPM) } & \text { CODE } \\ \text { SB } & & & \\ \text { TE } & 51 & 7.00 E-03 & 3 \\ \text { I } & 52 & 1.00 E-01 & 99 \\ \text { XE } & 53 & 2.00 E-01 & 4 \\ \text { CS } & 54 & 1.00 E-03 & 5 \\ \text { BA } & 55 & 1.80 E-02 & 3 \\ \text { LA } & 56 & 4.40 E-02 & 1 \\ \text { CE } & 57 & 7.00 E-05 & 3 \\ \text { PR } & 58 & 1.00 E-01 & 99 \\ \text { ND } & 59 & 6.70 E+02 & 5 \\ \text { PM } & 60 & 2.50 E+03 & 5 \\ \text { SM } & 61 & 5.00 E-21 & 99 \\ \text { EU } & 62 & 1.00 E-02 & 99 \\ \text { GD } & 63 & 1.00 E-02 & 99 \\ \text { TB } & 64 & 5.00 E-02 & 99 \\ \text { DY } & 65 & 5.00 E-03 & 99 \\ \text { HO } & 66 & 3.00 E+02 & 5 \\ \text { ER } & 67 & 7.00 E+01 & 5 \\ \text { TM } & 68 & 8.00 E+01 & 5 \\ \text { YB } & 69 & 1.00 E-03 & 99 \\ \text { LU } & 70 & 5.00 E-03 & 99 \\ \text { HF } & 71 & 7.00 E+01 & 5 \\ \text { TA } & 72 & 5.00 E-01 & 99 \\ \text { W } & 73 & 5.00 E+00 & 99 \\ \text { RE } & 74 & 1.00 E+02 & 5 \\ \text { OS } & 75 & 1.00 E-01 & 5 \\ \text { IR } & 76 & 1.00 E-01 & 5 \\ \text { PT } & 77 & 1.00 E-03 & 5 \\ \text { AU } & 78 & 1.00 E+00 & 5 \\ \text { HG } & 79 & 1.10 E-01 & 1 \\ \text { TL } & 80 & 4.00 E-02 & 3 \\ \text { PB } & 81 & 1.00 E-01 & 4 \\ \text { B1 } & 82 & 2.40 E+00 & 1 \\ \text { PO } & 84 & 4.70 E-01 & 1 \\ \text { AT } & 85 & 1.00 E-09 & 5 \\ \text { RN } & 86 & 1.00 E-17 & 5 \\ \text { FR } & 87 & 1.00 E-11 & 5 \\ \text { RA } & 88 & 8.00 E-05 & 5 \\ \text { AC } & 89 & 1.00 E-08 & 5 \\ \text { TH } & 90 & 6.00 E+02 & 5 \\ \text { PA } & 91 & 1.00 E-05 & 5 \\ \text { U } & 92 & 1.00 E+02 & 5 \\ \text { NP } & 93 & 1.00 E-10 & 5 \\ \text { PU } & 94 & 1.00 E-11 & 5 \\ \text { AM } & 95 & 1.00 E-28 & 5 \\ \text { CM } & 96 & 1.00 E-28 & 5 \\ \text { BK } & 97 & 1.00 E-28 & 5 \\ \text { CF } & 98 & 1.00 E-28 & 5 \\ \text { ES } & 99 & 1.00 E-28 & 5 \\ \text { FM } & 100 & 1.00 E-28 & 5 \\ & & & \end{array}$

REFERENCE (S)

893

893

1798
893 


\begin{tabular}{|c|c|c|c|c|}
\hline $\begin{array}{l}\text { EL EMENT } \\
\text { SYMBDL }\end{array}$ & $\begin{array}{l}\text { ATOMIC } \\
\text { NO. }(Z)\end{array}$ & $\begin{array}{l}C O N C \\
C_{B}(P P M)\end{array}$ & CODE & REFERE \\
\hline $\mathrm{H}$ & 1 & $1.00 E+05$ & 6 & \\
\hline $\mathrm{HE}$ & 2 & $1.00 E-02$ & 5 & \\
\hline LI & 3 & $6.00 E-03$ & 4 & \\
\hline $\mathrm{BE}$ & 4 & $3.00 E-02$ & 4 & \\
\hline B & 5 & $1.30 E-01$ & 1 & 1798 \\
\hline c & 6 & $2.40 E+05$ & 6 & \\
\hline $\mathbf{N}$ & 7 & $2.90 E+04$ & 6 & \\
\hline 0 & 8 & $6.00 E+05$ & 6 & \\
\hline$F$ & 9 & 2. $00 E+04$ & 5 & \\
\hline NE & 10 & 1. $00 \mathrm{E}-01$ & 5 & \\
\hline NA & 11 & $1.40 E+03$ & 4 & \\
\hline MG & 12 & $2.30 E+02$ & 1 & 1798 \\
\hline$A L$ & 13 & $1.60 E+00$ & I & 1798 \\
\hline SI & 14 & $2.40 E+01$ & 4 & \\
\hline $\mathbf{p}$ & 15 & $3.30 E+03$ & 1 & 1798 \\
\hline 5 & 16 & $1.00 E+04$ & 99 & \\
\hline $\mathrm{CL}$ & 17 & $1.25 E+03$ & 4 & \\
\hline $\mathbf{A R}$ & 18 & $1.00 E+02$ & 5 & \\
\hline$k$ & 19 & $3.30 E+03$ & 1 & 1798 \\
\hline CA & 20 & $9.10 E+01$ & 1 & 1798 \\
\hline SC & 21 & $5.00 E-02$ & 99 & \\
\hline T I & 22 & $2.10 E-01$ & 1 & 1798 \\
\hline v & 23 & $7.00 E-02$ & 2 & 1774 \\
\hline CR & 24 & $4.80 E-02$ & 1 & 1798 \\
\hline$M N$ & 25 & 2. $90 E+00$ & 1 & 1798 \\
\hline $\mathrm{FE}$ & 26 & $3.10 E+02$ & 1 & 1798 \\
\hline $\mathrm{CO}$ & 27 & $1.30 E-01$ & 1 & 1798 \\
\hline N I & 28 & $1.40 E-01$ & 1 & 1798 \\
\hline$C U$ & 29 & $1.69 E+01$ & 1 & 1798 \\
\hline $2 N$ & 30 & $7.70 E+01$ & 1 & 1798 \\
\hline GA & 31 & $1.10 E-02$ & 4 & \\
\hline GE & 32 & $1.00 E+02$ & 5 & \\
\hline AS & 33 & $1.60 E-02$ & 2 & 893 \\
\hline SE & 34 & $3.10 E-01$ & 2 & 893 \\
\hline$B R$ & 35 & $4.40 E+00$ & 2 & 893 \\
\hline KR & 36 & 1. $00 E-02$ & 5 & \\
\hline RB & 37 & 3. $70 E+01$ & 2 & 2805 \\
\hline$S R$ & 38 & $4.60 E-02$ & 1 & 1798 \\
\hline$Y$ & 39 & $1.00 E+00$ & 99 & \\
\hline ZR & 40 & 1. $00 E+00$ & 4 & \\
\hline NB & 41 & $1.10 E+00$ & 4 & \\
\hline MO & 42 & $1.82 E+01$ & 1 & 1798 \\
\hline TC & 43 & $1.00 E-11$ & 5 & \\
\hline RU & 44 & $5.00 E-03$ & 99 & \\
\hline RH & 45 & $1.00 E-01$ & 5 & \\
\hline PD & 46 & $1.00 E-01$ & 5 & \\
\hline$A G$ & 47 & $4.20 E-02$ & 1 & 1798 \\
\hline$C D$ & 48 & $4.40 E+00$ & 1 & 1798 \\
\hline IN & 49 & $1.0 O E+01$ & 5 & \\
\hline SN & 50 & $9.60 E-01$ & 1 & 1798 \\
\hline
\end{tabular}

$\begin{array}{crrr}\text { ELEMENT } & \text { ATOMIC } & \text { CONC } \\ \text { SYMBOL } & \text { NO. (Z) } & \text { CB (PPM) } & \text { CODE } \\ \text { SB } & 51 & 1.80 E-02 & 2 \\ \text { TE } & 52 & 1.00 E-02 & 99 \\ \text { I } & 53 & 1.10 E+00 & 4 \\ \text { XE } & 54 & 1.00 E-03 & 5 \\ \text { CS } & 55 & 1.20 E-02 & 2 \\ \text { BA } & 56 & 2.70 E-02 & 1 \\ \text { LA } & 57 & 1.50 E-03 & 2 \\ \text { CE } & 58 & 1.00 E-01 & 99 \\ \text { PR } & 59 & 6.70 E+02 & 5 \\ \text { ND } & 60 & 2.50 E+03 & 5 \\ \text { PM } & 61 & 1.00 E-21 & 99 \\ \text { SM } & 62 & 1.00 E-02 & 99 \\ \text { EU } & 63 & 1.00 E-03 & 99 \\ \text { GD } & 64 & 1.00 E-02 & 99 \\ \text { TB } & 65 & 5.00 E-04 & 99 \\ \text { DY } & 66 & 3.00 E+02 & 5 \\ \text { HO } & 67 & 7.00 E+01 & 5 \\ \text { ER } & 68 & 8.00 E+01 & 5 \\ \text { TM } & 69 & 5.00 E-05 & 99 \\ \text { YB } & 70 & 1.00 E-04 & 99 \\ \text { LU } & 71 & 7.00 E+01 & 5 \\ \text { HF } & 72 & 5.00 E+00 & 99 \\ \text { TA } & 73 & 1.00 E+01 & 99 \\ \text { W } & 74 & 1.00 E+02 & 5 \\ \text { RE } & 75 & 1.00 E-01 & 5 \\ \text { OS } & 76 & 1.00 E-01 & 5 \\ \text { IR } & 77 & 1.00 E-03 & 5 \\ \text { PT } & 78 & 1.00 E+00 & 5 \\ \text { AU } & 79 & 1.70 E-01 & 2 \\ \text { HG } & 80 & 3.00 E-02 & 2 \\ \text { TL } & 81 & 1.10 E-01 & 4 \\ \text { PB } & 82 & 3.30 E+00 & 1 \\ \text { BI } & 83 & 9.10 E-02 & 1 \\ \text { PO } & 84 & 1.00 E-09 & 5 \\ \text { AT } & 85 & 1.00 E-17 & 5 \\ \text { RN } & 86 & 1.00 E-11 & 5 \\ \text { FR } & 87 & 1.00 E-16 & 5 \\ \text { RA } & 88 & 8.00 E-05 & 5 \\ \text { AC } & 89 & 1.00 E-08 & 5 \\ \text { TH } & 90 & 6.00 E+02 & 5 \\ \text { PA } & 91 & 1.00 E-05 & 5 \\ \text { U } & 92 & 1.00 E+02 & 5 \\ \text { NP } & 93 & 1.00 E-10 & 5 \\ \text { PU } & 94 & 1.00 E-11 & 5 \\ \text { AM } & 95 & 1.00 E-28 & 5 \\ \text { CM } & 96 & 1.00 E-28 & 5 \\ \text { BK } & 97 & 1.00 E-28 & 5 \\ \text { CF } & 98 & 1.00 E-28 & 5 \\ \text { ES } & 99 & 1.00 E-28 & 5 \\ \text { FM } & 100 & 1.00 E-28 & 5 \\ & & & \end{array}$

REFERENCE (S)

893

\section{3}

1798

893 


$\begin{array}{lrrrr}\text { ELEMENT } & \text { ATOMIC } & \text { CONC } & & \\ \text { SYMBOL } & \text { NO. }(2) & \text { CB (PPM) } & \text { CODE } & \text { REFERENC } \\ \text { H } & & & & \\ \text { HE } & 1 & 1.00 E+05 & 6 & \\ \text { LI } & 2 & 1.00 E-02 & 5 & \\ \text { BE } & 3 & 1.10 E-02 & 4 & \\ \text { B } & 4 & 3.00 E-02 & 4 & \\ \text { C } & 5 & 1.00 E-01 & 1 & 1798 \\ \text { N } & 6 & 2.40 E+05 & 6 & \\ \text { O } & 7 & 2.90 E+04 & 6 & \\ \text { F } & 8 & 6.00 E+05 & 6 & \\ \text { NE } & 9 & 2.00 E+04 & 5 & \\ \text { NA } & 10 & 1.00 E-01 & 5 & \\ \text { MG } & 11 & 2.00 E+03 & 6 & \\ \text { AL } & 12 & 1.20 E+02 & 1 & 1798 \\ \text { SI } & 13 & 1.50 E+00 & 1 & 1798 \\ \text { P } & 14 & 1.70 E+01 & 4 & \\ \text { S } & 15 & 1.20 E+03 & 1 & 1798 \\ \text { CL } & 16 & 1.00 E+04 & 99 & \\ \text { AR } & 17 & 2.00 E+03 & 6 & \\ \text { K } & 18 & 1.00 E+02 & 5 & \\ \text { CA } & 19 & 1.70 E+03 & 1 & 1798 \\ \text { SC } & 20 & 3.50 E+02 & 1 & 1798 \\ \text { TI } & 21 & 5.00 E+00 & 99 & \\ \text { V } & 22 & 1.30 E-01 & 1 & 1798 \\ \text { CR } & 23 & 1.00 E-02 & 1 & 1798 \\ \text { MN } & 24 & 1.50 E+00 & 1 & 1798 \\ \text { FE } & 25 & 3.20 E-01 & 1 & 1798 \\ \text { CO } & 26 & 9.40 E+01 & 1 & 1798 \\ \text { NI } & 27 & 4.00 E-02 & 2 & 2805 \\ \text { CU } & 28 & 1.70 E-01 & 1 & 1798 \\ \text { ZN } & 29 & 1.80 E+00 & 1 & 1798 \\ \text { GA } & 30 & 2.70 E+01 & 1 & 1798 \\ \text { GE } & 31 & 9.00 E-03 & 4 & \\ \text { AS } & 32 & 1.00 E+02 & 5 & \\ \text { SE } & 34 & 1.00 E-02 & 4 & \\ \text { BR } & 35 & 1.00 E+01 & 5 & \\ \text { KR } & 36 & 1.00 E-01 & 4 & \\ \text { RB } & 37 & 4.00 E-02 & 5 & \\ \text { SR } & 38 & 3.30 E-01 & 2 & 2805 \\ \text { Y } & 39 & 5.00 E+00 & 1 & 1798 \\ \text { ZR } & 40 & 9.00 E-01 & 4 & \\ \text { NB } & 41 & 9.00 E-01 & 4 & \\ \text { MD } & 42 & 4.00 E-02 & 1 & 1798 \\ \text { TC } & 43 & 1.00 E-11 & 5 & \\ \text { RU } & 44 & 5.00 E-03 & 99 & \\ \text { RH } & 45 & 1.00 E-01 & 5 & \\ \text { PD } & 46 & 1.00 E-01 & 5 & \\ \text { AG } & 47 & 1.10 E-02 & 1 & 1798 \\ \text { CD } & 48 & 7.80 E-01 & 1 & 1798 \\ \text { IN } & 49 & 1.00 E+01 & 5 & \\ \text { SN } & 50 & 2.10 E+00 & 1 & 1798\end{array}$

$\begin{array}{lrrr}\text { ELEMENT } & \text { ATOMIC } & \text { CONC } & \\ \text { SYMBOL } & \text { NO.121 } & \text { CB (PPM) } & \text { CODE } \\ \text { SB } & & & \\ \text { TE } & 51 & 3.00 E-02 & 4 \\ \text { I } & 52 & 5.00 E-02 & 99 \\ \text { XE } & 53 & 9.00 E-01 & 4 \\ \text { CS } & 54 & 1.00 E-03 & 5 \\ \text { BA } & 55 & 1.30 E-01 & 4 \\ \text { LA } & 56 & 8.70 E-02 & 1 \\ \text { CE } & 57 & 9.00 E-01 & 4 \\ \text { PR } & 58 & 5.00 E+00 & 99 \\ \text { ND } & 59 & 6.70 E+02 & 5 \\ \text { PM } & 60 & 2.50 E+03 & 5 \\ \text { SM } & 61 & 5.00 E-20 & 99 \\ \text { EU } & 62 & 5.00 E-02 & 99 \\ \text { GD } & 63 & 5.00 E-03 & 99 \\ \text { TB } & 64 & 5.00 E-01 & 99 \\ \text { OY } & 65 & 5.00 E-02 & 99 \\ \text { HO } & 66 & 3.00 E+02 & 5 \\ \text { ER } & 67 & 7.00 E+01 & 5 \\ \text { TM } & 68 & 8.00 E+01 & 5 \\ \text { YB } & 69 & 5.00 E-02 & 99 \\ \text { LU } & 70 & 5.00 E-02 & 99 \\ \text { HF } & 71 & 7.00 E+01 & 5 \\ \text { TA } & 72 & 1.00 E+02 & 99 \\ \text { W } & 73 & 1.00 E+02 & 99 \\ \text { RE } & 74 & 1.00 E+02 & 5 \\ \text { OS } & 75 & 1.00 E-01 & 5 \\ \text { IR } & 76 & 1.00 E-01 & 5 \\ \text { PT } & 77 & 1.00 E-03 & 5 \\ \text { AU } & 78 & 1.00 E+00 & 5 \\ \text { HG } & 80 & 2.30 E-01 & 1 \\ \text { TL } & 81 & 1.00 E+00 & 5 \\ \text { PB } & 82 & 2.00 E-02 & 4 \\ \text { BI } & 83 & 2.00 E-01 & 1 \\ \text { PO } & 84 & 1.00 E-09 & 1 \\ \text { AT } & 85 & 1.00 E-17 & 5 \\ \text { RN } & 86 & 1.00 E-11 & 5 \\ \text { FR } & 87 & 1.00 E-16 & 5 \\ \text { RA } & 88 & 8.00 E-05 & 5 \\ \text { AC } & 89 & 1.00 E-08 & 5 \\ \text { TH } & 90 & 6.00 E+02 & 5 \\ \text { PA } & 91 & 1.00 E-05 & 5 \\ \text { U } & 92 & 1.00 E+02 & 5 \\ \text { NP } & 93 & 1.00 E-10 & 5 \\ \text { PU } & 94 & 1.00 E-11 & 5 \\ \text { AM } & 95 & 1.00 E-28 & 5 \\ \text { CM } & 96 & 1.00 E-28 & 5 \\ \text { BK } & 97 & 1.00 E-28 & 5 \\ \text { CF } & 98 & 1.00 E-28 & 5 \\ \text { ES } & 99 & 1.00 E-28 & 5 \\ \text { FM } & 100 & 1.00 E-28 & 5 \\ & & & \end{array}$

\section{REFERENCE (S)}

1798

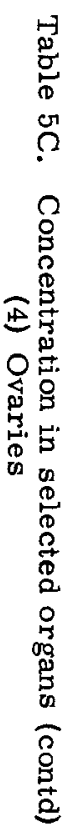




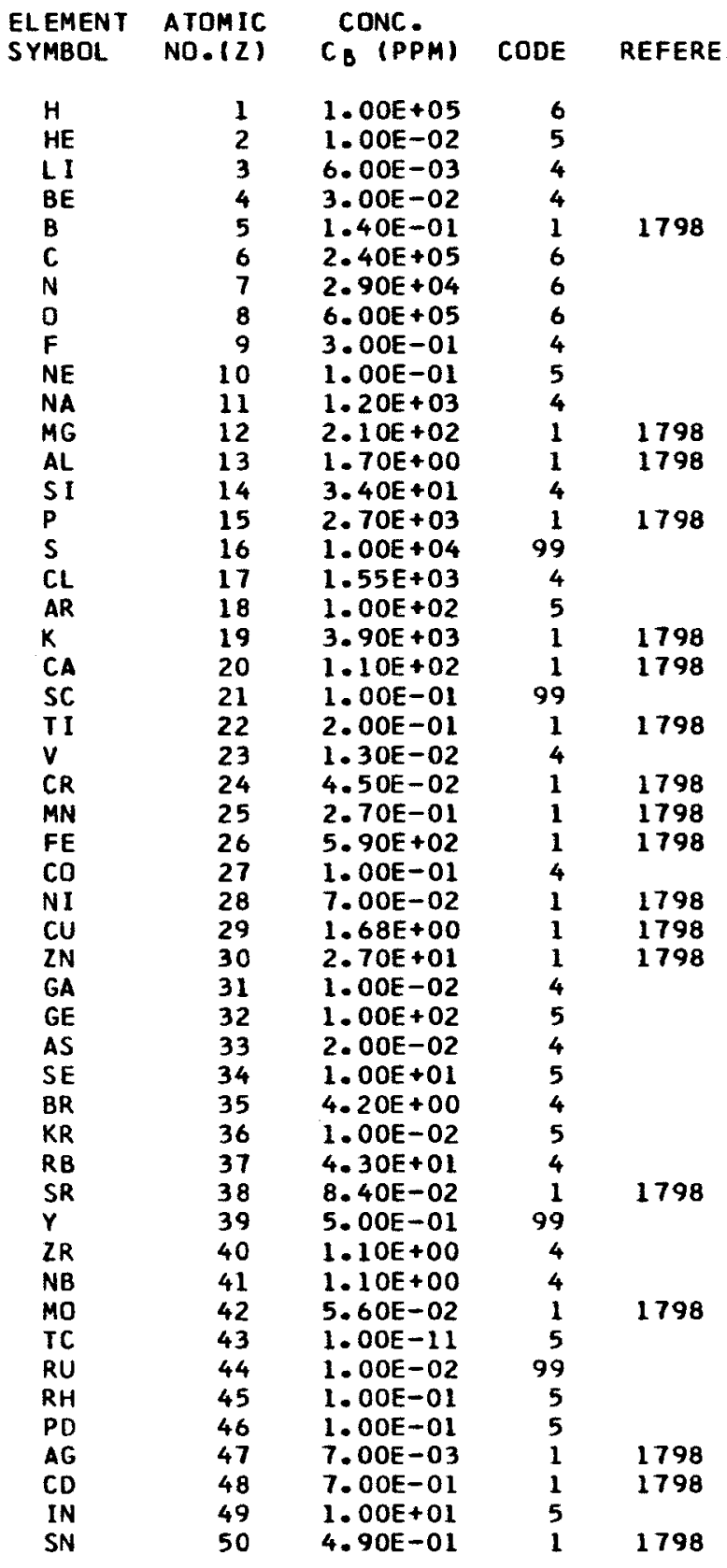

$\begin{array}{lrrr}\text { ELEMENT } & \text { ATOMIC } & \text { CONC } & \\ \text { SYMBOL } & \text { NO. } 2 \text { ( ) } & \text { CB (PPM) } & \text { CODE } \\ & & & \\ \text { SB } & 51 & 1.00 E-01 & 4 \\ \text { IE } & 52 & 5.00 E-02 & 99 \\ \text { I } & 53 & 5.00 E+02 & 5 \\ \text { XE } & 54 & 1.00 E-03 & 5 \\ \text { CS } & 55 & 6.00 E-01 & 4 \\ \text { BA } & 56 & 4.60 E-02 & 1 \\ \text { LA } & 57 & 1.10 E+00 & 4 \\ \text { CE } & 58 & 1.00 E-02 & 99 \\ \text { PR } & 59 & 6.70 E+02 & 5 \\ \text { ND } & 60 & 2.50 E+03 & 5 \\ \text { PM } & 61 & 5.00 E-22 & 99 \\ \text { SM } & 62 & 5.00 E-03 & 99 \\ \text { EU } & 63 & 5.00 E-04 & 99 \\ \text { GD } & 64 & 5.00 E-03 & 99 \\ \text { IB } & 65 & 5.00 E-04 & 99 \\ \text { DY } & 66 & 3.00 E+02 & 5 \\ \text { HO } & 67 & 7.00 E+01 & 5 \\ \text { ER } & 68 & 8.00 E+01 & 5 \\ \text { IM } & 69 & 5.00 E-03 & 99 \\ \text { YB } & 70 & 5.00 E-04 & 99 \\ \text { LU } & 71 & 7.00 E+01 & 5 \\ \text { HF } & 72 & 5.00 E+00 & 99 \\ \text { TA } & 73 & 5.00 E+00 & 99 \\ \text { W } & 74 & 1.00 E+02 & 5 \\ \text { RE } & 75 & 1.00 E-01 & 5 \\ \text { OS } & 76 & 1.00 E-01 & 5 \\ \text { IR } & 77 & 1.00 E-03 & 5 \\ \text { PT } & 78 & 1.00 E+00 & 5 \\ \text { AU } & 79 & 1.00 E-01 & 4 \\ \text { HG } & 80 & 1.30 E-01 & 4 \\ \text { TL } & 81 & 1.10 E-01 & 4 \\ \text { PB } & 82 & 1.30 E+00 & 1 \\ \text { BI } & 83 & 5.20 E-02 & 4 \\ \text { PO } & 84 & 1.00 E-09 & 5 \\ \text { AT } & 85 & 1.00 E-17 & 5 \\ \text { RN } & 86 & 1.00 E-11 & 5 \\ \text { FR } & 87 & 1.00 E-16 & 5 \\ \text { RA } & 88 & 8.00 E-05 & 5 \\ \text { AC } & 89 & 1.00 E-08 & 5 \\ \text { TH } & 90 & 6.00 E+02 & 5 \\ \text { PA } & 91 & 1.00 E-05 & 5 \\ \text { U } & 92 & 1.00 E+02 & 5 \\ \text { NP } & 93 & 1.00 E-10 & 5 \\ \text { PU } & 94 & 1.00 E-11 & 5 \\ \text { AM } & 95 & 1.00 E-28 & 5 \\ \text { CM } & 96 & 1.00 E-28 & 5 \\ \text { BK } & 97 & 1.00 E-28 & 5 \\ \text { CF } & 98 & 1.00 E-28 & 5 \\ \text { ES } & 99 & 1.00 E-28 & 5 \\ \text { FM } & 100 & 1.00 E-28 & 5\end{array}$

REFERENCE ( $S$ )

1798

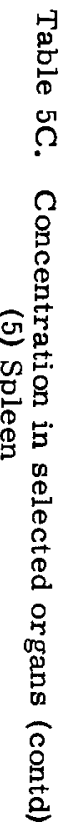

1798 


\begin{tabular}{|c|c|c|c|c|}
\hline $\begin{array}{l}\text { EL EMENT } \\
\text { SYMBOL }\end{array}$ & $\begin{array}{l}\text { ATOMIC } \\
\text { NO. }(Z)\end{array}$ & $\begin{array}{c}C O N C \\
C_{B}(P P M)\end{array}$ & CODE & REFERE \\
\hline$H$ & 1 & $1.00 E+05$ & 6 & \\
\hline $\mathrm{HE}$ & 2 & $1.00 E-02$ & 5 & \\
\hline LI & 3 & $9.00 E-03$ & 4 & \\
\hline$B E$ & 4 & $3.00 E-02$ & 4 & \\
\hline B & 5 & $5.60 E+00$ & 2 & 1774 \\
\hline$c$ & 6 & $2.40 E+05$ & 6 & \\
\hline $\mathrm{N}$ & 7 & $2.90 E+04$ & 6 & \\
\hline 0 & 8 & $6.00 E+05$ & 6 & \\
\hline$F$ & 9 & $2.00 E+04$ & 5 & \\
\hline$N E$ & 10 & $1.00 E-01$ & 5 & \\
\hline NA & 11 & $2.10 E+03$ & 4 & \\
\hline MG & 12 & $1.70 E+02$ & 1 & 1798 \\
\hline$A L$ & 13 & $8.80 E-01$ & 1 & 1798 \\
\hline SI & 14 & 3. $10 E+01$ & 4 & \\
\hline $\mathbf{P}$ & 15 & $1.80 E+03$ & 1 & 1798 \\
\hline$S$ & 16 & $1.00 E+04$ & 99 & \\
\hline $\mathrm{CL}$ & 17 & $2.33 E+03$ & 4 & \\
\hline$A R$ & 18 & $1.00 E+02$ & 5 & \\
\hline K & 19 & $2.60 E+03$ & 1 & 1798 \\
\hline CA & 20 & $1.50 E+02$ & 1 & 1798 \\
\hline SC & 21 & $5.00 E-01$ & 99 & \\
\hline TI & 22 & $1.10 E-01$ & 1 & 1798 \\
\hline$v$ & 23 & $4.00 E-02$ & 2 & 1774 \\
\hline CR & 24 & $6.90 E-02$ & 1 & 1798 \\
\hline MN & 25 & $2.30 E-01$ & 1 & 1798 \\
\hline FE & 26 & $5.00 E+01$ & 1 & 1798 \\
\hline $\mathrm{CO}$ & 27 & $2.80 E+00$ & 2 & 1774 \\
\hline NI & 28 & $1.80 E-01$ & 1 & 1798 \\
\hline $\mathrm{Cu}$ & 29 & $1.21 E+00$ & 1 & 1798 \\
\hline$Z N$ & 30 & $2.40 E+01$ & $i$ & 1798 \\
\hline GA & 31 & $9.00 E-03$ & 4 & \\
\hline GE & 32 & $1.00 E+02$ & 5 & \\
\hline AS & 33 & $4.00 E-02$ & 4 & \\
\hline SE & 34 & $1.00 E+01$ & 5 & \\
\hline$B R$ & 35 & $3.30 E-01$ & 4 & \\
\hline KR & 36 & $1.00 \mathrm{E}-02$ & 5 & \\
\hline RB & 37 & $1.20 E+01$ & 4 & \\
\hline SR & 38 & $1.40 E-01$ & $i$ & 1798 \\
\hline$Y$ & 39 & $5.00 E-01$ & 99 & \\
\hline ZR & 40 & $8.00 E-01$ & 4 & \\
\hline NB & 41 & $9.00 E-01$ & 4 & \\
\hline MO & 42 & $4.40 E-02$ & 1 & 1798 \\
\hline TC & 43 & $1.00 E-11$ & 5 & \\
\hline RU & 44 & $5.00 E-03$ & 99 & \\
\hline RH & 45 & $1.00 E-01$ & 5 & \\
\hline PD & 46 & $1.00 E-01$ & 5 & \\
\hline AG & 47 & $5.50 E-03$ & 1 & 1798 \\
\hline CD & 48 & $5.50 \mathrm{E}-01$ & 1 & 1798 \\
\hline IN & 49 & $1.00 E+01$ & 5 & \\
\hline SN & 50 & $3.60 E-01$ & 1 & 1798 \\
\hline
\end{tabular}

$\begin{array}{crrr}\text { ELEMENT } & \text { ATOMIC } & \text { CONC } & \\ \text { SYMBOL } & \text { NO. (2) } & \text { C } B(P P M) & \text { CODE } \\ & & & \\ \text { SB } & 51 & 5.00 E-02 & 4 \\ \text { TE } & 52 & 5.00 E-02 & 99 \\ \text { I } & 53 & 5.00 E+02 & 5 \\ \text { XE } & 54 & 1.00 E-03 & 5 \\ \text { CS } & 55 & 3.00 E-01 & 4 \\ \text { BA } & 56 & 4.00 E-02 & 1 \\ \text { LA } & 57 & 9.00 E-01 & 4 \\ \text { CE } & 58 & 5.00 E-01 & 99 \\ \text { PR } & 59 & 6.70 E+02 & 5 \\ \text { ND } & 60 & 2.50 E+03 & 5 \\ \text { PM } & 61 & 5.00 E-21 & 99 \\ \text { SM } & 62 & 5.00 E-03 & 99 \\ \text { EU } & 63 & 5.00 E-04 & 99 \\ \text { GD } & 64 & 5.00 E-02 & 99 \\ \text { TB } & 65 & 5.00 E-03 & 99 \\ \text { DY } & 66 & 3.00 E+02 & 5 \\ \text { HO } & 67 & 7.00 E+01 & 5 \\ \text { ER } & 68 & 8.00 E+01 & 5 \\ \text { IM } & 69 & 1.00 E-02 & 99 \\ \text { YB } & 70 & 1.00 E-02 & 99 \\ \text { LU } & 71 & 7.00 E+01 & 5 \\ \text { HF } & 72 & 5.00 E-02 & 99 \\ \text { TA } & 73 & 1.00 E+01 & 99 \\ \text { W } & 74 & 1.00 E+02 & 5 \\ \text { RE } & 75 & 1.00 E-01 & 5 \\ \text { OS } & 76 & 1.00 E-01 & 5 \\ \text { IR } & 77 & 1.00 E-03 & 5 \\ \text { PT } & 78 & 1.00 E+00 & 5 \\ \text { AU } & 79 & 1.10 E-01 & 1 \\ \text { HG } & 80 & 1.00 E+00 & 5 \\ \text { TL } & 81 & 8.00 E-02 & 4 \\ \text { PB } & 82 & 3.00 E-01 & 1 \\ \text { BI } & 83 & 2.00 E-01 & 1 \\ \text { PO } & 84 & 1.00 E-09 & 5 \\ \text { AT } & 85 & 1.00 E-17 & 5 \\ \text { RN } & 86 & 1.00 E-11 & 5 \\ \text { FR } & 87 & 1.00 E-16 & 5 \\ \text { RA } & 88 & 8.00 E-05 & 5 \\ \text { AC } & 89 & 1.00 E-08 & 5 \\ \text { TH } & 90 & 6.00 E+02 & 5 \\ \text { PA } & 91 & 1.00 E-05 & 5 \\ \text { U } & 92 & 1.00 E+02 & 5 \\ \text { NP } & 93 & 1.00 E-10 & 5 \\ \text { PU } & 94 & 1.00 E-11 & 5 \\ \text { AM } & 95 & 1.00 E-28 & 5 \\ \text { CM } & 96 & 1.00 E-28 & 5 \\ \text { BK } & 97 & 1.00 E-28 & 5 \\ \text { CF } & 98 & 1.00 E-28 & 5 \\ \text { ES } & 99 & 1.00 E-28 & 5 \\ \text { FM } & 100 & 1.00 E-28 & 5 \\ & & & 5\end{array}$

REFERENCE(S)

1798

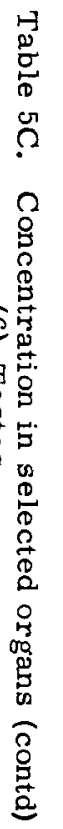


ELEMENT ATOMIC CONC. SYMBOL

ATOMIC

\begin{tabular}{|c|c|c|}
\hline $\begin{array}{l}H \\
H E\end{array}$ & 1 & $\begin{array}{l}1.00 E+05 \\
1.00 E-02\end{array}$ \\
\hline L I & 3 & $1.30 E-02$ \\
\hline$B E$ & 4 & $1.30 E-04$ \\
\hline B & 5 & 2. $90 \mathrm{E}-01$ \\
\hline C & 6 & $2.40 E+05$ \\
\hline $\mathbf{N}$ & 7 & $2.90 E+04$ \\
\hline 0 & 8 & $6.00 E+05$ \\
\hline $\mathrm{F}$ & 9 & $2.00 E+01$ \\
\hline NE & 10 & $1.00 E-01$ \\
\hline NA & 11 & $2.00 E+03$ \\
\hline$M G$ & 12 & 2. $90 \mathrm{E}+02$ \\
\hline AL & 13 & $1.00 E+00$ \\
\hline SI & 14 & 3. $00 E+01$ \\
\hline $\mathbf{P}$ & 15 & $1.15 E+04$ \\
\hline $\mathbf{s}$ & 16 & $2.50 E+03$ \\
\hline $\mathrm{CL}$ & 17 & 2. $00 E+03$ \\
\hline AR & 18 & $1.00 E+02$ \\
\hline K & 19 & $2.00 E+03$ \\
\hline CA & 20 & $1.26 E+04$ \\
\hline SC & 21 & $1.00 E-02$ \\
\hline TI & 22 & 3. $90 \mathrm{E}-01$ \\
\hline v & 23 & $1.50 \mathrm{E}-02$ \\
\hline CR & 24 & $2.00 E-01$ \\
\hline$M N$ & 25 & $3.75 E-01$ \\
\hline $\mathrm{FE}$ & 26 & $6.75 E+01$ \\
\hline $\mathrm{CO}$ & 27 & $2.45 E-02$ \\
\hline N I & 28 & $2.00 E-01$ \\
\hline CU & 29 & $1.30 E+00$ \\
\hline $\mathbf{Z N}$ & 30 & $3.15 E+01$ \\
\hline GA & 31 & $3.00 \mathrm{E}-05$ \\
\hline GE & 32 & $1.00 E+02$ \\
\hline AS & 33 & $1.40 E+00$ \\
\hline SE & 34 & $5.00 E-01$ \\
\hline BR & 35 & 3. $90 \mathrm{E}-01$ \\
\hline KR & 36 & $1.00 E-02$ \\
\hline RB & 37 & $4.75 E+00$ \\
\hline SR & 38 & $4.00 E+00$ \\
\hline$Y$ & 39 & 5. $00 \mathrm{~F}-01$ \\
\hline ZR & 40 & $8.60 E-02$ \\
\hline NB & 41 & 7. $00 \mathrm{E}-01$ \\
\hline MO & 42 & $1.75 E-01$ \\
\hline TC & 43 & $5.00 E-13$ \\
\hline RU & 44 & $1.00 E-03$ \\
\hline RH & 45 & $1.00 \mathrm{E}-01$ \\
\hline PD & 46 & i. OOE-OL \\
\hline AG & 47 & $1.90 \mathrm{E}-02$ \\
\hline$C D$ & 48 & $7.20 E-01$ \\
\hline IN & 49 & $1.00 E+01$ \\
\hline SN & 50 & $2.90 \mathrm{E}-01$ \\
\hline
\end{tabular}

CODE REFERENCE(S)

$$
5280
$$

5280

5280

5280

5280

5280

5280

5280

5280

5280

5280

5280

5280
5280

5280

5280

5280
5280

5280

5280

5280

5280

5280

5280

5280

5280

5280

5280

5280

5280

5280
ELEMENT ATOMIC CONC.

$\begin{array}{llll}\text { ELEMENT } & \text { ATOMIC } \\ \text { SYMBOL } & \text { ND. }(2) & C_{B} \text { (PPM) CODE REFERENCE(S) }\end{array}$

$\begin{array}{lrrr}\text { SB } & 51 & 5.00 E-02 & 99 \\ \text { TE } & 52 & 5.00 E-03 & 99 \\ \text { I } & 53 & 5.20 E-01 & 8 \\ \text { XE } & 54 & 1.00 E-03 & 5 \\ \text { CS } & 55 & 1.45 E-02 & 8 \\ \text { BA } & 56 & 7.20 E-01 & 8 \\ \text { LA } & 57 & 7.00 E-01 & 4 \\ \text { CE } & 58 & 5.00 E-02 & 99 \\ \text { PR } & 59 & 6.70 E+02 & 5 \\ \text { ND } & 60 & 2.50 E+03 & 5 \\ \text { PM } & 61 & 5.00 E-22 & 99 \\ \text { SM } & 62 & 5.00 E-03 & 99 \\ \text { EU } & 63 & 5.00 E-04 & 99 \\ \text { GD } & 64 & 5.00 E-03 & 99 \\ \text { TB } & 65 & 5.00 E-04 & 99 \\ \text { OY } & 66 & 3.00 E+02 & 5 \\ \text { HO } & 67 & 5.00 E-04 & 99 \\ \text { ER } & 68 & 8.00 E+01 & 5 \\ \text { TM } & 69 & 5.00 E-04 & 99 \\ \text { YB } & 70 & 5.00 E-04 & 99 \\ \text { LU } & 71 & 5.00 E-04 & 99 \\ \text { HF } & 72 & 1.00 E-01 & 99 \\ \text { TA } & 73 & 5.00 E-01 & 99 \\ \text { W } & 74 & 1.00 E+02 & 5 \\ \text { RE } & 75 & 1.00 E-01 & 5 \\ \text { OS } & 76 & 1.00 E-01 & 5 \\ \text { IR } & 77 & 1.00 E-05 & 99 \\ \text { PT } & 78 & 1.00 E+00 & 5 \\ \text { AU } & 79 & 1.45 E-01 & 8 \\ \text { HG } & 80 & 5.00 E-02 & 99 \\ \text { TL } & 81 & 8.60 E-02 & 4 \\ \text { PB } & 82 & 1.75 E+00 & 8 \\ \text { BI } & 83 & 1.45 E-02 & 8 \\ \text { PD } & 84 & 1.00 E-09 & 5 \\ \text { AT } & 85 & 1.00 E-17 & 5 \\ \text { RN } & 86 & 1.00 E-11 & 5 \\ \text { FR } & 87 & 1.00 E-16 & 5 \\ \text { RA } & 88 & 4.30 E-10 & 8 \\ \text { AC } & 89 & 1.00 E-08 & 5 \\ \text { TH } & 90 & 6.00 E+02 & 5 \\ \text { PA } & 91 & 1.00 E-05 & 5 \\ \text { U } & 92 & 2.90 E-04 & 8 \\ \text { NP } & 93 & 1.00 E-10 & 5 \\ \text { PU } & 94 & 1.00 E-11 & 5 \\ \text { AM } & 95 & 1.00 E-28 & 5 \\ \text { CM } & 96 & 1.00 E-28 & 5 \\ \text { BK } & 97 & 1.00 E-28 & 5 \\ \text { CF } & 98 & 1.00 E-28 & 5 \\ \text { ES } & 99 & 1.00 E-28 & 5 \\ \text { FM } & 100 & 1.00 E-28 & 5 \\ & & & \end{array}$

5280

5280

5280

5280

5280

5280

5280

5280 


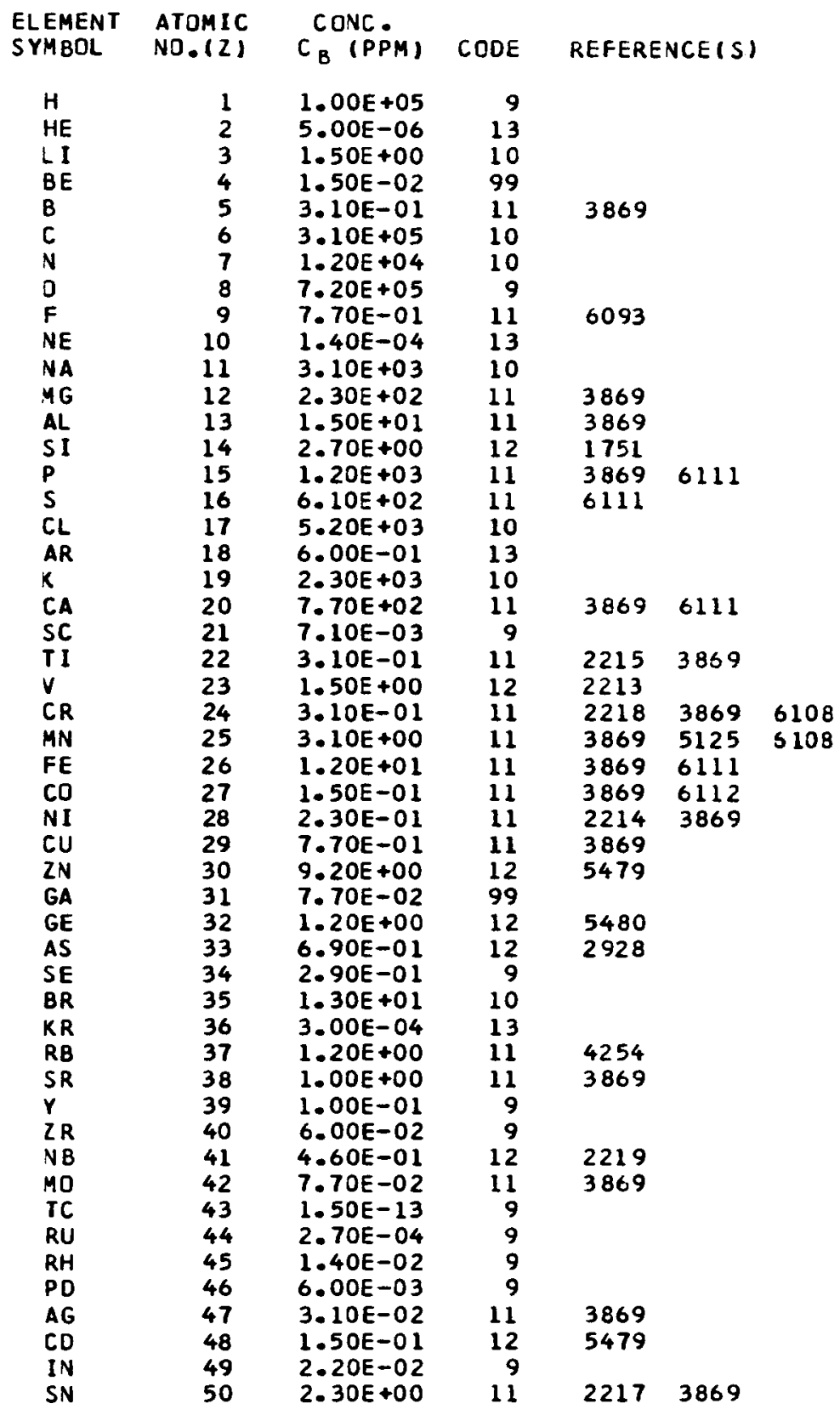

\section{ELEMENT ATOMIC SYMBOL NO.12I}

$\begin{array}{ll}\text { SB } & 51 \\ \text { TE } & 52 \\ \text { I } & 53 \\ \text { XE } & 54 \\ \text { CS } & 55 \\ \text { BA } & 56 \\ \text { LA } & 57 \\ \text { CE } & 58 \\ \text { PR } & 59 \\ \text { ND } & 60 \\ \text { PM } & 61 \\ \text { SM } & 62 \\ \text { EJ } & 63 \\ \text { GD } & 64 \\ \text { TB } & 65 \\ \text { DY } & 66 \\ \text { HO } & 67 \\ \text { ER } & 68 \\ \text { TM } & 69 \\ \text { YB } & 70 \\ \text { LU } & 71 \\ \text { HF } & 72 \\ \text { TA } & 73 \\ \text { W } & 74 \\ \text { RE } & 75 \\ \text { OS } & 76 \\ \text { IR } & 77 \\ \text { PT } & 78 \\ \text { AU } & 79 \\ \text { HG } & 80 \\ \text { TL } & 81 \\ \text { PB } & 82 \\ \text { BI } & 83 \\ \text { PD } & 84 \\ \text { AT } & 85 \\ \text { RN } & 86 \\ \text { FR } & 87 \\ \text { RA } & 88 \\ \text { AC } & 89 \\ \text { TH } & 90 \\ \text { PA } & 91 \\ \text { U } & 92 \\ \text { NP } & 93 \\ \text { PU } & 94 \\ \text { AM } & 95 \\ \text { CM } & 96 \\ \text { BK } & 97 \\ \text { CF } & 98 \\ \text { ES } & 99 \\ \text { FM } & 100 \\ & \end{array}$

CONC.

$C_{B}$ (PPM) CODE REFERENCE(S)

2.20E-02 $2.20 \mathrm{E}-03$ 7.70E-02 1. $00 \mathrm{E}-04$

7.70E-03

1. $30 \mathrm{E}+00$

7. $70 \varepsilon-02$ $1.00 E-01$ $1.40 E-02$ 5. $10 E-02$ $2.00 E-21$ $1.30 E-02$ 2.10E-03 $8.50 E-03$ $1.50 E-03$ 6.20E-03 $1.50 E-03$ $1.60 \mathrm{E}-03$ $1.50 E-03$ $1.60 E-03$ $1.50 E-03$ $5.40 \mathrm{E}-03$ $2.20 E-02$ 1. $40 E-02$ 2. $20 E-04$ 2. $70 \mathrm{E}-04$ 1. $20 \mathrm{E}-04$ 1. $20 E-04$
$4.90 E-03$ $4.90 E-03$
$2.50 E-04$ 2.5OE-04 $1.50 \mathrm{E}-02$ $3.10 E-02$ $3.10 E-01$ $1.40 \mathrm{E}-02$ $1.00 \overline{0}-09$ $1.00 E-17$ 1.5OE 13 $1.00 E-16$ $1.50 E-O S$ $1.00 E-08$ $6.00 E+02$ $1.00 E-05$ $1.50 \bar{c}-03$ $1.00 E-10$ 1. OOE- 11 $1.00 E-28$ $1.005-28$ $1.00 E-28$ $1.00 E-28$ $1.00 E-28$ 1. OOE-28
6111

4254

3869 
$\bullet$

•

- 


\section{6. $\mathrm{F}_{1}:$ UNIT-RAD DEPOSITION}

Unit-rad depositions $\left(\mathrm{F}_{1}\right)$ were calculated for the tissues of Table 1 and the isotopes of Table 2 (5574). The $F_{1}$ values via the forage-to-cow-to-milk pathway are listed in Table 6A; those via the soil-root pathway are listed in Table 6B. $F_{1}$ is the minimum deposition of each isotope in $\mu \mathrm{Ci} / \mathrm{m}^{2}$ that could result in a 30-year internal dose of $1 \mathrm{rad}$ to the tissue in question. It is calculated as follows:

\section{Forage-to-Cow-to-Milk Pathway}

$$
\begin{aligned}
F_{1}=\frac{\left(7.04 \times 10^{-8}\right)}{(U A F) Q f T_{P} T_{E}} & \left(1-T_{P} / T_{E}\right) \\
& \times \frac{\left(1-T_{P} / T_{E}-e^{-20.8 / T_{E}}\right)}{\left(1-T^{-20.5}\right.}
\end{aligned}
$$

The expression above yields $F_{1}$ via milk when (UAF) is expressed in $\mathrm{m}^{2} /$ day, $Q$ is expressed in $\mathrm{MeV}$, and $\mathrm{T}_{\mathrm{P}}$ and $\mathrm{T}_{\mathrm{E}}$ are expressed in yr. (UAF) was assumed to be $45 \mathrm{~m}^{2} /$ day (1759), and the halfresidence time on forage which determines $\mathrm{T}_{\mathrm{P}}$ was assumed to be 14 days (1762). The f term is calculated as

$$
f=f_{M} f_{B} / m
$$

The $F_{1}$ values are listed for the adult and the one-year child. 


\begin{tabular}{|c|c|c|c|c|}
\hline $\begin{array}{l}\text { ELEMENT } \\
\text { SYMBOL }\end{array}$ & $\begin{array}{l}\text { ATOMIC } \\
\text { NO. }(Z)\end{array}$ & $\begin{array}{l}\text { ISOTOPE } \\
\text { MASS(A) }\end{array}$ & $\begin{array}{l}\text { ADULT } \\
\text { UC / } / M * * 2\end{array}$ & $\begin{array}{c}\text { CHILD } \\
U C / M * * 2\end{array}$ \\
\hline H & 1 & 3 & 7. $0 E+02$ & $1.0 E+02$ \\
\hline$B E$ & 4 & 7 & 7. $9 E+05$ & $1.1 E+05$ \\
\hline $\mathrm{BE}$ & 4 & 10 & $1.1 E+04$ & $1.6 E+03$ \\
\hline C & 6 & 14 & 1. $O E+02$ & $1.5 E+01$ \\
\hline NA & 11 & 22 & $8 \cdot 2 E-01$ & $1-2 E-01$ \\
\hline NA & 11 & 24 & $1.7 E+02$ & $2.4 E+01$ \\
\hline MG & 12 & 28 & $6.5 E+02$ & $9.3 E+01$ \\
\hline$A L$ & 13 & 26 & 8. $0 E-02$ & $1.2 E-02$ \\
\hline SI & 14 & 32 & $1.4 E-01$ & $2.0 E-02$ \\
\hline $\mathbf{P}$ & 15 & 32 & $1.3 E+00$ & $1.9 E-01$ \\
\hline P & 15 & 33 & $6.8 E+00$ & $9.7 E-01$ \\
\hline $\mathrm{s}$ & 16 & 35 & $4.7 E+01$ & $6.7 E+00$ \\
\hline $\mathrm{Cl}$ & 17 & 36 & $2 \cdot 2 E+00$ & $3.1 E-01$ \\
\hline$A R$ & 18 & 37 & $7.4 E+02$ & $1.1 E+02$ \\
\hline AR & 18 & 39 & $2.8 E-02$ & $4.1 E-03$ \\
\hline$A R$ & 18 & 42 & $3.1 E-03$ & $4.4 E-04$ \\
\hline K & 19 & 40 & $2.2 E+00$ & 3. $1 E-01$ \\
\hline K & 19 & 42 & $2.1 E+03$ & $2.9 E+02$ \\
\hline $\mathrm{k}$ & 19 & 43 & $1.3 E+03$ & $1.8 \mathrm{E}+02$ \\
\hline CA & 20 & 41 & 4. $9 E-01$ & 7. $1 \mathrm{E}-02$ \\
\hline CA & 20 & 45 & $8.1 E-01$ & $1.2 E-01$ \\
\hline$C A$ & 20 & 47 & 4. $9 E+00$ & $7.0 E-01$ \\
\hline CA & 20 & 48 & 4. $9 E-04$ & $6.9 E-05$ \\
\hline SC & 21 & $44 M$ & $9.2 E+08$ & $1 \cdot 3 E+08$ \\
\hline SC & 21 & 46 & $6.5 E+06$ & $9.3 E+05$ \\
\hline SC & 21 & 47 & $4.5 E+09$ & $6.5 E+08$ \\
\hline SC & 21 & 48 & $1.2 E+09$ & $1.8 E+08$ \\
\hline TI & 22 & 44 & $1.1 E+04$ & $1.6 E+03$ \\
\hline v & 23 & 48 & 1. $5 E+03$ & $2.1 E+02$ \\
\hline$v$ & 23 & 49 & $7.4 E+04$ & $1.1 E+04$ \\
\hline v & 23 & 50 & $2.0 E+02$ & $2 \cdot 9 E+01$ \\
\hline$C R$ & 24 & 48 & $4.2 E+04$ & $5.9 E+03$ \\
\hline$C R$ & 24 & 51 & $1.4 E+04$ & $2.0 E+03$ \\
\hline MN & 25 & 52 & $7.7 E+03$ & $1.1 E+03$ \\
\hline$M N$ & 25 & 53 & $2 \cdot 1 E+04$ & $3.0 E+03$ \\
\hline$M N$ & 25 & 54 & $3.4 E+02$ & $4.8 E+01$ \\
\hline FE & 26 & 55 & 2. $0 E+03$ & $2.8 E+02$ \\
\hline $\mathrm{FE}$ & 26 & 59 & $1.8 E+02$ & $2.6 E+01$ \\
\hline FE & 26 & 60 & $1.8 E+00$ & $2.5 E-01$ \\
\hline $\mathrm{CO}$ & 27 & 55 & $5 \cdot 3 E+04$ & $7.5 E+03$ \\
\hline $\mathrm{CO}$ & 27 & 56 & $1.5 E+02$ & $2.1 E+01$ \\
\hline $\mathrm{CO}$ & 27 & 57 & $1.6 E+03$ & $2 \cdot 3 E+02$ \\
\hline $\mathrm{CO}$ & 27 & 58 & $3.1 E+02$ & $4.5 E+01$ \\
\hline $\mathrm{CO}$ & 27 & 60 & $7.7 E+01$ & $1 \cdot 1 E+01$ \\
\hline N I & 28 & 56 & $1.3 E+01$ & $1.9 E+00$ \\
\hline NI & 28 & 57 & $3 \cdot 3 E+02$ & 4. $7 E+O L$ \\
\hline NI & 28 & 59 & $1.5 E+01$ & $2.2 E+00$ \\
\hline N I & 28 & 63 & $6.8 E+00$ & $9.8 E-01$ \\
\hline N I & 28 & 66 & $1.6 E+02$ & $2.3 E+01$ \\
\hline $\mathrm{CU}$ & 29 & 64 & $4 \cdot 1 E+03$ & $5.9 E+02$ \\
\hline
\end{tabular}

$\begin{array}{lcc}\text { ELEMENT } & \text { ATOMIC } & \text { I SOTOPE } \\ \text { SYMBOL } & \text { NO. I }) & \text { MASS(A) } \\ \text { CU } & & \\ \text { ZN } & 29 & 67 \\ \text { ZN } & 30 & 65 \\ \text { ZN } & 30 & 69 M \\ \text { GA } & 30 & 72 \\ \text { GA } & 31 & 67 \\ \text { GE } & 31 & 72 \\ \text { GE } & 32 & 68 \\ \text { GE } & 32 & 69 \\ \text { AS } & 32 & 71 \\ \text { AS } & 33 & 71 \\ \text { AS } & 33 & 72 \\ \text { AS } & 33 & 73 \\ \text { AS } & 33 & 74 \\ \text { AS } & 33 & 76 \\ \text { SE } & 33 & 77 \\ \text { SE } & 34 & 72 \\ \text { SE } & 34 & 75 \\ \text { BR } & 34 & 79 \\ \text { BR } & 35 & 76 \\ \text { BR } & 35 & 77 \\ \text { KR } & 35 & 82 \\ \text { KR } & 36 & 76 \\ \text { KR } & 36 & 79 \\ \text { KR } & 36 & 81 \\ \text { RB } & 36 & 85 \\ \text { RB } & 37 & 83 \\ \text { RB } & 37 & 84 \\ \text { RB } & 37 & 86 \\ \text { SR } & 37 & 87 \\ \text { SR } & 38 & 82 \\ \text { SR } & 38 & 83 \\ \text { SR } & 38 & 85 \\ \text { SR } & 38 & 89 \\ \text { Y } & 38 & 90 \\ \text { Y } & 39 & 86 \\ Y & 39 & 87 \\ Y & 39 & 87 M \\ Y & 39 & 88 \\ Y & 39 & 90 \\ \text { ZR } & 39 & 91 \\ \text { ZR } & 40 & 86 \\ \text { ZR } & 40 & 88 \\ \text { ZR } & 40 & 89 \\ \text { ZR } & 40 & 93 \\ \text { ZR } & 40 & 95 \\ \text { NB } & 40 & 97 \\ \text { NB } & 41 & 90 \\ \text { NB } & 41 & 91 \\ \text { NB } & 41 & 91 M \\ & 41 & 92\end{array}$

AOULT $U C / M * * 2$

\section{$2 \cdot 5 E+02$}

8. $1 E+03$

1. $5 E+02$

6. $5 E+07$

9. $O E+07$

4. $4 E+01$

$5.8 E+04$

3. $8 E+05$

2. $0 E+03$

2. $2 \mathrm{E}+03$

$7.5 \mathrm{E}+02$

2. $9 E+01$

2. $9 E+03$

$1.1 \mathrm{E}+04$

2. $4 E-01$

3. $7 E-02$

$6.7 E-03$

$3.3 E+02$

$7.5 E+01$

7. $9 E+02$

4. $5 \mathrm{E}+02$

2. $8 \mathrm{E}-01$

$5.5 E-02$

1. $7 E+00$

$1.1 E+00$

3. $8 E+00$

$5.1 E+00$

4. $0 E+00$

1. $7 E+03$

8. $2 E+00$
$7.5 E+00$

7. $5 \mathrm{E}+00$

1. $2 \mathrm{E}+09$

$2.2 E+08$

4. $3 E+09$

4. $9 E+05$

2. $2 E+08$

$3.3 E+06$

$3.4 E+09$

2. $5 E+06$

4. $6 \mathrm{E}+08$

$2.6 E+07$

6. $3 E+06$

5. $0 E+09$

1. $5 \mathrm{E}+04$

1. $1 E+02$

1. $8 E+02$

4. $9 E+02$
CHILO

UCIM**2

3. $5 E+01$

2. $3 E-01$

1. $2 E+03$

2. $2 E+01$

9. $2 E+08$

1. $3 E+07$

$6.2 E+00$

$8.2 E+03$

$5.4 E+04$

$2.8 E+02$

$3.1 \mathrm{E}+02$

$1.1 E+02$

$4.1 E+00$

$4.2 E+02$

1. $5 E+03$

$3.4 E-02$

$5.3 E-03$

$9.6 E-04$

4. $7 E+01$

$1.1 E+01$

G. $O E+00$

.

$1.1 E+02$

6. $\mathrm{DE}+01$

7. BE- 03

2. $5 E-01$

1. $6 E-01$

5. $5 E-01$

7. $2 \mathrm{E}-01$

5. $7 E-01$

$2.4 E+02$

$1.2 E+00$

$1.1 E+00$

4. $6 E-03$

1. $7 E+08$

3. $2 E+07$

6. $1 E+08$

6. $9 E+04$

3. IE+07

3.1E+O

4. TE+OS

4. $9 E+08$

$3.6 E+05$

$6.6 E+07$

3. $8 E+05$

9. $0 E+05$

7. $1 E+08$

2. $2 E+03$

$1.6 E+01$

$2.6 E+01$ 
TABLE 6A. UNIT-RAD DEPOSITION FOR BONE

$\begin{array}{ccccc}\text { ELEMENT } & \text { ATOMIC } & \text { ISOTOPE } & \text { ADULT } & \text { CHILD } \\ \text { SYMBOL } & \text { ND.12) } & \text { MASS }(A) & \text { UC /M*2 } & \text { UC/M**2 } \\ \text { NB } & 41 & 93 M & 1.2 E+02 & 1.7 E+01 \\ \text { NB } & 41 & 94 & 1.1 E+00 & 1.6 E-01 \\ \text { NB } & 41 & 95 & 1.0 E+02 & 1.5 E+01 \\ \text { NB } & 41 & 95 M & 2.4 E+03 & 3.5 E+02 \\ \text { NB } & 41 & 96 & 1.2 E+04 & 1.7 E+03 \\ \text { MO } & 42 & 93 & 3.2 E+01 & 4.6 E+00 \\ \text { MO } & 42 & 99 & 3.3 E+02 & 4.7 E+01 \\ \text { TC } & 43 & 95 & 5.2 E+04 & 7.4 E+03 \\ \text { TC } & 43 & 95 M & 1.2 E+02 & 1.8 E+01 \\ \text { TC } & 43 & 96 & 8.8 E+02 & 1.3 E+02 \\ \text { TC } & 43 & 97 & 3.8 E+03 & 5.4 E+02 \\ \text { TC } & 43 & 97 M & 9.7 E+02 & 1.4 E+02 \\ \text { TC } & 43 & 98 & 5.1 E+01 & 7.3 E+00 \\ \text { TC } & 43 & 99 & 8.5 E+02 & 1.2 E+02 \\ \text { RU } & 44 & 97 & 9.6 E+06 & 1.4 E+06 \\ \text { RU } & 44 & 103 & 1.8 E+05 & 2.5 E+04 \\ \text { RU } & 44 & 106 & 6.0 E+03 & 8.5 E+02 \\ \text { RH } & 45 & 99 & 4.5 E+02 & 6.5 E+01 \\ \text { RH } & 45 & 100 & 1.3 E+04 & 1.8 E+03 \\ \text { RH } & 45 & 101 & 1.7 E+02 & 2.5 E+01 \\ \text { RH } & 45 & 101 M & 3.3 E+03 & 4.7 E+02 \\ \text { RH } & 45 & 102 & 3.9 E+01 & 5.5 E+00 \\ \text { RH } & 45 & 105 & 3.0 E+04 & 4.2 E+03 \\ \text { PD } & 46 & 100 & 2.9 E+03 & 4.2 E+02 \\ \text { PD } & 46 & 103 & 1.3 E+04 & 1.9 E+03 \\ \text { PD } & 46 & 107 & 3.0 E+05 & 4.3 E+04 \\ \text { PD } & 46 & 109 & 2.2 E+05 & 3.1 E+04 \\ \text { PD } & 46 & 112 & 1.7 E+04 & 2.4 E+03 \\ \text { AG } & 47 & 105 & 1.3 E+00 & 1.9 E-01 \\ \text { AG } & 47 & 106 M & 4.8 E+00 & 6.9 E-01 \\ \text { AG } & 47 & 108 M & 6.8 E-02 & 9.8 E-03 \\ \text { AG } & 47 & 110 M & 1.1 E-01 & 1.6 E-02 \\ \text { AG } & 47 & 111 & 3.2 E+01 & 4.6 E+00 \\ \text { CD } & 48 & 109 & 8.1 E+05 & 1.2 E+05 \\ \text { CD } & 48 & 113 M & 3.4 E+05 & 4.9 E+04 \\ \text { CD } & 48 & 115 & 2.1 E+07 & 3.0 E+06 \\ \text { CD } & 48 & 115 M & 3.3 E+05 & 4.8 E+04 \\ \text { IN } & 49 & 111 & 1.4 E+07 & 2.0 E+06 \\ \text { IN } & 49 & 114 M & 1.1 E+05 & 1.5 E+04 \\ \text { IN } & 49 & 115 & 2.2 E+05 & 3.2 E+04 \\ \text { SN } & 50 & 113 & 5.0 E+01 & 7.2 E+00 \\ \text { SN } & 50 & 117 M & 8.7 E+02 & 1.2 E+02 \\ \text { SN } & 50 & 119 M & 1.2 E+02 & 1.7 E+01 \\ \text { SN } & 50 & 121 & 1.9 E+05 & 2.7 E+04 \\ \text { SN } & 50 & 121 M & 2.1 E+01 & 3.0 E+00 \\ \text { SN } & 50 & 123 M & 2.9 E+01 & 4.1 E+00 \\ \text { SN } & 50 & 125 & 2.7 E+02 & 3.9 E+01 \\ \text { SN } & 50 & 126 & 8.1 E-01 & 1.2 E-01 \\ \text { SB } & 51 & 119 & 2.5 E+06 & 3.6 E+05 \\ \text { SB } & 51 & 120 M & 5.2 E+03 & 7.4 E+02 \\ & & & & \end{array}$

VIA MILK.

05/09/68 PAGE 2

$\begin{array}{lcc}\text { ELEMENT } & \text { ATOMIC } & \text { ISOTOPE } \\ \text { SYNBOL } & \text { NO. (Z) } & \text { MASSIA) } \\ & & \\ \text { SB } & 51 & 122 \\ \text { SB } & 51 & 124 \\ \text { SB } & 51 & 125 \\ \text { SB } & 51 & 126 \\ \text { SB } & 51 & 127 \\ \text { TE } & 52 & 118 \\ \text { TE } & 52 & 119 \\ \text { TE } & 52 & 119 M \\ \text { TE } & 52 & 121 \\ \text { TE } & 52 & 121 M \\ \text { TE } & 52 & 123 \\ \text { TE } & 52 & 123 M \\ \text { TE } & 52 & 125 M \\ \text { TE } & 52 & 127 M \\ \text { TE } & 52 & 129 M \\ \text { TE } & 52 & 131 M \\ \text { TE } & 52 & 132 \\ \text { I } & 53 & 123 \\ \text { I } & 53 & 124 \\ \text { I } & 53 & 125 \\ \text { I } & 53 & 126 \\ \text { I } & 53 & 129 \\ \text { I } & 53 & 130 \\ \text { I } & 53 & 131 \\ \text { I } & 53 & 133 \\ \text { XE } & 54 & 122 \\ \text { XE } & 54 & 125 \\ \text { XE } & 54 & 127 \\ \text { XE } & 54 & 129 M \\ \text { XE } & 54 & 131 M \\ \text { XE } & 54 & 133 \\ \text { XE } & 54 & 133 M \\ \text { CS } & 55 & 129 \\ \text { CS } & 55 & 131 \\ \text { CS } & 55 & 132 \\ \text { CS } & 55 & 134 \\ \text { CS } & 55 & 135 \\ \text { CS } & 55 & 136 \\ \text { CS } & 55 & 137 \\ \text { BA } & 56 & 128 \\ \text { BA } & 56 & 131 \\ \text { BA } & 56 & 133 \\ \text { BA } & 56 & 133 M \\ \text { BA } & 56 & 135 M \\ \text { BA } & 56 & 140 \\ \text { LA } & 57 & 135 \\ \text { LA } & 57 & 137 \\ \text { LA } & 57 & 138 \\ \text { LA } & 57 & 140 \\ \text { CE } & 58 & 134 \\ & & \end{array}$

ADULT CHILD $U C / M * 2 \quad U C / M * 2$

4. $1 E+04 \quad 5.9 E+03$

2. $9 E+02 \quad 4.1 E+01$

4. $4 E+02 \quad 6.3 E+01$

$1.4 E+03 \quad 2.1 E+02$

2. $0 E+03 \quad 2.8 E+02$

7. $3 E+04 \quad 1.0 E+04$

1. $9 E+03 \quad 2.7 E+02$

1. $2 E+03 \quad 1.7 E+02$

2. $9 E+01$

6. $5 \mathrm{E}+02$

1. $0 E+02$

$2.6 \mathrm{E}+02$

$8 \cdot 3 E+01$

5. $7 \mathrm{E}+01$

2.6E+03

3. $4 E+02$

2. $2 E+03$

5. $7 E+00$

1. $5 \mathrm{E}+01$

5. $5 \mathrm{E}+00$

.

$2.2 E+02$

$2.2 E+02$
$8.5 E+00$

$1.6 E+02$

$6.4 E+01$

$3 \cdot 5 E+02$

1. $2 E+00$

$1.1 E+01$

$8.2 E+00$

$2.8 E+01$

$5.5 E+01$

1. $6 E+02$

$8.4 E+01$

7. $8 \mathrm{E}+00$

5. $9 E-02$

$1.8 \mathrm{E}+00$

8. $2 \mathrm{E}-01$

$1.2 \mathrm{E}-01$

$7.5 \mathrm{E}+02$

$1.3 \mathrm{E}+02$

$2.6 E+01$

$5.8 E+03$

$2.6 E+04$

$\begin{array}{ll}2.4 E+02 & 3.4 E+01 \\ 1.2 E+10 & 1.7 E+09\end{array}$

$\begin{array}{ll}2.4 E+02 & 3.4 E+01 \\ 1.2 E+10 & 1.7 E+09\end{array}$

1. $3 E+07 \quad 1.8 E+06$

3. $7 E+05$

5. $3 E+04$

$\begin{array}{ll}8.5 E+08 & 1.2 E+08 \\ 2.2 E+08 & 3.1 E+07\end{array}$
2. $1 E+04 \quad 2.9 E+03$ 


$\begin{array}{lcccc}\text { ELEMENT } & \text { ATOMIC } & \text { ISOTOPE } & \text { ADULT } & \text { CHILD } \\ \text { SYMBOL } & \text { NO.12) } & \text { MASS(A) } & \text { UC /M**2 } & \text { UC/M**2 } \\ \text { CE } & 58 & 135 & 4.4 E+09 & 6.3 E+08 \\ \text { CE } & 58 & 137 M & 3.3 E+09 & 4.7 E+08 \\ \text { CE } & 58 & 139 & 6.6 E+06 & 9.5 E+05 \\ \text { CE } & 58 & 141 & 2.8 E+07 & 4.0 E+06 \\ \text { CE } & 58 & 142 & 9.3 E+04 & 1.3 E+04 \\ \text { CE } & 58 & 143 & 1.1 E+09 & 1.5 E+08 \\ \text { CE } & 58 & 144 & 1.9 E+05 & 2.7 E+04 \\ \text { PR } & 59 & 142 & 9.3 E+09 & 1.3 E+09 \\ \text { PR } & 59 & 143 & 1.9 E+08 & 2.7 E+07 \\ \text { ND } & 60 & 140 & 7.0 E+08 & 1.0 E+08 \\ \text { ND } & 60 & 144 & 2.0 E+05 & 2.9 E+04 \\ \text { ND } & 60 & 147 & 4.1 E+07 & 5.8 E+06 \\ \text { PM } & 61 & 143 & 6.7 E+06 & 9.6 E+05 \\ \text { PM } & 61 & 144 & 6.9 E+05 & 9.8 E+04 \\ \text { PM } & 61 & 145 & 7.7 E+06 & 1.1 E+06 \\ \text { PM } & 61 & 146 & 8.1 E+05 & 1.2 E+05 \\ \text { PM } & 61 & 147 & 4.0 E+05 & 5.8 E+04 \\ \text { PM } & 61 & 148 & 2.3 E+08 & 3.3 E+07 \\ \text { PM } & 61 & 148 M & 3.2 E+06 & 4.5 E+05 \\ \text { PM } & 61 & 149 & 4.1 E+09 & 5.8 E+08 \\ \text { PM } & 61 & 151 & 5.4 E+09 & 7.7 E+08 \\ \text { SM } & 62 & 141 & 1.1 E+07 & 1.6 E+06 \\ \text { SM } & 62 & 145 & 1.3 E+07 & 1.8 E+06 \\ \text { SM } & 62 & 146 & 1.4 E+05 & 2.0 E+04 \\ \text { SM } & 62 & 147 & 1.6 E+05 & 2.3 E+04 \\ \text { SM } & 62 & 148 & 9.3 E+04 & 1.3 E+04 \\ \text { SM } & 62 & 149 & 2.0 E+05 & 2.8 E+04 \\ \text { SM } & 62 & 151 & 2.5 E+07 & 3.6 E+06 \\ \text { SM } & 62 & 153 & 6.4 E+09 & 9.2 E+08 \\ \text { EU } & 63 & 145 & 3.7 E+08 & 5.4 E+07 \\ \text { EU } & 63 & 146 & 9.1 E+07 & 1.3 E+07 \\ \text { EU } & 63 & 146 M & 9.4 E+08 & 1.4 E+08 \\ \text { EU } & 63 & 147 & 1.1 E+07 & 1.5 E+06 \\ \text { EU } & 63 & 148 & 2.1 E+07 & 3.0 E+06 \\ \text { EU } & 63 & 149 & 9.7 E+06 & 1.4 E+06 \\ \text { EU } & 63 & 150 & 5.8 E+05 & 8.3 E+04 \\ \text { EU } & 63 & 150 M & 5.8 E+09 & 8.2 E+08 \\ \text { EU } & 63 & 152 & 3.4 E+05 & 4.9 E+04 \\ \text { EU } & 63 & 154 & 2.8 E+05 & 4.0 E+04 \\ \text { EU } & 63 & 155 & 9.9 E+06 & 1.4 E+06 \\ \text { EU } & 63 & 156 & 3.7 E+07 & 5.3 E+06 \\ \text { EU } & 63 & 157 & 1.6 E+10 & 2.2 E+09 \\ \text { GD } & 64 & 146 & 2.1 E+06 & 3.1 E+05 \\ \text { GD } & 64 & 147 & 1.3 E+09 & 1.8 E+08 \\ \text { GD } & 64 & 148 & 1.4 E+05 & 2.0 E+04 \\ \text { GD } & 64 & 149 & 1.0 E+08 & 1.4 E+07 \\ \text { GD } & 64 & 150 & 8.1 E+04 & 1.2 E+04 \\ \text { GD } & 64 & 151 & 1.2 E+07 & 1.8 E+06 \\ \text { GD } & 64 & 152 & 2.0 E+05 & 2.8 E+04 \\ \text { GD } & 64 & 153 & 1.5 E+07 & 2.2 E+06\end{array}$

$\begin{array}{cc}\text { ELEMENT } & \text { ATDMI } \\ \text { SYMBOL } & \text { ND. I } \\ \text { GD } & \\ \text { GD } & 64 \\ \text { TB } & 64 \\ \text { TB } & 65 \\ \text { TB } & 65 \\ \text { TB } & 65 \\ \text { TB } & 65 \\ \text { TB } & 65 \\ \text { TB } & 65 \\ \text { TB } & 65 \\ \text { TB } & 65 \\ \text { TB } & 65 \\ \text { TB } & 65 \\ \text { DY } & 65 \\ \text { DY } & 66 \\ \text { DY } & 66 \\ \text { DY } & 66 \\ \text { DY } & 66 \\ \text { HO } & 66 \\ \text { HO } & 67 \\ \text { HO } & 67 \\ \text { ER } & 67 \\ \text { ER } & 68 \\ \text { ER } & 68 \\ \text { TM } & 68 \\ \text { TM } & 69 \\ \text { TM } & 69 \\ \text { TM } & 69 \\ \text { TM } & 69 \\ \text { TM } & 69 \\ \text { YB } & 69 \\ \text { YB } & 70 \\ \text { YB } & 70 \\ \text { LU } & 70 \\ \text { LU } & 71 \\ \text { LU } & 71 \\ \text { LU } & 71 \\ \text { LU } & 71 \\ \text { LU } & 71 \\ \text { LU } & 71 \\ \text { LU } & 71 \\ \text { LU } & 71 \\ \text { LU } & 71 \\ \text { HF } & 71 \\ \text { HF } & 72 \\ \text { HF } & 72 \\ \text { HF } & 72 \\ \text { HF } & 72 \\ \text { HF } & 72 \\ \text { HF } & 72 \\ & \end{array}$

I SOTOPE
MASS (A)

ADULT UC $/ M * 2$

CHILD

$\begin{array}{ll}64 & 159 \\ 64 & 162 \\ 65 & 151 \\ 65 & 152\end{array}$

2. $7 E+1$ $1.1 E+06$

$2.4 \mathrm{E}+09$

$1.8 \mathrm{E}+09$

8. $8 \mathrm{E}+09$

$4.8 E+0 B$

$1.4 E+08$

$6.5 E+06$

2. $8 E+05$

$4.1 E+06$

$7.2 E+08$

$1.2 E+10$

3. $9 E+04$

4. $7 E+09$

6. $2 E+04$

4. $2 E+07$

$4.2 E+08$

$5.5 E+06$

3. $8 E+09$

2. $5 E+05$

1. $2 E+09$

9. $0 E+08$

9. $6 \mathrm{E}+08$

1. $2 \mathrm{E}+09$

$1.3 E+08$

2. $8 \mathrm{E}+06$

7. $9 E+06$

$3.7 E+07$

$7.4 E+0 B$

1. $8 \mathrm{E}+09$

4. $2 E+07$

$1.9 E+09$

$1.1 E+09$

3. $7 E+08$

6. $8 E+08$

$1.4 E+08$

$4.0 E+05$

$4.0 E+06$

$4.0 E+06$

7. $6 E+08$

7. $6 E+08$
$1.1 E+07$

1. $1 E+07$
$1.7 E+10$

$3.4 E+10$

2. $5 E+06$

1. $1 E+10$

8. $5 E+05$

4. $9 E+07$

5. $6 E+07$
3. $9 E+09$ $1.6 E+05$ 3. $4 E+08$ 2. $5 E+08$ 1. $3 E+09$ 6. $8 \mathrm{E}+07$ $6.8 E+07$
$2.1 E+07$ $9.3 E+03$ 4. $0 E+04$ $5.9 E+05$

$1.0 E+08$

1. $7 E+09$

$5.6 \mathrm{E}+03$ $6.7 E+0 B$

$8.8 E+03$

$6.0 E+O S$

$6.0 E+07$

$4 E+08$

$3.5 E+04$

$1.7 E+0 B$

1. $3 E+08$

1. $4 \mathrm{E}+08$

1. $7 E+08$

1. $8 E+07$

4. $0 E+0$ S

$1.15+06$

$5.2 E+06$

$1.1 E+08$

2. $5 E+08$

$5.9 E+00$

2. $8 E+08$

$1.5 E+08$

5. $3 E+07$

9. $8 \mathrm{E}+07$

1. $9 E+07$

1. $7 E+05$

$5.7 \mathrm{E}+04$

5.
$5.7 E+04$
$5.7 E+05$

5 .

S.6E+04

$1.6 \mathrm{E}+06$

2. $5 E+09$

4. $9 E+09$

$3.5 E+05$

l. $2 E+05$

$7.0 E+05$

$8.0 E+06$ 


\begin{tabular}{|c|c|c|c|c|}
\hline $\begin{array}{l}\text { EL EMENT } \\
\text { SYMBOL }\end{array}$ & $\begin{array}{l}\text { ATOMIC } \\
\text { NO. }(Z)\end{array}$ & $\begin{array}{l}\text { I SOTOPE } \\
\text { MASS (A) }\end{array}$ & $\begin{array}{c}\text { ADULT } \\
\text { UC / M**2 }\end{array}$ & $\begin{array}{l}\text { CHILD } \\
U C / M * * 2\end{array}$ \\
\hline HF & 72 & 182 & $1.3 E+06$ & $1.8 E+05$ \\
\hline TA & 73 & 177 & $1.0 E+03$ & $1.5 E+02$ \\
\hline TA & 73 & 179 & $3.0 E+01$ & $4.3 E+00$ \\
\hline TA & 73 & 182 & $3.4 E+00$ & $4.9 \mathrm{E}-01$ \\
\hline TA & 73 & 183 & $4.6 E+02$ & $6.6 E+01$ \\
\hline$W$ & 74 & 178 & $4.1 E+03$ & $5.8 E+02$ \\
\hline$W$ & 74 & 181 & $5.2 E+04$ & $7.4 E+03$ \\
\hline$W$ & 74 & 185 & 3. $3 E+04$ & 4. $7 E+03$ \\
\hline$W$ & 74 & 187 & $5.4 E+05$ & $7.8 E+04$ \\
\hline$w$ & 74 & 188 & $3.1 E+03$ & $4.4 E+02$ \\
\hline RE & 75 & 180 & 1. $1 E+04$ & $1.6 E+03$ \\
\hline RE & 75 & 181 & $1.2 E+04$ & $1.7 E+03$ \\
\hline RE & 75 & 182 & 2. $1 E+03$ & $3.0 E+02$ \\
\hline RE & 75 & $182 M$ & 2. $9 E+04$ & $4.1 E+03$ \\
\hline RE & 75 & 183 & $8.3 E+02$ & $1.2 E+02$ \\
\hline RE & 75 & 184 & 3. $3 E+02$ & $4.8 E+01$ \\
\hline RE & 75 & $184 M$ & 2. $O E+02$ & 2. $8 E+01$ \\
\hline RE & 75 & 186 & $4.5 E+03$ & $6.4 E+02$ \\
\hline RE & 75 & 187 & 1. $7 E+06$ & $2.4 E+05$ \\
\hline RE & 75 & 188 & $2.5 E+04$ & $3.6 E+03$ \\
\hline $\mathrm{RE}$ & 75 & 189 & 3. $2 E+04$ & $4.6 E+03$ \\
\hline OS & 76 & 182 & $1.6 E+03$ & $2.3 E+02$ \\
\hline OS & 76 & 183 & $1.2 E+04$ & 1. $8 E+03$ \\
\hline os & 76 & 185 & $3.1 E+00$ & $4.4 E-01$ \\
\hline OS & 76 & 191 & 1. $2 E+02$ & $1.8 E+01$ \\
\hline OS & 76 & $191 \mathrm{M}$ & 3. $0 E+04$ & $4.3 E+03$ \\
\hline OS & 76 & 193 & $3.4 E+03$ & $4.9 E+02$ \\
\hline OS & 76 & 194 & 4. $2 E-02$ & $6.0 E-03$ \\
\hline IR & 77 & 185 & $6.6 E+03$ & $9.4 E+02$ \\
\hline IR & 77 & 186 & $3.8 E+03$ & $5.5 E+02$ \\
\hline IR & 77 & 187 & 1. $.0 E+04$ & $1.5 E+03$ \\
\hline IR & 77 & 188 & 4. $5 E+02$ & $6.5 E+01$ \\
\hline IR & 77 & 189 & $8.4 E+01$ & $1.2 E+01$ \\
\hline IR & 77 & 190 & $2.5 E+01$ & $3.5 E+00$ \\
\hline IR & 77 & 192 & $2.9 E+00$ & $4.2 E-01$ \\
\hline IR & 77 & $192 \mathrm{M}$ & 2. $2 E-02$ & 3. $1 \mathrm{E}-03$ \\
\hline IR & 77 & $193 M$ & 4. $3 E+02$ & $6.2 E+01$ \\
\hline IR & 77 & 194 & 3. $6 E+03$ & 5. $1 E+02$ \\
\hline PT & 78 & 188 & $1.6 E+01$ & $2.3 E+00$ \\
\hline PT & 78 & 190 & 8. $2 E-03$ & $1.2 \mathrm{E}-03$ \\
\hline PT & 78 & 191 & 6. $7 E+02$ & $9.6 E+01$ \\
\hline PT & 78 & 192 & $9.8 E-03$ & $1.4 E-03$ \\
\hline PT & 78 & 193 & 3. $4 E-01$ & $4.9 E-02$ \\
\hline PT & 78 & $193 M$ & $8.1 E+02$ & $1.2 E+02$ \\
\hline PT & 78 & $195 \mathrm{M}$ & 7. $9 E+02$ & 1. $1 E+02$ \\
\hline PT & 78 & 197 & $1.4 E+04$ & $2.0 E+03$ \\
\hline AU & 79 & 193 & $8.4 E+03$ & $1.2 E+03$ \\
\hline AU & 79 & 194 & $5.4 E+02$ & $7.7 E+01$ \\
\hline$A U$ & 79 & 195 & 5. $4 E+00$ & $7.7 \mathrm{E}-01$ \\
\hline$A U$ & 79 & 196 & $1.4 E+02$ & 2. $.0 E+O 1$ \\
\hline
\end{tabular}

ELEMENT
SYMBOL
AU
AU
$H G$
$H G$
HG
HG
HG
TL
TL
$T L$
$T L$
TL
$P B$
$P B$
$P B$
$P B$
$P B$
$P B$
$B I$
$B I$
$B I$
$B I$
$B I$
$B I$
$P O$
$P O$
$P O$
$P O$
$P O$
$R N$
$R N$
$R A$
$R A$
$R A$
$R A$
$R A$
$R A$
$A C$
$A C$
$T H$
$T H$
$T H$
$T H$
$T H$
$T H$
$T H$
$P A$
$P A$
$P A$
$P A$
$P A$
$P A$
$U$

\section{$\begin{array}{ll}\text { ATOMIC } & \text { ISOTOPE } \\ \text { NO. }(Z) & \text { MASS(A) }\end{array}$}

\section{8}

154

$195 \mathrm{M}$

197

$197 M$

203

200

202

$2 \mathrm{C} 4$

200

$2 \mathrm{C2}$

203

$2 \mathrm{C} 4$

205
210

205

$2 \mathrm{C} 6$

$2 \mathrm{CB}$

$2 \mathrm{Cg}$

$210 \mathrm{M}$

$2 \mathrm{C6}$

$2 \mathrm{C} 8$

2 C9

210

222

222

224

224

225

228

225

227

227

228

229

230

231

232
234

234
228

229

230

231

232

233
230
$05 / 09 / 58$

$\begin{array}{cc}\text { ADULT } & \text { CHILD } \\ U C / M * * 2 & U C / M * * 2\end{array}$

$5.8 E+02 \quad 8.3 E+01$

$1.4 E+03 \quad 2.0 E+02$

$1.2 \mathrm{E}-02$

1. $2 E+01 \quad 1.6 E+00$

$\begin{array}{ll}2.2 E+01 & 3.2 E+00 \\ 7.9 E+01 & 1.1 E+01\end{array}$

$\begin{array}{ll}\text { 7. } 9 E+01 & 1.1 E+01 \\ 2.9 E-01 & 4.1 E-02\end{array}$

$1.6 E+03 \quad 2.2 E+02$

$1.4 E+03 \quad 1.9 E+02$

8. $1 E+01 \quad 1.2 E+01$

1. $6 E+01$ 2. $3 E+00$

$8.4 E+04 \quad 1.2 E+04$

4. $1 E+00 \quad 5.8 E-01$

3. $5 E+04$

9. $3 E-01$

2. $8 E+01$

5. $6 E-01$

8. $8 E+04$

2. $7 E+05$

$1.8 E+04$

1. $2 E+05$

1. $2 E+05$

8. $6 E+03$

6. $9 E+02$

3. $0 E+01$

4. $4 \mathrm{E}+01$

$5.4 E+01$

$1.6 E+02$

2. $2 E+00$

2. $9 E-01$

1. $9 E+00$

2. $0 E-01$

2. 3E-04

6. $6 \mathrm{E}-04$

$5.4 E+06$

4. $1 E+03$

7. $8 E+05$

1. $2 E+04$

1. $1 E+03$

1. $1 E+03$

1. $1 E+03$

$9.2 E+07$

9. $5 E+02$

4. $2 E+05$

2. $1 E+O B$

8. $7 E+07$

1. $2 E+06$

1. $5 E+03$

9. $2 E+07$

$6.1 E+05$

3. $8 E+02$

5. $0 E+03$

1. $3 E-01$

4. $O E+O O$

8. $0 E-02$

1. $3 E+04$

3. $8 E+04$

3. $7 E+03$

2. $5 E+03$

1. $7 E+04$

1. $2 E+03$

$9.9 E+01$

4. $2 E+00$

6. $4 E+00$

7. $7 \mathrm{E}+00$

2. $3 E+01$

3. $2 \mathrm{E}-01$

4. $1 \mathrm{E}-02$

$2.8 E-01$

2. $8 E-02$

3. $2 E-05$

9. $4 \mathrm{E}-0 \mathrm{~S}$

7. $7 E+05$

5. $8 E+02$

1. $1 E+05$

1. $7 \mathrm{E}+03$

1. $6 E+02$

1. $5 E+02$

$1.5 E+02$

1. $3 E+07$

$1.4 E+02$

$6.1 E+04$

3. $0 E+07$

1. $2 E+07$

1. $7 \mathrm{E}+05$

$2.2 E+02$

1. $3 E+07$

$8-7 E+04$

$5.4 E+01$ 


\begin{tabular}{|c|c|c|c|c|}
\hline $\begin{array}{l}\text { ELEMENT } \\
\text { SYMBOL }\end{array}$ & $\begin{array}{l}\text { ATOMIC } \\
\text { NO. }(Z)\end{array}$ & $\begin{array}{l}\text { I SOTOPE } \\
\text { MASS (A) }\end{array}$ & $\begin{array}{c}\text { ADULT } \\
\text { UC } / M * * 2\end{array}$ & $\begin{array}{c}\text { CHILD } \\
U C / M * * 2\end{array}$ \\
\hline$u$ & 92 & 231 & $4.6 E+03$ & $6.6 E+02$ \\
\hline u & 92 & 232 & $1.5 E+01$ & $2.1 E+00$ \\
\hline u & 92 & 233 & $1.5 E+01$ & $2.2 \mathrm{E}+00$ \\
\hline$u$ & 92 & 234 & $1.4 E+01$ & $2.0 E+00$ \\
\hline$u$ & 92 & 235 & $1 \cdot 3 E+01$ & $1.9 E+00$ \\
\hline$u$ & 92 & 236 & $1 \cdot 3 E+01$ & $1.9 E+00$ \\
\hline$u$ & 92 & 237 & 1. $8 E+03$ & $2.6 E+02$ \\
\hline u & 92 & 238 & $1.2 E+01$ & $1.8 E+00$ \\
\hline$u$ & 92 & 240 & $1.4 E+05$ & $2.1 E+04$ \\
\hline NP & 93 & 234 & 7. $9 E+06$ & $1.1 E+06$ \\
\hline NP & 93 & 235 & 2. $O E+04$ & $2.9 E+03$ \\
\hline NP & 93 & 236 & 1. $1 E+03$ & 1. $5 E+02$ \\
\hline NP & 93 & $236 M$ & $1.4 E+08$ & $2.0 E+07$ \\
\hline NP & 93 & 237 & 1. $1 E+03$ & $1.6 E+02$ \\
\hline NP & 93 & 238 & 2. $8 E+07$ & $3.9 E+06$ \\
\hline NP & 93 & 239 & $2.1 E+07$ & $3.0 E+06$ \\
\hline PU & 94 & 236 & 5. $7 E+04$ & $8.2 E+03$ \\
\hline PU & 94 & 237 & $1.7 E+06$ & $2.5 E+05$ \\
\hline PU & 94 & 238 & 8. $9 E+03$ & $1.3 E+03$ \\
\hline PU & 94 & 239 & $7.5 E+03$ & $1.1 E+03$ \\
\hline PU & 94 & 240 & $7.4 E+03$ & $1.1 E+03$ \\
\hline PU & 94 & 241 & $1.5 E+04$ & $2.1 E+03$ \\
\hline PU & 94 & 242 & $7.2 E+03$ & $1.0 E+03$ \\
\hline PU & 94 & 244 & $6.4 E+03$ & $9.2 E+02$ \\
\hline PU & 94 & 246 & $9.6 E+06$ & $1.4 E+06$ \\
\hline AM & 95 & 239 & $9.0 E+08$ & $1.3 E+0 B$ \\
\hline AM & 95 & 240 & $5 \cdot 3 E+07$ & $7.6 E+06$ \\
\hline AM & 95 & 241 & $2.2 E+03$ & $3.2 E+02$ \\
\hline AM & 95 & 242 & 4. $8 E+08$ & $6.9 E+07$ \\
\hline$A M$ & 95 & $242 M$ & $2.2 E+03$ & $3.1 E+02$ \\
\hline$A M$ & 95 & 243 & $2.0 E+03$ & $2.8 E+02$ \\
\hline
\end{tabular}

$\begin{array}{lr}\text { ELEMENT } & \text { ATOMIC } \\ \text { SYMBOL } & \text { NO. } 12 \\ \text { CM } & 96 \\ \text { CM } & 96 \\ \text { CM } & 96 \\ \text { CM } & 96 \\ \text { CM } & 96 \\ \text { CM } & 96 \\ \text { CM } & 96 \\ \text { CM } & 96 \\ \text { CM } & 96 \\ \text { BK } & 97 \\ \text { BK } & 97 \\ \text { BK } & 97 \\ \text { BK } & 97 \\ \text { BK } & 97 \\ \text { CF } & 98 \\ \text { CF } & 98 \\ \text { CF } & 98 \\ \text { CF } & 98 \\ \text { CF } & 98 \\ \text { CF } & 98 \\ \text { CF } & 98 \\ \text { ES } & 99 \\ \text { ES } & 99 \\ \text { ES } & 99 \\ \text { ES } & 99 \\ \text { ES } & 99 \\ \text { ES } & 99 \\ \text { FM } & 100 \\ \text { FM } & 100 \\ \text { FM } & 100 \\ \text { FM } & 100 \\ & \end{array}$

ISOTOPE

240

241

242

243
244

244
245

245
246

247

248

245

246

247

248

249

246

248

249

250

251

253

253
251

252

253

254

$254 M$

255

252

253
255

255
257
ADULT
UC/M**

$7 . O E+05$

7. $O E+05$
5. $1 E+05$

5. $1 E+05$
8. $1 E+04$

2. $2 E+03$

2. $7 E+03$

1. $6 E+03$

1. $6 E+03$

1. $5 E+03$

1. $4 E+03$

$E+07$

2. $2 E+08$

7. $0 E+08$

1. $5 \mathrm{E}+05$

3. $1 E+08$

1. $3 E+05$

6. $2 E+03$

$1.4 E+04$

5. $6 E+03$

4. $3 E+04$

3. $6 E+06$

2. $7 \mathrm{E}+08$

3. $0 E+05$

3. $3 E+06$

8. $1 E+04$

$2.2 E+0 B$

2. $4 E+06$

6. $3 E+08$

3. $6 E+07$

3. $6 E+07$
7. $7 E+08$

5. $3 E+05$
CHILD
$U C / M * * 2$

1. $O E+05$

7. $3 E+04$

l. $2 \mathrm{E}+04$

1. $2 E+04$
3. $1 E+02$

3. $8 \mathrm{E}+02$

2. $3 E+02$

$2.2 E+02$

$2.1 E+02$

2. $0 E+02$

5. $3 E+06$

3. $2 E+07$

$8.8 E+02$

1. $0 E+08$

$2.1 E+04$

4. $5 E+07$

1. $8 \mathrm{E}+04$

8. $9 E+02$

2. $0 E+03$

8. $O E+D 2$

$6.1 E+03$

5. $1 E+05$

3. $9 E+07$

$4 \cdot 3 E+04$

4. $8 E+05$

1. $2 E+04$

$3.4 E+05$

9. $0 E+07$

9. $0 E+07$

5. $1 E+06$
1. $1 E+08$

1. $1 E+08$
$7.6 E+04$ 
TABLE 6A. UNIT-RAD DEPOSITION FOR KIDNEYS VIA MILK.

05/09/58 PAGE 0

\begin{tabular}{|c|c|c|c|c|}
\hline $\begin{array}{l}\text { ELEMENT } \\
\text { SYMBOL }\end{array}$ & $\begin{array}{l}\text { ATOMIC } \\
\text { NO. }(2)\end{array}$ & $\begin{array}{l}\text { I SOTOPE } \\
\text { MASS (A) }\end{array}$ & $\begin{array}{c}\text { ADULT } \\
\text { UC/M**2 }\end{array}$ & $\begin{array}{c}\text { CHILD } \\
\text { UC/M**2 }\end{array}$ \\
\hline $\mathrm{H}$ & 1 & 3 & 7. $0 E+02$ & $1.4 E+02$ \\
\hline BE & 4 & 7 & $5.3 E+05$ & $1.0 E+05$ \\
\hline$B E$ & 4 & 10 & $2-2 E+04$ & $4.3 E+03$ \\
\hline$C$ & 6 & 14 & $1-1 E+02$ & $2.2 E+01$ \\
\hline NA & 11 & 22 & $8.2 E-01$ & $1.6 E-01$ \\
\hline NA & 11 & 24 & 1. $7 E+02$ & $3.2 E+01$ \\
\hline MG & 12 & 28 & $1.6 E+01$ & $3.0 E+0 O$ \\
\hline$A L$ & 13 & 26 & $3.9 E-03$ & $7.6 \mathrm{E}-04$ \\
\hline SI & 14 & 32 & $6.6 E-03$ & $1.3 E-03$ \\
\hline $\mathbf{P}$ & 15 & 32 & $2.1 E+00$ & $4.1 E-01$ \\
\hline $\mathbf{P}$ & 15 & 33 & $1.4 E+01$ & $2.8 E+00$ \\
\hline$S$ & 16 & 35 & $2.4 E+01$ & $4.6 E+00$ \\
\hline $\mathrm{CL}$ & 17 & 36 & $2.2 E+00$ & 4. $2 \mathrm{E}-01$ \\
\hline$A R$ & 18 & 37 & $7.4 E+02$ & $1.4 E+02$ \\
\hline AR & 18 & 39 & $2.8 E-02$ & $5.5 E-03$ \\
\hline AR & 18 & 42 & 3. $1 E-03$ & $6.0 E-04$ \\
\hline K & 19 & 40 & $2.2 E+00$ & 4. $3 E-01$ \\
\hline K & 19 & 42 & $2 \cdot 1 E+03$ & $4.0 E+02$ \\
\hline K & 19 & 43 & $1 \cdot 3 E+03$ & $2.5 E+02$ \\
\hline$C A$ & 20 & 41 & $1.6 E+03$ & $3.0 E+02$ \\
\hline CA & 20 & 45 & $7.5 E+01$ & $1.4 E+01$ \\
\hline CA & 20 & 47 & $5.5 E+01$ & $1 \cdot 1 E+O L$ \\
\hline CA & 20 & 48 & $1.5 E+00$ & $3.0 \mathrm{E}-0 \mathrm{I}$ \\
\hline SC & 21 & $44 M$ & $4.4 E+08$ & $8.6 E+07$ \\
\hline SC & 21 & 46 & 3. $1 E+06$ & $6.1 E+05$ \\
\hline SC & 21 & 47 & $2 \cdot 2 E+09$ & $4.2 E+0 B$ \\
\hline SC & 21 & 48 & $6.0 E+08$ & $1 \cdot 2 E+08$ \\
\hline TI & 22 & 44 & 5. $3 E+02$ & 1. $O E+02$ \\
\hline v & 23 & 48 & 2. $7 E+02$ & $5 \cdot 2 E+01$ \\
\hline v & 23 & 49 & 2. $0 E+04$ & $3.9 E+03$ \\
\hline$v$ & 23 & 50 & $6.2 E+01$ & $1.2 E+01$ \\
\hline CR & 24 & 48 & $7.8 E+05$ & $1.5 E+05$ \\
\hline CR & 24 & 51 & 2. $7 E+05$ & $5.2 E+04$ \\
\hline MN & 25 & 52 & $7.7 E+02$ & $1.5 E+02$ \\
\hline MN & 25 & 53 & $2.5 E+04$ & $4.8 E+03$ \\
\hline$M N$ & 25 & 54 & $2.0 E+02$ & $3.9 E+01$ \\
\hline FE & 26 & 55 & 4. $8 E+02$ & $9.3 E+01$ \\
\hline FE & 26 & 59 & 3. $0 E+01$ & $5.8 E+00$ \\
\hline FE & 26 & 60 & $6.6 E-01$ & $1.3 E-01$ \\
\hline $\mathrm{CO}$ & 27 & 55 & 1. $7 E+02$ & $3.3 E+01$ \\
\hline $\mathrm{CO}$ & 27 & 56 & $4.7 E-01$ & $9.2 E-02$ \\
\hline $\mathrm{CO}$ & 27 & 57 & 5. $1 E+00$ & $1.0 E+00$ \\
\hline CO & 27 & 58 & 1. $O E+O O$ & $2.0 E-01$ \\
\hline $\mathrm{CO}$ & 27 & 60 & 2. $5 E-01$ & $4.8 E-02$ \\
\hline NI & 28 & 56 & $3.2 E-01$ & $6 \cdot 3 E-02$ \\
\hline NI & 28 & 57 & $7.9 E+00$ & $1.5 E+00$ \\
\hline NI & 28 & 59 & 4. $0 E-02$ & $7.7 \mathrm{E}-03$ \\
\hline NI & 28 & 63 & 1. $9 E-02$ & $3.7 E-03$ \\
\hline NI & 28 & 66 & 3. $9 E+00$ & $7.5 \mathrm{E}-01$ \\
\hline $\mathrm{CU}$ & 29 & 64 & $4.8 E+03$ & $9.3 E+02$ \\
\hline
\end{tabular}

\begin{tabular}{|c|c|c|c|c|c|}
\hline $\begin{array}{l}\text { EL.EMENT } \\
\text { SYMBOL }\end{array}$ & $\begin{array}{l}\text { ATOMIC } \\
\text { NO. }(Z)\end{array}$ & $\begin{array}{l}\text { ISOTOPE } \\
\text { MASS (A) }\end{array}$ & $\begin{array}{c}\text { ADULT } \\
U C / M * 2\end{array}$ & $\begin{array}{c}C H I L D \\
U C / M * * 2\end{array}$ & \\
\hline $\mathrm{CU}$ & 29 & 67 & 2. $9 E+02$ & 5. $7 E+01$ & \\
\hline$Z N$ & 30 & 65 & 6. $5 E-01$ & $1.3 E-01$ & \\
\hline $\mathrm{ZN}$ & 30 & $69 M$ & $1.5 E+03$ & $2.9 E+02$ & \\
\hline$Z N$ & 30 & 72 & $2.8 E+01$ & $5.5 E+00$ & \\
\hline GA & 31 & 67 & 5. $0 E+07$ & $9.8 E+0 S$ & \\
\hline GA & 31 & 72 & 6. $6 E+07$ & $1.3 E+07$ & $\mapsto$ \\
\hline GE & 32 & 68 & 1. $7 E+03$ & $3.3 E+02$ & ڤँ \\
\hline $\mathrm{GE}$ & 32 & 69 & 1. $1 E+05$ & $2.1 E+04$ & $\breve{D}$ \\
\hline GE & 32 & 71 & $1.2 E+06$ & $2.3 E+05$ & $g$ \\
\hline AS & 33 & 71 & $9.6 E+03$ & $1.9 E+03$ & $?$ \\
\hline AS & 33 & 72 & $1.1 E+04$ & $2.1 E+03$ & \\
\hline AS & 33 & 73 & 4. $1 E+03$ & $8.0 E+02$ & S \\
\hline AS & 33 & 74 & $1.4 E+02$ & $2.8 E+01$ & 孛. \\
\hline AS & 33 & 76 & 1. $4 E+04$ & $2.8 E+03$ & 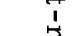 \\
\hline AS & 33 & 77 & $5.2 E+04$ & $1.0 E+04$ & गे \\
\hline SE & 34 & 72 & 4. $5 E-01$ & $8.8 E-02$ & \\
\hline SE & 34 & 75 & $4.8 E-01$ & $9.3 E-02$ & $\frac{2}{0}$ \\
\hline SE & 34 & 79 & 5. $O E+00$ & $9.7 E-01$ & \\
\hline$B R$ & 35 & 76 & $3.3 E+02$ & $6.4 E+01$ & $\stackrel{\varpi}{=}$ \\
\hline BR & 35 & 77 & $7.6 E+01$ & $1.5 E+01$ & N \\
\hline$B R$ & 35 & 82 & $6.3 E+01$ & $1 \cdot 2 E+01$ & $x^{5}$ \\
\hline$K R$ & 36 & 76 & 7. $9 E+02$ & 1. $5 E+02$ & $\overrightarrow{0} 0$ \\
\hline KR & 36 & 79 & $4.5 E+02$ & $8.8 E+01$ & \\
\hline KR & 36 & 81 & $2.8 E-01$ & $5.4 E-02$ & 40 \\
\hline$K R$ & 36 & 85 & 5. $5 E-02$ & $1.1 E-02$ & $\frac{\pi}{0}$ \\
\hline RB & 37 & 83 & 1. $7 E+00$ & 3. $4 E-01$ & \\
\hline RB & 37 & 84 & $1.1 E+00$ & $2.2 E-01$ & 8 \\
\hline RB & 37 & 86 & 3. $8 E+00$ & $7.5 E-01$ & \\
\hline RB & 37 & 87 & $5.1 E+00$ & $9.8 E-01$ & \\
\hline$S R$ & 38 & 82 & $8.1 E+01$ & $1.6 E+01$ & \\
\hline SR & 38 & 83 & $1.4 E+04$ & $2.7 E+03$ & 品 \\
\hline$S R$ & 38 & 85 & 3. $2 E+02$ & $6.2 E+01$ & $\leq$ \\
\hline$S R$ & 38 & 89 & $2.4 E+02$ & 4. $7 E+01$ & 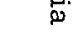 \\
\hline$S R$ & 38 & 90 & 7. $1 E+01$ & 1. $4 E+01$ & $\xi$ \\
\hline$Y$ & 39 & 86 & $1.4 E+09$ & 2. $7 E+08$ & $E$ \\
\hline $\mathbf{Y}$ & 39 & 87 & 2. $7 E+08$ & $5.3 E+07$ & $\sqrt{n}$ \\
\hline$Y$ & 39 & $87 M$ & $5.2 E+09$ & 1. $O E+09$ & $\widehat{8}$ \\
\hline$y$ & 39 & 88 & 6. $0 E+05$ & 1. $2 E+05$ & 8 \\
\hline Y & 39 & SO & $2.6 E+08$ & $5.1 E+07$ & $\tilde{2}$ \\
\hline Y & 39 & 91 & 4. $0 E+06$ & $7.8 E+05$ & \\
\hline $2 R$ & 40 & 86 & 3. $0 E+09$ & $5.7 E+08$ & \\
\hline$Z R$ & 40 & 88 & $2.2 E+06$ & 4. $3 E+05$ & \\
\hline $2 R$ & 40 & 89 & 4. $0 E+08$ & 7. $8 E+07$ & \\
\hline$Z R$ & 40 & 93 & $2.5 E+07$ & $4.9 E+06$ & \\
\hline ZR & 40 & 95 & 5. $5 E+06$ & $1.1 E+06$ & \\
\hline ZR & 40 & 97 & $4.4 E+09$ & 8. $5 E+08$ & \\
\hline NB & 41 & 90 & $1.4 E+04$ & $2.7 E+03$ & \\
\hline NB & 41 & 91 & 1. $3 E+02$ & $2.6 E+01$ & \\
\hline NB & 41 & $91 M$ & 1. $7 E+02$ & 3. $3 E+01$ & \\
\hline NB & 41 & 92 & $4 \cdot 5 E+02$ & 8. $8 E+01$ & \\
\hline
\end{tabular}




\begin{tabular}{|c|c|c|c|c|}
\hline $\begin{array}{l}\text { EL EMEN T } \\
\text { SYMBOL }\end{array}$ & $\begin{array}{l}\text { ATOMIC } \\
\text { NO. }(Z)\end{array}$ & $\begin{array}{l}\text { ISOTOPE } \\
\text { MASS (A) }\end{array}$ & $\begin{array}{c}\text { ADULT } \\
U C / M * 2\end{array}$ & $\begin{array}{l}\text { CHILD } \\
U C / M * * 2\end{array}$ \\
\hline NB & 41 & $93 M$ & $1.3 E+02$ & $2.6 E+01$ \\
\hline NB & 41 & 94 & $1.4 E+00$ & $2.6 \mathrm{E}-01$ \\
\hline NB & 41 & 95 & 9. $5 \mathrm{E}+0 \mathrm{l}$ & $1.9 E+01$ \\
\hline NB & 41 & $95 M$ & $2.3 E+03$ & $4.4 E+02$ \\
\hline NB & 41 & 96 & 1. $1 E+04$ & $2.1 E+03$ \\
\hline MO & 42 & 93 & 2. $8 E+01$ & $5.5 E+00$ \\
\hline MO & 42 & 99 & $3.6 E+01$ & $7.0 E+00$ \\
\hline TC & 43 & 95 & $5.1 \mathrm{E}+02$ & $9.8 \mathrm{E}+01$ \\
\hline TC & 43 & $95 M$ & $1.4 E+00$ & 2.7E-01 \\
\hline $\mathrm{TC}$ & 43 & 96 & 8. $9 E+00$ & 1.7E+OO \\
\hline TC & 43 & 97 & 4. $6 E+01$ & $9.0 E+O O$ \\
\hline TC & 43 & $97 M$ & $1.1 E+01$ & $2.2 E+00$ \\
\hline$T C$ & 43 & 98 & $6.2 \mathrm{E}-01$ & $1.2 \mathrm{E}-01$ \\
\hline TC & 43 & 99 & $1.0 E+01$ & 2. $.0 E+00$ \\
\hline RU & 44 & 97 & $8.6 E+05$ & $1.7 E+05$ \\
\hline RU & 44 & 103 & $1.8 E+04$ & $3.4 E+03$ \\
\hline RU & 44 & 106 & 8. $9 E+02$ & 1. $7 E+02$ \\
\hline RH & 45 & 99 & 2. $9 E+01$ & $5.7 E+00$ \\
\hline RH & 45 & 100 & 9. $9 E+02$ & 1. $9 E+02$ \\
\hline RH & 45 & 101 & $8.4 E+00$ & $1.6 E+00$ \\
\hline RH & 45 & $101 M$ & $2.4 E+02$ & $4.7 E+01$ \\
\hline $\mathbf{R H}$ & 45 & 102 & 1. $9 E+00$ & $3.8 E-01$ \\
\hline RH & 45 & 105 & $2.3 E+03$ & $4.5 E+02$ \\
\hline PD & 46 & 100 & $2.0 E+01$ & $3.9 E+00$ \\
\hline PD & 46 & 103 & $5.7 \mathrm{E}+01$ & $1.1 E+01$ \\
\hline PD & 46 & 107 & $6.3 E+02$ & $1.2 E+02$ \\
\hline$P D$ & 46 & 109 & $2.0 E+03$ & $3.8 E+02$ \\
\hline PD & 46 & 112 & $1 \cdot 5 \bar{E}+02$ & $2.8 E+01$ \\
\hline $\mathbf{A G}$ & 47 & 105 & $1.5 E+00$ & $3.0 E-01$ \\
\hline $\mathbf{A G}$ & 47 & $106 M$ & 1. $7 E+00$ & $3.4 \mathrm{E}-01$ \\
\hline $\mathbf{A G}$ & 47 & $108 M$ & 4. $5 E-01$ & $8.8 E-02$ \\
\hline $\mathbf{A G}$ & 47 & $110 \mathrm{M}$ & $4.4 E-01$ & $8.6 E-02$ \\
\hline $\mathbf{A G}$ & 47 & 111 & $1.1 E+01$ & $2.1 E+00$ \\
\hline$C D$ & 48 & 109 & $7.3 E+03$ & $1.4 E+03$ \\
\hline$C D$ & 48 & $113 M$ & 2. $5 E+03$ & $4.8 E+02$ \\
\hline$C D$ & 48 & 115 & $3.6 E+05$ & $7.0 E+04$ \\
\hline$C D$ & 48 & $115 \mathrm{M}$ & 4. $9 E+03$ & $9.5 E+02$ \\
\hline IN & 49 & 111 & $2.9 E+06$ & $5.6 E+05$ \\
\hline IN & 49 & $114 M$ & $2.1 E+04$ & $4.1 E+03$ \\
\hline IN & 49 & 115 & $4.4 E+04$ & $8.5 E+03$ \\
\hline SN & 50 & 113 & 3. $3 E+01$ & $6.4 E+00$ \\
\hline SN & 50 & $117 \mathrm{M}$ & $5.6 E+02$ & $1.1 E+02$ \\
\hline SN & 50 & $119 M$ & 8. $1 E+01$ & $1.6 E+01$ \\
\hline$S N$ & 50 & 121 & 1. $2 E+05$ & $2.4 E+04$ \\
\hline SN & 50 & $121 M$ & $1.5 E+01$ & $2.8 E+00$ \\
\hline SN & 50 & $123 M$ & $1.9 E+01$ & $3.6 E+00$ \\
\hline SN & 50 & 125 & 1. $7 E+02$ & $3.4 E+01$ \\
\hline SN & 50 & 126 & $5.6 \mathrm{E}-01$ & $1.1 E-01$ \\
\hline SB & 51 & 119 & $7.2 E+06$ & $1.4 E+0 B$ \\
\hline$S B$ & 51 & $120 \mathrm{M}$ & $1.4 E+04$ & $2.8 E+03$ \\
\hline
\end{tabular}

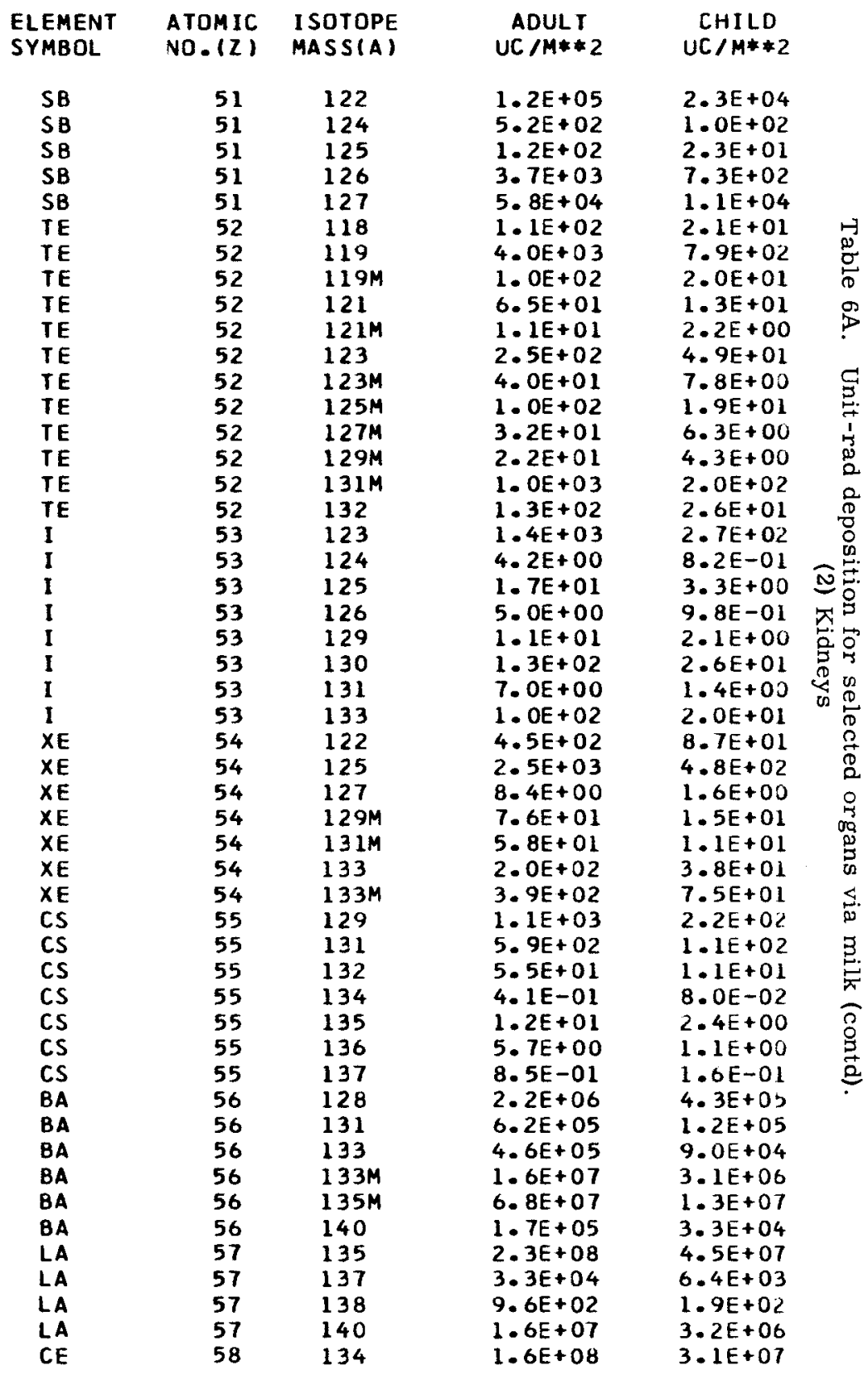




\begin{tabular}{|c|c|c|c|c|}
\hline $\begin{array}{l}\text { ELEMENT } \\
\text { SYMBOL }\end{array}$ & $\begin{array}{l}\text { ATOMIC } \\
\text { NO. (Z) }\end{array}$ & $\begin{array}{l}\text { I SOTOPE } \\
\text { MASS (A) }\end{array}$ & $\begin{array}{c}\text { ADULT } \\
\text { UC } / M * * 2\end{array}$ & $\begin{array}{l}\text { CHILD } \\
U C / M * * 2\end{array}$ \\
\hline CE & 58 & 135 & $3.2 E+09$ & $6.2 E+08$ \\
\hline$C E$ & 58 & $137 \mathrm{M}$ & $2.4 E+09$ & 4. $7 E+08$ \\
\hline$C E$ & 58 & 139 & $5 \cdot 5 E+06$ & $1.1 E+06$ \\
\hline$C E$ & 58 & 141 & $2.1 E+07$ & $4.0 E+06$ \\
\hline CE & 58 & 142 & 1. $8 E+05$ & $3.5 E+04$ \\
\hline $\mathrm{CE}$ & 58 & 143 & 7. $8 E+08$ & $1.5 E+08$ \\
\hline$C E$ & 58 & 144 & $1.7 E+05$ & $3.3 E+04$ \\
\hline$P R$ & 59 & 142 & $9.0 E+09$ & $1.7 E+09$ \\
\hline PR & 59 & 143 & $1.9 E+08$ & $3.6 E+07$ \\
\hline ND & 60 & 140 & $2.4 E+08$ & $4.6 E+07$ \\
\hline ND & 60 & 144 & $1.6 E+05$ & $3.0 E+04$ \\
\hline ND & 60 & 147 & $1.4 E+07$ & $2.7 E+06$ \\
\hline PM & 61 & 143 & $6.8 E+06$ & $1.3 E+06$ \\
\hline PM & 61 & 144 & $7.3 E+05$ & $1.4 E+05$ \\
\hline PM & 61 & 145 & $1.3 E+07$ & $2.6 E+06$ \\
\hline PM & 61 & 146 & $1.1 E+06$ & $2.2 E+05$ \\
\hline PM & 61 & 147 & $5.2 E+05$ & $1.0 E+05$ \\
\hline PM & 61 & 148 & $2.0 E+08$ & $3.8 E+07$ \\
\hline PM & 61 & $148 \mathrm{M}$ & $2.8 E+06$ & $5.4 E+05$ \\
\hline PM & 61 & 149 & $3.5 E+09$ & $6.7 E+08$ \\
\hline PM & 61 & 151 & $4.6 E+09$ & $8.9 E+08$ \\
\hline SM & 62 & 141 & 9. $9 E+06$ & $1.9 E+06$ \\
\hline SM & 62 & 145 & $1.3 E+07$ & $2.6 E+06$ \\
\hline SM & 62 & 146 & $2.8 E+05$ & $5.4 E+04$ \\
\hline SM & 62 & 147 & $3.1 E+05$ & $6.1 E+04$ \\
\hline SM & 62 & 148 & 1. $8 E+05$ & $3.5 E+04$ \\
\hline SM & 62 & 149 & $3.8 E+05$ & $7.4 E+04$ \\
\hline SM & 62 & 151 & 4. $7 E+07$ & $9.1 \mathrm{E}+06$ \\
\hline$S M$ & 62 & 153 & $5.4 E+09$ & $1.1 E+09$ \\
\hline EU & 63 & 145 & $2.2 E+08$ & 4. $2 E+07$ \\
\hline EU & 63 & 146 & $5.3 E+07$ & $1.0 E+07$ \\
\hline EU & 63 & $146 M$ & $5.5 E+08$ & $1.1 E+08$ \\
\hline EU & 63 & 147 & $6.3 E+06$ & $1.2 E+06$ \\
\hline $\mathrm{EU}$ & 63 & 148 & 1. $2 E+07$ & $2.4 E+06$ \\
\hline EU & 63 & 149 & $5.6 E+06$ & $1.1 E+06$ \\
\hline EU & 63 & 150 & $3.4 E+05$ & $6.6 E+04$ \\
\hline EU & 63 & $150 M$ & $3.3 E+09$ & $6.5 E+08$ \\
\hline EU & 63 & 152 & 2. $0 E+05$ & $3.9 E+04$ \\
\hline EU & 63 & 154 & $1.7 E+05$ & $3.2 E+04$ \\
\hline EU & 63 & 155 & $5.8 E+06$ & $1.1 E+06$ \\
\hline $\mathrm{EU}$ & 63 & 156 & $2.2 E+07$ & $4.2 E+06$ \\
\hline EU & 63 & 157 & $9.1 E+09$ & $1.8 E+09$ \\
\hline GD & 64 & 146 & $2.3 E+06$ & $4.5 E+05$ \\
\hline GD & 64 & 147 & $1.4 E+09$ & $2.7 E+08$ \\
\hline GD & 64 & 148 & $1.2 E+05$ & $2.2 E+04$ \\
\hline GD & 64 & 149 & $1.1 E+0 B$ & $2.1 E+07$ \\
\hline GD & 64 & 150 & $6.8 E+04$ & $1.3 E+04$ \\
\hline GD & 64 & 151 & 1. $3 E+07$ & $2.6 E+06$ \\
\hline GD & 64 & 152 & 1. $7 E+05$ & $3.2 E+04$ \\
\hline
\end{tabular}

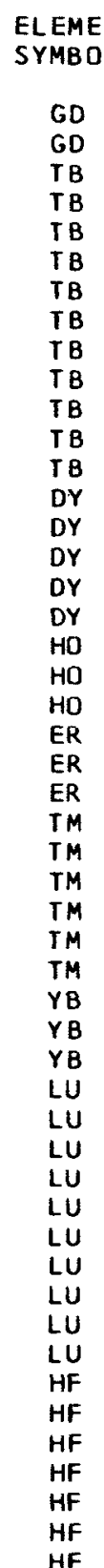

ATOMIC

I SOTOPE

ADULT
UC $/ M * * 2$

CHILD
$U C / M * * 2$

Go

64
64
65
65
65
65
65
65
65
65
65
65
65

159
162

162
151
152

152
153

153
154
155

155
156

156
157

158
160

160
161

161
164
154

154
$154 M$

$154 M$
156

159

$1 \in 6$
$1 \in 3$

$1 \in 6$
$1 \in 6$

$166 \mathrm{M}$

160
169

169
172

165
167

167
168

170

171
172

$1 \in 6$

166
169

169
175

175
$1 \in 9$

170

171
172

172
173

174

$174 M$
176

176
177

177
$177 M$

$177 M$
170

171
172

172
173

173
174

175

$3.0 E+10$
$1.1 E+06$
$7.9 E+09$
$2.3 E+09$
$1.7 E+09$
$8.6 E+09$
$4.6 E+08$
$1.4 E+08$
$8.9 E+06$
$3.9 E+05$
$4.1 E+06$
$7.0 E+08$
$1.2 E+10$
$1.5 E+02$
$1.4 E+08$
$2.4 E+02$
$1.1 E+06$
$1.2 E+07$
$1.1 E+07$
$5.9 E+09$
$4.8 E+05$
$1.7 E+09$
$1.3 E+09$
$1.4 E+09$
$1.9 E+09$
$2.1 E+08$
$5.2 E+06$
$1.5 E+07$
$1.0 E+08$
$1.2 E+09$
$1.0 E+09$
$2.4 E+07$
$1.1 E+09$
$3.5 E+09$
$1.2 E+09$
$2.3 E+09$
$4.5 E+08$
$4.5 E+06$
$1.6 E+06$
$1.4 E+07$
$1.7 E+06$
$2.5 E+09$
$3.9 E+07$
$6.3 E+09$
$1.2 E+10$
$9.4 E+05$
$3.9 E+09$
$3.3 E+05$
$1.8 E+07$
$2.0 E+07$

5. $7 E+09$

2. $2 E+05$

1. $5 E+09$

4. $5 E+08$
$3.3 E+08$

1. $7 E+09$

$9.0 E+07$

2. $7 E+07$

1. $7 E+06$

7. $5 E+04$

1. $E$ t 08

$2.3 E+09$

2. $9 E+01$

2. $6 E+07$

4. $6 \mathrm{E}+01$

$2.1 E+05$

$2.4 E+06$

2. $1 E+06$

9.1E+09

3.

3. $3 E+0 B$

2. $5 E+08$

$2.7 E+08$
$3.6 E+08$

4. $0 E+07$

1. $0 \mathrm{E}+06$

2. $9 E+06$

$2.0 E+07$

2. $3 E+08$

1. $9 E+08$

4. $6 E+06$

2. $1 E+O B$

6. $8 E+0 B$

$2.4 E+08$

4. $4 E+08$

8. $7 E+07$

8. $7 \mathrm{E}+05$

3. $O E+O S$

2. $7 E+06$

4. $9 E+08$

4. $6 E+08$

$1.2 E+09$

$2.4 E+09$

$1.8 E+05$

7. $6 E+08$

$6.4 E+04$

$3.5 E+06$

3. $9 E+06$ 


\begin{tabular}{|c|c|c|c|c|}
\hline $\begin{array}{l}\text { ELEMENT } \\
\text { SYMBOL }\end{array}$ & $\begin{array}{l}\text { ATOMIC } \\
\text { ND. }(Z)\end{array}$ & $\begin{array}{l}\text { ISOTOPE } \\
\text { MASS (A) }\end{array}$ & $\begin{array}{l}\text { ADULT } \\
\text { UC/M**2 }\end{array}$ & $\begin{array}{c}\text { CHILD } \\
U C / M * * 2\end{array}$ \\
\hline HF & 72 & 182 & 5. $O E+05$ & $9.7 E+04$ \\
\hline TA & 73 & 177 & $3.4 E+02$ & $6.5 E+01$ \\
\hline TA & 73 & 179 & $8.2 E+00$ & $1.6 E+00$ \\
\hline TA & 73 & 182 & $1.0 E+00$ & $2.0 E-01$ \\
\hline TA & 73 & 183 & $1.5 E+02$ & $2.9 E+01$ \\
\hline$W$ & 74 & 178 & 4. $.0 E+00$ & $7.8 E-01$ \\
\hline$w$ & 74 & 181 & $1.1 E+01$ & $2.2 E+00$ \\
\hline$w$ & 74 & 185 & 1. $2 E+01$ & $2.4 E+00$ \\
\hline$w$ & 74 & 187 & 1. $7 E+03$ & $3.2 E+02$ \\
\hline$w$ & 74 & 188 & $1.2 E+00$ & $2.3 E-01$ \\
\hline RE & 75 & 180 & $4.3 E+00$ & $8.3 E-01$ \\
\hline RE & 75 & 181 & 4. $7 E+00$ & $9.1 E-01$ \\
\hline RE & 75 & 182 & 5.7E-01 & $1.1 E-01$ \\
\hline RE & 75 & $182 M$ & $1.2 E+01$ & $2.4 E+00$ \\
\hline RE & 75 & 183 & $1.9 E-02$ & $3.7 \mathrm{E}-03$ \\
\hline RE & 75 & 184 & $1.4 E-02$ & $2.6 E-03$ \\
\hline RE & 75 & $184 M$ & $1.9 E-03$ & $3.7 E-04$ \\
\hline RE & 75 & 186 & 1. $O E+00$ & $2.0 E-01$ \\
\hline RE & 75 & 187 & 3. $8 E-01$ & $7.4 \mathrm{E}-02$ \\
\hline RE & 75 & 188 & $1.0 E+01$ & $2.0 E+00$ \\
\hline RE & 75 & 189 & $1 \cdot 2 \mathrm{E}+01$ & $2.4 E+00$ \\
\hline OS & 76 & 182 & $1.9 E+03$ & $3.6 E+02$ \\
\hline os & 76 & 183 & $1.3 E+04$ & $2.5 E+03$ \\
\hline os & 76 & 185 & 5. $9 E+01$ & $1.1 E+01$ \\
\hline OS & 76 & 191 & $4.8 E+02$ & $9.2 E+O I$ \\
\hline OS & 76 & $191 M$ & $3.2 E+04$ & $6.3 E+03$ \\
\hline OS & 76 & 193 & $4 \cdot 2 E+03$ & $8.2 E+02$ \\
\hline as & 76 & 194 & $1.7 E+01$ & $3.2 E+00$ \\
\hline IR & 77 & 185 & $7.1 E+03$ & $1.4 E+03$ \\
\hline IR & 77 & 186 & 4. $2 E+03$ & $8.1 E+02$ \\
\hline IR & 77 & 187 & $1.1 E+04$ & $2.2 E+03$ \\
\hline IR & 77 & 188 & $5.0 E+02$ & $9.7 E+D 1$ \\
\hline IR & 77 & 189 & $1 \cdot 1 E+02$ & $2.2 E+01$ \\
\hline IR & 77 & 190 & 3. $3 E+01$ & $6.3 E+00$ \\
\hline IR & 77 & 192 & 7. $9 E+00$ & $1.5 E+00$ \\
\hline IR & 77 & $192 M$ & 3. $4 E+00$ & $6.6 E-01$ \\
\hline IR & 77 & $193 M$ & 5. $7 E+02$ & $1.1 E+02$ \\
\hline IR & 77 & 194 & 3. $9 E+03$ & $7.6 E+02$ \\
\hline PT & 78 & 188 & $9.2 E+00$ & $1.8 E+00$ \\
\hline PT & 78 & 190 & 4. $9 E-01$ & $9.6 E-02$ \\
\hline PT & 78 & 191 & $3.4 E+02$ & $6.6 E+01$ \\
\hline PT & 78 & 192 & 5. $9 E-01$ & $1.1 E-01$ \\
\hline PT & 78 & 193 & 2. $0 E+01$ & $3.9 E+00$ \\
\hline PT & 78 & $193 M$ & $4.2 E+02$ & B. $2 E+01$ \\
\hline PT & 78 & $195 M$ & 4. $1 E+02$ & 8. $0 E+01$ \\
\hline PT & 78 & 197 & 6. $7 E+03$ & $1.3 E+03$ \\
\hline$A U$ & 79 & 193 & $1.4 E+04$ & $2.6 E+03$ \\
\hline$A U$ & 79 & 194 & $8.8 E+02$ & $1.7 E+02$ \\
\hline$A U$ & 79 & 195 & $1.4 E+01$ & $2.8 E+00$ \\
\hline$A U$ & 79 & 196 & 2. $3 E+02$ & $4.4 E+01$ \\
\hline
\end{tabular}

$\begin{array}{lcc}\text { ELEMENT } & \text { ATOMIC } & \text { ISOTOPE } \\ \text { SYMBDL } & \text { NO. IZ) } & \text { MASS(A) } \\ \text { AU } & & \\ \text { AU } & 79 & 198 \\ \text { HG } & 79 & 199 \\ \text { HG } & 80 & 154 \\ \text { HG } & 80 & 195 M \\ \text { HG } & 80 & 197 \\ \text { HG } & 80 & 197 M \\ \text { TL } & 80 & 2 C 3 \\ \text { TL } & 81 & 200 \\ \text { TL } & 81 & 201 \\ \text { TL } & 81 & 202 \\ \text { PB } & 81 & 2 C 4 \\ \text { PB } & 82 & 2 C 0 \\ \text { PB } & 82 & 202 \\ \text { PB } & 82 & 203 \\ \text { PB } & 82 & 2 C 4 \\ \text { PB } & 82 & 205 \\ \text { BI } & 82 & 210 \\ \text { BI } & 83 & 2 C 5 \\ \text { BI } & 83 & 2 C 6 \\ \text { BI } & 83 & 207 \\ \text { BI } & 83 & 208 \\ \text { BI } & 83 & 210 \\ \text { PO } & 83 & 210 M \\ \text { PO } & 84 & 206 \\ \text { PO } & 84 & 208 \\ \text { PO } & 84 & 209 \\ \text { RN } & 84 & 210 \\ \text { RN } & 86 & 211 \\ \text { RA } & 86 & 222 \\ \text { RA } & 88 & 223 \\ \text { RA } & 88 & 224 \\ \text { RA } & 88 & 225 \\ \text { RA } & 88 & 226 \\ \text { AC } & 89 & 228 \\ \text { AC } & 89 & 225 \\ \text { TH } & 90 & 227 \\ \text { TH } & 90 & 228 \\ \text { TH } & 90 & 229 \\ \text { TH } & 90 & 230 \\ \text { TH } & 90 & 231 \\ \text { TH } & 90 & 232 \\ \text { TH } & 90 & 234 \\ \text { PA } & 91 & 228 \\ \text { PA } & 91 & 229 \\ \text { PA } & 91 & 230 \\ \text { PA } & 91 & 231 \\ \text { PA } & 91 & 232 \\ \text { PA } & 91 & 233 \\ \text { U } & 92 & 230\end{array}$

ADULT $U C / M * * 2$

9. $5 \mathrm{E}+02$

2. $3 E+03$

1. $8 \mathrm{E}-02$

1. $8 E+00$

3. $7 E+0 O$

1. $2 E+01$

1. $7 E-01$

8. $2 E+01$

7. $1 E+01$

4. $3 E+00$

$8.6 E-01$

2. $4 E+00$

2. $4 E+00$

$5.4 E-01$

1. $6 E+01$

2. $6 \mathrm{E}-01$

7. $0 E+02$

1. $8 \mathrm{E}+03$

2. $8 \mathrm{E}+02$

1. $9 E+02$

7. $5 E+02$

9. $2 E+01$

7. $7 E-O I$

1. $1 E+00$

1. $7 E+00$

1. $8 \mathrm{E}+02$

2. $4 E+00$

7. $5 E+00$

$3.2 E+01$

5. $9 E+00$

1. $7 E+00$

1. $4 E+00$

7. $8 E+06$

$6.4 E+03$

5. $3 E+05$

8. $1 E+03$

8. $6 E+02$

8. $1 E+02$

$6.2 E+07$

7. $2 E+02$

2. 9E+ 05

1. $1 E+08$

6. $6 E+05$

6. $6 E+05$

8. $4 E+02$

3. $3 E+05$

4. $1 E+01$
CHILO

$U C / M * * 2$

$1.8 E+02$

3. $5 E+02$

$3.5 E-01$

7. $1 \mathrm{E}-01$

2. $3 E+00$

3. 3E-02

$1.6 E+01$

$1.4 E+01$

8. $3 E-01$

1. $7 \mathrm{E}-01$

1. $6 E+03$

4. $6 \mathrm{E}-01$

$6.5 E+02$

1. $O E-01$

3. $2 E+0 U$
5. $0 E-02$

$1.4 E+02$

$3.5 E+02$

$5.4 E+01$

$3.7 E+01$

1. $4 \mathrm{E}+02$

1. $8 E+01$

$8.0 E+0$

1. $5 E-01$

2. $2 E-01$

3. $3 E-01$

3. $5 E+01$

4. $7 E-01$

1. $5 E+00$

$6.2 E+00$

$1.1 E+00$

3. $2 E-01$

3.

2. 8E-O1

1. $2 E+03$

$1.0 E+05$

1. $6 E+03$

1. $7 E+02$

$1.6 E+02$

1. $2 E+07$

$1.4 E+02$

$5.6 E+04$

$2.2 E+07$

9. $2 \mathrm{E}+06$

1. $3 E+05$

$1.6 E+02$

$9.8 E+06$

$6.4 E+04$ 
TABLE 6A. UNIT-RAD DEPOSITION FOR KI DNEYS

VIA MILK.

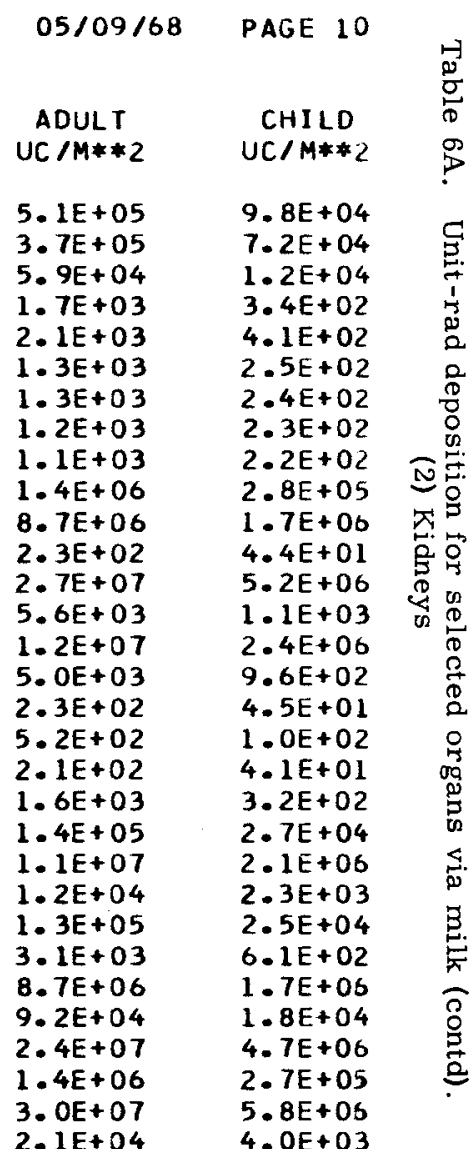

\begin{tabular}{|c|c|c|c|c|}
\hline $\begin{array}{l}\text { ELEMENT } \\
\text { SYMBOL }\end{array}$ & $\begin{array}{l}\text { ATOMIC } \\
\text { NO. }(Z)\end{array}$ & $\begin{array}{l}\text { ISOTOPE } \\
\text { MASS (A) }\end{array}$ & $\begin{array}{c}\text { ADULT } \\
\text { UC / } / * * 2\end{array}$ & $\begin{array}{c}C H I L D \\
U C / M * * 2\end{array}$ \\
\hline U & 92 & 231 & $2.8 E+02$ & $5.5 E+01$ \\
\hline$u$ & 92 & 232 & $1.4 E+01$ & $2.7 E+00$ \\
\hline u & 92 & 233 & $1.5 E+01$ & $2.8 E+00$ \\
\hline u & 92 & 234 & $1.4 E+01$ & $2.7 E+00$ \\
\hline U & 92 & 235 & $1 \cdot 3 E+01$ & $2.5 E+00$ \\
\hline$u$ & 92 & 236 & 1. $3 E+01$ & $2.4 E+00$ \\
\hline$u$ & 92 & 237 & $1.3 E+02$ & $2.5 E+01$ \\
\hline u & 92 & 238 & $1.2 \mathrm{E}+01$ & $2.3 E+00$ \\
\hline$u$ & 92 & 240 & $7.2 E+03$ & $1.4 E+03$ \\
\hline NP & 93 & 234 & $6.9 E+06$ & $1.3 E+06$ \\
\hline NP & 93 & 235 & $1.8 E+04$ & $3.5 E+03$ \\
\hline NP & 93 & 236 & $9.4 E+02$ & $1.8 E+02$ \\
\hline NP & 93 & $236 M$ & 1. $2 E+08$ & $2.3 E+07$ \\
\hline NP & 93 & 237 & 9. $9 E+02$ & $1.9 E+02$ \\
\hline NP & 93 & 238 & $2.4 E+07$ & $4.7 E+06$ \\
\hline NP & 93 & 239 & 1. $9 E+07$ & $3.6 E+06$ \\
\hline PU & 94 & 236 & $1.1 E+05$ & $2.2 E+04$ \\
\hline PU & 94 & 237 & $3.4 E+06$ & $6.5 E+05$ \\
\hline PU & 94 & 238 & $1.8 E+04$ & $3.6 E+03$ \\
\hline PU & 94 & 239 & $1.6 E+04$ & $3.0 E+03$ \\
\hline PU & 94 & 240 & $1.5 E+04$ & $3.0 E+03$ \\
\hline PU & 94 & 241 & $3.0 E+04$ & $5.9 E+03$ \\
\hline PU & 94 & 242 & 1. $5 E+04$ & $2.9 E+03$ \\
\hline PU & 94 & 244 & $1.3 E+04$ & $2.6 E+03$ \\
\hline PU & 94 & 246 & $1.9 E+07$ & $3.6 E+06$ \\
\hline$A M$ & 95 & 239 & $3.6 E+08$ & 7. $O E+07$ \\
\hline$A M$ & 95 & 240 & 2. $2 E+07$ & $4.2 E+06$ \\
\hline$A M$ & 95 & 241 & $9.7 E+02$ & $1.9 E+02$ \\
\hline$A M$ & 95 & 242 & $1.9 E+08$ & $3.8 E+07$ \\
\hline$A M$ & 95 & $242 M$ & $9.5 E+02$ & $1.8 E+02$ \\
\hline AM & 95 & 243 & $8.6 E+02$ & $1.7 E+02$ \\
\hline
\end{tabular}

$\begin{array}{lcc}\text { ELEMENT } & \text { ATOMIC } & \text { ISOTOPE } \\ \text { SYMBOL } & \text { NO. (Z) } & \text { MASS(A) } \\ \text { CM } & 96 & 240 \\ \text { CM } & 96 & 241 \\ \text { CM } & 96 & 242 \\ \text { CM } & 96 & 243 \\ \text { CM } & 96 & 244 \\ \text { CM } & 96 & 245 \\ \text { CM } & 96 & 246 \\ \text { CM } & 96 & 247 \\ \text { CM } & 96 & 248 \\ \text { BK } & 97 & 245 \\ \text { BK } & 97 & 246 \\ \text { BK } & 97 & 247 \\ \text { BK } & 97 & 248 \\ \text { BK } & 97 & 249 \\ \text { CF } & 98 & 246 \\ \text { CF } & 98 & 248 \\ \text { CF } & 98 & 249 \\ \text { CF } & 98 & 250 \\ \text { CF } & 98 & 251 \\ \text { CF } & 98 & 252 \\ \text { CF } & 98 & 253 \\ \text { ES } & 99 & 251 \\ \text { ES } & 99 & 252 \\ \text { ES } & 99 & 253 \\ \text { ES } & 99 & 254 \\ \text { ES } & 99 & 254 M \\ \text { ES } & 99 & 255 \\ \text { FM } & 100 & 252 \\ \text { FM } & 100 & 253 \\ \text { FM } & 100 & 255 \\ \text { FM } & 100 & 257 \\ & & \end{array}$




\begin{tabular}{|c|c|c|c|c|}
\hline $\begin{array}{l}\text { ELEMENT } \\
\text { SYMBOL }\end{array}$ & $\begin{array}{l}\text { ATOMIC } \\
\text { NO. }(Z)\end{array}$ & $\begin{array}{l}\text { ISOTOPE } \\
\text { MASS (A) }\end{array}$ & $\begin{array}{l}\text { ADULT } \\
\text { UC } / M * * 2\end{array}$ & $\begin{array}{c}C H I L D \\
U C / M * * 2\end{array}$ \\
\hline H & 1 & 3 & $6.9 E+02$ & $1.1 E+02$ \\
\hline$B E$ & 4 & 7 & $7.7 E+05$ & $1.2 E+05$ \\
\hline BE & 4 & 10 & $1.7 E+04$ & $2.8 E+03$ \\
\hline C & 6 & 14 & $1.1 E+02$ & $1.8 E+01$ \\
\hline NA & 11 & 22 & $8.1 E-01$ & $1.3 E-01$ \\
\hline NA & 11 & 24 & $1.6 E+02$ & $2.6 E+01$ \\
\hline MG & 12 & 28 & $9.1 E+01$ & $1.5 E+01$ \\
\hline$A L$ & 13 & 26 & $2.3 E-02$ & $3.6 E-03$ \\
\hline SI & 14 & 32 & $3.8 E-02$ & $6.2 E-03$ \\
\hline P & 15 & 32 & $2.0 E+00$ & $3.1 \mathrm{E}-01$ \\
\hline $\mathbf{p}$ & 15 & 33 & $1.2 E+01$ & 2. $0 E+00$ \\
\hline$s$ & 16 & 35 & $6.9 E+01$ & $1.1 E+01$ \\
\hline $\mathrm{CL}$ & 17 & 36 & $2.1 E+00$ & $3.4 E-01$ \\
\hline AR & 18 & 37 & $7.3 E+02$ & 1. $2 E+02$ \\
\hline AR & 18 & 39 & 2. $8 E-02$ & $4.5 E-03$ \\
\hline AR & 18 & 42 & 3. $O E-03$ & $4.9 E-04$ \\
\hline 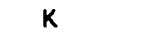 & 19 & 40 & $2 \cdot 2 E+00$ & 3. $5 E-01$ \\
\hline K & 19 & 42 & $2.0 E+03$ & $3.3 E+02$ \\
\hline K & 19 & 43 & $1.3 E+03$ & $2.0 E+02$ \\
\hline CA & 20 & 41 & $2.9 E+03$ & 4. $7 E+02$ \\
\hline CA & 20 & 45 & $1.3 E+02$ & $2.1 E+01$ \\
\hline CA & 20 & 47 & $5.9 E+01$ & $9.5 E+00$ \\
\hline CA & 20 & 48 & $2.9 E+00$ & $4.6 \mathrm{E}-01$ \\
\hline SC & 21 & $44 M$ & $3.4 E+0 B$ & $5.5 E+07$ \\
\hline SC & 21 & 46 & $2.4 E+06$ & $3.9 E+05$ \\
\hline SC & 21 & 47 & $1.7 E+09$ & $2.7 E+08$ \\
\hline SC & 21 & 48 & 4. $6 E+08$ & $7.5 E+07$ \\
\hline TI & 22 & 44 & $3.1 E+03$ & $5.0 E+02$ \\
\hline$v$ & 23 & 48 & 1. $0 E+03$ & $1.7 \mathrm{E}+02$ \\
\hline$v$ & 23 & 49 & $8.1 E+04$ & $1.3 E+04$ \\
\hline v & 23 & 50 & $2.6 E+02$ & $4.1 E+01$ \\
\hline CR & 24 & 48 & 1. $2 E+04$ & $1.9 E+03$ \\
\hline$C R$ & 24 & 51 & $3.9 E+03$ & $6.3 E+02$ \\
\hline MN & 25 & 52 & $9.8 E+02$ & $1.6 E+02$ \\
\hline MN & 25 & 53 & 1. $3 E+04$ & $2.0 E+03$ \\
\hline MN & 25 & 54 & 1. $2 E+02$ & $1.9 E+01$ \\
\hline FE & 26 & 55 & $7.5 E+02$ & $1.2 E+02$ \\
\hline FE & 26 & 59 & $4.1 E+01$ & $6.6 E+00$ \\
\hline $\mathrm{FE}$ & 26 & 60 & $1.1 E+00$ & $1.8 \mathrm{E}-01$ \\
\hline $\mathrm{CO}$ & 27 & 55 & 4. $2 E+04$ & $6.8 E+03$ \\
\hline $\mathrm{CO}$ & 27 & 56 & $1.2 E+02$ & $1.9 E+01$ \\
\hline $\mathrm{CO}$ & 27 & 57 & 1. $3 E+03$ & $2.1 E+02$ \\
\hline $\mathrm{CO}$ & 27 & 58 & $2.5 E+02$ & $4.1 E+01$ \\
\hline $\mathrm{CO}$ & 27 & 60 & $6.2 E+01$ & $1.0 E+O 1$ \\
\hline NI & 28 & 56 & $2.8 E+01$ & $4.6 E+00$ \\
\hline NI & 28 & 57 & $6.9 E+02$ & $1.1 E+02$ \\
\hline NI & 28 & 59 & 5. $2 E+01$ & $8.4 E+00$ \\
\hline NI & 28 & 63 & $2 \cdot 3 E+01$ & 3. $7 E+00$ \\
\hline NI & 28 & 66 & $3.4 E+02$ & $5.4 E+01$ \\
\hline cu & 29 & 64 & $1.4 E+04$ & $2.2 E+03$ \\
\hline
\end{tabular}

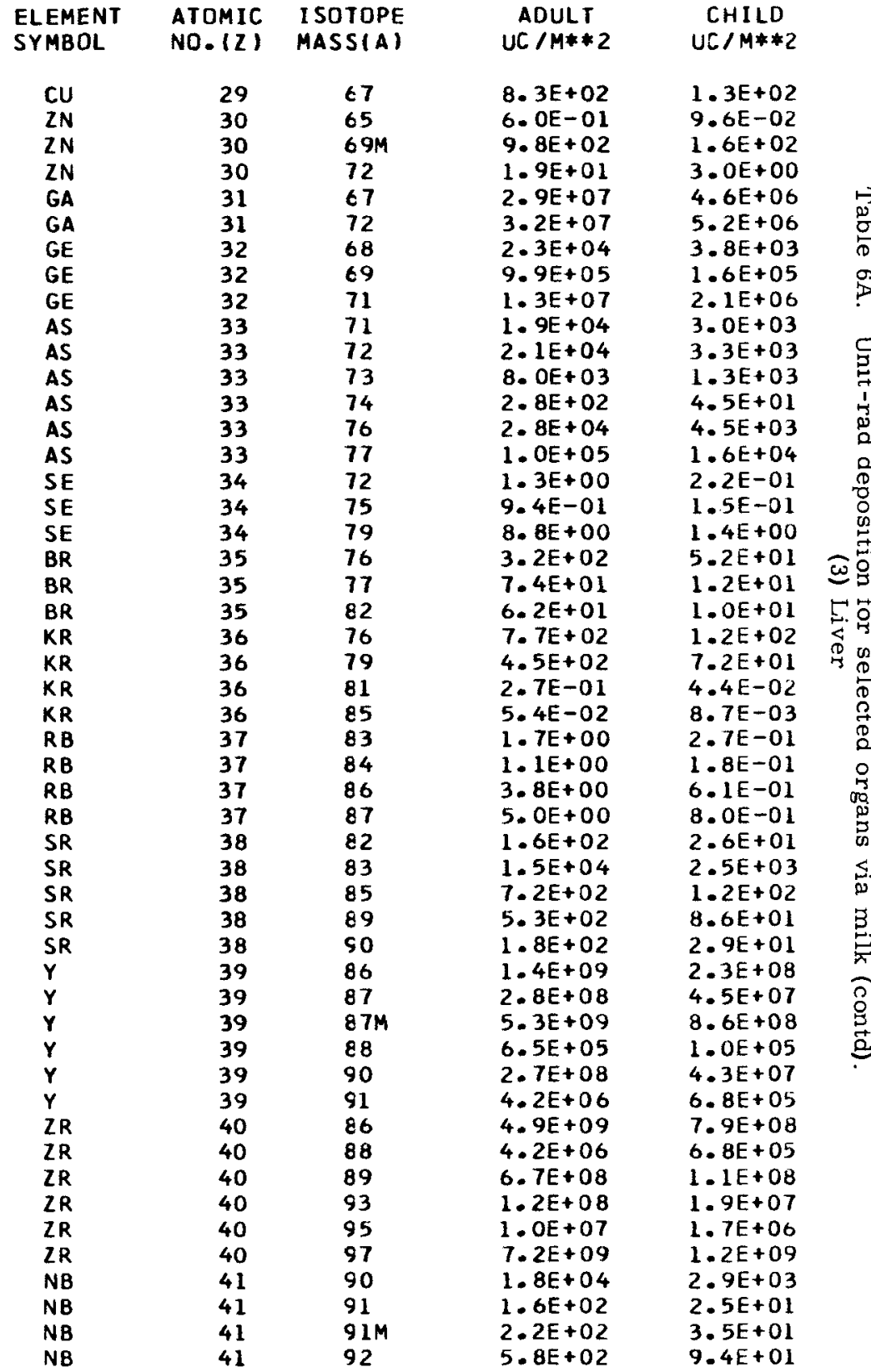




\begin{tabular}{|c|c|c|c|c|}
\hline $\begin{array}{l}\text { EL EMEN T } \\
\text { SYMBDL }\end{array}$ & $\begin{array}{l}\text { ATOMIC } \\
\text { NO. }(Z)\end{array}$ & $\begin{array}{l}\text { ISOTOPE } \\
\text { MASS (A) }\end{array}$ & $\begin{array}{l}\text { ADULT } \\
\text { UC/M**2 }\end{array}$ & $\begin{array}{c}\text { CHILD } \\
U C / M * * 2\end{array}$ \\
\hline NB & 41 & $93 M$ & $1.6 E+02$ & $2.6 E+01$ \\
\hline NB & 41 & 94 & $1.6 E+00$ & $2.5 \mathrm{E}-01$ \\
\hline NB & 41 & 95 & 1. $2 E+02$ & 2. $0 E+01$ \\
\hline NB & 41 & $95 M$ & $2.9 E+03$ & $4.7 E+02$ \\
\hline NB & 41 & 96 & $1.4 E+04$ & $2.3 E+03$ \\
\hline MO & 42 & 93 & $8.2 E+00$ & $1.3 E+0 D$ \\
\hline MO & 42 & 99 & 3. $9 E+01$ & $6.3 E+00$ \\
\hline TC & 43 & 95 & $9.7 E+03$ & $1.6 E+03$ \\
\hline$T C$ & 43 & $95 M$ & 2. $1 E+01$ & $3 \cdot 3 E+00$ \\
\hline TC & 43 & 96 & $1.6 E+02$ & $2.6 E+01$ \\
\hline TC & 43 & 97 & 6. $0 E+02$ & $9.6 \mathrm{E}+01$ \\
\hline $\mathrm{TC}$ & 43 & $97 M$ & $1.6 E+02$ & $2.5 E+01$ \\
\hline TC & 43 & 98 & 8. $0 E+00$ & $1.3 E+00$ \\
\hline TC & 43 & 99 & $1.3 E+02$ & $2.1 E+01$ \\
\hline RU & 44 & 97 & $1.5 E+06$ & $2.4 E+05$ \\
\hline RU & 44 & 103 & $3.8 E+04$ & $6.1 E+03$ \\
\hline RU & 44 & 106 & $3.0 E+03$ & $4.9 E+02$ \\
\hline RH & 45 & 99 & 1. $5 E+02$ & $2.5 E+01$ \\
\hline RH & 45 & 100 & $4.4 E+03$ & $7.1 E+02$ \\
\hline RH & 45 & 101 & $5.6 E+01$ & $9.0 E+00$ \\
\hline RH & 45 & $101 \mathrm{M}$ & $1.1 E+03$ & $1.8 E+02$ \\
\hline RH & 45 & 102 & $1.2 E+01$ & $2.0 E+00$ \\
\hline RH & 45 & 105 & $1.0 E+04$ & $1.7 E+03$ \\
\hline PD & 46 & 100 & $1.2 E+02$ & $2.0 E+01$ \\
\hline PD & 46 & 103 & 4. $0 E+02$ & $6.4 E+01$ \\
\hline$P D$ & 46 & 107 & 5. $8 E+03$ & $9.3 E+02$ \\
\hline PD & 46 & 109 & $1.2 E+04$ & $1.9 E+03$ \\
\hline PD & 46 & 112 & $8.6 E+02$ & $1.4 E+02$ \\
\hline$A G$ & 47 & 105 & $9.5 \bar{E}-01$ & $1.5 \mathrm{E}-01$ \\
\hline$A G$ & 47 & $106 \mathrm{M}$ & $2.5 E+00$ & $4.1 E-01$ \\
\hline AG & 47 & $108 M$ & $1.4 E-01$ & $2.2 E-02$ \\
\hline$A G$ & 47 & $110 M$ & $1.6 \mathrm{E}-01$ & $2.5 E-02$ \\
\hline $\mathbf{A G}$ & 47 & 111 & $1.7 E+01$ & $2.7 E+00$ \\
\hline$C D$ & 48 & 109 & $7.3 E+03$ & $1.2 E+03$ \\
\hline$C D$ & 48 & $113 M$ & $2.8 E+03$ & $4.5 E+02$ \\
\hline$C D$ & 48 & 115 & $2.8 E+05$ & $4.5 E+04$ \\
\hline$C D$ & 48 & $115 M$ & 4. $0 E+03$ & $6.4 E+02$ \\
\hline IN & 49 & 111 & 4. $8 E+06$ & $7.7 E+05$ \\
\hline IN & 49 & $114 M$ & $3.6 E+04$ & $5.8 E+03$ \\
\hline IN & 49 & 115 & $7.5 E+04$ & $1.2 E+04$ \\
\hline SN & 50 & 113 & $5.2 E+02$ & $8.4 E+01$ \\
\hline SN & 50 & $117 \mathrm{M}$ & 9. $7 E+03$ & $1.6 E+03$ \\
\hline SN & 50 & $119 M$ & $1 \cdot 2 E+03$ & $1.9 E+02$ \\
\hline SN & 50 & 121 & $2.1 E+06$ & $3.4 E+05$ \\
\hline SN & 50 & $121 \mathrm{M}$ & 1. $2 E+02$ & $1.9 E+01$ \\
\hline SN & 50 & $123 M$ & 3. $0 E+02$ & $4.8 E+01$ \\
\hline SN & 50 & 125 & 3. $0 E+03$ & $4.8 E+02$ \\
\hline SN & 50 & 126 & 4. $1 E+00$ & $6.6 E-01$ \\
\hline SB & 51 & 119 & 3. $6 \mathrm{E}+07$ & $5.9 E+06$ \\
\hline SB & 51 & $120 M$ & 8. $0 E+04$ & $1.3 E+04$ \\
\hline
\end{tabular}

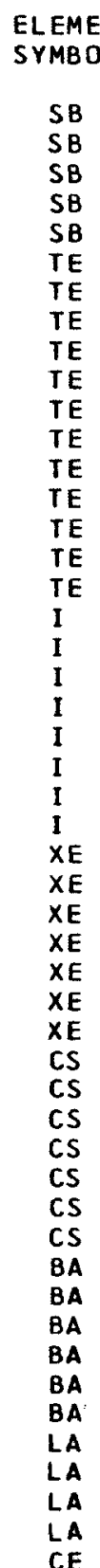

\section{ATOMIC ISOTOPE \\ ND. (Z) MASS(A)}

$\begin{array}{ll}51 & 1 \\ 51 & 1 \\ 51 & 12 \\ 51 & 12 \\ 51 & 1 \\ 52 & 11 \\ 52 & 11 \\ 52 & 11\end{array}$

122
124
125
126

ADULT
UC $/ M * * 2$

6. $0 E+05$ 6. $6 E+03$

1. $5 E+04$

2. $4 E+04$
$3.1 E+05$

1. $3 E+03$

4. $7 E+04$

1. $2 E+03$

7. $6 E+02$

1. $3 E+02$

2. $9 E+03$

4. $7 E+02$

1. $2 E+03$
$3.7 E+02$

2. $6 E+02$

1. $2 E+04$

1. $6 \mathrm{E}+03$

2. $7 E+03$

8. $2 E+00$

3. $3 E+01$

9. $7 E+00$

2. $1 E+01$

2. $6 E+02$

1. $4 \mathrm{E}+01$

1. $9 E+02$

4. $4 E+02$
2. $4 E+03$

8. $3 E+00$

7. $5 E+01$

5. $7 E+01$

1. $9 E+02$

3. $8 E+02$

$1.1 E+03$

$5.8 E+02$

5. $4 E+01$

4. $0 E-01$

1. $2 \mathrm{E}+01$

5. $6 E+00$

8. $4 E-01$

1. $7 E+06$

2. $6 E+05$

5. $3 E+03$

1. $3 E+07$

5. $8 E+07$

6. $6 \mathrm{E}+04$

9. $0 E+09$

2. $4 E+07$

6. $9 E+05$

6. $4 E+08$

7. $4 \mathrm{E}+07$
CHILD
$U C / M * * 2$

9. $7 E+04$

1. $1 E+03$

2. $5 E+03$

3. $9 E+03$

$5.0 E+04$

$2.1 E+02$

7. $6 E+03$

1. $9 E+02$

$1.2 E+02$

$1.2 E+02$

4. $7 E+02$

7. $5 \mathrm{E}+01$

1. $9 E+02$

$6.0 E+01$

4. $2 E+01$

1. $9 E+03$

$2.5 E+02$

4. $3 E+02$

1. $3 E+0 D$

$5.3 E+00$

1. $6 E+00$ जै

$3.3 E+00$ E.

$2.2 E+00$ D

$3.1 \mathrm{E}+01$

7 IE

3. $9 E+02$

1. $3 E+00$

$1 \cdot 2 E+01$

$9.1 E+0 J$

3. $1 E+01$

$6.1 \mathrm{E}+01$

1. $8 E+02$

$9 \cdot 3 E+01$

8. $6 \mathrm{E}+00$

6. $5 E-02$

2. $0 E+00$

9. $1 E-01$

1. $3 E-01$

2. $7 E+05$

4. $2 E+04$

$8.6 E+02$

2. $1 E+06$

9. $3 E+06$

1. $1 E+04$

1. $5 E+09$

3. $8 E+06$

1. $1 \mathrm{E}+05$

1. $O E+O B$

1. $2 E+07$ 


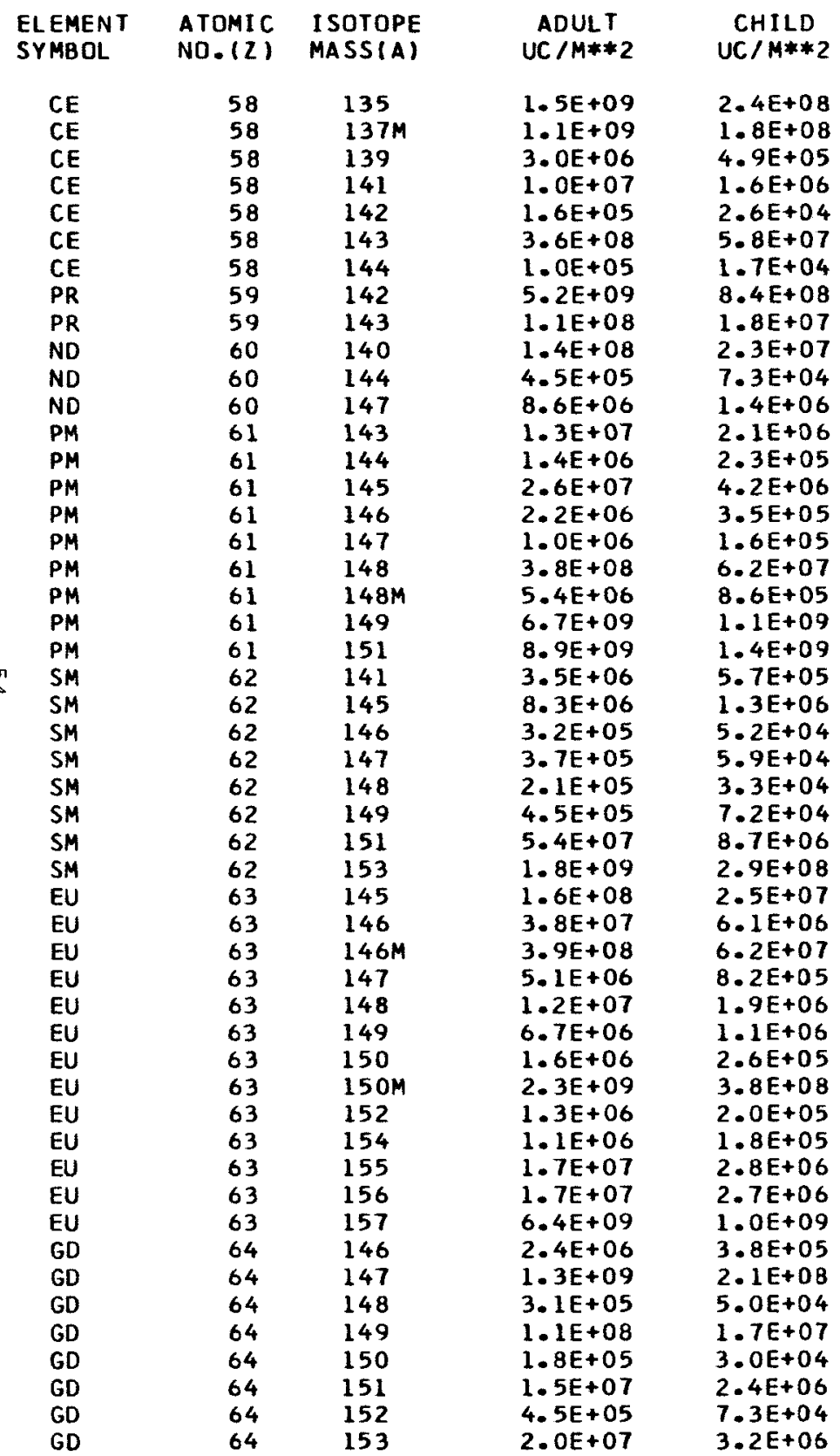

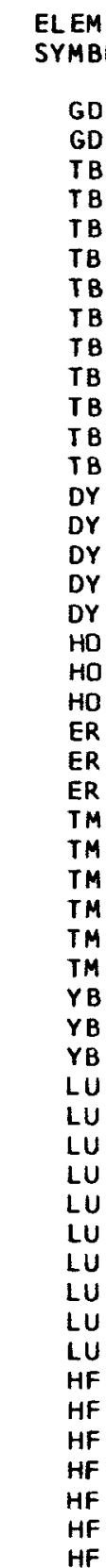

ATOMIC

I SOTOPE
MASS(A)

ADULT
UC $/ M * 2 * 2$

CHILD

SYMBOL

$\begin{array}{ll}64 & 159 \\ 64 & 162 \\ 65 & 151 \\ 65 & 152\end{array}$

2. $9 E+10$
1. $5 E+06$
7. $0 E+09$

7. $O E+09$

2. $0 E+09$
$1.5 E+09$

7. $5 E+09$

4. $3 E+08$

1. $3 E+08$

3. $5 E+06$

3. $5 E+06$
6. $7 E+06$

6. $7 E+06$

1. $O E+10$

2. $2 E+05$

1. $3 E+10$

3. $5 E+05$

1. $3 E+08$

1. $2 E+09$
1. $9 E+07$

1. $9 E+07$
1. $1 E+10$

8. $4 E+05$

6. $5 E+09$

5. $1 E+09$

5. $4 E+09$

3. $0 E+08$

3. $0 E+08$
1. $2 E+07$

1. $2 E+07$
4. $0 E+07$

4. $8 E+08$

1. $6 E+09$

5. $0 E+09$

1. $6 E+0 B$

5. $6 E+09$

7. $1 E+07$

1. $3 E+08$

2. $6 E+07$

1. $5 E+05$

3. $3 E+04$

6. $6 E+05$

9. $9 E+03$

1. $4 \mathrm{E}+08$

1. $9 E+06$

1. $6 E+09$
3. $2 E+09$

3. $2 E+09$
2. $2 E+05$

1. $0 E+09$

7. $6 E+04$

4. $6 E+06$

4. $6 E+09$

2. $5 E+05$

1. $1 \mathrm{E}+09$

1. $1 E+09$

$3.2 E+08$
$2.5 E+08$

1. $2 E+09$

7. $0 E+07$

$2 \cdot 1 E+07$

1. $3 E+07$

5. $6 E+05$

1. $1 \mathrm{E}+06$

1. $1 E+08$
1. $7 E+09$

1. $7 E+09$
3. $6 E+04$

$2.1 \mathrm{E}+09$

5. $6 E+04$

2. $1 E+07$

1. $9 E+08$

$3.1 E+06$

$1.8 E+09$

$1.4 E+05$
$1.0 E+09$

8. $2 E+08$

8. $7 E+08$

$3.9 E+08$

4. $8 E+07$

1. $9 E+06$

$6.4 E+05$

$7.7 E+07$
$2.5 E+08$

8. $0 E+08$

2. $6 E+07$

9. $O E+08$

3. $3 E+07$

1. $1 E+07$

. $1 E+07$

4. $1 E+06$

2. $5 E+04$

$5.4 E+03$

$1.1 E+05$

$1.6 E+03$

2. $3 E+07$

3. $0 E+05$

$2.6 E+08$

$5.1 E+08$

$3.6 E+04$

1. $6 E+08$

$1.2 E+04$

7. $4 E+05$

8. $4 E+05$ 


\begin{tabular}{|c|c|c|c|c|}
\hline $\begin{array}{l}\text { ELEMENT } \\
\text { SYMBOL }\end{array}$ & $\begin{array}{l}\text { ATOMIC } \\
\text { NO. }(Z)\end{array}$ & $\begin{array}{l}\text { I SOTOPE } \\
\text { MASS (A) }\end{array}$ & $\begin{array}{c}\text { ADUL T } \\
U C / M * * 2\end{array}$ & $\begin{array}{c}\text { CHILD } \\
U C / M * * 2\end{array}$ \\
\hline HF & 72 & 182 & $1.2 E+05$ & $1.9 E+04$ \\
\hline TA & 73 & 177 & 2. $O E+02$ & 3. $1 E+01$ \\
\hline TA & 73 & 179 & 4. $7 E+00$ & $7.6 \mathrm{E}-01$ \\
\hline TA & 73 & 182 & $6.0 E-01$ & $9.7 E-02$ \\
\hline TA & 73 & 183 & $8.6 E+01$ & $1.4 E+01$ \\
\hline$w$ & 74 & 178 & $2.5 E+03$ & $4.1 E+02$ \\
\hline$w$ & 74 & 181 & $3.7 E+04$ & $5.9 E+03$ \\
\hline$w$ & 74 & 185 & 2. $3 E+04$ & $3.7 E+03$ \\
\hline$W$ & 74 & 187 & $2.0 E+05$ & $3.2 E+04$ \\
\hline$W$ & 74 & 188 & $2.1 E+03$ & $3.4 E+02$ \\
\hline RE & 75 & 180 & $2.6 E+03$ & $4.2 E+02$ \\
\hline RE & 75 & 181 & 2. $9 E+03$ & $4.6 E+02$ \\
\hline RE & 75 & 182 & 3. $9 E+02$ & $6.3 E+01$ \\
\hline RE & 75 & $182 M$ & $7.3 E+03$ & $1.2 E+03$ \\
\hline RE & 75 & 183 & $6.3 E+01$ & $1.0 E+01$ \\
\hline RE & 75 & 184 & $2.8 E+01$ & $4.5 \mathrm{E}+00$ \\
\hline RE & 75 & $184 M$ & $1.4 E+01$ & $2.2 E+00$ \\
\hline RE & 75 & 186 & $7.6 E+02$ & $1.2 E+02$ \\
\hline RE & 75 & 187 & $1.1 E+05$ & 1. $8 E+04$ \\
\hline RE & 75 & 188 & $6.2 E+03$ & $9.9 E+02$ \\
\hline RE & 75 & 189 & $7.5 E+03$ & $1.2 E+03$ \\
\hline OS & 76 & 182 & $1.3 E+04$ & $2.1 E+03$ \\
\hline OS & 76 & 183 & $9.4 E+04$ & $1.5 E+04$ \\
\hline OS & 76 & 185 & $3.9 E+02$ & $6.3 E+01$ \\
\hline OS & 76 & 191 & $3.2 E+03$ & $5.2 E+02$ \\
\hline OS & 76 & $191 \mathrm{M}$ & $2.3 E+05$ & $3.7 E+04$ \\
\hline os & 76 & 193 & 3. $0 E+04$ & $4.8 E+03$ \\
\hline OS & 76 & 194 & 1. $1 E+02$ & $1.8 E+01$ \\
\hline IR & 77 & 185 & $8.2 E+03$ & $1.3 E+03$ \\
\hline IR & 77 & 186 & $4.8 E+03$ & $7.8 E+02$ \\
\hline IR & 77 & 187 & $1.3 E+04$ & $2.1 E+03$ \\
\hline IR & 77 & 188 & 5. $9 E+02$ & $9.4 E+01$ \\
\hline IR & 77 & 189 & $1.5 E+02$ & $2.5 E+01$ \\
\hline IR & 77 & 190 & 4. $3 E+01$ & 7. $0 E+00$ \\
\hline IR & 77 & 192 & 1. $3 E+01$ & $2.2 E+00$ \\
\hline IR & 77 & $192 M$ & $7.2 \mathrm{E}+00$ & $1.2 E+00$ \\
\hline IR & 77 & $193 M$ & $7.6 E+02$ & $1.2 E+02$ \\
\hline IR & 77 & 294 & $4.5 E+03$ & $7.2 E+02$ \\
\hline PT & 78 & 188 & $8.6 E+02$ & $1.4 E+02$ \\
\hline PT & 78 & 190 & $1 \cdot 1 E+02$ & $1.7 E+01$ \\
\hline PT & 78 & 191 & $2.7 E+04$ & $4.4 E+03$ \\
\hline PT & 78 & 192 & 1. $3 E+02$ & $2.1 E+01$ \\
\hline PT & 78 & 193 & 4. $4 E+03$ & 7. $1 \mathrm{E}+02$ \\
\hline PT & 78 & $193 M$ & $3.5 E+04$ & $5.6 E+03$ \\
\hline PT & 78 & $195 \mathrm{M}$ & $3.4 E+04$ & $5.4 E+03$ \\
\hline PT & 78 & 197 & $5.0 E+05$ & $8.1 E+04$ \\
\hline$A U$ & 79 & 193 & 5. $9 E+04$ & $9.5 E+03$ \\
\hline$A U$ & 79 & 194 & $3.8 E+03$ & $6.1 E+02$ \\
\hline AU & 79 & 195 & $6.1 E+01$ & $9.9 \varepsilon+00$ \\
\hline$A U$ & 79 & 196 & 9. $9 E+02$ & $1.6 E+02$ \\
\hline
\end{tabular}

\begin{tabular}{c} 
ELEME \\
SYMBO \\
\\
$A U$ \\
$A U$ \\
HG \\
$H G$ \\
$H G$ \\
$H G$ \\
$H G$ \\
$H G$ \\
$T L$ \\
$T L$ \\
$T L$ \\
$T L$ \\
$P B$ \\
$P B$ \\
$P B$ \\
$P B$ \\
$P B$ \\
$P B$ \\
$P B$ \\
$B I$ \\
$B I$ \\
$B I$ \\
$B I$ \\
$B I$ \\
$B I$ \\
$P O$ \\
$P O$ \\
$P O$ \\
$P O$ \\
$P N$ \\
$R N$ \\
$R N$ \\
$R A$ \\
$R A$ \\
$R A$ \\
$R A$ \\
$R A$ \\
$A C$ \\
$A C$ \\
$T H$ \\
$T H$ \\
TH \\
TH \\
IH \\
TH \\
IH \\
$T H$ \\
$P A$ \\
$P A$ \\
$P A$ \\
$P A$ \\
$P A$ \\
$P A$ \\
\hline
\end{tabular}

\section{ATOMIC ISOTOPE}

ADULT

$U C / M * * 2$

4. $1 E+03$

1. $O E+04$
$2.7 E-01$

2. $5 E+01$

$5.1 E+01$

$1.6 E+02$

2. $5 E+00$

$5.6 E+02$

4. $9 E+02$

8. $0 E+00$

8. $0 E+00$
7. $3 E+04$

7. $3 E+04$

3. $0 E+04$

1. $4 E+00$

4. $1 E+01$

7. $5 \mathrm{E}-01$

4. $6 E+03$

1. $5 E+04$
1. $3 E+03$

8. $8 E+02$

6. $3 E+03$

4. $3 E+02$

1. $0 E+02$

3. $O E+00$

5. $9 E+00$

1. $8 E+02$

1. $8 E+02$

2. $4 E+00$

$2.2 E+02$

9. $3 E+02$

1. $7 E+02$

4. $B E+01$

4. $2 E+01$

9. $1 E+05$

1. $8 \mathrm{E}+03$

4. $6 E+04$

4. $6 E+04$
4. $5 E+03$

4. $5 E+03$

4. $6 E+03$

$3.8 E+03$

1. $7 E+06$

5. $2 E+O B$

2. $E+08$

2. $2 E+08$

3. $1 E+06$

$3.9 E+03$
$2.3 E+08$

1 - $5 E+06$

1. $1 E+01$
CHILD

$6.7 E+02$

1. $6 \mathrm{E}+03$ 4. $3 E-02$ $4.0 E+00$ 8. $2 E+00$ $2.6 E+01$ 4. $0 E-01$ $9.0 E+01$ 7. $9 E+01$ 5. $1 E+00$ 1. $3 E+00$ 1. $2 E+04$ 4. $9 E+03$ $2.2 E-01$ $6.6 E+00$ 1. $\mathrm{E}+01$ $7.5 E+02$ 7. $5 E+02$ $2 \cdot 3 E+03$ $2.1 E+02$ ढ $1.4 E+02$ 1. $0 E+03$ E. 6. $9 E+01$ 1. $7 E+01$ 4. $8 E-01$ -1E-OI $2.9 E+01$ 3. $9 E-01$ $3.5 E+01$ $1.5 \mathrm{E}+02$ 2. $8 \mathrm{E}+0 \mathrm{O}$ 7. $7 E+00$ 6. $8 \mathrm{E}+00$ 1. $5 E+05$ 1. 2. E +02 7. $4 E+03$ $7.3 E+02$ $6.8 E+02$ $5.8 E+07$ $6.1 E+02$ $2.7 E+05$ 8. $4 E+07$ 3. $5 E+07$ 5. $0 E+05$ $6.2 E+02$ $3.8 E+07$ 2. $5 E+05$ 1. $8 E+00$ 
$05 / 09 / 68$

ELEMEN
SYMBOL
$U$
$U$
$U$
$U$
$U$
$U$
$U$
$U$
$U$
$N P$
$N P$
$N P$
$N P$
$N P$
$N P$
$N P$
$P U$
$P U$
$P U$
$P U$
$P U$
$P U$
$P U$
$P U$
$P U$
$A M$
$A M$
$A M$
$A M$
$A M$
$A M$

$\begin{array}{cc}\text { ATOMIC } & \text { ISOTOPE } \\ \text { NO. (Z) } & \text { MASS (A) } \\ 92 & 231 \\ 92 & 232 \\ 92 & 233 \\ 92 & 234 \\ 92 & 235 \\ 92 & 236 \\ 92 & 237 \\ 92 & 238 \\ 92 & 240 \\ 92 & 234 \\ 93 & 235 \\ 93 & 236 \\ 93 & 236 M \\ 93 & 237 \\ 93 & 238 \\ 93 & 239 \\ 93 & 236 \\ 94 & 237 \\ 94 & 238 \\ 94 & 239 \\ 94 & 240 \\ 94 & 241 \\ 94 & 242 \\ 94 & 244 \\ 94 & 246 \\ 94 & 239 \\ 95 & 240 \\ 95 & 241 \\ 95 & 242 \\ 95 & 242 M \\ 95 & 243 \\ 95 & \end{array}$

$\begin{array}{cc}\text { ADULT } & \text { CHILD } \\ U C / M * * 2 & U C / M * * 2\end{array}$

$1.4 E+02$

2. $1 E-02$

1. $9 E-02$

1. $8 \mathrm{E}-02$

$1.6 \mathrm{E}-02$

$1.6 \mathrm{E}-02$

$5.6 \mathrm{E}+01$

$1.5 E-02$

4. $4 E+03$

2. $4 E+07$

$6.2 E+04$

3. $3 E+03$

4. $2 E+08$

3. $5 \mathrm{E}+03$

$8.4 E+07$

$6.5 E+07$

8. $8 E+04$

2. $6 E+06$

$1.4 E+04$

1. $2 E+04$

1. $2 E+04$

2. $4 E+04$

1. $2 E+04$

1. $0 E+04$

$1.4 E+07$

1. $8 \mathrm{E}+08$

1. $1 E+07$

1. $\mathrm{OE}+03$

9.7E+07

9. $9 E+02$

9. $1 E+02$
$2 \cdot 3 E+01$

3. $3 E-03$

3. $0 \mathrm{E}-03$

2. $8 E-03$

2.6E-03

2. $6 \mathrm{E}-03$

$9.0 E+00$

2. $5 \mathrm{E}-03$

7. $1 E+02$

$3.9 E+06$

1. $0 E+04$

5. $3 E+02$

$6.8 E+07$

$5.6 E+02$

1. $3 E+07$

1. $0 E+07$

$1.4 E+04$

4. $2 E+05$

2. $3 E+03$

2. $0 E+03$

1. $9 E+03$

$3.8 E+03$

1. $9 E+03$

1. $7 E+03$

2. $3 E+06$

2. $9 E+07$

1. $7 E+06$

$1.7 E+02$

$1.7 E+02$

1. $E$ E 02

$1.6 E+02$
$1.5 E+02$

$\begin{array}{lrc}\text { ELEMENT } & \text { ATOMIC } & \text { I SOTOPE } \\ \text { SYMBOL } & \text { NO. }(Z) & \text { MASS(A) } \\ \text { CM } & 96 & 240 \\ \text { CM } & 96 & 241 \\ \text { CM } & 96 & 242 \\ \text { CM } & 96 & 243 \\ \text { CM } & 96 & 244 \\ \text { CM } & 96 & 245 \\ \text { CM } & 96 & 246 \\ \text { CM } & 96 & 247 \\ \text { CM } & 96 & 248 \\ \text { BK } & 97 & 245 \\ \text { BK } & 97 & 246 \\ \text { BK } & 97 & 247 \\ \text { BK } & 97 & 248 \\ \text { BK } & 97 & 249 \\ \text { CF } & 98 & 246 \\ \text { CF } & 98 & 248 \\ \text { CF } & 98 & 249 \\ \text { CF } & 98 & 250 \\ \text { CF } & 98 & 251 \\ \text { CF } & 98 & 252 \\ \text { CF } & 98 & 253 \\ \text { ES } & 99 & 251 \\ \text { ES } & 99 & 252 \\ \text { ES } & 99 & 253 \\ \text { ES } & 99 & 254 \\ \text { ES } & 99 & 254 M \\ \text { ES } & 99 & 255 \\ \text { FM } & 100 & 252 \\ \text { FM } & 100 & 253 \\ \text { FM } & 100 & 255 \\ \text { FM } & 100 & 257\end{array}$

\begin{tabular}{|c|c|}
\hline $\begin{array}{l}\text { ADULT } \\
U C / M * * 2\end{array}$ & $\begin{array}{l}\text { CHILD } \\
U C / M * * 2\end{array}$ \\
\hline 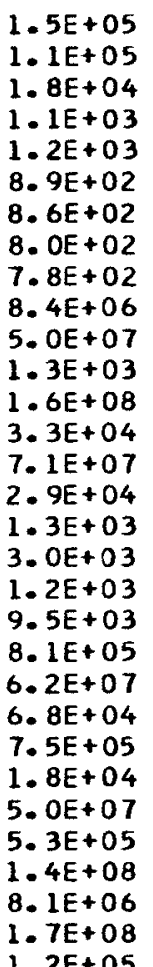 & $\begin{array}{l}2 \cdot 4 E+04 \\
1 \cdot 8 E+04 \\
2 \cdot 9 E+03 \\
1 \cdot 7 E+02 \\
1 \cdot 9 E+02 \\
1 \cdot 4 E+02 \\
1 \cdot 4 E+02 \\
1 \cdot 3 E+02 \\
1 \cdot 3 E+02 \\
1 \cdot 3 E+06 \\
8 \cdot 1 E+06 \\
2 \cdot 1 E+02 \\
2 \cdot 5 E+07 \\
5 \cdot 3 E+03 \\
1 \cdot 1 E+07 \\
4 \cdot 6 E+03 \\
2 \cdot 2 E+02 \\
4 \cdot 9 E+02 \\
2 \cdot 0 E+02 \\
1 \cdot 5 E+03 \\
1 \cdot 3 E+05 \\
9 \cdot 9 E+05 \\
1 \cdot 1 E+04 \\
1 \cdot 2 E+05 \\
2 \cdot 9 E+03 \\
8 \cdot 1 E+06 \\
8 \cdot 6 E+04 \\
2 \cdot 3 E+07 \\
1 \cdot 3 E+06 \\
2 \cdot 8 E+07 \\
19 E+07\end{array}$ \\
\hline
\end{tabular}


TABLE 6A. UNIT-RAD DEPOSITION FOR OVARIES

VIA MILK.

$\begin{array}{lcccc}\text { ELEMENT } & \text { ATOMIC } & \text { 1 SOTOPE } & \text { ADULT } & \text { CHILD } \\ \text { SYMBOL } & \text { NO.12) } & \text { MASS }(A) & \text { UC IM**2 } & \text { UC/M**2 } \\ & & & & \\ \text { H } & 1 & 3 & 7.2 E+02 & 6.8 E+01 \\ \text { BE } & 4 & 7 & 2.8 E+02 & 2.7 E+01 \\ \text { BE } & 4 & 10 & 2.7 E-01 & 2.6 E-02 \\ \text { C } & 6 & 14 & 1.2 E+02 & 1.1 E+01 \\ \text { NA } & 11 & 22 & 8.5 E-01 & 8.0 E-02 \\ \text { NA } & 11 & 24 & 1.7 E+02 & 1.6 E+01 \\ \text { MG } & 12 & 28 & 4.1 E-01 & 3.8 E-02 \\ \text { AL } & 13 & 26 & 1.0 E-04 & 9.5 E-06 \\ \text { SI } & 14 & 32 & 3.6 E+02 & 3.4 E+01 \\ \text { P } & 15 & 32 & 2.7 E+00 & 2.5 E-01 \\ \text { P } & 15 & 33 & 1.9 E+01 & 1.8 E+00 \\ \text { S } & 16 & 35 & 7.7 E+00 & 7.2 E-01 \\ \text { CL } & 17 & 36 & 2.2 E+00 & 2.1 E-01 \\ \text { AR } & 18 & 37 & 7.7 E+02 & 7.2 E+01 \\ \text { AR } & 18 & 39 & 2.9 E-02 & 2.8 E-03 \\ \text { AR } & 18 & 42 & 3.2 E-03 & 3.0 E-04 \\ \text { K } & 19 & 40 & 2.3 E+00 & 2.1 E-01 \\ \text { K } & 19 & 42 & 2.1 E+03 & 2.0 E+02 \\ \text { K } & 19 & 43 & 1.3 E+03 & 1.2 E+02 \\ \text { CA } & 20 & 41 & 8.0 E+02 & 7.5 E+01 \\ \text { CA } & 20 & 45 & 4.2 E+01 & 4.0 E+00 \\ \text { CA } & 20 & 47 & 5.3 E+01 & 5.0 E+00 \\ \text { CA } & 20 & 48 & 7.8 E-01 & 7.4 E-02 \\ \text { SC } & 21 & 44 M & 8.5 E+06 & 8.0 E+05 \\ \text { SC } & 21 & 46 & 5.7 E+04 & 5.4 E+03 \\ \text { SC } & 21 & 47 & 4.2 E+07 & 3.9 E+06 \\ \text { SC } & 21 & 48 & 1.1 E+07 & 1.1 E+06 \\ \text { TI } & 22 & 44 & 1.4 E+01 & 1.3 E+00 \\ \text { V } & 23 & 48 & 2.3 E-01 & 2.1 E-02 \\ \text { V } & 23 & 49 & 3.8 E+00 & 3.5 E-01 \\ \text { V } & 23 & 50 & 6.4 E-04 & 6.0 E-05 \\ \text { CR } & 24 & 48 & 5.2 E+01 & 4.9 E+00 \\ \text { CR } & 24 & 51 & 1.7 E+01 & 1.6 E+00 \\ \text { MN } & 25 & 52 & 5.4 E+02 & 5.1 E+01 \\ \text { MN } & 25 & 53 & 7.9 E+04 & 7.5 E+03 \\ \text { MN } & 25 & 54 & 5.9 E+02 & 5.6 E+01 \\ \text { FE } & 26 & 55 & 5.0 E+02 & 4.7 E+01 \\ \text { FE } & 26 & 59 & 2.4 E+01 & 2.3 E+00 \\ \text { FE } & 26 & 60 & 8.2 E-01 & 7.7 E-02 \\ \text { CD } & 27 & 55 & 4.4 E+04 & 4.1 E+03 \\ \text { CO } & 27 & 56 & 1.2 E+02 & 1.2 E+01 \\ \text { CO } & 27 & 57 & 1.3 E+03 & 1.2 E+02 \\ \text { CO } & 27 & 58 & 2.6 E+02 & 2.5 E+01 \\ \text { CO } & 27 & 60 & 6.4 E+01 & 6.1 E+00 \\ \text { NI } & 28 & 56 & 8.3 E-03 & 7.8 E-04 \\ \text { NI } & 28 & 57 & 2.0 E-01 & 1.9 E-02 \\ \text { NI } & 28 & 59 & 1.0 E-03 & 9.6 E-05 \\ \text { NI } & 28 & 63 & 5.0 E-04 & 4.7 E-05 \\ \text { NI } & 28 & 66 & 1.0 E-01 & 9.4 E-03 \\ \text { CU } & 29 & 64 & 1.4 E+03 & 1.3 E+02\end{array}$

$05 / 09 / 68$

PAGE 15

$\begin{array}{lllcc}\text { ELEMENT } & \text { ATOMIC } & \text { ISOTOPE } & \text { ADULT } & \text { CHILD } \\ \text { SYMBOL } & \text { NO. }(Z) & \text { MASSIA) } & \text { UC } / M * * 2 & U C / M * * 2\end{array}$

\begin{tabular}{|c|c|c|}
\hline $\begin{array}{l}C U \\
Z N\end{array}$ & $\begin{array}{l}29 \\
30\end{array}$ & $\begin{array}{l}67 \\
65\end{array}$ \\
\hline $\mathrm{ZN}$ & 30 & $69 M$ \\
\hline$Z N$ & 30 & 72 \\
\hline GA & 31 & 67 \\
\hline GA & 31 & 72 \\
\hline GE & 32 & 68 \\
\hline GE & 32 & 69 \\
\hline GE & 32 & 71 \\
\hline AS & 33 & 71 \\
\hline AS & 33 & 72 \\
\hline AS & 33 & 73 \\
\hline AS & 33 & 74 \\
\hline AS & 33 & 76 \\
\hline AS & 33 & 77 \\
\hline$S E$ & 34 & 72 \\
\hline SE & 34 & 75 \\
\hline SE & 34 & 79 \\
\hline$B R$ & 35 & 76 \\
\hline BR & 35 & 77 \\
\hline BR & 35 & 82 \\
\hline KR & 36 & 76 \\
\hline KR & 36 & 79 \\
\hline$K R$ & 36 & 81 \\
\hline KR & 36 & 85 \\
\hline RB & 37 & 83 \\
\hline RB & 37 & 84 \\
\hline$R B$ & 37 & 86 \\
\hline RB & 37 & 87 \\
\hline SR & 38 & 82 \\
\hline SR & 38 & 83 \\
\hline SR & 38 & 85 \\
\hline SR & 38 & 89 \\
\hline SR & 38 & 90 \\
\hline$Y$ & 39 & 86 \\
\hline $\mathbf{Y}$ & 39 & 87 \\
\hline$Y$ & 39 & $87 M$ \\
\hline$\gamma$ & 39 & 88 \\
\hline$Y$ & 39 & 90 \\
\hline Y & 39 & 91 \\
\hline$Z R$ & 40 & 86 \\
\hline$Z R$ & 40 & 88 \\
\hline$Z R$ & 40 & 89 \\
\hline$Z R$ & 40 & 93 \\
\hline $2 R^{\prime}$ & 40 & $\$ 5$ \\
\hline$Z R$ & 40 & 97 \\
\hline NB & 41 & 90 \\
\hline NB & 41 & 91 \\
\hline NB & 41 & S1M \\
\hline NB & 41 & 92 \\
\hline
\end{tabular}

8. $9 E+00$ 2. $0 E-01$ $3.6 E+02$ $6.9 E+00$ 1. $8 \mathrm{E}+03$ $3.0 E+03$ $5.1 E-03$ $6.8 E+00$ 4. $4 E+01$ $2.3 E-01$ $2.6 E-01$ $8.8 E-02$ $3.4 \mathrm{E}-03$ 3. $5 \mathrm{E}-01$ $1.3 E+00$ 2. $8 E-05$ 4. $3 E-06$

7. $9 \mathrm{E}-07$

$3 \cdot 2 \mathrm{E}+01$ 正垔

7. $3 E+00$ O

7. $7 E+01$

4. $4 E+01$

$2.7 \mathrm{E}-02$ i

5. $3 \mathrm{E}-03$

1.7E-01

3. $7 E-01$

4. $9 E-01$

$4.4 E+00$

$1.2 E+03$

1. $4 \mathrm{E}+01$

1. $1 E+01$ $2.3 E+00$ $1.0 E+08$ 2. $0 E+07$

3. $8 E+08$

4. $3 E+04$
1. $9 E+07$ 2. $9 E+05$ $1.4 E+05$ 9. $7 E+01$ 1. $9 E+04$ 1. $5 \mathrm{E}+02$ 2. $5 E+02$ 2. $1 E+05$ 3. $4 E+02$ 3. $3 \mathrm{E}-01$ 1. $1 E+01$ 


\begin{tabular}{|c|c|c|c|c|}
\hline $\begin{array}{l}\text { ELEMENT } \\
\text { SYMBOL }\end{array}$ & $\begin{array}{l}\text { ATOMIC } \\
\text { NO. }(Z)\end{array}$ & $\begin{array}{l}\text { I SOTOPE } \\
\text { MASS (A) }\end{array}$ & $\begin{array}{l}\text { ADULT } \\
\text { UC / } M * * 2\end{array}$ & $\begin{array}{c}C H I L D \\
U C / M * 2\end{array}$ \\
\hline NB & 41 & $93 M$ & $1 \cdot 2 E+01$ & $1.2 E+00$ \\
\hline NB & 41 & 94 & $3.6 E-02$ & 3. $4 E-03$ \\
\hline NB & 41 & 95 & $2 \cdot 4 E+01$ & $2.2 E+00$ \\
\hline NB & 41 & $95 M$ & 5. $8 E+02$ & $5.4 E+01$ \\
\hline NB & 41 & 96 & $2.8 E+03$ & $2.6 E+02$ \\
\hline MO & 42 & 93 & 3. $8 E+02$ & $3.6 E+01$ \\
\hline MO & 42 & 99 & $3.6 E+02$ & $3.4 E+01$ \\
\hline TC & 43 & 95 & 2. $5 E-01$ & $2.4 \mathrm{E}-02$ \\
\hline$T C$ & 43 & $95 M$ & $1.8 E-04$ & $1.7 E-05$ \\
\hline TC & 43 & 96 & 3. $8 E-03$ & $3.5 E-04$ \\
\hline TC & 43 & 97 & $6.4 E-05$ & $6.0 \mathrm{E}-06$ \\
\hline TC & 43 & $97 M$ & $1.0 E-03$ & $9.8 \mathrm{E}-05$ \\
\hline TC & 43 & 98 & 8. $5 E-07$ & 8. $0 E-O B$ \\
\hline IC & 43 & 99 & $1.4 E-05$ & $1.3 E-06$ \\
\hline RU & 44 & 97 & $4.6 E+06$ & $4.3 E+05$ \\
\hline RU & 44 & 103 & $1.2 E+05$ & $1.1 E+04$ \\
\hline RU & 44 & 106 & $9.4 E+03$ & $8.8 E+02$ \\
\hline RH & 45 & 99 & 4. $8 \mathrm{E}-01$ & $4.5 E-02$ \\
\hline RH & 45 & 100 & $2.5 E+01$ & $2.3 E+00$ \\
\hline RH & 45 & 101 & $3.3 E-03$ & $3.1 \mathrm{E}-04$ \\
\hline RH & 45 & $101 M$ & $5.4 E+00$ & $5.0 E-01$ \\
\hline RH & 45 & 102 & $6.0 E-03$ & $5.6 E-04$ \\
\hline RH & 45 & 105 & $5.6 E+01$ & $5.3 E+00$ \\
\hline PD & 46 & 100 & $1.5 E+01$ & $1.5 E+00$ \\
\hline PD & 46 & 103 & $3.1 E+01$ & 2. $9 E+00$ \\
\hline PD & 46 & 107 & $2.2 E+00$ & $2.0 \mathrm{E}-01$ \\
\hline PD & 46 & 109 & 1. $7 E+03$ & $1.6 E+02$ \\
\hline PD & 46 & 112 & $1.2 E+02$ & $1.1 \mathrm{E}+01$ \\
\hline$A G$ & 47 & 105 & 1. $7 \mathrm{E}+00$ & $1.6 E-01$ \\
\hline$A G$ & 47 & $106 \mathrm{M}$ & $1.8 E+00$ & $1.7 \mathrm{E}-01$ \\
\hline$A G$ & 47 & $108 M$ & $5.2 E-01$ & $4.9 E-02$ \\
\hline$A G$ & 47 & $110 \mathrm{M}$ & $5.0 E-01$ & $4.7 \mathrm{E}-02$ \\
\hline$A G$ & 47 & 111 & $1.1 E+01$ & $1.1 E+00$ \\
\hline$C D$ & 48 & 109 & $8.0 E+04$ & $7.5 E+03$ \\
\hline$C D$ & 48 & $113 M$ & 3. $7 E+04$ & $3.5 E+03$ \\
\hline$C D$ & 48 & 115 & $1.3 E+06$ & $1.2 E+05$ \\
\hline$C D$ & 48 & $115 M$ & $2.4 E+04$ & $2.3 E+03$ \\
\hline IN & 49 & 111 & $2 \cdot 8 E+03$ & $2.7 E+02$ \\
\hline IN & 49 & $114 \mathrm{M}$ & $1.2 E+01$ & $1.1 E+0 D$ \\
\hline IN & 49 & 115 & $3.6 E-01$ & $3.4 E-02$ \\
\hline SN & 50 & 113 & 1. $9 E+01$ & $1.8 E+00$ \\
\hline SN & 50 & $117 M$ & $1.4 E+02$ & $1.3 E+01$ \\
\hline SN & 50 & $119 \mathrm{M}$ & $7.4 E+01$ & 7. $O E+O O$ \\
\hline SN & 50 & 121 & $2.4 E+04$ & $2.3 E+03$ \\
\hline SN & 50 & $121 M$ & 3. $9 E+01$ & $3.6 E+0 D$ \\
\hline SN & 50 & $123 M$ & $1.1 E+01$ & $1.1 E+00$ \\
\hline SN & 50 & 125 & 4. $0 E+01$ & $3.8 E+00$ \\
\hline SN & 50 & 126 & $1.6 E+00$ & $1.5 E-01$ \\
\hline SB & 51 & 119 & $1.2 E+06$ & $1.1 E+05$ \\
\hline SB & 51 & $120 M$ & $2.3 E+03$ & $2.2 E+02$ \\
\hline
\end{tabular}

$\begin{array}{lcc}\text { ELEMENT } & \text { ATOMIC } & \text { I SOTOP } \\ \text { SYMBOL } & \text { NO. }(2) & \text { MASS(A } \\ & & \\ \text { SB } & 51 & 122 \\ \text { SB } & 51 & 124 \\ \text { SB } & 51 & 125 \\ \text { SB } & 51 & 126 \\ \text { SB } & 51 & 127 \\ \text { TE } & 52 & 118 \\ \text { TE } & 52 & 119 \\ \text { TE } & 52 & 119 M \\ \text { TE } & 52 & 121 \\ \text { TE } & 52 & 121 M \\ \text { TE } & 52 & 123 \\ \text { TE } & 52 & 123 M \\ \text { TE } & 52 & 125 M \\ \text { TE } & 52 & 127 M \\ \text { TE } & 52 & 129 M \\ \text { TE } & 52 & 131 M \\ \text { TE } & 52 & 132 \\ \text { I } & 53 & 123 \\ \text { I } & 53 & 124 \\ \text { I } & 53 & 125 \\ \text { I } & 53 & 126 \\ \text { I } & 53 & 129 \\ \text { I } & 53 & 130 \\ \text { I } & 53 & 131 \\ \text { I } & 53 & 133 \\ \text { XE } & 54 & 122 \\ \text { XE } & 54 & 125 \\ \text { XE } & 54 & 127 \\ \text { XE } & 54 & 129 M \\ \text { XE } & 54 & 131 M \\ \text { XE } & 54 & 133 \\ \text { XE } & 54 & 133 M \\ \text { CS } & 55 & 129 \\ \text { CS } & 55 & 131 \\ \text { CS } & 55 & 132 \\ \text { CS } & 55 & 134 \\ \text { CS } & 55 & 135 \\ \text { CS } & 55 & 136 \\ \text { CS } & 55 & 137 \\ \text { BA } & 56 & 128 \\ \text { BA } & 56 & 131 \\ \text { BA } & 56 & 133 \\ \text { BA } & 56 & 133 M \\ \text { BA } & 56 & 135 M \\ \text { BA } & 56 & 140 \\ \text { LA } & 57 & 135 \\ \text { LA } & 57 & 137 \\ \text { LA } & 57 & 138 \\ \text { LA } & 57 & 140 \\ \text { CE } & 58 & 134\end{array}$

ADULT

CHILD

1. $9 E+04$

1. $8 E+03$

8. $5 E+01 \quad 8.0 E+00$

1. $9 E+01 \quad 1.8 E+00$

6. $O E+02 \quad 5.7 E+01$

9. $3 E+03 \quad 8.7 E+02$

2. $9 E+02 \quad 2.7 E+01$

1. $0 E+04$

2. $7 E+02$

9. $8 E+02$

2. $5 E+01$

1. $7 E+02$

2. $9 E+01$

6. $5 \mathrm{E}+02$

1. $0 \mathrm{E}+02$

2. $6 E+02$
8. $3 E+01$

5. $7 E+01$

2. $6 E+03$

3. $5 \mathrm{E}+02$

1. $3 E+00$

2. $7 \mathrm{E}-03$

1. $8 \mathrm{E}-03$

1. $8 \mathrm{E}-03$

1. $0 E-05$

1. $3 E-01$

3. $4 \mathrm{E}-03$

9. $2 \mathrm{E}-02$

2. $5 \mathrm{E}+03$

8. $7 E+00$

7. $9 E+01$

6. $O E+01$

2. $O E+02$

4. $0 E+02$

$1.6 E+01$

2. $7 E+00$

6. $1 E+01$

2. $4 E+01$

7. $8 E+00$

$5.4 E+00$

$2.4 E+02$

$3.3 E+01$

1. $2 E-01$

2. $6 \mathrm{E}-04$

1. $7 E-04$

1. $7 \mathrm{E}-04$

9. $7 \mathrm{E}-07$

1. $2 E-02$

8. $7 E-03$

4. $3 E+01$

$2.4 E+02$

2. $E$ T 02

7. $4 E+00$

$5.6 E+00$

1. $9 E+01$

3. $7 E+01$

1. $2 \mathrm{E}+03$

5. $6 \mathrm{E}+01$

$4.2 E-01$

4. $2 E-01$

5. $9 E+00$

8. $8 \mathrm{E}-01$

1. $6 E+04$

5. $7 E+03$

1. $1 E+02$

5. $7 E+01$

5. $3 E+00$
4. $O E-02$

4. $0 E-02$

$1.2 E+00$

5. $6 \mathrm{E}-01$

8. $3 E-02$

1. $5 \mathrm{E}+03$

$5.4 E+02$

4. $9 \mathrm{E}+02$

5. $2 E+03$

1. $1 E+05$

4. $6 E+05$

1. $6 E+03$

6. $0 E+06$

8. $5 E+02$

2. $5 \mathrm{E}+01$

4. $2 E+05$

1. $1 E+04$

4. $5 E+04$

1. $5 E+02$

$5.6 E+05$

$8.0 E+01$

2. $3 E+00$

4. $0 E+04$

3. $8 E+06$ 
TABLE 6A. UNIT-RAD DEPOSITION FOR OVARIES

VIA MILK.

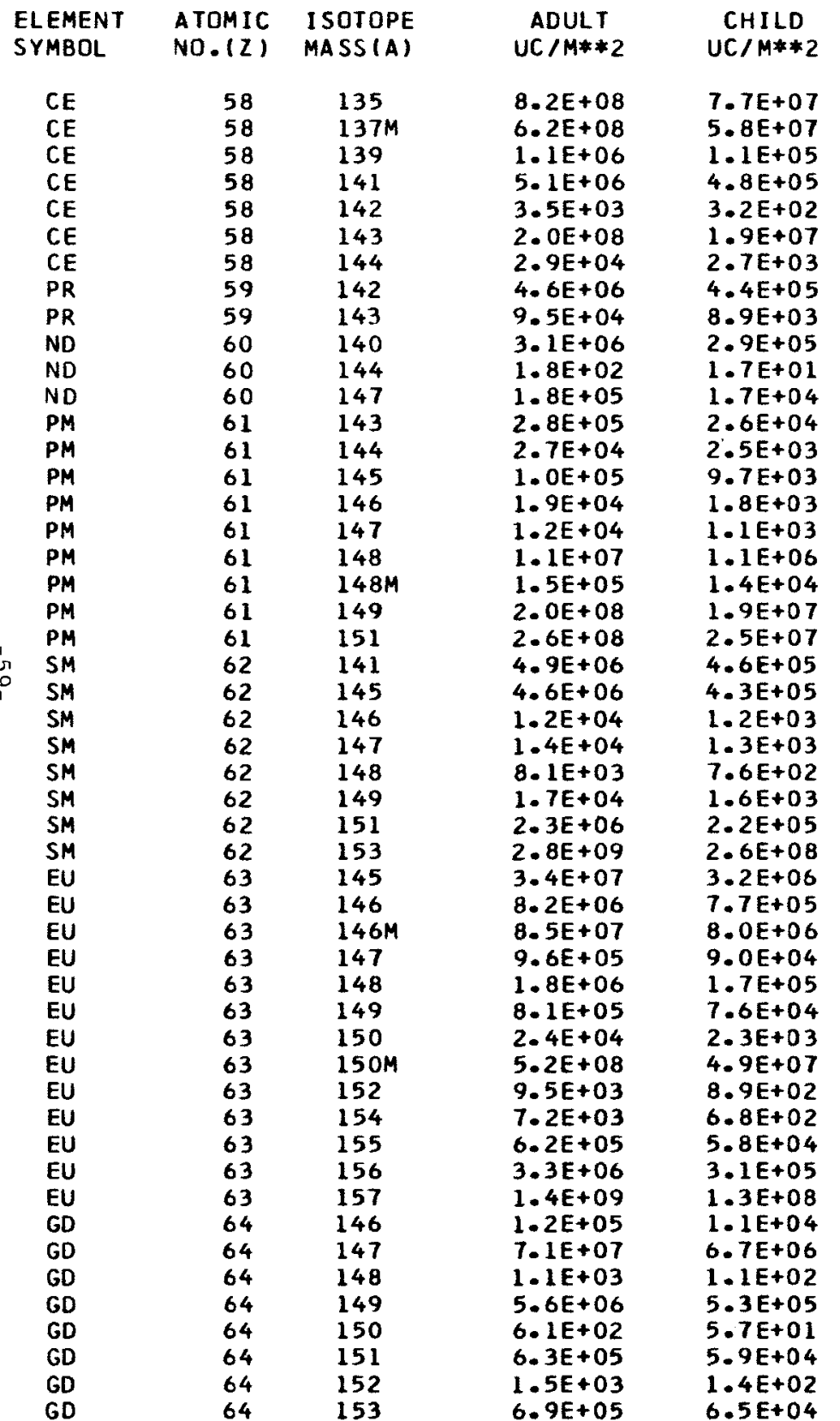

$05 / 09 / 58$

PAGE 18

$\begin{array}{lcc}\text { ELEMENT } & \text { ATOMIC } & \text { ISOTOP } \\ \text { SYMBOL } & \text { NO. }(2) & \text { MASS(A) } \\ \text { GD } & 64 & \\ \text { GD } & 64 & 159 \\ \text { TB } & 65 & 162 \\ \text { TB } & 65 & 152 \\ \text { TB } & 65 & 153 \\ \text { TB } & 65 & 154 \\ \text { TB } & 65 & 155 \\ \text { TB } & 65 & 156 \\ \text { TB } & 65 & 157 \\ \text { IB } & 65 & 158 \\ \text { TB } & 65 & 160 \\ \text { TB } & 65 & 161 \\ \text { IB } & 65 & 164 \\ \text { DY } & 66 & 154 \\ \text { DY } & 66 & 154 M \\ \text { DY } & 66 & 156 \\ \text { DY } & 66 & 159 \\ \text { DY } & 66 & 166 \\ \text { HO } & 67 & 163 \\ \text { HO } & 67 & 166 \\ \text { HO } & 67 & 166 M \\ \text { ER } & 68 & 160 \\ \text { ER } & 68 & 169 \\ \text { ER } & 68 & 172 \\ \text { TM } & 69 & 165 \\ \text { TM } & 69 & 167 \\ \text { TM } & 69 & 168 \\ \text { TM } & 69 & 170 \\ \text { TM } & 69 & 171 \\ \text { TM } & 69 & 172 \\ \text { YB } & 70 & 166 \\ \text { YB } & 70 & 169 \\ \text { YB } & 70 & 175 \\ \text { LU } & 71 & 169 \\ \text { LU } & 71 & 170 \\ \text { LU } & 71 & 171 \\ \text { LU } & 71 & 172 \\ \text { LU } & 71 & 173 \\ \text { LU } & 71 & 174 \\ \text { LU } & 71 & 174 M \\ \text { LU } & 71 & 176 \\ \text { LU } & 71 & 177 \\ \text { LU } & 71 & 177 M \\ \text { HF } & 72 & 170 \\ \text { HF } & 72 & 171 \\ \text { HF } & 72 & 172 \\ \text { HF } & 72 & 173 \\ \text { HF } & 72 & 174 \\ \text { HF } & 72 & 175 \\ \text { HF } & 72 & 181 \\ & & \end{array}$

ADULT
$U C / M * 2$
$1.5 E+09$
$4.5 E+04$
$6.1 E+08$
$1.8 E+08$
$1.3 E+08$
$6.6 E+08$
$3.5 E+07$
$1.1 E+07$
$6.8 E+04$
$3.0 E+03$
$2.9 E+05$
$5.4 E+07$
$9.0 E+08$
$3.9 E+00$
$3.5 E+06$
$6.2 E+00$
$2.7 E+04$
$3.2 E+05$
$5.9 E+02$
$3.0 E+06$
$2.6 E+01$
$8.6 E+05$
$6.7 E+05$
$7.2 E+05$
$1.3 E+08$
$1.4 E+07$
$2.8 E+05$
$7.6 E+05$
$2.4 E+06$
$8.0 E+07$
$8.6 E+07$
$1.9 E+06$
$9.4 E+07$
$9.0 E+05$
$3.2 E+05$
$5.7 E+05$
$1.1 E+05$
$6.9 E+02$
$1.5 E+02$
$2.9 E+03$
$4.4 E+01$
$6.4 E+05$
$8.3 E+03$
$3.2 E+07$
$6.4 E+07$
$4.9 E+03$
$2.0 E+07$
$1.7 E+03$
$9.3 E+04$
$1.0 E+05$

CHILD

UC/ $M * * 2$

$1.4 E+08$ 4. $3 E+03$ $5.8 \mathrm{E}+0 \mathrm{l}$ 1. $7 E+07$ 1. $2 E+07$ 6. $2 E+07$ $3.3 E+06$ 1. $0 E+06$ $6.4 E+03$ $2.8 E+02$ 2. $7 E+04$ 5. $0 E+06$ 8. $5 E+07$ 3. $7 E-01$ $3.3 E+05$ $5.8 \mathrm{E}-01$ $2.6 E+03$ 2.6E+03 3. $0 E+04$ $5.6 E+01$ 2. $9 E+05$ 2. $5 E+00$ 8. $1 E+04$ $6.3 E+04$ 6. $8 E+04$ 1. $2 \mathrm{E}+07$ 1. $3 E+06$ 1. $3 E+06$ 2. $7 E+04$ $7.2 E+04$ $2.2 \mathrm{E}+05$ 7. $5 E+0 S$ 8. $1 E+05$ 1. $8 E+05$ 8. $8 E+06$ $8.5 E+04$ 3. $0 E+04$ $5.4 E+04$ $5.4 E+04$ $1.1 E+04$ $6.5 E+01$ $1.4 \mathrm{E}+01$ $2.7 E+02$ 4. $1 E+00$ 6. $0 E+04$ $7.8 E+02$ 3. $1 E+0 S$ 6. $O E+O B$ 4. $6 E+02$ 1. $9 E+06$ 1. $6 E+02$ 8. $7 E+03$
$9.8 E+03$ 


\begin{tabular}{|c|c|c|c|c|}
\hline $\begin{array}{l}\text { ELEMENT } \\
\text { SYMBOL }\end{array}$ & $\begin{array}{l}\text { ATOMIC } \\
\text { NO. }(Z)\end{array}$ & $\begin{array}{l}\text { I SOTOPE } \\
\text { MASS(A) }\end{array}$ & $\begin{array}{l}\text { ADULT } \\
\text { UC } / M * * 2\end{array}$ & $\begin{array}{l}\text { CHILD } \\
U C / M * * 2\end{array}$ \\
\hline HF & 72 & 182 & $2.6 E+03$ & $2.4 E+02$ \\
\hline TA & 73 & 177 & $5.2 E+00$ & $4.9 E-01$ \\
\hline TA & 73 & 179 & $1.2 \mathrm{E}-01$ & $1.1 E-02$ \\
\hline TA & 73 & 182 & $1.6 E-02$ & 1. $5 E-03$ \\
\hline TA & 73 & 183 & $2.3 E+00$ & $2.1 \mathrm{E}-01$ \\
\hline$W$ & 74 & 178 & $1.0 E-01$ & $9.7 \mathrm{E}-03$ \\
\hline$w$ & 74 & 181 & 2. $9 E-01$ & $2.8 E-02$ \\
\hline$W$ & 74 & 185 & $3.1 \mathrm{E}-01$ & $3.0 \mathrm{E}-02$ \\
\hline W & 74 & 187 & $4.3 E+01$ & 4. $O E+00$ \\
\hline$W$ & 74 & 188 & 3. $1 E-02$ & $2.9 E-03$ \\
\hline RE & 75 & 180 & 1. $1 E-01$ & 1. $0 E-02$ \\
\hline RE & 75 & 181 & $1 \cdot 2 E-01$ & $1.1 \mathrm{E}-02$ \\
\hline RE & 75 & 182 & $1.5 E-02$ & $1.4 \mathrm{E}-03$ \\
\hline RE & 75 & $182 M$ & 3. $1 E-01$ & $2.9 E-02$ \\
\hline RE & 75 & 183 & 4. $9 E-04$ & $4.6 E-05$ \\
\hline RE & 75 & 184 & $3.5 E-04$ & $3.3 E-05$ \\
\hline RE & 75 & $184 M$ & 4. $9 E-05$ & $4.6 E-06$ \\
\hline RE & 75 & 186 & $2.7 E-02$ & $2.5 E-03$ \\
\hline RE & 75 & 187 & $9.8 E-03$ & $9.3 E-04$ \\
\hline RE & 75 & 188 & $2.6 E-01$ & $2.5 E-02$ \\
\hline RE & 75 & 189 & $3.1 E-01$ & $2.9 E-02$ \\
\hline OS & 76 & 182 & $2.0 E+00$ & $1.9 E-01$ \\
\hline OS & 76 & 183 & $1.5 E+01$ & $1.4 E+00$ \\
\hline DS & 76 & 185 & $3.8 \mathrm{E}-03$ & $3.6 E-04$ \\
\hline OS & 76 & 191 & 1. $5 \mathrm{E}-01$ & $1.4 \mathrm{E}-02$ \\
\hline OS & 76 & $191 \mathrm{M}$ & $3.7 E+O 1$ & $3.5 E+00$ \\
\hline OS & 76 & 193 & 4. $3 E+00$ & $4.0 E-01$ \\
\hline os & 76 & 194 & $5.2 E-05$ & $4.9 E-06$ \\
\hline IR & 77 & 185 & $8.2 E+00$ & $7.7 \mathrm{E}-01$ \\
\hline IR & 77 & 186 & $4.8 E+00$ & $4.5 E-01$ \\
\hline IR & 77 & 187 & $1.3 E+01$ & $1.2 E+00$ \\
\hline IR & 77 & 188 & $5.6 E-01$ & $5.3 E-02$ \\
\hline IR & 77 & 189 & $1.1 E-01$ & $9.9 E-03$ \\
\hline IR & 77 & 190 & 3. $1 E-02$ & 2. $9 E-03$ \\
\hline IR & 77 & 192 & $3.7 E-03$ & $3.5 E-04$ \\
\hline IR & 77 & $192 \mathrm{M}$ & $2.7 \mathrm{E}-05$ & $2.5 E-06$ \\
\hline IR & 77 & $193 M$ & $5.4 E-01$ & $5.1 \mathrm{E}-02$ \\
\hline IR & 77 & 194 & $4.5 E+00$ & $4.2 E-01$ \\
\hline PT & 78 & 188 & $2.0 E-02$ & $1.9 E-03$ \\
\hline pT & 78 & 190 & $1.0 \mathrm{E}-05$ & $9.6 \mathrm{E}-07$ \\
\hline PT & 78 & 191 & $8.4 E-01$ & $7.9 E-02$ \\
\hline PT & 78 & 192 & 1. $2 \mathrm{E}-05$ & $1.1 E-06$ \\
\hline PT & 78 & 193 & $4.3 E-04$ & $4.0 E-05$ \\
\hline $\mathrm{PT}$ & 78 & $193 \mathrm{M}$ & 1. $O E+O 0$ & $9.5 E-02$ \\
\hline PT & 78 & $195 \mathrm{M}$ & 9. $9 E-01$ & $9.3 E-02$ \\
\hline Pr & 78 & 197 & 1. $7 E+01$ & $1.6 \mathrm{E}+00$ \\
\hline$A U$ & 79 & 193 & $1.1 E+01$ & $9.9 E-01$ \\
\hline AU & 79 & 194 & $6.8 \mathrm{E}-01$ & $6.3 E-02$ \\
\hline $\mathrm{AU}$ & 79 & 195 & $6.8 E-03$ & $6.4 E-04$ \\
\hline AU & 79 & 196 & $1.7 \mathrm{E}-01$ & $1.6 E-02$ \\
\hline
\end{tabular}

\begin{tabular}{|c|c|c|c|c|}
\hline $\begin{array}{l}\text { ELEMENT } \\
\text { SYMBOL }\end{array}$ & $\begin{array}{l}\text { ATOMIC } \\
\text { NO. }(Z)\end{array}$ & $\begin{array}{l}\text { I SOTOPE } \\
\text { MASS (A) }\end{array}$ & $\begin{array}{l}\text { ADULT } \\
\text { UC } / M * * 2\end{array}$ & $\begin{array}{c}C H I L D \\
U C / M * * 2\end{array}$ \\
\hline$A U$ & 79 & 198 & $7.3 \mathrm{E}-01$ & $6.8 E-02$ \\
\hline$A U$ & 79 & 199 & 1. $8 E+00$ & 1.7E-01 \\
\hline$H G$ & 80 & 194 & $1.5 \mathrm{E}-05$ & $1.4 E-06$ \\
\hline$H G$ & 80 & $195 \mathrm{M}$ & $1.4 E-02$ & $1.4 E-03$ \\
\hline$H G$ & 80 & 197 & $2.8 E-02$ & $2.6 E-03$ \\
\hline HG & 80 & $197 M$ & 9. $9 E-02$ & $9.3 E-03$ \\
\hline$H G$ & 80 & 203 & $3.6 \mathrm{E}-04$ & $3.4 E-05$ \\
\hline $\mathrm{TL}$ & 81 & 200 & $3.4 E+01$ & $3.2 E+00$ \\
\hline $\mathrm{TL}$ & 81 & $2 \mathrm{Cl}$ & $2.8 E+01$ & $2.6 E+00$ \\
\hline$T L$ & 81 & 202 & $1.4 E+00$ & 1. $3 \mathrm{E}-01$ \\
\hline TL & 81 & 204 & $9.1 E-03$ & $8.5 E-04$ \\
\hline PB & 82 & 200 & $2.6 E+01$ & $2.5 E+00$ \\
\hline PB & 82 & $2 \mathrm{C} 2$ & $5.4 E-04$ & $5.1 E-05$ \\
\hline PB & 82 & 203 & $1.1 E+01$ & 1. $O E+00$ \\
\hline PB & 82 & 204 & 1. $2 \mathrm{E}-04$ & $1.2 E-05$ \\
\hline PB & 82 & 205 & 3. $7 \mathrm{E}-03$ & $3.5 E-04$ \\
\hline PB & 82 & 210 & $8.6 E-05$ & 8. OE -06 \\
\hline$B \bar{I}$ & 83 & 205 & 1. $5 E+00$ & $1.4 E-01$ \\
\hline B I & 83 & 206 & $6.8 E+00$ & $6.4 \mathrm{E}-01$ \\
\hline B I & 83 & 207 & $2.4 E-03$ & $2.2 E-04$ \\
\hline BI & 83 & $2 \mathrm{CB}$ & 1. $2 E-03$ & $1.1 E-04$ \\
\hline BI & 83 & 210 & $3.2 E+00$ & $3.0 E-01$ \\
\hline BI & 83 & $210 \mathrm{M}$ & $5.7 E-04$ & $5.4 \mathrm{E}-05$ \\
\hline PO & 84 & 206 & $6.3 E-02$ & $6.0 E-03$ \\
\hline PO & 84 & $2 \mathrm{CB}$ & 8. $2 E-05$ & $7.8 \mathrm{E}-0 \mathrm{~S}$ \\
\hline PO & 84 & 209 & $2.0 E-05$ & $1.8 E-06$ \\
\hline PO & 84 & 210 & 9. $9 E-04$ & $9.3 E-05$ \\
\hline RN & 86 & 211 & 1. $9 E+02$ & 1. $8 E+01$ \\
\hline RN & 86 & 222 & $2.5 E+00$ & $2.4 E-01$ \\
\hline RA & 88 & 223 & 1. $8 \mathrm{E}-04$ & $1.7 \mathrm{E}-05$ \\
\hline RA & 88 & 224 & 1. $2 \mathrm{E}-03$ & $1.1 E-04$ \\
\hline RA & 88 & 225 & $1.2 E-04$ & $1.2 E-05$ \\
\hline RA & 88 & 226 & $1.1 E-07$ & 1. $1 \mathrm{E}-\mathrm{OB}$ \\
\hline RA & 88 & 228 & 3. $7 E-07$ & $3.5 E-08$ \\
\hline$A C$ & 89 & 225 & $2.0 E+03$ & $1.9 E+02$ \\
\hline$A C$ & 89 & 227 & 1. $5 E+00$ & $1.4 E-01$ \\
\hline $\mathrm{TH}$ & 90 & 227 & $6.8 E+02$ & $6.4 E+01$ \\
\hline TH & 90 & 228 & $1.0 E+01$ & $9.5 \mathrm{E}-01$ \\
\hline $\mathrm{TH}$ & 90 & 229 & $9.5 E-01$ & $9.0 E-02$ \\
\hline TH & 90 & 230 & 8. $9 E-01$ & $8.4 E-02$ \\
\hline TH & 90 & 231 & $8.1 E+04$ & $7.6 \mathrm{E}+03$ \\
\hline TH & 90 & 232 & 8. $0 E-01$ & $7.5 E-02$ \\
\hline TH & 90 & 234 & 3. $7 E+02$ & $3.5 E+01$ \\
\hline PA & 91 & 228 & $1.2 E+03$ & $1.1 E+02$ \\
\hline PA & 91 & 229 & 4. $9 E+02$ & $4.6 E+01$ \\
\hline PA & 91 & 230 & $6.9 E+00$ & $6.4 E-01$ \\
\hline PA & 91 & 231 & $8.2 E-03$ & 7. $7 E-04$ \\
\hline PA & 91 & 232 & $5.2 E+02$ & 4. $9 E+01$ \\
\hline PA & 91 & 233 & $3.4 E+00$ & $3.2 E-01$ \\
\hline$u$ & 92 & 230 & 4. $9 E+00$ & $4.6 \mathrm{E}-01$ \\
\hline
\end{tabular}


TABLE GA. UNIT-RAD DEPOSITION FOR OVARIES VIA MILK.

05/09/68 PAGE 20

\section{ELEMENT}

ATOMIC

NO. (Z)

I SOTOPE

240

5. $9 E+00$

8. $6 E-04$

7. $8 \mathrm{E}-04$

$7.4 E-04$

$6.8 E-04$

6. $7 E-04$

$2.3 E+00$

$6.5 E-04$

1. $9 E+02$

5. $0 E+02$
$1.3 E+00$

1. $3 E+00$
$6.5 E-02$

6. $5 E-02$
$8.8 E+03$

6. 9 E-02

1. $7 \mathrm{E}+03$

1. $4 E+03$

$5.3 E+01$

1. $6 E+03$

$8.1 E+00$

$6.8 E+00$

$6.7 E+D O$

1. $4 E+01$

$6.5 E+00$

5. $8 \mathrm{E}+00$

9. $0 E+03$

2. $6 E+04$

2. $6 E+04$

$1.6 E+03$
$6.2 E-02$

6. $2 E-02$
$1.4 E+04$

1. $4 E+04$
$6.1 E-02$

5. $5 \mathrm{E}-02$

$\begin{array}{lrl}\text { CM } & 96 & 240 \\ \text { CM } & 96 & 241 \\ \text { CM } & 96 & 242 \\ \text { CM } & 96 & 243 \\ \text { CM } & 96 & 244 \\ \text { CM } & 96 & 245 \\ C M & 96 & 246 \\ C M & 96 & 247 \\ \text { CM } & 96 & 248 \\ \text { BK } & 97 & 245 \\ \text { BK } & 97 & 246 \\ \text { BK } & 97 & 247 \\ \text { BK } & 97 & 248 \\ \text { BK } & 97 & 249 \\ C F & 98 & 246 \\ C F & 98 & 248 \\ C F & 98 & 249 \\ C F & 98 & 250 \\ C F & 98 & 251 \\ \text { CF } & 98 & 252 \\ \text { CF } & 98 & 253 \\ \text { ES } & 99 & 251 \\ \text { ES } & 99 & 252 \\ \text { ES } & 99 & 253 \\ \text { ES } & 99 & 254 \\ \text { ES } & 99 & 254 M \\ \text { ES } & 99 & 255 \\ \text { FM } & 100 & 252 \\ \text { FM } & 100 & 253 \\ \text { FM } & 100 & 255 \\ \text { FM } & 100 & 257\end{array}$

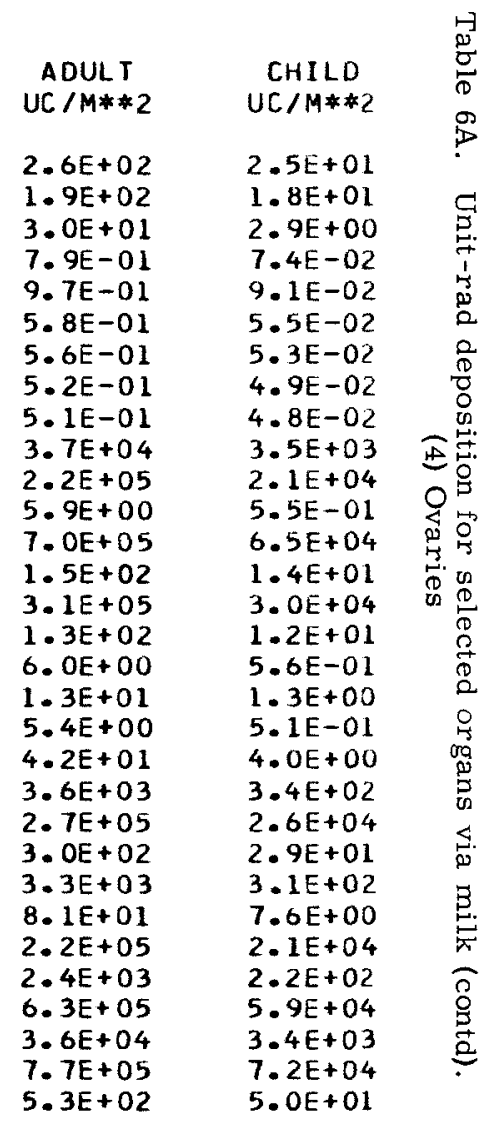




\begin{tabular}{|c|c|c|c|c|}
\hline $\begin{array}{l}\text { ELEMENT } \\
\text { SYMBOL }\end{array}$ & $\begin{array}{l}\text { ATOMIC } \\
\text { NO. }(Z)\end{array}$ & $\begin{array}{l}\text { I SOTOPE } \\
\text { MASS (A) }\end{array}$ & $\begin{array}{c}\text { ADULT } \\
\text { UC/M**2 }\end{array}$ & $\begin{array}{c}\text { CHILD } \\
\text { UC/M**2 }\end{array}$ \\
\hline$H$ & 1 & 3 & $6.9 E+02$ & $1.1 E+02$ \\
\hline $\mathrm{BE}$ & 4 & 7 & 3. $4 E+06$ & $5.3 E+05$ \\
\hline $\mathrm{BE}$ & 4 & 10 & $4.2 E+04$ & $6.4 E+03$ \\
\hline$C$ & 6 & 14 & $1.1 E+02$ & $1.8 E+01$ \\
\hline NA & 11 & 22 & $8.2 E-01$ & $1.3 E-01$ \\
\hline NA & 11 & 24 & $1.7 \mathrm{E}+02$ & $2.6 E+01$ \\
\hline MG & 12 & 28 & 8. $9 E+00$ & $1.4 E+00$ \\
\hline$A L$ & 13 & 26 & $1.6 E+01$ & $2.5 E+00$ \\
\hline SI & 14 & 32 & $3.7 E-03$ & $5.8 \mathrm{E}-04$ \\
\hline $\mathbf{P}$ & 15 & 32 & 2. $1 E+00$ & $3.2 E-01$ \\
\hline $\mathbf{P}$ & 15 & 33 & $1.4 E+01$ & $2.1 E+00$ \\
\hline 5 & 16 & 35 & $1.1 E+02$ & $1.7 E+01$ \\
\hline $\mathrm{CL}$ & 17 & 36 & $2.2 E+00$ & $3.3 E-01$ \\
\hline AR & 18 & 37 & $7.4 E+02$ & $1.1 E+02$ \\
\hline AR & 18 & 39 & $2.8 \mathrm{E}-02$ & $4.4 E-03$ \\
\hline AR & 18 & 42 & $3.1 E-03$ & $4 \cdot 7 E-04$ \\
\hline K & 19 & 40 & $2.2 E+00$ & $3.4 E-01$ \\
\hline $\mathrm{k}$ & 19 & 42 & 2. $1 E+03$ & $3.2 E+02$ \\
\hline K & 19 & 43 & 1. $3 E+03$ & $1.9 E+02$ \\
\hline$C A$ & 20 & 41 & $2.5 E+03$ & $3.9 E+02$ \\
\hline CA & 20 & 45 & 1. $1 E+02$ & $1.8 E+01$ \\
\hline CA & 20 & 47 & 5. $5 E+01$ & $8.5 E+00$ \\
\hline CA & 20 & 48 & $2.5 E+00$ & $3.8 E-01$ \\
\hline SC & 21 & $44 M$ & $9.5 E+07$ & $1.5 E+07$ \\
\hline SC & 21 & 46 & $6.7 E+05$ & $1.0 E+05$ \\
\hline SC & 21 & 47 & 4. $7 E+08$ & $7.2 \mathrm{E}+07$ \\
\hline SC & 21 & 48 & $1.3 E+08$ & 2. $0 E+07$ \\
\hline TI & 22 & 44 & $5.8 E+05$ & $9.0 \mathrm{E}+04$ \\
\hline$v$ & 23 & 48 & 5. $8 E+02$ & $9.0 E+01$ \\
\hline $\mathbf{v}$ & 23 & 49 & 3. $8 E+04$ & $5.9 E+03$ \\
\hline v & 23 & 50 & $1 \cdot 2 \mathrm{E}+02$ & $1.8 E+01$ \\
\hline CR & 24 & 48 & 1. $1 E+03$ & $1.7 E+02$ \\
\hline CR & 24 & 51 & 3. $8 E+02$ & $5.8 E+01$ \\
\hline MN & 25 & 52 & 4. $0 E+03$ & $6.2 E+02$ \\
\hline NN & 25 & 53 & $1.8 E+05$ & $2.8 E+04$ \\
\hline MN & 25 & 54 & $1.4 E+03$ & $2.2 E+02$ \\
\hline$F E$ & 26 & 55 & 4. $5 E+02$ & $7.0 E+01$ \\
\hline $\mathrm{FE}$ & 26 & 59 & $2.6 E+01$ & 4. $. O E+O D$ \\
\hline FE & 26 & 60 & $6.7 E-01$ & $1.0 E-01$ \\
\hline $\mathrm{CO}$ & 27 & 55 & 6. $8 E+04$ & $1.1 E+04$ \\
\hline $\mathrm{CO}$ & 27 & 56 & 1. $9 E+02$ & $2.9 E+01$ \\
\hline $\mathrm{CO}$ & 27 & 57 & $2.1 E+03$ & $3 \cdot 2 E+02$ \\
\hline $\mathrm{CO}$ & 27 & 58 & 4. $1 E+02$ & $6.3 E+01$ \\
\hline $\mathrm{CO}$ & 27 & 60 & 1. $0 E+02$ & $1.5 E+01$ \\
\hline NI & 28 & 56 & $1.8 E-01$ & $2.8 E-02$ \\
\hline NI & 28 & 57 & 4. $4 E+00$ & $6.8 E-01$ \\
\hline N I & 28 & 59 & $2.2 \mathrm{E}-02$ & $3.5 \mathrm{E}-03$ \\
\hline NI & 28 & 63 & 1. $1 \mathrm{E}-02$ & $1.7 \mathrm{E}-03$ \\
\hline NI & 28 & 66 & $2 \cdot 2 \mathrm{E}+00$ & $3.4 E-01$ \\
\hline $\mathrm{CU}$ & 29 & 64 & 1. $4 E+03$ & $2.2 E+02$ \\
\hline
\end{tabular}

\begin{tabular}{c} 
ELEM \\
SYMB \\
\\
$C U$ \\
$Z N$ \\
$Z N$ \\
$Z N$ \\
GA \\
$G A$ \\
$G E$ \\
$G E$ \\
$G E$ \\
$A S$ \\
$A S$ \\
$A S$ \\
$A S$ \\
$A S$ \\
$A S$ \\
SE \\
SE \\
$S E$ \\
$B R$ \\
$B R$ \\
$B R$ \\
$K R$ \\
KR \\
$K R$ \\
$K R$ \\
$R B$ \\
$R B$ \\
$R B$ \\
$R B$ \\
SR \\
SR \\
$S R$ \\
$S R$ \\
$S R$ \\
SR \\
$Y$ \\
$Y$ \\
$Y$ \\
$Y$ \\
$Y$ \\
$Y$ \\
$Z R$ \\
$Z R$ \\
$Z R$ \\
$Z R$ \\
$Z R$ \\
$Z R$ \\
$N B$ \\
$N B$ \\
$N B$ \\
$N B$ \\
\hline
\end{tabular}

ATOMIC ISOTOPE
NO. (Z) MASS(A)

ADULT

CHILD YMB

$\begin{array}{ll}29 & 67 \\ 30 & 65 \\ 30 & 69 M \\ 30 & 72 \\ 31 & 67 \\ 31 & 72 \\ 32 & 68 \\ 32 & 69 \\ 32 & 71 \\ 33 & 71 \\ 33 & 72 \\ 33 & 73 \\ 33 & 74 \\ 33 & 76 \\ 33 & 77 \\ 34 & 72 \\ 34 & 75 \\ 34 & 79 \\ 35 & 76 \\ 35 & 77 \\ 35 & 82 \\ 36 & 76 \\ 36 & 79 \\ 36 & 81 \\ 36 & 85 \\ 37 & 83 \\ 37 & 84 \\ 37 & 86 \\ 37 & 87 \\ 38 & 82 \\ 38 & 83 \\ 38 & 85 \\ 38 & 89 \\ 38 & 90 \\ 39 & 86 \\ 39 & 87 \\ 39 & 87 M \\ 39 & 88 \\ 39 & 90 \\ 39 & 91 \\ 40 & 86 \\ 40 & 88 \\ 40 & 89 \\ 40 & 93 \\ 40 & 95 \\ 40 & 97 \\ 41 & 90 \\ 41 & 91 \\ 41 & 91 M \\ 41 & 92\end{array}$

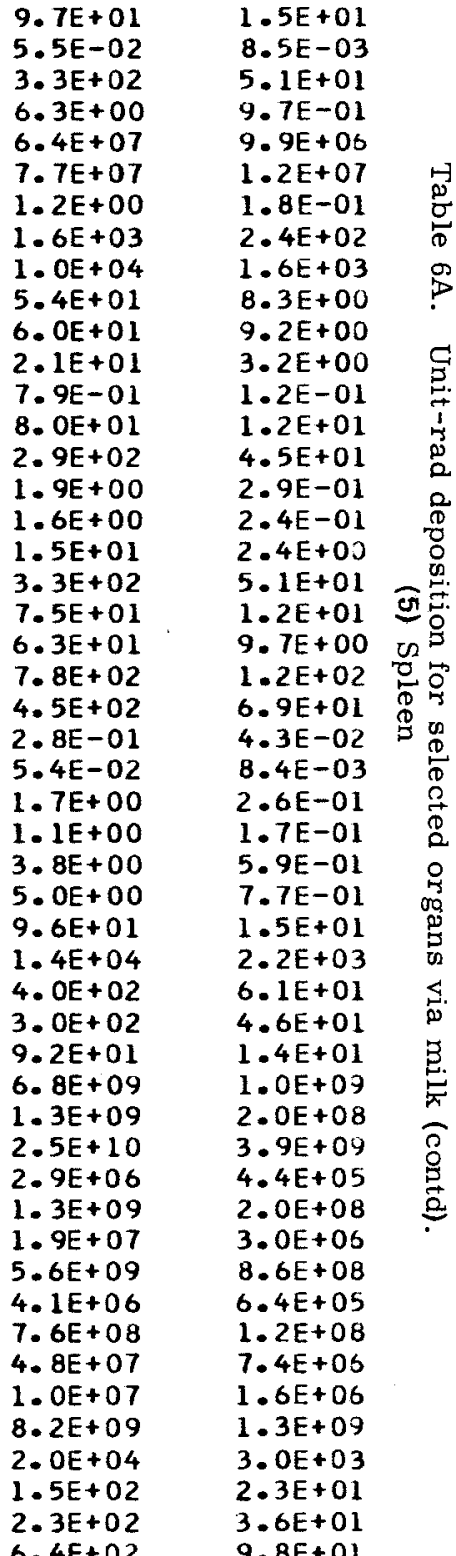

.


TABLE 6A. UNIT-RAD DEPOSITION FOR SPLEEN VIA MILK.

05/09/58 PAGE 22

$\begin{array}{lcccc}\text { ELEMENT } & \text { ATOMIC } & \text { I SOTOPE } & \text { ADULT } & \text { CHILD } \\ \text { SYMBDL } & \text { NO. (2) } & \text { MASS IA) } & \text { UCIM*2 } & \text { UC/M**2 } \\ \text { NB } & 41 & 93 M & 1.6 E+02 & 2.5 E+01 \\ \text { NB } & 41 & 94 & 1.5 E+00 & 2.4 E-01 \\ \text { NB } & 41 & 95 & 1.3 E+02 & 2.1 E+01 \\ \text { NB } & 41 & 95 M & 3.2 E+03 & 4.9 E+02 \\ \text { NB } & 41 & 96 & 1.5 E+04 & 2.4 E+03 \\ \text { MO } & 42 & 93 & 2.9 E+02 & 4.4 E+01 \\ \text { MD } & 42 & 99 & 1.5 E+02 & 2.3 E+01 \\ \text { TC } & 43 & 95 & 5.5 E+00 & 8.5 E-01 \\ \text { TC } & 43 & 95 M & 4.0 E-03 & 6.2 E-04 \\ \text { TC } & 43 & 96 & 8.2 E-02 & 1.3 E-02 \\ \text { TC } & 43 & 97 & 1.4 E-03 & 2.1 E-04 \\ \text { TC } & 43 & 97 M & 2.3 E-02 & 3.5 E-03 \\ \text { TC } & 43 & 98 & 1.9 E-05 & 2.9 E-06 \\ \text { TC } & 43 & 99 & 3.1 E-04 & 4.7 E-05 \\ \text { RU } & 44 & 97 & 2.2 E+06 & 3.5 E+05 \\ \text { RU } & 44 & 103 & 4.7 E+04 & 7.3 E+03 \\ \text { RU } & 44 & 106 & 2.5 E+03 & 3.9 E+02 \\ \text { RH } & 45 & 99 & 5.6 E+01 & 8.6 E+00 \\ \text { RH } & 45 & 100 & 1.7 E+03 & 2.6 E+02 \\ \text { RH } & 45 & 101 & 1.9 E+01 & 2.9 E+00 \\ \text { RH } & 45 & 101 M & 4.3 E+02 & 6.6 E+01 \\ \text { RH } & 45 & 102 & 4.3 E+00 & 6.6 E-01 \\ \text { RH } & 45 & 105 & 4.0 E+03 & 6.1 E+02 \\ \text { PD } & 46 & 100 & 1.3 E+02 & 1.9 E+01 \\ \text { PD } & 46 & 103 & 4.4 E+02 & 6.7 E+01 \\ \text { PD } & 46 & 107 & 7.1 E+03 & 1.1 E+03 \\ \text { PD } & 46 & 109 & 1.1 E+04 & 1.7 E+03 \\ \text { PD } & 46 & 112 & 8.5 E+02 & 1.3 E+02 \\ \text { AG } & 47 & 105 & 4.0 E+00 & 6.2 E-01 \\ \text { AG } & 47 & 106 M & 8.0 E+00 & 1.2 E+00 \\ \text { AG } & 47 & 108 M & 8.6 E-01 & 1.3 E-01 \\ \text { AG } & 47 & 110 M & 8.8 E-01 & 1.4 E-01 \\ \text { AG } & 47 & 111 & 5.2 E+01 & 8.0 E+00 \\ \text { CD } & 48 & 109 & 1.4 E+05 & 2.1 E+04 \\ \text { CD } & 48 & 113 M & 5.0 E+04 & 7.8 E+03 \\ \text { CD } & 48 & 115 & 5.5 E+06 & 8.5 E+05 \\ \text { CD } & 48 & 115 M & 7.8 E+04 & 1.2 E+04 \\ \text { IN } & 49 & 111 & 3.3 E+06 & 5.1 E+05 \\ \text { IN } & 49 & 114 M & 2.7 E+04 & 4.1 E+03 \\ \text { IN } & 49 & 115 & 6.2 E+04 & 9.5 E+03 \\ \text { SN } & 50 & 113 & 5.9 E+02 & 9.1 E+01 \\ \text { SN } & 50 & 117 M & 1.1 E+04 & 1.6 E+03 \\ \text { SN } & 50 & 119 M & 1.4 E+03 & 2.2 E+02 \\ \text { SN } & 50 & 121 & 2.3 E+06 & 3.5 E+05 \\ \text { SN } & 50 & 121 M & 2.1 E+02 & 3.2 E+01 \\ \text { SN } & 50 & 123 M & 3.4 E+02 & 5.2 E+01 \\ \text { SN } & 50 & 125 & 3.3 E+03 & 5.0 E+02 \\ \text { SN } & 50 & 126 & 8.0 E+00 & 1.2 E+00 \\ \text { SB } & 51 & 119 & 2.4 E+07 & 3.7 E+06 \\ \text { SB } & 51 & 120 M & 4.7 E+04 & 7.3 E+03\end{array}$

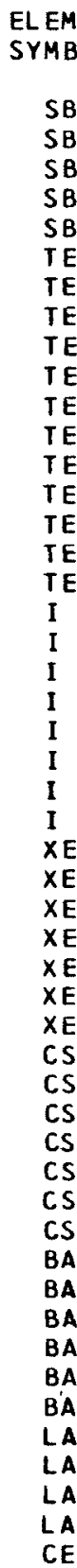

$\begin{array}{ll}\text { ATOMIC } & \text { ISOTOPE } \\ \text { NO. }(Z) & \text { MASSIA) }\end{array}$

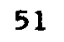

51

SB

SB

$S B$

TE
ADULT $U C / M * 2$

CHILD UC $/ M * 2$

3. $9 E+05 \quad 5.9 E+04$ 1. $7 E+03$ 3. $9 E+02$ 1. $2 E+04$ 1. $9 E+05$
5. $0 E+02$ 5. $0 E+02$ 4. $7 E+02$ 2. $9 E+02$ $5.1 E+01$ $1.1 E+03$ $1.8 E+02$ 1. $8 E+02$ 4. $5 E+02$ 1. $0 E+02$ 1. $0 E+02$
4. $6 E+03$ 6. $1 E+02$ $6.3 E+03$ 1. $9 E+01$ 7. $6 E+01$ $2.3 E+01$ 4. $8 E+01$ 6. $0 E+02$ 3. $2 E+01$ 4. $5 E+02$ 4. $4 \mathrm{E}+02$ $2.4 E+03$ 8. $4 E+00$ 7. $6 E+01$ 5. $7 E+01$ 1. $9 E+02$ 3. $8 E+02$ 1. $1 E+03$ 5. $8 E+02$ $5.4 E+01$ 4. $1 E-01$ 1. $2 E+01$ 5. $7 E+00$ 8. $4 E-01$ 2. $3 E+06$ 5. $6 E+05$ 3. $4 E+05$ 1. $7 E+07$ 7. $4 E+07$ 1. $5 E+05$ 1. $3 E+08$ 1. $9 E+04$ 5. $4 E+02$ 9. $2 E+06$ 4. $9 E+07$ $2.7 E+02$ 6. $0 E+01$ 2. $9 E+04$ 2. $9 E+04$ 7. $7 E+01$ 2. $8 E+03$ 7. $2 E+01$ 4. $5 E+01$ 7. $9 E+00$ $1.8 E+02$ 2. $8 \mathrm{E}+01$ $6.9 E+01$ . 1. $5 E+01$ 7. $0 E+02$ $9.3 E+01$ $9.6 E+02$ 2. $9 E+00$ 4. $9 E+00$ $7.0 E+01$ $6.8 E+01$ $3.8 E+02$ $1.3 E+0 J$ 1. $2 E+01$ 8. $8 \mathrm{E}+00$ $1.7 E+02$ 9. $0 E+01$ 8. $4 E+00$ $6.3 E-02$ 1. $9 E+00$ 8. $8 \mathrm{E}-01$ 1. $3 E-01$ 3. $5 E+05$ 8. $7 \mathrm{E}+04$ 5. $3 E+04$ 2. $6 E+06$ 1. $1 E+07$ 2. $3 E+04$ 2. $0 E+07$ 2. $9 E+03$ 8. $3 E+01$ 1. $4 E+06$ 7. $6 E+06$ $2.9 E+00$
$1.2 E+01$
$3.5 E+00$ ज् $7.5 E+00$ Z 9.3E+01 3. $0 E+01$ $5.9 E+01$
$1.7 E+02$ 


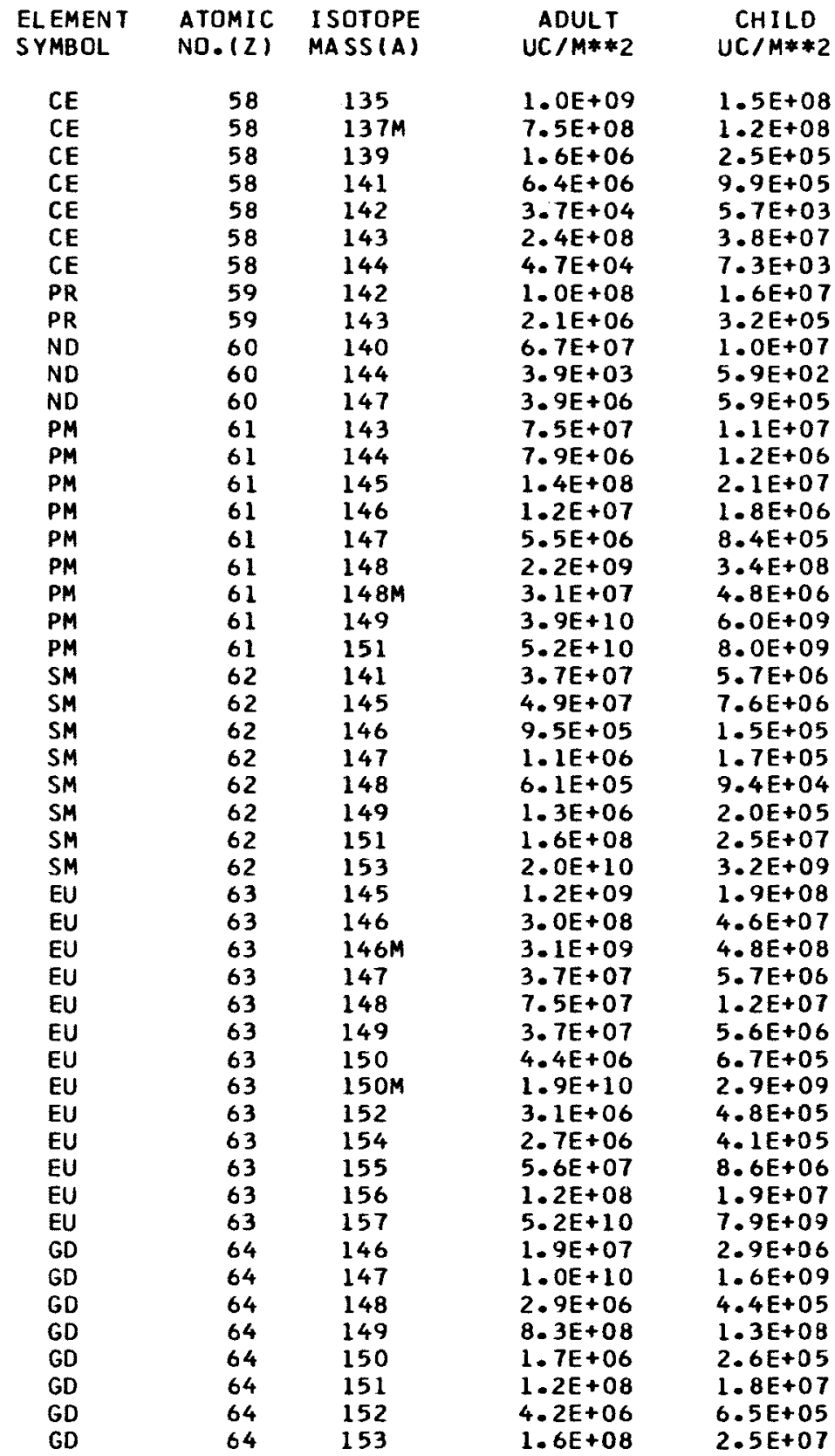

$\begin{array}{ccc}\text { ELEMENT } & \text { ATOMIC } & \text { ISOTOPE } \\ \text { SYMBOL } & \text { NO. }(Z) & \text { MASS(A) } \\ \text { GD } & 64 & 159 \\ \text { GD } & 64 & 162 \\ \text { TB } & 65 & 151 \\ \text { TB } & 65 & 152 \\ \text { TB } & 65 & 153 \\ \text { TB } & 65 & 154 \\ \text { TB } & 65 & 155 \\ \text { TB } & 65 & 156 \\ \text { TB } & 65 & 157 \\ \text { TB } & 65 & 158 \\ \text { TB } & 65 & 160 \\ \text { TB } & 65 & 161 \\ \text { TB } & 65 & 164 \\ \text { DY } & 66 & 154 \\ \text { DY } & 66 & 154 M \\ \text { DY } & 66 & 156 \\ \text { DY } & 66 & 159 \\ \text { DY } & 66 & 166 \\ \text { HO } & 67 & 163 \\ \text { HO } & 67 & 166 \\ \text { HO } & 67 & 166 M \\ \text { ER } & 68 & 160 \\ \text { ER } & 68 & 169 \\ \text { ER } & 68 & 172 \\ \text { TM } & 69 & 165 \\ \text { TM } & 69 & 167 \\ \text { TM } & 69 & 168 \\ \text { TM } & 69 & 170 \\ \text { TM } & 69 & 171 \\ \text { TM } & 69 & 172 \\ \text { YB } & 70 & 166 \\ \text { YB } & 70 & 169 \\ \text { YB } & 70 & 175 \\ \text { LU } & 71 & 169 \\ \text { LU } & 71 & 170 \\ \text { LU } & 71 & 171 \\ \text { LU } & 71 & 172 \\ \text { LU } & 71 & 173 \\ \text { LU } & 71 & 174 \\ \text { LU } & 71 & 174 M \\ \text { LU } & 71 & 176 \\ \text { LU } & 71 & 177 \\ \text { LU } & 71 & 177 M \\ \text { HF } & 72 & 170 \\ \text { HF } & 72 & 171 \\ \text { HF } & 72 & 172 \\ \text { HF } & 72 & 173 \\ \text { HF } & 72 & 174 \\ \text { HF } & 72 & 175 \\ \text { HF } & 72 & 181\end{array}$

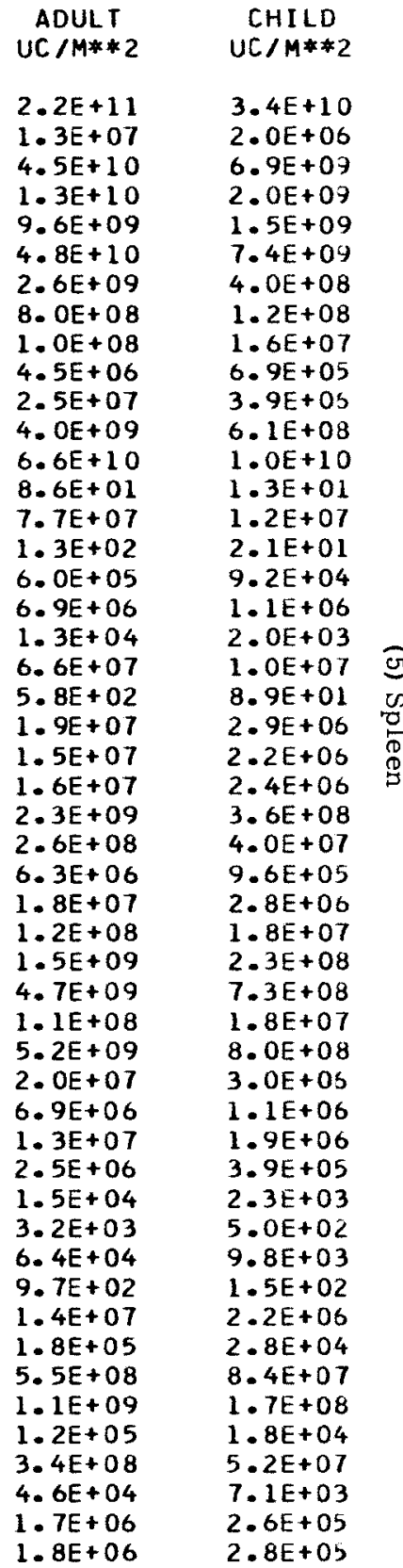


TABLE 6A. UNIT-RAD DEPOSITION FOR SPLEEN

\begin{tabular}{|c|c|c|c|c|}
\hline $\begin{array}{l}\text { ELEMEN T } \\
\text { SYMBOL }\end{array}$ & $\begin{array}{l}\text { ATOMIC } \\
\text { NO. }(Z)\end{array}$ & $\begin{array}{l}\text { I SOTOPE } \\
\text { MASS (A) }\end{array}$ & $\begin{array}{c}\text { ADULT } \\
\text { UC } / M * * 2\end{array}$ & $\begin{array}{l}\text { CHILD } \\
\text { UC } / M * * 2\end{array}$ \\
\hline HF & 72 & 182 & 7. $0 E+04$ & 1. $1 E+04$ \\
\hline TA & 73 & 177 & 5. $7 E+02$ & $8.8 E+01$ \\
\hline TA & 73 & 179 & $1.9 E+01$ & 3. $O E+O O$ \\
\hline TA & 73 & 182 & 2. $0 E+00$ & $3.1 \mathrm{E}-01$ \\
\hline TA & 73 & 183 & $2.5 E+02$ & $3.9 E+01$ \\
\hline$W$ & 74 & 178 & $2.3 E+00$ & 3. $5 \mathrm{E}-0 \mathrm{I}$ \\
\hline$w$ & 74 & 181 & $6.4 E+00$ & $9.9 E-01$ \\
\hline $\mathbf{W}$ & 74 & 185 & $6.9 E+00$ & $1.1 E+00$ \\
\hline$w$ & 74 & 187 & $9.3 E+02$ & $1.4 E+02$ \\
\hline$W$ & 74 & 188 & $6.8 \mathrm{E}-01$ & $1.0 E-01$ \\
\hline RE & 75 & 180 & $2.4 E+00$ & 3. $7 \mathrm{E}-01$ \\
\hline$R E$ & 75 & 181 & 2. $7 E+00$ & $4 \cdot 1 E-01$ \\
\hline $\mathrm{RE}$ & 75 & 182 & $3.2 E-01$ & $5.0 \mathrm{E}-02$ \\
\hline RE & 75 & $182 \mathrm{M}$ & $6.8 E+00$ & $1.1 \mathrm{E}+0 \mathrm{D}$ \\
\hline RE & 75 & 183 & $1.1 \mathrm{E}-02$ & $1.7 E-03$ \\
\hline RE & 75 & 184 & 7. $7 E-03$ & $1.2 \mathrm{E}-03$ \\
\hline RE & 75 & $184 M$ & $1.1 E-03$ & $1.7 \mathrm{E}-04$ \\
\hline RE & 75 & 186 & 5. $9 E-01$ & $9.1 \mathrm{E}-02$ \\
\hline RE & 75 & 187 & $2.2 \mathrm{E}-01$ & $3.3 E-02$ \\
\hline RE & 75 & 188 & 5. $7 E+00$ & $8.8 E-01$ \\
\hline RE & 75 & 189 & $6.8 E+00$ & $1.1 E+00$ \\
\hline OS & 76 & 182 & 4. $4 E+01$ & $6.8 E+00$ \\
\hline OS & 76 & 183 & $3.4 E+02$ & $5.2 E+01$ \\
\hline OS & 76 & 185 & $8.4 E-02$ & $1.3 \mathrm{E}-02$ \\
\hline OS & 76 & 191 & $3.4 E+00$ & $5.2 \mathrm{E}-01$ \\
\hline os & 76 & $191 M$ & 8. $2 E+02$ & $1.3 E+02$ \\
\hline OS & 76 & 193 & $9.4 E+01$ & $1.4 E+01$ \\
\hline OS & 76 & 194 & $1.1 E-03$ & $1.8 \mathrm{E}-04$ \\
\hline IR & 77 & 185 & $9.1 E+03$ & $1.4 E+03$ \\
\hline IR & 77 & 186 & $5.3 E+03$ & 8. $2 E+02$ \\
\hline IR & 77 & 187 & $1.4 E+04$ & $2.2 E+03$ \\
\hline IR & 77 & 188 & $6.4 E+02$ & $9.8 E+01$ \\
\hline IR & 77 & 189 & $1.5 E+02$ & $2.2 E+01$ \\
\hline IR & 77 & 190 & $4.2 E+01$ & $6.4 E+00$ \\
\hline IR & 77 & 192 & $1.0 E+01$ & $1.5 E+00$ \\
\hline IR & 77 & $192 \mathrm{M}$ & $4.3 E+00$ & $6.7 \mathrm{E}-01$ \\
\hline IR & 77 & $193 M$ & 7. $3 E+02$ & $1.1 E+02$ \\
\hline IR & 77 & 194 & $5.0 E+03$ & $7.6 E+02$ \\
\hline PT & 78 & 188 & $6.5 E+01$ & 1. $0 E+01$ \\
\hline PT & 78 & 190 & $3.5 E+00$ & $5.4 \mathrm{E}-01$ \\
\hline PT & 78 & 191 & $2.4 E+03$ & $3.7 E+02$ \\
\hline PT & 78 & 192 & 4. $2 E+00$ & $6.4 E-01$ \\
\hline PT & 78 & 193 & $1.4 E+02$ & $2.2 E+01$ \\
\hline PT & 78 & $193 M$ & 3. $0 E+03$ & $4.6 E+02$ \\
\hline PT & 78 & $195 M$ & $2.9 E+03$ & $4.5 E+02$ \\
\hline $\mathrm{PT}$ & 78 & 197 & 4. $8 E+04$ & $7.3 E+03$ \\
\hline$A U$ & 79 & 193 & $4.6 E+04$ & $7.1 E+03$ \\
\hline$A U$ & 79 & 194 & $3.0 E+03$ & $4.6 E+02$ \\
\hline$A U$ & 79 & 195 & 5. $2 E+01$ & 8. $O E+O D$ \\
\hline AU & 79 & 196 & 7. $7 \mathrm{E}+02$ & $1.2 E+02$ \\
\hline
\end{tabular}

VIA MILK.

05/09/68 PAGE 24

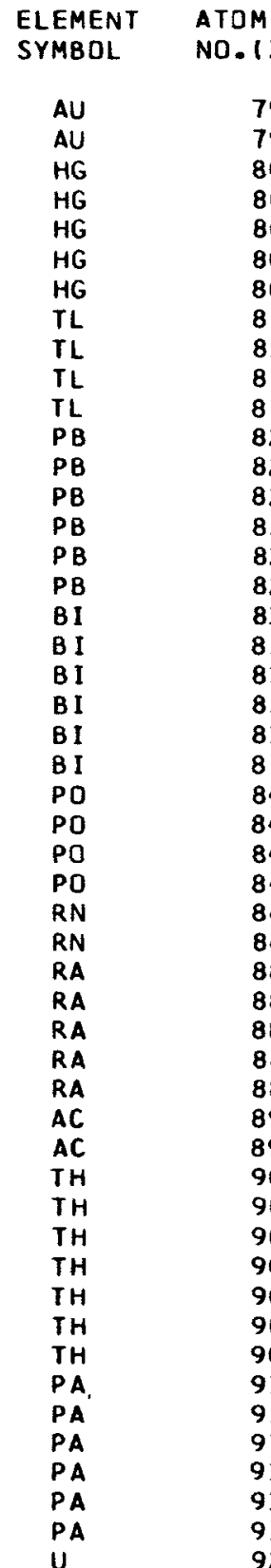

\section{ADULT CHILD} UC $/ M * 2$ UC/M**2

3. $2 E+03 \quad 5.0 E+02$ 7. $8 E+03 \quad 1.2 E+03$ 1. $9 \mathrm{E}-01 \quad 2.9 \mathrm{E}-02$

$1.4 E+01$

$2 \cdot 9 E+01$

8. $9 E+01 \quad 1.4 E+01$

$\begin{array}{ll}\text { 1. } 7 E+00 & 2.6 E-01 \\ 5.4 E+02 & 8.3 E+01\end{array}$

4. $8 \mathrm{E}+02 \quad 7.4 \mathrm{E}+01$

3. $1 E+01 \quad 4.7 E+00$

7. $8 \mathrm{E}+00 \quad 1.2 \mathrm{E}+0.0$

1. $2 E-02 \quad 8.8 E+01$

$1.8 E-03$

2. $4 E+02 \quad 3.6 E+01$

$\begin{array}{ll}\text { 2. } \\ \text { 8. } 1 E-03 & \text { 4. } 1 E-02 \\ 1.3 E-02 & 1.3 E-02\end{array}$

1. $9 E-03 \quad 2.9 E-04$

$2.4 E+04 \quad 3.7 E+03$

2. $8 E+03 \quad 4.3 E+02$

1. $9 E+03 \quad 3.0 E+02$

$1.0 \mathrm{E}+04$

1. $0 E+04$

9. $3 E+02$

4. $8 E+01$

1. $O E+00$

2. $2 E+00$

$1 \cdot 8 \mathrm{E}+\mathrm{O2}$

2. $4 \mathrm{E}+00$

3. $9 \mathrm{E}-03$

2. $7 E-02$

2. $7 E-03$

2. $5 \mathrm{E}-06$

8. $1 E-06$

4. $4 E+04$

3. $2 \mathrm{E}+01$

1. $5 E+04$

2. $2 \mathrm{E}+02$

2. $1 E+01$

1. $9 E+01$

$1.8 \mathrm{E}+06$

$1.7 E+01$

2. $5 E+06$

2. $5 E+06$
$1.1 E+06$

1. $5 E+04$

1. $8 \mathrm{E}+01$

1. $1 E+06$

7. $5 E+03$

1. $1 E+00$

1. $4 E+02$

7. $3 E+00$

1. $5 E-01$

2. $2 \mathrm{E}-01$

3. $3 E-01$

2. $8 E+01$

3. $7 E-01$

$4.1 E-03$

$4.1 E-04$

4.

3. $9 E-07$

6. $8 E+03$

4. $9 E+00$

$2.3 E+03$

$3.4 E+01$

3. $2 E+00$

3. $0 E+00$

2. $7 E+05$

2. $7 E+00$

1. $3 E+03$

3. $9 E+05$

1. $6 E+05$

2. $3 E+03$
8. $5 E+03 \quad 1.3 E+03$

$1.6 E+03$ 
TABLE 6A. UNIT-RAD DEPOSITION FOR SPLEEN

\begin{tabular}{|c|c|c|c|c|}
\hline $\begin{array}{l}\text { ELEMENT } \\
\text { SYMBOL }\end{array}$ & $\begin{array}{l}\text { ATOMIC } \\
\text { NO. }(Z)\end{array}$ & $\begin{array}{l}\text { ISOTOPE } \\
\text { MASS (A) }\end{array}$ & $\begin{array}{c}\text { ADULT } \\
\text { UC/M**2 }\end{array}$ & $\begin{array}{c}C H I L D \\
U C / M * * 2\end{array}$ \\
\hline$u$ & 92 & 231 & $1.4 E+01$ & $2.1 E+00$ \\
\hline u & 92 & 232 & 2. $0 E-03$ & $3.1 E-04$ \\
\hline u & 92 & 233 & $1.8 \mathrm{E}-03$ & $2.8 E-04$ \\
\hline u & 92 & 234 & 1. $7 \mathrm{E}-03$ & $2.6 E-04$ \\
\hline u & 92 & 235 & $1.6 \mathrm{E}-03$ & $2.4 E-04$ \\
\hline u & 92 & 236 & $1.6 E-03$ & $2.4 E-04$ \\
\hline u & 92 & 237 & $5.4 E+00$ & $8.4 E-01$ \\
\hline u & 92 & 238 & $1.5 E-03$ & $2.3 E-04$ \\
\hline u & 92 & 240 & 4. $3 E+02$ & $6.6 E+01$ \\
\hline NP & 93 & 234 & $1.2 E+05$ & $1.8 E+04$ \\
\hline NP & 93 & 235 & $3.0 E+02$ & $4.6 E+01$ \\
\hline NP & 93 & 236 & 1. $5 E+01$ & $2.3 E+00$ \\
\hline NP & 93 & $236 M$ & 2. $0 E+06$ & $3.2 E+05$ \\
\hline NP & 93 & 237 & $1.6 E+01$ & $2.5 E+00$ \\
\hline NP & 93 & 238 & $4.1 E+05$ & $6.3 E+04$ \\
\hline NP & 93 & 239 & 3. $1 E+05$ & $4.8 E+04$ \\
\hline PU & 94 & 236 & $1.2 E+04$ & $1.9 E+03$ \\
\hline PU & 94 & 237 & 3. $8 E+05$ & $5.9 E+04$ \\
\hline PU & 94 & 238 & $1.9 E+03$ & $2.9 E+02$ \\
\hline PU & 94 & 239 & $1.6 E+03$ & $2.4 E+02$ \\
\hline PU & 94 & 240 & $1.6 E+03$ & $2.4 E+02$ \\
\hline PU & 94 & 241 & 3. $2 E+03$ & $4.9 E+02$ \\
\hline PU & 94 & 242 & $1.5 E+03$ & $2.3 E+02$ \\
\hline PU & 94 & 244 & $1.4 E+03$ & $2.1 E+02$ \\
\hline PU & 94 & 246 & $2.1 E+06$ & $3.2 E+05$ \\
\hline AM & 95 & 239 & $6.1 E+06$ & $9.5 E+05$ \\
\hline AM & 95 & 240 & $3.6 E+05$ & $5.6 E+04$ \\
\hline AM & 95 & 241 & $1.5 E+01$ & $2.2 E+00$ \\
\hline AM & 95 & 242 & $3.3 E+06$ & $5.1 E+05$ \\
\hline AM & 95 & $242 M$ & $1.4 E+01$ & $2.2 E+00$ \\
\hline AM & 95 & 243 & $1.3 E+01$ & $2 . O E+D D$ \\
\hline
\end{tabular}

VIA MILK.

$05 / 09 / 68$

PAGE 25

$\begin{array}{lrc}\text { ELEMENT } & \text { ATOMIC } & \text { ISOTOPE } \\ \text { SYMBOL } & \text { NO. (Z) } & \text { MASS }(A) \\ \text { CM } & 96 & 240 \\ \text { CM } & 96 & 241 \\ \text { CM } & 96 & 242 \\ \text { CM } & 96 & 243 \\ \text { CM } & 96 & 244 \\ \text { CM } & 96 & 245 \\ \text { CM } & 96 & 246 \\ \text { CM } & 96 & 247 \\ \text { CM } & 96 & 248 \\ \text { BK } & 97 & 245 \\ \text { BK } & 97 & 246 \\ \text { BK } & 97 & 247 \\ \text { BK } & 97 & 248 \\ \text { BK } & 97 & 249 \\ \text { CF } & 98 & 246 \\ \text { CF } & 98 & 248 \\ \text { CF } & 98 & 249 \\ \text { CF } & 98 & 250 \\ \text { CF } & 98 & 251 \\ \text { CF } & 98 & 252 \\ \text { CF } & 98 & 253 \\ \text { ES } & 99 & 251 \\ \text { ES } & 99 & 252 \\ \text { ES } & 99 & 253 \\ \text { ES } & 99 & 254 \\ \text { ES } & 99 & 254 M \\ \text { ES } & 99 & 255 \\ \text { FM } & 100 & 252 \\ \text { FM } & 100 & 253 \\ \text { FM } & 100 & 255 \\ \text { FM } & 100 & 257\end{array}$

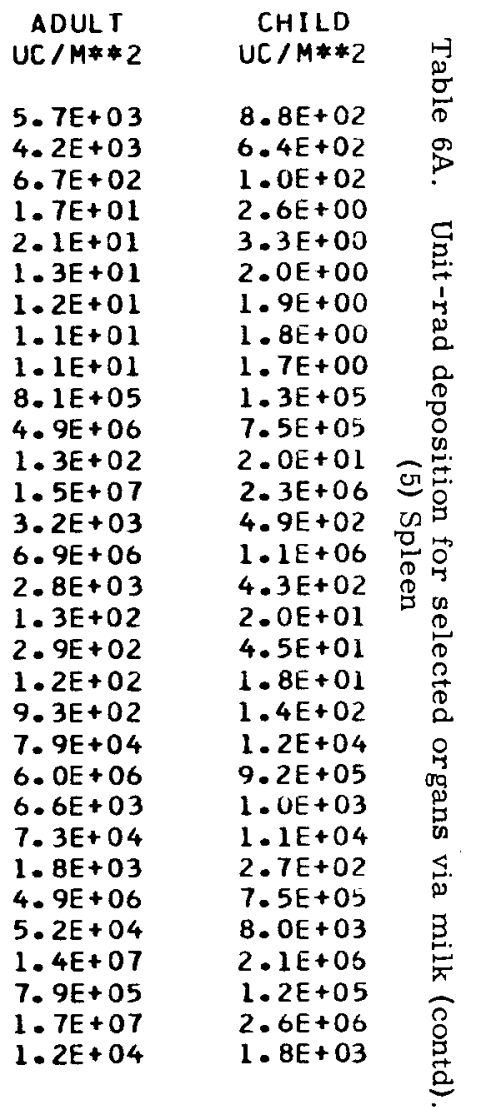


TABLE 6A. UNIT-RAD DEPOSITION FOR TESTES

VIA MILK.

$\begin{array}{lcccc}\text { ELEMENT } & \text { ATOMIC } & \text { ISOTOPE } & \text { ADULT } & \text { CHILD } \\ \text { SYMBOL } & \text { NO.12) } & \text { MASS (A) } & \text { UC/M**2 } & \text { UC/M**2 } \\ \text { H } & & & & \\ \text { BE } & 1 & 3 & 6.9 E+02 & 1.6 E+01 \\ \text { BE } & 4 & 7 & 2.1 E+03 & 4.9 E+01 \\ \text { C } & 4 & 10 & 2.1 E+00 & 4.7 E-02 \\ \text { NA } & 6 & 14 & 1.1 E+02 & 2.6 E+00 \\ \text { NA } & 11 & 22 & 8.2 E-01 & 1.9 E-02 \\ \text { MG } & 11 & 24 & 1.7 E+02 & 3.8 E+00 \\ \text { AL } & 12 & 28 & 3.0 E+00 & 7.0 E-02 \\ \text { SI } & 13 & 26 & 7.5 E-04 & 1.7 E-05 \\ \text { P } & 14 & 32 & 3.1 E+02 & 7.2 E+00 \\ \text { P } & 15 & 32 & 2.1 E+00 & 4.7 E-02 \\ \text { S } & 15 & 33 & 1.4 E+01 & 3.2 E-01 \\ \text { CL } & 16 & 35 & 1.0 E+01 & 2.3 E-01 \\ \text { AR } & 17 & 36 & 2.2 E+00 & 4.9 E-02 \\ \text { AR } & 18 & 37 & 7.3 E+02 & 1.7 E+01 \\ \text { AR } & 18 & 39 & 2.8 E-02 & 6.5 E-04 \\ \text { K } & 18 & 42 & 3.0 E-03 & 7.0 E-05 \\ \text { K } & 19 & 40 & 2.2 E+00 & 5.0 E-02 \\ \text { K } & 19 & 42 & 2.0 E+03 & 4.7 E+01 \\ \text { CA } & 19 & 43 & 1.3 E+03 & 2.9 E+01 \\ \text { CA } & 20 & 41 & 1.8 E+03 & 4.2 E+01 \\ \text { CA } & 20 & 45 & 8.7 E+01 & 2.0 E+00 \\ \text { CA } & 20 & 47 & 6.3 E+01 & 1.5 E+00 \\ \text { SC } & 20 & 48 & 1.8 E+00 & 4.1 E-02 \\ \text { SC } & 21 & 44 M & 6.3 E+07 & 1.5 E+06 \\ \text { SC } & 21 & 46 & 4.3 E+05 & 9.8 E+03 \\ \text { SC } & 21 & 47 & 3.1 E+08 & 7.2 E+06 \\ \text { TI } & 21 & 48 & 8.6 E+07 & 2.0 E+06 \\ \text { V } & 22 & 44 & 1.0 E+02 & 2.4 E+00 \\ \text { V } & 23 & 48 & 1.7 E+00 & 3.9 E-02 \\ \text { V } & 23 & 49 & 2.8 E+01 & 6.5 E-01 \\ \text { CR } & 23 & 50 & 4.8 E-03 & 1.1 E-04 \\ \text { CR } & 24 & 48 & 3.9 E+02 & 9.0 E+00 \\ \text { MN } & 24 & 51 & 1.3 E+02 & 3.0 E+00 \\ \text { MN } & 25 & 52 & 7.4 E+02 & 1.7 E+01 \\ \text { MN } & 25 & 53 & 2.3 E+04 & 5.3 E+02 \\ \text { FE } & 26 & 54 & 1.9 E+02 & 4.3 E+00 \\ \text { FE } & 26 & 55 & 8.0 E+02 & 1.8 E+01 \\ \text { FE } & 26 & 60 & 2.6 E+01 & 6.0 E-01 \\ \text { CO } & 27 & 55 & 1.5 E+00 & 3.5 E-02 \\ \text { CO } & 27 & 56 & 4.9 E+04 & 1.1 E+03 \\ \text { CO } & 27 & 57 & 1.4 E+02 & 3.2 E+00 \\ \text { CO } & 27 & 58 & 1.5 E+03 & 3.4 E+01 \\ \text { CO } & 27 & 60 & 2.9 E+02 & 6.8 E+00 \\ \text { NI } & 28 & 56 & 7.2 E+01 & 1.7 E+00 \\ \text { NI } & 28 & 57 & 6.2 E-02 & 1.4 E-03 \\ \text { NI } & 28 & 59 & 1.5 E+00 & 3.5 E-02 \\ \text { NI } & 28 & 63 & 3.7 E-03 & 1.8 E-04 \\ \text { NI } & 28 & 66 & 7.5 E-03 & 8.6 E-05 \\ \text { CU } & 29 & 64 & 1.4 E+03 & 1.7 E-02 \\ & & & & 3.2 E+01\end{array}$

$\begin{array}{lll}\text { ELEMENT } & \text { ATOMIC } & \text { ISOTOPE } \\ \text { SYMBOL } & \text { NO. (2) } & \text { MASS(A) }\end{array}$

PAGE 26

NO. 12

67

65

72

72
67
72

68

71

71

73

74

76

77

72

75
79

79
76

77

82

76

79

CHILO

UC/M**2 UC/M**2

9. $8 E+01 \quad 2.3 E+00$

4. $0 E+00 \quad 9.2 E-02$

1. $3 E+04 \quad 2.9 E+02$

$2.4 E+02 \quad 5.5 E+00$

$1.4 E+05 \quad 3.3 E+03$

2. $4 E+05 \quad 5.5 E+03$

4.1E-01 9.4E-03

$5.4 E+02$

$3.5 E+03$

1. $8 \mathrm{E}+01$

1. $2 E+01$

B. $2 E+01$

4. 3E-01

4. 7E-01

$1.6 E-01$

7. $O E+00$

2. $7 \mathrm{E}-01$

2. $8 \mathrm{E}+01$

1. $0 E+02$

2. $2 E-03$

3. $5 E-04$

6. $3 E-05$

3. $3 E+02$

7. $5 E+01$

6. $3 E+01$

7. $8 E+02$

4. $5 E+02$

2. $8 E-01$

5. $4 \mathrm{E}-02$

1. $7 E+00$

1. $1 E+00$

3. $8 E+00$

5. $0 E+00$

6. $9 E+01$

1. $3 E+04$

$2.6 E+02$

2. $0 E+02$

5. $5 \mathrm{E}+01$

5. $8 E+09$

1. $1 E+09$

2. $2 E+10$

2. $5 E+06$

1. $1 E+09$

1. $6 E+07$

5. $7 E+08$

4. $3 E+05$

7. $8 E+07$

5. $5 E+06$

1. $1 E+06$

8. $4 E+08$

2. $7 E+04$

2. $6 E+01$

3. $0 E+02$

$.2 E-03$

$2.3 E+00$

2. $3 E+00$

8. $0 E-0 S$

1. $4 E-06$

7. $5 E+00$ के

$1.4 E+00 \mathrm{H}$

$1.8 \mathrm{E}+01$ \&

$1.0 E+01 \vec{D}$

$1.0 E+01$ \&
$6.4 E-03$

$6.4 E-03$
$1.2 E-03$

3. $9 \mathrm{E}-02$

$2.6 E-02$

8. $8 E-02$

$1.2 E-01$

1. $6 E+00$

$3.1 E+02$

. $0 E+00$

$4.6 E+00$

1. $3 E+00$

1. $3 E+08$

2. $6 E+07$

5. $0 E+08$

5. $7 E+04$

2. $5 E+07$

$3.8 E+05$

1. $3 E+0$

9. $9 E+03$

1. $8 E+06$

1. $3 E+05$

$2.5 E+04$

1. $9 E+07$

$6.2 \mathrm{E}+02$

6. IE- 01

7. $0 E+00$

2. $0 E+01$ 


\begin{tabular}{|c|c|c|c|c|}
\hline $\begin{array}{l}\text { ELEMENT } \\
\text { SYMBOL }\end{array}$ & $\begin{array}{l}\text { ATOMIC } \\
\text { NO. }(Z)\end{array}$ & $\begin{array}{l}\text { I SOTOPE } \\
\text { MASS (A) }\end{array}$ & $\begin{array}{l}\text { ADULT } \\
\text { UC } / M * * 2\end{array}$ & $\begin{array}{l}\text { CHILD } \\
U C / M * * 2\end{array}$ \\
\hline NB & 41 & $93 M$ & $9.3 E+01$ & $2.1 E+00$ \\
\hline NB & 41 & 94 & $2.7 E-01$ & $6.2 \mathrm{E}-03$ \\
\hline NB & 41 & 95 & $1.8 \mathrm{E}+02$ & 4. $1 E+00$ \\
\hline NB & 41 & $95 \mathrm{M}$ & $4.3 E+03$ & $1.0 E+02$ \\
\hline NB & 41 & 96 & $2.1 E+04$ & $4.8 E+02$ \\
\hline MO & 42 & 93 & $3.5 E+02$ & $8.0 E+00$ \\
\hline MO & 42 & 99 & $3.5 E+02$ & $8.0 E+00$ \\
\hline$T C$ & 43 & 95 & $1.9 E+00$ & $4.3 E-02$ \\
\hline TC & 43 & $95 M$ & $1.4 \mathrm{E}-03$ & $3.2 E-05$ \\
\hline TC & 43 & 96 & $2.8 E-02$ & $6.5 E-04$ \\
\hline TC & 43 & 97 & $4.8 E-04$ & $1.1 \mathrm{E}-05$ \\
\hline TC & 43 & $97 M$ & 7. $8 \mathrm{E}-03$ & $1.8 E-04$ \\
\hline TC & 43 & 98 & $6.4 E-06$ & $1.5 \mathrm{E}-07$ \\
\hline TC & 43 & 99 & $1.1 \mathrm{E}-04$ & $2.4 \mathrm{E}-06$ \\
\hline RU & 44 & 97 & $1.2 E+06$ & $2.7 E+04$ \\
\hline$R U$ & 44 & 103 & $2.4 E+04$ & $5.5 E+02$ \\
\hline RU & 44 & 106 & $1.2 E+03$ & $2.7 E+01$ \\
\hline RH & 45 & 99 & $3.6 E+00$ & $8.3 E-02$ \\
\hline RH & 45 & 100 & $1.9 E+02$ & $4 \cdot 3 E+00$ \\
\hline RH & 45 & 101 & $2.5 \bar{E}-02$ & $5.8 E-04$ \\
\hline RH & 45 & $101 \mathrm{M}$ & 4. $0 E+01$ & $9.2 \mathrm{E}-01$ \\
\hline RH & 45 & 102 & $4.5 E-02$ & $1.0 \mathrm{E}-03$ \\
\hline RH & 45 & 105 & $4.2 E+02$ & $9.7 E+00$ \\
\hline PD & 46 & 100 & $1.2 E+02$ & 2. $7 E+00$ \\
\hline PD & 46 & 103 & $2.3 E+02$ & $5.4 E+00$ \\
\hline PD & 46 & 107 & $1.6 E+01$ & $3.7 \mathrm{E}-01$ \\
\hline PD & 46 & 109 & 1. $3 E+04$ & $2.9 E+02$ \\
\hline PD & 46 & 112 & $9.2 E+02$ & 2. $1 E+01$ \\
\hline$A G$ & 47 & 105 & $3.6 E+00$ & $8.3 E-02$ \\
\hline$A G$ & 47 & $106 M$ & $4.8 E+00$ & $1.1 \mathrm{E}-01$ \\
\hline $\mathbf{A G}$ & 47 & $108 M$ & $1.0 E+00$ & $2.3 E-02$ \\
\hline$A G$ & 47 & $110 M$ & $9.9 E-01$ & $2.3 E-02$ \\
\hline$A G$ & 47 & 111 & 3. $0 E+01$ & $6.9 E-01$ \\
\hline$C D$ & 48 & 109 & $2.2 E+03$ & $5.0 E+01$ \\
\hline$C D$ & 48 & $113 M$ & $1.4 E+02$ & $3.2 E+00$ \\
\hline$C D$ & 48 & 115 & 2. $8 E+05$ & $6.3 E+03$ \\
\hline$C D$ & 48 & $115 \mathrm{M}$ & $3.3 E+03$ & $7.6 E+01$ \\
\hline IN & 49 & 111 & $2.1 E+04$ & $4.9 E+02$ \\
\hline IN & 49 & $114 M$ & 9. $0 E+01$ & $2.1 E+00$ \\
\hline IN & 49 & 115 & 2. $7 E+00$ & $6.2 \mathrm{E}-02$ \\
\hline SN & 50 & 113 & $1.2 E+02$ & $2.8 E+00$ \\
\hline SN & 50 & $117 M$ & $9.8 E+02$ & $2.3 E+01$ \\
\hline SN & 50 & $119 M$ & 4. $4 E+02$ & $1.0 E+01$ \\
\hline SN & 50 & 121 & $1.8 E+05$ & $4.1 E+03$ \\
\hline SN & 50 & $121 \mathrm{M}$ & $2.2 E+02$ & $5.0 E+00$ \\
\hline SN & 50 & $123 M$ & $7.0 E+01$ & $1.6 E+00$ \\
\hline SN & 50 & 125 & $2.9 E+02$ & $6.6 E+00$ \\
\hline SN & 50 & 126 & $9.0 E+00$ & $2.1 E-01$ \\
\hline SB & 51 & 119 & $8.7 E+06$ & $2.0 E+05$ \\
\hline & & & & $4 O F+\cap ?$ \\
\hline
\end{tabular}

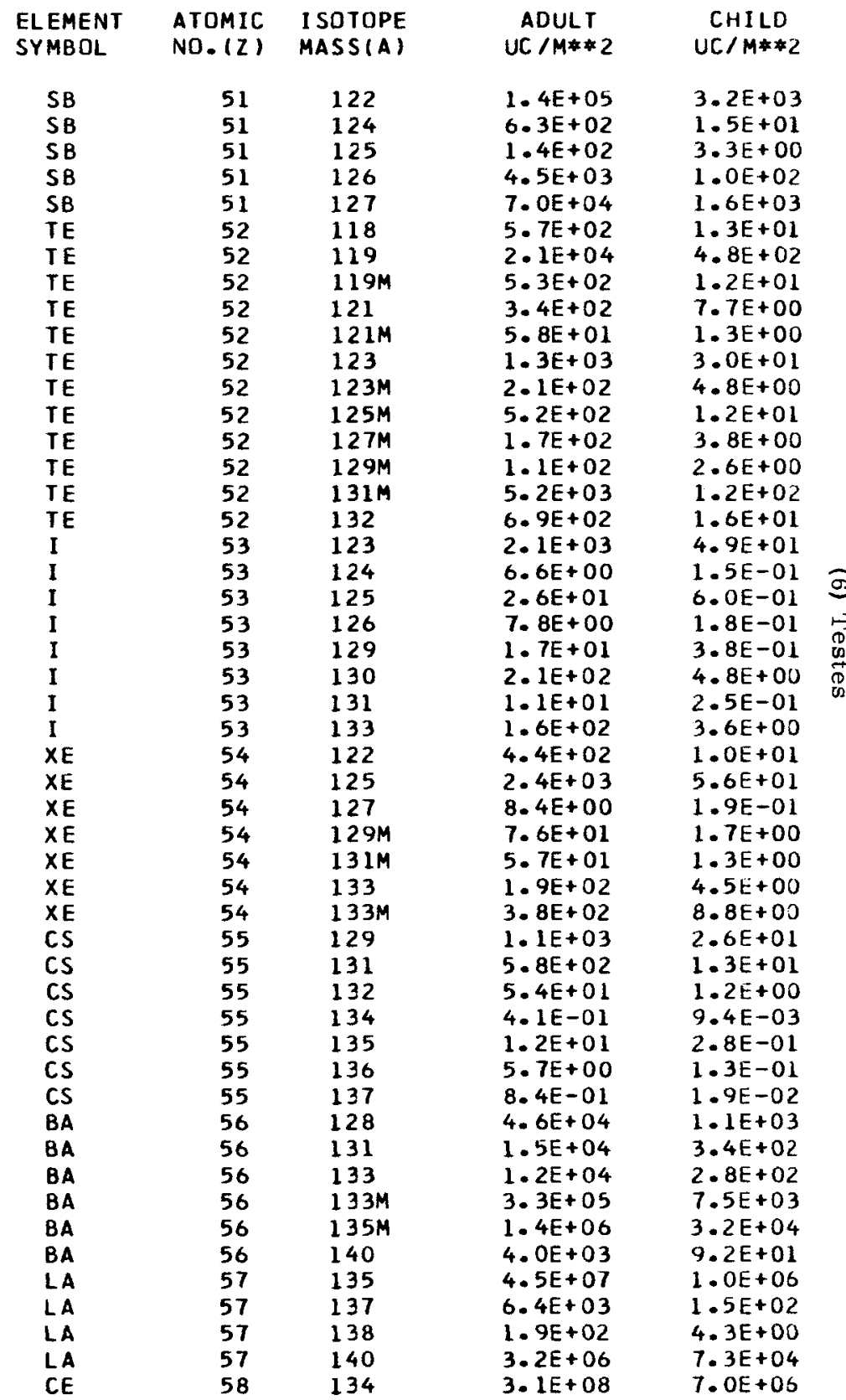




$\begin{array}{lcc}\text { ELEMENT } & \text { ATOMIC } & \text { ISOTOPE } \\ \text { SYMBOL } & \text { NO. (Z) } & \text { MASS(A) } \\ & & \\ \text { CE } & 58 & 135 \\ \text { CE } & 58 & 137 M \\ \text { CE } & 58 & 139 \\ \text { CE } & 58 & 141 \\ \text { CE } & 58 & 142 \\ \text { CE } & 58 & 143 \\ \text { CE } & 58 & 144 \\ \text { PR } & 59 & 142 \\ \text { PR } & 59 & 143 \\ \text { ND } & 60 & 140 \\ \text { ND } & 60 & 144 \\ \text { ND } & 60 & 147 \\ P M & 61 & 143 \\ \text { PM } & 61 & 144 \\ \text { PM } & 61 & 145 \\ \text { PM } & 61 & 146 \\ \text { PM } & 61 & 147 \\ \text { PM } & 61 & 148 \\ \text { PM } & 61 & 148 M \\ \text { PM } & 61 & 149 \\ \text { PM } & 61 & 151 \\ \text { SM } & 62 & 141 \\ \text { SM } & 62 & 145 \\ \text { SM } & 62 & 146 \\ \text { SM } & 62 & 147 \\ \text { SM } & 62 & 148 \\ \text { SM } & 62 & 149 \\ \text { SM } & 62 & 151 \\ \text { SM } & 62 & 153 \\ \text { EU } & 63 & 145 \\ \text { EU } & 63 & 146 \\ \text { EU } & 63 & 146 M \\ \text { EU } & 63 & 147 \\ \text { EU } & 63 & 148 \\ \text { EU } & 63 & 149 \\ \text { EU } & 63 & 150 \\ \text { EU } & 63 & 150 M \\ \text { EU } & 63 & 152 \\ \text { EU } & 63 & 154 \\ \text { EU } & 63 & 155 \\ \text { EU } & 63 & 156 \\ \text { EU } & 63 & 157 \\ \text { GD } & 64 & 146 \\ \text { GD } & 64 & 147 \\ \text { GD } & 64 & 148 \\ \text { GD } & 64 & 149 \\ \text { GD } & 64 & 150 \\ \text { GD } & 64 & 151 \\ \text { GD } & 64 & 152 \\ \text { GD } & 64 & 153 \\ & & \end{array}$

$\begin{array}{ll}\text { ADULT } & \text { CHILD } \\ \text { UC } / M * 2 & \text { UC } 1 M+2 \\ & \\ 6.2 E+09 & 1.4 E+08 \\ 4.7 E+09 & 1.1 E+08 \\ 1.2 E+07 & 2.8 E+05 \\ 4.2 E+07 & 9.6 E+05 \\ 5.7 E+05 & 1.3 E+04 \\ 1.5 E+09 & 3.5 E+07 \\ 4.0 E+05 & 9.3 E+03 \\ 3.5 E+07 & 8.0 E+05 \\ 7.1 E+05 & 1.6 E+04 \\ 2.3 E+07 & 5.3 E+05 \\ 1.3 E+03 & 3.0 E+01 \\ 1.3 E+06 & 3.0 E+04 \\ 3.5 E+06 & 8.0 E+04 \\ 3.9 E+05 & 8.9 E+03 \\ 9.2 E+06 & 2.1 E+05 \\ 7.2 E+05 & 1.7 E+04 \\ 3.1 E+05 & 7.1 E+03 \\ 8.6 E+07 & 2.0 E+06 \\ 1.2 E+06 & 2.8 E+04 \\ 1.5 E+09 & 3.4 E+07 \\ 2.0 E+09 & 4.6 E+07 \\ 3.9 E+07 & 9.0 E+05 \\ 6.4 E+07 & 1.5 E+06 \\ 1.8 E+06 & 4.1 E+04 \\ 2.0 E+06 & 4.6 E+04 \\ 1.1 E+06 & 2.6 E+04 \\ 2.5 E+06 & 5.7 E+04 \\ 3.0 E+08 & 6.9 E+05 \\ 2.1 E+10 & 4.9 E+08 \\ 2.6 E+08 & 5.9 E+06 \\ 6.2 E+07 & 1.4 E+06 \\ 6.4 E+08 & 1.5 E+07 \\ 7.6 E+06 & 1.8 E+05 \\ 1.6 E+07 & 3.6 E+05 \\ 7.8 E+06 & 1.8 E+05 \\ 1.0 E+06 & 2.4 E+04 \\ 3.9 E+09 & 8.9 E+07 \\ 7.4 E+05 & 1.7 E+04 \\ 6.4 E+05 & 1.5 E+04 \\ 1.3 E+07 & 2.9 E+05 \\ 2.6 E+07 & 5.9 E+05 \\ 1.1 E+10 & 2.4 E+08 \\ 9.9 E+05 & 2.3 E+04 \\ 5.3 E+08 & 1.2 E+07 \\ 1.7 E+05 & 3.9 E+03 \\ 4.3 E+07 & 9.9 E+05 \\ 1.0 E+05 & 2.4 E+03 \\ 6.4 E+06 & 1.5 E+05 \\ 2.5 E+05 & 5.8 E+03 \\ 9.0 E+06 & 2.1 E+05\end{array}$

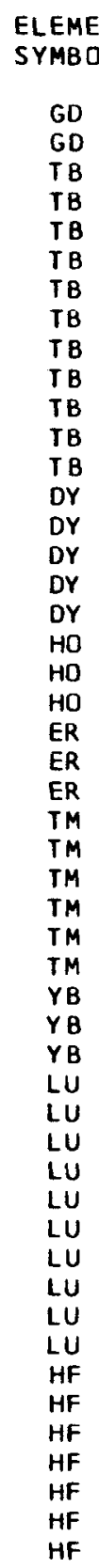

\section{ATOMIC ISOTOPE NO. (Z) MASS(A)}

UC $/ M * * 2$

1. $1 E+10$ 7. $2 E+05$

4. $6 E+09$

$1.3 E+09$

5. $0 E+09$

5. $2 E+09$
2. $7 E+08$

8. $2 E+07$

9. $0 E+06$

3. $9 E+05$

2. $5 E+06$

4. $1 E+08$

6. $8 E+09$

2. $9 E+01$

2. $6 E+07$

4. $6 E+01$

2. $0 E+05$

2. $4 E+06$

4. $5 E+03$

2. $3 E+07$

2. $0 E+02$

6. $5 E+06$

5. $0 E+06$

$5.4 E+06$

9. $6 E+08$

1. $1 E+0 B$

2. $6 E+06$

7. $5 E+06$

4. $8 E+07$

6. $0 E+08$

6. $5 E+08$

1. $6 \mathrm{E}+07$

7. $1 E+08$

6. $8 E+06$

2. $4 E+06$

4. $3 E+06$

8. $6 E+05$

5. $2 E+03$

1. $1 E+03$

2. $2 \mathrm{E}+04$

3. $3 E+02$

4. $8 E+06$

6. $3 E+04$

9. $O E+10$

1. $8 E+11$

1. $4 E+07$

5. $6 E+10$

4. $7 E+06$

2. $6 E+08$
$2.9 E+08$
CHILD

2. $6 E+08$ 1. $6 E+04$ 1. $I E+08$ 3. $1 E+07$

2. $3 E+07$

2. $3 E+07$

1. $1 \mathrm{E}+08$

$6.2 E+06$

1. $9 E+06$

2. $1 E+05$

9. $O E+03$

5. $9 E+04$

9. $4 E+0 S$

1. $6 E+08$

6. $8 \mathrm{E}-01$

$6.1 E+05$

1. $1 E+00$

4. $7 E+03$

5. $5 E+04$

1. $0 E+02$

5. $2 E+05$

4. $6 E+00$

1. $5 E+05$

1. $E+05$

1. $2 E+05$

$1.2 E+05$
$2.2 E+07$

2. $4 E+0 S$

5. $9 E+04$

1. $7 E+05$

1. $1 E+06$

1. $4 E+07$

1. $5 E+07$

3. $6 E+05$

$1.6 E+07$

$1.6 E+05$

5. $4 E+04$

9. $9 E+04$

2. $O E+04$

1. $2 \mathrm{E}+02$

2. $5 E+01$

5. $O E+02$

7. $6 \mathrm{E}+00$

1. $1 E+05$

1. $4 E+03$

2. $1 E+09$

4. $1 E+09$

3. $1 E+05$

1. $3 E+09$

1. $1 E+05$

5. $9 E+05$ 


$\begin{array}{lcccc}\text { ELEMENT } & \text { ATOMIC } & \text { I SOTOPE } & \text { ADULT } & \text { CHILD } \\ \text { SYMBOL } & \text { NO.12) } & \text { MASS(A) } & \text { UC } / M * * 2 & \text { UC/M**2 } \\ & & & & \\ \text { HF } & 72 & 182 & 7.2 E+06 & 1.6 E+05 \\ \text { TA } & 73 & 177 & 3.9 E+01 & 9.0 E-01 \\ \text { TA } & 73 & 179 & 8.7 E-01 & 2.0 E-02 \\ \text { TA } & 73 & 182 & 1.2 E-01 & 2.7 E-03 \\ \text { TA } & 73 & 183 & 1.7 E+01 & 3.9 E-01 \\ \text { W } & 74 & 178 & 7.8 E-01 & 1.8 E-02 \\ \text { W } & 74 & 181 & 2.2 E+00 & 5.1 E-02 \\ \text { W } & 74 & 185 & 2.4 E+00 & 5.4 E-02 \\ \text { W } & 74 & 187 & 3.2 E+02 & 7.4 E+00 \\ \text { W } & 74 & 188 & 2.3 E-01 & 5.3 E-03 \\ \text { RE } & 75 & 180 & 8.3 E-01 & 1.9 E-02 \\ \text { RE } & 75 & 181 & 9.1 E-01 & 2.1 E-02 \\ \text { RE } & 75 & 182 & 1.1 E-01 & 2.6 E-03 \\ \text { RE } & 75 & 182 M & 2.3 E+00 & 5.4 E-02 \\ \text { RE } & 75 & 183 & 3.7 E-03 & 8.5 E-05 \\ \text { RE } & 75 & 184 & 2.6 E-03 & 6.1 E-05 \\ \text { RE } & 75 & 184 M & 3.7 E-04 & 8.5 E-06 \\ \text { RE } & 75 & 186 & 2.0 E-01 & 4.7 E-03 \\ \text { RE } & 75 & 187 & 7.4 E-02 & 1.7 E-03 \\ \text { RE } & 75 & 188 & 2.0 E+00 & 4.5 E-02 \\ \text { RE } & 75 & 189 & 2.3 E+00 & 5.4 E-02 \\ \text { OS } & 76 & 182 & 1.5 E+01 & 3.5 E-01 \\ \text { OS } & 76 & 183 & 1.2 E+02 & 2.7 E+00 \\ \text { OS } & 76 & 185 & 2.9 E-02 & 6.6 E-04 \\ \text { OS } & 76 & 191 & 1.2 E+00 & 2.7 E-02 \\ \text { OS } & 76 & 191 M & 2.8 E+02 & 6.4 E+00 \\ \text { OS } & 76 & 193 & 3.2 E+01 & 7.4 E-01 \\ \text { OS } & 76 & 194 & 3.9 E-04 & 9.0 E-06 \\ \text { IR } & 77 & 185 & 6.1 E+01 & 1.4 E+00 \\ \text { IR } & 77 & 186 & 3.6 E+01 & 8.3 E-01 \\ \text { IR } & 77 & 187 & 9.7 E+01 & 2.2 E+00 \\ \text { IR } & 77 & 188 & 4.2 E+00 & 9.7 E-02 \\ \text { IR } & 77 & 189 & 7.9 E-01 & 1.8 E-02 \\ \text { IR } & 77 & 190 & 2.3 E-01 & 5.3 E-03 \\ \text { IR } & 77 & 192 & 2.8 E-02 & 6.3 E-04 \\ \text { IR } & 77 & 192 M & 2.0 E-04 & 4.6 E-06 \\ \text { IR } & 77 & 193 M & 4.0 E+00 & 9.3 E-02 \\ \text { IR } & 77 & 194 & 3.3 E+01 & 7.7 E-01 \\ \text { PT } & 78 & 188 & 1.5 E-01 & 3.5 E-03 \\ \text { PT } & 78 & 190 & 7.7 E-05 & 1.8 E-06 \\ \text { PT } & 78 & 191 & 6.3 E+00 & 1.4 E-01 \\ \text { PT } & 78 & 192 & 9.2 E-05 & 2.1 E-06 \\ \text { PT } & 78 & 193 & 3.2 E-03 & 7.4 E-05 \\ \text { PT } & 78 & 193 M & 7.6 E+00 & 1.7 E-01 \\ \text { PT } & 78 & 195 M & 7.4 E+00 & 1.7 E-01 \\ \text { PT } & 78 & 197 & 1.3 E+02 & 3.0 E+00 \\ \text { AU } & 79 & 193 & 7.9 E+01 & 1.8 E+00 \\ \text { AU } & 79 & 194 & 5.1 E+00 & 1.2 E-01 \\ \text { AU } & 79 & 195 & 5.1 E-02 & 1.2 E-03 \\ \text { AU } & 79 & 196 & 1.3 E+00 & 3.0 E-02 \\ & & & & \end{array}$

\begin{tabular}{|c|c|c|c|c|}
\hline $\begin{array}{l}\text { ELEMENT } \\
\text { SYMBOL }\end{array}$ & $\begin{array}{l}\text { A TOMIC } \\
\text { NO. }(Z)\end{array}$ & $\begin{array}{l}\text { I SOTOPE } \\
\text { MASS(A) }\end{array}$ & $\begin{array}{c}\text { ADULT } \\
U C / M * * 2\end{array}$ & $\begin{array}{c}\text { CHILD } \\
U C / M * * 2\end{array}$ \\
\hline$A U$ & 79 & 198 & $5 \cdot 5 E+00$ & $1.3 E-01$ \\
\hline$A U$ & 79 & 199 & $1.3 E+01$ & $3.0 E-01$ \\
\hline HG & 80 & 194 & 1. $1 E-04$ & $2.5 E-06$ \\
\hline HG & 80 & $195 \mathrm{M}$ & 1. $1 E-01$ & $2.5 E-03$ \\
\hline HG & 80 & 197 & 2. $1 E-01$ & $4.8 E-03$ \\
\hline HG & 80 & $197 \mathrm{M}$ & $7.4 E-01$ & $1.7 \mathrm{E}-02$ \\
\hline HG & 80 & $2 \mathrm{C3}$ & $2.7 E-03$ & $6.2 \mathrm{E}-05$ \\
\hline$T L$ & 81 & $2 \mathrm{co}$ & $2.5 E+02$ & $5.8 E+00$ \\
\hline TL & 81 & 201 & $2.1 E+02$ & $4.8 E+00$ \\
\hline TL & 81 & 202 & 1. $O E+O 1$ & $2.3 E-01$ \\
\hline $\mathrm{TL}$ & 81 & 204 & $6.8 E-02$ & $1.6 \mathrm{E}-03$ \\
\hline PB & 82 & $2 \mathrm{CO}$ & 2. $O E+02$ & $4.5 E+00$ \\
\hline$P B$ & 82 & 202 & 4. $1 E-03$ & $9.3 E-05$ \\
\hline PB & 82 & 203 & 8. $1 E+01$ & $1.9 E+00$ \\
\hline PB & 82 & $2 \mathrm{C4}$ & $9.2 E-04$ & $2 \cdot 1 E-05$ \\
\hline PB & 82 & $2 C 5$ & $2.8 \mathrm{E}-02$ & $6.4 E-04$ \\
\hline PB & 82 & 210 & $6.4 E-04$ & $1.5 E-05$ \\
\hline B I & 83 & 205 & 1. $1 E+01$ & $2.6 E-01$ \\
\hline B I & 83 & $2 \mathrm{Cb}$ & 5. $1 E+01$ & $1.2 E+00$ \\
\hline BI & 83 & 207 & 1. $8 E-02$ & $4-1 E-04$ \\
\hline B I & 83 & 208 & $8.8 E-03$ & $2.0 E-04$ \\
\hline B I & 83 & 210 & 2. $4 E+01$ & $5.4 E-01$ \\
\hline B I & 83 & $210 M$ & $4.3 E-03$ & $9.8 E-05$ \\
\hline PO & 84 & 206 & $4.8 \mathrm{E}-01$ & $1.1 E-02$ \\
\hline PO & 84 & 208 & $6.2 \mathrm{E}-04$ & $1.4 E-05$ \\
\hline PO & 84 & 209 & $1.5 E-04$ & $3.4 E-06$ \\
\hline PO & 84 & 210 & 7. $5 E-03$ & $1.7 E-04$ \\
\hline RN & 86 & 211 & $1.8 E+02$ & $4 \cdot 1 E+00$ \\
\hline RN & 86 & 222 & $2.4 E+00$ & $5.5 E-02$ \\
\hline RA & 88 & 223 & $1.3 E-03$ & $3.1 E-05$ \\
\hline RA & 88 & 224 & $9.1 E-03$ & $2.1 E-04$ \\
\hline RA & 88 & 225 & $9.2 E-04$ & $2.1 E-05$ \\
\hline RA & 88 & 226 & $8.6 E-07$ & $2.0 E-08$ \\
\hline RA & 88 & 228 & $2.8 E-06$ & $6.4 E-08$ \\
\hline$A C$ & 89 & 225 & $1.5 E+04$ & $3.5 E+02$ \\
\hline$A C$ & 89 & 227 & 1. $1 E+01$ & $2.5 E-01$ \\
\hline $\mathrm{TH}$ & 90 & 227 & $5.1 E+03$ & $1.2 E+02$ \\
\hline TH & 90 & 228 & $7.6 E+01$ & 1. $7 E+00$ \\
\hline $\mathrm{TH}$ & 90 & 229 & $7.1 E+00$ & $1.6 E-01$ \\
\hline$T H$ & 90 & 230 & $6.7 E+00$ & $1.5 E-01$ \\
\hline TH & 90 & 231 & 6. $0 E+05$ & $1.4 E+04$ \\
\hline TH & 90 & 232 & 6. $O E+00$ & $1.4 E-01$ \\
\hline$T H$ & 90 & 234 & $2.8 E+03$ & $6.4 E+01$ \\
\hline PA & 91 & 228 & 8. $7 E+05$ & $2.0 E+04$ \\
\hline PA & 91 & 229 & 3. $7 E+05$ & $8.4 E+03$ \\
\hline PA & 91 & 230 & $5.1 E+03$ & $1 \cdot 2 E+02$ \\
\hline$P A$ & 91 & 231 & $6.1 E+00$ & $1.4 E-01$ \\
\hline PA & 91 & 232 & $3.9 E+05$ & $9.0 E+03$ \\
\hline PA & 91 & 233 & $2.6 E+03$ & $5.9 E+01$ \\
\hline$u$ & 92 & 230 & 3. $7 E+01$ & $8.4 E-01$ \\
\hline
\end{tabular}


ELEMENT SYMBOL

\section{ATOMIC ISOTOPE}

NO. (Z) MASS(A)

ADULT
$U C / M * 22$
$4.7 E+02$
$6.9 E-02$
$6.2 E-02$
$5.9 E-02$
$5.5 E-02$
$5 \cdot 4 E-02$
$1.9 E+02$
$5.2 E-02$
$1.5 E+04$
$4.0 E+04$
$1.0 E+02$
$5.2 E+00$
$7.0 E+05$
$5.5 E+00$
$1.4 E+05$
$1.1 E+05$
$4 \cdot 3 E+03$
$1.3 E+05$
$6.5 E+02$
$5.4 E+02$
$5.4 E+02$
$1.1 E+03$
$5.2 E+02$
$4.6 E+02$
$7.2 E+05$
$2.1 E+06$
$1.3 E+05$
$5.0 E+00$
$1.1 E+06$
$4.9 E+00$
$4.4 E+00$

CHILD
UC $/ M * * 2$

$1.1 E+01$

$1.6 \mathrm{E}-03$

$1.4 \mathrm{E}-03$

1. $3 E-03$

$1.2 \mathrm{E}-03$

4. $3 E+00$

1. $2 \mathrm{E}-03$

$3.4 E+02$

$9.2 \mathrm{E}+02$

$2.4 E+00$

$1.2 E-01$

1. $6 E+04$

$1.3 E-01$

3. $2 E+03$

$2.5 E+03$

9. $8 \mathrm{E}+01$

3. $0 E+03$

1. $2 \mathrm{E}+01$

1. $2 \mathrm{E}+01$

$2.5 E+01$

$1.2 E+01$

$1.1 \mathrm{E}+01$

1. $7 E+04$

4. $B E+04$

2. $9 E+03$

$1.1 \mathrm{E}-01$

$2.6 E+04$

1. $1 E-01$

1. OE-OI
VIA MILK.

ELEMENT
SYMBOL

ATOMIC

NO. (2) MASSIAI

$C M$
$C M$
$C M$
$C M$
$C M$
$C M$
$C M$
$C M$
$C M$
$B K$
$B K$
$B K$
$B K$
$B K$
$C F$
$C F$
$C F$
$C F$
$C F$
$C F$
$C F$
$E S$
$E S$
$E S$
$E S$
$E S$
$E S$
$F M$
$F M$
$F M$
$F M$

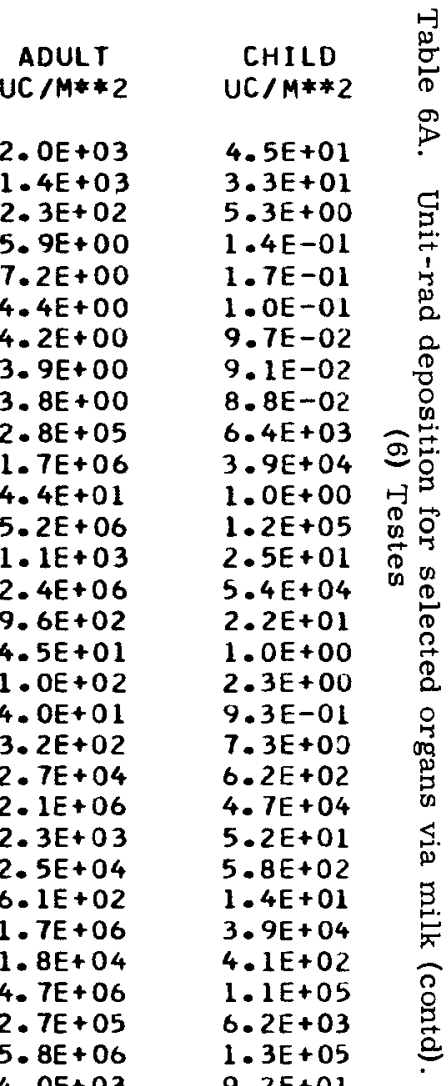




\begin{tabular}{|c|c|}
\hline $\begin{array}{l}\text { ELEMENT } \\
\text { SYMBOL }\end{array}$ & $\begin{array}{l}\text { ATOMI } \\
\text { NO. } 12\end{array}$ \\
\hline$H$ & \\
\hline$B E$ & \\
\hline$B E$ & \\
\hline C & \\
\hline NA & 11 \\
\hline NA & 11 \\
\hline MG & 12 \\
\hline AL & 13 \\
\hline$\$ 1$ & 14 \\
\hline P & 15 \\
\hline$P$ & 15 \\
\hline$S$ & 16 \\
\hline $\mathrm{CL}$ & 17 \\
\hline AR & 18 \\
\hline AR & 18 \\
\hline AR & 18 \\
\hline K & 19 \\
\hline$K$ & 19 \\
\hline K & 19 \\
\hline$C A$ & 20 \\
\hline$C A$ & 20 \\
\hline$C A$ & 20 \\
\hline$C A$ & 20 \\
\hline SC & 21 \\
\hline SC & 21 \\
\hline SC & 21 \\
\hline SC & 21 \\
\hline TI & 22 \\
\hline v & 23 \\
\hline V & 23 \\
\hline v & 23 \\
\hline CR & 26 \\
\hline$C R$ & 26 \\
\hline MN & 2 \\
\hline MN & \\
\hline$M N$ & 25 \\
\hline $\mathrm{FE}$ & \\
\hline FE & \\
\hline$F E$ & \\
\hline $\mathrm{CO}$ & \\
\hline $\mathrm{CO}$ & \\
\hline $\mathrm{CO}$ & \\
\hline $\mathrm{CO}$ & \\
\hline $\mathrm{CO}$ & \\
\hline NI & \\
\hline NI & \\
\hline NI & \\
\hline N I & \\
\hline N I & 8 \\
\hline $\mathrm{CU}$ & \\
\hline
\end{tabular}

\begin{tabular}{|c|c|}
\hline $\begin{array}{l}\text { ADULT } \\
U C / M * 2\end{array}$ & $\begin{array}{l}\text { CHILD } \\
U C / M * * 2\end{array}$ \\
\hline $\begin{array}{l}6.9 E+02 \\
3.2 E+06 \\
1.0 E+05 \\
1.1 E+02 \\
8.2 E-01 \\
1.7 E+02 \\
3.6 E+03 \\
1.2 E+01 \\
1.8 E+02 \\
7.5 E+00 \\
4.0 E+01 \\
2.6 E+01 \\
2.2 E+00 \\
7.4 E+02 \\
2.8 E-02 \\
3.1 E-03 \\
2.2 E+00 \\
2.1 E+03 \\
1.3 E+03 \\
5.0 E+00 \\
8.0 E+00 \\
4.8 E+01 \\
4.9 E-03 \\
2.0 E+09 \\
1.4 E+07 \\
9.9 E+09 \\
2.7 E+09 \\
2.3 E+06 \\
2.7 E+03 \\
2.9 E+05 \\
9.9 E+02 \\
4.6 E+05 \\
1.6 E+05 \\
8.2 E+03 \\
1.9 E+05 \\
1.6 E+03 \\
3.0 E+03 \\
2.0 E+02 \\
4.0 E+00 \\
3.8 E+04 \\
1.1 E+02 \\
1.2 E+03 \\
2.3 E+02 \\
5.6 E+01 \\
7.3 E+01 \\
1.8 E+03 \\
1.0 E+02 \\
4.5 E+01 \\
8.8 E+02 \\
3.8 E+04\end{array}$ & $\begin{array}{l}9.9 E+01 \\
4.6 E+05 \\
1.4 E+04 \\
1.6 E+01 \\
1.2 E-01 \\
2.4 E+01 \\
5.1 E+02 \\
1.7 E+00 \\
2.6 E+01 \\
1.1 E+00 \\
5.7 E+00 \\
3.8 E+00 \\
3.1 E-01 \\
1.1 E+02 \\
4.0 E-03 \\
4.4 E-04 \\
3.1 E-01 \\
2.9 E+02 \\
1.8 E+02 \\
7.1 E-01 \\
1.1 E+00 \\
6.9 E+00 \\
7.0 E-04 \\
2.9 E+08 \\
2.0 E+06 \\
1.4 E+09 \\
3.9 E+08 \\
3.3 E+05 \\
3.9 E+02 \\
4.2 E+04 \\
1.4 E+02 \\
6.5 E+04 \\
2.3 E+04 \\
1.2 E+03 \\
2.8 E+04 \\
2.3 E+02 \\
4.4 E+02 \\
2.9 E+01 \\
5.7 E-01 \\
5.5 E+03 \\
1.5 E+01 \\
1.7 E+02 \\
3.3 E+01 \\
8.1 E+00 \\
1.1 E+01 \\
2.5 E+02 \\
1.4 E+01 \\
6.4 E+00 \\
1.3 E+02 \\
5.5 F+03\end{array}$ \\
\hline
\end{tabular}

ELEMENT

ATOMIC

I SOTDPE

ADULT

CHILD

$C U$
$Z N$
$Z N$

29
30
30
30

$\begin{array}{ll}67 & 2 \\ 65 & 2 \\ 69 M & 1 \\ 72 & 2 \\ 67 & 2 \\ 72 & 3 \\ 68 & 1 \\ 69 & 1 \\ 71 & 5\end{array}$

2. $8 E+00$

1. $3 E+04$

2. $5 E+02$

. $1 E+08$

$1.3 E+05$

1. $7 E+06$

5. $O E+07$

2. $2 E+04$

$2.4 E+04$

1. $0 E+04$

3. $4 E+02$

$3.2 E+04$

1. $2 E+05$

4. $8 E+00$

5. $O E+01$

$3.3 E+02$

7. $5 E+01$

6. $3 E+01$

7. $8 \mathrm{E}+02$

4. $5 E+02$

2. $8 E-01$

$5.4 E-02$

1. $7 E+00$

1. $1 E+00$

3. $8 E+00$

5. $O E+00$

3. $1 E+01$

1. $3 E+04$

6. $3 E+01$

5. $8 \mathrm{E}+01$

2. $6 E-01$

$9.5 \mathrm{E}+09$

..5E+O

1. $8 \mathrm{E}+09$

3. $5 E+10$

4. $O E+06$

2. $7 E+0$

1. $3 E+10$

- $1 E+0$

$1.8 E+09$

2. $3 E+08$

2. $O E+07$

6. $3 E+0$

6. $1 E+02$

7. $6 E+02$

$3 E+02$

4. $0 E-01$

$1.9 E+03$

$3.6 E+01$

3. $7 E+07$

4. $4 E+07$

1. $9 E+04$

$2.4 E+05$

7. $1 E+06$

. $1 E+03$

$3.4 E+03$

$1.5 E+03$

4. $8 E+01$

1. $7 \mathrm{E}+04$

6. $5 E-01$

$6.9 E-01$

7. $2 E+00$

4. $7 E+01$

1. $1 E+01$

9. $0 E+00$

$1.1 E+02$

6. $4 E+01$

7. $8 E-03$

2. $5 E-01$

$1.6 E-01$

5.5E-01

7. $2 \mathrm{E}-01$

4. $4 E+00$

1. $8 E+03$

9. $0 E+00$

8. $2 E+00$

3. $8 \mathrm{E}-02$

$1.4 E+09$

$2.6 \mathrm{E}+08$

$5.0 E+09$

5. $7 E+05$

$2.5 E+08$

$3.8 E+06$

1. $9 E+09$

1. $5 E+06$

. $6 E+08$

$3.3 E+07$

$3.8 E+06$

2. $8 E+09$

$8.9 E+03$

8. $7 E+01$

1. $1 E+02$

2. $9 E+02$ 
TABLE 6A. UNIT-RAD DEPOSITION FOR WHOLE BODY VIA MILK.

05/09/68 PAGE 32

\begin{tabular}{|c|c|c|c|c|}
\hline $\begin{array}{l}\text { ELEMENT } \\
\text { SYMBDL }\end{array}$ & $\begin{array}{l}\text { ATOMIC } \\
\text { NO. }(2)\end{array}$ & $\begin{array}{l}\text { I SOTOPE } \\
\text { MASS (A) }\end{array}$ & $\begin{array}{l}\text { ADULT } \\
\text { UC } / M * * 2\end{array}$ & $\begin{array}{c}\text { CHILD } \\
U C / M * * 2\end{array}$ \\
\hline NB & 41 & $93 M$ & $6.0 E+02$ & $8.5 E+01$ \\
\hline NB & 41 & 94 & $6.2 E+00$ & 8. $8 \mathrm{E}-01$ \\
\hline NB & 41 & 95 & 4. $3 E+02$ & $6.2 E+01$ \\
\hline NB & 41 & $95 \mathrm{M}$ & 1. $0 E+04$ & $1.5 E+03$ \\
\hline NB & 41 & 96 & $4.9 E+04$ & $7.0 E+03$ \\
\hline MO & 42 & 93 & $9.1 E+01$ & $1.3 E+01$ \\
\hline MO & 42 & 99 & $4.5 E+02$ & $6.5 E+01$ \\
\hline TC & 43 & 95 & $1.4 E+03$ & $2.0 E+02$ \\
\hline TC & 43 & $95 \mathrm{M}$ & $1.7 E+01$ & $2.4 E+00$ \\
\hline TC & 43 & 96 & 4. $0 E+01$ & $5.7 E+00$ \\
\hline TC & 43 & 97 & $6.9 E+02$ & $9.9 E+01$ \\
\hline TC & 43 & $97 M$ & $1.4 E+02$ & $2.0 E+O 1$ \\
\hline TC & 43 & 98 & $9.3 E+00$ & $1.3 E+00$ \\
\hline TC & 43 & 99 & $1.5 E+02$ & $2.2 E+O 1$ \\
\hline RU & 44 & 97 & 1. $2 E+07$ & $1.7 E+06$ \\
\hline RU & 44 & 103 & $2.6 E+05$ & $3.7 E+04$ \\
\hline RU & 44 & 106 & 1. $5 E+04$ & $2.2 E+03$ \\
\hline RH & 45 & 99 & $3.2 E+02$ & $4.6 E+01$ \\
\hline $\mathrm{RH}$ & 45 & 100 & 7. $0 E+03$ & $1.0 E+03$ \\
\hline $\mathrm{RH}$ & 45 & 101 & $1.5 E+02$ & $2.2 E+01$ \\
\hline RH & 45 & $101 \mathrm{M}$ & 2. $0 E+03$ & $2.9 E+02$ \\
\hline RH & 45 & 102 & $3.3 E+01$ & $4.7 E+00$ \\
\hline RH & 45 & 105 & 1. $7 E+04$ & $2.4 E+03$ \\
\hline PD & 46 & 100 & $7.1 E+02$ & 1. $0 E+02$ \\
\hline PD & 46 & 103 & $3.6 \mathrm{E}+03$ & $5.1 E+02$ \\
\hline PD & 46 & 107 & $8.5 E+04$ & $1.2 E+04$ \\
\hline PD & 46 & 109 & 4. $9 E+04$ & 7. $0 E+03$ \\
\hline PD & 46 & 112 & $3.8 E+03$ & $5.4 E+02$ \\
\hline$A G$ & 47 & 105 & 1. $1 E+00$ & $1.6 \mathrm{E}-01$ \\
\hline$A G$ & 47 & $106 \mathrm{M}$ & 2. $9 E+00$ & $4.2 \mathrm{E}-01$ \\
\hline$A G$ & 47 & $108 M$ & $1.6 \mathrm{E}-01$ & $2.3 \mathrm{E}-02$ \\
\hline$A G$ & 47 & $110 \mathrm{M}$ & $1.8 E-01$ & $2.6 \mathrm{E}-02$ \\
\hline$A G$ & 47 & 111 & $1.9 E+01$ & $2.8 E+0$ \\
\hline$C D$ & 48 & 109 & $2.2 E+05$ & $3.1 E+04$ \\
\hline$C D$ & 48 & $113 M$ & 8. $2 E+04$ & 1. $2 E+04$ \\
\hline $\mathrm{CD}$ & 48 & 115 & $8.2 E+06$ & $1.2 E+06$ \\
\hline$C D$ & 48 & $125 \mathrm{M}$ & $1.2 E+05$ & $1.7 E+04$ \\
\hline IN & 49 & 111 & $2.6 E+07$ & $3.8 E+0$ \\
\hline IN & 49 & $114 M$ & $2.1 E+05$ & $3.1 E+04$ \\
\hline IN & 49 & 115 & $4.9 E+05$ & 7. $1 E+04$ \\
\hline SN & 50 & 113 & $3.0 E+02$ & $4.3 E+01$ \\
\hline SN & 50 & $117 M$ & $4.0 E+03$ & $5.7 E+02$ \\
\hline SN & 50 & $119 M$ & 8. $9 E+02$ & $1.3 E+02$ \\
\hline SN & 50 & 121 & $8.3 E+05$ & $1.2 E+05$ \\
\hline SN & 50 & $121 \mathrm{M}$ & 3. $1 E+02$ & $4.4 E+01$ \\
\hline SN & 50 & $123 M$ & 1. $7 E+02$ & $2.5 E+0$ \\
\hline SN & 50 & 125 & $1.2 E+03$ & 1. $7 \mathrm{E}+02$ \\
\hline SN & 50 & 126 & 1. $3 E+01$ & $1.8 E+00$ \\
\hline SB & 51 & 119 & $2.8 E+06$ & $4.0 E+05$ \\
\hline SB & 51 & $120 \mathrm{M}$ & $6.2 E+03$ & $8.8 E+02$ \\
\hline
\end{tabular}

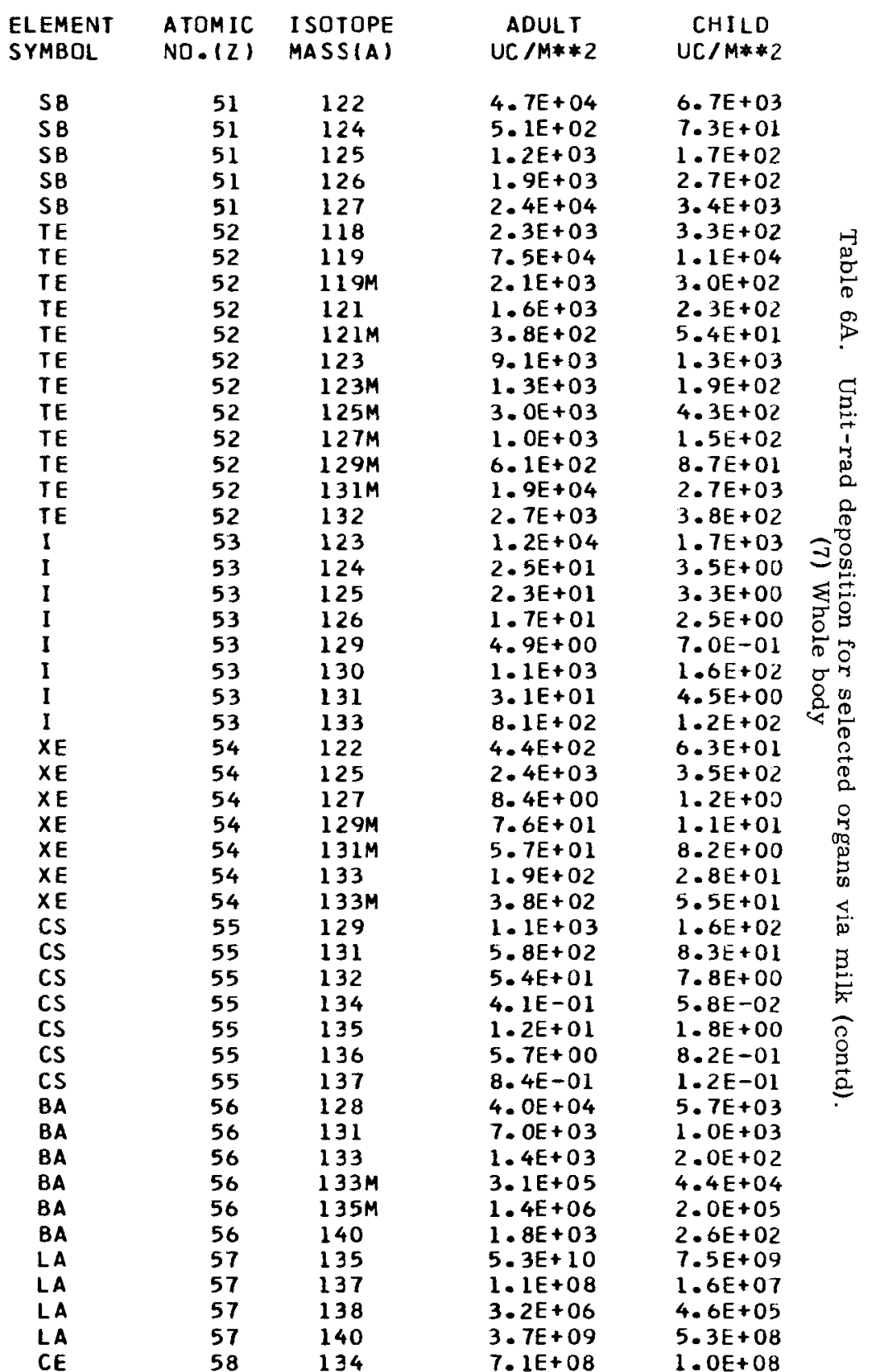


TABLE 6A. UNIT-RAD DEPOSITION FOR WHOLE BODY VIA MILK.

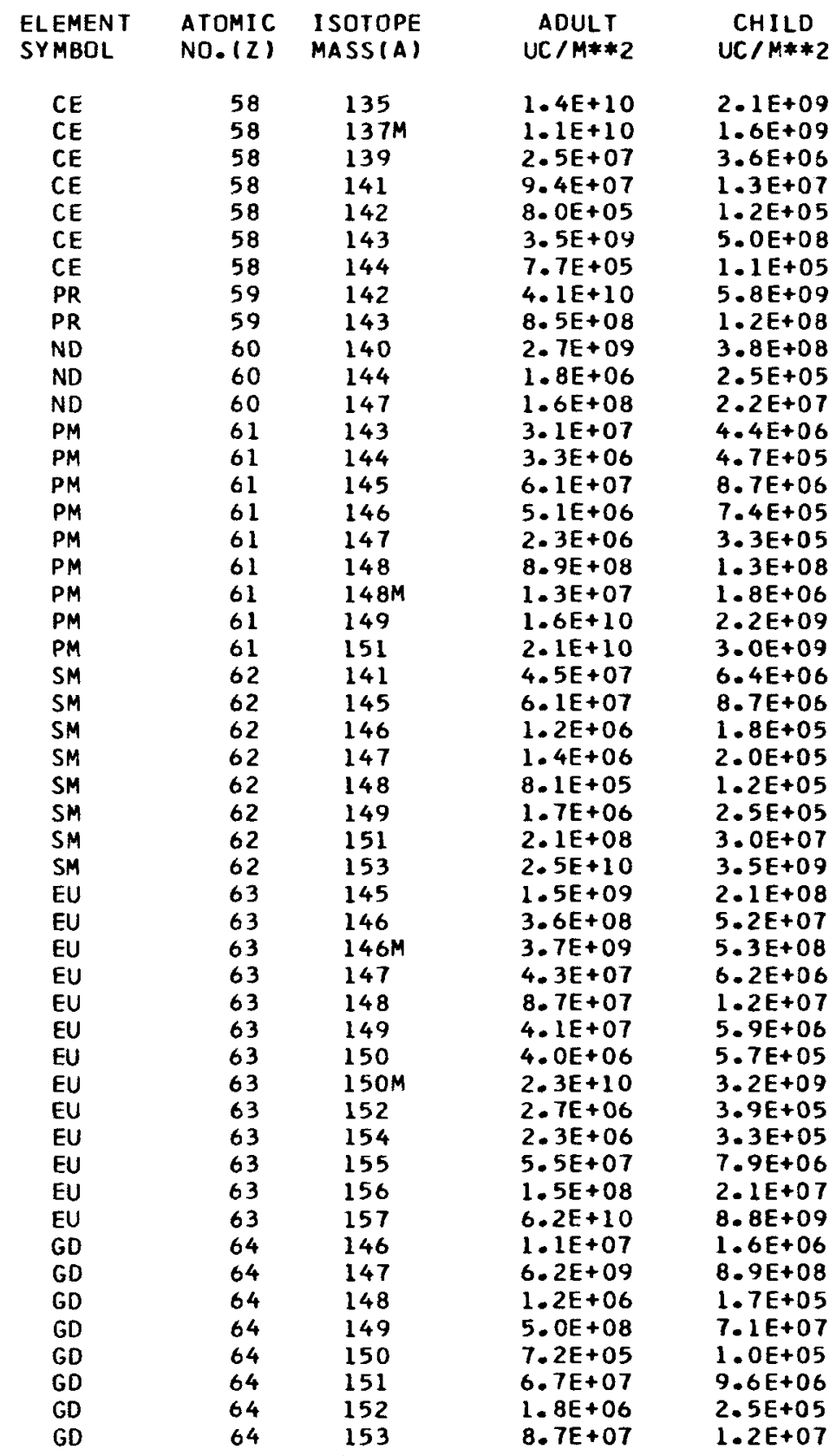

$\begin{array}{lcc}\text { ELEMENT } & \text { ATOMIC } & \text { ISOTOPE } \\ \text { SYMBOL } & \text { NO. }(2) & \text { MASS A } \\ \text { GD } & & \\ \text { GD } & 64 & 159 \\ \text { TB } & 64 & 162 \\ \text { TB } & 65 & 151 \\ \text { TB } & 65 & 152 \\ \text { TB } & 65 & 153 \\ \text { TB } & 65 & 154 \\ \text { TB } & 65 & 155 \\ \text { TB } & 65 & 156 \\ \text { TB } & 65 & 157 \\ \text { TB } & 65 & 158 \\ \text { TB } & 65 & 160 \\ \text { TB } & 65 & 161 \\ \text { DY } & 65 & 164 \\ \text { DY } & 66 & 154 \\ \text { DY } & 66 & 154 M \\ \text { DY } & 66 & 156 \\ \text { DY } & 66 & 159 \\ \text { HO } & 66 & 166 \\ \text { HO } & 67 & 163 \\ \text { HD } & 67 & 166 \\ \text { ER } & 67 & 166 M \\ \text { ER } & 68 & 160 \\ \text { ER } & 68 & 169 \\ \text { TM } & 68 & 172 \\ \text { TM } & 69 & 165 \\ \text { TM } & 69 & 167 \\ \text { TM } & 69 & 168 \\ \text { TM } & 69 & 170 \\ \text { TM } & 69 & 171 \\ \text { YB } & 69 & 172 \\ \text { YB } & 70 & 166 \\ \text { YB } & 70 & 169 \\ \text { LU } & 70 & 175 \\ \text { LU } & 71 & 169 \\ \text { LU } & 71 & 170 \\ \text { LU } & 71 & 171 \\ \text { LU } & 71 & 172 \\ \text { LU } & 71 & 173 \\ \text { LU } & 71 & 174 \\ \text { LU } & 71 & 174 M \\ \text { LU } & 71 & 176 \\ \text { LU } & 71 & 177 \\ \text { HF } & 71 & 177 M \\ \text { HF } & 72 & 170 \\ \text { HF } & 72 & 171 \\ \text { HF } & 72 & 172 \\ \text { HF } & 72 & 173 \\ \text { HF } & 72 & 174 \\ \text { HF } & 72 & 175 \\ & & 181\end{array}$

ADULT
$U C / M * 2$

1. $3 E+1$

$6.6 E+06$

5. $4 E+10$

1. $6 E+10$

1. $2 E+10$

5. $8 E+10$

3. $1 E+09$

9. $5 E+08$

6. $3 E+07$

$2.7 E+06$

2. $8 E+07$

4. $7 E+09$

7. $9 E+10$

3. $7 E+05$

3. $1 E+10$

5. $8 E+05$

2. $9 E+08$

2. $8 E+09$

5. $2 E+07$

2. $7 E+10$

2. $3 E+06$

7. $6 E+09$

5. $9 E+09$

6. $3 E+09$

8. $4 E+09$

9. $2 E+08$

2. $1 E+07$

5. $9 E+07$

3. $1 E+08$

5. $2 E+09$

- $1 E+10$

2. $7 E+08$

1. $2 E+10$

7. $9 E+09$

2. $8 E+09$

5. $1 E+09$

1. $0 E+09$

1. $O E+07$

3. $5 E+06$

. $1 E+07$

3. $9 E+06$

5. $6 E+09$

8. $8 \mathrm{E}+07$

2. $8 E+10$

5. $6 E+10$

4. $3 E+06$

1. $8 \mathrm{E}+10$

1. $5 E+06$

8. $1 E+0$

9. $2 E+07$
CHILD

$U C / M * \$ 2$

- $9 E+10$

$9.4 E+05$

$2.2 E+09$

- $6 E+09$

$8.3 E+07$

4. $5 E+08$

1. $4 E+08$

$9.0 E+0 S$

3. $9 E+05$

4. OE + OS

6. $8 E+0 B$

$1.1 E+10$

5. $3 E+04$

4. $4 E+09$

$8 \cdot 3 E+04$

$4.1 E+07$

4. $0 E+08=$

$3.8 E+09$

$3.3 E+05$ S

$1.1 E+07$ क

8. $5 E+08$ G

$9.1 E+08$ ह 용

$1.3 E+08$

3. $0 E+06$

8. $5 E+0 S$

4. $5 E+07$

7. $5 E+08$

7. $5 E+08$

1. $6 E+09$

3. $8 E+0$ ?

1. $8 E+07$

1. $1 E+07$

4. $0 E+08$

7. $3 E+08$

$1.4 E+08$

1. $4 E+D O$

$5.0 E+05$

$4.5 E+05$

$4.5 E+05$
$5.5 E+05$

$8.1 E+08$

1. $3 E+07$

4. $1 E+09$

8. $0 E+09$

6. $1 E+0 b$

2. $5 E+09$

$2.1 E+05$

$1.2 E+07$ 


\begin{tabular}{|c|c|c|c|c|}
\hline $\begin{array}{l}\text { ELEMENT } \\
\text { SYMBOL }\end{array}$ & $\begin{array}{l}\text { ATOMIC } \\
\text { NO. }(2)\end{array}$ & $\begin{array}{l}\text { ISOTOPE } \\
\text { MASS (A) }\end{array}$ & $\begin{array}{l}\text { ADULT } \\
U C / M * * 2\end{array}$ & $\begin{array}{c}\text { CHILD } \\
\text { UC/M**2 }\end{array}$ \\
\hline $\mathrm{HF}$ & 72 & 182 & $2.3 E+06$ & $3.2 E+05$ \\
\hline TA & 73 & 177 & $2.3 E+03$ & $3.3 E+02$ \\
\hline TA & 73 & 179 & 7. $7 \mathrm{E}+01$ & $1.1 E+O 1$ \\
\hline TA & 73 & 182 & 8. $1 E+00$ & $1.2 E+0 J$ \\
\hline TA & 73 & 183 & $1.0 E+03$ & $1.4 E+02$ \\
\hline$w$ & 74 & 178 & $2.1 E+04$ & $3.0 E+03$ \\
\hline w & 74 & 181 & $3.4 E+05$ & 4. $8 E+04$ \\
\hline$w$ & 74 & 185 & $2.1 E+05$ & $3.0 E+04$ \\
\hline$w$ & 74 & 187 & $7.5 E+05$ & $1.1 E+05$ \\
\hline$w$ & 74 & 188 & 1. $9 E+04$ & $2.7 E+03$ \\
\hline RE & 75 & 180 & $1.1 E+03$ & $1.5 E+02$ \\
\hline RE & 75 & 181 & 1. $2 \mathrm{E}+03$ & $1.7 E+02$ \\
\hline RE & 75 & 182 & 1. $8 E+02$ & $2.6 E+01$ \\
\hline RE & 75 & $182 M$ & 2. $9 E+03$ & $4.2 E+02$ \\
\hline RE & 75 & 183 & 4. $8 E+01$ & $6.8 E+00$ \\
\hline $\mathrm{RE}$ & 75 & 184 & $2.0 E+01$ & $2.8 E+00$ \\
\hline RE & 75 & $184 \mathrm{M}$ & $1 \cdot 1 E+01$ & $1.6 E+00$ \\
\hline$R E$ & 75 & 186 & $3.6 E+02$ & $5.2 E+01$ \\
\hline RE & 75 & 187 & $9.2 E+04$ & $1.3 E+04$ \\
\hline RE & 75 & 188 & $2.5 E+03$ & $3.6 E+02$ \\
\hline RE & 75 & 189 & $3.1 E+03$ & 4. $5 E+02$ \\
\hline OS & 76 & 182 & $2.6 E+04$ & $3.7 E+03$ \\
\hline os & 76 & 183 & $1.7 E+05$ & $2.4 E+04$ \\
\hline OS & 76 & 185 & $1.6 E+03$ & $2.3 E+02$ \\
\hline OS & 76 & 191 & $1.1 E+04$ & $1.6 E+03$ \\
\hline OS & 76 & $191 M$ & 4. $2 E+05$ & $6.0 E+04$ \\
\hline os & 76 & 193 & $6.2 E+04$ & $8.9 E+03$ \\
\hline OS & 76 & 194 & 4. $7 E+02$ & $6.7 E+01$ \\
\hline IR & 77 & 185 & $7.4 E+04$ & 1. $1 E+04$ \\
\hline IR & 77 & 186 & 4. $3 E+04$ & $6.2 E+03$ \\
\hline IR & 77 & 187 & $1.2 E+05$ & $1.7 E+04$ \\
\hline IR & 77 & 188 & $5.4 E+03$ & $7.7 E+02$ \\
\hline IR & 77 & 189 & $1.5 E+03$ & $2.2 E+02$ \\
\hline IR & 77 & 190 & $4.3 E+02$ & $6.1 E+01$ \\
\hline IR & 77 & 192 & $1.5 E+02$ & 2. $2 E+01$ \\
\hline IR & 77 & $192 \mathrm{M}$ & 8. $7 E+01$ & $1.2 \mathrm{E}+01$ \\
\hline IR & 77 & $193 M$ & $7.5 E+03$ & $1.1 E+03$ \\
\hline IR & 77 & 194 & $4.1 E+04$ & $5.8 E+03$ \\
\hline PT & 78 & 188 & $2.5 E+02$ & $3.6 E+01$ \\
\hline PT & 78 & 190 & 2. $8 E+01$ & $4.0 E+00$ \\
\hline PT & 78 & 191 & $8.3 E+03$ & $1.2 E+03$ \\
\hline PT & 78 & 192 & 3. $3 E+01$ & 4. $8 E+O D$ \\
\hline PT & 78 & 193 & $1.1 E+03$ & $1.6 E+02$ \\
\hline $\mathrm{PT}$ & 78 & $193 M$ & 1. $0 E+04$ & 1. $5 E+03$ \\
\hline PT & 78 & $195 M$ & $1.0 E+04$ & $1.5 E+03$ \\
\hline PT & 78 & 197 & $1.6 E+05$ & $2.2 E+04$ \\
\hline$A U$ & 79 & 193 & 9. $3 E+04$ & $1.3 E+04$ \\
\hline$A U$ & 79 & 194 & $6.0 E+03$ & $8.6 E+02$ \\
\hline$A U$ & 79 & 195 & $1.5 E+02$ & $2.1 E+01$ \\
\hline$A U$ & 79 & 196 & $1.6 E+03$ & $2.3 E+02$ \\
\hline
\end{tabular}

$\begin{array}{lcc}\text { ELEMENT } & \text { ATOMIC } & \text { ISOTOPE } \\ \text { SYMBOL } & \text { NO. }(2) & \text { MASS(A) } \\ \text { AU } & & \\ \text { AU } & 79 & 198 \\ \text { HG } & 79 & 199 \\ \text { HG } & 80 & 194 \\ \text { HG } & 80 & 195 M \\ \text { HG } & 80 & 197 \\ \text { HG } & 80 & 197 M \\ \text { TL } & 80 & 2 C 3 \\ \text { TL } & 81 & 200 \\ \text { TL } & 81 & 201 \\ \text { TL } & 81 & 2 C 2 \\ \text { PB } & 81 & 2 C 4 \\ \text { PB } & 82 & 2 C 0 \\ \text { PB } & 82 & 2 C 2 \\ \text { PB } & 82 & 2 C 3 \\ \text { PB } & 82 & 204 \\ \text { PB } & 82 & 2 C 5 \\ \text { BI } & 82 & 210 \\ \text { BI } & 83 & 2 C 5 \\ \text { BI } & 83 & 2 C 6 \\ \text { BI } & 83 & 2 C 7 \\ \text { BI } & 83 & 208 \\ \text { BI } & 83 & 210 \\ \text { PO } & 83 & 210 M \\ \text { PD } & 84 & 206 \\ \text { PO } & 84 & 208 \\ \text { PO } & 84 & 2 C 9 \\ \text { RN } & 84 & 210 \\ \text { RN } & 86 & 211 \\ \text { RA } & 86 & 222 \\ \text { RA } & 88 & 223 \\ \text { RA } & 88 & 224 \\ \text { RA } & 88 & 225 \\ \text { RA } & 88 & 226 \\ \text { AC } & 88 & 228 \\ \text { AC } & 89 & 225 \\ \text { TH } & 89 & 227 \\ \text { TH } & 90 & 227 \\ \text { TH } & 90 & 228 \\ \text { TH } & 90 & 229 \\ \text { TH } & 90 & 230 \\ \text { TH } & 90 & 231 \\ \text { TH } & 90 & 232 \\ \text { PA } & 90 & 234 \\ \text { PA } & 91 & 228 \\ \text { PA } & 91 & 229 \\ \text { PA } & 91 & 230 \\ \text { PA } & 91 & 231 \\ \text { PA } & 91 & 232 \\ \text { H } & 91 & 233 \\ & 92 & 230\end{array}$

ADULT TC
UC $/ M * * 2$

CHILD

6. $5 E+03 \quad 9.3 E+02$

$1-1 \mathrm{E}+03$

$1.0 E+02$

3. $7 \mathrm{E}+00$ 5. $6 E+00$ 7. $0 E+00$ $2.6 E+01$ 3. 5E-01 2. $2 E-01$ $1.5 E+00$

1. $5 E-01$

1. $5 E-01$
2. $2 E-04$ 2. $5 E+06$

1. $4 E+04$

6. $0 E+06$

9. $O E+04$

8. $8 \mathrm{E}+03$

8. $2 E+03$

$2.1 E+03$
$8.6 E+05$

8. $6 E+05$

1. $3 E+03$

7. $1 E+08$

1. $2 E+03$

7. $4 E+03 \quad 1.0 E+08$ 1. $1 E+03$

3. $3 E+06$ 4. $7 E+05$

1. $0 E+09$

$1.5 E+08$

4. $3 E+08$

6. $0 E+06$

$6.1 E+07$

1. $1 E+03$

4. $6 E+08$

$6.5 E+07$

3. $0 E+06$

4. $3 E+05$ 
TABLE 6A. UNIT-RAD DEPOSITION FOR WHOLE BODY VIA MILK.

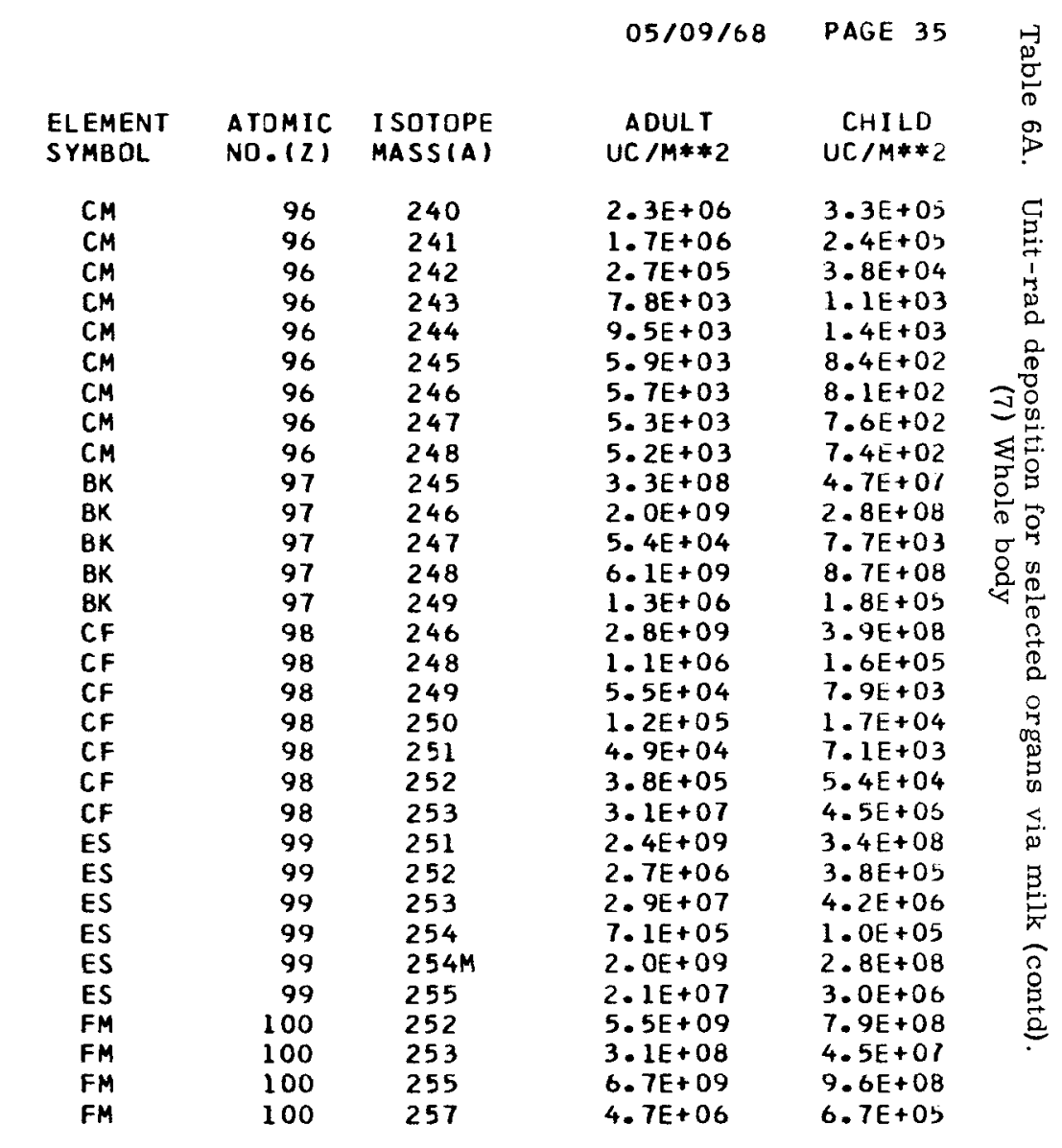

\begin{tabular}{|c|c|c|c|c|}
\hline $\begin{array}{l}\text { EL EMENT } \\
\text { SYMBOL }\end{array}$ & $\begin{array}{l}\text { ATOMIC } \\
\text { NO. (Z) }\end{array}$ & $\begin{array}{l}\text { I SOTOPE } \\
\text { MASS (A) }\end{array}$ & $\begin{array}{l}\text { ADULT } \\
U C / M * * 2\end{array}$ & $\begin{array}{l}\text { CHILD } \\
U C / M * * 2\end{array}$ \\
\hline U & 92 & 231 & 5. $7 E+05$ & $8.2 E+04$ \\
\hline$u$ & 92 & 232 & $5.3 E+03$ & $7.5 E+02$ \\
\hline$u$ & 92 & 233 & $5.5 E+03$ & $7.8 E+02$ \\
\hline$u$ & 92 & 234 & $5.1 E+03$ & $7.4 E+02$ \\
\hline U & 92 & 235 & $4.8 E+03$ & $6.8 E+02$ \\
\hline$u$ & 92 & 236 & 4. $7 E+03$ & $6.7 E+02$ \\
\hline$U$ & 92 & 237 & $2.3 E+05$ & 3. $3 E+04$ \\
\hline U & 92 & 238 & $4.5 E+03$ & $6.4 E+02$ \\
\hline U & 92 & 240 & 1. $7 E+07$ & $2.5 E+06$ \\
\hline NP & 93 & 234 & 4. $7 E+07$ & $6.7 E+06$ \\
\hline NP & 93 & 235 & 1. $2 E+05$ & $1.7 E+04$ \\
\hline NP & 93 & 236 & $6.6 E+03$ & $9.4 E+02$ \\
\hline NP & 93 & $236 \mathrm{M}$ & $8.2 E+08$ & 1. $2 E+08$ \\
\hline NP & 93 & 237 & 7. $0 E+03$ & $1.0 E+03$ \\
\hline NP & 93 & 238 & $1.6 E+08$ & $2.3 E+07$ \\
\hline NP & 93 & 239 & $1.3 E+08$ & $1.8 E+07$ \\
\hline PU & 94 & 236 & $5.0 E+05$ & $7.2 E+04$ \\
\hline PU & 94 & 237 & 1. $5 E+07$ & $2.2 E+06$ \\
\hline$P U$ & 94 & 238 & 7. $9 E+04$ & $1.1 E+04$ \\
\hline PU & 94 & 239 & $6.6 E+04$ & $9.5 E+03$ \\
\hline PU & 94 & 240 & $6.6 E+04$ & $9.4 E+03$ \\
\hline PU & 94 & 241 & 1. $3 E+05$ & $1.9 E+04$ \\
\hline PU & 94 & 242 & $6.3 E+04$ & $9.1 E+03$ \\
\hline PU & 94 & 244 & $5.7 E+04$ & $8.1 E+03$ \\
\hline PU & 94 & 246 & $8.4 E+07$ & $1.2 E+07$ \\
\hline$A M$ & 95 & 239 & 2. $5 E+09$ & $3.5 E+08$ \\
\hline AM & 95 & 240 & $1 \cdot 5 E+08$ & $2.1 E+07$ \\
\hline AM & 95 & 241 & $6.9 E+03$ & $9.9 E+02$ \\
\hline AM & 95 & 242 & $1.3 E+09$ & 1. $9 E+08$ \\
\hline$A M$ & 95 & $242 M$ & 6. $7 E+03$ & $9.6 E+02$ \\
\hline AM & 95 & 243 & $6.1 E+03$ & $8.7 E+02$ \\
\hline
\end{tabular}


TABLE 6A. UNIT-RAD DEPJSITION FOR GI TRACT

\begin{tabular}{|c|c|c|c|c|}
\hline $\begin{array}{l}\text { ELEMENT } \\
\text { SYMBOL }\end{array}$ & $\begin{array}{l}\text { ATOMIC } \\
\text { NO. }(Z)\end{array}$ & $\begin{array}{l}\text { ISOTOPE } \\
\text { MASS(A) }\end{array}$ & $\begin{array}{c}\text { ADULT } \\
\text { UC/M**2 }\end{array}$ & $\begin{array}{l}\text { CHILD } \\
\text { UC } / M * *\end{array}$ \\
\hline $\begin{array}{l}H \\
B E \\
B E \\
C \\
N A \\
N A \\
M G \\
A L \\
S I \\
P \\
P \\
S \\
C L \\
A R \\
A R \\
A R \\
K \\
K \\
K \\
C A \\
C A \\
C A \\
C A \\
S C \\
S C \\
S C \\
S C \\
T I \\
V \\
V \\
V \\
C R \\
C R \\
M N \\
M N \\
M N \\
F E \\
F E \\
F E \\
C O \\
C D \\
C D \\
C O \\
C O \\
N I \\
N I \\
N I \\
N I \\
N I \\
C U\end{array}$ & $\begin{array}{l}1 \\
4 \\
4 \\
6 \\
11 \\
11 \\
12 \\
13 \\
14 \\
15 \\
15 \\
16 \\
17 \\
18 \\
18 \\
18 \\
19 \\
19 \\
19 \\
20 \\
20 \\
20 \\
20 \\
21 \\
21 \\
21 \\
21 \\
22 \\
23 \\
23 \\
23 \\
24 \\
24 \\
25 \\
25 \\
25 \\
26 \\
26 \\
26 \\
27 \\
27 \\
27 \\
27 \\
27 \\
28 \\
28 \\
28 \\
28 \\
28 \\
29\end{array}$ & $\begin{array}{l}3 \\
7 \\
10 \\
14 \\
22 \\
24 \\
28 \\
26 \\
32 \\
32 \\
33 \\
35 \\
36 \\
37 \\
39 \\
42 \\
40 \\
42 \\
43 \\
41 \\
45 \\
47 \\
48 \\
44 M \\
46 \\
47 \\
48 \\
44 \\
48 \\
49 \\
50 \\
48 \\
51 \\
52 \\
53 \\
54 \\
55 \\
59 \\
60 \\
55 \\
56 \\
57 \\
58 \\
60 \\
56 \\
57 \\
59 \\
63 \\
66 \\
64\end{array}$ & $\begin{array}{l}7.9 E+02 \\
2.6 E+03 \\
3.4 E+02 \\
1.1 E+02 \\
8.5 E-01 \\
3.2 E+01 \\
9.0 E+00 \\
7.0 E+00 \\
6.0 E+02 \\
3.2 E+00 \\
2.8 E+01 \\
1.1 E+02 \\
6.0 E+00 \\
2.6 E+03 \\
1.9 E+01 \\
1.6 E+00 \\
1.2 E+01 \\
3.9 E+02 \\
2.9 E+02 \\
4.3 E+02 \\
1.9 E+01 \\
4.3 E+00 \\
4.2 E-01 \\
7.4 E+03 \\
1.1 E+03 \\
4.6 E+04 \\
8.6 E+03 \\
7.0 E+02 \\
6.7 E+00 \\
2.1 E+03 \\
7.9 E+00 \\
5.3 E+01 \\
2.1 E+02 \\
5.4 E+01 \\
7.4 E+03 \\
5.5 E+01 \\
1.4 E+03 \\
9.4 E+00 \\
3.4 E+00 \\
3.4 E+02 \\
8.2 E+00 \\
1.0 E+02 \\
1.7 E+01 \\
5.5 E+00 \\
2.3 E+00 \\
2.2 E+01 \\
2.7 E+02 \\
1.2 E+02 \\
1.4 E+01 \\
2.9 E+02\end{array}$ & $\begin{array}{l}1.1 E+02 \\
3.7 E+02 \\
4.9 E+01 \\
1.6 E+01 \\
1.2 E-01 \\
4.5 E+00 \\
1.3 E+D J \\
1.0 E+0 J \\
8.5 E+01 \\
4.6 E-01 \\
3.9 E+00 \\
1.6 E+01 \\
8.5 E-01 \\
3.7 E+02 \\
2.8 E+0 J \\
2.3 E-01 \\
1.7 E+00 \\
5.6 E+01 \\
4.1 E+01 \\
6.1 E+01 \\
2.7 E+00 \\
6.1 E-01 \\
6.0 E-02 \\
1.1 E+03 \\
1.6 E+02 \\
6.6 E+03 \\
1.2 E+03 \\
1.0 E+02 \\
9.5 E-01 \\
3.0 E+02 \\
1.1 E+00 \\
7.6 E+00 \\
3.0 E+01 \\
7.7 E+00 \\
1.1 E+03 \\
7.9 E+00 \\
2.0 E+02 \\
1.3 E+0 J \\
4.9 E-01 \\
4.9 E+01 \\
1.2 E+0 D \\
1.5 E+01 \\
2.4 E+00 \\
7.8 E-01 \\
3.2 E-01 \\
3.1 E+00 \\
3.9 E+01 \\
1.7 E+01 \\
1.9 E+00 \\
4.1 E+01\end{array}$ \\
\hline
\end{tabular}

VIA MILK.

$10 / 09 / 68$

PAGE 36
AOULT

3. $4 E+01$

$3.4 E+01$
$5.8 E-01$

2. $9 E+01$

9. $1 E-01$

7. $6 E+03$

5. $6 E+03$

1. $3 \mathrm{E}+0 \mathrm{I}$

1. $9 E+02$

2. $5 E+01$

2. $5 E+0$

1. $8 E+01$

1. $8 E+02$

2. $4 E+01$

1. $O E+O$

2. $2 \mathrm{E}+0 \mathrm{O}$

4. $2 E+00$

4. $7 E+01$

6. $3 E+01$

2. $2 E+01$

1. $5 E+01$

1. $6 E+02$

1. $3 E+02$

2. $0 E+02$

1. $7 E+0$

6. $8 E+00$

2. $6 E+00$

$6.0 E+00$

3. $8 E+01$

$3.4 E+00$

$3.4 E+00$
$1.5 E+02$

$1.5 E+02$
$1.7 E+01$

1. $2 E+01$

4. $5 E+00$

1. $9 E+04$

8. $4 E+03$

7. $0 E+04$

4. $1 E+02$

7. $1 E+03$

1. $5 E+03$

. $8 E+04$

7. $5 E+02$

8. $2 E+03$

9. $9 E+04$

1. $5 E+03$

4. $1 E+04$

6. $3 E+0$

2. $2 \mathrm{E}+02$

$2.2 E+02$

1. $1 E+01$

\begin{tabular}{|c|c|c|c|c|c|}
\hline $\begin{array}{l}\text { ELEMENT } \\
\text { SYMBOL }\end{array}$ & $\begin{array}{l}\text { ATOMIC } \\
\text { NO. (Z) }\end{array}$ & $\begin{array}{l}\text { I SOTOPE } \\
\text { MASSIA) }\end{array}$ & $\begin{array}{l}\text { AOULT } \\
\text { UC/M**2 }\end{array}$ & $\begin{array}{l}\text { CHILD } \\
U C / M * * 2\end{array}$ & \\
\hline $\begin{array}{l}C U \\
Z N \\
Z N \\
Z N \\
G A \\
G A \\
G E \\
G E \\
G E \\
A S \\
A S \\
A S \\
A S \\
A S \\
A S \\
S E \\
S E \\
S E \\
B R \\
B R \\
\text { SR } \\
K R \\
K R \\
K R \\
K R \\
R E \\
R E \\
R B \\
R B \\
S R \\
S R \\
S R \\
S R \\
S R \\
Y \\
Y \\
Y \\
Y \\
Y \\
Y \\
Z R \\
Z R \\
Z R \\
Z R \\
Z R \\
Z R \\
N E \\
N B \\
N B \\
N B\end{array}$ & $\begin{array}{l}29 \\
30 \\
30 \\
30 \\
31 \\
31 \\
32 \\
32 \\
32 \\
33 \\
33 \\
33 \\
33 \\
33 \\
33 \\
34 \\
34 \\
34 \\
35 \\
35 \\
35 \\
36 \\
36 \\
36 \\
36 \\
37 \\
37 \\
37 \\
37 \\
38 \\
38 \\
38 \\
38 \\
38 \\
39 \\
39 \\
39 \\
39 \\
30 \\
39 \\
40 \\
40 \\
40 \\
40 \\
40 \\
40 \\
41 \\
41 \\
41 \\
41\end{array}$ & 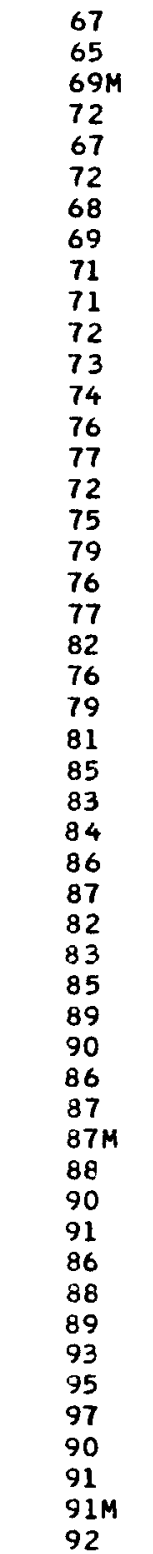 & 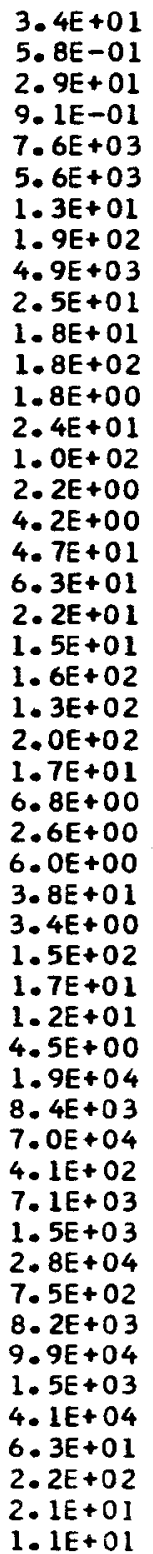 & 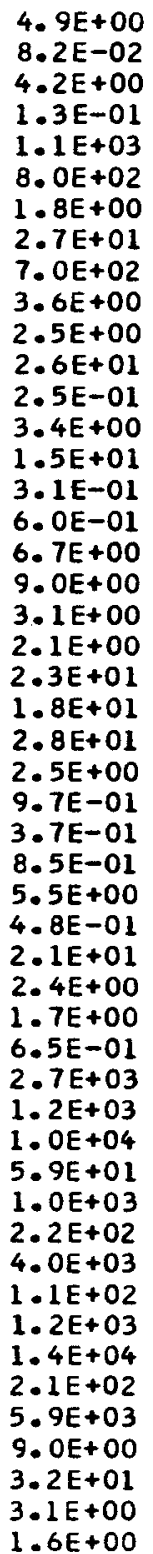 & 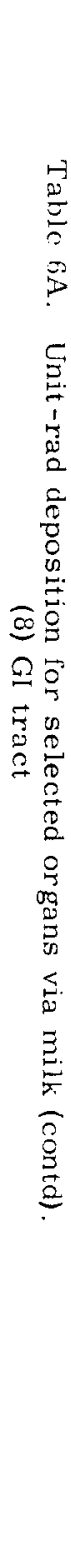 \\
\hline
\end{tabular}

CHILD
$U C / M * * 2$

4. $9 E+00$ 8. 2E-02

$4.2 E+00$
$1.3 E-01$

$1.3 E-01$
$1.1 E+03$

8. $0 E+02$

1. $8 \mathrm{E}+00$

2. $7 E+01$

7. $0 E+02$

3. $6 E+00$

. $5 E+00$

$2.6 E+01$

2. $5 E+01$

$3.4 E+00$

$1.5 E+01$

3. $1 E-01$

6. $0 E-01$

6. $7 E+00$

$0 E+00$

3.1E+00

$2.1 E+00$

$2.3 E+01$

$1.8 E+01$

2. $8 \mathrm{E}+01$

2. $5 \mathrm{E}+00$

.7E-O

$8.5 \mathrm{E}-0$

5. $5 E+00$

4. $8 E-01$

2. $8 E-01$

2. $4 E+00$

1. $7 E+00$

$6.5 E-01$

2. $7 E+03$

1. $2 E+03$

. $0 E+04$

5. $9 E+01$

1. $0 \mathrm{E}+03$

2. $2 \mathrm{E}+02$

$4.0 E+03$

$1.1 E+02$

1. $2 E+03$

1. $4 \mathrm{E}+04$

$2.1 E+02$

5. $9 E+03$

9. $O E+00$

3. $2 E+01$

3. $1 E+00$
$1.6 E+00$ 
TABLE 6A. UNIT-RAD DEPOSITION FOR GI TRACT

VIA MILK.

10/09/68 PAGE 37

\begin{tabular}{|c|c|c|c|c|}
\hline $\begin{array}{l}\text { ELEMENT } \\
\text { SYMBOL }\end{array}$ & $\begin{array}{l}\text { ATOMIC } \\
\text { NO. }(Z)\end{array}$ & $\begin{array}{l}\text { ISOTOPE } \\
\text { MASS (A) }\end{array}$ & $\begin{array}{l}\text { ADULT } \\
\text { UC / M**2 }\end{array}$ & $\begin{array}{l}\text { CHILD } \\
U C / M * * 2\end{array}$ \\
\hline $\begin{array}{l}N B \\
N B \\
N B \\
N B \\
N B \\
M O \\
M O \\
M C \\
T C \\
T C \\
T C \\
T C \\
T C \\
T C \\
I C \\
R U \\
R U \\
R U \\
R H \\
R H \\
R H \\
R H \\
R H \\
R H \\
R H \\
C H \\
P D \\
P D \\
P D \\
P D \\
P D \\
A G \\
A G \\
A G \\
A G \\
A G \\
A G \\
C D \\
C D \\
C D \\
C D \\
I N \\
I N \\
I N \\
S N \\
S N \\
S N \\
S N \\
S N \\
S N \\
S N \\
S N \\
S B \\
S B\end{array}$ & $\begin{array}{l}41 \\
41 \\
41 \\
41 \\
41 \\
42 \\
42 \\
43 \\
43 \\
43 \\
43 \\
43 \\
43 \\
43 \\
44 \\
44 \\
44 \\
45 \\
45 \\
45 \\
45 \\
45 \\
45 \\
46 \\
46 \\
46 \\
46 \\
46 \\
47 \\
47 \\
47 \\
47 \\
47 \\
48 \\
48 \\
48 \\
48 \\
49 \\
49 \\
49 \\
50 \\
50 \\
50 \\
50 \\
50 \\
50 \\
50 \\
50 \\
51 \\
51\end{array}$ & $\begin{array}{l}93 M \\
94 \\
95 \\
95 M \\
96 \\
93 \\
99 \\
95 \\
95 M \\
96 \\
97 \\
97 M \\
98 \\
99 \\
97 \\
103 \\
106 \\
99 \\
100 \\
101 \\
101 M \\
102 \\
105 \\
100 \\
103 \\
107 \\
109 \\
112 \\
105 \\
106 M \\
108 M \\
110 M \\
111 \\
109 \\
113 M \\
115 \\
115 M \\
111 \\
114 M \\
115 \\
113 \\
117 M \\
119 M \\
121 \\
121 M \\
123 M \\
125 \\
126 \\
119 \\
120 M\end{array}$ & $\begin{array}{l}1.4 E+02 \\
2.2 E+00 \\
7.2 E+00 \\
2.5 E+01 \\
5.8 E+01 \\
3.5 E+02 \\
7.1 E+01 \\
2.4 E+02 \\
4.7 E+00 \\
9.1 E+00 \\
2.0 E+02 \\
4.0 E+01 \\
2.7 E+00 \\
4.4 E+01 \\
2.1 E+05 \\
3.3 E+04 \\
5.9 E+03 \\
5.3 E+00 \\
3.7 E+01 \\
3.7 E+00 \\
2.0 E+01 \\
7.8 E-01 \\
1.1 E+02 \\
5.0 E+00 \\
3.6 E+01 \\
1.0 E+03 \\
2.1 E+02 \\
1.8 E+01 \\
2.5 E+00 \\
2.4 E+00 \\
7.8 E-01 \\
7.5 E-01 \\
1.5 E+01 \\
7.3 E+02 \\
3.8 E+02 \\
7.3 E+02 \\
1.0 E+02 \\
2.0 E+03 \\
1.0 E+02 \\
4.5 E+02 \\
1.1 E+01 \\
2.7 E+01 \\
4.7 E+01 \\
1.0 E+03 \\
2.9 E+01 \\
6.4 E+00 \\
6.0 E+00 \\
1.2 E+00 \\
2.4 E+03 \\
1.1 E+01\end{array}$ & $\begin{array}{l}2.0 E+01 \\
3.2 E-01 \\
1.0 E+00 \\
3.6 E+00 \\
8.2 E+00 \\
5.0 E+01 \\
1.0 E+01 \\
3.5 E+01 \\
6.7 E-01 \\
1.3 E+00 \\
2.8 E+01 \\
5.7 E+00 \\
3.8 E-01 \\
6.3 E+00 \\
2.9 E+04 \\
4.8 E+03 \\
8.5 E+02 \\
7.5 E-01 \\
5.3 E+00 \\
5.4 E-01 \\
2.9 E+00 \\
1.1 E-01 \\
1.5 E+01 \\
7.2 E-01 \\
5.1 E+00 \\
1.4 E+02 \\
3.1 E+01 \\
2.6 E+00 \\
3.5 E-01 \\
3.4 E-01 \\
1.1 E-01 \\
1.1 E-01 \\
2.1 E+00 \\
1.0 E+02 \\
5.4 E+01 \\
1.0 E+02 \\
1.5 E+01 \\
2.9 E+02 \\
1.5 E+01 \\
6.5 E+01 \\
1.5 E+00 \\
3.9 E+00 \\
6.8 E+00 \\
1.5 E+02 \\
4.1 E+00 \\
9.2 E-01 \\
8.6 E-01 \\
1.7 E-01 \\
3.4 E+02 \\
1.6 E+00\end{array}$ \\
\hline
\end{tabular}

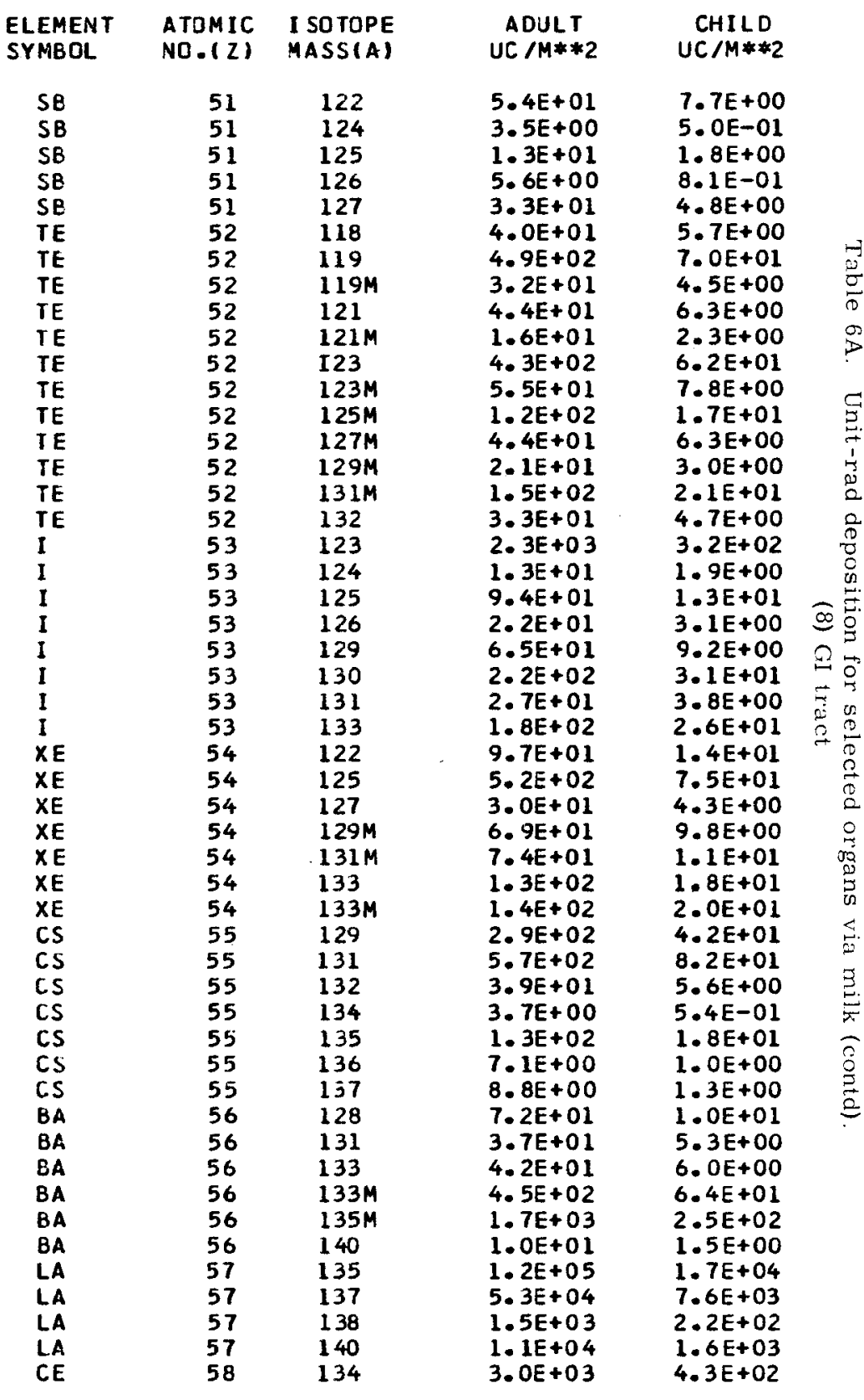


TABLE 6A. UNIT-RAD DEPOSITION FOR GI TRACT

$\begin{array}{llccc}\text { ELEMENT } & \text { ATOMIC } & \text { ISOTOPE } & \text { ADULT } & \text { CHILD } \\ \text { SYMBOL } & \text { NO.(Z) } & \text { MASS(A) } & \text { UC/M**2 } & U C / M * * 2\end{array}$

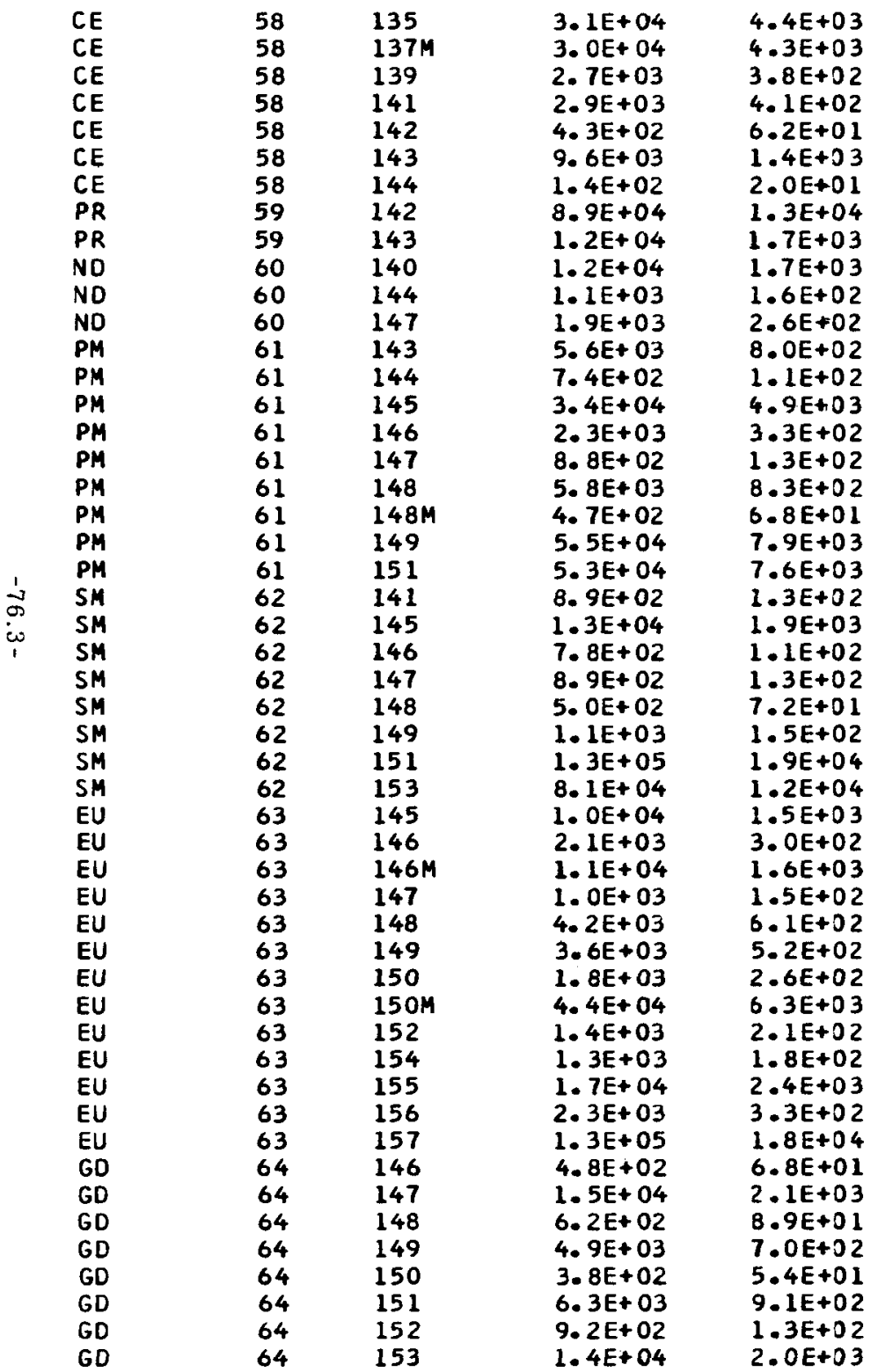

VIA MILK.

10/09/68 PAGE 38

SYMBOL ATOMIC ISOTOPE

GD

TB

TB

TE

T B

$T B$

TB

T B

TB

or

DY

DY

DY

HO

HO

\section{D. (2) MASSIA)}

$\begin{array}{ll}64 & 15 \\ 64 & 16 \\ 65 & 15 \\ 65 & 15 \\ 65 & 15 \\ 65 & 15 \\ 65 & 155 \\ 65 & 156 \\ 65 & 15 \\ 65 & 15 \\ 65 & 16 \\ 65 & 16 \\ 65 & 164\end{array}$

159
162
151
152
153
154
155
156
157

ADULT UC / M* *2

2. $9 E+05$ 1. $4 E+03$

1. $1 E+05$

3. $3 E+04$

4. $5 E+04$

2. $1 E+04$

6. $2 E+03$

4. $0 E+04$

1. $7 E+03$

1. $8 E+03$

3. $8 E+04$

1. $9 E+05$

2. $5 E+02$

6. $0 E+04$

3. $9 E+02$

3. $3 E+04$

1. $3 E+04$
3. $7 E+04$

3. $7 E+04$

6. $7 E+04$
$1.6 E+03$

1. $6 E+03$
2. $0 E+04$

6. $1 E+04$

2. $1 E+04$

2. $2 E+04$

$9.6 E+03$

$1.5 E+03$

$6.0 E+03$

1. $0 E+05$

2. $1 E+04$

4. $2 E+04$

8. $1 E+03$

6. $7 E+04$

2. $3 E+04$

9. $2 E+03$

4. $6 E+04$

7. $8 E+03$

2. $9 E+03$

1. $6 E+03$

4. $1 E+03$

2. $8 E+03$

4. $4 E+04$

1. $1 E+04$

5. $4 E+04$

l. $1 E+05$

1. $7 E+03$

4. $2 E+04$

4. $9 E+03$

4. $9 E+03$
$3.7 E+03$
$C H I L D$
$U C / M * * 2$

4. $1 E+04$ 2. $0 E+02$

1. $6 E+04$

4. $8 E+03$

$6.4 E+03$

1. $9 E+04$

3. $0 E+03$

8. $9 E+02$

$5.7 E+03$

$2.5 E+02$
$2.5 E+02$

$2.5 E+02$

$5.4 E+03$
$2.7 E+04$

2. $7 E+04$
$3.5 E+01$

$8.6 E+03$

5. $5 E+01$

4. $7 E+03$

1. $9 E+03$

$5.3 E+03$

$9.5 \mathrm{E}+03$

2. $3 E+02$

$2.8 E+03$

$8.7 E+03$

3. $0 E+03$

$3.1 \mathrm{E}+03$

$2.2 E+02$

$8.6 E+02$

8. $6 E+02$

1 . $5 E+04$

3. $0 E+03$
$6.0 E+03$

6. $\mathrm{OE}+03$

1. $2 \mathrm{E}+03$

$9.5 E+03$

$3.2 E+03$

$1.3 E+03$

$6.6 E+03$

$1.1 E+03$

$4.2 E+02$

2. $3 E+02$

$5.8 E+02$

3. $9 E+02$

$6.3 E+03$

$1.6 E+03$

7. $7 \mathrm{E}+03$

$1.6 E+04$

$2.5 E+02$

7. $0 E+02$ 


$\begin{array}{lcccc}\text { ELEMENT } & \text { ATOMIC } & \text { ISOTOPE } & \text { ADULT } & \text { CHILD } \\ \text { SYMBOL } & \text { NO.1Z1 } & \text { MASSIAI } & \text { UC/M**2 } & \text { UC/M**2 } \\ \text { HF } & 72 & 182 & 1.2 E+03 & 1.7 E+02 \\ \text { TA } & 73 & 177 & 4.1 E+00 & 5.9 E-01 \\ \text { IA } & 73 & 179 & 6.3 E+00 & 9.0 E-01 \\ \text { TA } & 73 & 182 & 3.0 E-01 & 4.3 E-02 \\ \text { TA } & 73 & 183 & 3.1 E+00 & 4.4 E-01 \\ \text { H } & 74 & 178 & 1.0 E+02 & 1.4 E+01 \\ \text { W } & 74 & 181 & 1.6 E+03 & 2.3 E+02 \\ \text { W } & 74 & 185 & 9.9 E+02 & 1.4 E+02 \\ \text { W } & 74 & 187 & 4.4 E+03 & 6.4 E+02 \\ \text { W } & 74 & 188 & 9.1 E+01 & 1.3 E+01 \\ \text { RE } & 75 & 180 & 2.1 E+01 & 3.1 E+00 \\ \text { RE } & 75 & 181 & 2.4 E+01 & 3.4 E+0 J \\ \text { RE } & 75 & 182 & 5.1 E+00 & 7.3 E-01 \\ \text { RE } & 75 & 182 M & 5.3 E+01 & 7.6 E+00 \\ \text { RE } & 75 & 183 & 2.9 E+00 & 4.2 E-01 \\ \text { RE } & 75 & 184 & 1.2 E+00 & 1.7 E-01 \\ \text { RE } & 75 & 184 M & 7.1 E-01 & 1.0 E-01 \\ \text { RE } & 75 & 186 & 1.2 E+01 & 1.7 E+00 \\ \text { RE } & 75 & 187 & 6.2 E+03 & 8.8 E+02 \\ \text { RE } & 75 & 188 & 4.8 E+01 & 6.9 E+0 J \\ \text { RE } & 75 & 189 & 6.4 E+01 & 9.2 E+00 \\ \text { OS } & 76 & 182 & 4.5 E+01 & 6.5 E+00 \\ \text { OS } & 76 & 183 & 2.8 E+02 & 4.1 E+01 \\ \text { OS } & 76 & 185 & 3.4 E+00 & 4.8 E-01 \\ \text { OS } & 76 & 191 & 2.3 E+01 & 3.4 E+00 \\ \text { OS } & 76 & 191 M & 7.2 E+02 & 1.0 E+02 \\ \text { OS } & 76 & 193 & 1.1 E+02 & 1.6 E+01 \\ \text { OS } & 76 & 194 & 9.9 E-01 & 1.4 E-01 \\ \text { IR } & 77 & 185 & 1.6 E+02 & 2.3 E+01 \\ \text { IR } & 77 & 186 & 9.5 E+01 & 1.4 E+01 \\ \text { IR } & 77 & 187 & 2.4 E+02 & 3.4 E+01 \\ \text { IR } & 77 & 188 & 1.7 E+01 & 2.4 E+00 \\ \text { IR } & 77 & 189 & 1.4 E+01 & 2.1 E+00 \\ \text { IR } & 77 & 190 & 3.8 E+00 & 5.5 E-01 \\ \text { IR } & 77 & 192 & 2.6 E+00 & 3.7 E-01 \\ \text { IR } & 77 & 192 M & 1.8 E+00 & 2.6 E-01 \\ \text { IR } & 77 & 193 M & 6.7 E+01 & 9.6 E+00 \\ \text { IR } & 77 & 194 & 9.5 E+01 & 1.4 E+01 \\ \text { PI } & 78 & 188 & 2.2 E+00 & 3.1 E-01 \\ \text { PT } & 78 & 190 & 7.1 E-01 & 1.0 E-01 \\ \text { PT } & 78 & 191 & 3.5 E+01 & 5.0 E+00 \\ \text { PT } & 78 & 192 & 8.5 E-01 & 1.2 E-01 \\ \text { PT } & 78 & 193 & 2.9 E+01 & 4.2 E+0 J \\ \text { PT } & 78 & 193 M & 5.5 E+01 & 7.9 E+00 \\ \text { PT } & 78 & 195 M & 5.1 E+01 & 7.3 E+00 \\ \text { PT } & 78 & 197 & 3.7 E+02 & 5.3 E+01 \\ \text { AU } & 79 & 193 & 2.2 E+02 & 3.1 E+01 \\ \text { AU } & 79 & 194 & 2.0 E+01 & 2.8 E+00 \\ \text { AU } & 79 & 195 & 1.2 E+01 & 1.7 E+00 \\ \text { AU } & 79 & 196 & 1.2 E+01 & 1.6 E+00\end{array}$

\begin{tabular}{|c|c|c|}
\hline $\begin{array}{l}\text { ELEMENT } \\
\text { SYMBOL }\end{array}$ & $\begin{array}{l}\text { ATOMIC } \\
\text { NO. }(Z)\end{array}$ & $\begin{array}{l}\text { I SOTOPE } \\
\text { MASSIAI) }\end{array}$ \\
\hline$A U$ & 79 & 198 \\
\hline AU & 79 & 199 \\
\hline HG & 80 & 194 \\
\hline HG & 80 & $195 M$ \\
\hline HG & 80 & 197 \\
\hline$H G$ & 80 & $197 \mathrm{M}$ \\
\hline$H G$ & 80 & 203 \\
\hline TL & 81 & 200 \\
\hline IL & 81 & 201 \\
\hline TL & 81 & 202 \\
\hline$T L$ & 81 & 204 \\
\hline$P B$ & 82 & 200 \\
\hline PB & 82 & 202 \\
\hline PB & 82 & 203 \\
\hline PE & 82 & 204 \\
\hline PB & 82 & 205 \\
\hline PB & 82 & 210 \\
\hline$B 1$ & 83 & 205 \\
\hline e I & 83 & 206 \\
\hline BI & 83 & 207 \\
\hline 81 & 83 & 208 \\
\hline BI & 83 & 210 \\
\hline BI & 83 & $210 M$ \\
\hline PO & 84 & 206 \\
\hline PO & 84 & 208 \\
\hline PO & 84 & 209 \\
\hline$P C$ & 84 & 210 \\
\hline RN & 86 & 211 \\
\hline RN & 86 & 222 \\
\hline RA & $8 B$ & 223 \\
\hline RA & 88 & 224 \\
\hline RA & 88 & 225 \\
\hline KA & 88 & 226 \\
\hline RA & 88 & 228 \\
\hline$A C$ & 89 & 225 \\
\hline$A C$ & 89 & 227 \\
\hline TH & 90 & 227 \\
\hline TH & 90 & 228 \\
\hline$T H$ & 90 & 229 \\
\hline TH & 90 & 230 \\
\hline TH & 90 & 231 \\
\hline TH & 90 & 232 \\
\hline TH & 90 & 234 \\
\hline$P A$ & 91 & 228 \\
\hline PA & 91 & 229 \\
\hline PA & 91 & 230 \\
\hline PA & 91 & 231 \\
\hline PA & 91 & 232 \\
\hline PA & 91 & 233 \\
\hline U & 92 & 230 \\
\hline
\end{tabular}

ADULT
UC $/ M * * 2$

2. $8 E+01$

$7.6 E+01$

5. $4 E-01$

$1.1 E+01$

2. $9 E+01$

6. $2 \mathrm{E}+01$

4. $4 E+00$

1. $9 E+01$

2. $7 E+01$

3. $9 E+00$

2. $7 E+00$

4. $5 E+02$

2. $9 E+01$

2. $9 E+02$

6. $7 E+00$

2. $0 E+02$

3. $O E+0 O$

2. $1 E+01$

4. $4 E+01$

1. $1 E+01$

7. $3 E+00$

1. $7 \mathrm{E}+01$

3. $6 E+00$

$3.5 E+00$

4. $6 E-01$

7. $1 E-01$

7. $4 \mathrm{E}-01$

$3.7 E+01$

1. $2 E+00$

$1.2 E+00$

2. $2 E-01$

2. $2 E-01$

3. $0 E-02$

2. $7 E-02$

1. $9 E+02$

5. $9 E+01$

1. $1 E+02$

5. $9 E+01$

5. $9 E+01$

5. $9 E+01$

5. $6 E+01$

1. $7 E+03$

5. $O E+01$

7. $9 E+01$

2. $3 E+03$

1. $2 E+03$

$1.1 E+02$

$5.1 E+01$

1. $2 E+03$

9. $0 E-01$
CHILD

$U C / M * * 2$

4. $0 \mathrm{E}+00$

$1.1 E+01$

7. $7 E-02$

1. $6 E+00$

4. $1 E+00$

8. $9 E+00$

6. $2 E-01$

3. $8 E+00$

5. 5E-01

3. $8 E-01$

$6.4 E+01$

$4.2 E+00$

4. $1 E+01$

9. $6 E-01$

2. $9 E+01$

4. $3 E-01$

3. $1 E+00$

$6.3 E+00$

1. $5 \mathrm{E}+00$

$1.1 E+00$

2. $4 E+00$

5.1E-01

5. OE-01

1. OE-01

1. $1 E-01$

$5.3 E+00$

1. $8 E-01$

1 . 2E-02

$3.2 E-02$

1. OE-02

4. $4 E-03$

3. $8 E-03$

2. $8 E+01$

$8.4 E+00$

1. $6 E+01$

$8.4 E+00$

8. $5 E+00$

7. $9 E+00$

2. $5 E+02$

$7.1 E+00$

$1.1 E+01$

3. $3 E+02$

1. $8 E+02$

1. $5 E+01$

7. $3 E+00$

1. $7 E+02$

$1.2 E+01$
$1.3 E-01$ 
TABLE 6A. UNIT-RAD DEPOSITION FOR

GI TRACF

VIA MILK.

$10 / 09 / 68$

PAGE 40

\begin{tabular}{|c|c|c|c|c|}
\hline $\begin{array}{l}\text { ELEMENT } \\
\text { SYMBOL }\end{array}$ & $\begin{array}{l}\text { ATOMIC } \\
\text { NO. (Z) }\end{array}$ & $\begin{array}{l}\text { I SOTOPE } \\
\text { MASS(A) }\end{array}$ & $\begin{array}{l}\text { ADULT } \\
U C / M * 2\end{array}$ & $\begin{array}{l}\text { CHILD } \\
U C / M * * 2\end{array}$ \\
\hline $\begin{array}{l}U \\
U \\
U \\
U \\
U \\
U \\
U \\
U \\
U \\
N P \\
N P \\
N P \\
N P \\
N P \\
N P \\
N P \\
P U \\
P U \\
P U \\
P U \\
P U \\
P U \\
P U \\
P U \\
P U \\
A M \\
A M \\
A M \\
A M \\
A M \\
A M\end{array}$ & $\begin{array}{l}92 \\
92 \\
92 \\
92 \\
92 \\
92 \\
92 \\
92 \\
92 \\
93 \\
93 \\
93 \\
93 \\
93 \\
93 \\
93 \\
94 \\
94 \\
94 \\
94 \\
94 \\
94 \\
94 \\
94 \\
94 \\
95 \\
95 \\
95 \\
95 \\
95 \\
95\end{array}$ & $\begin{array}{l}231 \\
232 \\
233 \\
234 \\
235 \\
236 \\
237 \\
238 \\
240 \\
234 \\
235 \\
236 \\
236 M \\
237 \\
238 \\
239 \\
236 \\
237 \\
238 \\
239 \\
240 \\
241 \\
242 \\
244 \\
246 \\
239 \\
240 \\
241 \\
242 \\
242 M \\
243\end{array}$ & $\begin{array}{l}\text { 3. } 0 E+00 \\
5.0 E-01 \\
5.2 E-01 \\
4.9 E-01 \\
4.5 E-01 \\
4.5 E-01 \\
1.7 E+00 \\
4.3 E-01 \\
3.4 E+01 \\
2.6 E+02 \\
4.7 E+01 \\
4.3 E+01 \\
1.9 E+03 \\
4.6 E+01 \\
5.6 E+02 \\
4 \cdot 6 E+02 \\
1.5 E+02 \\
2.0 E+02 \\
1.4 E+02 \\
1.4 E+02 \\
1.3 E+02 \\
1.4 E+02 \\
1.3 E+02 \\
1.2 E+02 \\
3.0 E+02 \\
4.7 E+03 \\
5.0 E+02 \\
4.1 E+01 \\
2.7 E+03 \\
3.3 E+01 \\
3.7 E+0 .\end{array}$ & $\begin{array}{l}4.3 \mathrm{E}-01 \\
7.1 \mathrm{E}-02 \\
7.4 \mathrm{E}-02 \\
7.0 \mathrm{E}-02 \\
6.5 \mathrm{E}-02 \\
6.4 \mathrm{E}-02 \\
2.4 \mathrm{E}-01 \\
6.1 \mathrm{E}-02 \\
4.9 \mathrm{E}+00 \\
3.8 \mathrm{E}+01 \\
6.7 \mathrm{E}+00 \\
6.2 \mathrm{E}+00 \\
2.7 \mathrm{E}+02 \\
6.5 \mathrm{E}+00 \\
8.0 \mathrm{E}+01 \\
6.6 \mathrm{E}+01 \\
2.1 \mathrm{E}+01 \\
2.9 \mathrm{E}+01 \\
2.1 \mathrm{E}+01 \\
1.9 \mathrm{E}+01 \\
1.9 \mathrm{E}+01 \\
1.9 \mathrm{E}+01 \\
1.9 \mathrm{E}+01 \\
1.7 \mathrm{E}+01 \\
4.2 \mathrm{E}+01 \\
6.7 \mathrm{E}+02 \\
7.2 \mathrm{E}+01 \\
5.8 \mathrm{E}+00 \\
3.9 \mathrm{E}+02 \\
5.4 \mathrm{E}+00 \\
5.2 \mathrm{E}+00\end{array}$ \\
\hline
\end{tabular}

\begin{tabular}{lcc} 
ELEMENT & ATOMIC & I SOIOPE \\
SYMBOL & ND.IZI & MASS(A) \\
CM & 96 & 240 \\
CM & 96 & 241 \\
CM & 96 & 242 \\
CM & 96 & 243 \\
CM & 96 & 244 \\
CM & 96 & 245 \\
CM & 96 & 246 \\
CM & 96 & 247 \\
CM & 96 & 248 \\
EK & 97 & 245 \\
UK & 97 & 246 \\
EK & 97 & 247 \\
EK & 97 & 248 \\
BK & 97 & 249 \\
CF & 98 & 246 \\
CF & 98 & 248 \\
CF & 98 & 249 \\
CF & 98 & 250 \\
CF & 98 & 251 \\
CF & 98 & 252 \\
CF & 98 & 253 \\
ES & 99 & 251 \\
ES & 99 & 252 \\
ES & 99 & 253 \\
ES & 99 & 254 \\
ES & 99 & $254 M$ \\
ES & 99 & 255 \\
FM & 100 & 252 \\
FM & 100 & 253 \\
FM & 100 & 255 \\
FM & 100 & 257 \\
\hline & &
\end{tabular}

ADULT
$U C, M * * 2$

$\underset{U C / M * * 2}{C H I L D}$

$6 \cdot 2 E+01$

$5 \cdot 8 \mathrm{E}+01$

4. $2 E+01$

3. $6 E+01$

3. $6 E+01$

3. $7 E+01$

3. $5 E+01$

3. $2 E+01$

6. $0 E+02$

1. $8 E+03$

1. $1 \mathrm{E}+02$

4. $3 E+03$

1. $1 E+02$

2. $4 E+03$

$1.1 E+02$

1. $1 E+02$

1. $O E+02$

$9.8 E+01$

1. $8 E+02$

2. $1 E+03$

1. $1 E+02$

1. $8 E+02$

9. $7 E+01$

1. $7 E+03$

1. $5 E+02$

$5.4 E+02$

$4.5 E+03$

4. $5 E+03$
1. $1 E+02$

8. $8 E+00$

$8.3 E+00$

6. $O E+00$

$5.2 E+00$
$5.1 E+00$

$5.2 E+00$

5. $O E+00$

4. $7 E+00$

4. $6 E+00$

A. $6 E+01$

. $6 E+01$

$1.6 E+01$

$6.1 E+02$

$1.6 \mathrm{E}+01$

3. $4 E+02$

1. $6 E+01$

$1.6 E+01$

1. $5 E+01$

1. $4 E+01$

$1.4 E+01$

$2.6 E+01$

$2.9 E+02$

1. $5 E+01$

2. $6 E+01$

$2.5 E+02$

2. $2 E+01$

$5.5 E+02$

7. $7 E+01$

$6.4 E+02$

6. $5 E+02$
$1.5 E+01$ 
-

•

- 
6. $\mathrm{F}_{1}:$ UNIT-RAD DEPOSITION
(Continued)

\section{Soil-Root Pathway}

$\mathrm{F}_{1}=\frac{3.71 \times 10^{-1} \rho \mathrm{d}}{\mathrm{Q}}\left(\frac{\mathrm{C}_{\mathrm{S}}}{\mathrm{C}_{\mathrm{B}}}\right)$

$\times \frac{1}{\left[T_{R}\left(1-e^{-20.8 / T_{R}}\right)-T_{E}\left(1-e^{-20.8 / T_{E}}\right)\right]}$

The expression above yields $\mathrm{F}_{1}$ via soil when $\rho$ is expressed in $\mathrm{g} / \mathrm{cm}^{3}, d$ is expressed in $\mathrm{cm}, \mathrm{Q}$ is expressed in $\mathrm{MeV}$, and $T_{R}$ and $T_{E}$ are expressed in yr. The $\rho$ was assumed to be $2 \mathrm{~g} / \mathrm{cm}^{3}$, and d was assumed to be $20 \mathrm{~cm} . F_{1}$ values are listed for the adult and the infant.

In calculating the $F_{1}$ via soil for infants, the $\mathrm{T}_{\mathrm{E}}$ for all radionuclides was set equal to zero. Since the newborn experiences rapid growth, he will come into equilibrium with his diet and environment (soil) much more rapidly than an adult. Setting $\mathrm{T}_{E}=0$ simply accounts for this rapid equilibration. 


\begin{tabular}{|c|c|c|c|c|}
\hline $\begin{array}{l}\text { ELEMENT } \\
\text { SYMBOL }\end{array}$ & $\begin{array}{l}\text { A TOMIC } \\
\text { ND. (Z) }\end{array}$ & $\begin{array}{l}\text { I SOTOPE } \\
\text { MASSIA) }\end{array}$ & $\begin{array}{l}\text { ADULT } \\
U C / M * 2\end{array}$ & $\begin{array}{l}\text { INFANT } \\
\text { UC } / M * * 2\end{array}$ \\
\hline $\mathrm{H}$ & 1 & 3 & $4.7 E+01$ & $4.7 E+01$ \\
\hline$B E$ & 4 & 7 & 1. $9 E+02$ & $2.0 E+01$ \\
\hline $\mathrm{BE}$ & 4 & 10 & $2.6 E-02$ & $2.5 E-02$ \\
\hline $\mathrm{C}$ & 6 & 14 & $1.8 E+00$ & $1.8 E+00$ \\
\hline NA & 11 & 22 & 1. $5 E+00$ & 1. $4 \mathrm{E}+00$ \\
\hline AL & 13 & 26 & $6.2 E+02$ & $6.2 E+02$ \\
\hline SI & 14 & 32 & $6.5 E+02$ & $6.5 E+02$ \\
\hline 5 & 16 & 35 & $3.0 E+03$ & $3.7 E+02$ \\
\hline $\mathrm{CL}$ & 17 & 36 & $1.3 E-01$ & $1.3 E-01$ \\
\hline$A R$ & 18 & 37 & $5.5 E+02$ & $5.5 E+02$ \\
\hline$A R$ & 18 & 39 & 2. $9 \mathrm{E}-02$ & $2.9 E-02$ \\
\hline$A R$ & 18 & 42 & 3.1E-03 & $3.1 \mathrm{E}-03$ \\
\hline $\mathrm{K}$ & 19 & 40 & $1.9 E+00$ & $1.9 E+00$ \\
\hline CA & 20 & 41 & $1.6 E+02$ & 2. $9 E+01$ \\
\hline$C A$ & 20 & 45 & $5.8 E+03$ & $5.3 E+01$ \\
\hline$C A$ & 20 & 48 & $1.5 \mathrm{E}-01$ & $2.8 \mathrm{E}-02$ \\
\hline SC & 21 & 46 & $8.4 E+04$ & $4.2 E+03$ \\
\hline T I & 22 & 44 & $1.4 E+03$ & $1.4 E+03$ \\
\hline$v$ & 23 & 49 & $3.1 E+05$ & $2.2 E+05$ \\
\hline$v$ & 23 & 50 & 3. $9 E+01$ & $3.8 E+01$ \\
\hline$M N$ & 25 & 53 & 4. $4 E+04$ & 4. $2 E+04$ \\
\hline$M N$ & 25 & 54 & $1.6 E+04$ & $7.5 E+03$ \\
\hline FE & 26 & 55 & $1.4 E+05$ & $5.3 E+04$ \\
\hline FE & 26 & 59 & $2.2 E+05$ & $5.8 E+03$ \\
\hline FE & 26 & 60 & 2. $1 E+01$ & $1.7 E+01$ \\
\hline $\mathrm{CO}$ & 27 & 56 & 7. $0 E+01$ & $5.4 E+01$ \\
\hline CO & 27 & 57 & $2.5 E+02$ & $2.3 E+02$ \\
\hline CO & 27 & 58 & $1.6 E+02$ & $1.2 E+02$ \\
\hline CO & 27 & 60 & 1. $8 E+00$ & 1. $8 \mathrm{E}+00$ \\
\hline NI & 28 & 59 & $2.5 E+02$ & $2.2 \mathrm{E}+02$ \\
\hline $\mathrm{NI}$ & 28 & 63 & 1. $2 E+02$ & $1.1 E+02$ \\
\hline$Z N$ & 30 & 65 & $1.2 E+02$ & $2.0 E+01$ \\
\hline $\mathrm{GE}$ & 32 & 68 & $1.2 E-01$ & 1. $2 E-01$ \\
\hline AS & 33 & 73 & $3.2 E+06$ & $3.2 E+06$ \\
\hline SE & 34 & 75 & $7.6 E-01$ & $7.6 \mathrm{E}-01$ \\
\hline SE & 34 & 79 & $1.5 \mathrm{E}-01$ & $1.5 \mathrm{E}-01$ \\
\hline$K R$ & 36 & 81 & 2. $9 E-01$ & $2.9 \mathrm{E}-01$ \\
\hline$K R$ & 36 & 85 & $5.6 E-02$ & $5.6 E-02$ \\
\hline RB & 37 & 83 & $2.2 E+03$ & $1.1 \mathrm{E}+03$ \\
\hline RB & 37 & 84 & 3. $0 E+03$ & $8.7 E+02$ \\
\hline RB & 37 & 87 & 8. $3 E+01$ & $8 \cdot 3 E+01$ \\
\hline$S R$ & 38 & 85 & $1.6 E+05$ & $5.6 E+02$ \\
\hline$S R$ & 38 & 89 & 1. $7 E+05$ & $4.9 E+02$ \\
\hline$S R$ & 38 & 90 & $1.4 E+01$ & $2.2 E+00$ \\
\hline$Y$ & 39 & 88 & 3. $1 E+04$ & $1.8 E+02$ \\
\hline$Y$ & 39 & 91 & $3.4 E+05$ & $1.1 E+03$ \\
\hline ZR & 40 & 88 & $7.7 E+04$ & $6.1 E+03$ \\
\hline $2 R$ & 40 & 93 & $1.2 E+04$ & L. $1 E+04$ \\
\hline ZR & 40 & 95 & 2. $4 E+05$ & $1.5 E+04$ \\
\hline NB & 41 & 91 & $4.6 E-01$ & $4.0 E-01$ \\
\hline
\end{tabular}

ELEMENT
SYMBO

NB
NB
NB
NB
$M O$
$T C$
$T C$
$T C$
$T C$
$T C$
$T C$
$R U$
$R U$
$R H$
$R H$
$R H$
$P D$
$A G$
$A G$
$A G$
$C D$
$C D$
$C D$
$I N$
$I N$
$S N$
$S N$
$S N$
$S N$
$S N$
$S N$
$S B$
$S B$
$T E$
$T E$
$T E$
$T E$
$T E$
$T E$
$I$
$I$
$X E$
$C S$
$C S$
$C S$
$B A$
$L A$
$L A$
$C E$
$C E$
$C E$
$C E$
$N D$

\section{ATOMIC ISOTOPE \\ NO. (Z) MASSIA)}

$\begin{array}{ll}41 & 91 M \\ 41 & 93 M \\ 41 & 94 \\ 41 & 95 \\ 42 & 93 \\ 43 & 95 M \\ 43 & 57 \\ 43 & 97 M \\ 43 & 98 \\ 43 & 99 \\ 44 & 1 C 3 \\ 44 & 1 C 6 \\ 45 & 1 C 1 \\ 45 & 1 C 2 \\ 46 & 1 C 7 \\ 47 & 105 \\ 47 & 108 M \\ 47 & 110 M \\ 48 & 1 C 9 \\ 48 & 113 M \\ 48 & 115 M \\ 49 & 114 M \\ 49 & 115 \\ 50 & 113 \\ 50 & 119 M \\ 50 & 121 M \\ 50 & 123 M \\ 50 & 126 \\ 51 & 124 \\ 51 & 125 \\ 52 & 121 M \\ 52 & 123 \\ 52 & 123 M \\ 52 & 125 M \\ 52 & 127 M \\ 52 & 129 M \\ 53 & 125 \\ 53 & 129 \\ 54 & 127 \\ 55 & 134 \\ 55 & 135 \\ 55 & 137 \\ 56 & 133 \\ 57 & 137 \\ 57 & 138 \\ 58 & 139 \\ 58 & 141 \\ 58 & 142 \\ 58 & 144 \\ 60 & 144 \\ & \\ 53\end{array}$

ADULT
UC /M**2

$6.4 E+01$

$2.4 E+00$

4. $6 E-03$

5. $7 E+01$

$1.6 E+02$

1. $2 E+00$

3. $6 E-01$

4. $8 \mathrm{E}-03$

7. $9 \mathrm{E}-02$

5. $6 \mathrm{E}+02$

2. $7 E+00$

9. $1 E-02$

1. $7 E-01$

5. $8 \mathrm{E}+00$

8. $7 E+02$

1. $3 E+00$

1. $5 \mathrm{E}+01$

1. $3 E+02$

6. $7 E+00$

4. $5 E+02$

1. $8 \mathrm{E}+00$

3. 3E-02

$1.6 E+03$

1. $9 E+03$

1. $8 \mathrm{E}+01$

8. $7 E+02$

5. $0 E-01$

1. $1 E+03$

1. $3 E+02$

9. $7 E+00$

4. $7 E+00$

4. $8 \mathrm{E}+01$

2. $0 E+02$

3. $8 \mathrm{E}+01$

6. $7 E+01$

2. $8 E+04$

1. $6 E+02$

$6 \cdot 3 E+00$

3. $5 E+00$

1. $1 E+01$

1. $O E+O O$

1. $8 E+03$

2. $2 E-01$

$6.4 E-03$

2. $3 E+05$

3. $2 E+06$

$8.2 E+01$

3. $4 E+03$
INFANT

UC/M**2

$3.7 E+00$

$1.4 E+03$

4. $0 E-03$

1. $9 E+00$

1. $6 \mathrm{E}+02$

8. $4 \mathrm{E}-01$

3. $6 E-01$

$5.1 E+00$

4. $8 \mathrm{E}-03$

7. $9 E-02$

4. $5 E+01$

1. $2 E+0 U$

$9.0 E-02$

1. $5 E-01$

5. $8 \mathrm{E}+00$

1. $2 E+02$

1. $2 \mathrm{E}+00$

7. $4 E+00$

1. $0 E+02$

$6.5 \mathrm{E}+00$

1. $2 E+02$
8. $5 E-01$

8. $5 E-01$
3. $3 E-02$

$2.3 E+02$

$5.1 E+02$

1. $6 E+01$

1. $3 E+02$

$4.5 E-01$

4. $5 E-01$

$4.3 E+02$
$1.2 E+02$

8. $1 E+00$

4. $7 E+00$

3. $7 E+O I$

1. $3 E+02$

3. $0 E+01$

2. $2 E+04$

1. $5 \mathrm{E}+02$

$6 \cdot 3 E+00$

3. $1 E+00$

1. $1 E+O I$

1. $0 E+0 J$

1. $9 E-01$

$5.5 E-03$

$5.5 E-03$
$2.0 E+04$

2. $0 E+04$

6. $8 \mathrm{E}+04$

$6.6 \mathrm{E}+01$

$5.4 E+02$
$4.0 E-03$ 


$\begin{array}{ccccc}\text { ELEMENT } & \text { ATOMIC } & \text { I SOTOPE } & \text { ADULT } & \text { INFANT } \\ \text { SYMBOL } & \text { NO. (Z) } & \text { MASS(A) } & \text { UC/M**2 } & \text { UC/M**2 } \\ \text { PM } & & & & \\ \text { PM } & 61 & 143 & 7.2 E+04 & 1.1 E+04 \\ \text { PM } & 61 & 144 & 5.4 E+03 & 1.1 E+03 \\ \text { PM } & 61 & 145 & 5.7 E+03 & 4.2 E+03 \\ \text { PM } & 61 & 146 & 1.5 E+03 & 7.8 E+02 \\ \text { PM } & 61 & 147 & 1.2 E+03 & 4.8 E+02 \\ \text { SM } & 61 & 148 M & 1.7 E+05 & 4.6 E+03 \\ \text { SM } & 62 & 145 & 6.9 E+04 & 1.3 E+04 \\ \text { SM } & 62 & 146 & 4.5 E+01 & 3.6 E+01 \\ \text { SM } & 62 & 147 & 5.1 E+01 & 4.1 E+01 \\ \text { SM } & 62 & 148 & 2.9 E+01 & 2.3 E+01 \\ \text { SM } & 62 & 149 & 6.2 E+01 & 5.0 E+01 \\ \text { EU } & 62 & 151 & 8.5 E+03 & 6.7 E+03 \\ \text { EU } & 63 & 148 & 9.5 E+05 & 3.3 E+04 \\ \text { EU } & 63 & 149 & 2.5 E+05 & 1.6 E+04 \\ \text { EU } & 63 & 150 & 1.0 E+03 & 5.4 E+02 \\ \text { EU } & 63 & 152 & 3.1 E+02 & 2.1 E+02 \\ \text { EU } & 63 & 154 & 2.3 E+02 & 1.6 E+02 \\ \text { GD } & 63 & 155 & 4.5 E+04 & 1.4 E+04 \\ \text { GD } & 64 & 146 & 3.7 E+04 & 1.7 E+03 \\ \text { GD } & 64 & 148 & 2.5 E+01 & 2.1 E+01 \\ \text { GD } & 64 & 150 & 1.3 E+01 & 1.1 E+01 \\ \text { GD } & 64 & 151 & 1.0 E+05 & 1.1 E+04 \\ \text { GD } & 64 & 152 & 3.2 E+01 & 2.8 E+01 \\ \text { GD } & 64 & 153 & 6.5 E+04 & 1.2 E+04 \\ \text { TB } & 64 & 162 & 3.1 E+03 & 8.3 E+02 \\ \text { TB } & 65 & 157 & 2.5 E+03 & 2.1 E+03 \\ \text { TB } & 65 & 158 & 1.1 E+02 & 9.3 E+01 \\ \text { DY } & 65 & 160 & 1.1 E+05 & 7.7 E+03 \\ \text { DY } & 66 & 154 & 1.0 E-03 & 8.8 E-04 \\ \text { DY } & 66 & 156 & 1.6 E-03 & 1.4 E-03 \\ \text { HO } & 66 & 159 & 4.5 E+01 & 5.7 E+00 \\ \text { HO } & 67 & 163 & 1.5 E-01 & 1.3 E-01 \\ \text { TM } & 67 & 166 M & 6.8 E-03 & 5.9 E-03 \\ \text { TM } & 69 & 168 & 7.3 E+04 & 5.8 E+03 \\ \text { TM } & 69 & 170 & 1.5 E+05 & 1.6 E+04 \\ \text { YB } & 69 & 171 & 1.3 E+05 & 5.5 E+04 \\ \text { LU } & 70 & 169 & 2.4 E+06 & 7.4 E+04 \\ \text { LU } & 71 & 173 & 4.5 E-01 & 1.5 E-01 \\ \text { LU } & 71 & 174 & 5.9 E-02 & 3.3 E-02 \\ \text { LU } & 71 & 174 M & 4.3 E+00 & 6.1 E-01 \\ \text { LU } & 71 & 176 & 1.1 E-02 & 9.9 E-03 \\ \text { HF } & 71 & 177 M & 1.3 E+01 & 1.7 E+00 \\ \text { HF } & 72 & 172 & 4.2 E+01 & 3.2 E+01 \\ \text { HF } & 72 & 174 & 3.7 E+00 & 3.4 E+00 \\ \text { HF } & 72 & 175 & 1.8 E+04 & 1.9 E+03 \\ \text { HF } & 72 & 181 & 2.9 E+04 & 2.0 E+03 \\ \text { TA } & 73 & 182 & 5.7 E+00 & 5.2 E+00 \\ \text { TA } & 73 & 179 & 2.2 E+02 & 1.4 E+02 \\ \text { W } & 74 & 181 & 1.1 E+02 & 3.2 E+01 \\ & & & 6.4 E+00 & 6.0 E+00\end{array}$

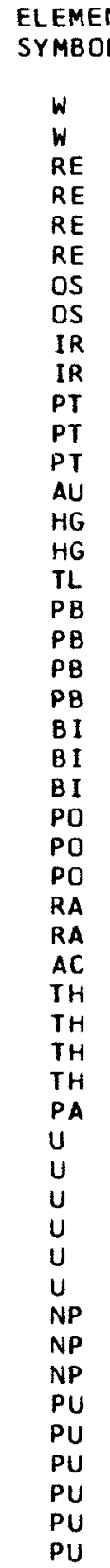

ATOMIC ISOTOPE
NO. (Z) MASS(A)

ADULT
UC $/ M * 2$

6. $7 E+00$ $6.6 \mathrm{E}-01$ 2. $5 E+00$ 1. $6 E+00$ 2. $6 \mathrm{E}-01$ $5.5 E+01$

7. $6 E-01$

1. $2 \mathrm{E}-02$

7. $\mathrm{OE}-01$

6. $0 E-03$

2. $3 E-03$

2. $7 E-03$

9. $6 E-02$

5. $7 E+00$

3. $5 E+00$

1. $3 E+01$

6. 2E-01

1. $4 \mathrm{E}-01$

$-3 E+00$

1. $1 E-01$

$5.3 E-03$
$2.6 E-03$

1. $3 E-03$

6. $8 \mathrm{E}-03$

1. $6 \mathrm{E}-03$

8. $7 E-02$

5. $5 E+00$

3. $4 E+01$

7. $8 \mathrm{E}-03$

2. $4 E-01$

4. $3 E-03$

4. $O E-03$

3. $6 E-03$

$3.6 E-03$

2. $2 E+01$

1. $9 E+01$

1. $8 \mathrm{E}+01$

1. $7 E+01$

1. $7 E+01$

1. $6 E+01$

5. $4 E-01$

3. $3 E-03$

8. $2 E-02$

$4.4 E+01$

3. $6 E-03$

3.

2. $9 E-03$

2. $9 \mathrm{E}-03$
7. $8 \mathrm{E}-03$

INFANT
UC /M**2

6. $0 E+00$ 5. $8 E-01$ 2. $3 E+00$ 1. $5 E+00$ 2. $6 E-01$ $5.5 E+01$

7. $6 E-01$

$1.2 E-02$

$7.0 E-01$

$6.0 E-03$

2.3E-03

2. $7 \mathrm{E}-03$

$9.6 E-02$

$5.7 E+00$

1. $7 E-01$

3. $5 E+00$

1. $3 E+01$

3. $6 \mathrm{E}-01$

$8.2 \mathrm{E}-02$

2. $5 E+00$

5. $7 E-02$

$5.3 E-03$

$2.6 E-03 \quad \square$

$1.3 E-03$ \%े

$6.6 \mathrm{E}-03$

$1.6 E-03$

$7.4 E-02$

1. $1 E+00$

3. $5 E+00$

$3.3 E-04$
$2.3 E-03$

2. $1 E-04$

2. $0 E-04$

1. $8 E-04$

1. $8 \mathrm{E}-04$

2. $1 E+01$

1. $9 E+01$

1. $8 E+01$

$1.6 E+01$

1. $6 \mathrm{E}+01$

1. $5 E+01$

3. $0 E-03$

1. $6 E-04$

$1.6 E-04$

1. $1 \mathrm{E}-03$

2. $7 E-02$

1.7E-04

1. $5 \mathrm{E}-04$

1. $4 E-04$
$2.9 E-04$ 
TABLE 6B. UNIT-RAD DEPOSITION FOR BONE

\begin{tabular}{|c|c|c|c|c|}
\hline $\begin{array}{l}\text { ELEMENT } \\
\text { SYMBOL }\end{array}$ & $\begin{array}{l}\text { ATOMIC } \\
\text { NO. }(2)\end{array}$ & $\begin{array}{l}\text { I SOTOPE } \\
\text { MASS }(A)\end{array}$ & $\begin{array}{l}\text { ADULT } \\
U C / M * * 2\end{array}$ & $\begin{array}{l}\text { INFANT } \\
\text { UC } / M * * 2\end{array}$ \\
\hline $\begin{array}{l}P U \\
P U \\
A M \\
A M \\
A M \\
C M \\
C M \\
C M \\
C M \\
C M \\
C M \\
C M\end{array}$ & $\begin{array}{l}94 \\
94 \\
95 \\
95 \\
95 \\
96 \\
96 \\
96 \\
96 \\
96 \\
96 \\
96\end{array}$ & $\begin{array}{l}242 \\
244 \\
241 \\
242 M \\
243 \\
241 \\
242 \\
243 \\
244 \\
245 \\
246 \\
247\end{array}$ & $\begin{array}{l}2.8 E-03 \\
2.5 E-03 \\
3.0 E-03 \\
3.0 E-03 \\
2.6 E-03 \\
6.5 E+01 \\
2.9 E+00 \\
3.9 E-03 \\
5.3 E-03 \\
2.6 E-03 \\
2.5 E-03 \\
2.3 E-03\end{array}$ & $\begin{array}{l}1.4 \mathrm{E}-04 \\
1.2 \mathrm{E}-04 \\
1.5 \mathrm{E}-04 \\
1.5 \mathrm{E}-04 \\
1.3 \mathrm{E}-04 \\
3.1 \mathrm{E}-02 \\
6.4 \mathrm{E}-03 \\
1.8 \mathrm{E}-04 \\
2.2 \mathrm{E}-04 \\
1.3 \mathrm{E}-04 \\
1.3 \mathrm{E}-04 \\
1.2 \mathrm{E}-04\end{array}$ \\
\hline
\end{tabular}

VIA TYPICAL AGRICULTURAL SOIL.

$\begin{array}{lrc}\text { ELEMENT } & \text { ATJMIC } & \text { ISOTOPE } \\ \text { SYMBOL } & \text { NO. (Z) } & \text { MASS(A) } \\ \text { CM } & 96 & 248 \\ \text { BK } & 97 & 247 \\ \text { BK } & 97 & 249 \\ \text { CF } & 98 & 248 \\ \text { CF } & 98 & 249 \\ \text { CF } & 98 & 250 \\ \text { CF } & 98 & 251 \\ \text { CF } & 98 & 252 \\ \text { ES } & 99 & 252 \\ \text { ES } & 99 & 254 \\ \text { FM } & 100 & 257\end{array}$


TABLE 6B. UNIT-RAD DEPOSITION FOR KIDNEYS

$\begin{array}{lrr}\text { ELEMENT } & \text { ATOMIC } & \text { ISOTOPE } \\ \text { SYMBOL } & \text { NO. (Z) } & \text { MASS }(A) \\ & & \\ \text { H } & 1 & 3 \\ \text { BE } & 4 & 7 \\ \text { BE } & 4 & 10 \\ \text { C } & 6 & 14 \\ \text { NA } & 11 & 22 \\ \text { AL } & 13 & 26 \\ \text { SI } & 14 & 32 \\ \text { S } & 16 & 35 \\ \text { CL } & 17 & 36 \\ \text { AR } & 18 & 37 \\ \text { AR } & 18 & 39 \\ \text { AR } & 18 & 42 \\ \text { K } & 19 & 40 \\ \text { CA } & 20 & 41 \\ \text { CA } & 20 & 45 \\ \text { CA } & 20 & 48 \\ \text { SC } & 21 & 46 \\ \text { TI } & 22 & 44 \\ \text { V } & 23 & 49 \\ \text { V } & 23 & 50 \\ \text { MN } & 25 & 53 \\ \text { MN } & 25 & 54 \\ \text { FE } & 26 & 55 \\ \text { FE } & 26 & 59 \\ \text { FE } & 26 & 60 \\ \text { CO } & 27 & 56 \\ \text { CO } & 27 & 57 \\ \text { CO } & 27 & 58 \\ \text { CO } & 27 & 60 \\ \text { NI } & 28 & 59 \\ \text { NI } & 28 & 63 \\ \text { ZN } & 30 & 65 \\ \text { GE } & 32 & 68 \\ \text { AS } & 33 & 73 \\ \text { SE } & 34 & 75 \\ \text { SE } & 34 & 79 \\ \text { KR } & 36 & 81 \\ \text { KR } & 36 & 85 \\ \text { RB } & 37 & 83 \\ \text { RB } & 37 & 84 \\ \text { RB } & 37 & 87 \\ \text { SR } & 38 & 85 \\ \text { SR } & 38 & 89 \\ \text { SR } & 38 & 90 \\ \text { Y } & 39 & 88 \\ \text { Y } & 39 & 91 \\ \text { ZR } & 40 & 88 \\ \text { ZR } & 40 & 93 \\ \text { ZR } & 40 & 95 \\ \text { NB } & 41 & 91\end{array}$

VIA TYPICAL AGRICULTURAL SOIL.

TYPICAL AGRICULTURAL SOIL.

$05 / 09 / 58$

PAGE \&

\begin{tabular}{|c|c|c|}
\hline $\begin{array}{l}\text { ELEMENT } \\
\text { SYMBOL }\end{array}$ & $\begin{array}{l}\text { ATOMIC } \\
\text { NO. }(2)\end{array}$ & $\begin{array}{l}\text { I SOTOPE } \\
\text { MASSIA) }\end{array}$ \\
\hline NB & 41 & $91 \mathrm{M}$ \\
\hline NB & 41 & S3M \\
\hline NB & 41 & $\$ 4$ \\
\hline NB & 41 & 95 \\
\hline MO & 42 & 93 \\
\hline TC & 43 & $95 \mathrm{M}$ \\
\hline TC & 43 & 97 \\
\hline TC & 43 & $97 M$ \\
\hline$T C$ & 43 & 98 \\
\hline TC & 43 & 99 \\
\hline RU & 44 & 103 \\
\hline RU & 44 & 106 \\
\hline RH & 45 & $1 \mathrm{Cl}$ \\
\hline RH & 45 & 102 \\
\hline PD & 46 & 107 \\
\hline$A G$ & 47 & 105 \\
\hline$A G$ & 47 & $108 M$ \\
\hline AG & 47 & $110 M$ \\
\hline$C D$ & 48 & 109 \\
\hline$C D$ & 48 & $113 M$ \\
\hline $\mathrm{CO}$ & 48 & $115 \mathrm{M}$ \\
\hline IN & 49 & $114 M$ \\
\hline IN & 49 & 115 \\
\hline SN & 50 & 113 \\
\hline SN & 50 & $119 M$ \\
\hline SN & 50 & $121 \mathrm{M}$ \\
\hline SN & 50 & $123 M$ \\
\hline SN & 50 & 126 \\
\hline SB & 51 & 124 \\
\hline SB & 51 & 125 \\
\hline TE & 52 & $121 \mathrm{M}$ \\
\hline TE & 52 & 123 \\
\hline TE & 52 & $123 \mathrm{M}$ \\
\hline TE & 52 & $125 \mathrm{M}$ \\
\hline TE & 52 & $127 M$ \\
\hline TE & 52 & $129 \mathrm{M}$ \\
\hline I & 53 & 125 \\
\hline I & 53 & 129 \\
\hline$X E$ & 54 & 127 \\
\hline CS & 55 & 134 \\
\hline CS & 55 & 135 \\
\hline cs & 55 & 137 \\
\hline$B A$ & 56 & 133 \\
\hline LA & 57 & 137 \\
\hline LA & 57 & 138 \\
\hline$C E$ & 58 & 139 \\
\hline$C E$ & 58 & 141 \\
\hline$C E$ & 58 & 142 \\
\hline$C E$ & 58 & 144 \\
\hline ND & 60 & 144 \\
\hline
\end{tabular}

ADULT INFANT

$2 \cdot 6 E+04$

1. $2 E+03$

2. $3 E+04$

1. $4 E+02$

$1.1 E+00$

$3.6 E-01$

$6.3 E+00$

4. $8 \mathrm{E}-03$

7. $9 \mathrm{E}-02$

$2 \cdot 5 \mathrm{E}+01$

$1.8 \mathrm{E}-01$

1. $7 E-01$

5. $8 \mathrm{E}+00$

1. $2 E+03$

1. $1 E+01$

6. $9 E+01$

6. $2 E-02$

$8.4 E+00$

$8.4 E+00$

3. $3 E-02$

3. $3 E-02$
1. $3 E+04$

1. $3 E+04$

1. $6 \mathrm{E}+04$

6. $9 E+03$

4. $1 E+00$

1. $2 E+04$

$3.4 E+03$

4. $9 E-01$

2. 3E-OI

2. $4 E+00$

9. $8 E+00$

1. $9 \mathrm{E}+00$

3. $3 E+00$

3. $7 E+04$

$2 \cdot 3 E+02$

6. $3 E+00$

1. $2 E+03$

3. $7 \mathrm{E}+03$

3. $5 E+02$

6. $3 E+04$

3. $2 E+05$

5. $0 E+05$

$6.3 E+06$

3. $6 E+02$

8. $O E+03$

4. $3 E-03$
INFANT
UC/ $M * * 2$

4. $7 E+01$

- $9 E+02$

$8 E-01$

$6.9 E+00$

$1.8 E+04$

1. $0 E+04$

9. $0 E+01$

$5.0 E-01$

$2.9 E-02$

3. $1 E-03$

5. $1 E+00$

$1.5 E+04$

$2.8 E+04$

- $5 E+01$

. $1 E+03$

4. $1 E+06$

7. $1 E+02$

$5.9 E+04$

$1.1 E+04$

$2.7 E+05$

2. $4 E+01$

$1.2 E+04$

5. $E+04$

$2.7 E+04$

4. $0 E+02$

- $1 E+04$

. $5 E+04$

. $2 \mathrm{E}-0$

3. $2 E+06$

$4.2 E+01$

8. $5 E+00$

.6E-02

$8.0 E+03$

6. $2 E+03$

$5.9 E+02$

$3.5 E+05$

- $6 E+03$

$1.8 E+02$

$6.1 E+03$

1. $1 E+04$

2. $1 E+02$
$U C / M * \# 2$

2. $0 E+03$

7. $4 \mathrm{E}+02$

$2.1 E+00$

1. $0 E+03$

$1.4 E+02$

$8.4 E-01$

3. $6 E-01$

$.1 E+00$

4. $8 \mathrm{E}-03$

7. $9 E-02$

$4.5 E+00$

1. $2 E-01$

1. $5 E-01$

$5.8 \mathrm{E}+00$

. $1 E+03$

1. $1 E+01$

6. $8 E+01$

9. $0 E-01$

$8 E-02$

- $1 E+00$

8. $5 E-01$

3. $3 E-02$

1. $9 E+03$

4. $3 E+03$

1. $3 E+02$

1. $1 E+03$

3. $8 E+00$

1. $2 E+04$

3. $4 E+03$

. $1 E-01$

2. 3E-01

1. $9 E+0 J$

$6.4 E+00$

$1.5 E+00$

1. $7 E+00$

3. $4 E+04$

2. $3 E+02$ $6.3 E+00$

1. $0 E+03$

3. $6 E+03$

3. $4 E+02$

$6.3 E+04$

1. $1 E+07$

3. $2 E+05$

1. $0 E+05$

$3.4 E+0 D$

$3.3 E+02$

2. $7 E+03$

4. $O E-03$ 
TABLE 6B. UNIT-RAD DEPOSITION FOR KIDNEYS

$\begin{array}{lcccc}\text { ELEMENT } & \text { ATOMIC } & \text { I SOTOPE } & \text { ADULT } & \text { INFANT } \\ \text { SYMBOL } & \text { NO. }(2) & \text { MASSIA } & \text { UC /M**2 } & \text { UC/M**2 } \\ \text { PM } & & & & \\ \text { PM } & 61 & 143 & 3.8 E+04 & 1.1 E+04 \\ \text { PM } & 61 & 144 & 3.0 E+03 & 1.1 \mathrm{E}+03 \\ \text { PM } & 61 & 145 & 4.8 E+03 & 4.2 E+03 \\ \text { PM } & 61 & 146 & 1.1 E+03 & 7.8 E+02 \\ \text { PM } & 61 & 147 & 8.0 E+02 & 4.8 E+02 \\ \text { SM } & 61 & 148 M & 7.8 E+04 & 4.6 E+03 \\ \text { SM } & 62 & 145 & 1.9 E+05 & 6.4 E+04 \\ \text { SM } & 62 & 146 & 2.0 E+02 & 1.8 E+02 \\ \text { SM } & 62 & 147 & 2.2 E+02 & 2.0 E+02 \\ \text { SM } & 62 & 148 & 1.3 E+02 & 1.2 E+02 \\ \text { SM } & 62 & 149 & 2.7 E+02 & 2.5 E+02 \\ \text { EU } & 62 & 151 & 3.7 E+04 & 3.4 E+04 \\ \text { EU } & 63 & 148 & 4.7 E+05 & 1.7 E+04 \\ \text { EU } & 63 & 149 & 1.2 E+05 & 8.1 E+03 \\ \text { EU } & 63 & 150 & 5.0 E+02 & 2.7 E+02 \\ \text { EU } & 63 & 152 & 1.5 E+02 & 1.1 E+02 \\ \text { EU } & 63 & 154 & 1.1 E+02 & 8.1 E+01 \\ \text { GD } & 63 & 155 & 2.2 E+04 & 6.9 E+03 \\ \text { GD } & 64 & 146 & 4.8 E+04 & 1.7 E+03 \\ \text { GD } & 64 & 148 & 2.6 E+01 & 2.1 E+01 \\ \text { GD } & 64 & 150 & 1.4 E+01 & 1.1 E+01 \\ \text { GD } & 64 & 151 & 1.3 E+05 & 1.1 E+04 \\ \text { GD } & 64 & 152 & 3.4 E+01 & 2.8 E+01 \\ \text { GD } & 64 & 153 & 8.0 E+04 & 1.2 E+04 \\ \text { TB } & 64 & 162 & 3.8 E+03 & 8.3 E+02 \\ \text { TB } & 65 & 157 & 2.4 E+03 & 2.1 E+03 \\ \text { TB } & 65 & 158 & 1.0 E+02 & 9.3 E+01 \\ \text { DY } & 65 & 160 & 8.2 E+04 & 7.7 E+03 \\ \text { DY } & 66 & 154 & 8.8 E-04 & 8.8 E-04 \\ \text { DY } & 66 & 156 & 1.4 E-03 & 1.4 E-03 \\ \text { HD } & 66 & 159 & 5.7 E+00 & 5.7 E+00 \\ \text { HO } & 67 & 163 & 1.5 E-01 & 1.3 E-01 \\ \text { TM } & 67 & 166 M & 6.6 E-03 & 5.9 E-03 \\ \text { TM } & 69 & 168 & 1.4 E+05 & 2.9 E+04 \\ \text { TM } & 69 & 170 & 3.0 E+05 & 8.2 E+04 \\ \text { YB } & 69 & 171 & 4.1 E+05 & 2.7 E+05 \\ \text { LU } & 70 & 169 & 1.7 E+06 & 7.4 E+04 \\ \text { LU } & 71 & 173 & 3.7 E-01 & 1.5 E-01 \\ \text { LU } & 71 & 174 & 5.2 E-02 & 3.3 E-02 \\ \text { LU } & 71 & 174 M & 3.4 E+00 & 6.1 E-01 \\ \text { LU } & 71 & 176 & 1.1 E-02 & 9.9 E-03 \\ \text { HF } & 71 & 177 M & 1.0 E+01 & 1.7 E+00 \\ \text { HF } & 72 & 172 & 4.1 E+01 & 3.2 E+01 \\ \text { HF } & 72 & 174 & 3.7 E+00 & 3.4 E+00 \\ \text { HF } & 72 & 175 & 1.7 E+04 & 1.9 E+03 \\ \text { HF } & 72 & 181 & 2.7 E+04 & 2.0 E+03 \\ \text { TA } & 73 & 179 & 5.6 E+00 & 5.2 E+00 \\ \text { TA } & 73 & 182 & 4.8 E+01 & 2.9 E+01 \\ \text { W } & 74 & 181 & 2.8 E+01 & 6.3 E+00 \\ & & & 6.0 E+00 & 6.0 E+00\end{array}$

VIA TYPICAL AGRICULTURAL SOIL.

\begin{tabular}{|c|c|c|}
\hline $\begin{array}{l}\text { ELEMENT } \\
\text { SYMBOL }\end{array}$ & $\begin{array}{l}\text { A TOMIC } \\
\text { NO. }(2)\end{array}$ & $\begin{array}{l}\text { I SOTOPE } \\
\text { MASS(A) }\end{array}$ \\
\hline$W$ & 74 & 185 \\
\hline$w$ & 74 & 188 \\
\hline RE & 75 & 183 \\
\hline RE & 75 & 184 \\
\hline RE & 75 & $184 M$ \\
\hline RE & 75 & 187 \\
\hline DS & 76 & 185 \\
\hline OS & 76 & 194 \\
\hline IR & 77 & 192 \\
\hline IR & 77 & $192 \mathrm{M}$ \\
\hline PT & 78 & 190 \\
\hline PI & 78 & 192 \\
\hline PT & 78 & 193 \\
\hline$A U$ & 79 & 195 \\
\hline$H G$ & 80 & 194 \\
\hline HG & 80 & $2 C 3$ \\
\hline$T L$ & 81 & $2 C_{4}$ \\
\hline PB & 82 & $2 \mathrm{C2}$ \\
\hline PB & 82 & 204 \\
\hline PB & 82 & 205 \\
\hline PB & 82 & 210 \\
\hline BI & 83 & $2 \mathrm{C7}$ \\
\hline B I & 83 & 208 \\
\hline B I & 83 & $210 M$ \\
\hline PO & 84 & $2 \mathrm{CB}$ \\
\hline PO & 84 & 209 \\
\hline$P O$ & 84 & 210 \\
\hline RA & 88 & 226 \\
\hline RA & 88 & 228 \\
\hline$A C$ & 89 & 227 \\
\hline TH & 90 & 228 \\
\hline TH & 90 & 229 \\
\hline $\mathrm{TH}$ & 90 & 230 \\
\hline TH & 90 & 232 \\
\hline PA & 91 & 231 \\
\hline U & 92 & 232 \\
\hline$u$ & 92 & 233 \\
\hline U & 92 & 234 \\
\hline U & 92 & 235 \\
\hline u & 92 & 236 \\
\hline$U$ & 92 & 238 \\
\hline NP & 93 & 235 \\
\hline NP & 93 & 236 \\
\hline NP & 93 & 237 \\
\hline PU & 94 & 236 \\
\hline PU & 94 & 237 \\
\hline PU & 94 & 238 \\
\hline PU & 94 & 239 \\
\hline PU & 94 & 240 \\
\hline & 94 & \\
\hline
\end{tabular}

ADULT

INFANT $U C / M * 2 \quad U C / M * 2$

6. $O E+00$

5. $8 \mathrm{E}-01$

2. $3 E+00$

$1.5 E+00$

2. $6 \mathrm{E}-01$

8. $O E-01$

1. $2 \mathrm{E}-02$

1. $2 \mathrm{E}+00$

6. $1 E-03$

2. $3 E-03$

2. $8 E-03$

9. $7 \mathrm{E}-02$

4. $7 E+00$

1. $2 E+02$

1. $3 E+01$

5. $4 E+00$

3. $7 E+01$

B. 8E-01

1. $1 E-01$

5. $6 \mathrm{E}-02$

2. $7 \mathrm{E}-02$

7. $1 E-03$

1. $6 E-03$

1. $1 E-01$

2. 3E-04

7. $5 E-04$

2. $7 E-03$

7. $3 E-02$

1. $4 E-03$

1. $3 E-03$

1. $2 E-03$

2. $6 E-03$

2. $1 E-04$

1. $9 E-04$

1. $8 \mathrm{E}-04$

1. $6 \mathrm{E}-04$

1. $6 E-04$

1. $5 \mathrm{E}-04$

4. $7 E-01$

2. $7 E-03$

2. $9 E-03$

3. $7 E-02$

1. $9 E+01$

1. $6 E-03$

1. $3 E-03$

1. $3 E-03$

$6.0 E+00$

$5.8 \mathrm{E}-01$

$2.3 E+00$

1. $5 E+00$

$2.6 E-01$

$5.5 E+01$

7. $6 E-01$

1. $2 \mathrm{E}-02$

7. $0 E-01$

6. $O E-03$

2. $3 E-03$

2. $7 \mathrm{E}-03$

$9.6 \mathrm{E}-02$

$1.3 E+02$

$4 \cdot 3 E+00$

8. $9 E+01$

$1.3 E+01$

1. $1 E+00$

$3.5 E+01$

7. $9 E-01$

1. $1 E-01$

5.6E-02

2. $7 E-02$

$6.6 E-03$

$1.6 \mathrm{E}-03$

7. $4 E-02$

2. $3 E-04$

7. $5 E-04$

$3.3 E-04$

$2.3 E-03$

2. $1 E-04$

2. $0 E-04$

1. $8 E-04$

1. $8 \mathrm{E}-04$

2. $1 E-04$

1. $9 E-04$

1. $8 \mathrm{E}-04$

1. $6 \mathrm{E}-04$

1. $6 E-04$

1. $5 E-04$

3. $0 \mathrm{E}-03$

1. $6 E-04$

1. $6 E-04$

1. $1 E-03$

2. $7 E-02$

$1.7 E-04$

1. $5 \mathrm{E}-04$

1. $4 E-04$

2. $9 E-04$ 
TABLE 6B. UNIT-RAD DEPOSITION FOR KIDNEYS

ELEMENT SYMBOL

ATOMIC ISOTOPE

NO. (Z) MASS(A)

ADULT
UC/M**2

I NF ANT

INFANT
UC/M**2

$P U$
$P U$
$A M$
$A M$
$A M$
$C M$
$C M$
$C M$
$C M$
$C M$
$C M$
$C M$

94
94
95
95
95
96
96
96
96
96
96
96

242

241

$242 M$

243

241

242

243

244

245

247

1. $3 E-03$

1. $1 \mathrm{E}-03$

1. $2 \mathrm{E}-03$

1. $2 \mathrm{E}-03$

1. $0 E-03$

2. $1 E+01$

$9.5 E-01$

$1.4 \mathrm{E}-03$

$1.9 E-03$

$9.2 \mathrm{E}-04$

8. $9 E-04$

8. 3E-04

1. 4 E- 04

1. $2 E-04$

1. $5 E-04$

$1.5 E-04$

1. 3E-04

3.1 E-02

$6.4 E-03$

1. 8E-04

2. $2 E-04$

1. $3 \mathrm{E}-04$

$1.3 E-04$

1. $2 \mathrm{E}-04$
VIA TYPICAL AGRICULTURAL SOIL.

$\begin{array}{ccc}\text { ELEMENT } & \text { ATOMIC } & \text { ISOTOPE } \\ \text { SYMBOL } & \text { NO. }(Z) & \text { MASS(A) } \\ \text { CM } & 96 & 248 \\ \text { BK } & 97 & 247 \\ \text { BK } & 97 & 249 \\ \text { CF } & 98 & 248 \\ \text { CF } & 98 & 249 \\ \text { CF } & 98 & 250 \\ \text { CF } & 98 & 251 \\ \text { CF } & 98 & 252 \\ \text { ES } & 99 & 252 \\ \text { ES } & 99 & 254 \\ \text { FM } & 100 & 257\end{array}$




\begin{tabular}{|c|c|c|c|c|}
\hline $\begin{array}{l}\text { EL EMENT } \\
\text { SYMBOL }\end{array}$ & $\begin{array}{l}\text { ATOMIC } \\
\text { NO. }(Z)\end{array}$ & $\begin{array}{l}\text { I SOTOPE } \\
\text { MASS (A) }\end{array}$ & $\begin{array}{c}\text { ADULT } \\
U C / M * 2\end{array}$ & $\begin{array}{l}\text { INFANT } \\
\text { UC/M**2 }\end{array}$ \\
\hline$H$ & 1 & 3 & 4. $7 E+01$ & $4.7 E+01$ \\
\hline BE & 4 & 7 & 2. $5 E+06$ & 4.1E+05 \\
\hline$B E$ & 4 & 10 & $5.1 E+02$ & $4.9 E+02$ \\
\hline C & 6 & 14 & 9. $8 \mathrm{E}-01$ & $9.8 E-01$ \\
\hline NA & 11 & 22 & $1 \cdot 1 E+01$ & $1 \cdot 1 E+O 1$ \\
\hline$A L$ & 13 & 26 & 1. $0 E+04$ & 1. $0 E+04$ \\
\hline SI & 14 & 32 & $9.0 E+03$ & $9.0 E+03$ \\
\hline $\mathrm{s}$ & 16 & 35 & $9.0 E+01$ & $9.0 E+01$ \\
\hline$C L$ & 17 & 36 & $1.7 \mathrm{E}-01$ & $1 . T E-01$ \\
\hline $\mathrm{AR}$ & 18 & 37 & $5.5 E+02$ & $5.5 E+02$ \\
\hline$A R$ & 18 & 39 & 2. $9 E-02$ & $2.9 E-02$ \\
\hline AR & 18 & 42 & 3. $1 E-03$ & $3.1 \mathrm{E}-03$ \\
\hline $\mathrm{K}$ & 19 & 40 & $3.6 E+00$ & $3.6 E+00$ \\
\hline$C A$ & 20 & 41 & 3. $0 E+04$ & $3.0 E+04$ \\
\hline CA & 20 & 45 & 5. $7 E+04$ & $5.5 E+04$ \\
\hline CA & 20 & 48 & 2. $9 E+01$ & $2.9 E+01$ \\
\hline SC & 21 & 46 & 8. $9 E+04$ & $4.2 E+03$ \\
\hline $\mathrm{TI}$ & 22 & 44 & $6.8 E+03$ & $6.8 E+03$ \\
\hline$v$ & 23 & 49 & 5. $7 E+06$ & $4.7 E+06$ \\
\hline$v$ & 23 & 50 & $8.2 E+02$ & $8.2 E+02$ \\
\hline MN & 25 & 53 & $3.5 E+04$ & $3.5 E+04$ \\
\hline MN & 25 & 54 & 7. $7 E+03$ & $6.2 E+03$ \\
\hline $\mathrm{FE}$ & 26 & 55 & $1.6 E+05$ & $1.0 \mathrm{E}+05$ \\
\hline $\mathrm{FE}$ & 26 & 59 & 1. $5 E+05$ & $1 \cdot 1 E+04$ \\
\hline FE & 26 & 60 & $3.5 E+01$ & $3.2 E+01$ \\
\hline $\mathrm{CO}$ & 27 & 56 & 2. $7 E+03$ & $2.1 E+03$ \\
\hline CO & 27 & 57 & $9.5 E+03$ & $8.7 E+03$ \\
\hline $\mathrm{CO}$ & 27 & 58 & $6.2 E+03$ & $4.6 E+03$ \\
\hline CO & 27 & 60 & $6.8 E+01$ & $6.8 E+01$ \\
\hline NI & 28 & 59 & $2.8 E+04$ & $2.6 E+04$ \\
\hline NI & 28 & 63 & 1. $4 E+04$ & $1.3 E+04$ \\
\hline$Z N$ & 30 & 65 & $3.8 E+01$ & $2.8 E+01$ \\
\hline GE & 32 & 68 & $1.2 \mathrm{E}-01$ & $1.2 E-01$ \\
\hline AS & 33 & 73 & $1.7 E+07$ & $2.0 E+06$ \\
\hline SE & 34 & 75 & 3. $0 E+01$ & $2.5 E+01$ \\
\hline$S E$ & 34 & 79 & 4. $9 E+00$ & $4.9 E+00$ \\
\hline$K R$ & 36 & 81 & $2.9 E-01$ & $2.9 E-01$ \\
\hline$K R$ & 36 & 85 & $5.6 E-02$ & $5.6 E-02$ \\
\hline RB & 37 & 83 & 6. $0 E+02$ & $3.0 E+02$ \\
\hline$R B$ & 37 & 84 & $8.1 E+02$ & $2.4 E+02$ \\
\hline RB & 37 & 87 & 2. $3 E+01$ & $2.2 E+01$ \\
\hline SR & 38 & 85 & 1. $1 E+06$ & $1.0 E+06$ \\
\hline$S R$ & 38 & 89 & 1. $0 E+06$ & $9.1 E+05$ \\
\hline$S R$ & 38 & 90 & $4 \cdot 2 E+03$ & $4.2 E+03$ \\
\hline$Y$ & 39 & 88 & $1 \cdot 3 E+04$ & $9.1 E+02$ \\
\hline Y & 39 & 91 & $1.4 E+05$ & $5.6 E+03$ \\
\hline$Z R$ & 40 & 88 & 2. $9 E+04$ & $6.1 E+03$ \\
\hline $2 R$ & 40 & 93 & $1.1 E+04$ & $1.1 E+04$ \\
\hline $2 R$ & 40 & 95 & 8. $8 E+04$ & $1.5 E+04$ \\
\hline NB & 41 & 91 & $2.1 E+02$ & $1.9 E+02$ \\
\hline
\end{tabular}

\begin{tabular}{|c|c|c|}
\hline $\begin{array}{l}\text { ELEMENT } \\
\text { SYMBOL }\end{array}$ & $\begin{array}{l}\text { ATOMIC } \\
\text { NO. (Z) }\end{array}$ & $\begin{array}{l}\text { I SOTOP } \\
\text { MASSIA }\end{array}$ \\
\hline NB & 41 & $91 M$ \\
\hline NB & 41 & S3M \\
\hline NB & 41 & 94 \\
\hline NB & 41 & 95 \\
\hline MO & 42 & 93 \\
\hline$T C$ & 43 & S5M \\
\hline TC & 43 & 97 \\
\hline TC & 43 & $97 M$ \\
\hline$T C$ & 43 & 98 \\
\hline TC & 43 & 59 \\
\hline RU & 44 & 103 \\
\hline RU & 44 & 106 \\
\hline RH & 45 & 101 \\
\hline RH & 45 & $1 C 2$ \\
\hline PD & 46 & 107 \\
\hline $\mathbf{A G}$ & 47 & 105 \\
\hline $\mathbf{A G}$ & 47 & $108 M$ \\
\hline $\mathbf{A G}$ & 47 & $110 M$ \\
\hline$C D$ & 48 & 109 \\
\hline$C D$ & 48 & $113 M$ \\
\hline$C D$ & 48 & $115 \mathrm{M}$ \\
\hline IN & 49 & $114 M$ \\
\hline IN & 49 & 115 \\
\hline SN & 50 & 113 \\
\hline SN & 50 & $119 M$ \\
\hline SN & 50 & $121 \mathrm{M}$ \\
\hline SN & 50 & $123 \mathrm{M}$ \\
\hline SN & 50 & 126 \\
\hline SB & 51 & 124 \\
\hline SB & 51 & 125 \\
\hline TE & 52 & $121 \mathrm{M}$ \\
\hline TE & 52 & 123 \\
\hline TE & 52 & $123 \mathrm{M}$ \\
\hline TE & 52 & $125 M$ \\
\hline TE & 52 & $127 \mathrm{M}$ \\
\hline TE & 52 & $129 M$ \\
\hline I & 53 & 125 \\
\hline I & 53 & 129 \\
\hline$X E$ & 54 & 127 \\
\hline CS & 55 & 134 \\
\hline CS & 55 & 135 \\
\hline CS & 55 & 137 \\
\hline$B A$ & 56 & 133 \\
\hline LA & 57 & 137 \\
\hline LA & 57 & 138 \\
\hline CE & 58 & 139 \\
\hline CE & 58 & 141 \\
\hline CE & 58 & 142 \\
\hline$C E$ & 58 & 144 \\
\hline
\end{tabular}

ADULT
$U C / M * 2$
$2.6 E+04$
$1.1 E+03$
$2.2 E+00$
$2.3 E+04$
$4.2 E+00$
$1.3 E+00$
$3.6 E-01$
$6.8 E+00$
$4.8 E-03$
$7.9 E-02$
$1.3 E+02$
$1.4 E+00$
$9.1 E-02$
$1.7 E-01$
$5.8 E+00$
$6.6 E+02$
$2.9 E+00$
$2.1 E+01$
$1.6 E+01$
$7.7 E-01$
$7.6 E+01$
$1.8 E+00$
$3.3 E-02$
$1.7 E+04$
$1.9 E+04$
$1.1 E+02$
$8.9 E+03$
$2.8 E+00$
$7.8 E+03$
$1.4 E+03$
$4.9 E+00$
$2.3 E+00$
$2.4 E+01$
$9.8 E+01$
$1.9 E+01$
$3.3 E+01$
$6.8 E+03$
$4.2 E+01$
$6.3 E+00$
$1.7 E+03$
$5.5 E+03$
$5.2 E+02$
$1.4 E+05$
$5.4 E+05$
$1.6 E+04$
$3.1 E+05$
$3.4 E+06$
$3.4 E+02$
$5.4 E+03$
$4.0 E-03$
1.75

I NFANT $U C / M * * 2$

$1.8 \mathrm{E}+03$ 6. $7 E+02$ 1. $9 E+00$ 9. $2 E+02$ 4. $1 E+00$ 8. $4 \mathrm{E}-01$ $3 \cdot 6 E-01$ $5.1 E+00$ 4. $8 \mathrm{E}-03$ 7. $9 E-02$ 4. $5 \mathrm{E}+01$ 1. $2 E+00$ 9. $0 E-02$ 1. $5 \mathrm{E}-01$ 5. $8 E+00$ $2.9 E+02$ 2. $8 E+00$ 1. $8 \mathrm{E}+01$ $1.1 E+01$
$7.4 E-01$ 1. $3 E+01$ 8. $5 E-01$ $1.1 E+03$ 2. $5 E+03$ 8. $0 E+01$ $6.5 E+02$ $2.2 E+00$ 4. $8 E+03$ 1. $3 E+03$ 4. $1 E+00$ 2. $3 E+0 J$ 1. $9 E+01$ $6.4 E+01$ 1. $5 E+01$ 1. $7 E+01$ $6.1 E+03$ $4.2 E+01$ $6.3 E+00$ 1. $5 E+03$ $5.4 E+03$ 5. $1 E+02$ 1. $0 E+05$ 5. $1 E+05$ 1. $5 E+04$ 1. $0 E+05$ 3. $4 E+05$ 3. $3 E+02$ 2. $7 E+03$ 4. $0 E-03$ 
SOIL.

$05 / 09 / 68$

PAGE 8

$\begin{array}{ccccc}\text { ELEMENT } & \text { ATOMIC } & \text { I SOTOPE } & \text { ADULT } & \text { INFANT } \\ \text { SYMBOL } & \text { NO. (Z) } & \text { MASS(A) } & \text { UC IM**2 } & \text { UCIM**2 } \\ & & & & \\ \text { PM } & 61 & 143 & 1.9 E+05 & 5.4 E+04 \\ \text { PM } & 61 & 144 & 1.5 E+04 & 5.3 E+03 \\ \text { PM } & 61 & 145 & 2.4 E+04 & 2.1 E+04 \\ \text { PM } & 61 & 146 & 5.5 E+03 & 3.9 E+03 \\ \text { PM } & 61 & 147 & 4.0 E+03 & 2.4 E+03 \\ \text { PM } & 61 & 148 M & 3.9 E+05 & 2.3 E+04 \\ \text { SM } & 62 & 145 & 9.9 E+04 & 6.4 E+04 \\ \text { SM } & 62 & 146 & 1.8 E+02 & 1.8 E+02 \\ \text { SM } & 62 & 147 & 2.1 E+02 & 2.0 E+02 \\ \text { SM } & 62 & 148 & 1.2 E+02 & 1.2 E+02 \\ \text { SM } & 62 & 149 & 2.5 E+02 & 2.5 E+02 \\ \text { SM } & 62 & 151 & 3.5 E+04 & 3.4 E+04 \\ \text { EU } & 63 & 148 & 5.5 E+05 & 1.7 E+05 \\ \text { EU } & 63 & 149 & 1.8 E+05 & 8.1 E+04 \\ \text { EU } & 63 & 150 & 2.9 E+03 & 2.7 E+03 \\ \text { EU } & 63 & 152 & 1.1 E+03 & 1.1 E+03 \\ \text { EU } & 63 & 154 & 8.3 E+02 & 8.1 E+02 \\ \text { EU } & 63 & 155 & 8.2 E+04 & 6.9 E+04 \\ \text { GD } & 64 & 146 & 9.0 E+04 & 8.5 E+03 \\ \text { GD } & 64 & 148 & 1.1 E+02 & 1.1 E+02 \\ \text { GD } & 64 & 150 & 6.1 E+01 & 5.7 E+01 \\ \text { GD } & 64 & 151 & 2.6 E+05 & 5.4 E+04 \\ \text { GD } & 64 & 152 & 1.5 E+02 & 1.4 E+02 \\ \text { GO } & 64 & 153 & 1.8 E+05 & 6.2 E+04 \\ \text { GD } & 64 & 162 & 9.4 E+03 & 4.2 E+03 \\ \text { TB } & 65 & 157 & 2.2 E+04 & 2.1 E+04 \\ \text { TB } & 65 & 158 & 9.4 E+02 & 9.3 E+02 \\ \text { TB } & 65 & 160 & 1.5 E+05 & 7.7 E+04 \\ \text { DY } & 66 & 154 & 9.4 E-04 & 8.8 E-04 \\ \text { DY } & 66 & 156 & 1.5 E-03 & 1.4 E-03 \\ \text { DY } & 66 & 159 & 2.5 E+01 & 5.7 E+00 \\ \text { HO } & 67 & 163 & 1.5 E-01 & 1.3 E-01 \\ \text { HO } & 67 & 166 M & 6.7 E-03 & 5.9 E-03 \\ \text { TM } & 69 & 168 & 1.1 E+06 & 5.8 E+05 \\ \text { TM } & 69 & 170 & 2.5 E+06 & 1.6 E+06 \\ \text { TM } & 69 & 171 & 6.0 E+06 & 5.5 E+06 \\ \text { YB } & 70 & 169 & 1.2 E+07 & 3.7 E+06 \\ \text { LU } & 71 & 173 & 1.5 E-01 & 1.5 E-01 \\ \text { LU } & 71 & 174 & 3.3 E-02 & 3.3 E-02 \\ \text { LU } & 71 & 174 M & 6.1 E-01 & 6.1 E-01 \\ \text { LU } & 71 & 176 & 9.9 E-03 & 9.9 E-03 \\ \text { LU } & 71 & 177 M & 1.7 E+00 & 1.7 E+00 \\ \text { HF } & 72 & 172 & 4.3 E+00 & 3.2 E+00 \\ \text { HF } & 72 & 174 & 3.7 E-01 & 3.4 E-01 \\ \text { HF } & 72 & 175 & 1.9 E+03 & 1.9 E+02 \\ \text { HF } & 72 & 181 & 3.0 E+03 & 2.0 E+02 \\ \text { HF } & 72 & 182 & 5.7 E-01 & 5.2 E-01 \\ \text { TA } & 73 & 179 & 2.4 E+01 & 1.4 E+01 \\ \text { TA } & 73 & 182 & 1.4 E+01 & 3.2 E+00 \\ \text { W } & 74 & 181 & 6.2 E+00 & 6.0 E+00\end{array}$

ELEMENT ATOMIC ISOTOPE ADULT INFANT

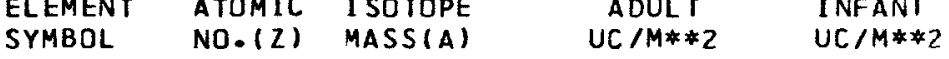

\begin{tabular}{|c|c|c|c|c|}
\hline$w$ & 74 & 185 & $6.3 E+00$ & $6.0 E+00$ \\
\hline$w$ & 74 & 188 & $6.2 E-01$ & $5.8 E-01$ \\
\hline RE & 75 & 183 & $2.8 E+00$ & $2.3 E+00$ \\
\hline RE & 75 & 184 & 2. $O E+00$ & $1.5 E+00$ \\
\hline$R E$ & 75 & $184 M$ & $2.8 E-01$ & $2.6 E-01$ \\
\hline RE & 75 & 187 & $5.5 E+01$ & $5.5 E+01$ \\
\hline DS & 76 & 185 & 8. $O E-01$ & $7.6 \mathrm{E}-01$ \\
\hline OS & 76 & 194 & $1.2 E-02$ & $1.2 \mathrm{E}-02$ \\
\hline IR & 77 & 192 & $9.6 E-01$ & $7.0 \mathrm{E}-01$ \\
\hline IR & 77 & $192 M$ & $6.1 E-03$ & $6.0 E-03$ \\
\hline PT & 78 & 190 & $2.3 E-03$ & $2.3 E-03$ \\
\hline PT & 78 & 192 & $2.8 E-03$ & $2.7 E-03$ \\
\hline PT & 78 & 193 & $9.6 E-02$ & $9.6 \mathrm{E}-02$ \\
\hline$A U$ & 79 & 195 & $2.2 E+02$ & $8.4 E+01$ \\
\hline$H G$ & 80 & 194 & $6.2 E+00$ & $5.7 E+00$ \\
\hline$H G$ & 80 & $2 \mathrm{C3}$ & $1 \cdot 5 E+02$ & $1 \cdot 2 E+02$ \\
\hline$T L$ & 81 & 204 & $1.2 E+01$ & $1.2 E+01$ \\
\hline PB & 82 & 202 & 4. $9 E+00$ & $3.7 E+00$ \\
\hline PB & 82 & 204 & 1. $1 E+00$ & $8.3 E-01$ \\
\hline PB & 82 & $2 C 5$ & $3.4 E+01$ & $2.5 E+01$ \\
\hline PB & 82 & 210 & 8. $5 E-01$ & $5.8 E-01$ \\
\hline BI & 83 & 207 & 5. $9 E-01$ & $5.9 E-01$ \\
\hline B I & 83 & 208 & $2.9 E-01$ & 2. $9 E-01$ \\
\hline BI & 83 & $210 M$ & $1.4 E-01$ & $1.4 E-01$ \\
\hline PO & 84 & 208 & $6.9 E-03$ & $6.6 \mathrm{E}-03$ \\
\hline PO & 84 & 209 & $1.6 E-03$ & $1.6 \mathrm{E}-03$ \\
\hline PO & 84 & 210 & $9.6 E-02$ & $7.4 E-02$ \\
\hline RA & 88 & 226 & $2 \cdot 3 E-04$ & $2.3 E-04$ \\
\hline RA & 88 & 228 & 7. $5 E-04$ & $7.5 E-04$ \\
\hline$A C$ & 89 & 227 & 5. $3 E-04$ & $3.3 E-04$ \\
\hline$T H$ & 90 & 228 & 1. $9 E-01$ & $2.3 E-03$ \\
\hline $\mathrm{TH}$ & 90 & 229 & 3. $4 E-03$ & $2.1 E-04$ \\
\hline TH & 90 & 230 & 3. $1 E-03$ & $2.0 E-04$ \\
\hline$T H$ & 90 & 232 & $2.8 E-03$ & $1.8 E-04$ \\
\hline PA & 91 & 231 & $2.9 E-03$ & $1.8 E-04$ \\
\hline $\mathrm{u}$ & 92 & 232 & $2.1 E-04$ & $2.1 E-04$ \\
\hline u & 92 & 233 & 1. $9 E-04$ & 1. $9 E-04$ \\
\hline U & 92 & 234 & $1.8 E-04$ & $1.8 E-04$ \\
\hline U & 92 & 235 & $1.6 E-04$ & $1.6 E-04$ \\
\hline u & 92 & 236 & $1.6 E-04$ & $1.6 E-04$ \\
\hline U & 92 & 238 & $1.5 E-04$ & $1.5 E-04$ \\
\hline NP & 93 & 235 & 4. $0 E-01$ & 3. $0 E-03$ \\
\hline NP & 93 & 236 & $2.3 E-03$ & $1.6 \mathrm{E}-04$ \\
\hline NP & 93 & 237 & $2.4 E-03$ & $1.6 \mathrm{E}-04$ \\
\hline PU & 94 & 236 & $3.4 E-02$ & $1.1 E-03$ \\
\hline PU & 94 & 237 & $1.8 E+01$ & $2.7 \mathrm{E}-02$ \\
\hline PU & 94 & 238 & $1.6 E-03$ & 1. $7 E-04$ \\
\hline PU & 94 & 239 & $1.3 E-03$ & $1.5 E-04$ \\
\hline PU & 94 & 240 & $1.2 \mathrm{E}-03$ & $1.4 \mathrm{E}-04$ \\
\hline PU & 94 & 241 & 3. $3 E-03$ & $2.9 E-04$ \\
\hline
\end{tabular}


TABLE 6B. UNIT-RAD DEPOSITION FOR LIVER

$\begin{array}{ccccc}\begin{array}{l}\text { ELEMENT } \\ \text { SYMBOL }\end{array} & \begin{array}{c}\text { ATOMIC } \\ \text { NO. }(Z)\end{array} & \begin{array}{c}\text { ISOTOPE } \\ \text { MASS(A) }\end{array} & \begin{array}{c}\text { ADULT } \\ \text { UC } / M * * 2\end{array} & \begin{array}{l}\text { INFANT } \\ \text { UC/M**2 }\end{array} \\ \text { PU } & 94 & 242 & 1.2 E-03 & 1.4 \mathrm{E}-04 \\ \text { PU } & 94 & 244 & 1.1 \mathrm{E}-03 & 1.2 \mathrm{E}-04 \\ \text { AM } & 95 & 241 & 2.5 \mathrm{E}-04 & 1.5 \mathrm{E}-04 \\ \text { AM } & 95 & 242 M & 2.5 \mathrm{E}-04 & 1.5 \mathrm{E}-04 \\ \text { AM } & 95 & 243 & 2.2 \mathrm{E}-04 & 1.3 \mathrm{E}-04 \\ \text { CM } & 96 & 241 & 2.7 \mathrm{E}+00 & 3.1 \mathrm{E}-02 \\ \text { CM } & 96 & 242 & 1.2 \mathrm{E}-01 & 6.4 \mathrm{E}-03 \\ \text { CM } & 96 & 243 & 3.0 \mathrm{E}-04 & 1.8 \mathrm{E}-04 \\ \text { CM } & 96 & 244 & 3.9 \mathrm{E}-04 & 2.2 \mathrm{E}-04 \\ \text { CM } & 96 & 245 & 2.1 \mathrm{E}-04 & 1.3 \mathrm{E}-04 \\ \text { CM } & 96 & 246 & 2.0 \mathrm{E}-04 & 1.3 \mathrm{E}-04 \\ \text { CM } & 96 & 247 & 1.9 \mathrm{E}-04 & 1.2 \mathrm{E}-04\end{array}$

VIA TYPICAL AGRICULTURAL SOIL.

ELEMENT SYMBOL

ATDMIC ISDTOPE NO. $(Z)$ MASS(A)

CM
$B K$
$B K$
$C F$
CF
CF
CF
$C F$
ES
ES
$F M$

$\begin{array}{rl}96 & 248 \\ 97 & 247 \\ 97 & 249 \\ 98 & 248 \\ 98 & 249 \\ 98 & 250 \\ 98 & 251 \\ 98 & 252 \\ 99 & 252 \\ 99 & 254 \\ 100 & 257\end{array}$


TABLE 6B. UNIT-RAD DEPOSITION FOR OVARIES

$\begin{array}{lcc}\text { ELEMEN I } & \text { ATOMIC } & \text { I SOTOPE } \\ \text { SYMBOL } & \text { NO. IZI } & \text { MASS }(A) \\ & & \\ \text { H } & 1 & 3 \\ \text { GE } & 4 & 7 \\ \text { BE } & 4 & 10 \\ \text { C } & 6 & 14 \\ \text { NA } & 11 & 22 \\ \text { AL } & 13 & 26 \\ \text { SI } & 14 & 32 \\ \text { S } & 16 & 35 \\ \text { CL } & 17 & 36 \\ \text { AR } & 18 & 37 \\ \text { AR } & 18 & 39 \\ \text { AR } & 18 & 42 \\ \text { K } & 19 & 40 \\ \text { CA } & 20 & 41 \\ \text { CA } & 20 & 45 \\ \text { CA } & 20 & 48 \\ \text { SC } & 21 & 46 \\ \text { TI } & 22 & 44 \\ V & 23 & 49 \\ V & 23 & 50 \\ \text { MN } & 25 & 53 \\ \text { MN } & 25 & 54 \\ \text { FE } & 26 & 55 \\ \text { FE } & 26 & 59 \\ \text { FE } & 26 & 60 \\ \text { CO } & 27 & 56 \\ \text { CO } & 27 & 57 \\ \text { CO } & 27 & 58 \\ \text { CO } & 27 & 60 \\ \text { NI } & 28 & 59 \\ \text { NI } & 28 & 63 \\ \text { ZN } & 30 & 65 \\ \text { GE } & 32 & 68 \\ \text { AS } & 33 & 73 \\ \text { SE } & 34 & 75 \\ \text { SE } & 34 & 79 \\ \text { KR } & 36 & 81 \\ \text { KR } & 36 & 85 \\ \text { RB } & 37 & 83 \\ \text { RB } & 37 & 84 \\ \text { RB } & 37 & 87 \\ \text { SR } & 38 & 85 \\ \text { SR } & 38 & 89 \\ \text { SR } & 38 & 90 \\ \text { Y } & 39 & 88 \\ \text { Y } & 39 & 91 \\ \text { ZR } & 40 & 88 \\ \text { ZR } & 40 & 93 \\ \text { ZR } & 40 & 95 \\ \text { NB } & 41 & 91 \\ & & \end{array}$

$U C / M * * 2$

4. $7 E+01$
VIA TYPICAL AGRICULTURAL

SOIL.

4. $7 E+0$

4. $9 E+02$

$9.8 \mathrm{E}-01 \quad 9.8 \mathrm{E}-01$

1.6E+00

1. $3 E+04 \quad 1.3 E+04$

9. $0 E+01 \quad 9.0 E+01$

$1.1 E-01 \quad 1.1 E-01$

$5.5 E+02 \quad 5.5 E+02$

2. $9 E-02 \quad 2.9 E-02$

$3.1 E-03 \quad 3.1 E-03$

7. $0 E+00 \quad 6.9 E+00$

1. $7 E+04 \quad 1.4 E+04$

7. $6 E+00 \quad 7.6 E+00$

4. $2 E+01 \quad 4.2 E+01$

$1.1 E+04 \quad 1.1 E+04$

3. $3 E+07 \quad 3.3 E+07$

$5.7 E+03 \quad 5.7 E+03$

$3.2 E+05 \quad 3.2 E+05$

5. $7 E+04 \quad 5.6 E+04$

4. $1 E+05 \quad 3.7 E+04$

$1.1 E+02 \quad 1.1 E+02$

8. $8 E+03 \quad .7 E+03$

3. $1 E+04 \quad 2.8 E+04$

$2.0 E+04 \quad 1.5 E+04$

$2.2 E+02 \quad 2.2 E+02$

$2.2 E+04$

$1.1 E+02 \quad 7.8 E+01$

$1.2 E-01 \quad 1.2 E-01$

3. $2 E+06 \quad 3.2 E+06$

7.6E-01 7.6E-01

$1.5 \mathrm{E}-01 \quad 1.5 \mathrm{E}-01$

2.9E-01 2.9E-01

5. $6 E-02 \quad 5.6 E-02$

4. $7 E+04 \quad 2.4 E+04$

$6.3 E+04 \quad 1.9 E+04$

$1.8 \mathrm{E}+03 \quad 1.8 \mathrm{E}+03$

2. $5 E+05 \quad 1.5 E+05$

$2.4 E+05 \quad 1.3 E+05$

$5.9 E+02$

$1.8 \mathrm{E}+02$

$\begin{array}{ll}1.1 E+03 & 1.1 E+03 \\ 6.7 E+03 & 6.7 E+03\end{array}$

$1.2 E+04 \quad 1.2 E+04$

$1.7 E+04 \quad 1.7 E+04$

$\begin{array}{lll}\text { ELEMENT } & \text { ATOMIC } & \text { ISOTOPE } \\ \text { SYMBOL } & \text { NO.(Z) } & \text { MASS(A) }\end{array}$

$\begin{array}{lll}\text { ELEMENT } & \text { ATOMIC } & \text { ISOTOPE } \\ \text { SYMBOL } & \text { NO.(Z) } & \text { MASS(A) }\end{array}$

NB

N

NB

NB

MO

TC

TC

TC

TC

RU

RH

RH

PD

AG

AG

CD

CD

IN

IN

SN

SN

SN

SN

$S B$

TE

TE

TE
TE
TE

41
41
41
41
42
43
43
43
43
43
44
44
45
45
46
47
47
47
48
48
48
49
49
50
50
50
50
50
51
51
52
52
52
52
52
52
53
53
54
55
55
55
56
57
57
58
58
58
58
60

ADULT

UC $/ M * * 2$

INF ANT

$05 / 09 / 68$

PAGE 13

$25+03$

8. $2 E+02$

2. $4 E+00$

1. $1 E+03$

1. $9 E+03$

8. $4 E-01$

3. $6 E-01$

5. $1 E+00$

4. $8 E-03$

7. $9 E-02$

1. $3 E+02$

1. $4 E+00$

9. $0 E-02$

$1.5 E-01$

5. $8 E+00$

1. $1 E+01$

1. $9 E+01$

7. $4 \mathrm{E}+01$

4. $2 E+00$

1. $9 E+02$

8. $5 \mathrm{E}-01$

3. $3 E-02$

$7.2 E+02$

$1.4 E+03$

3. $7 E+01$

4. $0 \mathrm{E}+02$

1. $O E+0 O$

2. $9 E+03$

7. $B E+02$

9. $7 E-01$

4. $7 E-01$

4. $8 \mathrm{E}+00$

2. $0 E+01$

3. $8 \mathrm{E}+00$

6. $7 E+00$

7. $5 E+03$

5. $2 E+01$

6. $3 E+00$

1. $6 E+02$

5. $1 E+02$

4. $8 \mathrm{E}+01$

3. $2 E+04$

8. $5 \mathrm{E}+02$

2. $5 \mathrm{E}+01$

2. $0 E+03$

6. $8 E+03$

$6.6 E+00$

5. $4 E+01$
$2 \cdot 2 E+03$

8. $2 E+02$

$2.4 E+00$

1. $1 \mathrm{E}+03$

$1.9 E+03$

$8.4 E-01$

$5.1 E+00$

5. $1 E+00$

4. $8 \mathrm{E}-03$
7. $9 \mathrm{E}-02$

4. $5 E+01$

1. $2 \mathrm{E}+0 \mathrm{~J}$

9. $0 E-02$

1. $5 E-01$

5. $8 \mathrm{E}+00$

$1.1 E+03$

$1.1 E+01$

$6.8 E+01$

6. $5 E+01$

4. $2 E+00$

3. $3 E-02$

$5.2 E+02$

l. $2 \mathrm{E}+03$

$3.6 E+01$

$3.6 E+01$

3. $0 E+02$

1. $O E+00$

2. $9 E+03$

7. $8 E+02$

4. $7 E-01$

3. $7 E+00$

$1.3 E+01$

3. $O E+0 D$

3. $5 E+00$

7. $5 \mathrm{E}+03$

$5.2 E+01$

6. $3 E+00$

1. $4 E+02$

5. $0 E+02$

4. $7 E+01$

3. $2 E+04$

$8.5 E+02$

- $5 E+02$

2. $5 E+01$

2. $0 E+03$

6. $8 E+03$

$6.6 E+00$

$5.4 E+01$ 


\begin{tabular}{|c|c|c|c|c|}
\hline $\begin{array}{l}\text { ELEMENT } \\
\text { SYMBOL }\end{array}$ & $\begin{array}{l}\text { ATOMIC } \\
\text { NO. }(Z)\end{array}$ & $\begin{array}{l}\text { ISOTOPE } \\
\text { MASS (A) }\end{array}$ & $\begin{array}{l}\text { ADULT } \\
\text { UC } / M * * 2\end{array}$ & $\begin{array}{l}\text { INFANT } \\
\text { UC/M* } / 2\end{array}$ \\
\hline PM & 61 & 143 & $1 \cdot 1 E+03$ & $1.1 E+03$ \\
\hline PM & 61 & 144 & $1 \cdot 1 E+02$ & $1.1 E+02$ \\
\hline PM & 61 & 145 & $4 \cdot 2 E+02$ & $4.2 E+02$ \\
\hline PM & 61 & 146 & $7.8 E+01$ & 7. $8 E+01$ \\
\hline PM & 61 & 147 & $4.8 E+01$ & $4.8 E+01$ \\
\hline PM & 61 & $148 M$ & $4.6 E+02$ & $4.6 E+02$ \\
\hline SM & 62 & 145 & $1 \cdot 3 E+04$ & $1.3 E+04$ \\
\hline SM & 62 & 146 & $3.6 E+01$ & $3.6 E+01$ \\
\hline SM & 62 & 147 & 4. $I E+01$ & 4. $1 E+01$ \\
\hline SM & 62 & 148 & $2 \cdot 3 E+01$ & $2.3 E+01$ \\
\hline SM & 62 & 149 & $5.0 E+01$ & $5.0 E+01$ \\
\hline SM & 62 & 151 & $6.7 E+03$ & $6.7 E+03$ \\
\hline EU & 63 & 148 & 3. $3 E+04$ & $3.3 E+04$ \\
\hline EU & 63 & 149 & $1.6 E+04$ & $1.6 E+04$ \\
\hline EU & 63 & 150 & $5.4 E+02$ & $5.4 E+02$ \\
\hline EU & 63 & 152 & $2.1 E+02$ & $2.1 E+02$ \\
\hline EU & 63 & 154 & $1.6 E+02$ & $1.6 E+02$ \\
\hline EU & 63 & 155 & $1.4 E+04$ & $1.4 E+04$ \\
\hline GD & 64 & 146 & 1. $7 E+02$ & 1. $7 E+02$ \\
\hline GD & 64 & 148 & 2. $1 E+00$ & $2 \cdot 1 E+00$ \\
\hline GD & 64 & 150 & 1. $1 E+00$ & $1.1 E+00$ \\
\hline GD & 64 & 151 & $1.1 E+03$ & $1.1 E+03$ \\
\hline GD & 64 & 152 & $2.8 E+00$ & $2.8 E+00$ \\
\hline GD & 64 & 153 & $1.2 E+03$ & 1. $2 E+03$ \\
\hline GD & 64 & 162 & 8. $3 E+01$ & $8 \cdot 3 E+01$ \\
\hline TB & 65 & 157 & $2 \cdot 1 E+02$ & $2.1 E+02$ \\
\hline TB & 65 & 158 & 9. $3 E+00$ & $9.3 E+00$ \\
\hline TB & 65 & 160 & 7. $7 E+02$ & $7.7 E+02$ \\
\hline or & 66 & 154 & 8. $8 E-04$ & $8.8 E-04$ \\
\hline DY & 66 & 156 & $1.4 E-03$ & $1.4 \mathrm{E}-03$ \\
\hline DY & 66 & 159 & $5.7 E+00$ & $5.7 E+00$ \\
\hline HO & 67 & 163 & $1.3 E-01$ & $1.3 E-01$ \\
\hline HO & 67 & $166 M$ & 5. $9 E-03$ & $5.9 E-03$ \\
\hline TM & 69 & 168 & $5 \cdot 8 E+02$ & $5.8 E+02$ \\
\hline TM & 69 & 170 & $1.6 E+03$ & $1.6 E+03$ \\
\hline TM & 69 & 171 & $5.5 E+03$ & $5.5 E+03$ \\
\hline YB & 70 & 169 & $7.4 E+03$ & $7.4 E+03$ \\
\hline LU & 71 & 173 & $1.5 E-01$ & $1.5 \mathrm{E}-01$ \\
\hline LU & 71 & 174 & 3. $3 E-02$ & $3.3 E-02$ \\
\hline LU & 71 & $174 M$ & $6.1 E-01$ & $6.1 E-01$ \\
\hline LU & 71 & 176 & 9. $9 E-03$ & $9.9 E-03$ \\
\hline LU & 71 & $177 M$ & 1. $7 E+00$ & $1.7 E+00$ \\
\hline HF & 72 & 172 & $2.1 E-01$ & $1.6 \mathrm{E}-01$ \\
\hline HF & 72 & 174 & $1.8 E-02$ & $1.7 E-02$ \\
\hline$H F$ & 72 & 175 & 8. $5 E+01$ & $9.4 E+00$ \\
\hline $\mathrm{HF}$ & 72 & 181 & $1.4 E+02$ & $1.0 E+01$ \\
\hline $\mathrm{HF}$ & 72 & 182 & $2.8 \mathrm{E}-02$ & $2.6 E-02$ \\
\hline TA & 73 & 179 & $2.6 E+00$ & $1.4 E+00$ \\
\hline TA & 73 & 182 & 1. $6 E+00$ & 3. $2 \mathrm{E}-01$ \\
\hline & 74 & 181 & $6.0 E+00$ & $6.0 E+00$ \\
\hline
\end{tabular}

$\begin{array}{lccc}\text { ELEMENT } & \text { ATOMIC } & \text { I SOTOPE } & \text { ADULT } \\ \text { SYMBOL } & \text { NO. (Z) } & \text { MASSIA) } & \text { UC IM**2 } \\ \text { W } & & & \\ \text { W } & 74 & 185 & 6.0 E+00 \\ \text { RE } & 74 & 188 & 5.8 E-01 \\ \text { RE } & 75 & 183 & 2.3 E+00 \\ \text { RE } & 75 & 184 & 1.5 E+00 \\ \text { RE } & 75 & 184 M & 2.6 E-01 \\ \text { OS } & 75 & 187 & 5.5 E+01 \\ \text { OS } & 76 & 185 & 7.6 E-01 \\ \text { IR } & 76 & 194 & 1.2 E-02 \\ \text { IR } & 77 & 192 & 7.0 E-01 \\ \text { PT } & 77 & 192 M & 6.0 E-03 \\ \text { PT } & 78 & 190 & 2.3 E-03 \\ \text { PT } & 78 & 192 & 2.7 E-03 \\ \text { AU } & 78 & 193 & 9.6 E-02 \\ \text { HG } & 79 & 195 & 6.2 E+01 \\ \text { HG } & 80 & 154 & 1.7 E-01 \\ \text { TL } & 80 & 203 & 3.5 E+00 \\ \text { PB } & 81 & 204 & 1.4 E+01 \\ \text { PB } & 82 & 202 & 4.5 E+01 \\ \text { PB } & 82 & 2 C 4 & 1.0 E+01 \\ \text { PB } & 82 & 2 C 5 & 3.1 E+02 \\ \text { BI } & 82 & 210 & 7.1 E+00 \\ \text { BI } & 83 & 207 & 2.7 E+00 \\ \text { BI } & 83 & 2 C 8 & 1.3 E+00 \\ \text { PO } & 83 & 210 M & 6.4 E-01 \\ \text { PO } & 84 & 2 C 8 & 6.6 E-03 \\ \text { PO } & 84 & 209 & 1.6 E-03 \\ \text { RA } & 84 & 210 & 7.4 E-02 \\ \text { RA } & 88 & 226 & 2.3 E-04 \\ \text { AC } & 88 & 228 & 7.5 E-04 \\ \text { TH } & 89 & 227 & 3.3 E-04 \\ \text { TH } & 90 & 228 & 2.3 E-03 \\ \text { TH } & 90 & 229 & 2.1 E-04 \\ \text { TH } & 90 & 230 & 2.0 E-04 \\ \text { PA } & 90 & 232 & 1.8 E-04 \\ U & 91 & 231 & 1.8 E-04 \\ U & 92 & 232 & 2.1 E-04 \\ \text { U } & 92 & 233 & 1.9 E-04 \\ \text { U } & 92 & 234 & 1.8 E-04 \\ \text { U } & 92 & 235 & 1.6 E-04 \\ \text { U } & 92 & 238 & 1.6 E-04 \\ \text { NP } & 93 & 235 & 1.5 E-04 \\ \text { NP } & 93 & 236 & 1.0 E-03 \\ \text { NP } & 93 & 237 & 1.6 E-04 \\ \text { PU } & 94 & 236 & 1.1 E-04 \\ \text { PU } & 94 & 237 & 2.7 E-02 \\ \text { PU } & 94 & 238 & 1.7 E-04 \\ \text { PU } & 94 & 239 & 1.5 E-04 \\ \text { PU } & 94 & 240 & 1.4 E-04 \\ \text { PU } & 94 & 241 & 2.9 E-04 \\ & & & \end{array}$

INFANT
UC/M**2

$6.0 E+00$ $5.8 \mathrm{E}-01$ 2. $3 E+00$ $1.5 \mathrm{E}+00$ 2.6E-01 5. $5 E+01$ 7. $6 \mathrm{E}-01$ 1. $2 E-02$ 7. $O E-01$ 6. $0 E-03$ 2. $3 E-03$ 2. $7 E-03$ $9.6 \mathrm{E}-02$ 6. $2 \mathrm{E}+01$ 1. $7 \mathrm{E}-01$ 3. $5 E+00$ 1. $4 E+01$ 4. $5 E+01$ 1. $0 E+01$ $3.1 E+02$ 7. $1 E+00$ 2. $7 E+00$ 1. $3 E+00$ $6.4 E-01$ $6.6 E-03$ 1. $6 \mathrm{E}-03$ $7.4 E-02$ $2.3 E-04$ 7. $5 E-04$ 3. $3 E-04$ 2. $3 E-03$ $2.1 \mathrm{E}-04$ 2. $0 E-04$ 1. $8 E-04$ 1. $8 E-04$ 2. $1 E-04$ 1. $9 E-04$ 1. $8 E-04$ $1.6 E-04$ $1.6 E-04$ 1. $5 \mathrm{E}-04$ 3. $0 E-03$ 1. $6 E-04$ 1. $6 E-04$ 1. $E-03$ 1. $7 E-02$ 1. $7 E-04$ 1. $5 E-04$ $1.4 E-04$ 2. $9 E-04$ 


\author{
TABLE 6B. UNIT-RAD DEPOSITION FOR OVARIES
}

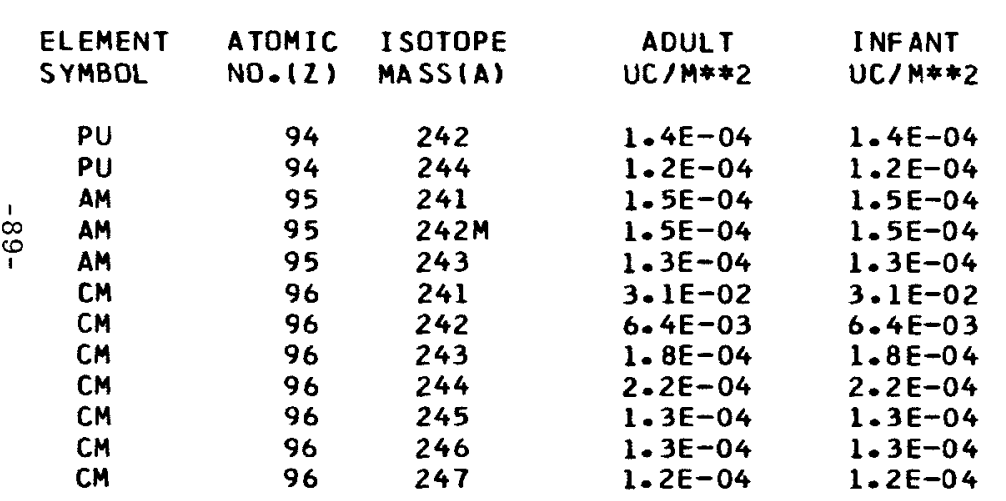

VIA TYPICAL AGRICULTURAL

$\begin{array}{lrc}\text { ELEMENT } & \text { ATOMIC } & \text { ISOTOPE } \\ \text { SYMBDL } & \text { NO. (Z) } & \text { MASS(A) } \\ \text { CM } & 96 & 248 \\ \text { BK } & 97 & 247 \\ \text { BK } & 97 & 249 \\ \text { CF } & 98 & 248 \\ \text { CF } & 98 & 249 \\ \text { CF } & 98 & 250 \\ \text { CF } & 98 & 251 \\ \text { CF } & 98 & 252 \\ \text { ES } & 99 & 252 \\ \text { ES } & 99 & 254 \\ \text { FM } & 100 & 257\end{array}$




$\begin{array}{ccc}\text { ELEMENT } & \text { ATOMIC } & \text { I SOTOPE } \\ \text { SYMBOL } & \text { NO. }(2) & \text { MASS (A) } \\ \text { H } & & \\ \text { BE } & 1 & 3 \\ \text { BE } & 4 & 7 \\ \text { C } & 4 & 10 \\ \text { NA } & 6 & 14 \\ \text { AL } & 11 & 22 \\ \text { SI } & 13 & 26 \\ \text { S } & 14 & 32 \\ \text { CL } & 16 & 35 \\ \text { AR } & 17 & 36 \\ \text { AR } & 18 & 37 \\ \text { AR } & 18 & 39 \\ \text { K } & 18 & 42 \\ \text { CA } & 19 & 40 \\ \text { CA } & 20 & 41 \\ \text { CA } & 20 & 45 \\ \text { SC } & 20 & 48 \\ \text { TI } & 21 & 46 \\ \text { V } & 22 & 44 \\ V & 23 & 49 \\ \text { MN } & 23 & 50 \\ \text { MN } & 25 & 53 \\ \text { FE } & 25 & 54 \\ \text { FE } & 26 & 55 \\ \text { FE } & 26 & 59 \\ \text { CO } & 26 & 60 \\ \text { CO } & 27 & 56 \\ \text { CO } & 27 & 57 \\ \text { CD } & 27 & 58 \\ \text { NI } & 27 & 60 \\ \text { NI } & 28 & 59 \\ \text { ZN } & 28 & 63 \\ \text { GE } & 30 & 65 \\ \text { AS } & 32 & 68 \\ \text { SE } & 33 & 73 \\ \text { SE } & 34 & 75 \\ \text { KR } & 34 & 79 \\ \text { KR } & 36 & 81 \\ \text { RB } & 36 & 85 \\ \text { RB } & 37 & 83 \\ \text { RB } & 37 & 84 \\ \text { SR } & 37 & 87 \\ \text { SR } & 38 & 85 \\ \text { SR } & 38 & 89 \\ \text { Y } & 38 & 90 \\ \text { Y } & 39 & 88 \\ \text { ZR } & 39 & 91 \\ \text { ZR } & 40 & 88 \\ \text { ZR } & 40 & 93 \\ \text { NB } & 41 & 95 \\ & & 91\end{array}$

$\begin{array}{ll}\begin{array}{c}\text { ADULT } \\ \text { UC } / M * 2\end{array} & \text { INFANT } \\ & \text { UC/M**2 } \\ 4.7 E+01 & 4.7 E+01 \\ 4.5 E+06 & 4.1 E+05 \\ 5.3 E+02 & 4.9 E+02 \\ 9.8 E-01 & 9.8 E-01 \\ 1.3 E+01 & 1.3 E+01 \\ 1.0 E+04 & 9.5 E+03 \\ 6.3 E+03 & 6.3 E+03 \\ 9.0 E+01 & 9.0 E+01 \\ 1.4 E-01 & 1.4 E-01 \\ 5.5 E+02 & 5.5 E+02 \\ 2.9 E-02 & 2.9 E-02 \\ 3.1 E-03 & 3.1 E-03 \\ 3.0 E+00 & 3.0 E+00 \\ 2.5 E+04 & 2.5 E+04 \\ 4.8 E+04 & 4.5 E+04 \\ 2.4 E+01 & 2.4 E+01 \\ 3.8 E+04 & 2.1 E+03 \\ 7.5 E+03 & 7.1 E+03 \\ 3.2 E+07 & 2.5 E+07 \\ 4.4 E+03 & 4.4 E+03 \\ 3.8 E+05 & 3.7 E+05 \\ 7.0 E+04 & 6.7 E+04 \\ 8.7 E+04 & 5.4 E+04 \\ 8.4 E+04 & 5.9 E+03 \\ 1.8 E+01 & 1.7 E+01 \\ 3.5 E+03 & 2.7 E+03 \\ 1.2 E+04 & 1.1 E+04 \\ 8.1 E+03 & 6.0 E+03 \\ 8.9 E+01 & 8.8 E+01 \\ 5.3 E+04 & 5.3 E+04 \\ 2.6 E+04 & 2.6 E+04 \\ 7.8 E+01 & 7.8 E+01 \\ 1.2 E-01 & 1.2 E-01 \\ 1.6 E+06 & 1.6 E+06 \\ 8.8 E-01 & 7.6 E-01 \\ 1.5 E-01 & 1.5 E-01 \\ 2.9 E-01 & 2.9 E-01 \\ 5.6 E-02 & 5.6 E-02 \\ 5.1 E+02 & 2.6 E+02 \\ 6.9 E+02 & 2.0 E+02 \\ 1.9 E+01 & 1.9 E+01 \\ 6.8 E+05 & 5.7 E+05 \\ 6.2 E+05 & 5.0 E+05 \\ 2.3 E+03 & 2.3 E+03 \\ 1.7 E+05 & 1.8 E+03 \\ 1.9 E+06 & 1.1 E+04 \\ 6.4 E+04 & 5.5 E+03 \\ 1.1 E+04 & 9.7 E+03 \\ 2.0 E+05 & 1.4 E+04 \\ 2.2 E+02 & 1.9 E+02\end{array}$

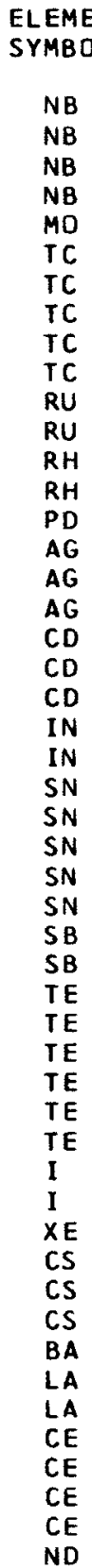

\section{ATOMIC I SOTOPE}

ADULT

INFANT SYMBOL

NO. (Z) MASS(A)

UC $/ M * * 2$

UC $/ M * * 2$

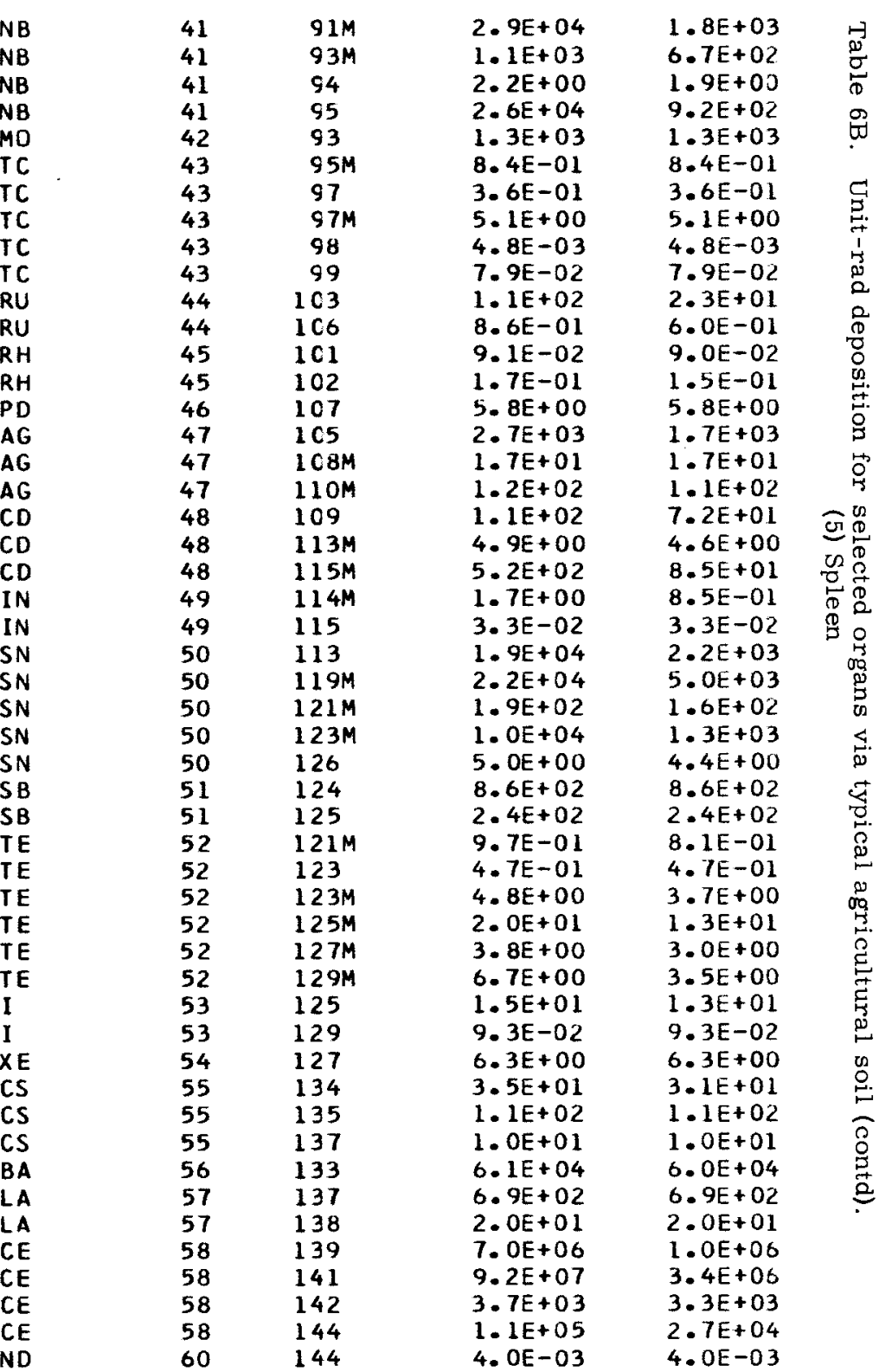




\begin{tabular}{|c|c|c|c|c|}
\hline $\begin{array}{l}\text { ELEMEN T } \\
\text { SYMBOL }\end{array}$ & $\begin{array}{l}\text { ATOMIC } \\
\text { NO. }(Z)\end{array}$ & $\begin{array}{l}\text { ISOTOPE } \\
\text { MASS (A) }\end{array}$ & $\begin{array}{c}\text { ADULT } \\
\text { UC/M } / M=2\end{array}$ & $\begin{array}{l}\text { INFANT } \\
\text { UC/M* } / 2\end{array}$ \\
\hline PM & 61 & 143 & 4. $1 E+05$ & $1.1 E+05$ \\
\hline$P M$ & 61 & 144 & 3. $2 E+04$ & $1.1 E+04$ \\
\hline$P M$ & 61 & 145 & 4. $9 E+04$ & $4.2 E+04$ \\
\hline PM & 61 & 146 & 1. $1 E+04$ & $7.8 E+03$ \\
\hline$P M$ & 61 & 147 & 8. $3 E+03$ & $4.8 E+03$ \\
\hline PM & 61 & $148 M$ & $8.6 E+05$ & $4.6 E+04$ \\
\hline$S M$ & 62 & 145 & $4.0 E+05$ & $1.3 E+05$ \\
\hline$S M$ & 62 & 146 & $4.0 E+02$ & $3.6 E+02$ \\
\hline$S M$ & 62 & 147 & 4. $5 E+02$ & $4.1 E+02$ \\
\hline SM & 62 & 148 & $2.6 E+02$ & $2.3 E+02$ \\
\hline SM & 62 & 149 & $5.5 E+02$ & $5.0 E+02$ \\
\hline SM & 62 & 151 & $7.5 E+04$ & $6.7 E+04$ \\
\hline EU & 63 & 149 & $3.1 E+06$ & $3.3 E+05$ \\
\hline EU & 63 & 149 & $8.5 E+05$ & $1.6 E+05$ \\
\hline EU & 63 & 150 & $6.7 E+03$ & $5.4 E+03$ \\
\hline EU & 63 & 152 & $2.4 E+03$ & $2.1 E+03$ \\
\hline EU & 63 & 154 & $1.8 E+03$ & $1.6 E+03$ \\
\hline EU & 63 & 155 & $2.3 E+05$ & $1.4 E+05$ \\
\hline GD & 64 & 146 & $1.5 E+05$ & 1. $7 E+04$ \\
\hline GD & 64 & 148 & 2. $2 E+02$ & $2.1 E+02$ \\
\hline GD & 64 & 150 & $1.2 E+02$ & $1 \cdot 1 E+02$ \\
\hline GD & 64 & 151 & $4.5 E+05$ & 1. $1 E+05$ \\
\hline GO & 64 & 152 & 2. $9 E+02$ & $2.8 E+02$ \\
\hline GD & 64 & 153 & $3.3 E+05$ & $1.2 E+05$ \\
\hline GD & 64 & 162 & 1. $7 E+04$ & $8.3 E+03$ \\
\hline TB & 65 & 157 & $2.2 E+04$ & $2.1 E+04$ \\
\hline TB & 65 & 158 & $9.7 E+02$ & $9.3 E+02$ \\
\hline TB & 65 & 160 & $4.4 E+05$ & $7.7 E+04$ \\
\hline DY & 66 & 154 & $8.8 E-04$ & $8.8 \mathrm{E}-04$ \\
\hline DY & 66 & 156 & $1.4 \mathrm{E}-03$ & $1.4 \mathrm{E}-03$ \\
\hline DY & 66 & 159 & $5.7 E+00$ & $5.7 E+00$ \\
\hline HO & 67 & 163 & 1. $3 E-01$ & $1.3 E-01$ \\
\hline HO & 67 & $166 \mathrm{M}$ & $5.9 E-03$ & $5.9 E-03$ \\
\hline TM & 69 & 168 & $3.3 E+04$ & $5.8 E+03$ \\
\hline TM & 69 & 170 & $6.9 E+04$ & $1.6 E+04$ \\
\hline TM & 69 & 171 & 8. $7 E+04$ & $5.5 E+04$ \\
\hline$Y B$ & 70 & 169 & $1.0 E+07$ & $7.4 E+05$ \\
\hline LU & 71 & 173 & $1.5 \mathrm{E}-01$ & $1.5 \mathrm{E}-01$ \\
\hline LU & 71 & 174 & 3. $3 E-02$ & $3.3 E-02$ \\
\hline LU & 71 & $174 M$ & $6.1 E-01$ & $6.1 E-01$ \\
\hline LU & 71 & 176 & $9.9 E-03$ & $9.9 E-03$ \\
\hline LU & 71 & $177 M$ & 1. $7 E+00$ & 1. $7 E+00$ \\
\hline $\mathrm{HF}$ & 72 & 172 & 3. $8 E+00$ & $3.2 E+00$ \\
\hline $\mathrm{HF}$ & 72 & 174 & $3.6 E-01$ & $3.4 \mathrm{E}-01$ \\
\hline $\mathrm{HF}$ & 72 & 175 & $1.1 E+03$ & 1. $9 \mathrm{E}+02$ \\
\hline $\mathrm{HF}$ & 72 & 181 & 1. $8 E+03$ & $2.0 E+02$ \\
\hline $\mathrm{HF}$ & 72 & 182 & 5. $5 \mathrm{E}-01$ & $5.2 E-01$ \\
\hline TA & 73 & 179 & 4. $0 E+01$ & $2.9 E+01$ \\
\hline TA & 73 & 182 & 1. $9 E+01$ & $6.3 E+00$ \\
\hline$w$ & 74 & 181 & $6.0 E+00$ & $6.0 E+00$ \\
\hline
\end{tabular}

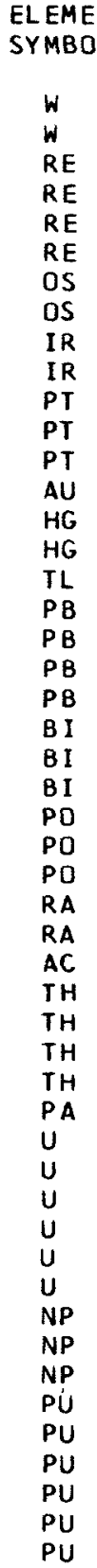

ATOMIC

ISOTOPE

$74 \quad 185$

$\begin{array}{ll}74 & 185 \\ 74 & 188 \\ 75 & 183 \\ 75 & 184 \\ 75 & 184 M \\ 75 & 187 \\ 76 & 185\end{array}$

185

194

192

190

192

193

195
194

194

203

204

$2 \mathrm{C} 2$
$2 \mathrm{C} 4$

204
205
210

210

$2 \mathrm{CB}$

$210 M$

208
209

210

226

228

227

228

229
230
232

232

231

232
233
234

234
235

236

238

235

236

236

237
238

238
239

240

241
AOULT

INFANT

$6.0 E+00$

. $8 E-01$

- $3 E+00$

1. $5 E+00$

2. $6 E-01$

5. $5 E+01$

7. $2 \mathrm{E}-01$

1. $2 E+00$

6. $1 E-03$

2. $2 \mathrm{E}-03$

2. $3 E-03$
$2.8 E-03$

9. $7 E-02$

3. $3 E+02$

1. $4 E+00$

3. $3 E+01$

1. $2 E+01$

2. $1 E+00$

2. $1 E+00$

1. $5 \mathrm{E}+00$

1. $O E+0 O$

5. $1 E-01$

2. $5 \mathrm{E}-01$

7. $0 E-03$

1. $6 E-03$

1. $1 \mathrm{E}-0$

2. $3 E-04$

7. $5 E-04$

3. $3 E-04$

2. $3 E-03$

2. $0 E-04$

1. $8 E-04$

1. $8 \mathrm{E}-04$

2. $1 \mathrm{E}-04$

1. $9 \mathrm{E}-04$

1. $8 E-04$

1. $6 \mathrm{E}-04$

1. $6 E-04$

1. $5 E-04$

3. $0 E-03$

$1.6 E-04$

$1.6 E-04$

2. $7 E-02$

1. $7 E-04$

1. $5 \mathrm{E}-04$

1. $4 E-04$
$2.9 E-04$

$6.0 E+00$

5. $8 E-01$

$2.3 E+00$

$1.5 E+00$

$2.6 E-01$

5. $5 E+01$

$1.2 E-0$

1.

7. $0 E-01$

2. $3 E-03$

$2.3 \mathrm{E}-03$
$2.7 \mathrm{E}-03$

$9.6 \mathrm{E}-02$

1. $4 E+02$

$1.3 E+00$

$2.7 E+0$

1. $2 E+01$

9. $3 E+00$

$2.1 E+00$

$6.4 E+01$

1. $5 E+00$

1. $0 E+00$

5. $1 E-01$

$2.5 E-01$

$6.6 \mathrm{E}-03$

1. $6 E-03$

7. $4 \mathrm{E}-02$

2. $3 E-04$

7. $5 \mathrm{E}-04$

3. $3 E-04$

2. $3 E-03$

2. $1 E-04$

2. OE-04

1. 8E-04

1. $8 \mathrm{E}-04$

2. $1 E-04$

$1.9 E-04$

1. $8 \mathrm{E}-04$

$1.6 E-04$

1. $6 E-04$

1. $5 E-04$

3. $0 E-03$

1. $6 E-04$

1. $6 E-04$

1. $1 \mathrm{E}-03$

2. $7 E-02$

2.7E-02

1.7E-04

1. $5 E-04$

1. $4 E-04$
2. $9 E-04$ 
TABLE 6B. UNIT-RAD DEPOSITION FOR SPLEEN

ELEMENT ATOMIC ISOTOPE $\quad$ ADULT INFANT

$\begin{array}{ll} & P U \\ & P U \\ C & A M \\ O & A M \\ & A M \\ C M \\ C M \\ C M \\ C M \\ C M \\ C M \\ C M\end{array}$

$\begin{array}{ll}94 & 242 \\ 94 & 244 \\ 95 & 241 \\ 95 & 242 M \\ 95 & 243 \\ 96 & 241 \\ 96 & 242 \\ 96 & 243 \\ 96 & 244 \\ 96 & 245 \\ 96 & 246 \\ 96 & 247\end{array}$

$1.4 \mathrm{E}-04$

1. $2 E-04$

1. $5 \mathrm{E}-04$

1. $5 E-04$

1. $3 E-04$

3. $1 E-02$

1. $8 \mathrm{E}-04$

2. $2 E-04$

1. $3 E-04$

1. $3 E-04$

1. $2 E-04$

$1.4 E-04$
$1.2 E-04$

$1.2 E-04$
$1.5 E-04$

1. $5 \mathrm{E}-04$

1. $3 E-04$

3. $1 \mathrm{E}-02$

$6.4 E-03$

$6.4 E-03$

2.2E-04

1. 3E-04

1. $3 E-04$

1. $2 \mathrm{E}-04$

VIA TYPICAL AGRICULTURAL

SOIL.

$05 / 09 / 68$

PAGE 15

ELEMENT

SYMBDL

ATOMIC ISOTOPE

ND. (Z) MASS(A)

ADULT

IE 04

1. $1 E-04$
$1.2 E-04$

2. $8 E-03$

2. $5 E-03$

1. $2 E-04$

2. $7 E-04$

1. $1 E-04$

8. 5E-04

$5.6 E-03$

1. $6 E-03$

9. $3 E-03$

I NF ANT

$U C / M * 2$

1. $1 E-04$ S

1. $2 E-04$

2.8E-03

2.5E-03

1. $2 E-04$

$2.7 E-04$

1. $1 E-04$

8. $5 E-04$

5. $6 E-03$

$1.6 E-03$

9. $3 E-03$ 


$\begin{array}{lcr}\text { ELEMENT } & \text { ATOMIC } & \text { I SOTOP } \\ \text { SYMBOL } & \text { NO. }(Z) & \text { MASS }(A) \\ \text { H } & & \\ \text { BE } & 1 & 3 \\ \text { BE } & 4 & 7 \\ \text { C } & 4 & 10 \\ \text { NA } & 6 & 14 \\ \text { AL } & 11 & 22 \\ \text { SI } & 13 & 26 \\ \text { S } & 14 & 32 \\ \text { CL } & 16 & 35 \\ \text { AR } & 17 & 36 \\ \text { AR } & 18 & 37 \\ \text { AR } & 18 & 39 \\ \text { K } & 18 & 42 \\ \text { CA } & 19 & 40 \\ \text { CA } & 20 & 41 \\ \text { CA } & 20 & 45 \\ \text { SC } & 20 & 48 \\ \text { TI } & 21 & 46 \\ \text { V } & 22 & 44 \\ \text { V } & 23 & 49 \\ \text { MN } & 23 & 50 \\ \text { MN } & 25 & 53 \\ \text { FE } & 25 & 54 \\ \text { FE } & 26 & 55 \\ \text { FE } & 26 & 59 \\ \text { CD } & 26 & 60 \\ \text { CO } & 27 & 56 \\ \text { CO } & 27 & 57 \\ \text { CO } & 27 & 58 \\ \text { NI } & 27 & 60 \\ \text { NI } & 28 & 59 \\ \text { ZN } & 28 & 63 \\ \text { GE } & 30 & 65 \\ \text { AS } & 32 & 68 \\ \text { SE } & 33 & 73 \\ \text { SE } & 34 & 75 \\ \text { KR } & 34 & 79 \\ \text { KR } & 36 & 81 \\ \text { RB } & 36 & 85 \\ \text { RB } & 37 & 83 \\ \text { RB } & 37 & 84 \\ \text { SR } & 37 & 87 \\ \text { SR } & 38 & 85 \\ \text { SR } & 38 & 89 \\ \text { Y } & 38 & 90 \\ \text { Y } & 39 & 88 \\ \text { ZR } & 39 & 91 \\ \text { ZR } & 40 & 88 \\ \text { ZR } & 40 & 93 \\ \text { NB } & 40 & 95 \\ & 41 & 91 \\ & & \end{array}$

$\begin{array}{cl}\text { ADULT } & \text { INFANT } \\ \text { UC } / M * 2 & \text { UC } / M * * 2 \\ 4.7 E+01 & 4.7 E+01 \\ 4.1 E+05 & 4.1 E+05 \\ 4.9 E+02 & 4.9 E+02 \\ 9.8 E-01 & 9.8 E-01 \\ 7.4 E+00 & 7 \cdot 3 E+00 \\ 1.8 E+04 & 1.8 E+04 \\ 7.0 E+03 & 6.9 E+03 \\ 7.3 E+02 & 9.0 E+01 \\ 9.2 E-02 & 9.2 E-02 \\ 5.5 E+02 & 5.5 E+02 \\ 2.9 E-02 & 2.9 E-02 \\ 3.1 E-03 & 3.1 E-03 \\ 4.6 E+00 & 4.5 E+00 \\ 1.8 E+04 & 1.8 E+04 \\ 3.6 E+04 & 3.3 E+04 \\ 1.8 E+01 & 1.8 E+01 \\ 4.2 E+02 & 4.2 E+02 \\ 1.3 E+04 & 1.3 E+04 \\ 8.2 E+06 & B .2 E+06 \\ 1.4 E+03 & 1.4 E+03 \\ 4.4 E+05 & 4.4 E+05 \\ 8.5 E+04 & 7.9 E+04 \\ 7.9 E+05 & 6.4 E+05 \\ 4.4 E+05 & 6.9 E+04 \\ 2.1 E+02 & 2.0 E+02 \\ 1.3 E+02 & 9.6 E+01 \\ 4.4 E+02 & 4.1 E+02 \\ 2.9 E+02 & 2.1 E+02 \\ 3.2 E+00 & 3.1 E+00 \\ 2.1 E+04 & 2.1 E+04 \\ 1.0 E+04 & 1.0 E+04 \\ 1.9 E+02 & 8.8 E+01 \\ 1.2 E-01 & 1.2 E-01 \\ 8.1 E+05 & 8.1 E+05 \\ 7.6 E-01 & 7.6 E-01 \\ 1.5 E-01 & 1.5 E-01 \\ 2.9 E-01 & 2.9 E-01 \\ 5.6 E-02 & 5.6 E-02 \\ 1.8 E+03 & 9.4 E+02 \\ 2.5 E+03 & 7.3 E+02 \\ 7.0 E+01 & 6.9 E+01 \\ 4.5 E+05 & 3.4 E+05 \\ 4.2 E+05 & 3.0 E+05 \\ 1.4 E+03 & 1.4 E+03 \\ 1.4 E+05 & 1.8 E+03 \\ 1.6 E+06 & 1.1 E+04 \\ 7.9 E+04 & 7.6 E+03 \\ 1.5 E+04 & 1.3 E+04 \\ 2.5 E+05 & 1.9 E+04 \\ 2.3 E+02 & 2.3 E+02\end{array}$

$\begin{array}{lcc}\text { ELEMENT } & \text { ATOMIC } & \text { ISOTOPE } \\ \text { SYMBOL } & \text { NO. (Z) } & \text { MASS }(A) \\ & & \\ \text { NB } & 41 & 91 M \\ \text { NB } & 41 & 93 M \\ \text { NB } & 41 & 94 \\ \text { NB } & 41 & 95 \\ \text { MO } & 42 & 93 \\ \text { TC } & 43 & 95 M \\ \text { TC } & 43 & 97 \\ \text { TC } & 43 & 97 M \\ \text { TC } & 43 & 98 \\ \text { TC } & 43 & 99 \\ \text { RU } & 44 & 1 C 3 \\ \text { RU } & 44 & 1 C 6 \\ \text { RH } & 45 & 1101 \\ \text { RH } & 45 & 1 C 2 \\ \text { PD } & 46 & 1 C 7 \\ \text { AG } & 47 & 1 C 5 \\ \text { AG } & 47 & 108 M \\ \text { AG } & 47 & 110 M \\ \text { CD } & 48 & 1 C 9 \\ \text { CD } & 48 & 113 M \\ \text { CD } & 48 & 115 M \\ \text { IN } & 49 & 114 M \\ \text { IN } & 49 & 115 \\ \text { SN } & 50 & 113 \\ \text { SN } & 50 & 119 M \\ \text { SN } & 50 & 121 M \\ \text { SN } & 50 & 123 M \\ \text { SN } & 50 & 126 \\ \text { SB } & 51 & 124 \\ \text { SB } & 51 & 125 \\ \text { TE } & 52 & 121 M \\ \text { TE } & 52 & 123 \\ \text { TE } & 52 & 123 M \\ \text { TE } & 52 & 125 M \\ \text { TE } & 52 & 127 M \\ \text { TE } & 52 & 129 M \\ \text { I } & 53 & 125 \\ \text { I } & 53 & 129 \\ \text { XE } & 54 & 127 \\ \text { CS } & 55 & 134 \\ \text { CS } & 55 & 135 \\ \text { CS } & 55 & 137 \\ \text { BA } & 56 & 133 \\ \text { LA } & 57 & 137 \\ \text { LA } & 57 & 138 \\ \text { CE } & 58 & 139 \\ \text { CE } & 58 & 141 \\ \text { CE } & 58 & 142 \\ \text { CE } & 58 & 144 \\ \text { ND } & 60 & 144\end{array}$

ADULT UC $/ M * * 2$

I NFANT $U C / M * * 2$

$2.2 E+03 \quad 2.2 E+03$

(2E+02

1. $1 E+03$

$1.1 E+03$

8. $4 \mathrm{E}-01$

3. $6 \mathrm{E}-01$

5. $1 E+00$

4. $8 \mathrm{E}-03$

7. $9 E-02$

2. $5 E+02$

1. $8 E+00$

9. $0 E-02$

1. $5 E-01$

5. $8 \mathrm{E}+00$

2. $6 E+03$

2. $1 E+01$

1. $4 \mathrm{E}+02$

9. $2 E+01$

$5.9 E+00$

1. $1 E+02$

8. $5 E-01$

3. $3 E-02$

$4.6 E+03$

$8.4 E+03$

2. $2 E+02$

$2 \cdot 6 E+03$

6. $O E+00$

1. $7 E+03$

4. $7 E+02$

. $7 E-01$

4.7E-O

4.7E- 1

2. $0 E+01$

3. $8 E+00$

6. $7 E+00$

1. $5 E+01$

9. $3 E-02$

$6.3 E+00$

7. $0 \mathrm{E}+01$

2. $2 \mathrm{E}+02$

. $1 E+01$

- $9 E+04$

8. $5 \mathrm{E}+02$

2. $5 E+01$

6. $8 E+04$

7. $8 E+05$

6. $9 E+01$

1. $2 E+0$

4. $0 E-03$

- $2 E+02$

$2.4 E+00$
$1.1 E+03$

$1.7 \mathrm{E}+03$

$1.7 E+03$

3. $6 \mathrm{E}-01$

$5.1 E+00$

4. $8 \mathrm{E}-03$

$7.9 E-02$

4. $5 E+01$

$1.2 E+00$

$9.0 \mathrm{E}-02$

1. $5 E-01$

5. $8 \mathrm{E}+00$

$2.2 E+03$

$2.1 E+01$
$1.4 E+02$

$1 E+02$

$3.0 E+03$

$6.8 E+03$

$2.1 E+02$

1. $7 E+03$

$6.0 E+00$

- $7 E+03$

- $7 E+02$

4.7E-01

$3.7 E+00$

$3.7 E+00$
$1.3 E+01$

3. $0 E+00$

$3.5 E+00$

1. $3 E+01$

$9.3 E-02$

$6.3 E+00$

$6.1 E+01$

2. $E+02$

2. $0 E+01$

$6.9 E+04$

8. $5 \mathrm{E}+02$

$2.5 E+01$

2. $0 E+04$

6. $8 E+04$

$6.6 E+01$

$5.4 E+02$

4. $0 E-03$

$9.2 \mathrm{E}+01$ क

$8.5 E-01$ o $\vec{D}$

$3.3 E-02$ D
$3.0 E+03$ O 


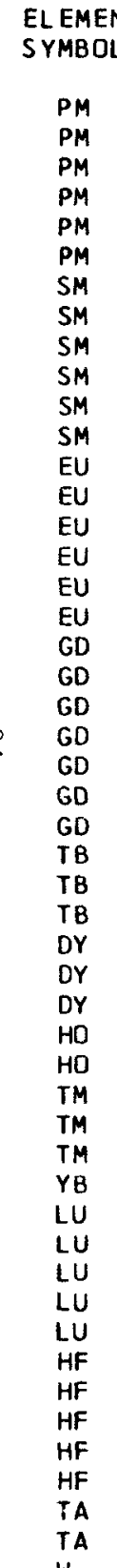

\begin{tabular}{|c|c|c|c|}
\hline $\begin{array}{l}\text { ATOMIC } \\
\text { NO. }(Z)\end{array}$ & $\begin{array}{l}\text { I SOTOPE } \\
\text { MASS (A) }\end{array}$ & $\begin{array}{c}\text { ADULT } \\
\text { UC/M**2 }\end{array}$ & $\begin{array}{l}\text { INFANT } \\
\text { UC/M**2 }\end{array}$ \\
\hline 61 & 143 & $2.7 E+04$ & $1.1 E+04$ \\
\hline 61 & 144 & $2.2 E+03$ & $1.1 E+03$ \\
\hline 61 & 145 & $4.5 E+03$ & $4.2 E+03$ \\
\hline 61 & 146 & $9.7 E+02$ & $7.8 E+02$ \\
\hline 61 & 147 & $6.7 E+02$ & $4.8 E+02$ \\
\hline 61 & $148 M$ & 4. $9 E+04$ & $4.6 E+03$ \\
\hline 62 & 145 & $2.8 E+05$ & $1.3 E+05$ \\
\hline 62 & 146 & $3.8 E+02$ & $3.6 E+02$ \\
\hline 62 & 147 & $4 \cdot 3 E+02$ & $4.1 E+02$ \\
\hline 62 & 148 & $2.4 E+02$ & $2.3 E+02$ \\
\hline 62 & 149 & $5.2 E+02$ & $5.0 E+02$ \\
\hline 62 & 151 & $7.1 E+04$ & $6.7 E+04$ \\
\hline 63 & 148 & $2.7 E+06$ & $3.3 E+05$ \\
\hline 63 & 149 & $7.5 E+05$ & $1.6 E+05$ \\
\hline 63 & 150 & $6.5 E+03$ & $5.4 E+03$ \\
\hline 63 & 152 & $2.4 E+03$ & $2.1 E+03$ \\
\hline 63 & 154 & 1. $8 E+03$ & $1.6 E+03$ \\
\hline 63 & 155 & $2.2 E+05$ & $1.4 E+05$ \\
\hline 64 & 146 & $1.3 E+04$ & $1.7 E+03$ \\
\hline 64 & 148 & $2.2 E+01$ & $2.1 E+01$ \\
\hline 64 & 150 & $1.2 E+01$ & $1 \cdot 1 E+01$ \\
\hline 64 & 151 & 4. $0 E+04$ & $1.1 E+04$ \\
\hline 64 & 152 & 2. $9 E+01$ & $2.8 E+01$ \\
\hline 64 & 153 & $3.0 E+04$ & $1 \cdot 2 E+04$ \\
\hline 64 & 162 & $1.6 E+03$ & $8.3 E+02$ \\
\hline 65 & 157 & $2.3 E+03$ & $2.1 E+03$ \\
\hline 65 & 158 & 9. $8 E+01$ & $9.3 E+01$ \\
\hline 65 & 160 & 5. $0 E+04$ & 7. $7 E+03$ \\
\hline 66 & 154 & $8.8 E-04$ & $8.8 E-04$ \\
\hline 66 & 156 & $1.4 E-03$ & $1.4 E-03$ \\
\hline 66 & 159 & 5. $7 E+00$ & $5.7 E+00$ \\
\hline 67 & 163 & $1 \cdot 3 E-01$ & $1.3 E-01$ \\
\hline 67 & $166 M$ & 5. $9 E-03$ & $5.9 E-03$ \\
\hline 69 & 168 & $1.6 E+04$ & $2.9 E+03$ \\
\hline 69 & 170 & $3.4 E+04$ & $8.2 E+03$ \\
\hline 69 & 171 & $4.3 E+04$ & 2. $7 E+04$ \\
\hline 70 & 169 & $5.1 E+05$ & $3.7 E+04$ \\
\hline 71 & 173 & $1.5 E-01$ & $1.5 E-01$ \\
\hline 71 & 174 & 3. $3 E-02$ & 3. $3 E-02$ \\
\hline 71 & $174 M$ & $6.1 E-01$ & $6.1 E-01$ \\
\hline 71 & 176 & 9. $9 E-03$ & $9.9 E-03$ \\
\hline 71 & $177 M$ & 1. $7 E+00$ & $1.7 \mathrm{E}+00$ \\
\hline 72 & 172 & 4. $1 E+02$ & $3.2 E+02$ \\
\hline 72 & 174 & 3. $7 E+01$ & $3.4 E+01$ \\
\hline 72 & 175 & 1. $7 E+05$ & $1.9 E+04$ \\
\hline 72 & 181 & 2. $7 E+05$ & $2.0 E+04$ \\
\hline 72 & 182 & $5.6 E+01$ & $5.2 E+01$ \\
\hline 73 & 179 & $2.6 E+01$ & $1.4 E+01$ \\
\hline 73 & 182 & $1.6 E+01$ & $3.2 E+00$ \\
\hline 74 & 181 & $6.0 E+00$ & $6.0 E+00$ \\
\hline
\end{tabular}

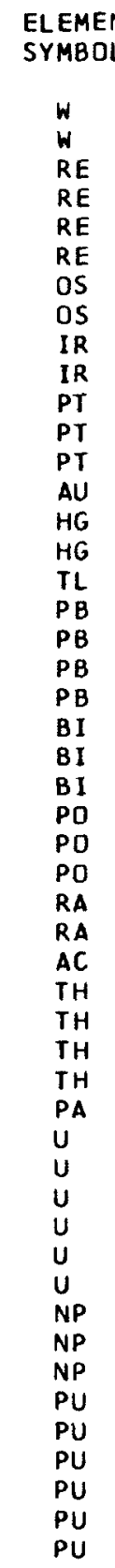

ATOMIC ISOTOPE
VD. $(Z)$ MASSIA)

\begin{tabular}{ll}
74 & 185 \\
74 & 188 \\
75 & 183 \\
75 & 184 \\
75 & $184 M$ \\
75 & 187 \\
76 & 185 \\
76 & 194 \\
77 & 192 \\
77 & $192 M$ \\
78 & 190 \\
78 & 192 \\
78 & 193 \\
79 & 195 \\
80 & 194 \\
80 & 203 \\
81 & $2 C 4$ \\
82 & 202 \\
82 & 204 \\
82 & 205 \\
82 & 210 \\
83 & $2 C 7$ \\
83 & $2 C 8$ \\
83 & $210 M$ \\
84 & $2 C 8$ \\
84 & 209 \\
84 & 210 \\
88 & 226 \\
88 & 228 \\
89 & 227 \\
90 & 228 \\
90 & 229 \\
90 & 230 \\
90 & 232 \\
91 & 231 \\
92 & 232 \\
92 & 233 \\
92 & 234 \\
92 & 235 \\
92 & 236 \\
92 & 238 \\
93 & 235 \\
93 & 236 \\
93 & 237 \\
94 & 236 \\
94 & 237 \\
94 & 238 \\
94 & 239 \\
94 & 240 \\
94 & 241 \\
\hline
\end{tabular}

ADULT INFANT $U C / M * * 2 \quad U C / M * * 2$

\begin{tabular}{|c|c|}
\hline $\begin{array}{l}\text { 6. } 0 E+00 \\
5.8 E-01 \\
2.3 E+00 \\
1.5 E+00 \\
2.6 E-01 \\
5.5 E+01 \\
7.6 E-01 \\
1.2 E-02 \\
7.0 E-01 \\
6.0 E-03 \\
2.3 E-03 \\
2.7 E-03 \\
9.6 E-02 \\
1.3 E+02 \\
1.7 E-01 \\
3.5 E+00 \\
1.6 E+01 \\
4.0 E+01 \\
9.1 E+00 \\
2.8 E+02 \\
6.4 E+00 \\
2.7 E-01 \\
1.3 E-01 \\
6.4 E-02 \\
6.6 E-03 \\
1.6 E-03 \\
7.4 E-02 \\
2.3 E-04 \\
7.5 E-04 \\
3.3 E-04 \\
2.3 E-03 \\
2.1 E-04 \\
2.0 E-04 \\
1.8 E-04 \\
1.8 E-04 \\
2.1 E-04 \\
1.9 E-04 \\
1.8 E-04 \\
1.6 E-04 \\
1.6 E-04 \\
1.5 E-04 \\
3.0 E-03 \\
1.6 E-04 \\
1.6 E-04 \\
1.1 E-03\end{array}$ & $\begin{array}{l}6.0 E+00 \\
5.8 E-01 \\
2.3 E+00 \\
1.5 E+00 \\
2.6 E-01 \\
5.5 E+01 \\
7.6 E-01 \\
1.2 E-02 \\
7.0 E-01 \\
6.0 E-03 \\
2.3 E-03 \\
2.7 E-03 \\
9.6 E-02 \\
1.3 E+02 \\
1.7 E-01 \\
3.5 E+00 \\
1.6 E+01 \\
4.0 E+01 \\
9.1 E+00 \\
2.8 E+02 \\
6.4 E+00 \\
2.7 E-01 \\
1.3 E-01 \\
6.4 E-02 \\
6.6 E-03 \\
1.6 E-03 \\
7.4 E-02 \\
2.3 E-04 \\
7.5 E-04 \\
3.3 E-04 \\
2.3 E-03 \\
2.1 E-04 \\
2.0 E-04 \\
1.8 E-04 \\
1.8 E-04 \\
2.1 E-04 \\
1.9 E-04 \\
1.8 E-04 \\
1.6 E-04 \\
1.6 E-04 \\
1.5 E-04 \\
3.0 E-03 \\
1.6 E-04 \\
1.6 E-04 \\
1.1 E-03\end{array}$ \\
\hline
\end{tabular}


TABLE 68. UNIT-RAD DEPOSITION FOR TESTES

\begin{tabular}{|c|c|c|c|c|}
\hline $\begin{array}{l}\text { ELEMENT } \\
\text { SYMBOL }\end{array}$ & $\begin{array}{l}\text { ATOMIC } \\
\text { NO. }(Z)\end{array}$ & $\begin{array}{l}\text { I SOTOPE } \\
\text { MASS (A) }\end{array}$ & $\begin{array}{c}\text { ADULT } \\
\text { UC/M**2 }\end{array}$ & $\begin{array}{l}\text { INFANT } \\
\text { UC } / M * * 2\end{array}$ \\
\hline $\begin{array}{l}P U \\
P U \\
A M \\
A M \\
A M \\
C M \\
C M \\
C M \\
C M \\
C M \\
C M \\
C M\end{array}$ & $\begin{array}{l}94 \\
94 \\
95 \\
95 \\
95 \\
96 \\
96 \\
96 \\
96 \\
96 \\
96 \\
96\end{array}$ & $\begin{array}{l}242 \\
244 \\
241 \\
242 M \\
243 \\
241 \\
242 \\
243 \\
244 \\
245 \\
246 \\
247\end{array}$ & $\begin{array}{l}1.4 E-04 \\
1.2 E-04 \\
1.5 E-04 \\
1.5 E-04 \\
1.3 E-04 \\
3.1 E-02 \\
6.4 E-03 \\
1.8 E-04 \\
2.2 E-04 \\
1.3 E-04 \\
1.3 E-04 \\
1.2 E-04\end{array}$ & $\begin{array}{l}1.4 E-04 \\
1.2 E-04 \\
1.5 E-04 \\
1.5 E-04 \\
1.3 E-04 \\
3.1 E-02 \\
6.4 E-03 \\
1.8 E-04 \\
2.2 E-04 \\
1.3 E-04 \\
1.3 E-04 \\
1.2 E-04\end{array}$ \\
\hline
\end{tabular}

VIA TYPICAL AGRICULTURAL SOIL

$\begin{array}{crc}\begin{array}{c}\text { ELEMENT } \\ \text { SYMBOL }\end{array} & \begin{array}{r}\text { ATOMIC } \\ \text { NO. }(Z)\end{array} & \begin{array}{c}\text { I SOTOPE } \\ \text { MASSIA) }\end{array} \\ \text { CM } & 96 & 248 \\ \text { BK } & 97 & 247 \\ \text { BK } & 97 & 249 \\ \text { CF } & 98 & 248 \\ \text { CF } & 98 & 249 \\ \text { CF } & 98 & 250 \\ \text { CF } & 98 & 251 \\ \text { CF } & 98 & 252 \\ \text { ES } & 99 & 252 \\ \text { ES } & 99 & 254 \\ \text { FM } & 100 & 257\end{array}$




\begin{tabular}{|c|c|c|c|c|}
\hline $\begin{array}{l}\text { ELEMENT } \\
\text { SYMBOL }\end{array}$ & $\begin{array}{l}\text { ATOMIC } \\
\text { NO. }(Z)\end{array}$ & $\begin{array}{l}\text { I SOTOPE } \\
\text { MASS (A) }\end{array}$ & $\begin{array}{c}\text { ADULT } \\
\text { UC/M**2 }\end{array}$ & $\begin{array}{l}\text { INFANT } \\
\text { UC/M**2 }\end{array}$ \\
\hline$H$ & 1 & 3 & 4. $7 E+01$ & 4. $7 E+01$ \\
\hline$B E$ & 4 & 7 & $4.1 E+08$ & $9.5 E+07$ \\
\hline$B E$ & 4 & 10 & $1.2 E+05$ & $1.1 E+05$ \\
\hline$c$ & 6 & 14 & $9.8 \mathrm{E}-01$ & $9.8 E-01$ \\
\hline NA & 11 & 22 & $7.7 E+00$ & $7.6 E+00$ \\
\hline$A L$ & 13 & 26 & $1.7 E+04$ & $1.6 E+04$ \\
\hline SI & 14 & 32 & $7.2 E+03$ & 7. $2 \mathrm{E}+03$ \\
\hline$S$ & 16 & 35 & $7.3 E+02$ & $3.6 E+02$ \\
\hline $\mathrm{CL}$ & 17 & 36 & $1.1 E-01$ & $1.1 \mathrm{E}-01$ \\
\hline AR & 18 & 37 & $5.5 E+02$ & $5.5 E+02$ \\
\hline AR & 18 & 39 & $2.9 E-02$ & $2.9 E-02$ \\
\hline AR & 18 & 42 & $3.1 E-03$ & $3.1 E-03$ \\
\hline K & 19 & 40 & $5 \cdot 9 E+00$ & 5. $9 E+00$ \\
\hline CA & 20 & 41 & $1.1 E+03$ & $2.1 E+02$ \\
\hline CA & 20 & 45 & $4.0 E+04$ & $3.9 E+02$ \\
\hline CA & 20 & 48 & $1.1 E+00$ & $2.1 E-01$ \\
\hline SC & 21 & 46 & $3.8 E+05$ & $2.1 E+04$ \\
\hline TI & 22 & 44 & $3.9 E+03$ & $3.7 E+03$ \\
\hline$v$ & 23 & 49 & $2.5 E+07$ & $2.2 E+07$ \\
\hline v & 23 & 50 & 3. $8 E+03$ & $3.8 E+03$ \\
\hline MN & 25 & 53 & $2 \cdot 7 E+05$ & $2.7 E+05$ \\
\hline MN & 25 & 54 & $5.4 E+04$ & $4.8 E+04$ \\
\hline $\mathrm{FE}$ & 26 & 55 & $8.6 E+05$ & $4.7 E+05$ \\
\hline FE & 26 & 59 & $9.6 E+05$ & $5.1 E+04$ \\
\hline $\mathrm{FE}$ & 26 & 60 & 1. $7 E+02$ & $1.5 E+02$ \\
\hline $\mathrm{CO}$ & 27 & 56 & $1.4 E+04$ & $1.1 E+04$ \\
\hline $\mathrm{CO}$ & 27 & 57 & $5.0 E+04$ & $4.6 E+04$ \\
\hline $\mathrm{CO}$ & 27 & 58 & $3.3 E+04$ & $2.5 E+04$ \\
\hline $\mathrm{CO}$ & 27 & 60 & $3.6 E+02$ & $3.6 E+02$ \\
\hline NI & 28 & 59 & $2.0 E+04$ & $1.9 E+04$ \\
\hline NI & 28 & 63 & 9. $9 E+03$ & $9.0 E+03$ \\
\hline$Z N$ & 30 & 65 & 3. $2 E+02$ & $6.7 E+01$ \\
\hline GE & 32 & 68 & $1 \cdot 2 E-01$ & $1.2 \mathrm{E}-01$ \\
\hline AS & 33 & 73 & $1.1 E+05$ & $2.3 E+04$ \\
\hline SE & 34 & 75 & $1.7 \mathrm{E}+01$ & $1.5 E+01$ \\
\hline SE & 34 & 79 & $3.1 E+00$ & $3.1 E+00$ \\
\hline$K R$ & 36 & 81 & $2.9 E-01$ & $2.9 E-01$ \\
\hline$K R$ & 36 & 85 & $5.6 E-02$ & $5.6 E-02$ \\
\hline$R B$ & 37 & 83 & 4. $7 E+03$ & $2.4 E+03$ \\
\hline RB & 37 & 84 & $6.3 E+03$ & $1.8 E+03$ \\
\hline$R B$ & 37 & 87 & $1.8 E+02$ & $1.7 E+02$ \\
\hline SR & 38 & 85 & $2.5 E+06$ & 1. $2 E+04$ \\
\hline$S R$ & 38 & 89 & $2.7 E+06$ & $1.0 E+04$ \\
\hline SR & 38 & 90 & $2.2 E+02$ & $4.8 E+01$ \\
\hline$Y$ & 39 & 88 & $2.4 E+05$ & $1.8 E+03$ \\
\hline$Y$ & 39 & 91 & $2.7 E+06$ & 1. $1 \mathrm{E}+04$ \\
\hline$Z R$ & 40 & 88 & $4.4 E+05$ & 7. $0 E+04$ \\
\hline $2 R$ & 40 & 93 & $1.3 E+05$ & $1.2 E+05$ \\
\hline$Z R$ & 40 & 95 & $1.4 E+06$ & 1. $7 E+05$ \\
\hline NB & 41 & 91 & $3.3 E+02$ & $3.0 E+02$ \\
\hline
\end{tabular}

\begin{tabular}{|c|c|c|c|c|}
\hline $\begin{array}{l}\text { ELEMENT } \\
\text { SYMBOL }\end{array}$ & $\begin{array}{l}\text { ATOMIC } \\
\text { NO. (Z) }\end{array}$ & $\begin{array}{l}\text { I SOTOPE } \\
\text { MASSI(A) }\end{array}$ & $\begin{array}{l}\text { ADULT } \\
\text { UC / } / M * * 2\end{array}$ & $\begin{array}{l}\text { INFANT } \\
\text { UC/M\# } / M 2\end{array}$ \\
\hline NB & 41 & $91 M$ & 3. $8 E+04$ & $2.8 E+03$ \\
\hline NB & 41 & $93 M$ & $1.6 E+03$ & $1.1 E+03$ \\
\hline NB & 41 & 94 & $3.4 E+00$ & $3.0 E+00$ \\
\hline NB & 41 & 95 & $3.3 E+04$ & $1.4 E+03$ \\
\hline MO & 42 & 93 & 4. $4 E+02$ & $4.3 E+02$ \\
\hline TC & 43 & S5M & $1.8 E+01$ & 1. $7 E+01$ \\
\hline TC & 43 & 97 & 7. $1 E+00$ & $7.1 E+00$ \\
\hline$T C$ & 43 & $97 M$ & 1. $1 E+02$ & $1.0 E+02$ \\
\hline TC & 43 & 98 & $9.6 \mathrm{E}-02$ & $9.6 E-02$ \\
\hline TC & 43 & s9 & $1.6 E+00$ & $1.6 E+00$ \\
\hline RU & 44 & 103 & 9. $7 E+02$ & $2 \cdot 3 E+02$ \\
\hline RU & 44 & 106 & $8.1 E+00$ & $6.0 E+00$ \\
\hline RH & 45 & 101 & $9.1 E-02$ & 9. $0 \mathrm{E}-02$ \\
\hline RH & 45 & $1 C 2$ & $1.6 E-01$ & $1.5 E-01$ \\
\hline PD & 46 & 107 & 5. $8 E+00$ & $5.8 E+00$ \\
\hline AG & 47 & $1 C 5$ & $1.5 E+03$ & $6.3 E+02$ \\
\hline AG & 47 & $108 M$ & $6.4 E+00$ & $6.2 E+00$ \\
\hline$A G$ & 47 & $110 M$ & 4. $7 E+O L$ & $3.9 E+01$ \\
\hline$C D$ & 48 & 109 & $1.0 E+02$ & 7. $0 E+01$ \\
\hline CD & 48 & $113 M$ & 4. $7 E+00$ & $4.5 E+00$ \\
\hline CD & 48 & $115 \mathrm{M}$ & 4. $7 E+02$ & $8.2 E+01$ \\
\hline IN & 49 & $114 M$ & 1. $7 E+00$ & $8.5 E-01$ \\
\hline IN & 49 & 115 & 3. $3 E-02$ & $3.3 E-02$ \\
\hline SN & 50 & 113 & 1. $0 E+04$ & $3.8 E+03$ \\
\hline SN & 50 & $119 M$ & 1. $5 E+04$ & $8.4 E+03$ \\
\hline SN & 50 & $121 \mathrm{M}$ & 2. $7 E+02$ & $2.6 E+02$ \\
\hline SN & 50 & $123 M$ & $5.6 E+03$ & $2.1 E+03$ \\
\hline SN & 50 & 126 & $7.6 E+00$ & $7.4 \mathrm{E}+00$ \\
\hline SB & 51 & 124 & $2.8 E+03$ & 1. $7 E+03$ \\
\hline SB & 51 & 125 & $4 \cdot 9 E+02$ & 4. $7 E+02$ \\
\hline$T E$ & 52 & $121 M$ & 8. $9 E+00$ & $8.1 E+00$ \\
\hline$T E$ & 52 & 123 & 4. $7 E+00$ & $4.7 E+00$ \\
\hline TE & 52 & $123 M$ & 4. $3 E+01$ & 3. $7 E+01$ \\
\hline$T E$ & 52 & $125 M$ & $1.6 E+02$ & $1.3 E+02$ \\
\hline TE & 52 & $127 M$ & $3.4 E+01$ & $3.0 E+01$ \\
\hline TE & 52 & $129 M$ & 5. $1 E+01$ & $3 \cdot 5 E+01$ \\
\hline I & 53 & 125 & 4. $3 E+04$ & 1. $3 E+04$ \\
\hline 1 & 53 & 129 & 9. $1 E+01$ & $8.9 E+01$ \\
\hline$X E$ & 54 & 127 & $6.3 E+00$ & $6.3 E+00$ \\
\hline CS & 55 & 134 & $1.4 E+03$ & $1 \cdot 3 E+03$ \\
\hline CS & 55 & 135 & $4.5 E+03$ & 4. $5 E+03$ \\
\hline CS & 55 & 137 & $4 \cdot 3 E+02$ & $4 \cdot 2 E+02$ \\
\hline BA & 56 & 133 & 3. $9 E+03$ & $3.8 E+03$ \\
\hline LA & 57 & 137 & 1. $2 E+03$ & $1.1 E+03$ \\
\hline LA & 57 & 138 & $3.4 E+01$ & $3.2 E+01$ \\
\hline $\mathrm{CE}$ & 58 & 139 & 1. $0 E+06$ & 2. $0 E+05$ \\
\hline CE & 58 & 141 & 1. $3 E+07$ & $6.8 E+05$ \\
\hline$C E$ & 58 & 142 & 7. $1 E+02$ & $6.6 E+02$ \\
\hline$C E$ & 58 & 144 & 1. $6 E+04$ & $5.4 E+03$ \\
\hline ND & 60 & 144 & $4 \cdot 3 E-03$ & $4.0 E-03$ \\
\hline
\end{tabular}

\section{ATOMIC ISOTOPE}

UC IM**2 


$\begin{array}{cccc}\text { ATOMIC } & \text { ISOTOPE } & \text { ADULT } & \text { INFANT } \\ \text { NO. } 121 & \text { MASS }(A) & \text { UCIM**2 } & \text { UC } / M *+2 \\ 61 & 143 & 3.8 E+05 & 1.1 E+05 \\ 61 & 144 & 3.0 E+04 & 1.1 E+04 \\ 61 & 145 & 4.8 E+04 & 4.2 E+04 \\ 61 & 146 & 1.1 E+04 & 7.8 E+03 \\ 61 & 147 & 8.0 E+03 & 4.8 E+03 \\ 61 & 148 M & 7.8 E+05 & 4.6 E+04 \\ 62 & 145 & 3.8 E+05 & 1.3 E+05 \\ 62 & 146 & 3.9 E+02 & 3.6 E+02 \\ 62 & 147 & 4.5 E+02 & 4.1 E+02 \\ 62 & 148 & 2.5 E+02 & 2.3 E+02 \\ 62 & 149 & 5.4 E+02 & 5.0 E+02 \\ 62 & 151 & 7.4 E+04 & 6.7 E+04 \\ 63 & 148 & 4.2 E+06 & 3.3 E+05 \\ 63 & 149 & 1.1 E+06 & 1.6 E+05 \\ 63 & 150 & 7.3 E+03 & 5.4 E+03 \\ 63 & 152 & 2.5 E+03 & 2.1 E+03 \\ 63 & 154 & 1.9 E+03 & 1.6 E+03 \\ 63 & 155 & 2.7 E+05 & 1.4 E+05 \\ 64 & 146 & 2.1 E+05 & 1.7 E+04 \\ 64 & 148 & 2.3 E+02 & 2.1 E+02 \\ 64 & 150 & 1.2 E+02 & 1.1 E+02 \\ 64 & 151 & 6.0 E+05 & 1.1 E+05 \\ 64 & 152 & 3.0 E+02 & 2.8 E+02 \\ 64 & 153 & 4.1 E+05 & 1.2 E+05 \\ 64 & 162 & 2.1 E+04 & 8.3 E+03 \\ 65 & 157 & 2.4 E+04 & 2.1 E+04 \\ 65 & 158 & 1.0 E+03 & 9.3 E+02 \\ 65 & 160 & 7.9 E+05 & 7.7 E+04 \\ 66 & 154 & 9.7 E-04 & 8.8 E-04 \\ 66 & 156 & 1.5 E-03 & 1.4 E-03 \\ 66 & 159 & 3.3 E+01 & 5.7 E+00 \\ 67 & 163 & 2.1 E+04 & 1.9 E+04 \\ 67 & 166 M & 9.2 E+02 & 8.3 E+02 \\ 69 & 168 & 5.1 E+05 & 5.8 E+04 \\ 69 & 170 & 1.0 E+06 & 1.6 E+05 \\ 69 & 171 & 1.1 E+06 & 5.5 E+05 \\ 70 & 169 & 1.7 E+07 & 7.4 E+05 \\ 71 & 173 & 5.2 E+04 & 2.1 E+04 \\ 71 & 174 & 7.3 E+03 & 4.6 E+03 \\ 71 & 174 M & 4.8 E+05 & 8.6 E+04 \\ 71 & 176 & 1.5 E+03 & 1.4 E+03 \\ 71 & 177 M & 1.4 E+06 & 2.4 E+05 \\ 72 & 172 & 2.1 E+02 & 1.6 E+02 \\ 72 & 174 & 1.8 E+01 & 1.7 E+01 \\ 72 & 175 & 8.5 E+04 & 9.4 E+03 \\ 72 & 181 & 1.4 E+05 & 1.0 E+04 \\ 72 & 182 & 2.8 E+01 & 2.6 E+01 \\ 73 & 179 & 4.0 E+02 & 2.9 E+02 \\ 73 & 182 & 1.9 E+02 & 6.3 E+01 \\ 74 & 181 & 6.1 E+00 & 6.0 E+00\end{array}$

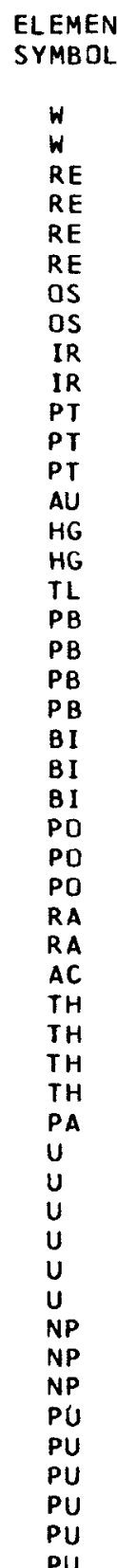

$\begin{array}{ll}\text { ATOMIC } & \text { ISOTOPE } \\ \text { NO.(Z) } & \text { MASS(A) }\end{array}$

ADULT

I NF ANT

$\begin{array}{ll}74 & 185 \\ 74 & 188 \\ 75 & 183\end{array}$

$6.1 E+00$ $U C / M * 2$ 5.8E-01 $2.3 E+03$ $1.7 E+00$ 2.7E-01 $7.5 E+01$

7. $7 E-01$
$1.2 E-02$ 1. $2 E-02$ 8. $9 E+01$ 6. $1 E-01$ 2. $3 E-03$ 2. $8 \mathrm{E}-03$

9. $6 \mathrm{E}-02$

$1.6 \mathrm{E}+02$ 3. $7 E+00$ 8. $6 E+01$ 1. $5 E+01$ 8. $5 E+00$ $1.9 E+00$ 5. $9 E+01$ $1.5 E+00$ 3. $7 E+00$ $1.8 E+00$ 8. $8 E-01$ 8. $8 E-01$ $6.8 E-03$
$1.6 E-03$ 1. $6 E-03$ 9. $\mathrm{OE}-02$ 1. $2 \mathrm{E}+02$ 7. $3 E+02$ 2. 7E-03 1. $9 E-01$ 3. $4 E-03$ 3. $1 E-03$ $2.8 \mathrm{E}-03$ 2.1E-03 $7.2 E+01$ $6.5 E+01$ $6.2 E+01$ 5. $7 E+01$ 5. $6 E+01$ 5. $4 E+01$ $2.9 E-01$ 1. $7 E-03$ 1. $\mathrm{BE}-03$ 1. $8 E-03$ 7. $3 E-02$ 3. $9 E+01$ 2. $6 E-03$ $2.6 E-03$ 7. $\mathrm{OE}-03$ $1.5 E+00$ 2. $6 E-01$ $5.5 E+01$ $7.6 E-01$ $1.2 \mathrm{E}-02$ 7. $0 E+01$ $6.0 E-01$ 2. $3 E-03$ 2. $7 E-03$ $9.6 E-02$ $9.9 E+01$ $3.4 E+00$ $7.1 E+01$ 1. $5 E+01$ $6.9 E+00$ 1. $6 \mathrm{E}+00$ 4. $7 E+01$ $1.1 E+00$ $3 \cdot 7 \mathrm{E}+00$ 1. $8 E+00$ 8. 8E-01 $6.6 E-03$ $1.6 \mathrm{E}-03$ 7.4E-02 4. $3 E+01$ $1.4 E+02$ $3 \cdot 3 E-04$ 2. 3E-03 $2.1 E-04$ 2. $0 E-04$ 1. $8 E-04$ 1. $8 E-04$ 7. $1 E+01$ $6.4 E+01$ 6. $1 E+01$ 5. $6 E+01$ $5.6 E+01$ $5.3 E+01$ $3.0 E-03$ $1.6 E-04$ 1. $6 \mathrm{E}-0$ 1. $1 \mathrm{E}-03$ 2.7E-02 1. $7 \mathrm{E}-04$ 1. $5 E-04$ $1.4 E-04$ 2. $9 E-04$ 
TABLE 6B. UNIT-RAD DEPOSITION FOR WHOLE BODY

VIA TYPICAL AGRICULTURAL SOIL.

\begin{tabular}{|c|c|c|c|c|}
\hline $\begin{array}{l}\text { ELEMENT } \\
\text { SYMBOL }\end{array}$ & $\begin{array}{l}\text { A TоMIC } \\
\text { NO. }(Z)\end{array}$ & $\begin{array}{l}\text { I SOTOPE } \\
\text { MASS(A) }\end{array}$ & $\begin{array}{c}\text { ADULT } \\
\text { UC / M**2 }\end{array}$ & $\begin{array}{l}\text { INFANT } \\
\text { UC/M**2 }\end{array}$ \\
\hline $\begin{array}{l}P U \\
P U \\
A M \\
A M \\
A M \\
C M \\
C M \\
C M \\
C M \\
C M \\
C M \\
C M\end{array}$ & $\begin{array}{l}94 \\
94 \\
95 \\
95 \\
95 \\
96 \\
96 \\
96 \\
96 \\
96 \\
96 \\
96\end{array}$ & $\begin{array}{l}242 \\
244 \\
241 \\
242 M \\
243 \\
241 \\
242 \\
243 \\
244 \\
245 \\
246 \\
247\end{array}$ & $\begin{array}{l}2.5 E-03 \\
2.2 E-03 \\
8.9 E-04 \\
8.9 E-04 \\
7.8 E-04 \\
2.1 E+01 \\
9.5 E-01 \\
1.4 E-03 \\
1.9 E-03 \\
9.2 E-04 \\
8.9 E-04 \\
8.3 E-04\end{array}$ & $\begin{array}{l}1.4 \mathrm{E}-04 \\
1.2 \mathrm{E}-04 \\
1.5 \mathrm{E}-04 \\
1.5 \mathrm{E}-04 \\
1.3 \mathrm{E}-04 \\
3.1 \mathrm{E}-02 \\
6.4 \mathrm{E}-03 \\
1.8 \mathrm{E}-04 \\
2.2 \mathrm{E}-04 \\
1.3 \mathrm{E}-04 \\
1.3 \mathrm{E}-04 \\
1.2 \mathrm{E}-04\end{array}$ \\
\hline
\end{tabular}

$\begin{array}{lrc}\text { ELEMENT } & \text { ATOMIC } & \text { ISOTOPE } \\ \text { SYMBOL } & \text { NO. (Z) } & \text { MASSIA) } \\ \text { CM } & 96 & 248 \\ \text { BK } & 97 & 247 \\ \text { BK } & 97 & 249 \\ \text { CF } & 98 & 248 \\ \text { CF } & 98 & 249 \\ \text { CF } & 98 & 250 \\ \text { CF } & 98 & 251 \\ \text { CF } & 98 & 252 \\ \text { ES } & 99 & 252 \\ \text { ES } & 99 & 254 \\ \text { FM } & 100 & 257\end{array}$

$05 / 09 / 58$

ADULT UC $/ M * * 2$

8. $O E-04$ 2. $1 E-03$

5. $9 E-01$

4. $7 E-01$

2. $2 E-03$

7. $1 E-03$

1. $9 E-03$

6. $\mathrm{OE}-02$

2. $6 E+00$

2. $2 E-01$

I NFANT $U C / M * * 2$

1. 1E-04 1. $2 E-04$ 2. $8 E-03$ 2. $5 E-03$ 1. $2 \mathrm{E}-04$ $1.2 E-04$ 1. $1 \mathrm{E}-04$ 8. $5 E-04$ 5. $6 E-03$ 1. $6 E-03$
$9.3 E-03$ 
$\begin{array}{ll}\text { Al:ulT } & \text { INFANT } \\ U C / M * * 2 & \text { UC } / M * * 2\end{array}$

4. $7 E+01$

8. $4 E+05$

9. $9 E+02$

7. $6 \mathrm{E}-01$

4. $9 E+00$

$1.1 \mathrm{E}+03$

8. $O E+04$

1. $5 E+03$

4. $1 \mathrm{E}-02$

9. $5 E+04$

4. $8 E+00$

5. $2 E-01$

3. $5 E+03$

6. $5 E+03$

3. $5 \mathrm{E}+00$

3. $0 E+04$

4. $6 E+03$

2. $2 E+05$

3. $8 \mathrm{E}+01$

3. $3 E+04$

5. $9 E+03$

2. $7 E+06$

3. $0 E+05$

8. $4 E+02$

1. $8 E+03$

7. $6 E+03$

4. $1 E+03$

5. $9 E+01$

1. $6 E+04$

7. $8 \mathrm{E}+03$

2. $3 E+02$

9. $9 E+00$

4. $8 E+04$

2. $7 E+01$

5. $3 E+00$

9. $5 E+00$

9. $5 E+03$

7. $6 E+03$

6. $9 E+02$

4. $9 E+04$

4. $3 E+04$

1. $9 E+02$

9. $2 E+03$

5. $8 E+04$

1. $0 E+05$

1. $8 E+05$

2. $5 E+05$
4. $7 E+01$

8. $2 E+05$

$9.9 E+02$

7. $6 \mathrm{E}-01$

4. $9 E+00$

1. $1 E+03$

8. $0 E+54$

1. $5 E+03$

4. $1 E-02$

$9.1 E+04$

4. $8 E+00$

5. $2 E-01$

$5.1 E+00$

$3.5 E+03$

$6.4 E+03$

3. $5 E+00$

3. $0 E+04$

$4.6 E+03$

$2.2 E+05$

3. $8 \mathrm{E}+0 \mathrm{O}$

$3.3 E+04$

$5.8 E+03$

$2.7 E+06$

2. $9 E+05$

$8.4 E+02$

$1.8 E+03$

$7.6 E+03$

$4.0 E+03$

5. $9 E+01$

$1.6 E+04$

7. $8 E+03$

$2.3 E+02$

$9.8 E+00$

$4.7 E+04$

2. $6 E+01$

$5.3 E+00$

$9.5 E+00$

$1.9 E+35$

9. $4 E+03$

$7.3 E+03$

6. $9 E+02$

4. $2 E+04$

$1.9 E+02$

5. $6 E+04$

1. $0 E+05$

$1.8 E+05$

$2.5 E+05$

\section{ELEMENT}

ATOAIC

I SOTOPE

AOULT

INFANT

SYMBC

NB
NE
NG
NE
MO
TC
TC
TC
TC
TC
RU

$\begin{array}{ll}41 & \\ 41 & \\ 41 & \\ 41 & \\ 42 & 9 \\ 43 & 9 \\ 43 & \\ 43 & \\ 43 & 9 \\ 43 & 9 \\ 44 & 10\end{array}$

$91 M$
$93 M$

94

93

$95 M$

97

98
99

99
103

106

101

101
102
107

107
105

$108 \mathrm{M}$

$110 M$

109

$113 M$

$114 \mathrm{M}$

115

113

$119 M$

$121 M$

$123 M$

126

124

125

$121 \mathrm{M}$

123

$123 \mathrm{M}$

$125 M$

$127 M$

$129 M$
125
129

129
127

134

135

137

133

137

138
139

139

142

144
144

$U C / M * 2 \quad U C / M * * 2$

4. $4 E+03 \quad 4.3 E+03$

1. $6 E+03$

4. $6 E+00$

2. $3 E+03$

5. $8 E+01$

2. $4 E+01$

3. $5 E+02$

3. $2 \mathrm{E}-01$

5. $3 E+002$

2. $2 E+01$

6. $5 E-01$

1. $1 E+00$

9.7E+O1

4. $O E+02$

3. $8 \mathrm{E}+00$

$2.4 E+01$

$3.4 E+0$

2. $2 E+01$

4. $1 E+02$

4. $0 E+02$

1. $5 E+01$

4. $8 E+02$

1. $1 E+03$

2. $7 E+02$

2. $7 E+02$

4. $O E+03$

1. $1 \mathrm{E}+03$

1. $9 E+01$

1. $1 E+01$

8. $6 E+01$

3. $O E+02$

6. $8 E+01$

8. $3 E+01$

8. $9 E+04$

6. $0 E+02$

$6.6 E+01$

2. $4 E+03$

8. $4 E+03$

7. $9 E+02$

2. $1 E+03$

9. $9 E+03$

2. $9 E+02$

1. $O E+05$

3. $6 E+05$

3. $3 E+02$

2. $7 E+03$

1. $6 E+03$
$4.6 E+00$

$2.2 E+03$

9. $8 E+02$

5. $6 E+01$

$2.4 E+01$

$3.4 E+02$

3. 2E-01

$8.4 E+02$

2. $2 E+01$

6. $4 E-01$

$1.1 E+00$

9. $7 E+01$

3. $9 E+02$

3. $8 E+00$

$2.4 E+01$

$3.4 E+02$

2. $2 E+01$

4. $0 E+02$

3. $9 E+02$

4. $8 E+02$

$1.1 E+03$

3. $3 E+01$

$2.7 \mathrm{E}+02$

9. $4 E-01$

3. $9 E+03$

1. $1 E+03$

1. $8 E+01$

$1.1 E+01$

8. $5 \mathrm{E}+01$

2. $9 E+02$

6. $8 E+01$

7. $9 E+01$

8. $7 E+04$

$6.0 \mathrm{E}+02$

6. $3 E+01$

2. $4 E+03$

$8.4 E+03$

7. $9 E+02$

2. E+03

2.1E+03

$2.9 E+02$

2. $9 E+02$

1. $O E+05$

3. $4 E+05$

$3.3 E+02$

- $9 E+02$

1. $9 E+02$ 
TABLE 6B. UNIT-RAD DEPOSITION FOR GI TRACT

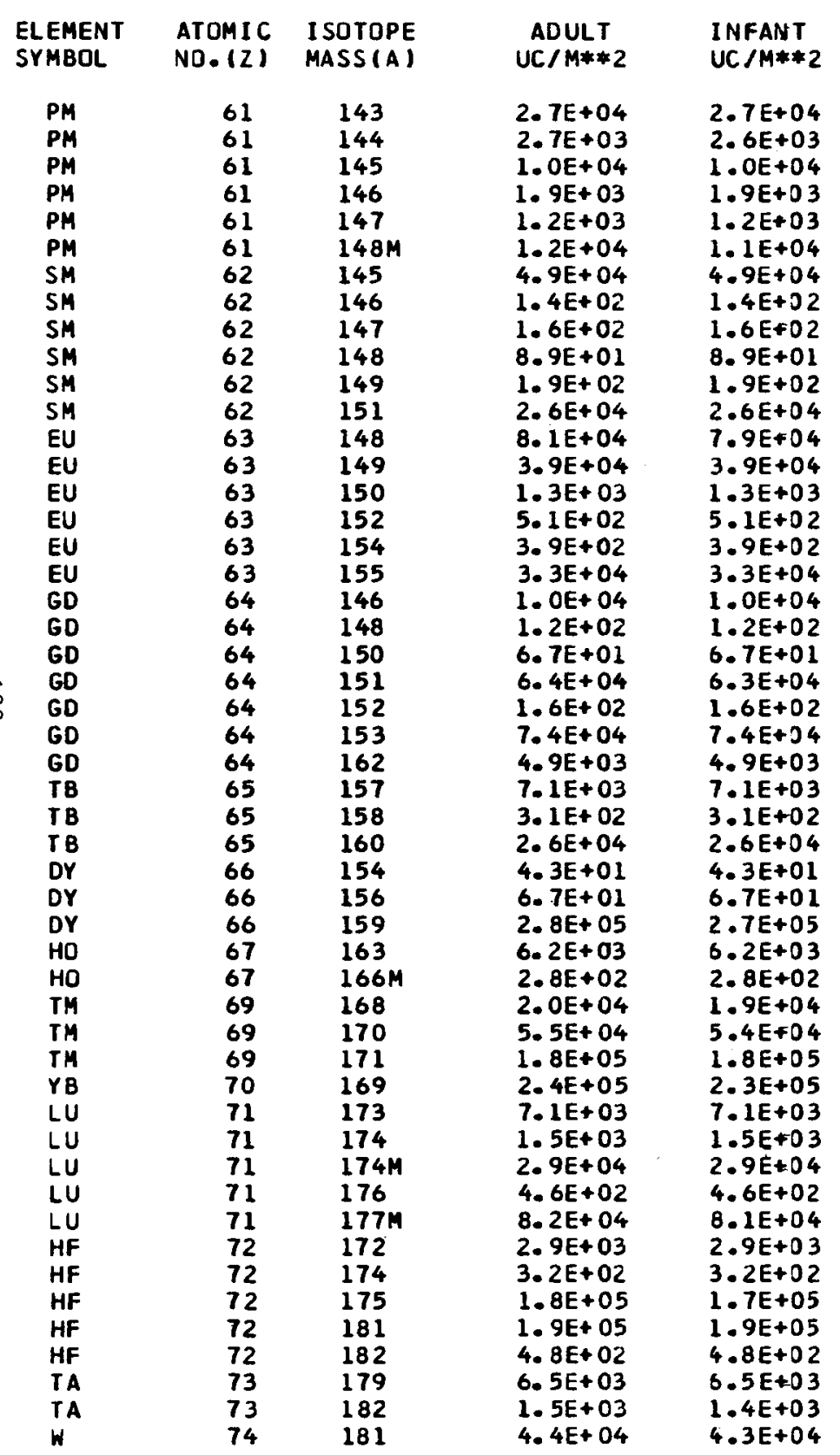

VIA TYPICAL AGRICULTURAL SOIL.

ELEMENT
SYMBOL

ATOMIC I SOTOPE
ND. (Z) MASSIA)

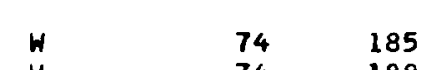

W

RE

RE

RE

OS

OS

IR

PT

PT

AU

HG

TL

PB
PB

PB

PE

BI

PO

PQ

RA

RA
RA
AC

AC

TH

TH

PA

U

U

U

NP

NP
PU

PU

PU

PU

ADULT

I NFANT

4. $4 E+04 \quad 403 E+04$

$4.3 E+04$ 4. $2 E+03$ $1.1 \mathrm{E}+03$ 6. $7 E+02$ $1.2 E+02$ $2.5 E+04$ 2. $8 E+02$ 4. $4 E+00$ 5. $9 E+00$ 4. 7E-01 5. $6 E-01$ $2.0 E+01$ $5.7 E+04$ $5.7 E+04$
$1.1 E+01$ $2.4 E+02$ $4.1 E+01$ 3. $9 E+01$ 8. $9 E+00$ 2. $7 E+02$ $6.2 E+00$ 3. $8 \mathrm{E}+00$ $1.9 E+00$ 9.1E-01 $1.6 E-03$ ह 7. $4 E-02$ 1. $2 E+01$ 4. $0 E+01$ 3. $3 E-04$ 2. $3 E-03$ $2.1 E-04$ $2.1 E-04$
$2.0 E-04$ 1. $8 E-04$ 1. $8 E-04$ $1.4 E+01$ $1.2 E+01$ 1. $2 E+01$ 1. $1 E+01$ 1. $1 E+01$ 1. $0 E+01$ 3. $0 E-03$ 3. $0 E-03$
$1.6 E-04$ $1.6 E-04$
$1.6 E-04$ 1. $1 E-03$ 2. $7 E-02$ $1.7 E-04$ $1.5 \mathrm{E}-04$ 1. $4 \mathrm{E}-04$ 2. $9 E-04$ 
TABLE 6B. UNIT-RAD DEPOSITION FOR GI TRACT

\begin{tabular}{|c|c|c|c|c|}
\hline $\begin{array}{l}\text { ELEMENT } \\
\text { SYMBOL }\end{array}$ & $\begin{array}{l}\text { ATOMIC } \\
\text { NO. }(2)\end{array}$ & $\begin{array}{l}\text { ISOTOPE } \\
\text { MASS (A) }\end{array}$ & $\begin{array}{l}\text { ADULT } \\
U C / M * * 2\end{array}$ & $\begin{array}{l}\text { INFANT } \\
\text { UC/M**2 }\end{array}$ \\
\hline $\begin{array}{l}P U \\
P U \\
A M \\
A M \\
A M \\
C M \\
C M \\
C M \\
C M \\
C M \\
C M \\
C M\end{array}$ & $\begin{array}{l}94 \\
94 \\
95 \\
95 \\
95 \\
96 \\
96 \\
96 \\
96 \\
96 \\
96 \\
96\end{array}$ & $\begin{array}{l}242 \\
244 \\
241 \\
242 M \\
243 \\
241 \\
242 \\
243 \\
244 \\
245 \\
246 \\
247\end{array}$ & $\begin{array}{l}1.4 \mathrm{E}-04 \\
1.2 \mathrm{E}-04 \\
1.5 \mathrm{E}-04 \\
1.5 \mathrm{E}-04 \\
1.3 \mathrm{E}-04 \\
3.2 \mathrm{E}-02 \\
6.4 \mathrm{E}-03 \\
1.8 \mathrm{E}-04 \\
2.2 \mathrm{E}-04 \\
1.3 \mathrm{E}-04 \\
1.3 \mathrm{E}-04 \\
1.2 \mathrm{E}-04\end{array}$ & $\begin{array}{l}1.4 \mathrm{E}-04 \\
1.2 \mathrm{E}-04 \\
1.5 \mathrm{E}-04 \\
1.5 \mathrm{E}-04 \\
1.3 \mathrm{E}-04 \\
2.1 \mathrm{E}-02 \\
3.4 \mathrm{E}-03 \\
1.8 \mathrm{E}-04 \\
2.2 \mathrm{E}-04 \\
1.3 \mathrm{E}-04 \\
1.3 \mathrm{E}-04 \\
1.2 \mathrm{E}-04\end{array}$ \\
\hline
\end{tabular}

VIA TYPICAL AGRICULTURAL

SJIL.

ELEMENT SYMBOL

ATOMIC
VD. I
96
97
97
98
98
96
98
98
99
99
100

$10 / 09 / 68$

ADULT $U C / M * * 2$

1. $1 E-04$ 1. $2 E-04$ 2. $9 E-03$ 2. $5 \mathrm{E}-03$ 1. $2 E-04$

2. $7 E-04$

1. $1 E-04$

8. $5 E-04$

5. $7 E-03$

$1.6 E-03$

9. $5 E-03$ INFANT
UC/M**2

1. 1 E- 04 $1.2 E-04$ 2. 8E-03 $2.5 \mathrm{E}-03$ $1.2 E-04$ 2. $7 E-04$ 1.1E-04

8. $5 E-04$ 5. $6 E-03$ 1. $6 E-03$ $1.6 E-03$
$9.3 E-03$ 
-

•

- 
10/22/68: Insert after $C_{A}$ divider page.

\section{7. $\mathrm{C}_{\mathrm{A}}$ : CONCENTRATIONS IN WATER}

$\underline{\mathrm{C}}_{\mathrm{A}}:$ Seawater

The concentrations of various elements in the ocean as reported in the literature often vary over orders of magnitude. Much of this difference can often be accounted for as a difference between the open ocean and the continental shelf or an estuarine area. The higher concentrations are reported for the latter which are abundant with suspended material. Thus, this difference is often accounted for as the difference between the "element in solution" and the total concentration. At the same time, in those cases where there is a wide range in the concentration in the water, the literature also demonstrates a proportionate range in the concentration in sea food. In other words, the "element in solution" appears to be in equilibrium with suspended material; hence, the total concentration is representative of the biological exchangeable pool. Therefore, in each case we have selected the higher values, since in any peaceful application of nuclear explosives, such as the construction of harbors or canals, the continental shelf or estuarine situation will be the area of interest. The $\mathrm{C}_{\mathrm{A}}$ values were assigned according to the procedures and code numbers shown below.

Rules for estimating $\mathrm{C}_{\mathrm{A}}$ of seawater

Code \#

1 The $\mathrm{C}_{\mathrm{A}}$ was taken from the measured values in the literature in accordance with the above paragraph.

4 The $\mathrm{C}_{\mathrm{A}}$ for scandium, yttrium, lanthanum and the rare earths were based upon a composite of all the data on these elements as discussed in the Appendix under cerium.

8 The $\mathrm{C}_{\mathrm{A}}$ for the platinum metals were based upon ruthenium as described in the Appendix under rhodium.

9 The $\mathrm{C}_{\mathrm{A}}$ for the actinide elements were based upon plutonium as described in the Appendix under actinium.

99 The $\mathrm{C}_{\mathrm{A}}$ was estimated from collateral data as described in the Appendix. 


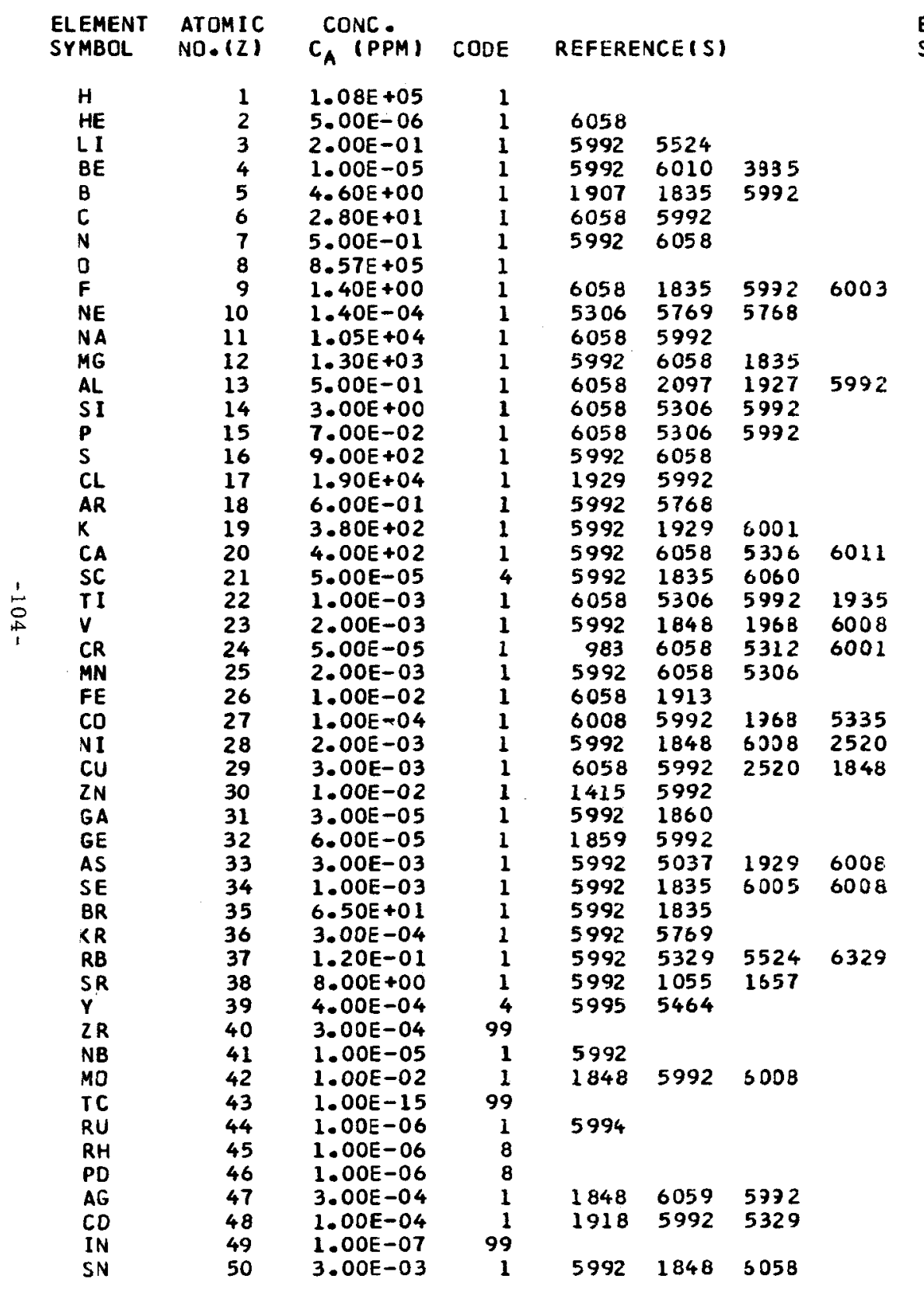

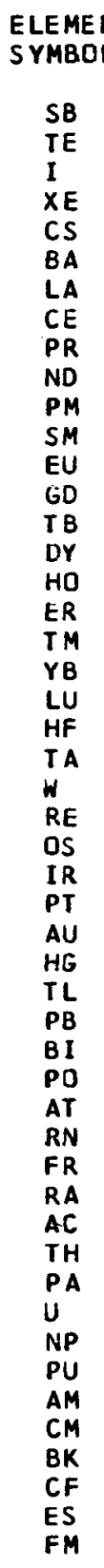

ATOMIC
NC. $(Z)$

51
52
53
54
55
56
57
58
59
60
61
62
63
64
65
66
67
68
69
70
71
72
73
74
75
76
77
78
79
80
81
82
83
84
85
86
87
88
89
90
91
92
93
94
95
96
97
98
99
100
7

CONC. CA (PPM) CODE

5.00E-04 $1.00 E-05$ $.00 E-02$ $1.00 E-04$ 5. 00 E- 04 $.00 E-02$ $.00 E-04$ .00E-04 5. OOE-05 $2.00 E-04$ 4. $00 E-24$ 5.00E-05 .0E-06 $3.00 E-05$ .00E-05 .0DE $5.00 \mathrm{E}-06$ $6.00 E-06$ $6.00 E-06$ 5. OOE-06
$6.00 E-06$ $5.00 E-06$ $6.00 E-06$ 2.00E-06 1.00E-04 1. 0 E 04 $1.00 \mathrm{E}-06$ $1.00 \mathrm{E}-08$ $1.00 E-05$ .00E-05 $3.00 E-05$ $1.00 E-06$ 5.00E-03 $2.00 E-04$

$1.00 \mathrm{E}-13$

$1.00 E-21$

$1.40 E-14$ $1.00 \mathrm{E}-22$

$1.00 E-10$

.00E-O9

$3.00 E-05$

$7.00 E-06$ $3.00 \mathrm{E}-0 \mathrm{~S}$ $7.00 E-11$

7.00E- 12

$7.00 E-29$

$7.00 E-29$

$7.00 E-29$

7.00E-29

$7.00 \mathrm{E}-29$
$7.00 \mathrm{E}-29$
REFERENCE (S)

$\begin{array}{llll}5992 & 5306 & 6060 & 6059 \\ 5992 & 6058 & 4400 & 5034 \\ 5992 & 5769 & & \\ 5992 & 1930 & 6060 & 2151 \\ 5992 & 1657 & & \\ 5995 & 5992 & 1835 & \\ 5995 & 6026 & 5992 & 1835\end{array}$

6008

6219

$\begin{array}{llll}6000 & 1922 & 1852 \quad 6059\end{array}$ $1968 \quad 1835$

$1935 \quad 1848$

$1935 \quad 5992$

$1989506 \quad 5230$

19895314

6256

$\begin{array}{llll}1911 & 1920 \quad 5992 \quad 3885\end{array}$

60491299 


\section{CONCENTRATIONS IN WATER (contd)}

\section{$\underline{\mathrm{C}}_{\mathrm{A}}:$ Freshwater}

In the case of freshwater, the differences in concentration often represent differences in the source of the water. Therefore, average values were selected for freshwater concentrations. The $\mathrm{C}_{\mathrm{A}}$ values were assigned according to the procedures and code numbers shown below.

Rules for estimating $\mathrm{C}_{\mathrm{A}}$ of freshwater

\section{Code \#}

1 The $\mathrm{C}_{\mathrm{A}}$ was taken from measured values in the literature.
4 The $\mathrm{C}_{\mathrm{A}}$ for scandium, yttrium, lanthanum and the rare earths were based upon a composite of all the data on these elements as discussed in the Appendix under cerium.

7 The $\mathrm{C}_{\mathrm{A}}$ was assumed to be the same as that of seawater.

8 The $\mathrm{C}_{\mathrm{A}}$ for the platinum metals were based upon ruthenium as discussed in the Appendix under rhodium.

9 The $\mathrm{C}_{\mathrm{A}}$ for the actinide elements were based upon plutonium as dis cussed in the Appendix under actinium.

99 The $\mathrm{C}_{\mathrm{A}}$ was estimated from collateral data as described in the Appendix. 


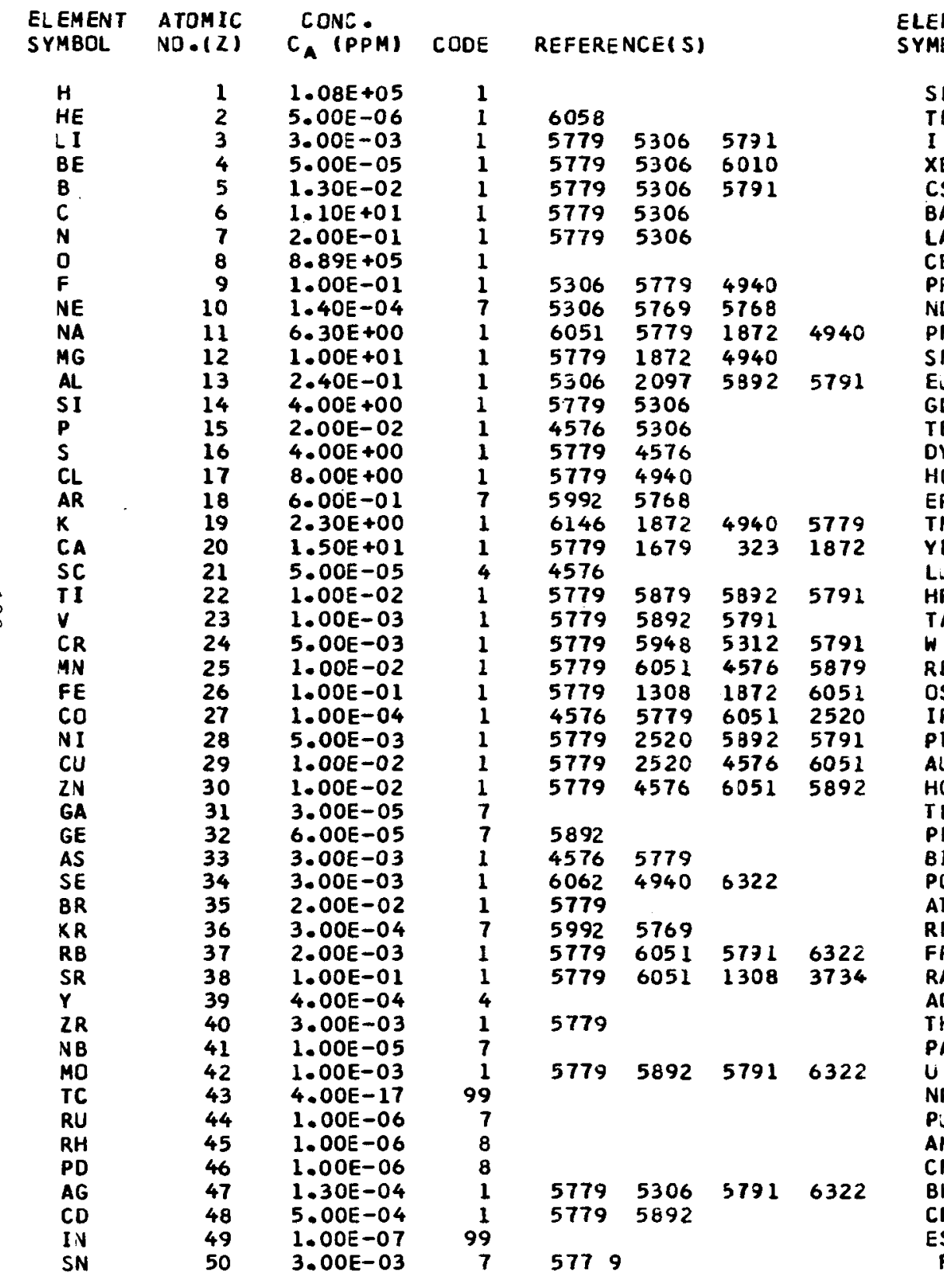

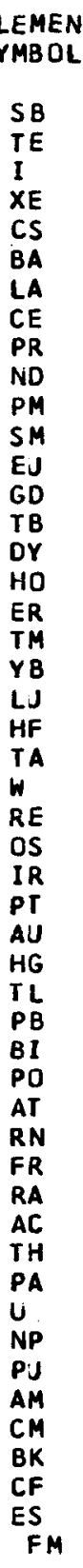

ATOMIC
NO.(2)

CONC.

C. (PPM) CODE

REFERENCE (S)

$\begin{array}{rrrrrr}5.00 E-04 & 1 & 6322 & & & \\ 3.00 E-05 & 99 & & & & \\ 2.00 E-03 & 1 & 928 & 5779 & & \\ 1.00 E-04 & 7 & 5992 & 5769 & & \\ 2.00 E-05 & 1 & 6051 & 5779 & 6146 & 6322 \\ 5.00 E-02 & 1 & 5779 & 5791 & & \\ 3.00 E-04 & 4 & 4576 & & & \end{array}$

$4.00 E-04$

$5.00 E-05$

2. $00 \mathrm{E}-04$

4. $00 E-24$

$5.00 E-05$

8.00E-06

3. $00 E-05$

$5.00 E-06$

$2.00 E-05$

5. $00 E-06$

6. $00 E-06$

$5.00 E-06$

$6.00 E-06$

5.00E-06

$6.00 E-05$

$2.00 E-06$

$1.00 E-04$

$8.40 E-06$

$1.00 E-06$

$1.00 E-08$

$1.00 E-05$

$3.00 E-05$

1. 0 E 04

$5.00 \mathrm{E}-03$

$5.00 E-03$
$2.00 E-04$

$2.00 E-04$
$3.00 E-13$

3. $00 \mathrm{E}-13$

4. $00 E-23$

$3.50 E-13$

4. $00 \mathrm{E}-24$

4. $00 \mathrm{E}-10$

$7.00 E-09$

1.0OE-06

$7.00 E-11$

7. $00 \mathrm{E}-12$

$7.00 \mathrm{E}-12$

$7.00 \mathrm{E}-29$

$7.00 E-29$
$7.00 E-29$

$7.00 \mathrm{E}-29$
$7.00 \mathrm{E}-29$

$7.00 E-29$

$7.00 E-29$
57795306

$\begin{array}{lll}5779 & 5892 \quad 5791\end{array}$

5892

$5306 \quad 5779 \quad 4930$

$\begin{array}{llll}5779 & 4939 & 1301 \quad 4930\end{array}$

$\begin{array}{llll}5779 & 5306 & 5314 & 6256\end{array}$

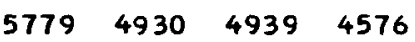

5780 


\section{8. $C^{*}$ : RATIO OF AQUATIC DIET TO TERRESTRIAL DIET}

This ratio is used to correct the $C_{B}$ values where a trace element might be present in higher concentration in a totally aquatic diet than in a terrestrial diet. It was assumed that if this occurs, the $C_{B}$ value would increase in direct proportion to the intake.

The concentrations in the terrestrial diet are those listed in Table $5 \mathrm{C}$ for the GI tract. The concentrations in the aquatic diet were determined by assuming that the diet is composed of $60 \%$ plants, $20 \%$ invertebrates, and $20 \%$ fish. These percentages together with the concentrations listed in Tables 11 and 12 were then used to calculate the aquatic diet concentrations.

If the calculated ratio of the concentrations was less than 1, it was set equal to 1. This was done to account for those situations where the trace element might be essential and the body could compensate for a lowered intake.

Rules for estimating $C^{*}$

Code \#

1 The $C^{*}$ was set equal to 1.

2 The $C^{*}$ was set equal to the calculated value. 


\begin{tabular}{|c|c|c|c|c|c|c|c|c|}
\hline $\begin{array}{l}\text { EL EMENT } \\
\text { SYMBOL }\end{array}$ & $\begin{array}{l}\text { ATOMIC } \\
\text { NO. }(Z)\end{array}$ & $c^{*}$ & CODE & $\begin{array}{l}\text { ELEMENT } \\
\text { SYMBOL }\end{array}$ & $\begin{array}{l}\text { ATOMIC } \\
\text { NO. }(Z)\end{array}$ & $c^{*}$ & CODE & \\
\hline $\begin{array}{l}H \\
H E \\
L I \\
B E \\
B \\
C \\
N \\
D \\
F \\
N E \\
N A \\
M G \\
A L \\
S I \\
P \\
S \\
C L \\
A R \\
R \\
C A \\
S C \\
T I \\
V \\
C R \\
M N \\
F E \\
C D \\
N I \\
C U \\
Z N \\
G A \\
G E \\
A S \\
S E \\
B R \\
X R \\
R B \\
S R \\
Y \\
Z R \\
N B \\
M O \\
I C \\
R U \\
R H \\
P D \\
A G \\
C D \\
I N \\
S N\end{array}$ & $\begin{array}{l}1 \\
2 \\
3 \\
4 \\
5 \\
6 \\
7 \\
8 \\
9 \\
10 \\
11 \\
12 \\
13 \\
14 \\
15 \\
16 \\
17 \\
18 \\
19 \\
20 \\
21 \\
22 \\
23 \\
24 \\
25 \\
26 \\
27 \\
28 \\
29 \\
30 \\
31 \\
32 \\
33 \\
34 \\
35 \\
36 \\
37 \\
38 \\
39 \\
40 \\
41 \\
42 \\
43 \\
44 \\
45 \\
46 \\
47 \\
48 \\
49 \\
50\end{array}$ & $\begin{array}{l}1.00 E+00 \\
1.00 E+00 \\
1.00 E+00 \\
1.00 E+00 \\
2.10 E+01 \\
1.00 E+00 \\
1.00 E+00 \\
1.00 E+00 \\
1.80 E+00 \\
1.00 E+00 \\
1.00 E+00 \\
4.30 E+00 \\
1.20 E+01 \\
5.20 E+01 \\
1.00 E+00 \\
1.00 E+00 \\
1.00 E+00 \\
1.00 E+00 \\
1.00 E+00 \\
1.00 E+00 \\
4.40 E+01 \\
5.20 E+00 \\
1.00 E+00 \\
1.00 E+00 \\
4.50 E+01 \\
1.00 E+00 \\
1.00 E+00 \\
1.90 E+00 \\
4.20 E+00 \\
2.40 E+01 \\
1.00 E+00 \\
1.00 E+00 \\
1.00 E+00 \\
2.20 E+00 \\
1.80 E+01 \\
1.70 E+00 \\
1.50 E+00 \\
7.10 E+01 \\
2.50 E+01 \\
1.00 E+00 \\
1.00 E+00 \\
1.30 E+00 \\
1.00 E+00 \\
1.60 E+00 \\
1.00 E+00 \\
1.00 E+00 \\
5.40 E+00 \\
3.40 E+01 \\
1.00 E+00 \\
1.00 E+00\end{array}$ & $\begin{array}{l}2 \\
2 \\
1 \\
1 \\
2 \\
1 \\
2 \\
2 \\
2 \\
2 \\
2 \\
2 \\
2 \\
2 \\
2 \\
2 \\
2 \\
2 \\
1 \\
1 \\
2 \\
2 \\
1 \\
1 \\
2 \\
1 \\
2 \\
2 \\
2 \\
2 \\
1 \\
1 \\
2 \\
2 \\
2 \\
2 \\
2 \\
2 \\
2 \\
2 \\
1 \\
2 \\
1 \\
2 \\
1 \\
1 \\
2 \\
2 \\
1 \\
1\end{array}$ & $\begin{array}{l}\text { SB } \\
\text { TE } \\
I \\
X E \\
C S \\
B A \\
\text { LA } \\
C E \\
P R \\
N D \\
P M \\
S M \\
E U \\
\text { GD } \\
T B \\
D Y \\
H D \\
E R \\
I M \\
Y B \\
\text { LU } \\
H F \\
T A \\
W \\
R E \\
O S \\
I R \\
P T \\
A U \\
H G \\
T L \\
P B \\
B I \\
P D \\
A T \\
R N \\
F R \\
R A \\
A C \\
T H \\
P A \\
U \\
N P \\
P U \\
A M \\
C M \\
B K \\
C F \\
E S \\
F M\end{array}$ & $\begin{array}{l}51 \\
52 \\
53 \\
54 \\
55 \\
56 \\
57 \\
58 \\
59 \\
60 \\
61 \\
62 \\
63 \\
64 \\
65 \\
66 \\
67 \\
68 \\
69 \\
70 \\
71 \\
72 \\
73 \\
74 \\
75 \\
76 \\
77 \\
78 \\
79 \\
80 \\
81 \\
82 \\
83 \\
84 \\
85 \\
86 \\
87 \\
88 \\
89 \\
90 \\
91 \\
92 \\
93 \\
94 \\
95 \\
96 \\
97 \\
98 \\
99 \\
100\end{array}$ & $\begin{array}{l}1.40 E+03 \\
4.50 E+01 \\
1.00 E+00 \\
3.00 E+00 \\
1.40 E+00 \\
2.60 E+00 \\
2.50 E+01 \\
2.50 E+01 \\
2.50 E+01 \\
2.50 E+01 \\
2.50 E+01 \\
2.50 E+01 \\
2.50 E+01 \\
2.50 E+01 \\
2.50 E+01 \\
2.50 E+01 \\
2.50 E+01 \\
2.50 E+01 \\
2.50 E+01 \\
2.50 E+01 \\
2.50 E+01 \\
6.90 E+00 \\
1.00 E+00 \\
1.00 E+00 \\
1.70 E+00 \\
5.80 E+00 \\
1.00 E+00 \\
3.30 E+01 \\
4.00 E+00 \\
4.20 E+01 \\
2.30 E+01 \\
2.80 E+00 \\
1.10 E+03 \\
1.00 E+00 \\
1.00 E+00 \\
1.00 E+00 \\
1.00 E+00 \\
5.30 E+00 \\
4.60 E+02 \\
1.00 E+00 \\
4.60 E+02 \\
1.30 E+03 \\
4.60 E+02 \\
4.60 E+02 \\
4.60 E+02 \\
4.60 E+02 \\
4.60 E+02 \\
4.60 E+02 \\
4.60 E+02 \\
4.60 E+02\end{array}$ & $\begin{array}{l}2 \\
2 \\
1 \\
2 \\
2 \\
2 \\
2 \\
2 \\
2 \\
2 \\
2 \\
2 \\
2 \\
2 \\
2 \\
2 \\
2 \\
2 \\
2 \\
2 \\
2 \\
2 \\
1 \\
1 \\
2 \\
2 \\
1 \\
2 \\
2 \\
2 \\
2 \\
2 \\
2 \\
1 \\
1 \\
1 \\
1 \\
2 \\
2 \\
1 \\
2 \\
2 \\
2 \\
2 \\
2 \\
2 \\
2 \\
2 \\
2 \\
2\end{array}$ & 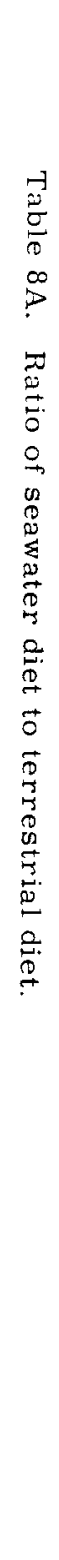 \\
\hline
\end{tabular}


TABLE 8B. RATIO OF FRESTWATER DIET TO TERRESTRIAL DIET

$10 / 09 / 68$

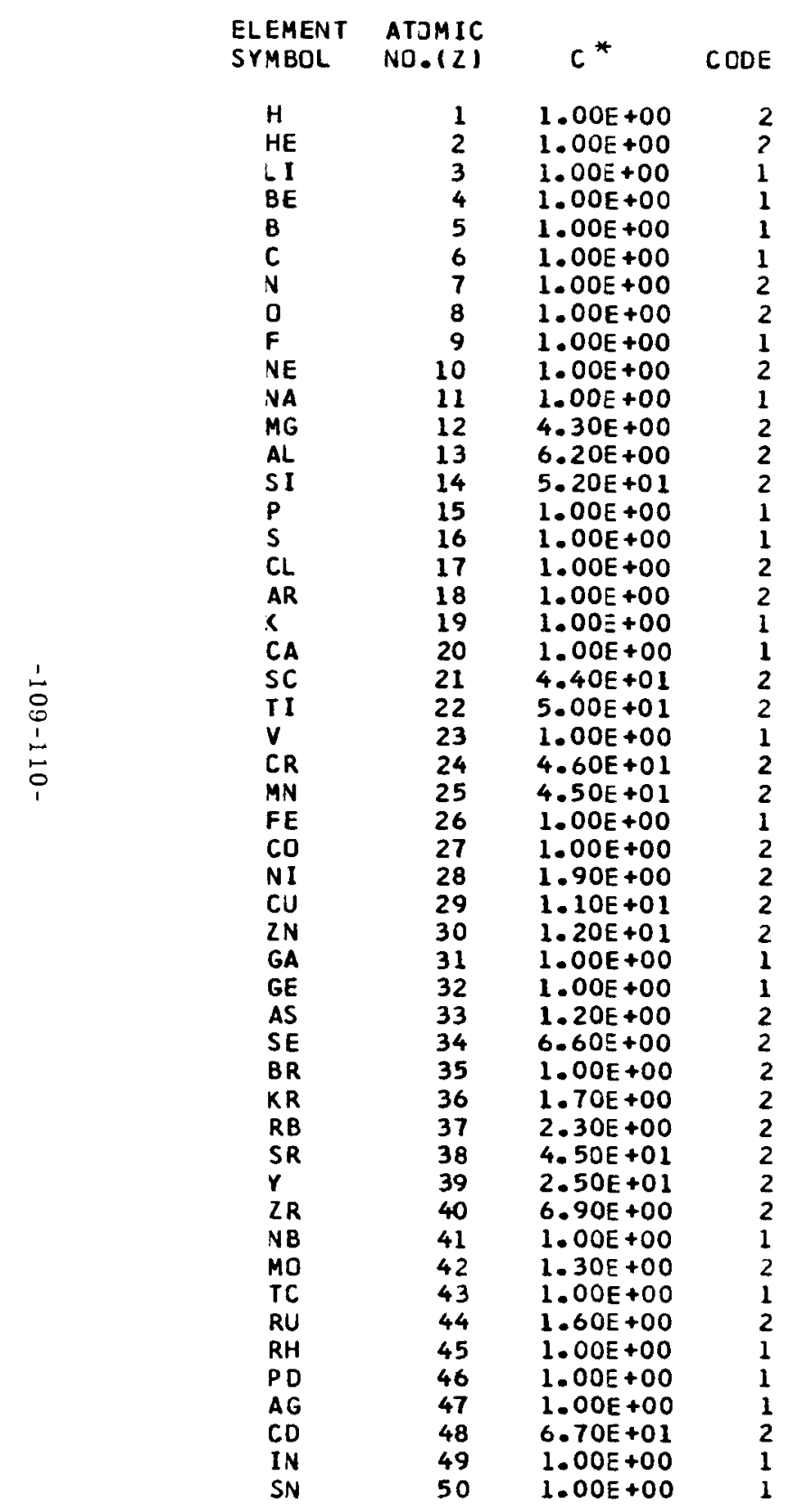

ELEMENT

ATOMIC

SYMBOL NO.IZI

$c^{*}$

COOE

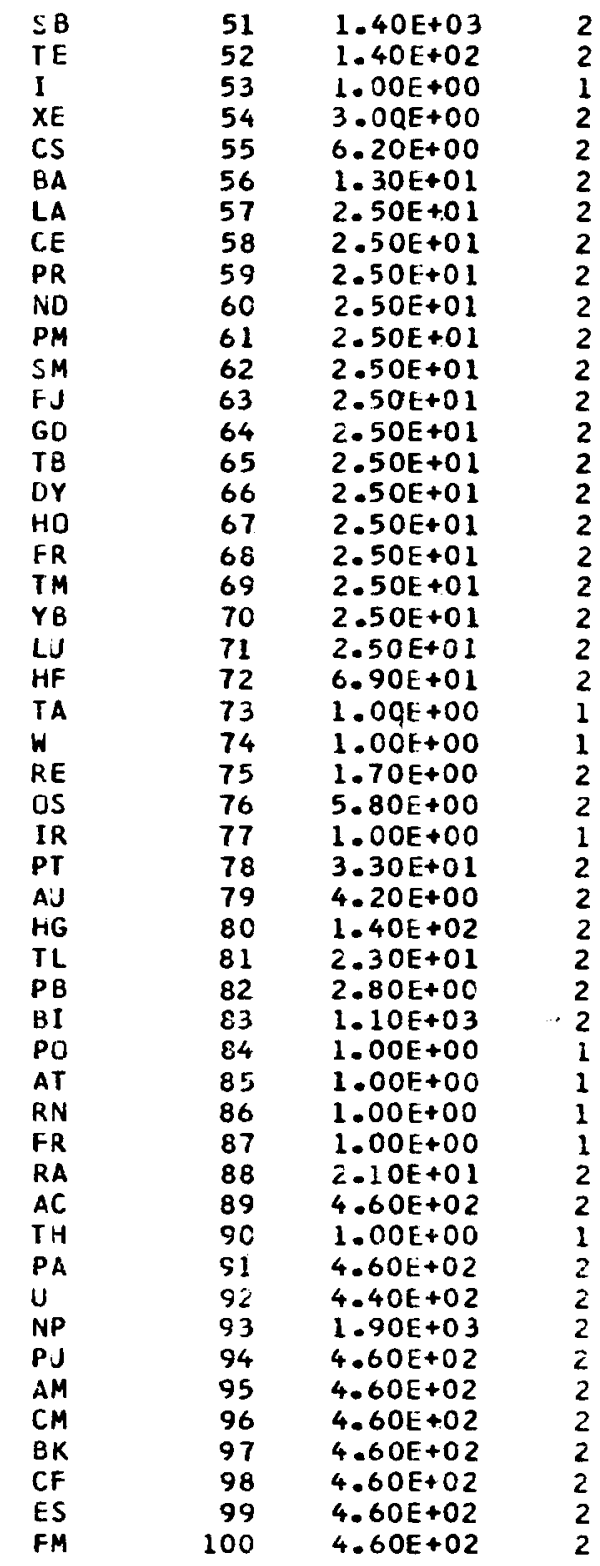


-

-

- 
10/22/68: Insert after "F $\mathrm{A}$ sea" divider page.

\section{9. $\mathrm{F}_{\mathrm{A}}$ : UNIT-RAD CONTAMINATION}

The unit-rad contamination $\mathrm{F}_{\mathrm{A}}$ is the initial concentration of a radionuclide in water that could result in a 30 -year integrated dose of $1 \mathrm{rad}$ to the tissue in question (6354). The equation for $\mathrm{F}_{\mathrm{A}}$ is similar to that for $F_{1}$ via soil. In converting the soil $F_{1}$ to $F_{A}$, all that is required is to divide through by $d$. This converts the unit to $\mu \mathrm{Ci} / \mathrm{m}^{3} / \mathrm{rad}$. Then $\rho$ is set equal to $10^{6} \mathrm{~g} / \mathrm{m}^{3}$, the density of water, and $\mathrm{C}_{\mathrm{S}}$ is replaced by $\mathrm{C}_{\mathrm{A}}$, the concentration of the stable element in water.

One additional modification of the equation is also made. The $\mathrm{C}_{B}$ value is replaced by $\left(\mathrm{C}_{B} \mathrm{C}^{*}\right)$, where $\mathrm{C}^{*}$ is the ratio of the concentrations (aquatic diet/ terrestrial diet). Thus, $\mathrm{C}^{*}$ corrects the $C_{B}$ values for those cases where the aquatic diet supplies more of an element than does a terrestrial diet. The assumption here is that if a larger quantity of a trace element is consumed, the body burden will increase proportionately.

The resultant equation for $\mathrm{F}_{\mathrm{A}}$ for the adult is:

$$
\begin{aligned}
& F_{A}=\left(\frac{37.1}{Q}\right)\left(\frac{C_{A}}{C_{B} C^{*}}\right) \cdot \\
& {\left[\frac{T_{R}\left(1-e^{-20.8 / T_{R}}\right)-T_{E}\left(1-e^{-20.8 / T_{E}}\right)}{1}\right] .}
\end{aligned}
$$

Again in the case of the infant, we assume instantaneous equilibrium with the environment and the following equation applies:

$F_{A}=\left(\frac{37.1}{Q}\right)\left(\frac{C_{A}}{C_{B} C^{* *}}\right)\left[\frac{1}{T_{R}\left(1-e^{-20.8 / T_{R}}\right)}\right]$ 


\begin{tabular}{|c|c|c|c|c|}
\hline $\begin{array}{l}\text { EL EMENT } \\
\text { SYMBOL }\end{array}$ & $\begin{array}{l}\text { ATOMIC } \\
\text { NO. }(2)\end{array}$ & $\begin{array}{l}\text { ISOTJPE } \\
\text { MASS (A) }\end{array}$ & $\begin{array}{l}\text { ADULT } \\
\text { UC } / M * * 3\end{array}$ & $\begin{array}{l}\text { INFANT } \\
\text { UC/M**3 }\end{array}$ \\
\hline $\begin{array}{l}H \\
B E \\
B E \\
C \\
N A \\
N A \\
M G \\
A L \\
S I \\
P \\
P \\
S \\
C L \\
A R \\
A R \\
A R \\
X \\
X \\
K \\
C A \\
C A \\
C A \\
C A \\
S C \\
S C \\
S C \\
S C \\
T I \\
V \\
V \\
V \\
C R \\
C R \\
M N \\
M N \\
M N \\
F E \\
F E \\
F E \\
C D \\
C O \\
C O \\
C O \\
C D \\
V I \\
N I \\
V I \\
V I \\
V I \\
C U\end{array}$ & $\begin{array}{l}1 \\
4 \\
4 \\
6 \\
11 \\
11 \\
12 \\
13 \\
14 \\
15 \\
15 \\
16 \\
17 \\
18 \\
18 \\
18 \\
19 \\
19 \\
19 \\
20 \\
20 \\
20 \\
20 \\
21 \\
21 \\
21 \\
21 \\
22 \\
23 \\
23 \\
23 \\
24 \\
24 \\
25 \\
25 \\
25 \\
26 \\
26 \\
26 \\
27 \\
27 \\
27 \\
27 \\
27 \\
28 \\
28 \\
28 \\
28 \\
28 \\
29\end{array}$ & $\begin{array}{l}3 \\
7 \\
10 \\
14 \\
22 \\
24 \\
28 \\
26 \\
32 \\
32 \\
33 \\
33 \\
35 \\
36 \\
37 \\
39 \\
42 \\
40 \\
42 \\
43 \\
41 \\
45 \\
47 \\
48 \\
4\end{array}$ & $\begin{array}{l}6.4 E+02 \\
8.0 E-04 \\
1.1 E-07 \\
6.4 E-03 \\
6.1 E+00 \\
8.0 E+04 \\
1.2 E+05 \\
9.1 E-04 \\
2.8 E-04 \\
6.3 E-02 \\
2.4 E-01 \\
7.8 E+03 \\
6.0 E+01 \\
8.2 E+02 \\
4.4 E-02 \\
4.7 E-03 \\
1.3 E-01 \\
6.5 E+04 \\
3.9 E+04 \\
1.1 E+01 \\
4.3 E+02 \\
2.5 E+04 \\
1.1 E-02 \\
2.9 E+01 \\
3.4 E-02 \\
1.3 E+02 \\
4.0 E+01 \\
1.5 E-04 \\
3.5 E+00 \\
1.5 E+01 \\
1.9 E-03 \\
4.2 E-02 \\
1.5 E-01 \\
7.9 E-01 \\
5.8 E-03 \\
2.2 E-03 \\
9.5 E-02 \\
1.5 E-01 \\
1.4 E-05 \\
4.9 E+00 \\
2.2 E-03 \\
7.7 E-03 \\
5.0 E-03 \\
5.6 E-05 \\
4.8 E+00 \\
1.5 E+02 \\
1.6 E-02 \\
8.0 E-03 \\
7.1 E+01 \\
2.1 E+03\end{array}$ & $\begin{array}{l}6.4 \mathrm{E}+02 \\
8.5 \mathrm{E}-05 \\
1.0 \mathrm{E}-07 \\
6.3 \mathrm{E}-03 \\
6.0 \mathrm{E}+00 \\
4.3 \mathrm{E}+03 \\
5.9 \mathrm{E}+02 \\
9.1 \mathrm{E}-04 \\
2.8 \mathrm{E}-04 \\
7.7 \mathrm{E}-04 \\
5.0 \mathrm{E}-03 \\
9.9 \mathrm{E}+02 \\
6.0 \mathrm{E}+01 \\
9.2 \mathrm{E}+02 \\
4.4 \mathrm{E}-02 \\
4.7 \mathrm{E}-03 \\
1.3 \mathrm{E}-01 \\
5.7 \mathrm{E}+02 \\
6.2 \mathrm{E}+02 \\
2.1 \mathrm{E}+00 \\
3.9 \mathrm{E}+03 \\
6.5 \mathrm{E}+00 \\
2.1 \mathrm{E}-03 \\
4.3 \mathrm{E}-02 \\
1.7 \mathrm{E}-03 \\
2.8 \mathrm{E}-01 \\
4.6 \mathrm{E}-02 \\
1.5 \mathrm{E}-04 \\
3.7 \mathrm{E}-01 \\
1.1 \mathrm{E}+01 \\
1.9 \mathrm{E}-03 \\
4.2 \mathrm{E}-02 \\
1.5 \mathrm{E}-01 \\
1.2 \mathrm{E}-02 \\
5.5 \mathrm{E}-03 \\
9.8 \mathrm{E}-04 \\
3.5 \mathrm{E}-02 \\
3.8 \mathrm{E}-03 \\
1.1 \mathrm{E}-05 \\
1.5 \mathrm{E}-01 \\
1.7 \mathrm{E}-03 \\
7.1 \mathrm{E}-03 \\
3.8 \mathrm{E}-03 \\
5.5 \mathrm{E}-05 \\
3.7 \mathrm{E}-02 \\
2.9 \mathrm{E}-01 \\
1.5 \mathrm{E}-02 \\
7.1 \mathrm{E}-03 \\
2.0 \mathrm{E}-01 \\
6.1 \mathrm{E}+00\end{array}$ \\
\hline
\end{tabular}

$\begin{array}{lcc}\text { ELEMENT } & \text { ATOMIC } & \text { ISOTOPE } \\ \text { SYMBOL } & \text { NO. (Z) } & \text { MASS (A) } \\ & & \\ \text { CU } & 29 & 67 \\ \text { ZN } & 30 & 65 \\ \text { ZN } & 30 & 69 M \\ \text { ZN } & 30 & 72 \\ \text { GA } & 31 & 67 \\ \text { GA } & 31 & 72 \\ \text { GE } & 32 & 68 \\ \text { GE } & 32 & 69 \\ \text { GE } & 32 & 71 \\ \text { AS } & 33 & 71 \\ \text { AS } & 33 & 72 \\ \text { AS } & 33 & 73 \\ \text { AS } & 33 & 74 \\ \text { AS } & 33 & 76 \\ \text { AS } & 33 & 77 \\ \text { SE } & 34 & 72 \\ \text { SE } & 34 & 75 \\ \text { SE } & 34 & 79 \\ \text { BR } & 35 & 76 \\ \text { BR } & 35 & 77 \\ \text { BR } & 35 & 82 \\ \text { KR } & 36 & 76 \\ \text { KR } & 36 & 79 \\ \text { KR } & 36 & 81 \\ \text { KR } & 36 & 85 \\ \text { RB } & 37 & 83 \\ \text { RB } & 37 & 84 \\ \text { RB } & 37 & 86 \\ \text { RB } & 37 & 87 \\ \text { SR } & 38 & 82 \\ \text { SR } & 38 & 83 \\ \text { SR } & 38 & 85 \\ \text { SR } & 38 & 89 \\ \text { SR } & 38 & 90 \\ \text { Y } & 39 & 86 \\ \text { Y } & 39 & 87 \\ Y & 39 & 87 M \\ \text { Y } & 39 & 88 \\ \text { Y } & 39 & 90 \\ \text { Y } & 39 & 91 \\ \text { ZR } & 40 & 86 \\ \text { ZR } & 40 & 88 \\ \text { ZR } & 40 & 89 \\ \text { ZR } & 40 & 93 \\ \text { ZR } & 40 & 95 \\ \text { ZR } & 40 & 97 \\ \text { NB } & 41 & 90 \\ \text { NB } & 41 & 91 \\ \text { NB } & 41 & 91 M \\ \text { NB } & 41 & 92 \\ \text { SR } & & \end{array}$

$A D U L T$
$U C / M * 3$

INF ANT

$6.4 E+02$

1. $0 \mathrm{E}-07$

$6.0 E+D 0$

$4.3 E+03$

$9.1 E-04$

$2.8 \mathrm{E}-04$

7. $7 E-04$
$5.0 E-03$

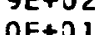

(E)

$6.2 E+02$

$3.9 E+0 J$

$2.1 \mathrm{E}-03$

$4.3 E-02$

$1.7 E-03$

4. $6 \mathrm{E}-02$

3.7E-01

1. $9 E-03$

$4.2 E-02$
$1.5 E-31$

$1.2 E-02$

$5 E-02$

$1 E-05$

$7 E-03$

1E-03

$.5 E-05$

-9E-01

7. $1 E-03$

$6.1 E+O D$
UC $/ M * * 3$

2. $6 \mathrm{E}-03 \quad 4.1 \mathrm{E}-04$

$\begin{array}{ll}2.3 E+02 & 1.0 E-01\end{array}$

2. $9 E+00$ 6.1E-01

4. $7 E+00$ 2.2E-01

$1.8 E-05 \quad 1.8 E-05$

2. $6 E-03 \quad 2.6 E-03$

7. $1 E-02 \quad 7.1 E-02$

$2.4 E+03 \quad 2.4 E+03$

1. $2 E+03 \quad 1.2 E+03$

4. $9 E+03 \quad 4.9 E+03$

$1.2 E+02 \quad 1.2 E+02$

1. $7 E+03 \quad 1.7 E+03$

$8.5 E+03$

2. $3 E-02$

1. $7 E-03$

$1.4 E+06$

$2.9 E+05$

2. $5 E+05$

1. $5 E+02$

1. $9 E+02$

$1.3 E+00$

2. $5 E-01$

4. $4 E+00$

6. $O E+00$

2. $9 E+01$

1. $7 E-01$

$1.4 E+02$

1. $5 E+05$

1. $5 E+02$

1. $6 E+02$

1. $3 E-02$

4. $9 E+02$

7. $9 E+01$

1. $8 \mathrm{E}+03$

2. $4 E-02$

$8.0 E+01$

2. $7 E-01$

1. $8 E+03$

$1.9 E-01$

2. $0 E+02$

3. $1 E-02$

6. $1 E-01$

2. $6 E+03$

1. $3 E-01$

2. $2 E-06$

3. $O E-04$
$2.6 E-03$

$8.5 E+03$

2. $3 E-02$

8. $7 E-03$

$1.7 E-03$

1.1. E+05

$6.6 E+04$

3. $9 E+04 \equiv$

$1.5 E+02$

1. $9 \mathrm{E}+02$

1. $3 E+00$

2. $5 E-01$

1. $7 E+00$

$5.5 E+00$

$1.7 \mathrm{E}-01$

2. OE-01

1. $2 E+01$

5.2E-01

4.6E-01

2.1E-03

1.7 E-02

1. $5 \mathrm{E}-02$

5. $9 E-02$

1.5E-04

1. $2 E-02$

9. $0 \mathrm{E}-04$

$1.3 E+00$

1. $5 \mathrm{E}-02$

$6.7 E-01$

2.7E-02

2.7E-02
$3.7 \mathrm{E}-02$

3.7E-O2

1. $8 E+00$

8. $0 \mathrm{E}-05$

1. $9 \mathrm{E}-06$

$1.8 \mathrm{E}-05$
$2.6 \mathrm{E}-05$
$1.1 E+02 \quad 1.5 E+00$ 
TABLE 9A. UNIT-RAD EGUTAMINATION FOR BONE

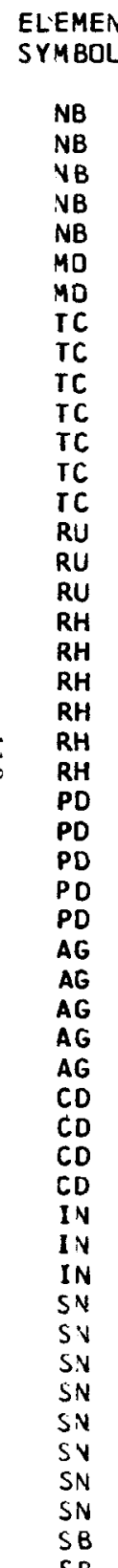

ATOMIC ISOTOPE

ADULT
VIA SEAWAIER.

INFAN T

$U C / M * 3$

6. $5 E-C S$

$1.9 E-08$

$9.0 E-36$

$6.6 E-05$

$9.7 E-05$

1. $-1 E+D 1$
$-1 E+00$

2. $0 E+3 J$

2. $1 E-02$

1.3E-01

$8.9 E-03$

1. 3E-OL

$1.2 E-04$

2. $0 E-03$

$9.6 \mathrm{E}-01$

7. $1 \mathrm{E}-02$

1. $9 E-03$

1. $7 E-02$

$9.9 E-32$

2. $3 E-04$

8. $9 E-02$

$3.8 \mathrm{E}-04$

3.7E-OI

$2.2 E-02$

$1.2 \mathrm{E}-01$

$1.5 E-02$

$4.4 E-J 1$

4. $9 \mathrm{E}-02$

1. $7 E-01$

$3.4 E-01$

$1.6 \mathrm{E}-03$

1. $0 E-02$

2. $1 E+0 D$

$1.5 E-33$

9.5E-05

2. $7 E-02$

$1.7 E-03$

1. $1 E-04$

2. $1 E-06$

$8.1 E-03$

1.7E-01

$1.9 E+O D$

3. $8 E-01$

1. $2 \mathrm{E}-02$

$9.7 \mathrm{E}-02$

4. $7 E-01$

3. $4 \mathrm{E}-04$

2. $9 \mathrm{E}-01$

$1.6 \mathrm{E}-03$
ELEMENT SYMBOL

ATOMIC

ISOTOPE

$51 \quad 122$

$\begin{array}{ll}S B & 51 \\ S B & 51 \\ S E & 51 \\ S E & 51 \\ S B & 51 \\ T E & 52 \\ T E & 52\end{array}$

125

126

127
118

119
$119 \mathrm{M}$

$119 M$
121

$121 \mathrm{M}$

123

$125 \mathrm{M}$

$127 \mathrm{M}$

$129 M$

$131 \mathrm{M}$

132
123

123

125

126

129

130

131

133

122

125
127

$129 M$

$131 \mathrm{M}$

133

$133 \mathrm{M}$

129
131

131
132

132
134

135

136

128

131
133

$133 \mathrm{M}$

$135 \mathrm{M}$

140

135
137

137

138

134
$10 / 09 / 68$

PAGE 2

ADULT IVFAVT

2. $8 E-01$

4. $5 E-04$

5. $O E-05$
$6.2 E-03$

1. $3 E-01$

4. $3 E-01$

2. $2 E+01$

4. 3E-O1

1. $6 E-01$

$5.4 E-03$

$2.6 E-03$

.7E-02

1. $1 E-01$

2. $1 E-02$

3. $7 E-02$

5. $2 E+00$

6. $1 E-01$

$1.1 E+03$

7. $0 E+02$

. $1 E+02$

3. $9 E+00$

5. $2 E+04$

- $3 E+0$

3. $7 E+04$

2. $1 E+02$

1. $1 E+03$

5. $3 E+01$

2. $4 E+02$

2. $3 E+02$

$4.6 E+02$

$4.7 E+02$

8. $7 E+0$

2. $9 E+0$

3. $1 E+00$

6. $2 E-04$

2. $0 E-03$

2. $5 E-01$

1. $9 E-04$

1. $5 E+02$

$7 E+01$

1. $7 E+0$

3. $4 E-02$

1. $2 E+0 ?$
5. $5 E+0 ?$

4. $2 E+0$

6. $9 E-0$.

$1.6 E-07$

4. $8 E-09$

4. $6 E-03$

5. $2 E+O L$
UC $/ M * * 3$

7. $6 \mathrm{E}-03$

. $7 E-04$

$6.9 E-04$

4. $9 \mathrm{E}-03$

7.2E-02

4. $7 E-01$

$5.9 E-02$
$5.9 E-02$

4. 5E-03

$2.6 E-03$

2.1E-02

7. $2 \mathrm{E}-02$

1. $7 E-02$

$2.9 E-02$

5. $9 E-02$

$1.9 E+04$

$2.4 E+02$

.6E+02

3. $9 E+00=$

$1.7 E+03 \pi$

$4.7 \mathrm{E}+02$

2.1 E+03

2. $1 E+02$

$1.1 E+03$

5. $3 E+01$

$2.4 E+02$

$2.3 E+02$

4. $6 E+02$

1. $0 E+00$

$2.4 E+00$

$1.8 \mathrm{E}-01$

$5.4 E-04$

1. $9 E-03$

$2.7 E-02$

1. $8 \mathrm{E}-04$

5. $3 E+00$

2. $5 E+00$

2. $3 E+02$

3.

$\therefore 9 E+01$

i. $O E+02$

$0.8 E-01$

$5.6 E-05$

1. $4 \mathrm{E}-07$

4. $2 E-09$

7. $7 \mathrm{E}-06$

1. OE - OI 


\begin{tabular}{|c|c|c|c|c|}
\hline $\begin{array}{l}\text { EL EMENT } \\
\text { SYMBOL }\end{array}$ & $\begin{array}{l}\text { ATOMIC } \\
\text { NO. }(Z)\end{array}$ & $\begin{array}{l}\text { ISOTOPE } \\
\text { MASS (A) }\end{array}$ & $\begin{array}{l}\text { ADULT } \\
\text { UC } / M * * 3\end{array}$ & $\begin{array}{l}\text { INFANT } \\
\text { UC } / M * * 3\end{array}$ \\
\hline 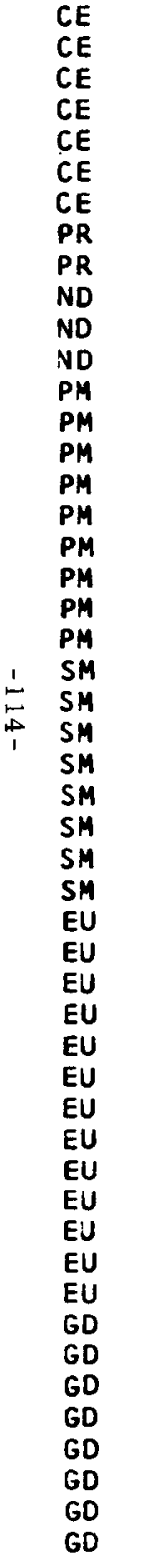 & $\begin{array}{l}58 \\
58 \\
58 \\
58 \\
58 \\
58 \\
58 \\
59 \\
59 \\
60 \\
60 \\
60 \\
61 \\
61 \\
61 \\
61 \\
61 \\
61 \\
61 \\
61 \\
61 \\
62 \\
62 \\
62 \\
62 \\
62 \\
62 \\
62 \\
62 \\
63 \\
63 \\
63 \\
63 \\
63 \\
63 \\
63 \\
63 \\
63 \\
63 \\
63 \\
63 \\
63 \\
64 \\
64 \\
64 \\
64 \\
64 \\
64 \\
64 \\
64\end{array}$ & $\begin{array}{l}135 \\
137 M \\
139 \\
141 \\
142 \\
143 \\
144 \\
142 \\
143 \\
140 \\
144 \\
147 \\
143 \\
144 \\
145 \\
146 \\
147 \\
148 \\
148 M \\
149 \\
151 \\
141 \\
145 \\
146 \\
147 \\
148 \\
149 \\
151 \\
153 \\
145 \\
146 \\
146 M \\
147 \\
148 \\
149 \\
150 \\
150 M \\
152 \\
154 \\
155 \\
156 \\
157 \\
146 \\
147 \\
148 \\
149 \\
150 \\
151 \\
152 \\
153\end{array}$ & $\begin{array}{l}1.2 E+03 \\
8.8 E+02 \\
1.8 E-01 \\
2.4 E+00 \\
6.2 E-05 \\
2.8 E+02 \\
2.5 E-03 \\
8.0 E-02 \\
8.8 E-04 \\
4.8 E-03 \\
3.9 E-09 \\
1.9 E-04 \\
2.9 E-02 \\
2.2 E-03 \\
2.3 E-03 \\
6.1 E-04 \\
4.8 E-04 \\
1.4 E+01 \\
6.9 E-02 \\
3.0 E+02 \\
4.3 E+02 \\
5.0 E-01 \\
5.4 E-02 \\
3.5 E-05 \\
4.0 E-05 \\
2.3 E-05 \\
4.8 E-05 \\
6.6 E-03 \\
6.0 E+02 \\
4.6 E+01 \\
1.2 E+01 \\
1.5 E+02 \\
7.0 E-01 \\
7.6 E-01 \\
2.0 E-01 \\
8.0 E-04 \\
9.7 E+02 \\
2.5 E-04 \\
1.8 E-04 \\
3.6 E-02 \\
3.1 E+00 \\
2.6 E+03 \\
2.7 E-02 \\
6.4 E+01 \\
1.8 E-05 \\
3.3 E+00 \\
9.4 E-06 \\
7.2 E-02 \\
2.3 E-05 \\
4.6 E-02\end{array}$ & $\begin{array}{l}6.1 \mathrm{E}-01 \\
8.4 \mathrm{E}-01 \\
1.5 \mathrm{E}-02 \\
5.2 \mathrm{E}-02 \\
5.0 \mathrm{E}-05 \\
2.6 \mathrm{E}-01 \\
4.1 \mathrm{E}-04 \\
4.3 \mathrm{E}-05 \\
8.0 \mathrm{E}-06 \\
1.1 \mathrm{E}-05 \\
3.2 \mathrm{E}-09 \\
1.4 \mathrm{E}-05 \\
4.3 \mathrm{E}-03 \\
4.2 \mathrm{E}-04 \\
1.7 \mathrm{E}-03 \\
3.1 \mathrm{E}-04 \\
1.9 \mathrm{E}-04 \\
5.1 \mathrm{E}-02 \\
1.8 \mathrm{E}-03 \\
4.4 \mathrm{E}-01 \\
3.4 \mathrm{E}-01 \\
6.6 \mathrm{E}-03 \\
1.0 \mathrm{E}-02 \\
2.8 \mathrm{E}-05 \\
3.2 \mathrm{E}-05 \\
1.8 \mathrm{E}-05 \\
3.9 \mathrm{E}-05 \\
5.2 \mathrm{E}-03 \\
7.8 \mathrm{E}-01 \\
1.8 \mathrm{E}-01 \\
3.7 \mathrm{E}-02 \\
1.6 \mathrm{E}-01 \\
1.1 \mathrm{E}-02 \\
2.6 \mathrm{E}-02 \\
1.3 \mathrm{E}-02 \\
4.3 \mathrm{E}-04 \\
3.5 \mathrm{E}-01 \\
1.7 \mathrm{E}-04 \\
1.3 \mathrm{E}-04 \\
1.1 \mathrm{E}-02 \\
3.1 \mathrm{E}-02 \\
1.1 \mathrm{E}+00 \\
1.2 \mathrm{E}-03 \\
6.7 \mathrm{E}-02 \\
1.5 \mathrm{E}-05 \\
3.0 \mathrm{E}-02 \\
8.2 \mathrm{E}-03 \\
7.7 \mathrm{E}-03 \\
2.0 \mathrm{E}-05 \\
8.9 \mathrm{E}-03\end{array}$ \\
\hline
\end{tabular}

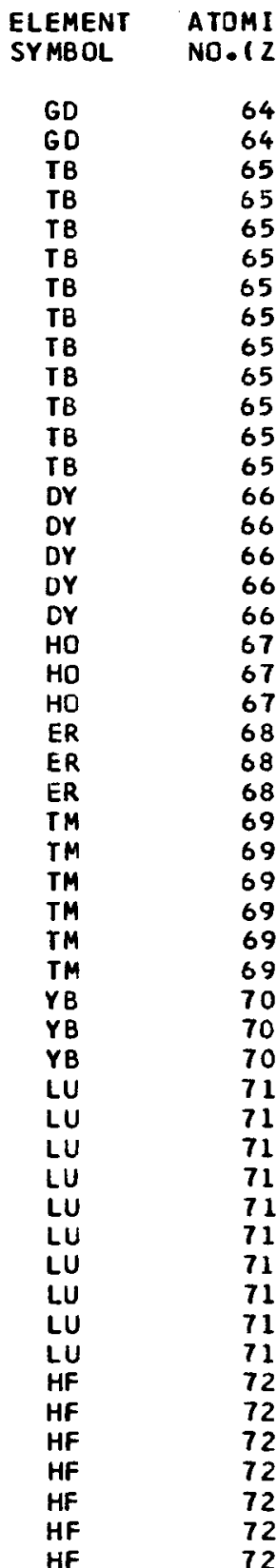

ISOTOPE
MASS(A)
159
162
151
152
153
154
155
156
157
158
160
161
164
154
$154 M$
156
159
166
163
166
$166 M$
160
169
172
165
167
168
170
171
172
166
169
175
169
170
171
172
173
174
$174 M$
176
177
$177 M$
170
171
172
173
174
175
181
19

ADULT

$1.4 E+03$

2. $2 \mathrm{E}-03$

9. $5 \mathrm{E}+02$

2. $7 E+02$

1. $8 E+02$

1. $O E+03$

4. $1 E+01$

1. $8 \mathrm{E}-03$

7.7E-05

$8.2 E-02$

5. $9 E+01$

5. $9 E+01$
$1.4 E+03$

1. $4 E+03$
$6.8 E-10$

6. $8 E-10$
3. $7 E-02$

1. $1 E-09$

3. 0 E- 05

2. $8 \mathrm{E}-03$

1. $1 E-07$

3. 3E-02

4. $9 E-09$

9.7E-03

4. $9 E-03$

7. $7 E-03$

1. $4 E+02$

1. $O E+01$

5. $2 E-02$

1. $0 E-01$

9. $6 E-02$

8. $2 E+01$

2. $1 E+02$

1. $8 E+00$

2. $1 E+02$

9. $5 E-03$

3. $E-03$

3. $2 E-03$

4. $2 E-03-04$

9. $O E-04$
3. $2 E-07$

4. $2 E-08$

3. $1 E-06$

8. $2 E-09$

5. $0 E-03$

9. 3E-06

1. $3 E+01$

1. $3 E+01$

1. $5 E-05$

7. $9 E+00$

1. 3E-06

6. $5 E-03$
INF ANT

UC $/ M * * 3$

1. $1 E+00$

6. OE-04

7. $1 E-01$

2.1E-01

8. $8 \mathrm{E}-01$

2. $3 E-01$

6. $8 E-02$

1. 5E-03

$6.6 E-05$

$5,5 E-03$

4. OE-O1

1. $4 E+00$

5. $9 E-10$

2. OE-05

9.2E-10

. 8E-06

$9.5 \mathrm{E}-08$

$3.7 E-05$

3. TE-OS

4.2E-09

1.2E-05

4. $6 E-05$

$1.6 E-05$

1.7E-01

$9.6 E-02$

4. $1 E-03$

$1.2 E-02$

3. $9 E-02$

2. 2E-01

5. 2E-01

$5.5 E-02$

8. 8E-01

1. $4 E-05$

6. $4 E-06$

3. $4 \mathrm{E}-05$

6. OE-06

1. 1 E-07

2. $4 \mathrm{E}-08$

4. $4 \mathrm{E}-07$

$7.1 \mathrm{E}-09$

3. $4 E-05$

1. $2 \mathrm{E}-06$

1.1E-02

1.

2.5E-02

$1.1 \mathrm{E}-05$

1. 3E-02

1. $2 \mathrm{E}-06$

6. $8 E-04$ 
TABLE 9A. - UNIT-RAD LONTAMINATION FCR BCNE

\begin{tabular}{|c|c|c|c|c|}
\hline $\begin{array}{l}\text { ELEMENT } \\
\text { SYMBOL }\end{array}$ & $\begin{array}{l}\text { ATOMIC } \\
\text { VO. }(Z)\end{array}$ & $\begin{array}{l}\text { ISOIOPE } \\
\text { MASSIA) }\end{array}$ & $\begin{array}{l}\text { ADULT } \\
U C / M * * 3\end{array}$ & $\begin{array}{l}\text { INFANT } \\
U C / M * 3\end{array}$ \\
\hline HF & 72 & 182 & 2. $0 E-06$ & $1.9 E-05$ \\
\hline TA & 73 & 177 & 1. $4 E+00$ & $1.0 E-02$ \\
\hline TA & 73 & 179 & $1.1 E-03$ & $7.1 E-34$ \\
\hline TA & 73 & 182 & 5. $7 \mathrm{E}-04$ & $1.6 E-04$ \\
\hline TA & 73 & 183 & $5.2 \mathrm{E}-0 \mathrm{I}$ & $8.4 E-03$ \\
\hline$W$ & 74 & 178 & 5. $1 \mathrm{E}-04$ & $3.6 E-04$ \\
\hline$W$ & 74 & 181 & $1.6 E-03$ & $1.5 E-03$ \\
\hline$H$ & 74 & 185 & 1. $7 E-03$ & $1.5 E-03$ \\
\hline W & 74 & 187 & $1.6 E-01$ & $1.6 \mathrm{E}-02$ \\
\hline$W$ & 74 & 188 & 1. $7 \mathrm{E}-04$ & $1.5 E-04$ \\
\hline RE & 75 & 180 & 2. $3 E+00$ & $4.4 E-01$ \\
\hline RE & 75 & 181 & 2. $5 E+00$ & 4. $8 E-01$ \\
\hline RE & 75 & 182 & 3. $8 E-01$ & $1.7 E-O 1$ \\
\hline RE & 75 & $182 \mathrm{M}$ & $6.1 E+00$ & $8.2 E-01$ \\
\hline RE & 75 & 183 & 3. $0 E-02$ & $2.9 E-02$ \\
\hline RE & 75 & 184 & 2. OE -02 & $1.8 E-02$ \\
\hline RE & 75 & $184 M$ & 3. $3 E-03$ & $3.2 \mathrm{E}-03$ \\
\hline RE & 75 & 186 & $7.8 E-01$ & $4.0 E-01$ \\
\hline RE & 75 & 187 & $6.8 E-01$ & $6.8 E-01$ \\
\hline RE & 75 & 188 & 5. $3 E+00$ & $8 \cdot 9 E-J 1$ \\
\hline RE & 75 & 189 & $6.6 E+00$ & $1.4 E+00$ \\
\hline os & 76 & 182 & $1.2 E-02$ & $1.2 E-02$ \\
\hline OS & 76 & 183 & 5. $2 E-02$ & $5.2 \mathrm{E}-02$ \\
\hline js & 76 & 185 & $3.3 E-04$ & $3.3 E-04$ \\
\hline OS & 76 & 191 & 7. $8 \mathrm{E}-03$ & $7.8 E-03$ \\
\hline os & 76 & $191 M$ & 1. $5 \mathrm{E}-01$ & $1.5 E-01$ \\
\hline os & 76 & 193 & 3. $7 \mathrm{E}-02$ & $3.7 \mathrm{E}-02$ \\
\hline OS & 76 & 194 & $5.1 E-06$ & $5.1 E-05$ \\
\hline IR & 77 & 185 & 2. $0 E-01$ & 2.OE-O1 \\
\hline IR & 77 & 186 & $1.2 E-01$ & $1.2 \mathrm{E}-01$ \\
\hline IR & 77 & 187 & $2.5 E-01$ & $2.5 \mathrm{E}-01$ \\
\hline IR & 77 & 188 & $3.5 E-02$ & $3.5 E-02$ \\
\hline I R & 77 & 189 & 2. $9 E-02$ & $2.9 E-02$ \\
\hline IR & 77 & 190 & 8. $0 E-03$ & $8.0 E-33$ \\
\hline IR & 77 & 192 & $1.8 E-03$ & $1.8 E-03$ \\
\hline IR & 77 & $192 M$ & $\begin{array}{l}1.0 E-05 \\
1.5 E-05\end{array}$ & $1.05-05$ \\
\hline I $R$ & 77 & $193 M$ & $1.4 \mathrm{E}-01$ & $1-4 E-01$ \\
\hline IR & 77 & 194 & $1.4 \mathrm{E}-01$ & $1.4 E-01$ \\
\hline PT & 78 & 188 & 1. $5 E-04$ & $1.5 E-04$ \\
\hline PI & 78 & 190 & $1.7 E-07$ & $1.7 \mathrm{E}-07$ \\
\hline PI & 78 & 191 & $2.6 E-03$ & $2.6 E-03$ \\
\hline $\mathrm{Pr}$ & 78 & 192 & 2.1E-07 & 2. $1 E-J 7$ \\
\hline PT & 78 & 193 & $7.3 \mathrm{E}-06$ & $7.3 \mathrm{E}-06$ \\
\hline PT & 78 & $193 M$ & $4.2 \mathrm{E}-03$ & $4.2 E-03$ \\
\hline PT & 78 & $195 M$ & 3. $9 E-03$ & $3.9 E-03$ \\
\hline PT & 78 & 197 & 1. $7 E-02$ & $1.7 E-02$ \\
\hline$A U$ & 79 & 193 & $9.1 E-02$ & $9.1 E-02$ \\
\hline$\Delta U$ & 79 & 194 & $1.2 \mathrm{E}-02$ & $1.2 \mathrm{E}-02$ \\
\hline A. & 79 & 195 & $1.1 \mathrm{E}-03$ & $1.1 E-03$ \\
\hline A. $J$ & 79 & 196 & 9. $O E-03$ & $9.0 \mathrm{E}-03$ \\
\hline
\end{tabular}

VIA SEAWATER.

$\begin{array}{lcc}\text { ELEMENT } & \text { ATOMIC } & \text { ISOTOPE } \\ \text { SYMBOL } & \text { NO. }(2) & \text { MASSIA } \\ \text { AU } & & \\ \text { AU } & 79 & 198 \\ \text { HG } & 79 & 199 \\ \text { HG } & 80 & 194 \\ \text { HG } & 80 & 195 M \\ \text { HG } & 80 & 197 \\ \text { HG } & 80 & 197 M \\ \text { IL } & 80 & 203 \\ \text { TL } & 81 & 200 \\ \text { TL } & 81 & 201 \\ \text { TL } & 81 & 202 \\ \text { PB } & 81 & 204 \\ \text { PE } & 82 & 200 \\ \text { PB } & 82 & 202 \\ \text { PB } & 82 & 203 \\ \text { PE } & 82 & 204 \\ \text { PB } & 82 & 205 \\ \text { BI } & 82 & 210 \\ \text { BI } & 83 & 205 \\ \text { HI } & 83 & 206 \\ \text { BI } & 83 & 207 \\ \text { BI } & 83 & 208 \\ \text { BI } & 83 & 210 \\ \text { PO } & 83 & 210 M \\ \text { PO } & 84 & 206 \\ \text { PO } & 84 & 208 \\ \text { PO } & 84 & 209 \\ \text { RN } & 84 & 210 \\ \text { RN } & 86 & 211 \\ \text { RA } & 86 & 222 \\ \text { RA } & 88 & 223 \\ \text { RA } & 88 & 224 \\ \text { RA } & 88 & 225 \\ \text { RA } & 88 & 226 \\ \text { AC } & 88 & 228 \\ \text { AC } & 89 & 225 \\ \text { TH } & 89 & 227 \\ \text { TH } & 90 & 227 \\ \text { TH } & 90 & 228 \\ \text { TH } & 90 & 229 \\ \text { TH } & 90 & 230 \\ \text { TH } & 90 & 231 \\ \text { TH } & 90 & 232 \\ \text { PA } & 90 & 234 \\ \text { PA } & 91 & 228 \\ \text { PA } & 91 & 229 \\ \text { PA } & 91 & 230 \\ \text { PA } & 91 & 231 \\ \text { PA } & 91 & 233 \\ \text { U } & 92 & 230\end{array}$

10/09/68 PAGE $U C / M * * 3$

2. $0 E-02$

3. $1 E-05$

$3.5 \mathrm{E}-03$

1. OE-O2

$1.5 E-02$

$6.3 \mathrm{E}-04$

$3.8 E-03$

7. $7 \mathrm{E}-03$

$9.7 E-04$

$1.4 E-05$

4.6E-01

. 6E-04

4.3E-01

$3.6 E-05$

$1.1 E-03$

2. 5E-05

B. 3E-06

$2.2 \mathrm{E}-05$

2. $4 \mathrm{E}-0 \mathrm{~B}$

$1.2 E-08=$

$8.6 E-06=$

$5.8 \mathrm{E}-09$ bo

5. $0 \mathrm{E}-02 \bigcirc$

1. 7E-04

4. $0 E-05$

$1.8 \mathrm{E}-03$

3. $O E+00$

1. $9 E-01$

4.6E-02

$1.4 \mathrm{E}-01$

$3.6 \mathrm{E}-02$

$6.4 E-05$

$2.1 \mathrm{E}-04$

$7.3 \mathrm{E}-02$

$1.3 \mathrm{E}-04$

$1.1 E-06$

$2.8 \mathrm{E}-08$

2.7E-09

2. 5E-09

1. 6 E-05

2. 2 E-O9

$6.7 E-07$

$6.2 E-01$

4.1E-01

$3.3 E-02$

7. $0 E-05$

3. $9 E-01$

2. $O E-02$

2. $1 E+04$

5. $2 E+01$

3. $8 \mathrm{E}-02$ 
TABLE 9A. UNIT-RAD CONTAMINATION FOR BONE

\begin{tabular}{|c|c|c|c|c|}
\hline $\begin{array}{l}\text { ELEMENT } \\
\text { SYMBOL }\end{array}$ & $\begin{array}{l}\text { ATOMIC } \\
\text { NO. }(Z)\end{array}$ & $\begin{array}{l}\text { ISOTOPE } \\
\text { MASS(A) }\end{array}$ & $\begin{array}{l}\text { ADULT } \\
\text { UC/M**3 }\end{array}$ & $\begin{array}{l}\text { INFANT } \\
\text { UC } / M * * 3\end{array}$ \\
\hline $\begin{array}{l}U \\
U \\
U \\
U \\
U \\
U \\
U \\
U \\
U \\
N P \\
N P \\
N P \\
N P \\
N P \\
N P \\
N P \\
P U \\
P U \\
P U \\
P U \\
P U \\
P U \\
P U \\
P U \\
P U \\
A M \\
A M \\
A M \\
A M \\
A M \\
A M\end{array}$ & $\begin{array}{l}92 \\
92 \\
92 \\
92 \\
92 \\
92 \\
92 \\
92 \\
92 \\
93 \\
93 \\
93 \\
93 \\
93 \\
93 \\
93 \\
94 \\
94 \\
94 \\
94 \\
94 \\
94 \\
94 \\
94 \\
94 \\
95 \\
95 \\
95 \\
95 \\
95 \\
95\end{array}$ & $\begin{array}{l}231 \\
232 \\
233 \\
234 \\
235 \\
236 \\
237 \\
238 \\
240 \\
234 \\
235 \\
236 \\
236 M \\
237 \\
238 \\
239 \\
236 \\
237 \\
238 \\
239 \\
240 \\
241 \\
242 \\
244 \\
246 \\
239 \\
240 \\
241 \\
242 \\
242 M \\
243\end{array}$ & $\begin{array}{l}1.4 E+01 \\
1.2 E-04 \\
1.1 E-04 \\
1.1 E-04 \\
9.8 E-05 \\
9.7 E-05 \\
4.8 E+00 \\
9.3 E-05 \\
5.3 E+02 \\
1.8 E+03 \\
2.0 E-01 \\
1.2 E-03 \\
4.0 E+04 \\
1.2 E-03 \\
7.3 E+03 \\
5.6 E+03 \\
3.1 E-02 \\
1.7 E+01 \\
1.4 E-03 \\
1.1 E-03 \\
1.1 E-03 \\
3.0 E-03 \\
1.1 E-03 \\
9.5 E-04 \\
2.2 E+02 \\
1.2 E+05 \\
6.5 E+03 \\
1.1 E-03 \\
6.5 E+04 \\
1.1 E-03 \\
1.0 E-03\end{array}$ & $\begin{array}{l}1.9 E-01 \\
1.2 E-04 \\
1.1 E-04 \\
1.0 E-04 \\
9.4 E-05 \\
9.3 E-05 \\
1.1 E-01 \\
8.9 E-05 \\
1.0 E+00 \\
1.1 E-01 \\
1.1 E-03 \\
5.9 E-05 \\
5.0 E-01 \\
6.2 E-05 \\
2.1 E-01 \\
1.8 E-01 \\
4.4 E-04 \\
1.0 E-02 \\
6.6 E-05 \\
5.6 E-05 \\
5.5 E-05 \\
1.1 E-04 \\
5.3 E-05 \\
4.7 E-05 \\
3.3 E-02 \\
8.4 E-01 \\
1.9 E-01 \\
5.7 E-05 \\
5.9 E-01 \\
5.6 E-05 \\
5.0 E-05\end{array}$ \\
\hline
\end{tabular}

VIA SEAWATER.

$\begin{array}{lcc}\text { ELEMENT } & \text { ATOMIC } & \text { I SOTOPE } \\ \text { SYMBOL } & \text { NO. (Z) } & \text { MASS(A) } \\ \text { CM } & 96 & 240 \\ \text { CM } & 96 & 241 \\ \text { CM } & 96 & 242 \\ \text { CM } & 96 & 243 \\ \text { CM } & 96 & 244 \\ \text { CM } & 96 & 245 \\ \text { CM } & 96 & 246 \\ \text { CM } & 96 & 247 \\ \text { CM } & 96 & 248 \\ \text { BK } & 97 & 245 \\ \text { BK } & 97 & 246 \\ \text { BK } & 97 & 247 \\ \text { BK } & 97 & 248 \\ \text { BK } & 97 & 249 \\ \text { CF } & 98 & 246 \\ \text { CF } & 98 & 248 \\ \text { CF } & 98 & 249 \\ \text { CF } & 98 & 250 \\ \text { CF } & 98 & 251 \\ \text { CF } & 98 & 252 \\ \text { CF } & 98 & 253 \\ \text { ES } & 99 & 251 \\ \text { ES } & 99 & 252 \\ \text { ES } & 99 & 253 \\ \text { ES } & 99 & 254 \\ \text { ES } & 99 & 254 M \\ \text { ES } & 99 & 255 \\ \text { FM } & 100 & 252 \\ \text { FM } & 100 & 253 \\ \text { FM } & 100 & 255 \\ \text { FM } & 100 & 257 \\ \text { F } & & \end{array}$

\begin{tabular}{|c|c|}
\hline $10 / 09 / 68$ & PAGE 5 \\
\hline $\begin{array}{l}\text { ADULT } \\
\text { UC } / M * * 3\end{array}$ & $\begin{array}{l}\text { INFANT } \\
U C / M * * 3\end{array}$ \\
\hline $\begin{array}{l}4.0 E+01 \\
2.5 E+01 \\
1.1 E+00 \\
1 \cdot 5 E-03 \\
2.0 E-03 \\
1.0 E-03 \\
9.6 E-04 \\
8.9 E-04 \\
8.7 E-04 \\
1.1 E+03 \\
8.0 E+03 \\
9.0 E-04 \\
2.6 E+04 \\
2.5 E-01 \\
1.2 E+04 \\
2.0 E-01 \\
9.3 E-04 \\
3.0 E-03 \\
8.3 E-04 \\
2.6 E-02 \\
6.2 E+01 \\
1.0 E+04 \\
1.1 E+00 \\
5.6 E+01 \\
9.4 E-02 \\
8.1 E+03 \\
3.6 E+01 \\
2.4 E+04 \\
1.1 E+03 \\
2.9 E+04 \\
3.3 E+00\end{array}$ & $\begin{array}{l}1.5 E-02 \\
1.2 E-02 \\
2.4 E-03 \\
6.7 E-05 \\
8.3 E-05 \\
5.0 E-05 \\
4.8 E-05 \\
4.5 E-05 \\
4.4 E-05 \\
7.6 E-02 \\
2.0 E-01 \\
4.5 E-05 \\
3.5 E-01 \\
1.1 E-03 \\
2.4 E-01 \\
9.6 E-04 \\
4.6 E-05 \\
1.0 E-04 \\
4.1 E-05 \\
3.2 E-04 \\
1.6 E-02 \\
2.1 E-01 \\
2.1 E-03 \\
1.5 E-02 \\
6.1 E-04 \\
1.8 E-01 \\
1.2 E-02 \\
3.1 E-01 \\
6.8 E-02 \\
3.4 E-01 \\
3.5 E-03\end{array}$ \\
\hline
\end{tabular}


TABLE 9A. UNIT-RAD CONTAMINATION FOR KIONEYS

\begin{tabular}{|c|c|c|}
\hline $\begin{array}{l}\text { ELEMENT } \\
\text { SYMBOL }\end{array}$ & $\begin{array}{l}\text { ATOMIC } \\
\text { ND. }(2)\end{array}$ & $\begin{array}{l}\text { ISOTOPE } \\
\text { MASS (A) }\end{array}$ \\
\hline H & 1 & 3 \\
\hline$B E$ & 4 & 7 \\
\hline $\mathrm{BE}$ & 4 & 10 \\
\hline C & 6 & 14 \\
\hline VA & 11 & 22 \\
\hline VA & 11 & 24 \\
\hline MG & 12 & 28 \\
\hline AL & 13 & 26 \\
\hline SI & 14 & 32 \\
\hline $\mathbf{P}$ & 15 & 32 \\
\hline $\mathbf{P}$ & 15 & 33 \\
\hline$S$ & 16 & 35 \\
\hline$C L$ & 17 & 36 \\
\hline AR & 18 & 37 \\
\hline AR & 18 & 39 \\
\hline AR & 18 & 42 \\
\hline K & 19 & 40 \\
\hline$K$ & 19 & 42 \\
\hline$K$ & 19 & 43 \\
\hline CA & 20 & 41 \\
\hline CA & 20 & 45 \\
\hline CA & 20 & 47 \\
\hline $\begin{array}{l}\text { CA } \\
\text { SC }\end{array}$ & $\begin{array}{l}20 \\
21\end{array}$ & $\begin{array}{l}48 \\
44 M\end{array}$ \\
\hline SC & 21 & 46 \\
\hline SC & 21 & 47 \\
\hline sc & 21 & 48 \\
\hline T I & 22 & 44 \\
\hline$v$ & 23 & 48 \\
\hline$v$ & 23 & 49 \\
\hline$v$ & 23 & 50 \\
\hline CR & 24 & 48 \\
\hline CR & 24 & 51 \\
\hline MN & 25 & 52 \\
\hline MN & 25 & 53 \\
\hline MN & 25 & 54 \\
\hline FE & 26 & 55 \\
\hline FE & 26 & 59 \\
\hline FE & 26 & 60 \\
\hline $\mathrm{CO}$ & 27 & 55 \\
\hline $\mathrm{CJ}$ & 27 & 56 \\
\hline $\mathrm{CO}$ & 27 & 37 \\
\hline CO & 27 & 58 \\
\hline $\mathrm{CO}$ & 27 & 60 \\
\hline VI & 28 & 56 \\
\hline NI & 28 & 57 \\
\hline NI & 28 & 59 \\
\hline VI & 28 & 63 \\
\hline NI & 28 & 66 \\
\hline cu & 29 & 64 \\
\hline
\end{tabular}

VIA SEAWATER.

INFANT
UC $/ M * 3$

UC $/ M * * 3$

$5.4 E+32$

6. $4 E+02$

$\begin{array}{ll}5.5 E+00 & 1.7 E+03 \\ 2.1 E-03 & 2.1 E-03\end{array}$

3. $4 E-03 \quad 3.4 E-03$

2. $9 E+01 \quad 2.9 E+01$

3. $9 E+05 \quad 2.1 E+04$

4. $4 E+03 \quad 4.4 E+03$

$4.5 \mathrm{E}-03$

4. $4.5 E-03$ TE

6. $9 E-02 \quad 3 E-01 \quad 2.0 E-01$

$\begin{array}{ll}\text { 3. } 4 E-01 & 2.0 E-01 \\ 2.4 E+02 & 2.4 E+02\end{array}$

4. $9 E+01 \quad 4.8 E+01$

8. $2 E+02 \quad 8.2 E+02$

4. $4 \mathrm{E}-02$ 4. $4 \mathrm{E}-02$

4.7E-03 4.7E-03

$3.6 \mathrm{E}-01 \quad 3.6 \mathrm{E}-01$

$1.6 E+03$

$1.1 E+05 \quad 1.7 E+03$

2. $2 E+03 \quad 2.0 E+33$

1. $6 E+04 \quad 3.4 E+03$

1. $1 E+00 \quad 1.1 E+D O$

1. $4 E+01 \quad 2.2 E-02$

1.7E-02 $\quad 8.5 E-04$

$2.3 \mathrm{E}-02$

2. $7 \mathrm{E}-03 \quad 2.3 \mathrm{E}-02$

3. $9 E+01 \quad 6.9 E+0 J$

2. $5 E+02.2 .1 E+02$

3. $6 E-02 \quad 3.6 E-02$

2. $6 E+03 \quad 4.1 E+J J$

3. $3 E+02 \quad 1.4 E+0$

9. $4 \mathrm{E}-02 \quad 1.7 \mathrm{E}-02$

$7.8 \mathrm{E}-03$
$1.5 \mathrm{E}-03$

3. $0 t-01$

3. $2 E-01$

$6.1 E-05 \quad 5.5 E-05$

$1.1 E+03 \quad 3.4 E+31$

5. $2+.01$ 3.5E

1. $1.00 \quad i .6 E+00$

$1.1 t+00 \quad 9.5 E-01$

$1.2 E-02$

3. $9 E+01 \quad 3.9 E+01$

2. $O E+00$ 2. $O E+O D$

O. $3 t-01$

2. $8 E+01$

2. $3 E+03$

$9.8 \mathrm{E}-01$

2. $8 E+01$

$1.4 E+01$

$\begin{array}{lllll}\text { ELEMENT } & \text { ATOMIC } & \text { ISOTOPE } & \text { ADULT } & \text { INFANT } \\ \text { SYMBOL } & \text { NO.(Z) } & \text { MASS(A) } & \text { UC } / M * * 3 & \text { UC/M**3 }\end{array}$

SYMBOL $\because \mathrm{C} .(Z)$ MASS (A) UC/M**3 UCIM*\#3

1. $2 E+02 \quad 3.4 E+00$

8. $8 E-04 \quad 5.4 E-04$

3. $5 E+01$

6. $2 \mathrm{E}-01$

2. $3 E+02$

3. $6 \mathrm{E}+02$

$1.8 \mathrm{E}-05$

2. $1 E-02$

1. $5 E-01$

5. $0 E+05$

6. $1 E+05$

. $0 E+04$

3. $9 E+03$

8. $2 E+05$

2. $9 E+06$

3. $O E+O O$

5. 3E- 01

9. $7 E-02$

$1.4 E+05$

1. $4 E+05$

2. $2 E+04$

1. $5 E+02$

1. $9 E+02$

1. $3 E+0 O$

2. $5 \mathrm{E}-01$

3. $2 E+01$

4. $3 E+01$

2. $1 E+02$

$1.2 E+00$

2. $4 E+02$

2. $4 E+02$
$1.0 E+05$

1. $O E+05$

4. $7 E+02$

4. $3 E+02$

1. $5 E+00$

1. $1 E+02$

1. $8 E+01$

4. $2 E+02$

6. $5 E-02$

$1.6 E+03$

1. $8 \mathrm{E}-01$

1. $8 E+02$

3. $0 E-02$

5. 5E-01

2. $3 E+03$

5. $3 E+01$

1. $1 E-03$

1. $2 E-01$

$5.4 E-04$
$1.4 E-01$

8. $O E-03$

6. $1 E+01$

2. $2 E+01$

$1.8 E-05$

2. $6 E-03$

7. $1 E-02$

$2.4 E+03$

1. $2 E+03$

4. $9 E+03$

$1.2 E+02$

1. $7 E+03$

8. $5 \mathrm{E}+03$

$1.3 E+00$

4. $8 \mathrm{E}-01$

$9.6 \mathrm{E}-02$

$1.1 E+04$

$6.6 E+03$

$3.9 \mathrm{E}+03$

1. $5 E+02$

1. $9 E+02$

$1.3 E+00$

$2.5 E-01$ क

$1.6 \mathrm{E}+01$

1. $2 E+01$

1.2E+01

3. $9 E+01$

1. $4 E+02$

8. $3 E+03$

3. $8 E+02$

$3.3 E+02$

1. $5 E+00$

1. $7 \mathrm{E}-02$

1. $5 \mathrm{E}-02$

5. $9 E-02$

1. $5 \mathrm{E}-04$

$1.2 \mathrm{E}-02$

9. $0 E-04$

$1.3 E+00$
$1.5 E-02$

1. $5 \mathrm{E}-02$

6. 7E-01

2. $7 E-02$

3. $7 \mathrm{E}-02$

1. $8 E+00$

$4.2 E-02$

ใ.

$9.4 E-03$

$9.4 \mathrm{E}-03$
$1.4 \mathrm{E}-02$ 


\begin{tabular}{|c|c|c|c|c|}
\hline $\begin{array}{l}\text { ELEMENT } \\
\text { SYMBOL }\end{array}$ & $\begin{array}{l}\text { ATJMIC } \\
\text { VO. }(Z)\end{array}$ & $\begin{array}{l}\text { ISOTOPE } \\
\text { MASS (A) }\end{array}$ & $\begin{array}{c}\text { ADULT } \\
\text { UC/M**3 }\end{array}$ & $\begin{array}{l}\text { INFANT } \\
\text { UC } / M * * 3\end{array}$ \\
\hline $\begin{array}{l}N B \\
N B \\
V B \\
V B \\
N B \\
M D \\
M D \\
T C \\
T C \\
T C \\
T C \\
T C \\
T C \\
T C \\
T C \\
R U \\
R U \\
R U \\
R H \\
R H \\
R H \\
R H \\
R H \\
R H \\
R H \\
P D \\
P D \\
P D \\
P D \\
P D \\
P D \\
A G \\
A G \\
A G \\
A G \\
A G \\
C D \\
C D \\
C D \\
C D \\
I V \\
I N \\
I N \\
S N \\
S N \\
S N \\
S N \\
S N \\
S N \\
S N \\
S N \\
S B \\
S B\end{array}$ & $\begin{array}{l}41 \\
41 \\
41 \\
41 \\
41 \\
42 \\
42 \\
43 \\
43 \\
43 \\
43 \\
43 \\
43 \\
43 \\
44 \\
44 \\
44 \\
45 \\
45 \\
45 \\
45 \\
45 \\
45 \\
46 \\
46 \\
46 \\
46 \\
46 \\
47 \\
47 \\
47 \\
47 \\
47 \\
48 \\
48 \\
48 \\
48 \\
49 \\
49 \\
49 \\
50 \\
50 \\
50 \\
50 \\
50 \\
50 \\
50 \\
50 \\
51 \\
51\end{array}$ & $\begin{array}{l}93 M \\
94 \\
95 \\
95 M \\
96 \\
93 \\
99 \\
95 \\
95 M \\
96 \\
97 \\
97 M \\
98 \\
99 \\
97 \\
103 \\
106 \\
99 \\
100 \\
101 \\
101 M \\
102 \\
105 \\
100 \\
103 \\
107 \\
109 \\
112 \\
105 \\
106 M \\
108 M \\
110 M \\
111 \\
109 \\
113 M \\
115 \\
115 M \\
111 \\
114 M \\
115 \\
113 \\
117 M \\
119 M \\
121 \\
121 M \\
123 M \\
125 \\
126 \\
119 \\
120 M\end{array}$ & $\begin{array}{l}5.4 E-03 \\
1.1 E-05 \\
1.1 E-01 \\
7.1 E+00 \\
4.0 E+01 \\
1.4 E+00 \\
8.0 E+02 \\
5.0 E+01 \\
2.8 E-02 \\
7.1 E-01 \\
8.9 E-03 \\
1.6 E-01 \\
1.2 E-04 \\
2.0 E-03 \\
6.1 E+00 \\
3.9 E-02 \\
2.8 E-04 \\
4.8 E-02 \\
3.3 E+00 \\
2.3 E-04 \\
6.4 E-01 \\
4.4 E-04 \\
7.3 E+00 \\
2.0 E-01 \\
3.2 E-01 \\
1.5 E-02 \\
2.4 E+01 \\
1.7 E+00 \\
1.7 E+00 \\
4.6 E+00 \\
1.5 E-02 \\
9.6 E-02 \\
3.0 E+01 \\
2.2 E-05 \\
9.2 E-07 \\
3.2 E-02 \\
1.2 E-04 \\
2.4 E-03 \\
4.7 E-06 \\
8.2 E-08 \\
9.5 E+00 \\
7.7 E+02 \\
1.2 E+01 \\
3.1 E+05 \\
1.1 E-01 \\
5.1 E+00 \\
2.8 E+02 \\
3.1 E-03 \\
8.2 E+00 \\
4.7 E-02\end{array}$ & $\begin{array}{l}3.5 E-03 \\
1.0 E-05 \\
4.8 E-03 \\
3.5 E-02 \\
5.2 E-02 \\
1.4 E+00 \\
8.2 E+01 \\
2.0 E+00 \\
2.1 E-02 \\
1.3 E-01 \\
8.9 E-03 \\
1.3 E-01 \\
1.2 E-04 \\
2.0 E-03 \\
9.6 E-02 \\
7.1 E-03 \\
1.9 E-04 \\
1.7 E-02 \\
9.9 E-02 \\
2.3 E-04 \\
8.9 E-02 \\
3.8 E-04 \\
3.7 E-01 \\
2.2 E-02 \\
1.2 E-01 \\
1.5 E-02 \\
4.4 E-01 \\
4.9 E-02 \\
1.5 E+00 \\
3.1 E+00 \\
1.5 E-02 \\
9.4 E-02 \\
1.9 E+01 \\
1.3 E-05 \\
8.5 E-07 \\
2.4 E-04 \\
1.6 E-05 \\
1.1 E-04 \\
2.1 E-06 \\
8.1 E-08 \\
1.4 E+00 \\
1.6 E+01 \\
3.2 E+000 \\
5.2 E+02 \\
1.0 E-01 \\
8.2 E-01 \\
4.0 E+00 \\
2.8 E-03 \\
8.2 E+00 \\
4.7 E-02\end{array}$ \\
\hline
\end{tabular}

ELEMENT
SYMBOL

SB
SB
SB
SB
SB
$T E$
$T E$
$T E$
$T E$
$T E$
$T E$
$T E$
$T E$
$T E$
$T E$
$T E$
$T E$
$T E$
$T E$
$I$
$I$
$I$
$I$
$I$
$I$
$I$
$I$
$I$
$X E$
$X E$
$X E$
$X E$
$X E$
$X E$
$X E$
$C S$
$C S$
$C S$
$C S$
$C S$
$C S$
$C S$
$B A$
$B A$
$B A$
$B A$
$B A$
$B A$
$L A$
$L A$
$L A$
$L A$
$C E$

ATOMIC ISOTOPE
ND. (Z) MASS(A)

$U C / M * * 3$

2. $2 \mathrm{E}-01$

1. $3 \mathrm{E}-03$

2. $0 E-02$

1. $4 \mathrm{E}-01$

2. $2 E-02$

1. $1 E+00$

8. $2 \mathrm{E}-03$

2. $7 E-04$

1. $3 \mathrm{E}-04$

1. $3 E-03$

5. $4 E-03$

1. $1 E-03$

1. $8 \mathrm{E}-03$

2. 6E-01

3. $0 E-02$

3. $9 E+05$

$9.6 E+02$

9. $4 E+02$

7. $6 E+02$

5. $8 E+00$

3. $8 E+04$

1. $3 E+03$

2. $8 E+04$

2. $1 E+02$

1. $1 E+03$

5. $3 E+01$

2. $4 E+02$

2. $3 E+02$

4. $6 E+02$

$4.7 E+02$

2. $9 E+04$

9. $0 E+03$

2. 1E-01

6. 5E-01

8. $4 E+01$

6. 2E-02

1. $6 E+02$

1. $2 E+00$

$6.7 E+03$

6. $7 E+03$

2. $9 E+04$

4. $1 E+01$

3. $2 E+03$
8. $2 E+00$

2. $4 E-01$

4. $4 E+02$

$9.8 E+01$
INFAN T

$U C / M * * 3$

2. 2 E-01

$1.3 E-03$

$2.0 E-02$

$1.4 E-01$

$1.4 \mathrm{E}-01$

$3.6 E-03$

2. $3 E-02$

3. 0 E-03

2. 3E-04

1. 3 E-04

1. 0 E-03

3. $6 E-03$

8. 3E-04

$9.7 E-04$

1. OE-02

3. $0 E-03$

2. $8 E+04$

$3.6 E+02$

$8.4 E+02$

5. $0 E+02$

$5.8 E+00$

$2.6 \mathrm{E}+03$

7. $0 E+02$

$3.1 E+03$

$2 \cdot 1 E+02$

$1.1 E+03$

5. $3 E+01$

$2.4 E+02$

2. $3 E+02$

4. $6 E+02$

4. $7 E+02$

$3.4 E+02$

7. $9 E+02$

1. $B E-01$

$6.4 E-01$

8. $9 E+00$

6. $1 E-02$

1. $9 E+02$

9. $2 E+01$

$1 \cdot 2 E+00$

1. $1 E+03$

3. $7 \mathrm{E}+03$

2. $5 E+01$

$3.2 E+03$

8. $2 E+00$

2. $4 E-01$

4. $4 E+02$
$5.2 E-01$ 
TABLE 9A. UNIT-RAD CONTAMINATION FOR KIDNEYS

\begin{tabular}{|c|c|c|c|c|}
\hline $\begin{array}{l}\text { E.EMENT } \\
\text { S/MBOL }\end{array}$ & $\begin{array}{l}\text { A TOMIC } \\
\text { NO. }(Z)\end{array}$ & $\begin{array}{l}\text { ISOTDPE } \\
\text { MASS (A) }\end{array}$ & $\begin{array}{l}\text { ADULT } \\
U C / M * * 3\end{array}$ & $\begin{array}{l}\text { INFANT } \\
\text { UC } / M * * 3\end{array}$ \\
\hline $\begin{array}{l}C E \\
C E \\
C E \\
C E \\
C E \\
C E \\
C E \\
P R \\
P R \\
N D \\
N D \\
N D \\
P M \\
P M \\
P M \\
P M \\
P M \\
P M \\
P M \\
P M \\
P M \\
S M \\
S M \\
S M \\
S M \\
S M \\
S M \\
S M \\
S M \\
E U \\
E U \\
E U \\
E U \\
E U \\
E U \\
E U \\
E U \\
E U \\
E U \\
E U \\
E U \\
E U \\
G D \\
G D \\
G D \\
G D \\
G D \\
G D \\
G D \\
G D\end{array}$ & $\begin{array}{l}58 \\
58 \\
58 \\
58 \\
58 \\
58 \\
58 \\
59 \\
59 \\
60 \\
60 \\
60 \\
61 \\
61 \\
61 \\
61 \\
61 \\
61 \\
61 \\
61 \\
61 \\
62 \\
62 \\
62 \\
62 \\
62 \\
62 \\
62 \\
62 \\
63 \\
63 \\
63 \\
63 \\
63 \\
63 \\
63 \\
63 \\
63 \\
63 \\
63 \\
63 \\
63 \\
64 \\
64 \\
64 \\
64 \\
64 \\
64 \\
64 \\
64\end{array}$ & $\begin{array}{l}135 \\
137 M \\
139 \\
141 \\
142 \\
143 \\
144 \\
142 \\
143 \\
140 \\
144 \\
147 \\
143 \\
144 \\
145 \\
146 \\
147 \\
148 \\
148 M \\
149 \\
151 \\
141 \\
145 \\
146 \\
147 \\
148 \\
149 \\
151 \\
153 \\
145 \\
146 \\
146 M \\
147 \\
148 \\
149 \\
150 \\
150 \\
152 \\
154 \\
155 \\
156 \\
157 \\
146 \\
147 \\
148 \\
149 \\
150 \\
151 \\
152 \\
153\end{array}$ & $\begin{array}{l}2.3 E+03 \\
1.6 E+03 \\
3.8 E-01 \\
4.7 E+00 \\
2.7 E-04 \\
5.3 E+02 \\
6.0 E-03 \\
4.0 E-02 \\
4.4 E-04 \\
2.1 E-03 \\
3.5 E-09 \\
8.5 E-05 \\
1.5 E-02 \\
1.2 E-03 \\
1.9 E-03 \\
4.4 E-04 \\
3.2 E-04 \\
6.3 E+00 \\
3.1 E-02 \\
1.3 E+02 \\
1.9 E+02 \\
1.1 E+00 \\
1.5 E-01 \\
1.5 E-04 \\
1.7 E-04 \\
9.9 E-05 \\
2.1 E-04 \\
2.9 E-02 \\
1.3 E+03 \\
2.3 E+01 \\
5.9 E+00 \\
7.3 E+01 \\
3.4 E-01 \\
3.8 E-01 \\
9.8 E-02 \\
4.0 E-04 \\
4.8 E+02 \\
1.2 E-04 \\
9.0 E-05 \\
1.8 E-02 \\
1.5 E+00 \\
1.3 E+03 \\
3.4 E-02 \\
8.4 E+01 \\
1.9 E-05 \\
4.3 E+00 \\
9.8 E-06 \\
9.1 E-02 \\
2.4 E-05 \\
5.7 E-02\end{array}$ & $\begin{array}{l}3.0 \mathrm{E}+00 \\
4.2 \mathrm{E}+00 \\
7.5 \mathrm{E}-02 \\
2.6 \mathrm{E}-01 \\
2.5 \mathrm{E}-04 \\
1.3 \mathrm{E}+00 \\
2.0 \mathrm{E}-03 \\
4.3 \mathrm{E}-05 \\
8.0 \mathrm{E}-06 \\
1.1 \mathrm{E}-05 \\
3.2 \mathrm{E}-09 \\
1.4 \mathrm{E}-06 \\
4.3 \mathrm{E}-03 \\
4.2 \mathrm{E}-04 \\
1.7 \mathrm{E}-03 \\
3.1 \mathrm{E}-04 \\
1.9 \mathrm{E}-04 \\
5.1 \mathrm{E}-02 \\
1.8 \mathrm{E}-03 \\
4.4 \mathrm{E}-01 \\
3.4 \mathrm{E}-01 \\
3.3 \mathrm{E}-02 \\
5.0 \mathrm{E}-02 \\
1.4 \mathrm{E}-04 \\
1.6 \mathrm{E}-04 \\
9.1 \mathrm{E}-05 \\
1.9 \mathrm{E}-04 \\
2.6 \mathrm{E}-02 \\
3.9 \mathrm{E}+00 \\
9.1 \mathrm{E}-02 \\
1.8 \mathrm{E}-02 \\
7.8 \mathrm{E}-02 \\
5.5 \mathrm{E}-03 \\
1.3 \mathrm{E}-02 \\
6.5 \mathrm{E}-03 \\
2.2 \mathrm{E}-04 \\
1.7 \mathrm{E}-01 \\
8.5 \mathrm{E}-05 \\
6.5 \mathrm{E}-05 \\
5.5 \mathrm{E}-03 \\
1.6 \mathrm{E}-02 \\
5.6 \mathrm{E}-01 \\
1.2 \mathrm{E}-03 \\
6.7 \mathrm{E}-02 \\
1.5 \mathrm{E}-05 \\
3.0 \mathrm{E}-02 \\
8.2 \mathrm{E}-06 \\
7.7 \mathrm{E}-03 \\
2.0 \mathrm{E}-05 \\
8.9 \mathrm{E}-03\end{array}$ \\
\hline
\end{tabular}

VIA SEAWATER.

$\begin{array}{lcc}\text { ELEMENT } & \text { ATOMIC } & \text { ISOTOP } \\ \text { SYMBOL } & \text { NO. I I } & \text { MASS IA } \\ \text { GD } & & \\ \text { GD } & 64 & 159 \\ \text { TB } & 64 & 162 \\ \text { TB } & 65 & 151 \\ \text { TB } & 65 & 152 \\ \text { TB } & 65 & 153 \\ \text { TB } & 65 & 154 \\ \text { TB } & 65 & 155 \\ \text { TB } & 65 & 156 \\ \text { TB } & 65 & 157 \\ \text { TB } & 65 & 158 \\ \text { TB } & 65 & 160 \\ \text { TB } & 65 & 161 \\ \text { DY } & 65 & 164 \\ \text { DY } & 66 & 154 \\ \text { DY } & 66 & 154 M \\ \text { DY } & 66 & 156 \\ \text { DY } & 66 & 159 \\ \text { HO } & 66 & 166 \\ \text { HO } & 67 & 163 \\ \text { HU } & 67 & 166 \\ \text { ER } & 67 & 166 M \\ \text { ER } & 68 & 160 \\ \text { ER } & 68 & 169 \\ \text { TM } & 68 & 172 \\ \text { TM } & 69 & 165 \\ \text { TM } & 69 & 167 \\ \text { TM } & 69 & 168 \\ \text { TM } & 69 & 170 \\ \text { TM } & 69 & 171 \\ \text { YB } & 69 & 172 \\ \text { YB } & 70 & 166 \\ \text { YB } & 70 & 169 \\ \text { LU } & 70 & 175 \\ \text { LU } & 71 & 169 \\ \text { LU } & 71 & 170 \\ \text { LU } & 71 & 171 \\ \text { LU } & 71 & 172 \\ \text { LU } & 71 & 173 \\ \text { LU } & 71 & 174 \\ \text { LU } & 71 & 174 M \\ \text { LU } & 71 & 176 \\ \text { LU } & 71 & 177 \\ \text { HF } & 71 & 177 M \\ \text { HF } & 72 & 170 \\ \text { HF } & 72 & 171 \\ \text { HF } & 72 & 172 \\ \text { HF } & 72 & 173 \\ \text { HF } & 72 & 174 \\ \text { HF } & 72 & 175 \\ & 72 & 181\end{array}$

$10 / 09 / 68$

PAGE 8

INFANT UC $/ M * * 3$

$U C / M * * 3$

1. $8 E+03$

1. $1 E+00$ 6. $0 E-04$ 7. $1 \mathrm{E}-01$ $2.1 E-01$ 4.6E-01 8. 8E-01 2. 3E-01 $6.8 E-02$ 1.5E-03 $6.6 \mathrm{E}-05$ 6. $5 E-03$ 5. $E$ E 03 4. $0 E-01$ $1.4 E+00$ 5. $9 E-10$ 2. $0 \mathrm{E}-05$ 9. $2 E-10$ 3. 8E-06 $9.4 E-06$ $9.5 \mathrm{E}-08$ 3. $7 E-05$
$4.2 E-09$ 4.2E-09 N 1.2E-05 $1.6 E-05$

$8.6 \mathrm{E}-01$

4. $8 \mathrm{E}-01$ $2.1 E-02$ $5.8 E-02$ 2. $0 E-01$ 2.0E-01 1. $1 E+00$ $5.5 E-02$ 8. 8E-01 $1.4 \mathrm{E}-05$ $6.4 E-06$ 3. $E-05$ 3.4E-05 6. $0 E-06$
$1.1 E-07$ $1.1 E-07$
$2.4 E-08$ 4. $4 E-07$ 7. $1 E-09$ 3. $4 E-05$ 1. $2 E-06$ 1. $1 \mathrm{E}-02$ $2.5 E-02$ 1. $1 \mathrm{E}-05$ $1.3=-02$ 1. $2 \mathrm{E}-06$ 6. $8 E-04$
$7.3 E-04$ 


\begin{tabular}{|c|c|c|c|c|}
\hline $\begin{array}{l}\text { ELEMENT } \\
\text { SYMBOL }\end{array}$ & $\begin{array}{l}\text { ATJMIC } \\
N D .(Z)\end{array}$ & $\begin{array}{l}\text { ISOTOPE } \\
\text { MASS (A) }\end{array}$ & $\begin{array}{l}\text { ADULT } \\
U C / M * * 3\end{array}$ & $\begin{array}{l}\text { INFANT } \\
\text { UC } / M * * 3\end{array}$ \\
\hline 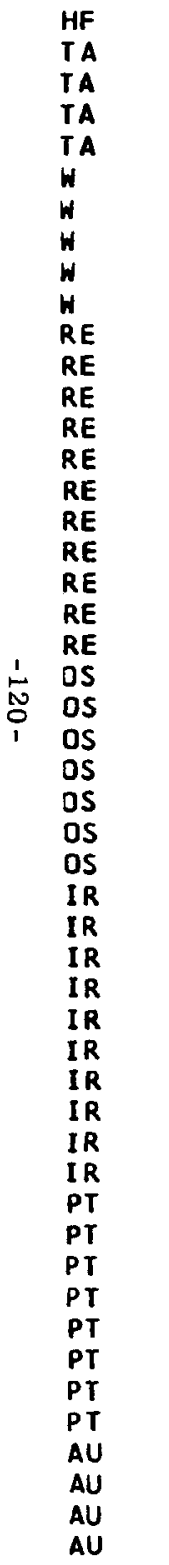 & $\begin{array}{l}72 \\
73 \\
73 \\
73 \\
73 \\
74 \\
74 \\
74 \\
74 \\
74 \\
75 \\
75 \\
75 \\
75 \\
75 \\
75 \\
75 \\
75 \\
75 \\
75 \\
75 \\
76 \\
76 \\
76 \\
76 \\
76 \\
76 \\
76 \\
77 \\
77 \\
77 \\
77 \\
77 \\
77 \\
77 \\
77 \\
77 \\
77 \\
78 \\
78 \\
78 \\
78 \\
78 \\
78 \\
78 \\
78 \\
79 \\
79 \\
79 \\
79\end{array}$ & $\begin{array}{l}182 \\
177 \\
179 \\
182 \\
183 \\
178 \\
181 \\
185 \\
187 \\
188 \\
180 \\
181 \\
182 \\
182 M \\
183 \\
184 \\
184 M \\
186 \\
187 \\
188 \\
189 \\
182 \\
183 \\
185 \\
191 \\
191 M \\
193 \\
194 \\
185 \\
186 \\
187 \\
188 \\
189 \\
190 \\
192 \\
192 M \\
193 M \\
194 \\
188 \\
190 \\
191 \\
192 \\
193 \\
193 M \\
195 M \\
197 \\
193 \\
194 \\
195 \\
196\end{array}$ & $\begin{array}{l}\text { 2. } 0 E-06 \\
3.6 E-01 \\
2.4 E-04 \\
1.4 E-04 \\
1.4 E-01 \\
3.6 E-04 \\
1.5 E-03 \\
1.5 E-03 \\
1.6 E-02 \\
1.5 E-04 \\
4.4 E-01 \\
4.8 E-01 \\
1.7 E-01 \\
8.2 E-01 \\
2.9 E-02 \\
1.8 E-02 \\
3.2 E-03 \\
4.0 E-01 \\
6.8 E-01 \\
8.9 E-01 \\
1.4 E+00 \\
7.9 E-02 \\
5.7 E-01 \\
3.4 E-04 \\
1.0 E-02 \\
1.4 E+00 \\
1.7 E-01 \\
5.1 E-06 \\
1.6 E+01 \\
9.5 E+00 \\
2.6 E+01 \\
1.1 E+00 \\
1.4 E-01 \\
4.2 E-02 \\
2.9 E-03 \\
1.5 E-05 \\
7.3 E-01 \\
8.7 E+00 \\
1.0 E-03 \\
1.8 E-07 \\
5.4 E-02 \\
2.1 E-07 \\
7.3 E-06 \\
6.1 E-02 \\
6.0 E-02 \\
1.2 E+00 \\
7.8 E+02 \\
4.7 E+01 \\
6.2 E-02 \\
9.4 E+00\end{array}$ & $\begin{array}{l}1.9 E-05 \\
2.1 \mathrm{E}-03 \\
1.4 \mathrm{E}-04 \\
3.2 \mathrm{E}-05 \\
1.7 \mathrm{E}-03 \\
3.6 \mathrm{E}-04 \\
1.5 \mathrm{E}-03 \\
1.5 \mathrm{E}-03 \\
1.6 \mathrm{E}-02 \\
1.5 \mathrm{E}-04 \\
4.4 \mathrm{E}-01 \\
4.8 \mathrm{E}-01 \\
1.7 \mathrm{E}-01 \\
8.2 \mathrm{E}-01 \\
2.9 \mathrm{E}-02 \\
1.8 \mathrm{E}-02 \\
3.2 \mathrm{E}-03 \\
4.0 \mathrm{E}-01 \\
6.8 \mathrm{E}-01 \\
8.9 \mathrm{E}-01 \\
1.4 \mathrm{E}+00 \\
1.2 \mathrm{E}-02 \\
5.2 \mathrm{E}-02 \\
3.3 \mathrm{E}-04 \\
7.8 \mathrm{E}-03 \\
1.5 \mathrm{E}-01 \\
3.7 \mathrm{E}-02 \\
5.1 \mathrm{E}-06 \\
2.0 \mathrm{E}-01 \\
1.2 \mathrm{E}-01 \\
2.5 \mathrm{E}-01 \\
3.5 \mathrm{E}-02 \\
2.9 \mathrm{E}-02 \\
8.0 \mathrm{E}-03 \\
1.8 \mathrm{E}-03 \\
1.5 \mathrm{E}-05 \\
1.4 \mathrm{E}-01 \\
1.4 \mathrm{E}-01 \\
1.5 \mathrm{E}-04 \\
1.7 \mathrm{E}-01 \\
2.6 \mathrm{E}-03 \\
2.1 \mathrm{E}-07 \\
7.3 \mathrm{E}-05 \\
4.2 \mathrm{E}-03 \\
3.9 \mathrm{E}-03 \\
1.7 \mathrm{E}-02 \\
2.1 \mathrm{E}+00 \\
2.7 \mathrm{E}-01 \\
2.4 \mathrm{E}-02 \\
2.0 \mathrm{E}-01\end{array}$ \\
\hline
\end{tabular}

\begin{tabular}{lcc} 
ELEMENT & ATOMIC & ISOTOPE \\
SYMBOL & VD. IZI & MASS(A) \\
AU & 79 & 198 \\
AU & 79 & 199 \\
HG & 80 & 194 \\
HG & 80 & $195 M$ \\
HG & 80 & 197 \\
HG & 80 & $197 M$ \\
HG & 80 & 203 \\
TL & 81 & 200 \\
TL & 81 & 201 \\
TL & 81 & 202 \\
TL & 81 & 204 \\
PB & 82 & 200 \\
PB & 82 & 202 \\
PB & 82 & 203 \\
PB & 82 & 204 \\
PB & 82 & 205 \\
PB & 82 & 210 \\
BI & 83 & 205 \\
BI & 83 & 206 \\
BI & 83 & 207 \\
BI & 83 & 208 \\
BI & 83 & 210 \\
BI & 83 & $210 M$ \\
PO & 84 & 206 \\
PO & 84 & 208 \\
PO & 84 & 209 \\
PO & 84 & 210 \\
RN & 86 & 211 \\
RN & 86 & 222 \\
RA & 88 & 223 \\
RA & 88 & 224 \\
RA & 88 & 225 \\
RA & 88 & 226 \\
RA & 88 & 228 \\
AC & 89 & 225 \\
AC & 89 & 227 \\
TH & 90 & 227 \\
TH & 90 & 228 \\
TH & 90 & 229 \\
TH & 90 & 230 \\
TH & 90 & 231 \\
TH & 90 & 232 \\
TH & 90 & 234 \\
PA & 91 & 228 \\
PA & 91 & 229 \\
PA & 91 & 230 \\
PA & 91 & 231 \\
PA & 91 & 232 \\
PA & 91 & 233 \\
U & 92 & 230 \\
\hline
\end{tabular}

ADULT $U C / M * * 3$

INFANT $U C / M * * 3$

4. $8 \mathrm{E}+01 \quad 4.6 \mathrm{E}-01$ $1.1 E+02 \quad 1.3 E+00$ 8.4E-04 7.6E-04 8.5E-01 8.8E-02 1. $6 E+00 \quad 2.6 E-01$ 5. $9 \mathrm{E}+00 \quad 3.8 \mathrm{E}-01$ 2. $1 E-02 \quad 1.6 E-02$ 1. 3E-01 3.8E-03 9.7E-02 7.7E-03

3. $8 E-03$

1. $4 E-05$ 3. $9 E+03$ 2. $4 E-03$

1. $5 E+03$ 5. 5E-04

1. $7 E-02$

3. $9 E-04$

2. $4 E-04$

9. $1 E-04$ 2. $6 E-07$

2. $0 E-07$

4. $0 E-04$
1. $2 E-07$

4. $5 E-01$

1. $8 \mathrm{E}-04$

4. $0 E-05$

2. $8 \mathrm{E}-03$

3. $0 E+00$

1. $9 E-01$

1. $8 \mathrm{E}-05$

1. $1 E-04$

1. $3 E-05$

4. $4 \mathrm{E}-08$

1. $8 E+02$

1. $O E-03$

1. $3 \mathrm{E}-03$

9. $2 E-07$

1.7E-08

1. $6 E-08$

3. $3 E-01$

1. $5 \mathrm{E}-08$

6. $1 E-04$

3. $4 E+04$

1. $4 E+04$

9. $8 E+01$

9. $8 E-04$

1. $5 E+04$

3. $6 E+01$
$6.6 E-07$

9. $7 E-04$

$1.4 E-05$

$6.4 E+00$

2. $2 E-03$

$6.1 E+00$

5.1E-04

1. $5 \mathrm{E}-02$

3. 5E-04

$1.8 E-04$

$4.6 E-04$

5. 2E-07

2.6E-07

$1.8 E-04$
$1.2 E-07$

5. 0E-02

1. $7 \mathrm{E}-04$

4. OE-05

1. $8 E-03$

3. $O E+00$

1. $9 E-01$

9. $8 E-06$

3. 0 E-05

7. $6 \mathrm{E}-06$

$1.4 E-08$

$4.4 E-08$

7. 3E-02

1. $3 E-04$

$1.1 \mathrm{E}-06$

$2.8 \mathrm{E}-08$

$2.7 \mathrm{E}-0$

2. 5E-09

1. $6 E-05$

$2.2 E-09$

6. $7 E-07$

$6.2 E-01$

4. $1 E-01$

$3.3 \mathrm{E}-02$

3. $3 E-02$

. OE OS

3. $9 E-01$

2. $0 E-02$
$3.8 E-07$ 
TABLE 9A. UNIT-RAD CONTAMINATION FOR KIDNEYS

\begin{tabular}{|c|c|c|c|c|}
\hline $\begin{array}{l}\text { ELEMENT } \\
\text { SYMBOL }\end{array}$ & $\begin{array}{l}\text { ATOMIC } \\
\text { VO. (Z) }\end{array}$ & $\begin{array}{l}\text { ISOTOPE } \\
\text { MASS (A) }\end{array}$ & $\begin{array}{l}\text { ADULT } \\
\text { UC/M**3 }\end{array}$ & $\begin{array}{l}\text { INFANT } \\
\text { UC } / M * * 3\end{array}$ \\
\hline $\begin{array}{l}U \\
U \\
U \\
U \\
U \\
U \\
U \\
U \\
U \\
U \\
N P \\
D \\
N P \\
N \text { NP } \\
N P \\
N P \\
N P \\
N P \\
P U \\
P U \\
P U \\
P U \\
P U \\
P U \\
P U \\
P U \\
P U \\
P U \\
A M \\
A M \\
A M \\
A M \\
A M \\
A M\end{array}$ & $\begin{array}{l}92 \\
92 \\
92 \\
92 \\
92 \\
92 \\
92 \\
92 \\
92 \\
93 \\
93 \\
93 \\
93 \\
93 \\
93 \\
93 \\
94 \\
94 \\
94 \\
94 \\
94 \\
94 \\
94 \\
94 \\
94 \\
95 \\
95 \\
95 \\
95 \\
95 \\
95\end{array}$ & $\begin{array}{l}231 \\
232 \\
233 \\
234 \\
235 \\
236 \\
237 \\
238 \\
240 \\
234 \\
235 \\
236 \\
236 M \\
237 \\
238 \\
239 \\
236 \\
237 \\
238 \\
239 \\
240 \\
241 \\
242 \\
244 \\
246 \\
239 \\
240 \\
241 \\
242 \\
242 M \\
243\end{array}$ & $\begin{array}{l}\text { 8. } 7 E-06 \\
1.2 E-09 \\
1.1 E-09 \\
1.0 E-09 \\
9.4 E-10 \\
9.3 E-10 \\
3.4 E-06 \\
8.9 E-10 \\
2.8 E-04 \\
1.6 E+03 \\
1.8 E-01 \\
1.0 E-03 \\
3.5 E+04 \\
1.1 E-03 \\
6.4 E+03 \\
4.9 E+03 \\
1.4 E-02 \\
7.3 E+00 \\
6.3 E-04 \\
5.1 E-04 \\
5.0 E-04 \\
1.3 E-03 \\
4.8 E-04 \\
4.3 E-04 \\
9.6 E+01 \\
4.5 E+04 \\
2.4 E+03 \\
4.5 E-04 \\
2.4 E+04 \\
4.4 E-04 \\
3.9 E-04\end{array}$ & $\begin{array}{l}1.9 \mathrm{E}-06 \\
1.2 \mathrm{E}-09 \\
1.1 \mathrm{E}-07 \\
1.0 \mathrm{E}-03 \\
9.4 \mathrm{E}-13 \\
9.3 \mathrm{E}-10 \\
1.1 \mathrm{E}-05 \\
8.9 \mathrm{E}-10 \\
1.0 \mathrm{E}-05 \\
1.1 \mathrm{E}-01 \\
1.1 \mathrm{E}-03 \\
5.9 \mathrm{E}-05 \\
5.0 \mathrm{E}-01 \\
6.2 \mathrm{E}-05 \\
2.1 \mathrm{E}-01 \\
1.8 \mathrm{E}-01 \\
4.4 \mathrm{E}-04 \\
1.0 \mathrm{E}-02 \\
6.6 \mathrm{E}-05 \\
5.6 \mathrm{E}-05 \\
5.5 \mathrm{E}-05 \\
1.1 \mathrm{E}-04 \\
5.3 \mathrm{E}-05 \\
4.7 \mathrm{E}-05 \\
3.3 \mathrm{E}-02 \\
8.4 \mathrm{E}-01 \\
1.9 \mathrm{E}-01 \\
5.7 \mathrm{E}-05 \\
5.9 \mathrm{E}-01 \\
5.6 \mathrm{E}-05 \\
5.0 \mathrm{E}-05\end{array}$ \\
\hline
\end{tabular}

VIA SEAWATER.

ELEME
SYMBO
$C M$
$C M$
$C M$
$C M$
$C M$
$C M$
$C M$
$C M$
$C M$
$C M$
$B K$
$B K$
$B K$
$B K$
$B K$
$C F$
$C F$
$C F$
$C F$
$C F$
$C F$
$C F$
$E S$
$E S$
$E S$
$E S$
$E S$
$E S$
$F M$
$F M$
$F M$
$F M$




$\begin{array}{ccc}\text { ELEMENT } & \text { ATOMIC } & \text { ISOTOPE } \\ \text { SYMBOL } & \text { NO. }(2) & \text { MASS }(A) \\ \text { H } & & \\ \text { BE } & 1 & 3 \\ \text { BE } & 4 & 7 \\ \text { C } & 4 & 10 \\ \text { NA } & 6 & 14 \\ \text { VA } & 11 & 22 \\ \text { MG } & 11 & 24 \\ \text { AL } & 12 & 28 \\ \text { SI } & 13 & 26 \\ \text { P } & 14 & 32 \\ \text { P } & 15 & 32 \\ \text { S } & 15 & 33 \\ \text { CL } & 16 & 35 \\ \text { AR } & 17 & 36 \\ \text { AR } & 18 & 37 \\ \text { AR } & 18 & 39 \\ \text { X } & 18 & 42 \\ \text { K } & 19 & 40 \\ \text { K } & 19 & 42 \\ \text { CA } & 19 & 43 \\ \text { CA } & 20 & 41 \\ \text { CA } & 20 & 45 \\ \text { CA } & 20 & 47 \\ \text { SC } & 20 & 48 \\ \text { SC } & 21 & 44 M \\ \text { SC } & 21 & 46 \\ \text { SC } & 21 & 47 \\ \text { TI } & 21 & 48 \\ \text { V } & 22 & 44 \\ \text { V } & 23 & 48 \\ \text { V } & 23 & 49 \\ \text { CR } & 23 & 50 \\ \text { CR } & 24 & 48 \\ \text { MN } & 24 & 51 \\ \text { MN } & 25 & 52 \\ \text { MN } & 25 & 53 \\ \text { FE } & 25 & 54 \\ \text { FE } & 26 & 55 \\ \text { FE } & 26 & 59 \\ \text { CO } & 26 & 60 \\ \text { CO } & 27 & 55 \\ \text { CO } & 27 & 56 \\ \text { CO } & 27 & 57 \\ \text { CO } & 27 & 58 \\ \text { NI } & 27 & 60 \\ \text { NI } & 28 & 56 \\ \text { NI } & 28 & 57 \\ \text { NI } & 28 & 59 \\ \text { NI } & 28 & 63 \\ \text { CU } & 28 & 66 \\ & 29 & 64\end{array}$

$\begin{array}{ll}\text { ADULT } & \text { INFANT } \\ \text { UC } / M * 3 & \text { UC } / M * * 3 \\ & \\ 6.4 E+02 & 6.4 E+02 \\ 1.0 E+01 & 1.7 E+00 \\ 2.1 E-03 & 2.1 E-03 \\ 3.4 E-03 & 3.4 E-03 \\ 4.6 E+01 & 4.5 E+01 \\ 6.1 E+05 & 3.3 E+04 \\ 3.6 E+03 & 3.6 E+03 \\ 1.5 E-02 & 1.5 E-02 \\ 3.9 E-03 & 3.9 E-03 \\ 6.3 E-02 & 1.9 E-02 \\ 2.8 E-01 & 1.2 E-01 \\ 2.4 E+02 & 2.4 E+02 \\ 8.2 E+01 & 8.1 E+01 \\ 8.2 E+02 & 8.2 E+02 \\ 4.4 E-02 & 4.4 E-02 \\ 4.7 E-03 & 4.7 E-03 \\ 2.5 E-01 & 2.5 E-01 \\ 1.2 E+05 & 1.1 E+03 \\ 7.3 E+04 & 1.2 E+03 \\ 2.2 E+03 & 2.2 E+03 \\ 4.2 E+03 & 4.0 E+03 \\ 1.8 E+04 & 6.7 E+03 \\ 2.1 E+00 & 2.1 E+00 \\ 3.1 E+01 & 4.3 E-02 \\ 3.6 E-02 & 1.7 E-03 \\ 1.4 E+02 & 2.8 E-01 \\ 4.3 E+01 & 4.6 E-02 \\ 7.1 E-04 & 7.1 E-04 \\ 4.2 E+01 & 7.9 E+00 \\ 2.8 E+02 & 2.3 E+02 \\ 4.1 E-02 & 4.1 E-02 \\ 4.2 E+00 & 4.2 E+00 \\ 1.5 E+01 & 1.5 E+01 \\ 1.4 E-01 & 1.0 E-02 \\ 4.6 E-03 & 4.6 E-03 \\ 1.0 E-03 & 8.1 E-04 \\ 1.1 E-01 & 6.8 E-02 \\ 9.8 E-02 & 7.4 E-03 \\ 2.3 E-05 & 2.1 E-05 \\ 1.9 E+02 & 5.7 E+00 \\ 8.5 E-02 & 6.4 E-02 \\ 3.0 E-01 & 2.7 E-01 \\ 1.9 E-01 & 1.4 E-01 \\ 2.1 E-03 & 2.1 E-03 \\ 3.6 E+02 & 4.3 E+00 \\ 1.1 E+04 & 3.4 E+01 \\ 1.9 E+00 & 1.7 E+00 \\ 9.1 E-01 & 8.4 E-01 \\ 5.3 E+03 & 2.4 E+01 \\ 6.8 E+03 & 3.0 E+00\end{array}$

ATOMIC

I SOTOPE

UC $/ M * * 3$

3. $6 E+02$

7. $9 E-04$

2. $3 E+01$

4. $0 E-01$

1. $4 E+02$
1. $8 E+02$

1. $8 E-05$

1. $4 E-02$

1. $2 \mathrm{E}-01$

3. $2 E+05$

3. $8 E+05$

2. $5 E+04$

2. $5 \mathrm{E}+03$

5. $2 E+05$

1. $8 E+06$

2. $9 E+00$

3. $4 E-01$

5.6E-02

9. $5 E+04$
$2.0 E+04$

2. $0 E+04$
1. $7 E+04$

1. $7 E+04$

1. $5 E+02$

$1.3 E+00$

2. $5 \mathrm{E}-01$

1. $2 E+00$

1. $6 E+00$

7. $9 E+00$

4. 5E-02

4. $7 E+02$

1. $2 E+05$

1. $1 E+03$

3. $9 E+00$

$1.9 E+02$

1. $9 E+02$

3. $1 E+01$
7. $O E+02$

1. $0 E-02$

3. $1 E+01$

1. $1 E-01$

5. $7 E+02$

7. $2 \mathrm{E}-02$

6. $6 E+01$

2. $8 \mathrm{E}-02$

2. $2 E-01$

8. $4 E+02$

5. $3 E+01$

1. $0 \mathrm{E}-03$

1. $2 E-01$
$1.1 E+00$

7. $6 \mathrm{E}-01$

5.7E-04

1. $5 E-01$

5. $6 E+01$

2. $0 E+01$

1. 8E-05

$2.6 \mathrm{E}-03$

$7.1 E-02$

$1.5 E+03$

$7.5 \mathrm{E}+02$

7. $5 E+02$

7. $8 E+01$

1. $0 E+03$

5. $3 E+03$

7. $5 \mathrm{E}-01$

2.8E-01

$5.6 E-02$

7. $5 E+03$

4. $5 E+03$

2. $7 \mathrm{E}+03$

$1.5 \mathrm{E}+02$

1. $9 E+02$

$1.3 E+00$

2.5E-01

6. 1E-01

4. $7 E-01$

$1.5 E+00$

4. $5 E-02$
$3.8 E+02$

$2.2 E+04$

9. $8 E+02$

$8.6 E+02$

3. $9 E+00$

8. 3E-02

$7.3 \mathrm{E}-02$

2. $9 \mathrm{E}-01$

7. $3 E-04$

5. 9E-02

4. $5 E-03$

1. $3 E+00$

1. $5 \mathrm{E}-02$

$6.7 E-01$

2. 7E-02

3. $7 E-02$

1. $8 \mathrm{E}+00$

3. $8 E-02$

$9.0 E-04$

8. 5 E-03 
TABLE 9A. UNIT-RAD CONTAMINATION FOR LIVER

\begin{tabular}{|c|c|c|c|c|}
\hline $\begin{array}{l}\text { ELEMENT } \\
\text { SYMBOL }\end{array}$ & $\begin{array}{l}\text { ATJMIC } \\
\text { VJ. (Z) }\end{array}$ & $\begin{array}{l}\text { ISOTDPE } \\
\text { MASS (A) }\end{array}$ & $\begin{array}{l}\text { ADULT } \\
\text { UC/M**3 }\end{array}$ & $\begin{array}{l}\text { INFANT } \\
\text { UC/M**3 }\end{array}$ \\
\hline $\begin{array}{l}N B \\
N B \\
N B \\
N B \\
N B \\
\text { NO } \\
M D \\
M C \\
T C \\
T C \\
T C \\
T C \\
T C \\
T C \\
T C \\
T C \\
R U \\
R U \\
R U \\
R H \\
R H \\
R H \\
R H \\
R H \\
R H \\
R H \\
R H \\
P D \\
P D \\
P D \\
P D \\
P D \\
P D \\
A G \\
A G \\
A G \\
A G \\
A G \\
C D \\
C D \\
C D \\
C D \\
I N \\
I N \\
I N \\
S N \\
S N \\
S N \\
S N \\
S N \\
S N \\
S N \\
S N \\
S B \\
S B\end{array}$ & $\begin{array}{l}41 \\
41 \\
41 \\
41 \\
41 \\
42 \\
42 \\
43 \\
43 \\
43 \\
43 \\
43 \\
43 \\
43 \\
44 \\
44 \\
44 \\
45 \\
45 \\
45 \\
45 \\
45 \\
45 \\
46 \\
46 \\
46 \\
46 \\
46 \\
47 \\
47 \\
47 \\
47 \\
47 \\
48 \\
48 \\
48 \\
48 \\
49 \\
49 \\
49 \\
50 \\
50 \\
50 \\
50 \\
50 \\
50 \\
50 \\
50 \\
51 \\
51\end{array}$ & $\begin{array}{l}93 M \\
94 \\
95 \\
95 M \\
96 \\
93 \\
99 \\
95 \\
95 M \\
96 \\
97 \\
97 M \\
98 \\
99 \\
97 \\
103 \\
106 \\
99 \\
100 \\
101 \\
101 M \\
102 \\
105 \\
100 \\
103 \\
107 \\
109 \\
112 \\
105 \\
106 M \\
108 M \\
110 M \\
111 \\
109 \\
113 M \\
115 \\
115 M \\
111 \\
114 M \\
115 \\
113 \\
117 M \\
119 M \\
121 \\
121 M \\
123 M \\
125 \\
126 \\
119 \\
120 M\end{array}$ & $\begin{array}{l}\text { 5.1E-03 } \\
1.0 E-05 \\
1.1 E-01 \\
7.1 E+00 \\
4.1 E+01 \\
4.0 E-02 \\
8.5 E+01 \\
7.4 E+01 \\
3.2 E-02 \\
1.0 E+00 \\
9.0 E-03 \\
1.7 E-01 \\
1.2 E-04 \\
2.0 E-03 \\
2.4 E+01 \\
2.0 E-01 \\
2.2 E-03 \\
3.7 E-02 \\
2.2 E+00 \\
2.3 E-04 \\
4.5 E-01 \\
4.2 E-04 \\
4.9 E+00 \\
1.4 E-01 \\
2.5 E-01 \\
1.5 E-02 \\
1.5 E+01 \\
1.1 E+00 \\
9.2 E-01 \\
5.9 E+00 \\
4.0 E-03 \\
3.0 E-02 \\
4.0 E+01 \\
2.4 E-04 \\
1.1 E-05 \\
2.7 E-01 \\
1.1 E-03 \\
2.3 E-03 \\
4.6 E-06 \\
8.2 E-08 \\
1.2 E+01 \\
1.1 E+03 \\
1.4 E+01 \\
4.4 E+05 \\
8.1 E-02 \\
6.7 E+00 \\
4.1 E+02 \\
2.1 E-03 \\
8.0 E+01 \\
1.4 E-01\end{array}$ & $\begin{array}{l}3.2 \mathrm{E}-03 \\
9.1 \mathrm{E}-05 \\
4.3 \mathrm{E}-03 \\
3.2 \mathrm{E}-02 \\
4.7 \mathrm{E}-02 \\
4.0 \mathrm{E}-02 \\
2.4 \mathrm{E}+00 \\
2.0 \mathrm{E}+00 \\
2.1 \mathrm{E}-02 \\
1.3 \mathrm{E}-01 \\
8.9 \mathrm{E}-03 \\
1.3 \mathrm{E}-01 \\
1.2 \mathrm{E}-04 \\
2.0 \mathrm{E}-03 \\
9.6 \mathrm{E}-01 \\
7.1 \mathrm{E}-02 \\
1.9 \mathrm{E}-03 \\
1.7 \mathrm{E}-02 \\
9.9 \mathrm{E}-02 \\
2.3 \mathrm{E}-04 \\
8.9 \mathrm{E}-02 \\
3.8 \mathrm{E}-04 \\
3.7 \mathrm{E}-01 \\
2.2 \mathrm{E}-02 \\
1.2 \mathrm{E}-01 \\
1.5 \mathrm{E}-02 \\
4.4 \mathrm{E}-01 \\
4.9 \mathrm{E}-02 \\
4.0 \mathrm{E}-01 \\
8.1 \mathrm{E}-01 \\
3.9 \mathrm{E}-03 \\
2.5 \mathrm{E}-02 \\
5.1 \mathrm{E}+00 \\
1.7 \mathrm{E}-04 \\
1.1 \mathrm{E}-05 \\
3.1 \mathrm{E}-03 \\
2.0 \mathrm{E}-04 \\
1.1 \mathrm{E}-04 \\
2.1 \mathrm{E}-06 \\
8.1 \mathrm{E}-08 \\
8.5 \mathrm{E}-01 \\
9.4 \mathrm{E}+00 \\
1.9 \mathrm{E}+00 \\
3.1 \mathrm{E}+02 \\
6.0 \mathrm{E}-02 \\
4.9 \mathrm{E}-01 \\
2.4 \mathrm{E}+00 \\
1.7 \mathrm{E}-03 \\
3.2 \mathrm{E}+00 \\
1.8 \mathrm{E}-02\end{array}$ \\
\hline
\end{tabular}

VIA SEAWATER.

$\begin{array}{lcc}\text { ELEMENI } & \text { ATOMIC } & \text { ISOTOP } \\ \text { SYMBOL } & \text { ND. }(Z) & \text { MASSIA } \\ & & \\ \text { SB } & 51 & 122 \\ \text { SE } & 51 & 124 \\ \text { SB } & 51 & 125 \\ \text { SB } & 51 & 126 \\ \text { SB } & 51 & 127 \\ \text { TE } & 52 & 118 \\ \text { TE } & 52 & 119 \\ \text { TE } & 52 & 119 M \\ \text { TE } & 52 & 121 \\ \text { TE } & 52 & 121 M \\ \text { TE } & 52 & 123 \\ \text { TE } & 52 & 123 M \\ \text { TE } & 52 & 125 M \\ \text { TE } & 52 & 127 M \\ \text { TE } & 52 & 129 M \\ \text { TE } & 52 & 131 M \\ \text { TE } & 52 & 132 \\ \text { I } & 53 & 123 \\ \text { I } & 53 & 124 \\ \text { I } & 53 & 125 \\ \text { I } & 53 & 126 \\ \text { I } & 53 & 129 \\ \text { I } & 53 & 130 \\ \text { I } & 53 & 131 \\ \text { I } & 53 & 133 \\ \text { XE } & 54 & 122 \\ \text { XE } & 54 & 125 \\ \text { XE } & 54 & 127 \\ \text { XE } & 54 & 129 M \\ \text { XE } & 54 & 131 M \\ \text { XE } & 54 & 133 \\ \text { XE } & 54 & 133 M \\ \text { CS } & 55 & 129 \\ \text { CS } & 55 & 131 \\ \text { CS } & 55 & 132 \\ \text { CS } & 55 & 134 \\ \text { CS } & 55 & 135 \\ \text { CS } & 55 & 136 \\ \text { CS } & 55 & 137 \\ \text { BA } & 56 & 128 \\ \text { BA } & 56 & 131 \\ \text { BA } & 56 & 133 \\ \text { BA } & 56 & 133 M \\ \text { BA } & 56 & 135 M \\ \text { EA } & 56 & 140 \\ \text { LA } & 57 & 135 \\ \text { LA } & 57 & 137 \\ \text { LA } & 57 & 138 \\ \text { LA } & 57 & 140 \\ \text { CE } & 58 & 134\end{array}$

$10 / 09 / 68$

PAGE 12

ADULT INFANT

$1.2 E+00$

1. $2 E+00$

5. $3 E-04$

3. $1 \mathrm{E}-02$

$5.9 E-01$

2. $2 E-01$

1. $1 E+01$

8. $2 \mathrm{E}-02$

2. $7 E-03$

1. $3 \mathrm{E}-03$

1. $3 \mathrm{E}-02$

1. $3 E-02$

5. $4 E-02$
$1.1 E-02$

1. 8E-02

2. $6 E+00$

3. $0 E-01$

7. $1 E+04$

1. $7 E+02$

1. $7 E+02$

1. $4 E+02$

1. $1 E+00$

6. $9 E+03$

2. $4 E+02$

5. $1 E+03$

2. $1 E+02$

1. $1 E+03$

5. $3 E+01$

2. $4 E+02$

2. $3 E+02$

4. $6 \mathrm{E}+02$

4. $7 E+02$

4. $3 E+04$

1. $5 E+04$

1. $6 \mathrm{E}+03$

3. $1 E-01$

9. $8 E-01$

1. $3 E+02$

9. $3 E-02$

1. $3 E+05$

1. $3 E+04$

2. $8 E+00$
$1.0 E+06$

1. $0 E+06$

4. $8 E+06$

3. $1 E+03$

7. $4 E+04$

4. $0 \mathrm{E}-01$

1. $2 E-02$

5. $1 E+01$
UC/M**3

8. $4 E-02$

1. $9 E-03$

5. $1 E-04$
$7.7 E-03$

5. 5E-02

3. $6 E-02$

2. $3 E-01$

2. $9 E-02$

2. $3 E-03$

1. $3 E-03$

1. OE-02

3. $6 E-02$

8.3E-03

9. $7 E-03$

1. $0 E-01$

3. $0 E-02$

$5.1 E+03$

$6.5 E+01$

1. $5 E+02$

9. $O E+01$

$1.1 E+00$

4. $8 E+02$

1. $3 E+02$

5. $6 \mathrm{E}+02$

$2.1 \mathrm{E}+02$

$1.1 E+03$

5. $3 E+01$

2.4E 02

$2.3 E+02$

4. $7 E+02$

5. $0 E+02$

1. $2 E+03$

8. $9 E+01$

2. 7E-01

9. $6 E-01$

$1.3 E+01$

$3.1 E+02$

. 1 Et 02

2. $0 \mathrm{E}+00$

1. $7 E+03$

6. $0 E+03$

4. $1 E+01$

$1.5 E+02$

3. $8 \mathrm{E}-01$

1. $1 E-02$

2. $1 E+01$

$5.2 E-01$ 


\begin{tabular}{|c|c|c|c|c|}
\hline $\begin{array}{l}\text { ELEMENT } \\
\text { SYMBOL }\end{array}$ & $\begin{array}{l}\text { ATOMIC } \\
\text { NO. }(Z)\end{array}$ & $\begin{array}{l}\text { ISOTOPE } \\
\text { MASS (A) }\end{array}$ & $\begin{array}{c}\text { AOULT } \\
\text { UC } / M * 3\end{array}$ & $\begin{array}{l}\text { INFANT } \\
\text { UC } / M * * 3\end{array}$ \\
\hline $\begin{array}{l}\text { CE } \\
C E \\
C E \\
C E \\
C E \\
C E \\
C E \\
P R \\
P R \\
N D \\
N D \\
N D \\
P H \\
P M \\
P M \\
P M \\
P M \\
P M \\
P M \\
P M \\
P M \\
D \\
S M \\
S M \\
S M \\
S M \\
S M \\
S M \\
S M \\
S M \\
S M \\
E U \\
E U \\
E U \\
E U \\
E U \\
E U \\
E U \\
E U \\
E U \\
E U \\
E U \\
E U \\
E U \\
G D \\
G D \\
G D \\
G D \\
G D \\
G D \\
G D \\
G D\end{array}$ & $\begin{array}{l}58 \\
58 \\
58 \\
58 \\
58 \\
58 \\
58 \\
59 \\
59 \\
60 \\
60 \\
60 \\
61 \\
61 \\
61 \\
61 \\
61 \\
61 \\
61 \\
61 \\
61 \\
62 \\
62 \\
62 \\
62 \\
62 \\
62 \\
62 \\
62 \\
63 \\
63 \\
63 \\
63 \\
63 \\
63 \\
63 \\
63 \\
63 \\
63 \\
63 \\
63 \\
63 \\
64 \\
64 \\
64 \\
64 \\
64 \\
64 \\
64 \\
64\end{array}$ & 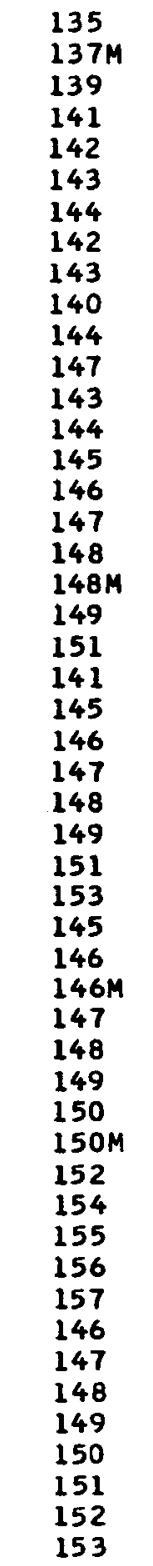 & $\begin{array}{l}1.2 E+03 \\
8.6 E+02 \\
2.3 E-01 \\
2.6 E+00 \\
2.6 E-04 \\
2.8 E+02 \\
4.1 E-03 \\
2.0 E-02 \\
2.3 E-04 \\
4.3 E-04 \\
3.2 E-09 \\
1.8 E-05 \\
7.5 E-02 \\
5.9 E-03 \\
9.6 E-03 \\
2.2 E-03 \\
1.6 E-03 \\
3.1 E+01 \\
1.6 E-01 \\
6.6 E+02 \\
9.3 E+02 \\
3.4 E-01 \\
7.8 E-02 \\
1.4 E-04 \\
1.6 E-04 \\
9.3 E-05 \\
2.0 E-04 \\
2.7 E-02 \\
3.8 E+02 \\
2.0 E+01 \\
5.3 E+00 \\
6.4 E+01 \\
3.5 E-01 \\
4.4 E-01 \\
1.4 E-01 \\
2.3 E-03 \\
4.1 E+02 \\
8.8 E-04 \\
6.7 E-04 \\
6.6 E-02 \\
1.5 E+00 \\
1.1 E+03 \\
6.4 E-02 \\
1.5 E+02 \\
8.1 E-05 \\
7.8 E+00 \\
4.3 E-05 \\
1.9 E-01 \\
1.1 E-04 \\
1.3 E-01\end{array}$ & $\begin{array}{l}3.0 E+00 \\
4.2 E+00 \\
7.5 E-02 \\
2.6 E-01 \\
2.5 E-04 \\
1.3 E+00 \\
2.0 E-03 \\
4.3 E-05 \\
8.0 E-06 \\
1.1 E-05 \\
3.2 E-09 \\
1.4 E-06 \\
2.2 E-02 \\
2.1 E-03 \\
8.3 E-03 \\
1.6 E-03 \\
9.6 E-04 \\
2.6 E-01 \\
9.1 E-03 \\
2.2 E+00 \\
1.7 E+00 \\
3.3 E-02 \\
5.0 E-02 \\
1.4 E-04 \\
1.6 E-04 \\
9.1 E-05 \\
1.9 E-04 \\
2.6 E-02 \\
3.9 E+00 \\
9.1 E-01 \\
1.8 E-01 \\
7.8 E-01 \\
5.5 E-02 \\
1.3 E-01 \\
6.5 E-02 \\
2.2 E-03 \\
1.7 E+00 \\
8.5 E-04 \\
6.5 E-04 \\
5.5 E-02 \\
1.6 E-01 \\
5.6 E+00 \\
6.1 E-03 \\
3.3 E-01 \\
7.5 E-05 \\
1.5 E-01 \\
4.1 E-05 \\
3.8 E-02 \\
1.0 E-04 \\
4.5 E-02\end{array}$ \\
\hline
\end{tabular}

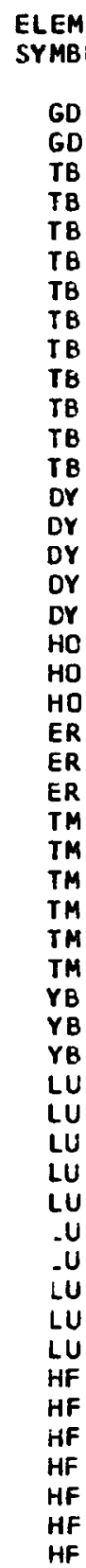

ATOMIC ISOTOPE
NO. $(2)$ MASS(A) UC $/ M * * 3$

3. $2 E+03$ 6. $7 E-03$ 6. $4 E+02$

1. $9 E+02$

1. $3 E+02$

$6.9 E+02$

3. $0 E+01$

9. $1 E+00$

6.7E-04

1. $1 E-01$

1. $1 E-01$

4. $3 E+01$

9. $3 E+02$

6. $3 E-10$

1. $8 E-02$

$1.7 E-05$

1. $4 E-03$

1. $1 E-07$

2.

2. $8 E-09$

4. $2 E-03$

4. $2 E-03$
2. $1 E-03$

3. 3E- 03

1. $0 E+03$

8. $0 E+01$

7. $5 E-01$

1. $8 E+00$

$4.3 E+00$

4.

5. $9 E+02$

7. $7 E+02$

8. $8 E+00$

$7.8 E+02$

1. $4 E-05$

E -06

3. $4 E-05$

6. OE-06

1. $1 E-07$

2. $E-08$

4. $4 \mathrm{E}-07$

7. $1 E-09$

7. $1 E-09$
$3.4 E-05$

1. $2 E-06$

1. $4 E+00$

2. $7 E+00$

1. $5 E-06$

8. 3E-01

1. $4 E-07$

1. $4 E-07$

6. $7 E-04$
1. $1 E-03$
INFANT

UC $/ M * * 3$

$5.3 E+00$

3. $0 \mathrm{E}-03$

7. $1 \mathrm{E}+00$

$2.1 E+00$

4. $6 E+00$

8. $8 \mathrm{E}+00$

2. $3 E+00$

$1.5 E-02$

6. $6 E-04$

5. 5E-02

$4.0 E+00$

$4.0 E+00$

5. $9 E-10$

2. OE-05

9. $2 E-10$

3. $8 E-06$

9. $4 E-06$

9. $5 \mathrm{E}-08$

3. $7 E-05$

4.2E-09

1. $2 \mathrm{E}-05$

4. $6 E-05$

$1.6 E-05$

1. $7 \mathrm{E}+01$

9. $6 E+00$

4. $1 E-01$

1. $2 E+00$

1.

2. $2 E+01$

$2.6 E+01$

$2.8 E+00$

4. $4 E+01$

$1.4 E-05$

$6.4 E-06$

$3.4 \mathrm{E}-05$

6. $0 E-06$

$1.1 E-07$

$2.4 E-08$

4. $4 \mathrm{E}-07$

7. $1 E-09$

1. $2 \mathrm{E}-06$

$1.1 \mathrm{E}-03$

2. $5 \mathrm{E}-03$

1. 1 E-06

1. $3 E-03$

1. $2 \mathrm{E}-07$

6. $8 E-05$
7. $3 E-05$ 
TABLE 9A. UNIT-RAD CONTAMINATION FOR LIVER

$\begin{array}{lcccc}\text { ELEMENT } & \text { ATOMIC } & \text { ISOTOPE } & \text { ADULT } & \text { INFANT } \\ \text { SYMBOL } & \text { NO.121 } & \text { MASSIAI } & \text { UC /M**3 } & \text { UCIM**3 } \\ \text { HF } & & & & \\ \text { TA } & 72 & 182 & 2.1 E-07 & 1.9 E-07 \\ \text { IA } & 73 & 177 & 1.8 E-01 & 1.0 E-03 \\ \text { TA } & 73 & 179 & 1.2 E-04 & 7.1 E-05 \\ \text { TA } & 73 & 182 & 7.1 E-05 & 1.6 E-05 \\ \text { W } & 73 & 183 & 6.8 E-02 & 8.4 E-04 \\ \text { W } & 74 & 178 & 4.2 E-04 & 3.6 E-04 \\ \text { W } & 74 & 181 & 1.6 E-03 & 1.5 E-03 \\ \text { W } & 74 & 185 & 1.6 E-03 & 1.5 E-03 \\ \text { H } & 74 & 187 & 8.1 E-02 & 1.6 E-02 \\ \text { RE } & 74 & 188 & 1.5 E-04 & 1.5 E-04 \\ \text { RE } & 75 & 180 & 8.3 E+00 & 4.4 E-01 \\ \text { RE } & 75 & 181 & 9.1 E+00 & 4.8 E-01 \\ \text { RE } & 75 & 182 & 1.1 E+00 & 1.7 E-01 \\ \text { RE } & 75 & 182 M & 2.3 E+01 & 8.2 E-01 \\ \text { RE } & 75 & 183 & 3.5 E-02 & 2.9 E-02 \\ \text { RE } & 75 & 184 & 2.5 E-02 & 1.8 E-02 \\ \text { RE } & 75 & 184 M & 3.5 E-03 & 3.2 E-03 \\ \text { RE } & 75 & 186 & 2.0 E+00 & 4.0 E-01 \\ \text { RE } & 75 & 187 & 6.8 E-01 & 6.8 E-01 \\ \text { RE } & 75 & 188 & 2.0 E+01 & 8.9 E-01 \\ \text { OS } & 75 & 189 & 2.3 E+01 & 1.4 E+00 \\ \text { OS } & 76 & 182 & 8.5 E-02 & 1.2 E-02 \\ \text { OS } & 76 & 183 & 6.2 E-01 & 5.2 E-02 \\ \text { OS } & 76 & 185 & 3.5 E-04 & 3.3 E-04 \\ \text { OS } & 76 & 191 & 1.1 E-02 & 7.8 E-03 \\ \text { OS } & 76 & 191 M & 1.5 E+00 & 1.5 E-01 \\ \text { OS } & 76 & 193 & 1.9 E-01 & 3.7 E-02 \\ \text { IR } & 76 & 194 & 5.1 E-06 & 5.1 E-05 \\ \text { IR } & 77 & 185 & 8.8 E+00 & 2.0 E-01 \\ \text { IR } & 77 & 186 & 5.2 E+00 & 1.2 E-01 \\ \text { IR } & 77 & 187 & 1.4 E+01 & 2.5 E-01 \\ \text { IR } & 77 & 189 & 5.9 E-01 & 3.5 E-02 \\ \text { IR } & 77 & 190 & 8.8 E-02 & 2.9 E-02 \\ \text { IR } & 77 & 192 & 2.6 E-02 & 8.0 E-03 \\ \text { IR } & 77 & 192 M & 2.4 E-03 & 1.8 E-03 \\ \text { IR } & 77 & 193 M & 1.5 E-05 & 1.5 E-05 \\ \text { IR } & 77 & 194 & 4.6 E-01 & 1.4 E-01 \\ \text { PT } & 78 & 188 & 4.8 E+00 & 1.4 E-01 \\ \text { PT } & 78 & 190 & 4.4 E-04 & 1.5 E-04 \\ \text { PT } & 78 & 191 & 1.7 E-07 & 1.7 E-07 \\ \text { PT } & 78 & 192 & 2.0 E-02 & 2.6 E-03 \\ \text { PT } & 78 & 193 & 2.1 E-07 & 2.1 E-07 \\ \text { PI } & 78 & 193 M & 7.3 E-06 & 7.3 E-06 \\ \text { PT } & 78 & 195 M & 2.3 E-02 & +.2 E-03 \\ \text { PT } & 78 & 197 & 2.3 E-02 & 3.9 E-03 \\ \text { AU } & 79 & 193 & 4.2 E-01 & 1.7 E-02 \\ \text { AU } & 79 & 194 & 5.4 E+02 & 1.3 E+00 \\ \text { AU } & 79 & 195 & 3.3 E+01 & 1.8 E-01 \\ \text { AU } & 79 & 196 & 4.2 E-02 & 1.6 E-02 \\ & & & 6.5 E+00 & 1.3 E-01\end{array}$

VIA SEAHATER.

\begin{tabular}{|c|c|c|}
\hline $\begin{array}{l}\text { ELEMENT } \\
\text { SYMBOL }\end{array}$ & $\begin{array}{l}\text { ATOMIC } \\
\text { NO. }(Z)\end{array}$ & $\begin{array}{l}\text { I SOTOPE } \\
\text { MASS(A) }\end{array}$ \\
\hline$A U$ & 79 & 198 \\
\hline$A U$ & 79 & 199 \\
\hline$H G$ & 80 & 194 \\
\hline HG & 80 & $195 M$ \\
\hline HG & 80 & 197 \\
\hline$H G$ & 80 & $197 M$ \\
\hline HG & 80 & 203 \\
\hline $\mathrm{TL}$ & 81 & 200 \\
\hline rL & 81 & 201 \\
\hline $\mathrm{TL}$ & 81 & 202 \\
\hline $\mathrm{TL}$ & 81 & 204 \\
\hline PB & 82 & 200 \\
\hline PB & 82 & 202 \\
\hline PB & 82 & 203 \\
\hline PE & 82 & 204 \\
\hline PB & 82 & 205 \\
\hline PB & 82 & 210 \\
\hline B! & 83 & 205 \\
\hline BI & 83 & 206 \\
\hline BI & 83 & 207 \\
\hline B I & 83 & 208 \\
\hline BI & 83 & 210 \\
\hline BI & 83 & $210 M$ \\
\hline PO & 84 & 206 \\
\hline PO & 84 & 208 \\
\hline PO & 84 & 209 \\
\hline PO & 84 & 210 \\
\hline RN & 86 & 211 \\
\hline RN & 86 & 222 \\
\hline RA & 88 & 223 \\
\hline RA & 88 & 224 \\
\hline RA & 88 & 225 \\
\hline RA & 88 & 226 \\
\hline RA & 88 & 228 \\
\hline$A C$ & 89 & 225 \\
\hline$A C$ & 89 & 227 \\
\hline TH & 90 & 227 \\
\hline TH & 90 & 228 \\
\hline TH & 90 & 229 \\
\hline TH & 90 & 230 \\
\hline TH & 90 & 231 \\
\hline $\mathrm{TH}$ & 90 & 232 \\
\hline TH & 90 & 234 \\
\hline PA & 91 & 228 \\
\hline PA & 91 & 229 \\
\hline$P A$ & 91 & 230 \\
\hline PA & 91 & 231 \\
\hline PA & 91 & 232 \\
\hline PA & 91 & 233 \\
\hline$u$ & 92 & 230 \\
\hline
\end{tabular}

$10 / 09 / 68$

PAGE 14

INFANT

$U C / M * 3$

\section{3E+01 3. $0 E-01$}

7. $8 E+01 \quad 8.1 E-01$

1. $1 \mathrm{E}-03 \quad 1.0 \mathrm{E}-03$

$\begin{array}{ll}1.1 E+00 & 1.2 E-01 \\ 2.0 E+00 & 3.4 E-01\end{array}$

7. $3 E+00$

2. 7E-02

8. $3 E-02$

6. $5 E-02$

1. $3 E-05$

1. $0 E+04$

2. $2 E-03$

4. $0 E+03$

5. $0 E-04$

1. $5 E-02$

3. $8 \mathrm{E}-04$

1. $8 \mathrm{E}-03$

8. $2 E-03$

2. $7 E-06$

1. 3E-06

3. $8 \mathrm{E}-03$

6. $4 E-07$

2. $8 \mathrm{E}-01$

1. $7 E-04$

4. $0 E-05$

2. $4 E-03$

3. $O E+0 O$

1. $9 E-01$

1. $8 E-05$

1. $1 E-04$

1. 3E-05

1. $4 E-08$
4. $4 E-08$

4. $4 E-08$

1. $8 E+01$

2. $0 E-04$

$3.4 E-03$

2. $3 E-06$

3. $9 E-08$

3. $9 E-08$

3. $5 E-08$

3. $5 E-08$

1. $6 E-03$

3. $9 E+04$

1. $6 E+04$

1. $1 E+02$

1. $1 \mathrm{E}-03$

1. $7 E+04$

4. $1 E+01$

3. $4 E-01$
5. $0 E-01$

2. 1 E-02

3. $4 E-03$

7. $O E-03$

8. 8E-04

1. 3E-05

4. $7 E+00$

1. $6 E-03$

$4.4 E+00$

3. 7 E-04

$2.6 E-04$

$9.1 E-04$

2. $4 E-03$

2.7E-06

$9.4 E-04$ -

$6.4 E-07$

5. OE-02

1. $7 E-04$

4. OE-05

1. $8 \mathrm{E}-03$

3. $0 E+00$

1. $9 E-01$

9. $8 E-06$

3. 0E-05

7. $6 E-06$

$1.4 E-08$

$4.4 E-08$

$7.3 E-02$

1. $3 E-04$

$1.1 E-06$

2. 8 E-08

2. $7 E-09$

2. $5 E-09$

1 . $6 E-05$

2. 2E-09

$6.7 E-07$

$6.2 E-01$

4.1E-01

3. 3E-02

7. $0 E-05$

3. $9 E-01$

2. OE-02

3. 8E-07 
TABLE 9A. UNIT-RAD JOVTAMINATION FOR LIVER

\begin{tabular}{|c|c|c|c|c|}
\hline $\begin{array}{l}\text { ELEMENT } \\
\text { SYMBOL }\end{array}$ & $\begin{array}{l}\text { ATOMIC } \\
\text { NO. (Z) }\end{array}$ & $\begin{array}{l}\text { ISOTOPE } \\
\operatorname{MASS}(A)\end{array}$ & $\begin{array}{l}\text { ADULT } \\
\text { UC/M**3 }\end{array}$ & $\begin{array}{l}\text { INFANT } \\
\text { UC/M**3 }\end{array}$ \\
\hline $\begin{array}{l}U \\
U \\
U \\
U \\
U \\
U U \\
U \\
U \\
U \\
N P \\
N P \\
N P \\
N P \\
N P \\
N P \\
N P \\
P U \\
P U \\
P U \\
P U \\
P U \\
P U \\
P U \\
P U \\
P U \\
A M \\
A M \\
A M \\
A M \\
A M \\
A M\end{array}$ & $\begin{array}{l}92 \\
92 \\
92 \\
92 \\
92 \\
92 \\
92 \\
92 \\
92 \\
93 \\
93 \\
93 \\
93 \\
93 \\
93 \\
93 \\
94 \\
94 \\
94 \\
94 \\
94 \\
94 \\
94 \\
94 \\
94 \\
95 \\
95 \\
95 \\
95 \\
95 \\
95\end{array}$ & $\begin{array}{l}231 \\
232 \\
233 \\
234 \\
235 \\
236 \\
237 \\
238 \\
240 \\
234 \\
235 \\
236 \\
236 M \\
237 \\
238 \\
239 \\
236 \\
237 \\
238 \\
239 \\
240 \\
241 \\
242 \\
244 \\
246 \\
239 \\
240 \\
241 \\
242 \\
242 M \\
243\end{array}$ & $\begin{array}{l}1.9 E-06 \\
1.2 E-09 \\
1.1 E-09 \\
1.0 E-09 \\
9.4 E-10 \\
9.3 E-10 \\
1.1 E-06 \\
8.9 E-10 \\
1.0 E-05 \\
1.4 E+03 \\
1.5 E-01 \\
8.8 E-04 \\
2.9 E+04 \\
9.3 E-04 \\
5.4 E+03 \\
4.1 E+03 \\
1.3 E-02 \\
6.8 E+00 \\
5.9 E-04 \\
4.8 E-04 \\
4.7 E-04 \\
1.3 E-03 \\
4.6 E-04 \\
4.1 E-04 \\
9.0 E+01 \\
5.8 E+03 \\
3.1 E+02 \\
9.6 E-05 \\
3.1 E+03 \\
9.5 E-05 \\
8.4 E-05\end{array}$ & $\begin{array}{l}1.9 E-06 \\
1.2 E-09 \\
1.1 E-09 \\
1.0 E-07 \\
9.4 E-10 \\
9.3 E-10 \\
1.1 E-05 \\
8.9 E-10 \\
1.0 E-05 \\
1.1 E-01 \\
1.1 E-03 \\
5.9 E-05 \\
5.0 E-01 \\
6.2 E-05 \\
2.1 E-01 \\
1.8 E-01 \\
4.4 E-04 \\
1.0 E-02 \\
6.6 E-05 \\
5.6 E-05 \\
5.5 E-05 \\
1.1 E-04 \\
5.3 E-05 \\
4.7 E-05 \\
3.3 E-02 \\
8.4 E-01 \\
1.9 E-01 \\
5.7 E-05 \\
5.9 E-01 \\
5.6 E-05 \\
5.0 E-05\end{array}$ \\
\hline
\end{tabular}

VIA SEAHATER.

$\begin{array}{lcc}\text { ELEMENT } & \text { ATOMIC } & \text { I SOTOPE } \\ \text { SYMBOL } & \text { NO. (Z) } & \text { MASS(A) } \\ & & \\ \text { CM } & 96 & 240 \\ \text { CM } & 96 & 241 \\ \text { CM } & 96 & 242 \\ \text { CM } & 96 & 243 \\ \text { CM } & 96 & 244 \\ \text { CM } & 96 & 245 \\ \text { CM } & 96 & 246 \\ \text { CM } & 96 & 247 \\ \text { CM } & 96 & 248 \\ \text { BK } & 97 & 245 \\ \text { BK } & 97 & 246 \\ \text { BK } & 97 & 247 \\ \text { BK } & 97 & 248 \\ \text { BK } & 97 & 249 \\ \text { CF } & 98 & 246 \\ \text { CF } & 98 & 248 \\ \text { CF } & 98 & 249 \\ \text { CF } & 98 & 250 \\ \text { CF } & 98 & 251 \\ \text { CF } & 98 & 252 \\ \text { CF } & 98 & 253 \\ \text { ES } & 99 & 251 \\ \text { ES } & 99 & 252 \\ \text { ES } & 99 & 253 \\ \text { ES } & 99 & 254 \\ \text { ES } & 99 & 254 M \\ \text { ES } & 99 & 255 \\ \text { FM } & 100 & 252 \\ \text { FM } & 100 & 253 \\ \text { FM } & 100 & 255 \\ \text { FM } & 100 & 257\end{array}$

10/09/68 PAGE 15

\section{ADULT} $U C / M * * 3$

1. $7 E+00$

1. $0 \mathrm{E}+00$

4. $7 E-02$
$1.1 E-04$

$1.1 E-04$
$1.5 E-04$

7. $9 E-05$

7. $6 E-05$

7. OE-O5

6. $9 E-05$

7. $6 E-02$

2. $0 E-01$

4. 5E-05

3. $5 \mathrm{E}-01$

1. 1E-03

2. $4 E-01$

9. $6 E-04$

4. $6 E-05$

1. $0 E-04$

4. $1 E-05$

3. $2 \mathrm{E}-04$

1. 6 E-02

2. $1 E-01$

2. $1 E-03$

1. 5E-02

6. $1 E-04$

1. $8 E-01$

1. $2 E-02$

6. $8 E-02$

3. $4 E-01$

3. 5E- 03
INFAN T UC $/ M * * 3$

1. 5E-02

1. $2 \mathrm{E}-02$

2. $4 E-03$

6.7E-05

8. 3E-05

5. 0E-05

4. 8E-05

4.5E-05

4. 4E-05

7. $6 \mathrm{E}-02$ कै

2. 0E-01

$4.5 \mathrm{E}-05$

3.5E-01

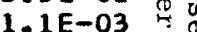

2. 4E-01

$9.6 \mathrm{E}-04$

$4.6 E-05$

1. 0E-04

4. $1 E-05$

3. $2 \mathrm{E}-04$

$1.6 \mathrm{E}-02$

2. $1 \mathrm{E}-01$

$2.1 E-03$

$1.5 \mathrm{E}-02$

$6.1 \mathrm{E}-04$

1. $8 E-01$
$1.2 E-02$

1.2E-02

6. $8 \mathrm{E}-02$

3. $4 E-01$

$3.5 E-03$ 
TABLE 9A. UNIT-RAD CONTAMINATION FOR OVIRIES

VIA SEAWATER.

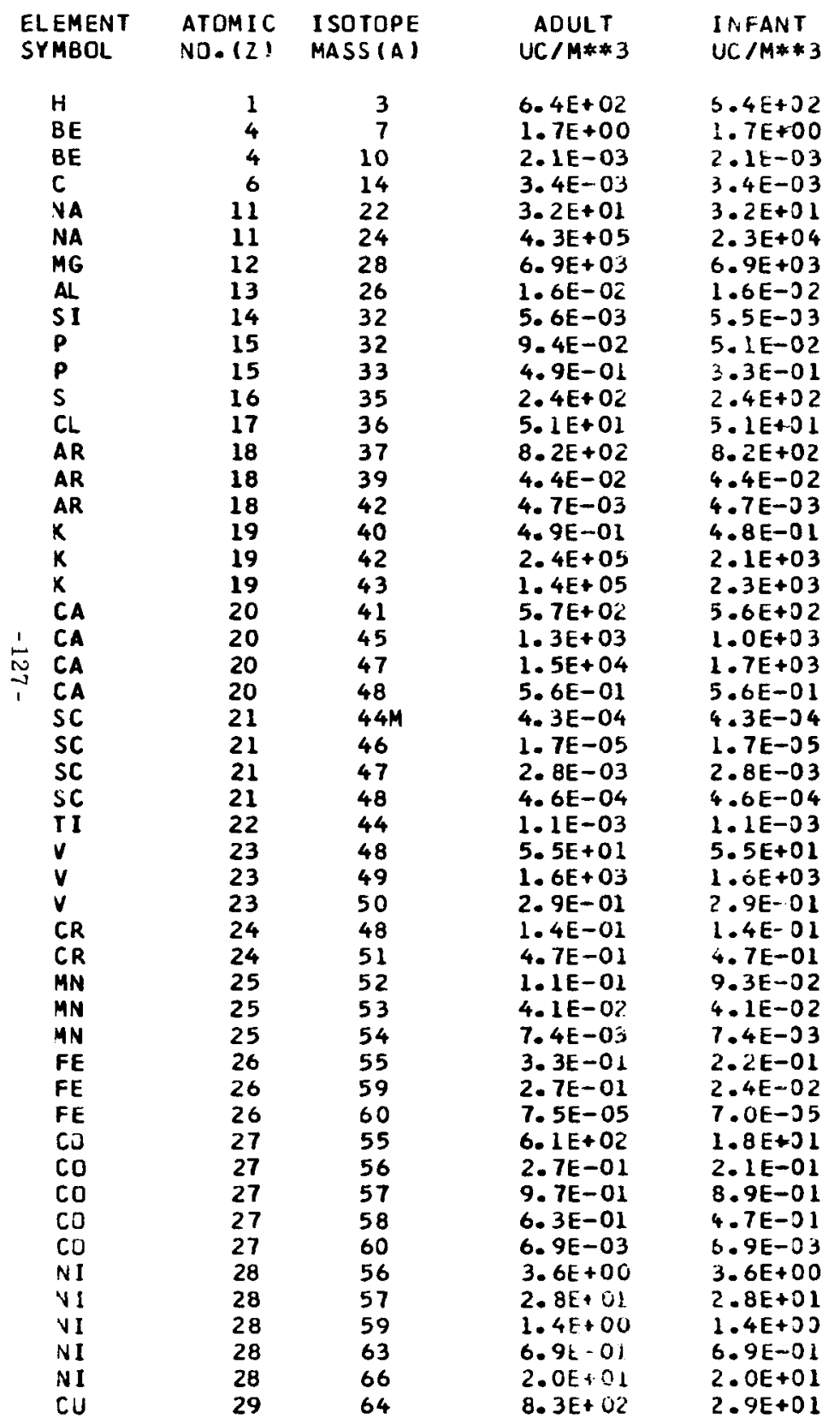

\begin{tabular}{|c|c|c|}
\hline $\begin{array}{l}\text { ELEMENT } \\
\text { SYMBOL }\end{array}$ & $\begin{array}{l}\text { ATOMIC } \\
\text { NO. (Z) }\end{array}$ & $\begin{array}{l}\text { I SOTCPE } \\
\text { MASS (A) }\end{array}$ \\
\hline $\mathrm{CW}^{\prime}$ & 29 & $6 i$ \\
\hline 21. & 30 & 65 \\
\hline $2 N$ & 30 & $69 \mathrm{M}$ \\
\hline$\angle N$ & 30 & 72 \\
\hline GA & 31 & 67 \\
\hline GA & 31 & 72 \\
\hline GE & 32 & 68 \\
\hline GL & 32 & 69 \\
\hline GE & 32 & 71 \\
\hline$A S$ & 33 & 71 \\
\hline AS & 33 & 72 \\
\hline AS & 33 & 73 \\
\hline$A S$ & 33 & 74 \\
\hline AS & 33 & 76 \\
\hline AS & 33 & 77 \\
\hline SE & 34 & 72 \\
\hline SE & 34 & 75 \\
\hline SE & 34 & 79 \\
\hline ER & 35 & 76 \\
\hline GR & 35 & 77 \\
\hline BR & 35 & 82 \\
\hline$K R$ & 30 & 70 \\
\hline KE & 36 & 79 \\
\hline KR & 36 & 81 \\
\hline KR & 36 & 85 \\
\hline$K B$ & 37 & 83 \\
\hline KB & 37 & 84 \\
\hline RB & 37 & 86 \\
\hline RB & 37 & 87 \\
\hline Sk & 38 & 82 \\
\hline SR & 38 & 83 \\
\hline SR & 38 & 85 \\
\hline SP & 38 & 89 \\
\hline$S K$ & 38 & 90 \\
\hline$Y$ & 39 & 86 \\
\hline$Y$ & 39 & 87 \\
\hline$Y$ & 33 & $87 M$ \\
\hline$Y$ & 313 & 88 \\
\hline$Y$ & 39 & 90 \\
\hline$Y$ & 39 & 91 \\
\hline$Z R$ & 40 & 86 \\
\hline $2 R$. & 40 & 88 \\
\hline $2 R$ & 40 & 89 \\
\hline$i^{5}$ & 40 & 53 \\
\hline if & 40 & 95 \\
\hline $2 k$ & 40 & 97 \\
\hline$N E$ & 41 & 90 \\
\hline $\mathrm{NE}$ & 41 & 91 \\
\hline Ne & 41 & $91 \mathrm{M}$ \\
\hline Ivt & 41 & 92 \\
\hline
\end{tabular}

$10 / 09 / 68$

PAGE IO

ADULT INFANT

$U C / M * * 3 \quad U C / M * * 3$

4. $9 E+01 \quad 7.1 E+00$

2. $3 E-03 \quad 1.6 E-03$

7. $6 E+01$ 4. $1 E-01$

1. $3 E+00 \quad 2.4 E-02$

$6.9 E+01$

2. $4 E+01$

1. $8 E-05$

2. $6 E-03$

7. $1 E-02$

$2.4 E+03$

1. $2 E+03$

1. $2 \mathrm{E}+02$

1. $2 E+02$

1. $7 E+03$

8. $5 E+03$
2. $3 E-02$

$8.7 E-03$

1. $7 E-03$

1. $3 E+06$

2. $6 E+05$

2. $6 E+05$

1. $5 E+02$

1. $9 E+02$

1. $3 E+00$

2. $5 E-01$

9. $4 E+O 1$

1. $3 E+02$

6. $2 E+02$

$3.6 E+00$

1. $5 E+02$

1. $0 E+05$

2. $3 E+02$

2. $3 E+02$

5. 5E-01

1. $5 \mathrm{E}-02$

5. $9 E-02$

1. $5 \mathrm{E}-04$

1. $2 E-02$

9. $O E-04$

1. $4 E+00$

1. $7 E-02$

7. $4 E-01$

3. $\mathrm{OE}-02$

4. $2 E-02$

2. $1 E+00$

4. $7 E-02$

1. $1 E-03$

1. $0 E-02$

$2.4 E-02$
$6.8 E+01$

$2.4 E+01$

1. $8 E-05$

$2.6 E-03$

7. $1 E-02$

$2.4 E+03$

1. $2 E+03$

4. $9 E+03$

1. $2 E+02$

1. $7 E+03$

$8.5 E+03$

$2.3 \mathrm{~L} \quad 02$

8.7E-03

1. $7 E-03$

1. $0 E+05$

$6.0 E+04$

$3.6 \mathrm{E}+04$

1. $5 E+02$

1. $9 E+02$

1. $3 E+00$

2. $5 E-01$

4. $8 E+01$

3. $7 \mathrm{E}+01$

$1.2 E+02$

3. $5 E+00$

5. $3 E+U 1$

3. $0 E+03$

$1.4 E+02$

1. $2 E+02$

5. 5E-01

1. $7 E-02$

1. $5 \mathrm{E}-02$

5. $9 \mathrm{E}-02$

1. $5 \mathrm{E}-04$

1. 2 E-02

9. $0 E-04$

$1.4 E+00$

1. $7 E-02$

7. $4 \mathrm{E}-01$

3. $0 \mathrm{E}-02$

$4.2 t-02$

2. $1 \leq+00$

4.150 ?

1. $0 E-02$
1. $5 E-02$

$\therefore t=0$

$\mathrm{L}, \mathrm{CE}-\mathrm{C}$
$\mathrm{l} .5 \mathrm{~S}-02$ 


\begin{tabular}{|c|c|c|c|c|}
\hline $\begin{array}{l}\text { ELEMENT } \\
\text { SYMBOL }\end{array}$ & $\begin{array}{l}\text { ATOMIC } \\
\text { NO. }(Z)\end{array}$ & $\begin{array}{l}\text { ISOTOPE } \\
\text { MASS (A) }\end{array}$ & $\begin{array}{c}\text { ADULT } \\
\text { UC } / M * * 3\end{array}$ & $\begin{array}{l}\text { INFANT } \\
\text { UC } / M * * 3\end{array}$ \\
\hline 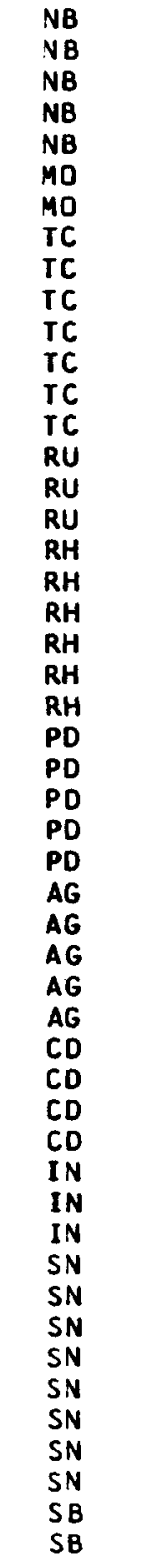 & $\begin{array}{l}41 \\
41 \\
41 \\
41 \\
41 \\
42 \\
42 \\
43 \\
43 \\
43 \\
43 \\
43 \\
43 \\
43 \\
44 \\
44 \\
44 \\
45 \\
45 \\
45 \\
45 \\
45 \\
45 \\
46 \\
46 \\
46 \\
46 \\
46 \\
47 \\
47 \\
47 \\
47 \\
47 \\
48 \\
48 \\
48 \\
48 \\
49 \\
49 \\
49 \\
50 \\
50 \\
50 \\
50 \\
50 \\
50 \\
50 \\
50 \\
51 \\
51\end{array}$ & $\begin{array}{l}93 M \\
94 \\
95 \\
95 M \\
96 \\
93 \\
99 \\
95 \\
95 M \\
96 \\
97 \\
97 M \\
98 \\
99 \\
97 \\
103 \\
106 \\
99 \\
100 \\
101 \\
101 M \\
102 \\
105 \\
100 \\
103 \\
107 \\
109 \\
112 \\
105 \\
106 M \\
108 M \\
110 M \\
111 \\
109 \\
113 M \\
115 \\
115 M \\
111 \\
114 M \\
115 \\
113 \\
117 M \\
119 M \\
121 \\
121 M \\
123 M \\
125 \\
126 \\
119 \\
120 M\end{array}$ & $\begin{array}{l}3.9 E-03 \\
1.1 E-05 \\
5.3 E-03 \\
3.9 E-02 \\
5.7 E-02 \\
1.8 E+01 \\
7.8 E+03 \\
2.0 E+00 \\
2.1 E-02 \\
1.3 E-01 \\
8.9 E-03 \\
1.3 E-01 \\
1.2 E-04 \\
2.0 E-03 \\
2.4 E+01 \\
2.0 E-01 \\
2.2 E-03 \\
1.7 E-02 \\
9.9 E-02 \\
2.3 E-04 \\
6.9 E-02 \\
3.8 E-04 \\
3.7 E-01 \\
2.2 E-02 \\
1.2 E-01 \\
1.5 E-02 \\
4.4 E-01 \\
4.9 E-02 \\
1.6 E+00 \\
4.2 E+00 \\
1.5 E-02 \\
9.5 E-02 \\
2.7 E+01 \\
1.1 E-03 \\
6.2 E-05 \\
5.1 E-01 \\
2.8 E-03 \\
1.1 E-04 \\
2.1 E-06 \\
8.1 E-08 \\
5.4 E-01 \\
1.8 E+01 \\
1.0 E+00 \\
5.8 E+03 \\
2.8 E-02 \\
3.0 E-01 \\
6.3 E+00 \\
7.7 E-04 \\
1.9 E+00 \\
1.1 E-02\end{array}$ & $\begin{array}{l}3.9 \mathrm{E}-03 \\
1.1 \mathrm{E}-05 \\
5.3 \mathrm{E}-03 \\
3.9 \mathrm{E}-02 \\
5.7 \mathrm{E}-02 \\
1.8 \mathrm{E}+01 \\
1.1 \mathrm{E}+03 \\
2.0 \mathrm{E}+00 \\
2.1 \mathrm{E}-02 \\
1.3 \mathrm{E}-01 \\
8.9 \mathrm{E}-03 \\
1.3 \mathrm{E}-01 \\
1.2 \mathrm{E}-04 \\
2.0 \mathrm{E}-03 \\
9.6 \mathrm{E}-01 \\
1.1 \mathrm{E}-02 \\
1.9 \mathrm{E}-03 \\
1.7 \mathrm{E}-02 \\
9.9 \mathrm{E}-02 \\
2.3 \mathrm{E}-04 \\
8.9 \mathrm{E}-02 \\
3.8 \mathrm{E}-04 \\
3.7 \mathrm{E}-01 \\
2.2 \mathrm{E}-02 \\
1.2 \mathrm{E}-01 \\
1.5 \mathrm{E}-02 \\
4.4 \mathrm{E}-01 \\
4.9 \mathrm{E}-02 \\
1.5 \mathrm{E}+00 \\
3.1 \mathrm{E}+00 \\
1.5 \mathrm{E}-02 \\
9.4 \mathrm{E}-02 \\
1.9 \mathrm{E}+01 \\
9.5 \mathrm{E}-04 \\
6.1 \mathrm{E}-05 \\
1.7 \mathrm{E}-02 \\
1.1 \mathrm{E}-03 \\
1.1 \mathrm{E}-04 \\
2.1 \mathrm{E}-06 \\
8.1 \mathrm{E}-03 \\
3.9 \mathrm{E}-01 \\
4.3 \mathrm{E}+00 \\
8.7 \mathrm{E}-01 \\
1.4 \mathrm{E}+02 \\
2.7 \mathrm{E}-02 \\
2.2 \mathrm{E}-01 \\
1.1 \mathrm{E}+00 \\
7.7 \mathrm{E}-04 \\
1.9 \mathrm{E}+00 \\
1.1 \mathrm{E}-02\end{array}$ \\
\hline
\end{tabular}

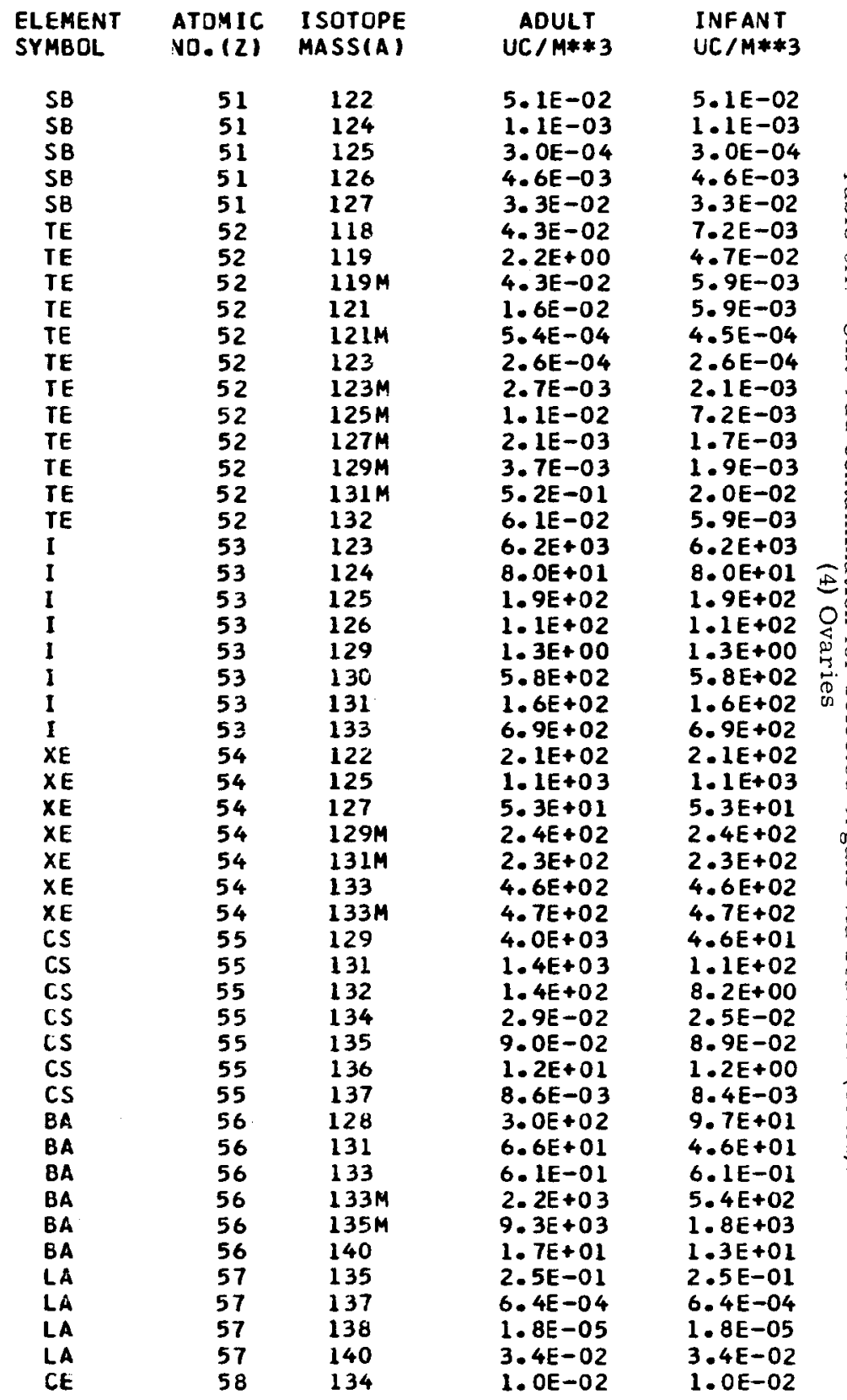


TABLE 9A. UNIT-RAD CONTAMINATION FOR OVARIES

\begin{tabular}{|c|c|c|c|c|}
\hline $\begin{array}{l}\text { ELEMENT } \\
\text { SYMBOL }\end{array}$ & $\begin{array}{l}\text { ATOMIC } \\
\text { VD. (Z) }\end{array}$ & $\begin{array}{l}\text { ISOTOPE } \\
\text { MASS (A) }\end{array}$ & $\begin{array}{l}\text { ADULT } \\
U C / M * \#=3\end{array}$ & $\begin{array}{l}\text { INFANT } \\
\text { UC } / M * * 3\end{array}$ \\
\hline $\begin{array}{l}C E \\
C E \\
C E \\
C E \\
C E \\
C E \\
C E \\
P R \\
P R \\
N D \\
N D \\
N D \\
P M \\
P M \\
P M \\
P M \\
P M \\
P M \\
P M \\
P M \\
P M \\
S M \\
S M \\
S M \\
S M \\
S M \\
S M \\
S M \\
S M \\
E U \\
E U \\
E U \\
E U \\
E U \\
E U \\
E U \\
E U \\
E U \\
E U \\
E U \\
E U \\
E U \\
G D \\
G D \\
G D \\
G D \\
G D \\
G D \\
G D \\
G D\end{array}$ & $\begin{array}{l}58 \\
58 \\
58 \\
58 \\
58 \\
58 \\
58 \\
59 \\
59 \\
60 \\
60 \\
60 \\
61 \\
61 \\
61 \\
61 \\
61 \\
61 \\
61 \\
61 \\
61 \\
62 \\
62 \\
62 \\
62 \\
62 \\
62 \\
62 \\
62 \\
63 \\
63 \\
63 \\
63 \\
63 \\
63 \\
63 \\
63 \\
63 \\
63 \\
63 \\
63 \\
63 \\
64 \\
64 \\
64 \\
64 \\
64 \\
64 \\
64 \\
64\end{array}$ & $\begin{array}{l}135 \\
137 M \\
139 \\
141 \\
142 \\
143 \\
144 \\
142 \\
143 \\
140 \\
144 \\
147 \\
143 \\
144 \\
145 \\
146 \\
147 \\
148 \\
148 M \\
149 \\
151 \\
141 \\
145 \\
146 \\
147 \\
148 \\
149 \\
151 \\
153 \\
145 \\
146 \\
146 M \\
147 \\
148 \\
149 \\
150 \\
150 M \\
152 \\
154 \\
155 \\
156 \\
157 \\
146 \\
147 \\
148 \\
149 \\
150 \\
151 \\
152 \\
153\end{array}$ & $\begin{array}{l}\text { 6.1E-02 } \\
8.4 E-02 \\
1.5 E-03 \\
5.2 E-03 \\
5.0 E-06 \\
2.6 E-02 \\
4.1 E-05 \\
4.3 E-05 \\
8.0 E-06 \\
1.1 E-05 \\
3.2 E-09 \\
1.4 E-06 \\
4.3 E-04 \\
4.2 E-05 \\
1.7 E-04 \\
3.1 E-05 \\
1.9 E-05 \\
5.1 E-03 \\
1.8 E-04 \\
4.4 E-02 \\
3.4 E-02 \\
6.6 E-03 \\
1.0 E-02 \\
2.8 E-05 \\
3.2 E-05 \\
1.8 E-05 \\
3.9 E-05 \\
5.2 E-03 \\
7.8 E-01 \\
1.8 E-01 \\
3.7 E-02 \\
1.6 E-01 \\
1.1 E-02 \\
2.6 E-02 \\
1.3 E-02 \\
4.3 E-04 \\
3.5 E-01 \\
1.7 E-04 \\
1.3 E-04 \\
1.1 E-06 \\
3.1 E-02 \\
1.1 E+00 \\
1.2 E-04 \\
6.7 E-03 \\
1.5 E-06 \\
3.0 E-03 \\
8.2 E-07 \\
7.7 E-04 \\
2.0 E-06 \\
8.9 E-04\end{array}$ & $\begin{array}{l}6.1 \mathrm{E}-02 \\
8.4 \mathrm{E}-02 \\
1.5 \mathrm{E}-03 \\
5.2 \mathrm{E}-03 \\
5.0 \mathrm{E}-06 \\
2.6 \mathrm{E}-02 \\
4.1 \mathrm{E}-05 \\
4.3 \mathrm{E}-05 \\
8.0 \mathrm{E}-06 \\
1.1 \mathrm{E}-05 \\
3.2 \mathrm{E}-09 \\
1.4 \mathrm{E}-06 \\
4.3 \mathrm{E}-04 \\
4.2 \mathrm{E}-05 \\
1.7 \mathrm{E}-04 \\
3.1 \mathrm{E}-05 \\
1.9 \mathrm{E}-05 \\
5.1 \mathrm{E}-03 \\
1.8 \mathrm{E}-04 \\
4.4 \mathrm{E}-02 \\
3.4 \mathrm{E}-02 \\
6.6 \mathrm{E}-03 \\
1.0 \mathrm{E}-02 \\
2.8 \mathrm{E}-05 \\
3.2 \mathrm{E}-05 \\
1.8 \mathrm{E}-05 \\
3.9 \mathrm{E}-05 \\
5.2 \mathrm{E}-03 \\
7.8 \mathrm{E}-01 \\
1.8 \mathrm{E}-01 \\
3.7 \mathrm{E}-02 \\
1.6 \mathrm{E}-01 \\
1.1 \mathrm{E}-02 \\
2.6 \mathrm{E}-02 \\
1.3 \mathrm{E}-02 \\
4.3 \mathrm{E}-04 \\
3.5 \mathrm{E}-01 \\
1.7 \mathrm{E}-04 \\
1.3 \mathrm{E}-04 \\
1.1 \mathrm{E}-02 \\
3.1 \mathrm{E}-02 \\
1.1 \mathrm{E}+00 \\
1.2 \mathrm{E}-04 \\
6.7 \mathrm{E}-03 \\
\therefore .5 \mathrm{E}-06 \\
3.0 \mathrm{E}-03 \\
9.2 \mathrm{E}-07 \\
7.7 \mathrm{E}-04 \\
2.0 \mathrm{E}-06 \\
8.9 \mathrm{E}-04\end{array}$ \\
\hline
\end{tabular}

VIA SEAWATER.

ELEMENT

ATOMIC I SOTOPE

$10 / 09 / 68$

PAGE 18 SYMEOL ND. (Z) MASS(A)

ADULT

INFANT

GD

GO

TB

$\begin{array}{ll}64 & 159\end{array}$

162

1. $1 E-01$

$J C / M * * 3$

$1.1 E-01$ 7. $I E-02$

2. $1 E-02$

4. $6 \mathrm{E}-02$

$8.8 E-02$

2. $3 E-02$

6. $8 E-03$
$1.5 E-04$

6. $6 E-06$

5. $5 E-04$

4. $0 E-02$

1. $4 \mathrm{E}-01$

5. $9 E-10$

2. 0E-05

9. $2 E-10$

3. $8 E-06$

9. $4 E-06$

9. $5 E-08$

3. $7 E-05$

4. $2 E-09$

1. $2 E-05$

4. $6 E-05$

$1.6 E-05$

$1.7 E-02$

$9.6 E-03$

4. $1 E-04$
1. $2 E-03$

3. $9 E-03$

2. $2 E-02$

5. $2 E-02$

$5.5 E-03$

8. 8E-02

1. $4 E-05$

6. $4 E-06$

$3.4 E-05$

$6.0 E-06$

1. $1 E-07$

2. $4 E-08$

4. $4 E-07$

7. $1 E-09$

3. $4 E-05$

1. $2 E-06$

$6 \cdot 2 E \cdot 02$

1. $2 \mathrm{E} \cdot 01$

3. $7 E-02$

3. $T E-02$

6.7E-09

3. $1 E-0 S$

6. OE-05

2.1E-02

4.6E-02

$8.8 E-02$

$2.3 E-02$

$6.8 E-03$
$1.5 E-04$

$1.5 \mathrm{E}-04$

$6.6 E-06$

$5.5 E-04$

4. $0 E-02$

$1.4 E-01$

$2.0 E-05$

9. $E-10$

3.8E-06

$9.4 E-06$

$9.5 \mathrm{E}-08$

3.7E-05

4. 2E-09

1.2E-05

.6E-05

$1.6 E-05$ :

$9.6 E-03$

4. $1 E-04$

1. $2 E-03$

3. $9 E-03$

2. $2 E-02$

$5.2 E-02$

$5.5 E-03$

8. 8E-02

1. $4 E-05$

$6.4 E-06$

$6.4 E-06$

$3.4 E-05$

6. OE-06

$1.1 E-07$

$2.4 E-08$

$4.4 E-07$

7. $1 \mathrm{E}-09$

3. $4 E-05$

$1.2 E-06$

$5.5 E-05$

1. 3E-04

5.

$6.6 E-05$

$6.2 E-09$

3. $4 E-06$
3. $7 E-06$ 


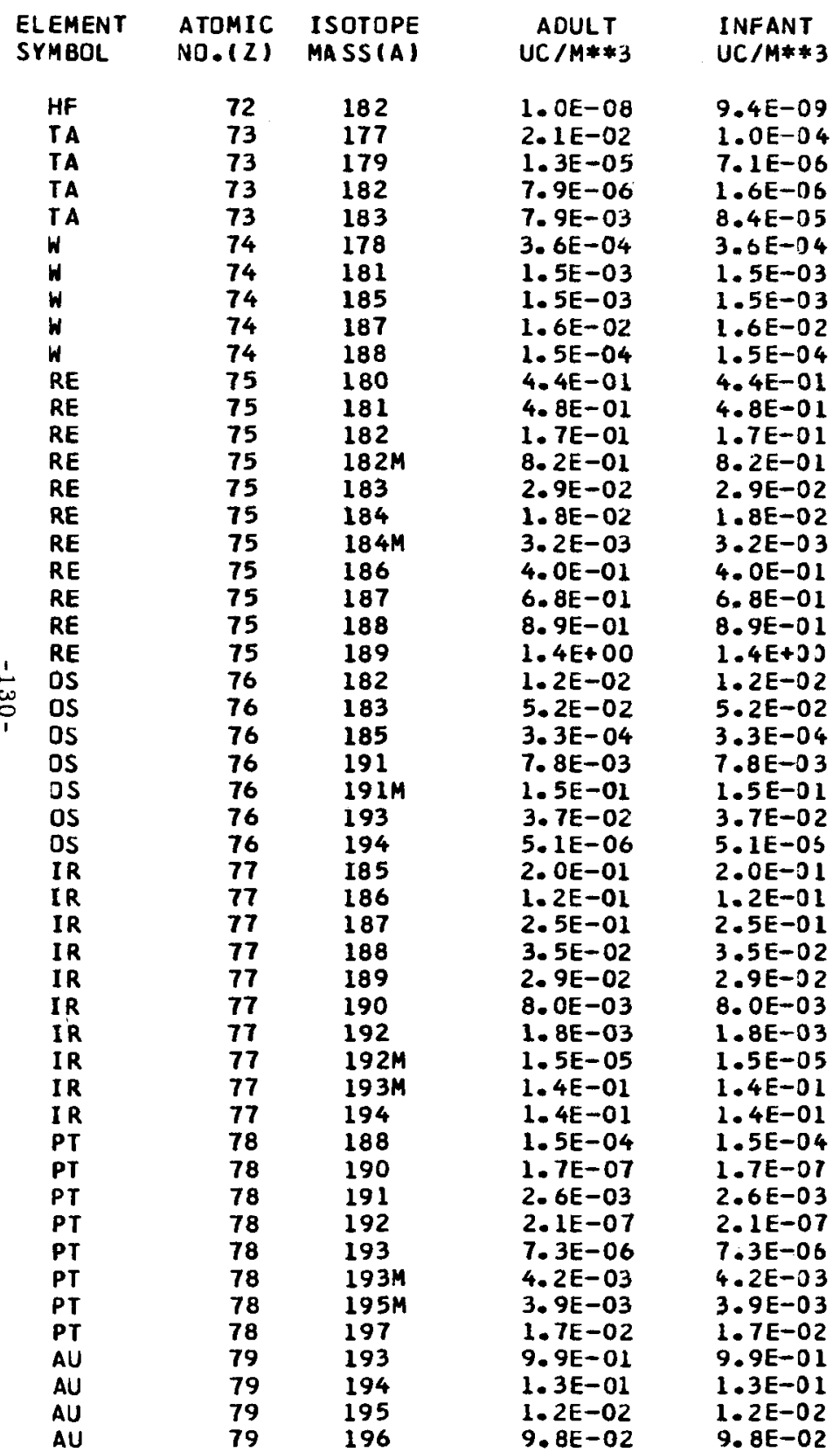

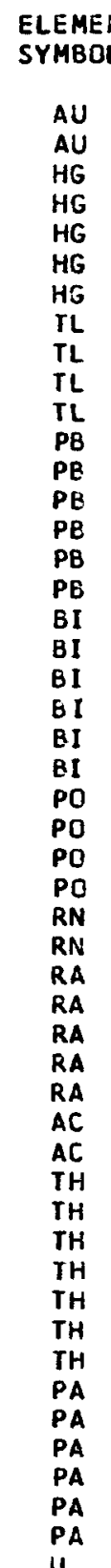

ATOMIC

I SOTOPE

$\underset{U C / M * * 3}{\text { ADULT }}$

INFANT

$\cos 20-00$

\section{9}

198
199
194
$195 \mathrm{M}$
197
$197 \mathrm{M}$
203
200
201
202

2. $2 E-01$ 6. OE-OI 3. 1E-05 3. $5 E-03$
$1.0 E-02$ 1. $0 E-02$
1. $5 E-02$ 6. $3 E-04$ 4. $2 E-03$ 8. $6 E-03$ 1. IE- 03 1. 1E-03 1. $6 E-05$ 5. $7 E+01$
$2.0 E-02$ 2. $0 E-02$ 5. $4 E+01$
$4.5 E-03$ 4. 5E-03 3. $2 E-03$ 4. $1 E-03$ 1. $1 E-02$ 1. $25-05$ 1. $2 E-05$ 6. $0 E-06$
4. 3E-03 4. $3 E-03$
2. $9 E-06$ 5. OE-02 1. $7 E-04$ 4. $0 E-05$ 1. $8 \mathrm{E}-03$ 3. $0 E+0 O$ 1. $9 E-01$ 9.8E-06 3. $0 E-05$ 3. $0 E-05$
7. $6 E-06$ 7. $6 E-06$
$1.4 E-08$ 1. $4 \mathrm{E}-08$
4. $4 \mathrm{E}-08$ 7. $3 \mathrm{E}-02$ 1. 3E-04 1. $1 E-06$ 2. $8 E-08$ 2.7E-09 2. $5 E-09$ 1. $6 \mathrm{E}-05$ 2. 2E-09 2. $2 E-09$
$6.7 E-07$ 6. 2E-01 4. $1 E-01$ 3. 3E-02 7. $0 E-05$ 3. $9 E-01$ 2. $0 E-02$ 3. 8E-0 7

$2.2 E-01$
$6.0 E-01$
$3.1 E-05$
$3.5 E-03$
$1.0 E-02$
$1.5 E-02$
$6.3 E-04$
$4.2 E-03$
$8.6 E-03$
$1.1 E-03$
$1.6 E-05$
$5.7 E+01$
$2.0 E-02$
$5.4 E+01$
$4.5 E-03$
$1.4 E-01$
$3.2 E-03$
$4.1 E-03$
$1.1 E-02$
$1.2 E-05$
$6.0 E-06$
$4.3 E-03$
$2.9 E-06$
$5.0 E-02$
$1.7 E-04$
$4.0 E-05$
$1.8 E-03$
$3.0 E+00$
$1.9 E-01$
$9.8 E-06$
$3.0 E-05$
$7.6 E-06$
$1.4 E-08$
$4.4 E-08$
$7.3 E-02$
$1.3 E-04$
$1.1 E-06$
$2.8 E-08$
$2.7 E-09$
$2.5 E-09$
$1.6 E-05$
$2.2 E-09$
$6.7 E-07$
$6.2 E-01$
$4.1 E-01$
$3.3 E-02$
$7.0 E-05$
$3.9 E-01$
$2.0 E-02$
$3.8 E-07$


TABLE 9A. UNIT-RAD EONTAMINATION FUR OVARIES

INFANT

$U C / M * * 3$

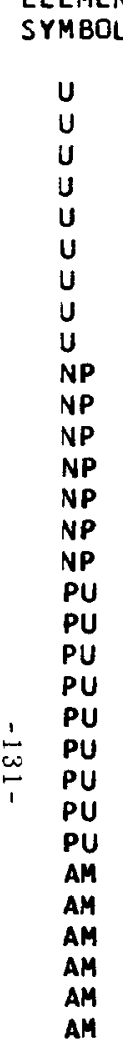

V.. (Z) MASS (A)

ADULT

$U C / M * 3$

1. 9E-06

1. $2 E-09$

$1.1 E-09$

1. $0 E-07$

9. $4 E-10$

9. $3 E-10$

$8.9 E-10$

1. OE-05

1. $1 E-01$

1. $1 E-03$

5. $9 E-05$

5. OE-O1

$6.2 \mathrm{E}-05$

2. 1E-O 1

1. $8 E-01$

4. $4 E-04$
$1.0 E-02$

$6.6 E-05$

$6.6 E-05$

5.5E-05

1. 1 E-04

$1.1 \mathrm{E}-04$
$5.3 \mathrm{E}-05$

4. 7E-05

3. 3E-02

8. $4 \mathrm{E}-01$

1. $9 E-01$

5. 7E-05

$5.9 E-01$

$5.9 E-01$

$5.6 E-05$
$5.0 E-05$
VIA SEANATER.

$\begin{array}{lcc}\text { ELEMENT } & \text { ATOMIC } & \text { I SOTOPE } \\ \text { SYMB OL } & \text { NO. (Z) } & \text { MASS (A } \\ \text { CM } & 96 & 240 \\ \text { CM } & 96 & 241 \\ \text { CM } & 96 & 242 \\ \text { CM } & 96 & 243 \\ \text { CM } & 96 & 244 \\ \text { CM } & 96 & 245 \\ \text { CM } & 96 & 246 \\ \text { CM } & 96 & 247 \\ \text { CM } & 96 & 248 \\ \text { BK } & 97 & 245 \\ \text { BK } & 97 & 246 \\ \text { BK } & 97 & 247 \\ \text { BK } & 97 & 248 \\ \text { BK } & 97 & 249 \\ \text { CF } & 98 & 246 \\ \text { CF } & 98 & 246 \\ \text { CF } & 98 & 249 \\ \text { CF } & 98 & 250 \\ \text { CF } & 98 & 251 \\ \text { CF } & 98 & 252 \\ \text { CF } & 98 & 253 \\ \text { ES } & 99 & 251 \\ \text { ES } & 99 & 252 \\ \text { ES } & 99 & 253 \\ \text { ES } & 99 & 254 \\ \text { ES } & 99 & 254 M \\ \text { ES } & 99 & 255 \\ \text { FM } & 100 & 252 \\ \text { FM } & 100 & 253 \\ \text { FM } & 100 & 255 \\ \text { FM } & 100 & 257 \\ \text { F } & & \end{array}$

$10 / 09 / 68$

PAGE 20

ADULT

1. $5 E-02$

1. $5 E-02$
$1.2 E-02$

2. $4 E-03$

8. 3E-0 5

5. OE-05

4. $8 E-05$

4. 5E-05

4. $4 \mathrm{E}-05$

7. $6 E-02$

2. $0 E-01$

4. 5E-0

3. 5E-01

1. $1 E-03$

2. $4 E-01$

$9.6 E-04$

4. $6 E-05$

1. 0E-04

4. 1E-05

3. $2 \mathrm{E}-04$

1. $6 \mathrm{E}-02$

2. 1E-01

2. $1 E-03$

1. 5E-02

6. $1 E-04$

1. $8 \mathrm{E}-01$

1. $2 \mathrm{E}-02$

3. 1E-01

$6.8 E-02$

3. $4 E-01$

3. $5 E-03$
I NFAN T

$U C / M * * 3$

1. $5 \mathrm{E}-02$

$1.2 \mathrm{E}-02$

$6.7 E-05$

8.7E-05

5. OE-05

4. 8E-05

4. $5 E-05$

4. $4 E-05$

7. $6 \mathrm{E}-02$

2. $0 E-01$

$4.5 \mathrm{E}-05$

3.5E-01

1.1E-03

$2.4 E-01$

9.6E-04

4. 6E-05

1. $0 E-04$

3. $2 E-04$

1.

$2.1 \mathrm{E}-01$

2.1E-01

1.5E-02

6. $1 E-04$

1. $8 \mathrm{E}-01$

1. $2 E-02$

3. $1 E-01$

6. 8E-02

$3.4 E-01$

$3.5 E-03$ 


\section{ATOMIC ISOTOPE}

$\underset{U C, M * * 3}{A D U L T}$

INFANT

$U C / M * * 3$

\begin{tabular}{|c|c|c|}
\hline $\begin{array}{l}H \\
B E\end{array}$ & $\begin{array}{l}1 \\
4\end{array}$ & $\begin{array}{l}3 \\
7\end{array}$ \\
\hline BE & $\begin{array}{l}4 \\
4\end{array}$ & 10 \\
\hline$C$ & 6 & 14 \\
\hline VA & 11 & 22 \\
\hline NA & 11 & 24 \\
\hline 16 & 12 & 28 \\
\hline$A L$ & 13 & 26 \\
\hline SI & 14 & 32 \\
\hline$p$ & 15 & 32 \\
\hline $\mathbf{P}$ & 15 & 33 \\
\hline$S$ & 16 & 35 \\
\hline $\mathrm{CL}$ & 17 & 36 \\
\hline$A R$ & 18 & 37 \\
\hline$A R$ & 18 & 39 \\
\hline AR & 18 & 42 \\
\hline K & 19 & 40 \\
\hline$K$ & 19 & 42 \\
\hline K & 19 & 43 \\
\hline$C A$ & 20 & 41 \\
\hline CA & 20 & 45 \\
\hline CA & 20 & 47 \\
\hline CA & 20 & 48 \\
\hline $\begin{array}{l}\text { SC } \\
\text { SC }\end{array}$ & $\begin{array}{l}21 \\
21\end{array}$ & $\begin{array}{l}44 M \\
46\end{array}$ \\
\hline SC & 21 & 47 \\
\hline SC & 21 & 48 \\
\hline T I & 22 & 44 \\
\hline$v$ & 23 & 48 \\
\hline V & 23 & 49 \\
\hline V & 23 & 50 \\
\hline CR & 24 & 48 \\
\hline CR & 24 & 51 \\
\hline MN & 25 & 52 \\
\hline MN & 25 & 53 \\
\hline MN & 25 & 54 \\
\hline $\mathbf{F E}$ & 26 & 55 \\
\hline $\mathrm{FE}$ & 26 & 59 \\
\hline $\mathrm{FE}$ & 26 & 60 \\
\hline$C D$ & 27 & 55 \\
\hline $\mathrm{CO}$ & 27 & 56 \\
\hline$C D$ & 27 & 57 \\
\hline $\mathrm{CO}$ & 27 & 58 \\
\hline $\mathrm{CO}$ & 27 & 60 \\
\hline NI & 28 & 56 \\
\hline VI & 28 & 57 \\
\hline VI & 28 & 59 \\
\hline NI & 28 & 63 \\
\hline NI & 28 & 66 \\
\hline CU & 29 & 64 \\
\hline
\end{tabular}

$\begin{array}{ll}6.4 E+02 & 6.4 E+02 \\ 1.9 E+01 & 1.7 E+00 \\ 2.2 E-03 & 2.1 E-03 \\ 3.4 E-03 & 3.4 E-03 \\ 5.4 E+01 & 5.3 E+01 \\ 7.1 E+05 & 3.8 E+04 \\ 4.0 E+03 & 4.0 E+03 \\ 1.5 E-02 & 1.4 E-02 \\ 2.8 E-03 & 2.8 E-03 \\ 6.3 E-02 & 2.3 E-02 \\ 3.0 E-01 & 1.5 E-01 \\ 2.4 E+02 & 2.4 E+02 \\ 6.6 E+01 & 5.6 E+01 \\ 8.2 E+02 & 8.2 E+02 \\ 4.4 E-02 & 4.4 E-02 \\ 4.7 E-03 & 4.7 E-03 \\ 2.1 E-01 & 2.1 E-01 \\ 1.0 E+05 & 9.2 E+02 \\ 6.2 E+04 & 9.8 E+02 \\ 1.8 E+03 & 1.8 E+03 \\ 3.5 E+03 & 3.3 E+03 \\ 1.6 E+04 & 5.5 E+03 \\ 1.8 E+00 & 1.8 E+00 \\ 1.3 E+01 & 2.2 E-02 \\ 1.5 E-02 & 8.5 E-04 \\ 6.0 E+01 & 1.4 E-01 \\ 1.8 E+01 & 2.3 E-02 \\ 7.8 E-04 & 7.4 E-04 \\ 2.8 E+02 & 4.2 E+01 \\ 1.6 E+03 & 1.3 E+03 \\ 2.2 E-01 & 2.2 E-01 \\ 4.5 E+00 & 4.5 E+00 \\ 1.6 E+01 & 1.6 E+01 \\ 4.2 E-01 & 1.1 E-01 \\ 4.9 E-02 & 4.9 E-02 \\ 9.2 E-03 & 8.7 E-03 \\ 5.7 E-02 & 3.6 E-02 \\ 5.5 E-02 & 3.9 E-03 \\ 1.2 E-05 & 1.1 E-05 \\ 2.4 E+02 & 7.4 E+00 \\ 1.1 E-01 & 8.4 E-02 \\ 3.9 E-01 & 3.5 E-01 \\ 2.5 E-01 & 1.9 E-01 \\ 2.8 E-03 & 2.7 E-03 \\ 8.7 E+00 & 8.7 E+00 \\ 6.8 E+01 & 6.8 E+01 \\ 3.5 E+00 & 3.5 E+03 \\ 1.7 E+00 & 1.7 E+00 \\ 4.8 E+01 & 4.8 E+01 \\ 7.1 E+02 & 3.1 E+01 \\ & \end{array}$

$\begin{array}{lcc}\text { ELEMENT } & \text { ATOMIC } & \text { I SOTOPE } \\ \text { SYMBOL } & \text { NO. I I } & \text { MASS A I } \\ \text { CU } & & \\ \text { ZN } & 29 & 67 \\ \text { ZN } & 30 & 65 \\ \text { ZN } & 30 & 69 M \\ \text { GA } & 30 & 72 \\ \text { GA } & 31 & 67 \\ \text { GE } & 31 & 72 \\ \text { GE } & 32 & 68 \\ \text { GE } & 32 & 69 \\ \text { AS } & 32 & 71 \\ \text { AS } & 33 & 71 \\ \text { AS } & 33 & 72 \\ \text { AS } & 33 & 73 \\ \text { AS } & 33 & 74 \\ \text { AS } & 33 & 76 \\ \text { SE } & 33 & 77 \\ \text { SE } & 34 & 72 \\ \text { SE } & 34 & 75 \\ \text { BR } & 34 & 79 \\ \text { BR } & 35 & 76 \\ \text { GR } & 35 & 77 \\ \text { KR } & 35 & 82 \\ \text { KR } & 36 & 76 \\ \text { KR } & 36 & 79 \\ \text { KR } & 36 & 81 \\ \text { RB } & 36 & 85 \\ \text { RB } & 37 & 83 \\ \text { RB } & 37 & 84 \\ \text { RB } & 37 & 86 \\ \text { SR } & 37 & 87 \\ \text { SR } & 38 & 82 \\ \text { SR } & 38 & 83 \\ \text { SR } & 38 & 85 \\ \text { SR } & 38 & 89 \\ Y & 38 & 90 \\ Y & 39 & 86 \\ Y & 39 & 87 \\ Y & 39 & 87 M \\ Y & 39 & 88 \\ Y & 39 & 90 \\ \text { ZR } & 39 & 91 \\ \text { ZR } & 40 & 86 \\ \text { ZR } & 40 & 88 \\ \text { ZR } & 40 & 89 \\ \text { ZR } & 40 & 93 \\ \text { ZR } & 40 & 95 \\ \text { NB } & 40 & 97 \\ \text { NB } & 41 & 90 \\ \text { NB } & 41 & 91 \\ \text { NB } & 41 & 91 M \\ & 41 & 92\end{array}$

ADULT
UC $/ M * * 3$

INFANT

UC $/ M * * 3$

4. $4 E+01$

1. $6 E-03$

4. $1 E-01$

2. $4 E-02$

1. $7 E+02$

2. $5 E+02$

2. $6 \mathrm{E}-03$

7. 1 E- 02

1. $2 \mathrm{E}+03$

6. $0 E+02$

2. $4 E+03$

6. $2 E+01$

8. $3 E+02$

4. $3 E+03$

7. $3 E-02$

1. OE-02

1. $7 E-03$

1. $0 E+05$

2. $0 E+04$

1. $8 E+04$

1. $5 E+02$

1. $9 E+02$

1. $3 E+00$

2. $5 \mathrm{E}-01$

1. $4 E+00$

$6.8 \mathrm{E}+00$

3. $9 E-02$

3. $0 E+02$

1. $2 E+05$

$6.4 E+02$

$5.8 E+02$

2. $2 E+00$

2. $7 E+03$

4. $4 E+02$

1. $0 \mathrm{E}+04$

1. $4 E-01$

4. $4 E+02$

1. $4 E+03$

1. $4 E+03$

1. $6 E-01$

1. $7 E+02$

5. $0 E-01$

2. $1 E+03$

6. $O E+01$

1. $O E-03$

1. $4 E-01$
$7.6 E+00$

$1.6 \mathrm{E}-03$

4.1E-01

$2.4 E-02$

6. $1 E+01$

2. $2 E+01$

$2.6 \mathrm{E}-03$

7. $1 \mathrm{E}-02$

$1.2 E+03$

6. $0 E+02$

$2.4 E+03$

$6.2 \mathrm{E}+01$

8. $3 E+02$

4. $3 E+03$

$2.3 E-02$

8.7E-03

1. $7 E-03$

7. $9 E+03$

4. $7 E+03$

2. $8 E+03$

1. $5 E+02$

1. $9 E+02$

$1.3 E+00$

2. $5 E-01$

5. $2 E-01$

4. $1 E-01$

$1.3 E+00$

3. $8 \mathrm{E}-02$

2. $1 E+02$

1. $2 E+04$

$5.4 E+02$

4. $7 \mathrm{E}+02$

2. $2 E+00$

1. $7 E-01$

1. $5 \mathrm{E}-01$

5. 9E-01

1. $5 E-03$

1. $2 E-01$

1. $1 E+00$

$1.1 E+00$
$1.4 E-02$

$6.1 \mathrm{E}-01$

$2.4 \mathrm{E}-02$

3. $4 \mathrm{E}-02$

1. $7 E+00$

3. $8 \mathrm{E}-02$

9. OE-O4

8. $5 E-03$
1. $3 E-02$ 


\begin{tabular}{|c|c|c|c|c|}
\hline $\begin{array}{l}\text { ELEMENT } \\
\text { SYMBOL }\end{array}$ & $\begin{array}{l}\text { ATOMIC } \\
\text { ND. }(Z)\end{array}$ & $\begin{array}{l}\text { ISOTOPE } \\
\text { MASS (A) }\end{array}$ & $\begin{array}{l}\text { ADULT } \\
\text { UC } / M * * 3\end{array}$ & $\begin{array}{l}\text { INFANT } \\
\text { UC/M**3 }\end{array}$ \\
\hline $\begin{array}{l}N B \\
V B \\
N B \\
N B \\
N B \\
M D \\
M D \\
T C \\
T C \\
T C \\
T C \\
T C \\
T C \\
T C \\
T C \\
R U \\
R U \\
R U \\
R H \\
R H \\
R H \\
R H \\
R H \\
W \\
C H \\
R H \\
P D \\
P D \\
P D \\
P D \\
P D \\
A G \\
A G \\
A G \\
A G \\
A G \\
A G \\
C D \\
C D \\
C D \\
C D \\
I N \\
I N \\
I N \\
S N \\
S N \\
S N \\
S N \\
S N \\
S N \\
S N \\
S N \\
S B \\
S B\end{array}$ & $\begin{array}{l}41 \\
41 \\
41 \\
41 \\
41 \\
42 \\
42 \\
43 \\
43 \\
43 \\
43 \\
43 \\
43 \\
43 \\
44 \\
44 \\
44 \\
45 \\
45 \\
45 \\
45 \\
45 \\
45 \\
46 \\
46 \\
46 \\
46 \\
46 \\
47 \\
47 \\
47 \\
47 \\
47 \\
48 \\
48 \\
48 \\
48 \\
49 \\
49 \\
49 \\
50 \\
50 \\
50 \\
50 \\
50 \\
50 \\
50 \\
50 \\
51 \\
51\end{array}$ & $\begin{array}{l}93 M \\
94 \\
95 \\
95 M \\
96 \\
93 \\
99 \\
95 \\
95 M \\
96 \\
97 \\
97 M \\
98 \\
99 \\
97 \\
103 \\
106 \\
99 \\
100 \\
101 \\
101 M \\
102 \\
105 \\
100 \\
103 \\
107 \\
109 \\
112 \\
105 \\
106 M \\
108 M \\
110 M \\
111 \\
109 \\
113 M \\
115 \\
115 M \\
111 \\
114 M \\
115 \\
113 \\
117 M \\
119 M \\
121 \\
121 M \\
123 M \\
125 \\
126 \\
119 \\
120 M\end{array}$ & $\begin{array}{l}5.4 E-03 \\
1.0 E-05 \\
1.2 E-01 \\
8.0 E+00 \\
4.6 E+01 \\
1.3 E+01 \\
3.0 E+03 \\
2.0 E+00 \\
2.1 E-02 \\
1.3 E-01 \\
8.9 E-03 \\
1.3 E-01 \\
1.2 E-04 \\
2.0 E-03 \\
2.7 E+01 \\
1.8 E-01 \\
1.3 E-03 \\
4.0 E-02 \\
2.5 E+00 \\
2.3 E-04 \\
5.0 E-01 \\
4.2 E-04 \\
5.5 E+00 \\
1.1 E-01 \\
2.2 E-01 \\
1.5 E-02 \\
1.2 E+01 \\
8.9 E-01 \\
3.7 E+00 \\
1.8 E+01 \\
2.4 E-02 \\
1.6 E-01 \\
1.2 E+02 \\
1.6 E-03 \\
7.2 E-05 \\
1.9 E+00 \\
7.6 E-03 \\
2.0 E-03 \\
4.2 E-06 \\
8.2 E-08 \\
1.4 E+01 \\
1.2 E+03 \\
1.7 E+01 \\
4.8 E+05 \\
1.4 E-01 \\
7.7 E+00 \\
4.4 E+02 \\
3.7 E-03 \\
5.8 E-01 \\
3.3 E-03\end{array}$ & $\begin{array}{l}3.2 \mathrm{E}-03 \\
9.1 \mathrm{E}-06 \\
4.3 \mathrm{E}-03 \\
3.2 \mathrm{E}-02 \\
4.7 \mathrm{E}-02 \\
1.3 \mathrm{E}+01 \\
7.8 \mathrm{E}+02 \\
2.0 \mathrm{E}+00 \\
2.1 \mathrm{E}-02 \\
1.3 \mathrm{E}-01 \\
8.9 \mathrm{E}-03 \\
1.3 \mathrm{E}-01 \\
1.2 \mathrm{E}-04 \\
2.0 \mathrm{E}-03 \\
4.8 \mathrm{E}-01 \\
3.6 \mathrm{E}-02 \\
9.3 \mathrm{E}-04 \\
1.7 \mathrm{E}-02 \\
9.9 \mathrm{E}-02 \\
2.3 \mathrm{E}-04 \\
8.9 \mathrm{E}-02 \\
3.8 \mathrm{E}-04 \\
3.7 \mathrm{E}-01 \\
2.2 \mathrm{E}-02 \\
1.2 \mathrm{E}-01 \\
1.5 \mathrm{E}-02 \\
4.4 \mathrm{E}-01 \\
4.9 \mathrm{E}-02 \\
2.4 \mathrm{E}+00 \\
4.9 \mathrm{E}+00 \\
2.3 \mathrm{E}-02 \\
1.5 \mathrm{E}-01 \\
3.1 \mathrm{E}+01 \\
1.1 \mathrm{E}-03 \\
6.8 \mathrm{E}-05 \\
1.9 \mathrm{E}-02 \\
1.2 \mathrm{E}-03 \\
1.1 \mathrm{E}-04 \\
2.1 \mathrm{E}-00 \\
8.1 \mathrm{E}-03 \\
1.7 \mathrm{E}+00 \\
1.9 \mathrm{E}+01 \\
3.7 \mathrm{E}+00 \\
6.1 \mathrm{E}+02 \\
1.2 \mathrm{E}-01 \\
9.5 \mathrm{E}-01 \\
4.7 \mathrm{E}+00 \\
3.3 \mathrm{E}-03 \\
5.8 \mathrm{E}-01 \\
3.3 \mathrm{E}-03\end{array}$ \\
\hline
\end{tabular}

ELEMENT
SYMBOL

SE
$S B$
$S B$
$S B$
$S B$
$T E$
$T E$
$T E$
$T E$
$T E$
$T E$
$T E$
$T E$
$T E$
$T E$
$T E$
$T E$
$T E$
$I$
$I$
$I$
$I$
$I$
$I$
$I$
$I$
$I$
$X E$
$X E$
$X E$
$X E$
$X E$
$X E$
$X E$
$C S$
$C S$
$C S$
$C S$
$C S$
$C S$
$C S$
TA
$B A$
$B A$
$G A$
$6 A$
$B A$
$L A$
$L A$
LA
LA
$C E$

ATOMIC

ADULT

INFANT

1. $5 \mathrm{E}-02$

3. $3 E-04$

9. $1 E-05$

1. $4 E-03$

4. 3E-02

2. $2 E+00$

4. $3 E-02$

1. $6 E-02$

5. $4 E-04$

2. $6 E-04$

2. $7 E-03$

2. $1 E-03$

3. $7 E-03$

5. $2 E-01$

6. 1E-02

$1.6 E+02$

3. $8 E-01$

3. $7 E-01$

3. $0 E-01$

2. $3 E-03$

1. $5 E+01$

5. $2 E-01$

1 . $1 E+01$

2. $1 E+02$

1. $1 E+03$

5. $3 E+01$

2. $4 E+02$

2. $3 E+02$

4. $6 E+02$

4. $7 E+02$

8. $7 E+02$

2. $9 E+02$

3. $1 E+01$

6. $2 E-03$

2. $\mathrm{EE}-02$

2.

2. $5 E+00$

1. $9 E-03$

1. $9 E+02$

1. $2 E+00$

9. $2 E+03$

4. $1 E+04$

4. $8 E+01$

2. $O E-01$

$5.2 E-04$

1. $5 E-05$

1.5E- 05

2. $8 E-02$
1. $5 E+03$

1. 5E-02

3. 3E-04

$9.1 E-05$

1. $4 E-03$

9. $9 E-03$

7. $2 E-03$

4.7E-02

5. 9E-03

5. 9E-03

$4.5 \mathrm{E}-04$

2.6E-04

2.1 E-03

7. $2 E-03$

$1.7 \mathrm{E}-03$

1. $9 E-03$

2. $0 \mathrm{E}-02$

5. $9 E-03$

$1.1 \mathrm{E}+01$

1. $4 \mathrm{E}-01$

3. $4 \mathrm{E}-01$

2. 0 E-01 ड

2.3E-03 D

2. $8 E-01$

$1.2 E+00$

$2.1 E+02$

1. $1 E+03$

5. $3 E+01$

$2.4 E+02$

$2.3 E+02$

4. $6 E+02$

4. $7 E+02$

1. $0 E+01$

$2.4 E+01$

1. $8 E+00$

5. $4 \mathrm{E}-03$

1.9E-O2

$2.7 E-01$

1. $8 \mathrm{E}-03$

1. $8 E+02$

8. $8 E+01$

1. $2 E+00$

1. $0 E+03$

3. $5 E+03$

$2.4 E+01$

$5.2 E-04$

1. $5 \mathrm{E}-05$

2.8E-O?

2. $8 \mathrm{E}-02$
5. $2 \mathrm{E}+0 \mathrm{OO}$ 


\begin{tabular}{|c|c|c|c|c|}
\hline $\begin{array}{l}\text { ELEMENT } \\
\text { SYMBOL }\end{array}$ & $\begin{array}{l}\text { ATOMIC } \\
\text { VO. }(Z)\end{array}$ & $\begin{array}{l}\text { ISOTOPE } \\
\text { MASS (A) }\end{array}$ & $\begin{array}{l}\text { ADULT } \\
U C / M * * 3\end{array}$ & $\begin{array}{l}\text { INFANT } \\
U C / M * * 3\end{array}$ \\
\hline $\begin{array}{l}C E \\
C E \\
C E \\
C E \\
C E \\
C E \\
C E \\
P R \\
P R \\
N D \\
N D \\
N D \\
P M \\
P M \\
P M \\
P M \\
P M \\
P M \\
P M \\
P M \\
P M \\
S M \\
S M \\
S M \\
S M \\
S M \\
S M \\
S M \\
S M \\
E U \\
E U \\
E U \\
E U \\
E U \\
E U \\
E U \\
E U \\
E U \\
E U \\
E U \\
E U \\
E U \\
G D \\
G D \\
G D \\
G D \\
G D \\
G D \\
G D \\
G D\end{array}$ & $\begin{array}{l}58 \\
58 \\
58 \\
58 \\
58 \\
58 \\
58 \\
59 \\
59 \\
60 \\
60 \\
60 \\
61 \\
61 \\
61 \\
61 \\
61 \\
61 \\
61 \\
61 \\
61 \\
62 \\
62 \\
62 \\
62 \\
62 \\
62 \\
62 \\
62 \\
63 \\
63 \\
63 \\
63 \\
63 \\
63 \\
63 \\
63 \\
63 \\
63 \\
63 \\
63 \\
63 \\
64 \\
64 \\
64 \\
64 \\
64 \\
64 \\
64 \\
64\end{array}$ & $\begin{array}{l}135 \\
137 M \\
139 \\
141 \\
142 \\
143 \\
144 \\
142 \\
143 \\
140 \\
144 \\
147 \\
143 \\
144 \\
145 \\
146 \\
147 \\
148 \\
148 M \\
149 \\
151 \\
141 \\
145 \\
146 \\
147 \\
148 \\
149 \\
151 \\
153 \\
145 \\
146 \\
146 M \\
147 \\
148 \\
149 \\
150 \\
150 M \\
152 \\
154 \\
155 \\
156 \\
157 \\
146 \\
147 \\
148 \\
149 \\
150 \\
151 \\
152 \\
153\end{array}$ & $\begin{array}{l}3.4 E+04 \\
2.5 E+04 \\
5.3 E+00 \\
7.0 E+01 \\
2.8 E-03 \\
8.0 E+03 \\
8.0 E-02 \\
4.3 E-05 \\
8.0 E-06 \\
1.1 E-05 \\
3.2 E-09 \\
1.4 E-06 \\
1.6 E-01 \\
1.3 E-02 \\
1.9 E-02 \\
4.5 E-03 \\
3.3 E-03 \\
7.0 E+01 \\
3.5 E-01 \\
1.5 E+03 \\
2.1 E+03 \\
2.4 E+00 \\
3.1 E-01 \\
3.1 E-04 \\
3.5 E-04 \\
2.0 E-04 \\
4.3 E-04 \\
5.9 E-02 \\
2.9 E+03 \\
1.4 E+02 \\
3.6 E+01 \\
4.4 E+02 \\
2.1 E+00 \\
2.4 E+00 \\
6.8 E-01 \\
5.4 E-03 \\
2.9 E+03 \\
1.9 E-03 \\
1.4 E-03 \\
1.8 E-01 \\
9.5 E+00 \\
7.8 E+03 \\
1.1 E-01 \\
2.5 E+02 \\
1.6 E-04 \\
1.3 E+01 \\
8.6 E-05 \\
3.2 E-01 \\
2.1 E-04 \\
2.3 E-01\end{array}$ & $\begin{array}{l}3.0 E+01 \\
4.2 E+01 \\
7.5 E-01 \\
2.6 E+00 \\
2.5 E-03 \\
1.3 E+01 \\
2.0 E-02 \\
4.3 E-05 \\
8.0 E-06 \\
1.1 E-05 \\
3.2 E-09 \\
1.4 E-06 \\
4.3 E-02 \\
4.2 E-03 \\
1.7 E-02 \\
3.1 E-03 \\
1.9 E-03 \\
5.1 E-01 \\
1.8 E-02 \\
4.4 E+00 \\
3.4 E+00 \\
6.6 E-02 \\
1.0 E-01 \\
2.8 E-04 \\
3.2 E-04 \\
1.8 E-04 \\
3.9 E-04 \\
5.2 E-02 \\
7.8 E+00 \\
1.8 E+00 \\
3.7 E-01 \\
1.6 E+00 \\
1.1 E-01 \\
2.6 E-01 \\
1.3 E-01 \\
4.3 E-03 \\
3.5 E+00 \\
1.7 E-03 \\
1.3 E-03 \\
1.1 E-01 \\
3.1 E-01 \\
1.1 E+01 \\
1.2 E-02 \\
6.7 E-01 \\
1.5 E-04 \\
3.0 E-01 \\
8.2 E-05 \\
7.7 E-02 \\
2.0 E-04 \\
8.9 E-02\end{array}$ \\
\hline
\end{tabular}

\section{ELEMENT} SY MBOL

ATOMIC I SOTOPE

ADULT

I NFANT

\section{GO}

GD
TB
TB
TB
TB
TB
TB
TE
TB
T6

TB

TB

TE

T6

TB

OY

DY

DY

DY

HO
HO

HO

$E R$
$E R$
$E R$

ER

TM

TM

$Y B$
$Y B$

YB
YB

LU

LU

LU

LU

LU

LU

LU

LU

HF

HF

HF
HF
HF

HF
HF UC $/ M * * 3$

NO. (Z) MASSIA)

$5 \cdot 4 E+03$

1. $2 E-02$

9. $3 E+02$

6. $2 E+02$

3. $4 E+03$

1. $4 E+02$

4. $4 E+01$

1. $6 E-02$

7. $0 E-04$

3. $1 E-01$

2. $0 E+02$

4. $7 E+03$

5. $9 \mathrm{E}-10$

2. $0 E-05$

9. $2 E-10$

3. $8 E-06$

9. $4 E-06$

$9.5 E-08$

3. $7 E-05$

4. $2 E-09$

1. 2E-05

4. $6 E-05$

1. $6 E-05$

5. $7 E+01$

4. $1 E+00$

2. $3 E-02$

4. $9 E-02$

6. $2 E-02$

3. $3 E+01$

8. $8 E+02$

7. $6 E+00$

8. $7 E+02$

1. $4 E-05$

$6.4 E-06$

3. $4 E-05$

6. $0 E-06$

1. $L E-07$

2. $4 E-08$

4. $4 E-07$

7. $1 E-09$

3. $4 E-05$

1. 2E-06

7. 8E-01

1. $5 E+00$

1. $4 E-06$

4. $6 E-01$

1. $3 E-07$

4. $1 E-04$
1.1E+01 6. $0 E-03$ 7. $1 E+00$ $2.1 E+00$ 4. $6 E+00$ 8. $8 E+00$ $2.3 E+00$ 6. $8 \mathrm{E}-01$ 1. $5 \mathrm{E}-02$ $6.6 \mathrm{E}-04$ $5.5 E-02$ 4. $O E+00$ 1. $4 E+01$ 5. $9 E-10$ 2. OE-05 9. $2 \mathrm{E}-10$ 3. $8 \mathrm{E}-06$ $9.4 E-06$ 9. $5 E-08$ 3. $7 E-05$ 4. 2E-09

$4.6 E-05$

1.6E-05

$1.7 \mathrm{E}-01$ $9.6 E-02$

$4.1 E-03$

1. 2E-02 3. $9 E-02$ 2. $2 E-01$ $5.2 E+00$ 5. $5 E-01$ 8. $8 E+00$ . $4 E-05$ . $4 E-06$ 3. $4 E-05$ 6. $0 \mathrm{E}-06$ 1. $1 \mathrm{E}-07$ $2.4 E-08$ 4. $4 \mathrm{E}-07$ 7. 1 E- 09 3. $4 E-05$ 1. 2E-06 1. $1 E-03$ $2.5 E-03$ $1.1 \mathrm{E}-06$ 1. $3 E-03$ 1. $2 E-07$ 6. $8 E-05$ 7. $3 E-05$ 
TABLE 9A. UNIT-RAD CONTAMINATION FOR

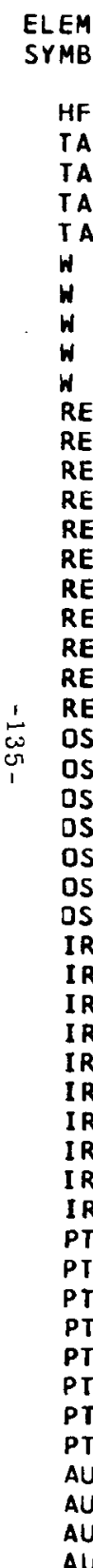
NO. (Z) ISOTOPE

$\begin{array}{ll}72 & 182 \\ 73 & 177 \\ 73 & 179 \\ 73 & 182 \\ 73 & 183 \\ 74 & 178 \\ 74 & 181 \\ 74 & 185 \\ 74 & 187 \\ 74 & 188 \\ 75 & 180 \\ 75 & 181 \\ 75 & 182 \\ 75 & 182 M \\ 75 & 183 \\ 75 & 184 \\ 75 & 184 M \\ 75 & 186 \\ 75 & 187 \\ 75 & 188 \\ 75 & 189 \\ 76 & 182 \\ 76 & 183 \\ 76 & 185 \\ 76 & 191 \\ 76 & 191 M \\ 76 & 193 \\ 76 & 194 \\ 77 & 185 \\ 77 & 186 \\ 77 & 187 \\ 77 & 188 \\ 77 & 189 \\ 77 & 190 \\ 77 & 192 \\ 77 & 192 M \\ 77 & 193 M \\ 77 & 194 \\ 78 & 188 \\ 78 & 190 \\ 78 & 191 \\ 78 & 192 \\ 78 & 193 \\ 78 & 193 M \\ 78 & 195 M \\ 78 & 197 \\ 79 & 193 \\ 79 & 194 \\ 79 & 195 \\ 79 & 196 \\ & \\ 75 \\ 79\end{array}$

SPLEEN

ADULT $U C / M * * 3$

INFANT

UC $/ M * * 3$

2. $0 E-07$

2. $2 E-01$

2.

8. $3 E-02$

3. $6 E-04$

1. $5 \mathrm{E}-03$

1. $5 \mathrm{E}-03$

1. $6 E-02$

1. $5 \mathrm{E}-04$

4. 4E-

1. $7 \mathrm{E}-01$

8. $2 E-01$

2. $9 E-02$

1. $8 \mathrm{E}-02$

3. $2 \mathrm{E}-03$

4. $O E-0 I$

6. 8E-01

8. 9E-01

- $4 E+00$

1. $2 E-02$

$5.2 E-02$
$3.3 E-04$

3. $3 E-04$
7. $8 E-03$

7. $8 \mathrm{E}-03$

1. $5 \mathrm{E}-01$

5. $1 E-06$

1. $6 E+01$

9. $5 E+00$

2. $6 E+01$

1. $1 E+00$

1. $4 \mathrm{E}-01$

4. $2 E-02$

2. $9 E-03$

1. $5 \mathrm{E}-05$
7. $3 \mathrm{E}-01$

7. $3 E-01$

1. $0 \mathrm{E}-03$

1. $8 E-07$

5. $4 \mathrm{E}-02$

2. $1 E-07$

7. $3 E-00$

6. $1 E-02$

6. $0 E-02$

1. $2 \mathrm{E}+00$

7. $3 E+02$

4. $5 E+01$

6. $2 E-0 \overrightarrow{2}$

- 9E-07

2.1E-0

उ. $2 E-04$

. 2 E

3.6E-04

1. $5 E-03$

$1.5 \mathrm{E}-03$

$1.6 \mathrm{E}-02$

1. 5E-04

4. $4 E-01$

4.8E-01

$1.7 E-01$

8. $2 \mathrm{E}-0$

2. $9 E-02$

1. $8 \mathrm{E}-02$

$3.2 \mathrm{E}-03$

4. OE-O 1

6. $8 E-01$

1. $2 \mathrm{E}-02$

5. 2E-02

$3.3 E-04$
$7.8 E-03$

1. 5E-OI

3. $7 E-02$

5.1E-OS

1. $2 \mathrm{E}-01$

2. $5 \mathrm{E}-01$

$3.5 \mathrm{E}-02$

2. $9 E-02$

8. OE-03

1. $8 E-03$

$1.5 \mathrm{E}-05$

$1.4 \mathrm{E}-0$

$1.4 E-01$

$1.5 E-04$

1.7E-O

2.6E-03

2.1E-07

7. $3 \mathrm{E}-\mathrm{O}$

4. $2 E-03$

3. $9 \mathrm{E}-03$

1. 7E-02

$2.3 E+00$

$3.0 \mathrm{E}-5$

2.7E-02
VIA SEAHATER.

8. $9 E-01$

$1.4 E+5 J$

2. OE-O 1

ELEMENT
SYMBOL

ATJMIC ISOTUPE NO. (Z) MASS (A)

\begin{tabular}{|c|c|c|}
\hline$A U$ & 79 & 190 \\
\hline$A U$ & 79 & 199 \\
\hline HÉ & 80 & 194 \\
\hline HG & 80 & $195 \mathrm{M}$ \\
\hline HG & 80 & 197 \\
\hline HG & 80 & $197 \mathrm{M}$ \\
\hline HG & 80 & 203 \\
\hline $\mathrm{Tl}$ & 81 & 200 \\
\hline TL & 81 & 201 \\
\hline$T L$ & 81 & 202 \\
\hline$T L$ & 31 & 204 \\
\hline PB & 82 & 200 \\
\hline PB & 82 & 202 \\
\hline PB & 82 & 20.3 \\
\hline$P B$ & 82 & 204 \\
\hline$P E$ & 82 & 205 \\
\hline PE & 82 & 210 \\
\hline BI & 83 & 205 \\
\hline 61 & 83 & 206 \\
\hline B I & 83 & 207 \\
\hline BI & 83 & 208 \\
\hline BI & 83 & 210 \\
\hline BI & 83 & $210 \mathrm{M}$ \\
\hline PO & 84 & 206 \\
\hline $\mathbf{P C}$ & 84 & 208 \\
\hline PO & 84 & 209 \\
\hline$P O$ & 84 & 210 \\
\hline RN & 86 & 211 \\
\hline $\mathbf{k}$ & 86 & 222 \\
\hline KA & 88 & 223 \\
\hline RA & 88 & 224 \\
\hline RA & 88 & 225 \\
\hline RA & 88 & 226 \\
\hline RA & 88 & $22 \varepsilon$ \\
\hline$A C$ & 89 & 225 \\
\hline$A C$ & 89 & 227 \\
\hline ب & 90 & 227 \\
\hline TH & 90 & 228 \\
\hline TH & 90 & 229 \\
\hline TH & $\because 0$ & 230 \\
\hline TH & 90 & 231 \\
\hline TH & 90 & 232 \\
\hline TH & 90 & 234 \\
\hline PA & 91 & 228 \\
\hline PA & $\because$ & 229 \\
\hline PA & $\Rightarrow$ & $\therefore 30$ \\
\hline PA & $0 !$ & 231 \\
\hline PA & 91 & 232 \\
\hline PA & 91 & 233 \\
\hline U & 92 & \\
\hline
\end{tabular}

10/09/68 PAGE 24

ADULT

TE+01

4. $5 E+01$

2. $5 E-04$

1. $9 E-01$

3. $7 E-01$

1. $3 E+00$

5. $9 E-03$

8. 3E-02

6. $5 E-02$

2.7E-03

1. $2 E+01$

1. $2 E+01$

4. $1 E-03$

9. $4 E-04$

2. $8 E-02$

$6.5 E-04$

2. $6 E-03$

1. $1 E-02$

4. $7 E-06$

2. $3 E-06$

4. $9 E-03$

1. $1 E-06$

3. $9 E-01$

1. $8 E-04$

4. $O E-05$

2. $6 E-03$

3. $0 E+00$

1. $9 E-01$

$9.8 E-06$

3. $0 E-05$

7. $6 E-06$

1. $4 E-08$

4. $4 E-08$

7. $3 E-02$

1. $3 E-04$

1. $1 E-06$

2. $8 \mathrm{E}-08$

2. $7 E-09$

2. $5 E-09$

1. $6 \mathrm{E}-05$

2. $2 E-09$

6. $7 E-07$

$0.2 E-01$

4. $1 E-01$

3. $3 E-02$

7. $O E-O E$ :

3. $9 E-01$

2. $O E-O Z$

3. $8 E-07$
INFANT

UC/M**3

5. $0 E-01$

. $4 E+00$

2. 3E-04

- $7 E-02$

$1.2 E-01$

4. $9 E-03$

7. $0 E-03$

8. 8E-04

1. $3 E-05$

$1.2 E+01$

1. $1 E+01$

$9.4 E-04$

2. 8E-02

6.5E-04

$1.6 E-03$

4. $2 \mathrm{E}-03$

4.7E-06 $\mathrm{G}$

.3E-06

$1.6 E-03$ 巳

1.1E-06 8

1.7E-04

4. $0 E-05$

1. $8 E-03$

3. $O E+00$

1. $9 E-01$

9. $8 \mathrm{E}-06$

. 0E-05

7. 6 E-06

. $4 \mathrm{E}-0 \mathrm{~B}$

$4.4 E-08$

7. $3 \mathrm{E}-02$

$1.3 E-04$

1. $1 E-06$

2. $8 \mathrm{E}-08$

2.7E-09

$2.5 E-09$

$.6 E-05$

2. $2 E-09$

$6.7 E-07$

$6.2 E-01$

$4.1 E-01$

$3.3 E-02$

7. $0 E-05$

$3.9 E-01$

2. 0E-02

3. $8 \mathrm{E}-07$ 
TABLE 9A. UNIT-RAD IONTAMINATION FOR SPLEEN

\begin{tabular}{|c|c|c|c|c|}
\hline $\begin{array}{l}\text { ELEMENT } \\
\text { SYMBOL }\end{array}$ & $\begin{array}{l}\text { ATDMIC } \\
\text { NO. ( } 2)\end{array}$ & $\begin{array}{l}\text { ISOTOPE } \\
\text { MASS (A) }\end{array}$ & $\begin{array}{c}\text { ADULT } \\
\text { UC/M**3 }\end{array}$ & $\begin{array}{l}\text { INFANT } \\
\text { UC } / M * * 3\end{array}$ \\
\hline $\begin{array}{l}U \\
U \\
U \\
U \\
U \\
U \\
U \\
U \\
U \\
N P \\
N P \\
U P \\
W P \\
N P \\
N P \\
N P \\
N P \\
P U \\
P U \\
P U \\
P U \\
P U \\
P U \\
P U \\
P U \\
P U \\
A M \\
A M \\
A M \\
A M \\
A M \\
A M \\
A M\end{array}$ & $\begin{array}{l}92 \\
92 \\
92 \\
92 \\
92 \\
92 \\
92 \\
92 \\
92 \\
93 \\
93 \\
93 \\
93 \\
93 \\
93 \\
93 \\
94 \\
94 \\
94 \\
94 \\
94 \\
94 \\
94 \\
94 \\
94 \\
95 \\
95 \\
95 \\
95 \\
95 \\
95\end{array}$ & $\begin{array}{l}231 \\
232 \\
233 \\
234 \\
235 \\
236 \\
237 \\
238 \\
240 \\
234 \\
235 \\
236 \\
236 M \\
237 \\
238 \\
239 \\
236 \\
237 \\
238 \\
239 \\
240 \\
241 \\
242 \\
244 \\
246 \\
239 \\
240 \\
241 \\
242 \\
242 M \\
243\end{array}$ & $\begin{array}{l}1.9 E-06 \\
1.2 E-09 \\
1.1 E-09 \\
1.0 E-09 \\
9.4 E-10 \\
9.3 E-10 \\
1.1 E-06 \\
8.9 E-10 \\
1.0 E-05 \\
1.1 E-01 \\
1.1 E-03 \\
5.9 E-05 \\
5.0 E-01 \\
6.2 E-05 \\
2.1 E-01 \\
1.8 E-01 \\
4.4 E-04 \\
1.0 E-02 \\
6.6 E-05 \\
5.6 E-05 \\
5.5 E-05 \\
1.1 E-04 \\
5.3 E-05 \\
4.7 E-05 \\
3.3 E-02 \\
8.4 E-01 \\
1.9 E-01 \\
5.7 E-05 \\
5.9 E-01 \\
5.6 E-05 \\
5.0 E-05\end{array}$ & $\begin{array}{l}1.9 E-06 \\
1.2 \mathrm{E}-09 \\
1.1 \mathrm{E}-09 \\
1.0 \mathrm{E}-09 \\
9.4 \mathrm{E}-10 \\
9.3 \mathrm{E}-10 \\
1.1 \mathrm{E}-0 . \\
8.9 \mathrm{E}-10 \\
1.0 \mathrm{E}-05 \\
1.1 \mathrm{E}-01 \\
1.1 \mathrm{E}-03 \\
5.9 \mathrm{E}-05 \\
5.0 \mathrm{E}-01 \\
6.2 \mathrm{E}-05 \\
2.1 \mathrm{E}-01 \\
1.8 \mathrm{E}-01 \\
4.4 \mathrm{E}-04 \\
1.0 \mathrm{E}-02 \\
6.6 \mathrm{E}-05 \\
5.6 \mathrm{E}-05 \\
5.5 \mathrm{E}-05 \\
1.1 \mathrm{E}-04 \\
5.3 \mathrm{E}-05 \\
4.7 \mathrm{E}-05 \\
3.3 \mathrm{E}-02 \\
8.4 \mathrm{E}-01 \\
1.9 \mathrm{E}-01 \\
5.7 \mathrm{E}-05 \\
5.9 \mathrm{E}-01 \\
5.6 \mathrm{E}-05 \\
5.0 \mathrm{E}-05\end{array}$ \\
\hline
\end{tabular}

VIA SEANATER.

ELEMENT
SYMBOL

ATOMIC

$C M$
$C M$
$C M$
$C M$
$C M$
$C M$
$C M$
$C M$
$C M$
$B K$
$B K$
$B K$
$B K$
$E K$
$C F$
$C F$
$C F$
$C F$
$C F$
$C F$
$C F$
$E S$
$E S$
$E S$
$E S$
$E S$
$E S$
$F M$
$F M$
$F M$
$F M$
$10 / 09 / 68$

ADULT UC $/ M * * 3$

1. $5 \mathrm{E}-02$

2. $4 E-03$

$6.7 E-05$

6. $7 E-05$

$8.3 E-05$

$5.0 E-05$

4. $8 E-05$

4. $5 E-05$
4. $4 E-05$

7. $6 E-02$

2. OE-OI

4. $5 E-05$

3. $5 \mathrm{E}-01$

1. $1 E-03$

2. $4 E-01$

$9.6 E-04$

4. $6 E-05$

$1.0 E-04$

4. $1 E-05$

3. $2 E-04$

1. $6 E-02$

2. $1 E-01$

2. $1 E-03$

1. 5E-02

6. $1 E-04$

1. $8 E-01$

1. 2E-02

3. $1 E-01$

6. $8 E-02$

3. $4 \mathrm{E}-01$
PAGE 25

INFAN T UC $/ M * * 3$

$1.5 \mathrm{E}-02$
$1.2 \mathrm{E}-02$
$2.4 \mathrm{E}-03$
$6.7 \mathrm{E}-05$
$8.3 \mathrm{E}-05$
$5.0 \mathrm{E}-05$
$4.8 \mathrm{E}-05$
$4.5 \mathrm{E}-05$
$4.4 \mathrm{E}-05$
$7.6 \mathrm{E}-02$
$2.0 \mathrm{E}-01$
$4.5 \mathrm{E}-05$
$3.5 \mathrm{E}-01$
$1.1 \mathrm{E}-03$
$2.4 \mathrm{E}-01$
$9.6 \mathrm{E}-04$
$4.6 \mathrm{E}-05$
$1.0 \mathrm{E}-04$
$4.1 \mathrm{E}-05$
$3.2 \mathrm{E}-04$
$1.6 \mathrm{E}-02$
$2.1 \mathrm{E}-01$
$2.1 \mathrm{E}-03$
$1.5 \mathrm{E}-02$
$6.1 \mathrm{E}-04$
$1.8 \mathrm{E}-01$
$1.2 \mathrm{E}-02$
$3.1 \mathrm{E}-01$
$6.8 \mathrm{E}-02$
$3.4 \mathrm{E}-01$
$3.5 \mathrm{E}-03$




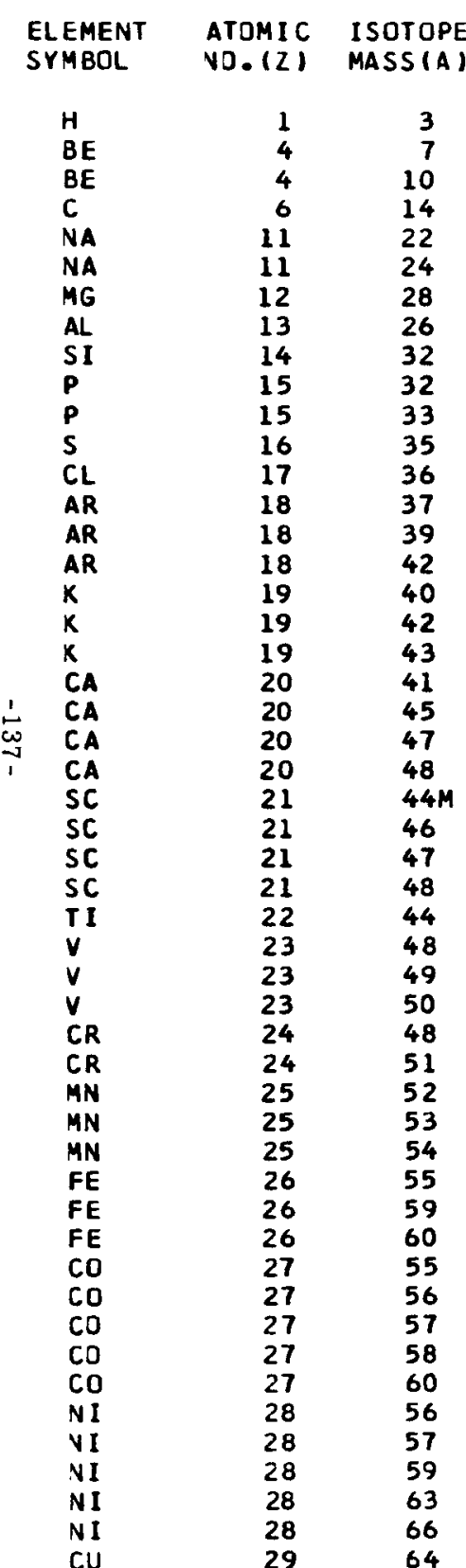

\begin{tabular}{|c|c|}
\hline $\begin{array}{l}\text { ADULT } \\
U C / M * * 3\end{array}$ & $\begin{array}{l}\text { INFANT } \\
U C / M * * 3\end{array}$ \\
\hline $\begin{array}{l}\text { 6. } 4 E+02 \\
1.7 E+00 \\
2.1 E-03 \\
3.4 E-03 \\
3.1 E+01 \\
4.0 E+05 \\
4.9 E+03 \\
2.7 E-02 \\
3.1 E-03 \\
7.7 E-02 \\
3.8 E-01 \\
1.9 E+03 \\
4.4 E+01 \\
8.2 E+02 \\
4.4 E-02 \\
4.7 E-03 \\
3.2 E-01 \\
1.6 E+05 \\
9.3 E+04 \\
1.3 E+03 \\
2.7 E+03 \\
1.9 E+04 \\
1.3 E+00 \\
4.3 E-03 \\
1.7 E-04 \\
2.8 E-02 \\
4.6 E-03 \\
1.4 E-03 \\
1.4 E+01 \\
4.1 E+02 \\
7.1 E-02 \\
2.9 E+00 \\
1.0 E+01 \\
7.2 E-01 \\
5.8 E-02 \\
1.1 E-02 \\
5.2 E-01 \\
2.9 E-01 \\
1.4 E-04 \\
8.7 E+00 \\
3.9 E-03 \\
1.4 E-02 \\
9.0 E-03 \\
9.9 E-05 \\
3.4 E+00 \\
2.6 E+01 \\
1.4 E+00 \\
6.5 E-0 I \\
1.9 E+01 \\
8.3 E+02\end{array}$ & $\begin{array}{l}6.4 E+02 \\
1.7 E+00 \\
2.1 E-03 \\
3.4 E-03 \\
3.0 E+01 \\
2.2 E+04 \\
4.9 E+03 \\
2.7 E-02 \\
3.0 E-03 \\
3.4 E-02 \\
2.2 E-01 \\
2.4 E+02 \\
4.4 E+01 \\
8.2 E+02 \\
4.4 E-02 \\
4.7 E-03 \\
3.2 E-01 \\
1.4 E+03 \\
1.5 E+03 \\
1.3 E+03 \\
2.4 E+03 \\
4.0 E+03 \\
1.3 E+00 \\
4.3 E-03 \\
1.7 E-04 \\
2.8 E-02 \\
4.6 E-03 \\
1.4 E-03 \\
1.4 E+01 \\
4.1 E+02 \\
1.1 E-02 \\
2.9 E+00 \\
1.0 E+01 \\
1.3 E-01 \\
5.7 E-02 \\
1.0 E-02 \\
4.2 E-01 \\
4.6 E-02 \\
1.3 E-04 \\
2.6 E-01 \\
3.0 E-03 \\
1.3 E-02 \\
6.7 E-03 \\
9.8 E-05 \\
3.4 E+00 \\
2.6 E+01 \\
1.4 E+00 \\
6.5 E-01 \\
1.9 E+01 \\
4.3 E+01\end{array}$ \\
\hline
\end{tabular}

ELEME
$S Y M B O$

$C U$
$Z N$
$Z N$
$Z N$
$G A$
$G A$
$G E$
$G E$
$G E$
$A S$
$A S$
$A S$
$A S$
$A S$
$A S$
$S E$
$S E$
$S E$
$B R$
$B R$
$B R$
$K R$
$K R$
$K R$
$K R$
$R E$
$R B$
$R B$
$R B$
$S R$
$S R$
$S R$
$S R$
$S R$
$Y$
$Y$
$Y$
$Y$
$Y$
$Y$
$Z R$
$Z R$
$Z R$
$Z R$
$Z R$
$Z R$
$N B$
$N B$
$N B$
$N B$

$\begin{array}{ll}\text { ATOMIC } & \text { ISOTOPE } \\ \text { NO.IZ) } & \text { MASS(A) }\end{array}$

AOULT
UC $/ M * * 3$

INFANT

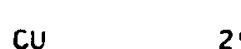

30

67
65

65

$69 M$
72
67

7

68

69

71

5. $2 E+01$ 3. $9 E-03$

$2.2 E+02$

3. $8 E+00$ 6. $8 E+01$ 2. $4 E+01$

1. $8 E-05$

2. $6 E-03$

7. $1 E-02$

3. $0 E+02$

$2 E+03$

3. $1 E+01$

4. $1 E+02$

2. $1 E+03$

2. $3 E-02$

8. $7 E-03$

1. $7 E-03$

1. $3 E+06$

$2.6 E+05$

2. $3 E+05$

1. $5 E+02$

1. $9 E+02$

2. 5E-O

3. $7 E+00$

5. $0 E+00$

2. $4 E+01$

1. $4 E-01$

$2.2 E+02$

1. $1 E+05$

4. $2 E+02$

3. $9 E+02$

1. $3 E+00$

2. $3 E+03$

3. $7 E+02$

8. $4 E+03$

1. $1 E-01$

3. $7 E+02$

1. $3 E+00$

1. $8 E+03$

2. $O E-01$

2. $1 E+02$

3. $7 E-02$

. 2E-01

2. $6 E+03$

4. $7 \mathrm{E}-02$

1. $1 E-03$

1. $0 E-02$

UC $/ N * * 3$

$1.1 E+01$

1. $8 E-03$

4. $7 E-01$

. $8 E+01$

2. $4 E+01$

$2.4 E+01$
$1.8 E-05$

$2.6 E-03$

7. $1 E-02$

5. $9 E+02$

$3.0 E+02$

. $2 E+03$

$3.1 E+01$

$4.1 E+02$

2. $3 E-02$

8. $7 \mathrm{E}-03$

1. $7 E-03$

1. $0 E+05$

6. $O E+04$

$3.6 E+04$

$1.5 \mathrm{E}+02$

- $9 E+02$

$1.3 E+00$

2.5E-01

1. $9 E+00$

$1.5 E+00$

4. $6 E+00$

1. $4 E-01$

$1.2 E+02$

7. $1 E+03$

$3.2 E+02$

2. $8 E+02$

- $3 E+00$

1. $7 E-01$

1.5E-01

5. $9 E-01$

1. 5E-03

1 . $2 E-01$

9. $0 E-03$

1. $6 E+00$

1. $9 i-02$

1. $=L-02$

- $3 \mathrm{E}-01$

3. $3 E-02$

C. $7 E-02$

$2.3 E+00$

4.7E-02

1. $O E-02$

1. $5 E-02$ 


\begin{tabular}{|c|c|c|c|c|}
\hline $\begin{array}{l}\text { ELEMENT } \\
\text { SYMBOL }\end{array}$ & $\begin{array}{l}\text { ATOMIC } \\
\text { NO. }(Z)\end{array}$ & $\begin{array}{l}\text { ISOTDPE } \\
\text { MASSIA) }\end{array}$ & $\begin{array}{c}\text { ADULT } \\
\text { UC / } / M * * 3\end{array}$ & $\begin{array}{l}\text { INFANT } \\
\text { UC } / M * * 3\end{array}$ \\
\hline $\begin{array}{l}N B \\
N B \\
N B \\
N B \\
N B \\
M D \\
M O \\
\text { TC } \\
T C \\
T C \\
T C \\
T C \\
T C \\
T C \\
T C \\
R U \\
R U \\
R U \\
R H \\
R H \\
R H \\
R H \\
R H \\
R H \\
R H \\
R H \\
P D \\
P D \\
P D \\
P D \\
P D \\
A G \\
A G \\
A G \\
A G \\
A G \\
C D \\
C D \\
C D \\
C D \\
C D \\
I N \\
I N \\
I N \\
S N \\
S N \\
S N \\
S N \\
S N \\
S N \\
S N \\
S N \\
S B \\
S B\end{array}$ & $\begin{array}{l}41 \\
41 \\
41 \\
41 \\
41 \\
42 \\
42 \\
43 \\
43 \\
43 \\
43 \\
43 \\
43 \\
43 \\
44 \\
44 \\
44 \\
45 \\
45 \\
45 \\
45 \\
45 \\
45 \\
46 \\
46 \\
46 \\
46 \\
46 \\
47 \\
47 \\
47 \\
47 \\
47 \\
48 \\
48 \\
48 \\
48 \\
49 \\
49 \\
49 \\
50 \\
50 \\
50 \\
50 \\
50 \\
50 \\
50 \\
50 \\
51 \\
51\end{array}$ & $\begin{array}{l}93 M \\
94 \\
95 \\
95 M \\
96 \\
93 \\
99 \\
95 \\
95 M \\
96 \\
97 \\
97 M \\
98 \\
99 \\
97 \\
103 \\
106 \\
99 \\
100 \\
101 \\
101 M \\
102 \\
105 \\
100 \\
103 \\
107 \\
109 \\
112 \\
105 \\
106 M \\
108 M \\
110 M \\
111 \\
109 \\
113 M \\
115 \\
115 M \\
111 \\
114 M \\
115 \\
113 \\
117 M \\
119 M \\
121 \\
121 M \\
123 M \\
125 \\
126 \\
119 \\
120 M\end{array}$ & $\begin{array}{l}\text { 3. } 9 \mathrm{E}-03 \\
1.1 \mathrm{E}-05 \\
5.3 \mathrm{E}-03 \\
3.9 \mathrm{E}-02 \\
5.7 \mathrm{E}-02 \\
1.6 \mathrm{E}+01 \\
7.5 \mathrm{E}+03 \\
2.0 \mathrm{E}+00 \\
2.1 \mathrm{E}-02 \\
1.3 \mathrm{E}-01 \\
8.9 \mathrm{E}-03 \\
1.3 \mathrm{E}-01 \\
1.2 \mathrm{E}-04 \\
2.0 \mathrm{E}-03 \\
6.1 \mathrm{E}+01 \\
3.9 \mathrm{E}-01 \\
2.8 \mathrm{E}-03 \\
1.7 \mathrm{E}-02 \\
9.9 \mathrm{E}-02 \\
2.3 \mathrm{E}-04 \\
8.9 \mathrm{E}-02 \\
3.8 \mathrm{E}-04 \\
3.7 \mathrm{E}-01 \\
2.2 \mathrm{E}-02 \\
1.2 \mathrm{E}-01 \\
1.5 \mathrm{E}-02 \\
4.4 \mathrm{E}-01 \\
4.9 \mathrm{E}-02 \\
3.6 \mathrm{E}+00 \\
1.1 \mathrm{E}+01 \\
3.0 \mathrm{E}-02 \\
1.9 \mathrm{E}-01 \\
7.5 \mathrm{E}+01 \\
1.4 \mathrm{E}-03 \\
8.7 \mathrm{E}-05 \\
2.5 \mathrm{E}-02 \\
1.6 \mathrm{E}-03 \\
1.1 \mathrm{E}-04 \\
2.1 \mathrm{E}-00 \\
8.1 \mathrm{E}-08 \\
3.4 \mathrm{E}+00 \\
1.3 \mathrm{E}+02 \\
6.3 \mathrm{E}+00 \\
4.5 \mathrm{E}+04 \\
1.6 \mathrm{E}-01 \\
1.9 \mathrm{E}+00 \\
4.7 \mathrm{E}+01 \\
4.5 \mathrm{E}-03 \\
1.2 \mathrm{E}+00 \\
6.6 \mathrm{E}-03\end{array}$ & $\begin{array}{l}3.9 E-03 \\
1.1 \mathrm{E}-05 \\
5.3 \mathrm{E}-03 \\
3.9 \mathrm{E}-02 \\
5.7 \mathrm{E}-02 \\
1.6 \mathrm{E}+01 \\
9.9 \mathrm{E}+02 \\
2.0 \mathrm{E}+00 \\
2.1 \mathrm{E}-02 \\
1.3 \mathrm{E}-01 \\
8.9 \mathrm{E}-03 \\
1.3 \mathrm{E}-01 \\
1.2 \mathrm{E}-04 \\
2.0 \mathrm{E}-03 \\
9.6 \mathrm{E}-01 \\
7.1 \mathrm{E}-02 \\
1.9 \mathrm{E}-03 \\
1.7 \mathrm{E}-02 \\
9.9 \mathrm{E}-02 \\
2.3 \mathrm{E}-04 \\
8.9 \mathrm{E}-02 \\
3.8 \mathrm{E}-04 \\
3.7 \mathrm{E}-01 \\
2.2 \mathrm{E}-02 \\
1.2 \mathrm{E}-01 \\
1.5 \mathrm{E}-02 \\
4.4 \mathrm{E}-01 \\
4.9 \mathrm{E}-02 \\
3.0 \mathrm{E}+00 \\
6.2 \mathrm{E}+00 \\
3.0 \mathrm{E}-02 \\
1.9 \mathrm{E}-01 \\
3.9 \mathrm{E}+01 \\
1.4 \mathrm{E}-03 \\
8.7 \mathrm{E}-05 \\
2.5 \mathrm{E}-02 \\
1.6 \mathrm{E}-03 \\
1.1 \mathrm{E}-04 \\
2.1 \mathrm{E}-05 \\
8.1 \mathrm{E}-08 \\
2.3 \mathrm{E}+00 \\
2.5 \mathrm{E}+01 \\
5.1 \mathrm{E}+00 \\
8.3 \mathrm{E}+02 \\
1.6 \mathrm{E}-01 \\
1.3 \mathrm{E}+00 \\
6.3 \mathrm{E}+00 \\
4.5 \mathrm{E}-03 \\
1.2 \mathrm{E}+00 \\
6.6 \mathrm{E}-03\end{array}$ \\
\hline
\end{tabular}

\begin{tabular}{|c|c|c|}
\hline $\begin{array}{l}\text { ELEMENT } \\
\text { SYMBOL }\end{array}$ & $\begin{array}{l}\text { ATOMIC } \\
\text { NO. }(Z)\end{array}$ & $\begin{array}{l}\text { ISOTOPE } \\
\text { MASS(A) }\end{array}$ \\
\hline$S B$ & 51 & 122 \\
\hline SB & 51 & 124 \\
\hline SB & 51 & 125 \\
\hline 58 & 51 & 126 \\
\hline SB & 51 & 127 \\
\hline$T E$ & 52 & 118 \\
\hline TE & 52 & 119 \\
\hline TE & 52 & $119 M$ \\
\hline$T E$ & 52 & 121 \\
\hline$T E$ & 52 & $121 M$ \\
\hline TE & 52 & 123 \\
\hline TE & 52 & $123 M$ \\
\hline TE & 52 & $125 M$ \\
\hline TE & 52 & $127 M$ \\
\hline$T E$ & 52 & $129 \mathrm{M}$ \\
\hline TE & 52 & $131 M$ \\
\hline TE & 52 & 132 \\
\hline I & 53 & 123 \\
\hline I & 53 & 124 \\
\hline I & 53 & 125 \\
\hline I & 53 & 126 \\
\hline I & 53 & 129 \\
\hline I & 53 & 130 \\
\hline$I$ & 53 & 131 \\
\hline I & 53 & 133 \\
\hline$X E$ & 54 & 122 \\
\hline$X E$ & 54 & 125 \\
\hline$X E$ & 54 & 127 \\
\hline$X E$ & 54 & $129 M$ \\
\hline$X E$ & 54 & $131 M$ \\
\hline$X E$ & 54 & 133 \\
\hline$X E$ & 54 & $133 M$ \\
\hline CS & 55 & 129 \\
\hline CS & 55 & 131 \\
\hline CS & 55 & 132 \\
\hline cS & 55 & 134 \\
\hline CS & 55 & 135 \\
\hline CS & 55 & 136 \\
\hline CS & 55 & 137 \\
\hline$B A$ & 56 & 128 \\
\hline BA & 56 & 131 \\
\hline$B A$ & 56 & 133 \\
\hline BA & 56 & $133 M$ \\
\hline EA & 56 & $135 \mathrm{M}$ \\
\hline BA & 56 & 140 \\
\hline LA & 57 & 135 \\
\hline LA & 57 & 137 \\
\hline LA & 57 & 138 \\
\hline LA & 57 & 140 \\
\hline CE & 58 & 134 \\
\hline
\end{tabular}

ADULT
UC $1 M * * 3$

INFANT

$.9 E-03$

$1 E-05$
$3 E-03$

$9 E-02$

$6 E+01$

$9 E+02$

$3 \mathrm{E}-\mathrm{J} 1$

. $3 E-01$

OE-O3

. $1 E-01$

$.9 E-03$

. $9 E-02$

$3 E-04$

$8 \mathrm{E}-04$

$.7 E-01$
$.2 E-02$

. 2E-0 1

. $4 E-01$

政 02

$2 E+00$

$9 E-01$

$4 E-03$

.7E-05

$1 E-04$

. $1 E-08$

$5 E+01$

作

. $6 E-01$

$6.6 E-03$
3. $O E-02$

6. $7 E-04$

1. $8 E-04$

2. $8 E-03$

4. $3 \mathrm{E}-02$

4. $3 E-02$

4. 3E- 02

1. $6 E-02$

5. $4 E-04$

2. $6 E-04$

2. $7 E-03$

1. $1 E-02$

2. IE-03

3. $7 E-03$

5. $2 E-01$

6. $1 E-02$

1. $6 E+02$

3. $8 E-01$

3. $7 E-01$

3. $0 E-01$

1. $5 \mathrm{E}+01$

5. $2 E-01$

1. $1 E+01$

2. $1 E+02$

1. $1 E+03$

5. $3 E+01$

$2.4 E+02$

$2.3 E+02$

4. $6 E+02$

4. $7 E+02$

1. $7 E+03$

5. $9 E+02$

6. $3 E+01$

1. $2 E-02$

3. $9 E-02$

$5.0 E+O O$

3. $7 E-03$
7. $6 E+02$

1. $6 E+02$

1. $3 E+00$

2. $5 E+04$

4. $1 E+01$

2. 5E-0 1

6. $4 E-04$

1. $8 E-05$

3. $4 E-02$

1. $2 \mathrm{E}+01$
3. $0 \mathrm{E}-02$

6. $7 E-04$

1. $8 E-04$

2. $8 E-03$

2. $0 \mathrm{E}-02$

$7.2 \mathrm{E}-03$

4. $7 \mathrm{E}-02$

5. $9 E-03$

5. $9 E-03$

4. $5 \mathrm{E}-04$

2.6E-04

2. $1 \mathrm{E}-03$

7. $2 E-03$

1. $7 \mathrm{E}-03$

1. $9 E-03$

5. $9 E-03$

1. $1 E+0 I$

1. $4 \mathrm{E}-01$

3. $4 E-01$

2. $0 E-01$

$2.3 E-03$

1. $0 E+00$

2. $8 E-01$

$1.2 \mathrm{E}+00$

2. IE+02

1. $1 E+03$

$5.3 E+01$

$2.4 E+02$

$2.3 E+02$

4. $6 E+02$

4. $7 E+02$

2. $0 E+01$

4. $7 \mathrm{E}+01$

3. $5 \mathrm{E}+00$

1. $1 \mathrm{E}-02$

3. $9 E-02$

5. $3 \mathrm{E}-01$

3. $6 E-03$

2. $1 E+02$

1. $\mathrm{OE}+02$

1. $3 E+00$

1. $2 E+03$

4. $O E+03$

2. $7 E+01$

$2.5 E-01$

$6.4 \mathrm{E}-04$

6. $4 E-04$
1. $8 E-05$

1. $4 E-05$

3. $4 \mathrm{E}-02$
1. $0 \mathrm{E}-01$ 
TABLE 9A. UNIT-RAD LONTAMINATI ON FOR TESTES

$\begin{array}{ccccc}\text { ELEMENT } & \text { ATOMIC } & \text { ISOTOPE } & \text { ADULT } & \text { INFANT } \\ \text { SYMBOL } & \text { V. } 121 & \text { MASS1A } & \text { UC/M**3 } & \text { UC/M**3 } \\ \text { CE } & 58 & 135 & 2.8 E+02 & 6.1 E-01 \\ \text { CE } & 58 & 137 M & 2.0 E+02 & 8.4 E-01 \\ \text { CE } & 58 & 139 & 5.2 E-02 & 1.5 E-02 \\ \text { CE } & 58 & 141 & 5.9 E-01 & 5.2 E-02 \\ \text { CE } & 58 & 142 & 5.2 E-05 & 5.0 E-05 \\ \text { CE } & 58 & 143 & 6.5 E+01 & 2.6 E-01 \\ \text { CE } & 58 & 144 & 8.9 E-04 & 4.1 E-04 \\ \text { PR } & 59 & 142 & 4.3 E-05 & 4.3 E-05 \\ \text { PR } & 59 & 143 & 8.0 E-06 & 8.0 E-06 \\ \text { ND } & 60 & 140 & 1.1 E-05 & 1.1 E-05 \\ \text { ND } & 60 & 144 & 3.2 E-09 & 3.2 E-09 \\ \text { ND } & 60 & 147 & 1.4 E-06 & 1.4 E-06 \\ \text { PM } & 61 & 143 & 1.1 E-02 & 4.3 E-03 \\ \text { PM } & 61 & 144 & 8.8 E-04 & 4.2 E-04 \\ \text { PM } & 61 & 145 & 1.8 E-03 & 1.7 E-03 \\ \text { PM } & 61 & 146 & 3.9 E-04 & 3.1 E-04 \\ \text { PM } & 61 & 147 & 2.7 E-04 & 1.9 E-04 \\ \text { PM } & 61 & 148 & 3.8 E+00 & 5.1 E-02 \\ \text { PM } & 61 & 148 M & 2.0 E-02 & 1.8 E-03 \\ \text { PM } & 61 & 149 & 8.0 E+01 & 4.4 E-01 \\ \text { PM } & 61 & 151 & 1.1 E+02 & 3.4 E-01 \\ \text { SM } & 62 & 141 & 1.4 E+00 & 6.6 E-02 \\ \text { SM } & 62 & 145 & 2.2 E-01 & 1.0 E-01 \\ \text { SM } & 62 & 146 & 3.0 E-04 & 2.8 E-04 \\ \text { SM } & 62 & 147 & 3.4 E-04 & 3.2 E-04 \\ \text { SM } & 62 & 148 & 1.9 E-04 & 1.8 E-04 \\ \text { SM } & 62 & 149 & 4.1 E-04 & 3.9 E-04 \\ \text { SM } & 62 & 151 & 5.6 E-02 & 5.2 E-02 \\ \text { SM } & 62 & 153 & 1.6 E+03 & 7.8 E+00 \\ \text { EU } & 63 & 145 & 1.2 E+02 & 1.8 E+00 \\ \text { EU } & 63 & 146 & 3.1 E+01 & 3.7 E-01 \\ \text { EU } & 63 & 146 M & 3.8 E+02 & 1.6 E+00 \\ \text { EU } & 63 & 147 & 1.8 E+00 & 1.1 E-01 \\ \text { EU } & 63 & 148 & 2.1 E+00 & 2.6 E-01 \\ \text { EU } & 63 & 149 & 6.0 E-01 & 1.3 E-01 \\ \text { EU } & 63 & 150 & 5.2 E-03 & 4.3 E-03 \\ \text { EU } & 63 & 150 M & 2.5 E+03 & 3.5 E+00 \\ \text { EU } & 63 & 152 & 1.9 E-03 & 1.7 E-03 \\ \text { EU } & 63 & 154 & 1.4 E-03 & 1.3 E-03 \\ \text { EU } & 63 & 155 & 1.7 E-01 & 1.1 E-01 \\ \text { EU } & 63 & 156 & 8.1 E+00 & 3.1 E-01 \\ \text { EU } & 63 & 157 & 6.7 E+03 & 1.1 E+01 \\ \text { GD } & 64 & 146 & 9.6 E-03 & 1.2 E-03 \\ \text { GD } & 64 & 147 & 2.1 E+01 & 6.7 E-02 \\ \text { GD } & 64 & 148 & 1.6 E-05 & 1.5 E-05 \\ \text { GD } & 64 & 149 & 1.1 E+00 & 3.0 E-02 \\ \text { GD } & 64 & 150 & 8.5 E-06 & 8.2 E-06 \\ \text { GD } & 64 & 151 & 2.9 E-02 & 7.7 E-03 \\ \text { GD } & 64 & 152 & 2.1 E-05 & 2.0 E-05 \\ \text { GD } & 64 & 153 & 2.1 E-02 & 8.9 E-03\end{array}$

VIA SEAHATER.

$\begin{array}{lcc}\text { ELEMENT } & \text { ATOMIC } & \text { ISOTOP } \\ \text { SYMBOL } & \text { NO. }(Z) & \text { MASS(A } \\ \text { GO } & & \\ \text { GD } & 64 & 159 \\ \text { TB } & 64 & 152 \\ \text { TB } & 65 & 151 \\ \text { TB } & 65 & 152 \\ \text { TB } & 65 & 153 \\ \text { TE } & 65 & 154 \\ \text { TB } & 65 & 155 \\ \text { TB } & 65 & 156 \\ \text { TB } & 65 & 157 \\ \text { TB } & 65 & 158 \\ \text { TB } & 65 & 160 \\ \text { TE } & 65 & 161 \\ \text { DY } & 65 & 164 \\ \text { DY } & 66 & 154 \\ \text { DY } & 66 & 154 M \\ \text { DY } & 66 & 156 \\ \text { DY } & 66 & 159 \\ \text { HO } & 66 & 166 \\ \text { HC } & 67 & 163 \\ \text { HO } & 67 & 166 \\ \text { ER } & 67 & 166 M \\ \text { ER } & 68 & 160 \\ \text { ER } & 68 & 169 \\ \text { TM } & 68 & 172 \\ \text { TM } & 69 & 165 \\ \text { TM } & 69 & 167 \\ \text { TM } & 69 & 168 \\ \text { TM } & 69 & 170 \\ \text { TM } & 69 & 171 \\ \text { YB } & 69 & 172 \\ \text { YB } & 70 & 166 \\ \text { YB } & 70 & 169 \\ \text { LU } & 70 & 175 \\ \text { LU } & 71 & 169 \\ \text { LU } & 71 & 170 \\ \text { LU } & 71 & 171 \\ \text { LU } & 71 & 172 \\ \text { LU } & 71 & 173 \\ \text { LU } & 71 & 174 \\ \text { LU } & 71 & 174 M \\ \text { LU } & 71 & 176 \\ \text { LU } & 71 & 177 \\ \text { HF } & 71 & 177 M \\ \text { HF } & 72 & 170 \\ \text { HF } & 72 & 171 \\ \text { HF } & 72 & 172 \\ \text { HF } & 72 & 173 \\ \text { HF } & 72 & 174 \\ \text { HF } & 72 & 175 \\ & 72 & 181\end{array}$

$10 / 09 / 68$

PAGE 28

INFAN T

$U C / M * * 3$

UC $/ M * * 3$

4. $7 E+02$

1. $1 E-03$

3. $8 E+02$

1. $1 E+02$

7. $2 E+01$

4. $1 E+02$

1. $7 E+01$

$1.6 E-03$

7. $0 E-05$

3. $6 \mathrm{E}-02$

2. $4 E+01$

$5.5 E+02$

5. $9 E-10$

2. $0 E-05$

9. 2E-10

3. 8E-0 6

9. $4 E-06$

9. $5 E-08$

3. $7 E-05$

4. 2E-09

1. 2E-05

4. $6 E-05$

1. $6 E-05$

2. $9 E+01$

1. 2E-02

2. $4 E-02$

3. $1 E-02$

1. $6 E+01$

4. $4 E+01$

3. $8 E-01$

4. $4 E+01$

1. $4 E-05$

6. $4 E-06$
$3.4 E-05$

3. $4 E-05$

6. $0 E-06$

2. $4 E-08$

4. $4 E-07$

7. 1E-09

3. $4 E-05$

1. 2E-06

1. $2 E+02$

2. $4 E+02$

1. $5 E-04$

7. $5 E+01$

1. $3 E-05$

6. $1 E-02$

1. $1 \mathrm{E}+00$

6. $0 E-04$

7. $1 E-01$

2.1E-01

4. $6 \mathrm{E}-01$

8. $8 E-01$

$6.8 E-02$

1. 5E-03

6. $6 E-05$

$5.5 E-03$

4. $O E-01$

1. $4 E+00$

5. $9 E-10$

2. $0 E-05$

$9.2 \mathrm{E}-10$

3. 8E-06

9. $4 E-06$

$9.5 E-08$

.7E-05

4.2E-09 बㅎ

1.2E-05
$4.6 E-05$

$1.6 E-05$

$1.6 E-05$ D

8.6E-02

$2.1 E-03$

$5.8 E-03$

2. $0 E-02$

$1.1 E-01$

$2.6 E-01$

.8E-02

$4.4 \mathrm{E}-01$

$1.4 E-05$

$6.4 E-06$
$3.4 E-05$

3. $4 \mathrm{E}-05$

6. $0 E-06$

$1.1 E-07$

$2.4 E-08$

4. $4 \mathrm{E}-07$

7.1E-09

$3.4 E-05$

$1.2 E-06$

$1.2 E-06$

2. $5 \mathrm{E}-01$

$1.1 E-04$

$1.3 E-01$

1. $2 E-05$

6. $8 E-03$ 


\begin{tabular}{|c|c|c|c|c|}
\hline $\begin{array}{l}\text { ELEMENT } \\
\text { SYMBOL }\end{array}$ & $\begin{array}{l}\text { ATOMIC } \\
\text { NO. }(Z)\end{array}$ & $\begin{array}{l}\text { ISOTOPE } \\
\text { MASSIA) }\end{array}$ & $\begin{array}{l}\text { ADULLT } \\
\text { UC/M**3 }\end{array}$ & $\begin{array}{l}\text { INFANT } \\
\text { UC } / M * * 3\end{array}$ \\
\hline 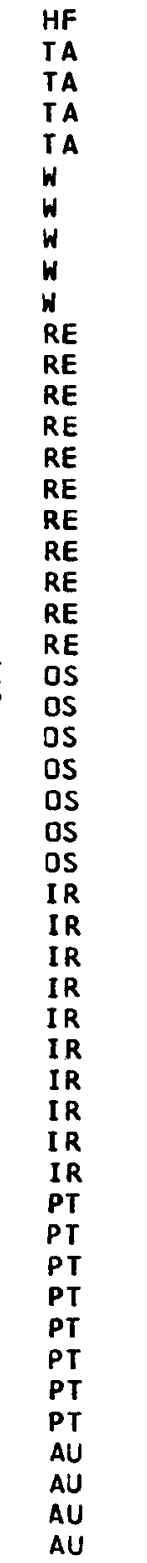 & $\begin{array}{l}72 \\
73 \\
73 \\
73 \\
73 \\
74 \\
74 \\
74 \\
74 \\
74 \\
75 \\
75 \\
75 \\
75 \\
75 \\
75 \\
75 \\
75 \\
75 \\
75 \\
75 \\
76 \\
76 \\
76 \\
76 \\
76 \\
76 \\
76 \\
77 \\
77 \\
77 \\
77 \\
77 \\
77 \\
77 \\
77 \\
77 \\
77 \\
78 \\
78 \\
78 \\
78 \\
78 \\
78 \\
78 \\
78 \\
79 \\
79 \\
79 \\
79\end{array}$ & $\begin{array}{l}182 \\
177 \\
179 \\
182 \\
183 \\
178 \\
181 \\
185 \\
187 \\
188 \\
180 \\
181 \\
182 \\
182 M \\
183 \\
184 \\
184 M \\
186 \\
187 \\
188 \\
189 \\
182 \\
183 \\
185 \\
191 \\
191 M \\
193 \\
194 \\
185 \\
186 \\
187 \\
188 \\
189 \\
190 \\
192 \\
192 M \\
193 M \\
194 \\
188 \\
190 \\
191 \\
192 \\
193 \\
193 M \\
195 M \\
197 \\
193 \\
194 \\
195 \\
196\end{array}$ & $\begin{array}{l}\text { 2. } 0 \mathrm{E}-05 \\
2.1 \mathrm{E}-01 \\
1.3 \mathrm{E}-04 \\
\text { 7. } 9 \mathrm{E}-05 \\
7.9 \mathrm{E}-02 \\
3.6 \mathrm{E}-04 \\
1.5 \mathrm{E}-03 \\
1.5 \mathrm{E}-03 \\
1.6 \mathrm{E}-02 \\
1.5 \mathrm{E}-04 \\
4.4 \mathrm{E}-01 \\
4.8 \mathrm{E}-01 \\
1.7 \mathrm{E}-01 \\
8.2 \mathrm{E}-01 \\
2.9 \mathrm{E}-02 \\
1.8 \mathrm{E}-02 \\
3.2 \mathrm{E}-03 \\
4.0 \mathrm{E}-01 \\
6.8 \mathrm{E}-01 \\
8.9 \mathrm{E}-01 \\
1.4 \mathrm{E}+00 \\
1.2 \mathrm{E}-02 \\
5.2 \mathrm{E}-02 \\
3.3 \mathrm{E}-04 \\
7.8 \mathrm{E}-03 \\
1.5 \mathrm{E}-01 \\
3.7 \mathrm{E}-02 \\
5.1 \mathrm{E}-06 \\
2.0 \mathrm{E}-01 \\
1.2 \mathrm{E}-01 \\
2.5 \mathrm{E}-01 \\
3.5 \mathrm{E}-02 \\
2.9 \mathrm{E}-02 \\
8.0 \mathrm{E}-03 \\
1.8 \mathrm{E}-03 \\
1.5 \mathrm{E}-05 \\
1.4 \mathrm{E}-01 \\
1.4 \mathrm{E}-01 \\
1.5 \mathrm{E}-04 \\
1.7 \mathrm{E}-07 \\
2.6 \mathrm{E}-03 \\
2.1 \mathrm{E}-07 \\
7.3 \mathrm{E}-06 \\
4.2 \mathrm{E}-03 \\
3.9 \mathrm{E}-03 \\
1.7 \mathrm{E}-02 \\
2.1 \mathrm{E}+00 \\
2.7 \mathrm{E}-01 \\
2.4 \mathrm{E}-02 \\
2.0 \mathrm{E}-01\end{array}$ & $\begin{array}{l}1.9 \mathrm{E}-05 \\
1.0 \mathrm{E}-03 \\
7.1 \mathrm{E}-05 \\
1.6 \mathrm{E}-05 \\
8.4 \mathrm{E}-04 \\
3.6 \mathrm{E}-04 \\
1.5 \mathrm{E}-03 \\
1.5 \mathrm{E}-03 \\
1.6 \mathrm{E}-02 \\
1.5 \mathrm{E}-04 \\
4.4 \mathrm{E}-01 \\
4.8 \mathrm{E}-01 \\
1.7 \mathrm{E}-01 \\
8.2 \mathrm{E}-01 \\
2.9 \mathrm{E}-02 \\
1.8 \mathrm{E}-02 \\
3.2 \mathrm{E}-03 \\
4.0 \mathrm{E}-01 \\
6.8 \mathrm{E}-01 \\
8.9 \mathrm{E}-01 \\
1.4 \mathrm{E}+00 \\
1.2 \mathrm{E}-02 \\
5.2 \mathrm{E}-02 \\
3.3 \mathrm{E}-04 \\
7.8 \mathrm{E}-03 \\
1.5 \mathrm{E}-01 \\
3.7 \mathrm{E}-02 \\
5.1 \mathrm{E}-06 \\
2.0 \mathrm{E}-01 \\
1.2 \mathrm{E}-01 \\
2.5 \mathrm{E}-01 \\
3.5 \mathrm{E}-02 \\
2.9 \mathrm{E}-02 \\
8.0 \mathrm{E}-03 \\
1.8 \mathrm{E}-03 \\
1.5 \mathrm{E}-05 \\
1.4 \mathrm{E}-01 \\
1.4 \mathrm{E}-01 \\
1.5 \mathrm{E}-04 \\
1.7 \mathrm{E}-07 \\
2.6 \mathrm{E}-03 \\
2.1 \mathrm{E}-07 \\
7.3 \mathrm{E}-05 \\
4.2 \mathrm{E}-03 \\
3.9 \mathrm{E}-03 \\
1.7 \mathrm{E}-02 \\
2.1 \mathrm{E}+00 \\
2.7 \mathrm{E}-01 \\
2.4 \mathrm{E}-02 \\
2.0 \mathrm{E}-01\end{array}$ \\
\hline
\end{tabular}

$\begin{array}{lcc}\text { ELEMENT } & \text { ATOMIC } & \text { I SOTCPE } \\ \text { SYMEOL } & \text { NO. }(Z) & \text { MASS (A) } \\ & & \\ \text { AU } & 79 & 198 \\ \text { AU } & 79 & 199 \\ \text { HG } & 80 & 194 \\ \text { HG } & 80 & 195 M \\ \text { HG } & 80 & 197 \\ \text { HG } & 80 & 197 M \\ \text { HG } & 80 & 203 \\ \text { TL } & 81 & 200 \\ \text { TL } & 81 & 201 \\ \text { TL } & 81 & 202 \\ \text { TL } & 81 & 204 \\ \text { PB } & 82 & 200 \\ \text { PB } & 82 & 202 \\ \text { PB } & 82 & 203 \\ \text { PE } & 82 & 204 \\ \text { PB } & 82 & 205 \\ \text { PB } & 82 & 210 \\ \text { BI } & 83 & 205 \\ \text { EI } & 83 & 206 \\ \text { BI } & 83 & 207 \\ \text { BI } & 83 & 208 \\ \text { BI } & 83 & 210 \\ \text { BI } & 83 & 210 M \\ \text { PO } & 84 & 206 \\ \text { PO } & 84 & 208 \\ \text { PO } & 84 & 209 \\ \text { PO } & 84 & 210 \\ \text { RN } & 86 & 211 \\ \text { RN } & 86 & 222 \\ \text { RA } & 88 & 223 \\ \text { RA } & 88 & 224 \\ \text { RA } & 88 & 225 \\ \text { RA } & 88 & 226 \\ \text { RA } & 88 & 228 \\ \text { AC } & 89 & 225 \\ \text { AC } & 89 & 227 \\ \text { TH } & 90 & 227 \\ \text { TH } & 90 & 228 \\ \text { IH } & 90 & 229 \\ \text { TH } & 90 & 230 \\ \text { TH } & 90 & 231 \\ \text { TH } & 90 & 232 \\ \text { TH } & 90 & 234 \\ \text { PA } & 91 & 228 \\ \text { PA } & 91 & 229 \\ \text { PA } & 91 & 230 \\ \text { PA } & 91 & 231 \\ \text { PA } & 91 & 232 \\ \text { PA } & 91 & 233 \\ \text { U } & 92 & 230\end{array}$

$\underset{U C, M * * 3}{A D U L T}$

INF ANT

4. $6 E-01$

4.6E-01

$1.3 E+00 \quad 1.3 E+00$

3. $1 E-05 \quad 3.1 E-05$

3. $5 E-03 \quad 3.5 E-03$

$1.0 E-02 \quad 1.0 E-02$

6. $3 E-04 \quad 6.3 E-04$

4. $7 E-03 \quad 4.7 E-03$

9. $7 E-03$ 9.7E-03

1. $2 \mathrm{E}-03 \quad 1.2 \mathrm{E}-03$

1. $7 E-05 \quad 1.7 E-05$

5. $2 E+01 \quad 5.2 E+01$

1. $8 \mathrm{E}-02 \quad 1.8 \mathrm{E}-02$

4. $9 E+01$ 4. $9 E+01$

4. $1 E-03 \quad 4.1 E-03$

1. $2 E-01 \quad 1.2 E-01$

2. $8 \mathrm{E}-03$ 2.8E-03

4. $1 E-04 \quad 4.1 E-04$

1. $1 E-03$ 1.1E-03

1. $2 E-06$

6. $0 E-07$

4. 3E-04

2. $9 E-07$

5. $O E-02$

1.7E-04

4. $0 E-05$

1. $3 E-03$

1. $9 E-01$

9.8E-06

3. $0 E-05$

7. $6 E-06$

1. $4 E-08$

4. $4 \mathrm{E}-08$

7. $3 E-02$

1. $3 E-04$

1. $1 E-06$

2. $8 \mathrm{E}-08$

2. $7 E-09$

2. 5E-09

$1.6 E-05$

2. $2 \mathrm{E}-09$

6. $7 E-07$

$1.1 \mathrm{E}-03$
$1.2 \mathrm{E}-06$

6. $0 E-07$

4. 3E-04

2. $9 E-07$

5. OE-02

1. $7 E-04$

4. $0 \mathrm{E}-05$

$1.8 \mathrm{E}-03$

$3.0 E+00$

1. $9 E-01$

9. 8E-06

3. $0 E-05$

7. $6 \mathrm{E}-06$

$1.4 \mathrm{E}-08$

4. $4 \mathrm{E}-08$

7. $3 E-02$

$1.3 E-04$

$1.1 \mathrm{E}-06$

2. $8 \mathrm{E}-08$

2. $7 E-09$

2. 5 E-09

$1.6 \mathrm{E}-05$

2. $2 \mathrm{E}-09$

6. $2 \mathrm{E}-01$

6. 7E-07

4. 1E-O1

3. $3 E-02$

7. $0 \mathrm{E}-05$

4.1E-OI

3. 3E-02

7. $0 \mathrm{E}-05$

2. $0 E-02$

3. $9 \mathrm{E}-01$

3. $8 \mathrm{E}-07$

3. $8 \mathrm{E}-07$ 
TABLE 9A. UNIT-RAD CONTAMINATION FOR TESTES

VIA SEAWATER.

\begin{tabular}{|c|c|c|c|c|}
\hline $\begin{array}{l}\text { ELEMENT } \\
\text { SYMBOL }\end{array}$ & $\begin{array}{l}\text { ATOMIC } \\
\text { VD. }(Z)\end{array}$ & $\begin{array}{l}\text { ISOTOPE } \\
\text { MASS(A) }\end{array}$ & $\begin{array}{l}\text { ADULT } \\
\text { UC } / M * * 3\end{array}$ & $\begin{array}{l}\text { INFANT } \\
\text { UC } / M * * 3\end{array}$ \\
\hline $\begin{array}{l}U \\
U \\
U \\
U \\
U \\
U \\
U \\
U \\
U \\
N P \\
N P \\
N P \\
N P \\
N P \\
N P \\
N P \\
P U \\
P U \\
P U \\
P U \\
P U \\
P U \\
P U \\
P U \\
P U \\
A M \\
A M \\
A M \\
A M \\
A M \\
A M\end{array}$ & $\begin{array}{l}92 \\
92 \\
92 \\
92 \\
92 \\
92 \\
92 \\
92 \\
92 \\
93 \\
93 \\
93 \\
93 \\
93 \\
93 \\
93 \\
94 \\
94 \\
94 \\
94 \\
94 \\
94 \\
94 \\
94 \\
94 \\
95 \\
95 \\
95 \\
95 \\
95 \\
95\end{array}$ & $\begin{array}{l}231 \\
232 \\
233 \\
234 \\
235 \\
236 \\
237 \\
238 \\
240 \\
234 \\
235 \\
236 \\
236 M \\
237 \\
238 \\
239 \\
236 \\
237 \\
238 \\
239 \\
240 \\
241 \\
242 \\
244 \\
246 \\
239 \\
240 \\
241 \\
242 \\
242 M \\
243\end{array}$ & $\begin{array}{l}1.9 \mathrm{E}-06 \\
1.2 \mathrm{E}-09 \\
1.1 \mathrm{E}-09 \\
1.0 \mathrm{E}-09 \\
9.4 \mathrm{E}-10 \\
9.3 \mathrm{E}-10 \\
1.1 \mathrm{E}-06 \\
8.9 \mathrm{E}-10 \\
1.0 \mathrm{E}-05 \\
1.1 \mathrm{E}-01 \\
1.1 \mathrm{E}-03 \\
5.9 \mathrm{E}-05 \\
5.0 \mathrm{E}-01 \\
6.2 \mathrm{E}-05 \\
2.1 \mathrm{E}-01 \\
1.8 \mathrm{E}-01 \\
4.4 \mathrm{E}-04 \\
1.0 \mathrm{E}-02 \\
6.6 \mathrm{E}-05 \\
5.6 \mathrm{E}-05 \\
5.5 \mathrm{E}-05 \\
1.1 \mathrm{E}-04 \\
5.3 \mathrm{E}-05 \\
4.7 \mathrm{E}-05 \\
3.3 \mathrm{E}-02 \\
8.4 \mathrm{E}-01 \\
1.9 \mathrm{E}-01 \\
5.7 \mathrm{E}-05 \\
5.9 \mathrm{E}-01 \\
5.6 \mathrm{E}-05 \\
5.0 \mathrm{E}-05\end{array}$ & $\begin{array}{l}1.9 \mathrm{E}-05 \\
1.2 \mathrm{E}-09 \\
1.1 \mathrm{E}-07 \\
1.0 \mathrm{E}-09 \\
9.4 \mathrm{E}-10 \\
9.3 \mathrm{E}-10 \\
1.1 \mathrm{E}-06 \\
8.9 \mathrm{E}-10 \\
1.0 \mathrm{E}-05 \\
1.1 \mathrm{E}-01 \\
1.1 \mathrm{E}-03 \\
5.9 \mathrm{E}-05 \\
5.0 \mathrm{E}-01 \\
6.2 \mathrm{E}-05 \\
2.1 \mathrm{E}-01 \\
1.8 \mathrm{E}-01 \\
4.4 \mathrm{E}-04 \\
1.0 \mathrm{E}-02 \\
6.6 \mathrm{E}-05 \\
5.6 \mathrm{E}-05 \\
5.5 \mathrm{E}-05 \\
1.1 \mathrm{E}-04 \\
5.3 \mathrm{E}-05 \\
4.7 \mathrm{E}-05 \\
3.3 \mathrm{E}-02 \\
8.4 \mathrm{E}-01 \\
1.9 \mathrm{E}-01 \\
5.7 \mathrm{E}-05 \\
5.9 \mathrm{E}-01 \\
5.6 \mathrm{E}-05 \\
5.0 \mathrm{E}-05\end{array}$ \\
\hline
\end{tabular}

\begin{tabular}{lcc} 
ELEMENT & ATOMIC & I SOTOPE \\
SYMBOL & NO. $(Z)$ & MASS(A) \\
CM & 96 & 240 \\
CM & 96 & 241 \\
CM & 96 & 242 \\
CM & 96 & 243 \\
CM & 96 & 244 \\
CM & 96 & 245 \\
CM & 96 & 246 \\
CM & 96 & 247 \\
CM & 96 & 248 \\
BK & 97 & 245 \\
BK & 97 & 246 \\
BK & 97 & 247 \\
BK & 97 & 248 \\
BK & 97 & 249 \\
CF & 98 & 246 \\
CF & 98 & 248 \\
CF & 98 & 249 \\
CF & 98 & 250 \\
CF & 98 & 251 \\
CF & 98 & 252 \\
CF & 98 & 253 \\
ES & 99 & 251 \\
ES & 99 & 252 \\
ES & 99 & 253 \\
ES & 99 & 254 \\
ES & 99 & $254 M$ \\
ES & 99 & 255 \\
FM & 100 & 252 \\
FM & 100 & 253 \\
FM & 100 & 255 \\
FM & 100 & 257 \\
\hline & &
\end{tabular}

$10 / 09 / 68$ PAGE 30

AOULT UC/ M**3

INFANT UC $/ M * * 3$

1. $5 E-02$

1. $2 E-02$

2. $4 E-03$

6. $7 E-05$

8. $3 E-05$

5. $0 E-05$

4. $8 \mathrm{E}-05$

4. 5E-05

4. $4 E-05$

7. $6 E-02$

2. OE-OI

4. $5 E-05$

3. $5 \mathrm{E}-01$

1. IE-OS

2. $4 E-01$

$9.6 E-04$

4. $6 E-05$

1. OE-04

4. $1 E-05$

3. $2 E-04$

1. $6 E-02$

2. $1 E-01$

2. IE-03

1. $5 \mathrm{E}-02$

6. $1 E-04$

1. $8 \mathrm{E}-01$

1. $2 E-02$

3. $1 E-01$

6. 8E-02

3. $4 E-01$

3. $5 E-03$

8. 3E-05

5. OE-05

4. 8E-05

4. 5E-05

4. $4 \mathrm{E}-05$

7. $6 \mathrm{E}-02$

2.0E-01

4.5E-05

3. $5 E-01$

$1.1 E-0$

$2.4 E-01$

$9.6 \mathrm{E}-04$

4. $6 E-05$

1. OE-04

4. 1E-05

3. 2E-04

$1.6 \mathrm{E}-02$

2. $1 E-01$

$2.1 E-03$

1. $5 E-02$

6.1E-04

$1.8 E-01$

$1.2 \mathrm{E}-02$

3.1E-OI

6. 8E-02

$3.4 \mathrm{E}-01$

$3.5 E-03$

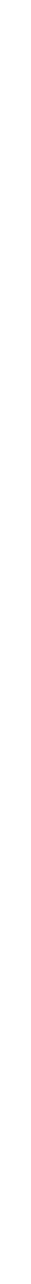


TABLE 9A. UNIT-RAD LOVTAMINATION FOR WHOLE BOOY VIA SEAHATER.

\begin{tabular}{|c|c|c|c|c|}
\hline $\begin{array}{l}\text { ELEMENT } \\
\text { SYMBOL }\end{array}$ & $\begin{array}{l}\text { ATOMIC } \\
\text { VD. (Z) }\end{array}$ & $\begin{array}{l}\text { ISOTOPE } \\
\text { MASS(A) }\end{array}$ & $\begin{array}{c}\text { ADULT } \\
\text { UC } / M * * 3\end{array}$ & $\begin{array}{l}\text { INFANT } \\
U C / M * * 3\end{array}$ \\
\hline $\begin{array}{l}H \\
B E \\
B E \\
C \\
N A \\
N A \\
M G \\
A L \\
S I \\
P \\
P \\
S \\
C L \\
A R \\
A R \\
A R \\
K \\
X \\
K \\
C A \\
C A \\
C A \\
C A \\
S C \\
S C \\
S C \\
S C \\
T I \\
V \\
V \\
V \\
C R \\
C R \\
M N \\
M N \\
M N \\
F E \\
F E \\
F E \\
C O \\
C O \\
C D \\
C D \\
C O \\
V I \\
N I \\
N I \\
N I \\
V I \\
C U\end{array}$ & $\begin{array}{l}1 \\
4 \\
4 \\
6 \\
11 \\
11 \\
12 \\
13 \\
14 \\
15 \\
15 \\
16 \\
17 \\
18 \\
18 \\
18 \\
19 \\
19 \\
19 \\
20 \\
20 \\
20 \\
20 \\
21 \\
21 \\
21 \\
21 \\
22 \\
23 \\
23 \\
23 \\
24 \\
24 \\
25 \\
25 \\
25 \\
26 \\
26 \\
26 \\
27 \\
27 \\
27 \\
27 \\
27 \\
28 \\
28 \\
28 \\
28 \\
28 \\
29\end{array}$ & $\begin{array}{l}3 \\
7 \\
10 \\
14 \\
22 \\
24 \\
28 \\
26 \\
32 \\
32 \\
33 \\
35 \\
36 \\
37 \\
39 \\
42 \\
40 \\
42 \\
43 \\
41 \\
45 \\
47 \\
48 \\
44 M \\
46 \\
47 \\
48 \\
48 \\
44 \\
48 \\
49 \\
50 \\
48 \\
51 \\
51\end{array}$ & $\begin{array}{l}\text { 6. } 4 E+02 \\
1.7 E+03 \\
4.9 E-01 \\
3.4 E-03 \\
3.2 E+01 \\
4.3 E+05 \\
5.8 E+05 \\
2.5 E-02 \\
3.2 E-03 \\
1.0 E-01 \\
3.9 E-01 \\
1.9 E+03 \\
5.1 E+01 \\
8.2 E+02 \\
4.4 E-02 \\
4.7 E-03 \\
4.1 E-01 \\
2.0 E+05 \\
1.2 E+05 \\
7.9 E+01 \\
2.9 E+03 \\
1.7 E+05 \\
7.7 E-02 \\
1.3 E+02 \\
1.5 E-01 \\
6.0 E+02 \\
1.8 E+02 \\
4.0 E-04 \\
1.3 E+02 \\
1.2 E+03 \\
1.9 E-01 \\
6.5 E+02 \\
8.2 E+01 \\
5.8 E-01 \\
3.5 E-02 \\
7.0 E-03 \\
5.6 E-01 \\
6.3 E-01 \\
1.1 E-04 \\
9.9 E+02 \\
4.5 E-01 \\
1.6 E+00 \\
1.0 E+00 \\
1.1 E-02 \\
3.4 E+02 \\
1.1 E+04 \\
1.3 E+00 \\
6.5 E-01 \\
4.9 E+03 \\
1.8 E+04\end{array}$ & $\begin{array}{l}6.4 E+02 \\
3.9 E+02 \\
4.7 E-01 \\
3.4 E-03 \\
3.2 E+01 \\
2.3 E+04 \\
2.9 E+03 \\
2.4 E-02 \\
3.1 E-03 \\
5.3 E-03 \\
3.5 E-02 \\
9.5 E+02 \\
5.1 E+01 \\
8.2 E+02 \\
4.4 E-02 \\
4.7 E-03 \\
4.1 E-01 \\
1.8 E+03 \\
1.9 E+03 \\
1.6 E+01 \\
2.9 E+01 \\
4.8 E+01 \\
1.5 E-02 \\
2.2 E-01 \\
8.5 E-03 \\
1.4 E+00 \\
2.3 E-01 \\
3.8 E-04 \\
3.7 E+01 \\
1.1 E+03 \\
1.9 E-01 \\
1.0 E+00 \\
3.5 E+00 \\
7.9 E-02 \\
3.5 E-02 \\
6.3 E-03 \\
3.1 E-01 \\
3.4 E-02 \\
9.8 E-05 \\
3.0 E+01 \\
3.4 E-01 \\
1.4 E+03 \\
7.7 E-01 \\
1.1 E-02 \\
3.0 E+00 \\
2.4 E+01 \\
1.2 E+00 \\
5.9 E-01 \\
1.7 E+01 \\
4.0 E+01\end{array}$ \\
\hline
\end{tabular}

\begin{tabular}{|c|c|c|}
\hline $\begin{array}{l}\text { ELEMENT } \\
\text { SYMB OL }\end{array}$ & $\begin{array}{l}\text { ATOMIC } \\
\text { NO. (Z) }\end{array}$ & $\begin{array}{l}\text { I SOTOPE } \\
\text { MASS(A) }\end{array}$ \\
\hline $\mathrm{CU}$ & 29 & 67 \\
\hline$Z N$ & 30 & 65 \\
\hline $2 N$ & 30 & $69 M$ \\
\hline $2 N$ & 30 & 72 \\
\hline GA & 31 & 67 \\
\hline GA & 31 & 72 \\
\hline GE & 32 & 68 \\
\hline $\mathbf{G E}$ & 32 & 69 \\
\hline GE & 32 & 71 \\
\hline AS & 33 & 71 \\
\hline AS & 33 & 72 \\
\hline AS & 33 & 73 \\
\hline AS & 33 & 74 \\
\hline$A S$ & 33 & 76 \\
\hline AS & 33 & 77 \\
\hline SE & 34 & 72 \\
\hline SE & 34 & 75 \\
\hline SE & 34 & 79 \\
\hline GR & 35 & 76 \\
\hline$B R$ & 35 & 77 \\
\hline$B R$ & 35 & 82 \\
\hline KR & 36 & 76 \\
\hline$K R$ & 36 & 79 \\
\hline$K R$ & 36 & 81 \\
\hline$K R$ & 36 & 85 \\
\hline$R B$ & 37 & 83 \\
\hline RB & 37 & 84 \\
\hline RB & 37 & 86 \\
\hline RE & 37 & 87 \\
\hline$S R$ & 38 & 82 \\
\hline$S R$ & 38 & 83 \\
\hline$S R$ & 38 & 85 \\
\hline SR & 38 & 89 \\
\hline SR & 36 & 90 \\
\hline Y & 39 & 86 \\
\hline$Y$ & 39 & 87 \\
\hline$Y$ & 39 & $87 M$ \\
\hline$Y$ & 39 & 88 \\
\hline$Y$ & 39 & 90 \\
\hline Y & 39 & 91 \\
\hline ZR & 40 & 86 \\
\hline $2 \mathrm{R}$ & 40 & 88 \\
\hline $\mathrm{ZR}$ & 40 & 89 \\
\hline ZR & 40 & 93 \\
\hline $2 R$ & 40 & 95 \\
\hline $2 R$ & 40 & 97 \\
\hline NB & 41 & 90 \\
\hline NB & 41 & 91 \\
\hline NB & 41 & $91 M$ \\
\hline NE & 41 & 92 \\
\hline
\end{tabular}

$\underset{U C / M * * 3}{\text { ADULT }}$

$9.8 E+02$ 6. $7 E-03$ 5. $7 E+02$ 9. $9 E+00$ $5.8 E+04$

8. $2 E+04$

1. $8 E-05$

4. $2 E-03$

7. TE-02

1. $8 E+03$

2. $2 E+03$

1. $6 E+02$

1. $5 \mathrm{E}+01$

3. $0 E+03$

1. $1 E+0$

1. $1 E+00$

1. $9 E-01$

3. $5 E-02$

$1.1 E+06$

. $0 E+05$

1. $5 E+0$

1. $9 E+02$

1. $3 E+00$

2. $5 E-0$

9. $3 E+00$

1. $3 E+O I$

6. $1 E+01$

. $5 E-O$

2. $2 E+0$

2. $4 E+06$

2. $3 E+03$

2. $5 E+03$

2. $1 E-01$

3. $8 \mathrm{E}+03$

6. $2 E+02$

6. $2 E+02$

1. $4 E+04$

6. $2 E+02$

6. $2 E+02$

2. $1 E+00$

9. $3 E+03$

1. $1 E+03$

3. $3 E-01$

3. $4 E+00$

1. $4 E+04$

$1.4 E+04$

1. $6 E-0$

1. $8 E-01$

1. $5 E+00$
I NFAN T

UC $/ M * * 3$

9. $8 E+00$

1. $4 \mathrm{E}-03$

3. $5 E-01$

2. $0 E-02$

$2.0 E+04$

7. $3 E+03$

1. $8 E-05$

$2.6 E-03$

7. $1 E-02$

1. $7 E+01$

$8.6 E+00$

$3.5 E+01$

8. $9 E-01$

1. $2 E+01$

$6.1 E+01$

4. $6 E-01$

. $7 E-01$

8. $5 E+04$

5.1E+04

- OE+04

1. $5 E+02$

$1.5 E+02$

$1.9 E+02$

2.5E-01

4. $7 \mathrm{E}+00$

3. $7 E+00$

$1.2 E+01$

. $5 E-01$

. $3 E+00$

$2.5 E+02$

1. $1 E+01$

9. $8 E+00$

4. 5E-02

1.7E-01

1. $5 \mathrm{E}-01$

5. $9 E-01$

5.

$1.5 E-03$

1. $2 E-01$

$9.0 E-03$

1. $5 E+01$

1. $8 E-01$

7. $8 \mathrm{E}+00$

3. $1 E-01$

4. $3 E-01$

. $1 E+01$

$6.0 E-02$

1. $4 \mathrm{E}-03$

1. $3 E-02$ 
TABLE 9A. UNIT-RAD CONTAMINATION FOR WHOLE BODY VIA SEAWATER.

$10 / 09 / 68$ PAGE 32

\begin{tabular}{|c|c|c|c|c|}
\hline $\begin{array}{l}\text { ELEMENT } \\
\text { SYMBOL }\end{array}$ & $\begin{array}{l}\text { ATOMIC } \\
\text { NO. }(Z)\end{array}$ & $\begin{array}{l}\text { ISOTOPE } \\
\operatorname{MASS}(A)\end{array}$ & $\begin{array}{l}\text { ADULT } \\
\text { UC } / M * * 3\end{array}$ & $\begin{array}{l}\text { INFANT } \\
\text { UC } / M * * 3\end{array}$ \\
\hline $\begin{array}{l}\text { NB } \\
N B \\
N B \\
N B \\
N B \\
M D \\
M O \\
\text { TC } \\
\text { TC } \\
T C \\
T C \\
T C \\
T C \\
T C \\
\text { TU } \\
R U \\
R U \\
R H \\
R H \\
R H \\
R H \\
R H \\
C H \\
R H \\
R H \\
P D \\
P D \\
P D \\
P D \\
P D \\
A G \\
A G \\
A G \\
A G \\
A G \\
C D \\
C D \\
C D \\
C D \\
I N \\
I N \\
I N \\
S N \\
S N \\
S N \\
S N \\
S N \\
S N \\
S N \\
S N \\
S B \\
S B\end{array}$ & $\begin{array}{l}41 \\
41 \\
41 \\
41 \\
42 \\
42 \\
43 \\
43 \\
43 \\
43 \\
43 \\
43 \\
43 \\
44 \\
44 \\
44 \\
45 \\
45 \\
45 \\
45 \\
45 \\
45 \\
46 \\
46 \\
46 \\
46 \\
46 \\
47 \\
47 \\
47 \\
47 \\
47 \\
48 \\
48 \\
48 \\
48 \\
49 \\
49 \\
49 \\
50 \\
50 \\
50 \\
50 \\
50 \\
50 \\
50 \\
50 \\
51 \\
51\end{array}$ & $\begin{array}{l}93 M \\
94 \\
95 \\
95 M \\
96 \\
93 \\
99 \\
95 \\
95 M \\
96 \\
97 \\
97 M \\
98 \\
99 \\
97 \\
103 \\
106 \\
99 \\
100 \\
101 \\
101 M \\
102 \\
105 \\
100 \\
103 \\
107 \\
109 \\
112 \\
105 \\
106 M \\
108 M \\
110 M \\
111 \\
109 \\
113 M \\
115 \\
115 M \\
111 \\
114 M \\
115 \\
113 \\
117 M \\
119 M \\
121 \\
121 M \\
123 M \\
125 \\
126 \\
119 \\
120 M\end{array}$ & $\begin{array}{l}\text { 7. } 8 E-03 \\
1.6 E-05 \\
1.5 E-01 \\
1.0 E+01 \\
5.8 E+01 \\
4.2 E+00 \\
9.3 E+03 \\
1.8 E+02 \\
4.4 E-01 \\
4.3 E+00 \\
1.8 E-01 \\
2.7 E+00 \\
2.4 E-03 \\
4.0 E-02 \\
2.2 E+02 \\
1.5 E+00 \\
1.3 E-02 \\
2.9 E-02 \\
1.3 E+00 \\
2.3 E-04 \\
2.9 E-01 \\
4.0 E-04 \\
3.0 E+00 \\
5.2 E-02 \\
1.5 E-01 \\
1.5 E-02 \\
4.3 E+00 \\
3.3 E-01 \\
2.0 E+00 \\
1.3 E+01 \\
8.9 E-03 \\
6.5 E-02 \\
8.9 E+01 \\
1.5 E-03 \\
7.0 E-05 \\
1.7 E+00 \\
6.8 E-03 \\
2.0 E-03 \\
4.2 E-06 \\
8.2 E-08 \\
7.6 E+00 \\
4.8 E+02 \\
1.1 E+01 \\
1.8 E+05 \\
2.1 E-01 \\
4.2 E+00 \\
1.8 E+02 \\
5.7 E-03 \\
2.9 E+01 \\
5.0 E-02\end{array}$ & $\begin{array}{l}5.0 E-03 \\
1.4 E-05 \\
6.8 E-03 \\
5.0 E-02 \\
7.4 E-02 \\
4.1 E+00 \\
2.5 E+02 \\
4.0 E+01 \\
4.2 E-01 \\
2.5 E+00 \\
1.8 E-01 \\
2.6 E+00 \\
2.4 E-03 \\
4.0 E-02 \\
4.8 E+00 \\
3.6 E-01 \\
9.3 E-03 \\
1.7 E-02 \\
9.9 E-02 \\
2.3 E-04 \\
8.9 E-02 \\
3.8 E-04 \\
3.7 E-01 \\
2.2 E-02 \\
1.2 E-01 \\
1.5 E-02 \\
4.4 E-01 \\
4.9 E-02 \\
8.8 E-01 \\
1.8 E+00 \\
8.6 E-03 \\
5.4 E-02 \\
1.1 E+01 \\
1.0 E-03 \\
6.6 E-05 \\
1.9 E-02 \\
1.2 E-03 \\
1.1 E-04 \\
2.1 E-03 \\
8.1 E-03 \\
2.8 E+00 \\
3.1 E+01 \\
6.3 E+00 \\
1.0 E+03 \\
2.0 E-01 \\
1.6 E+00 \\
7.9 E+00 \\
5.6 E-03 \\
1.2 E+00 \\
6.6 E-03\end{array}$ \\
\hline
\end{tabular}

$\begin{array}{lcc}\text { ELEMENT } & \text { ATOMIC } & \text { I SOTOP } \\ \text { SYMBOL } & \text { NO.IZI } & \text { MASSIA } \\ & & \\ \text { SB } & 51 & 122 \\ \text { SB } & 51 & 124 \\ \text { SB } & 51 & 125 \\ \text { SB } & 51 & 126 \\ \text { SB } & 51 & 127 \\ \text { TE } & 52 & 118 \\ \text { TE } & 52 & 119 \\ \text { TE } & 52 & 119 M \\ \text { TE } & 52 & 121 \\ \text { IE } & 52 & 121 M \\ \text { TE } & 52 & 123 \\ \text { TE } & 52 & 123 M \\ \text { TE } & 52 & 125 M \\ \text { TE } & 52 & 127 M \\ \text { TE } & 52 & 129 M \\ \text { TE } & 52 & 131 M \\ \text { TE } & 52 & 132 \\ \text { I } & 53 & 123 \\ \text { I } & 53 & 124 \\ \text { I } & 53 & 125 \\ \text { I } & 53 & 126 \\ \text { I } & 53 & 129 \\ \text { I } & 53 & 130 \\ \text { I } & 53 & 131 \\ \text { I } & 53 & 133 \\ \text { XE } & 54 & 122 \\ \text { XE } & 54 & 125 \\ \text { XE } & 54 & 127 \\ \text { XE } & 54 & 129 M \\ \text { XE } & 54 & 131 M \\ \text { XE } & 54 & 133 \\ \text { XE } & 54 & 133 M \\ \text { CS } & 55 & 129 \\ \text { CS } & 55 & 131 \\ \text { CS } & 55 & 132 \\ \text { CS } & 55 & 134 \\ \text { CS } & 55 & 135 \\ \text { CS } & 55 & 136 \\ \text { CS } & 55 & 137 \\ \text { BA } & 56 & 128 \\ \text { BA } & 56 & 131 \\ \text { BA } & 56 & 133 \\ \text { BA } & 56 & 133 M \\ \text { BA } & 56 & 135 M \\ \text { BA } & 56 & 140 \\ \text { LA } & 57 & 135 \\ \text { LA } & 57 & 137 \\ \text { LA } & 57 & 138 \\ \text { LA } & 57 & 140 \\ \text { CE } & 58 & 134 \\ & & \end{array}$

ADULT
$U C / M * 3$

INF ANT

4. $4 E-01$

1. $1 E-03$

3. $0 E-02$

1. $1 E-02 \quad 2.8 E-03$

2. $1 E-012.0 E-02$

. 5E-01

1. $1 E+01$

- 5E-01

7.2E-02

4. $7 E-01$

5. $9 E-02$

5. $9 E-02$

4.5E-0

5. OE-O

2. $6 E-03$

. $4 \mathrm{E}-02$

$2.6 E-03$
$2.1 E-02$

7.2E-02

. $9 E-02$

2. $8 E-02$

2. $7 E+00$

3. 3E- 01

2. $8 E+06$

4. $7 E+03$

1. $1 E+03$

$2.2 E+03$
$2.3 E+00$

2. $7 E+05$

4. $9 E+03$

1. $9 E+05$

2. $1 E+02$

1. $1 E+03$

5. $3 E+01$

2. $4 E+02$

2. $3 E+02$

4. $6 E+02$

4. $7 E+02$

$3.6 E+04$
$1.2 E+04$

1. $2 E+04$

1. $3 E+03$

1.7E-02

1. $9 E-02$

5. $9 E-02$

1. $1 E+04$

1. $4 \mathrm{E}+02$

$3.2 E+02$

1. $9 E+02$

2. $2 E+00$

1. $0 E+03$

2. $7 E+02$

$1.2 \mathrm{E}+03$

$2.1 E+02$

$1.1 E+03$

$5.3 E+01$

$2.4 E+02$

$2.3 E+02$

4. $6 E+02$

4. $7 \mathrm{E}+02$

4. $2 E+02$

9. $8 \mathrm{E}+02$

7. $3 E+01$

2. $E E-O$

2. 3E-01

8. 1E-OI

7. $7 E-02$

3. $3 E+02$

3. $7 \mathrm{E}+01$

8. $0 E-01$

1. $1 \mathrm{E}+01$

7. $5 \mathrm{E}-02$

1. $2 E+0$

$5.6 \mathrm{E}+00$

7. $6 E-02$

7. $4 \mathrm{E}-02$

2. $7 E+03$

$6.5 \mathrm{E}+01$

1. $2 E+04$

$2.2 E+02$

9. $2 \mathrm{E}+00$

2. $O E+02$

1. $5 \mathrm{E}+00$

3. $2 E-01$

8. 8E- 04

2. $5 E-05$

$2.4 E-05$

2. $0 E+02$

1. $O E+00$ 


\begin{tabular}{|c|c|c|c|c|}
\hline $\begin{array}{l}\text { ELEMENT } \\
\text { SYMBOL }\end{array}$ & $\begin{array}{l}\text { ATOMIC } \\
N D \cdot(Z)\end{array}$ & $\begin{array}{l}\text { ISOTOPE } \\
\text { MASS(A) }\end{array}$ & $\begin{array}{l}\text { ADULT } \\
\text { UC/M**3 }\end{array}$ & $\begin{array}{l}\text { INFANT } \\
\text { UC } / M * * 3\end{array}$ \\
\hline $\begin{array}{l}\text { CE } \\
C E \\
C E \\
C E \\
C E \\
C E \\
C E \\
P R \\
P R \\
N D \\
\text { ND } \\
N D \\
P M \\
P M \\
P M \\
P M \\
P M \\
P M \\
P M \\
P M \\
P M \\
S M \\
S M \\
S M \\
S M \\
S M \\
S M \\
S M \\
S M \\
E U \\
E U \\
E U \\
E U \\
E U \\
E U \\
E U \\
E U \\
E U \\
E U \\
E U \\
E U \\
E U \\
G D \\
G D \\
G D \\
G D \\
G D \\
G D \\
G D \\
G D\end{array}$ & $\begin{array}{l}58 \\
58 \\
58 \\
58 \\
58 \\
58 \\
58 \\
59 \\
59 \\
60 \\
60 \\
60 \\
61 \\
61 \\
61 \\
61 \\
61 \\
61 \\
61 \\
61 \\
61 \\
62 \\
62 \\
62 \\
62 \\
62 \\
62 \\
62 \\
62 \\
63 \\
63 \\
63 \\
63 \\
63 \\
63 \\
63 \\
63 \\
63 \\
63 \\
63 \\
63 \\
63 \\
64 \\
64 \\
64 \\
64 \\
64 \\
64 \\
64 \\
64\end{array}$ & $\begin{array}{l}135 \\
137 M \\
139 \\
141 \\
142 \\
143 \\
144 \\
142 \\
143 \\
140 \\
144 \\
147 \\
143 \\
144 \\
145 \\
146 \\
147 \\
148 \\
148 M \\
149 \\
151 \\
141 \\
145 \\
146 \\
147 \\
148 \\
149 \\
151 \\
153 \\
145 \\
146 \\
1469 \\
147 \\
148 \\
149 \\
150 \\
150 M \\
152 \\
154 \\
155 \\
156 \\
157 \\
146 \\
147 \\
148 \\
149 \\
150 \\
151 \\
152 \\
153\end{array}$ & $\begin{array}{l}\text { 4. } 6 E+03 \\
3.3 E+03 \\
7.5 E-01 \\
9.5 E+00 \\
5.4 E-04 \\
1.1 E+03 \\
1.2 E-02 \\
4.0 E-02 \\
4.4 E-04 \\
2.1 E-03 \\
3.5 E-09 \\
8.5 E-05 \\
1.5 E-01 \\
1.2 E-02 \\
1.9 E-02 \\
4.4 E-03 \\
3.2 E-03 \\
6.3 E+01 \\
3.1 E-01 \\
1.3 E+03 \\
1.9 E+03 \\
2.2 E+00 \\
2.9 E-01 \\
3.1 E-04 \\
3.5 E-04 \\
2.0 E-04 \\
4.2 E-04 \\
5.8 E-02 \\
2.6 E+03 \\
2.0 E+02 \\
5.1 E+01 \\
6.3 E+02 \\
3.0 E+00 \\
3.4 E+00 \\
9.1 E-01 \\
5.9 E-03 \\
4.1 E+03 \\
2.0 E-03 \\
1.5 E-03 \\
2.2 E-01 \\
1.3 E+01 \\
1.1 E+04 \\
1.5 E-01 \\
3.5 E+02 \\
1.6 E-04 \\
1.9 E+01 \\
8.8 E-05 \\
4.3 E-01 \\
2.1 E-04 \\
2.9 E-01\end{array}$ & $\begin{array}{l}6.1 \mathrm{E}+00 \\
8.4 \mathrm{E}+00 \\
1.5 \mathrm{E}-01 \\
5.2 \mathrm{E}-01 \\
5.0 \mathrm{E}-04 \\
2.6 \mathrm{E}+00 \\
4.1 \mathrm{E}-03 \\
4.3 \mathrm{E}-05 \\
8.0 \mathrm{E}-06 \\
1.1 \mathrm{E}-05 \\
3.2 \mathrm{E}-09 \\
1.4 \mathrm{E}-06 \\
4.3 \mathrm{E}-02 \\
4.2 \mathrm{E}-03 \\
1.7 \mathrm{E}-02 \\
3.1 \mathrm{E}-03 \\
1.9 \mathrm{E}-03 \\
5.1 \mathrm{E}-01 \\
1.8 \mathrm{E}-02 \\
4.4 \mathrm{E}+00 \\
3.4 \mathrm{E}+00 \\
6.6 \mathrm{E}-02 \\
1.0 \mathrm{E}-01 \\
2.8 \mathrm{E}-04 \\
3.2 \mathrm{E}-04 \\
1.8 \mathrm{E}-04 \\
3.9 \mathrm{E}-04 \\
5.2 \mathrm{E}-02 \\
7.8 \mathrm{E}+00 \\
1.8 \mathrm{E}+00 \\
3.7 \mathrm{E}-01 \\
1.6 \mathrm{E}+00 \\
1.1 \mathrm{E}-01 \\
2.6 \mathrm{E}-01 \\
1.3 \mathrm{E}-01 \\
4.3 \mathrm{E}-03 \\
3.5 \mathrm{E}+00 \\
1.7 \mathrm{E}-03 \\
1.3 \mathrm{E}-03 \\
1.1 \mathrm{E}-01 \\
3.1 \mathrm{E}-01 \\
1.1 \mathrm{E}+01 \\
1.2 \mathrm{E}-02 \\
6.7 \mathrm{E}-01 \\
1.5 \mathrm{E}-04 \\
3.0 \mathrm{E}-01 \\
8.2 \mathrm{E}-05 \\
7.7 \mathrm{E}-02 \\
2.0 \mathrm{E}-04 \\
8.9 \mathrm{E}-02\end{array}$ \\
\hline
\end{tabular}
ELEMENT
SYMBOL

ATOMIC I SOTOPE

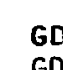

GD

TB

TB

TB

TE
T B

TB

TB

TB

TB

DY

DY

DY

DY

HO

HO

HO

ER

ER
ER

TM

TM

TM

TM

YM

$Y B$
$Y B$

$Y B$

LU

LU

LU

LU

LU

LU

LU

LU

HF

HF

HF

HF
HF
HF

ND. (Z) MASSIA)
7. $8 \mathrm{E}+03$

1. $5 E-02$

6. $3 E+03$

$1.8 \mathrm{E}+03$

1. $2 E+03$

6. $8 E+03$

2. $8 E+02$

8. $5 \mathrm{E}+01$

1. $7 \mathrm{E}-02$

7. $3 E-04$

5. $6 \mathrm{E}-01$

4. $O E+02$

$9.2 E+03$

6. $5 E-10$

2. $6 \mathrm{E}-02$

1. $0 E-09$

2. $2 E-05$

1. $9 E-03$

1. $5 \mathrm{E}-02$

$3.4 E+03$

6. $6 E-04$

6. $3 E-03$

3. $2 E-03$

5. OE-03

9. $7 E+02$

6. $9 E+01$

3. 7E-01

7. $5 E-01$

7. $7 E-01$

5. $5 E+02$

1. $5 E+03$

1. $2 E+01$

1. $5 E+03$

1. $0 E+03$

3. $4 E+02$

4. $5 E+02$

9. $5 E+01$

3. $7 E-02$

5. $2 E-03$

3. $4 E-01$

1. $1 E-03$

. $3 E+02$

1. $0 E+00$

6. $2 E+01$

1. $2 E+02$

7. $5 \mathrm{E}-05$

3. $7 E+01$

6. $7 E-06$

3. $1 E-02$

5. $0 E-02$
INFAN T

$U C / M * \$ 3$

1. $1 E+01$

6. $0 E-03$

2. $1 E+00$

4. $6 E+00$

8. $8 E+00$

$2.3 E+00$

$6.8 E-01$

1. $5 \mathrm{E}-02$

6. 6E-04

$5.5 E-02$

4. $O E+00$

1. $4 E+01$

5. $9 E-10$

2. OE-05

9. $2 \mathrm{E}-10$

3. 8E-06

$9.4 E-06$

1. $3 E-02$

5. $2 E+00$

5. $9 E-04$

4.6E-05

$1.6 \mathrm{E}-05$

1. $7 E+00$

9. $6 E-01$

$4.1 E-02$

1. $2 E-01$

3. $9 E-01$

2. $2 E+00$

$5.2 \mathrm{E}+00$

5. $5 \mathrm{E}-01$

8. $8 E+00$

. $0 E+00$

9. 0 E-01

4. $8 E+00$

8. $4 E-01$

1. $5 \mathrm{E}-02$

$3.3 E-03$

$6.1 \mathrm{E}-02$

4 . +00

1.

1. $7 E-01$

5. $5 E-02$

1. $3 E-01$

$5.7 E-05$

3. $4 E-03$

$3.7 E-03$ 
TABLE 9A. UNIT-RAD CONTAMINATION FOR WHOLE BODY VIA SEAWATER.

$10 / 09 / 68$ PAGE 34

\begin{tabular}{|c|c|c|c|c|}
\hline $\begin{array}{l}\text { ELEMENT } \\
\text { SYMBOL }\end{array}$ & $\begin{array}{l}\text { ATOMIC } \\
\text { ND. }(Z)\end{array}$ & $\begin{array}{l}\text { ISOTOPE } \\
\text { MASS(A) }\end{array}$ & $\begin{array}{l}\text { ADULT } \\
\text { UC } / M * 3\end{array}$ & $\begin{array}{l}\text { INFANT } \\
U C / M * 3\end{array}$ \\
\hline 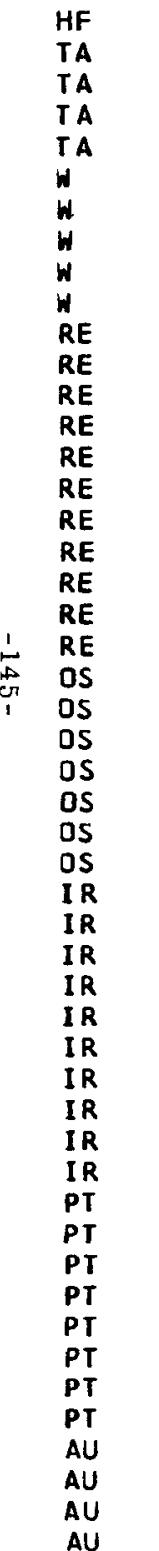 & $\begin{array}{l}72 \\
73 \\
73 \\
73 \\
73 \\
74 \\
74 \\
74 \\
74 \\
74 \\
75 \\
75 \\
75 \\
75 \\
75 \\
75 \\
75 \\
75 \\
75 \\
75 \\
75 \\
76 \\
76 \\
76 \\
76 \\
76 \\
76 \\
76 \\
77 \\
77 \\
77 \\
77 \\
77 \\
77 \\
77 \\
77 \\
77 \\
77 \\
78 \\
78 \\
78 \\
78 \\
78 \\
78 \\
78 \\
78 \\
79 \\
79 \\
79 \\
79\end{array}$ & $\begin{array}{l}182 \\
177 \\
179 \\
182 \\
183 \\
178 \\
181 \\
185 \\
187 \\
188 \\
180 \\
181 \\
182 \\
182 M \\
183 \\
184 \\
184 M \\
186 \\
187 \\
188 \\
189 \\
182 \\
183 \\
185 \\
191 \\
191 M \\
193 \\
194 \\
185 \\
186 \\
187 \\
188 \\
189 \\
190 \\
192 \\
192 M \\
193 M \\
194 \\
188 \\
190 \\
191 \\
192 \\
193 \\
193 M \\
195 M \\
197 \\
193 \\
194 \\
195 \\
196\end{array}$ & $\begin{array}{l}1.0 E-05 \\
2.2 E+00 \\
2.0 E-03 \\
9.7 E-04 \\
8.3 E-01 \\
3.8 E-04 \\
1.5 E-03 \\
1.5 E-03 \\
3.2 E-02 \\
1.5 E-04 \\
4.1 E+00 \\
4.5 E+00 \\
6.0 E-01 \\
1.1 E+01 \\
3.2 E-02 \\
2.1 E-02 \\
3.3 E-03 \\
1.2 E+00 \\
6.8 E-01 \\
9.6 E+00 \\
1.2 E+01 \\
3.9 E-02 \\
2.6 E-01 \\
3.3 E-04 \\
8.8 E-03 \\
6.5 E-01 \\
9.1 E-02 \\
5.1 E-06 \\
6.6 E+02 \\
3.9 E+02 \\
1.0 E+03 \\
4.4 E+01 \\
7.3 E+00 \\
2.1 E+00 \\
2.2 E-01 \\
1.5 E-03 \\
3.8 E+01 \\
3.6 E+02 \\
4.9 E-04 \\
1.7 E-07 \\
2.3 E-02 \\
2.1 E-07 \\
7.3 E-00 \\
2.7 E-02 \\
2.6 E-02 \\
4.9 E-01 \\
2.5 E+02 \\
1.5 E+01 \\
3.1 E-02 \\
3.2 E+00\end{array}$ & $\begin{array}{l}9.4 E-06 \\
2.1 E-02 \\
1.4 E-03 \\
3.2 E-04 \\
1.7 E-02 \\
3.6 E-04 \\
1.5 E-03 \\
1.5 E-03 \\
1.6 E-02 \\
1.5 E-04 \\
4.4 E-01 \\
4.8 E-01 \\
1.7 E-01 \\
8.2 E-01 \\
2.9 E-02 \\
1.8 E-02 \\
3.2 E-03 \\
4.0 E-01 \\
6.8 E-01 \\
8.9 E-01 \\
1.4 E+00 \\
1.2 E-02 \\
5.2 E-02 \\
3.3 E-04 \\
7.8 E-03 \\
1.5 E-01 \\
3.7 E-02 \\
5.1 E-06 \\
2.0 E+01 \\
1.2 E+01 \\
2.5 E+01 \\
3.5 E+00 \\
2.9 E+00 \\
8.0 E-01 \\
1.8 E-01 \\
1.5 E-03 \\
1.4 E+01 \\
1.4 E+01 \\
1.5 E-04 \\
1.7 E-07 \\
2.6 E-03 \\
2.1 E-007 \\
7.3 E-00 \\
4.2 E-03 \\
3.9 E-03 \\
1.7 E-02 \\
1.6 E+00 \\
2.1 E-01 \\
1.8 E-02 \\
1.6 E-01\end{array}$ \\
\hline
\end{tabular}

\begin{tabular}{lcc} 
ELEMENT & ATOMIC & I SOTOP \\
SYMBOL & NO. I I & MASSIA \\
& & \\
AU & 79 & 198 \\
AU & 79 & 199 \\
HC & 80 & 194 \\
HG & 80 & $195 M$ \\
HG & 80 & 197 \\
HG & 80 & $197 M$ \\
HG & 80 & 203 \\
TL & 81 & 200 \\
TL & 81 & 201 \\
TL & 81 & 202 \\
TL & 81 & 204 \\
PB & 82 & 200 \\
PB & 82 & 202 \\
PB & 82 & 203 \\
PB & 82 & 204 \\
PB & 82 & 205 \\
PB & 82 & 210 \\
BI & 83 & 205 \\
BI & 83 & 206 \\
BI & 83 & 207 \\
BI & 83 & 208 \\
BI & 83 & 210 \\
BI & 83 & $210 M$ \\
PO & 84 & 206 \\
PO & 84 & 208 \\
PO & 84 & 209 \\
PO & 84 & 210 \\
RN & 86 & 211 \\
RN & 86 & 222 \\
RA & 88 & 223 \\
RA & 88 & 224 \\
RA & 88 & 225 \\
RA & 88 & 226 \\
RA & 88 & 228 \\
AC & 89 & 225 \\
AC & 89 & 227 \\
TH & 90 & 227 \\
TH & 90 & 228 \\
TH & 90 & 229 \\
TH & 90 & 230 \\
TH & 90 & 231 \\
TH & 90 & 232 \\
TH & 90 & 234 \\
PA & 91 & 228 \\
PA & 91 & 229 \\
PA & 91 & 230 \\
PA & 91 & 231 \\
PA & 91 & 232 \\
PA & 91 & 233 \\
U & 92 & 230 \\
\hline & & \\
\hline
\end{tabular}

ADULT

$U C / M * * 3$

$1.6 E+01$

3. $7 E+01$

6. $5 \mathrm{E}-04$

4. $9 E-01$

3. $3 E+00$

1. $5 E-02$

1. $1 E-01$

8. 3E-02

3. $5 E-03$

1. $7 E-05$

1. $5 E+04$

3. $8 E-03$

$5.6 E+03$

2. $6 E-02$

2. 6E-02

$6.5 E-04$
$7.6 E-03$

2. $7 E-02$

2. 7E-02

1. 7E-05

1. 2E-02

4. $0 E-06$

2. 2E-01

$1.7 E-04$

4. OE-0 5

2. 2E- 03

3. $O E+O$

1. $9 E-01$

1. $3 E+03$

$1.3 E+04$

7. $8 E+02$

7. 2E-03

4. $3 E-02$

1. $8 \mathrm{E}+02$

1. $0 E-03$

3. $4 \mathrm{E}-03$

2. $3 E-0$

4. $2 E-08$

3. $9 \mathrm{E}-08$

8.7E-01

3. $5 E-08$

1. $6 E-03$

2. $8 E+04$

1. $1 E+04$

7. $9 E+0$

8. $0 E-04$

$1.2 E+04$

2. $9 E+01$
INFANT

$U C / M * * 3$

3. $5 E-01$

9.5E-01

7. OE -02

2. $O E-01$

3. OE-O1

1. 3E-02

4. $4 E-03$

9. $0 E-03$

1. 1E-03

1. $6 E-05$

3. $1 E-03$

$8.3 E+00$

7. OE-04

$2.1 E-02$

4. $9 \mathrm{E}-04$

5.7E-03

1. 5E-02

1. 7E-05

5. $9 E-03$

4. OE-06

5. OE-02 $\sigma$ in

$1.7 \mathrm{E}-04$ 过

4. OE-05

$1.8 E-03$

3. $0 E+00$

1. $9 \mathrm{E}-01$

1. $8 E+00$

5. $6 E+00$

$1.4 E+00$

$2.5 E-03$

8. $2 \mathrm{E}-03$

7. $3 E-02$

$1.3 \mathrm{E}-04$

1.1 E-06

2. $8 E-08$

$2.7 E-09$
$2.5 E-09$

2.5E-09

2. $2 \mathrm{E}-0$

6. 7E-07

6.2E-01

4. 1E-O1

3. 3E-02

7. $0 E-05$

3. $9 E-01$

2. $0 E-02$

2. $0 E-02$ 
TABLE 9A. UNIT-RAD CONTAMINATION FOR WHOLE BODY VIA SEANATER.

\begin{tabular}{|c|c|c|c|c|}
\hline $\begin{array}{l}\text { ELEMENT } \\
\text { SYMBOL }\end{array}$ & $\begin{array}{l}\text { ATOMIC } \\
\text { NO. }(2)\end{array}$ & $\begin{array}{l}\text { ISOTOPE } \\
\text { MASS (A) }\end{array}$ & $\begin{array}{l}\text { ADULT } \\
\text { UC } / M * * 3\end{array}$ & $\begin{array}{l}\text { INFANT } \\
\text { UC/M**3 }\end{array}$ \\
\hline $\begin{array}{l}U \\
U \\
U \\
U \\
U \\
U \\
U \\
U \\
U \\
N P \\
1 \\
N P \\
N P \\
\text { IP } \\
N P \\
N P \\
N P \\
P U \\
P U \\
P U \\
P U \\
P U \\
P U \\
P U \\
P U \\
P U \\
A M \\
A M \\
A M \\
A M \\
A M \\
A M\end{array}$ & $\begin{array}{l}92 \\
92 \\
92 \\
92 \\
92 \\
92 \\
92 \\
92 \\
92 \\
93 \\
93 \\
93 \\
93 \\
93 \\
93 \\
93 \\
94 \\
94 \\
94 \\
94 \\
94 \\
94 \\
94 \\
94 \\
94 \\
95 \\
95 \\
95 \\
95 \\
95 \\
95\end{array}$ & $\begin{array}{l}231 \\
232 \\
233 \\
234 \\
235 \\
236 \\
237 \\
238 \\
240 \\
234 \\
235 \\
236 \\
236 M \\
237 \\
238 \\
239 \\
236 \\
237 \\
238 \\
239 \\
240 \\
241 \\
242 \\
244 \\
246 \\
239 \\
240 \\
241 \\
242 \\
242 M \\
243\end{array}$ & $\begin{array}{l}1.6 E+01 \\
4.2 E-04 \\
3.8 E-04 \\
3.6 E-04 \\
3.3 E-04 \\
3.2 E-04 \\
5.8 E+00 \\
3.1 E-04 \\
6.1 E+02 \\
9.8 E+02 \\
1.1 E-01 \\
6.5 E-04 \\
2.1 E+04 \\
6.8 E-04 \\
3.9 E+03 \\
3.0 E+03 \\
2.8 E-02 \\
1.5 E+01 \\
1.2 E-03 \\
9.9 E-04 \\
9.8 E-04 \\
2.6 E-03 \\
9.4 E-04 \\
8.5 E-04 \\
2.0 E+02 \\
3.3 E+04 \\
1.8 E+03 \\
3.4 E-04 \\
1.8 E+04 \\
3.4 E-04 \\
3.0 E-04\end{array}$ & $\begin{array}{l}6.5 \mathrm{E}-01 \\
4.1 \mathrm{E}-04 \\
3.7 \mathrm{E}-04 \\
3.5 \mathrm{E}-04 \\
3.2 \mathrm{E}-04 \\
3.2 \mathrm{E}-04 \\
3.6 \mathrm{E}-01 \\
3.1 \mathrm{E}-04 \\
3.6 \mathrm{E}+00 \\
1.1 \mathrm{E}-01 \\
1.1 \mathrm{E}-03 \\
5.9 \mathrm{E}-05 \\
5.0 \mathrm{E}-01 \\
6.2 \mathrm{E}-05 \\
2.1 \mathrm{E}-01 \\
1.8 \mathrm{E}-01 \\
4.4 \mathrm{E}-04 \\
1.0 \mathrm{E}-02 \\
6.6 \mathrm{E}-05 \\
5.6 \mathrm{E}-05 \\
5.5 \mathrm{E}-05 \\
1.1 \mathrm{E}-04 \\
5.3 \mathrm{E}-05 \\
4.7 \mathrm{E}-05 \\
3.3 \mathrm{E}-02 \\
8.4 \mathrm{E}-01 \\
1.9 \mathrm{E}-01 \\
5.7 \mathrm{E}-05 \\
5.9 \mathrm{E}-01 \\
5.6 \mathrm{E}-05 \\
5.0 \mathrm{E}-05\end{array}$ \\
\hline
\end{tabular}

$\begin{array}{lr}\text { ELEMENT } & \text { ATOMIC } \\ \text { SYMBOL } & \text { YD. (Z) } \\ \text { CM } & \\ \text { CM } & 96 \\ \text { CM } & 96 \\ \text { CM } & 96 \\ \text { CM } & 96 \\ \text { CM } & 96 \\ \text { CM } & 96 \\ \text { CM } & 96 \\ \text { CM } & 96 \\ \text { BK } & 96 \\ \text { BK } & 97 \\ \text { BK } & 97 \\ \text { BK } & 97 \\ \text { BK } & 97 \\ \text { CF } & 97 \\ \text { CF } & 98 \\ \text { CF } & 98 \\ \text { CF } & 98 \\ \text { CF } & 98 \\ \text { CF } & 98 \\ \text { CF } & 98 \\ \text { ES } & 98 \\ \text { ES } & 99 \\ \text { ES } & 99 \\ \text { ES } & 99 \\ \text { ES } & 99 \\ \text { ES } & 99 \\ \text { FM } & 99 \\ \text { FM } & 100 \\ \text { FM } & 100 \\ \text { FM } & 100 \\ & \end{array}$

10/09/68 PAGE 35

ADULT INFANT

ISOTOPE

$U C / M * * 3$

$U C / M * * 3$

1. $3 E+01$

8. $1 E+00$

3. $6 E-01$

5. $2 E-04$
7. $0 E-04$

3. 5E- 04

3. $4 \mathrm{E}-04$

3. $1 E-04$

3. $1 E-04$

9. $9 E+02$

7. $2 E+03$

8. $1 E-04$

2. $4 E+04$

2. $3 E-01$

1. $0 E+04$

1. $8 \mathrm{E}-01$

8. 3E-04

2. $7 E-03$

7. $4 E-04$

2. 3E-02

5. $5 E+01$

1. OE +0

1. OE+OO

8. $3 E-02$

7. $2 E+03$

3. $2 E+01$

2. $1 E+04$

9. $8 \mathrm{E}+02$

2. $6 E+04$

$1.5 E-02$
$1.2 E-02$

$2.4 E-03$

$6.7 E-05$

8. 3E-05

5. OE-05

4.8E-05

4. 5E-05

4. $4 \mathrm{E}-05$

7.6E-02 $\Rightarrow$

2. OE-01

3. 5E-01

2.4 E-01

$9.6 \mathrm{E}-04$

4. 6E-05

1. $0 \mathrm{E}-04$

4. 1 E-OS

3. 2E-04

1. $6 E-02$

2. $1 E-01$

2. $1 E-03$

1. $5 \mathrm{E}-02$

6.1E-04

1. 8E-01

1. $2 \mathrm{E}-02$

3. IE-01

6. $8 \mathrm{E}-02$

3. $4 \mathrm{E}-01$

3. $5 E-03$ 
TABLE 9A. UNIT-RAD CONTAMINATION FOR GI TRACT

\begin{tabular}{|c|c|c|}
\hline $\begin{array}{l}\text { ELEMENT } \\
\text { SYMBOL }\end{array}$ & $\begin{array}{l}\text { ATOMIC } \\
\text { NO. (Z) }\end{array}$ & $\begin{array}{l}\text { ISOTOPE } \\
\operatorname{MASS}(A)\end{array}$ \\
\hline H & 1 & 3 \\
\hline BE & 4 & 7 \\
\hline $\mathrm{BE}$ & 4 & 10 \\
\hline$c$ & 6 & 14 \\
\hline NA & 11 & 22 \\
\hline NA & 11 & 24 \\
\hline MG & 12 & 28 \\
\hline AL & 13 & 26 \\
\hline SI & 14 & 32 \\
\hline $\mathbf{P}$ & 15 & 32 \\
\hline P & 15 & 33 \\
\hline$S$ & 16 & 35 \\
\hline $\mathrm{CL}$ & 17 & 36 \\
\hline AR & 18 & 37 \\
\hline AR & 18 & 39 \\
\hline AR & 18 & 42 \\
\hline K & 19 & 40 \\
\hline$K$ & 19 & 42 \\
\hline $\mathrm{K}$ & 19 & 43 \\
\hline CA & 20 & 41 \\
\hline CA & 20 & 45 \\
\hline CA & 20 & 47 \\
\hline CA & 20 & 48 \\
\hline SC & 21 & $44 M$ \\
\hline SC & 21 & 46 \\
\hline SC & 21 & 47 \\
\hline SC & 21 & 48 \\
\hline TI & 22 & 44 \\
\hline$v$ & 23 & 48 \\
\hline$v$ & 23 & 49 \\
\hline$v$ & 23 & 50 \\
\hline CR & 24 & 48 \\
\hline CR & 24 & 51 \\
\hline MN & 25 & 52 \\
\hline$M N$ & 25 & 53 \\
\hline$M N$ & 25 & 54 \\
\hline FE & 26 & 55 \\
\hline FE & 26 & 59 \\
\hline FE & 26 & 60 \\
\hline $\mathrm{CO}$ & 27 & 55 \\
\hline CO & 27 & 56 \\
\hline CO & 27 & 57 \\
\hline $\mathrm{CO}$ & 27 & 58 \\
\hline co & 27 & 60 \\
\hline NI & 28 & 56 \\
\hline VI & 28 & 57 \\
\hline VI & 28 & 59 \\
\hline N I & 28 & 63 \\
\hline NI & 28 & 66 \\
\hline $\mathrm{CU}$ & 29 & 64 \\
\hline
\end{tabular}

$\begin{array}{ll}\text { ADULT } & \text { INFANT } \\ \text { UC } / M * 3 & \text { UC } / M * 3 \\ & \\ 6.4 E+02 & 6.4 E+02 \\ 3.5 E+00 & 3.4 E+00 \\ 4.1 E-03 & 4.1 E-03 \\ 2.7 E-03 & 2.7 E-03 \\ 2.1 E+01 & 2.1 E+01 \\ 5.0 E+04 & 1.5 E+04 \\ 9.7 E+03 & 3.6 E+03 \\ 1.6 E-03 & 1.6 E-03 \\ 3.5 E-02 & 3.5 E-02 \\ 5.7 E-02 & 5.1 E-02 \\ 3.5 E-01 & 3.3 E-01 \\ 4.0 E+03 & 3.9 E+03 \\ 2.0 E+01 & 2.0 E+01 \\ 1.4 E+05 & 1.4 E+05 \\ 7.3 E+00 & 7.3 E+00 \\ 7.9 E-01 & 7.9 E-01 \\ 3.6 E-01 & 3.6 E-01 \\ 6.1 E+03 & 1.6 E+03 \\ 4.4 E+03 & 1.7 E+03 \\ 2.6 E+02 & 2.6 E+02 \\ 4.7 E+02 & 4.7 E+02 \\ 1.0 E+03 & 7.9 E+02 \\ 2.5 E-01 & 2.5 E-01 \\ 4.9 E-01 & 3.0 E-01 \\ 1.2 E-02 & 1.2 E-02 \\ 2.9 E+00 & 2.0 E+00 \\ 5.9 E-01 & 3.3 E-01 \\ 4.8 E-04 & 4.8 E-04 \\ 4.0 E-01 & 3.7 E-01 \\ 1.1 E+01 & 1.1 E+01 \\ 1.9 E-03 & 1.9 E-03 \\ 1.7 E+00 & 6.6 E-01 \\ 2.4 E+00 & 2.3 E+00 \\ 1.2 E-02 & 9.6 E-03 \\ 4.3 E-03 & 4.3 E-03 \\ 7.7 E-04 & 7.6 E-04 \\ 1.8 E+00 & 1.8 E+00 \\ 2.0 E-01 & 1.9 E-01 \\ 5.5 E-04 & 5.5 E-04 \\ 1.5 E+01 & 4.9 E+00 \\ 5.7 E-02 & 5.6 E-02 \\ 2.4 E-01 & 2.4 E-01 \\ 1.3 E-01 & 1.3 E-01 \\ 1.8 E-03 & 1.8 E-03 \\ 3.3 E+00 & 2.6 E+00 \\ 4.1 E+01 & 2.1 E+01 \\ 1.1 E+00 & 1.1 E+00 \\ 5.1 E-01 & 5.1 E-01 \\ 2.4 E+01 & 1.5 E+01 \\ 2.5 E+02 & 6.7 E+01 \\ & \\ 3.9 & \end{array}$

VIA SEAWATER.

$\begin{array}{lcc}\text { ELEMENT } & \text { ATOMIC } & \text { I SOTOPE } \\ \text { SYMBOL } & \text { NO. }(2) & \text { MASS (A) } \\ & & \\ \text { CU } & 29 & 67 \\ \text { ZN } & 30 & 65 \\ \text { ZN } & 30 & 69 M \\ \text { ZN } & 30 & 72 \\ \text { GA } & 31 & 67 \\ \text { GA } & 31 & 72 \\ \text { GE } & 32 & 68 \\ \text { GE } & 32 & 69 \\ \text { GE } & 32 & 71 \\ \text { AS } & 33 & 71 \\ \text { AS } & 33 & 72 \\ \text { AS } & 33 & 73 \\ \text { AS } & 33 & 74 \\ \text { AS } & 33 & 76 \\ \text { AS } & 33 & 77 \\ \text { SE } & 34 & 72 \\ \text { SE } & 34 & 75 \\ \text { SE } & 34 & 79 \\ \text { BR } & 35 & 76 \\ \text { ER } & 35 & 77 \\ \text { BR } & 35 & 82 \\ \text { KR } & 36 & 76 \\ \text { KR } & 36 & 79 \\ \text { KR } & 36 & 81 \\ \text { KR } & 36 & 85 \\ \text { RB } & 37 & 83 \\ \text { RB } & 37 & 84 \\ \text { RE } & 37 & 86 \\ \text { RB } & 37 & 87 \\ \text { SR } & 38 & 82 \\ \text { SR } & 38 & 83 \\ \text { SR } & 38 & 85 \\ \text { SR } & 38 & 89 \\ \text { SR } & 38 & 90 \\ Y & 39 & 86 \\ Y & 39 & 87 \\ Y & 39 & 87 M \\ Y & 39 & 88 \\ Y & 39 & 90 \\ Y & 39 & 91 \\ \text { ZR } & 40 & 86 \\ Z R & 40 & 88 \\ \text { ZR } & 40 & 89 \\ \text { ZR } & 40 & 93 \\ \text { ZR } & 40 & 95 \\ \text { ZR } & 40 & 97 \\ \text { NS } & 41 & 90 \\ \text { NB } & 41 & 91 \\ \text { NB } & 41 & 91 M \\ \text { NB } & 41 & 92\end{array}$

$10 / 09 / 68$

FAGE 36
ADULT INFANT

$U C / M * * 3 \quad U C / M * * 3$

2. $6 E+01 \quad 1.7 E+01$

4. $8 \mathrm{E}-03 \quad 4.8 \mathrm{E}-03$

4. $3 E+00 \quad 1.2 E+00$

1. 2E-01 7.0E-02

1. $2 E+01 \quad 8.0 E+00$

1. $0 E+01 \quad 2.9 E+00$

4. 1E-01 1.5E-03

6. $7 E+00 \quad 5.9 E+00$

$5.4 E+01 \quad 3.4 E+01$

4. $2 E+01 \quad 1.7 E+01$

7. $2 E+01 \quad 7.0 E+01$

$\begin{array}{ll}7.2 E+01 & 7.0 E+01 \\ 2.0 E+00 & 1.8 E+00\end{array}$

$5.6 E+01 \quad 2.4 E+01$

2. $4 E+02 \quad 1.2 E+02$

9. $4 E-01$ 8. $0 E-01$

3. $O E-01$ 3. $0 E-01$

6. $0 E-02$

8. $1 E+03$

8.1E+03 2.6E+03

1. $8 E+0$

1. $7 E+04$

1. $3 E+04$

4. $2 E+01$

8. $2 E+00$

1. $9 E+01$

1. $5 E+01$

5. $O E+01$

1. $4 E+00$

1. $8 E+01$

2. $1 E+03$

4. $6 E+01$

4. $1 E+01$

$1.8 E-01$

2. $9 E+00$

$1.1 E+00$

1. $0 E+01$

7. $4 E-03$

9. $3 E-01$

6. $5 E+01$

2. $6 E-01$

$1.6 E+01$

4. $4 E-01$

$6.4 E-01$

9. $6 E+01$

3. $2 E-01$

2. $2 \mathrm{E}-03$

2. $1 E-02$

1. $5 E+03$

$9.1 E+02$

$5.1 E+03$

$6.4 E+03$

4. $2 E+01$

政

1. $9 E+01$

$1.5 E+01$

4.6E+01

1. $7 \mathrm{E}+01$

9. $9 E+02$

$4.5 E+01$

3. $9 E+01$

1. $8 \mathrm{E}-01$

8. $3 E-01$

7. $3 E-01$

2. $9 E+00$

$5.9 E-01$

. $5 \mathrm{E}-02$

4. $5 \mathrm{E}-02$

2. $5 E+01$

1. $I E+01$

$4.4 \mathrm{E}-01$

6. 2E-01

3. $1 E+01$

$9.2 E-02$

2. $2 \mathrm{E}-03$

2. $0 \mathrm{E}-02$

3. $0 E-02$ 


$\begin{array}{llcl}\text { ATOMIC } & \text { ISOTOPE } & \text { ADULT } & \text { INFANT } \\ \text { NO.( } Z \text { ) } & \text { MASS(A) } & U C / M * * 3 & \text { UC } / M * * 3\end{array}$

$$
\begin{aligned}
& 41 \\
& 41 \\
& 41 \\
& 41 \\
& 41 \\
& 42 \\
& 42 \\
& 43 \\
& 43 \\
& 43 \\
& 43 \\
& 43 \\
& 43 \\
& 43 \\
& 44 \\
& 44 \\
& 44 \\
& 45 \\
& 45 \\
& 45 \\
& 45 \\
& 45 \\
& 45 \\
& 46 \\
& 46 \\
& 46 \\
& 46 \\
& 46 \\
& 47 \\
& 47 \\
& 47 \\
& 47 \\
& 47 \\
& 48 \\
& 48 \\
& 48 \\
& 48 \\
& 49 \\
& 49 \\
& 49 \\
& 50 \\
& 50 \\
& 50 \\
& 50 \\
& 50 \\
& 50 \\
& 50 \\
& 50 \\
& 51 \\
& 51 \\
&
\end{aligned}
$$

$\begin{array}{ll}7.6 E-03 & 7.5 E-03 \\ 2.2 E-05 & 2.2 E-05 \\ 1.1 E-02 & 1.0 E-02 \\ 1.1 E-01 & 7.5 E-02 \\ 2.9 E-01 & 1.1 E-01 \\ 9.4 E+00 & 9.4 E+00 \\ 8.7 E+02 & 5.6 E+02 \\ 3.7 E+02 & 1.3 E+02 \\ 1.4 E+00 & 1.4 E+00 \\ 1.1 E+01 & 8.4 E+00 \\ 5.9 E-01 & 5.9 E-01 \\ 8.7 E+00 & 8.6 E+00 \\ 8.0 E-03 & 8.0 E-03 \\ 1.3 E-01 & 1.3 E-01 \\ 2.7 E+01 & 1.8 E+01 \\ 1.4 E+00 & 1.3 E+00 \\ 3.5 E-02 & 3.4 E-02 \\ 1.4 E-01 & 1.2 E-01 \\ 1.9 E+00 & 7.1 E-01 \\ 1.6 E-03 & 1.6 E-03 \\ 8.5 E-01 & 6.3 E-01 \\ 2.8 E-03 & 2.7 E-03 \\ 5.3 E+00 & 2.7 E+00 \\ 5.2 E-01 & 3.7 E-01 \\ 2.1 E+00 & 1.9 E+00 \\ 2.4 E-01 & 2.4 E-01 \\ 2.7 E+01 & 7.3 E+00 \\ 2.2 E+00 & 8.2 E-01 \\ 5.6 E-01 & 5.4 E-01 \\ 1.3 E+00 & 1.1 E+00 \\ 5.3 E-03 & 5.3 E-03 \\ 3.4 E-02 & 3.3 E-02 \\ 8.3 E+00 & 5.9 E+00 \\ 5.0 E-03 & 5.0 E-03 \\ 3.2 E-04 & 3.2 E-04 \\ 1.5 E-01 & 9.1 E-02 \\ 6.0 E-03 & 5.8 E-03 \\ 7.5 E-02 & 4.9 E-02 \\ 1.0 E-03 & 9.7 E-04 \\ 3.7 E-05 & 3.7 E-05 \\ 3.6 E-01 & 3.6 E-01 \\ 4.4 E+00 & 3.9 E+00 \\ 8.0 E-01 & 7.9 E-01 \\ 3.0 E+02 & 1.3 E+02 \\ 2.5 E-02 & 2.5 E-02 \\ 2.1 E-01 & 2.0 E-01 \\ 1.1 E+00 & 9.9 E-01 \\ 7.0 E-04 & 7.0 E-04 \\ 5.1 E+00 & 2.6 E+00 \\ 1.9 E-02 & 1.5 E-02 \\ 1.90\end{array}$

96

97

\section{ELEMENT} SYMBOL ATOMIC ISOTOPE
NO. (Z) MASS(A)

$\begin{array}{lll}\text { SB } & 51 & 1 \\ \text { SB } & 51 & 12 \\ \text { SB } & 51 & 12 \\ \text { SB } & 51 & 12 \\ \text { SB } & 51 & 1 \\ \text { TE } & 52 & 1 \\ \text { TE } & 52 & 1 \\ \text { TE } & 52 & 1\end{array}$

$7.5 E-03$

$1.0 E-02$

$.5 E-02$
$1.1 E-01$

$4 E+D J$

. $3 E+02$

. $4 E+0 J$

8. $6 E+0 D$

8. $0 E-03$

$1.8 E+O 1$

3.4E-02

$1.2 \mathrm{E}-01$

$.6 E-03$

6.3E-01

2. $7 E+00$

3.7E-01

$2.4 E-01$

$8.2 E-01$

5. $3 \mathrm{E}-03$

$6.9 E+D J$

3. $2 \mathrm{E}-04$

$1 E-02$

$9 E-02$

$7 E-05$

$6 E-01$

$9 E-01$

5E-02

$9.9 E-01$

2. $6 E+0 D$

$1.5 E-02$

122
124
125
126
127
118
119
$119 M$
121
$121 M$
123
$123 M$
$125 M$
$127 M$
$129 M$
$131 M$
132
123
124
125
126
129
130
131
133
122
125
127
$129 M$
$131 M$
133
$133 M$
129
131
132
134
135
136
137
128
131
133
$133 M$
$135 M$
140
135
137
138
140
134

ADULT UC $/ M * * 3$

1. 1E-01 1. $6 E-03$

4. 2E-04

7. 1E-0 3

2. IE-01

2. $1 E-01$

1. $8 \mathrm{E}-01$

1. 5E-01

1. $O E-02$

5. $9 E-03$

4. $8 E-02$

1. $7 E-01$

3. $8 \mathrm{E}-02$

4. $6 E-02$

2. $0 E-01$

2. $8 E+05$

1. $3 E+03$

2. $2 \mathrm{E}+03$

1. $4 E+03$

1. $5 E+01$

2. $2 E+03$

2. $2 E+03$

5. $9 E+03$

3. $2 E+04$

5. $5 E+02$

2. $8 E+03$

$2.6 E+03$

5. $9 E+03$

7. $7 E+03$

1. $7 E+03$

2. $1 E+03$

1. $7 E+02$

4. 3E- 01

1. $5 E+00$

2. $3 E+O 1$

1. $4 \mathrm{E}-01$

1. $1 E+01$

4. $1 E-02$

$7.0 E+01$

7. $0 E+01$

2. $8 E+02$

8. $3 E+01$

7. $4 E-03$

2. 2E-04

7. $6 \mathrm{E}-01$
INF AN T

6. $9 E-02$ 1. $5 E-03$ 4. $2 E-04$ $6.3 E-03$ 4. 5E-02

$1.6 E-01$ $1.1 E+00$ 1. 3E-01

1. $4 \mathrm{E}-01$

1. $0 E-02$

5. $9 \mathrm{E}-03$

4. 7E-02

1. $6 \mathrm{E}-01$

3. 8E-02

4. $4 E-02$

4. $5 E-01$

$1.4 \mathrm{E}-01$

$7.3 E+04$

$9.3 E+02$

2. $2 E+03$

1. $3 E+03$

1. $5 E+01$

$6.8 E+03$

1. $8 E+03$

8. $0 E+03$

2. $1 E+03$

1. $1 E+04$

5. $3 E+02$

$2.4 E+03$

2. $3 E+03$

$4.6 E+03$

4. $7 E+03$

7. $8 E+02$

1. $9 E+03$

$1.4 E+02$

4. 2 E-01

1. $5 E+00$

2. $1 E+01$

1. $4 E-01$

$6.5 E+00$

$3.1 E+00$

4. $1 E-02$

3. $6 E+01$

3. $6 E+01$

$1.2 E+02$

8. $4 E-01$

2. $9 E+00$

7. $4 \mathrm{E}-03$

2. $2 \mathrm{E}-04$

4. OE-O1 
TABLE 9A. UNIT-RAD LOVTAMINATION FOR GI TRACT VIA SEAWATER.

$\begin{array}{lcccc}\text { ELEMENT } & \text { ATOMIC } & \text { ISOTOPE } & \text { ADULT } & \text { INFANT } \\ \text { SYMBOL } & \text { VO. } 121 & \text { MASS (A) } & \text { UC/M**3 } & \text { UC/M**3 } \\ \text { CE } & 58 & 135 & 9.1 E+00 & 3.0 E+00 \\ \text { CE } & 58 & 137 M & 8.6 E+00 & 4.2 E+00 \\ \text { CE } & 58 & 139 & 7.6 E-02 & 7.5 E-02 \\ \text { CE } & 58 & 141 & 2.7 E-01 & 2.6 E-01 \\ \text { CE } & 58 & 142 & 2.5 E-04 & 2.5 E-04 \\ \text { CE } & 58 & 143 & 2.7 E+00 & 1.3 E+00 \\ \text { CE } & 58 & 144 & 2.0 E-03 & 2.0 E-03 \\ \text { PR } & 59 & 142 & 5.9 E+00 & 2.0 E+00 \\ \text { PR } & 59 & 143 & 4.2 E-01 & 3.8 E-01 \\ \text { ND } & 60 & 140 & 7.6 E-01 & 5.2 E-01 \\ \text { ND } & 60 & 144 & 1.6 E-04 & 1.6 E-04 \\ \text { ND } & 60 & 147 & 7.9 E-02 & 7.0 E-02 \\ \text { PM } & 61 & 143 & 1.1 E-02 & 1.1 E-02 \\ \text { PM } & 61 & 144 & 1.1 E-03 & 1.1 E-03 \\ \text { PM } & 61 & 145 & 4.2 E-03 & 4.2 E-03 \\ \text { PM } & 61 & 146 & 7.8 E-04 & 7.8 E-04 \\ \text { PM } & 61 & 147 & 4.8 E-04 & 4.8 E-04 \\ \text { PM } & 61 & 148 & 1.6 E-01 & 1.3 E-01 \\ \text { PM } & 61 & 148 M & 4.7 E-03 & 4.6 E-03 \\ \text { PM } & 61 & 149 & 1.9 E+00 & 1.1 E+00 \\ \text { PM } & 61 & 151 & 1.9 E+00 & 8.4 E-01 \\ \text { SM } & 62 & 141 & 2.7 E-02 & 2.5 E-02 \\ \text { SM } & 62 & 145 & 3.9 E-02 & 3.8 E-02 \\ \text { SM } & 62 & 146 & 1.1 E-04 & 1.1 E-04 \\ \text { SM } & 62 & 147 & 1.2 E-04 & 1.2 E-04 \\ \text { SM } & 62 & 148 & 7.0 E-05 & 7.0 E-05 \\ \text { SM } & 62 & 149 & 1.5 E-04 & 1.5 E-04 \\ \text { SM } & 62 & 151 & 2.0 E-02 & 2.0 E-02 \\ \text { SM } & 62 & 153 & 5.3 E+00 & 3.0 E+00 \\ \text { EU } & 63 & 145 & 5.4 E-01 & 4.3 E-01 \\ \text { EU } & 63 & 146 & 1.2 E-01 & 8.8 E-02 \\ \text { EU } & 63 & 146 M & 7.3 E-01 & 3.7 E-01 \\ \text { EU } & 63 & 147 & 2.8 E-02 & 2.6 E-02 \\ \text { EU } & 63 & 148 & 6.5 E-02 & 6.3 E-02 \\ \text { EU } & 63 & 149 & 3.1 E-02 & 3.1 E-02 \\ \text { EU } & 63 & 150 & 1.0 E-03 & 1.0 E-03 \\ \text { EU } & 63 & 150 M & 3.1 E+00 & 8.2 E-01 \\ \text { EU } & 63 & 152 & 4.1 E-04 & 4.1 E-04 \\ \text { EU } & 63 & 154 & 3.1 E-04 & 3.1 E-04 \\ \text { EU } & 63 & 155 & 2.6 E-02 & 2.6 E-02 \\ \text { EU } & 63 & 156 & 8.2 E-02 & 7.5 E-02 \\ \text { EU } & 63 & 157 & 8.9 E+00 & 2.6 E+00 \\ \text { GD } & 64 & 146 & 7.4 E-03 & 7.2 E-03 \\ \text { GD } & 64 & 147 & 9.6 E-01 & 3.9 E-01 \\ \text { GD } & 64 & 148 & 8.9 E-05 & 8.9 E-05 \\ \text { GD } & 64 & 149 & 2.0 E-01 & 1.8 E-01 \\ \text { GD } & 64 & 150 & 4.8 E-05 & 4.8 E-05 \\ \text { GD } & 64 & 151 & 4.6 E-02 & 4.5 E-02 \\ \text { GD } & 64 & 152 & 1.2 E-04 & 1.2 E-04 \\ \text { GD } & 64 & 153 & 5.3 E-02 & 5.3 E-02\end{array}$

$\begin{array}{lllll}\text { ELEMENT } & \text { ATOMIC } & \text { I SOTOPE } & \text { ADULT } & \text { INFANT } \\ \text { SYMBOL } & \text { ND. }(Z) & \text { MASSIA) } & \text { UC/M**3 } & \text { UC } / M * * 3\end{array}$

GO $159-64 \quad 162$

1. $9 E+01$

7. $1 E+00$

2. $1 E+00$

2. $4 \mathrm{E}+00$

8 . $0 E+00$

9. $7 E-01$

2. $9 E-01$

2. $2 E-04$

1. $9 E-02$

1. $6 E+00$

1. $1 E+01$

2. $8 E-05$

3. $6 E+00$

4.5E-05

1. $8 E-01$

6. 5E- $5 E-03$

4. $O E+00$

2. $O E-04$

1. $3 E+00$

2. $6 E+00$

1. $4 E+00$

1. $3 E+00$

3. $7 E-01$

1. $4 \mathrm{E}-02$

3. 9E-02

1. $3 E-01$

1. $1 E+00$

2. $6 E+00$

1. 8E-01

3. $7 E+00$

1. $3 E+00$

5. $2 E-01$

1. $9 E+00$

3. $4 E-01$

5. $1 E-03$

1. 1E-03

2. $1 E-02$

3. $3 \mathrm{E}-04$

1. $9 E+00$
$5.9 E-02$

4. $1 E+00$

8. $3 E+00$

1. $1 E-03$

$1.1 E-04$

6. $4 E-02$

6. $2 E+00$
$3.5 E-03$

$2.4 E+00$

$6.8 E-01$

$1.5 \mathrm{E}+00$

2. $9 E+00$

7. 7E-01

2.3E-01

2. 2 E-04

$2.2 E-04$
$1.8 E-02$

$1.3 E+00$

$4.6 E+00$

2. $8 \mathrm{E}-05$

9. $6 \mathrm{E}-01$

$4.5 E-05$

1. $8 \mathrm{E}-01$

4. 5E-01

4. 5E-03

$1.7 E+00$

1.7E+00

5. $9 E-01$

5. $9 E-01$
$2.3 E+00$

2. $3 E+00$

7. $8 \mathrm{E}-01$

5. $8 \mathrm{E}-01$

3. $2 \mathrm{E}-01$

1. $4 E-02$

3. $9 \mathrm{E}-02$

7. $2 \mathrm{E}-01$

$1.6 E+00$

1. $7 \mathrm{E}-01$

2. $8 E+00$

$6.6 \mathrm{E}-01$

3. $0 E-01$

$1.6 E+00$

2. 8E-01

$5.1 \mathrm{E}-03$

$1.1 \mathrm{E}-03$

2. OE-02

3. $3 E-04$

1. $6 E+00$

5. $8 \mathrm{E}-02$

1. $O E+00$

2. $3 E+00$

1. $1 E-03$

1. $2 E+00$

1. $1 \mathrm{E}-04$

6. $3 E-02$

$6.8 E-02$ 


\begin{tabular}{|c|c|c|c|c|}
\hline $\begin{array}{l}\text { ELEMENT } \\
\text { SYMBOL }\end{array}$ & $\begin{array}{l}\text { ATOM IC } \\
\text { ND. }(Z)\end{array}$ & $\begin{array}{l}\text { ISOTOPE } \\
\text { MASS (A) }\end{array}$ & $\begin{array}{l}\text { ADULT } \\
\text { UC/M**3 }\end{array}$ & $\begin{array}{l}\text { INFANT } \\
\text { UC } / M * * 3\end{array}$ \\
\hline 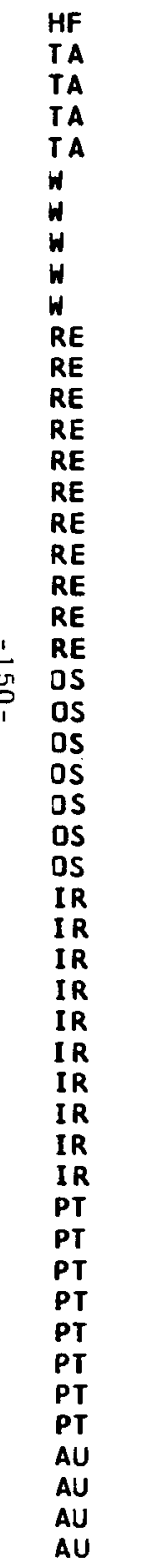 & $\begin{array}{l}72 \\
73 \\
73 \\
73 \\
73 \\
74 \\
74 \\
74 \\
74 \\
74 \\
75 \\
75 \\
75 \\
75 \\
75 \\
75 \\
75 \\
75 \\
75 \\
75 \\
75 \\
76 \\
76 \\
76 \\
76 \\
76 \\
76 \\
76 \\
77 \\
77 \\
77 \\
77 \\
77 \\
77 \\
77 \\
77 \\
77 \\
77 \\
78 \\
78 \\
78 \\
78 \\
78 \\
78 \\
78 \\
78 \\
79 \\
79 \\
79 \\
79\end{array}$ & $\begin{array}{l}182 \\
177 \\
179 \\
182 \\
183 \\
178 \\
181 \\
185 \\
187 \\
188 \\
180 \\
181 \\
182 \\
182 M \\
183 \\
184 \\
184 M \\
186 \\
187 \\
188 \\
189 \\
182 \\
183 \\
185 \\
191 \\
191 M \\
193 \\
194 \\
185 \\
186 \\
187 \\
188 \\
189 \\
190 \\
192 \\
192 M \\
193 M \\
194 \\
188 \\
190 \\
191 \\
192 \\
193 \\
193 M \\
195 M \\
197 \\
193 \\
194 \\
195 \\
196\end{array}$ & $\begin{array}{l}1.7 \mathrm{E}-04 \\
7.8 \mathrm{E}-01 \\
3.3 \mathrm{E}-02 \\
7.3 \mathrm{E}-03 \\
5.0 \mathrm{E}-01 \\
2.7 \mathrm{E}+00 \\
1.1 \mathrm{E}+01 \\
1.1 \mathrm{E}+01 \\
2.9 \mathrm{E}+02 \\
1.1 \mathrm{E}+00 \\
5.5 \mathrm{E}+02 \\
6.1 \mathrm{E}+02 \\
1.2 \mathrm{E}+02 \\
1.4 \mathrm{E}+03 \\
1.3 \mathrm{E}+01 \\
8 \cdot 5 \mathrm{E}+00 \\
1.5 \mathrm{E}+00 \\
2.6 \mathrm{E}+02 \\
3.1 \mathrm{E}+02 \\
1.3 \mathrm{E}+03 \\
1.6 \mathrm{E}+03 \\
1.2 \mathrm{E}+01 \\
7.7 \mathrm{E}+01 \\
1.2 \mathrm{E}-01 \\
3.2 \mathrm{E}+00 \\
1.9 \mathrm{E}+02 \\
2.9 \mathrm{E}+01 \\
1.9 \mathrm{E}-03 \\
5.6 \mathrm{E}+00 \\
3.3 \mathrm{E}+00 \\
8.5 \mathrm{E}+00 \\
5.5 \mathrm{E}-01 \\
2.7 \mathrm{E}-01 \\
7.5 \mathrm{E}-02 \\
1.5 \mathrm{E}-02 \\
1.3 \mathrm{E}-04 \\
1.3 \mathrm{E}+00 \\
3.3 \mathrm{E}+00 \\
3.4 \mathrm{E}-02 \\
3.5 \mathrm{E}-05 \\
7.8 \mathrm{E}-01 \\
4.2 \mathrm{E}-05 \\
1.5 \mathrm{E}-03 \\
1.1 \mathrm{E}+00 \\
1.1 \mathrm{E}+00 \\
9.5 \mathrm{E}+00 \\
2.7 \mathrm{E}+03 \\
2.3 \mathrm{E}+02 \\
1.1 \mathrm{E}+01 \\
1.1 \mathrm{E}+02\end{array}$ & $\begin{array}{l}1.7 E-04 \\
4.7 E-01 \\
3.2 E-02 \\
7.2 E-03 \\
3.8 E-01 \\
2.6 E+00 \\
1.1 E+01 \\
1.1 E+01 \\
1.2 E+02 \\
1.0 E+00 \\
2.0 E+02 \\
2.2 E+02 \\
7.6 E+01 \\
3.7 E+02 \\
1.3 E+01 \\
8.2 E+00 \\
1.5 E+00 \\
1.8 E+02 \\
3.1 E+02 \\
4.0 E+02 \\
6.4 E+02 \\
4.5 E+00 \\
1.9 E+01 \\
1.2 E-01 \\
2.9 E+03 \\
5.4 E+01 \\
1.4 E+01 \\
1.9 E-003 \\
1.7 E+00 \\
9.7 E-01 \\
2.1 E+00 \\
2.9 E-01 \\
2.4 E-01 \\
6.7 E-02 \\
1.5 E-02 \\
1.3 E-04 \\
1.2 E+00 \\
1.1 E+00 \\
3.0 E-02 \\
3.5 E-05 \\
5.2 E-01 \\
4.2 E-00 \\
1.5 E-03 \\
8.5 E-01 \\
7.9 E-01 \\
3.4 E+00 \\
9.1 E+02 \\
1.2 E+02 \\
1.1 E+01 \\
9.0 E+01\end{array}$ \\
\hline
\end{tabular}

\begin{tabular}{|c|c|c|}
\hline $\begin{array}{l}\text { ELEMENT } \\
\text { SYMEOL }\end{array}$ & $\begin{array}{l}\text { A TOM IC } \\
\text { NO. }(Z)\end{array}$ & $\begin{array}{l}\text { I SOTOP } \\
\text { MASSIA }\end{array}$ \\
\hline$A U$ & 79 & 198 \\
\hline$A U$ & 79 & 199 \\
\hline HG & 80 & 194 \\
\hline HG & 80 & $195 \mathrm{M}$ \\
\hline HG & 80 & 197 \\
\hline HG & 80 & $197 M$ \\
\hline$H G$ & 80 & 203 \\
\hline $\mathrm{TL}$ & 81 & 200 \\
\hline $\mathrm{TL}$ & 81 & 201 \\
\hline TL & 81 & 202 \\
\hline TL & 81 & 204 \\
\hline $\mathrm{PE}$ & 82 & 200 \\
\hline PE & 82 & 202 \\
\hline PB & 82 & 203 \\
\hline PB & 82 & 204 \\
\hline PB & 82 & 205 \\
\hline PB & 82 & 210 \\
\hline BI & 83 & 205 \\
\hline BI & 83 & 206 \\
\hline BI & 83 & 207 \\
\hline BI & 83 & 208 \\
\hline BI & 83 & 210 \\
\hline BI & 83 & $210 \mathrm{M}$ \\
\hline PO & 84 & 206 \\
\hline PO & 84 & 208 \\
\hline PO & 84 & 209 \\
\hline PC & 84 & 210 \\
\hline RN & 86 & 211 \\
\hline RN & 86 & 222 \\
\hline RA & 88 & 223 \\
\hline RA & 88 & 224 \\
\hline RA & 88 & 225 \\
\hline RA & 88 & 226 \\
\hline RA & 88 & 228 \\
\hline$A C$ & 89 & 225 \\
\hline$A C$ & 89 & 227 \\
\hline TH & 90 & 227 \\
\hline TH & 90 & 228 \\
\hline $\mathrm{TH}$ & 90 & 229 \\
\hline TH & 90 & 230 \\
\hline $\mathrm{TH}$ & 90 & 231 \\
\hline TH & 90 & 232 \\
\hline TH & 90 & 234 \\
\hline PA & 91 & 228 \\
\hline PA & 91 & 229 \\
\hline PA & 91 & 230 \\
\hline PA & 91 & 231 \\
\hline PA & 91 & 232 \\
\hline PA & 91 & 233 \\
\hline U & 92 & 230 \\
\hline
\end{tabular}

ADULT
UC $\triangle M * * 3$

UC $/ M * 3$

3. $1 E+02 \quad 2.0 E+02$

B. $2 E+02 \quad 5.5 E+02$

2. $1 E-03-03$

4. $4 E-01$

1.

4. 4E-02 4.2E-02

- 2E-02

$2.9 E-02$

$2.5 \mathrm{E}-02$

$3.1 E-03$

4. $5 E-05$

1. $4 E+02$

4.5E-05

5. $O E+01$

1. $7 E-02$

4.7E+01

8. $0 E+01$

4. $0 E-03$

1. $2 E-01$

2. $7 E-03$

$1.2 E-01$

2. $7 E-03$

$6.5 E-03$

1. $9 E-02$

. $7 E-05$

8. $6 E-06$

8. $0 E-03$

4. $2 E-06$

5. $9 E-02$

1. $0 E-04$

1. $9 E-03$

1. $8 \mathrm{E}+02$

7. $5 E+02$

5. $9 E-01$

2. $3 E+00$

4. $5 \mathrm{E}-01$

7. $3 E-04$

2. $3 E-03$

8. $4 E-02$

1. $3 E-04$

1. $2 E-06$

2. $8 E-08$

2. $7 E-09$

2. 5E-09

3. $9 E-05$

2. $2 \mathrm{E}-09$

7. $1 E-07$

5. $9 E-03$

1. $6 E-02$

1. $6 E-02$

8. $6 \mathrm{E}-06$

6. $6 E-06$

4. $2 \mathrm{E}-06$

5. $0 \mathrm{E}-02$

1. $7 \mathrm{E}-04$

4. OE-05

1. 8E-03

$5.4 E+00$

$5.2 E-01$
$1.6 E+00$

$1.6 \mathrm{E}+00$

7.3E-04

2. 3E-03

7. $3 \mathrm{E}-02$

$1.3 E-04$

1. $8 \mathrm{E}-08$

2.7E-09

2.7E-09

$2.5 E-09$
$1.6 E-05$

$1.6 \mathrm{E}-05$
$2.2 \mathrm{E}-09$

6. 7E-07

- $6 E+00$

6. $2 E-01$

. 2E-01

3. 5E-02

7. $O E-05$

$2.1 E-02$
$2.7 E-02$

4.1E-O

7. OE-O5

3. $9 E-01$

2. $5 \mathrm{E}-02$ 
TABLE 9A. UNIT-RAD CONTAMINATION FOR GI TRACT VIA SEAWATER.

\begin{tabular}{lcccc}
$\begin{array}{l}\text { ELEMENT } \\
\text { SYMBOL }\end{array}$ & $\begin{array}{c}\text { ATOMIC } \\
\text { ND. }(2)\end{array}$ & $\begin{array}{c}\text { ISOTOPE } \\
\text { MASS(A) }\end{array}$ & $\begin{array}{c}\text { ADULT } \\
\text { UC } / M * * 3\end{array}$ & $\begin{array}{c}\text { INFANT } \\
\text { UC } / M * * 3\end{array}$ \\
$U$ & & & & \\
$U$ & 92 & 231 & $1.7 E-01$ & $1.3 E-01$ \\
$U$ & 92 & 232 & $7.9 E-05$ & $7.9 E-05$ \\
$U$ & 92 & 233 & $7.2 E-05$ & $7.2 E-05$ \\
$U$ & 92 & 234 & $6.8 E-05$ & $6.8 E-05$ \\
$U$ & 92 & 235 & $6.3 E-05$ & $6.3 E-05$ \\
$U$ & 92 & 236 & $6.2 E-05$ & $6.2 E-05$ \\
$U$ & 92 & 237 & $8.6 E-02$ & $7.1 E-02$ \\
$U$ & 92 & 238 & $5.9 E-05$ & $5.9 E-05$ \\
$U$ & 92 & 240 & $2.5 E+00$ & $6.9 E-01$ \\
NP & 93 & 234 & $1.5 E-01$ & $1.1 E-01$ \\
NP & 93 & 235 & $1.1 E-03$ & $1.1 E-03$ \\
\hline NP & 93 & 236 & $5.9 E-05$ & $5.9 E-05$ \\
NP & 93 & $236 M$ & $1.3 E+00$ & $5.0 E-01$ \\
$N$ & 93 & 237 & $6.3 E-05$ & $6.2 E-05$ \\
NP & 93 & 238 & $3.6 E-01$ & $2.1 E-01$ \\
NP & 93 & 239 & $2.9 E-01$ & $1.8 E-01$ \\
NP & 94 & 236 & $4.4 E-04$ & $4.4 E-04$ \\
PU & 94 & 237 & $1.1 E-02$ & $1.0 E-02$ \\
PU & 94 & 238 & $6.6 E-05$ & $6.6 E-05$ \\
PU & 94 & 239 & $5.6 E-05$ & $5.6 E-05$ \\
PU & 94 & 240 & $5.5 E-05$ & $5.5 E-05$ \\
PU & 94 & $1.1 E-04$ & $1.1 E-04$ \\
PU & 94 & 241 & $5.3 E-05$ & $5.3 E-05$ \\
PU & 94 & 242 & $4.8 E-05$ & $4.7 E-05$ \\
PU & 94 & 244 & $3.7 E-02$ & $3.3 E-02$ \\
PU & 94 & 246 & $3.3 E+00$ & $8.4 E-01$ \\
AM & 95 & 239 & $3.2 E-01$ & $1.9 E-01$ \\
AM & 95 & 240 & $5.7 E-05$ & $5.7 E-05$ \\
AM & 95 & 241 & $1.9 E+00$ & $5.9 E-01$ \\
AM & 95 & 242 & $5.6 E-05$ & $5.6 E-05$ \\
AM & 95 & $242 M$ & $5.0 E-05$ & $5.0 E-05$ \\
AM & 95 & 243 & & \\
& & & &
\end{tabular}

$10 / 09 / 68$

\section{ELEMENT} SYMB OL

ATOMIC

ISUTOPE MASSIA)

$C M$
$C M$
$C M$
$C M$
$C M$
$C M$
$C M$
$C M$
$C M$
$B K$
$B K$
$B K$
EK
BK
$C F$
$C F$
$C F$
$C F$
$C F$
$C F$
$C F$
CS
ES
ES
$E S$
ES
ES
FM
FM
FM
FM

$\begin{array}{cc}96 & 240 \\ 96 & 241 \\ 96 & 242 \\ 96 & 243 \\ 96 & 244 \\ 96 & 245 \\ 96 & 246 \\ 96 & 247 \\ 96 & 248 \\ 97 & 245 \\ 97 & 246 \\ 97 & 247 \\ 97 & 248 \\ 97 & 249 \\ 98 & 246 \\ 98 & 248 \\ 98 & 249 \\ 98 & 250 \\ 98 & 251 \\ 98 & 252 \\ 98 & 253 \\ 99 & 251 \\ 99 & 252 \\ 99 & 253 \\ 99 & 254 \\ 99 & 254 M \\ 99 & 255 \\ 100 & 252 \\ 100 & 253 \\ 100 & 255 \\ 100 & 257\end{array}$

ADULT UC $/ M * * 3$

$1.6 \mathrm{E}-02$

1. $2 E-02$

$2.4 E-03$

8. 3E-0

5. $0 E-05$

4. $8 E-05$

$4.5 E-05$

4.

4. $9 E-02$

9. $9 E-02$

4. $5 \mathrm{E}-05$

8. $9 E-01$

1. $1 E-03$

4. $7 E-01$

$9.6 \mathrm{E}-04$

4. $6 E-05$

1. $0 E-04$

4. $1 E-05$

3. $2 E-04$

1. $7 E-02$

4. $1 E-01$

2. 2E-03

1. $6 E-02$

6. $1 E-04$

3. $5 \mathrm{E}-01$

1. $2 E-02$

8. $O E-01$

9. 1E-02

9. $4 E-01$
PAGE 40

INFAN T UC $/ M * * 3$

$1.5 \mathrm{E}-02$
$1.2 \mathrm{E}-02$

1. $2 E-02$
$2.4 E-03$

6. $7 E-05$

8. 3E-05

5. $0 \mathrm{E}-05$

4. $8 \mathrm{E}-05$

$4.5 E-05$

$4.4 \mathrm{E}-05$

7. $6 \mathrm{E}-02$

2. $0 E-01$

4.5E-05

3. 5 E-01

$2.4 E-01$

$9.6 \mathrm{E}-04$

4. $6 E-05$

1. OE-04

4. 1 E-05

3. $2 \mathrm{E}-04$

1. $6 E-02$

$2.1 E-01$

2.1E-03

1.5E-02

6.1 E-04

1. $8 E-01$

$1.2 E-02$

3. $1 E-01$

6. $8 \mathrm{E}-02$

$3.4 E-01$ 
-

-

- 


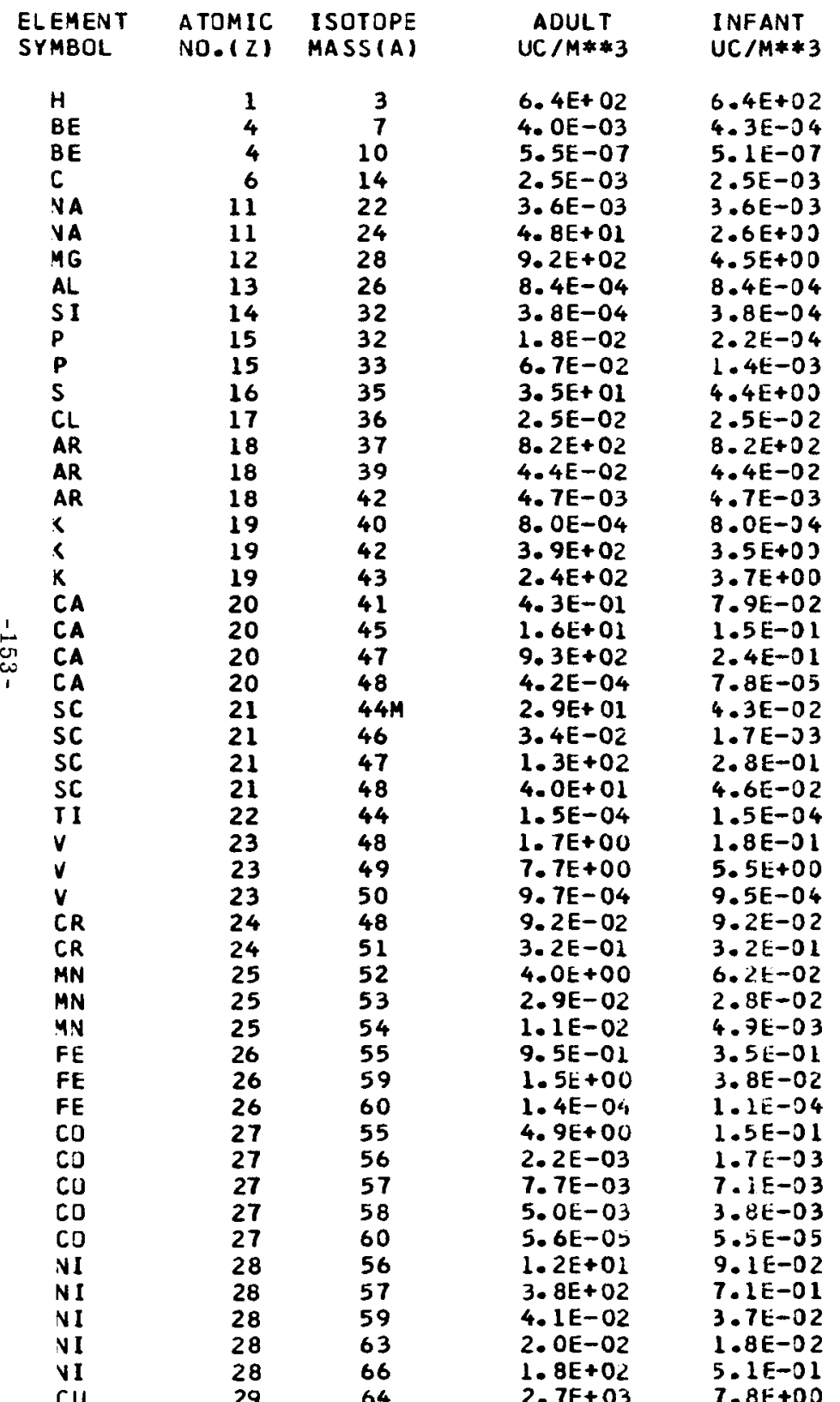

\begin{tabular}{|c|c|c|}
\hline $\begin{array}{l}\text { ELEMENT } \\
\text { SYMBOL }\end{array}$ & $\begin{array}{l}\text { ATJMIC } \\
N O-(Z)\end{array}$ & $\begin{array}{l}\text { I SOTOPE } \\
\text { MASS(A) }\end{array}$ \\
\hline $\mathrm{CU}$ & 29 & 67 \\
\hline $2 N$ & 30 & 65 \\
\hline $2 N$ & 30 & $69 M$ \\
\hline $2 N$ & 30 & 72 \\
\hline GA & 31 & 67 \\
\hline GA & 31 & 72 \\
\hline GE & 32 & 68 \\
\hline $\mathrm{GE}$ & 32 & 69 \\
\hline $\mathrm{GE}$ & 32 & 71 \\
\hline AS & 33 & 71 \\
\hline AS & 33 & 72 \\
\hline AS & 33 & 73 \\
\hline AS & 33 & 74 \\
\hline AS & 33 & 70 \\
\hline AS & 33 & 77 \\
\hline SE & 34 & 72 \\
\hline SE & 34 & 75 \\
\hline SE & 34 & 79 \\
\hline$B R$ & 35 & 76 \\
\hline BR & 35 & 77 \\
\hline BR & 35 & 82 \\
\hline$K R$ & 36 & 76 \\
\hline KR & 36 & 79 \\
\hline KR & 36 & 81 \\
\hline KR & 36 & 85 \\
\hline$R B$ & 37 & 83 \\
\hline RB & 37 & 84 \\
\hline RB & 37 & 86 \\
\hline RB & 37 & 87 \\
\hline$S R$ & 38 & 82 \\
\hline$S R$ & 38 & 83 \\
\hline$S R$ & 38 & 85 \\
\hline SR & 38 & 89 \\
\hline$S R$ & 38 & 90 \\
\hline$Y$ & 39 & 86 \\
\hline$Y$ & 39 & 87 \\
\hline$Y$ & $3 !$ & $87 \mathrm{M}$ \\
\hline$Y$ & 39 & 38 \\
\hline$Y$ & 39 & 50 \\
\hline$Y$ & 39 & 91 \\
\hline $2 R$ & 40 & 86 \\
\hline$Z R$ & 40 & 88 \\
\hline$Z R$ & 40 & 89 \\
\hline ZR & 40 & 95 \\
\hline$Z R$ & 40 & 95 \\
\hline $2 R$ & 40 & 97 \\
\hline NE & 41 & 90 \\
\hline NG & 41 & 91 \\
\hline NB & 41 & $91 M$ \\
\hline NB & 41 & 92 \\
\hline
\end{tabular}

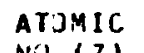

I SOTOPE
MASSIA)

ADULT

INFANT

$U C / M * * 3$

$1.4 E+02$
$5.2 E-03$
$4.6 E+02$
$8.0 E+00$
$2.9 E+00$
$4.7 E+00$
$1.8 E-05$
$2.6 E-03$
$7.1 E-02$
$2.0 E+03$
$1.0 E+03$
$4.0 E+03$
$1.0 E+02$
$1.4 E+03$
$7.1 E+03$
$2.3 E-02$
$8.7 E-03$
$1.7 E-03$
$7.7 E+03$
$1.6 E+03$
$1.4 E+03$
$1.5 E+02$
$1.9 E+02$
$1.3 E+00$
$2.5 E-01$
$4.8 E-02$
$6.5 E-02$
$3.2 E-01$
$1.8 E-03$
$2.8 E+00$
$3.0 E+03$
$2.9 E+00$
$3.2 E+00$
$2.6 E-04$
$4.9 E+02$
$7.9 E+01$
$1.8 E+03$
$2.4 E-02$
$8.0 E+01$
$2.7 E-02$
$2.6 E+03$
$2.8 E-01$
$3.0 E+02$
$4.4 E-02$
$8.9 E-01$
$3.8 E+03$
$1.3 E-01$
$2.2 E-06$
$3.0 E-04$
$2.6 E-03$
1.903

1. $9 E+00$

8. $2 E-04$

$2.1 E-01$
$1.2 E-02$

6. $1 E-01$

2. $2 E-01$

$1.8 E-05$

$2.6 E-03$

$7.1 \mathrm{E}-02$

.

$4 . O E+03$

4. $O E+03$

1. $0 E+02$

7. $1 E+03$

$2.3 E-02$

8. $7 E-03$

1. $7 E-03$

$6.1 E+02$

3. $7 E+02$

$2.2 \mathrm{E}+02$

$15 E+02$

$1.5 E+02$

$1.3 E+00$

$1.3 E+00$
$2.5 E-01$

$2.4 E-02$

1. $9 E-02$

6. $0 E-02$

$1.8 E-03$

4. OE-03

2. $3 E-01$

$1.0 E-02$

. $0 E-03$

4. $2 \mathrm{E}-05$

1. $7 \mathrm{E}-02$

1. $5 E-02$

5. $9 E-02$

1. $5 E-04$

1. $2 \mathrm{E}-02$

$9.0 E-04$

1. $8 \mathrm{E}+00$

2. 2E-02

9. $7 E-01$

3. $9 E-02$

$5.4 E-02$

2. $7 E+00$

8. OE-05

1. $9 E-06$

$1.8 E-05$ 


\begin{tabular}{|c|c|c|c|c|}
\hline $\begin{array}{l}\text { ELEMENT } \\
\text { SYMBOL }\end{array}$ & $\begin{array}{l}\text { ATJMIC } \\
\text { NJ. }(Z)\end{array}$ & $\begin{array}{l}\text { ISOTOPE } \\
\text { MASS (A) }\end{array}$ & $\begin{array}{c}\text { ADULT } \\
\text { UC/M**3 }\end{array}$ & $\begin{array}{l}\text { INFANT } \\
\text { UC } / M * 3\end{array}$ \\
\hline $\begin{array}{l}\text { NB } \\
\text { NB } \\
N B \\
\text { VB } \\
N B \\
M D \\
M D \\
\text { TC } \\
T C \\
T C \\
T C \\
T C \\
T C \\
T C \\
R U \\
R U \\
R U \\
R H \\
R H \\
R H \\
R H \\
R H \\
R H \\
P D \\
P D \\
P D \\
P D \\
P D \\
A G \\
A G \\
A G \\
A G \\
A G \\
C D \\
C D \\
C D \\
C D \\
I N \\
I N \\
I N \\
S N \\
S N \\
S N \\
S N \\
\text { SN } \\
\text { SN } \\
\text { SN } \\
\text { SN } \\
S B \\
S B\end{array}$ & $\begin{array}{l}41 \\
41 \\
41 \\
41 \\
41 \\
42 \\
42 \\
43 \\
43 \\
43 \\
43 \\
43 \\
43 \\
43 \\
44 \\
44 \\
44 \\
45 \\
45 \\
45 \\
45 \\
45 \\
45 \\
46 \\
46 \\
46 \\
46 \\
46 \\
47 \\
47 \\
47 \\
47 \\
47 \\
48 \\
48 \\
48 \\
48 \\
49 \\
49 \\
49 \\
50 \\
50 \\
50 \\
50 \\
50 \\
50 \\
50 \\
50 \\
51 \\
51\end{array}$ & $\begin{array}{l}93 M \\
94 \\
95 \\
95 M \\
96 \\
93 \\
99 \\
95 \\
95 M \\
96 \\
97 \\
97 M \\
98 \\
99 \\
97 \\
103 \\
106 \\
99 \\
100 \\
101 \\
101 M \\
102 \\
105 \\
100 \\
103 \\
107 \\
109 \\
112 \\
105 \\
106 M \\
108 M \\
110 M \\
111 \\
109 \\
113 M \\
115 \\
115 M \\
111 \\
114 M \\
115 \\
113 \\
117 M \\
119 M \\
121 \\
121 M \\
123 M \\
125 \\
126 \\
119 \\
120 M\end{array}$ & 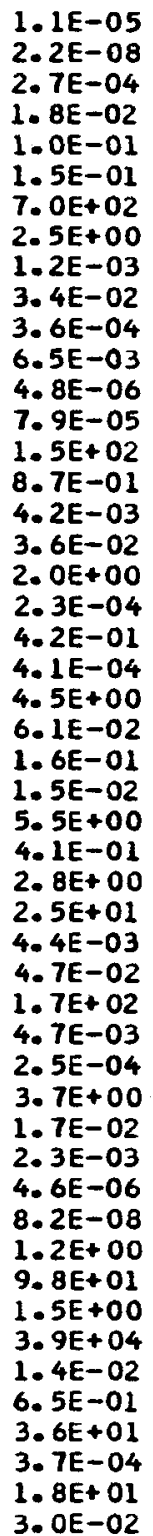 & $\begin{array}{l}6.5 E-06 \\
1.9 E-03 \\
9.0 E-06 \\
6.6 E-05 \\
9.7 E-05 \\
1.5 E-01 \\
9.1 E+00 \\
8.0 E-02 \\
8.4 E-04 \\
5.0 E-03 \\
3.6 E-04 \\
5.1 E-03 \\
4.8 E-05 \\
7.9 E-05 \\
9.6 E-01 \\
7.1 E-02 \\
1.9 E-03 \\
1.7 E-02 \\
9.9 E-02 \\
2.3 E-04 \\
8.9 E-02 \\
3.8 E-04 \\
3.7 E-01 \\
2.2 E-02 \\
1.2 E-01 \\
1.5 E-02 \\
4.4 E-01 \\
4.9 E-02 \\
3.9 E-01 \\
8.0 E-01 \\
3.8 E-03 \\
2.4 E-02 \\
5.0 E+00 \\
3.8 E-03 \\
2.4 E-04 \\
6.9 E-02 \\
4.4 E-03 \\
1.1 E-04 \\
2.1 E-06 \\
8.1 E-08 \\
1.7 E-01 \\
1.9 E+00 \\
3.8 E-01 \\
6.2 E+01 \\
1.2 E-02 \\
9.7 E-02 \\
4.7 E-01 \\
3.4 E-04 \\
2.9 E-01 \\
1.6 E-03\end{array}$ \\
\hline
\end{tabular}

$\begin{array}{lcc}\text { ELEMENT } & \text { ATOMIC } & \text { I SOTO } \\ \text { SYMBOL } & \text { NO. }(Z) & \text { MASS1 } \\ & & \\ \text { SB } & 51 & 122 \\ \text { SB } & 51 & 124 \\ \text { SB } & 51 & 125 \\ \text { SB } & 51 & 126 \\ \text { SB } & 51 & 127 \\ \text { TE } & 52 & 118 \\ \text { TE } & 52 & 119 \\ \text { TE } & 52 & 119 M \\ \text { TE } & 52 & 121 \\ \text { TE } & 52 & 121 \\ \text { TE } & 52 & 123 \\ \text { TE } & 52 & 1234 \\ \text { TE } & 52 & 1254 \\ \text { TE } & 52 & 127 \\ \text { TE } & 52 & 1294 \\ \text { TE } & 52 & 131 M \\ \text { TE } & 52 & 132 \\ \text { I } & 53 & 123 \\ \text { I } & 53 & 124 \\ \text { I } & 53 & 125 \\ \text { I } & 53 & 126 \\ \text { I } & 53 & 129 \\ \text { I } & 53 & 130 \\ \text { I } & 53 & 131 \\ \text { I } & 53 & 133 \\ \text { XE } & 54 & 122 \\ \text { XE } & 54 & 125 \\ \text { XE } & 54 & 127 \\ \text { XE } & 54 & 129 M \\ \text { XE } & 54 & 131 M \\ \text { XE } & 54 & 133 \\ \text { XE } & 54 & 133 M \\ \text { CS } & 55 & 129 \\ \text { CS } & 55 & 131 \\ \text { CS } & 55 & 132 \\ \text { CS } & 55 & 134 \\ \text { CS } & 55 & 135 \\ \text { CS } & 55 & 136 \\ \text { CS } & 55 & 137 \\ \text { BA } & 56 & 128 \\ \text { BA } & 56 & 131 \\ \text { BA } & 56 & 133 \\ \text { BA } & 56 & 133 M \\ \text { BA } & 56 & 135 M \\ \text { BA } & 56 & 140 \\ \text { LA } & 57 & 135 \\ \text { LA } & 57 & 137 \\ \text { LA } & 57 & 138 \\ \text { LA } & 57 & 140 \\ \text { CE } & 58 & 134 \\ & & \end{array}$

ADULT
$U C / M * * 3$

$2.8 E-01$
$4.5 E-04$
$5.0 E-05$
$6.2 E-03$
$1.3 E-01$
$4.2 E-01$
$2.1 E+01$
$4.2 E-01$
$1.6 E-01$
$5.2 E-03$
$2.5 E-03$
$2.6 E-02$
$1.0 E-01$
$2.0 E-02$
$3.6 E-02$
$5.0 E+00$
$5.9 E-01$
$2.2 E+04$
$4.4 E+01$
$2.8 E+01$
$2.8 E+01$
$1.6 E-01$
$2.1 E+03$
$5.3 E+01$
$1.5 E+03$
$2.1 E+02$
$1.1 E+03$
$5.3 E+01$
$2.4 E+02$
$2.3 E+02$
$4.6 E+02$
$4.7 E+02$
$7.8 E-01$
$2.6 E-01$
$2.8 E-02$
$5.6 E-06$
$1.8 E-05$
$2.3 E-03$
$1.7 E-06$
$1.5 E+02$
$1.7 E+01$
$3.4 E-02$
$1.2 E+03$
$5.5 E+03$
$4.2 E+00$
$6.9 E-02$
$1.6 E-07$
$4.8 E-09$
$4.6 E-03$
$5.2 E+01$

INFANT

7. $6 E-03$

1. $7 \mathrm{E}-04$

4. $6 E-05$

6. $9 E-04$

4. $9 E-03$

7. 0 E-02

4. $5 \mathrm{E}-01$

.7E-02

. $7 E-02$

.3E-03

2.5E-03

2. $0 E-02$
$6.9 E-02$

$1.6 E-02$

1. $9 \mathrm{E}-02$

1. $9 E-01$

5.7E-02

$7.5 \mathrm{E}+02$

$9-6 E+00$

$2.2 E+01$

$1.3 E+01$

1. $5 E-01$

7. $0 E+01$

7. $0 E+01$

$1.9 E+01$

8. $2 E+01$

1. $1 E+03$

5. 3E+01

2. $4 E+02$

$2.3 E+02$

$4.6 E+02$

4. $7 E+02$

$2.1 E-02$

$2.1 E-02$
$1.6 E-03$

4. $9 E-06$

1. $7 E-05$

2. $4 E-04$

$1.6 E-06$

5. $3 E+00$

2. $5 E+00$

3.3E-02

2. $9 E+01$

1. $0 E+02$

6. $8 E-01$

$5.6 \mathrm{E}-05$

1. $4 E-07$

4. $2 E-09$

7. $7 E-06$

1. OE-01 
TABLE 9B. UNIT-RAD CONTAMINATION FOR BONE

\begin{tabular}{|c|c|c|c|c|}
\hline $\begin{array}{l}\text { ELEMENT } \\
\text { SYMBOL }\end{array}$ & $\begin{array}{l}\text { ATOMIC } \\
\text { NO. }(Z)\end{array}$ & $\begin{array}{l}\text { ISOTOPE } \\
\text { MASS (A) }\end{array}$ & $\begin{array}{l}\text { ADULT } \\
\text { UC/M**3 }\end{array}$ & $\begin{array}{l}\text { INFAN T } \\
\text { UC/M**3 }\end{array}$ \\
\hline $\begin{array}{l}C E \\
C E \\
C E \\
C E \\
C E \\
C E \\
C E \\
P R \\
P R \\
\text { ND } \\
N D \\
N D \\
P M \\
P M \\
P M \\
P M \\
P M \\
P M \\
P M \\
P M \\
P M \\
P S \\
S M \\
S M \\
S M \\
S M \\
S M \\
S M \\
S M \\
S M \\
E U \\
E U \\
E U \\
E U \\
E U \\
E U \\
E U \\
E U \\
E U \\
E U \\
E U \\
E U \\
E U \\
G D \\
G D \\
G D \\
G D \\
G D \\
G D \\
G D \\
G D\end{array}$ & $\begin{array}{l}58 \\
58 \\
58 \\
58 \\
58 \\
58 \\
58 \\
59 \\
59 \\
60 \\
60 \\
60 \\
61 \\
61 \\
61 \\
61 \\
61 \\
61 \\
61 \\
61 \\
61 \\
62 \\
62 \\
62 \\
62 \\
62 \\
62 \\
62 \\
62 \\
63 \\
63 \\
63 \\
63 \\
63 \\
63 \\
63 \\
63 \\
63 \\
63 \\
63 \\
63 \\
63 \\
64 \\
64 \\
64 \\
64 \\
64 \\
64 \\
64 \\
64\end{array}$ & $\begin{array}{l}135 \\
137 M \\
139 \\
141 \\
142 \\
143 \\
144 \\
142 \\
143 \\
140 \\
144 \\
147 \\
143 \\
144 \\
145 \\
146 \\
147 \\
148 \\
148 M \\
149 \\
151 \\
141 \\
145 \\
146 \\
147 \\
148 \\
149 \\
151 \\
153 \\
145 \\
146 \\
1464 \\
147 \\
148 \\
149 \\
150 \\
150 M \\
152 \\
154 \\
155 \\
156 \\
157 \\
146 \\
147 \\
148 \\
149 \\
150 \\
151 \\
152 \\
153\end{array}$ & $\begin{array}{l}1.2 E+03 \\
8.8 E+02 \\
1.8 E-01 \\
2.4 E+00 \\
6.2 E-05 \\
2.8 E+02 \\
2.5 E-03 \\
8.0 E-02 \\
8.8 E-04 \\
4.8 E-03 \\
3.9 E-09 \\
1.9 E-04 \\
2.9 E-02 \\
2.2 E-03 \\
2.3 E-03 \\
6.1 E-04 \\
4.8 E-04 \\
1.4 E+01 \\
6.9 E-02 \\
3.0 E+02 \\
4.3 E+02 \\
5.0 E-01 \\
5.4 E-02 \\
3.5 E-05 \\
4.0 E-05 \\
2.3 E-05 \\
4.8 E-05 \\
6.6 E-03 \\
6.0 E+02 \\
4.6 E+01 \\
1.2 E+01 \\
1.5 E+02 \\
7.0 E-01 \\
7.6 E-01 \\
2.0 E-01 \\
8.0 E-04 \\
9.7 E+02 \\
2.5 E-04 \\
1.8 E-04 \\
3.6 E-02 \\
3.1 E+00 \\
2.6 E+03 \\
2.7 E-02 \\
6.4 E+01 \\
1.8 E-05 \\
3.3 E+00 \\
9.4 E-06 \\
7.2 E-02 \\
2.3 E-05 \\
4.6 E-02\end{array}$ & $\begin{array}{l}6.1 \mathrm{E}-01 \\
8.4 \mathrm{E}-01 \\
1.5 \mathrm{E}-02 \\
5.2 \mathrm{E}-02 \\
5.0 \mathrm{E}-05 \\
2.6 \mathrm{E}-01 \\
4.1 \mathrm{E}-04 \\
4.3 \mathrm{E}-05 \\
8.0 \mathrm{E}-06 \\
1.1 \mathrm{E}-05 \\
3.2 \mathrm{E}-09 \\
1.4 \mathrm{E}-05 \\
4.3 \mathrm{E}-03 \\
4.2 \mathrm{E}-04 \\
1.7 \mathrm{E}-03 \\
3.1 \mathrm{E}-04 \\
1.9 \mathrm{E}-04 \\
5.1 \mathrm{E}-02 \\
1.8 \mathrm{E}-03 \\
4.4 \mathrm{E}-01 \\
3.4 \mathrm{E}-01 \\
6.6 \mathrm{E}-03 \\
1.0 \mathrm{E}-02 \\
2.8 \mathrm{E}-05 \\
3.2 \mathrm{E}-05 \\
1.8 \mathrm{E}-05 \\
3.9 \mathrm{E}-05 \\
5.2 \mathrm{E}-03 \\
7.8 \mathrm{E}-01 \\
1.8 \mathrm{E}-01 \\
3.7 \mathrm{E}-02 \\
1.6 \mathrm{E}-01 \\
1.1 \mathrm{E}-02 \\
2.6 \mathrm{E}-02 \\
1.3 \mathrm{E}-02 \\
4.3 \mathrm{E}-04 \\
3.5 \mathrm{E}-01 \\
1.7 \mathrm{E}-04 \\
1.3 \mathrm{E}-04 \\
1.1 \mathrm{E}-02 \\
3.1 \mathrm{E}-02 \\
1.1 \mathrm{E}+00 \\
1.2 \mathrm{E}-03 \\
6.7 \mathrm{E}-02 \\
1.5 \mathrm{E}-05 \\
3.0 \mathrm{E}-02 \\
8.2 \mathrm{E}-03 \\
7.7 \mathrm{E}-03 \\
2.0 \mathrm{E}-05 \\
8.9 \mathrm{E}-03\end{array}$ \\
\hline
\end{tabular}

VIA FRESHWATER.

10/09/68 PAGE 3

\begin{tabular}{|c|c|c|}
\hline $\begin{array}{l}\text { ELEMENT } \\
\text { SYMBOL }\end{array}$ & $\begin{array}{l}\text { ATOMIC } \\
\text { NO. ( Z) }\end{array}$ & $\begin{array}{l}\text { I SOTUPE } \\
\text { MASS(A) }\end{array}$ \\
\hline GD & 64 & 159 \\
\hline GD & 64 & 162 \\
\hline TE & 65 & 151 \\
\hline TB & 65 & 152 \\
\hline TE & 65 & 153 \\
\hline TB & 65 & 154 \\
\hline TB & 65 & 155 \\
\hline TE & 65 & 156 \\
\hline TB & 65 & 157 \\
\hline$T B$ & 65 & 158 \\
\hline TB & 65 & 160 \\
\hline TB & 65 & 161 \\
\hline TB & 65 & 164 \\
\hline DY & 66 & 154 \\
\hline DY & 60 & $154 M$ \\
\hline or & 66 & 156 \\
\hline DY & 66 & 159 \\
\hline DY & 66 & 166 \\
\hline HO & 67 & 163 \\
\hline HO & 67 & 166 \\
\hline HO & 67 & $166 \mathrm{M}$ \\
\hline ER & 68 & 160 \\
\hline ER & 68 & 169 \\
\hline ER & 68 & 172 \\
\hline$T M$ & 69 & 165 \\
\hline$T M$ & 69 & 167 \\
\hline$T M$ & 69 & 168 \\
\hline$T M$ & 69 & 170 \\
\hline$T M$ & 69 & 171 \\
\hline$T M$ & 69 & 172 \\
\hline$Y B$ & 70 & 166 \\
\hline YB & 70 & 169 \\
\hline YB & 70 & 175 \\
\hline LU & 71 & 169 \\
\hline LU & 71 & 170 \\
\hline LU & 71 & 171 \\
\hline LU & 71 & 172 \\
\hline LU & 71 & 173 \\
\hline LU & 71 & 174 \\
\hline LU & 71 & $174 M$ \\
\hline LU & 71 & 176 \\
\hline LU & 71 & 177 \\
\hline LU & 71 & $177 M$ \\
\hline $\mathrm{HF}$ & 72 & 170 \\
\hline HF & 72 & 171 \\
\hline HF & 72 & 172 \\
\hline HF & 72 & 173 \\
\hline HF & 72 & 174 \\
\hline HF & 72 & 175 \\
\hline HF & 72 & 181 \\
\hline
\end{tabular}

ADULT INFANT

$U C / M * * 3 \quad U C / M * * 3$

$\begin{array}{ll}1.4 E+03 & 1.1 E+00 \\ 2.2 E-03 & 6.0 E-04\end{array}$

9. $5 E+02 \quad 7.1 E-01$

2. $7 E+02$ 2.1E-01

1. $8 E+02 \quad 4.6 E-01$

1. $\mathrm{OE}+03$ 8. $8 \mathrm{E}-01$

4. $1 E+01$ 2. $3 E-01$

$1.3 E+01$ 6.8E-02

1. $8 \mathrm{E}-03 \quad 1.5 \mathrm{E}-03$

$\begin{array}{ll}7.7 E-05 & 6.6 E-05 \\ 8.2 E-02 & 5.5 E-03\end{array}$

8. $2 E-02$

5. $9 E+01$

$1.4 E+03$

$6.8 E-10$

3. $7 E-02$

1. $1 E-09$

3. $0 E-05$

2. $8 \mathrm{E}-03$

1. $1 E-07$

3. 3E-02

4. $9 E-09$

9. $7 E-03$

4. $9 E-03$

7. $7 E-03$

$1.4 E+02$

1. $0 E+01$

5. $2 E-02$

1. $0 E-01$

$9.6 E-02$

8. $2 \mathrm{E}+01$

2. $1 E+02$

1. $8 E+00$

2. $1 E+02$

9. $5 E-03$

3. $2 \mathrm{E}-03$

4. $2 E-03$

9. $\mathrm{OE}-04$

3. $2 E-07$

4. $2 E-08$

3. $1 E-06$

8. $2 E-09$

5. $O E-03$

1. $3 E+01$

$2.6 E+01$

1. $5 \mathrm{E}-05$

7. $9 E+00$

1. $3 E-06$

1. $1 \mathrm{E}-02$
5. 5E-03 $1.4 E+00$

5. $9 E-10$

2. $0 E-05$

9. $2 E-10$

$3.8 \mathrm{E}-06$

$9.4 E-06$

9. $5 E-08$

3. $7 E-05$

4. 2E-09

1. 2E-05

4. $6 E-05$

1. $6 E-05$

1. $7 E-01$

$9.6 E-02$

4. $1 E-03$

1.2E-02

3. $9 E-02$

2. $2 E-01$

$5.2 \mathrm{E}-01$

$5.5 \mathrm{E}-02$

8. 8E-01

1. $4 \mathrm{E}-05$

6. $4 E-06$

$3.4 E-05$

6. $0 E-06$

1. 1 E-07

$2.4 \mathrm{E}-08$

4.4E-07

7.1 E-09

3. $4 \mathrm{E}-05$

1. $2 \mathrm{E}-06$

$1.1 \mathrm{E}-02$

2.5E-02

$1.1 E-05$

$1.3 E-02$

1. $2 \mathrm{E}-06$

$1.2 \mathrm{E}-06$

6. $8 \mathrm{E}-04$
$7.3 \mathrm{E}-04$ 


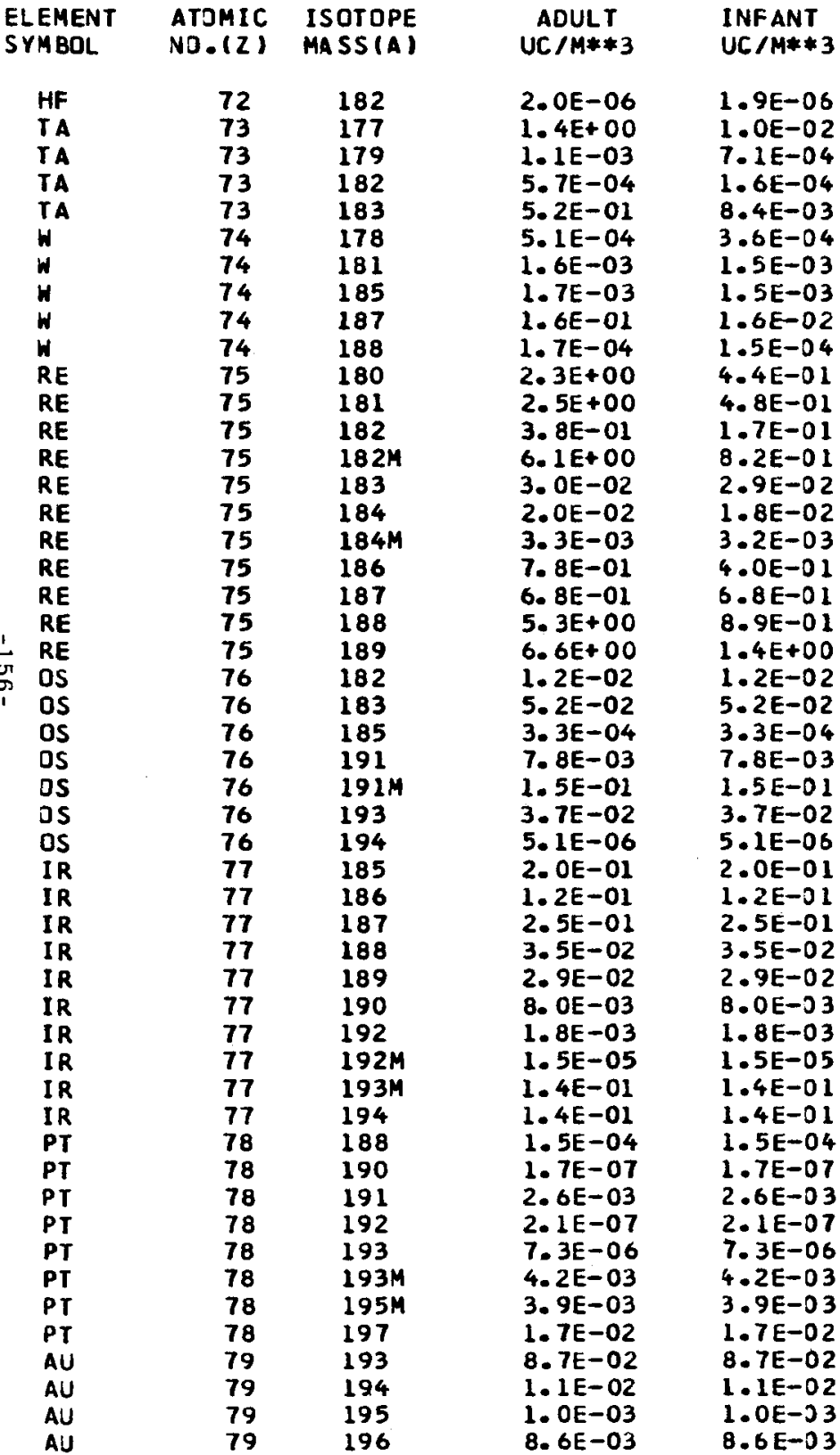

$\begin{array}{ccc}\text { ELEMENT } & \text { ATOMIC } & \text { ISOTOP } \\ \text { SYMBOL } & \text { NO. }(2) & \text { MASS(A } \\ & & \\ \text { AU } & 79 & 198 \\ \text { AU } & 79 & 199 \\ \text { HG } & 80 & 194 \\ \text { HG } & 80 & 195 M \\ \text { HG } & 80 & 197 \\ \text { HG } & 80 & 197 M \\ \text { HG } & 80 & 203 \\ \text { TL } & 81 & 200 \\ \text { TL } & 81 & 201 \\ \text { TL } & 81 & 202 \\ \text { TL } & 81 & 204 \\ \text { PE } & 82 & 200 \\ \text { PB } & 82 & 202 \\ \text { PB } & 82 & 203 \\ \text { PB } & 82 & 204 \\ \text { PB } & 82 & 205 \\ \text { PB } & 82 & 210 \\ \text { BI } & 83 & 205 \\ \text { BI } & 83 & 206 \\ \text { BI } & 83 & 207 \\ \text { BI } & 83 & 208 \\ \text { BI } & 83 & 210 \\ \text { BI } & 83 & 210 M \\ \text { PO } & 84 & 206 \\ \text { PO } & 84 & 208 \\ \text { PO } & 84 & 209 \\ \text { PO } & 84 & 210 \\ \text { RN } & 86 & 211 \\ \text { RN } & 86 & 222 \\ \text { RA } & 88 & 223 \\ \text { RA } & 88 & 224 \\ \text { RA } & 88 & 225 \\ \text { RA } & 88 & 226 \\ \text { RA } & 88 & 228 \\ \text { AC } & 89 & 225 \\ \text { AC } & 89 & 227 \\ \text { TH } & 90 & 227 \\ \text { TH } & 90 & 228 \\ \text { TH } & 90 & 229 \\ \text { TH } & 90 & 230 \\ \text { TH } & 90 & 231 \\ \text { TH } & 90 & 232 \\ \text { TH } & 90 & 234 \\ \text { PA } & 91 & 228 \\ \text { PA } & 91 & 229 \\ \text { PA } & 91 & 230 \\ \text { PA } & 91 & 231 \\ \text { PA } & 91 & 232 \\ \text { PA } & 91 & 233 \\ \text { U } & 92 & 230 \\ & & \end{array}$

ADULT

INFANT

1. $9 E-02$ 1.9E-02 $5.3 E-02 \quad 5.3 E-02$ 3.1E-05 3. $5 \mathrm{E}-03 \quad 3.5 \mathrm{E}-03$ $1.0 E-02$ 1. $0 E-02$ 1. $5 \mathrm{E}-02$ 1.5E-02 $6.3 E-04 \quad 6.3 E-04$ 1. $3 E-01$ 3. $8 E-03$ 9. $9 E-02$ 7. $7 E-03$ 3. $9 E-03$ 9. $7 E-04$ $1.4 E-05$ $1.4 E-05$ 1.9E+03 4.6E-OI

2. $8 E-04$

$7.3 E+02$

6. $3 E-05$

1. $9 E-03$

4. $9 E-05$

1. $5 E-05$

6. $8 \mathrm{E}-05$

2. $4 E-08$

1. $2 E-08$

3. $1 E-05$ 5. $8 E-09$

5. $6 \mathrm{E}-01$

5. $1 \mathrm{E}-04$

1. $2 \mathrm{E}-04$

6. $5 E-03$

7. $4 \mathrm{E}+01$

4. $7 E+00$

$6.5 E+01$

$6.5 E+02$

4. $0 E+01$

3. $3 E-04$

2. $0 \mathrm{E}-03$

5. $3 E+02$

3. $0 E-03$

2. $9 E-03$

2. $0 E-06$

3. $6 E-08$

3. $3 \mathrm{E}-08$

7. $4 \mathrm{E}-01$

3. $0 E-08$

1. $3 E-03$

4. $9 E+04$

2. $0 E+04$

1. $4 E+02$

1. $4 E-03$

$\begin{array}{ll}5.2 E+01 & 2.0 E-02 \\ 5.8 E-01 & 3.8 E-02\end{array}$ 1. $6 E-04$

4. $3 E-01$
3. $6 \mathrm{E}-05$

1. $1 E-03$ 2. 5E-05 8. 3E-06

2.2E-05

2. $4 \mathrm{E}-08$

1. $E-08$

$1.2 E-08=$

8.6E-06 00

1. $5 \mathrm{E}-01$

5. $0 \mathrm{E}-04$

1. $2 E-04$

5. $5 E-03$

7. $4 E+01$

4. $7 E+00$

4.6E-02

$1.4 E-01$

3.6E-02 $6.5 E-05$ 6. 2.1E-04

1. $3 E-04$

1. $3 E-04$
7. $3 E-07$

1. $9 \mathrm{E}-08$ 1. $8 E-09$ 1. $7 \mathrm{E}-09$

$1.1 E-05$

1. $5 E-09$

4. 5E-07 6. 6. E 01 4.1E-O1 $3.3 E-02$

7. OE-05 
TABLE 9B. UNIT-RAD CONTAMINATION FOR BDNE

\begin{tabular}{|c|c|c|c|c|}
\hline $\begin{array}{l}\text { ELEMENT } \\
\text { SYMBOL }\end{array}$ & $\begin{array}{l}\text { ATOMIC } \\
\text { NO. }(Z)\end{array}$ & $\begin{array}{l}\text { ISOTOPE } \\
\text { MASS (A) }\end{array}$ & $\begin{array}{c}\text { ADULT } \\
U C / M * * 3\end{array}$ & $\begin{array}{l}\text { INFANT } \\
\text { UC } / M * * 3\end{array}$ \\
\hline 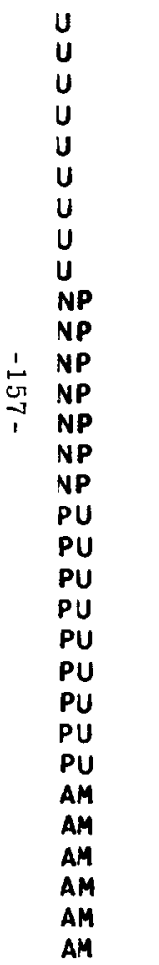 & $\begin{array}{l}92 \\
92 \\
92 \\
92 \\
92 \\
92 \\
92 \\
92 \\
92 \\
93 \\
93 \\
93 \\
93 \\
93 \\
93 \\
93 \\
94 \\
94 \\
94 \\
94 \\
94 \\
94 \\
94 \\
94 \\
94 \\
95 \\
95 \\
95 \\
95 \\
95 \\
95\end{array}$ & $\begin{array}{l}231 \\
232 \\
233 \\
234 \\
235 \\
236 \\
237 \\
238 \\
240 \\
234 \\
235 \\
236 \\
236 M \\
237 \\
238 \\
239 \\
236 \\
237 \\
238 \\
239 \\
240 \\
241 \\
242 \\
244 \\
246 \\
239 \\
240 \\
241 \\
242 \\
242 M \\
243\end{array}$ & $\begin{array}{l}1.4 E+01 \\
1.2 E-04 \\
1.1 E-04 \\
1.0 E-04 \\
9.7 E-05 \\
9.5 E-05 \\
4.7 E+00 \\
9.1 E-05 \\
5.2 E+02 \\
4.5 E+02 \\
5.0 E-02 \\
2.9 E-04 \\
9.6 E+03 \\
3.0 E-04 \\
1.8 E+03 \\
1.3 E+03 \\
3.1 E-02 \\
1.7 E+01 \\
1.4 E-03 \\
1.1 E-03 \\
1.1 E-03 \\
3.0 E-03 \\
1.1 E-03 \\
9.5 E-04 \\
2.2 E+02 \\
1.2 E+05 \\
6.5 E+03 \\
1.1 E-03 \\
6.5 E+04 \\
1.1 E-03 \\
1.0 E-03\end{array}$ & $\begin{array}{l}1.9 E-01 \\
1.2 E-04 \\
1.1 E-04 \\
1.0 E-34 \\
9.3 E-05 \\
9.1 E-05 \\
1.0 E-01 \\
8.8 E-05 \\
1.0 E+0 J \\
2.7 E-02 \\
2.8 E-04 \\
1.4 E-05 \\
1.2 E-01 \\
1.5 E-05 \\
5.1 E-02 \\
4.3 E-02 \\
4.4 E-04 \\
1.0 E-02 \\
6.6 E-05 \\
5.6 E-05 \\
5.5 E-05 \\
1.1 E-04 \\
5.3 E-05 \\
4.7 E-05 \\
3.3 E-02 \\
8.4 E-01 \\
1.9 E-01 \\
5.7 E-05 \\
5.9 E-01 \\
5.6 E-05 \\
5.0 E-05\end{array}$ \\
\hline
\end{tabular}

VIA FRFSHWATER.

$\begin{array}{lcc}\text { ELEMENT } & \text { ATOMIC } & \text { I SOTOPE } \\ \text { SYMBOL } & \text { NO. I } 2) & \text { MASS(A) } \\ \text { CM } & 96 & 240 \\ \text { CM } & 96 & 241 \\ \text { CM } & 96 & 242 \\ \text { CM } & 96 & 243 \\ \text { CM } & 96 & 244 \\ \text { CM } & 96 & 245 \\ \text { CH } & 96 & 246 \\ \text { CM } & 96 & 247 \\ \text { CM } & 96 & 248 \\ \text { GK } & 97 & 245 \\ \text { EK } & 97 & 246 \\ \text { GK } & 97 & 247 \\ \text { BK } & 97 & 248 \\ \text { BK } & 97 & 249 \\ \text { CF } & 98 & 246 \\ \text { CF } & 98 & 248 \\ \text { CF } & 98 & 249 \\ \text { CF } & 98 & 250 \\ \text { CF } & 98 & 251 \\ \text { CF } & 98 & 252 \\ \text { CF } & 98 & 253 \\ \text { ES } & 99 & 251 \\ \text { ES } & 99 & 252 \\ \text { ES } & 99 & 253 \\ \text { ES } & 99 & 254 \\ \text { ES } & 99 & 254 M \\ \text { ES } & 99 & 255 \\ \text { FM } & 100 & 252 \\ \text { FM } & 100 & 253 \\ \text { FM } & 100 & 255 \\ \text { FM } & 100 & 257 \\ & & \end{array}$

$\begin{array}{ll}10 / 09 / 68 & \text { PAGE } \\ \text { ADULT } & \text { INFANT } \\ \text { UC/M**3 } & \text { UC/M*\$3 } \\ & \\ 4.0 E+01 & 1.5 E-02 \\ 2.5 E+01 & 1.2 E-02 \\ 1.1 E+00 & 2.4 E-03 \\ 1.5 E-03 & 6.7 E-05 \\ 2.0 E-03 & 8.3 E-05 \\ 1.0 E-03 & 5.0 E-05 \\ 9.6 E-04 & 4.8 E-05 \\ 8.9 E-04 & 4.5 E-05 \\ 8.7 E-04 & 4.4 E-05 \\ 1.1 E+03 & 7.6 E-02 \\ 8.0 E+03 & 2.0 E-01 \\ 9.0 E-04 & 4.5 E-05 \\ 2.6 E+04 & 3.5 E-01 \\ 2.5 E-01 & 1.1 E-03 \\ 1.2 E+04 & 2.4 E-01 \\ 2.0 E-01 & 9.6 E-04 \\ 9.3 E-04 & 4.6 E-05 \\ 3.0 E-03 & 1.0 E-04 \\ 8.3 E-04 & 4.1 E-05 \\ 2.6 E-02 & 3.2 E-04 \\ 6.2 E+01 & 1.6 E-02 \\ 1.0 E+04 & 2.1 E-01 \\ 1.1 E+00 & 2.1 E-03 \\ 5.6 E+01 & 1.5 E-02 \\ 9.4 E-02 & 6.1 E-04 \\ 8.1 E+03 & 1.8 E-01 \\ 3.6 E+01 & 1.2 E-02 \\ 2.4 E+04 & 3.1 E-01 \\ 1.1 E+03 & 6.8 E-02 \\ 2.9 E+04 & 3.4 E-01 \\ 3.3 E+00 & 3.5 E-03 \\ & \\ 1.01 & \end{array}$




\begin{tabular}{ll} 
ADULT & INFANT \\
UC $/ M * * 3$ & UC $/ M * * 3$ \\
& \\
$6.4 E+02$ & $6.4 E+02$ \\
$2.8 E+01$ & $8.5 E+00$ \\
$1.0 E-02$ & $1.0 E-02$ \\
$1.3 E-03$ & $1.3 E-03$ \\
$1.8 E-02$ & $1.7 E-02$ \\
$2.3 E+02$ & $1.2 E+01$ \\
$3.4 E+01$ & $3.4 E+01$ \\
$2.4 E-02$ & $2.4 E-02$ \\
$6.0 E-03$ & $6.0 E-03$ \\
$2.0 E-02$ & $8.8 E-03$ \\
$9.8 E-02$ & $5.7 E-02$ \\
$1.1 E+00$ & $1.1 E+00$ \\
$2.0 E-02$ & $2.0 E-02$ \\
$8.2 E+02$ & $8.2 E+02$ \\
$4.4 E-02$ & $4.4 E-02$ \\
$4.7 E-03$ & $4.7 E-03$ \\
$2.2 E-03$ & $2.2 E-03$ \\
$1.1 E+03$ & $9.4 E+00$ \\
$6.4 E+02$ & $1.0 E+01$ \\
$4.1 E+01$ & $4.1 E+01$ \\
$8.3 E+01$ & $7.5 E+01$ \\
$5.8 E+02$ & $1.3 E+02$ \\
$4.1 E-02$ & $4.0 E-02$ \\
$1.4 E+01$ & $2.2 E-02$ \\
$1.7 E-02$ & $8.5 E-04$ \\
$6.7 E+01$ & $1.4 E-01$ \\
$2.0 E+01$ & $2.3 E-02$ \\
$2.8 E-03$ & $2.8 E-03$ \\
$1.9 E+01$ & $3.4 E+00$ \\
$1.3 E+02$ & $1.0 E+02$ \\
$1.8 E-02$ & $1.8 E-02$ \\
$5.7 E+03$ & $8.8 E+00$ \\
$7.1 E+02$ & $3.1 E+01$ \\
$4.7 E-01$ & $8.7 E-02$ \\
$3.9 E-02$ & $3.9 E-02$ \\
$7.5 E-03$ & $5.9 E-03$ \\
$3.0 E+00$ & $1.8 E+00$ \\
$3.2 E+00$ & $1.9 E-01$ \\
$6.1 E-04$ & $5.5 E-04$ \\
$1.1 E+03$ & $3.4 E+01$ \\
$5.0 E-01$ & $3.8 E-01$ \\
$1.8 E+00$ & $1.6 E+00$ \\
$1.1 E+00$ & $8.5 E-01$ \\
$1.3 E-02$ & $1.2 E-02$ \\
$1.3 E+01$ & $1.3 E+01$ \\
$9.9 E+01$ & $9.9 E+01$ \\
$5.1 E+00$ & $5.1 E+00$ \\
$2.5 E+00$ & $2.5 E+0 J$ \\
$7.0 E+01$ & $7.0 E+01$ \\
$2.9 E+03$ & $1.8 E+01$ \\
& \\
\hline .02 &
\end{tabular}

\begin{tabular}{|c|c|c|}
\hline $\begin{array}{l}\text { ELEMENT } \\
\text { SYMBOL }\end{array}$ & $\begin{array}{l}\text { ATDMIC } \\
\text { NO. }(Z)\end{array}$ & $\begin{array}{l}\text { I SOTOPE } \\
\text { MASSIAI }\end{array}$ \\
\hline $\mathrm{CU}$ & $\begin{array}{l}29 \\
30\end{array}$ & $\begin{array}{l}67 \\
65\end{array}$ \\
\hline $\begin{array}{l}Z N \\
Z N\end{array}$ & $\begin{array}{l}30 \\
30\end{array}$ & $\begin{array}{l}65 \\
69 M\end{array}$ \\
\hline$Z N$ & 30 & 72 \\
\hline GA & 31 & 67 \\
\hline GA & 31 & 72 \\
\hline GE & 32 & 68 \\
\hline GE & 32 & 69 \\
\hline GE & 32 & 71 \\
\hline AS & 33 & 71 \\
\hline AS & 33 & 72 \\
\hline AS & 33 & 73 \\
\hline AS & 33 & 74 \\
\hline AS & 33 & 76 \\
\hline AS & 33 & 77 \\
\hline SE & 34 & 72 \\
\hline SE & 34 & 75 \\
\hline SE & 34 & 79 \\
\hline$B R$ & 35 & 76 \\
\hline$B R$ & 35 & 77 \\
\hline BR & 35 & 82 \\
\hline KR & 36 & 76 \\
\hline KR & 36 & 79 \\
\hline KR & 36 & 81 \\
\hline KR & 36 & 85 \\
\hline RB & 37 & 83 \\
\hline RB & 37 & 84 \\
\hline $\mathrm{RB}$ & 37 & 86 \\
\hline RB & 37 & 87 \\
\hline$S R$ & 38 & 82 \\
\hline$S R$ & 38 & 83 \\
\hline$S R$ & 38 & 85 \\
\hline SR & 38 & 89 \\
\hline SR & 38 & 90 \\
\hline$r$ & 39 & 86 \\
\hline$Y$ & 39 & 87 \\
\hline$Y$ & 39 & $87 M$ \\
\hline$Y$ & 39 & 88 \\
\hline$\gamma$ & 39 & 90 \\
\hline$r$ & 39 & 91 \\
\hline $2 R$ & 40 & 86 \\
\hline ZR & 40 & 88 \\
\hline $2 R$ & 40 & 89 \\
\hline $2 R$ & 40 & 93 \\
\hline$Z R$ & 40 & 95 \\
\hline $2 R$ & 40 & 97 \\
\hline NB & 41 & 90 \\
\hline NB & 41 & 91 \\
\hline NB & 41 & $91 M$ \\
\hline NB & 41 & 92 \\
\hline
\end{tabular}

ADULT $\mathrm{UC} / M * * 3$

1. $6 E+02$ 1. $8 E-03$

7. $1 E+01$

2. $3 E+02$

3. $6 E+02$

1. $8 E-05$

2. $1 E-02$

1. $5 E-01$

4. $2 E+05$

5. $1 E+05$

3. $3 E+04$

3. $3 E+03$

6. $9 E+05$

2. $4 E+06$

3. $0 E+00$

5. $3 E-01$

9.7E-02

7. $7 E+02$

1. $6 E+02$

1. $4 E+02$

1. $5 E+02$

1. $9 E+02$

1. $3 E+00$

2. 5E-01

3. $4 E-01$

4. $6 E-01$

2. $3 E+00$

1. $3 E-02$

4. $6 E+00$

2. $1 E+03$

9. $3 E+00$

8. $5 E+00$

3. $O E-02$

1. $1 E+02$

1. $8 E+01$

4. $2 E+02$

1. $9 E+01$

6. $5 E-02$

2. $5 E-01$

2. $5 E-01$

2. $7 E+02$

4. $4 E-02$

5. $3 E+01$

1. $1 E-03$

1. $2 E-01$

1. $O E+O D$
INFANT

$4.4 E+C C$

1. $1 E-03$

2. $8 E-01$

1. $6 \vec{E}-02$

6. $1 E+01$

$2.2 E+01$

1. $8 \mathrm{E}-05$

$2.6 E-03$

$7.1 \mathrm{E}-02$

2. $0 E+03$

$1.0 E+03$

4. $G E+03$

1. $0 E+02$

1. $4 E+03$

7. $1 E+03$

1. $3 E+00$

$4.8 E-01$

$4.8 E-01$

$9.6 E-02$

$6.1 E+01$

$3.7 E+01 \widehat{N}$

2. $2 \mathrm{E}+\mathrm{Cl}$

1. $5 E+02$

$1.3 E+00$ D

$2.5 E-01$ in

$1.7 E-01$

$1.4 E-O$

1. $E-01$

4. $3 E-01$

1. $3 E-02$

2. $9 E+00$

$1.6 E+02$

$7.4 E+C D$

$6.5 E+0 u$

3. $0 E-02$

1. $7 E-02$

$1.5 \mathrm{E}-\mathrm{C}$

5. $9 E-02$

1. $5 E-C 4$

1. $2 E-02$

$1.2 E-02$

. E-O4

1. $8 E+C 0$

2. $25-02$

S. $7 E-0$

3. $9 E-02$

5. $4 E-02$

2. TE + CO

$4.2 E-C$

$9.9 E-04$

1. $4 E-1$ ? 


\begin{tabular}{|c|c|c|c|c|}
\hline $\begin{array}{l}\text { ELEMENT } \\
\text { SYMBOL }\end{array}$ & $\begin{array}{l}\text { ATJMIC } \\
N J \cdot(Z)\end{array}$ & $\begin{array}{l}\text { ISOTOPE } \\
\text { MASS (A) }\end{array}$ & $\begin{array}{l}\text { ADULT } \\
\text { UC/M**3 }\end{array}$ & $\begin{array}{l}\text { INFANT } \\
\text { UC } / M * * 3\end{array}$ \\
\hline 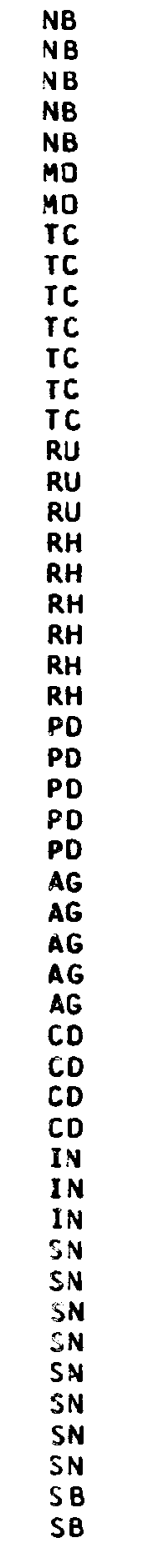 & $\begin{array}{l}41 \\
41 \\
41 \\
41 \\
41 \\
42 \\
42 \\
43 \\
43 \\
43 \\
43 \\
43 \\
43 \\
43 \\
44 \\
44 \\
44 \\
45 \\
45 \\
45 \\
45 \\
45 \\
45 \\
46 \\
46 \\
46 \\
46 \\
46 \\
47 \\
47 \\
47 \\
47 \\
47 \\
48 \\
48 \\
48 \\
48 \\
49 \\
49 \\
49 \\
50 \\
50 \\
50 \\
50 \\
50 \\
50 \\
50 \\
50 \\
51 \\
51\end{array}$ & $\begin{array}{l}93 M \\
94 \\
95 \\
95 M \\
96 \\
93 \\
99 \\
95 \\
95 M \\
96 \\
97 \\
97 M \\
98 \\
99 \\
97 \\
103 \\
106 \\
99 \\
100 \\
101 \\
101 M \\
102 \\
105 \\
100 \\
103 \\
107 \\
109 \\
112 \\
105 \\
106 M \\
108 M \\
110 M \\
111 \\
109 \\
113 M \\
115 \\
115 M \\
111 \\
114 M \\
115 \\
113 \\
117 M \\
119 M \\
121 \\
121 M \\
123 M \\
125 \\
126 \\
119 \\
120 M\end{array}$ & $\begin{array}{l}\text { 5. } 4 E-03 \\
1.1 \mathrm{E}-05 \\
1.1 \mathrm{E}-01 \\
7.1 \mathrm{E}+00 \\
4.0 \mathrm{E}+01 \\
1.4 \mathrm{E}-01 \\
8.0 \mathrm{E}+01 \\
2.0 \mathrm{E}+00 \\
1.1 \mathrm{E}-03 \\
2.8 \mathrm{E}-02 \\
3.6 \mathrm{E}-04 \\
6.3 \mathrm{E}-03 \\
4.8 \mathrm{E}-06 \\
7.9 \mathrm{E}-05 \\
6.1 \mathrm{E}+00 \\
3.9 \mathrm{E}-02 \\
2.8 \mathrm{E}-04 \\
4.8 \mathrm{E}-02 \\
3.3 \mathrm{E}+00 \\
2.3 \mathrm{E}-04 \\
6.4 \mathrm{E}-01 \\
4.4 \mathrm{E}-04 \\
7.3 \mathrm{E}+00 \\
2.0 \mathrm{E}-01 \\
3.2 \mathrm{E}-01 \\
1.5 \mathrm{E}-02 \\
2.4 \mathrm{E}+01 \\
1.7 \mathrm{E}+00 \\
3.9 \mathrm{E}+00 \\
1.1 \mathrm{E}+01 \\
3.5 \mathrm{E}-02 \\
2.2 \mathrm{E}-01 \\
7.0 \mathrm{E}+01 \\
5.5 \mathrm{E}-05 \\
2.3 \mathrm{E}-06 \\
8.1 \mathrm{E}-02 \\
3.2 \mathrm{E}-04 \\
2.4 \mathrm{E}-03 \\
4.7 \mathrm{E}-06 \\
8.2 \mathrm{E}-08 \\
9.5 \mathrm{E}+00 \\
7.7 \mathrm{E}+02 \\
1.2 \mathrm{E}+01 \\
3.1 \mathrm{E}+05 \\
1.1 \mathrm{E}-01 \\
5.1 \mathrm{E}+00 \\
2.8 \mathrm{E}+02 \\
3.1 \mathrm{E}-03 \\
8.2 \mathrm{E}+00 \\
4.7 \mathrm{E}-02\end{array}$ & $\begin{array}{l}3.5 E-03 \\
1.0 E-05 \\
4.8 E-03 \\
3.5 E-02 \\
5.2 E-02 \\
1.4 E-01 \\
8.2 E+0 J \\
8.0 E-02 \\
8.4 E-04 \\
5.0 E-03 \\
3.6 E-04 \\
5.1 E-03 \\
4.8 E-05 \\
7.9 E-05 \\
9.6 E-02 \\
7.1 E-03 \\
1.9 E-04 \\
1.7 E-02 \\
9.9 E-02 \\
2.3 E-04 \\
8.9 E-02 \\
3.8 E-04 \\
3.7 E-01 \\
2.2 E-02 \\
1.2 E-01 \\
1.5 E-02 \\
4.4 E-01 \\
4.9 E-02 \\
3.6 E+00 \\
7.3 E+00 \\
3.5 E-02 \\
2.2 E-01 \\
4.5 E+01 \\
3.4 E-05 \\
2.2 E-06 \\
6.2 E-04 \\
3.9 E-05 \\
1.1 E-04 \\
2.1 E-06 \\
8.1 E-0 B \\
1.4 E+00 \\
1.6 E+01 \\
3.2 E+00 \\
5.2 E+02 \\
1.0 E-01 \\
8.2 E-01 \\
4.0 E+00 \\
2.8 E-03 \\
8.2 E+00 \\
4.7 E-02\end{array}$ \\
\hline
\end{tabular}

$\begin{array}{lcc}\text { ELEMENT } & \text { ATOMIC } & \text { I SOTOPE } \\ \text { SYMBOL } & \text { NO. }(Z) & \text { MASSIA } \\ \text { SE } & & \\ \text { SE } & 51 & 122 \\ \text { SB } & 51 & 124 \\ \text { SB } & 51 & 125 \\ \text { SB } & 51 & 126 \\ \text { TE } & 51 & 127 \\ \text { TE } & 52 & 118 \\ \text { TE } & 52 & 119 \\ \text { TE } & 52 & 119 M \\ \text { TE } & 52 & 121 \\ \text { TE } & 52 & 121 M \\ \text { TE } & 52 & 123 \\ \text { TE } & 52 & 123 M \\ \text { TE } & 52 & 125 M \\ \text { TE } & 52 & 127 M \\ \text { TE } & 52 & 129 M \\ \text { TE } & 52 & 131 M \\ \text { I } & 52 & 132 \\ \text { I } & 53 & 123 \\ \text { I } & 53 & 124 \\ \text { I } & 53 & 125 \\ \text { I } & 53 & 126 \\ \text { I } & 53 & 129 \\ \text { I } & 53 & 130 \\ \text { I } & 53 & 131 \\ \text { XE } & 53 & 133 \\ \text { XE } & 54 & 122 \\ \text { XE } & 54 & 125 \\ \text { XE } & 54 & 127 \\ \text { XE } & 54 & 129 M \\ \text { XE } & 54 & 131 M \\ \text { XE } & 54 & 133 \\ \text { CS } & 54 & 133 M \\ \text { CS } & 55 & 129 \\ \text { CS } & 55 & 131 \\ \text { CS } & 55 & 132 \\ \text { CS } & 55 & 134 \\ \text { CS } & 55 & 135 \\ \text { CS } & 55 & 136 \\ \text { BA } & 55 & 137 \\ \text { BA } & 56 & 128 \\ \text { BA } & 56 & 131 \\ \text { BA } & 56 & 133 \\ \text { BA } & 56 & 133 M \\ \text { BA } & 56 & 135 M \\ \text { LA } & 56 & 140 \\ \text { LA } & 57 & 135 \\ \text { LA } & 57 & 137 \\ \text { LA } & 57 & 138 \\ \text { CE } & 57 & 140 \\ & 58 & 134 \\ & & \end{array}$

ADULT

INFANT

2. $2 \mathrm{E}-01$

4. $8 E-03$

1. $3 E-03$

2. $0 E-02$

1. $4 E-01$

2.1E-02

1.

2. $1 E-02$

$7.9 E-03$
$2.6 E-04$

1. $3 E-04$

1. $3 E-03$

5. $2 E-03$

1. $O E-03$

$1.8 E-03$

2. $5 E-01$

2. $9 E-02$

1. $6 E+04$

3. $8 E+01$

3. $7 E+01$

3. $0 E+01$

2. $3 E-01$

1. $5 E+03$

5. $2 E+01$

1. $1 E+03$

2. $1 E+02$

1. $1 E+03$

5. $3 E+01$

2. $4 E+02$

$2 \cdot 3 E+02$

4. $6 E+02$

4. $7 E+02$

2. $6 E+02$

8. $8 E+01$

9. $5 E+00$

1. $9 E-03$

5. $9 E-03$

7. $6 E-01$

$5.6 E-04$

8. $8 E+02$

1. $6 E+02$

. $2 E+00$

$6.7 E+03$

2. $9 E+04$

4. $1 E+01$

3. $2 E+03$

8. $2 E+0 O$

2. $4 E-01$

4. $4 E+02$

$4.8 E-03$

$1.3 E-03$

2. $0 E-02$

$1.4 E-01$

. $5 E-03$

.3E-02

.

. $9 E-03$

$2.2 E-04$

$1.3 E-04$

1. $0 \mathrm{E}-03$

3. 5E-03

8. OE-04

$9.3 E-04$

$.6 E-03$

$9 E-03$

$-1 E+03$

$1.4 E+01$

$3.4 E+01$ 으

2. $0 E+01$

$2.3 E-01$

2.8E+01

$1.2 \mathrm{E}+02$

$2.1 E+02$

$1.1 E+03$

$5.3 E+01$

2. $4 E+02$

$2.3 E+02$

$4.6 E+02$

4. $7 \mathrm{E}+02$

3. $O E+00$

$7.1 E+00$

5. 3E-01

$1.6 E-03$

$8 E-03$

$5.5 E-04$

.

1. $9 E+02$

$9.2 E+01$

$1.2 E+00$

$1.1 E+03$

3. $7 E+03$

2. $5 E+01$

$3.2 E+03$

8. $2 E+00$

2. $4 E-01$

2. $4 E-01$

5. $2 E-01$ 


$\begin{array}{ccccc}\text { ELEMENT } & \text { ATOMIC } & \text { ISOTOPE } & \text { AOULT } & \text { INFANT } \\ \text { SYMBOL } & \text { NO. }(Z 1 & \text { MASSIA } & \text { UC M } * * 3 & \text { UC JM**3 } \\ \text { CE } & 58 & 135 & & \\ \text { CE } & 58 & 137 M & 2.3 E+03 & 3.0 E+00 \\ \text { CE } & 58 & 139 & 1.6 E+03 & 4.2 E+00 \\ \text { CE } & 58 & 141 & 3.8 E-01 & 7.5 E-02 \\ \text { CE } & 58 & 142 & 4.7 E+00 & 2.6 E-01 \\ \text { CE } & 58 & 143 & 2.7 E-04 & 2.5 E-04 \\ \text { CE } & 58 & 144 & 5.3 E+02 & 1.3 E+00 \\ \text { PR } & 59 & 142 & 6.0 E-03 & 2.0 E-03 \\ \text { PR } & 59 & 143 & 4.0 E-02 & 4.3 E-05 \\ \text { ND } & 60 & 140 & 4.4 E-04 & 8.0 E-06 \\ \text { ND } & 60 & 144 & 2.1 E-03 & 1.1 E-05 \\ \text { ND } & 60 & 147 & 3.5 E-09 & 3.2 E-09 \\ \text { PM } & 61 & 143 & 8.5 E-05 & 1.4 E-06 \\ \text { PM } & 61 & 144 & 1.5 E-02 & 4.3 E-03 \\ \text { PM } & 61 & 145 & 1.2 E-03 & 4.2 E-04 \\ \text { PM } & 61 & 146 & 1.9 E-03 & 1.7 E-03 \\ \text { PM } & 61 & 147 & 4.4 E-04 & 3.1 E-04 \\ \text { PM } & 61 & 148 & 3.2 E-04 & 1.9 E-04 \\ \text { PM } & 61 & 148 M & 6.3 E+00 & 5.1 E-02 \\ \text { PM } & 61 & 149 & 3.1 E-02 & 1.8 E-03 \\ \text { PM } & 61 & 151 & 1.3 E+02 & 4.4 E-01 \\ \text { SM } & 62 & 141 & 1.9 E+02 & 3.4 E-01 \\ \text { SM } & 62 & 145 & 1.1 E+00 & 3.3 E-02 \\ \text { SM } & 62 & 146 & 1.5 E-01 & 5.0 E-02 \\ \text { SM } & 62 & 147 & 1.5 E-04 & 1.4 E-04 \\ \text { SM } & 62 & 148 & 1.7 E-04 & 1.6 E-04 \\ \text { SM } & 62 & 149 & 9.9 E-05 & 9.1 E-05 \\ \text { SM } & 62 & 151 & 2.1 E-04 & 1.9 E-04 \\ \text { SM } & 62 & 153 & 2.9 E-02 & 2.6 E-02 \\ \text { EU } & 63 & 145 & 1.3 E+03 & 3.9 E+00 \\ \text { EU } & 63 & 146 & 2.3 E+01 & 9.1 E-02 \\ \text { EU } & 63 & 146 M & 5.9 E+00 & 1.8 E-02 \\ \text { EU } & 63 & 147 & 7.3 E+01 & 7.8 E-02 \\ \text { EU } & 63 & 148 & 3.4 E-01 & 5.5 E-03 \\ \text { EU } & 63 & 149 & 9.8 E-01 & 1.3 E-02 \\ \text { EU } & 63 & 150 & 4.0 E-04 & 6.5 E-03 \\ \text { EU } & 63 & 150 M & 4.8 E+02 & 2.2 E-04 \\ \text { EU } & 63 & 152 & 1.2 E-04 & 8.5 E-01 \\ \text { EU } & 63 & 154 & 9.0 E-05 & 6.5 E-05 \\ \text { EU } & 63 & 155 & 1.8 E-02 & 5.5 E-03 \\ \text { EU } & 63 & 156 & 1.5 E+00 & 1.6 E-02 \\ \text { EU } & 63 & 157 & 1.3 E+03 & 5.6 E-01 \\ \text { GD } & 64 & 146 & 3.4 E-02 & 1.2 E-03 \\ \text { GD } & 64 & 147 & 8.4 E+01 & 6.7 E-02 \\ \text { GD } & 64 & 148 & 1.9 E-05 & 1.5 E-05 \\ \text { GD } & 64 & 149 & 4.3 E+00 & 3.0 E-02 \\ \text { GD } & 64 & 150 & 9.8 E-06 & 8.2 E-06 \\ \text { GD } & 64 & 151 & 9.1 E-02 & 7.7 E-03 \\ \text { GD } & 64 & 152 & 2.4 E-05 & 2.0 E-05 \\ \text { GD } & 64 & 153 & 5.7 E-02 & 8.9 E-03\end{array}$

\begin{tabular}{ccc} 
ELEMENT & ATOMIC & I SOTOPE \\
SYMBOL & NO.IZI & MASS(A) \\
GD & & \\
GO & 64 & 159 \\
TB & 64 & 162 \\
TB & 65 & 151 \\
TB & 65 & 152 \\
IB & 65 & 153 \\
IB & 65 & 154 \\
TB & 65 & 155 \\
TE & 65 & 156 \\
TB & 65 & 157 \\
TB & 65 & 158 \\
TB & 65 & 160 \\
TB & 65 & 161 \\
OY & 65 & 164 \\
CY & 66 & 154 \\
DY & 66 & $154 M$ \\
OY & 66 & 156 \\
DY & 66 & 159 \\
HO & 66 & 166 \\
HU & 67 & 163 \\
HO & 67 & 166 \\
ER & 67 & $166 M$ \\
ER & 68 & 160 \\
ER & 68 & 169 \\
TM & 68 & 172 \\
IM & 69 & 165 \\
TM & 69 & 167 \\
TH & 69 & 168 \\
TM & 69 & 170 \\
TM & 69 & 171 \\
YB & 69 & 172 \\
YB & 70 & 166 \\
YB & 70 & 169 \\
LU & 70 & 175 \\
LU & 71 & 169 \\
LU & 71 & 170 \\
LU & 71 & 171 \\
LU & 71 & 172 \\
LU & 71 & 173 \\
LU & 71 & 174 \\
LU & 71 & $174 M$ \\
LU & 71 & 176 \\
LU & 71 & 177 \\
HF & 71 & $177 M$ \\
HF & 72 & 170 \\
HF & 72 & 171 \\
HF & 72 & 172 \\
HF & 72 & 173 \\
HF & 72 & 174 \\
HF & 72 & 175 \\
& 181 \\
& & \\
\hline
\end{tabular}

AOULT
$U C / M * * 3$

I NFANT

$U C / M * * 3$

1. $8 E+03$

2. $7 E-03$

6. $6 E+02$

1. $9 E+02$

1. $3 E+02$

7. $1 E+02$

2. $9 E+01$

8. $9 E+00$

1. $7 E-03$

5. $9 E-02$

4. $1 E+01$

$9.6 E+02$

5. $9 E-10$

2. $0 E-05$

9. $2 E-10$

3. 8E-06

9. $4 E-06$

1. $1 E-07$

2. $6 E-02$

4. $7 E-09$

6. $3 E-03$

$3.2 E-03$

5. $0 E-03$

2. $4 E+02$

1. $0 E+01$

1. $0 E-01$

2. $1 E-01$

1. $4 E+02$

1. $5 E+02$

1. $2 E+00$

1. $5 E+02$

7. 1E-03

2. $4 E-03$

3. $2 E-03$

$6.8 E-04$

2. $7 E-07$
3. $7 E-08$

2. $4 E-06$

7. $9 E-09$

3. $8 E-03$

7. $3 E-06$

1. $2 E+01$

2. $4 E+01$

1. $5 E-05$

1. $3 E-06$

6. IE- 03

6. $1 E-03$
$1 . O E-02$

$1.1 E+00$

6. $0 E-04$

7. 1 E-01

$2.1 E-01$

4. $6 E-01$

8. $8 E-01$

2. 3E-01

$6.8 E-02$

$1.5 E-03$

6. $6 \mathrm{E}-05$

5. 5E-03

$1.4 E+00$

$5.9 E-10$

5.

2. $2 E-10$

3. BE-06

9. $4 E-06$

9. $5 E-08$

3. $7 E-05$

4.2E-09

1. 2 E-05

4. $6 E-05$

$1.6 E-05$

$8.6 E-01$

4. 8E-01

2. 1 E-02

5. $8 E-02$

1. $1 E+00$

5.2E-01

$5.5 E-02$

$8.8 \mathrm{E}-01$

1. $E-05$

$6.4 E-06$

6.

6. $0 \mathrm{E}-06$

1. 1 E-07

$2.4 E-08$

4. $4 E-07$

7. 1 E-09

3. $4 \mathrm{E}-05$

1. $2 E-06$

$1.1 \mathrm{E}-02$

2. 5E-02

1.1E-05

1 $E-02$

$1.3 E-02$

$6.8 \mathrm{E}-04$

7. $3 E-04$ 
TABLE 9B. UNIT-RAJ CONTAMINATION FOR KIDNEYS

VIA FRESHWATER.

$10 / 09 / 68$ PAGE 9

\begin{tabular}{|c|c|c|c|c|}
\hline $\begin{array}{l}\text { ELEMENT } \\
\text { SYMBOL }\end{array}$ & $\begin{array}{l}\text { ATOMIC } \\
\text { NO. }(Z)\end{array}$ & $\begin{array}{l}\text { ISOTOPE } \\
\text { MASS(A) }\end{array}$ & $\begin{array}{l}\text { ADULT } \\
\text { UC/M\#*3 }\end{array}$ & $\begin{array}{l}\text { INFANT } \\
U C / M * * 3\end{array}$ \\
\hline 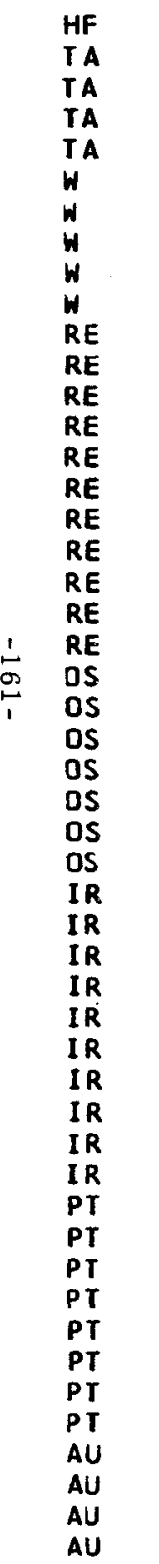 & $\begin{array}{l}72 \\
73 \\
73 \\
73 \\
73 \\
74 \\
74 \\
74 \\
74 \\
74 \\
75 \\
75 \\
75 \\
75 \\
75 \\
75 \\
75 \\
75 \\
75 \\
75 \\
75 \\
76 \\
76 \\
76 \\
76 \\
76 \\
76 \\
76 \\
77 \\
77 \\
77 \\
77 \\
77 \\
77 \\
77 \\
77 \\
77 \\
77 \\
78 \\
78 \\
78 \\
78 \\
78 \\
78 \\
78 \\
78 \\
79 \\
79 \\
79 \\
79\end{array}$ & $\begin{array}{l}182 \\
177 \\
179 \\
182 \\
183 \\
178 \\
181 \\
185 \\
187 \\
188 \\
180 \\
181 \\
182 \\
182 M \\
183 \\
184 \\
184 M \\
186 \\
187 \\
188 \\
189 \\
182 \\
183 \\
185 \\
191 \\
191 M \\
193 \\
194 \\
185 \\
186 \\
187 \\
188 \\
189 \\
190 \\
192 \\
192 M \\
193 M \\
194 \\
188 \\
190 \\
191 \\
192 \\
193 \\
193 M \\
195 M \\
197 \\
193 \\
194 \\
195 \\
196\end{array}$ & $\begin{array}{l}2.0 E-06 \\
3.6 E-01 \\
2.4 E-04 \\
1.4 E-04 \\
1.4 E-01 \\
3.6 E-04 \\
1.5 E-03 \\
1.5 E-03 \\
1.6 E-02 \\
1.5 E-04 \\
4.4 E-01 \\
4.8 E-01 \\
1.7 E-01 \\
8.2 E-01 \\
2.9 E-02 \\
1.8 E-02 \\
3.2 E-03 \\
4.0 E-01 \\
6.8 E-01 \\
8.9 E-01 \\
1.4 E+00 \\
7.9 E-02 \\
5.7 E-01 \\
3.4 E-04 \\
1.0 E-02 \\
1.4 E+00 \\
1.7 E-01 \\
5.1 E-06 \\
1.6 E+01 \\
9.5 E+00 \\
2.6 E+01 \\
1.1 E+00 \\
1.4 E-01 \\
4.2 E-02 \\
2.9 E-03 \\
1.5 E-05 \\
7.3 E-01 \\
8.7 E+00 \\
1.0 E-03 \\
1.8 E-07 \\
5.4 E-02 \\
2.1 E-07 \\
7.3 E-06 \\
6.1 E-02 \\
6.0 E-02 \\
1.2 E+00 \\
7.4 E+02 \\
4.5 E+01 \\
5.9 E-02 \\
9.0 E+00\end{array}$ & $\begin{array}{l}1.9 \mathrm{E}-06 \\
2.1 \mathrm{E}-03 \\
1.4 \mathrm{E}-04 \\
3.2 \mathrm{E}-05 \\
1.7 \mathrm{E}-03 \\
3.6 \mathrm{E}-04 \\
1.5 \mathrm{E}-03 \\
1.5 \mathrm{E}-03 \\
1.6 \mathrm{E}-02 \\
1.5 \mathrm{E}-04 \\
4.4 \mathrm{E}-01 \\
4.8 \mathrm{E}-01 \\
1.7 \mathrm{E}-01 \\
8.2 \mathrm{E}-01 \\
2.9 \mathrm{E}-02 \\
1.8 \mathrm{E}-02 \\
3.2 \mathrm{E}-03 \\
4.0 \mathrm{E}-01 \\
6.8 \mathrm{E}-01 \\
8.9 \mathrm{E}-01 \\
1.4 \mathrm{E}+00 \\
1.2 \mathrm{E}-02 \\
5.2 \mathrm{E}-02 \\
3.3 \mathrm{E}-04 \\
1.8 \mathrm{E}-03 \\
1.5 \mathrm{E}-01 \\
3.7 \mathrm{E}-02 \\
5.1 \mathrm{E}-00 \\
2.0 \mathrm{E}-01 \\
1.2 \mathrm{E}-01 \\
2.5 \mathrm{E}-01 \\
3.5 \mathrm{E}-02 \\
2.9 \mathrm{E}-02 \\
8.0 \mathrm{E}-03 \\
1.8 \mathrm{E}-03 \\
1.5 \mathrm{E}-05 \\
1.4 \mathrm{E}-01 \\
1.4 \mathrm{E}-01 \\
1.5 \mathrm{E}-04 \\
1.7 \mathrm{E}-07 \\
2.6 \mathrm{E}-03 \\
2.1 \mathrm{E}-07 \\
7.3 \mathrm{E}-06 \\
4.2 \mathrm{E}-03 \\
3.9 \mathrm{E}-03 \\
1.7 \mathrm{E}-02 \\
2.0 \mathrm{E}+00 \\
2.6 \mathrm{E}-01 \\
2.3 \mathrm{E}-02 \\
1.9 \mathrm{E}-01\end{array}$ \\
\hline
\end{tabular}

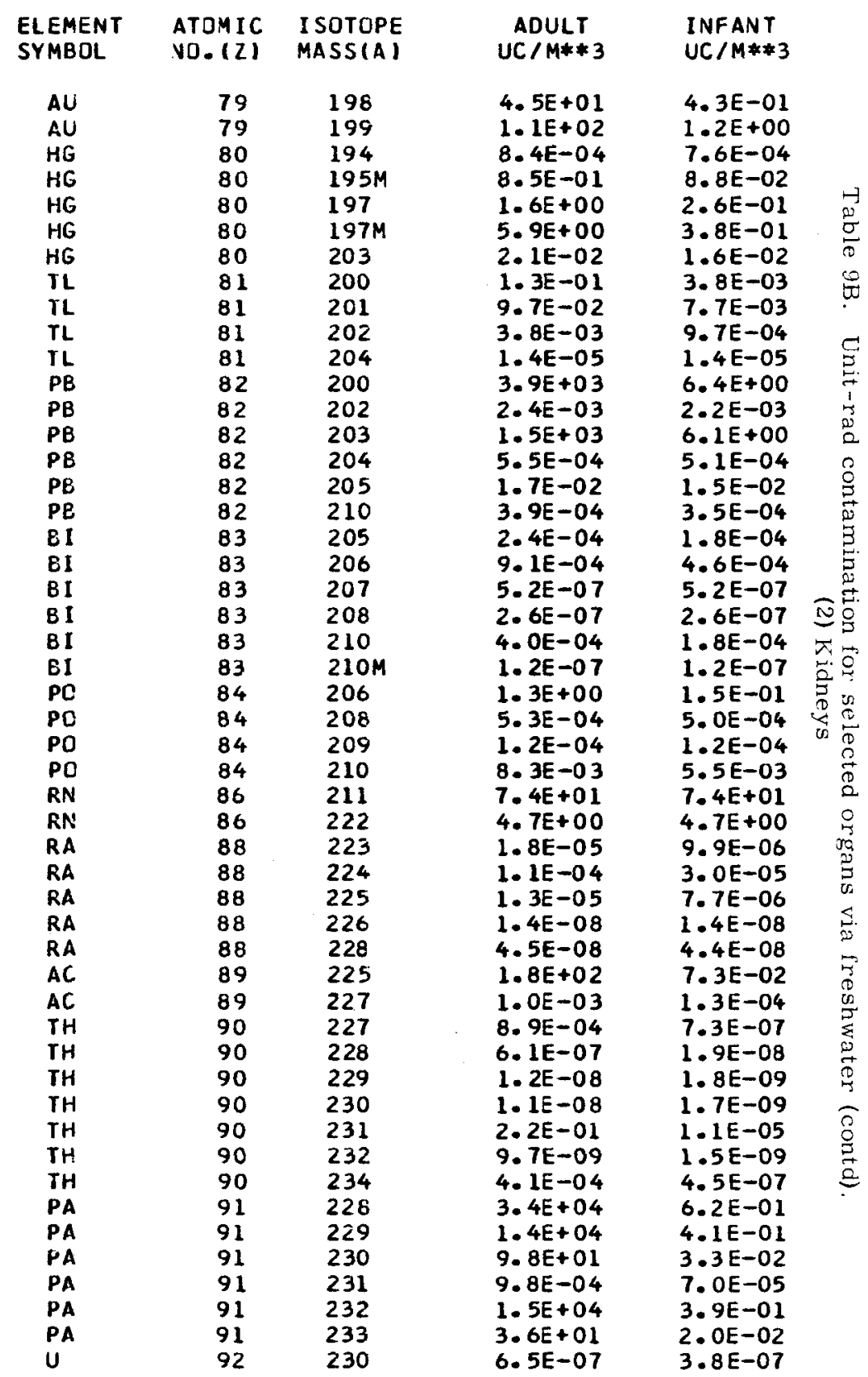


TABLE 98. UNIT-RAD CONTAMINATI ON FOR KIDNEYS

\begin{tabular}{|c|c|c|c|c|}
\hline $\begin{array}{l}\text { ELEMENT } \\
\text { SYMBOL }\end{array}$ & $\begin{array}{l}\text { ATOM IC } \\
\text { VO. (Z) }\end{array}$ & $\begin{array}{l}\text { ISOTOPE } \\
\text { MASS (A) }\end{array}$ & $\begin{array}{l}\text { ADULT } \\
\text { UC/M**3 }\end{array}$ & $\begin{array}{l}\text { INFANT } \\
\text { UC } / M * * 3\end{array}$ \\
\hline $\begin{array}{l}U \\
U \\
U \\
U \\
U \\
U \\
U \\
U \\
U \\
N P \\
N P \\
N P \\
\text { NP } \\
N P \\
N P \\
N P \\
P U \\
P U \\
P U \\
P U \\
P U \\
P U \\
P U \\
P U \\
P U \\
\text { AM } \\
\text { AM } \\
\text { AM } \\
\text { AM } \\
\text { AM } \\
\text { AM }\end{array}$ & $\begin{array}{l}92 \\
92 \\
92 \\
92 \\
92 \\
92 \\
92 \\
92 \\
92 \\
93 \\
93 \\
93 \\
93 \\
93 \\
93 \\
93 \\
94 \\
94 \\
94 \\
94 \\
94 \\
94 \\
94 \\
94 \\
94 \\
95 \\
95 \\
95 \\
95 \\
95 \\
95\end{array}$ & $\begin{array}{l}231 \\
232 \\
233 \\
234 \\
235 \\
236 \\
237 \\
238 \\
240 \\
234 \\
235 \\
236 \\
236 M \\
237 \\
238 \\
239 \\
236 \\
237 \\
238 \\
239 \\
240 \\
241 \\
242 \\
244 \\
246 \\
239 \\
240 \\
241 \\
242 \\
242 M \\
243\end{array}$ & $\begin{array}{l}\text { 8.5E-06 } \\
1.2 E-09 \\
1.1 E-09 \\
1.0 E-09 \\
9.3 E-10 \\
9.2 E-10 \\
3.4 E-06 \\
8.8 E-10 \\
2.7 E-04 \\
3.9 E+02 \\
4.4 E-02 \\
2.5 E-04 \\
8.4 E+03 \\
2.7 E-04 \\
1.5 E+03 \\
1.2 E+03 \\
1.4 E-02 \\
7.3 E+00 \\
6.3 E-04 \\
5.1 E-04 \\
5.0 E-04 \\
1.3 E-03 \\
4.8 E-04 \\
4.3 E-04 \\
9.6 E+01 \\
4.5 E+04 \\
2.4 E+03 \\
4.5 E-04 \\
2.4 E+04 \\
4.4 E-04 \\
3.9 E-04\end{array}$ & $\begin{array}{l}1.9 E-06 \\
1.2 \mathrm{E}-09 \\
1.1 \mathrm{E}-09 \\
1.0 \mathrm{E}-09 \\
9.3 \mathrm{E}-10 \\
9.1 \mathrm{E}-10 \\
1.0 \mathrm{E}-05 \\
8.8 \mathrm{E}-10 \\
1.0 \mathrm{E}-05 \\
2.7 \mathrm{E}-02 \\
2.8 \mathrm{E}-04 \\
1.4 \mathrm{E}-05 \\
1.2 \mathrm{E}-01 \\
1.5 \mathrm{E}-05 \\
5.1 \mathrm{E}-02 \\
4.3 \mathrm{E}-02 \\
4.4 \mathrm{E}-04 \\
1.0 \mathrm{E}-02 \\
6.6 \mathrm{E}-05 \\
5.6 \mathrm{E}-05 \\
5.5 \mathrm{E}-05 \\
1.1 \mathrm{E}-04 \\
5.3 \mathrm{E}-05 \\
4.7 \mathrm{E}-05 \\
3.3 \mathrm{E}-02 \\
8.4 \mathrm{E}-01 \\
1.9 \mathrm{E}-01 \\
5.7 \mathrm{E}-05 \\
5.9 \mathrm{E}-01 \\
5.6 \mathrm{E}-05 \\
5.0 \mathrm{E}-05\end{array}$ \\
\hline
\end{tabular}

VIA FRESHHATER.

\begin{tabular}{lcc} 
ELEMENT & ATOMIC & I SOTOPE \\
SYMBOL & NO. ( $Z)$ & MASS(A) \\
CM & 96 & 240 \\
CM & 96 & 241 \\
CM & 96 & 242 \\
CM & 96 & 243 \\
CM & 96 & 244 \\
CM & 96 & 245 \\
CM & 96 & 246 \\
CM & 96 & 247 \\
CM & 96 & 246 \\
BK & 97 & 245 \\
EK & 97 & 246 \\
BK & 97 & 247 \\
BK & 97 & 248 \\
BK & 97 & 249 \\
CF & 98 & 246 \\
CF & 98 & 248 \\
CF & 98 & 249 \\
CF & 98 & 250 \\
CF & 98 & 251 \\
CF & 98 & 252 \\
CF & 98 & 253 \\
ES & 99 & 251 \\
ES & 99 & 252 \\
ES & 99 & 253 \\
ES & 99 & 254 \\
ES & 99 & $254 M$ \\
ES & 99 & 255 \\
FM & 100 & 252 \\
FM & 100 & 253 \\
FM & 100 & 255 \\
FM & 100 & 257 \\
\hline & &
\end{tabular}

$10 / 09 / 68$

PAGE 10

ADULT

$U C / M * * 3$

1. $3 E+01$

3. $6 \mathrm{E}-01$

5. $2 E-04$

7. $O E-04$

3. $5 E-04$

3. $4 E-04$

3. $1 E-04$

3.1E-04

7. $6 E-02$

2. $0 E-01$

4. 5E- 05

3. $5 E-01$

1. $1 E-03$

2. $4 E-01$

$9.6 \mathrm{E}-04$

. $6 \mathrm{E}-0$

1. $0 E-04$
4. $1 E-05$

. 2E-0

1. $6 E-02$

2. $1 E-01$

2. $1 E-03$

1. 5E-02

6. 1E-04

1. $8 \mathrm{E}-01$

1. $2 E-02$

3. $1 E-01$

6. $8 E-02$

$3.4 E-01$
$3.5 E-03$
I NFAN T

$U C / M * * 3$

1. $5 E-02$

. $4 E-03$

$6.7 E-05$

8. E-05

5. $0 E-05$

4. $8 \mathrm{E}-05$

4. 4E-05

4. 4E-05

7.6E-02

2. 0E-01

$4.5 E-05$

1 E-03

2. 4E-01

$9.6 \mathrm{E}-04$

4.6E-05

4. 1 EE-04

1. $0 E-04$
4. $1 E-05$

3. 2E-0

$1.6 \mathrm{E}-02$

2. IE-01

2. IE-03

1.5E-02

6. $1 E-04$

1. $8 \mathrm{E}-01$

$1.2 E-02$

3.1E-01

6. 8E-02

$3.4 E-01$
$3.5 E-03$ 
TARI F 9B. UNIT-RAD CONTAMINATION FOR LIVER

\begin{tabular}{|c|c|c|}
\hline $\begin{array}{l}\text { ELEMENT } \\
\text { SYMBOL }\end{array}$ & $\begin{array}{l}\text { ATOMIC } \\
\text { NO. }(Z)\end{array}$ & $\begin{array}{l}\text { ISOTOP } \\
\text { MASS (A) }\end{array}$ \\
\hline$H$ & 1 & 3 \\
\hline$B E$ & 4 & 7 \\
\hline$B E$ & 4 & 10 \\
\hline C & 6 & 14 \\
\hline NA & 11 & 22 \\
\hline NA & 11 & 24 \\
\hline MG & 12 & 28 \\
\hline AL & 13 & 26 \\
\hline SI & 14 & 32 \\
\hline $\mathbf{P}$ & 15 & 32 \\
\hline $\mathbf{P}$ & 15 & 33 \\
\hline$S$ & 16 & 35 \\
\hline$C L$ & 17 & 36 \\
\hline$A R$ & 18 & 37 \\
\hline AR & 18 & 39 \\
\hline AR & 18 & 42 \\
\hline K & 19 & 40 \\
\hline K & 19 & 42 \\
\hline K & 19 & 43 \\
\hline CA & 20 & 41 \\
\hline CA & 20 & 45 \\
\hline$C A$ & 20 & 47 \\
\hline CA & 20 & 48 \\
\hline SC & 21 & $44 M$ \\
\hline SC & 21 & 46 \\
\hline $\begin{array}{l}\text { SC } \\
\text { SC }\end{array}$ & $\begin{array}{l}21 \\
21\end{array}$ & $\begin{array}{l}47 \\
48\end{array}$ \\
\hline T I & 22 & 44 \\
\hline v & 23 & 48 \\
\hline v & 23 & 49 \\
\hline v & 23 & 50 \\
\hline CR & 24 & 48 \\
\hline CR & 24 & 51 \\
\hline MN & 25 & 52 \\
\hline MN & 25 & 53 \\
\hline MN & 25 & 54 \\
\hline $\mathrm{FE}$ & 26 & 55 \\
\hline FE & 26 & 59 \\
\hline FE & 26 & 60 \\
\hline $\mathrm{CO}$ & 27 & 55 \\
\hline $\mathrm{CO}$ & 27 & 56. \\
\hline$C D$ & 27 & 57 \\
\hline CD & 27 & 58 \\
\hline CO & 27 & 60 \\
\hline NI & 28 & 56 \\
\hline NI & 28 & 57 \\
\hline NI & 28 & 59 \\
\hline NI & 28 & 63 \\
\hline NI & 28 & 66 \\
\hline $\mathrm{CU}$ & 29 & 64 \\
\hline
\end{tabular}

5. $2 E+01$

$1.1 E-02$

1. $8 \mathrm{E}-0 \mathrm{C}$

3. $6 E+02$

$5.2 E-03$

$1.8 E-02$

1. $1 \mathrm{E}+00$

3. $4 E-02$

A. $A E-02$

4. $7 E-03$

1. $5 \mathrm{E}-03$

7. $4 \mathrm{E}+02$

8. $2 E+0.1$

6. $7 \mathrm{E}+02$

8. OE-02

3. $6 E-02$

7. $4 E-04$

2. $1 E-02$

3. $2 \mathrm{E}+01$

6. $9 E-01$

2. $3 E-02$

1. $1 E+00$

9. $8 \mathrm{E}-01$

1. $9 E+02$

$9 E-0$

2. $1 E-03$

4. $7 E+00$

2. $3 E+00$

8. $7 E+03$
VIA FRESHIN ATER.

10/09/68 PAGE 11

INFAN T

UC / $M *:$ :

ELEMENT
SYMBOL

$6.4 E+J i$

8. $5 E+00$

1. $0 E-02$

1.3E-03

$2.7 E-J 2$

2.0E+D1

2.8E+0

$1.4 E-02$
$5.2 E-03$

$5.3 E-03$

3. $4 E-02$

1. $1 E+O J$

3. $4 E-52$

8. $2 E+02$

$4.4 \mathrm{E}-02$

$4.7 \mathrm{E}-03$

1.5 E-03

$6.6 E+00$

$7.0 E+00$

Q. $1 E+01$

$1.5 E+02$

$2.5 E+02$

8. OE-02

3. $1 E+01$ 4.3E-02

$4.3 E-02$
$1.7 E-03$

$2.8 E-01$

4. $3 E+01$

4.6E-02

$7.4 E-34$

3. $9 E+03$

$1 \cdot 2 E+02$

2. $0 E-02$

$9.2 E+5 J$

3. $2 E+01$

$5.1 E-02$

$2.3 E-02$

$4.1 E-03$

$6.8 E-01$

$7.4 E-02$

$2.1 E-04$
$5.7 E+33$

$5 . T E+33$

$6.4 \mathrm{E}-\mathrm{O}$

$2.7 \mathrm{E}-01$

$1.4 E-01$
$2.1 E-03$

B. $5 E+0$

$4.4 E+J$

4.

2. $1 E+50$

3. $9 E+00$

\begin{tabular}{l}
$C U$ \\
$Z N$ \\
$Z N$ \\
$Z N$ \\
$G A$ \\
$G A$ \\
$G E$ \\
$G E$ \\
$G E$ \\
$A S$ \\
$A S$ \\
$A S$ \\
$A S$ \\
$A S$ \\
$A S$ \\
$S E$ \\
$S E$ \\
$S E$ \\
$B R$ \\
$B R$ \\
$B R$ \\
$K R$ \\
$K R$ \\
$K R$ \\
$K R$ \\
$K R$ \\
$R B$ \\
$R B$ \\
$R B$ \\
$R B$ \\
$S R$ \\
$S R$ \\
$S R$ \\
$S R$ \\
$S R$ \\
$S R$ \\
$Y$ \\
$Y$ \\
$Y$ \\
$Y$ \\
$Y$ \\
$Y$ \\
$Y$ \\
$Z R$ \\
$Z R$ \\
$Z K$ \\
$I R$ \\
$Z R$ \\
$Z R$ \\
$N B$ \\
$N B$ \\
$N B$ \\
$N B$ \\
\hline \\
\hline
\end{tabular}

A ITMIC

I SOTOPE

ADULT UC $/ M * * 3$

INFANT

$U C / M * * 3$

CU $29 \quad 67$

$\begin{array}{ll}67 & 4 \\ 65 & 1 \\ 69 M & 4 \\ 72 & 8 \\ 67 & 1 \\ 72 & 1 \\ 68 & 1 \\ 69 & 1 \\ 71 & 1 \\ 71 & 2 \\ 72 & 3 \\ 73 & 2 \\ 74 & 2 \\ 76 & 2\end{array}$

4. $6 E+02$ $1.6 E-03$

4. $0 E-01$

$1.4 E+02$

$1.8 E+02$

1. $8 E-05$

- $4 E-0$

1. $2 E-01$

. $2 E+05$

2. $1 E+04$

2. $0 E+03$

4. $3 E+05$

1. $5 E+06$

2. $9 E+00$

3. $4 E-01$

$5.6 E-02$

5. $3 E+02$

$1 \cdot 1 E+02$

9. $6 E+01$

1. $5 E+02$

1. $9 E+02$

1. $3 E+00$

2. 5E-01

1. $3 E-02$

1. $8 E-02$

8. 5E-02

4. $9 E-04$

$9 \cdot 2 E+00$

2. $1 E+01$

1. $9 E+01$

7. $8 E-02$

1. $9 E+02$

1. $E+01$

3. $0 E+02$

7. OE+02

1. OE-O2

3. $1 E+01$

1. $1 E-01$

8. $2 E+02$

1. $O E-01$

9. $6 E+01$

4. $\mathrm{OE}-02$

3. $2 E-01$

1. $2 E+03$

5. $3 E+01$

1. OE-O3

1. $O E-03$

1. $2 E-01$
$1.1 E+00$

9.6E-01

$1.1 \mathrm{E}-03$

2. $9 E-01$

1. $7 E-02$

$5.6 E+01$

. OE+0I

. E 05

2. $6 E-03$

$7.1 E-02$

$1.2 E+03$

$6.3 E+02$

$2.5 E+03$

$6.5 E+01$

$8.6 E+02$

4. $4 E+03$

7. $5 E-01$

. 8E-01

$5.6 \mathrm{E}-02$

$4.2 E+01$

$2.5 E+01$

$1.5 E+01$

$1.5 E+02$

$1.9 E+02$

- $3 E+00$

$.5 E-01$

$6.6 E-03$

$5.1 E-03$

$1.6 E-02$

. $9 E-04$

7. $4 E+00$

4. $3 E+02$

1. $9 E+01$

1. $7 E+01$

. 8E-02

$8.3 E-02$

. $E-01$

2. $9 E-01$

7. $3 E-04$

5. $9 E-02$

$4.5 t-03$

1. $8 \mathrm{E}+0 \mathrm{OC}$

2. $2 E-02$

9. $7 E-01$

3. $9 E-02$

$5.4 \mathrm{E}-02$

2. $7 E+00$

$3.8 E-02$

9. $0 \mathrm{E}-04$

8. $5 E-03$
$1.3 E-02$ 


\begin{tabular}{|c|c|c|c|c|}
\hline $\begin{array}{l}\text { ELEMENT } \\
\text { SYMBOL }\end{array}$ & $\begin{array}{l}\text { ATOMIC } \\
\text { ND. (Z) }\end{array}$ & $\begin{array}{l}\text { ISOTOPE } \\
\text { MASS(A) }\end{array}$ & $\begin{array}{c}\text { ADULT } \\
U C / M * * 3\end{array}$ & $\begin{array}{l}\text { INFANT } \\
U C / M * * 3\end{array}$ \\
\hline 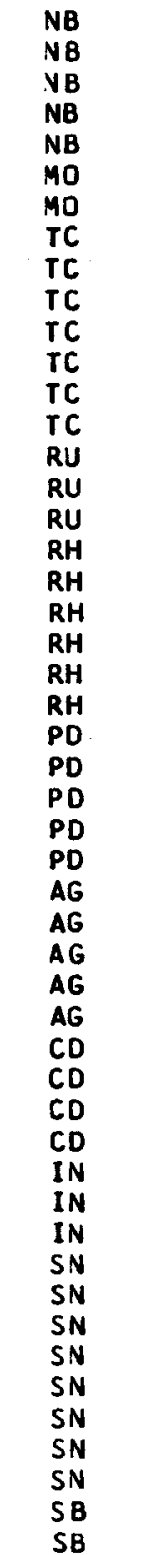 & $\begin{array}{l}41 \\
41 \\
41 \\
41 \\
41 \\
42 \\
42 \\
43 \\
43 \\
43 \\
43 \\
43 \\
43 \\
43 \\
44 \\
44 \\
44 \\
45 \\
45 \\
45 \\
45 \\
45 \\
45 \\
46 \\
46 \\
46 \\
46 \\
46 \\
47 \\
47 \\
47 \\
47 \\
47 \\
48 \\
48 \\
48 \\
48 \\
49 \\
49 \\
49 \\
50 \\
50 \\
50 \\
50 \\
50 \\
50 \\
50 \\
50 \\
51 \\
51\end{array}$ & $\begin{array}{l}93 M \\
94 \\
95 \\
95 M \\
96 \\
93 \\
99 \\
95 \\
95 M \\
96 \\
97 \\
97 M \\
98 \\
99 \\
97 \\
103 \\
106 \\
99 \\
100 \\
101 \\
101 M \\
102 \\
105 \\
100 \\
103 \\
107 \\
109 \\
112 \\
105 \\
106 M \\
108 M \\
110 M \\
111 \\
109 \\
113 M \\
115 \\
115 M \\
111 \\
114 M \\
115 \\
113 \\
117 M \\
119 M \\
121 \\
121 M \\
123 M \\
125 \\
126 \\
119 \\
120 M\end{array}$ & $\begin{array}{l}5.1 E-03 \\
1.0 E-05 \\
1.1 E-01 \\
7.1 E+00 \\
4.1 E+01 \\
4.0 E-03 \\
8.5 E+00 \\
3.0 E+00 \\
1.3 E-03 \\
4.0 E-02 \\
3.6 E-04 \\
6.8 E-03 \\
4.8 E-06 \\
7.9 E-05 \\
2.4 E+01 \\
2.0 E-01 \\
2.2 E-03 \\
3.7 E-02 \\
2.2 E+00 \\
2.3 E-04 \\
4.5 E-01 \\
4.2 E-04 \\
4.9 E+00 \\
1.4 E-01 \\
2.5 E-01 \\
1.5 E-02 \\
1.5 E+01 \\
1.1 E+00 \\
2.1 E+00 \\
1.4 E+01 \\
9.4 E-03 \\
6.9 E-02 \\
9.5 E+01 \\
6.1 E-04 \\
2.9 E-05 \\
6.9 E-01 \\
2.8 E-03 \\
2.3 E-03 \\
4.6 E-06 \\
8.2 E-08 \\
1.2 E+01 \\
1.1 E+03 \\
1.4 E+01 \\
4.4 E+05 \\
8.1 E-02 \\
6.7 E+00 \\
4.1 E+02 \\
2.1 E-03 \\
8.0 E+01 \\
1.4 E-01\end{array}$ & $\begin{array}{l}3.2 \mathrm{E}-03 \\
9.1 \mathrm{E}-03 \\
4.3 \mathrm{E}-03 \\
3.2 \mathrm{E}-02 \\
4.7 \mathrm{E}-02 \\
4.0 \mathrm{E}-03 \\
2.4 \mathrm{E}-01 \\
8.0 \mathrm{E}-02 \\
8.4 \mathrm{E}-04 \\
5.0 \mathrm{E}-03 \\
3.6 \mathrm{E}-04 \\
5.1 \mathrm{E}-03 \\
4.8 \mathrm{E}-05 \\
7.9 \mathrm{E}-05 \\
9.6 \mathrm{E}-01 \\
7.1 \mathrm{E}-02 \\
1.9 \mathrm{E}-03 \\
1.7 \mathrm{E}-02 \\
9.9 \mathrm{E}-02 \\
2.3 \mathrm{E}-04 \\
8.9 \mathrm{E}-02 \\
3.8 \mathrm{E}-04 \\
3.7 \mathrm{E}-01 \\
2.2 \mathrm{E}-02 \\
1.2 \mathrm{E}-01 \\
1.5 \mathrm{E}-02 \\
4.4 \mathrm{E}-01 \\
4.9 \mathrm{E}-02 \\
9.3 \mathrm{E}-01 \\
1.9 \mathrm{E}+00 \\
9.1 \mathrm{E}-03 \\
5.8 \mathrm{E}-02 \\
1.2 \mathrm{E}+01 \\
4.3 \mathrm{E}-04 \\
2.8 \mathrm{E}-05 \\
7.9 \mathrm{E}-03 \\
5.0 \mathrm{E}-04 \\
1.1 \mathrm{E}-04 \\
2.1 \mathrm{E}-06 \\
8.1 \mathrm{E}-0 \mathrm{~B} \\
8.5 \mathrm{E}-01 \\
9.4 \mathrm{E}+00 \\
1.9 \mathrm{E}+00 \\
3.1 \mathrm{E}+02 \\
6.0 \mathrm{E}-02 \\
4.9 \mathrm{E}-01 \\
2.4 \mathrm{E}+00 \\
1.7 \mathrm{E}-03 \\
3.2 \mathrm{E}+00 \\
1.8 \mathrm{E}-02\end{array}$ \\
\hline
\end{tabular}

$\begin{array}{lcc}\text { ELEMENT } & \text { ATOMIC } & \text { ISOTOPE } \\ \text { SYMBOL } & \text { NO. I I } & \text { MASSIA } \\ & & \\ \text { SB } & 51 & 122 \\ \text { SB } & 51 & 124 \\ \text { SB } & 51 & 125 \\ \text { SB } & 51 & 126 \\ \text { SB } & 51 & 127 \\ \text { TE } & 52 & 118 \\ \text { TE } & 52 & 119 \\ \text { TE } & 52 & 119 M \\ \text { TE } & 52 & 121 \\ \text { TE } & 52 & 121 M \\ \text { TE } & 52 & 123 \\ \text { TE } & 52 & 123 M \\ \text { TE } & 52 & 125 M \\ \text { TE } & 52 & 127 M \\ \text { TE } & 52 & 129 M \\ \text { TE } & 52 & 131 M \\ \text { TE } & 52 & 132 \\ \text { I } & 53 & 123 \\ \text { I } & 53 & 124 \\ \text { I } & 53 & 125 \\ \text { I } & 53 & 126 \\ \text { I } & 53 & 129 \\ \text { I } & 53 & 130 \\ \text { I } & 53 & 131 \\ \text { I } & 53 & 133 \\ \text { XE } & 54 & 122 \\ \text { XE } & 54 & 125 \\ \text { XE } & 54 & 127 \\ \text { XE } & 54 & 129 M \\ \text { XE } & 54 & 131 M \\ \text { XE } & 54 & 133 \\ \text { XE } & 54 & 133 M \\ \text { CS } & 55 & 129 \\ \text { CS } & 55 & 131 \\ \text { CS } & 55 & 132 \\ \text { CS } & 55 & 134 \\ \text { CS } & 55 & 135 \\ \text { CS } & 55 & 136 \\ \text { CS } & 55 & 137 \\ \text { BA } & 56 & 128 \\ \text { BA } & 56 & 131 \\ \text { BA } & 56 & 133 \\ \text { BA } & 56 & 133 M \\ \text { BA } & 56 & 135 M \\ \text { BA } & 56 & 140 \\ \text { LA } & 57 & 135 \\ \text { LA } & 57 & 137 \\ \text { LA } & 57 & 138 \\ \text { LA } & 57 & 140 \\ \text { CE } & 58 & 134\end{array}$

AOULT $U C / M * 3$

1. $2 E+00$

3. $0 E-03$

5. 3E- 04

5. $9 E-01$

2. $1 E-01$

1. $0 E+01$

2. $1 E-01$

7. $9 E-02$

2. $6 E-03$

1. 3E-03

1. 3E-02

5. $2 E-02$

1. $O E-02$

1. $8 \mathrm{E}-02$

2. $5 E+00$

2. 9E- 01

2. $8 E+03$

$6.8 E+00$

5. $5 E+00$

4. 2E-02

2. $8 \mathrm{E}+02$

9. $5 E+00$

2. $O E+02$

2. $1 E+02$

1. $1 E+03$

5. $3 E+01$

2. $3 E+02$

$4.6 E+02$

4. $7 E+02$

3. $9 E+02$

1. $3 E+02$

1. $4 E+01$

2. $8 E-03$
8. $8 E-03$

8. $8 E-03$

1. $1 E+00$

8. $4 E-04$

1. $3 E+05$

1. $3 E+04$

2. $8 \mathrm{E}+00$

1. $0 E+06$

4. $8 E+06$

3. $1 E+03$

7. $4 E+04$

4. $O E-01$

1. $2 \mathrm{E}-02$

4. $9 E+03$
INFANT

UC/M**3

8. 4 E-02

1. $9 E-03$

$5.1 E-04$
$7.7 E-03$

5. 5E-02

$3,5 E-02$

$2.3 \mathrm{E}-01$

$2.8 E-02$

2. 9E-02

2. $2 \mathrm{E}-03$

$1.3 \mathrm{E}-03$

1. $0 E-02$

3. 5E-02

8. $0 E-03$

$9.3 E-03$

$9.6 E-02$

2. 9E-02

2. $0 E+02$

$2.6 E+00$

$6.1 E+00$

$3.6 E+00$

4.2E-02

1.9E+01

$5.1 E+00$

$2.2 E+01$

$2.1 E+02$

1. $1 E+03$

2. $4 E+02$

$2.3 E+02$

4. $6 E+02$

4. $7 E+02$

4. $5 E+00$

$1.1 E+01$

8. OE-01

2. 5E-03

8. $7 E-03$

1. $2 \mathrm{E}-01$

8. 2E-04

$3.1 E+02$

$1.5 E+02$

2. $O E+00$

1. $7 E+03$

6. $0 E+03$

4. $1 E+01$

1. $5 E+02$

3. $8 \mathrm{E}-01$

1. $1 E-02$

2. $1 E+01$ 
TABLE 9B. UNIT-RAD COVTAMINATION FOR LIVER

\begin{tabular}{|c|c|c|c|c|}
\hline $\begin{array}{l}\text { ELEMENT } \\
\text { SYMBOL }\end{array}$ & $\begin{array}{l}\text { AromIC } \\
\text { NO. }(Z)\end{array}$ & $\begin{array}{l}\text { ISOTOPE } \\
\text { MASS (A) }\end{array}$ & $\begin{array}{l}\text { ADULT } \\
\text { UC/M**5 }\end{array}$ & $\begin{array}{l}\text { INFAN T } \\
\text { UC } / M * \div 3\end{array}$ \\
\hline $\begin{array}{l}C E \\
C E \\
C E \\
C E \\
C E \\
C E \\
C E \\
P R \\
P R \\
N D \\
N D \\
N D \\
P M \\
P M \\
P M \\
P M \\
P M \\
P M \\
P M \\
P M \\
P M \\
S M \\
S M \\
S M \\
S M \\
S M \\
S M \\
S M \\
S M \\
S M \\
E U \\
E U \\
E U \\
E U \\
E U \\
E U \\
E U \\
E U \\
E U \\
E U \\
E U \\
E U \\
E U \\
G D \\
G D \\
G D \\
G D \\
G D \\
G D \\
G D \\
G D\end{array}$ & $\begin{array}{l}58 \\
58 \\
58 \\
58 \\
58 \\
58 \\
58 \\
59 \\
59 \\
60 \\
60 \\
60 \\
61 \\
61 \\
61 \\
61 \\
61 \\
61 \\
61 \\
61 \\
61 \\
62 \\
62 \\
62 \\
62 \\
62 \\
62 \\
62 \\
62 \\
63 \\
63 \\
63 \\
63 \\
63 \\
63 \\
63 \\
63 \\
63 \\
63 \\
63 \\
63 \\
63 \\
64 \\
64 \\
64 \\
64 \\
64 \\
64 \\
64 \\
64\end{array}$ & $\begin{array}{l}135 \\
137 M \\
139 \\
141 \\
142 \\
143 \\
144 \\
142 \\
143 \\
140 \\
144 \\
147 \\
143 \\
144 \\
145 \\
146 \\
147 \\
148 \\
148 M \\
149 \\
151 \\
141 \\
145 \\
146 \\
147 \\
148 \\
149 \\
151 \\
153 \\
145 \\
146 \\
146 \\
147 \\
148 \\
149 \\
150 \\
150 \\
159 \\
152 \\
154 \\
155 \\
156 \\
157 \\
146 \\
147 \\
148 \\
149 \\
150 \\
151 \\
152 \\
153\end{array}$ & $\begin{array}{l}1.2 E+03 \\
8.6 E+02 \\
2.3 E-01 \\
2.6 E+00 \\
2.6 E-04 \\
2.8 E+02 \\
4.1 E-03 \\
2.0 E-02 \\
2.3 E-04 \\
4.3 E-04 \\
3.2 E-09 \\
1.8 E-05 \\
7.5 E-02 \\
5.9 E-03 \\
9.6 E-03 \\
2.2 E-03 \\
1.6 E-03 \\
3.1 E+01 \\
1.6 E-01 \\
6.6 E+02 \\
9.3 E+02 \\
3.4 E-01 \\
7.8 E-02 \\
1.4 E-04 \\
1.6 E-04 \\
9.3 E-05 \\
2.0 E-04 \\
2.7 E-02 \\
3.8 E+02 \\
2.0 E+01 \\
5.3 E+00 \\
6.4 E+01 \\
3.5 E-01 \\
4.4 E-01 \\
1.4 E-01 \\
2.3 E-03 \\
4.1 E+02 \\
8.8 E-04 \\
6.7 E-04 \\
6.6 E-02 \\
1.5 E+00 \\
1.1 E+03 \\
6.4 E-02 \\
1.5 E+02 \\
8.1 E-05 \\
7.8 E+00 \\
4.3 E-05 \\
1.9 E-01 \\
1.1 E-04 \\
1.3 E-01\end{array}$ & $\begin{array}{l}3.0 E+00 \\
4.2 E+00 \\
7.5 E-02 \\
2.6 E-01 \\
2.5 E-04 \\
1.3 E+00 \\
2.0 E-03 \\
4.3 E-05 \\
8.0 E-06 \\
1.1 E-05 \\
3.2 E-09 \\
1.4 E-06 \\
2.2 E-02 \\
2.1 E-03 \\
8.3 E-03 \\
1.6 E-03 \\
9.6 E-04 \\
2.6 E-01 \\
9.1 E-03 \\
2.2 E+03 \\
1.7 E+00 \\
3.3 E-02 \\
5.0 E-02 \\
1.4 E-04 \\
1.6 E-04 \\
9.1 E-05 \\
1.9 E-04 \\
2.6 E-02 \\
3.9 E+00 \\
9.1 E-01 \\
1.8 E-01 \\
7.8 E-01 \\
5.5 E-02 \\
1.3 E-01 \\
6.5 E-02 \\
2.2 E-03 \\
1.7 E+00 \\
8.5 E-04 \\
6.5 E-04 \\
3.5 E-02 \\
1.6 E-01 \\
5.6 E+00 \\
6.1 E-03 \\
3.3 E-01 \\
7.5 E-05 \\
1.5 E-01 \\
4.1 E-05 \\
3.8 E-02 \\
1.0 E-04 \\
4.5 E-02\end{array}$ \\
\hline
\end{tabular}

VIA FRESHWATER.

$10 / 09 / 68$

PAGE 13

\begin{tabular}{|c|c|c|}
\hline $\begin{array}{l}\text { ELEMENT } \\
\text { SYMEOL }\end{array}$ & $\begin{array}{l}\text { A TOMIC } \\
\text { NO. (Z) }\end{array}$ & $\begin{array}{l}\text { I SOTUP } \\
\text { MASSIA }\end{array}$ \\
\hline GD & 64 & 159 \\
\hline GD & 64 & 162 \\
\hline TB & 65 & 151 \\
\hline TE & 65 & 152 \\
\hline TB & 65 & 153 \\
\hline TB & 65 & 154 \\
\hline$T B$ & 65 & 155 \\
\hline TB & 65 & 150 \\
\hline TB & 65 & 157 \\
\hline TB & 65 & 158 \\
\hline TE & 65 & 160 \\
\hline$T_{B}$ & 65 & 161 \\
\hline TB & 65 & 164 \\
\hline DY & 66 & 154 \\
\hline or & 66 & $154 \mathrm{M}$ \\
\hline DY & 66 & 156 \\
\hline or & 66 & 159 \\
\hline or & 66 & 166 \\
\hline $\mathrm{HC}$ & 67 & 163 \\
\hline HO & 67 & 166 \\
\hline HO & 67 & $166 \mathrm{~N}$ \\
\hline$E R$ & 68 & 160 \\
\hline$E R$ & 68 & 169 \\
\hline ER & 68 & 172 \\
\hline$T M$ & 69 & 165 \\
\hline$T M$ & 69 & 167 \\
\hline$T M$ & 69 & 168 \\
\hline TM & 69 & 170 \\
\hline TM & 69 & 171 \\
\hline$T M$ & 69 & 172 \\
\hline $\mathrm{YH}_{\mathrm{B}}$ & 70 & 166 \\
\hline YB & 70 & 169 \\
\hline$Y_{B}$ & 70 & 175 \\
\hline$L U$ & 71 & 169 \\
\hline LU & 71 & 170 \\
\hline $\mathrm{LU}$ & 71 & 171 \\
\hline LU & $7 i$ & 172 \\
\hline $\mathrm{LU}$ & 71 & 173 \\
\hline 1.10 & 71 & 174 \\
\hline L: & 71 & $174 M$ \\
\hline LO & 71 & 176 \\
\hline LU & 71 & 177 \\
\hline LU & 71 & $177 \mathrm{~N}$ \\
\hline $\mathrm{HF}$ & 72 & 170 \\
\hline $\mathrm{HF}$ & 72 & 171 \\
\hline HF & 72 & 172 \\
\hline $\mathrm{HF}$ & 72 & 173 \\
\hline HF & 72 & 174 \\
\hline $\mathrm{HF}$ & 72 & 175 \\
\hline HiF & 72 & 181 \\
\hline
\end{tabular}

ADULT INFAVT US $/ M * * 3 \quad U C / M * * 3$

$3.2 E+03 \quad 5.3 E+00$ $6.7 E-03 \quad 3.0 E-03$ $6.4 E+02 \quad 7.1 E+00$ 1. $9 E+02 \quad 2.1 E+00$ 1. $3 E+02 \quad 4.6 E+00$ 6. $9 E+02$ $3.0 E+01$ 3. $0 E+01$ 1. $5 E-02$ $1.5 E-02$
$6.7 E-04$ 6. $7 E-04$

1. $1 E-01$ 4. $3 E+01$ $9.3 E+02$ 6. $3 E-10$

1. $8 E-02$

9. $9 E-10$

1. $7 E-05$

1. $4 E-03$

1. $1 E-07$

2. $9 E-02$

4. $8 E-09$

4. $2 E-03$

2. $1 E-03$

3. 3E-03

1. $0 E+03$

8. $0 E+O 1$

7. $5 E-01$

1. $8 E+00$

4. $3 E+00$

5. $9 E+02$

7. $7 E+02$

$8.8 E+00$

. $4 E-05$

1. $4 E-05$

$3.4 E-05$

$6.0 E-06$

1. $1 E-07$

2. $4 E-08$

4. $4 E-07$

7. $1 E-09$

1. $2 E-06$

1. $4 E+00$

2. $7 E+00$

. 5E-06

8. 3E-OI

8. $3 E-01$

1. $4 E-07$

6. $7 E-04$
$1.1 E-03$

8. $8 E+00$

$6.8 E-01$

$4 \mathrm{E}+01$

5. $9 E-10$

2. $0 E-05$

9. $2 E-10$

3. $8 E-06$

9. $4 E-06$

1.2E-05

$1.7 E+0$

$9.6 E+00$

4. $1 E-01$

2. $2 E+01$

$2.6 \mathrm{E}+01$

2. $8 E+00$

$4.4 E+01$

$1.4 E-05$

$6.4 E-06$

$3.4 \mathrm{E}-05$

6. $0 E-06$

1. $1 E-07$

2. $4 E-08$

$4.4 E-07$

7. 1 E-09

3. $4 E-05$

1. $2 E-06$

1. $1 \mathrm{E}-03$

2. 5E-03

$1.1 E-06$

$1.3 E-03$

6. $8 \mathrm{E}-05$
. $3 E+00$

. $5 E-02$

$6.6 E-04$

$5.5 E-02$

$9.5 E-08$

4. $6 \mathrm{E}-05$

1. $6 E-05$

1. $2 E+00$

3. $9 E+00$

$7.3 \mathrm{E}-05$

3.7E-05 ड0 
ELEMENT

ATOMIC ATOMIC TSE

ADULT

INFANT

UC/M**3

2. $1 E-07$ 1. $8 \mathrm{E}-01$

1. 2E-04

7. $1 E-05$
$6.8 E-02$

4. $2 E-04$

1. $6 E \div 03$

1. $6 \mathrm{E}-03$

8. $1 E-02$

1. $5 \mathrm{E}-04$

-

9. $1 E+00$

2. $3 E+O 1$

3. $5 E-02$

2. $5 \mathrm{E}-02$

3. $5 \mathrm{E}-03$

2. $O E+O O$

6. $8 E-01$

2. $0 E+01$

2. $3 E+01$

8. $5 E-02$

3. $5 E-04$

1. $1 E-02$

1. $5 E+00$

1. $9 E-01$

5. $1 E-06$

8. $8 E+00$

5. $2 E+00$

1. $4 E+01$

5. $9 E-01$

8. 8E-02

2. $6 E-02$

2. $4 E-03$

1. $5 E-05$

4. $6 \mathrm{E}-01$

4. $8 E+00$

4. $4 E-04$

1. $7 E-07$

2. $0 E-02$

2. $1 E-07$

7. 3E-06

2. 3E-02

2. 3E-02

4. $2 E-01$

5. $1 E+02$

3. $1 E+01$

4. $2 E+00$

1. $9 E-07$

1. OE-D 3

7. $1 E-05$

$1.6 E-05$

$8.4 E-04$

$.6 E-04$
$.5 E-03$

$1.5 E-03$

$1.6 E-02$

1.5E-0

4. $4 E-01$

$4.8 \mathrm{E}-01$

1. $7 \mathrm{E}-01$

8. $2 \mathrm{E}-01$

2. 9E-02

1. 8E-D2

$3.2 E-03$

4. OE-01

8. $E-01$

$1.4 E+0 J$

1. 2E- 02

5. 2E-02

$3.3 E-04$

$7.8 E-03$

1. $5 E-01$

3. 7E-02

$5.1 E-05$

2. OE-O 1

1. 2E-O 1

2.5E-01

$3.5 E-02$

2.9E-02

8. 0E-0 3

$1.8 E-03$

$1.5 E-05$

$1.4 E-01$

1. $4 \mathrm{E}-01$

1. $5 \mathrm{E}-04$

$1.7 \mathrm{E}-01$

2. $1 E-07$

7.3E-0S

4. $2 E-03$

3. $9 E-03$

1. $7 E-02$

$1.7 E-01$

$1.5 E-02$

1.3E-O1
ELEMENT

SYMBOL

ATOMIC

I SOTOPE

UC/M**3

2E+01

3. $2 E+01$

7. $4 E+01$
$1.1 E-03$

1. $1 E+00$

2. $0 E+00$

7. $3 E+00$

2.7E-02

8. 3E-02

6. $5 E-02$

2. $7 E-03$

1. $3 E-05$

1. $0 E+04$

2. $2 E-03$

4. $O E+03$

5. $0 E-04$

1. $5 E-02$

3. $8 E-04$

1. $8 \mathrm{E}-03$

8. $2 E-03$

2. $7 E-06$

1. $3 E-06$

3. $8 E-03$

6. $4 E-07$

8. 5E-01

5. $2 E-04$

1. 2E-04

7. $2 E-03$

$7.4 E+01$

4. $7 E+00$

1. 8E-05

1. $1 E-04$

1. 3E-05

1. $4 E-08$

4. $5 E-08$

1. $8 E+01$

2. $0 E-04$

2. $3 E-03$

1. $6 E-06$

2. $8 E-08$

2. $6 E-0 B$

5. $8 E-01$

2. $3 E-08$

1. $1 E-03$

3. $9 E+04$

1. $6 E+04$

1. $1 E+02$

1. $1 E-03$

1. $7 E+04$

4. $1 E+0$

3. 8E-0 7
INFANT
UC/M**3

2. $8 \mathrm{E}-01$ 7. $7 E-01$ 1. $0 E-03$

1. $2 E-01$

3. $4 \mathrm{E}-01$

5. $0 E-01$

2. $1 E-02$

7. $0 E-03$

8. 8E-04

1. 3E-05

4. $7 E+00$

1. $6 E-03$

4. $4 E+00$

3. $7 E-04$

1. 1 E-02

$2.6 E-04$

$9.1 E-04$

2. $7 E-06$

1.

$9.4 E-04$

$6.4 E-07$

1. $5 \mathrm{E}-01$

5. $0 E-04$

1. 2E-04

5. 5E-03

7. $4 E+01$

.

3. 0 E-05

3. $0 E-05$

$1.4 \mathrm{E}-08$

$4.4 E-08$

7. $3 E-02$

1. 3E-04

7. $3 E-07$

1. $9 E-08$

1. $8 E-09$

1. $7 E-09$

1. $1 E-05$

1. 5E-09

4. 5E-07

6. 2E-01

4. $1 E-01$

$3.3 E-02$

7. $0 E-05$

3. $9 E-01$

3. 8E-07

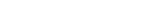


TABLE 9B. UNIT-RAD IONTAMINATI ON FOR LIVER

$\begin{array}{lcccc}\begin{array}{l}\text { ELEMENT } \\ \text { SYMBOL }\end{array} & \begin{array}{c}\text { ATOMIC } \\ \text { VJ. (Z) }\end{array} & \begin{array}{c}\text { ISOTOPE } \\ \text { MASSIA) }\end{array} & \begin{array}{c}\text { ADULT } \\ \text { UC } / M * 3\end{array} & \begin{array}{l}\text { INFANT } \\ \text { UC } / M * * 3\end{array} \\ U & 92 & 231 & 1.9 E-06 & 1.9 E-06 \\ U & 92 & 232 & 1.2 E-09 & 1.2 E-09 \\ U & 92 & 233 & 1.1 E-09 & 1.1 E-09 \\ U & 92 & 234 & 1.0 E-09 & 1.0 E-09 \\ U & 92 & 235 & 9.3 E-10 & 9.3 E-10 \\ U & 92 & 236 & 9.1 E-10 & 9.1 E-10 \\ U & 92 & 237 & 1.0 E-06 & 1.0 E-05 \\ U & 92 & 238 & 8.8 E-10 & 8.8 E-10 \\ U & 92 & 240 & 1.0 E-05 & 1.0 E-05 \\ \text { NP } & 93 & 234 & 3.3 E+02 & 2.7 E-02 \\ \text { NP } & 93 & 235 & 3.7 E-02 & 2.8 E-04 \\ \text { NP } & 93 & 236 & 2.1 E-04 & 1.4 E-05 \\ \text { NP } & 93 & 236 M & 7.1 E+03 & 1.2 E-01 \\ \text { NP } & 93 & 237 & 2.3 E-04 & 1.5 E-05 \\ \text { NP } & 93 & 238 & 1.3 E+03 & 5.1 E-02 \\ \text { NP } & 93 & 239 & 9.9 E+02 & 4.3 E-02 \\ \text { PU } & 94 & 236 & 1.3 E-02 & 4.4 E-04 \\ \text { PU } & 94 & 237 & 6.8 E+00 & 1.0 E-02 \\ \text { PU } & 94 & 238 & 5.9 E-04 & 6.6 E-05 \\ \text { PU } & 94 & 239 & 4.8 E-04 & 5.6 E-05 \\ \text { PU } & 94 & 240 & 4.7 E-04 & 5.5 E-05 \\ \text { PU } & 94 & 241 & 1.3 E-03 & 1.1 E-04 \\ \text { PU } & 94 & 242 & 4.6 E-04 & 5.3 E-05 \\ \text { PU } & 94 & 244 & 4.1 E-04 & 4.7 E-05 \\ \text { PU } & 94 & 246 & 9.0 E+01 & 3.3 E-02 \\ \text { AM } & 95 & 239 & 5.8 E+03 & 8.4 E-01 \\ \text { AM } & 95 & 240 & 3.1 E+02 & 1.9 E-01 \\ \text { AM } & 95 & 241 & 9.6 E-05 & 5.7 E-05 \\ \text { AM } & 95 & 242 & 3.1 E+03 & 5.9 E-01 \\ \text { AM } & 95 & 242 M & 9.5 E-05 & 5.6 E-05 \\ \text { AM } & 95 & 243 & 8.4 E-05 & 5.0 E-05 \\ & & & & \end{array}$

VIA FRESHWATER.

ELEMEN1 SY MEOL

ATOMIC NO. $(2)$

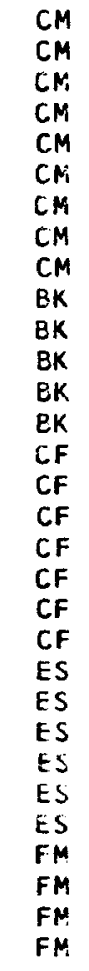

$10 / 09 / 68$

ADULT $U C / M * * 3$

1. $7 E+00$

1. $0 E+00$

4. $7 E-02$

1. $5 E-0$

7. $9 E-05$

7. $6 E-05$

7. $0 E-05$

$6.9 E-05$

6. $9 E-05$
7. $6 E-02$

7. $2 E-02$

2. $0 E-01$

3. $5 E-01$

1. $1 E-03$

2. $4 E-01$

9. $6 E-04$

4. $6 E-05$

1. $0 E-04$

4. $1 E-05$

3. $2 E-04$

1. $6 E-02$

2. $1 E-01$

2. $1 E-03$

1. $5 E-02$

6. $1 E-04$

1. $2 E-02$

3. $1 E-01$

3. $4 \mathrm{E}-02$

3. $5 \mathrm{E}-03$
PAGE 15

INFANT

UC $/ M * * 3$

1. $5 E-02$
$1.2 E-02$

1. $2 \mathrm{E}-02$

2. 6 . $7 E-03$

8. 3E-05

5. OE-05

4. 8E-05

$4.5 E-05$

$4.5 E-05$

4. $4 E-05$

2. $0 E-01$

2. $0 E-01$

4. $5 \mathrm{E}-05$

3. $5 \mathrm{E}-01$

1. 1 E-03

$2.4 E-01$

$9.6 \mathrm{E}-04$

4. $6 E-05$

1. OE-OA

4. $1 E-05$

$3.2 \mathrm{E}-04$

$1.6 E-02$

2.1E-0

2. $1 \mathrm{E}-03$

1. $5 \mathrm{E}-02$

6. $1 E-04$

1. $8 E-01$

1. 2E-02

3.1E-01

6. $8 \mathrm{E}-02$

$3.4 E-01$
$3.5 E-03$ 


$\begin{array}{ll}\text { ADULT } & \text { INFANT } \\ \text { UCIM**3 } & U C / M * * 3\end{array}$

ELEMENT
SYMBOL

\section{ATOMIC}

I SOTOPE

ADULT $U C / M * * 3$

INFANT 6. $4 E+02 \quad 6.4 E+02$ 8. $5 E+00 \quad 8.5 E+00$ 1. 3E-03

1. $9 \mathrm{E}-02$ 1. $O E-02$

$1.3 \mathrm{E}-03$

$5 E+01$

1. $5 E-02$

7. $4 \mathrm{E}-03$

2. $7 \mathrm{E}-02$

1. $4 E-01$

1. $1 E+00$

2. 2E-02

8. $2 E+02$

4. $4 E-02$

4. 7E-03

. $0 E-03$

1. $4 E+03$

. $1 E+01$

4. $7 E+01$

5. $8 E+02$

2. $1 E-02$

4. 3E-04

1. $7 E-05$

. $8 E-03$

1. $2 E-03$

2. $8 E+01$

B. $2 E+02$

$1.4 E-01$

2. 9E-01

1. $0 E+00$

5. $4 E-01$

2. $1 E-01$

3. $7 E-02$

3. $3 E+00$

7. $5 E-04$

6. $1 E+02$

2. $7 \mathrm{E}-01$

9. 7E-OI

6. 3E-01

6. 9E-03

7. $0 E+01$

7. OE+ OI

3. $6 E+00$

1. TE+00

$5.0 E+01$
$1.1 E+03$

$3 E+01$

$1.5 \mathrm{E}-02$

7. $4 \mathrm{E}-03$

$1.5 \mathrm{E}-02$
$9.5 \mathrm{E}-02$

1. $1 E+O J$

2. $1 E-02$

$8.2 E+02$

4. $4 \mathrm{E}-02$

4.7E- 03

2. $9 \mathrm{E}-03$

$1.3 E+01$

$1.4 \mathrm{E}+01$

2. $1 E+01$

3. $9 E+01$

$6.5 E+01$

2. $1 E-02$

4. 3E-D 4

1. 7E-05

2.8E-03

$4.6 \mathrm{E}-04$

1. $2 \mathrm{E}-03$

2. $8 E+01$

8. $2 \mathrm{E}+02$

$1.4 \mathrm{E}-01$

2. $9 E-01$

1. $O E+O D$

4.6E-01

2.1E-O 1

3. $7 E-02$

2. $2 \mathrm{E}+00$

2. $4 E-01$

7. $0 \mathrm{E}-04$

$1.8 \mathrm{E}+01$

2. $1 E-0$

8. $9 E-01$

4.7E-D 1

6. $9 E-03$

$8.9 E+00$

7. $O E+O 1$

7.

$3.6 E+00$

5. $0 E+01$

$3.6 E+01$

\begin{tabular}{|c|c|}
\hline 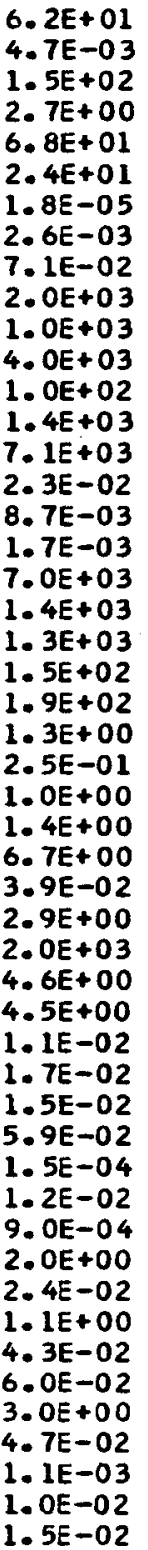 & 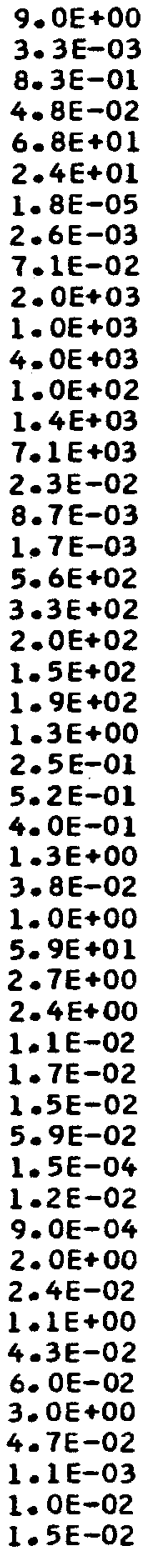 \\
\hline
\end{tabular}




\begin{tabular}{|c|c|c|c|c|}
\hline $\begin{array}{l}\text { ELEMENT } \\
\text { SYMBOL }\end{array}$ & $\begin{array}{l}\text { ATOMIC } \\
\text { NO. }(Z)\end{array}$ & $\begin{array}{l}\text { ISOT DPE } \\
\text { MASS (A) }\end{array}$ & $\begin{array}{l}\text { ADULT } \\
\text { UC } / M * * 3\end{array}$ & $\begin{array}{l}\text { INFANT } \\
\text { UC } / M * * 3\end{array}$ \\
\hline 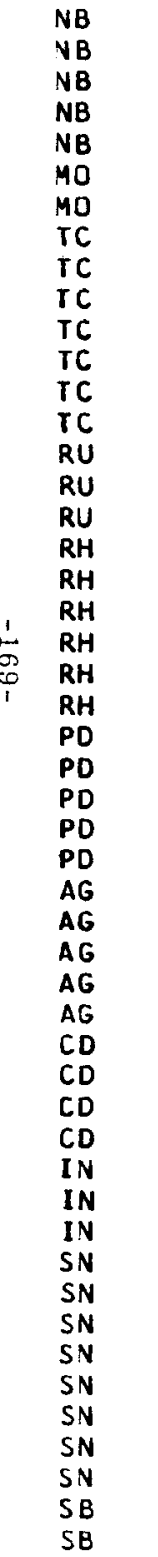 & $\begin{array}{l}41 \\
41 \\
41 \\
41 \\
41 \\
42 \\
42 \\
43 \\
43 \\
43 \\
43 \\
43 \\
43 \\
43 \\
44 \\
44 \\
44 \\
45 \\
45 \\
45 \\
45 \\
45 \\
45 \\
46 \\
46 \\
46 \\
46 \\
46 \\
47 \\
47 \\
47 \\
47 \\
47 \\
48 \\
48 \\
48 \\
48 \\
49 \\
49 \\
49 \\
50 \\
50 \\
50 \\
50 \\
50 \\
50 \\
50 \\
50 \\
51 \\
51\end{array}$ & $\begin{array}{l}93 M \\
94 \\
95 \\
95 M \\
96 \\
93 \\
99 \\
95 \\
95 M \\
96 \\
97 \\
97 M \\
98 \\
99 \\
97 \\
103 \\
106 \\
99 \\
100 \\
101 \\
101 M \\
102 \\
105 \\
100 \\
103 \\
107 \\
109 \\
112 \\
105 \\
106 M \\
108 M \\
110 M \\
111 \\
109 \\
113 M \\
115 \\
115 M \\
111 \\
114 M \\
115 \\
113 \\
117 M \\
119 M \\
121 \\
121 M \\
123 M \\
125 \\
126 \\
119 \\
120 M\end{array}$ & $\begin{array}{l}\text { 3. } 9 E-03 \\
1.1 E-05 \\
5.3 E-03 \\
3.9 E-02 \\
5.7 E-02 \\
1.8 E+00 \\
7.8 E+02 \\
8.0 E-02 \\
8.4 E-04 \\
5.0 E-03 \\
3.6 E-04 \\
5.1 E-03 \\
4.8 E-06 \\
7.9 E-05 \\
2.4 E+01 \\
2.0 E-01 \\
2.2 E-03 \\
1.7 E-02 \\
9.9 E-02 \\
2.3 E-04 \\
8.9 E-02 \\
3.8 E-04 \\
3.7 E-01 \\
2.2 E-02 \\
1.2 E-01 \\
1.5 E-02 \\
4.4 E-01 \\
4.9 E-02 \\
3.8 E+00 \\
9.9 E+00 \\
3.5 E-02 \\
2.2 E-01 \\
6.4 E+01 \\
2.7 E-03 \\
1.6 E-04 \\
1.3 E+00 \\
7.1 E-03 \\
1.1 E-04 \\
2.1 E-06 \\
8.1 E-08 \\
5.4 E-02 \\
1.8 E+01 \\
1.0 E+00 \\
5.8 E+0- \\
2.8 E-02 \\
3.0 E-01 \\
6.3 E+00 \\
7.7 E-04 \\
1.9 E+00 \\
1.1 E-02\end{array}$ & $\begin{array}{l}3.9 E-03 \\
1.1 \mathrm{E}-05 \\
5.3 \mathrm{E}-03 \\
3.9 \mathrm{E}-02 \\
5.7 \mathrm{E}-02 \\
1.8 \mathrm{E}+00 \\
1.1 \mathrm{E}+02 \\
8.0 \mathrm{E}-02 \\
8.4 \mathrm{E}-04 \\
5.0 \mathrm{E}-03 \\
3.6 \mathrm{E}-04 \\
5.1 \mathrm{E}-03 \\
4.8 \mathrm{E}-05 \\
7.9 \mathrm{E}-05 \\
9.6 \mathrm{E}-01 \\
7.1 \mathrm{E}-02 \\
1.9 \mathrm{E}-03 \\
1.7 \mathrm{E}-02 \\
9.9 \mathrm{E}-02 \\
2.3 \mathrm{E}-04 \\
8.9 \mathrm{E}-02 \\
3.8 \mathrm{E}-04 \\
3.7 \mathrm{E}-01 \\
2.2 \mathrm{E}-02 \\
1.2 \mathrm{E}-01 \\
1.5 \mathrm{E}-02 \\
4.4 \mathrm{E}-01 \\
4.9 \mathrm{E}-02 \\
3.6 \mathrm{E}+00 \\
7.3 \mathrm{E}+00 \\
3.5 \mathrm{E}-02 \\
2.2 \mathrm{E}-01 \\
4.5 \mathrm{E}+01 \\
2.4 \mathrm{E}-03 \\
1.6 \mathrm{E}-04 \\
4.4 \mathrm{E}-02 \\
2.8 \mathrm{E}-03 \\
1.1 \mathrm{E}-04 \\
2.1 \mathrm{E}-0 \mathrm{O} \\
8.1 \mathrm{E}-03 \\
3.9 \mathrm{E}-01 \\
4.3 \mathrm{E}+00 \\
8.7 \mathrm{E}-01 \\
1.4 \mathrm{E}+02 \\
2.7 \mathrm{E}-02 \\
2.2 \mathrm{E}-01 \\
1.1 \mathrm{E}+00 \\
7.7 \mathrm{E}-04 \\
1.9 \mathrm{E}+00 \\
1.1 \mathrm{E}-02\end{array}$ \\
\hline
\end{tabular}

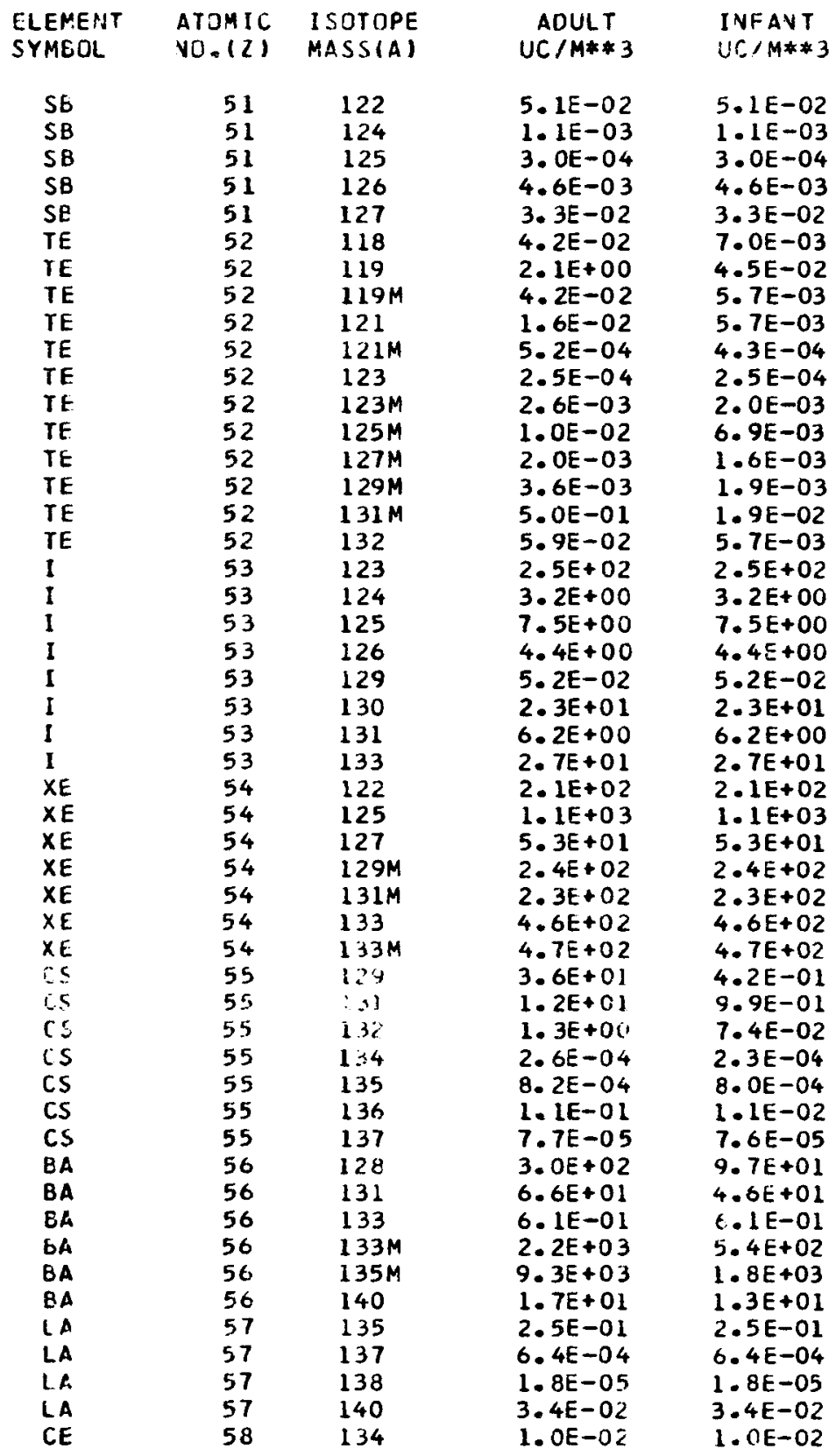




\begin{tabular}{|c|c|c|c|c|}
\hline $\begin{array}{l}\text { ELEMENT } \\
\text { SYMBOL }\end{array}$ & $\begin{array}{l}\text { ATOMIC } \\
\text { VD. (Z) }\end{array}$ & $\begin{array}{l}\text { ISOTOPE } \\
\text { MASS (A) }\end{array}$ & $\begin{array}{c}\text { ADULT } \\
\text { UC/M**3 }\end{array}$ & $\begin{array}{l}\text { INFANT } \\
\text { UC/M**3 }\end{array}$ \\
\hline $\begin{array}{l}C E \\
C E \\
C E \\
C E \\
C E \\
C E \\
C E \\
P R \\
P R \\
N D \\
N D \\
N D \\
P M \\
P M \\
P M \\
P M \\
P M \\
P M \\
P M \\
P M \\
P M \\
S M \\
S M \\
S M \\
S M \\
S M \\
S M \\
S M \\
S M \\
E U \\
E U \\
E U \\
E U \\
E U \\
E U \\
E U \\
E U \\
E U \\
E U \\
E U \\
E U \\
E U \\
G D \\
G D \\
G D \\
G D \\
G D \\
G D \\
G D \\
G D\end{array}$ & $\begin{array}{l}58 \\
58 \\
58 \\
58 \\
58 \\
58 \\
58 \\
59 \\
59 \\
60 \\
60 \\
60 \\
61 \\
61 \\
61 \\
61 \\
61 \\
61 \\
61 \\
61 \\
61 \\
62 \\
62 \\
62 \\
62 \\
62 \\
62 \\
62 \\
62 \\
63 \\
63 \\
63 \\
63 \\
63 \\
63 \\
63 \\
63 \\
63 \\
63 \\
63 \\
63 \\
63 \\
64 \\
64 \\
64 \\
64 \\
64 \\
64 \\
64 \\
64\end{array}$ & $\begin{array}{l}135 \\
137 M \\
139 \\
141 \\
142 \\
143 \\
144 \\
142 \\
143 \\
140 \\
144 \\
147 \\
143 \\
144 \\
145 \\
146 \\
147 \\
148 \\
148 M \\
149 \\
151 \\
141 \\
145 \\
146 \\
147 \\
148 \\
149 \\
151 \\
153 \\
145 \\
146 \\
146 M \\
147 \\
148 \\
149 \\
150 \\
150 M \\
152 \\
154 \\
155 \\
156 \\
157 \\
146 \\
147 \\
148 \\
149 \\
150 \\
151 \\
152 \\
153\end{array}$ & $\begin{array}{l}\text { 6.1E-02 } \\
8.4 \mathrm{E}-02 \\
1.5 \mathrm{E}-03 \\
5.2 \mathrm{E}-03 \\
5.0 \mathrm{E}-06 \\
2.6 \mathrm{E}-02 \\
4.1 \mathrm{E}-05 \\
4.3 \mathrm{E}-05 \\
8.0 \mathrm{E}-06 \\
1.1 \mathrm{E}-05 \\
3.2 \mathrm{E}-09 \\
1.4 \mathrm{E}-06 \\
4.3 \mathrm{E}-04 \\
4.2 \mathrm{E}-05 \\
1.7 \mathrm{E}-04 \\
3.1 \mathrm{E}-05 \\
1.9 \mathrm{E}-05 \\
5.1 \mathrm{E}-03 \\
1.8 \mathrm{E}-04 \\
4.4 \mathrm{E}-02 \\
3.4 \mathrm{E}-02 \\
6.6 \mathrm{E}-03 \\
1.0 \mathrm{E}-02 \\
2.8 \mathrm{E}-05 \\
3.2 \mathrm{E}-05 \\
1.8 \mathrm{E}-05 \\
3.9 \mathrm{E}-05 \\
5.2 \mathrm{E}-03 \\
7.8 \mathrm{E}-01 \\
1.8 \mathrm{E}-01 \\
3.7 \mathrm{E}-02 \\
1.6 \mathrm{E}-01 \\
1.1 \mathrm{E}-02 \\
2.6 \mathrm{E}-02 \\
1.3 \mathrm{E}-02 \\
4.3 \mathrm{E}-04 \\
3.5 \mathrm{E}-01 \\
1.7 \mathrm{E}-04 \\
1.3 \mathrm{E}-04 \\
1.1 \mathrm{E}-02 \\
3.1 \mathrm{E}-02 \\
1.1 \mathrm{E}+00 \\
1.2 \mathrm{E}-04 \\
6.7 \mathrm{E}-03 \\
1.5 \mathrm{E}-06 \\
3.0 \mathrm{E}-03 \\
8.2 \mathrm{E}-07 \\
7.7 \mathrm{E}-04 \\
2.0 \mathrm{E}-06 \\
8.9 \mathrm{E}-04\end{array}$ & $\begin{array}{l}6.1 \mathrm{E}-02 \\
8.4 \mathrm{E}-02 \\
1.5 \mathrm{E}-03 \\
5.2 \mathrm{E}-03 \\
5.0 \mathrm{E}-06 \\
2.6 \mathrm{E}-02 \\
4.1 \mathrm{E}-05 \\
4.3 \mathrm{E}-05 \\
8.0 \mathrm{E}-06 \\
1.1 \mathrm{E}-05 \\
3.2 \mathrm{E}-09 \\
1.4 \mathrm{E}-06 \\
4.3 \mathrm{E}-04 \\
4.2 \mathrm{E}-05 \\
1.7 \mathrm{E}-04 \\
3.1 \mathrm{E}-05 \\
1.9 \mathrm{E}-05 \\
5.1 \mathrm{E}-03 \\
1.8 \mathrm{E}-04 \\
4.4 \mathrm{E}-02 \\
3.4 \mathrm{E}-02 \\
6.6 \mathrm{E}-03 \\
1.0 \mathrm{E}-02 \\
2.8 \mathrm{E}-05 \\
3.2 \mathrm{E}-05 \\
1.8 \mathrm{E}-05 \\
3.9 \mathrm{E}-05 \\
5.2 \mathrm{E}-03 \\
7.8 \mathrm{E}-01 \\
1.8 \mathrm{E}-01 \\
3.7 \mathrm{E}-02 \\
1.6 \mathrm{E}-01 \\
1.1 \mathrm{E}-02 \\
2.6 \mathrm{E}-02 \\
1.3 \mathrm{E}-02 \\
4.3 \mathrm{E}-04 \\
3.5 \mathrm{E}-01 \\
1.7 \mathrm{E}-04 \\
1.3 \mathrm{E}-04 \\
1.1 \mathrm{E}-02 \\
3.1 \mathrm{E}-02 \\
1.1 \mathrm{E}+00 \\
1.2 \mathrm{E}-04 \\
6.7 \mathrm{E}-03 \\
1.5 \mathrm{E}-06 \\
3.0 \mathrm{E}-03 \\
3.2 \mathrm{E}-07 \\
7.7 \mathrm{E}-04 \\
2.0 \mathrm{E}-05 \\
8.9 \mathrm{E}-04\end{array}$ \\
\hline
\end{tabular}

ELEMENT SYMBOL

GD

GD

$T B$

TB

$T B$

TB

TB

TB

TB

or

DY
ATOMIC I SOTOPE NO. (Z) MASS(A)

$\begin{array}{ll}64 & 159 \\ 64 & 162 \\ 65 & 151 \\ 65 & 152 \\ 65 & 153 \\ 65 & 154\end{array}$
UC /M**3

I NFANT

1. 1E-01

6. $0 E-05$

7. $1 E-02$

2. $1 E-02$
$4.6 E-02$

$4.6 E-02$
$8.8 E-02$

2. $3 E-02$

6. $8 E-03$

1. 5E-04

6. $6 E-06$

5. 5E-04

4. $\mathrm{OE}-02$

1. $4 E-01$

5. 9E-10

2. OE-05

9. 2E-10

3. 8E-06

9. $4 E-06$

9. $5 E-08$

3. $7 E-05$
4. $2 E-09$

4. $2 E-09$
1. $2 E-05$

4. $6 \mathrm{E}-05$

1. 6 E- 05

1. $7 E-02$

$4.1 E-04$

4. $1 E-04$

1. $2 \mathrm{E}-03$

3. $9 E-03$
$2.2 E-02$

5. 2E-02

5. 5E-03

8. $8 \mathrm{E}-02$

1. $4 E-05$

6. $4 E-06$

3. $4 E-05$

$6.0 E-06$

1. $1 E-07$

2. $4 E-08$

4. $4 \mathrm{E}-07$

7. 1E-09

3. $4 E-05$

1. $2 \mathrm{E}-06$

6. 2E-02

1. $2 \mathrm{E}-01$

7. $5 \mathrm{E}-08$

3. $7 E-02$

6.7E-09

3. $1 E-05$

5. $0 E-05$

1. 1E-01 6. $0 \mathrm{E}-05$

7.1 E-02 2. $1 E-02$

4.6E-02

8. $8 \mathrm{E}-02$ 2. 3E-02 $6.8 \mathrm{E}-03$

1.5E-04

$6.6 E-06$

5. 5E-04

4. OE-02

1. 4 E-O1

5. $9 E-10$

2. $0 E-05$

9. $2 \mathrm{E}-10$

$3.8 E-06$

$9.4 E-06$

3. $7 E-05$

3.7E-OS

4. 2 E-09
$1.2 E-05$

4. $6 E-05$

$1.6 E-05$

1.7E-02

4. $1 E-04$

1. $2 E-03$

2. $2 E-02$

5. $2 E-02$

$5.5 E-03$

8. 8E-02

1. $4 \mathrm{E}-05$

$6.4 E-06$

$3.4 E-05$

6. $0 \mathrm{E}-06$

1. 1 E-07

2.4E-08

4. $4 \mathrm{E}-07$

7.1E-09

3. $4 E-05$

$1.2 \mathrm{E}-06$

5. 5E-05

1. $3 \mathrm{E}-04$

5. $7 E-08$

6.6E-05

$6.2 E-09$

$3.4 E-06$
$3.7 E-06$ 


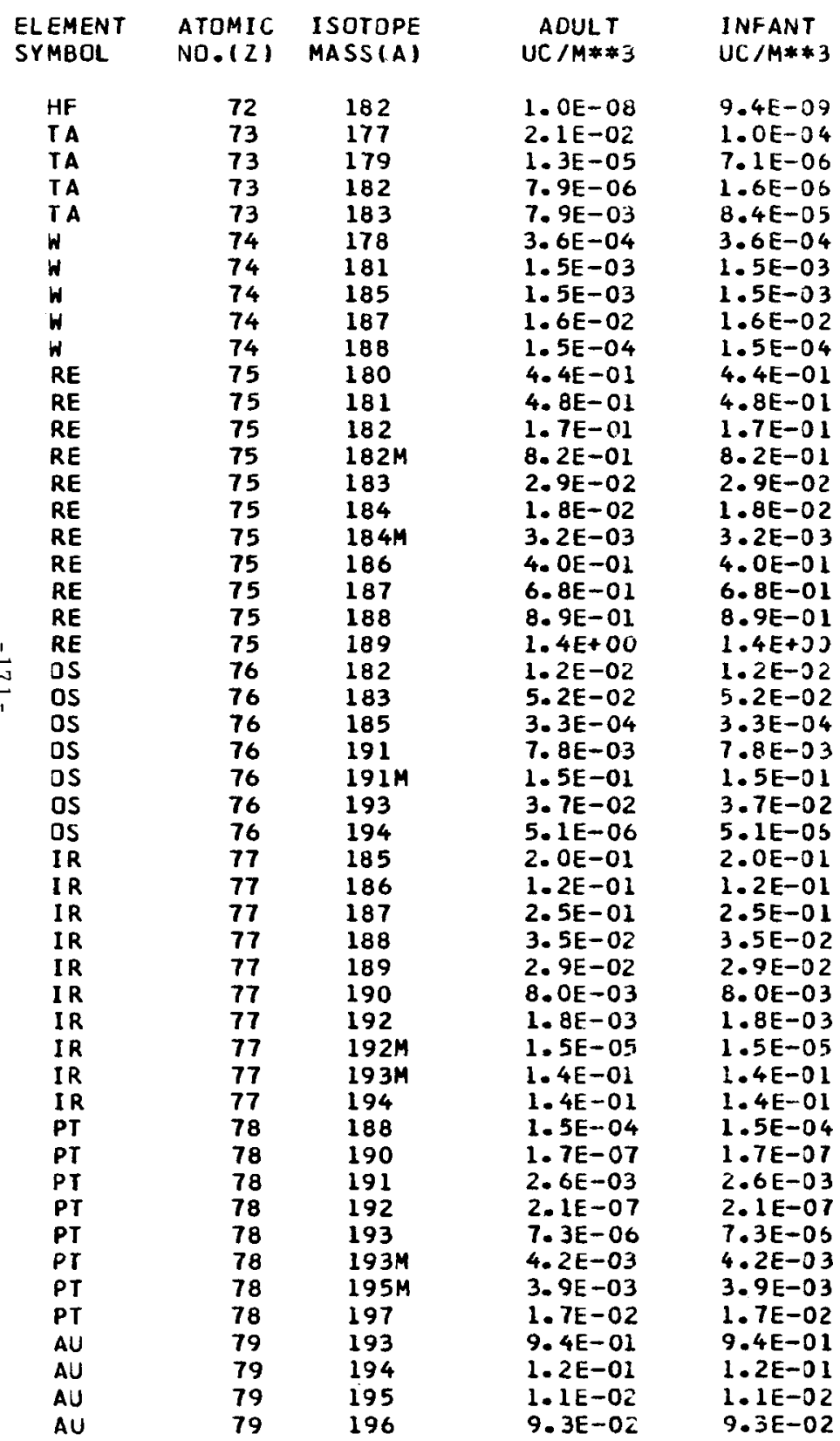

\begin{tabular}{ccc} 
ELEMENT & ATOMIC & ISOTOPE \\
SYMBOL & NO. $(Z)$ & MASSIAI \\
& & \\
AL & 79 & 198 \\
AU & 79 & 199 \\
HG & 80 & 194 \\
HG & 80 & $195 M$ \\
HG & 80 & 197 \\
HG & 80 & $197 M$ \\
HG & 80 & 203 \\
TL & 81 & 200 \\
TL & 81 & 201 \\
TL & 81 & 202 \\
TL & 81 & 204 \\
PB & 82 & 200 \\
PE & 82 & 202 \\
PE & 82 & 203 \\
PE & 82 & 204 \\
PB & 82 & 205 \\
PB & 82 & 210 \\
HI & 83 & 205 \\
BI & 83 & 206 \\
EI & 83 & 207 \\
BI & 83 & 208 \\
BI & 83 & 210 \\
EI & 83 & $210 M$ \\
PO & 84 & 206 \\
PO & 84 & 208 \\
PO & 84 & 209 \\
PO & 84 & 210 \\
RN & 86 & 211 \\
FN & 86 & 222 \\
RA & 88 & 223 \\
RA & 88 & 224 \\
RA & 88 & 225 \\
RA & 86 & 226 \\
RA & 88 & 228 \\
AC & 89 & 225 \\
AC & 89 & 227 \\
TH & 90 & 227 \\
TH & 90 & 228 \\
TH & 90 & 229 \\
TH & 90 & 230 \\
TH & 90 & 231 \\
TH & 90 & 232 \\
TH & 90 & 234 \\
PA & 91 & 228 \\
PA & 91 & 229 \\
PA & 91 & 230 \\
PA & 91 & 231 \\
PA & 91 & 232 \\
PA & 91 & 233 \\
U & 92 & 230 \\
\hline
\end{tabular}

ADULT
$U C, M * 3 * 3$

2. $1 E-01$

5. $7 E-01$

3. $1 E-05$

3. $5 E-03$
$1.0 E-02$

1. $0 E-02$

1. $5 \mathrm{E}-02$

6. $3 E-04$
$4.2 E-03$

8. $6 \mathrm{E}-03$

1. $1 E-03$

1. $6 E-05$

5. $7 \mathrm{E}+01$

2. $0 E-02$

$5.4 E+01$

4. 5E-0

1. $4 E-01$

3. $2 E-03$

4. $1 E-03$

1. $1 E-02$

1. 2E-05

6. $0 E-06$

4. $3 E-03$

2. $9 \mathrm{E}-06$

1. $5 E-01$

5. $0 E-04$

1. $2 E-04$

5. $5 E-03$

7. $4 E+01$

9. $9 E-06$

3. $O E-05$

7. $7 \mathrm{E}-06$

1. $4 \mathrm{E}-08$

4. $4 E-08$

7. $3 E-02$

7. $3 E-02$
$1.3 E-04$

7. $3 E-07$

1. $9 E-08$

1. $8 \mathrm{E}-09$

1. $7 E-09$

1. 1E-05

1. $5 E-09$

4. $5 E-07$

6. $2 E-01$

4. IE-OI

3. $3 E-02$

7. $O E-05$

3. $9 E-01$

2. $O E-02$

INF ANT

$2.1 E-C 1$ 5. 7E-01

3. 1 E-OS

3. $5 \mathrm{E}-03$

1. $0 \mathrm{E}-02$

1. $5 E-02$
$6.3 E-04$

$4.2 \mathrm{E}-03$

8. $6 \mathrm{E}-03$

1. 1E-03

1. $6 E-05$

5. $7 E+01$

2. $0 E-02$

$5.4 E+01$

4. 5E-03

$1.4 E-01$

3. $2 E-03$

4. $1 E-03$

1. IE-02

1. $2 E-05$

$6.0 E-06$

4. $3 E-03$

2. $9 E-06$

1. $5 \mathrm{E}-01$

5. $0 E-04$
$1.2 E-04$

5. $5 E-03$

7. $4 E+01$

4. $7 E+00$

9. $9 E-06$

3. OE-05

7. $7 \mathrm{E}-06$

$1.4 \mathrm{E}-08$

4. $4 E-08$

7. 3E-02

1. $3 E-04$

7. $3 E-07$

1. $9 E-08$

1. $8 \mathrm{E}-09$

1. 7E-09

1. $1 \mathrm{E}-05$

1. $5 E-09$

4. 5E-07

$6.2 E-01$

6. E-

4. 1E-O1

3. 3E-02

7. $0 \mathrm{E}-05$

3. $9 E-01$

2. $0 E-02$ 
TABLE 9B. UNIT-RAD CONTAMINATI ON FOR DVARIES

\begin{tabular}{|c|c|c|c|c|}
\hline $\begin{array}{l}\text { ELEMENT } \\
\text { SYMBOL }\end{array}$ & $\begin{array}{l}\text { ATOMIC } \\
\text { NO. (Z) }\end{array}$ & $\begin{array}{l}\text { ISOT OPE } \\
\text { MASS(A) }\end{array}$ & $\begin{array}{l}\text { ADULT } \\
\text { UC/M**3 }\end{array}$ & $\begin{array}{l}\text { INFANT } \\
\text { UC } / M * * 3\end{array}$ \\
\hline $\begin{array}{l}U \\
U \\
U \\
U \\
U \\
U \\
U \\
U \\
U \\
N P \\
N P \\
N P \\
N P \\
N P \\
N P \\
N P \\
P U \\
P U \\
P U \\
P U \\
P U \\
P U \\
P U \\
P U \\
P U \\
A M \\
A M \\
A M \\
A M \\
A M \\
A M\end{array}$ & $\begin{array}{l}92 \\
92 \\
92 \\
92 \\
92 \\
92 \\
92 \\
92 \\
92 \\
93 \\
93 \\
93 \\
93 \\
93 \\
93 \\
93 \\
94 \\
94 \\
94 \\
94 \\
94 \\
94 \\
94 \\
94 \\
94 \\
95 \\
95 \\
95 \\
95 \\
95 \\
95\end{array}$ & $\begin{array}{l}231 \\
232 \\
233 \\
234 \\
235 \\
236 \\
237 \\
238 \\
240 \\
234 \\
235 \\
236 \\
236 M \\
237 \\
238 \\
239 \\
236 \\
237 \\
238 \\
239 \\
240 \\
241 \\
242 \\
244 \\
246 \\
239 \\
240 \\
241 \\
242 \\
242 M \\
243\end{array}$ & $\begin{array}{l}1.9 E-06 \\
1.2 E-09 \\
1.1 E-09 \\
1.0 E-09 \\
9.3 E-10 \\
9.1 E-10 \\
1.0 E-06 \\
8.8 E-10 \\
1.0 E-05 \\
2.7 E-02 \\
2.8 E-04 \\
1.4 E-05 \\
1.2 E-01 \\
1.5 E-05 \\
5.1 E-02 \\
4.3 E-02 \\
4.4 E-04 \\
1.0 E-02 \\
6.6 E-05 \\
5.6 E-05 \\
5.5 E-05 \\
1.1 E-04 \\
5.3 E-05 \\
4.7 E-05 \\
3.3 E-02 \\
8.4 E-01 \\
1.9 E-01 \\
5.7 E-05 \\
5.9 E-01 \\
5.6 E-05 \\
5.0 E-05\end{array}$ & $\begin{array}{l}1.9 \mathrm{E}-06 \\
1.2 \mathrm{E}-09 \\
1.1 \mathrm{E}-09 \\
1.0 \mathrm{E}-09 \\
9.3 \mathrm{E}-10 \\
9.1 \mathrm{E}-10 \\
1.0 \mathrm{E}-05 \\
8.8 \mathrm{E}-10 \\
1.0 \mathrm{E}-05 \\
2.7 \mathrm{E}-02 \\
2.8 \mathrm{E}-04 \\
1.4 \mathrm{E}-05 \\
1.2 \mathrm{E}-01 \\
1.5 \mathrm{E}-05 \\
5.1 \mathrm{E}-02 \\
4.3 \mathrm{E}-02 \\
4.4 \mathrm{E}-04 \\
1.0 \mathrm{E}-02 \\
6.6 \mathrm{E}-05 \\
5.6 \mathrm{E}-05 \\
5.5 \mathrm{E}-05 \\
1.1 \mathrm{E}-04 \\
5.3 \mathrm{E}-05 \\
4.7 \mathrm{E}-05 \\
3.3 \mathrm{E}-02 \\
8.4 \mathrm{E}-01 \\
1.9 \mathrm{E}-01 \\
5.7 \mathrm{E}-05 \\
5.9 \mathrm{E}-01 \\
5.6 \mathrm{E}-05 \\
5.0 \mathrm{E}-05\end{array}$ \\
\hline
\end{tabular}

VIA FRESHHATER.

\begin{tabular}{|c|c|}
\hline $10 / 09 / 68$ & PAGE 20 \\
\hline $\begin{array}{l}\text { ADULT } \\
U C / M * * 3\end{array}$ & $\begin{array}{l}\text { INFANT } \\
\text { UC/M**3 }\end{array}$ \\
\hline $\begin{array}{l}1.5 E-02 \\
1.2 E-02 \\
2.4 E-03 \\
6.7 E-05 \\
8.3 E-05 \\
5.0 E-05 \\
4.8 E-05 \\
4.5 E-05 \\
4.4 E-05 \\
7.6 E-02 \\
2.0 E-01 \\
4.5 E-05 \\
3.5 E-01 \\
1.1 E-03 \\
2.4 E-01 \\
9.6 E-04 \\
4.6 E-05 \\
1.0 E-04 \\
4.1 E-05 \\
3.2 E-04 \\
1.6 E-02 \\
2.1 E-01 \\
2.1 E-03 \\
1.5 E-02 \\
6.1 E-04 \\
1.8 E-01 \\
1.2 E-02 \\
3.1 E-01 \\
6.8 E-02 \\
3.4 E-01 \\
3.5 E-03\end{array}$ & $\begin{array}{l}1.5 E-02 \\
1.2 E-02 \\
2.4 E-03 \\
6.7 E-05 \\
8.3 E-05 \\
5.0 E-05 \\
4.8 E-05 \\
4.5 E-05 \\
4.4 E-05 \\
7.6 E-02 \\
2.0 E-01 \\
4.5 E-05 \\
3.5 E-01 \\
1.1 E-03 \\
2.4 E-01 \\
9.6 E-04 \\
4.6 E-05 \\
1.0 E-04 \\
4.1 E-05 \\
3.2 E-04 \\
1.6 E-02 \\
2.1 E-01 \\
2.1 E-03 \\
1.5 E-02 \\
6.1 E-04 \\
1.8 E-01 \\
1.2 E-02 \\
3.1 E-01 \\
6.8 E-02 \\
3.4 E-01 \\
3.5 E-03\end{array}$ \\
\hline
\end{tabular}


TABLE 98. UNIT-RAD CONTAMINATION FOR SPLEEN

$\begin{array}{lcc}\text { ELEMENT } & \text { ATOMIC } & \text { ISOTOPE } \\ \text { SYMBOL } & \text { VD. I I } & \text { MASS (A) } \\ \text { H } & & \\ \text { BE } & 1 & 3 \\ \text { BE } & 4 & 7 \\ \text { C } & 4 & 10 \\ \text { NA } & 6 & 14 \\ \text { NA } & 11 & 22 \\ \text { MG } & 11 & 24 \\ \text { AL } & 12 & 28 \\ \text { SI } & 13 & 26 \\ \text { P } & 14 & 32 \\ \text { P } & 15 & 32 \\ \text { S } & 15 & 33 \\ \text { CL } & 16 & 35 \\ \text { AR } & 17 & 36 \\ \text { AR } & 18 & 37 \\ \text { AR } & 18 & 39 \\ \text { K } & 18 & 42 \\ \text { K } & 19 & 40 \\ \text { K } & 19 & 42 \\ \text { CA } & 19 & 43 \\ \text { CA } & 20 & 41 \\ \text { CA } & 20 & 45 \\ \text { CA } & 20 & 47 \\ \text { SC } & 20 & 48 \\ \text { SC } & 21 & 44 M \\ \text { SC } & 21 & 46 \\ \text { SC } & 21 & 47 \\ \text { TI } & 21 & 48 \\ \text { V } & 22 & 44 \\ \text { V } & 23 & 48 \\ \text { V } & 23 & 49 \\ \text { CR } & 23 & 50 \\ \text { CR } & 24 & 48 \\ \text { MN } & 24 & 51 \\ \text { MN } & 25 & 52 \\ \text { MN } & 25 & 53 \\ \text { FE } & 25 & 54 \\ \text { FE } & 26 & 55 \\ \text { FE } & 26 & 59 \\ \text { CO } & 26 & 60 \\ \text { CO } & 27 & 55 \\ \text { CO } & 27 & 56 \\ \text { CO } & 27 & 57 \\ \text { CO } & 27 & 58 \\ \text { NI } & 27 & 60 \\ \text { VI } & 28 & 56 \\ \text { VI } & 28 & 57 \\ \text { NI } & 28 & 59 \\ \text { NI } & 28 & 63 \\ \text { CU } & 28 & 66 \\ & 29 & 64\end{array}$

VIA FRESHWATER.

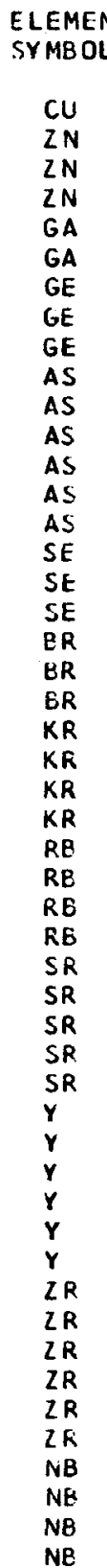

10/09/68 PAGE 21

\begin{tabular}{|c|c|}
\hline $\begin{array}{c}\text { ADULT } \\
U C / M * * 3\end{array}$ & $\begin{array}{l}\text { INFANT } \\
\text { UC } / M * 3\end{array}$ \\
\hline $\begin{array}{l}6.4 E+02 \\
9.5 E+01 \\
1.1 E-02 \\
1.3 E-03 \\
3.2 E-02 \\
4.3 E+02 \\
3.0 E+01 \\
1.4 E-02 \\
3.7 E-03 \\
1.8 E-02 \\
8.4 E-02 \\
1.1 E+00 \\
2.8 E-02 \\
8.2 E+02 \\
4.4 E-02 \\
4.7 E-03 \\
1.3 E-03 \\
6 \cdot 3 E+02 \\
3.8 E+02 \\
6.7 E+01 \\
1.3 E+02 \\
6.0 E+02 \\
6.6 E-02 \\
1.3 E+01 \\
1.5 E-02 \\
6.0 E+01 \\
1.8 E+01 \\
8.2 E-04 \\
1.4 E+02 \\
8.0 E+02 \\
1.1 E-01 \\
9.8 E+00 \\
3.4 E+01 \\
2.1 E+00 \\
2.5 E-01 \\
4.6 E-02 \\
5.7 E-01 \\
5.5 E-01 \\
1.2 E-04 \\
2.4 E+02 \\
1.1 E-01 \\
3.9 E-01 \\
2.5 E-01 \\
2.8 E-03 \\
2.2 E+01 \\
1.7 E+02 \\
8.7 E+00 \\
4.2 E+00 \\
1.2 E+02 \\
9.1 E+02\end{array}$ & $\begin{array}{l}6.4 E+02 \\
8.5 E+00 \\
1.0 E-02 \\
1.3 E-03 \\
3.2 E-02 \\
2.3 E+01 \\
3.0 E+01 \\
1.3 E-02 \\
3.7 E-03 \\
6.5 E-03 \\
4.2 E-02 \\
1.1 E+00 \\
2.8 E-02 \\
8.2 E+02 \\
4.4 E-02 \\
4.7 E-03 \\
1.3 E-03 \\
5.5 E+00 \\
6.0 E+00 \\
6.7 E+01 \\
1.2 E+02 \\
2.1 E+02 \\
6.6 E-02 \\
2.2 E-02 \\
8.5 E-04 \\
1.4 E-01 \\
2.3 E-02 \\
7.7 E-04 \\
2.1 E+01 \\
6.3 E+02 \\
1.1 E-01 \\
9.8 E+00 \\
3.4 E+01 \\
5.5 E-01 \\
2.4 E-01 \\
4.4 E-02 \\
3.6 E-01 \\
3.9 E-02 \\
1.1 E-04 \\
7.4 E+00 \\
8.4 E-02 \\
3.5 E-01 \\
1.9 E-01 \\
2.7 E-03 \\
2.2 E+01 \\
1.7 E+02 \\
8.7 E+00 \\
4.2 E+00 \\
1.2 E+02 \\
3.9 E+01\end{array}$ \\
\hline
\end{tabular}

\section{ATOMIC ISOTOPE} ADULT
UE /M**3

INFANT $29 \quad 67 \quad 5.5 E+01$

$\begin{array}{ll}29 & 67 \\ 30 & 65 \\ 30 & 69 M \\ 30 & 72 \\ 31 & 67 \\ 31 & 72\end{array}$

3. $3 E-03$ 8. $3 E-01$

4. $8 E-02$

2. $5 \mathrm{E}+02$

1. $8 E-05$

2. $6 E-03$

$2.6 \mathrm{E}-03$
$7.1 \mathrm{E}-02$

$9.8 E+02$

$5.0 E+02$

2. $O E+03$

5. $2 \mathrm{E}+01$

6. $9 E+02$

3. $5 E+03$

7. $3 \mathrm{E}-02$

1. $0 E-02$

1. $7 E-03$

5. $5 E+02$

1. $1 E+02$

1. $0 E+02$

1. $5 E+02$

1. $9 E+02$

1. $3 E+00$

2. 5E-0 1

1. $1 \mathrm{E}-02$

$1.5 \mathrm{E}-02$

7. $4 \mathrm{E}-02$

4. $2 E-04$

6. $O E+00$

2. $3 E+03$

1. $3 E+01$

1. $1 E+01$

4. $3 E-02$

2. $7 E+03$

4. $4 E+02$

1. $0 E+04$

1. $4 E-01$

$4.4 E+02$

$1.5 E+00$

2. $1 E+03$

2. $3 E-01$

2. $4 E+02$

4. $O E-02$

4. OE -02

3. $2 E+03$

6. $O E+01$

6. $O E+O 1$
1. $O E-03$

1. $4 E-01$

1. $2 E+00$
$U C / M * \# 3$

9. $7 \mathrm{E}+00$ 3. $3 E-03$ 8. $3 E-01$ 4. $8 \mathrm{E}-02$ 6.1E+01 $2.2 E+01$ 2. $6 \mathrm{E}-03$ 2. 1 EE-03 7. $1 \mathrm{E}-02$ $9.8 E+02$ 5. $0 E+02$ 2. $0 E+03$ $5.2 E+01$

6. $9 E+02$

$3.5 E+03$ 2. $3 E-02$ $8.7 E-03$ 8.7E 1. $7 E-03$ 4. $4 E+01$

$2.6 E+01$ ज

$1.6 \mathrm{E}+01$

1. $5 \mathrm{E}+02$

1. $9 E+02$

$1.3 E+00$

2. 5E-01 5. $7 \mathrm{E}-03$ 4. $4 E-03$ 1. $4 E-02$ 4. $2 E-04$ 4. $1 E+00$ $2 \cdot 3 E+02$ 1. $1 E+01$

9. $2 E+00$ 4. 3E-02 1. $7 E-01$ 1. $5 \mathrm{E}-01$ 5. $9 \mathrm{E}-01$ 1. 5E-03 $1.2 \mathrm{E}-01$ 9. $O E-03$ 1. $7 E+00$ 2. $0 E-02$ 8. $8 \mathrm{E}-01$ 3. $5 E-02$ 3.5E-02 4. $9 E-02$ 2. $4 E+00$ $3.8 \mathrm{E}-02$ $9.0 E-04$ 8. $5 E-03$ 


\begin{tabular}{|c|c|c|c|c|}
\hline $\begin{array}{l}\text { ELEMENT } \\
\text { SYMBOL }\end{array}$ & $\begin{array}{l}\text { ATOMIC } \\
\text { ND. }(Z)\end{array}$ & $\begin{array}{l}\text { ISOTOPE } \\
\text { MASSIA) }\end{array}$ & $\begin{array}{l}\text { ADULT } \\
\text { UC } / M * * 3\end{array}$ & $\begin{array}{l}\text { INFANT } \\
U C / M * * 3\end{array}$ \\
\hline $\begin{array}{l}\text { NB } \\
N B \\
N B \\
N B \\
N B \\
M O \\
M O \\
T C \\
T C \\
T C \\
\text { TC } \\
T C \\
T C \\
T C \\
R U \\
R U \\
R U \\
R H \\
R H \\
R H \\
R H \\
R H \\
R H \\
P D \\
P D \\
P D \\
P D \\
P D \\
A G \\
A G \\
A G \\
A G \\
A G \\
C D \\
C D \\
C D \\
C D \\
I N \\
I N \\
I N \\
S N \\
\text { SN } \\
\text { SN } \\
\text { SN } \\
\text { SN } \\
\text { SN } \\
S N \\
S N \\
\text { SB } \\
\text { SB }\end{array}$ & $\begin{array}{l}41 \\
41 \\
41 \\
41 \\
41 \\
42 \\
42 \\
43 \\
43 \\
43 \\
43 \\
43 \\
43 \\
43 \\
44 \\
44 \\
44 \\
45 \\
45 \\
45 \\
45 \\
45 \\
45 \\
46 \\
46 \\
46 \\
46 \\
46 \\
47 \\
47 \\
47 \\
47 \\
47 \\
48 \\
48 \\
48 \\
48 \\
49 \\
49 \\
49 \\
50 \\
50 \\
50 \\
50 \\
50 \\
50 \\
50 \\
50 \\
51 \\
51\end{array}$ & $\begin{array}{l}93 M \\
94 \\
95 \\
95 M \\
96 \\
93 \\
99 \\
95 \\
95 M \\
96 \\
97 \\
97 M \\
98 \\
99 \\
97 \\
103 \\
106 \\
99 \\
100 \\
101 \\
101 M \\
102 \\
105 \\
100 \\
103 \\
107 \\
109 \\
112 \\
105 \\
106 M \\
108 M \\
110 M \\
111 \\
109 \\
113 M \\
115 \\
115 M \\
111 \\
114 M \\
115 \\
113 \\
117 M \\
119 M \\
121 \\
121 M \\
123 M \\
125 \\
126 \\
119 \\
120 M\end{array}$ & $\begin{array}{l}5.4 E-03 \\
1.0 E-05 \\
1.2 E-01 \\
8.0 E+00 \\
4.6 E+01 \\
1.3 E+00 \\
3.0 E+02 \\
8.0 E-02 \\
8.4 E-04 \\
5.0 E-03 \\
3.6 E-04 \\
5.1 E-03 \\
4.8 E-06 \\
7.9 E-05 \\
2.7 E+01 \\
1.8 E-01 \\
1.3 E-03 \\
4.0 E-02 \\
2.5 E+00 \\
2.3 E-04 \\
5.0 E-01 \\
4.2 E-04 \\
5.5 E+00 \\
1.1 E-01 \\
2.2 E-01 \\
1.5 E-02 \\
1.2 E+01 \\
8.9 E-01 \\
8.7 E+00 \\
4.2 E+01 \\
5.5 E-02 \\
3.8 E-01 \\
2.8 E+02 \\
3.9 E-03 \\
1.8 E-04 \\
4.8 E+00 \\
1.9 E-02 \\
2.0 E-03 \\
4.2 E-06 \\
8.2 E-08 \\
1.4 E+01 \\
1.2 E+03 \\
1.7 E+01 \\
4.8 E+05 \\
1.4 E-01 \\
7.7 E+00 \\
4.4 E+02 \\
3.7 E-03 \\
5.8 E-01 \\
3.3 E-03\end{array}$ & $\begin{array}{l}3.2 E-03 \\
9.1 E-06 \\
4.3 E-03 \\
3.2 E-02 \\
4.7 E-02 \\
1.3 E+J 0 \\
7.8 E+01 \\
8.0 E-02 \\
8.4 E-04 \\
5.0 E-03 \\
3.6 E-04 \\
5.1 E-03 \\
4.8 E-06 \\
7.9 E-05 \\
4.8 E-01 \\
3.6 E-02 \\
9.3 E-04 \\
1.7 E-02 \\
9.9 E-02 \\
2.3 E-04 \\
8.9 E-02 \\
3.8 E-04 \\
3.7 E-01 \\
2.2 E-02 \\
1.2 E-01 \\
1.5 E-02 \\
4.4 E-01 \\
4.9 E-02 \\
5.6 E+00 \\
1.1 E+01 \\
5.5 E-02 \\
3.5 E-01 \\
7.1 E+01 \\
2.7 E-03 \\
1.7 E-04 \\
4.9 E-02 \\
3.2 E-03 \\
1.1 E-04 \\
2.1 E-00 \\
8.1 E-03 \\
1.7 E+00 \\
1.9 E+01 \\
3.7 E+00 \\
6.1 E+02 \\
1.2 E-01 \\
9.5 E-01 \\
4.7 E+00 \\
3.3 E-03 \\
5.8 E-01 \\
3.3 E-03\end{array}$ \\
\hline
\end{tabular}

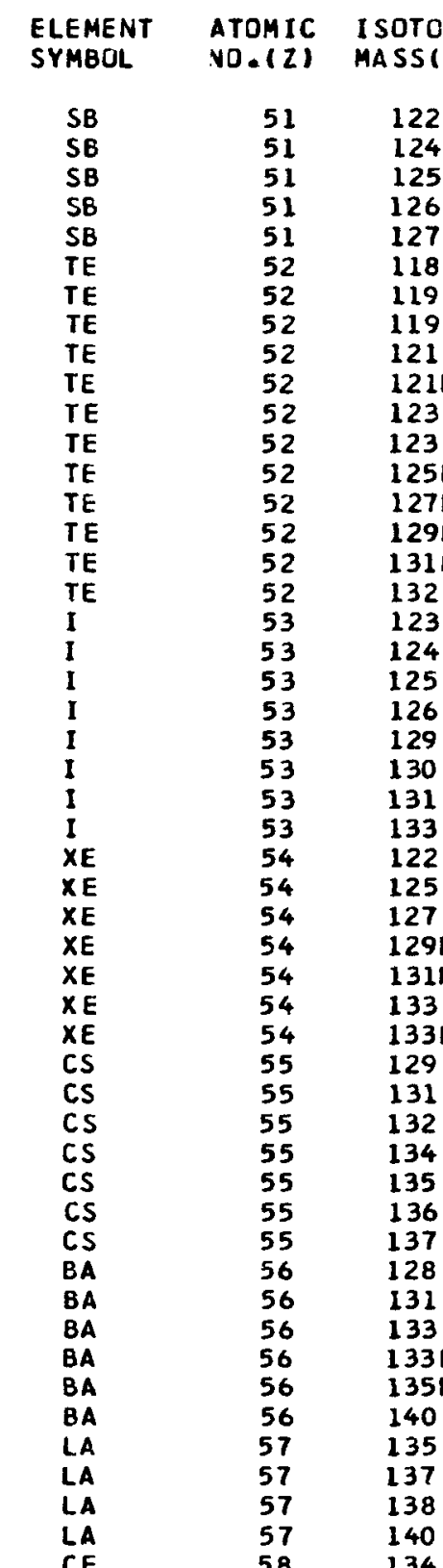

ADULT

INFANT

$C / M * * 3$

122

1. 5E-02

1. $5 \mathrm{E}-02$ 3. 3E-04 3. $3 E-04$ 9. $1 E-05$ $9.1 E-05$ $1.4 E-03$ 9. $9 E-03$ 7. $0 E-03$ 4. $5 E-02$ 5. $7 E-03$ 5. $7 E-03$ 4. 3E-04 2. $5 E-04$ 2.5E-04 6. $9 E-03$ $6.9 E-03$
$1.6 E-03$ 1. $9 E-03$ 1. $9 E-02$ $5.7 E-03$ $4.5 E-01$ 5. TE-03 $1.3 E-02$ $7.0 E-03$ $9.3 E-05$ 9. $3 E-05$
4. $2 E-02$ 4. 2E-02 $1.1 \mathrm{E}-02$ 4. $9 E-02$ $2.1 E+02$ $1.1 E+03$ 5. $3 E+01$ $2.4 E+02$ $2.3 E+02$ $4.6 \mathrm{E}+02$ 4. $7 E+02$ $9.1 E-02$ 2.1E-01 $1.6 E-02$ 4. $9 \mathrm{E}-05$ 1.7E-04 $2.4 E-03$

. $6 \mathrm{E}-05$

- $8 E+0$

1. $2 E+00$ $1.2 E+00$ 1. $0 E+03$
3. $5 E+03$ $2.4 E+01$ 2. $0 E-01$ 5. 2E-04 1. $5 E-05$

2. $8 \mathrm{E}-02$ 5. $2 E+00$ 


\begin{tabular}{|c|c|c|c|c|}
\hline $\begin{array}{l}\text { ELEMENT } \\
\text { SYMBOL }\end{array}$ & $\begin{array}{l}\text { ATOM IC } \\
\text { VO. }(Z)\end{array}$ & $\begin{array}{l}\text { ISOTOPE } \\
\text { MASS (A) }\end{array}$ & $\begin{array}{c}\text { ADULT } \\
\text { UC/M**3 }\end{array}$ & $\begin{array}{l}\text { INFANT } \\
\text { UC } / M * * 3\end{array}$ \\
\hline $\begin{array}{l}C E \\
C E \\
C E \\
C E \\
C E \\
C E \\
C E \\
P R \\
P R \\
N D \\
N D \\
N D \\
P M \\
P M \\
P M \\
P M \\
P M \\
P M \\
P M \\
P M \\
P M \\
S M \\
S M \\
S M \\
S M \\
S M \\
S M \\
S M \\
S M \\
E U \\
E U \\
E U \\
E U \\
E U \\
E U \\
E U \\
E U \\
E U \\
E U \\
E U \\
E U \\
E U \\
\text { GD } \\
G D \\
G D \\
G D \\
G D \\
G D \\
G D \\
G D\end{array}$ & $\begin{array}{l}58 \\
58 \\
58 \\
58 \\
58 \\
58 \\
58 \\
59 \\
59 \\
60 \\
60 \\
60 \\
61 \\
61 \\
61 \\
61 \\
61 \\
61 \\
61 \\
61 \\
61 \\
62 \\
62 \\
62 \\
62 \\
62 \\
62 \\
62 \\
62 \\
63 \\
63 \\
63 \\
63 \\
63 \\
63 \\
63 \\
63 \\
63 \\
63 \\
63 \\
63 \\
63 \\
64 \\
64 \\
64 \\
64 \\
64 \\
64 \\
64 \\
64\end{array}$ & $\begin{array}{l}135 \\
137 M \\
139 \\
141 \\
142 \\
143 \\
144 \\
142 \\
143 \\
140 \\
144 \\
147 \\
143 \\
144 \\
145 \\
146 \\
147 \\
148 \\
148 M \\
149 \\
151 \\
141 \\
145 \\
146 \\
147 \\
148 \\
149 \\
151 \\
153 \\
145 \\
146 \\
146 M \\
147 \\
148 \\
149 \\
150 \\
150 M \\
152 \\
154 \\
155 \\
156 \\
157 \\
146 \\
147 \\
148 \\
149 \\
150 \\
151 \\
152 \\
153\end{array}$ & $\begin{array}{l}3.4 E+04 \\
2.5 E+04 \\
5.3 E+00 \\
7.0 E+01 \\
2.8 E-03 \\
8.0 E+03 \\
8.0 E-02 \\
4.3 E-05 \\
8.0 E-06 \\
1.1 E-05 \\
3.2 E-09 \\
1.4 E-06 \\
1.6 E-01 \\
1.3 E-02 \\
1.9 E-02 \\
4.5 E-03 \\
3.3 E-03 \\
7.0 E+01 \\
3.5 E-01 \\
1.5 E+03 \\
2.1 E+03 \\
2.4 E+00 \\
3.1 E-01 \\
3.1 E-04 \\
3.5 E-04 \\
2.0 E-04 \\
4.3 E-04 \\
5.9 E-02 \\
2.9 E+03 \\
1.4 E+02 \\
3.6 E+01 \\
4.4 E+02 \\
2.1 E+00 \\
2.4 E+00 \\
6.8 E-01 \\
5.4 E-03 \\
2.9 E+03 \\
1.9 E-03 \\
1.4 E-03 \\
1.8 E-01 \\
9.5 E+00 \\
7.8 E+03 \\
1.1 E-01 \\
2.5 E+02 \\
1.6 E-04 \\
1.3 E+01 \\
8.6 E-05 \\
3.2 E-01 \\
2.1 E-04 \\
2.3 E-01\end{array}$ & $\begin{array}{l}3.0 E+01 \\
4.2 E+01 \\
7.5 E-01 \\
2.6 E+00 \\
2.5 E-03 \\
1.3 E+01 \\
2.0 E-02 \\
4.3 E-05 \\
8.0 E-06 \\
1.1 E-05 \\
3.2 E-03 \\
1.4 E-06 \\
4.3 E-02 \\
4.2 E-03 \\
1.7 E-02 \\
3.1 E-03 \\
1.9 E-03 \\
5.1 E-01 \\
1.8 E-02 \\
4.4 E+00 \\
3.4 E+00 \\
6.6 E-02 \\
1.0 E-01 \\
2.8 E-04 \\
3.2 E-04 \\
1.8 E-04 \\
3.9 E-04 \\
5.2 E-02 \\
7.8 E+00 \\
1.8 E+00 \\
3.7 E-01 \\
1.6 E+00 \\
1.1 E-01 \\
2.6 E-01 \\
1.3 E-01 \\
4.3 E-03 \\
3.5 E+00 \\
1.7 E-03 \\
1.3 E-03 \\
1.1 E-01 \\
3.1 E-01 \\
1.1 E+01 \\
1.2 E-02 \\
6.7 E-01 \\
1.5 E-04 \\
3.0 E-01 \\
8.2 E-05 \\
7.7 E-02 \\
2.0 E-04 \\
8.9 E-02\end{array}$ \\
\hline
\end{tabular}

$\begin{array}{lcc}\text { ELEMENT } & \text { ATOMIC } & \text { I SOTOPE } \\ \text { SYMEOL } & \text { NO. (Z) } & \text { MASS(A) } \\ \text { GD } & & \\ \text { GD } & 64 & 159 \\ \text { TB } & 64 & 162 \\ \text { TB } & 65 & 151 \\ \text { TE } & 65 & 152 \\ \text { TB } & 65 & 153 \\ \text { TB } & 65 & 154 \\ \text { TB } & 65 & 155 \\ \text { TB } & 65 & 156 \\ \text { TB } & 65 & 157 \\ \text { TE } & 65 & 158 \\ \text { TB } & 65 & 160 \\ \text { TB } & 65 & 161 \\ \text { DY } & 65 & 164 \\ \text { IY } & 66 & 154 \\ \text { DY } & 66 & 154 M \\ \text { DY } & 66 & 156 \\ \text { DY } & 66 & 159 \\ \text { HO } & 66 & 166 \\ \text { HO } & 67 & 163 \\ \text { HO } & 67 & 166 \\ \text { ER } & 67 & 166 M \\ \text { ER } & 68 & 160 \\ \text { ER } & 68 & 169 \\ \text { TM } & 68 & 172 \\ \text { TM } & 69 & 165 \\ \text { TM } & 69 & 167 \\ \text { TM } & 69 & 168 \\ \text { TM } & 69 & 170 \\ \text { TM } & 69 & 171 \\ \text { YB } & 69 & 172 \\ \text { YB } & 70 & 166 \\ \text { YB } & 70 & 169 \\ \text { LU } & 70 & 175 \\ \text { LU } & 71 & 169 \\ \text { LU } & 71 & 170 \\ \text { LU } & 71 & 171 \\ \text { LU } & 71 & 172 \\ \text { LU } & 71 & 173 \\ \text { LU } & 71 & 174 \\ \text { LU } & 71 & 174 M \\ \text { LU } & 71 & 170 \\ \text { LU } & 71 & 177 \\ \text { HF } & 71 & 177 M \\ \text { HF } & 72 & 170 \\ \text { HF } & 72 & 171 \\ \text { HF } & 72 & 172 \\ \text { HF } & 7: & 173 \\ \text { HF } & 72 & 174 \\ \text { HF } & 72 & 175 \\ & 72 & 181\end{array}$

ADULT
$U C / M+73$
$5.4 E+03$
$1.2 E-02$
$3.2 E+03$
$9.3 E+02$
$6.2 E+02$
$3.4 E+03$
$1.4 E+02$
$4.4 E+01$
$1.6 E-02$
$7.0 E-04$
$3.1 E-01$
$2.0 E+02$
$4.7 E+03$
$5.9 E-10$
$2.0 E-05$
$9.2 E-10$
$3.8 E-06$
$9.4 E-06$
$9.5 E-08$
$3.7 E-05$
$4.2 E-09$
$1.2 E-05$
$4.6 E-05$
$1.6 E-05$
$5.7 E+01$
$4.1 E+00$
$2.3 E-02$
$4.9 E-02$
$6.2 E-02$
$3.3 E+01$
$8.8 E+02$
$7.6 E+00$
$8.7 E+02$
$1.4 E-05$
$6.4 E-06$
$3.4 E-05$
$6.0 E-06$
$1.1 E-07$
$2.4 E-08$
$4.4 E-07$
$7.1 E-09$
$3.4 E-05$
$1.2 E-06$
$7.8 E-01$
$1.5 E+00$
$1.4 E-06$
$4.6 E-01$
$1.3 E-07$
$4.1 E-04$
$6.5 E-04$

INFANT

UC $/ M * * 3$

- $1 E+01$

6. $0 E-03$

7. $1 E+00$

$2.1 E+00$

$4.6 E+00$

8. $8 E+00$

$2.3 E+00$

$6.8 \mathrm{E}-01$

1. $5 E-02$

$6.6 E-04$
$5.5 E-02$

4. $0 E+00$

1. $4 E+01$

5. $9 E-10$

2. OE-05

9. $2 E-10$

3. $8 E-06$

$9.4 E-06$

9.5E-08

$3.7 E-05$ ज
$4.2 E-09$

$1.2 \mathrm{E}-05$ th

$4.6 E-05$ क

$1.6 E-05$

. $6 E-02$

$4.1 E-03$

1. $2 E-02$

3. $9 E-02$

$2.2 E-01$

$5.2 E+00$

$5.5 E-01$

8. $8 \mathrm{E}+00$

1. $4 E-05$

$6.4 E-06$

$3.4 E-05$

$6.4 E-05$

6. OE- 06

$2.4 E-08$

$4.4 E-07$

7.1E-09

3. $4 E-05$

1. $2 E-0 S$

- $1 E-03$

. $5 \mathrm{E}-03$

$1.1 E-06$

$1.3 E-03$

6. $8 E-05$

7.3E-05 


\begin{tabular}{|c|c|c|c|c|}
\hline $\begin{array}{l}\text { ELEMENT } \\
\text { SYMBOL }\end{array}$ & $\begin{array}{l}\text { ATOMIC } \\
\text { NO. }(Z)\end{array}$ & $\begin{array}{l}\text { ISOTOPE } \\
\text { MASS (A) }\end{array}$ & $\begin{array}{c}\text { ADULT } \\
U C / M * * 3\end{array}$ & $\begin{array}{l}\text { INFANT } \\
\text { UC } / M * * 3\end{array}$ \\
\hline 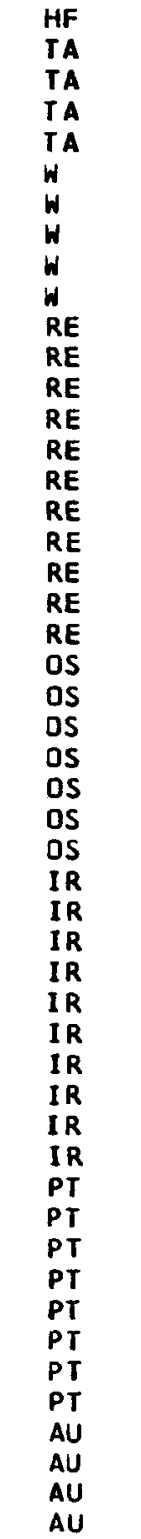 & $\begin{array}{l}72 \\
73 \\
73 \\
73 \\
73 \\
74 \\
74 \\
74 \\
74 \\
74 \\
75 \\
75 \\
75 \\
75 \\
75 \\
75 \\
75 \\
75 \\
75 \\
75 \\
75 \\
76 \\
76 \\
76 \\
76 \\
76 \\
76 \\
76 \\
77 \\
77 \\
77 \\
77 \\
77 \\
77 \\
77 \\
77 \\
77 \\
77 \\
78 \\
78 \\
78 \\
78 \\
78 \\
78 \\
78 \\
78 \\
79 \\
79 \\
79 \\
79\end{array}$ & $\begin{array}{l}182 \\
177 \\
179 \\
182 \\
183 \\
178 \\
181 \\
185 \\
187 \\
188 \\
180 \\
181 \\
182 \\
182 M \\
183 \\
184 \\
184 M \\
186 \\
187 \\
188 \\
189 \\
182 \\
183 \\
185 \\
191 \\
191 M \\
193 \\
194 \\
185 \\
186 \\
187 \\
188 \\
189 \\
190 \\
192 \\
192 M \\
193 M \\
194 \\
188 \\
190 \\
191 \\
192 \\
193 \\
193 M \\
195 M \\
197 \\
193 \\
194 \\
195 \\
196\end{array}$ & $\begin{array}{l}\text { 2. } 0 E-07 \\
2.2 \mathrm{E}-01 \\
2.0 \mathrm{E}-04 \\
9.7 \mathrm{E}-05 \\
8.3 \mathrm{E}-02 \\
3.6 \mathrm{E}-04 \\
1.5 \mathrm{E}-03 \\
1.5 \mathrm{E}-03 \\
1.6 \mathrm{E}-02 \\
1.5 \mathrm{E}-04 \\
4.4 \mathrm{E}-01 \\
4.8 \mathrm{E}-01 \\
1.7 \mathrm{E}-01 \\
8.2 \mathrm{E}-01 \\
2.9 \mathrm{E}-02 \\
1.8 \mathrm{E}-02 \\
3.2 \mathrm{E}-03 \\
4.0 \mathrm{E}-01 \\
6.8 \mathrm{E}-01 \\
8.9 \mathrm{E}-01 \\
1.4 \mathrm{E}+00 \\
1.2 \mathrm{E}-02 \\
5.2 \mathrm{E}-02 \\
3.3 \mathrm{E}-04 \\
7.8 \mathrm{E}-03 \\
1.5 \mathrm{E}-01 \\
3.7 \mathrm{E}-02 \\
5.1 \mathrm{E}-06 \\
1.6 \mathrm{E}+01 \\
9.5 \mathrm{E}+00 \\
2.6 \mathrm{E}+01 \\
1.1 \mathrm{E}+00 \\
1.4 \mathrm{E}-01 \\
4.2 \mathrm{E}-02 \\
2.9 \mathrm{E}-03 \\
1.5 \mathrm{E}-05 \\
7.3 \mathrm{E}-01 \\
8.7 \mathrm{E}+00 \\
1.0 \mathrm{E}-03 \\
1.8 \mathrm{E}-07 \\
5.4 \mathrm{E}-02 \\
2.1 \mathrm{E}-07 \\
7.3 \mathrm{E}-06 \\
6.1 \mathrm{E}-02 \\
6.0 \mathrm{E}-02 \\
1.2 \mathrm{E}+00 \\
7.0 \mathrm{E}+02 \\
4.2 \mathrm{E}+01 \\
5.9 \mathrm{E}-02 \\
8.5 \mathrm{E}+00\end{array}$ & $\begin{array}{l}1.9 \mathrm{E}-07 \\
2.1 \mathrm{E}-03 \\
1.4 \mathrm{E}-04 \\
3.2 \mathrm{E}-05 \\
1.7 \mathrm{E}-03 \\
3.6 \mathrm{E}-04 \\
1.5 \mathrm{E}-03 \\
1.5 \mathrm{E}-03 \\
1.6 \mathrm{E}-02 \\
1.5 \mathrm{E}-04 \\
4.4 \mathrm{E}-01 \\
4.8 \mathrm{E}-01 \\
1.7 \mathrm{E}-01 \\
8.2 \mathrm{E}-01 \\
2.9 \mathrm{E}-02 \\
1.8 \mathrm{E}-02 \\
3.2 \mathrm{E}-03 \\
4.0 \mathrm{E}-01 \\
6.8 \mathrm{E}-01 \\
8.9 \mathrm{E}-01 \\
1.4 \mathrm{E}+00 \\
1.2 \mathrm{E}-02 \\
5.2 \mathrm{E}-02 \\
3.3 \mathrm{E}-04 \\
7.8 \mathrm{E}-03 \\
1.5 \mathrm{E}-01 \\
3.7 \mathrm{E}-02 \\
5.1 \mathrm{E}-06 \\
2.0 \mathrm{E}-01 \\
1.2 \mathrm{E}-01 \\
2.5 \mathrm{E}-01 \\
3.5 \mathrm{E}-02 \\
2.9 \mathrm{E}-02 \\
8.0 \mathrm{E}-03 \\
1.8 \mathrm{E}-03 \\
1.5 \mathrm{E}-05 \\
1.4 \mathrm{E}-01 \\
1.4 \mathrm{E}-01 \\
1.5 \mathrm{E}-04 \\
1.7 \mathrm{E}-01 \\
2.6 \mathrm{E}-03 \\
2.1 \mathrm{E}-07 \\
7.3 \mathrm{E}-05 \\
4.2 \mathrm{E}-03 \\
3.9 \mathrm{E}-03 \\
1.7 \mathrm{E}-02 \\
2.2 \mathrm{E}+00 \\
2.9 \mathrm{E}-01 \\
2.6 \mathrm{E}-02 \\
2.1 \mathrm{E}-01\end{array}$ \\
\hline
\end{tabular}

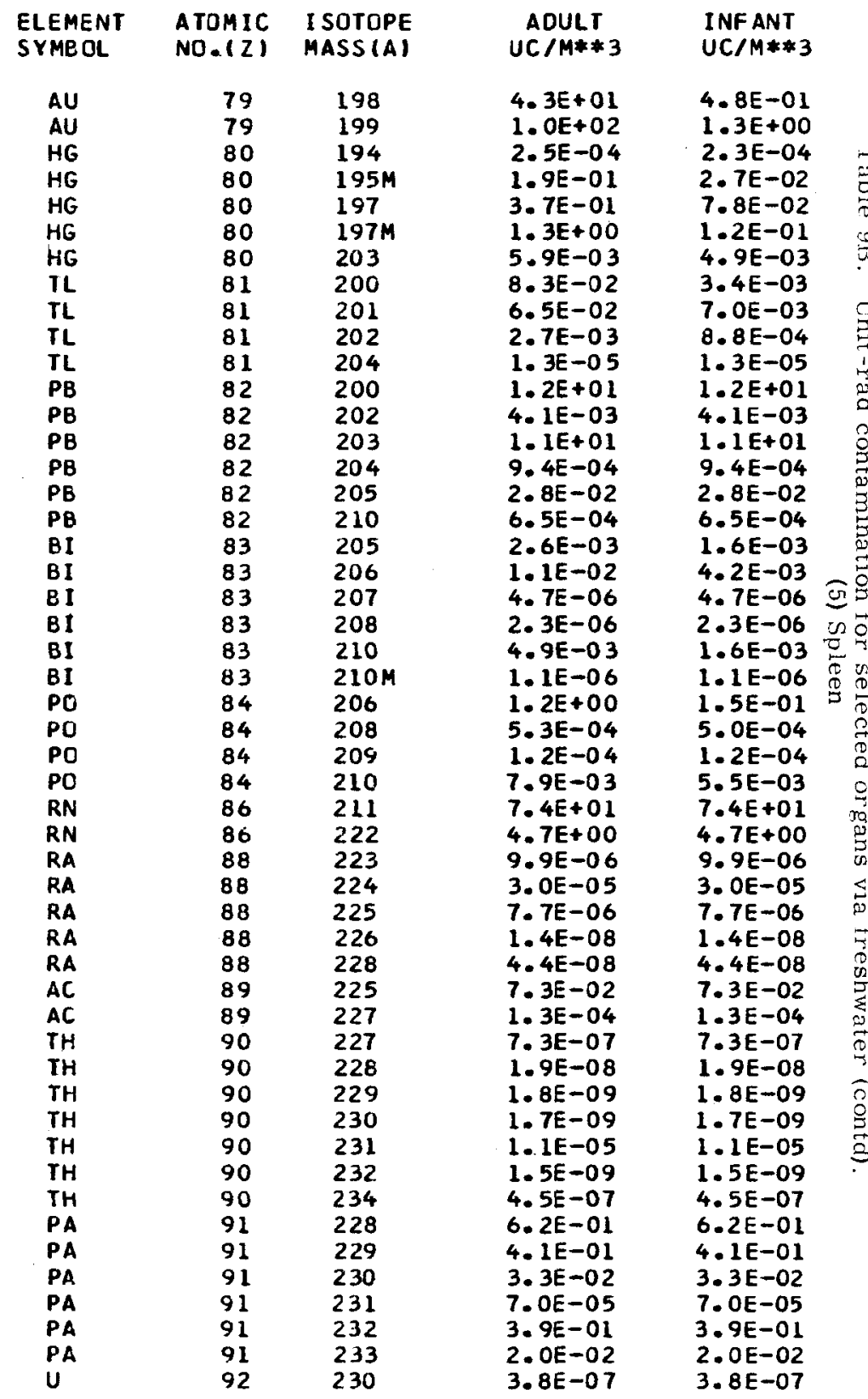


TABLE 9B. UNIT-RAD ZONTAMINATION FOR SPLEEN

\begin{tabular}{|c|c|c|c|c|}
\hline $\begin{array}{l}\text { ELEMENT } \\
\text { SYMBOL }\end{array}$ & $\begin{array}{l}\text { ATJMIC } \\
\text { VJ. }(Z)\end{array}$ & $\begin{array}{l}\text { ISOTOPE } \\
\text { MASSIA) }\end{array}$ & $\begin{array}{c}\text { ADULT } \\
\text { UC } / M * * 3\end{array}$ & $\begin{array}{l}\text { INFANT } \\
\text { UC } / M * * 3\end{array}$ \\
\hline $\begin{array}{l}U \\
U \\
U \\
U \\
U \\
U \\
U \\
U \\
U \\
N P \\
N P \\
N P \\
N P \\
N P \\
N P \\
N P \\
P U \\
P U \\
P U \\
P U \\
P U \\
P U \\
P U \\
P U \\
P U \\
A M \\
A M \\
A M \\
A M \\
A M \\
A M\end{array}$ & $\begin{array}{l}92 \\
92 \\
92 \\
92 \\
92 \\
92 \\
92 \\
92 \\
92 \\
93 \\
93 \\
93 \\
93 \\
93 \\
93 \\
93 \\
94 \\
94 \\
94 \\
94 \\
94 \\
94 \\
94 \\
94 \\
94 \\
95 \\
95 \\
95 \\
95 \\
95 \\
95\end{array}$ & $\begin{array}{l}231 \\
232 \\
233 \\
234 \\
235 \\
236 \\
237 \\
238 \\
240 \\
234 \\
235 \\
236 \\
236 M \\
237 \\
238 \\
239 \\
236 \\
237 \\
238 \\
239 \\
240 \\
241 \\
242 \\
244 \\
246 \\
239 \\
240 \\
241 \\
242 \\
242 M \\
243\end{array}$ & $\begin{array}{l}1.9 E-06 \\
1.2 E-09 \\
1.1 E-09 \\
1.0 E-09 \\
9.3 E-10 \\
9.1 E-10 \\
1.0 E-06 \\
8.8 E-10 \\
1.0 E-05 \\
2.7 E-02 \\
2.8 E-04 \\
1.4 E-05 \\
1.2 E-01 \\
1.5 E-05 \\
5.1 E-02 \\
4.3 E-02 \\
4.4 E-04 \\
1.0 E-02 \\
6.6 E-05 \\
5.6 E-05 \\
5.5 E-05 \\
1.1 E-04 \\
5.3 E-05 \\
4.7 E-05 \\
3.3 E-02 \\
8.4 E-01 \\
1.9 E-01 \\
5.7 E-05 \\
5.9 E-01 \\
5.6 E-05 \\
5.0 E-05\end{array}$ & $\begin{array}{l}1.9 E-06 \\
1.2 \mathrm{E}-09 \\
1.1 \mathrm{E}-09 \\
1.0 \mathrm{E}-09 \\
9.3 \mathrm{E}-10 \\
9.1 \mathrm{E}-10 \\
1.0 \mathrm{E}-06 \\
8.8 \mathrm{E}-10 \\
1.0 \mathrm{E}-05 \\
2.7 \mathrm{E}-02 \\
2.8 \mathrm{E}-04 \\
1.4 \mathrm{E}-05 \\
1.2 \mathrm{E}-01 \\
1.5 \mathrm{E}-05 \\
5.1 \mathrm{E}-02 \\
4.3 \mathrm{E}-02 \\
4.4 \mathrm{E}-04 \\
1.0 \mathrm{E}-02 \\
6.6 \mathrm{E}-05 \\
5.6 \mathrm{E}-05 \\
5.5 \mathrm{E}-05 \\
1.1 \mathrm{E}-04 \\
5.3 \mathrm{E}-05 \\
4.7 \mathrm{E}-05 \\
3.3 \mathrm{E}-02 \\
8.4 \mathrm{E}-01 \\
1.9 \mathrm{E}-01 \\
5.7 \mathrm{E}-05 \\
5.9 \mathrm{E}-01 \\
5.6 \mathrm{E}-05 \\
5.0 \mathrm{E}-05\end{array}$ \\
\hline
\end{tabular}

VIA FRESHWATER.

ELEMENT

SYMEOL

ATOMIC

I SOTOPE
MASSIA)

240

$C M$
$C M$
$C M$
$C M$

CM.

CM

CM

CM

EK

EK

BK

BK

CK

CF

$C F$
$C F$

CF

CF

CF

$E S$

ES

ES

ES

$F M$
$F M$
$F M$

$F M$
$10 / 09 / 68$

ADULT

1. 5E-02

1. $2 E-02$

$2.4 E-03$
$6.7 E-05$

6. $7 E-05$

8. 3E-05

5. $0 E-05$

4. $5 E-0$

4. $4 \mathrm{E}-05$

7. $6 E-02$

$2.0 E-02$

2. $0 \mathrm{E}-01$

3. $5 E-01$

1. $1 E-03$

2. $4 E-01$

$9.6 E-04$

4. $6 E-05$

1. $0 E-0$

4. $1 E-05$

3. $2 E-04$

1. $6 \mathrm{E}-02$

2. $1 E-01$

2. $1 E-03$

1. 5E-02

6. $1 E-04$

1. $8 E-01$

1. $2 E-02$

3. $1 E-01$

6. $8 E-02$

3. $4 E-01$

PAGE 25

INFAN T

$U C / M * * 3$

1. $5 \mathrm{E}-02$

$1.2 E-02$

$2.4 E-03$
$6.7 E-05$

8. 3E-05

5. OE-OS

4. 8E-05

4. 5E-05

4. 4E-05

$7.6 \mathrm{E}-02$

2. OE-01

4.5E-05

$3.5 \mathrm{E}-01$
$1.1 \mathrm{E}-03$

$1.1 E-03$
$2.4 E-01$

$4.6 E-05$

1. $0 E-04$

4.1E-05

3. 2E-04

1. $6 \mathrm{E}-02$

2.1E-0

2. $1 E-03$

1.5E-02

6.1E-04

$1.8 \mathrm{E}-01$
$1.2 \mathrm{E}-02$

$1.2 \mathrm{E}-02$

3. $1 E-01$

3. $4 E-0$

$3.5 \mathrm{E}-03$ 
ATOMIC ISOTOPE
NO. (Z) MASS(A)

ADULT

INFANT

$U C / M * * 3$

6. $4 E+02$ 8. $5 E+00$

1. $0 E-02$

1. $3 E-03$

1. $8 \mathrm{E}-02$

2. $4 E+02$

3. $8 \mathrm{E}+01$

2. $5 E-02$

2. 2E-02

1. $1 \mathrm{E}-01$

8. $6 E+00$

1. $8 \mathrm{E}-02$

8. $2 E+02$

4. 4E-02

4. $7 E-03$

1. 9E-03

9. $4 E+02$

5. $6 E+02$

5. $O E+01$

1. $0 E+02$

7. $0 E+02$

4. $3 E-03$

4. $3 E-03$

1. 7E-04

2. $8 \mathrm{E}-02$

1. $4 E-03$

6. $9 E+00$

2. $1 E+02$

3. $6 \mathrm{E}-02$

$6.4 E+00$

2. $2 E+01$

3. $6 \mathrm{E}+00$

2. $9 \mathrm{E}-01$

5. $6 E-02$

. 2E+ 00

2. $9 E+00$

1. $4 E-03$

8. $7 E+00$

3. $9 E-03$

1. $4 \mathrm{E}-02$

9. $0 E-03$

9. $9 E-05$

8. $4 E+00$

6. $6 E+01$

3. $4 E+00$

1. $6 E+00$

4. $7 E+01$
$1.1 E+03$
$6.4 E+02$

$8.5 E+00$

1. $0 E-02$

$1.3 E-03$

1. 8E-02

$1 \cdot 3 E+01$

3 - 8E+ 01

$2.5 E-02$

4. $0 E-03$

9. $8 \mathrm{E}-03$

$6.3 E-02$

1. $1 E+00$

$1.8 \mathrm{E}-02$

8. $2 \mathrm{E}+02$

4. 4E-D2

4. 7E-03

1. $9 E-03$

8. $3 E+O D$

$8.9 E+00$

4. $9 E+01$

$9.1 E+01$
$1.5 E+02$

4. $9 E-02$

4. 3E-03

2.8E-02

$4.6 E-03$

$1.4 E-03$

6. $9 E+D D$

$2.1 E+02$

$3.6 E-02$

6. $4 E+00$

2. $2 E+01$

6.4E-01

2. $9 E-01$

5.1 E-02

$4.2 E+00$

$4.6 E-01$

$1.3 E-03$

2. $6 \mathrm{E}-01$

3. $0 E-03$

$1.3 E-02$

6. $7 E-03$

9. $8 E-05$

$8.4 E+00$

$6.6 E+01$

3. $4 E+00$

1. $6 E+00$

4. $7 E+01$

$5.4 E+01$
ELEMENT
SYMGOL

ATOMIC I SOTOPE

MASSIA)

ADULT

UC $/ M * * 3$

I NFANT

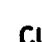

ZN

ZN

ZN

GA

GE

GE

AS

AS

AS

AS

AS

AS

SE

SE

BR

BR

KR

KR

$K R$

RB

$R B$

RB

SR

SR

67
65
$69 M$
72
67
72

$6.6 E+01 \quad 1.3 E+01$

7.7E-03 3.7E-03

4. $3 E+02 \quad 9.3 E-01$

7. $5 \mathrm{E}+00 \quad 5.4 \mathrm{E}-02$

$6.8 E+01 \quad 6.8 E+01$

2. $4 E+01 \quad 2.4 E+01$

$1.8 E-05 \quad 1.8 E-05$

2. $6 E-03 \quad 2.6 E-03$

7. $1 E-02$

4. $9 E+02$

2. $5 E+02$

7. $1 \mathrm{E}-02$

4. $9 E+02$

$2.5 E+02$

1. $0 E+03$

2. $6 E+01$

3. $4 E+02$

1. $8 E+03$

2. $3 E-02$

8. $7 E-03$

1. $7 \mathrm{E}-03$

7. $0 E+03$

$1.4 E+03$

1. $3 E+03$

1. $5 E+02$

1. $3 E+00$

$1.3 E+00$

2. $5 E-01$

5. $4 E-02$

2.6E-01

1. 5E-03

4. $4 E+00$

2. $2 E+03$

8. $3 E+00$

7. $7 E+00$

2. $6 E-02$

2. $3 E+03$

3. $7 E+02$

8. $4 E+03$

1. $1 E-01$

3. $7 E+02$

1. $3 E+00$

2. $6 E+03$

3. $0 E+02$

$5.4 E-02$

$1.0 E+03$

2. $6 E+01$

3. $4 E+02$

2. 3E-02

8. 7E-03

1. $7 E-03$

$5.6 E+02$

1. $9 E+02$

1. $3 E+00$

2. 5E-01

$2.0 E-02$

$1.6 E-02$

5. OE-02

1. 5E-03

$2.4 E+00$

$1.4 E+02$

$6.4 E+00$

5. $5 E+00$

2. $6 E-02$

1. $7 E-01$

$1.5 E-01$

5. $9 \mathrm{E}-01$

1. $5 E-03$

1. $2 \mathrm{E}-01$

9. $0 \mathrm{E}-03$

2. $3 E+00$

2. $7 E-02$

$1.2 E+00$

4. $8 \mathrm{E}-02$

3. $0 E-01$

6. $8 E-02$

3. $8 E+03$

$3.3 E+00$

4. .7E-02

1. $0 \mathrm{E}-02$

$4.7 E-02$

1. $1 E-03$

$1.1 E-03$

1. $5 E-02$

1. $5 \mathrm{E}-02$

$3.3 E+02$

2. $0 E+02$ 
TABLE 9B. UNIT-RAD CONTAMINATION FOR TESTES

VIA FRESHHATER.

$10 / 09 / 68$

PAGE 27

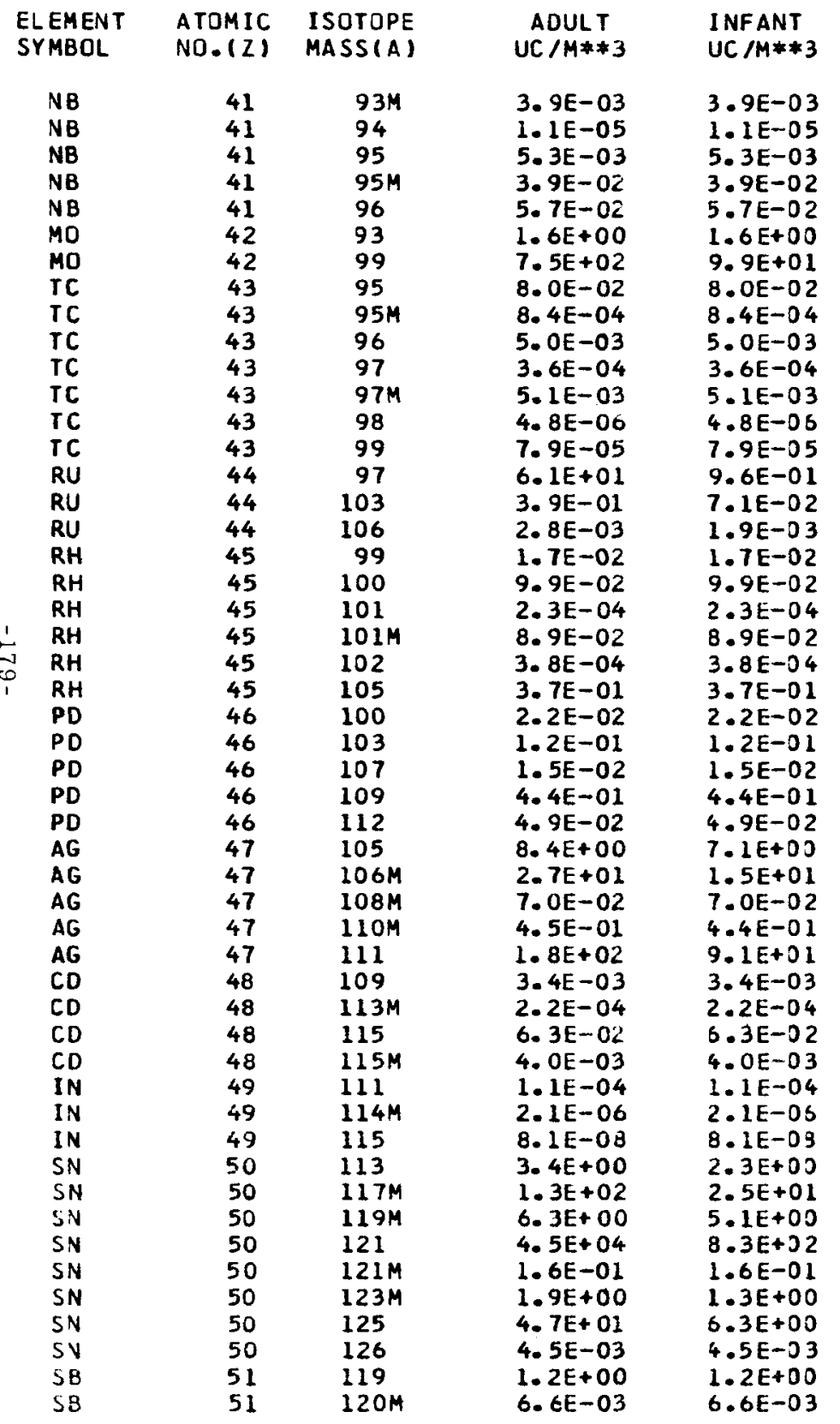

\begin{tabular}{lcc} 
ELEMENT & ATOMIC & ISOTUPE \\
SYMBOL & NO. (Z) & MA S S (A) \\
SB & & \\
SB & 51 & 122 \\
SE & 51 & 124 \\
SB & 51 & 125 \\
SB & 51 & 126 \\
TE & 51 & 127 \\
TE & 52 & 118 \\
TE & 52 & 119 \\
TE & 52 & $119 M$ \\
TE & 52 & 121 \\
TE & 52 & $121 M$ \\
TE & 52 & 123 \\
TE & 52 & $123 M$ \\
TE & 52 & $125 M$ \\
TE & 52 & $127 M$ \\
TE & 52 & $129 M$ \\
TE & 52 & $131 M$ \\
I & 52 & 132 \\
I & 53 & 123 \\
I & 53 & 124 \\
I & 53 & 125 \\
I & 53 & 126 \\
I & 53 & 129 \\
I & 53 & 130 \\
I & 53 & 131 \\
XE & 53 & 133 \\
XE & 54 & 122 \\
XE & 54 & 125 \\
XE & 54 & 127 \\
XE & 54 & $129 M$ \\
XE & 54 & $131 M$ \\
XE & 54 & 133 \\
CS & 54 & $133 M$ \\
CS & 55 & 129 \\
CS & 55 & 131 \\
CS & 55 & 132 \\
CS & 55 & 134 \\
CS & 55 & 135 \\
CS & 55 & 136 \\
BA & 55 & 137 \\
BA & 56 & 128 \\
BA & 56 & 131 \\
BA & 56 & 133 \\
BA & 56 & $133 M$ \\
BA & 56 & $135 M$ \\
LA & 56 & 140 \\
LA & 57 & 135 \\
LA & 57 & 137 \\
LA & 57 & 138 \\
CE & 57 & 140 \\
& 58 & 134 \\
\hline & &
\end{tabular}

ADULT
UC $/ M * * 3$

3. $O E-02$

6. $7 E-04$

1.8E-04

2. $0 E-02$

4. $2 \mathrm{E}-02$

2. $1 \mathrm{E}+00$

4. $2 E-02$

1. $6 E-02$

5. $2 \mathrm{E}-04$

2. $5 \mathrm{E}-0$

2. $6 E-03$

1. $O E-02$

2. $O E-03$

3. $6 E-03$

5. $0 E-01$

5. $9 E-02$

6. $3 E+00$

6. $3 E+00$
$1.5 E-02$

1. $5 E-02$

1. $2 \mathrm{E}-02$

9. $3 \mathrm{E}-05$

6. $1 E-01$

2. $1 E-02$

4. $4 E-01$

2. $I E+02$

$1.1 E+03$

5. $3 E+01$

2. $4 \mathrm{E}+02$

2. $3 E+02$

4. $6 E+02$

4. $7 E+02$

1. $6 E+01$

5. $3 E+00$

5. $7 E-01$

1. $1 E-04$

3. 5E-04

4. $6 E-02$

3. $3 E-05$

7. $6 E+02$

1. $6 E+02$

1. $3 E+00$

5. $7 E+03$

2. $5 E+04$

4. $1 E+01$

2. 5E-01

6. $4 E-04$

1. $8 E-05$

3. $4 E-02$
1. $2 E+01$
INFANT

UC/M**3

3. $0 E-02$

6. $7 E-04$

2. $8 E-03$

2. $0 E-02$

7. OE-03

4. $5 \mathrm{E}-02$

5. TE-03

5. $7 E-03$

4. $3 E-04$

2.5E-04

6. $9 E-03$

$1.6 \mathrm{E}-03$

1. $9 \mathrm{E}-03$

1. $9 E-02$

5. TE-03

4. $5 E-01$

5. $7 \mathrm{E}-03$

1. $3 E-02$

7. $9 E-03$

$9.3 \mathrm{E}-05$

4. $2 \mathrm{E}-02$

$1.1 E-02$

4. $9 \mathrm{E}-0$

2. $1 E+02$

1. $1 E+03$

5. $3 E+01$

4. $6 \mathrm{E}+02$

4. $7 E+02$

1. $8 E-01$

4. 3E-01

3. $2 \mathrm{E}-02$

9. $8 E-05$

3.5E-04

4. 8E-03

3. $3 E-05$

2. $1 E+02$

$1.0 E+02$

1. $3 E+00$

1. $2 \mathrm{E}+03$

4. $0 E+03$

2. $7 \mathrm{E}+01$

2. $5 E-01$

$6.4 E-04$

1. $8 E-05$

3. $4 E-02$
$1.0 E-01$ 
TABLE 9B. UNIT-RAD CONTAMINATION FOR TESTES

ELEMENT

ATDMIC ISOTOPE

$\underset{U C, M * * 3}{\text { ADULT }}$

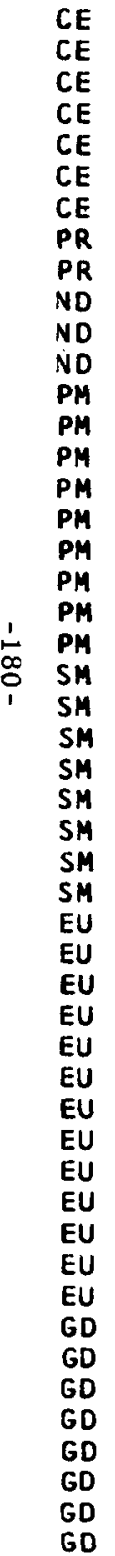

INFANT

VIA FRESHIATER.

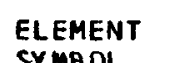

SYMBOL

6. $1 E-01$

$8.4 E-01$

$1.5 E-02$

$5.2 E-02$

5. 0E-05

$2.6 E-01$

4. $1 E-04$

4. 3E- 05

8. $0 \mathrm{E}-0 \mathrm{~S}$

1. $1 E-05$

$3.2 E-07$

$1.4 E-06$

4.3E- 03

$4.2 \mathrm{E}-04$

$1.7 \mathrm{E}-03$

3. 2 E-04

$1 \cdot 9 E-04$

$5.1 E-02$
$1.8 E-03$

$4.4 E-01$

4. $4 E-01$

$3.4 E-01$
$6.6 E-02$

$1.0 E-01$

2. 8E-04

$3.2 E-04$

$1.8 E-04$

3. $9 E-04$

5.2E-02

$7 . B E+00$

$1.8 E+0 J$

3.7E-01

$1.6 E+00$
$1.1 E-01$

$1.1 E-01$
$2.6 E-01$

1. 3E-O 1

4. $3 E-03$

$3.5 E+00$

1.7E-0 3

$1.1 E-01$

1.1E-01

1. $1 E+01$

1. $1 E+01$
$1.2 E-03$

6.7E-02

1. 5 E-O5

$3.0 E-02$

8. 2E-06

7.7E-03

$2.0 E-05$

$8.9 E-03$
ATOMIC

I SOTOPE

$10 / 09 / 68$

PAGE 28

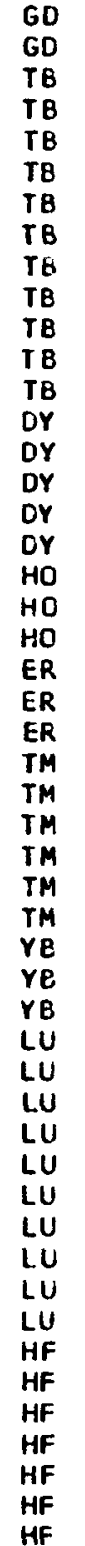

$\begin{array}{ll}64 & 159 \\ 64 & 162 \\ 65 & 151 \\ 65 & 152 \\ 65 & 153 \\ 65 & 154 \\ 65 & 155 \\ 65 & 156 \\ 65 & 157 \\ 65 & 158 \\ 65 & 160 \\ 65 & 161 \\ 65 & 164 \\ 66 & 154 \\ 66 & 154 M \\ 66 & 156 \\ 66 & 159 \\ 60 & 166 \\ 67 & 163 \\ 67 & 166 \\ 67 & 166 M \\ 68 & 160 \\ 68 & 169 \\ 68 & 172 \\ 69 & 165 \\ 69 & 167 \\ 69 & 168 \\ 69 & 170 \\ 69 & 171 \\ 69 & 172 \\ 70 & 166 \\ 70 & 169 \\ 70 & 175 \\ 71 & 169 \\ 71 & 170 \\ 71 & 171 \\ 71 & 172 \\ 71 & 173 \\ 71 & 174 \\ 71 & 174 M \\ 71 & 176 \\ 71 & 177 \\ 71 & 177 M \\ 72 & 170 \\ 72 & 171 \\ 72 & 172 \\ 72 & 173 \\ 72 & 174 \\ 72 & 175 \\ 72 & 181 \\ & \\ 69\end{array}$

AOULT

INFANT

$U C / M * * 3$

4. $7 E+02$ 1. $1 E-03 \quad 6.0 E-04$ 3. $8 E+02$

1. $1 E+02$

7. $2 E+01$

4. $1 E+02$

1. $7 E+01$

5. $1 E+00$

1. $6 E-03$

7. $0 E-05$

3. $6 E-02$

2. $4 E+01$

$5.5 E+02$

5. $9 E-10$

2. $0 E-05$

9. $2 E-10$

3. $8 E-06$

9. $4 E-06$

9. $5 \mathrm{E}-08$

3. $7 E-05$

4. $2 E-09$

1. $2 E-05$

4. $6 E-05$

$1.6 E-05$

2. $9 E+01$

2. $1 E+00$

1. 2E-02

2. $4 E-02$

3. $1 E-02$

1. $6 E+01$

4. $4 E+01$

$3.8 E-01$

4. $4 E+01$

1. $4 E-05$

6. $4 E-06$

3. $4 E-05$

6. $0 E-06$

1. $1 E-07$

2. $4 E-08$

4. $4 E-07$

7. $1 E-09$

3. $4 E-05$

1. $2 \mathrm{E}-06$

1. $2 E+02$

2. $4 E+02$

1. $5 E-04$

7. $5 E+01$

1. $3 E-05$

6. $1 E-02$

7. $1 E-01$

2.1E-01

8. $8 E-01$

$2 \cdot 3 E-01$

$6.8 E-02$

1. 5E-03

6. $6 \mathrm{E}-05$

$5.5 E-03$

4. 0E-01

$1.4 E+00$

5. $9 \mathrm{E}-10$

2. 0E-05

. $8 E-06$

9. $4 E-06$

9.5E-08

3.7E-05

3.7E 05

4. $2 E-09$

$1.2 E-05$
$4.6 E-05$

$1.6 E-05$

8. $6 E-02$

4. $8 E-02$

2.1E-03

$5.8 \mathrm{E}-03$

2. $0 E-02$

$1.1 E-01$

2.6E-01

$2.8 E-02$
$4.4 E-01$

$4.4 E-01$
$1.4 E-05$

$6.4 E-06$

$3.4 E-05$

6. OE-06

$1.1 E-07$

$2.4 E-08$

4. $4 E-07$

7.1E-09

3. $4 E-05$

$1.2 \mathrm{E}-06$

$1.1 \mathrm{E}-01$

$2.5 E-01$

1. $1 E-04$

1. 3E-01

1. $2 \mathrm{E}-05$

6. $8 E-03$

$7.3 E-03$ 
TABLE 9B. UNIT-RAD CONTAMINATION FOR TESTES

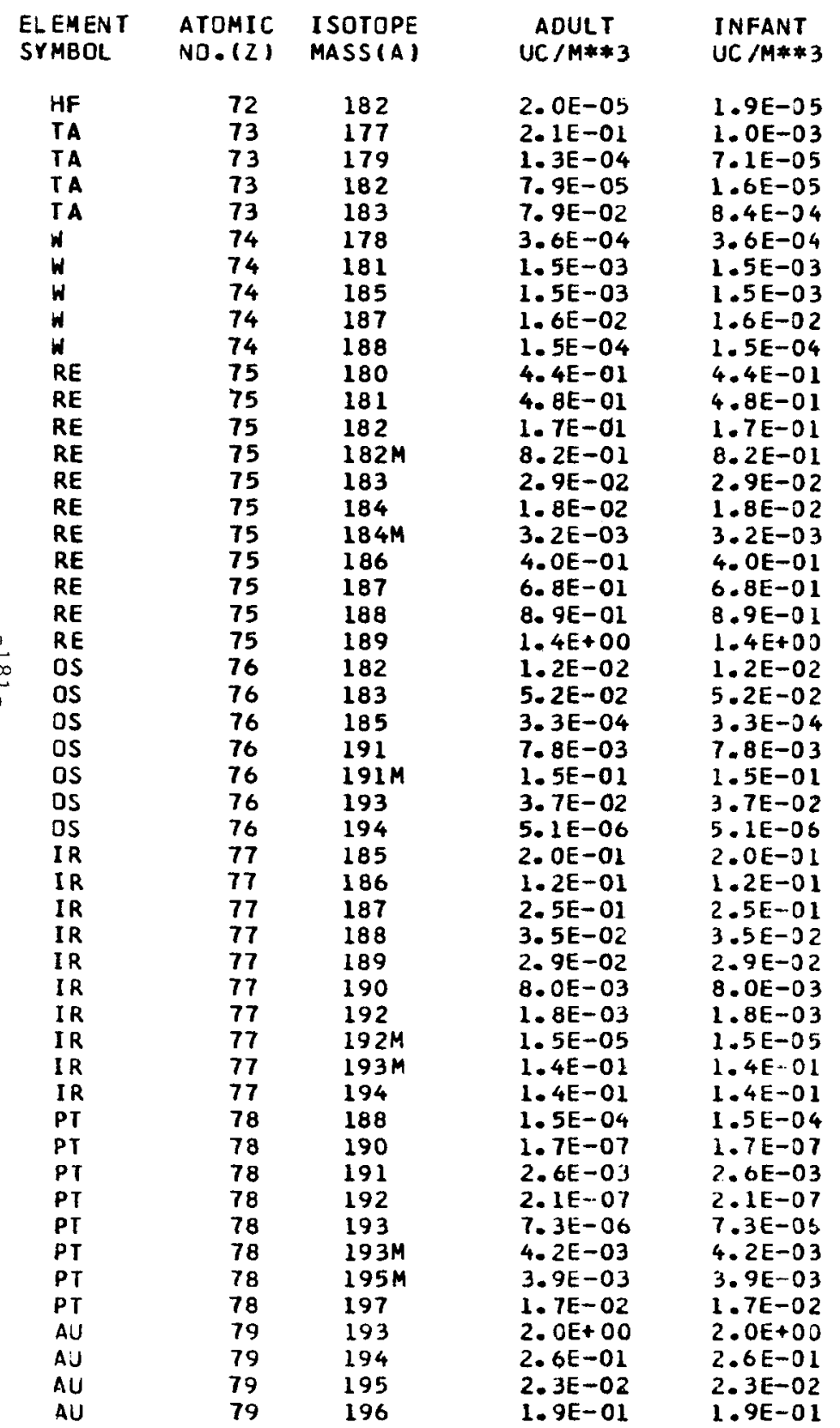

VIA FRESHWATER.

10/09/68 PAGE 29

ELEMENT
SYMBOL

ATOMIC I SOTOPE NO. (Z) MASS(A)

ADULT $U C / M * 3$

INF ANT $U C / M * * 3$

$\begin{array}{lll}\text { AU } & 79 & 198 \\ \text { AU } & 79 & 199 \\ \text { HG } & 80 & 194 \\ \text { HG } & 80 & 195 M \\ \text { HG } & 80 & 197 \\ \text { HG } & 80 & 197 M \\ \text { HG } & 80 & 203 \\ \text { TL } & 81 & 200 \\ \text { TL } & 81 & 201 \\ \text { TL } & 81 & 202 \\ \text { IL } & 81 & 204 \\ \text { PB } & 82 & 200 \\ \text { PB } & 82 & 202 \\ \text { PB } & 82 & 203 \\ \text { PE } & 82 & 204 \\ \text { PB } & 82 & 205 \\ \text { PB } & 82 & 210 \\ \text { GI } & 83 & 205 \\ \text { BI } & 83 & 206 \\ \text { BI } & 83 & 207 \\ \text { EI } & 83 & 208 \\ \text { EI } & 83 & 210 \\ \text { BI } & 83 & 210 M \\ \text { PO } & 84 & 206 \\ \text { PO } & 84 & 208 \\ \text { PO } & 84 & 209 \\ \text { PO } & 84 & 210 \\ \text { RN } & 86 & 211 \\ \text { RN } & 86 & 222 \\ \text { RA } & 88 & 223 \\ \text { RA } & 88 & 224 \\ \text { RA } & 88 & 225 \\ \text { TA } & 88 & 226 \\ \text { RA } & 88 & 228 \\ \text { AC } & 89 & 225 \\ \text { AC } & 89 & 227 \\ \text { TH } & 90 & 227 \\ \text { TH } & 90 & 228 \\ \text { IH } & 90 & 229 \\ \text { TH } & 90 & 230 \\ \text { IH } & 90 & 231 \\ \text { IH } & 90 & 232 \\ \text { IH } & 90 & 234 \\ \text { HA } & 91 & 228 \\ \text { PA } & 91 & 229 \\ \text { PA } & 91 & 230 \\ \text { PA } & 91 & 231 \\ \text { PA } & 91 & 232 \\ \text { PA } & 91 & 233 \\ \text { U } & 92 & 230\end{array}$

4. $3 \mathrm{E}-0$

1. $2 E+00$

3. $1 E-05$

3. $5 E-03$

1. $0 E-02$

1. 5E-02

6. 3E-04

4. $7 E-03$

9. $7 \mathrm{E}-03$

1. $2 E-03$

5. $2 E+01$

1. $8 E-02$

4. $1 E-03$

1. $2 E-01$

2. $8 \mathrm{E}-03$

4. $1 E-04$

1. $1 E-03$

1. $2 E-06$

6. $\mathrm{OE}-07$

4. $3 E-04$

2. $9 E-07$

1. 5E-01

5. OE-04

1. $2 E-04$

5. $5 E-03$

7. $4 E+01$

4. $7 \mathrm{E}+00$

9. $9 E-06$

3. $0 E-05$

7. $7 E-06$

1. $4 E-08$

4. $4 E-08$
7. $3 E-02$

7. $3 E-02$

7. $3 E-07$

1. $9 E-08$

1. $8 E-09$

1. $7 E-09$

$1.1 E-05$

1. $5 E-09$

1. $5 E-09$

4.

6. $2 E-01$

4. $1 E-01$

3. $3 E-02$

7. $O E-05$

3. $9 \mathrm{E}-01$

2. $0 E-02$

4. $3 E-01$

3. $1 E-05$

3. $5 \mathrm{E}-03$

1. $0 E-02$

$1.5 \mathrm{E}-02$

$6.3 E-04$

4. $7 E-03$

9. $7 \mathrm{E}-03$

$1.7 E-05$

$5.2 E+01$

1. 8E-02

4. $9 E+01$

4. $1 E-03$

$1 \cdot 2 E-01$

2. $8 E-03$

4. $1 E-04$

1. IE-03

$1.2 E-06$

$4.3 E-04$

2. $9 E-07$

1. $5 \mathrm{E}-01$

5. OE-04

1. $2 E-04$

5. $5 E-03$

7. $4 E+01$

9. $9 E-06$

3. OE-05

$7.0 E-O S$

$1.4 E-08$

4. $4 \mathrm{E}-08$

7. $3 \mathrm{E}-02$

1. $3 \mathrm{E}-04$

7. $3 E-07$

1. $9 \mathrm{E}-08$

1. $8 \mathrm{E}-09$

1. $7 E-09$

1. $1 \mathrm{E}-05$

1. $5 E-09$

4. $5 E-07$

$0.2 \mathrm{E}-01$

4. IE-O1

3. 3E-02

7. $0 E-05$

3. $9 E-01$

2. $0 \mathrm{E}-02$

3. $8 E-07$ 
TABLE 9B. UNIT-RAD CONTAMINATION FOR TESTES

\begin{tabular}{|c|c|c|c|c|}
\hline $\begin{array}{l}\text { ELEMENT } \\
\text { SYMBOL }\end{array}$ & $\begin{array}{l}\text { АTOMIC } \\
\text { VJ. }|Z|\end{array}$ & $\begin{array}{l}\text { ISOTOPE } \\
\text { MASS(A) }\end{array}$ & $\begin{array}{l}\text { ADULT } \\
U C / M * * 3\end{array}$ & $\begin{array}{l}\text { INFANT } \\
\text { UC/M**3 }\end{array}$ \\
\hline $\begin{array}{l}U \\
U \\
U \\
U \\
U \\
U \\
U \\
U \\
U \\
N P \\
N P \\
N P \\
\text { No } \\
N P \\
N P \\
N P \\
N P \\
P U \\
P U \\
P U \\
P U \\
P U \\
P U \\
P U \\
P U \\
P U \\
A M \\
A M \\
A M \\
A M \\
A M \\
A M\end{array}$ & $\begin{array}{l}92 \\
92 \\
92 \\
92 \\
92 \\
92 \\
92 \\
92 \\
92 \\
93 \\
93 \\
93 \\
93 \\
93 \\
93 \\
93 \\
94 \\
94 \\
94 \\
94 \\
94 \\
94 \\
94 \\
94 \\
94 \\
95 \\
95 \\
95 \\
95 \\
95 \\
95\end{array}$ & $\begin{array}{l}231 \\
232 \\
233 \\
234 \\
235 \\
236 \\
237 \\
238 \\
240 \\
234 \\
235 \\
236 \\
236 M \\
237 \\
238 \\
239 \\
236 \\
237 \\
238 \\
239 \\
240 \\
241 \\
242 \\
244 \\
246 \\
239 \\
240 \\
241 \\
242 \\
242 M \\
243\end{array}$ & $\begin{array}{l}1.9 E-06 \\
1.2 E-09 \\
1.1 E-09 \\
1.0 E-09 \\
9.3 E-10 \\
9.1 E-10 \\
1.0 E-06 \\
8.8 E-10 \\
1.0 E-05 \\
2.7 E-02 \\
2.8 E-04 \\
1.4 E-05 \\
1.2 E-01 \\
1.5 E-05 \\
5.1 E-02 \\
4.3 E-02 \\
4.4 E-04 \\
1.0 E-02 \\
6.6 E-05 \\
5.6 E-05 \\
5.5 E-05 \\
1.1 E-04 \\
5.3 E-05 \\
4.7 E-05 \\
3.3 E-02 \\
8.4 E-01 \\
1.9 E-01 \\
5.7 E-05 \\
5.9 E-01 \\
5.6 E-05 \\
5.0 E-05\end{array}$ & $\begin{array}{l}1.9 \mathrm{E}-06 \\
1.2 \mathrm{E}-09 \\
1.1 \mathrm{E}-09 \\
1.0 \mathrm{E}-09 \\
9.3 \mathrm{E}-10 \\
9.1 \mathrm{E}-10 \\
1.0 \mathrm{E}-06 \\
8.8 \mathrm{E}-10 \\
1.0 \mathrm{E}-05 \\
2.7 \mathrm{E}-02 \\
2.8 \mathrm{E}-04 \\
1.4 \mathrm{E}-05 \\
1.2 \mathrm{E}-01 \\
1.5 \mathrm{E}-05 \\
5.1 \mathrm{E}-02 \\
4.3 \mathrm{E}-02 \\
4.4 \mathrm{E}-04 \\
1.0 \mathrm{E}-02 \\
6.6 \mathrm{E}-05 \\
5.6 \mathrm{E}-05 \\
5.5 \mathrm{E}-05 \\
1.1 \mathrm{E}-04 \\
5.3 \mathrm{E}-05 \\
4.7 \mathrm{E}-05 \\
3.3 \mathrm{E}-02 \\
8.4 \mathrm{E}-01 \\
1.9 \mathrm{E}-01 \\
5.7 \mathrm{E}-05 \\
5.9 \mathrm{E}-01 \\
5.6 \mathrm{E}-05 \\
5.0 \mathrm{E}-05\end{array}$ \\
\hline
\end{tabular}

VIA FRESHWATER.

ELEMENT
SYMBOL

ATOM IC NO. (Z) MASS (A)

$\begin{array}{lr}\text { CM } & 96 \\ \text { CM } & 96 \\ \text { CM } & 96 \\ \text { CM } & 96 \\ \text { CM } & 96 \\ \text { CM } & 96 \\ \text { CM } & 96 \\ \text { CM } & 96 \\ \text { CM } & 96 \\ \text { BK } & 97 \\ \text { BK } & 97 \\ \text { BK } & 97 \\ \text { BK } & 97 \\ \text { BK } & 97 \\ \text { CF } & 98 \\ \text { CF } & 98 \\ \text { CF } & 98 \\ \text { CF } & 98 \\ \text { CF } & 98 \\ \text { CF } & 98 \\ \text { CF } & 98 \\ \text { ES } & 99 \\ \text { ES } & 99 \\ \text { ES } & 99 \\ \text { ES } & 99 \\ \text { ES } & 99 \\ \text { ES } & 99 \\ \text { FM } & 100 \\ \text { FM } & 100 \\ \text { FM } & 100 \\ \text { FM } & 100 \\ & \end{array}$

I SOTOPE

$10 / 09 / 68$

ADULT $U C / M * * 3$

\section{0}

241

242

244

245

246

247

248

245

246

247

248

249

246
248

249

250

251

252

253

251

252

253

254
$254 M$

252

253
255
257
1. $5 E-02$

2. $4 E-03$

2. $7 E-05$

6.7E-05

8. $3 E-05$
5. $0 E-05$

5. $0 E-05$
4. $8 E-05$

4. $8 E-05$
4. $5 E-05$

4. $4 E-05$

7. $6 E-02$

2. OE-O1

4. $5 E-05$
3. $5 E-01$

1. $1 E-03$

2. $4 E-01$

9. $6 E-04$

4. $6 E-05$

1. $0 E-04$

4. $1 E-05$

3. $2 E-04$

1. $6 E-02$

2. $1 E-01$

2. $1 E-03$

1. $5 E-02$

6. $1 E-04$

1. $8 \mathrm{E}-01$

1. $2 E-02$

3. $1 E-01$

$3.4 E-01$

3. $4 \mathrm{E}-01$
$3.5 \mathrm{E}-03$
PAGE 30

INFANT $U C / M * * 3$

1. $5 \mathrm{E}-02$

$2.4 E-03$

6.7 E-05

8. 3 E-05

5. $0 E-05$

4. BE-05

4. 5E-05

4. $4 E-05$

7. $6 E-02$

2. $0 E-01$

4. $5 \mathrm{E}-05$

3. $5 \mathrm{E}-01$

$1.1 E-03$

2. $4 E-01$

$9.6 E-04$

4. $6 E-05$

1. $0 E-04$

4. . $1 E-05$

3. $2 \mathrm{E}-04$

$1.6 E-02$

$2.1 E-01$

2. 1 E-03

1. $5 \mathrm{E}-02$

$6.1 E-04$

1. $8 \mathrm{E}-01$

1. $2 E-02$

3. 1 E-01

$6.8 \mathrm{E}-02$

$3.4 E-01$

$3.4 E-01$
$3.5 E-03$ 
TABLE 9B. UNIT-RAD ZONTAMINATION FOR WHOLE BODY VIA FRESHNATER.

10/09/68 PAGE 31

\begin{tabular}{|c|c|c|}
\hline $\begin{array}{l}\text { ELEMENT } \\
\text { SYMBOL }\end{array}$ & $\begin{array}{l}\text { ATOMIC } \\
\text { NO. }(Z)\end{array}$ & $\begin{array}{l}\text { IS OTOPE } \\
\text { MASSIA }\end{array}$ \\
\hline H & 1 & 3 \\
\hline$B E$ & 4 & 7 \\
\hline $\mathrm{BE}$ & 4 & 10 \\
\hline$C$ & 6 & 14 \\
\hline NA & 11 & 22 \\
\hline NA & 11 & 24 \\
\hline MG & 12 & 28 \\
\hline AL & 13 & 26 \\
\hline SI & 14 & 32 \\
\hline $\mathbf{P}$ & 15 & 32 \\
\hline$P$ & 15 & 33 \\
\hline 5 & 16 & 35 \\
\hline$C L$ & 17 & 36 \\
\hline $\overrightarrow{A R}$ & 18 & 37 \\
\hline$A R$ & 18 & 39 \\
\hline AR & 18 & 42 \\
\hline$k$ & 19 & 40 \\
\hline$x$ & 19 & 42 \\
\hline K & 19 & 43 \\
\hline$C_{A}$ & 20 & 41 \\
\hline$C A$ & 20 & 45 \\
\hline$C A$ & 20 & 47 \\
\hline$C A$ & 20 & 48 \\
\hline SC & 21 & $44 M$ \\
\hline SC & 21 & 46 \\
\hline SC & 21 & 47 \\
\hline SC & 21 & 48 \\
\hline TI & 22 & 44 \\
\hline$v$ & 23 & 48 \\
\hline$v$ & 23 & 49 \\
\hline$v$ & 23 & 50 \\
\hline$C R$ & 24 & 48 \\
\hline$C R$ & 24 & 51 \\
\hline$M N$ & 25 & 52 \\
\hline MN & 25 & 53 \\
\hline$M N$ & 25 & 54 \\
\hline FE & 26 & 55 \\
\hline $\mathrm{FE}$ & 26 & 59 \\
\hline$F E$ & 26 & 60 \\
\hline $\mathrm{CO}$ & 27 & 55 \\
\hline CD & 27 & 56 \\
\hline CO & 27 & 57 \\
\hline $\mathrm{CO}$ & 27 & 58 \\
\hline CO & 27 & 60 \\
\hline VI & 28 & 56 \\
\hline VI & 28 & 57 \\
\hline $\mathrm{NI}$ & 28 & 59 \\
\hline $\mathrm{NI}$ & 28 & 63 \\
\hline VI & 28 & 66 \\
\hline $\mathrm{CU}$ & 29 & 64 \\
\hline
\end{tabular}

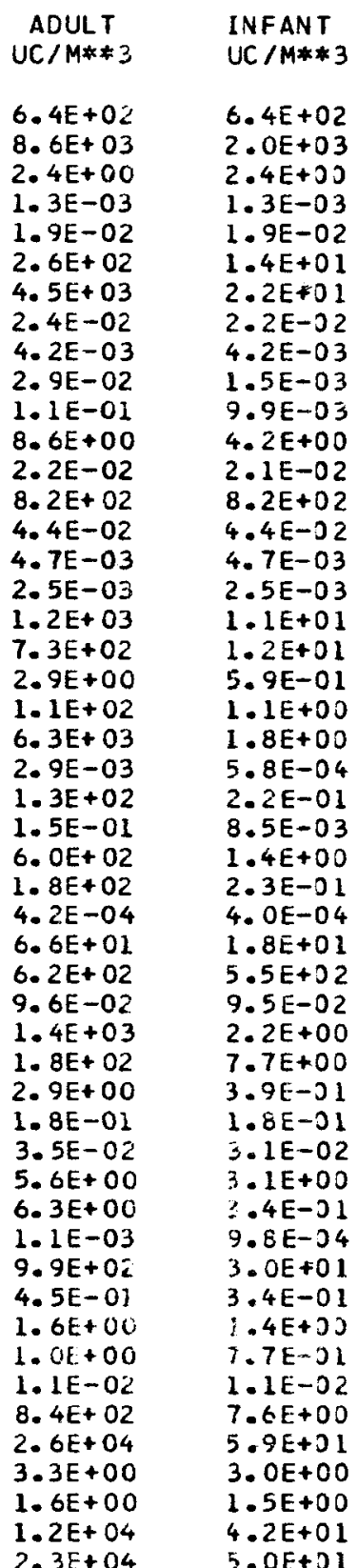

\begin{tabular}{|c|c|c|}
\hline $\begin{array}{l}\text { ELEMENY } \\
\text { SYME OL }\end{array}$ & $\begin{array}{l}\text { ATOMIC } \\
\text { NO. }(2)\end{array}$ & $\begin{array}{l}\text { ISOTOFE } \\
\text { MASS(A) }\end{array}$ \\
\hline $\mathrm{CU}$ & 29 & 67 \\
\hline$Z N$ & 30 & 65 \\
\hline$Z M$ & 30 & $69 \mathrm{M}$ \\
\hline $2 N$ & 30 & 72 \\
\hline GA & 31 & 67 \\
\hline GA & 31 & 72 \\
\hline GE & 32 & 68 \\
\hline $\mathrm{GE}$ & 32 & 69 \\
\hline GE & 32 & 71 \\
\hline AS & 33 & 71 \\
\hline$A S$ & 33 & 72 \\
\hline AS & 33 & 73 \\
\hline AS & 33 & 74 \\
\hline$A S$ & 33 & 76 \\
\hline AS & 33 & 77 \\
\hline SE & 34 & 72 \\
\hline$S E$ & 34 & 75 \\
\hline SE & 34 & 79 \\
\hline BR & 35 & 76 \\
\hline$B R$ & 35 & 77 \\
\hline ER & 35 & 82 \\
\hline$K R$ & 36 & 76 \\
\hline$K R$ & 36 & 79 \\
\hline KK & 36 & 81 \\
\hline KR & 36 & 85 \\
\hline $\mathrm{RE}$ & 37 & 83 \\
\hline $\mathrm{RB}$ & 37 & 84 \\
\hline$R B$ & 37 & 86 \\
\hline$R B$ & 37 & 87 \\
\hline$S R$. & 38 & 82 \\
\hline$S R$ & 38 & 83 \\
\hline$S R$ & 38 & 85 \\
\hline$S R$ & 38 & 89 \\
\hline$S K$ & 38 & 90 \\
\hline y & 39 & 86 \\
\hline$Y$ & 39 & 87 \\
\hline$Y$ & 39 & $87 M$ \\
\hline$Y$ & 39 & 88 \\
\hline$Y$ & 39 & 90 \\
\hline$Y$ & 39 & 91 \\
\hline $2 R$ & 40 & 86 \\
\hline $2 k$ & 40 & 88 \\
\hline ¿K & 40 & 89 \\
\hline $2 R$ & 40 & 93 \\
\hline ZR & 40 & 95 \\
\hline $2 R$ & 40 & 97 \\
\hline NB & 41 & 90 \\
\hline $\mathrm{NE}$ & 41 & 91 \\
\hline NB & 41 & $9 I M$ \\
\hline & & \\
\hline
\end{tabular}

$\underset{U C, M * * 3}{A D U L T}$

INFAVT

1. $2 E+03$

1. $3 E-02$

1. $1 E+03$

2. $0 E+01$

$5.8 E+04$

8. $2 E+04$

1. $8 E-05$

7. $7 \mathrm{E}-02$

1. $5 E+03$

1. $9 E+03$

1. $4 \mathrm{E}+02$

1. $2 E+01$

2. $5 E+03$

8. $8 E+03$

1. $1 E+00$

1. $9 E-01$

5E-02

1. $2 E+03$

1. $1 E+03$

1. $5 E+02$

1. $5 E+02$

1. $3 E+00$

2. $5 E-01$

1. $0 E-01$

1. $4 E-01$

6. $7 E-01$

3. $8 E-03$

4. $4 E+01$
$4.6 E+04$

4. $6 E+04$
4. $5 E+01$

5. $0 E+01$

4. $2 E-03$

3. $8 E+03$

6. $2 E+02$

1. $4 E+04$

1. $9 E-01$

6. 2E+02

2. $1 E+00$

1. $3 E+04$

$1.6 E+00$
$1.6 E+03$

1. $6 E+03$

5. $0 E+00$

2. $0 E+04$

7. $5 E+01$

1. $6 E-03$

1. $8 \mathrm{E}-01$

UCAM*3

1. $3 E+01$
2. $8 E-03$

7. $1 E-01$

4. $1 E-02$

2. $0 E+04$

7. $3 E+03$

1. $8 E-05$

$7.1 \mathrm{E}-02$

$1.4 E+01$

7. $2 E+00$

2. $9 E+01$

7. $4 E-01$

$5.1 E+01$

4. $6 E-01$

1. $7 E-01$

3. 5E-02.

$4.7 E+02$

2. $B E+02$

1. $7 \mathrm{E}+02$

1. $7 E+02$
$1.5 E+02$

1. $5 E+02$
$1.9 E+02$

1. $3 E+00$

2. $5 \mathrm{E}-01$

$5.1 E-02$

4. $0 \mathrm{E}-02$

1. $3 \mathrm{E}-01$

3. $8 E-03$

8. $6 E-02$

4. $9 E+00$

2. $2 E-01$

1. $9 E-01$
8. $9 E-04$

$1.7 \mathrm{E}-01$

1. $5 \mathrm{E}-01$

5. $9 E-01$

$1.5 E-03$

$1.2 \mathrm{E}-01$

9. $0 E-03$

2. $1 E+01$

$2.6 E-01$

1. $1 E+01$

6. $3 E-01$

3. $1 E+01$

6. OE-02

$1.4 E-03$

1. $5 E+00$

2. $0 E-02$ 


$\begin{array}{lcccc}\text { ELEMENT } & \text { ATOMIC } & \text { ISOTOPE } & \text { ADULT } & \text { INFANT } \\ \text { SYMBOL } & \text { ND. }(21 & \text { MASS(A) } & \text { UC/M**3 } & \text { UC /M**3 } \\ \text { NB } & & & & \\ \text { NB } & 41 & 93 M & 7.8 E-03 & 5.0 E-03 \\ \text { NB } & 41 & 94 & 1.6 E-05 & 1.4 E-05 \\ \text { NB } & 41 & 95 & 1.5 E-01 & 6.8 E-03 \\ \text { NB } & 41 & 95 M & 1.0 E+01 & 5.0 E-02 \\ \text { MO } & 41 & 96 & 5.8 E+01 & 7.4 E-02 \\ \text { MO } & 42 & 93 & 4.2 E-01 & 4.1 E-01 \\ \text { TC } & 42 & 99 & 9.3 E+02 & 2.5 E+01 \\ \text { TC } & 43 & 95 & 7.4 E+00 & 1.6 E+00 \\ \text { TC } & 43 & 95 M & 1.8 E-02 & 1.7 E-02 \\ \text { TC } & 43 & 97 & 1.7 E-01 & 1.0 E-01 \\ \text { TC } & 43 & 97 M & 7.1 E-03 & 7.1 E-03 \\ \text { TC } & 43 & 98 & 1.1 E-01 & 1.0 E-01 \\ \text { TC } & 43 & 99 & 9.6 E-05 & 9.6 E-05 \\ \text { RU } & 44 & 97 & 1.6 E-03 & 1.6 E-03 \\ \text { RU } & 44 & 103 & 2.2 E+02 & 4.8 E+00 \\ \text { RU } & 44 & 106 & 1.5 E+00 & 3.6 E-01 \\ \text { RH } & 45 & 99 & 1.3 E-02 & 9.3 E-03 \\ \text { RH } & 45 & 100 & 2.9 E-02 & 1.7 E-02 \\ \text { RH } & 45 & 101 & 1.3 E+00 & 9.9 E-02 \\ \text { RH } & 45 & 101 M & 2.3 E-04 & 2.3 E-04 \\ \text { RH } & 45 & 102 & 2.9 E-01 & 8.9 E-02 \\ \text { RH } & 45 & 105 & 4.0 E-04 & 3.8 E-04 \\ \text { PD } & 46 & 100 & 3.0 E+00 & 3.7 E-01 \\ \text { PD } & 46 & 103 & 5.2 E-02 & 2.2 E-02 \\ \text { PD } & 46 & 107 & 1.5 E-01 & 1.2 E-01 \\ \text { PD } & 46 & 109 & 1.5 E-02 & 1.5 E-02 \\ \text { PD } & 46 & 112 & 4.3 E+00 & 4.4 E-01 \\ \text { AG } & 47 & 105 & 3.3 E-01 & 4.9 E-02 \\ \text { AG } & 47 & 106 M & 4.7 E+00 & 2.1 E+00 \\ \text { AG } & 47 & 108 M & 3.1 E+01 & 4.2 E+00 \\ \text { AG } & 47 & 110 M & 2.1 E-02 & 2.0 E-02 \\ \text { AG } & 47 & 111 & 1.5 E-01 & 1.3 E-01 \\ \text { CD } & 48 & 109 & 2.1 E+02 & 2.6 E+01 \\ \text { CD } & 48 & 113 M & 3.7 E-03 & 2.6 E-03 \\ \text { CD } & 48 & 115 & 1.8 E-04 & 1.7 E-04 \\ \text { CD } & 48 & 115 M & 4.2 E+00 & 4.8 E-02 \\ \text { IN } & 49 & 111 & 1.7 E-02 & 3.1 E-03 \\ \text { IN } & 49 & 114 M & 2.0 E-03 & 1.1 E-04 \\ \text { IN } & 49 & 115 & 4.2 E-06 & 2.1 E-05 \\ \text { SN } & 50 & 113 & 8.2 E-08 & 8.1 E-03 \\ \text { SN } & 50 & 117 M & 7.6 E+00 & 2.8 E+00 \\ \text { SN } & 50 & 119 M & 4.8 E+02 & 3.1 E+01 \\ \text { SN } & 50 & 121 & 1.1 E+01 & 6.3 E+00 \\ \text { SN } & 50 & 121 M & 2.1 E-01 & 1.0 E+03 \\ \text { SN } & 50 & 123 M & 4.2 E+00 & 2.0 E-01 \\ \text { SN } & 50 & 125 & 1.8 E+02 & 7.6 E+00 \\ \text { SN } & 50 & 126 & 5.7 E-03 & 5.6 E+00 \\ \text { SB } & 51 & 119 & 2.9 E+01 & 1.2 E+00 \\ \text { SB } & 51 & 120 M & 5.0 E-02 & 6.6 E-03\end{array}$

\begin{tabular}{|c|c|c|c|}
\hline $\begin{array}{l}\text { ELEMENT } \\
\text { SYMBOL }\end{array}$ & $\begin{array}{l}\text { ATDMIC } \\
\text { NO. }(2)\end{array}$ & $\begin{array}{l}\text { I SOTOPE } \\
\text { MASS (A) }\end{array}$ & $\begin{array}{l}\text { ADULT } \\
\text { UC } / M * * 3\end{array}$ \\
\hline $\begin{array}{l}S B \\
S B \\
S B \\
S B \\
S B \\
T E \\
T E \\
T E \\
T E \\
T E \\
T E \\
T E \\
T E \\
T E \\
T E \\
T E \\
T E \\
I \\
I \\
I \\
I \\
I \\
I \\
I \\
I \\
X E \\
X E \\
X E \\
X E \\
X E \\
X E \\
X E \\
C S \\
C S \\
C S \\
C S \\
C S \\
C S \\
C S \\
B A \\
B A \\
B A \\
B A \\
B A \\
B A \\
L A \\
L A \\
L A \\
L A \\
C E\end{array}$ & $\begin{array}{l}51 \\
51 \\
51 \\
51 \\
51 \\
52 \\
52 \\
52 \\
52 \\
52 \\
52 \\
52 \\
52 \\
52 \\
52 \\
52 \\
52 \\
53 \\
53 \\
53 \\
53 \\
53 \\
53 \\
53 \\
53 \\
54 \\
54 \\
54 \\
54 \\
54 \\
54 \\
54 \\
55 \\
55 \\
55 \\
55 \\
55 \\
55 \\
55 \\
56 \\
56 \\
56 \\
56 \\
56 \\
56 \\
57 \\
57 \\
57 \\
57 \\
58\end{array}$ & $\begin{array}{l}122 \\
124 \\
125 \\
126 \\
127 \\
118 \\
119 \\
119 M \\
121 \\
121 M \\
123 \\
123 M \\
125 M \\
127 M \\
129 M \\
131 M \\
132 \\
123 \\
124 \\
125 \\
126 \\
129 \\
130 \\
131 \\
133 \\
122 \\
125 \\
127 \\
129 M \\
131 M \\
133 \\
133 M \\
129 \\
131 \\
132 \\
134 \\
135 \\
136 \\
137 \\
128 \\
131 \\
133 \\
133 M \\
135 M \\
140 \\
135 \\
137 \\
138 \\
140 \\
134\end{array}$ & 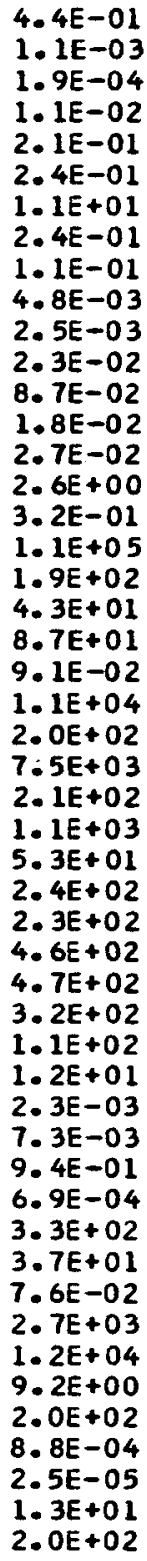 \\
\hline
\end{tabular}

INFANT

3. $0 E-02$

$6.7 E-04$

2.8E-03

2. $0 E-03$

$2.0 E-02$

7. $0 \mathrm{E}-02$

4. $5 \mathrm{E}-01$

5. $7 E-02$

$4.3 E-03$

2. 5E-03

2. $0 E-02$

6. $9 E-02$

$1.6 \mathrm{E}-02$

1. $9 \mathrm{E}-02$

$1.9 E-01$
$5.7 E-02$

4. $3 E+02$

5. $5 E+00$

$1.3 E+01$

7. $6 \mathrm{E}+00$

8. $9 \mathrm{E}-02$

4. $0 E+01$

$1.1 E+01$

4. $8 \mathrm{E}+01$

$2.1 E+02$

$1.1 E+03$

$5.3 E+01$
$2.4 E+02$

2. $3 E+02$

4. $6 E+02$

4. $7 E+02$

$3.8 E+00$

8. $9 E+00$

$6.6 E-01$

2. $0 E-03$

7. 2E-03

1. $0 E-01$

6. $8 E-04$

$1.2 E+01$

$5.6 E+00$

7. 4 E-02

$6.5 E+01$

$2.2 \mathrm{E}+02$

$1.5 E+00$

3. $2 E-01$

8. $2 \mathrm{E}-04$

$2.4 E-05$

1. $0 E+00$ 
TABLE YG. UNIT-RAD ZONTAMINATI ON FOR WHOLE BODY VIA FRESHWATER.

\begin{tabular}{|c|c|c|c|c|}
\hline $\begin{array}{l}\text { ELEMENT } \\
\text { SYMBOL }\end{array}$ & $\begin{array}{l}\text { АTJMIC } \\
\text { NO. }(2)\end{array}$ & $\begin{array}{l}\text { ISOTOPE } \\
\text { MASS (A) }\end{array}$ & 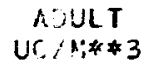 & $\begin{array}{l}\text { INFANT } \\
U C / M * * 3\end{array}$ \\
\hline $\begin{array}{l}C E \\
C E \\
C E \\
C E \\
C E \\
C E \\
C E \\
P R \\
P R \\
N D \\
N D \\
N D \\
P M \\
P M \\
P M \\
P M \\
P M \\
P M \\
P M \\
P M \\
P M \\
S M \\
S M \\
S M \\
S M \\
S M \\
S M \\
S M \\
S M \\
E U \\
E U \\
E U \\
E U \\
E U \\
E U \\
E U \\
E U \\
E U \\
E U \\
E U \\
E U \\
E U \\
G D \\
G D \\
G D \\
G D \\
G D \\
G D \\
G D \\
G D\end{array}$ & $\begin{array}{l}58 \\
58 \\
58 \\
58 \\
58 \\
58 \\
58 \\
59 \\
59 \\
60 \\
60 \\
60 \\
61 \\
61 \\
61 \\
61 \\
61 \\
61 \\
61 \\
61 \\
61 \\
62 \\
62 \\
62 \\
62 \\
62 \\
62 \\
62 \\
62 \\
63 \\
63 \\
63 \\
63 \\
63 \\
63 \\
63 \\
63 \\
63 \\
63 \\
63 \\
63 \\
63 \\
64 \\
64 \\
64 \\
64 \\
64 \\
64 \\
64 \\
64\end{array}$ & $\begin{array}{l}135 \\
137 M \\
139 \\
141 \\
142 \\
143 \\
144 \\
142 \\
143 \\
140 \\
144 \\
147 \\
143 \\
144 \\
145 \\
146 \\
147 \\
148 \\
148 M \\
149 \\
151 \\
141 \\
145 \\
146 \\
147 \\
148 \\
149 \\
151 \\
153 \\
145 \\
146 \\
146 M \\
147 \\
148 \\
149 \\
150 \\
150 M \\
152 \\
154 \\
155 \\
156 \\
157 \\
146 \\
147 \\
148 \\
149 \\
150 \\
151 \\
152 \\
153\end{array}$ & $\begin{array}{l}\text { 4. } 6 E+03 \\
3.3 E+03 \\
7.5 E-01 \\
9.5 E+00 \\
5.4 E-04 \\
1.1 E+03 \\
1.2 E-02 \\
4.0 E-02 \\
4.4 E-04 \\
2.1 E-03 \\
3.5 E-09 \\
8.5 E-05 \\
1.5 E-01 \\
1.2 E-02 \\
1.9 E-02 \\
4.4 E-03 \\
3.2 E-03 \\
6.3 E+01 \\
3.1 E-01 \\
1.3 E+03 \\
1.9 E+03 \\
2.2 E+00 \\
2.9 E-01 \\
3.1 E-04 \\
3.5 E-04 \\
2.0 E-04 \\
4.2 E-04 \\
5.8 E-02 \\
2.6 E+03 \\
2.0 E+02 \\
5.1 E+01 \\
6.3 E+02 \\
3.0 E+00 \\
3.4 E+00 \\
9.1 E-01 \\
5.9 E-03 \\
4.1 E+03 \\
2.0 E-03 \\
1.5 E-03 \\
2.2 E-01 \\
1.3 E+01 \\
1.1 E+04 \\
1.5 E-01 \\
3.5 E+02 \\
1.6 E-04 \\
1.3 E+01 \\
8.8 E-05 \\
4.3 E-01 \\
2.1 E-04 \\
2.9 E-01\end{array}$ & $\begin{array}{l}6.1 \mathrm{E}+00 \\
8.4 \mathrm{E}+00 \\
1.5 \mathrm{E}-01 \\
5.2 \mathrm{E}-01 \\
5.0 \mathrm{E}-04 \\
2.6 \mathrm{E}+00 \\
4.1 \mathrm{E}-03 \\
4.3 \mathrm{E}-05 \\
8.0 \mathrm{E}-05 \\
1.1 \mathrm{E}-05 \\
3.2 \mathrm{E}-09 \\
1.4 \mathrm{E}-0 \mathrm{0} \\
4.3 \mathrm{E}-02 \\
4.2 \mathrm{E}-03 \\
1.7 \mathrm{E}-02 \\
3.1 \mathrm{E}-03 \\
1.9 \mathrm{E}-03 \\
5.1 \mathrm{E}-01 \\
1.8 \mathrm{E}-02 \\
4.4 \mathrm{E}+00 \\
3.4 \mathrm{E}+00 \\
6.6 \mathrm{E}-02 \\
1.0 \mathrm{E}-01 \\
2.8 \mathrm{E}-04 \\
3.2 \mathrm{E}-04 \\
1.8 \mathrm{E}-04 \\
3.9 \mathrm{E}-04 \\
5.2 \mathrm{E}-02 \\
7.8 \mathrm{E}+00 \\
1.8 \mathrm{E}+00 \\
3.7 \mathrm{E}-01 \\
1.6 \mathrm{E}+00 \\
1.1 \mathrm{E}-01 \\
2.6 \mathrm{E}-01 \\
1.3 \mathrm{E}-01 \\
4.3 \mathrm{E}-03 \\
3.5 \mathrm{E}+00 \\
1.7 \mathrm{E}-03 \\
1.3 \mathrm{E}-03 \\
1.1 \mathrm{E}-01 \\
3.1 \mathrm{E}-01 \\
1.1 \mathrm{E}+01 \\
1.2 \mathrm{E}-02 \\
6.7 \mathrm{E}-01 \\
1.5 \mathrm{E}-04 \\
3.0 \mathrm{E}-01 \\
8.2 \mathrm{E}-05 \\
7.7 \mathrm{E}-02 \\
2.0 \mathrm{E}-04 \\
8.9 \mathrm{E}-02\end{array}$ \\
\hline
\end{tabular}

10/09/68 PAGE 33

\begin{tabular}{|c|c|c|}
\hline $\begin{array}{l}\text { ELEMENT } \\
\text { SYMBOL }\end{array}$ & $\begin{array}{l}\text { ATOMIC } \\
\text { NO. (Z) }\end{array}$ & $\begin{array}{l}\text { ISOTOPE } \\
\text { MASS(A) }\end{array}$ \\
\hline GD & 64 & 159 \\
\hline GD & 64 & 162 \\
\hline$T B$ & 65 & 151 \\
\hline T B & 65 & 152 \\
\hline TB & 65 & 153 \\
\hline TB & 65 & 154 \\
\hline$T_{B}$ & 65 & 155 \\
\hline$T B$ & 65 & 156 \\
\hline TB & 65 & 157 \\
\hline$T B$ & 65 & 158 \\
\hline$T B$ & 65 & 160 \\
\hline TB & 65 & 161 \\
\hline$T B$ & 65 & 164 \\
\hline DY & 66 & 154 \\
\hline or & 66 & $154 M$ \\
\hline OY & 66 & 156 \\
\hline DY & 66 & 159 \\
\hline DY & 66 & 166 \\
\hline HO & 67 & 163 \\
\hline $\mathrm{HO}$ & 67 & 166 \\
\hline HO & 67 & $166 \mathrm{M}$ \\
\hline$E R$ & 68 & 160 \\
\hline ER & 68 & 169 \\
\hline ER & 68 & 172 \\
\hline$T M$ & 69 & 165 \\
\hline$T M$ & 69 & 167 \\
\hline TM & 69 & 168 \\
\hline$T M$ & 69 & 170 \\
\hline$T M$ & 69 & 171 \\
\hline$T M$ & 69 & 172 \\
\hline$Y E$ & 70 & 166 \\
\hline$Y B$ & 70 & 169 \\
\hline$Y B$ & 70 & 175 \\
\hline LU & 71 & 169 \\
\hline LU & 71 & 170 \\
\hline LU & 71 & 171 \\
\hline LU & 71 & 172 \\
\hline LII & 71 & 173 \\
\hline LU & 71 & 174 \\
\hline LU & 71. & $174 \mathrm{M}$ \\
\hline LU & $7 \bar{l}$ & 176 \\
\hline LU & 71 & 177 \\
\hline LU & 71 & $177 M$ \\
\hline $\mathrm{HF}$ & 72 & 170 \\
\hline HF & 72 & 171 \\
\hline HF & 72 & 172 \\
\hline $\mathrm{HF}$ & 72 & 173 \\
\hline HF & 72 & 174 \\
\hline $\mathrm{HF}$ & 72 & 175 \\
\hline $\mathrm{HF}$ & 72 & 181 \\
\hline
\end{tabular}

ADULT $U C / M * 3$

7. $8 \mathrm{E}+03$

1. $5 E-02$

6. $3 E+03$

1. $8 \mathrm{E}+03$

$1.2 E+03$

6. $8 E+03$

2. $8 \mathrm{E}+02$

8. $5 E+01$

1. $7 \mathrm{E}-02$

7. $3 E-04$

5. $6 \mathrm{E}-01$

4. $0 E+02$

$9.2 E+03$

6. $5 E-10$

2. $6 \mathrm{E}-02$

1. $0 E-09$

2. 2E-05

1. $9 E-03$

1. 5E-02

3. $4 E+03$

6. $6 E-04$

$3.2 E-03$

5. $0 E-03$

9. $7 E+02$

6. $9 E+01$

3. $7 \mathrm{E}-01$

7. $5 E-01$

7. $7 E-01$

5. $5 \mathrm{E}+02$

1. $5 E+03$

1. $2 \mathrm{E}+01$

1. $5 E+03$

1. $0 E+03$

3. $4 E+02$

4. $5 E+102$

9. $5 E+01$

3. $7 E-02$
$5.2 E-03$

3. $4 t-01$

1. $1 E-03$

5. $3 E+02$

1. $O E+O O$

6. $2 E+01$
$1.2 E+02$

$7.5 E-05$

3. $7 E+01$

6. $7 E-06$

3. $1 \mathrm{E}-02$
5. $O E-02$
INFAN T

$U C / M * * 3$

1. $1 E+01$

6. $O E-03$

7.1E+0O

4. $6 E+00$

8. $8 E+00$

$2.3 E+00$

6. $8 E-01$

$1.5 \mathrm{E}-02$

$6.6 E-04$

$5.5 E-02$

4. $O E+00$

1. $4 E+01$

5. $9 E-10$

2. $0 E-05$

$9.2 \mathrm{E}-10$

3. $8 E-06$

$9.4 E-06$

$1.3 \mathrm{E}-02$

$5.2 \mathrm{E}+00 \mathrm{~J}$

5. 9E-04

1.2E-05

4.6E-05

$1.6 E+05$

$9.6 E-01$

$4.1 E-02$

1. $2 \mathrm{E}-01$

1. 2 E-01

$2.2 E+00$

$5.2 E+00$

$5.5 \mathrm{E}-01$

8. $8 E+00$

2. $O E+00$

9. OE-O1

8. $4 E-01$

. $4 E-01$

1. $5 E-02$

3. 3E-03

6. $1 E-02$

9. $9 E-04$

4. $8 E+00$

1. . FE-01

5. 5E-0?

$1.3 E-01$

5.7E-05

6. ÓE-02

3. $4 E-03$

$3.7 E-03$ 


\begin{tabular}{|c|c|c|c|c|}
\hline $\begin{array}{l}\text { ELEMENT } \\
\text { SYMBOL }\end{array}$ & $\begin{array}{l}\text { ATOMIC } \\
\text { NO. }(Z)\end{array}$ & $\begin{array}{l}\text { ISOTOPE } \\
\text { MASS (A) }\end{array}$ & $\begin{array}{l}\text { ADULT } \\
\text { UC/M**3 }\end{array}$ & $\begin{array}{l}\text { INFANT } \\
\text { UC/M**3 }\end{array}$ \\
\hline 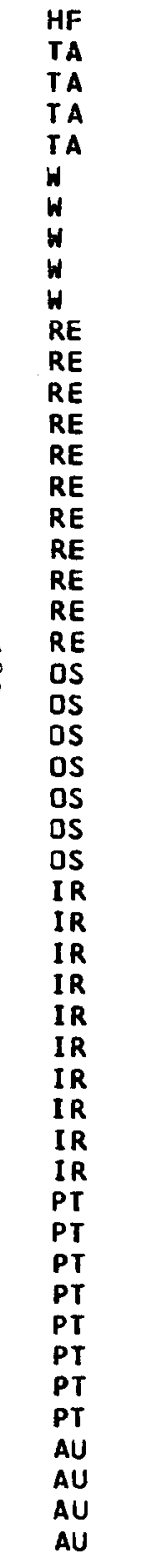 & $\begin{array}{l}72 \\
73 \\
73 \\
73 \\
73 \\
74 \\
74 \\
74 \\
74 \\
74 \\
75 \\
75 \\
75 \\
75 \\
75 \\
75 \\
75 \\
75 \\
75 \\
75 \\
75 \\
76 \\
76 \\
76 \\
76 \\
76 \\
76 \\
76 \\
77 \\
77 \\
77 \\
77 \\
77 \\
77 \\
77 \\
77 \\
77 \\
77 \\
78 \\
78 \\
78 \\
78 \\
78 \\
78 \\
78 \\
78 \\
79 \\
79 \\
79 \\
79\end{array}$ & $\begin{array}{l}182 \\
177 \\
179 \\
182 \\
183 \\
178 \\
181 \\
185 \\
187 \\
188 \\
180 \\
181 \\
182 \\
182 M \\
183 \\
184 \\
184 M \\
186 \\
187 \\
188 \\
189 \\
182 \\
183 \\
185 \\
191 \\
191 M \\
193 \\
194 \\
185 \\
186 \\
187 \\
188 \\
189 \\
190 \\
192 \\
192 M \\
193 M \\
194 \\
188 \\
190 \\
191 \\
192 \\
193 \\
193 M \\
195 M \\
197 \\
193 \\
194 \\
195 \\
196\end{array}$ & $\begin{array}{l}1.0 \mathrm{E}-05 \\
2.2 \mathrm{E}+00 \\
2.0 \mathrm{E}-03 \\
9.7 \mathrm{E}-04 \\
8.3 \mathrm{E}-01 \\
3.8 \mathrm{E}-04 \\
1.5 \mathrm{E}-03 \\
1.5 \mathrm{E}-03 \\
3.2 \mathrm{E}-02 \\
1.5 \mathrm{E}-04 \\
4.1 \mathrm{E}+00 \\
4.5 \mathrm{E}+00 \\
6.0 \mathrm{E}-01 \\
1.1 \mathrm{E}+01 \\
3.2 \mathrm{E}-02 \\
2.1 \mathrm{E}-02 \\
3.3 \mathrm{E}-03 \\
1.2 \mathrm{E}+00 \\
6.8 \mathrm{E}-01 \\
9.6 \mathrm{E}+00 \\
1.2 \mathrm{E}+01 \\
3.9 \mathrm{E}-02 \\
2.6 \mathrm{E}-01 \\
3.3 \mathrm{E}-04 \\
8.8 \mathrm{E}-03 \\
6.5 \mathrm{E}-01 \\
9.1 \mathrm{E}-02 \\
5.1 \mathrm{E}-06 \\
6.6 \mathrm{E}+02 \\
3.9 \mathrm{E}+02 \\
1.0 \mathrm{E}+03 \\
4.4 \mathrm{E}+01 \\
7.3 \mathrm{E}+00 \\
2.1 \mathrm{E}+00 \\
2.2 \mathrm{E}-01 \\
1.5 \mathrm{E}-03 \\
3.8 \mathrm{E}+01 \\
3.6 \mathrm{E}+02 \\
4.9 \mathrm{E}-04 \\
1.7 \mathrm{E}-07 \\
2.3 \mathrm{E}-02 \\
2.1 \mathrm{E}-07 \\
7.3 \mathrm{E}-00 \\
2.7 \mathrm{E}-02 \\
2.6 \mathrm{E}-02 \\
4.9 \mathrm{E}-01 \\
2.4 \mathrm{E}+02 \\
1.5 \mathrm{E}+01 \\
2.9 \mathrm{E}-02 \\
3.0 \mathrm{E}+00\end{array}$ & $\begin{array}{l}9.4 \mathrm{E}-06 \\
2.1 \mathrm{E}-02 \\
1.4 \mathrm{E}-03 \\
3.2 \mathrm{E}-04 \\
1.7 \mathrm{E}-02 \\
3.6 \mathrm{E}-04 \\
1.5 \mathrm{E}-03 \\
1.5 \mathrm{E}-03 \\
1.6 \mathrm{E}-02 \\
1.5 \mathrm{E}-04 \\
4.4 \mathrm{E}-01 \\
4.8 \mathrm{E}-01 \\
1.7 \mathrm{E}-01 \\
8.2 \mathrm{E}-01 \\
2.9 \mathrm{E}-02 \\
1.8 \mathrm{E}-02 \\
3.2 \mathrm{E}-03 \\
4.0 \mathrm{E}-01 \\
6.8 \mathrm{E}-01 \\
8.9 \mathrm{E}-01 \\
1.4 \mathrm{E}+00 \\
1.2 \mathrm{E}-02 \\
5.2 \mathrm{E}-02 \\
3.3 \mathrm{E}-04 \\
7.8 \mathrm{E}-03 \\
1.5 \mathrm{E}-01 \\
3.7 \mathrm{E}-02 \\
5.1 \mathrm{E}-06 \\
2.0 \mathrm{E}+01 \\
1.2 \mathrm{E}+01 \\
2.5 \mathrm{E}+01 \\
3.5 \mathrm{E}+00 \\
2.9 \mathrm{E}+00 \\
8.0 \mathrm{E}-01 \\
1.8 \mathrm{E}-01 \\
1.5 \mathrm{E}-03 \\
1.4 \mathrm{E}+01 \\
1.4 \mathrm{E}+01 \\
1.5 \mathrm{E}-04 \\
1.7 \mathrm{E}-07 \\
2.6 \mathrm{E}-03 \\
2.1 \mathrm{E}-01 \\
7.3 \mathrm{E}-06 \\
4.2 \mathrm{E}-03 \\
3.9 \mathrm{E}-03 \\
1.7 \mathrm{E}-02 \\
1.5 \mathrm{E}+00 \\
2.0 \mathrm{E}-01 \\
1.8 \mathrm{E}-02 \\
1.5 \mathrm{E}-01\end{array}$ \\
\hline
\end{tabular}

\begin{tabular}{lcc} 
ELEMENT & A TOMIC & I SOTO \\
SYMBOL & NO.I Z & MASSI \\
& & \\
AU & 79 & 198 \\
AU & 79 & 199 \\
HG & 80 & 194 \\
HG & 80 & 195 \\
HG & 80 & 197 \\
HG & 80 & 197 \\
HG & 80 & 203 \\
TL & 81 & 200 \\
TL & 81 & 201 \\
TL & 81 & 202 \\
TL & 81 & 204 \\
PB & 82 & 200 \\
PB & 82 & 202 \\
PB & 82 & 203 \\
PB & 82 & 204 \\
PB & 82 & 205 \\
PB & 82 & 210 \\
BI & 83 & 205 \\
BI & 83 & 206 \\
EI & 83 & 207 \\
BI & 83 & 208 \\
BI & 83 & 210 \\
BI & 83 & 210 \\
PO & 84 & 206 \\
PO & 84 & 208 \\
PO & 84 & 209 \\
PO & 84 & 210 \\
RN & 86 & 211 \\
RN & 86 & 222 \\
RA & 88 & 223 \\
RA & 88 & 224 \\
RA & 88 & 225 \\
RA & 88 & 226 \\
RA & 88 & 228 \\
AC & 89 & 225 \\
AC & 89 & 227 \\
TH & 90 & 227 \\
TH & 90 & 228 \\
TH & 90 & 229 \\
IH & 90 & 230 \\
TH & 90 & 231 \\
TH & 90 & 232 \\
TH & 90 & 234 \\
PA & 91 & 228 \\
PA & 91 & 229 \\
PA & 91 & 230 \\
PA & 91 & 231 \\
PA & 91 & 232 \\
PA & 91 & 233 \\
U & 92 & 230 \\
\hline & & \\
\hline
\end{tabular}

ADULT

INFANT

$U C / M * * 3$

1. $5 E+01$

$3.3 \mathrm{E}-01$ 3. $5 E+01$ 9.1E-01

$\begin{array}{lll}194 & 6.5 E-04 & 6.1 E-04 \\ 195 \mathrm{M} & 4.9 E-01 & 7.0 \mathrm{E}-02\end{array}$

$197 \quad 9.6 \mathrm{E}-01$

$197 M$

3. $3 E+00$

1. $5 E-02$

1. $1 E-01$

3. $0 \mathrm{E}-01$

$1.3 E-02$

$4.4 \mathrm{E}-03$

9. $0 \mathrm{E}-03$

3. $5 E-03$

$1.7 E-05$

$1.5 E+04$

$1.6 E-05$

8. $8 E+00$

3. $8 E-03$

$5.6 E+03$

B. TE-04

2. $6 E-02$

6. 5E-04

7. $6 E-03$

2. $7 E-02$

1. $7 E-05$

8. 3E-06

1. 2E-02

4. $0 E-06$

$6.6 E-01$

5. 1E-0 4

1. $2 E-04$

7. $4 E+01$

4. $7 E+00$

1. $3 E+03$

1. $3 E+04$

7. $8 E+02$

7. $3 E-03$

4. $3 E-02$

1. $8 E+02$

1. $0 E-03$

2. 3E-0 3

1. $6 E-06$

2. $8 E-08$

2. $6 E-08$

$5.8 E-01$

2. $3 E-08$

$1.1 E-03$

2. $8 E+04$

1. $1 E+04$

7. $9 E+01$

$3.1 E-03$

8. $3 E+00$

7. OE-O4

2.1E-02

4. $9 \mathrm{E}-04$

$1.5 E-02$

1. 7E-05

8. 3E-06

5. 9E-03

4. OE-06

1.5E-01

5. 0E-04

1. $2 E-04$
$5.5 E-03$

$7.4 E+01$

4. $7 E+00$

1. $8 E+00$

5. $7 E+00$

$1.4 E+00$

2.6E-03

8. $3 E-03$

7. $3 E-02$

$1.3 E-04$

7. $3 E-07$

1. $9 E-08$

1. $8 E-09$

1. $7 E-09$

1. 1E-05

1. 5E-09

4.5E-07

6.2E-01

4. $1 E-01$

3. 3E-02

8. $0 E-04$

7. OE-05

1. $2 E+04$

3. $9 \mathrm{E}-01$

2. $9 E+01$

2. $0 E-02$

1. $3 E-01$ 
TABLE 9B. UNIT-RAD CONTAMINATION FOR WHOLE BDDY VIA FRESHWATER.

10/09/68 PAGE 35

$\begin{array}{lcc}\text { ELEMENT } & \text { ATOMIC } & \text { ISOTOPE } \\ \text { SYMBOL } & \text { NO. ( } Z \text { ) } & \text { MASS (A) } \\ U & & \\ U & 92 & 231 \\ U & 92 & 232 \\ U & 92 & 233 \\ U & 92 & 234 \\ U & 92 & 235 \\ U & 92 & 236 \\ U & 92 & 237 \\ U & 92 & 238 \\ \text { NP } & 92 & 240 \\ \text { NP } & 93 & 234 \\ \text { NP } & 93 & 235 \\ \text { NP } & 93 & 236 \\ \text { NP } & 93 & 236 M \\ \text { NP } & 93 & 237 \\ \text { NP } & 93 & 238 \\ \text { PU } & 93 & 239 \\ \text { PU } & 94 & 236 \\ \text { PU } & 94 & 237 \\ \text { PU } & 94 & 238 \\ \text { PU } & 94 & 239 \\ \text { PU } & 94 & 240 \\ \text { PU } & 94 & 241 \\ \text { PU } & 94 & 242 \\ \text { PU } & 94 & 244 \\ \text { AM } & 94 & 246 \\ \text { AM } & 95 & 239 \\ \text { AM } & 95 & 240 \\ \text { AM } & 95 & 241 \\ \text { AM } & 95 & 242 \\ \text { AM } & 95 & 242 M \\ & 95 & 243\end{array}$

$\begin{array}{ll}\begin{array}{c}\text { ADULT } \\ \text { UC/M**3 }\end{array} & \text { INFANT } \\ \text { UC/M**3 } \\ 1.6 E+01 & 6.4 E-01 \\ 4.1 E-04 & 4.0 E-04 \\ 3.7 E-04 & 3.7 E-04 \\ 3.5 E-04 & 3.5 E-04 \\ 3.2 E-04 & 3.2 E-04 \\ 3.2 E-04 & 3.2 E-04 \\ 5.7 E+00 & 3.6 E-01 \\ 3.1 E-04 & 3.0 E-04 \\ 6.0 E+02 & 3.5 E+00 \\ 2.4 E+02 & 2.7 E-02 \\ 2.7 E-02 & 2.8 E-04 \\ 1.6 E-04 & 1.4 E-05 \\ 5.1 E+03 & 1.2 E-01 \\ 1.7 E-04 & 1.5 E-05 \\ 9.4 E+02 & 5.1 E-02 \\ 7.2 E+02 & 4.3 E-02 \\ 2.8 E-02 & 4.4 E-04 \\ 1.5 E+01 & 1.0 E-02 \\ 1.2 E-03 & 6.6 E-05 \\ 9.9 E-04 & 5.6 E-05 \\ 9.8 E-04 & 5.5 E-05 \\ 2.6 E-03 & 1.1 E-04 \\ 9.4 E-04 & 5.3 E-05 \\ 8.5 E-04 & 4.7 E-05 \\ 2.0 E+02 & 3.3 E-02 \\ 3.3 E+04 & 8.4 E-01 \\ 1.8 E+03 & 1.9 E-01 \\ 3.4 E-04 & 5.7 E-05 \\ 1.8 E+04 & 5.9 E-01 \\ 3.4 E-04 & 5.6 E-05 \\ 3.0 E-04 & 5.0 E-05\end{array}$

ELEMENT

ATOMIC ISOTOPE

SYMBOL

NO. (Z) MASSIA)

ADULT

INFANT

$U C / M * * 3$

$\begin{array}{lrl}\text { CM } & 96 & 240 \\ C M & 96 & 241 \\ C M & 96 & 242 \\ C M & 96 & 243 \\ C M & 96 & 244 \\ C M & 96 & 245 \\ C M & 96 & 246 \\ C M & 96 & 247 \\ C M & 96 & 248 \\ \text { BK } & 97 & 245 \\ \text { BK } & 97 & 246 \\ \text { BK } & 97 & 247 \\ \text { BK } & 97 & 248 \\ \text { BK } & 97 & 249 \\ C F & 98 & 246 \\ C F & 98 & 248 \\ C F & 98 & 249 \\ C F & 98 & 250 \\ C F & 98 & 251 \\ C F & 98 & 252 \\ C F & 98 & 253 \\ \text { ES } & 99 & 251 \\ \text { ES } & 99 & 252 \\ \text { ES } & 99 & 253 \\ \text { ES } & 99 & 254 \\ \text { ES } & 99 & 254 M \\ \text { ES } & 99 & 255 \\ \text { FM } & 100 & 252 \\ \text { FM } & 100 & 253 \\ \text { FM } & 100 & 255 \\ \text { FM } & 100 & 257\end{array}$

$1.3 E+01$
$8.1 E+00$
$3.6 E-01$
$5.2 E-04$
$7.0 E-04$
$3.5 E-04$
$3.4 E-04$
$3.1 E-04$
$3.1 E-04$
$9.9 E+02$
$7.2 E+03$
$8.1 E-04$
$2.4 E+04$
$2.3 E-01$
$1.0 E+04$
$1.8 E-01$
$8.3 E-04$
$2.7 E-03$
$7.4 E-04$
$2.3 E-02$
$5.5 E+01$
$9.0 E+03$
$1.0 E+00$
$5.0 E+01$
$8.3 E-02$
$7.2 E+03$
$3.2 E+01$
$2.1 E+04$
$9.8 E+02$
$2.6 E+04$
$2.9 E+00$

1. 5E-02

1. $2 E-02$

$2.4 E-03$

6. 7E-05

8. 3E-05

4. 8E-05

4. 5E-05

4. $4 E-05$

7.6E-02

2. $0 \mathrm{E}-01$

4. $5 \mathrm{E}-05$

3. $5 E-01$

$1.1 E-03$

$2.4 \mathrm{E}-01$

$9.6 E-04$
$4.6 E-05$

1. $0 E-05$

4. 1 E-05

$3.2 \mathrm{E}-04$

$1.6 E-02$

2. $1 E-01$

$2.1 E-01$
$2.1 E-03$

1. 5E-02

$6.1 E-04$

1. $8 E-01$

1. $2 E-02$

3.1E-01

$6.8 E-02$

3. $4 E-01$

3. $5 \mathrm{E}-03$

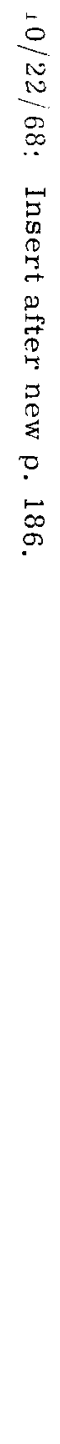




\begin{tabular}{|c|c|c|c|c|}
\hline $\begin{array}{l}\text { ELEMENT } \\
\text { SYMBOL }\end{array}$ & $\begin{array}{l}\text { ATOMIC } \\
\text { NO. (Z) }\end{array}$ & $\begin{array}{l}\text { ISOTOPE } \\
\text { MASS (A) }\end{array}$ & $\begin{array}{c}\text { ADULT } \\
U C / M * * 3\end{array}$ & $\begin{array}{l}\text { INFAN T } \\
\text { UC /M**3 }\end{array}$ \\
\hline $\begin{array}{l}H \\
B E \\
B E \\
C \\
V A \\
N A \\
M G \\
A L \\
S I \\
P \\
P \\
S \\
C L \\
A R \\
A R \\
A R \\
K \\
K \\
K \\
C A \\
C A \\
C A \\
C A \\
C A \\
C A \\
S C \\
S C \\
S C \\
S C \\
T I \\
V \\
V \\
V \\
C R \\
C R \\
C R \\
M N \\
M N \\
M N \\
F E \\
F E \\
F E \\
C O \\
C O \\
C O \\
C O \\
C O \\
N I \\
N I \\
N I \\
N I \\
N I \\
C U\end{array}$ & $\begin{array}{l}1 \\
4 \\
4 \\
6 \\
11 \\
11 \\
12 \\
13 \\
14 \\
15 \\
15 \\
16 \\
17 \\
18 \\
18 \\
18 \\
19 \\
19 \\
19 \\
20 \\
20 \\
20 \\
20 \\
21 \\
21 \\
21 \\
21 \\
22 \\
23 \\
23 \\
23 \\
24 \\
24 \\
25 \\
25 \\
25 \\
26 \\
26 \\
26 \\
27 \\
27 \\
27 \\
27 \\
27 \\
28 \\
28 \\
28 \\
28 \\
28 \\
29\end{array}$ & $\begin{array}{l}3 \\
7 \\
10 \\
14 \\
22 \\
24 \\
28 \\
26 \\
32 \\
32 \\
33 \\
35 \\
36 \\
37 \\
39 \\
42 \\
40 \\
42 \\
43 \\
41 \\
45 \\
47 \\
48 \\
44 M \\
46 \\
47 \\
48 \\
44 \\
48 \\
49 \\
50 \\
48 \\
51 \\
52 \\
53 \\
54 \\
55 \\
59 \\
60 \\
55 \\
56 \\
57 \\
58 \\
60 \\
56 \\
57 \\
59 \\
63 \\
66 \\
64\end{array}$ & $\begin{array}{l}\text { 6. } 4 E+02 \\
1.8 E+01 \\
2.1 E-02 \\
1.0 E-03 \\
1.2 E-02 \\
3.0 E+01 \\
7.5 E+01 \\
1.5 E-03 \\
4.6 E-02 \\
1.6 E-02 \\
1.0 E-01 \\
1.8 E+01 \\
8.2 E-03 \\
1.4 E+05 \\
7.3 E+00 \\
7.9 E-01 \\
2.2 E-03 \\
3.7 E+01 \\
2.6 E+01 \\
9.6 E+00 \\
1.8 E+01 \\
3.9 E+01 \\
9.5 E-03 \\
4.9 E-01 \\
1.2 E-02 \\
2.9 E+00 \\
5.9 E-01 \\
5.0 E-04 \\
2.0 E-01 \\
5.5 E+00 \\
9.5 E-04 \\
3.7 E+00 \\
5.2 E+00 \\
6.0 E-02 \\
2.1 E-02 \\
3.8 E-03 \\
1.8 E+01 \\
2.0 E+00 \\
5.5 E-03 \\
1.5 E+01 \\
5.7 E-02 \\
2.4 E-01 \\
1.3 E-01 \\
1.8 E-03 \\
8.2 E+00 \\
1.0 E+02 \\
2.7 E+00 \\
1.3 E+00 \\
6.1 E+01 \\
3.2 E+02\end{array}$ & $\begin{array}{l}6.4 \mathrm{E}+02 \\
1.7 \mathrm{E}+01 \\
2.1 \mathrm{E}-02 \\
1.0 \mathrm{E}-03 \\
1.2 \mathrm{E}-02 \\
8.8 \mathrm{E}+00 \\
2.6 \mathrm{E}+01 \\
1.5 \mathrm{E}-03 \\
4.6 \mathrm{E}-02 \\
1.5 \mathrm{E}-02 \\
9.5 \mathrm{E}-02 \\
1.7 \mathrm{E}+01 \\
8.2 \mathrm{E}-03 \\
1.4 \mathrm{E}+05 \\
7.3 \mathrm{E}+00 \\
7.9 \mathrm{E}-01 \\
2.2 \mathrm{E}-03 \\
9.4 \mathrm{E}+00 \\
1.0 \mathrm{E}+01 \\
9.6 \mathrm{E}+03 \\
1.8 \mathrm{E}+01 \\
3.0 \mathrm{E}+01 \\
9.5 \mathrm{E}-03 \\
3.0 \mathrm{E}-01 \\
1.2 \mathrm{E}-02 \\
2.0 \mathrm{E}+00 \\
3.3 \mathrm{E}-01 \\
5.0 \mathrm{E}-04 \\
1.8 \mathrm{E}-01 \\
5.5 \mathrm{E}+00 \\
9.5 \mathrm{E}-04 \\
1.4 \mathrm{E}+00 \\
4.9 \mathrm{E}+00 \\
4.8 \mathrm{E}-02 \\
2.1 \mathrm{E}-02 \\
3.8 \mathrm{E}-03 \\
1.8 \mathrm{E}+01 \\
1.9 \mathrm{E}+00 \\
5.5 \mathrm{E}-03 \\
4.9 \mathrm{E}+00 \\
5.6 \mathrm{E}-02 \\
2.4 \mathrm{E}-01 \\
1.3 \mathrm{E}-01 \\
1.8 \mathrm{E}-03 \\
6.6 \mathrm{E}+00 \\
5.1 \mathrm{E}+01 \\
2.7 \mathrm{E}+00 \\
1.3 \mathrm{E}+00 \\
3.7 \mathrm{E}+01 \\
8.5 \mathrm{E}+01\end{array}$ \\
\hline
\end{tabular}

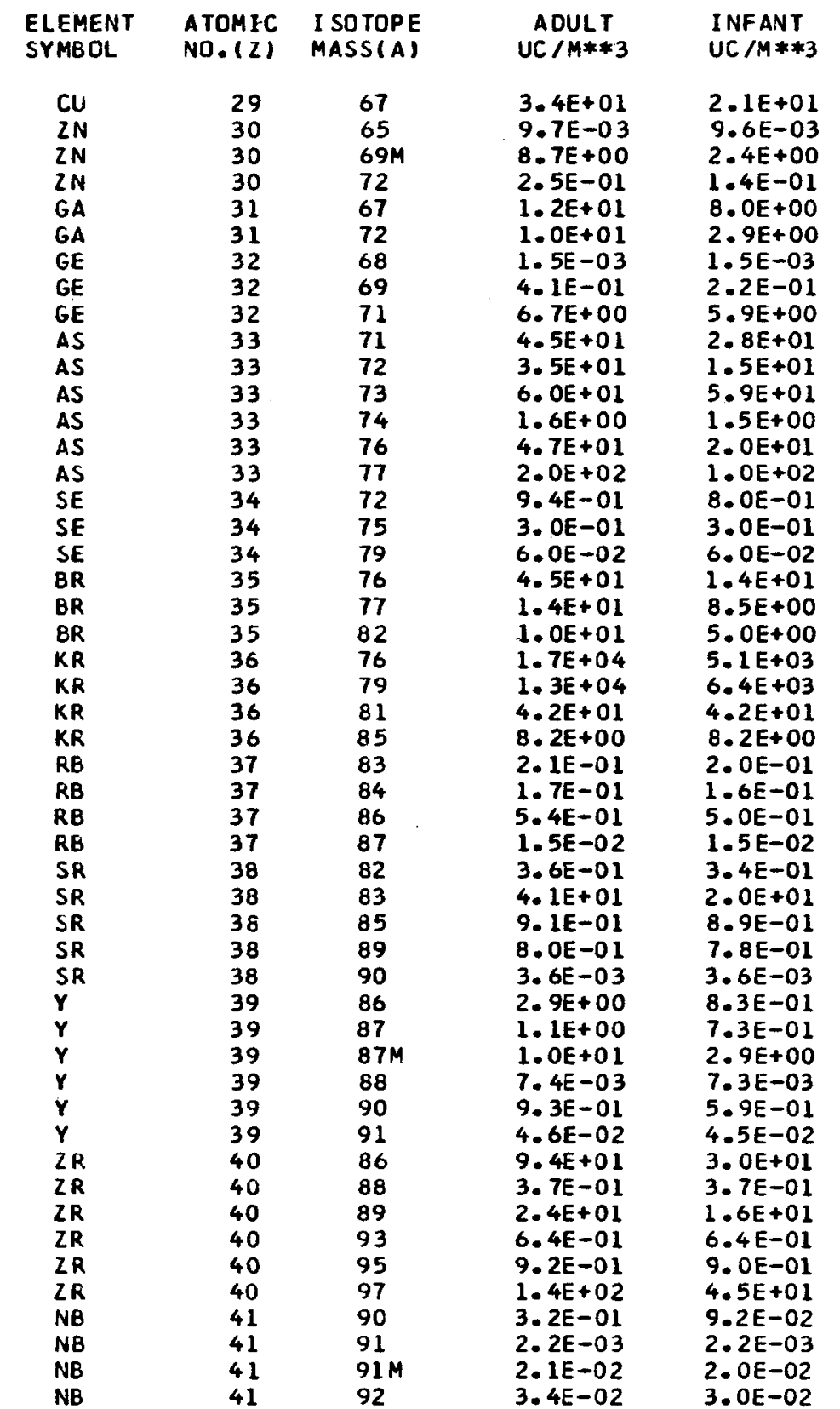


TABLE 9B. UNIT-RAD GONTAMINATION FOR GI TRACT VIA FRESHWATER.

$10 / 09 / 68$ PAGE 37

\begin{tabular}{|c|c|c|c|c|}
\hline $\begin{array}{l}\text { ELEMENT } \\
\text { SYMBOL }\end{array}$ & $\begin{array}{l}\text { ATOMIC } \\
\text { ND. (Z) }\end{array}$ & $\begin{array}{l}\text { ISOTOPE } \\
\text { MASSIA) }\end{array}$ & $\begin{array}{c}\text { ADULT } \\
U C / M \neq * 3\end{array}$ & $\begin{array}{l}\text { INF ANT } \\
\text { UC } / M * * 3\end{array}$ \\
\hline $\begin{array}{l}N B \\
N B \\
N B \\
N B \\
N B \\
M D \\
M O \\
M C \\
T C \\
T C \\
T C \\
T C \\
T C \\
T C \\
T C \\
R U \\
R U \\
R U \\
R H \\
R H \\
R H \\
\text { TH } \\
\text { RH } \\
R H \\
R H \\
P D \\
P D \\
P D \\
P D \\
P D \\
P D \\
A G \\
A G \\
A G \\
A G \\
A G \\
C D \\
C D \\
C D \\
C D \\
I N \\
I N \\
I N \\
S N \\
S N \\
S N \\
S N \\
S N \\
S N \\
S N \\
S N \\
S B \\
S B\end{array}$ & $\begin{array}{l}41 \\
41 \\
41 \\
41 \\
41 \\
42 \\
42 \\
43 \\
43 \\
43 \\
43 \\
43 \\
43 \\
43 \\
44 \\
44 \\
44 \\
45 \\
45 \\
45 \\
45 \\
45 \\
45 \\
46 \\
46 \\
46 \\
46 \\
46 \\
47 \\
47 \\
47 \\
47 \\
47 \\
48 \\
48 \\
48 \\
48 \\
49 \\
49 \\
49 \\
50 \\
50 \\
50 \\
50 \\
50 \\
50 \\
50 \\
50 \\
51 \\
51\end{array}$ & $\begin{array}{l}93 M \\
94 \\
95 \\
95 M \\
96 \\
93 \\
99 \\
95 \\
95 M \\
96 \\
97 \\
97 M \\
98 \\
99 \\
97 \\
103 \\
106 \\
99 \\
100 \\
101 \\
101 M \\
102 \\
105 \\
100 \\
103 \\
107 \\
109 \\
112 \\
105 \\
106 M \\
108 M \\
110 M \\
111 \\
109 \\
113 M \\
115 \\
115 M \\
111 \\
114 M \\
115 \\
113 \\
117 M \\
119 M \\
121 \\
121 M \\
123 M \\
125 \\
126 \\
119 \\
120 M\end{array}$ & $\begin{array}{l}\text { 7. } 6 E-03 \\
2.2 E-05 \\
1.1 E-02 \\
1.1 E-01 \\
2.9 E-01 \\
9.4 E-01 \\
8.7 E+01 \\
1.5 E+01 \\
5.8 E-02 \\
4.5 E-01 \\
2.4 E-02 \\
3.5 E-01 \\
3.2 E-04 \\
5.3 E-03 \\
2.7 E+01 \\
1.4 E+00 \\
3.5 E-02 \\
1.4 E-01 \\
1.9 E+00 \\
1.6 E-03 \\
8.5 E-01 \\
2.8 E-03 \\
5.3 E+00 \\
5.2 E-01 \\
2.1 E+00 \\
2.4 E-01 \\
2.7 E+01 \\
2.2 E+00 \\
1.3 E+00 \\
3.0 E+00 \\
1.2 E-02 \\
7.9 E-02 \\
1.9 E+01 \\
1.3 E-02 \\
8.1 E-04 \\
3.8 E-01 \\
1.5 E-02 \\
7.5 E-02 \\
1.0 E-03 \\
3.7 E-05 \\
3.6 E-01 \\
4.4 E+00 \\
8.0 E-01 \\
3.0 E+02 \\
2.5 E-02 \\
2.1 E-01 \\
1.1 E+00 \\
7.0 E-04 \\
5.1 E+00 \\
1.9 E-02\end{array}$ & $\begin{array}{l}7.5 E-03 \\
2.2 E-05 \\
1.0 E-02 \\
7.5 E-02 \\
1.1 E-01 \\
9.4 E-01 \\
5.6 E+01 \\
5.3 E+00 \\
5.6 E-02 \\
3.4 E-01 \\
2.4 E-02 \\
3.4 E-01 \\
3.2 E-04 \\
5.3 E-03 \\
1.8 E+01 \\
1.3 E+00 \\
3.4 E-02 \\
1.2 E-01 \\
7.1 E-01 \\
1.6 E-03 \\
6.3 E-01 \\
2.7 E-03 \\
2.7 E+00 \\
3.7 E-01 \\
1.9 E+00 \\
2.4 E-01 \\
7.3 E+00 \\
8.2 E-01 \\
1.3 E+00 \\
2.6 E+00 \\
1.2 E-02 \\
7.8 E-02 \\
1.6 E+01 \\
1.3 E-02 \\
8.1 E-04 \\
2.3 E-01 \\
1.5 E-02 \\
4.9 E-02 \\
9.7 E-04 \\
3.7 E-05 \\
3.6 E-01 \\
3.9 E+00 \\
7.9 E-01 \\
1.3 E+02 \\
2.5 E-02 \\
2.0 E-01 \\
9.9 E-01 \\
7.0 E-04 \\
2.6 E+00 \\
1.5 E-02\end{array}$ \\
\hline
\end{tabular}

$\begin{array}{lcc}\text { ELEMENT } & \text { ATOMIC } & \text { I SOTOP } \\ \text { SYMBOL } & \text { YO. IZ1 } & \text { MASSIA } \\ \text { SE } & & \\ \text { SE } & 51 & 122 \\ \text { SB } & 51 & 124 \\ \text { SB } & 51 & 125 \\ \text { SB } & 51 & 126 \\ \text { TE } & 51 & 127 \\ \text { TE } & 52 & 118 \\ \text { TE } & 52 & 119 \\ \text { TE } & 52 & 119 M \\ \text { TE } & 52 & 121 \\ \text { TE } & 52 & 121 M \\ \text { IE } & 52 & 123 \\ \text { TE } & 52 & 123 M \\ \text { TE } & 52 & 125 M \\ \text { IE } & 52 & 127 M \\ \text { TE } & 52 & 129 M \\ \text { TE } & 52 & 131 M \\ \text { I } & 52 & 132 \\ \text { I } & 53 & 123 \\ \text { I } & 53 & 124 \\ \text { I } & 53 & 125 \\ \text { I } & 53 & 126 \\ \text { I } & 53 & 129 \\ \text { I } & 53 & 130 \\ \text { I } & 53 & 131 \\ \text { XE } & 53 & 133 \\ \text { XE } & 54 & 122 \\ \text { XE } & 54 & 125 \\ \text { XE } & 54 & 127 \\ \text { XE } & 54 & 129 M \\ \text { XE } & 54 & 131 M \\ \text { XE } & 54 & 133 \\ \text { CS } & 54 & 133 M \\ \text { CS } & 55 & 129 \\ \text { CS } & 55 & 131 \\ \text { CS } & 55 & 132 \\ \text { CS } & 55 & 134 \\ \text { CS } & 55 & 135 \\ \text { CS } & 55 & 136 \\ \text { EA } & 55 & 137 \\ \text { EA } & 56 & 128 \\ \text { BA } & 56 & 131 \\ \text { BA } & 50 & 133 \\ \text { BA } & 56 & 133 M \\ \text { BA } & 56 & 135 M \\ \text { LA } & 56 & 140 \\ \text { LA } & 57 & 135 \\ \text { LA } & 57 & 137 \\ \text { LA } & 57 & 138 \\ \text { CE } & 57 & 140 \\ & 58 & 134\end{array}$

ADULT
UC $/ M * * 3$

1. $1 E-01$

1. $6 E-03$

4. $2 E-04$

7. $1 E-03$

6. $2 E-02$

2. $0 E-01$

3. $3 E+00$

1. $7 E-01$

1. $4 E-01$

$5.7 E-03$

4. $6 E-02$

4. $6 E-02$

$1.6 E-01$
$3.7 E-02$

4. $4 E-02$

4. $4 E-02$
$9.8 E-01$

1. $9 E-01$

1. $1 E+04$

5. $1 E+01$

8. $9 E+0$

5. $7 E+01$

6. $0 E-01$

- $1 E+0$

8. $6 E+01$

8. $7 E+02$

5. $9 E+03$

3. $2 \mathrm{E}+0$

5. $5 E+02$

2. $8 E+03$

2. $6 E+03$

$5.0 E+03$

7. $7 E+03$

1. $5 E+01$

1. $5 E+01$

1. $9 E+01$

1. $5 E+00$

3. $8 E-03$

2. $1 E-O$

1. $3 E-03$

1. $3 E-03$

$3.5 E+00$

$4.1 E-02$

4. $1 E-02$

7. $0 E+01$

2. $8 E+02$

$9.4 \mathrm{E}-01$

8. $3 E+00$

7. $4 E-03$

2. $2 \mathrm{E}-04$

7. $6 \mathrm{E}-01$

7. $8 \mathrm{E}-01$
INFANT

$U C / M * * 3$

6. $9 E-02$

. $5 E-03$

$4.2 \mathrm{E}-04$

6. 3E-03

4. 5E-02

$1.6 E-01$

1. $O E+00$

1. 3E-01

1. 3E-01

9. $9 E-03$

$5.7 \mathrm{E}-03$

4. $6 \mathrm{E}-02$

$1.6 E-01$

$3.6 \mathrm{E}-02$

$4.2 E-02$

4. $4 E-01$

1. $3 E-01$

2. $9 E+03$

. $7 E+01$

.

G.

2. $7 E+02$

7. $3 E+01$

$3.2 E+02$

$2.1 E+03$

$1.1 E+04$

$5.3 E+02$

2. $3 E+03$

$4.6 E+03$

$4.6 E+03$

4.7E+03

$1.1 E+00$

$1.2 \mathrm{E}+00$

3. $8 E-03$

$1.4 \mathrm{E}-02$

1. $9 E-01$

1. $3 E-03$

$6.5 E+00$

3. +00

$4.1 E-02$

4.1E-OZ

$3.6 E+01$

$1.2 E+02$

$8.4 \mathrm{E}-01$

2. $9 E+00$

7. $4 \mathrm{E}-03$

2. $2 E-04$

4. $0 \mathrm{E}-01$

5. $2 E-01$ 


\begin{tabular}{|c|c|c|c|c|c|c|c|c|c|}
\hline $\begin{array}{l}\text { ELEMENT } \\
\text { SYMBOL }\end{array}$ & $\begin{array}{l}\text { ATOMIC } \\
\text { VO. }(Z)\end{array}$ & $\begin{array}{l}\text { ISOTOPE } \\
\text { MASS (A) }\end{array}$ & $\begin{array}{l}\text { ADULT } \\
\text { UC/M**3 }\end{array}$ & $\begin{array}{l}\text { INFAHT } \\
\text { UC/M**3 }\end{array}$ & $\begin{array}{l}\text { ELEMENT } \\
\text { SYMBOL }\end{array}$ & $\begin{array}{l}\text { ATOM IC } \\
\text { NO. }(Z)\end{array}$ & $\begin{array}{l}\text { I SD TOPE } \\
\text { MASS }(A)\end{array}$ & $\begin{array}{l}\text { ADULT } \\
\text { UC/M**3 }\end{array}$ & $\begin{array}{l}\text { INFAN T } \\
\text { UC/M**3 }\end{array}$ \\
\hline $\begin{array}{l}C E \\
C E \\
C E \\
C E \\
C E \\
C E \\
C E \\
P R \\
P R \\
N D \\
N D \\
N D \\
P M \\
P M \\
P M \\
P M \\
P M \\
P M \\
P M \\
P M \\
P M \\
S M \\
S M \\
S M \\
S M \\
S M \\
S M \\
S M \\
S M \\
S M \\
E U \\
E U \\
E U \\
E U \\
E U \\
E U \\
E U \\
E U \\
E U \\
E U \\
E U \\
E U \\
E U \\
G D \\
G D \\
G D \\
G D \\
G D \\
G D \\
G D \\
G D\end{array}$ & $\begin{array}{l}58 \\
58 \\
58 \\
58 \\
58 \\
58 \\
58 \\
59 \\
59 \\
60 \\
60 \\
60 \\
61 \\
61 \\
61 \\
61 \\
61 \\
61 \\
61 \\
61 \\
61 \\
62 \\
62 \\
62 \\
62 \\
62 \\
62 \\
62 \\
62 \\
63 \\
63 \\
63 \\
63 \\
63 \\
63 \\
63 \\
63 \\
63 \\
63 \\
63 \\
63 \\
63 \\
64 \\
64 \\
64 \\
64 \\
64 \\
64 \\
64 \\
64\end{array}$ & $\begin{array}{l}135 \\
137 M \\
139 \\
141 \\
142 \\
143 \\
144 \\
142 \\
143 \\
140 \\
144 \\
147 \\
143 \\
144 \\
145 \\
146 \\
147 \\
148 \\
148 M \\
149 \\
151 \\
141 \\
145 \\
146 \\
147 \\
148 \\
149 \\
151 \\
153 \\
145 \\
146 \\
146 M \\
147 \\
148 \\
149 \\
150 \\
150 M \\
152 \\
154 \\
155 \\
156 \\
157 \\
146 \\
147 \\
148 \\
149 \\
150 \\
151 \\
152 \\
153\end{array}$ & $\begin{array}{l}\text { 9. } 1 \mathrm{E}+00 \\
8.6 \mathrm{E}+00 \\
7.6 \mathrm{E}-02 \\
2.7 \mathrm{E}-01 \\
2.5 \mathrm{E}-04 \\
2.7 \mathrm{~T}+00 \\
2.0 \mathrm{E}-03 \\
5.9 \mathrm{E}+00 \\
4.2 \mathrm{E}-01 \\
7.6 \mathrm{E}-01 \\
1.6 \mathrm{E}-04 \\
7.9 \mathrm{E}-02 \\
1.1 \mathrm{E}-02 \\
1.1 \mathrm{E}-03 \\
4.2 \mathrm{E}-03 \\
7.8 \mathrm{E}-04 \\
4.8 \mathrm{E}-04 \\
1.6 \mathrm{E}-01 \\
4.7 \mathrm{E}-03 \\
1.9 \mathrm{E}+00 \\
1.9 \mathrm{E}+00 \\
2.7 \mathrm{E}-02 \\
3.9 \mathrm{E}-02 \\
1.1 \mathrm{E}-04 \\
1.2 \mathrm{E}-04 \\
7.0 \mathrm{E}-05 \\
1.5 \mathrm{E}-04 \\
2.0 \mathrm{E}-02 \\
5.3 \mathrm{E}+00 \\
5.4 \mathrm{E}-01 \\
1.2 \mathrm{E}-01 \\
7.3 \mathrm{E}-01 \\
2.8 \mathrm{E}-02 \\
6.5 \mathrm{E}-02 \\
3.1 \mathrm{E}-02 \\
1.0 \mathrm{E}-03 \\
3.1 \mathrm{E}+00 \\
4.1 \mathrm{E}-04 \\
3.1 \mathrm{E}-04 \\
2.6 \mathrm{E}-02 \\
8.2 \mathrm{E}-02 \\
8.9 \mathrm{E}+00 \\
7.4 \mathrm{E}-03 \\
9.6 \mathrm{E}-01 \\
8.9 \mathrm{E}-05 \\
2.0 \mathrm{E}-01 \\
4.8 \mathrm{E}-05 \\
4.6 \mathrm{E}-02 \\
1.2 \mathrm{E}-04 \\
5.3 \mathrm{E}-02\end{array}$ & $\begin{array}{l}3.0 \mathrm{E}+00 \\
4.2 \mathrm{E}+00 \\
7.5 \mathrm{E}-02 \\
2.6 \mathrm{E}-01 \\
2.5 \mathrm{E}-04 \\
1.3 \mathrm{E}+00 \\
2.0 \mathrm{E}-03 \\
2.0 \mathrm{E}+00 \\
3.8 \mathrm{E}-01 \\
5.2 \mathrm{E}-01 \\
1.6 \mathrm{E}-04 \\
7.0 \mathrm{E}-02 \\
1.1 \mathrm{E}-02 \\
1.1 \mathrm{E}-03 \\
4.2 \mathrm{E}-03 \\
7.8 \mathrm{E}-04 \\
4.8 \mathrm{E}-04 \\
1.3 \mathrm{E}-01 \\
4.6 \mathrm{E}-03 \\
1.1 \mathrm{E}+00 \\
8.4 \mathrm{E}-01 \\
2.5 \mathrm{E}-02 \\
3.8 \mathrm{E}-02 \\
1.1 \mathrm{E}-04 \\
5.2 \mathrm{E}-04 \\
7.0 \mathrm{E}-05 \\
1.5 \mathrm{E}-04 \\
2.0 \mathrm{E}-02 \\
3.0 \mathrm{E}+00 \\
4.3 \mathrm{E}-01 \\
8.8 \mathrm{E}-02 \\
3.7 \mathrm{E}-01 \\
2.6 \mathrm{E}-02 \\
6.3 \mathrm{E}-02 \\
3.1 \mathrm{E}-02 \\
1.0 \mathrm{E}-03 \\
8.2 \mathrm{E}-01 \\
4.1 \mathrm{E}-04 \\
3.1 \mathrm{E}-04 \\
2.6 \mathrm{E}-02 \\
7.5 \mathrm{E}-02 \\
2.6 \mathrm{E}+00 \\
7.2 \mathrm{E}-03 \\
3.9 \mathrm{E}-01 \\
8.9 \mathrm{E}-05 \\
1.8 \mathrm{E}-01 \\
4.8 \mathrm{E}-05 \\
4.5 \mathrm{E}-02 \\
1.2 \mathrm{E}-04 \\
5.3 \mathrm{E}-02\end{array}$ & 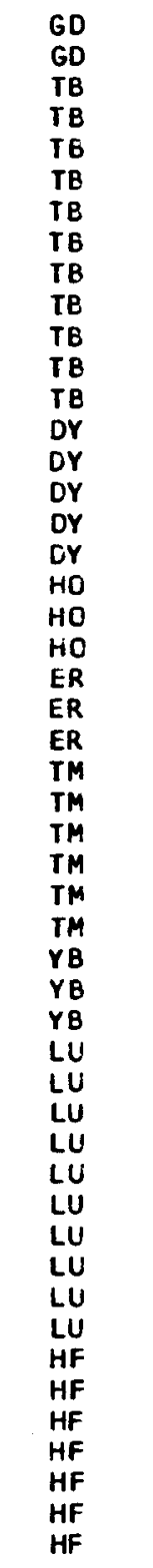 & $\begin{array}{l}64 \\
64 \\
65 \\
65 \\
65 \\
65 \\
65 \\
65 \\
65 \\
65 \\
65 \\
65 \\
65 \\
66 \\
66 \\
66 \\
66 \\
66 \\
67 \\
67 \\
67 \\
68 \\
68 \\
68 \\
69 \\
69 \\
69 \\
69 \\
69 \\
69 \\
70 \\
70 \\
70 \\
71 \\
71 \\
71 \\
71 \\
71 \\
71 \\
71 \\
71 \\
71 \\
71 \\
72 \\
72 \\
72 \\
72 \\
72 \\
72 \\
72\end{array}$ & $\begin{array}{l}159 \\
162 \\
151 \\
152 \\
153 \\
154 \\
155 \\
156 \\
157 \\
158 \\
160 \\
161 \\
164 \\
154 \\
154 M \\
156 \\
159 \\
166 \\
163 \\
166 \\
166 M \\
160 \\
169 \\
172 \\
165 \\
167 \\
168 \\
170 \\
171 \\
172 \\
166 \\
169 \\
175 \\
169 \\
170 \\
171 \\
172 \\
173 \\
174 \\
174 M \\
176 \\
177 \\
177 M \\
170 \\
171 \\
172 \\
173 \\
174 \\
175 \\
181\end{array}$ & $\begin{array}{l}1.9 E+01 \\
3.5 E-03 \\
7.1 E+00 \\
2.1 E+00 \\
2.4 E+00 \\
8.0 E+00 \\
9.7 E-01 \\
2.9 E-01 \\
5.1 E-03 \\
2.2 E-04 \\
1.9 E-02 \\
1.6 E+00 \\
1.1 E+01 \\
2.8 E-05 \\
3.6 E+00 \\
4.5 E-05 \\
1.8 E-01 \\
6.5 E-01 \\
4.5 E-03 \\
4.0 E+00 \\
2.0 E-04 \\
1.3 E+00 \\
2.6 E+00 \\
1.4 E+00 \\
1.3 E+00 \\
3.7 E-01 \\
1.4 E-02 \\
3.9 E-02 \\
1.3 E-01 \\
1.1 E+00 \\
2.6 E+00 \\
1.8 E-01 \\
3.7 E+00 \\
1.3 E+00 \\
5.2 E-01 \\
1.9 E+00 \\
3.4 E-01 \\
5.1 E-03 \\
1.1 E-03 \\
2.1 E-02 \\
3.3 E-04 \\
1.9 E+00 \\
5.9 E-02 \\
4.1 E+00 \\
8.3 E+00 \\
1.1 E-03 \\
3.1 E+00 \\
1.1 E-04 \\
6.4 E-02 \\
7.0 E-02\end{array}$ & $\begin{array}{l}6.2 E+00 \\
3.5 E-03 \\
2.4 E+00 \\
6.8 E-01 \\
1.5 E+00 \\
2.9 E+00 \\
7.7 E-01 \\
2.3 E-01 \\
5.1 E-03 \\
2.2 E-04 \\
1.8 E-02 \\
1.3 E+00 \\
4.6 E+00 \\
2.8 E-05 \\
9.6 E-01 \\
4.5 E-05 \\
1.8 E-01 \\
4.5 E-01 \\
4.5 E-03 \\
1.7 E+00 \\
2.0 E-04 \\
5.9 E-01 \\
2.3 E+00 \\
7.8 E-01 \\
5.8 E-01 \\
3.2 E-01 \\
1.4 E-02 \\
3.9 E-02 \\
1.3 E-01 \\
7.2 E-01 \\
1.6 E+00 \\
1.7 E-01 \\
2.8 E+00 \\
6.6 E-01 \\
3.0 E-01 \\
1.6 E+00 \\
2.8 E-01 \\
5.1 E-03 \\
1.1 E-03 \\
2.0 E-02 \\
3.3 E-04 \\
1.6 E+00 \\
5.8 E-02 \\
1.0 E+00 \\
2.3 E+00 \\
1.1 E-03 \\
1.2 E+00 \\
1.1 E-04 \\
6.3 E-02 \\
6.8 E-02\end{array}$ \\
\hline
\end{tabular}




\begin{tabular}{|c|c|c|c|c|}
\hline $\begin{array}{l}\text { ELEMENT } \\
\text { SYMBOL }\end{array}$ & $\begin{array}{l}\text { ATOMIC } \\
\text { NO. (Z) }\end{array}$ & $\begin{array}{l}\text { ISOTOPE } \\
\text { MASS(A) }\end{array}$ & $\begin{array}{l}\text { ADULT } \\
\text { UC } / M * * 3\end{array}$ & $\begin{array}{l}\text { INFANT } \\
\text { UC } / M * * 3\end{array}$ \\
\hline 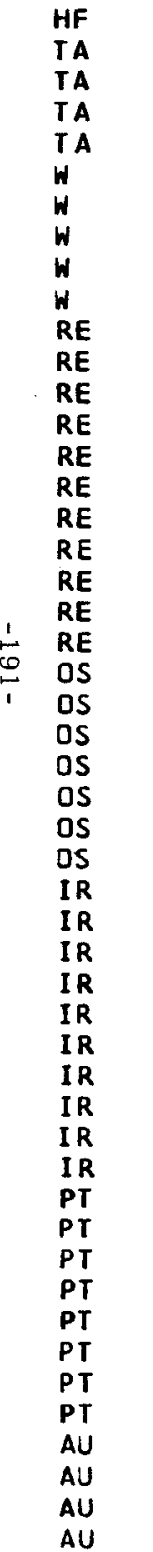 & $\begin{array}{l}72 \\
73 \\
73 \\
73 \\
73 \\
74 \\
74 \\
74 \\
74 \\
74 \\
75 \\
75 \\
75 \\
75 \\
75 \\
75 \\
75 \\
75 \\
75 \\
75 \\
75 \\
76 \\
76 \\
76 \\
76 \\
76 \\
76 \\
76 \\
77 \\
77 \\
77 \\
77 \\
77 \\
77 \\
77 \\
77 \\
77 \\
77 \\
78 \\
78 \\
78 \\
78 \\
78 \\
78 \\
78 \\
78 \\
79 \\
79 \\
79 \\
79\end{array}$ & $\begin{array}{l}182 \\
177 \\
179 \\
182 \\
183 \\
178 \\
181 \\
185 \\
187 \\
188 \\
180 \\
181 \\
182 \\
182 M \\
183 \\
184 \\
184 M \\
186 \\
187 \\
188 \\
189 \\
182 \\
183 \\
185 \\
191 \\
191 M \\
193 \\
194 \\
185 \\
186 \\
187 \\
188 \\
189 \\
190 \\
192 \\
192 M \\
193 M \\
194 \\
188 \\
190 \\
191 \\
192 \\
193 \\
193 M \\
195 M \\
197 \\
193 \\
194 \\
195 \\
196\end{array}$ & $\begin{array}{l}\text { 1. } 7 E-04 \\
7.8 E-01 \\
3.3 E-02 \\
7.3 E-03 \\
5.0 E-01 \\
2.7 E+00 \\
1.1 E+01 \\
1.1 E+01 \\
2.9 E+02 \\
1.1 E+00 \\
5.5 E+02 \\
6.1 E+02 \\
1.2 E+02 \\
1.4 E+03 \\
1.3 E+01 \\
8.5 E+00 \\
1.5 E+00 \\
2.6 E+02 \\
3.1 E+02 \\
1.3 E+03 \\
1.6 E+03 \\
1.2 E+01 \\
7.7 E+01 \\
1.2 E-01 \\
3.2 E+00 \\
1.9 E+02 \\
2.9 E+01 \\
1.9 E-03 \\
5.6 E+00 \\
3.3 E+00 \\
8.5 E+00 \\
5.5 E-01 \\
2.7 E-01 \\
7.5 E-02 \\
1.5 E-02 \\
1.3 E-04 \\
1.3 E+00 \\
3.3 E+00 \\
3.4 E-02 \\
3.5 E-05 \\
7.8 E-01 \\
4.2 E-05 \\
1.5 E-03 \\
1.1 E+00 \\
1.1 E+00 \\
9.5 E+00 \\
2.6 E+03 \\
2.2 E+02 \\
1.0 E+01 \\
1.1 E+02\end{array}$ & $\begin{array}{l}1.7 E-04 \\
4.7 E-01 \\
3.2 E-02 \\
7.2 E-03 \\
3.8 E-01 \\
2.6 E+00 \\
1.1 E+01 \\
1.1 E+01 \\
1.2 E+02 \\
1.0 E+0 D \\
2.0 E+02 \\
2.2 E+02 \\
7.6 E+01 \\
3.7 E+02 \\
1.3 E+01 \\
8.2 E+00 \\
1.5 E+00 \\
1.8 E+02 \\
3.1 E+02 \\
4.0 E+02 \\
6.4 E+02 \\
4.5 E+00 \\
1.9 E+01 \\
1.2 E-01 \\
2.9 E+0 J \\
5.4 E+01 \\
1.4 E+01 \\
1.9 E-03 \\
1.7 E+00 \\
9.7 E-01 \\
2.1 E+00 \\
2.9 E-01 \\
2.4 E-01 \\
6.7 E-02 \\
1.5 E-02 \\
1.3 E-04 \\
1.2 E+00 \\
1.1 E+00 \\
3.0 E-02 \\
3.5 E-05 \\
5.2 E-01 \\
4.2 E-05 \\
1.5 E-03 \\
8.5 E-01 \\
7.9 E-01 \\
3.4 E+00 \\
8.7 E+02 \\
1.1 E+02 \\
1.0 E+01 \\
8.6 E+01\end{array}$ \\
\hline
\end{tabular}

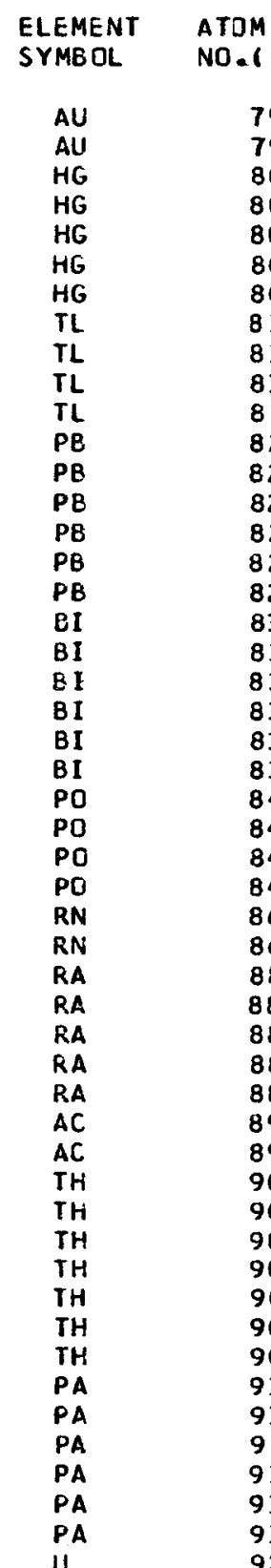

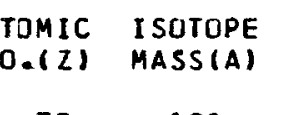

ADULT $U C / M * * 3$

3. $0 \mathrm{E}+02$ 7. $8 \mathrm{E}+02$

2. $1 E-03$

4. $4 \mathrm{E}-01$

$1.1 E+00$

2. $5 E+00$

4. $4 E-02$
2. $9 E-02$

3. $7 E-02$

3. $5 \mathrm{E}-03$

4. 5E-05

1. $7 E+02$

1. $7 E-02$
8. $0 E+01$

4. $0 E-03$

1. $2 E-01$

2. $7 E-03$

6. $5 \mathrm{E}-03$

1. $9 E-02$

1. $7 E-05$

8. 6E-06

4. $2 E-06$

4. $2 E-06$
1. $8 E-01$

1. $8 \mathrm{E}-01$

1. 2E-04

$5.6 E-03$

6. $9 E+03$

1. $9 E+02$

5. $9 E-01$

2. $3 E+00$

4. $5 E-01$

2. $4 E-03$

8. $4 E-02$

1. $3 E-04$

7. $9 E-07$

1. $9 \mathrm{E}-08$

1. 8E-09

1. $7 E-09$

2. $6 \mathrm{E}-05$

1. $5 \mathrm{E}-09$

4. $7 E-07$

1. $6 E+00$

8. $2 E-01$

3. $5 E-02$

7. OE- 05

8. 3E-01

2. $1 E-02$
INF ANT

$U C / M * * 3$

1. $9 E+02$

$5 \cdot 3 E+02$

2. $0 E-03$

2. 3E-01

6. $8 E-01$

1. $0 E+00$
$4.2 E-02$

$1.2 \mathrm{E}-02$

$2 \cdot 5 E-02$

3. $1 \mathrm{E}-03$

4. $5 \mathrm{E}-05$

5. $0 E+01$

4. $7 E+01$

4. $0 E-03$

1. $2 E-01$

2. 7E-03

$5.9 E-03$

$1.6 E-02$

1. $7 \mathrm{E}-05$

8.6E-06

$6.1 E-03$

4. $2 \mathrm{E}-06$
$1.5 \mathrm{E}-01$

1. $5 E-01$
5. $0 E-04$

1. 2E-04

5. 5E-03

2. $1 E+03$

1. $3 E+02$

5. $3 E-01$

1. $6 E+00$

4. $1 \mathrm{E}-01$

7. 3E-04

2. $4 E-03$
7. $3 E-02$

$1.3 E-04$

7.3E-07

1. $9 E-08$

1. $8 E-09$

1. $7 E-09$

1. $1 E-05$

$1.1 E-05$

1.

$6.2 \mathrm{E}-01$

4. 1 E- 01

3. $3 E-02$

7. $0 \mathrm{E}-05$

3. $9 E-01$

2. $0 E-02$

2. $5 \mathrm{E}-\mathrm{O} 2$ 
TABLE 98. UNIT-RAD CONTAMINATI ON FOR

SYMBOL

ATOMIC ISOTOPE
NO. (Z) MASS(A)

ADULT

$U C / M * * 3$

1. $7 E-01$

7. $1 E-05$

6. $T E-05$

6. $2 E-05$

6.1E-05

8. 5E-02

5. $8 \mathrm{E}-05$

2. $4 E+00$

3. $6 E-02$

2. $8 E-04$

1. $4 E-05$

3. 2E-01

1. $5 \mathrm{E}-05$

8. TE-02

. $1 E-02$

4. $4 E-04$

1. $1 E-02$

6. $6 E-05$

5. 5E-05

. $1 E-04$

5. 3E-05

4. $8 E-05$

. $7 E-02$

3. $3 E+00$

3. $2 E-01$

5. TE-05

1. $9 E+00$

5. $6 E-05$
GI TRACT

INFANT

$U C / M * * 3$

VIA FRESHWATER.

$.2 E-01$
$.8 E-05$

7.1E-05

6.7E-05

$6.2 E-05$

6. IE-05

$6.9 E-02$

$5.8 E-05$

$6.8 E-01$

2.7E-02

2.8E-0 4

1. $4 E-05$

1.2E-0

$1.5 \mathrm{E}-05$

1.5E-05

4. $3 E-02$

4. $4 \mathrm{E}-04$

1. OE-02

$6.6 E-05$

5. $6 E-0.5$

5.5E-05

1.1E-04

$5.3 E-05$

4. $7 E-05$

3.3E-02

B.4E-01

1. $9 E-01$

5. 7E-05

5. $9 E-01$

$5.6 E-05$

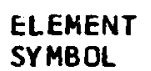

ATOMIC

ND. (Z) MASS(A)

$C M$
$C M$
$C M$
$C M$
$C M$
$C M$
$C M$
$C M$
$C M$
$B K$
$B K$
$B K$
$B K$
$B K$
$C F$
$C F$
$C F$
$C F$
$C F$
$C F$
$C F$
$E S$
$E S$
$E S$
$E S$
$E S$
$E S$
$F M$
$F M$
$F M$
$F M$
240
241

241

242

243

245

246

247

248

\begin{tabular}{|c|c|}
\hline $10 / 09 / 68$ & PAGE 40 \\
\hline $\begin{array}{l}\text { ADULT } \\
U C / M * * 3\end{array}$ & $\begin{array}{l}\text { INFANT } \\
\text { UC } / M * * 3\end{array}$ \\
\hline $\begin{array}{l}1.6 E-02 \\
1.2 E-02 \\
2.4 E-03 \\
6.7 E-05 \\
8.3 E-05 \\
5.0 E-05 \\
4.8 E-05 \\
4.5 E-05 \\
4.4 E-05 \\
9.9 E-02 \\
3.6 E-01 \\
4.5 E-05 \\
3.9 E-01 \\
1.1 E-03 \\
4.7 E-01 \\
9.6 E-04 \\
4.6 E-05 \\
1.0 E-04 \\
4.1 E-05 \\
3.2 E-04 \\
1.7 E-02 \\
4.1 E-01 \\
2.2 E-03 \\
1.6 E-02 \\
3.1 E-04 \\
3.5 E-01 \\
1.2 E-02 \\
3.0 E-01 \\
9.1 E-02 \\
9.4 E-01 \\
3.6 E-03\end{array}$ & $\begin{array}{l}1.5 E-02 \\
1.2 \mathrm{E}-02 \\
2.4 \mathrm{E}-03 \\
6.7 \mathrm{E}-05 \\
8.3 \mathrm{E}-05 \\
5.0 \mathrm{E}-05 \\
4.8 \mathrm{E}-05 \\
4.5 \mathrm{E}-05 \\
4.4 \mathrm{E}-05 \\
7.6 \mathrm{E}-02 \\
2.0 \mathrm{E}-01 \\
4.5 \mathrm{E}-05 \\
3.5 \mathrm{E}-01 \\
1.1 \mathrm{E}-03 \\
2.4 \mathrm{E}-01 \\
9.6 \mathrm{E}-04 \\
4.6 \mathrm{E}-05 \\
1.0 \mathrm{E}-04 \\
4.1 \mathrm{E}-05 \\
3.2 \mathrm{E}-04 \\
1.6 \mathrm{E}-02 \\
2.1 \mathrm{E}-01 \\
2.1 \mathrm{E}-03 \\
1.5 \mathrm{E}-02 \\
6.1 \mathrm{E}-04 \\
1.8 \mathrm{E}-01 \\
1.2 \mathrm{E}-02 \\
3.1 \mathrm{E}-01 \\
6.8 \mathrm{E}-02 \\
3.4 \mathrm{E}-01 \\
3.5 \mathrm{E}-03\end{array}$ \\
\hline
\end{tabular}


10/22/68: Insert after new "Foods" divider page.

\section{CONCENTRATION IN TERRESTRIAL FOODSTUFFS}

\section{$\underline{\mathrm{C}}_{\mathrm{P}}:$ Concentration in Plants}

The values listed in Table $10 \mathrm{~A}$ for $\mathrm{C}_{\mathrm{P}}$ are intended to approximate the average concentration in that portion of the human diet derived from plants. These $\mathrm{C}_{\mathrm{P}}$ values are not necessarily consistent $w$ ith the worst-case values derived in the Appendix. Although the concentrations are normally given as dry weight in the literature and are quoted as such in the Appendix, all values have been converted to wet weight in the table assuming the water concentration to be $75 \%$. The values were assigned according to the procedures and code numbers shown below.
Rules for estimating $\mathrm{C}_{\mathbf{P}}$

\section{Code \#}

1 The $C_{P}$ was assumed to be the average concentration in plants reported by Bowen (5306, 6105).

2 The $C_{P}$ was estimated from values reported in the literature.

3 The $\mathrm{C}_{\mathrm{P}}$ was estimated following the procedure described in the Appendix for actinium.

4 The $\mathrm{C}_{P}$ (of inert gases) was assumed to be the $\mathrm{C}_{A}$ for freshwater (Table 7B).

5 The $\mathrm{C}_{\mathrm{P}}$ was estimated following the procedures described in the Appendix for cerium.

99 The $C_{P}$ was estimated from collateral data as described in the Appendix 
$\begin{array}{llll}\text { ELEMENT ATOMIC } & \text { CONC. } \\ \text { SYMBOL } & \text { NO.(Z) } & C_{P}(P P M) & \text { CODE REFERENCE(S) }\end{array}$

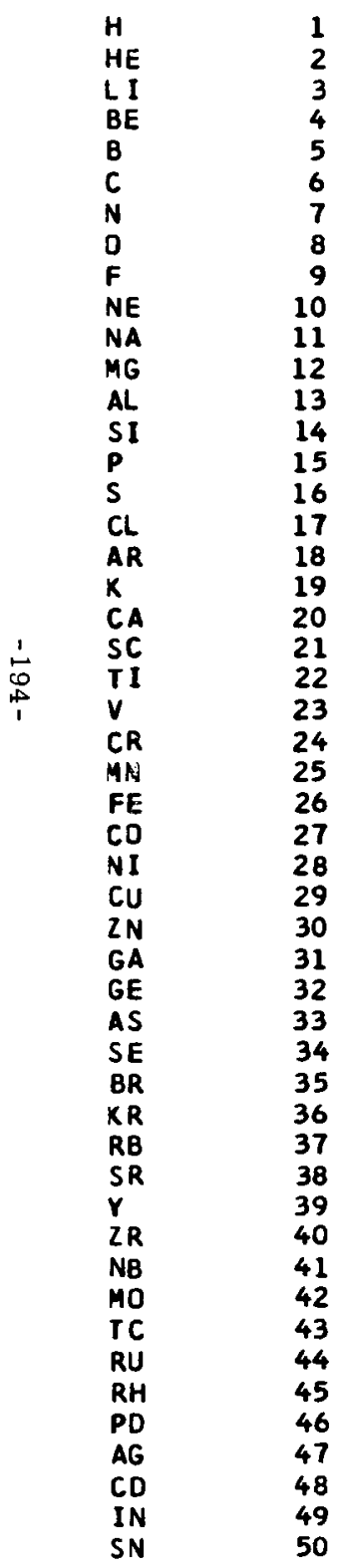

9.70E+04 15306

$5.00 E-06$

$2.50 \mathrm{E}-02$

$2.50 \mathrm{E}-03$

2. $50 E+00$

$1.10 E+05$

$7.50 E+03$

$7.60 E+05$

1. $30 E-01$

1. $40 E-04$

$3.30 E+02$

$8.00 E+02$

1. $30 E+01$

5. $00 E+01$

8. $80 E+02$

5. $00 E+02$

$5.00 E+02$

6. $00 E-01$

$5.00 E+03$

$5.00 E+02$

7. $50 \mathrm{E}-03$

$2.50 E-01$

1.30E-O1

2. $50 E+01$

$2.50 E+01$

$7.50 E-02$

7.50E-01

2. $50 E+00$

2. $00 E+01$

$7.50 E-03$

1. OOE 01

5. OOE-02

$1.30 E-01$

$3.80 E+00$

3. $00 E-04$

$1.30 E+01$

$5.00 E+00$

1.30E-01

5. OOE-02

5. 00E-02

2.50E-01

$2.50 E-14$

5. OOE-OS

1.30E-02

$5.00 E-03$

1.50E-02

1. $50 E-01$

2. 50E- 02

$2.50 E-02$
5. 0OE-02

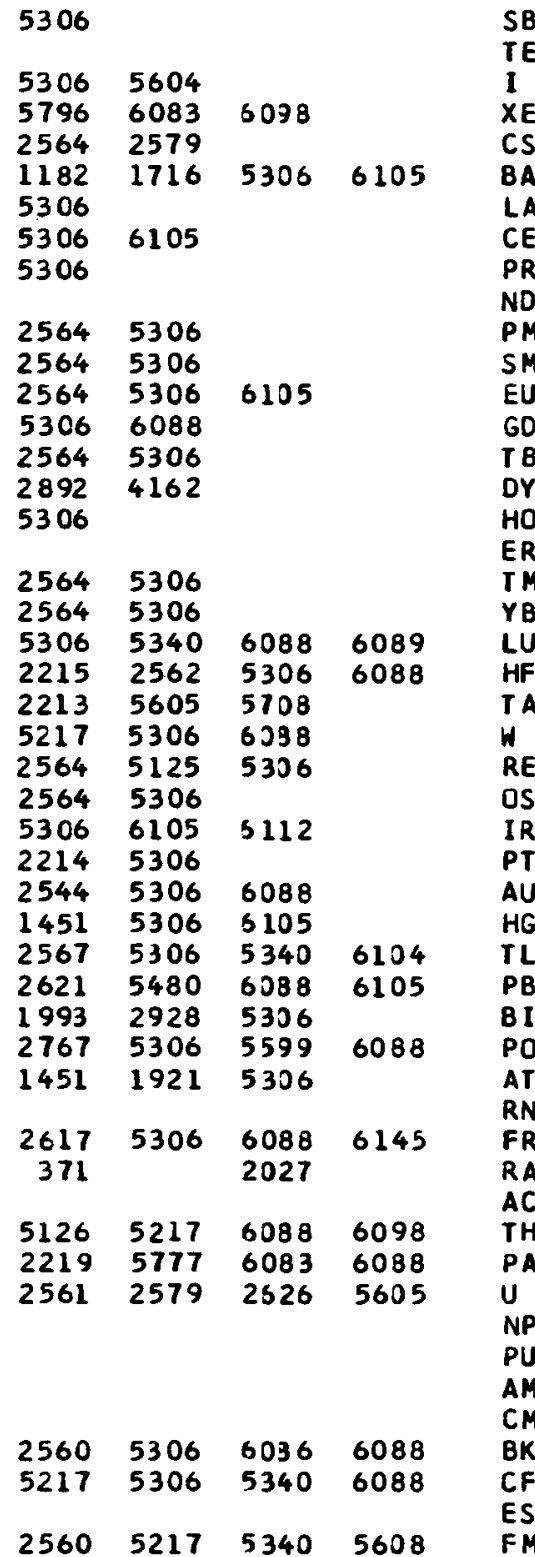

ELEMENT ATOMIC

SYMBOL NO. (2)

CONC.

CP (PPH) CODE

2.50E-02

$1.30 E-03$

$1.00 \mathrm{E}-01$

$1.00 E-04$

5. $00 E-02$

2. $50 E+00$

$1.00 E-01$

1. 30E-01

$1.70 E-02$

$6.00 E-02$

$2.50 E-21$

$1.60 E-02$

$2.50 E-03$

$1.10 E-02$

$1.80 E-03$

7. $50 E-03$

1. $80 E-03$

$2.00 E-03$

$1.80 E-03$

2. $00 E-03$

$1.80 E-03$

$1.00 E-03$

$6.30 E-03$

$1.80 E-02$

2.5OE- 04

5.00E-05

$1.30 E-04$

$5.00 E-03$

$2.50 E-04$

$3.80 E-03$

2.50E-02

$6.80 E-01$

1. $50 \mathrm{E}-02$

$1.00 E-11$

2.50E-20

3. $50 E-13$

$1.00 E-20$

$2.50 E-10$

2. $50 \mathrm{E}-13$

2.50E-02

$2.505-10$

2.5OE-O3

$2.50 \mathrm{E}-15$

2. $50 E-17$

$2.50 E-34$

$2.50 E-33$

$2.50 E-33$

2. $50 E-33$

$2.50 E-33$

2.5OE-33
REFERENCE (S)

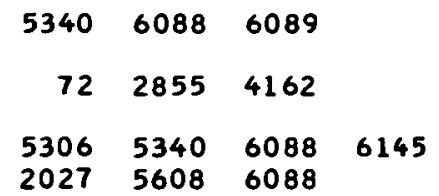

$\begin{array}{lll}5306 & 6088 & 6098\end{array}$

$6099 \quad 6243 \quad 6244$

$244 \quad 4947 \quad 5306$

60956097

$\begin{array}{llll}5306 & 5605 & 6095 & 6097\end{array}$ 
10. CONCENTRATION IN TERRESTRIAL FOODSTUFFS (contd)

$\mathrm{C}_{\text {meat }}:$ Concentration in Meat

The values of $\mathrm{C}_{\text {meat }}$ listed in Table $10 \mathrm{~B}$ give the average concentrations in meat.

The values were assigned according to the procedures and code numbers shown below.

Rules for estimating $\mathrm{C}_{\text {meat }}$

\section{Code \#}

1 The $\mathrm{C}_{\text {meat }}$ was estimated from the concentrations reported in the literature for meats (beef, pork, mutton, fowl, etc.).
2 The $\mathrm{C}_{\text {meat }}$ was assumed to be concentration in bovine heart.

3 The $\mathrm{C}_{\text {meat }}$ was assumed to be $\mathrm{C}_{\mathrm{B}}^{\mathrm{TB}}$.

4 The $\mathrm{C}_{\text {meat }}$ was assumed to be $100 \mathrm{C}_{\mathrm{S}}$.

5 The $\mathrm{C}_{\text {meat }}$ (of inert gases) was assumed to be $\mathrm{C}_{\mathrm{A}}$ for freshwater (Table 7B).

6 The $\mathrm{C}_{\text {meat }}$ was estimated following the procedure described in the Appendix for yttrium.

99 The $\mathrm{C}_{\text {meat }}$ was estimated from collateral data as described in the Appendix. 


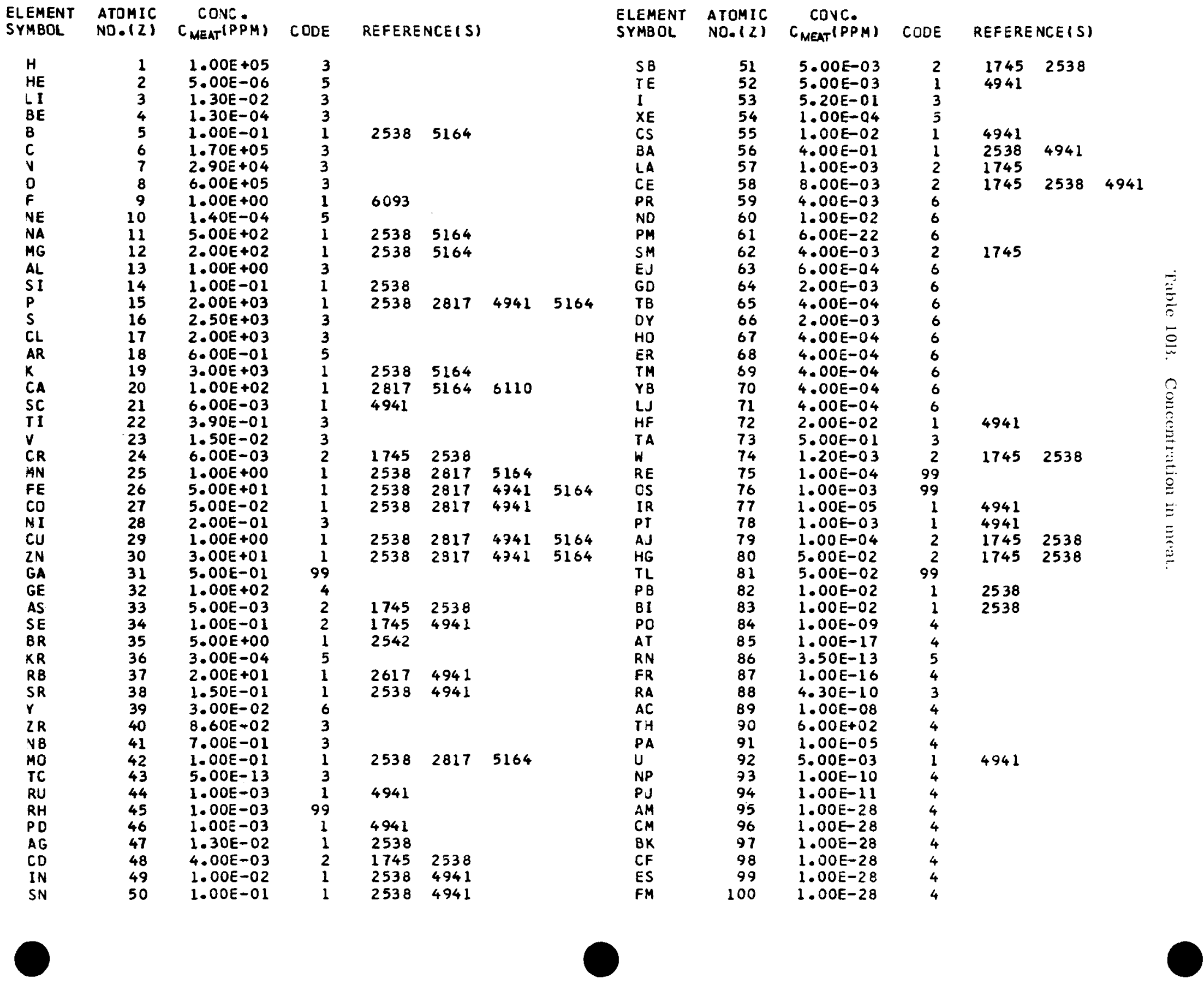




\section{CONCENTRATIONS IN SEAWATER FOODSTUFFS}

The concentrations in seawater foodstuffs are meant to be representative of the edible portions. Whenever one species tended to have a higher concentration than other species, this value was selected. Usually this occurred when oysters (which were higher) were compared with other invertebrates. The concentrations were assigned according to the procedures and code numbers shown below.

Rules for estimating concentrations in seawater foodstuffs

\section{Code \#}

1 The value was taken from the measured values in the literature.

2 The inert gas concentration was assumed to be the same as in seawater.

3 The concentration was assumed to be equal to $10^{5}\left(\mathrm{C}_{\mathrm{A}}\right)$.
4 The concentrations for scandium, yttrium, lanthanum and the rare earths were based upon a composite of all the data on these elements as discussed in the Appendix under cerium.

5 The concentration was determined by multiplying the seawater concentration by the concentration factors reported in the literature.

8 The concentrations for the platinum metals were based upon ruthenium as discussed in the Appendix under rhodium.

9 The concentrations for the actinide elements were based upon plutonium as discussed in the Appendix under actinium.

99 The concentration was estimated from collateral data as described in the Appendix. 
CONC:

ATOMIC
NO. $(2)$

(PPM)

CODE

REFERENCE (S)

\section{$1.00 E+05$} 5. $00 \mathrm{E}-06$

$6.00 E-01$

$1.00 E-03$

$5.00 E+04$

$5.00 E+03$

8. $20 E+05$

2. $00 E+00$

$1.40 E-04$

$6.00 E+03$

1. $00 E+03$

$3.00 E+02$

2. $00 E+02$

1. $50 E+02$

4. $00 E+02$

$6.00 E+03$

$6.00 E-01$

$5.50 E+03$

$2.00 E+03$

5. $00 E-01$

2. $00 E+00$

2. $00 E-01$

$1.00 E-01$

2. $00 E+01$

5. $00 E+02$

1. OOE -01

5. $00 \mathrm{E}-01$

3. $00 E+00$

$1.00 E+01$

5. $00 E-02$

$2.00 E-02$

$1.00 E+00$

$8.00 E-01$

1. $00 E+02$

3. $00 E-04$

2. $00 E+00$

1. $00 E+02$

4. $00 E+00$

. $00 E+00$

$1.00 E-02$

$1.00 E-01$

4. $00 E-12$

$2.00 E-03$

2. $00 \mathrm{E}-03$

2. $00 E-03$

$6.00 E-02$

$1.00 E-01$

$1.00 E-02$

$1.00 E-01$
ELEMENT ATOMIC

YYMBOL CONC

$\begin{array}{lrrrrrrrr}\text { SB } & 51 & 5.00 E+01 & 3 & & & & \\ \text { IE } & 52 & 1.00 E+00 & 3 & & & & \\ \text { I } & 53 & 2.00 E+02 & 1 & 6003 & 5998 & & \\ \text { XE } & 54 & 1.00 E-04 & 2 & & & & \\ \text { CS } & 55 & 1.00 E-02 & 1 & 1930 & 6012 & & \\ \text { BA } & 56 & 5.00 E+00 & 1 & 1848 & 6011 & 1657 & \\ \text { LA } & 57 & 3.00 E+00 & 4 & & & & \\ \text { CE } & 58 & 4.00 E+00 & 99 & 5335 & 506 & 2475 & 2715\end{array}$

$5.00 E-01$

2. $00 E+00$

$4.00 \mathrm{E}-20$

5. 00E-01

8.00E-02

$3.00 E-01$

5. $00 E-02$

2. OOE-01

$5.00 E-02$

$6.00 E-02$

$5.00 E-02$

$6.00 E-02$

$5.00 E-02$

$6.00 E-02$

. 00 E

3. $00 \mathrm{E}-03$

3. $00 \mathrm{E}-03$

2.00E-03

2. $00 \mathrm{E}-03$

$2.00 \mathrm{E}-05$

2. $00 E-02$

$1.0 O E-03$

3. 00 E-02

$1.00 E-0$

$1.00 E+O 0$

$2.00 E+01$

8.00 E- 11

4. $00 \mathrm{E}-18$

$1.40 E-14$

3. OOE- 22

$1.00 \leq-08$

$7.00 E-06$

3. OOE- 02

7.0OE-03

$3.00 E+00$

7. $00 \mathrm{E}-08$

7. OOE-09

$7.00 E-26$

7. $00 E-26$

$7.00 E-26$

$7.00 \mathrm{E}-26$

7. $00 \mathrm{E}-26$

7. OOE-26

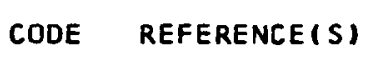

CODE REFERENCE (S)

6012

$1989 \quad 5998$

$1299 \quad 6012$ 
TABLE 11B. CONCENTRATION IN SEAWATER INVERTEBRATES

$10 / 09 / 68$

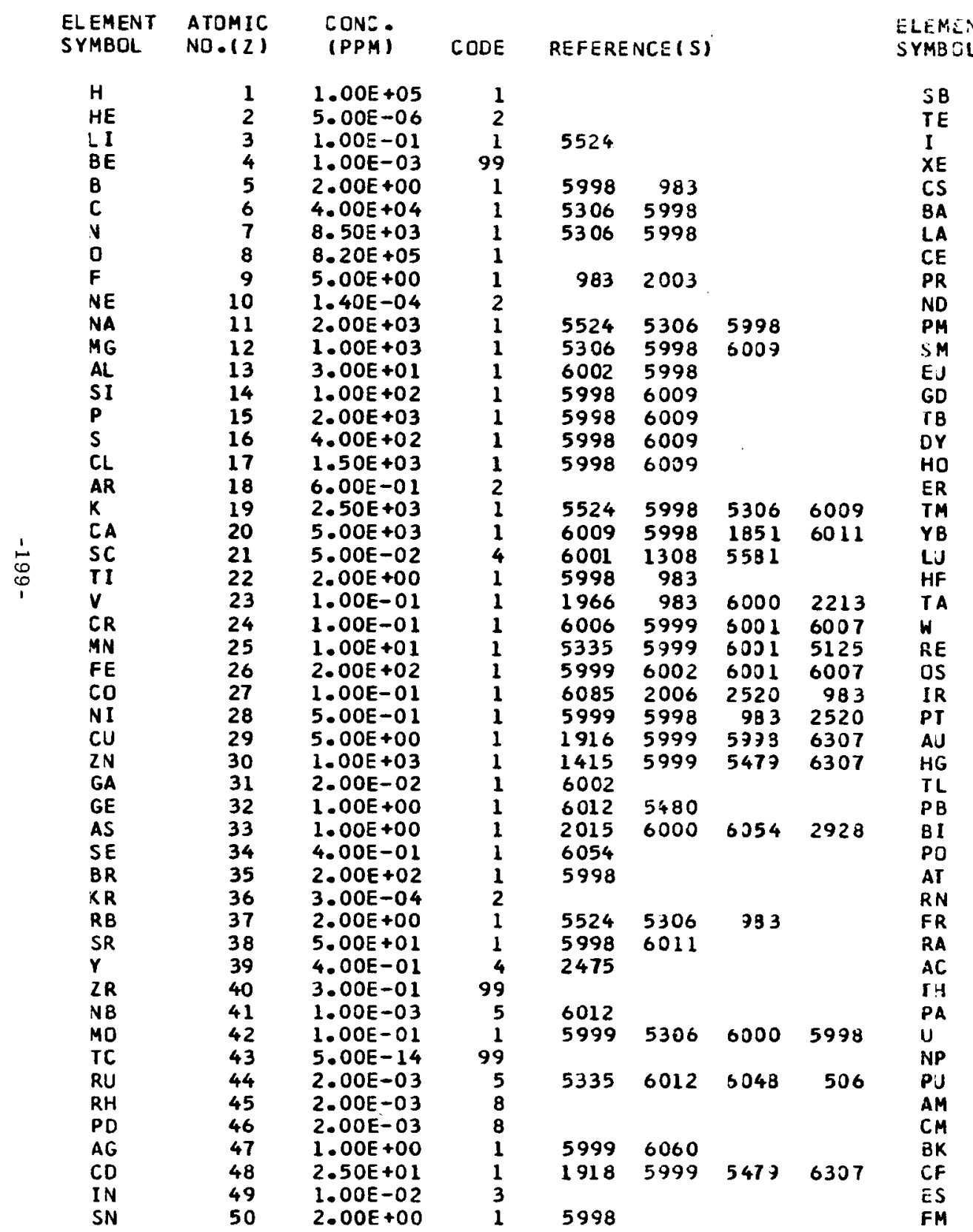

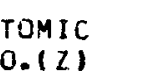

518.0

$\begin{array}{lrllllll}\text { SB } & 51 & 8.00 E+00 & 1 & 5999 & & 6060 & \\ \text { TE } & 52 & 1.00 E+00 & 3 & 5243 & & & \\ \text { I } & 53 & 2.50 E+00 & 1 & 5998 & 2843 & & \\ \text { XE } & 54 & 1.00 E-04 & 2 & & & & \\ \text { CS } & 55 & 1.00 E-02 & 1 & 5524 & 6012 & & \\ \text { BA } & 56 & 2.00 E+00 & 1 & 6011 & 1965 & & \\ \text { LA } & 57 & 3.00 E-01 & 4 & & & & \\ \text { CE } & 58 & 4.00 E-01 & 99 & 5335 & 2715 & 2475 & 6026\end{array}$

$5.00 E-02$

2. DOE-OI

$4.00 E-21$

$5.00 E-02$

8.00 E-0 3

3.00E-02

5. OOE 03

2. 00 E-02

$5.00 E-03$

$6.00 E-03$

$5.00 E-03$

$6.00 E-03$

$5.00 E-03$

6. 00 E -03

$2.00 E-04$

$3.00 E-03$

5. OOE-04

2. 00 E- 03

2. $00 E-05$

2. OOE-O2

1. $00 \mathrm{E}-03$

3. $00 E+00$

$1.50 E-01$

1. $00 E+00$

$2.00 E+01$

4. $00 \mathrm{E}-11$

$5.00 E-20$

$1.40 E-14$

2. $00 \mathrm{E}-21$

l. 00 E 08

$2.00 E-06$

$9.008-03$

$2.00 E-03$

$1.0 O E+O D$

$2.00 E-08$

2.00E-09

2. $00 E-26$

$2.00 E-26$

2. $00 E-26$

$2.00 \mathrm{E}-26$

$2.00 \mathrm{E}-26$

$2.00 \mathrm{E}-26$
5581

CODE REFERENCE(S)

E

\begin{tabular}{ll}
$H$ & $\stackrel{0}{\Sigma}$ \\
\multirow{2}{0}{} & $?$ \\
$\frac{0}{\sigma}$ & + \\
$ت$ & $\infty$
\end{tabular}




\begin{tabular}{|c|c|c|c|c|c|c|c|c|c|c|c|c|c|c|c|c|}
\hline $\begin{array}{l}\text { ELEMENT } \\
\text { SYMBOL }\end{array}$ & $\begin{array}{l}\text { ATOMIC } \\
\text { NO. }(Z)\end{array}$ & $\begin{array}{l}\text { CONC } \\
\text { (PPM) }\end{array}$ & CODE & REFERE & $N C E(S)$ & & & $\begin{array}{l}\text { ELEMENT } \\
\text { SYMBOL }\end{array}$ & $\begin{array}{l}\text { ATOMIC } \\
\text { NO. }(Z)\end{array}$ & $\begin{array}{l}\text { CONC. } \\
\text { (PPM) }\end{array}$ & CODE & REFERE & $\operatorname{NCE}(S)$ & & & \\
\hline $\begin{array}{l}H \\
H E \\
L I \\
B E \\
B \\
C \\
N \\
D \\
F \\
N E \\
N A \\
M G \\
A L \\
S I \\
P \\
S \\
C L \\
A R \\
K \\
C A \\
S C \\
r I \\
V \\
C R \\
M N \\
F E \\
C D \\
N I \\
C U \\
2 N \\
G A \\
G E \\
A S \\
S E \\
B R \\
K R \\
R B \\
S R \\
Y \\
Z R \\
N B \\
M O \\
T C \\
R U \\
R H \\
P D \\
A G \\
C D \\
I N \\
S N\end{array}$ & $\begin{array}{l}1 \\
2 \\
3 \\
4 \\
5 \\
6 \\
7 \\
8 \\
9 \\
10 \\
11 \\
12 \\
13 \\
14 \\
15 \\
16 \\
17 \\
18 \\
19 \\
20 \\
21 \\
22 \\
23 \\
24 \\
25 \\
26 \\
27 \\
28 \\
29 \\
30 \\
31 \\
32 \\
33 \\
34 \\
35 \\
36 \\
37 \\
38 \\
39 \\
40 \\
41 \\
42 \\
43 \\
44 \\
45 \\
46 \\
47 \\
48 \\
49 \\
50\end{array}$ & $\begin{array}{l}1.00 E+05 \\
5.00 E-06 \\
1.00 E-01 \\
1.00 E-03 \\
3.00 E+00 \\
5.00 E+04 \\
3.00 E+04 \\
8.20 E+05 \\
5.00 E+00 \\
1.40 E-04 \\
7.00 E+02 \\
1.00 E+03 \\
5.00 E+00 \\
7.00 E+00 \\
2.00 E+03 \\
1.50 E+03 \\
1.00 E+03 \\
6.00 E-01 \\
4.00 E+03 \\
6.00 E+02 \\
5.00 E-03 \\
1.00 E+00 \\
2.00 E-02 \\
2.00 E-02 \\
6.00 E-01 \\
3.00 E+01 \\
5.00 E-02 \\
2.00 E-01 \\
2.00 E+00 \\
2.00 E+01 \\
1.00 E-02 \\
2.00 E-01 \\
1.00 E+00 \\
4.00 E-01 \\
2.00 E+02 \\
3.00 E-04 \\
1.00 E+00 \\
4.00 E+00 \\
4.00 E-02 \\
3.00 E-02 \\
3.00 E-01 \\
1.00 E-01 \\
1.00 E-14 \\
1.00 E-04 \\
1.00 E-04 \\
1.00 E-04 \\
1.00 E+00 \\
3.00 E-01 \\
1.00 E-02 \\
3.00 E+00\end{array}$ & $\begin{array}{r}1 \\
2 \\
1 \\
99 \\
1 \\
1 \\
1 \\
1 \\
1 \\
2 \\
1 \\
1 \\
1 \\
1 \\
1 \\
1 \\
1 \\
2 \\
1 \\
1 \\
4 \\
1 \\
1 \\
1 \\
1 \\
1 \\
1 \\
1 \\
1 \\
1 \\
1 \\
1 \\
1 \\
1 \\
99 \\
2 \\
1 \\
1 \\
4 \\
99 \\
1 \\
1 \\
99 \\
5 \\
8 \\
8 \\
1 \\
1 \\
3 \\
1\end{array}$ & $\begin{array}{r}2702 \\
5306 \\
1307 \\
983 \\
5524 \\
1307 \\
5306 \\
5306 \\
2702 \\
5998 \\
5998 \\
1405 \\
5998 \\
6060 \\
1935 \\
2702 \\
983 \\
1935 \\
5236 \\
2006 \\
2702 \\
2702 \\
2716 \\
1935 \\
1935 \\
6000 \\
6054 \\
\\
5524 \\
2702 \\
6012 \\
2219 \\
6000 \\
5335\end{array}$ & $\begin{array}{l}5306 \\
5998 \\
2702 \\
5998 \\
5306 \\
\\
5306 \\
\\
5998 \\
2702 \\
2215 \\
5306 \\
6007 \\
2702 \\
2702 \\
5306 \\
5998 \\
5998 \\
2702 \\
\\
6012 \\
5998 \\
6353\end{array}$ & $\begin{array}{r}1766 \\
2752 \\
5125 \\
6007 \\
6085 \\
983 \\
983 \\
5479 \\
\\
5480 \\
6054\end{array}$ & $\begin{array}{l}2213 \\
6006 \\
1405 \\
6307 \\
2520 \\
2520 \\
6307 \\
6307 \\
2928\end{array}$ & $\begin{array}{l}\text { SB } \\
T E \\
I \\
X E \\
C S \\
B A \\
\text { LA } \\
C E \\
P R \\
\text { ND } \\
P M \\
\text { SM } \\
E U \\
\text { GD } \\
T B \\
\text { OY } \\
H D \\
E R \\
T M \\
Y B \\
\text { LU } \\
H F \\
T A \\
W \\
R E \\
O S \\
I R \\
P T \\
A U \\
H S \\
T L \\
P B \\
B I \\
P O \\
A T \\
R N \\
F R \\
R A \\
A C \\
\text { TH } \\
P A \\
U \\
N P \\
P U \\
A M \\
C M \\
B K \\
C F \\
E S \\
F M\end{array}$ & $\begin{array}{l}51 \\
52 \\
53 \\
54 \\
55 \\
56 \\
57 \\
58 \\
59 \\
60 \\
61 \\
62 \\
63 \\
64 \\
65 \\
66 \\
67 \\
68 \\
69 \\
70 \\
71 \\
72 \\
73 \\
74 \\
75 \\
76 \\
77 \\
78 \\
79 \\
80 \\
81 \\
82 \\
83 \\
84 \\
85 \\
86 \\
87 \\
88 \\
89 \\
90 \\
91 \\
92 \\
93 \\
94 \\
95 \\
96 \\
97 \\
98 \\
99 \\
100\end{array}$ & $\begin{array}{l}2.00 E-02 \\
1.00 E+00 \\
5.00 E-01 \\
1.00 E-04 \\
1.50 E-02 \\
1.00 E-01 \\
3.00 E-02 \\
4.00 E-02 \\
5.00 E-03 \\
2.00 E-02 \\
4.00 E-22 \\
5.00 E-03 \\
8.00 E-04 \\
3.00 E-03 \\
5.00 E-04 \\
2.00 E-03 \\
5.00 E-04 \\
6.00 E-04 \\
5.00 E-04 \\
6.00 E-04 \\
5.00 E-04 \\
6.00 E-04 \\
2.00 E-04 \\
3.00 E-03 \\
4.00 E-05 \\
1.00 E-04 \\
1.00 E-06 \\
1.00 E-03 \\
1.00 E-03 \\
5.00 E-02 \\
1.00 E-01 \\
3.00 E-01 \\
3.00 E-03 \\
4.00 E-11 \\
1.00 E-20 \\
1.40 E-14 \\
3.00 E-21 \\
5.00 E-09 \\
7.00 E-08 \\
3.00 E-04 \\
7.00 E-05 \\
3.00 E-02 \\
7.00 E-10 \\
7.00 E-11 \\
7.00 E-28 \\
7.00 E-28 \\
7.00 E-28 \\
7.00 E-28 \\
7.00 E-28 \\
7.00 E-28\end{array}$ & $\begin{array}{r}1 \\
3 \\
1 \\
2 \\
1 \\
1 \\
4 \\
99 \\
4 \\
4 \\
4 \\
4 \\
4 \\
4 \\
4 \\
4 \\
4 \\
4 \\
4 \\
4 \\
4 \\
99 \\
99 \\
1 \\
1 \\
8 \\
8 \\
8 \\
1 \\
1 \\
1 \\
1 \\
1 \\
99 \\
99 \\
2 \\
99 \\
1 \\
9 \\
9 \\
9 \\
9 \\
9 \\
5 \\
9 \\
9 \\
9 \\
9 \\
9 \\
9\end{array}$ & $\begin{array}{l}6000 \\
5998 \\
6054 \\
2702 \\
1935\end{array}$ & $\begin{array}{l}6060 \\
2843 \\
2151\end{array}$ & $\begin{array}{l}6012 \\
1935\end{array}$ & 6307 & 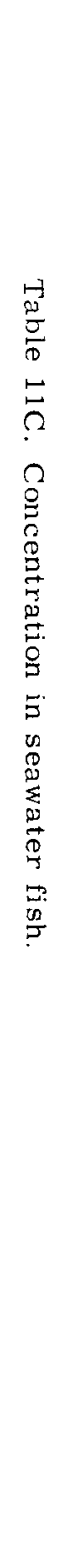 \\
\hline
\end{tabular}




\section{CONCENTRATION IN FRESHWATER FOODSTUFFS}

The concentrations in freshwater foodstuffs are meant to be representative of the edible portions. Whenever one species tended to have a higher concentration than other species, this value was selected. Usually this occurred when mollusks (which were higher) were compared with other invertebrates. The concentrations were assigned according to the procedures and code numbers shown below.

Rules for estimating concentrations in freshwater foodstuffs

\section{Code \#}

1 The value was taken from the measured values in the literature.

2 The inert gas concentration was assumed to be the same as in seawater.

3 The concentration was assumed to be equal to $10^{5}\left(\mathrm{C}_{\mathrm{A}}\right)$.

4 The concentrations for scandium, yttrium, lanthanum and the rare earths were based upon a composite of all the data on these elements as discussed in the Appendix under cerium.

5 The concentration was determined by multiplying the freshwater concentration by the concentration factors reported in the literature.

6 The ratio (foodstuff $/ \mathrm{C}_{A}$ ) was assumed to be the same in freshwater as in seawater.

7 The concentration in freshwater foodstuffs was assumed to be equal to the concentration in seawater foodstuffs.

8 The concentrations for the platinum metals were based upon ruthenium as discussed in the Appendix under rhodium.

9 The concentrations for the actinide elements were based upon plutonium as discussed in the Appendix under actinium.

99 The concentration was estimated from collateral data as described in the Appendix. 
TABLE 12A. CONCENTRATION IN FRESHWATER PLANTS

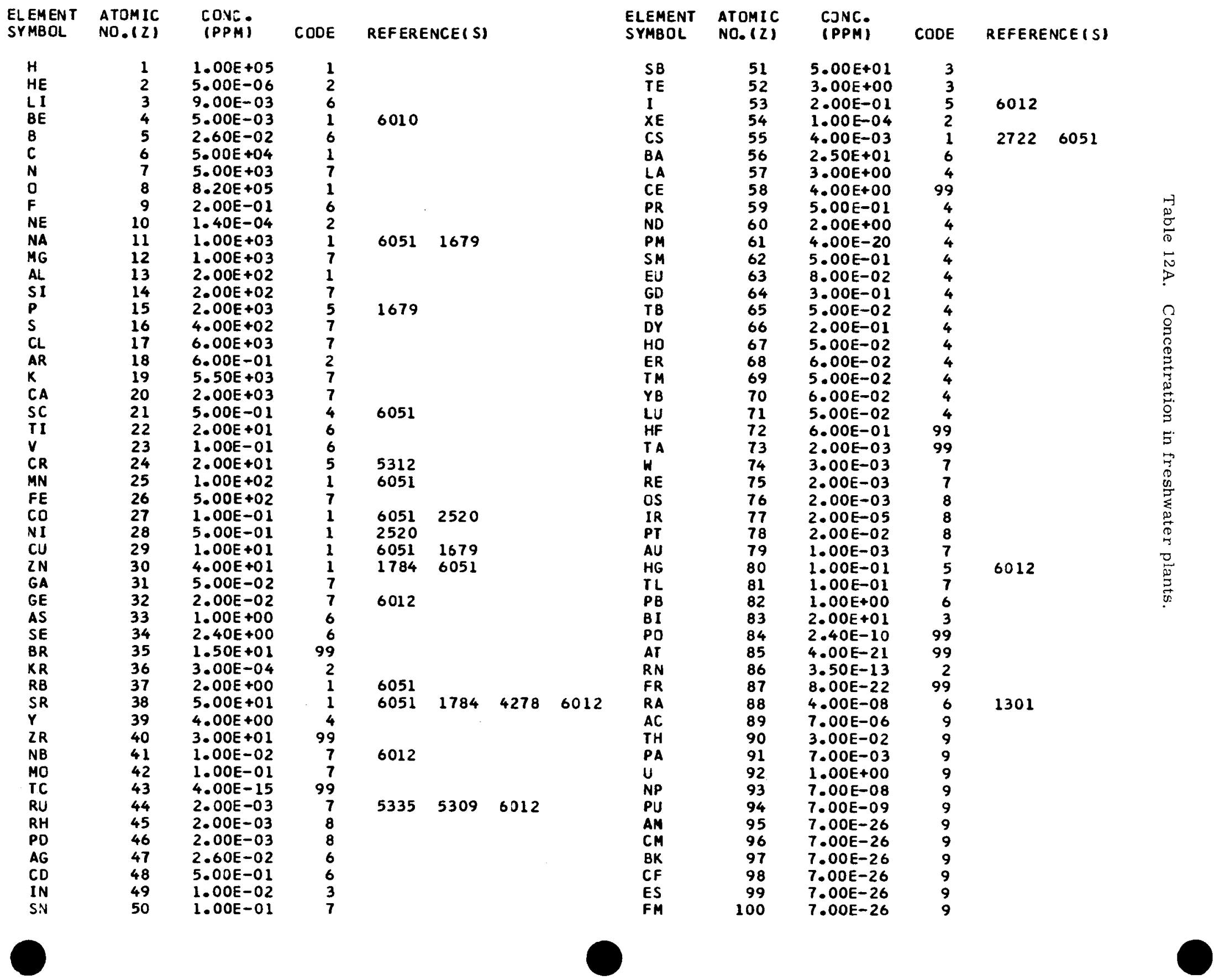


TABLE 128. CONCENTRATION IN FRESHWATER INVERTEBRATES

$10 / 09 / 68$

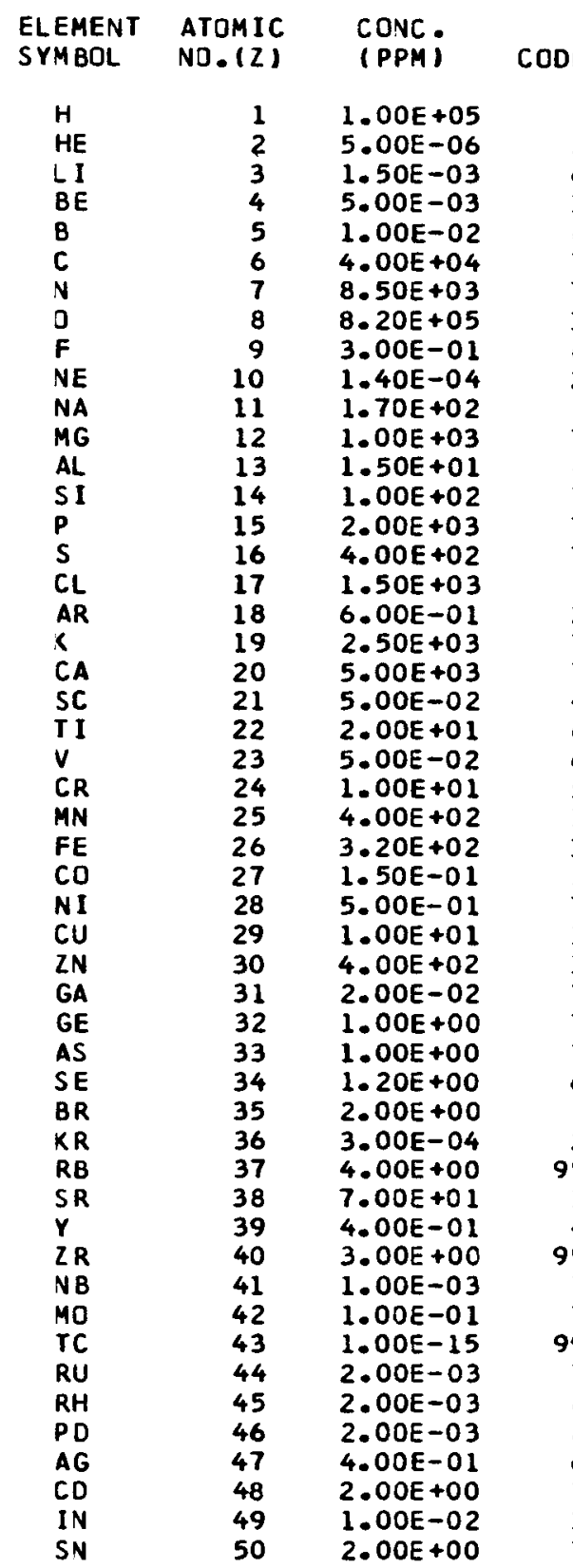

REFERENCE ( $S$ )

6010

6051

$6051 \quad 1308$

$1308 \quad 5312$

$6051 \quad 1308$

60511308

$\begin{array}{ll}6051 & 1308 \\ 6051 & 1308\end{array}$

$6051 \quad 1679$

$1308 \quad 6051 \quad 5743$

5998

$4278 \quad 6051 \quad 1308$

6012

$5309 \quad 6012$

1308

$1308 \quad 5999$

$\begin{array}{lccr}\text { ELEMENT } & \text { ATOMIC } & \text { CONCE } & \\ \text { SYMBOL } & \text { NO.1Z1 } & \text { (PPM) } & \text { CODE } \\ \text { SB } & & & \\ \text { TE } & 51 & 8.00 E+00 & 7 \\ \text { I } & 52 & 3.00 E+00 & 3 \\ \text { XE } & 53 & 5.00 E-02 & 5 \\ \text { CS } & 54 & 1.00 E-04 & 2 \\ \text { BA } & 55 & 2.00 E-02 & 5 \\ \text { LA } & 56 & 1.00 E+01 & 6 \\ \text { CE } & 57 & 3.00 E-01 & 4 \\ \text { PR } & 58 & 4.00 E-01 & 99 \\ \text { ND } & 59 & 5.00 E-02 & 4 \\ \text { PM } & 60 & 2.00 E-01 & 4 \\ \text { SM } & 61 & 4.00 E-21 & 4 \\ \text { EJ } & 62 & 5.00 E-02 & 4 \\ \text { GD } & 63 & 8.00 E-03 & 4 \\ \text { TB } & 64 & 3.00 E-02 & 4 \\ \text { DY } & 65 & 5.00 E-03 & 4 \\ \text { HO } & 66 & 2.00 E-02 & 4 \\ \text { ER } & 67 & 5.00 E-03 & 4 \\ \text { TM } & 68 & 6.00 E-03 & 4 \\ \text { YB } & 69 & 5.00 E-03 & 4 \\ \text { LJ } & 70 & 6.00 E-03 & 4 \\ \text { HF } & 71 & 5.00 E-03 & 4 \\ \text { TA } & 72 & 6.00 E-02 & 99 \\ \text { W } & 73 & 2.00 E-04 & 99 \\ \text { RE } & 74 & 3.00 E-03 & 7 \\ \text { OS } & 75 & 5.00 E-04 & 7 \\ \text { IR } & 76 & 2.00 E-03 & 8 \\ \text { PT } & 77 & 2.00 E-05 & 8 \\ \text { AU } & 78 & 2.00 E-02 & 8 \\ \text { HG } & 79 & 1.50 E-03 & 1 \\ \text { TL } & 80 & 1.00 E+01 & 99 \\ \text { PB } & 81 & 1.50 E-01 & 7 \\ \text { BI } & 83 & 1.00 E+00 & 6 \\ \text { PO } & 84 & 1.00 E+01 & 3 \\ \text { AT } & 85 & 1.00 E-10 & 99 \\ \text { RN } & 86 & 3.50 E-13 & 99 \\ \text { FR } & 87 & 4.00 E-21 & 99 \\ \text { RA } & 88 & 2.00 E-08 & 6 \\ \text { AC } & 89 & 2.00 E-06 & 9 \\ \text { TH } & 90 & 7.50 E-03 & 1 \\ \text { PA } & 91 & 2.00 E-03 & 9 \\ \text { U } & 92 & 3.00 E-01 & 9 \\ \text { NP } & 93 & 2.00 E-08 & 9 \\ \text { PJ } & 94 & 2.00 E-09 & 9 \\ \text { AM } & 95 & 2.00 E-26 & 9 \\ \text { CM } & 96 & 2.00 E-26 & 9 \\ \text { BK } & 97 & 2.00 E-26 & 9 \\ \text { CF } & 98 & 2.00 E-26 & 9 \\ \text { ES } & 99 & 2.00 E-26 & 9 \\ \text { FM } & 100 & 2.00 E-26 & 9 \\ & & & \end{array}$

REFERENCE (S)

6012

2722 
TABLE 12C. CDNCENTRATION IN FRESHWATER FISH

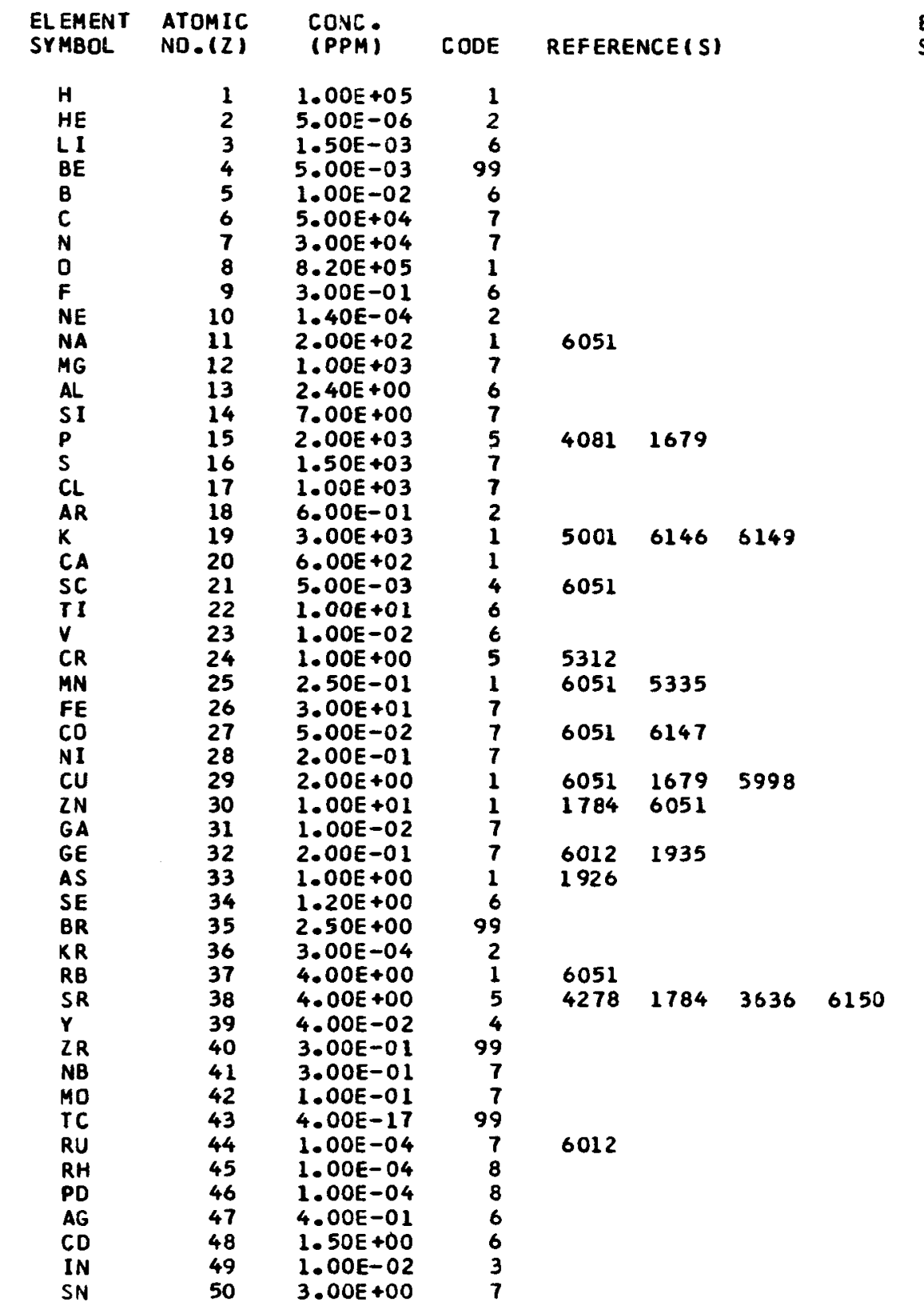

ATOMIC

CONC.

\subsection{E-02} $3.00 E+00$ $2.00 E-03$ $1.00 \mathrm{E}-04$
1.00 $1.00 E-04$ 2.00E-02 $5.00 E-01$ $3.00 E-02$ 4.00E-02 $5.00 \mathrm{E}-03$ 2. OOE-02 4.00E-22 $5.00 E-03$ $8.00 E-04$ $3.00 E-03$ $3.00 E-03$ $5.00 E-04$ 2.00E-03 5. $00 \mathrm{E}-04$ 6.00E-04 5.00E-04 $6.00 E-04$ 5.0OE-04 $6.00 E-03$ $6.00 E-02$ $3.00 E-03$ $4.00 E-05$ 4.00E-05 $1.00 \mathrm{E}-04$ $1.00 E-06$ $1.00 E-03$ 1. OOE-03 $1.00 \mathrm{E}-01$ 1. $00 E-01$ 3. $00 E-01$ 3. $00 \mathrm{E}-03$ $1.20 \mathrm{E}-10$ $4.00 E-23$ 3. $30 \mathrm{E}-13$ 3. 50E-13 4.00E-21 $2.00 E-08$ 7.00E-08 3. $00 \mathrm{E}-04$ 7. $00 \mathrm{EE}-05$ 1.00E-02 7.00 E-07 7. OOE-11 $7.00 E-28$ $7.00 E-28$ $7.00 E-28$ $7.00 E-28$ $7.0 O E-28$
$7.0 O E-28$ $7.00 E-28$
$7.00 E-28$
CODE REFERENCE (S)

\section{2}

6051 2722 $3636 \quad 6146$ 


\section{Appendix: Estimates of Input Parameters Using Collateral Data}

\section{INTRODUCTION}

Collateral data on the behavior of elements in the biological exchangeable pool have been utilized to make conservative estimates of input parameters that are more reasonable than the initially assumed worst-case values. This approach was attempted whenever a worst-case assumption led to a $\mathrm{m}^{2}$-rad estimate which was within the first three orders of magnitude (5574). It is important to note that this procedure was carried out only when sufficient data were available so that the estimates could be made with assurance, and that the resulting estimates, while lower and more reasonable than the initial ones, are still meant to be values which do not compromise the conservative aspects of the analysis. 


\section{General Equations}

The basic assumptions are (1) that the daily diet is constant, (2) that there is a steady state with respect to uptake and turnover, (3) that the turnover rate is firstorder so that retention can be described by a simple exponential function, and (4) that rates of uptake and turnover are basically similar in man and in other mammals that contribute to his diet or serve as subjects in experiments.

In the steady state the daily rate of ingestion $I$ is related to the concentration in an organ by Eq. (1) and to the concentration in the total body by Eq. (2):

$$
\begin{aligned}
& f_{B} I=\frac{0.693}{T_{B}} m_{B} \\
& f_{1} I=\frac{0.693}{T_{B}^{T B}} m^{T B} C_{B}^{T B}
\end{aligned}
$$

Eqs. (1) and (2) can be rearranged a number of ways:

$$
\begin{aligned}
& I=\frac{0.693}{f_{B} T_{B}} m_{B} \\
& I=\frac{0.693}{f_{1} T_{B}^{T B}} m^{T B} C_{B}^{T B} \\
& f_{B}=\frac{0.693}{\mathrm{IT}_{B}} \mathrm{mC}_{B} \\
& f_{1}=\frac{0.693}{\mathrm{IT}_{\mathrm{B}}^{\mathrm{TB}}} \mathrm{m}^{\mathrm{TB}} \mathrm{C}_{\mathrm{B}}^{\mathrm{TB}} \\
& \mathrm{T}_{\mathrm{B}}=\frac{0.693}{\mathrm{f}_{\mathrm{B}}} \mathrm{mC}_{\mathrm{B}} \\
& T_{B}^{T B}=\frac{0.693}{f_{1} \bar{I}} m^{T B} C_{B}^{T B} \\
& C_{B}=\frac{f_{B} I^{I T}}{0.693 m} \\
& \mathrm{C}_{\mathrm{B}}^{\mathrm{TB}}=\frac{\mathrm{f}_{1} \mathrm{IT}_{\mathrm{B}}^{\mathrm{TB}}}{0.693 \mathrm{~m}^{\mathrm{TB}}}
\end{aligned}
$$


2. Estimation of the Daily Intake

\section{Daily Intake of Man}

Man is assumed to ingest daily $300 \mathrm{~g}$ of meat, 1 liter of milk and $1 \mathrm{~kg}$ of food derived from plants (5219). In the absence of data on the concentration or uptake in plants contributing to man's diet, it is assumed hat cattle and man have a common dietary plant source and that the kilogram of food derived from plants is equivalent to $250 \mathrm{~g}$ dry weight of plants. Thus, if I is the daily intake in grams, $\mathrm{C}_{\text {meat }}$ the concentration in meat $(\mathrm{g} / \mathrm{g})$, $\mathrm{C}_{\mathrm{M}}$ the concentration in milk (g/liter), and $\mathrm{C}_{\mathrm{P}}$ the concentration in plants $(\mathrm{g} / \mathrm{g})$, $I=\left(3 \times 10^{2}\right) C_{\text {meat }}+C_{M}$

$$
+\left(2.5 \times 10^{2}\right) \mathrm{C}_{\mathrm{P}}
$$

Since a cow ingests $10 \mathrm{~kg}$ of dry forage daily (1759),

$$
\begin{aligned}
& C_{M}=f_{M} I_{\text {cow }}=f_{M} \times 10^{4} C_{P^{\prime}} \\
& I=\left(3 \times 10^{2}\right) C_{\text {meat }}+10^{4} f_{M} C_{P} \\
&+\left(2.5 \times 10^{2}\right) C_{P}
\end{aligned}
$$

\section{Daily Intake of Cattle}

Cattle are assumed to ingest $10 \mathrm{~kg}$ of dry forage daily. If $I_{\text {cattle }}$ is the daily intake in grams and $\mathrm{C}_{\mathrm{P}}$ is the concentration in forage $(\mathrm{g} / \mathrm{g})$,

$$
I_{\text {cattle }}=10^{4} \mathrm{C}_{\mathrm{P}} \text {. }
$$

\section{Concentration in Meat}

The concentration in meat $\mathrm{C}_{\text {meat, }}$, is assumed to the highest value found in meats (beef, pork, mutton, fowl, etc.). When concentrations in meats are deficient, $\mathrm{C}_{\text {meat }}$ is assumed to be a conservative estimate of $\mathrm{C}_{\text {beef }}$, the concentration in beef. The estimation of $\mathrm{C}_{\text {beef }}$ is based on a standard 500-kg steer whose muscle mass is $200 \mathrm{~kg}$.

\section{Concentration in Plants}

The concentration in plants $C_{P}$ can be estimated from the plant-to-soil concentration ratio $C_{P} / C_{S}$, which is obtained from tracer experiments:

$$
\mathrm{C}_{\mathrm{P}}=\left(\mathrm{C}_{\mathrm{P}} / \mathrm{C}_{\mathrm{S}}\right) \mathrm{C}_{\mathrm{S}}
$$

The concentration in plants can also be estimated from the daily intake of cattle using Eq. (13):

$$
\mathrm{C}_{\mathrm{P}}=\mathrm{I}_{\text {cattle }} / 10^{4} \text {. }
$$

The daily intake is determined by Eq. (3) or (4).

\footnotetext{
*In this section we have assumed implicitly that "beef" refers to steer skeletal muscle. Collateral data for other animal tissues are designated in the same way as in the original reference.
} 
3. Estimation of $f_{B}$ : Fractional Uptake by Ingestion

The uptake in an organ by ingestion $\mathrm{f}_{\mathrm{B}}$ is by definition the fraction absorbed $f_{1}$ times the uptake from blood $\mathrm{f}_{2}^{\prime}$ :

$$
f_{B}=f_{1} f_{2}^{\prime} \text {. }
$$

Data on uptake in organs of rats and other animals are often reported per gram of tissue. These data can sometimes be utilized for estimation of $\mathrm{f}_{2}^{\prime}$ and $\mathrm{f}_{\mathrm{B}}$. For example, if the $f_{2}^{\prime} / m$ in organ a were equal to the $f_{2}^{\prime} / m$ in organ $\underline{b}$, we assume that the $f_{2}^{\prime} / m$ are also equal in organs a and $\underline{b}$ of man. The $f_{2}^{\prime}$ in organ $\underline{a}$ of man can then be estimated from the $f_{2}^{\prime}$ in organ $\underline{\mathrm{b}}$ of man and the respective organ masses as follows:

$$
\left(f_{2}^{\prime}\right)^{a}=m^{a}\left(\frac{\left(f_{2}^{\prime}\right)^{b}}{m^{b}}\right) .
$$

Similarly

$$
\left(f_{B}\right)^{a}=m^{a}\left(\frac{\left(f_{B}\right)^{b}}{m^{b}}\right) \text {. }
$$

If the appropriate $f_{2}^{\prime}$ or $f_{B}$ for man is unknown, then we use the above equations together with the organ masses of the animal to calculate $f_{2}{ }^{\prime}$ or $f_{B}$ for the animal and assume this value for man.

Data have often been reported on uptake and distribution in the gonads of mixed groups of male and female subjects (usually rats). In keeping with our worst case approach, the $\mathrm{f}_{2}^{1}$ in the ovaries and testes are estimated assuming that the observed $f_{2}^{\prime}$ is attributable to males and females separately. Thus if there were $\mathrm{N}$ subjects composed of $\mathrm{M}$ males and $\mathrm{F}$ females and the average uptake from blood to the gonads was $\overline{\mathrm{f}_{2}^{1}}$,

$$
\begin{aligned}
& \mathrm{f}_{2}^{\prime} \text { (ovaries) }=\mathrm{N}_{2}^{\top} / \mathrm{F} \\
& \mathrm{f}_{2}^{\prime} \text { (testes) }=\mathrm{N} \mathrm{f}_{2}^{\top} / \mathrm{M} .
\end{aligned}
$$

If $M$ and $F$ were not stated, the $f_{2}^{\prime}$ in the ovaries and testes would each be

$$
\mathrm{f}_{2}^{\prime}=\mathrm{N}_{2}^{\top} \text {. }
$$

If $\mathrm{N}$ was not specified, it is assumed to be 5 . 
4. Estimation of $T_{B}$ : Biological Half-Life The biological half-life in an organ can be estimated from the fraction of the total body content in the organ $\left(f_{2}\right)$, the uptake in the organ following absorption $\left(f_{2}^{\prime}\right)$ and the biological half-life in the whole body $\left(\mathrm{T}_{\mathrm{B}}^{\mathrm{TB}}\right)$. The relationship below is obtained when Eq. (7) is divided by

Eq. (8):

$$
\frac{\mathrm{T}_{\mathrm{B}}}{\mathrm{T}_{\mathrm{B}}^{\mathrm{TB}}}=\frac{\mathrm{f}_{1}}{\mathrm{f}_{\mathrm{B}}} \frac{\mathrm{mC}_{\mathrm{B}}}{\mathrm{m}^{\mathrm{TB}} \mathrm{C}_{\mathrm{B}}^{\mathrm{TB}}} .
$$

Since $\frac{m_{C_{B}}}{m^{T B} C_{B}^{T B}}=f_{2}$ and $f_{B}=f_{1} f_{2}^{\prime}$,

$$
T_{B}=\left(f_{2} / f_{2}^{\prime}\right) T_{B}^{T B} .
$$

It follows from Eq. (19) that the biological half-life of organ a can be estimated from that of organ $\underline{b}$ and the $\left(f_{2} / f_{2}^{\prime}\right)$ ratios of organs $\underline{a}$ and $\underline{b}$, that is,

$$
T_{B}^{a}=\frac{\left(f_{2} / f_{2}^{\prime}\right)^{a}}{\left(f_{2} / f_{2}^{\prime}\right)^{b}} T_{B}^{b} .
$$




\section{Estimation of $\mathrm{C}_{\mathrm{B}}$ : Concentration}

The fraction of the total body content in an organ $f_{2}$ is related to the concentrations in the organ and in the total body by Eq. (21):

$$
f_{2}=\frac{m_{B}}{m^{T B} C_{B}^{T B}} .
$$

Equation (21) can be rearranged to yield expressions for $\mathrm{C}_{\mathrm{B}}$ and $\mathrm{C}_{\mathrm{B}}^{\mathrm{TB}}$ :

$$
\begin{aligned}
& C_{B}=\frac{f_{2} m^{T B} C_{B}^{T B}}{m}, \\
& C_{B}^{T B}=\frac{m_{B}}{f_{2} m^{T B}} .
\end{aligned}
$$

The $\mathrm{f}_{2}$ in the ovaries and testes can conservatively be estimated from distribution in the gonads of a mixed group of males and females. We follow an approach similar to that for derivation of Eqs. (1718). If there were $\mathrm{N}$ subjects composed of $F$ females and $M$ males and $\overline{f_{2}}$ was the average $f_{2}$ in the gonads,

$$
\begin{aligned}
& \mathrm{f}_{2} \text { (ovaries) }=\mathrm{N} \overline{\mathrm{f}_{2}} / \mathrm{F}, \\
& \mathrm{f}_{2} \text { (testes) }=\mathrm{N} \overline{\mathrm{f}_{2}} / \mathrm{M} .
\end{aligned}
$$

If $\mathrm{M}$ and $\mathrm{F}$ were not stated, the $\mathrm{f}_{2}$ in the ovaries and testes would each be

$$
\mathrm{f}_{2}=\mathrm{N} \overline{\mathrm{f}}_{2} \text {. }
$$

If $\mathrm{N}$ was not specified, it is assumed to be 5 .

The concentration in an organ can be estimated from the concentration in plasma and the tissue-to-plasma concentration ratio:

$$
\mathrm{C}_{\mathrm{B}}=\left(\mathrm{C}_{\mathrm{B}} / \mathrm{C}_{\text {plasma }}\right) \mathrm{C}_{\text {plasma }} .
$$

The tissue-to-plasma ratio can be determined from tracer experiments.

The concentration in the total body can be determined from the total body content estimated as the sum of the contents of each organ. Thus

$$
\mathrm{C}_{\mathrm{B}}^{\mathrm{TB}}=\frac{\sum\left(\mathrm{mC}_{\mathrm{B}}\right)}{\mathrm{m}^{\mathrm{TB}}} .
$$


6. Special Considerations in Estimation of $\mathrm{f}_{\mathrm{B}}-\mathrm{T}_{\mathrm{B}}$ and $\mathrm{C}_{\mathrm{B}}$

Functional Dependence of Unit-Rad Deposition on $\mathrm{f}_{\mathrm{B}^{2}} \mathrm{~T}_{\mathrm{B}}$ and $\mathrm{C}_{\mathrm{B}}$

Examination of the equations for the unit rad deposition $F_{1}$ (5571) shows that, if $\mathrm{T}_{\mathrm{B}}$ is less than a few years, the dependency on $\mathrm{f}_{B}, T_{B}$ and $C_{B}$ is as follows. Via milk:

$$
F_{1} \propto \frac{1}{f_{B} T_{E} T_{P}} \propto \frac{\left(T_{B}+T_{R}\right)}{f_{B} T_{B} T_{P} T_{R}},
$$

Via soil:

$$
\begin{array}{r}
\mathrm{F}_{1} \propto \frac{1}{\mathrm{C}_{\mathrm{B}}\left[\mathrm { T } _ { \mathrm { R } } \left(1-\mathrm{e}^{\left.\left.-20.8 / \mathrm{T}_{\mathrm{R}}\right)-\mathrm{T}_{\mathrm{E}}\right]}\right.\right.} \\
\propto \frac{\left(\mathrm{T}_{\mathrm{B}}+\mathrm{T}_{\mathrm{R}}\right)}{\mathrm{C}_{\mathrm{B}} \mathrm{T}_{\mathrm{R}}\left[\mathrm{T}_{\mathrm{R}}-\left(\mathrm{T}_{\mathrm{B}}+\mathrm{T}_{\mathrm{R}}\right) \mathrm{e}^{\left.-20.8 / \mathrm{T}_{\mathrm{R}}\right]}\right.} .
\end{array}
$$

For convenience in comparing the dependency on the biological parameters, let us also assume that $T_{R}$ is less than a few years. Then since by Eq. (9)

$$
\mathrm{C}_{\mathrm{B}} \propto \mathrm{f}_{\mathrm{B}} \mathrm{T}_{\mathrm{B}^{\prime}}
$$

Equation (29) can be written

$$
F_{1} \propto \frac{\left(T_{B}+T_{R}\right)}{f_{B} T_{B} T_{R}^{2}} .
$$

Thus, the functional dependence by the two pathways is the same.

The estimated maximum dose is greatest when $F_{1}$ is a minimum. In keeping with the conservative nature of these calculations, our estimates of $\mathrm{f}_{B}, T_{B}$ and $\mathrm{C}_{\mathrm{B}}$ must conform to the following rules: If $\mathrm{C}_{\mathrm{B}}$ is known, it sets an upper limit on the product $f_{B} T_{B}$; and then, $f_{B}$ must be maximized and $T_{B}$ minimized. If $C_{B}$ is not known, $f_{B}, T_{B}$ and $C_{B}$ must be maximized individually. Thus $f_{B}, T_{B}$ and $C_{B}$ are not necessarily consistent with each other in this Handbook.

When $f_{B}, T_{B}$ and $C_{B}$ are maximized independently, the resulting $F_{1}$ via the soil-root pathway may not be a minimum. However, the fetus, the newborn, and the infant should be regarded as the critical members of the population. This group experiences extremely rapid growth and development; accordingly, we have assumed that body tissues in this group very rapidly (instantaneously) equilibrate with the terrestrial environment (soil), from which the diet is derived. Thus, we estimate the dosage to this group via soil assuming that $\mathrm{T}_{\mathrm{B}}=0$ for all elements, which serves to minimize $F_{1}$ and maximize the estimate of dosage. 


\section{Estimation of $\mathrm{C}_{\mathrm{S}}$}

$\underline{\mathrm{C}}_{\mathrm{S}}$ of Naturally Occurring Radioisotopes

The concentration in soil $\left(\mathrm{C}_{\mathrm{S}}\right)$ of

naturally occurring radioisotopes can sometimes be estimated from that of the parent isotopes assuming secular equilibrium. Consider the general scheme:

1. Isotope $\mathrm{X}$ can decay into isotope $\mathrm{Y}$.

2. A fraction $f$ of the disintegrations of $\mathrm{X}$ results in the production of $\mathrm{Y}$.

3. $X$ and $Y$ represent the number of atoms of $\mathrm{X}$ and $\mathrm{Y}$ per gram of soil.

4. $\mathrm{T}_{\mathrm{X}}$ and $\mathrm{T}_{\mathrm{Y}}$ represent their halflives, $A_{X}$ and $A_{Y}$ represent their masses.

If $\mathrm{T}_{\mathrm{X}} \gg \mathrm{T}_{\mathrm{Y}}$, then $\mathrm{X}$ and $\mathrm{Y}$ can come to secular equilibrium, whence

$$
\mathrm{Y} / \mathrm{T}_{\mathrm{Y}}=\mathrm{fX} / \mathrm{T}_{\mathrm{X}}
$$

\section{Isotopes Produced by Spontaneous}

Fission-In the situation where $\mathrm{Y}$ is produced by spontaneous fission of $\mathrm{X}$, $\mathrm{f}$ is the fission yield. Since

$\left(C_{S}\right)_{Y}=Y A_{Y} / N_{O}$ and $\left(C_{S}\right)_{X}=X A_{X} / N_{O}$

$\left(C_{S}\right)_{Y}=\frac{f_{A_{Y} T_{Y}}}{A_{X} T_{X}}\left(C_{S}\right)_{X}$.

\section{Isotopes of Natural Radioactive}

Series - In the situation where $\mathrm{X}$ and $\mathrm{Y}$ are members of natural radioactive series, $f$ is the branching fraction. $X$ and $\mathrm{Y}$ are isotopes of high $\mathrm{Z}$ and high $\mathrm{A}$, so that $A_{Y} \approx A_{X}$. Since $A_{Y} / A_{X} \approx 1$, Eq. (32) reduces to

$$
\left(C_{S}\right)_{Y}=f\left(T_{Y} / T_{X}\right)\left(C_{S}\right)_{X}
$$

All half-lives used in the calculations were taken from the Chart of the Nuclides (5969) or the Table of Isotopes (5968). Unless otherwise indicated, the branching ratios are also from the Table of Isotopes (5968). The best known member of the chain was chosen as $X$.

In following this approach we focused on the daughter isotopes which would be expected to have the highest concentrations. Thus we considered those isotopes whose half-lives were relatively long and whose parents in the decay chain were relatively high in natural abundance.

When the contribution of one isotope to $\mathrm{C}_{\mathrm{S}}$ exceeded the sum of the contributions of the other isotopes by a factor of 10 or more, we considered only this isotope. (This is consistent with our conservative approach.)

In the absence of concentrations in soil, we assumed that the ratios of the concentrations of parent and daughter are similar in soils, rocks and ores. Thus the $\mathrm{C}_{\mathrm{S}}$ of $\mathrm{Y}$ can be estimated from the $C_{S}$ of $X$, the parent, and the ratio of the concentrations of $\mathrm{Y}$ and $\mathrm{X}$ in rock.

$$
\left(C_{S_{Y}}\right)_{Y}=\frac{\left(C_{\text {ore }}\right)_{Y}}{\left(C_{\text {ore }}\right)_{X}}\left(C_{S}\right)_{X}
$$


ESTIMATES OF INPUT PARAMETERS BY ELEMENT

This section describes the estimation of input parameters using collateral data (Code 99 entries). Data used in the calculations include values listed in the Handbook tables. It is to be noted that the data required in the calculations are listed as "Collateral Data "only if they are not found in the Handbook tables. The parameters are covered in the order of their appearance in the tables, i. e., $C_{S}$, $f_{B}, T_{B}, C_{B}$.

The estimates calculated by these procedures are rounded off in the direction yielding a more conservative $\mathrm{F}_{1}$. These final values are the values listed in the Handbook tables. 


\section{Estimation of $\mathrm{C}_{\mathrm{S}}$}

The $\mathrm{C}_{\mathrm{S}}$ of $\mathrm{H}$ was estimated by considering its presence in (1) liquid phase,

(2) organic matter, and (3) mineral matter. We assumed

1. Concentration of the liquid phase is $25 \%$ by volume (5804).

2. Density of soil water is $1 \mathrm{~g} / \mathrm{cm}^{3}$.

3. Bulk density of plow layer is

$$
1.5 \mathrm{~g} / \mathrm{cm}^{3} \text { (2062). }
$$

If $\mathrm{C}_{\mathrm{W}}$ is the concentration of $\mathrm{H}$ in water in $\mathrm{g} / \mathrm{cm}^{3}$, the $\mathrm{C}_{\mathrm{S}}$ from soil water is

$$
\mathrm{C}_{\mathrm{S}}=\frac{0.25 \mathrm{C}_{\mathrm{W}}}{1.5}=0.17 \mathrm{C}_{\mathrm{W}} \text {. }
$$

$2^{\mathrm{He}}$

\section{Estimation of $\mathrm{C}_{\mathrm{S}}$}

$\underline{C}_{\mathrm{S}}$ of Helium and Other Inert Gases

The $\mathrm{C}_{\mathrm{S}}$ of the inert gases were assumed to be the concentrations resulting from their presence in the soil atmosphere which is taken to be the normal atmosphere under STP. We assumed

1. Concentration of soil atmosphere is $25 \%$ by volume (5804).

2. Bulk density of plow layer is

$$
1.5 \mathrm{~g} / \mathrm{cm}^{3} \text { (2062). }
$$

Then if $\mathrm{C}_{\mathrm{a}}$ is the concentration in air in $\mathrm{g} / \mathrm{cm}^{3}$,

$$
\mathrm{C}_{\mathrm{S}}=0.25 \mathrm{C}_{\mathrm{a}} / 1.5=0.17 \mathrm{C}_{\mathrm{a}} \text {. }
$$

(1) Estimate of $\mathrm{C}_{\mathrm{S}}$ from soil water:

$$
\begin{array}{ll}
C_{W}=0.11 \quad \mathrm{~g} / \mathrm{cm}^{3}, \\
C_{\mathrm{S}}=1.8 \times 10^{-2} \mathrm{~g} / \mathrm{g} .
\end{array}
$$

(2) Estimate of $\mathrm{C}_{\mathrm{S}}$ from soil organic matter:

$$
\mathrm{C}_{\mathrm{S}}=2.5 \times 10^{-3} \mathrm{~g} / \mathrm{g}(5223) .
$$

(3) Estimate of $\mathrm{C}_{\mathrm{S}}$ from soil mineral matter:

$$
\mathrm{C}_{\mathrm{S}}=2.5 \times 10^{-3} \mathrm{~g} / \mathrm{g}(5223) .
$$

(4) The final estimate of $\mathrm{C}_{\mathrm{S}}$ was determined by summing the $\mathrm{C}_{\mathrm{S}}$ of (1), (2) and (3).

The $\mathrm{C}_{\mathrm{a}}$ of radon was estimated as that of $\mathrm{Rn}^{222}$ from the activity in the soil atmosphere (5770) and the specific activity (5938):

Concentration of $\mathrm{Rn}^{222}$ in soil atmosphere:

$$
5 \times 10^{-10} \mathrm{Ci} / \text { liter } .
$$

Specific activity of $\mathrm{Rn}^{222}$ :

$$
1.5 \times 10^{5} \mathrm{Ci} / \mathrm{g} .
$$

Hence

$$
\begin{aligned}
C_{a}=5 \times 10^{-10} / 1.5 \times 10^{5} \\
=3.3 \times 10^{-15} \mathrm{~g} / \text { liter. }
\end{aligned}
$$

(continued) 
$4^{\mathrm{Be}}$

\section{Estimation of $\mathrm{C}_{\mathrm{B}}$}

Individual Organs

(1) Estimation of daily intake by Eq. (4): $\mathrm{I}=2 \times 10^{-5} \mathrm{~g} / \mathrm{day}$

(2) $\mathrm{C}_{B}$ (of the gastrointestinal tract contents) was then estimated using a total intake of $1.3 \mathrm{~kg} /$ day.

\section{Estimation of Aquatic Data}

Estimation of Concentration in Foodstuffs

The concentration was assumed to be the same in plants, invertebrates, and fish. Plants and invertebrates are shown to be the same in reference 6010 . 
-

•

- 
Estimation of $\mathrm{C}_{\mathrm{S}}$ of inert gases

\begin{tabular}{lcll}
\hline Element & $\mathrm{C}_{\mathrm{a}}\left(\mu \mathrm{g} / \mathrm{m}^{3}, \mathrm{STP}\right)$ & Ref. & $\mathrm{C}_{\mathrm{S}}(\mathrm{g} / \mathrm{g})$ \\
\hline $2 \mathrm{He}$ & $9.2 \times 10^{2}$ & 5761 & $1.5 \times 10^{-10}$ \\
$10 \mathrm{Ne}$ & $1.6 \times 10^{4}$ & 5761 & $2.7 \times 10^{-9}$ \\
$18 \mathrm{Ar}$ & $1.6 \times 10^{7}$ & 5761 & $2.7 \times 10^{-6}$ \\
$36 \mathrm{Kr}$ & $4.1 \times 10^{3}$ & 5761 & $7.5 \times 10^{-10}$ \\
$54 \mathrm{Xe}$ & $5.0 \times 10^{2}$ & 5761 & $8.3 \times 10^{-11}$ \\
$86 \mathrm{Rn}$ & $3.3 \times 10^{-6}$ & & $5.4 \times 10^{-19}$ \\
\hline
\end{tabular}

$$
15^{\mathrm{P}}
$$

\section{Collateral Data}

\begin{tabular}{lllll}
\hline & Min & Max & Av & Ref. \\
\hline Uptake relationships & & & & \\
$\mathrm{f}_{2}^{\prime}$ & & & 0.5 & ICRP \\
Bone & 1.18 & 1.80 & & $3869 \mathrm{~A}$ \\
\hline I (g/day) & & & & \\
\hline
\end{tabular}

Estimation of $f_{B}$

\section{Individual Organs}

The uptake per gram in ovaries, kidneys, liver, spleen and testes of rats were shown to be less than or comparable to the uptake per gram in bone $(2413,5275,5295)$. Therefore we have assumed that $f_{2}^{\prime} / m$ in these tissues is the same as $\mathrm{f}_{2}^{\prime} / \mathrm{m}$ in bone. The $\mathrm{f}_{\mathrm{B}}$ in these organs were calculated using $f_{2}^{\prime} / \mathrm{m}$ in bone by Eqs. (16) and (16a).

Estimation of $T_{B}$

\section{Individual Organs}

The $T_{B}$ were estimated by Eq. (7) using an I equal to $1.2 \mathrm{~g} /$ day. 
Collateral Data

\begin{tabular}{|c|c|c|c|c|}
\hline & Min & $\operatorname{Max}$ & $\mathrm{Av}$ & Ref. \\
\hline \multicolumn{5}{|c|}{ Uptake relationships } \\
\hline \multicolumn{5}{|c|}{$f_{B}$} \\
\hline Bone & & & 0.03 & ICRP \\
\hline Testes & & & 0.0013 & ICRP \\
\hline Rat kidneys & & & 0.0025 & 5294 \\
\hline Rat liver & & & 0.005 & 5294 \\
\hline Rat spleen & & & 0.0003 & 5294 \\
\hline
\end{tabular}

\section{Estimation of $f_{B}$}

Individual Organs

In the absence of other data the $f_{B}$ for rat have been assumed for man. Sulfur is most concentrated in the structural and protective tissues (e.g., bone, connective tissue, skin, hair). Since the S-containing substances would be expected to have a general distribution among soft tissues, we have assumed that the uptake in ovaries is comparable to that in kidneys, liver, spleen and testes. The $\mathrm{f}_{\mathrm{B}} / \mathrm{m}$ in the testes, $2.2 \times 10^{-5}$, is the highest for the organs listed, and this was assumed for the ovaries. The $f_{B}$ in ovaries was estimated by Eq. (16b).

\section{Estimation of $\mathrm{C}_{B}$}

Individual Organs

Since sulfur is most concentrated in tissues such as skin, we have assumed values of $\mathrm{C}_{\mathrm{B}}$ comparable to that in skin, $10^{4} \mu \mathrm{g} / \mathrm{g}$ (ICRP).

${ }_{20} \mathrm{Ca}$

Collateral Data

\begin{tabular}{lllll}
\hline & Min & Max & Av & Ref. \\
\hline Uptake relationships & & & & \\
$\mathrm{f}_{2}^{\prime}$ & & & 0.9 & ICRP \\
Bone & 0.76 & 1.31 & & $3869 \mathrm{~A}$ \\
\hline
\end{tabular}

(continued) 
Estimation of $f_{B}$

\section{Individual Organs}

The $f_{2}^{1} / m$ in the soft tissues of the rat were shown to be more than a factor of ten lower than that in bone $(1798,3970$, 5171). Therefore, in estimating $f_{B}$, we have made the conservative assumption that the $\mathrm{f}_{2}^{1} / \mathrm{m}$ in organs other than bone is equal to $1.4 \times 10^{-5}$, which is $1 / 10$ of the $f_{2}^{\prime} / m$ in bone. The estimates of $f_{B}$ were then made using Eqs. (16a) and (16).

\section{Estimation of $\mathrm{T}_{\mathrm{B}}$}

\section{Individual Organs}

The $\mathrm{T}_{\mathrm{B}}$ were estimated by Eq. (7) using an I equal to $0.76 \mathrm{~g} /$ day.

$$
21^{\mathrm{Sc}}
$$

Collateral Data

\begin{tabular}{|c|c|c|c|c|}
\hline & Min & $\operatorname{Max}$ & $A v$ & Ref. \\
\hline \multicolumn{5}{|c|}{ Concentrations $(\mu \mathrm{g} / \mathrm{g})$} \\
\hline Human heart & $3.1 \times 10^{-6}$ & $1.08 \times 10^{-4}$ & & 1294 \\
\hline Cattle bone & $2 \times 10^{-3}$ & $5 \times 10^{-3}$ & & 4752 \\
\hline Cow heart & $1.8 \times 10^{-5}$ & $9.8 \times 10^{-5}$ & & 1745 \\
\hline Beef & & & $5.5 \times 10^{-3}$ & 4941 \\
\hline Pork & & & $2.6 \times 10^{-3}$ & 4941 \\
\hline Ham & & & $2.4 \times 10^{-3}$ & 4941 \\
\hline Chicken & & & $7.4 \times 10^{-3}$ & 4941 \\
\hline Kale tops & & & $8.35 \times 10^{-3}$ & 5340 \\
\hline \multicolumn{5}{|c|}{$\begin{array}{l}\text { Uptake relationships } \\
f_{2}^{\prime}\end{array}$} \\
\hline Rat spleen & 0.0077 & 0.053 & & 5180,5181 \\
\hline Rat gonads & 0.0008 & 0.0053 & & 5180,5181 \\
\hline \multicolumn{5}{|l|}{$\mathrm{f}_{2} / \mathrm{f}_{2}^{\prime}$} \\
\hline Bone & & & 1.1 & ICRP \\
\hline Liver & & & 1.2 & ICRP \\
\hline Rat kidneys & 0.7 & 1.1 & & 5181 \\
\hline Rat spleen & & & 0.85 & 5181 \\
\hline \multicolumn{5}{|l|}{$\mathrm{f}_{2}$} \\
\hline Bone & & & 0.22 & ICRP \\
\hline Kidneys & & & 0.05 & ICRP \\
\hline Liver & & & 0.18 & ICRP \\
\hline Rat spleen & 0.027 & 0.041 & & 5181 \\
\hline Rat gonads & 0.016 & 0.017 & & 5181 \\
\hline
\end{tabular}


Estimation of $\mathrm{f}_{\mathrm{B}}$

\section{Individual Organs}

The $f_{B}$ in the ovaries and testes were estimated from $f_{2}^{\prime}$ in the gonads by

Eqs. (18) and (16). The $\mathrm{f}_{B}$ in spleen was estimated by Eq. (16).

\section{Estimation of $\mathrm{T}_{\mathrm{B}}$}

\section{Individual Organs}

The $\mathrm{T}_{\mathrm{B}}$ were estimated by Eq. (19).

\section{Estimation of $\mathrm{C}_{\mathrm{B}}$}

\section{Whole Body}

Scandium is thought to be rather strongly excluded from plants with $\mathrm{C}_{\mathrm{P}} / \mathrm{C}_{\mathrm{S}}<0.01$ (5227, 5306). The $\mathrm{C}_{\mathrm{P}} / \mathrm{C}_{\mathrm{S}}$ of $\mathrm{Sc}^{46}$ in food crops irrigated with Columbia River water was less than 0.1 except in lettuce and beet tops, where the likelihood of foliar absorption was high (5237). In experiments on the uptake of $\mathrm{Sc}^{46}$ and $\mathrm{W}^{185}$ in leaves and stems of bush beans grown on various soils, the quotient of the $\mathrm{C}_{\mathrm{P}} / \mathrm{C}_{\mathrm{S}}$ ratio of scandium and that of tungsten varied from 0.03 to 0.23 , but for the most part the quotient was less than 0.1 (5229). In another series of experiments the greatest $\mathrm{C}_{\mathrm{P}} / \mathrm{C}_{\mathrm{S}}$ ratio of
$\mathrm{W}^{185}$ in beans or barley grown on different soils was $3.8(2697,5570)$; the other ratios were less than 1 . On the basis of these relationships a conservative estimate of the maximum $\mathrm{C}_{\mathrm{P}} / \mathrm{C}_{\mathrm{S}}$ ratio of scandium would be 3.8 times 0.23 , the product of the maximum $\mathrm{C}_{\mathrm{P}} / \mathrm{C}_{\mathrm{S}}$ ratio of tungsten and the maximum quotient of the $\mathrm{C}_{\mathrm{P}} / \mathrm{C}_{\mathrm{S}}$ ratios of scandium and tungsten. Accordingly

$$
\mathrm{C}_{\mathrm{P}} / \mathrm{C}_{\mathrm{S}}=1.0
$$

(1) Estimation of $\mathrm{C}_{\mathrm{P}}$ by Eq. (14):

$$
\mathrm{C}_{\mathrm{P}}=7 \times 10^{-6} \mathrm{~g} / \mathrm{g} \text {. }
$$

(2) Estimation of I by Eq. (12):

$$
\begin{aligned}
C_{\text {meat }} & =10^{-8} \mathrm{~g} / \mathrm{g}, \\
I & =1.8 \times 10^{-3} \mathrm{~g} / \text { day } .
\end{aligned}
$$

(3) $C_{B}^{T B}$ was then estimated by Eq. (10) using the I above.

\section{Individual Organs}

The $f_{2}$ in the ovaries and testes were estimated from $f_{2}$ in the gonads by Eq. (25) and are based on data from 3 rats (5181). The $\mathrm{C}_{\mathrm{B}}$ were estimated by Eq. (22). 
$25^{\mathrm{Mn}}$

Collateral Data

\begin{tabular}{|c|c|c|c|c|}
\hline & Min & $\operatorname{Max}$ & $\mathrm{Av}$ & Ref. \\
\hline \multicolumn{5}{|c|}{ Uptake relationships } \\
\hline \multicolumn{5}{|c|}{$\mathrm{f}_{2}^{1}$} \\
\hline Kidneys & & & 0.05 & ICRP \\
\hline Liver & & & 0.24 & ICRP \\
\hline Rat bone & 0.055 & 0.083 & & $5180,5181,5273$ \\
\hline Rat spleen & 0.0038 & 0.006 & & $5180,5181,5273$ \\
\hline Rat testes & & & 0.01 & 5273 \\
\hline$I(g /$ day $)$ & $3.45 \times 10^{-3}$ & $4.39 \times 10^{-3}$ & & $3869 \mathrm{~A}$ \\
\hline
\end{tabular}

\section{Estimation of $f_{B}$}

\section{Individual Organs}

In the absence of uptake data in humans we have estimated $f_{B}$ from uptake in rats. We have assumed further that the uptake in rat testes and ovaries are similar. This would be conservative since the mass of the testes in rat is a disproportionately large fraction of the total body mass. The $\mathrm{f}_{B}$ were estimated by Eq. (16).

\section{Estimation of $\mathrm{T}_{\mathrm{B}}$}

\section{Individual Organs}

The $T_{B}$ were estimated by Eq. (7) using an $I$ equal to $3.5 \times 10^{-5} \mathrm{~g} /$ day.

$$
26^{\mathrm{Fe}}
$$

Collateral Data

\begin{tabular}{|c|c|c|c|c|}
\hline & Min & $\operatorname{Max}$ & $\mathrm{Av}$ & Ref. \\
\hline \multicolumn{5}{|c|}{ Uptake relationships } \\
\hline \multicolumn{5}{|c|}{$\mathrm{f}_{2}^{\prime}$} \\
\hline Bone & & & 0.1 & ICRP \\
\hline Liver & & & 0.13 & ICRP \\
\hline Spleen & & & 0.02 & ICRP \\
\hline Rat kidneys & & & 0.03 & 5193 \\
\hline I (g/day) & $1.2 \times 10^{-2}$ & $1.5 \times 10^{-2}$ & & $3869 \mathrm{~A}$ \\
\hline
\end{tabular}


Estimation of $\mathrm{f}_{\mathrm{B}}$

\section{Individual Organs}

The $\mathrm{C}_{\mathrm{B}}$ of iron in bone, kidneys, liver, and spleen are greater than the $\mathrm{C}_{B}$ in ovaries or testes. This indicates that $f_{B} / m$ or $T_{B}$, or both, are smaller in the ovaries and testes. As the discussion of Eq. (30) indicates, we should maximize $\mathrm{f}_{\mathrm{B}}$ in this case. Accordingly we set $\mathrm{f}_{2}^{\prime} / \mathrm{m}$ in ovaries and testes equal to $1.2 \times 10^{-4}$, which is the $f_{2}^{\prime} / m$ for spleen and the highest of the tissues. Then $\mathrm{f}_{B}$ was estimated using Eqs. (16) and (16a). The $\mathrm{f}_{\mathrm{B}}$ in kidneys was estimated by Eq. (16).

\section{Estimation of $\mathrm{T}_{\mathrm{B}}$}

\section{Individual Organs}

The $\mathrm{T}_{\mathrm{B}}$ were calculated by Eq. (7) using an $I$ equal to $1.2 \times 10^{-2} \mathrm{~g} /$ day.

Collateral Data

\begin{tabular}{|c|c|c|c|c|}
\hline & Min & $\operatorname{Max}$ & $\mathrm{Av}$ & Ref. \\
\hline \multicolumn{5}{|c|}{ Uptake relationships } \\
\hline \multicolumn{5}{|c|}{$\mathrm{f}_{2}^{\prime}$} \\
\hline Kidneys & & & 0.002 & ICRP \\
\hline Liver & & & 0.04 & ICRP \\
\hline Spleen & & & 0.0014 & ICRP \\
\hline I (g/day) & & & $1.65 \times 10^{-4}$ & $3869 \mathrm{~A}$ \\
\hline
\end{tabular}

\section{Estimation of $f_{B}$}

\section{Individual Organs}

The $f_{2}^{\prime} / m$ in femur of rats was less than that in kidneys and liver by about an order of magnitude (2207, 5936). The $\mathrm{f}_{2}^{1} / \mathrm{m}$ in reproductive organs of young calves was less than that in kidneys, liver, and spleen. The $f_{2}^{1} / m$ in kidneys, liver, and spleen are approximately $10^{-5} \mathrm{~g}^{-1}$. We have used this value as a conservative estimate of the $f_{2}^{\prime} / m$ in bone, ovaries, and testes. The $f_{B}^{\prime}$ were estimated by Eqs. (16) and (16a).

\section{Estimation of $\mathrm{T}_{\mathrm{B}}$}

Whole Body

The $\mathrm{T}_{\mathrm{B}}^{\mathrm{TB}}$ was estimated by Eq. (8).

\section{Individual Organs}

The half-lives of cobalt in the whole body and various organs of rat were comparable $(3331,5936)$. Cobalt does not concentrate in bone, ovaries or testes (1798, 3868, 4756). We therefore feel justified in adopting $\mathrm{T}_{\mathrm{B}}^{\mathrm{TB}}$ as $\mathrm{T}_{\mathrm{B}}$ for all the organs of interest. 


$$
29^{\mathrm{Cu}}
$$

Collateral Data

\begin{tabular}{lllll}
\hline & Min & Max & Av & Ref. \\
\hline $\begin{array}{l}\text { Uptake relationships } \\
f_{2}^{\prime}\end{array}$ & & & \\
$\quad$ Brain & & 0.01 & ICRP \\
Heart & & 0.01 & ICRP \\
Kidneys & & 0.05 & ICRP \\
Liver & & 0.08 & ICRP \\
Spleen & & 0.07 & ICRP \\
I (g/day) & $9.1 \times 10^{-4}$ & $1.04 \times 10^{-3}$ & & $5869 \mathrm{~A}$ \\
\hline
\end{tabular}

\section{Estimation of $\mathrm{f}_{\mathrm{B}}$}

\section{Individual Organs}

Copper is most concentrated in liver, brain, heart and kidneys, and mean $\mathrm{C}_{B}$ in bone, ovaries, and testes $(1798,3868)$ are equal to or less than $\mathrm{C}_{\mathrm{B}}^{\mathrm{TB}}$. Thus copper does not normally concentrate in bone, ovaries, and testes. In accordance with the discussion of Eq. (30) we should maximize $f_{B}$. We therefore assumed that $f_{2}^{1} / m$ in ovaries and testes are equal to $4 \times 10^{-4} \mathrm{~g}^{-1}$, the $\mathrm{f}_{2}^{1} / \mathrm{m}$ in spleen, which is the greatest in any of the organs listed.
The $f_{B}$ were then estimated by Eqs. (16) and (16a). The $f_{2}^{\prime}$ in bone was assumed to be 0.8 , the fraction not known to be taken up in other organs of the rat (5220). The $f_{B}$ was then calculated by Eq. (16).

\section{Estimation of $\mathrm{T}_{\mathrm{B}}$}

Whole Body

The $T_{B}^{T B}$ was estimated by Eq. (8) using an $I$ equal to $9 \times 10^{-4} \mathrm{~g} /$ day.

Individual Organs

The $\mathrm{T}_{\mathrm{B}}$ were estimated by Eq. (7) using an $I$ equal to $9 \times 10^{-4} \mathrm{~g} /$ day. 
Estimation of $\mathrm{C}_{B}$

\section{Individual Organs}

The daily intake was assumed to be equal to the daily excretion of $110 \mu \mathrm{g} /$ day (6094). The $\mathrm{C}_{B}$ of the GI tract (contents) was estimated assuming a total intake of $1.3 \mathrm{~kg} /$ day.

\section{Estimation of $\mathrm{C}_{\text {meat }}$}

The daily intake of gallium was assumed to be the daily excretion, $110 \mu \mathrm{g}$ (6094). Since the dietary gallium is mostly of animal origin (6094), $\mathrm{C}_{\text {meat }}$ was set equal to $100 \mu \mathrm{g}$ divided by the daily intake of meat.

Collateral Data

\begin{tabular}{|c|c|c|c|c|}
\hline & Min & $\operatorname{Max}$ & $A v$ & Ref. \\
\hline \multicolumn{5}{|l|}{ Concentrations $(\mu \mathrm{g} / \mathrm{g})$} \\
\hline Human bone & $2.1 \times 10^{-2}$ & $9.8 \times 10^{-1}$ & & 2986 \\
\hline Human heart & $4 \times 10^{-3}$ & $2.5 \times 10^{-1}$ & & 1294,2986 \\
\hline Human kidneys & $1.4 \times 10^{-2}$ & $2.7 \times 10^{-1}$ & & 893,2986 \\
\hline $\begin{array}{l}\text { Human large } \\
\text { intestine }\end{array}$ & & & $4 \times 10^{-2}$ & 893 \\
\hline Human liver & $1.1 \times 10^{-2}$ & $3.1 \times 10^{-1}$ & & 893,2986 \\
\hline Human lungs & $6 \times 10^{-3}$ & $1.1 \times 10^{-2}$ & & 2986 \\
\hline Human muscle & $1.4 \times 10^{-2}$ & $2.3 \times 10^{-1}$ & & 2986 \\
\hline Human pancreas & $2.1 \times 10^{-2}$ & $2.6 \times 10^{-1}$ & & 2986 \\
\hline Human skin & $1.2 \times 10^{-2}$ & $1.2 \times 10^{-1}$ & & 2986 \\
\hline Human spleen & $1.4 \times 10^{-2}$ & $1.4 \times 10^{-1}$ & & 2986 \\
\hline
\end{tabular}

\section{Estimation of $\mathrm{C}_{\mathrm{B}}$}

Whole Body

The tissues whose concentrations are listed account for the major fraction of the total body mass. We therefore estimated $C_{B}^{T B}$ by Eq. (27) assuming a $\mathrm{C}_{\mathrm{B}}$ of $10^{-6} \mathrm{~g} / \mathrm{g}$ in bone and a $\mathrm{C}_{\mathrm{B}}$ of $3 \times 10^{-7} \mathrm{~g} / \mathrm{g}$ in all the other tissues. 


\section{$35^{\mathrm{Br}}$}

Estimation of Aquatic Data

Estimation of Concentration in Seawater Fish

The concentration in fish was assumed to be the same as in invertebrates.
Estimation of Concentration in Freshwater Foodstuffs

The ratio (foodstuffs $/ \mathrm{C}_{A}$ ) for chlorine was assumed to apply for bromine.

$$
37^{\mathrm{Rb}}
$$

\section{Estimation of Aquatic Data}

\section{Estimation of Concentration in Fresh-} water Invertebrates

The concentration in invertebrates was assumed to be the same as that in fish.

$$
38^{\mathrm{Sr}}
$$

Collateral Data

\begin{tabular}{lcccc}
\hline & Min & Max & Av & Ref. \\
\hline $\begin{array}{l}\text { Uptake relationships } \\
\text { I (g/day) }\end{array}$ & $1.24 \times 10^{-3}$ & $1.37 \times 10^{-3}$ & $3869 \mathrm{~A}$ \\
\hline
\end{tabular}

\section{Estimation of $\mathrm{f}_{\mathrm{B}}$}

\section{Individual Organs}

Clearance from the soft tissues of rat is very rapid; the uptake from blood to kidneys, liver, and spleen and other soft tissues is less than $\mathrm{m} / \mathrm{m}^{\mathrm{TB}}$ except at very early times (2873). We have there- fore simply assumed that $f_{2}^{\prime}=m / m^{T B}$. The $f_{B}$ were calculated using Eq. (16).

\section{Estimation of $T_{B}$}

\section{Individual Organs}

The $\mathrm{T}_{\mathrm{B}}$ were estimated by Eq. (7) using an I equal to $1.24 \times 10^{-3} \mathrm{~g} /$ day. 
-

-

- 


$$
39^{Y}
$$

Collateral Data

\begin{tabular}{|c|c|c|c|c|}
\hline & Min & $\operatorname{Max}$ & $\mathrm{Av}$ & Ref. \\
\hline \multicolumn{5}{|c|}{ Concentrations $(\mu \mathrm{g} / \mathrm{g})$} \\
\hline Cattle bone & $3 \times 10^{-3}$ & $10^{-2}$ & & 4752 \\
\hline Mouse ash & & & $2.4 \times 10^{-1}$ & 5225 \\
\hline \multicolumn{5}{|c|}{ Uptake relationships } \\
\hline \multicolumn{5}{|c|}{$f_{2}^{\prime}$} \\
\hline Rat kidneys & 0.026 & 0.028 & & $5166 \mathrm{~A}, 5173$ \\
\hline Rat liver & 0.096 & 0.17 & & $5166 \mathrm{~A}, 5173$ \\
\hline Rat spleen & 0.002 & 0.0035 & & $5166 \mathrm{~A}, 5173$ \\
\hline Rat testes & & & 0.0014 & $5166 \mathrm{~A}$ \\
\hline Rat gonads & & & $<0.0002$ & 5173 \\
\hline \multicolumn{5}{|l|}{$\mathrm{f}_{2} / \mathrm{f}_{2}^{\prime}$} \\
\hline Rat kidneys & & & 0.3 & $5166 \mathrm{~A}$ \\
\hline Rat liver & & & 0.1 & $5166 \mathrm{~A}$ \\
\hline Rat spleen & & & 0.75 & $5166 \mathrm{~A}$ \\
\hline Rat testes & & & 0.6 & $5166 \mathrm{~A}$ \\
\hline \multicolumn{5}{|l|}{$\mathrm{f}_{2}$} \\
\hline Bone & & & 0.97 & ICRP \\
\hline Rat kidneys & 0.007 & 0.013 & & $5166 \mathrm{~A}, 5173$ \\
\hline Rat liver & 0.0056 & 0.044 & & $5166 \mathrm{~A}, 5173$ \\
\hline Rat spleen & 0.0018 & 0.0027 & & $5166 \mathrm{~A}, 5173$ \\
\hline Rat testes & & & 0.00084 & $5166 \mathrm{~A}$ \\
\hline Rat gonads & & & $<0.00016$ & 5173 \\
\hline
\end{tabular}

\section{Estimation of $f_{B}$}

\section{Individual Organs}

The $f_{2}^{\prime}$ in ovaries was estimated from $f_{2}^{\prime}$ in the gonads by Eq. (18). The $f_{B}$ in the ovaries and other organs were estimated by Eq. (16).

\section{Estimation of $T_{B}$}

Individual Organs

\section{Estimation of $\mathrm{C}_{\mathrm{B}}$}

\section{Whole Body}

The maximum $\mathrm{C}_{\mathrm{P}} / \mathrm{C}_{\mathrm{S}}$ ratio of the lanthanides ${ }^{*}$ collectively is about 0.1 in crop plants (2540), and individual lanthanides appear to distribute in plants in essentially the same proportions as in

\footnotetext{
* Yttrium is included among the lanthanides.
}

(continued) 
soil (5225). The $\mathrm{C}_{\mathrm{P}} / \mathrm{C}_{\mathrm{S}}$ ratios of cerium in edible parts of plants have been found to vary between $10^{-5}$ and $1(1521,5219)$.

While the average and most probable value of $\mathrm{C}_{\mathrm{P}} / \mathrm{C}_{\mathrm{S}}$ would be less than 0.1 , we have adopted the upper limit

$$
\mathrm{C}_{\mathrm{P}} / \mathrm{C}_{\mathrm{S}}=1
$$

which is in keeping with the conservative aspects of the analysis.

Yttrium is a bone-seeker with $f_{2}^{\prime}$ and $f_{2}$ in bone both exceeding 0.5 (5593). Also the $f_{2}^{\prime}$ of lanthanides in muscle is relatively low (2114). Accordingly we have conservatively assumed that

$$
\mathrm{C}_{\text {beef }}=\mathrm{C}_{\text {steer }}^{\mathrm{TB}} \text {. }
$$

(1) Estimation of $C_{P}$ by Eq. (14):

$$
\mathrm{C}_{\mathrm{P}}=5 \times 10^{-5} \mathrm{~g} / \mathrm{g} \text {. }
$$

(2) Estimation of $I_{\text {steer }}$ by Eq. (13):

$$
I_{\text {steer }}=5 \times 10^{-1} \mathrm{~g} / \text { day } \text {. }
$$

(3) Estimation of $\mathrm{C}_{\text {steer }}^{\mathrm{TB}}$ by Eq. (10):

$$
\mathrm{C}_{\text {steer }}^{\mathrm{TB}}=2 \times 10^{-7} \mathrm{~g} / \mathrm{g} \text {. }
$$

(4) Estimation of I by Eq. (12):

$$
\begin{aligned}
\mathrm{C}_{\text {meat }} & =2 \times 10^{-7} \mathrm{~g} / \mathrm{g}, \\
I & =1.3 \times 10^{-2} \mathrm{~g} / \text { day } .
\end{aligned}
$$

(5) $C_{B}^{T B}$ was then estimated by Eq. (10).

\section{Individual Organs}

The $\mathrm{f}_{2}$ in ovaries was estimated from $f_{2}$ in the gonads by Eq. (25). The $C_{B}$ in the ovaries and other organs were then estimated by Eq. (22).

\section{Estimation of $\mathrm{C}_{\text {meat }}$}

Since no data for yttrium in meat have been found, we have assumed that $\mathrm{C}_{\text {meat }} / \mathrm{C}_{\mathrm{S}}$ is the same as $\mathrm{C}_{\text {meat }} / \mathrm{C}_{\mathrm{S}}$ of samarium, which is the highest value reported for the lanthanides. The value of $\mathrm{C}_{\text {meat }}$ was then estimated from the product of the ratio and $\mathrm{C}_{\mathrm{S}}$. 


$$
40^{\mathrm{Zr}}
$$

Collateral Data

\begin{tabular}{|c|c|c|c|c|}
\hline & Min & $\operatorname{Max}$ & $\mathrm{Av}$ & Ref. \\
\hline \multicolumn{5}{|c|}{$\begin{array}{l}\text { Uptake relationships } \\
\mathrm{f}_{2}^{\prime}\end{array}$} \\
\hline Rat testes & 0.01 & 0.02 & & $5166 \mathrm{~A}, 5170$ \\
\hline \multicolumn{5}{|l|}{$\mathrm{f}_{2}$} \\
\hline Bone & & & 0.8 & ICRP \\
\hline \multicolumn{5}{|l|}{$\mathrm{f}_{2} / \mathrm{f}_{2}^{\prime}$} \\
\hline Rat testes & & & 1.8 & 5170,5171 \\
\hline
\end{tabular}

Estimation of $f_{B}$

\section{Indi vidual Organs}

The $\mathrm{f}_{B}$ in testes was estimated by Eq. (16).

\section{Estimation of $\mathrm{T}_{\mathrm{B}}$}

\section{Individual Organs}

The $\mathrm{T}_{\mathrm{B}}$ in testes was estimated by Eq. (19)

\section{Estimation of $\mathrm{C}_{\mathrm{B}}$}

Individual Organs

The concentrations of zirconium in human tissues reported by Schroeder (5126) exceed other reported concentra- tions of zirconium in animals and man (2541, 4941, 5593). ${ }^{*}$ Schroeder's concentrations seem to be inconsistent with these others and with the uptake and retention behavior of zirconium. We therefore have chosen not to adopt Schroeder's values. If we had adopted his values, our estimate of $\mathrm{C}_{B}$ in bone would be greater by about a factor of 50 and $C_{B}$ in the other organs would be greater by about a factor of $10 . F_{1}$ values via soil would then decrease proportionately.

The $\mathrm{C}_{\mathrm{B}}$ in bone was estimated by Eq. (22).

\footnotetext{
*Also L. Anspaugh, Bio-Medical Division, Lawrence Radiation Laboratory, Livermore, Calif., unpublished data.
}

(continued) 
Estimation of Aquatic Data

Known Data

\begin{tabular}{|c|c|c|c|c|}
\hline & Min & $\operatorname{Max}$ & $\mathrm{Av}$ & Ref. \\
\hline \multicolumn{5}{|l|}{ Concentration } \\
\hline \multirow[t]{2}{*}{ Seawater } & & & $4 \times 10^{-3}$ & 6013 \\
\hline & & & $3 \times 10^{-5}$ & 5996 \\
\hline Freshwater & $5 \times 10^{-5}$ & $2 \times 10^{-2}$ & $3 \times 10^{-3}$ & 5779 \\
\hline Seawater plankton & & & 4.0 & 5997 \\
\hline Seawater invertebrates & $2 \times 10^{-1}$ & 1.3 & $6 \times 10^{-1}$ & 5126 \\
\hline \multirow[t]{2}{*}{ Seawater fish } & $5 \times 10^{-1}$ & $8 \times 10^{-1}$ & $7 \times 10^{-1}$ & 5126 \\
\hline & & & $2 \times 10^{1}$ & 2702 \\
\hline \multicolumn{5}{|l|}{ Concentration factors } \\
\hline \multirow[t]{4}{*}{ Seawater plants } & 400 & 1000 & & 5335 \\
\hline & 76 & 222 & 153 & 5318 \\
\hline & 200 & $>3000$ & & 6012 \\
\hline & & & 1000 & 506 \\
\hline \multirow[t]{2}{*}{ Freshwater plants } & 400 & 8000 & & 5335 \\
\hline & 200 & 3000 & 1000 & 6012 \\
\hline \multirow[t]{2}{*}{ Seawater invertebrates } & & & 1000 & 5335 \\
\hline & & & 1500 & 506 \\
\hline Freshwater invertebrates & 10 & 160 & 70 & 6012 \\
\hline \multirow[t]{2}{*}{ Seawater fish } & 1 & 30 & & 5335 \\
\hline & & & 10 & 1528 \\
\hline Freshwater fish & & & 6 & 6012 \\
\hline
\end{tabular}

\section{Estimation of $\mathrm{C}_{\mathrm{A}}$ for Seawater}

The $\mathrm{Ti} / \mathrm{Zr}$ ratio in soil and in freshwater is roughly equal to 10 . This value was also adopted for seawater. This leads to a value of $3 \times 10^{-4}$ which is in the middle of the range of measured values.

\section{Estimation of Concentration in Seawater} Foodstuffs

The following concentration factors are suggested by the known data

$\begin{array}{lr}\text { Plants } & 10,000 \\ \text { Invertebrates } & 1,000 \\ \text { Fish } & 100\end{array}$

The concentrations in seawater foodstuffs were determined by multiplying these factors by the seawater concentration. This leads to values that are lower than the Schroeder values (5126), particularly for fish. However, as stated under "Estimation for $\mathrm{C}_{\mathrm{B}}$ " above, there is reason to believe that his values are high by at least a factor of 10 . The concentration of $20 \mu \mathrm{g} / \mathrm{g}$ for fish in Ref. 2702 is based upon a single fish.

\section{Estimation of Concentration in Fresh-} water Foodstuffs

These concentrations were determined by using the concentration factors above and the concentration in freshwater. 
${ }_{41} \mathrm{Nb}$

Collateral Data

\begin{tabular}{|c|c|c|c|c|}
\hline & Min & $\operatorname{Max}$ & Av & Ref. \\
\hline \multicolumn{5}{|c|}{$\begin{array}{l}\text { Uptake relationships } \\
\mathrm{f}_{2}^{\prime}\end{array}$} \\
\hline Bone & & & 0.38 & ICRP \\
\hline Kidneys & & & 0.02 & ICRP \\
\hline Liver & & & 0.09 & ICRP \\
\hline Spleen & & & 0.008 & ICRP \\
\hline
\end{tabular}

\section{Estimation of $f_{B}$}

\section{Whole Body}

We should take into account that the $f_{1}$ of niobium may be greater than the ICRP value, $f_{1}=10^{-4}$. Niobium and tantalum are second- and third-transition group congeners with very similar atomic and ionic dimensions, and the two elements are very closely associated in nature. According to Schroeder (2219) over half the niobium present in the diet is absorbed. In other study $5 \%$ of an initial $\mathrm{Ta}^{182}$ deposition in the abdominal region had not been eliminated after 5 days (1485), so that the $f_{1}$ may be of the order of $5 \%$. We therefore have assumed that $f_{1}$ and $\mathrm{f}_{\mathrm{B}}^{\mathrm{TB}}$ are equal to 0.05 .

\section{Individual Organs}

The $f_{B}$ were estimated by Eq. (16). 
Collateral Data

\begin{tabular}{lllll}
\hline & Min & Max & Av & Ref. \\
\hline $\begin{array}{l}\text { Uptake relationship } \\
\text { I (g/day) }\end{array}$ & & $1.0 \times 10^{-4}$ & 3869 \\
\hline
\end{tabular}

\section{Estimation of $f_{B}$}

\section{Individual Organs}

A major fraction of molybdenum absorbed in the gastrointestinal tract is very rapidly excreted $(5181,5182,5275)$. We have estimated $f_{2}^{\prime}$ based on early deposition in rats (see below, 5181, 5182). Because of the very rapid excretion these values are over-conservative. These $f_{2}^{\prime}$ are assumed to apply to man.

Estimation of $f_{2}^{\prime}$ and $f_{2}^{\prime} / m$

\begin{tabular}{lll}
\hline Organs & $f_{2}^{\prime}$ & $\left.\mathrm{f}_{2}^{\prime} / \mathrm{m}^{-1} \mathrm{~g}^{-1}\right)$ \\
\hline Bone & 0.12 & $1.9 \times 10^{-5}$ \\
Kidneys & 0.06 & $1.9 \times 10^{-4}$ \\
Liver & 0.30 & $1.7 \times 10^{-5}$ \\
Spleen & 0.01 & $5.7 \times 10^{-5}$ \\
\hline
\end{tabular}

The $\mathrm{f}_{2}^{\prime} / \mathrm{m}$ in the ovaries and testes have been shown to be comparable and less than $f_{2}^{\prime} / m$ in bone, kidneys, liver, and spleen.* The $f_{2}^{\prime} / m$ in the ovaries and testes were therefore assumed to be $2 \times 10^{-5} \mathrm{~g}^{-1}$, which compares with the lowest values for the organs above. The $f_{2}^{\prime}$ in ovaries and testes were calculated by Eq. (16a); the $f_{B}$ in the organs of interest were then estimated by Eq. (16).

\section{Estimation of $T_{B}$}

\section{Whole Body}

The $\mathrm{T}_{B}^{\mathrm{TB}}$ estimated by Eq. (8) was about 100 days. The $T_{B}^{T B}$ was also estimated from excretion data of patients given intravenous $\mathrm{Mo}^{99}$ (5274). The $\mathrm{T}_{B}^{\mathrm{TB}}$ in 2 patients who were studied for 10 days was about 25 days. Our final choice of $\mathrm{T}_{\mathrm{B}}^{\mathrm{TB}}, 100$ days, minimizes the $\mathrm{F}_{1}$ of $\mathrm{Mo}^{93}$ via milk and influences only slightly its $F_{1}$ via soil, and has little effect on the $\mathrm{F}_{1}$ of $\mathrm{Mo}^{99}$ (via milk).

\section{Individual Organs}

The $\mathrm{T}_{\mathrm{B}}$ were estimated by Eq. (7).

*J. Barr and B. Shore, Bio-Medical Division, Lawrence Radiation Laboratory, Livermore, Calif., unpublished data. 


$$
43^{\mathrm{Tc}}
$$

\section{Collateral Data}

\begin{tabular}{|c|c|c|c|c|}
\hline & Min & $\operatorname{Max}$ & $\mathrm{Av}$ & Ref. \\
\hline \multicolumn{5}{|c|}{$\begin{array}{l}\text { Uptake relationships } \\
\mathrm{f}_{2}^{\prime}\end{array}$} \\
\hline Bone & & & 0.002 & ICRP \\
\hline Kidneys & & & 0.01 & ICRP \\
\hline Liver & & & 0.003 & ICRP \\
\hline
\end{tabular}

\section{Estimation of $\mathrm{C}_{\mathrm{S}}$}

The technetium occurring in nature is assumed to be the $\mathrm{Tc}^{99}$ derived from uranium. Other isotopes have not been found in nature. The $\mathrm{C}_{\mathrm{S}}$ was estimated by Eq. (34) from the following concentrations of $\mathrm{Tc}^{99}$ and $U$ in pitchblende (5763):

$$
\begin{array}{ll}
43 \mathrm{Tc}^{99} & 10^{-7} \mu \mathrm{g} / \mathrm{g} \\
92 \mathrm{U} & 4.2 \times 10^{5} \mu \mathrm{g} / \mathrm{g}
\end{array}
$$

\section{Estimation of $f_{B}$}

Whole Body

The fraction of technetium absorbed in man following ingestion was about 0.95 (5443). We therefore have assumed that $\mathrm{f}_{1}$ and $\mathrm{f}_{\mathrm{B}}^{\mathrm{TB}}$ are equal to 1 .

\section{Individual Organs}

The $f_{B}$ in bone, kidneys and liver were estimated by Eq. (16).

\section{Estimation of $\mathrm{T}_{\mathrm{B}}$}

\section{Whole Body}

The apparent biological half-life in man calculated from human retention data (5443) was about 3 days. The apparent biological half-life is the half-life associated with the simple exponential biological retention curve whose integral is equal to that of the experimental retention curve.

\section{Estimation of $\mathrm{C}_{B}$}

\section{Whole Body}

An analysis of studies on uptake into plants including those cited by Menzel (5227) and those in the table on p. 13 leads to the conclusion that the greatest $\mathrm{C}_{\mathrm{P}} / \mathrm{C}_{\mathrm{S}}$ ratio that can be assigned to an element in a broad spectrum of crop plants is about 100 . In the absence of data on the uptake of technetium into plants we have conservatively assumed that

$$
\mathrm{C}_{\mathrm{P}} / \mathrm{C}_{\mathrm{S}}=100
$$

The concentration in meat $\mathrm{C}_{\text {meat }}$ is assumed to be equal to a conservative $\mathrm{C}_{\text {beef }}$. The $\mathrm{f}_{2} / \mathrm{m}$ and $\mathrm{f}_{2} / \mathrm{m}$ of Tc in muscle are both relatively low (5170), so that conservatively

$$
\mathrm{C}_{\text {beef }}=\mathrm{C}_{\text {steer }}^{\mathrm{TB}} \text {. }
$$

(1) Estimation of $C_{P}$ by Eq. (14) : (continued) 


$$
\mathrm{C}_{\mathrm{P}}=10^{-17} \mathrm{~g} / \mathrm{g} \text {. }
$$

(2) Estimation of $I_{\text {steer }}$ by Eq. (13):

$$
I_{\text {steer }}=10^{-13} \mathrm{~g} / \text { day. }
$$

(3) Estimation of $C_{\text {steer }}^{\text {TB }}$ by Eq. (10):

$$
\mathrm{C}_{\text {steer }}^{\mathrm{TB}}=8.7 \times 10^{-19} \mathrm{~g} / \mathrm{g} \text {. }
$$

(4) Estimation of I by Eq. (12):

$$
I=7.8 \times 10^{-15} \mathrm{~g} / \text { day. }
$$

(5) $C_{B}^{T B}$ was then estimated by Eq. (10)

\section{Estimation of $C_{P}$}

The pertechnetate ion, a soluble and stable form of technetium, competes with the iodide ion in active transport to the thyroid $(947,6352)$. In the absence of plant data, we have assumed that the $\mathrm{C}_{\mathrm{P}} / \mathrm{C}_{\mathrm{S}}$ ratios of technetium and iodine are comparable. A similar $\mathrm{C}_{\mathrm{P}} / \mathrm{C}_{\mathrm{S}}$ ratio is noted for manganese, the first transition series element related to technetium. We therefore adopted a $\mathrm{C}_{\mathrm{P}} / \mathrm{C}_{\mathrm{S}}$ value of 1.0 . The $C_{P}$ was then estimated by Eq. (14).

\section{Estimation of Aquatic Data}

In all cases technetium was assumed to be similar to iodine and all values were determined by multiplying the iodine values by $2 \times 10^{-14}$, the $\mathrm{Tc} / \mathrm{I}$ ratio in soil. 
${ }_{44}^{\mathrm{Ru}}$

Collateral Data

\begin{tabular}{|c|c|c|c|c|}
\hline & Min & $\operatorname{Max}$ & Av & Ref. \\
\hline \multicolumn{5}{|c|}{ Concentrations $(\mu \mathrm{g} / \mathrm{g})$} \\
\hline Beef & & & $7 \times 10^{-4}$ & 4941 \\
\hline Pork & & & $5 \times 10^{-4}$ & 4941 \\
\hline Ham & & & $2 \times 10^{-3}$ & 4941 \\
\hline Chicken & & & $6 \times 10^{-4}$ & 4941 \\
\hline \multicolumn{5}{|c|}{$\begin{array}{l}\text { Uptake relationships } \\
\mathrm{f}_{2}^{\prime}\end{array}$} \\
\hline Rat bone & 0.05 & 0.11 & & $4293,5166,5182,5909$ \\
\hline Rat kidneys & 0.03 & 0.06 & & $4293,5166,5182,5909$ \\
\hline Rat liver & 0.027 & 0.21 & & $4293,5166,5182,5909$ \\
\hline Rat ovaries & & & 0.0003 & 5909 \\
\hline Rat spleen & 0.005 & 0.013 & & $4293,5166,5182,5909$ \\
\hline Rat testes & 0.0044 & 0.0085 & & 4293 \\
\hline
\end{tabular}

\section{Estimation of $\mathrm{C}_{\mathrm{S}}$}

The $f_{2}$ in muscle is equal to the ratio of organ to total body weight, so that

$$
\mathrm{C}_{\text {steer }}^{\mathrm{TB}}=\mathrm{C}_{\text {beef }} \text {. }
$$

(See "Estimation of $\mathrm{C}_{B}$ " for this element below.)

The $\mathrm{C}_{\mathrm{P}} / \mathrm{C}_{\mathrm{S}}$ ratio of ruthenium in leaves and stems of crop plants at or approaching maturity varied between 0.02 and $0.7(1521,5219)$. In order to minimize $\mathrm{C}_{\mathrm{S}}$ using Eq. (14), we assumed a $C_{P} / C_{S}$ ratio of 0.7 .

(1) Estimation of $I_{\text {steer }}$ by Eq. (4):

$$
\begin{aligned}
& C_{\text {steer }}^{\mathrm{TB}}=10^{-9} \mathrm{~g} / \mathrm{g}, \\
& \mathrm{I}_{\text {steer }}=8.9 \times 10^{-5} \mathrm{~g} / \text { day. }
\end{aligned}
$$

(2) Estimation of $\mathrm{C}_{\mathrm{P}}$ by Eq. (15):

$$
\mathrm{C}_{\mathrm{P}}=8.9 \times 10^{-9} \mathrm{~g} / \mathrm{g} \text {. }
$$

(3) $\mathrm{C}_{\mathrm{S}}$ was then estimated using Eq. (14).

The $\mathrm{C}_{\mathrm{S}}$ estimated by Eq. (14) is greater than the concentration in basic rocks (5799) by a factor of 10 . It is difficult to conceive of a mechanism which could result in a 10-fold increase in the concentration of $\mathrm{Ru}$ during the process of soil formation. The ICRP uptake parameters therefore may not be appropriate for cattle and man. This is further discussed in the next section, "Estimation of $\mathrm{f}_{\mathrm{B}}$." We have adopted the concentration in basic rocks as the value for $\mathrm{C}_{\mathrm{S}}$.

\section{Estimation of $f_{B}$}

Whole Body

As shown in the preceding section, the ICRP value of $f_{1}$ would lead to a 10 -fold overestimate of $\mathrm{C}_{\mathrm{S}}$. A similar discrepancy was noted by Iwashima (6048), who 
found that the ICRP uptake parameters led to underestimates of the $\mathrm{Ru}^{106}$ burden in human muscle by a factor of 10 to 100 . Part of this discrepancy observed by Iwashima could be accounted for by his use of the ICRP value of $T_{B}, 7.3$ days. As shown in the section, "Estimation of $T_{B}$," this appears to be low by a factor of 10 . Considering Iwashima's results and the results obtained from the estimation of $\mathrm{C}_{\mathrm{S}}$, it seems possible that the ICRP value for $f_{1}$ is in error by an order of magnitude. It may be that the $\mathrm{Ru}$ incorporated in food chains is more available than the $\mathrm{Ru}$ isotopes introduced in tracer experiments. We therefore have adopted a value for $f_{B}^{T B}$ and $f_{1}$ that is 10 times greater than the ICRP value.

\section{Individual Organs}

In the absence of human data the $f_{2}^{\prime}$ for rat organs are assumed to apply to man, and the $f_{B}$ were estimated by Eqs. (16) and (16a).

\section{Estimation of $T_{B}$}

\section{Whole Body}

In long-term measurements on the retention of $\mathrm{Ru}^{106}$, the apparent biological half-life in the total body was about 110 days in the rat (2396) and about 120 days in the dog (2397). The apparent biological half-life is the half-life associated with the simple exponential biological retention curve whose integral is equal to that of the experimental retention curve. The $T_{B}^{T B}$ in man would be expected to exceed that in dog and rat (4419). Therefore, a $T_{B}^{T B}$ of 130 days has been adopted.

\section{Individual Organs}

The $\mathrm{f}_{2} / \mathrm{f}_{2}^{\prime}$ ratios were calculated from the $\mathrm{f}_{2}$ adopted for estimating $\mathrm{C}_{B}$ and the $f_{2}^{\prime}$ adopted for estimating $f_{B}$. The $T_{B}$ were then estimated by Eq. (19).

\section{Estimation of $\mathrm{C}_{\mathrm{B}}$}

\section{Whole Body}

The $C_{B}^{T B}$ was estimated by Eq. (23) from the $f_{2}$ and $C_{B}$ in muscle. In the absence of human data the $C_{B}$ in muscle was assumed to be $10^{-3} \mu \mathrm{g} / \mathrm{g}$, the concentration in beef. The mass of muscle was $2.7 \times 10^{4} \mathrm{~g}(5307)$ and the $\mathrm{f}_{2}$ in muscle was the value derived below.

\section{Individual Organs}

Relative organ burdens in rats following chronic ruthenium feeding are shown below (5911).

Estimates of $\mathrm{f}_{2} / \mathrm{m}$ from studies on rats chronically fed Ru (5911)

$$
\mathrm{f}_{2} / \mathrm{m}
$$

(relative units)

\begin{tabular}{lr}
\hline Bone & 130 \\
Kidneys & 580 \\
Liver & 140 \\
Spleen & 200 \\
Testes & 43 \\
Muscle & 32 \\
\hline
\end{tabular}

(continued) 
In a similar set of experiments the $f_{2}$ in testes was shown to exceed that in ovaries by about a factor of 15 (5910). Since in rats weight of testes exceeds weight of ovaries by about the same factor $(3276)$, we have assumed that the $f_{2} / m$ are equal in ovaries and testes. In the absence of human data the relative $f_{2} / \mathrm{m}$ values from rat were assumed for man. The relative $f_{2}$ values below were determined as the product of $\mathrm{f}_{2} / \mathrm{m}$ and the respective human organ masses. The relative $f_{2}$ were then normalized to 1.0 using the sum of the relative $f_{2}$.

\footnotetext{
*Also, A. Silva and D. Fleshman, Bio-Medical Division, Lawrence Radiation Laboratory, Livermore, Calif., personal communication.
}

(1) Estimation of $f_{2}$ in man from $f_{2} / m$ :

\begin{tabular}{lll}
\hline & \multicolumn{1}{c}{$\mathrm{f}_{2}$} & \\
& (relative units) & \multicolumn{1}{c}{$\mathrm{f}_{2}$} \\
\hline Bone & $8.3 \times 10^{5}$ & 0.385 \\
Kidneys & $1.8 \times 10^{5}$ & 0.083 \\
Liver & $2.5 \times 10^{5}$ & 0.12 \\
Ovaries & $3.4 \times 10^{2}$ & 0.00016 \\
Spleen & $3.5 \times 10^{4}$ & 0.016 \\
Testes & $2.6 \times 10^{3}$ & 0.0012 \\
Muscle & $8.6 \times 10^{5}$ & 0.40 \\
\multicolumn{1}{c}{ Total } & $2.2 \times 10^{6}$ & $\mathbf{1 . 0}$ \\
\hline
\end{tabular}

(2) The $C_{B}$ were then estimated by Eq. (22).

\section{Estimation of $C_{P}$}

The $\mathrm{C}_{\mathrm{P}} / \mathrm{C}_{\mathrm{S}}$ ratio of ruthenium in crop plants was estimated to be $0.2(1521,5219)$. The $\mathrm{C}_{\mathrm{P}}$ was estimated by Eq. (14). 


$$
45^{R h}
$$

Collateral Data

\begin{tabular}{lcccc} 
& Min & Max & Av & Ref. \\
\hline $\begin{array}{l}\text { Uptake relationships } \\
f_{2}^{\prime}\end{array}$ & & & \\
Rat gonads & & 0.0055 & 5182 \\
\hline
\end{tabular}

\section{Estimation of $\mathrm{C}_{\mathrm{S}}$}

Rhodium has not been reported in soil. Its concentration in soil-forming rocks and shales ranges from $2 \times 10^{-4}$ to $2 \times 10^{-2} \mu \mathrm{g} / \mathrm{g}(5778,5799)$ averaging $4 \times 10^{-3} \mu \mathrm{g} / \mathrm{g}$ (5222). Rhodium is resis tant to weathering and may be expected to concentrate in some soils (5309).

\section{Estimation of $f_{B}$}

\section{Individual Organs}

In the absence of human values, the rat values are assumed to apply to man. The $f_{2}^{\prime}$ in the ovaries and testes were estimated from $f_{2}^{\prime}$ in the gonads by Eq. (18). The $f_{B}$ values were then estimated by Eq. (16).

\section{Estimation of $\mathrm{C}_{P}$}

Rhodium and iridium were assumed to behave similarly. The $\mathrm{C}_{\mathrm{P}} / \mathrm{C}_{\mathrm{S}}$ ratio of rhodium was assumed to be that of iridium. The $C_{P}$ was then estimated by Eq. (14).

\section{Estimation of $C_{\text {meat }}$}

In the absence of data, the $\mathrm{C}_{\text {meat }} / \mathrm{C}_{\mathrm{S}}$ ratios of rhodium and iridium were assumed to be comparable. The $\mathrm{C}_{\text {meat }} / \mathrm{C}_{\mathrm{S}}$ was assumed to be 1 so that the $C_{\text {meat }}$ equals $\mathrm{C}_{\mathrm{S}}$.

\section{Estimation of Aquatic Data}

The ratio of $X / R u$ in soil for the platinum metals is:

\begin{tabular}{ccccc}
$\mathrm{X}$ & Ratio & & $\mathrm{X}$ & Ratio \\
\cline { 1 - 2 } $\mathrm{Ru}$ & 1.0 & & $\mathrm{Os}$ & 1.0 \\
$\mathrm{Rh}$ & 1.0 & & $\mathrm{Ir}$ & 0.01 \\
$\mathrm{Pd}$ & 1.0 & & $\mathrm{Pt}$ & 10.0
\end{tabular}

These ratios were multiplied by the ruthenium values to obtain the values for the other elements. 
${ }_{46} \mathrm{Pd}$

Collateral Data

\begin{tabular}{llll}
\hline & Min & Max & Av \\
\hline $\begin{array}{l}\text { Uptake relationships } \\
\mathrm{f}_{2}^{\prime}\end{array}$ & & \\
$\quad$ Rat bone & 0.02 & 5182 \\
$\quad$ Rat gonads & 0.002 & 5182 \\
$\mathrm{f}_{2} / \mathrm{f}_{2}^{\prime}$ & & \\
$\quad$ Rat bone & 1.3 & 5182 \\
\hline
\end{tabular}

Estimation of $\mathrm{C}_{\mathrm{S}}$

Palladium has not been reported in soil. Its concentration in soil-forming rocks and shales ranges from $1 \times 10^{-4}$ to $4 \times 10^{-2} \mu \mathrm{g} / \mathrm{g}(5778,5779,5792)$ averaging $2 \times 10^{-3} \mu \mathrm{g} / \mathrm{g}$ (5222). Palladium is resistant to weathering and may be expected to concentrate in some soils (5309).

\section{Estimation of $f_{B}$}

\section{Individual Organs}

In the absence of human data, the rat values are assumed to apply to man. The $\mathrm{f}_{2}^{\prime}$ in the ovaries and testes were estimated from $f_{2}^{\prime}$ in the gonads by Eq. (18) and are based on data from 3 rats $(5182)$. The $\mathrm{f}_{B}$ in these organs and bone were then estimated by Eq. (16).

Estimation of $\mathrm{T}_{\mathrm{B}}$

Individual Organs

The $\mathrm{T}_{\mathrm{B}}$ in bone was estimated by Eq. (19) using the data for rat bone.

\section{Estimation of $\mathrm{C}_{\mathrm{P}}$}

Palladium and platinum were assumed to behave similarly. Their concentrations in meat were comparable (4941). The $\mathrm{C}_{\mathbf{P}}$ of palladium, therefore, was assumed to be that of platinum. 
${ }_{47}^{\mathrm{Ag}}$

Collateral Data

\begin{tabular}{|c|c|c|c|c|}
\hline & Min & $\operatorname{Max}$ & $\mathrm{Av}$ & Ref. \\
\hline \multicolumn{5}{|c|}{ Uptake relationships } \\
\hline \multicolumn{5}{|l|}{$f_{2}^{\prime}$} \\
\hline Bone & & & 0.05 & ICRP \\
\hline Kidneys & & & 0.02 & ICRP \\
\hline Liver & & & 0.03 & ICRP \\
\hline Rat ovaries & & & 0.0006 & 2324,5169 \\
\hline Rat spleen & & & 0.0011 & 2324,5169 \\
\hline I (g/day) & $3.5 \times 10^{-5}$ & $4.4 \times 10^{-5}$ & & 3869 \\
\hline
\end{tabular}

Estimation of $f_{B}$

\section{Whole Body}

There are data indicating that the $f_{1}$ in humans is greater than the ICRP value, $f_{1}=0.01$. A minimum value for $f_{1}$ in humans based on the ratio of the average daily urinary excretion and the average daily intake is 0.25 (3869A). The $f_{1}$ exceeds this minimum value, since the major fraction of the absorbed isotope is secreted in bile and excreted in feces (2324, 3869A, 5169). Therefore, we have assumed that $f_{1}$ and $f_{B}^{T B}$ are equal to 1.0 .

\section{Individual Organs}

In the absence of human data, the $\mathrm{f}_{2}^{\prime}$ in rat are assumed to apply to man. The uptake per gram in rat testes at 4 days was comparable to the uptake per gram in liver (2324). We therefore have assumed that the $f_{2}^{\prime} / m$ in human testes is equal to the $f_{2}^{\prime} / \mathrm{m}$ in liver and estimated $\mathrm{f}_{2}^{\prime}$ by Eq. (16a). The $\mathrm{f}_{\mathrm{B}}$ were estimated by Eq. (16).

\section{Estimation of $T_{B}$}

\section{Individual Organs}

The $T_{B}$ were estimated by Eq. (7) using an $I$ of $3.5 \times 10^{-5} \mathrm{~g} /$ day. 
${ }_{48} \mathrm{Cd}$

\begin{tabular}{|c|c|c|c|c|}
\hline \multicolumn{5}{|c|}{ Collateral Data } \\
\hline & Min & $\operatorname{Max}$ & $\mathrm{Av}$ & Ref. \\
\hline \multicolumn{5}{|c|}{ Uptake relationships } \\
\hline \multicolumn{5}{|c|}{$f_{2}^{\prime}$} \\
\hline Rat bone & & & 0.036 & 5170 \\
\hline Rat ovaries & & & 0.00076 & 5170 \\
\hline Rat spleen & & & 0.0037 & 5170 \\
\hline \multicolumn{5}{|l|}{$\mathrm{f}_{2}$} \\
\hline Rat bone & & & 0.022 & 5170 \\
\hline Rat ovaries & & & 0.00025 & 5170 \\
\hline Rat spleen & & & 0.0041 & 5170 \\
\hline
\end{tabular}

\section{Estimation of $f_{B}$}

\section{Individual Organs}

In the absence of data from humans we have assumed that the rat data apply to man. The $f_{B}$ in bone, ovaries, and spleen were estimated using Eq. (16).

The $C_{B}$ in testes is substantially lower than that in liver. Thus either $f_{2}^{\prime} / \mathrm{m}$ or $T_{B}$, or both, are lower in testes than in liver. In accordance with the discussion of Eq. (30), we have chosen to maximize $f_{B}$ and therefore set $f_{2}^{\prime} / m$ in the testes equal to that in liver. Then $f_{B}$ in the testes was estimated by Eqs. (16) and (16a). The $\mathrm{f}_{\mathrm{B}}$ in bone, ovaries and spleen were estimated by Eq. (16).

\section{Estimation of $T_{B}$}

Individual Organs

The $\mathrm{T}_{\mathrm{B}}$ were estimated by Eq. (19) using the rat data.

Estimation of $C_{B}$

\section{Individual Organs}

The $\mathrm{C}_{\mathrm{B}}$ in bone was estimated by Eq. (22). 
Estimation of $C_{S}$

Indium has not been reported in soil. Its concentration in soil-forming rocks and shales ranges from $3 \times 10^{-3}$ to $1.0 \mu \mathrm{g} / \mathrm{g}(5231,5792)$. Its average concentration in the earth's crust is $2.5 \times 10^{-1} \mu \mathrm{g} / \mathrm{g}(5801)$.

\section{Estimation of $\mathrm{C}_{\mathrm{P}}$}

The $\mathrm{C}_{\mathrm{P}} / \mathrm{C}_{\mathrm{S}}$ ratio of indium has been found to be less than 2.5 in mustard leaves (5206) and between 0.64 and 2.1 in citrus leaves (2826). The $\mathrm{C}_{\mathrm{P}}$ of indium in kale leaves was $<0.3 \mathrm{ppm}$ (6089). We have assumed a $C_{P} / C_{S}$ ratio for indium of 1. The $C_{P}$ was estimated from Eq. (14).

\section{Estimation of Aquatic Data}

\section{$\frac{\text { Estimation of } C_{A}}{\text { water }}$ for Seawater and Fresh-}

The ratio of $\mathrm{In} / \mathrm{Ga}$ in soil is $1 / 300$. This ratio was multiplied by the gallium concentration to obtain the indium concentration.

$50^{\mathrm{Sn}}$

Collateral Data

\begin{tabular}{lllll}
\hline & Min & Max & Av & Ref. \\
\hline $\begin{array}{l}\text { Uptake relationships } \\
f_{2}^{\prime}\end{array}$ & & & \\
Rat kidneys & & 0.022 & 5173 \\
Rat spleen & & 0.0009 & 5173 \\
Rat gonads & & 0.0007 & 5173 \\
I (g/day) & $1.49 \times 10^{-3}$ & $2.48 \times 10^{-3}$ & & $3869 \mathrm{~A}$ \\
\hline
\end{tabular}

\section{Estimation of $f_{B}$}

Individual Organs

In the absence of human data, the rat data are assumed to apply to man. The $f_{2}^{\prime}$ in the ovaries and testes were estimated from $f_{2}^{\prime}$ in the gonads by Eq. (18). The $f_{B}$ values were estimated by Eq. (16).

\section{Estimation of $T_{B}$}

Whole Body

The $\mathrm{T}_{\mathrm{B}}^{\mathrm{TB}}$ was calculated by Eq. (8) using an I equal to $1.5 \times 10^{-3} \mathrm{~g} /$ day.

\section{Individual Organs}

The $T_{B}$ values were estimated by Eq. (7) using an I equal to $1.5 \times 10^{-3}$ g/day. 


$$
51^{\mathrm{Sb}}
$$

\section{Collateral Data}

\begin{tabular}{|c|c|c|c|c|}
\hline & Min & $\operatorname{Max}$ & Av & Ref \\
\hline \multicolumn{5}{|c|}{ Concentrations $(\mu \mathrm{g} / \mathrm{g})$} \\
\hline Bovine heart & $1.7 \times 10^{-3}$ & $4.8 \times 10^{-3}$ & & 1745 \\
\hline \multicolumn{5}{|c|}{$\begin{array}{l}\text { Uptake relationships } \\
\mathrm{f}_{2}^{\prime}\end{array}$} \\
\hline Rat kidneys & & & $1.6 \times 10^{-3}$ & 5169 \\
\hline Rat spleen & & & $2.8 \times 10^{-4}$ & 5169 \\
\hline Rat gonads & & & $5.3 \times 10^{-5}$ & 5169 \\
\hline
\end{tabular}

\section{Estimation of $\mathrm{f}_{B}$}

\section{Indi vidual Organs}

In the absence of human data, we have assumed that the rat data apply to man. The $f_{2}^{\prime}$ in the ovaries and testes were estimated from $f_{2}^{\prime}$ in the gonads by
Eq. (18). The $\mathrm{f}_{\mathrm{B}}$ were estimated by Eq. (16).

Estimation of $C_{B}$

Whole Body

The $\mathrm{C}_{\mathrm{B}}^{\mathrm{TB}}$ was estimated by Eq. (27). The $C_{B}$ in muscle was assumed to be the $\mathrm{C}_{\mathrm{B}}$ in bovine heart. 


$$
52^{\mathrm{Te}}
$$

Collateral Data

\begin{tabular}{|c|c|c|c|c|}
\hline & Min & $\operatorname{Max}$ & $\mathrm{Av}$ & Ref. \\
\hline \multicolumn{5}{|c|}{ Concentrations $(\mu \mathrm{g} / \mathrm{g})$} \\
\hline Beef & & & $5 \times 10^{-3}$ & 4941 \\
\hline Chicken & & & $6 \times 10^{-3}$ & 4941 \\
\hline Ham & & & $2 \times 10^{-2}$ & 4941 \\
\hline Pork & & & $3 \times 10^{-3}$ & 4941 \\
\hline \multicolumn{5}{|c|}{ Uptake relationships } \\
\hline \multicolumn{5}{|l|}{$\mathrm{f}_{2}$} \\
\hline Bone & & & 0.18 & ICRP \\
\hline Kidneys & & & 0.14 & ICRP \\
\hline Liver & & & 0.1 & ICRP \\
\hline Spleen & & & 0.02 & ICRP \\
\hline Testes & & & 0.006 & ICRP \\
\hline
\end{tabular}

\section{Estimation of $\mathrm{C}_{\mathrm{S}}$}

Schroeder et al. (5243) report soil concentrations of tellurium ranging from 13.5 to $46.1 \mu \mathrm{g} / \mathrm{g}$. Vinogradov (2532) proposed that "its content is probably ten times less than that of selenium." Our more conservative estimate is based on the concentration in soil-forming rocks, which average $4 \times 10^{-3} \mu \mathrm{g} / \mathrm{g}(5801)$.

\section{Estimation of $f_{B}$}

\section{Individual Organs}

In sheep the $f_{2}^{1} / m$ in the ovaries was comparable to that in spleen (874). We have assumed that the same relationship applies to man and estimated the $f_{B}$ in ovaries using Eq. (16) and (16a).

\section{Estimation of $\mathrm{T}_{\mathrm{B}}$}

\section{Individual Organs}

The retention of tellurium in the ovaries was similar to that in bone, kidneys, liver, and spleen (874). We therefore assigned to the ovaries the same $T_{B}$ in these other organs.

\section{Estimation of $\mathrm{C}_{B}$}

Whole Body

Since tellurium does not localize significantly in the skeleton or in any other organ (4510), a reasonable assumption is that

$$
\mathrm{C}_{\text {steer }}^{\mathrm{TB}}=\mathrm{C}_{\text {beef }} \text {. }
$$


(1) Estimation of $\mathrm{I}_{\text {steer }}$ by Eq. (4):

$$
\begin{aligned}
& C_{\text {steer }}^{\mathrm{TB}}=10^{-8} \mathrm{~g} / \mathrm{g}, \\
& I_{\text {steer }}=9.2 \times 10^{-4} \mathrm{~g} / \text { day. }
\end{aligned}
$$

(2) Estimation of $C_{P}$ by Eq. (15):

$$
\mathrm{C}_{\mathrm{P}}=9.2 \times 10^{-8} \mathrm{~g} / \mathrm{g} \text {. }
$$

(3) Estimation of I by Eq. (12):

$$
\begin{aligned}
& \mathrm{C}_{\text {meat }}=10^{-8} \mathrm{~g} / \mathrm{g}, \\
& I=2.7 \times 10^{-5} \mathrm{~g} / \text { day. }
\end{aligned}
$$

(4) $C_{B}^{T B}$ was then estimated by Eq. (10).

A recent publication by Schroeder on tellurium in man should be noted (5243). Data were presented in this publication that suggest that the total tellurium content in the body is about $600 \mathrm{mg}$ and that concentrations in soil vary between 10 and $50 \mu \mathrm{g} / \mathrm{g}$. While the concentrations reported by Schroeder for tissues and crustal materials are much higher than those previously reported, it is noteworthy that our estimate of $\mathrm{C}_{\mathrm{B}}^{\mathrm{TB}} / \mathrm{C}_{\mathrm{S}}$ is greater than those that are based on his study. Since the $F_{1}$ via soil is inversely proportional to $C_{B} / C_{S}$, we have adopted, at least for the present, our more conservative set of values for $C_{B}^{T B}$ and $C_{S}$.

\section{Individual Organs}

The $\mathrm{f}_{2}$ in the ovaries was estimated by Eq. (19) from values derived in previous sections. The $\mathrm{C}_{B}$ in the ovaries and other tissues were then estimated by Eq. (22).

\section{Estimation of $\mathrm{C}_{\mathrm{P}}$}

Discrepancies exist between concentrations of tellurium in plants that have been reported in the literature (5243, 6088, 6089). A wide range of $\mathrm{C}_{\mathrm{P}} / \mathrm{C}_{\mathrm{S}}$ values have also been reported from $1 \times 10^{-3}$ to $5 \times 10^{1}(4105,5244)$. We have assumed a $C_{P} / C_{S}$ of tellurium equal to that of selenium, its congener. The $\mathrm{C}_{\mathrm{P}}$ was estimated by Eq. (14).

\section{Estimation of Aquatic Data}

\section{Estimation of $\mathrm{C}_{\mathrm{A}} \mathrm{fpr}$ Seawater and \\ Freshwater}

The ratio of $\mathrm{Te} / \mathrm{Se}$ in soils is 0.01 . This ratio was multiplied by the $\mathrm{C}_{\mathrm{A}}$ of selenium to obtain the $C_{A}$ for tellurium. 
-

-

○ 


$$
{ }_{58} \mathrm{Ce}
$$

Collateral Data

\begin{tabular}{|c|c|c|c|c|c|}
\hline & & Min & $\operatorname{Max}$ & $A v$ & Ref. \\
\hline \multicolumn{6}{|c|}{ Concentrations $^{*}(\mu \mathrm{g} / \mathrm{g})$} \\
\hline & Human heart & $1.2 \times 10^{-3}$ & $8.2 \times 10^{-3}$ & & 1294 \\
\hline & Cattle bone & & & $6.8 \times 10^{-3}$ & 4752 \\
\hline & Bovine heart & $2.5 \times 10^{-3}$ & $8 \times 10^{-3}$ & & 1745 \\
\hline & Beef & & & $3.8 \times 10^{-6}$ & 4941 \\
\hline & Pork & & & $1.8 \times 10^{-5}$ & 4941 \\
\hline & Ham & & & $1.5 \times 10^{-5}$ & 4941 \\
\hline & Chicken & & & $1.1 \times 10^{-5}$ & 4941 \\
\hline & Mouse ash & & & $5.8 \times 10^{-1}$ & 5225 \\
\hline \multicolumn{6}{|c|}{ Uptake relationships } \\
\hline & Mouse spleen & 0.001 & 0.03 & & 2325 \\
\hline & Rat spleen & 0.001 & 0.036 & & $\begin{array}{l}1185,2064, \\
2114,5166 \mathrm{~A}, \\
5172,5194, \\
5204\end{array}$ \\
\hline$(\mathrm{Y})$ & Rat testes & & & 0.0014 & $5165 \Lambda$ \\
\hline (La) & Rat ovaries & & & 0.00018 & $5166 \mathrm{~A}$ \\
\hline (La) & Rat testes & & & 0.0002 & $5166 \mathrm{~A}$ \\
\hline (La) & Rat gonads & & & 0.0016 & 5177 \\
\hline$(\mathrm{Pr})$ & Rat testes & & & $<0.0007$ & $5166 A$ \\
\hline (Pr) & Rat gonads & 0.0002 & 0.0017 & & 5171,5175 \\
\hline \multicolumn{6}{|c|}{$\mathrm{f}_{2} / \mathrm{f}_{2}^{\prime}$} \\
\hline & Rat spleen & & & 1.5 & 5194 \\
\hline$(\mathrm{Y})$ & Rat testes & & & 0.6 & $5166 \mathrm{~A}$ \\
\hline \multicolumn{6}{|c|}{$\mathrm{f}_{2}$} \\
\hline & Bone & & & 0.8 & ICRP \\
\hline & Kidneys & & & 0.02 & ICRP \\
\hline & Liver & & & 0.13 & ICRP \\
\hline & Rat spleen & & & 0.0015 & 5194 \\
\hline$(Y)$ & Rat testes & & & 0.00084 & $5166 \mathrm{~A}$ \\
\hline$(\mathrm{Pr})$ & Rat gonads & & & 0.0026 & 5175 \\
\hline
\end{tabular}

\footnotetext{
* See footnote on p. A. 3 .
}

(continued) 


\section{Estimation of $f_{B}$}

\section{Individual Organs}

In the absence of human data, the animal data are assumed to apply to man. The $f_{2}^{\prime}$ in the ovaries and testes are based on those of the neighboring lanthanides and were chosen not to be exceeded by the $\mathrm{f}_{2}^{\prime}$ in the gonads. The $\mathrm{f}_{2}^{\prime}$ in the testes was also chosen not to be exceeded by that of $\mathrm{Y}$, the only other lanthanide for which a value has been noted. The maximum value of $f_{2}^{\prime}$ in rat spleen was chosen for spleen. The $f_{B}$ were then estimated by Eq. (16).

\section{Estimation of $\mathrm{T}_{\mathrm{B}}$}

\section{Individual Organs}

The $\mathrm{f}_{2} / \mathrm{f}_{2}^{\prime}$ ratio in the testes is based on that of $\mathrm{Y}$, the only lanthanide for which an $f_{2} / f_{2}^{\prime}$ ratio in the testes has been noted. The $\mathrm{T}_{\mathbf{B}}$ in the testes and spleen were then estimated by Eq. (19).

\section{Estimation of $\mathrm{C}_{B}$}

Whole Body

The maximum $\mathrm{C}_{\mathrm{P}} / \mathrm{C}_{\mathrm{S}}$ ratio of the rare earth elements collectively is about 0.1 in crop plants (2540), and individual lanthanides appear to distribute in plants in essentially the same proportions as in soil (5225). In the case of cerium, $\mathrm{C}_{\mathrm{P}} / \mathrm{C}_{\mathrm{S}}$ ratios in edible parts of plants have been measured and found to vary between $10^{-5}$ and $1(1521,5219)$. While the average and most probable value of
$\mathrm{C}_{\mathrm{P}} / \mathrm{C}_{\mathrm{S}}$ would be less than 0.1 , our objectives require us to adopt the maximum, not-to-be-exceeded value,

$$
\mathrm{C}_{\mathrm{P}} / \mathrm{C}_{\mathrm{S}}=1 \text {. }
$$

(1) Estimation of $\mathrm{C}_{\mathrm{P}}$ by Eq. (14):

$$
C_{P}-5.3 \times 10^{-5} \mathrm{~g} / \mathrm{g} .
$$

(2) Estimation of I by Eq. (12):

$$
\begin{aligned}
& C_{\text {meat }}=10^{-8} \mathrm{~g} / \mathrm{g}, \\
& I=1.3 \times 10^{-2} \mathrm{~g} / \text { day. }
\end{aligned}
$$

(3) $C_{B}^{T B}$ was then estimated by Eq. (10)

\section{Estimation of $\mathrm{C}_{\mathrm{B}}$}

\section{Individual Organs}

The $f_{2}$ in the ovaries and iestes were estimated by Eq. (25) from the $f_{2}$ in the gonads for $\mathrm{Pr}$, the adjacent lanthanide. The $C_{B}$ in these and the other organs were then estimated by Eq. (22).

\section{Estimation of $\mathrm{C}_{\mathrm{P}}$}

The average $\mathrm{C}_{\mathrm{P}} / \mathrm{C}_{\mathrm{S}}$ ratio of radiocerium in crop plants is about $10^{-2}$ (1521, 1945, 5219, 5601). The $\mathrm{C}_{\mathrm{P}} / \mathrm{C}_{\mathrm{S}}$ ratios of $\mathrm{Y}^{91}(1521,6106)$ and $\mathrm{Pm}^{147^{S}}$ $(3493,5601)$ agree with this value. Available plant concentrations of the lanthanides $(2540,5306,6045,6098)$ are also consistent with this ratio. We have assumed that the $\mathrm{C}_{\mathrm{P}} / \mathrm{C}_{\mathrm{S}}$ ratios of the individual lanthanides (including yttrium) are also $10^{-2}$. The $C_{P}$ was then estimated by Eq. (14).

(continued) 


\section{Estimation of Aquatic Data}

\section{Known Data}

\begin{tabular}{|c|c|c|c|c|}
\hline & Min & $\operatorname{Max}$ & $\mathrm{Av}$ & Ref. \\
\hline \multicolumn{5}{|l|}{ Concentrations } \\
\hline \multicolumn{5}{|l|}{ Seawater } \\
\hline \multirow[t]{3}{*}{$\mathrm{Sc}$} & & & $4 \times 10^{-5}$ & 5992 \\
\hline & & & $2 \times 10^{-5}$ & 6001 \\
\hline & & & $1 \times 10^{-6}$ & 6060 \\
\hline \multirow[t]{3}{*}{$\mathrm{Y}$} & & & $3 \times 10^{-4}$ & 5992 \\
\hline & & & $1 \times 10^{-5}$ & 5995 \\
\hline & & & $6 \times 10^{-6}$ & 5464 \\
\hline \multirow[t]{4}{*}{$\mathrm{La}$} & & & $3 \times 10^{-6}$ & 6052 \\
\hline & & & $4 \times 10^{-6}$ & 5995 \\
\hline & & & $1 \times 10^{-5}$ & 5992 \\
\hline & & & $3 \times 10^{-4}$ & 1835,6058 \\
\hline \multirow[t]{5}{*}{$\mathrm{Ce}$} & & & $5 \times 10^{-6}$ & 5992 \\
\hline & & & $8 \times 10^{-6}$ & 5995 \\
\hline & & & $1 \times 10^{-6}$ & 6052 \\
\hline & & & $4 \times 10^{-4}$ & 6058 \\
\hline & $1 \times 10^{-5}$ & $8 \times 10^{-4}$ & & 6026 \\
\hline \multirow[t]{2}{*}{$\operatorname{Pr}$} & & & $6 \times 10^{-7}$ & 6052 \\
\hline & & & $3 \times 10^{-6}$ & 5992 \\
\hline \multirow[t]{2}{*}{$\mathrm{Nd}$} & & & $2 \times 10^{-6}$ & 6052 \\
\hline & & & $9 \times 10^{-6}$ & 5992 \\
\hline \multirow[t]{3}{*}{$\mathrm{Sm}$} & & & $4 \times 10^{-7}$ & 6052 \\
\hline & & & $2 \times 10^{-6}$ & 5992 \\
\hline & & & $7 \times 10^{-7}$ & 5995 \\
\hline \multirow[t]{3}{*}{$\mathrm{Eu}$} & & & $1 \times 10^{-7}$ & 6052 \\
\hline & & & $5 \times 10^{-7}$ & 5992 \\
\hline & & & $2 \times 10^{-7}$ & 5995 \\
\hline \multirow[t]{2}{*}{$\mathrm{Gd}$} & & & $6 \times 10^{-7}$ & 6052 \\
\hline & & & $2 \times 10^{-6}$ & 5992 \\
\hline Others & & & $10^{-6}-10^{-7}$ & $\begin{array}{l}5992,5995 \\
6052\end{array}$ \\
\hline
\end{tabular}

(continued) 
Known Data (cont.)

\begin{tabular}{|c|c|c|c|c|}
\hline & Min & $\operatorname{Max}$ & $\mathrm{Av}$ & Ref. \\
\hline \multicolumn{5}{|l|}{ Concentrations } \\
\hline \multicolumn{5}{|c|}{ Freshwater } \\
\hline $\mathrm{Sc}$ & $1 \times 10^{-6}$ & $4 \times 10^{-5}$ & $1 \times 10^{-5}$ & 4576 \\
\hline La & $1 \times 10^{-6}$ & $2 \times 10^{-3}$ & $6 \times 10^{-4}$ & 4576 \\
\hline \multicolumn{5}{|c|}{ Marine plants } \\
\hline \multirow[t]{2}{*}{$\mathrm{Sc}$} & $4 \times 10^{-1}$ & $7 \times 10^{-1}$ & $5 \times 10^{-1}$ & 6001 \\
\hline & & & $2 \times 10^{-4}$ & 6060 \\
\hline \multicolumn{5}{|c|}{ Freshwater plants } \\
\hline $\mathrm{Sc}$ & $1 \times 10^{-3}$ & $6 \times 10^{-2}$ & $2 \times 10^{-2}$ & 6051 \\
\hline \multicolumn{5}{|c|}{$\begin{array}{l}\text { Marine } \\
\text { invertebrates }\end{array}$} \\
\hline \multirow[t]{3}{*}{$\mathrm{Sc}$} & & & $1 \times 10^{-4}$ & 6060 \\
\hline & $2 \times 10^{-2}$ & $1 \times 10^{-1}$ & $5 \times 10^{-2}$ & 6001 \\
\hline & $1 \times 10^{-2}$ & $8 \times 10^{-2}$ & $5 \times 10^{-2}$ & 5581 \\
\hline \multicolumn{2}{|c|}{ La (includes shell) } & & $3 \times 10^{1}$ & 6056 \\
\hline $\mathrm{Ce}$ & $3 \times 10^{-2}$ & $5 \times 10^{-1}$ & & 6026 \\
\hline \multicolumn{5}{|c|}{$\begin{array}{l}\text { Freshwater } \\
\text { invertebrates }\end{array}$} \\
\hline \multirow[t]{2}{*}{$\mathrm{Sc}$} & $1 \times 10^{-2}$ & $1 \times 10^{-1}$ & $5 \times 10^{-2}$ & 6051 \\
\hline & $3 \times 10^{-3}$ & $3 \times 10^{-2}$ & $1 \times 10^{-2}$ & 1308 \\
\hline \multicolumn{5}{|c|}{ Marine fish } \\
\hline $\mathrm{Sc}$ & $6 \times 10^{-5}$ & $1.6 \times 10^{-3}$ & $5 \times 10^{-4}$ & 6060 \\
\hline La & $3 \times 10^{-3}$ & $4 \times 10^{-3}$ & $3 \times 10^{-3}$ & 6056 \\
\hline $\mathrm{Ce}$ & & & $<1 \times 10^{-2}$ & 6026 \\
\hline $\operatorname{Pr}$ & $2 \times 10^{-4}$ & $1 \times 10^{-3}$ & $6 \times 10^{-4}$ & 6056 \\
\hline $\mathrm{Eu}$ & & & $5 \times 10^{-4}$ & 6056 \\
\hline Dy & & & $4 \times 10^{-4}$ & 6056 \\
\hline \multicolumn{5}{|c|}{ Freshwater fish } \\
\hline $\mathrm{Sc}$ & $2 \times 10^{-4}$ & $4 \times 10^{-4}$ & $3 \times 10^{-4}$ & 6051 \\
\hline \multicolumn{5}{|c|}{ Concentration factors (foodstuff $/ \mathrm{C}_{\mathrm{A}}$ ) } \\
\hline \multicolumn{5}{|c|}{ Marine plants } \\
\hline $\mathrm{Sc}^{46}$ & $1.5 \times 10^{3}$ & $2.6 \times 10^{3}$ & $2 \times 10^{3}$ & 1406 \\
\hline $\mathrm{Y}^{91}$ & $1.6 \times 10^{2}$ & $9.0 \times 10^{2}$ & $5 \times 10^{2}$ & 6012,2475 \\
\hline La & & & $8 \times 10^{3}$ & 5306 \\
\hline \multirow[t]{6}{*}{$\mathrm{Ce}^{144}$} & $2 \times 10^{2}$ & $3 \times 10^{3}$ & $1 \times 10^{3}$ & 6012 \\
\hline & $3 \times 10^{2}$ & $9 \times 10^{2}$ & & 5335 \\
\hline & $2.5 \times 10^{3}$ & $4.5 \times 10^{3}$ & & 2715 \\
\hline & $2 \times 10^{3}$ & $4.5 \times 10^{3}$ & & 2717 \\
\hline & & & $7 \times 10^{2}$ & 506 \\
\hline & & & & tinued) \\
\hline
\end{tabular}


Kuown Dalta (conte)

\begin{tabular}{|c|c|c|c|c|}
\hline & Min & Max & $\Lambda v$ & Ref. \\
\hline \multicolumn{5}{|c|}{ ('oncerntaition lactors (foodsturf/ $\left.C_{\Lambda}\right)$ (contd) } \\
\hline \multicolumn{5}{|c|}{ Freshwater plants } \\
\hline$Y^{(3)}$ & $1 \times 10^{2}$ & $1 \times 10^{4}$ & $1 \times 10^{3}$ & (60) 12 \\
\hline \multirow[t]{4}{*}{$c^{1+4}$} & $1 \times 10^{2}$ & $4 \times 10^{3}$ & $1 \times 10^{3}$ & 6012 \\
\hline & $2 \times 10^{3}$ & $1 \times 10^{4}$ & & 53335 \\
\hline & $2 \times 10^{4}$ & $5 \times 10^{4}$ & $3 \times 10^{4}$ & 5395 \\
\hline & $4 \times 10^{3}$ & $1 \times 10^{4}$ & & 3606 \\
\hline \multirow[t]{2}{*}{$P_{11} 147$} & $1 \times 10^{4}$ & $3 \times 10^{4}$ & $2 \times 10^{4}$ & 59991 \\
\hline & $3 \times 10^{2}$ & $6 \times 10^{2}$ & $5 \times 10^{2}$ & (;0) 12 \\
\hline \multicolumn{5}{|c|}{ Mareine inverfebrates } \\
\hline$Y^{(9)}$ & & & 12 & 2475 \\
\hline \multirow[t]{5}{*}{$\mathrm{Ce}^{1+t}$} & & & $3 ! 00$ & 2475 \\
\hline & $1 \times 10^{2}$ & $1 \times 10^{3}$ & & 6026 \\
\hline & $1 \times 10^{2}$ & $2 \times 10^{3}$ & & 5335 \\
\hline & & & $3 \times 10^{3}$ & 2715 \\
\hline & & & $2 \times 10^{3}$ & 506 \\
\hline \multicolumn{5}{|c|}{$\begin{array}{l}\text { Freshwater } \\
\text { invertebrates }\end{array}$} \\
\hline $\mathrm{Y}^{91}$ & & & $2 \times 10^{2}$ & 6012 \\
\hline $\mathrm{Ce}^{144}$ & $4 \times 10^{1}$ & $7 \times 10^{2}$ & $4 \times 10^{2}$ & 6012 \\
\hline $\operatorname{Pm}^{147}$ & $3 \times 10^{1}$ & $2 \times 10^{2}$ & $1 \times 10^{2}$ & 6012 \\
\hline \multicolumn{5}{|c|}{ Mar.ine lish } \\
\hline $\mathrm{Y}$ & 2.0 & $1 \times 10^{1}$ & & 6012 \\
\hline $\mathrm{Ce}^{144}$ & & & $2 \times 10^{1}$ & 6026 \\
\hline \multicolumn{5}{|c|}{ J'reshwater lish } \\
\hline$c: e^{144}$ & & & $8 \times 10^{1}$ & 6012 \\
\hline Rare cal & & & $1 \times 10^{2}$ & 1679 \\
\hline \multicolumn{5}{|c|}{ Concentration ratios } \\
\hline \multicolumn{5}{|c|}{ Marine } \\
\hline \multicolumn{5}{|c|}{$\begin{array}{l}1 . \mathrm{a}^{140} \text { (plants/ } \\
\text { invertebrates) }\end{array}$} \\
\hline \multicolumn{2}{|c|}{$1 . a^{140}$ (plants/fish) } & & 100 & 6064 \\
\hline \multicolumn{2}{|c|}{ Sc (plants/fish) } & & 50 & 6051 \\
\hline
\end{tabular}

(continued) 
Estimation of $C_{A}$ for Seawater

As the known data demonstrate, there is a wide range in the values reported for the rare earths. Much of this is accounted for by the difference between the open ocean and estuarine areas. We selected the value of $4 \times 10^{-4}$ for the cerium concentration, since this is the middle of the range in the data presented in Ref. 6026. The concentrations of scandium, yttrium, lanthanum, and the other rare earths were then based upon this value and their abundance in soil relative to cerium.

\section{Estimation of $\mathrm{C}_{\mathrm{A}}$ in Freshwater}

The concentrations of scandium, yttrium, lanthanum, and the rare earths were assumed to be the same as in seawater. This assumption is supported by the Columbia River data reported in Ref. 4576 .
Estimation of Concentration in Seawater Foodstuffs

The following concentration factors were chosen for scandium, yttrium, lanthanum, and the rare earths:

$\begin{array}{ll}\text { Plants } & 10^{4} \\ \text { Invertebrates } & 10^{3} \\ \text { Fish } & 10^{2}\end{array}$

The values are at or near the top of the range reported in the literature. The concentrations in the foodstuffs were then determined as the product of these factors and the concentration in seawater. The values determined in this manner are consistent with the measured values.

Estimation of Concentration in Freshwater Foodstuffs

These were determined in the same manner as for seawater foodstuffs. 
$61^{\mathrm{Pm}}$

Collateral Data

\begin{tabular}{|c|c|c|c|c|}
\hline & Min & $\operatorname{Max}$ & Av & Ref \\
\hline \multicolumn{5}{|l|}{ Uptake relationships } \\
\hline \multicolumn{5}{|l|}{$\mathrm{f}_{2}^{\prime}$} \\
\hline Rat spleen & & & 0.0009 & 5195,5197 \\
\hline (Nd) Rat gonads & 0.0009 & 0.0018 & & 5174 \\
\hline \multicolumn{5}{|l|}{$f_{2} / f_{2}^{\prime}$} \\
\hline Rat spleen & & & 1.1 & 5197 \\
\hline \multicolumn{5}{|l|}{$\mathrm{f}_{2}$} \\
\hline Bone & & & 0.8 & ICRP \\
\hline Kidneys & & & 0.02 & ICRP \\
\hline Liver & & & 0.06 & ICRP \\
\hline Rat spleen & & & 0.001 & 5197 \\
\hline (Nd) Rat gonads & 0.00064 & 0.0018 & & 5174 \\
\hline
\end{tabular}

\section{Estimation of $\mathrm{C}_{\mathrm{S}}$}

No naturally occurring isotopes of promethium have been detected. The existence of $\mathrm{Pm}^{147}$ as a product of spontaneous fission of $U^{238}$ has been predicted by Kuroda and Edwards (5764). The $\mathrm{C}_{\mathrm{S}}$ was therefore estimated by Eq. (32) with $f=0.026$ (5764).

Estimation of $f_{B}$

\section{Individual Organs}

The $f_{2}^{\prime}$ in the ovaries and testes are assumed to be comparable to those of the neighboring lanthanide neodymium and were estimated by Eq. (18). The $\mathrm{f}_{\mathrm{B}}$ in ovaries, spleen and testes were estimated by Eq. (16).

\section{Estimation of $\mathrm{T}_{\mathrm{B}}$}

Individual Organs

The $\mathrm{f}_{2} / \mathrm{f}_{2}^{\prime}$ ratio in the testes is based on that of $Y$, the only lanthanide for which an $f_{2} / f_{2}^{\prime}$ ratio in the testes has been noted. The $\mathrm{T}_{\mathrm{B}}$ in the testes and spleen were estimated by Eq. (19).

\section{Estimation of $\mathrm{C}_{\mathrm{B}}$}

\section{Whole Body}

Since $\mathrm{C}_{\mathrm{B}}$ of promethium have not been reported, we have assumed a $\mathrm{C}_{\mathrm{B}}^{\mathrm{TB}} / \mathrm{C}_{\mathrm{S}}$ ratio for $\mathrm{Pm}$ equal to $4 \times 10^{-4}$, the maximum $\mathrm{C}_{\mathrm{B}}^{\mathrm{TB}} / \mathrm{C}_{\mathrm{S}}$ estimated for any lanthanide. The $\mathrm{C}_{\mathrm{B}}^{\mathrm{TB}}$ was obtained as the product of $\mathrm{C}_{\mathrm{S}}$ and the $\mathrm{C}_{\mathrm{B}}^{\mathrm{TB}} / \mathrm{C}_{\mathrm{S}}$ ratio.

\section{Individual Organs}

The $f_{2}$ in the ovaries and testes are assumed to be comparable to those of the neighboring lanthanide neodymium and were estimated by Eq. (25). The $C_{B}$ were estimated by Eq. (22). 


$$
62^{\mathrm{Sm}}
$$

Collateral Data

\begin{tabular}{|c|c|c|c|c|}
\hline & Min & $\operatorname{Max}$ & $\mathrm{Av}$ & Ref. \\
\hline \multicolumn{5}{|c|}{ Concentrations $(\mu \mathrm{g} / \mathrm{g})$} \\
\hline Human heart & $2 \times 10^{-4}$ & $2.3 \times 10^{-2}$ & & 1294 \\
\hline Cattle bone & & & $6 \times 10^{-4}$ & 4752 \\
\hline Cow heart & $1.9 \times 10^{-3}$ & $3.6 \times 10^{-3}$ & & 1745 \\
\hline Mouse ash & & & $4.3 \times 10^{-2}$ & 5225 \\
\hline \multicolumn{5}{|c|}{$\begin{array}{l}\text { Uptake relationships } \\
\mathrm{f}_{2}^{\prime}\end{array}$} \\
\hline Rat spleen & 0.0009 & 0.0026 & & 5173,5196 \\
\hline Rat gonads & & & 0.0002 & 5173 \\
\hline \multicolumn{5}{|l|}{$f_{2}$} \\
\hline Bone & & & 0.80 & ICRP \\
\hline Kidneys & & & 0.02 & ICRP \\
\hline Liver & & & 0.1 & ICRP \\
\hline
\end{tabular}

\section{Estimation of $f_{B}$}

\section{Individual Organs}

The $f_{2}^{\prime}$ in the ovaries and testes were estimated from $\mathrm{f}_{2}^{\prime}$ in the gonads by Eq. (18), and $f_{B}$ in these organs and spleen were estimated by Eq. (16).

\section{Estimation of $\mathrm{T}_{\mathrm{B}}$}

\section{Individual Organs}

The $\mathrm{f}_{2} / \mathrm{f}_{2}^{\prime}$ ratios and $T_{B}$ in spleen and testes are assumed to be comparable to those of the neighboring lanthanides promethium and europium. The $\mathrm{T}_{\mathrm{B}}$ were estimated using Eq. (19).

\section{Estimation of $C_{B}$}

\section{Whole Body}

The maximum $\mathrm{C}_{\mathrm{P}} / \mathrm{C}_{\mathrm{S}}$ ratio of the rare earth elements collectively is about 0.1 in crop plants (2540), and individual lanthanides appear to distribute in plants in essentially the same proportions as in soil (5225). The $\mathrm{C}_{\mathrm{P}} / \mathrm{C}_{\mathrm{S}}$ ratios of cerium in edible parts of plants have been found to vary between $10^{-5}$ and 1 (1521, 5219). While the average and most probable $\mathrm{C}_{\mathrm{P}} / \mathrm{C}_{\mathrm{S}}$ would be less than 0.1 , we have adopted the upper limit,

$$
\mathrm{C}_{\mathrm{P}} / \mathrm{C}_{\mathrm{S}}=1.0
$$

which is in keeping with the conservative aspects of the analysis. We have also assumed a $\mathrm{C}_{\text {meat }}$ equal to $5 \times 10^{-9} \mathrm{~g} / \mathrm{g}$, roughly the concentration in cow heart.

(1) Estimation of $C_{P}$ by Eq. (14):

$$
\mathrm{C}_{\mathrm{P}}=6.4 \times 10^{-6} \mathrm{~g} / \mathrm{g} \text {. }
$$

(2) Estimation of I by Eq. (12).

$$
I=1.6 \times 10^{-3} \mathrm{~g} / \mathrm{day} .
$$


(3) $C_{B}^{T B}$ Was then estimated by Eq. (10). lanthanides, promethium and europium;

Individual Organs the $\mathrm{f}_{2}$ in the ovaries and testes are

The $f_{2}$ in spleen is assumed to be comparable to those of the neighboring assumed to be comparable to those of europium. The $\mathrm{C}_{\mathrm{B}}$ were estimated by Eq. (22).

$$
63^{\mathrm{Eu}}
$$

Collateral Data

\begin{tabular}{|c|c|c|c|c|c|}
\hline & & Min & $\operatorname{Max}$ & $\mathrm{Av}$ & Ref \\
\hline \multicolumn{6}{|c|}{ Concentrations $(\mu \mathrm{g} / \mathrm{g})$} \\
\hline & Beef & & & 7. $\times 10^{-5}$ & 4941 \\
\hline & Pork & & & $5 \times 10^{-6}$ & 4941 \\
\hline & Ham & & & $2 \times 10^{-5}$ & 4941 \\
\hline & Chicken & & & $6 \times 10^{-5}$ & 4941 \\
\hline & Mouse ash & & & $1.6 \times 10^{-2}$ & 5225 \\
\hline \multicolumn{6}{|c|}{ Uptake relationships } \\
\hline \multicolumn{6}{|c|}{$\mathrm{f}_{2}^{\prime}$} \\
\hline & Rat spleen & 0.0015 & 0.0027 & & $5172,5173,5196$ \\
\hline & Rat gonads & 0.0006 & 0.0009 & & 5172,5173 \\
\hline \multicolumn{6}{|c|}{$f_{2} / f_{2}^{\prime}$} \\
\hline & Rat spleen & & & 0.7 & 5196 \\
\hline$(\mathrm{Y})$ & Rat testes & & & 0.6 & $5166 \mathrm{~A}$ \\
\hline \multicolumn{6}{|c|}{$\mathrm{f}_{2}$} \\
\hline & Bone & & & 0.85 & ICRP \\
\hline & Kidneys & & & 0.07 & ICRP \\
\hline & Liver & & & 0.05 & ICRP \\
\hline & Rat spleen & 0.0011 & 0.0018 & & 5175,5196 \\
\hline & Rat gonads & & & $<0.0003$ & 5175 \\
\hline
\end{tabular}

Estimation of $f_{B}$

Individual Organs

The $f_{2}^{\prime}$ in the ovaries and testes were estimated from $f_{2}^{\prime}$ in the gonads by Eq. (18), and $f_{B}$ in these organs and spleen were estimated by Eq. (16).
Estimation of $T_{B}$

Individual Organs

The $\mathrm{f}_{2} / \mathrm{f}_{2}^{\prime}$ ratio in the testes is based on that of yttrium, the only lanthanide for which an $\mathrm{f}_{2} / \mathrm{f}_{2}^{\prime}$ ratio in the testes has been noted. The $T_{B}$ in spleen and testes were estimated by Eq. (19). 


\section{Estimation of $\mathrm{C}_{\mathrm{B}}$}

\section{Whole Body}

The maximum $\mathrm{C}_{\mathrm{P}} / \mathrm{C}_{\mathrm{S}}$ ratio of the lanthanides collectively is about 0.1 in crop plants (2540), and individual lanthanides appear to distribute in plants in essentially the same proportions as in soil (5225). In the case of cerium, $\mathrm{C}_{\mathrm{P}} / \mathrm{C}_{\mathrm{S}}$ ratios in edible parts of plants have been found to vary between $10^{-5}$ and $1(1521,5219)$. While the average and most probable value of $\mathrm{C}_{\mathrm{P}} / \mathrm{C}_{\mathrm{S}}$ would be less than 0.1 , we have adopted the maximum, not to be exceeded values,

$$
\mathrm{C}_{\mathrm{P}} / \mathrm{C}_{\mathrm{S}}=1.0
$$

which is in keeping with the conservative aspects of the analysis.

(1) Estimation of $\mathrm{C}_{\mathrm{P}}$ by Eq. (14):

$$
\mathrm{C}_{\mathrm{P}}=10^{-6} \mathrm{~g} / \mathrm{g} \text {. }
$$

(2) Estimation of I by Eq. (12):

$$
\begin{aligned}
& C_{\text {meat }}=10^{-10} \mathrm{~g} / \mathrm{g}, \\
& I=2.5 \times 10^{-4} \mathrm{~g} / \text { day. }
\end{aligned}
$$

(3) $\mathrm{C}_{B}^{\mathrm{TB}}$ was then estimated by Eq. (10).

\section{Individual Organs}

The $f_{2}$ in the ovaries and testes were estimated from $f_{2}$ in the gonads by Eq. (25). The $\mathrm{C}_{\mathrm{B}}$ in these and the other organs were then estimated by Eq. (22).

$$
{ }_{64}^{\mathrm{Gd}}
$$

\section{Collateral Data}

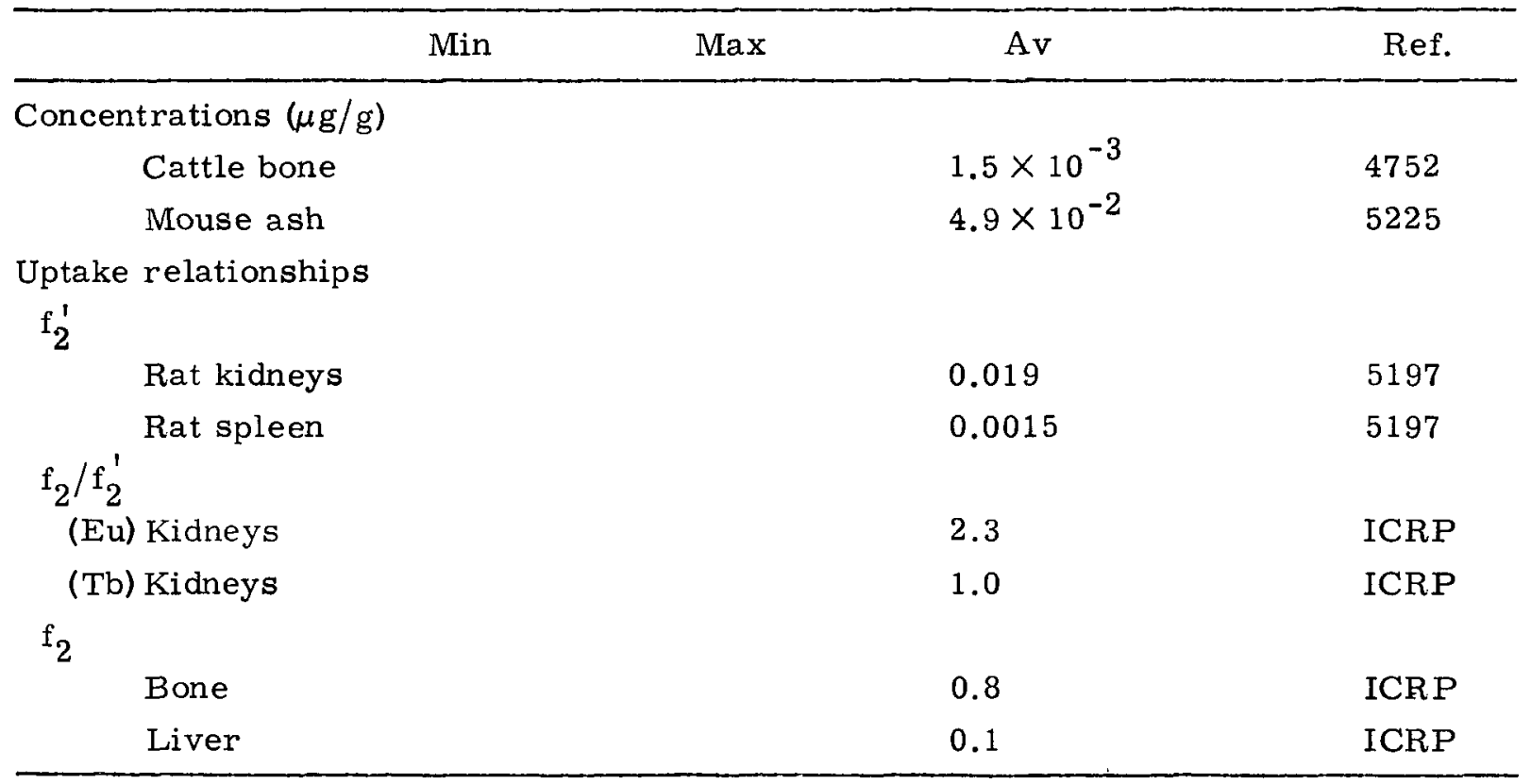


Estimation of $\mathrm{f}_{B}$

\section{Individual Organs}

The $f_{2}^{\prime}$ in the ovaries and testes are based on those of the neighboring lanthanides, Eu and $\mathrm{Tb}$, and were estimated by Eq. (18). The $\mathrm{f}_{\mathrm{B}}$ in these and the other organs were estimated by Eq. (16).

\section{Estimation of $\mathrm{T}_{\mathrm{B}}$}

\section{Individual Organs}

The $f_{2} / f_{2}^{\prime}$ ratios and $T_{B}$ are assumed to be comparable to those of the neighboring lanthanides europium and terbium. The $\mathrm{T}_{\mathrm{B}}$ were estimated by Eq. (19).
Estimation of $C_{B}$

\section{Whole Body}

Since concentrations of gadolinium have not been reported in tissues other than cattle bone and mouse whole body, we have assumed a $C_{B}^{T B} / C_{S}$ ratio for $G d$ equal to $4 \times 10^{-4}$, the maximum $\mathrm{C}_{\mathrm{B}}^{\mathrm{TB}} / \mathrm{C}_{\mathrm{S}}$ estimated for any lanthanide. The $\mathrm{C}_{\mathrm{B}}^{\mathrm{TB}}$ was obtained as the product of $\mathrm{C}_{\mathrm{S}}$ and the $C_{B}^{T B} / C_{S}$ ratio. The value thus selected compares with the concentrations in cattle bone and mouse whole body.

\section{Individual Organs}

The $f_{2}$ in kidneys, ovaries, spleen, and testes were adopted from those of the neighboring lanthanides europium and terbium. The $\mathrm{C}_{\mathrm{B}}$ for all the organs of interest were estimated by Eq. (22).

$65^{\mathrm{Tb}}$

Collateral Data

\begin{tabular}{|c|c|c|c|c|}
\hline & Min & $\operatorname{Max}$ & $\mathrm{Av}$ & Ref. \\
\hline \multicolumn{5}{|c|}{ Concentrations $(\mu \mathrm{g} / \mathrm{g})$} \\
\hline Mouse ash & & & $1.2 \times 10^{-2}$ & 5225 \\
\hline \multicolumn{5}{|c|}{ Uptake relationships } \\
\hline \multicolumn{5}{|l|}{$\mathrm{f}_{2}^{\prime}$} \\
\hline Rat Liver & 0.098 & 0.19 & & $5180,5197,5204$ \\
\hline Rat spleen & 0.0009 & 0.003 & & $5180,5197,5204$ \\
\hline Rat gonads & 0.0011 & 0.002 & & 5180 \\
\hline \multicolumn{5}{|l|}{$\mathrm{f}_{2} / \mathrm{f}_{2}^{\prime}$} \\
\hline Rat liver & & & 0.1 & 5197 \\
\hline Rat spleen & & & 0.5 & 5197 \\
\hline \multicolumn{5}{|l|}{$\mathrm{f}_{2}$} \\
\hline Bone & & & 0.9 & ICRP \\
\hline Kidneys & & & 0.03 & ICRP \\
\hline Rat liver & 0.0045 & 0.0066 & & 5182,5197 \\
\hline Rat spleen & 0.0006 & 0.0015 & & 5182,5197 \\
\hline Rat gonads & 0.0012 & 0.0043 & & 5182 \\
\hline
\end{tabular}




\section{Estimation of $f_{B}$}

\section{Individual Organs}

The $f_{2}^{\prime}$ in the ovaries and testes were estimated from $f_{2}^{\prime}$ in the gonads by

Eq. (18). The $\mathrm{f}_{B}$ in these and the other organs were estimated by Eq. (16).

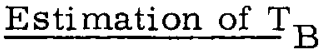

\section{Individual Organs}

The $f_{2} / f_{2}^{\prime}$ ratio in the testes is based on that found for yttrium, the only lanthanide for which an $\mathrm{f}_{2} / \mathrm{f}_{2}^{\prime}$ ratio in the testes has been noted. The $T_{B}$ in the testes and other organs were estimated by Eq. (19).

\section{Estimation of $\mathrm{C}_{\mathrm{B}}$}

Whole Body

Since concentrations of terbium have not been reported in tissues other than mouse whole body, we have assumed a $\mathrm{C}_{\mathrm{B}}^{\mathrm{TB}} / \mathrm{C}_{\mathrm{S}}$ ratio for $\mathrm{Tb}$ equal to $4 \times 10^{-4}$, the maximum $\mathrm{C}_{B}^{\mathrm{TB}} / \mathrm{C}_{\mathrm{S}}$ estimated for any lanthanide. The $C_{B}^{T B}$ was obtained as the product of $\mathrm{C}_{\mathrm{S}}$ and the $\mathrm{C}_{\mathrm{B}}^{\mathrm{TB}} / \mathrm{C}_{\mathrm{S}}$ ratio. The value thus selected compares with the concentration in mouse whole body.

\section{Individual Organs}

The $f_{2}$ in the ovaries and testes were estimated from $\mathrm{f}_{2}$ in the gonads by Eq. (25) and are based on data from 3 rats (5182). The $\mathrm{C}_{B}$ in the organs of interest were estimated by Eq. (22).

$67^{\text {Ho }}$

\section{Collateral Data}

\begin{tabular}{|c|c|c|c|c|}
\hline & Min & $\operatorname{Max}$ & $\mathrm{Av}$ & Ref. \\
\hline \multicolumn{5}{|c|}{ Concentrations $(\mu \mathrm{g} / \mathrm{g})$} \\
\hline Mouse ash & & & $1.7 \times 10^{-2}$ & 5225 \\
\hline
\end{tabular}

\section{Estimation of $\mathrm{C}_{\mathrm{B}}$}

Whole Body

Since concentrations of holmium have not been reported in tissues other than mouse whole body, we have assumed a
$\mathrm{C}_{\mathrm{B}}^{\mathrm{TB}} / \mathrm{C}_{\mathrm{S}}$ ratio for Ho equal to $4 \times 10^{-4}$, the maximum $\mathrm{C}_{\mathrm{B}}^{\mathrm{TB}} / \mathrm{C}_{\mathrm{S}}$ estimated for any lanthanide. The $\mathrm{C}_{\mathrm{B}}^{\mathrm{TB}}$ was obtained as the product of $\mathrm{C}_{S}$ and the $\mathrm{C}_{B}^{\mathrm{TB}} / \mathrm{C}_{\mathrm{S}}$ ratio. The value thus selected compares with the concentration in mouse whole body. 


$$
69^{\mathrm{Tm}}
$$

\section{Collateral Data}

\begin{tabular}{|c|c|c|c|c|}
\hline & Min & $\operatorname{Max}$ & Av & Ref. \\
\hline \multicolumn{5}{|c|}{ Concentrations $(\mu \mathrm{g} / \mathrm{g})$} \\
\hline Beef & & & $7 \times 10^{-6}$ & 4941 \\
\hline Pork & & & $5 \times 10^{-6}$ & 4941 \\
\hline Ham & & & $2 \times 10^{-5}$ & 4941 \\
\hline Chicken & & & $6 \times 10^{-6}$ & 4941 \\
\hline Mouse ash & & & $10^{-2}$ & 5225 \\
\hline \multicolumn{5}{|c|}{$\begin{array}{l}\text { Uptake relationships } \\
\mathrm{f}_{2}^{\prime}\end{array}$} \\
\hline Rat liver & 0.029 & 0.089 & & $5181,5195,5204$ \\
\hline Rat spleen & 0.0011 & 0.009 & & $5181,5195,5204$ \\
\hline Rat gonads & 0.0013 & 0.0025 & & 5181 \\
\hline \multicolumn{5}{|l|}{$\mathrm{f}_{2} / \mathrm{f}_{2}^{\prime}$} \\
\hline Rat liver & & & 0.6 & 5195 \\
\hline Rat spleen & & & 0.1 & 5195 \\
\hline \multicolumn{5}{|l|}{$\mathrm{f}_{2}$} \\
\hline Bone & & & 0.95 & ICRP \\
\hline Kidneys & & & 0.01 & ICRP \\
\hline Rat liver & 0.0025 & 0.005 & & 5184,5195 \\
\hline Rat spleen & 0.0007 & 0.0035 & & 5184,5195 \\
\hline Rat gonads & 0.0023 & 0.0035 & & 5184 \\
\hline
\end{tabular}

Estimation of $f_{B}$

\section{Individual Organs}

The $f_{2}^{1}$ in the ovaries and testes were estimated from $f_{2}^{\prime}$ in the gonads by

Eq. (18) and are based on data from 3 rats (5181). The $f_{B}$ in the various organs were estimated by Eq. (16).

\section{Estimation of $\mathrm{T}_{\mathrm{B}}$}

\section{Individual Organs}

The $f_{2} / f_{2}^{\prime}$ ratio in the testes is based on that found for yttrium, the only lanthanide for which an $\mathrm{f}_{2} / \mathrm{f}_{2}^{\prime}$ ratio in the testes has been noted. The $\mathrm{T}_{\mathrm{B}}$ in liver, spleen and testes were estimated by Eq. (19).

\section{Estimation of $\mathrm{C}_{\mathrm{B}}$}

\section{Whole body}

The maximum $\mathrm{C}_{\mathrm{P}} / \mathrm{C}_{\mathrm{S}}$ ratio of the lanthanides collectively is about 0.1 in crop plants (2540), and individual lanthanides appear to distribute in plants in essentially the same proportions as in soil (5225). The $\mathrm{C}_{\mathrm{P}} / \mathrm{C}_{\mathrm{S}}$ ratios of 
cerium in edible parts of plants have been found to vary between $10^{-5}$ and 1 (1521, 5219). While the average and most probable $C_{P} / C_{S}$ would be less than 0.1 , we have adopted the upper limit,

$$
\mathrm{C}_{\mathrm{P}} / \mathrm{C}_{\mathrm{S}}=1.0
$$

which is in keeping with the conservative aspects of the analysis.

(1) Estimation of $\mathrm{C}_{\mathrm{P}}$ by Eq. (14):

$$
C_{P}=7 \times 10^{-7} \mathrm{~g} / \mathrm{g} \text {. }
$$

(2) Estimation of I by Eq. (12):

$$
\begin{aligned}
& C_{\text {meat }}=2 \times 10^{-11} \mathrm{~g} / \mathrm{g}, \\
& I=1.8 \times 10^{-4} \mathrm{~g} / \text { day. }
\end{aligned}
$$

(3) $C_{B}^{T B}$ was then estimated by Eq. (10).

\section{Individual Organs}

The $\mathrm{f}_{2}$ in the ovaries and testes were estimated from $f_{2}$ in the gonads by Eq. (25). The $\mathrm{C}_{B}$ in the various organs

\begin{tabular}{|c|c|c|c|c|}
\hline & Min & $\operatorname{Max}$ & $\mathrm{Av}$ & Ref. \\
\hline \multicolumn{5}{|c|}{ Concentrations $(\mu \mathrm{g} / \mathrm{g})$} \\
\hline Beef & & & $7 \times 10^{-5}$ & 4941 \\
\hline Pork & & & $5 \times 10^{-6}$ & 4941 \\
\hline Ham & & & $2 \times 10^{-5}$ & 4941 \\
\hline Chicken & & & $6 \times 10^{-5}$ & 4941 \\
\hline Mouse ash & & & $2.6 \times 10^{-2}$ & 5225 \\
\hline \multicolumn{5}{|c|}{$\begin{array}{l}\text { Uptake relationships } \\
\mathrm{f}_{2}^{\prime}\end{array}$} \\
\hline Rat liver & & & 0.041 & 5196 \\
\hline Rat spleen & & & 0.0052 & 5196 \\
\hline (La) Rat gonads & 0.0015 & 0.003 & & 5177 \\
\hline \multicolumn{5}{|l|}{$f_{2}$} \\
\hline Bone & & & 0.85 & ICRP \\
\hline Kidneys & & & 0.05 & ICRP \\
\hline
\end{tabular}
were then estimated by Eq. (22).

${ }_{70} \mathrm{Yb}$

Collateral Data

\section{Estimation of $f_{B}$}

Individual Organs

The $f_{2}^{\prime}$ in the ovaries and testes are based on $f_{2}^{\prime}$ in the gonads for the neigh- boring lanthanides thulium and lutetium and were estimated by Eq. (18). The $f_{B}$ for the various organs were then estimated by Eq. (16). 
Estimation of $\mathrm{T}_{\mathrm{B}}$

Individual Organs

The $f_{2} / f_{2}^{\prime}$ ratios and $T_{B}$ in liver, spleen and testes are assumed to be comparable to those of the neighboring lanthanide thulium. The $\mathrm{T}_{\mathbf{B}}$ in the various organs were estimated by Eq. (19).

\section{Estimation of $C_{B}$}

Whole Body

The maximum $\mathrm{C}_{\mathrm{P}} / \mathrm{C}_{\mathrm{S}}$ ratio of the lanthanides collectively is about 0.1 in crop plants (2540), and individual lanthanides appear to distribute in plants in essentially the same proportions as in soil (5225). The $\mathrm{C}_{\mathrm{P}} / \mathrm{C}_{\mathrm{S}}$ ratios of cerium in edible parts of plants have been found to vary between $10^{-5}$ and 1 (1521, 5219). While the average and most probable $\mathrm{C}_{\mathrm{P}} / \mathrm{C}_{\mathrm{S}}$ would be less than 0.1 , we have adopted the upper limit,

$$
\mathrm{C}_{\mathrm{P}} / \mathrm{C}_{\mathrm{S}}=1.0
$$

which is in keeping with the conservative aspects of the analysis.

(1) Estimation of $\mathrm{C}_{\mathrm{P}}$ by Eq. (14):

$$
\mathrm{C}_{\mathrm{P}}=8 \times 10^{-7} \mathrm{~g} / \mathrm{g} \text {. }
$$

(2) Estimation of I by Eq. (12):

$$
\begin{aligned}
& C_{\text {meat }}=10^{-10} \mathrm{~g} / \mathrm{g}, \\
& I=2 \times 10^{-4} \mathrm{~g} / \text { day. }
\end{aligned}
$$

(3) $C_{B}^{T B}$ was then estimated by Eq. (10).

\section{Individual Organs}

The $\mathrm{f}_{2}$ in the liver, ovaries, spleen and testes are assumed to be comparable to those for the neighboring lanthanide thulium. The $\mathrm{C}_{\mathrm{B}}$ in the various organs were estimated by Eq. (22).

Collateral Data

\begin{tabular}{llll}
\hline & Min & Max & Av \\
\hline Concentrations $(\mu \mathrm{g} / \mathrm{g})$ & & & \\
Beef & $7 \times 10^{-5}$ & 4941 \\
Pork & $5 \times 10^{-6}$ & 4941 \\
Ham & $2 \times 10^{-5}$ & 4941 \\
Chicken & $6 \times 10^{-5}$ & 4941 \\
Mouse ash & $7.5 \times 10^{-3}$ & 5225 \\
\hline
\end{tabular}

\section{Estimation of $\mathrm{C}_{B}$}

\section{Whole Body}

The maximum $\mathrm{C}_{\mathrm{P}} / \mathrm{C}_{\mathrm{S}}$ ratio of the lanthanides collectively is about 0.1 in crop plants (2540), and individual lanthanides appear to distribute in plants in essentially the same proportions as in soil (5225). The $\mathrm{C}_{\mathrm{P}} / \mathrm{C}_{\mathrm{S}}$ ratios of cerium in edible parts of plants have 
been found to vary between $10^{-5}$ and 1

(1521, 5219). While the average and most probable $\mathrm{C}_{\mathrm{P}} / \mathrm{C}_{\mathrm{S}}$ would be less than 0.1 , we have adopted the upper limit,

$$
\mathrm{C}_{\mathrm{P}} / \mathrm{C}_{\mathrm{S}}=1.0
$$

which is in keeping with the conservative aspects of the analysis.
(1) Estimation of $C_{P}$ by Eq. (14):

$$
\mathrm{C}_{\mathrm{P}}=7 \times 10^{-7} \mathrm{~g} / \mathrm{g} \text {. }
$$

(2) Estimation of I by Eq. (12):

$$
\begin{aligned}
& C_{\text {meat }}=10^{-10} \mathrm{~g} / \mathrm{g}, \\
& I=1.8 \times 10^{-4} \mathrm{~g} / \text { day. }
\end{aligned}
$$

\begin{tabular}{|c|c|c|c|c|}
\hline & Min & $\operatorname{Max}$ & Av & Ref. \\
\hline \multicolumn{5}{|c|}{ Concentrations $(\mu \mathrm{g} / \mathrm{g})$} \\
\hline Beef & & & $2 \times 10^{-2}$ & 4941 \\
\hline Pork & & & $1.9 \times 10^{-2}$ & 4941 \\
\hline Ham & & & $1.2 \times 10^{-2}$ & 4941 \\
\hline Chicken & & & $1.9 \times 10^{-2}$ & 4941 \\
\hline \multicolumn{5}{|c|}{$\begin{array}{l}\text { Uptake relationships } \\
f_{2}^{\prime}\end{array}$} \\
\hline Bone & & & 0.15 & ICRP \\
\hline Kidneys & & & 0.02 & ICRP \\
\hline Liver & & & 0.45 & ICRP \\
\hline Spleen & & & 0.13 & ICRP \\
\hline \multicolumn{5}{|l|}{$\mathrm{f}_{2}$} \\
\hline Bone & & & 0.16 & ICRP \\
\hline Kidneys & & & 0.02 & ICRP \\
\hline Liver & & & 0.5 & ICRP \\
\hline Spleen & & & 0.08 & ICRP \\
\hline
\end{tabular}

(3) $C_{B}^{T B}$ was then estimated by Eq. (10).

$72^{\mathrm{Hf}}$

\section{Collateral Data}

\section{Estimation of $f_{B}$}

\section{Individual Organs}

The $\mathrm{f}_{2}^{1} / \mathrm{m}$ in testes of rats was noted to be exceeded by that in kidneys (5261). We have therefore assumed that the $\mathrm{f}_{2}^{1} / \mathrm{m}$ in testes equals the $\mathrm{f}_{2}^{\prime} / \mathrm{m}$ in kidneys and estimated $\mathrm{f}_{2}^{\prime}$ in testes by Eq. (16a). Since $90 \%$ of the halnium is taken up in tissues other than the gonads, we assumed an $\mathrm{f}_{2}^{\prime}$ in ovaries equal to 0.1 . The $f_{B}$ were then estimated by Eq. (16). 


\section{Estimation of $\mathrm{T}_{\mathrm{B}}$}

\section{Individual Organs}

The $f_{2} / f_{2}^{\prime}$ in the various organs are approximately unity. We therefore assumed an $\mathrm{f}_{2} / \mathrm{f}_{2}^{\prime}$ ratio of 1.0 for the ovaries and testes and estimated $\mathrm{T}_{\mathrm{B}}$ by Eq. (19).

\section{Estimation of $\mathrm{C}_{\mathrm{B}}$}

\section{Whole_Body}

Since hafnium is very poorly absorbed $\left(f_{1}<10^{-4}\right)$, a conservative assumption would be that concentrations in man and cattle are the same. We therefore estimated $C_{B}^{T B}$ in man from the concentration in beef. A conservative $f_{2}$ in muscle, 0.1 , was estimated from distribution in rats (5261). The $\mathrm{C}_{B}^{\mathrm{TB}}$ was estimated by Eq. (23) from the mass of muscle, $2.7 \times 10^{4} \mathrm{~g}$ (5307).

\section{Individual Organs}

The $C_{B}$ in the various organs were estimated by Eq. (22). The $f_{2}$ in the ovaries and testes were those used to estimate $T_{B}$.

\section{Estimation of $\mathrm{C}_{P}$}

The $C_{P}$ values of hafnium found in the literature were $3.5 \times 10^{-3} \mathrm{ppm}$ for "dry plant" (6105), <0.2 ppm for sugar beets (6088), and $<0.07 \mathrm{ppm}$ in kale leaves (6089). A value of $0.01 \mathrm{ppm}$ has been suggested for land plants (5306). We assume that hafnium and zirconium behave similarly. The $\mathrm{C}_{\mathrm{P}} / \mathrm{C}_{\mathrm{S}}$ of hafnium is assumed to be that of zirconium. The $\mathrm{C}_{P}$ was estimated by Eq. (14). The $\mathrm{C}_{P}$ estimated by this approach compares with the values cited above.

\section{Estimation of Aquatic Data}

The ratio of $\mathrm{Hf} / \mathrm{Zr}$ in soil is 0.02 . This value was multiplied by the zirconium concentrations to obtain the hafnium concentrations. 
Collateral Data

\begin{tabular}{|c|c|c|c|c|}
\hline & Min & $\operatorname{Max}$ & Av & Ref \\
\hline \multicolumn{5}{|c|}{ Uptake relationships } \\
\hline \multicolumn{5}{|l|}{$\mathrm{f}_{2}^{1}$} \\
\hline Bone & & & 0.2 & ICRP \\
\hline Kidneys & & & 0.03 & ICRP \\
\hline Liver & & & 0.3 & ICRP \\
\hline Spleen & & & 0.01 & ICRP \\
\hline Rat gonads & & & 0.01 & 5178 \\
\hline \multicolumn{5}{|l|}{$f_{2}$} \\
\hline Bone & & & 0.25 & ICRP \\
\hline Kidneys & & & 0.05 & ICRP \\
\hline Liver & & & 0.5 & ICRP \\
\hline Spleen & & & 0.01 & ICRP \\
\hline Rat gonads & 0.0013 & 0.032 & & 5181 \\
\hline
\end{tabular}

\section{Estimation of $\mathrm{C}_{\mathrm{S}}$}

Tantalum has not been reported in soils. Its concentration in Russian clays averaged $2.0 \mu \mathrm{g} / \mathrm{g}$ (5797). Soil-forming rocks average $2.5 \mu \mathrm{g} / \mathrm{g}(5801)$.

\section{Estimation of $f$}

\section{Whole Body}

We should take into account that the $f_{1}$ of tantalum may be greater than the ICRP value, $f_{1}=10^{-4}$. Niobium and tantalium are second-and thirdtransition-group congeners with very similar atomic and ionic dimensions, and the two elements are very closely associated in nature. According to Schroeder (2219), over half the niobium present in the diet is absorbed. In another study $5 \%$ of an initial $\mathrm{Ta}^{182}$ deposition in the abdominal region had not been eliminated after 5 days (1485), so that the $f_{1}$ may be of the order of $5 \%$. We therefore have assumed that $f_{1}$ and $\mathrm{f}_{\mathrm{B}}^{\mathrm{TB}}$ are equal to 0.05 .

\section{Individual Organs}

The $f_{2}^{\prime}$ in the ovaries and lestes were estimated from $\mathrm{f}_{2}^{\prime}$ in the gonads by Eq. (18). The $f_{B}$ were then estimated by Eq. (16).

\section{Estimation of $\mathrm{T}_{\mathrm{B}}$}

\section{Individual Organs}

The $\mathrm{f}_{2} / \mathrm{f}_{2}^{\prime}$ for the gonads was calculated using the higher $f_{2}$ value. The $T_{B}$ in the ovaries and testes were then estimated by Eq. (19) using this value.

(continued) 
Estimation of $\mathrm{C}_{\mathrm{B}}$

Whole Body

In experiments on the uptake of $\mathrm{Ta}^{182}$ and $W^{185}$ in leaves and stems of bush beans grown on various soils, the quotient of the $\mathrm{C}_{\mathrm{P}} / \mathrm{C}_{\mathrm{S}}$ ratios of tantalum and tungsten varied from 0.08 to 0.8 (5229). The uptake of tantalum did not vary in the 3 soils tested (pH $5.7-8.6$ ), while that of tungsten was greater in the more alkaline soils. In another series of experiments the greatest $\mathrm{C}_{\mathrm{P}} / \mathrm{C}_{\mathrm{S}}$ ratio of $\mathrm{W}^{185}$ in beans or barley grown on different soils was $3.8(2697,5570)$, which was obtained in the most alkaline soil. On the basis of the above relationships, we have estimated a conservative $\mathrm{C}_{\mathrm{P}} / \mathrm{C}_{\mathrm{S}}$ ratio of tantalum to be the greatest quotient of the $\mathrm{C}_{\mathrm{P}} / \mathrm{C}_{\mathrm{S}}$ ratios of tantalum and tungsten times the greatest $\mathrm{C}_{\mathrm{P}} / \mathrm{C}_{\mathrm{S}}$ ratio of tungsten, that is,

$$
\mathrm{C}_{\mathrm{P}} / \mathrm{C}_{\mathrm{S}}=0.8 \times 3.8=3
$$

The uptake of $\mathrm{Ta}^{182}$ in rats was less per gram of muscle than per gram of total body at times up to 256 days ( 5178 , $5179,5181)$. On this basis we can conservatively assume that

$$
\mathrm{C}_{\text {beef }}=\mathrm{C}_{\text {steer }}^{\mathrm{TB}} .
$$

(1) Estimation of $C_{P}$ by Eq. (14):

$$
C_{P}=9 \times 10^{-6} \mathrm{~g} / \mathrm{g} .
$$

(2) Estimation of $I_{\text {steer }}$ by Eq. (13):

$$
I_{\text {steer }}=9 \times 10^{-2} \mathrm{~g} / \text { day. }
$$

(3) Estimation of $\mathrm{C}_{\text {Steer }}^{\mathrm{TB}}$ by Eq. (10);

$$
\mathrm{C}_{\text {steer }}^{\mathrm{TB}}=3.1 \times 10^{-6} \mathrm{~g} / \mathrm{g} \text {. }
$$

(4) Estimation of I by Eq. (12):

$$
\mathrm{I}=3.4 \times 10^{-3} \mathrm{~g} / \text { day. }
$$

(5) The $C_{B}^{T B}$ was then estimated by Eq. (10).

\section{Individual Organs}

The $\mathrm{C}_{\mathrm{B}}$ in bone, kidneys, liver and spleen were estimated by Eq. (22).

\section{Estimation of $\mathrm{C}_{\mathrm{P}}$}

The $\mathrm{C}_{\mathrm{P}}$ of tantalum in kale leaves was given as $<0.1 \mathrm{ppm}$ (6089). The $\mathrm{C}_{\mathrm{P}} / \mathrm{C}_{\mathrm{S}}$ value of tantalum derived from tracer data was given as one-half to one-twelfth that of tungsten (5229). The $\mathrm{C}_{\mathrm{P}} / \mathrm{C}_{\mathrm{S}}$ of tungsten from tracer data is about 0.1 $(2697,5570)$. We assumed that $C_{P} / C_{S}$ of tantalum is one-fourth that of tungsten. The $C_{P}$ value was estimated by Eq. (14).

\section{Estimation of Aquatic Data}

$\mathrm{The} \mathrm{Ta} / \mathrm{Nb}$ ratio in soil is 0.2 . This ratio was multiplied by the niobium concentrations to obtain the tantalum concentrations. 
Estimation of $\mathrm{C}_{\mathrm{S}}$

Rhenium has not been reported in soil. Standard granite contains $6.7 \times 10^{-3} \mu \mathrm{g} / \mathrm{g}$ and standard diabase $5.6 \times 10^{-3} \mu \mathrm{g} / \mathrm{g}(5765)$.

\section{Estimation of $C_{P}$}

Perrhenate, a soluble and stable form of rhenium, like iodide, is actively transported to the thyroid $(947,6352)$. In the absence of definitive plant data, we assumed that the $\mathrm{C}_{\mathrm{P}} / \mathrm{C}_{\mathrm{S}}$ ratio of rhenium

${ }_{76}^{\text {Os }}$

\section{Estimation of $\mathrm{C}_{\mathrm{S}}$}

Osmium has not been reported in soils. It s concentration in standard granite is $6.5 \times 10^{-4}$ to $2 \times 10^{-3} \mu \mathrm{g} / \mathrm{g}$, and in standard diabase, $2.6 \times 10^{-3}$ to $4.6 \times 10^{-3}$

$\mu \mathrm{g} / \mathrm{g}$. Other soil forming rocks range from $4 \times 10^{-5}$ to $4 \times 10^{-2} \mu \mathrm{g} / \mathrm{g}$ (5799).

\section{Estimation of $\mathrm{C}_{\mathrm{P}}$}

Osmium and ruthenium were assumed to behave similarly. equals that of iodine. The $\mathrm{C}_{\mathrm{P}}$ value was estimated by Eq. (14).

\section{Estimation of $\mathrm{C}_{\text {meat }}$}

Rhenium does not concentrate in any particular tissue (5182) except skin and thyroid. It is therefore reasonable to assume that $\mathrm{C}_{\text {meat }}$ equals $\mathrm{C}_{\text {steer }}^{\mathrm{TB}}$.

(1) Estimation of $I_{\text {steer }}$ by Eq. (13): $\mathrm{I}_{\text {steer }}=10 \mu \mathrm{g} / \mathrm{day}$

(2) $\mathrm{C}_{\text {beef }}$ was then estimated by Eq. (10).
The $\mathrm{C}_{\mathrm{P}} / \mathrm{C}_{\mathrm{S}}$ of osmium was assumed to be that of ruthenium. The $\mathrm{C}_{\mathrm{P}}$ value was then estimated by Eq. (14.)

\footnotetext{
Estimation of $\mathrm{C}_{\text {meat }}$

The $\mathrm{C}_{\text {meat }} / \mathrm{C}_{\mathrm{S}}$ of osmium was assumed to be that of ruthenium. The $\mathrm{C}_{\text {meat }}$ was estimated from the product of the ratio and $\mathrm{C}_{\mathrm{S}}$.
} 


$$
77^{\text {Ir }}
$$

Collateral Data

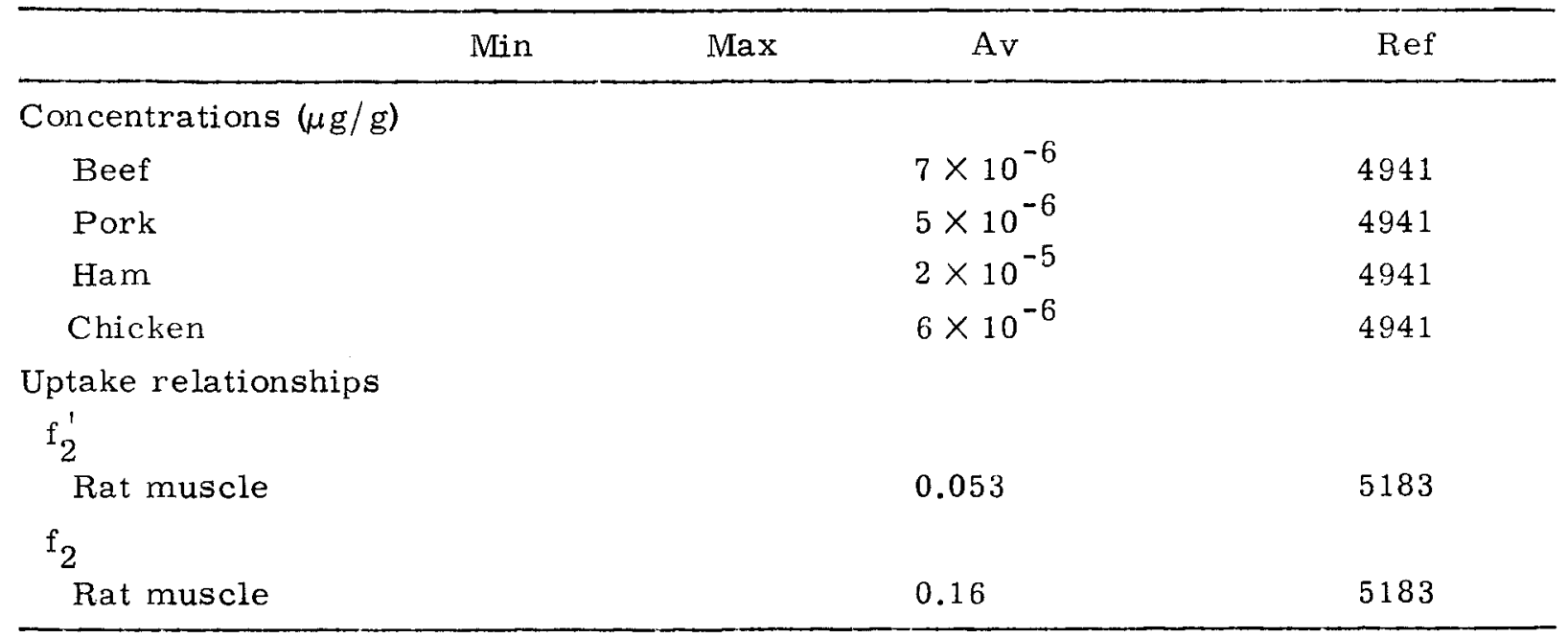

\section{Estimation of $C_{B}$}

Whole Body

The uptake parameters for rat muscle are assumed to apply to the steer.

(1) Estimation of $f_{B}$ and $T_{B}$ in beef (muscle) by Eqs. (16) and (19):

$$
\begin{aligned}
\mathrm{f}_{\mathrm{B}} & =5.3 \times 10^{-3}, \\
\mathrm{~T}_{\mathrm{B}} & =6 \times 10^{1} \text { day. }
\end{aligned}
$$

(2) Estimation of $I_{\text {steer }}$ by Eq. (3):

$$
\begin{aligned}
& \mathrm{C}_{\text {beef }}=10^{-11} \mathrm{~g} / \mathrm{g}, \\
& \mathrm{I}_{\text {steer }}=5.4 \times 10^{-6} \mathrm{~g} / \text { day. }
\end{aligned}
$$

(3) Estimation of $C_{P}$ by Eq. (15):

$$
C_{P}=5.4 \times 10^{-10} \mathrm{~g} / \mathrm{g} .
$$

(4) Estimation of I by Eq. (12):

$$
\begin{aligned}
& C_{\text {meat }}=2 \times 10^{-11} \mathrm{~g} / \mathrm{g} . \\
& I=1.7 \times 10^{-7} \mathrm{~g} / \text { day } .
\end{aligned}
$$

(5) $C_{B}^{\text {TB }}$ was then estimated by Eq. (10).

\section{Estimation of $\mathrm{C}_{\mathrm{P}}$}

The $C_{P}$ was assumed to be that derived above under Estimation of $C_{B}$ for Whole Body. 
Collateral Data

\begin{tabular}{|c|c|c|c|c|}
\hline & Min & $\operatorname{Max}$ & $\mathrm{Av}$ & Ref. \\
\hline \multicolumn{5}{|l|}{ Concentrations $(\mu \mathrm{g} / \mathrm{g})$} \\
\hline Beef & & & $7 \times 10^{-4}$ & 4941 \\
\hline \multicolumn{5}{|l|}{ Uptake relationships } \\
\hline $\mathrm{f}_{2}$ & & & & \\
\hline Rat muscle & & & 0.22 & 5183 \\
\hline
\end{tabular}

\section{Estimation of $\mathrm{C}_{P}$}

The uptake parameters for rat muscle are assumed to apply to the steer. The value of $\mathrm{C}_{\mathrm{P}}$ calculated below does not contradict the value for tea leaves (6087).

(1) Estimation of $\mathrm{C}_{\text {steer }}^{\mathrm{TB}}$ by Eq. (23):

$$
\mathrm{C}_{\text {steer }}^{\mathrm{TB}}=1.3 \times 10^{-9} \mathrm{~g} / \mathrm{g}
$$

(2) Estimation of $\mathrm{I}_{\text {steer }}$ by Eq. (4):

$$
\mathrm{I}_{\text {steer }}=1.84 \times 10^{-4} \mathrm{~g} / \text { day }
$$

(3) $\mathrm{C}_{\mathrm{P}}$ was then estimated by Eq. (15). 
$80^{\mathrm{Hg}}$

Collateral Data

\begin{tabular}{|c|c|c|c|c|}
\hline & Min & $\operatorname{Max}$ & Av & Ref. \\
\hline \multicolumn{5}{|l|}{ Concentrations $(\mu \mathrm{g} / \mathrm{g})$} \\
\hline Human adipose tissue & & & $\times 10^{-3}$ & 4756 \\
\hline Human heart & $4.3 \times 10^{-2}$ & $5.2 \times 10^{-2}$ & & 1294,4756 \\
\hline Human kidneys & & & $\times 10^{-2}$ & 893 \\
\hline Human liver & $\times 10^{-3}$ & $3.3 \times 10^{-2}$ & & 893,4756 \\
\hline Human muscle & & & $\times 10^{-2}$ & 4756 \\
\hline Bovine heart & $2.9 \times 10^{-3}$ & & $6.1 \times 10^{-2}$ & 1745 \\
\hline
\end{tabular}

Estimation of $\underline{C}_{B}$

\section{Whole Body}

The $\mathrm{f}_{2} / \mathrm{m}$ of mercury in the kidneys, liver, spleen and lungs of rat were much higher than the $f_{2} / m$ in other soft tissues and bone. We therefore estimated $\mathrm{C}_{\mathrm{B}}^{\mathrm{TB}}$ by Eq. (27) using a $C_{B}$ of $2 \times 10^{-2} \mu \mathrm{g} / \mathrm{g}$ for muscle and the maximum known $\mathrm{C}_{\mathrm{B}}$ of $5 \times 10^{-2} \mu \mathrm{g} / \mathrm{g}$ for the other soft tissues and bone.

\section{Estimation of Aquatic Data}

Estimation of Concentration in Invertebrates

Although Ref. 1308 gives a concentration of $4 \times 10^{-2} \mu \mathrm{g} / \mathrm{g}$ in molluscs, mercury was assumed to behave like zinc and cadmium where a concentration ratio over seawater of $10^{5}$ applies for oysters. The concentration in seawater and freshwater invertebrates was therefore set equal to $10^{5}\left(\mathrm{C}_{\underline{A}}\right)$. 
-

0

- 


$$
81^{\mathrm{T} l}
$$

\section{Collateral Data}

\begin{tabular}{|c|c|c|c|c|}
\hline & Min & $\operatorname{Max}$ & $\mathrm{Av}$ & Ref. \\
\hline \multicolumn{5}{|c|}{$\begin{array}{l}\text { Uptake relationships } \\
f_{2}^{\prime}\end{array}$} \\
\hline Rat spleen & & & 0.004 & $5168 \mathrm{~B}, 5184,5185$ \\
\hline Rat gonads & 0.0004 & 0.0006 & & 5184,5185 \\
\hline \multicolumn{5}{|l|}{$\mathrm{f}_{2} / \mathrm{f}_{2}^{\prime}$} \\
\hline Bone & & & 1.45 & ICRP \\
\hline Kidneys & & & 1.4 & ICRP \\
\hline Liver & & & 1 & ICRP \\
\hline Rat spleen & & & 1 & 5184,5185 \\
\hline \multicolumn{5}{|l|}{$\mathrm{f}_{2}$} \\
\hline Bone & & & 0.08 & ICRP \\
\hline \multicolumn{5}{|l|}{$\mathrm{T}_{\mathrm{B}}^{\mathrm{TB}}(\mathrm{dzy})$} \\
\hline Rat & 4.8 & 8.8 & 6 & 5416 \\
\hline Dog & 4.6 & 25 & & 5416 \\
\hline
\end{tabular}

\section{Estimation of $\mathrm{C}_{\mathrm{S}}$}

Thallium has not been reported in soils. Its average concentration in soilforming rocks is $7.0 \times 10^{-1} \mu \mathrm{g} / \mathrm{g}$ (5788).

\section{Estimation of $f_{B}$}

\section{Individual Organs}

The $f_{2}^{\prime}$ in the ovaries and testes were estimated from $f_{2}^{\prime}$ in the gonads by Eq. (18). The $f_{B}$ in these organs and spleen were then estimated by Eq. (16).

\section{Estimation of $\mathrm{T}_{\mathrm{B}}$}

\section{Whole Body}

In the absence of human data the maximum $T_{B}^{T B}$ observed in the dog is assumed to apply to man.

\section{Individual Organs}

The $\mathrm{T}_{\mathrm{B}}$ were estimated by Eq. (19).

Estimation of $\mathrm{C}_{B}$

Individual Organs

The $\mathrm{C}_{\mathrm{B}}$ in bone was estimated by

Eq. (22).

\section{Estimation of $C_{P}$}

We have assumed that the $\mathrm{C}_{\mathrm{P}} / \mathrm{C}_{\mathrm{S}}$ of thallium is comparable to that of potassium and rubidium $(5416,6352)$, $\mathrm{C}_{\mathrm{P}} / \mathrm{C}_{\mathrm{S}} \sim 1$. The value of $\mathrm{C}_{\mathrm{P}}$ was estimated by Eq. (14).

\section{Estimation of $C_{\text {meat }}$}

In the absence of any meat data, we have assumed that both the uptake and (continued) 


\section{$83^{\mathrm{Bi}} 84^{\mathrm{Po}}$}

retention are similar from one mammal to another. The $f_{B}$ in muscle is 0.24 (5593). Since $T_{B}$ in muscle is comparable to $T_{B}^{T B}(5593)$, we have assumed that $T_{B}$ in muscle is equal to $\mathrm{T}_{\mathrm{B}}^{\mathrm{TB}}$.

(1) Estimation of I steer by Eq. (13):

$$
I_{\text {steer }}=10^{-3} \mathrm{~g} / \text { day }
$$

(2) $\mathrm{C}_{\text {meat }}$ was then estimated by Eq. (9).

$83^{\mathrm{Bi}}$

\section{Estimation of $C_{S}$}

Bismuth has not been reported in

$$
84^{\mathrm{Po}}
$$

\section{Estimation of $\mathrm{C}_{\mathrm{S}}$}

Of the polonium isotopes, only those of the uranium decay chain have halflives greater than one second, so that we can regard uranium as the parent element of naturally occurring polonium (5840). The $\mathrm{C}_{\mathrm{S}}$ was estimated by Eq. (34) from the following concentrations of polonium and uranium in igneous rock (2532):

\section{Estimation of Aquatic Data}

Estimation of $\mathrm{C}_{\mathrm{A}}$ for Seawater

Reference 1935 states that thallium is present in less than $10^{-5} \mu \mathrm{g} / \mathrm{g}$.

Reference 6054 gives concentrations in fish and invertebrates of about $1 \times 10^{-1}$ $\mu \mathrm{g} / \mathrm{g}$. It was assumed that these foodstuff values represent a concentration ratio over seawater of $10^{5}$, and hence a seawater $\mathrm{C}_{\mathrm{A}}$ of $10^{-6} \mu \mathrm{g} / \mathrm{g}$.

soils. Its average concentration in soilforming rocks is $1.7 \times 10^{-1} \mu \mathrm{g} / \mathrm{g}$ (5788).

$$
\begin{aligned}
\left(c_{\text {rock }}\right)_{P_{O}} & =2 \times 10^{-10} \mu \mathrm{g} / \mathrm{g} . \\
\left(c_{\text {rock }}\right)_{U} & =4 \mu \mathrm{g} / \mathrm{g} .
\end{aligned}
$$

\section{Estimation of Aquatic Data}

The ratio of $\mathrm{Po} / \mathrm{Se}$ in soil is $10^{-10}$. This ratio was multiplied by the selenium concentrations to obtain the polonium values. 
Estimation of $\mathrm{C}_{\mathrm{S}}$

$\mathrm{At}^{218}$ from the decay of $\mathrm{U}^{238}$ is the major contribution to the $\mathrm{C}_{\mathrm{S}}$ of astatine. $\mathrm{C}_{\mathrm{S}}$ was estimated by Eq. (33) with $\mathrm{f}=0.02$.

\section{Estimation of $\mathrm{C}_{\mathrm{P}}$}

Astatide ion, like iodide, is concentrated well by thyroid tissue (947). We have assumed that the $\mathrm{C}_{\mathrm{P}} / \mathrm{C}_{\mathrm{S}}$ ratios of

\section{${ }_{87} \mathrm{Fr}$}

\section{Estimation of $C_{S}$}

The $\mathrm{C}_{\mathrm{S}}$ of francium was estimated as $\mathrm{Fr}^{223}$ derived from Ac ${ }^{227}(4 n+3$ series). $\mathrm{C}_{\mathrm{S}}$ was estimated by Eq. (33) with $\mathrm{f}=0.012$.

\section{Estimation of $C_{P}$}

Francium is assumed to behave like cesium. The $\mathrm{C}_{\mathrm{P}} / \mathrm{C}_{\mathrm{S}}$ of francium was assumed to be that of cesium. The $C_{P}$ value was estimated by Eq. (14).

\section{Estimation of Aquatic Data}

The ratio of $\mathrm{Fr} / \mathrm{Cs}$ is soil is $2 \times 10^{-19}$. This ratio was multiplied by the cesium concentrations to obtain the francium values.

\section{Estimation of $\mathrm{C}_{\mathrm{S}}$}

The $\mathrm{C}_{\mathrm{S}}$ of actinium was estimated as $\mathrm{Ac}^{227}$ derived from $\mathrm{Pa}^{231}(4 \mathrm{n}+3$ series $)$ and $\mathrm{Ac}^{228}$ derived from $\mathrm{Ra}^{228}$ (4n series). The $\mathrm{C}_{\mathrm{S}}$ were estimated by Eq. (33) with $f=1.0$ for both series, and then summed to yield the total $\mathrm{C}_{\mathrm{S}}$.

\section{Estimation of $\mathrm{C}_{\mathrm{P}}$}

The $\mathrm{C}_{\mathrm{P}} / \mathrm{C}_{\mathrm{S}}$ of actinium was assumed to be $10^{-2}$, the greatest $C_{P} / C_{S}$ ratio found for any actinide. The $\mathrm{C}_{\mathbf{P}}$ was estimated by Eq. (14). astatine and iodine are comparable and adopted a $\mathrm{C}_{\mathrm{P}} / \mathrm{C}_{\mathrm{S}}$ ratio of 1 . The $\mathrm{C}_{\mathrm{P}}$ value was estimated by Eq. (14).

\section{Estimation of Aquatic Data}

The ratio of $A t / I$ in soil is a $2 \times 10^{-20}$. This ratio was multiplied by the iodine concentration to obtain the astatine values. 
Estimation of $C_{S}$

The $\mathrm{C}_{\mathrm{S}}$ of protactinium was estimated as $\mathrm{Pa}^{231}$ derived from $\mathrm{U}^{235}(4 \mathrm{n}+3$ series). $\mathrm{C}_{\mathrm{S}}$ was estimated by Eq. (33) with $\mathrm{f}=1.0$.

\section{$93^{\mathrm{Np}}$}

\section{Estimation of $\mathrm{C}_{\mathrm{S}}$}

Neptunium isotopes have been isolated from uranium ores where they are

formed by neutron capture. $\mathrm{Np}^{237}$ is the most abundant (5759). The ratio of the concentrations of $\mathrm{Np}^{237}$ to $\mathrm{U}^{238}$ in uranium ore is $1.8 \times 10^{-12}$ (5757). The $\mathrm{C}_{\mathrm{S}}$ was estimated from this ratio by Eq. (34).

$$
{ }_{94}^{\mathrm{Pu}}
$$

\section{Estimation of $\mathrm{C}_{\mathrm{S}}$}

Plutonium has been qualitatively identified in soils (5780). Its concentration was estimated from the ratio of the concentrations of $\mathrm{Pu}^{239}$ to $\mathrm{U}$ in ores: $<4 \times 10^{-13}$ to $1.5 \times 10^{-11}$ (5758). The $\mathrm{C}_{\mathrm{S}}$

$$
95^{\mathrm{Am}}
$$

\section{Estimation of $\mathrm{C}_{P}$}

The $\mathrm{C}_{\mathrm{P}} / \mathrm{C}_{\mathrm{S}}$ of americium in crop plants was about $3 \times 10^{-3}$ (6079). was estimated by Eq. (34) assuming a concentration ratio of $10^{-13}$.

\section{Estimation of $\mathrm{C}_{\mathrm{P}}$}

The $\mathrm{C}_{\mathrm{P}} / \mathrm{C}_{\mathrm{S}}$ of plutonium ranged from $10^{-3}$ to $10^{-4}(2697,5570,6079)$. The $C_{P}$ value was estimated by Eq. (14) using a $\mathrm{C}_{\mathrm{P}} / \mathrm{C}_{\mathrm{S}}$ value of $10^{-3}$.
The $C_{P}$ value was estimated by Eq. (14) using a $C_{P} / C_{S}$ value of $10^{-3}$. 


\section{Bibliographic Citations}

\section{for Selected Accession Numbers}

Journal title abbreviations appear in the form used by Chemical Abstracts. A listing of the report series codes and the source agencies precedes the bibliographic citations 
$A / \triangle C .82 / G / R$.

$\triangle /$ CONF. $15 / \mathrm{P} /$

$A C-$

$A E-$

$\triangle E C-T R-$

$\triangle E C L-$

$\triangle E C L$ -

$\triangle E C U-$

$A E[-C D N F-(Y R)-$

AERE-R-

AERE-SPAR-

AHSE(RP)R-

GN hL -

ENWL-SA-

CAPE TOWN U THESIS

CEA-

CEA-TR-

CONF-

$\cos -$

CRER -

CPSPU-(YR)-
LNI TEO NATIONS. SECRETARIAT

UNI TED NATIONS. INTERNATICNAL CONFERENCE ON THE PEACEFUL USES OF ATOMIC ENERGY, 1958

DEFENSE DOCUMENTATION CENTER, ARLINGTON, VA.

AKTIEBOLAGET ATOMENERGI, STOCKHCLM

DIVISION OF TECHNICAL INFORMATION EXTENSION, AEC (TRANSLATIONS)

DIVISION OF TECHNICAL INFORMATION EXTENSION, AEC ICCNTINUATION OF MDDC-)

ATOMIC ENFRgY OF CANADA LTD. CHALK RIVER PROJECT, CHALK RIVER, ONT. DIVISION OF TECHEIICAL INFORMATION EXTENSION, AEC

ZENTRALSTELLE FUER ATOMKERNENERGIE-DOCUMENTATION, FRANKFURT AM MAIN (WEST GERMANY)

GT. BRIT. ATOMIC ENERGY RESEARCH ESTABLISHMENT, HARLELL, BERKSHIRE, ENGLAND

GT. BRIT. ATJMIC ENERGY RESEARCH ESTABLI SHMENT, HARWELL, BERKSHIRE, ENGLAND

UNI TED KINGDOM ATOMIC ENERGY AUTHORITY. AUTHORITY HEALTH AND SAFETY BRANCH, RADIOLOGICAL PROTECTION DIV., HARWELL, BERKSHIRE, ENGLAND

BATTELLE-NORTHWEST, RICHLAND, WASH.

BATTELLE-NORTHWEST, RICHLAND, WASH.

CAPE TOWN UNIVERSITY, CAPE TOWN, SOUTH AFRICA (THESIS)

FRANCE. COMMISSARIAT a L'ENERgIE atomique, PARIS

FRANCE. COMMISSARIAT A L'ENERGIE ATOMIQUE, PARIS ITHIS SEZIES USED BY THE AEC TO NUMBER TRANSLATIONS RECEIVED FROM THE CEA)

DIVISION OF TECHNICAL INFORMATION EXTENSION, AEC IASSIGNED TO ALL CONFERENCESI

CHICAGO OPERATIONS DFFICE, AEC

ATOMIC ENERGY OF CANADA LTD. CHALK RIVER PRDJECT, CHALK RIVER, ONT.

in pont de vemuurs (E. I.) and co. savanivah River lab., aiken, S. C. 
ERI-

EUR-

$F A C-\triangle E-$

FWE-

$\mathrm{HW}=$

HW-SA-

I CRP-

JPRS-

LA-

LAMS-

MOCC-

NAS-NRC/PUB-

NP-

NSEC-

ORINS-

CRAL -

ORNL-P-

ORO-

$\mathrm{PE}-$

$\mathbf{P} \mathrm{C}_{3}-$

Pr $\leq-L I V-$
ATOMIC ENERGY OF CANADA LTD., CHALK RIVER, ONT.

EUROPEAN ATOMIC FNERGY COMMUNITY

UNITED NATIONS, ROME. FOOD AND AGRICULTURE ORGAVIZATION IATOMIC SNERGY SERIESI

CIVISION OF TECHNICAL I INFURMATICN EXTENSION, AEC. FOREIGN WEAPONS EFFECTS REPQRTS ITHIS SERIES IS USED BY THE $\triangle E C$ TO IDENTIFY WEAPONS TEST REPORTS PREPARED BY VARIOUS BRITISH AND CANADIAN AGENEIES

RELEASEC TO THE U. S.I

HANFORD WORKS, RICHLAND, WASH.

HANFORD WORKS, P.ICHLAND, WASH.

INTERNATIONAL COMMISSIJN ON RADIOLOGICAL PROTECTION

JOINT PUBL ICATIONS RESEARCH SERVICE, NEW YORK

LOS ALAMOS SCIENTIFIC LAB., N. MEX.

LOS ALAMOS SCIENTIFIC LAB., N. MEX.

NANHATTAN DISTRICT, OAK RIDGE, TENN. (CONTINUED A? AECD-)

NATIONAL ACADEMY OF SCIENCES AND NATIONAL RESEARCH COUNCIL (PUBL ICATION)

UIVISION OF TECHNICAL INFORMATION EXTENSION, AEC INON-PROJEZT REPORT. INI TIALLY ASSIGNED TO ALL INON-AEC REPCRTS BUT LATER USED ONLY FOR THOSF WITHOUT OTHER NOS .1

NUCLEAR SCIENCE 410 ENGINEERING CORP., PITTSBURGH, PA.

DAK RIDGE IVST. OF NUCLEAR STUDIES, DAK RIDGE, TENN.

CAK RIDGE VATIONAL LAB̈, DAK RIOGE, TENN.

CAK RIDGE NATIUVAL LAE., OAK RIDGT, TENN.

CAK RIDGE (TENN.) LIPERATIOHS DFFICE, AEC

OFFICE OF TECHNICAL SERVICES, WASHINGTON, D. C.

UNI TED KINGUOM ATOMIC ENERGY AUTHORITY. PRODUCTION GROUP, RISLEY, LANCASHIRE, ENGLAVL

CALIFORNIA. UNIV., LIVERMURE RADIATION LAP. IASSIGNED FOR INTERNAL USE TO PLBLIC HEALTH SERVICE DOCUMENTS WITHOUT UTHER NOS.) 
PRNC-

RLC-

$S M-(N O \cdot) /(N O \cdot)$

SR I-

STI/PUB/

TI [-

UCLA-

UCRL -

USNRCL-TR- (YR)-

UWFL-

WT-

YALE-
PUERTO RICO NUCLEAK CENTER, SAN JIJAN

RICHLAND (WASHINGTUN) OPERATIONS OFFICE, $A E C$

INTERNATIONAL ATOMIC ENERGY AGENCY, VIENVA

STANFORD RESEARCH INST., MENLO PARK, CALIF.

INTERNATIONAL ATOMIC ENERGY AGENCY, VIENNA

DIVISION OF TECHNICAL INFORMATION EXTENSIOV, AEC

CALIFORNIA. UNIV., LOS A:VGELES

CALIFORNIA. UNIV., RADIATION LAB.

AAVAL RADIOLOGICAL DEFENSE LAB., SAN FRANCISCO, CALIF.

WASHINGTON. UNIV., SEATTLE. APPLIED FISHERIES LAB.

ASSIGNED TO REPURTS TO THE SCIENTIFIC DIRECTOR OF JOINT AEC-DOD HEAPON TESTS

YALE UNIVERSITY, NEW HAVEN, CONN. 
72 A JOHNSON J M+

72 B BUTLER G H+

244 ANDERSON E :

268 SQUIRE H M+

323 SEYMOUR A

371 BRYANT F Jt

426 MICHELSON I*

506 A MAUCHL INE $J$

506 B MAUCHLINE J+

874 A CASEY H H+

874 B CASEY H W+

874 C CASEY H H+
I ODINE CONTENT OF PASTURE PLANTS. 1. METHOD OF DETERMINATION ANO PRELI MINARY INVESTIGATION OF SPECIES AND STRAIN DIFFERENCES.

PHYSIOL. PLANTARUM

10. 100-111 (1957)

IODINE CONTENT DF PASTURE PLANTS. 2. INHERI TANCE OF LEAF IODINE CONTE NT OF PERENNIAL RYEGRASS (LOLIUM PERENNE 1.1. AUSTRALIAN J. BIOL. SCI.

$15, \quad 183-187(1962)$

CORRELATION OF CESIUM-137 IN MILK WITH RAINFALL.

LAMS-2780 144- 148 (1962)

THE METABOLISM BY DAIRY COWS OF FISSION PRODUCTS FROM OPERATION BUFFAL

FWE-229 , 103- 130 (1959)

SUMMARY OF REPORTS ON FALLOUT NUCLIDES IN THE MARINE ENVIRONMENT PRESE NTED AT THE 1961 RADIOECOLOGY SYMPOSIUM.

TID-7632 2, 348-359(1961)

STRONTIUM-90 IN THE AUSTRALIAN ENVIRONMENT, 1957 TO 1960. AUSTRAL I AN J. SCI

24. $397-409$ (1962)

RADIOACTIVITY IN TOTAL DIET.

J. NUTR.

78, $371-383(1962)$

THE BIOLOGICAL AND GEOGRAPHICAL DISTRIBUTION IN THE IR ISH SEA OF RADIO ACTIVE EFFLUENT FROM WINDSCALE WORKS 1959 TO 1960. AHSB(RP)R-2T (1963)

DISPERSION IN THE IRISH SEA OF THE RADIOACTIVE LIQUID EFFLUENT FROM WI NDSCALE WORKS OF THE U. K. ATOMIC ENERGY AUTHDRITY.

NATURE

198. 623-626 (1963)

METABOL ISM OF TE-132-I-132 IN LACTATING SHEEP.

HH-SA-3073 (1963)

METABOLI SM OF TE-132-I-132 IN LACTATING SHEEP. HEALTH PHYS

$$
\text { 9. 1223-1226(1963) }
$$

METABOLISM OF TE-132-I-132 IN LACTATING SHEEP. HW-80500 13- 16 (1964)

EXPERIMENTS DN THE METABOLISM OF CERTAIN FISSION PRODUCTS IN DAIRY COH

'RAOIOI SOTOPES IN SCIENTIFIC RESEARCH,'R. C. EXTERMANN, ED. PROCEEdING $S$ OF THE INTERNATIONAL CONFERENCE HELD IN PARIS, 1957. NEW YORK. PERGAM ON PRESS

4, 207- $220(1958)$ 
893 A SAMSAHL $K+$

893 B SAMSAHL $K+$

$928 \quad B O L I N B$

947 HOLFF $J$

983 FUKAI $R+$

1055 BOWEN V T+

1182 DESELM H R+

1185 SLOUKA $V$

1294 A WESTER P 0

1294 B WESTER P O

1299 PILLAI K C +

1301 TSIVOGLOU E C+
SIMUL TANEOUS DETERMINATION OF 30 TRACE ELEMENTS IN CANCEROUS AND NONCA NCEROUS HUMAN TISSUE SAMPLES WITH GAMMA-RAY SPECTROMETRY.

AE- 124 $(1963)$

SIMULTANEOUS DETERMINATION OF 30 TRACE ELEMENTS IN CANCEROUS AND NON-C ANCERDUS HUMAN TISSUE SAMPLES BY NEUTRON ACTIVATION ANALYSIS.

INTERN. J. APPL. RADIATION ISOTOPES

16. 273- 281 (1965)

NOTE ON THE EXCHANGE OF IODINE BETWEEN THE ATMOSPHERE, LAND AND SEA. INTERN. J. AIR POLLUTION

2. $127-131$ (1959)

TRANSPORT OF IODIDE AND OTHER ANIONS IN THE THYROID GLAND.

PHYSIOL. REV.

44. 45- 70 (1964)

TRACE ANALYSIS OF MARINE ORGANISMS. A COMPARISON OF ACTIVATION ANALYSI

$S$ AND CONYENTIONAL METHODS.

S AND CONVENTIONAL

4. 398- 408 (1959)

STRONTIUM-90 IN NORTH ATLANTIC SURFACE WATER.

PROC. NATL. ACAD. SCI. U. S.

43. $576-580(1957)$

ACCUMULATION AND CYCLING OF ORGANIC MATTER AND CHEMICAL CONSTITUENTS D URING EARLY VEGETATIONAL SUCCESSION ON A RADIOACTIVE HASTE DISPOSAL AR

ORO -580

(1962)

EXCRETION ANALYSIS OF INTERNAL CONTAMINATION BY RADIOCERIUM CE- 144 . FOLIA BIOL. (PRAGUE)

$$
\text { 6. } 248-256(1960)
$$

CONCENTRATI ON OF 24 TRACE ELEMENTS IN HUMAN HEART TISSUE DETERMINED BY NEUTRON ACTI VATION ANALYSIS.

AE-146

$$
\text { (1964) }
$$

CONCENTRATION OF 24 IRACE ELEMENTS IN HUMAN HEART TISSUE DETERMINED BY NEUTRON ACTI VATION ANALYSIS. SCAND. J. CLIN. LAB. INVEST

17. 357- 370 (1965)

PLUTONIUM IN THE MARINE ENVIRONMENT.

NATURE

203, 568- $571(1964)$

SURVEY OF INTERSTATE POLLUTION OF THE ANIMAS RIVER (COLORADO-NEH MEXIC

PHS-LIV-6 (1959) 


$\begin{array}{ll}1307 & \text { FONTAINE } Y \\ 1308 & \text { GIRARDI F+ } \\ 1405 & \text { FOLSOM T R+ } \\ 1406 & \text { GUTKNECHT J } \\ 1415 & \text { CHIPMAN H A+ } \\ 1651 & \text { BEESON K C } \\ 1528 & \text { RELUMHOLZ L A+ } \\ 1485 & \text { PALMER H E } \\ & \end{array}$

RADIOACTIVE CONTAMINATION OF AQUATIC MEDIA AND ORGANISMS. CEA-1588 $(1960)$ /AEC-TR-5358

STUDIES ON THE DISTRIBUTION OF TRACE ELEMENTS IN A MOLLUSK FROM A FRES HHATER ENVIRONMENT, BY ACTIVATION ANALYSIS.

EUR-474.E
ICDNF-384-1

MANGANESE-54 AND ZINC-65 IN COASTAL ORGANISMS OF CALIFORNIA. NATURE

200. $327-329(1963)$

ABSORPTION, ACCUMULATIDN AND LOSS OF RADIOACTIVE SCANDIUM BY MARINE MA CROALGAE.

BIOL. BULL.

$121.374-375(1961)$

UPtake and accumulation of radioactive zinC by maR INe PLANKTON, fish. AND SHELLFISH.

U. S. FISH WILOLIFE SERV. FISHERY BULL.

58, $279-292(1958)$

(BULL 135)

THE MINERAL COMPOSITION OF CROPS HITH PARTICULA? REFERENCE TO THE SOIL $S$ IN WHICH THEY WERE GROWN.

U. S. DEP T. AGR. MISC. PUBL. 369. $(1941)$

RETENTION OF RADIOISOTOPES.

$\mathrm{HW}-70050$ ? 42- 46 (1961)

PLANT UPTAKE OF RADIOELEMENTS FROM SOIL.

NP-13796 (1964)

/AD-434092 (1964)

/SRI-PROJ-IM-4536 (1964)

RADIONUCLIDE CONTENT DF OCEANIC FISH, PLANKTON, aNd hater IN THE VICIN

ITY OF CHRI STMAS ISLAND, MARCH THROUGH AUGUST, 1962.
UWFL-88

STRONTIUM AND BARIUM IN SEA WATER AND MARINE ORGANISMS.

J. MARINE BIOL. ASSOC. U. K.

35. 451- 460 (1956)

ACCUMULATION AND RETENTION OF RADIOACTIVITY FROM FISSION PRODUCTS AND OTHER RADIOMATERIALS BY FRESH-HATER ORGANISMS.

-EFFECTS OF ATOMIC RADIATION ON OCEANOGRAPHY AND FISHERIES, THE .' REPOR T OF THE COMMITTEE ON EFFECTS OF ATOMIC RADIATION ON OCEANOGRAPHY AND FI SHERIES OF THE NATIONAL ACADEMY OF SCIENCES STUDY OF THE BIOLOGICAL EFFE CTS OF ATOMIC RADIATION. WASHINGTON, D. C.. NATIONAL ACADEMY OF SCIENCE S-NATIONAL RESEARCH COJNCIL. B8- $95(1957)$

/NAS-NRC/PUB-551, 88- 95 (1957) 
$1716 \quad$ LIETH H

1745 A WESTER P

1745 B WESTER P

1751 SCHROEDER H A

1759 KORANDA J J

1762 THOMPSON $\mathrm{S}=$

1774 A TIPION I H+

1774 B TIPTON I H+

1784 A HARVEY R

1784 B HARVEY $R$ S

1794 TAMPLIV A R

1798 TIPTON I H+
THE RULE OF VEgETATION IN THE CARBON DIOXIDE CONTENT OF THE ATMOSPHERE

J. GEOPHYS. RES.

$68,3887-3898(1963)$

CONCENTRATION OF 17 ELEMENTS IN SUBCELLULAR FRACTIONS OF BEEF HEART TI SSUE DE TERMINED BY NEUTRON ACTIVATION ANALYSIS.

$\begin{array}{ll}A E-169 & (1964)\end{array}$

CONCENTRATION OF 17 ELEMENTS IN SUBCELLULAR FRACTIONS SF BEEF HEART TI SSUE DETERMINED BY NEUTRON ACTIVATION ANALYSIS.

BIOCHIM. BIOPHYS. ACTA

109, $268-283(1965)$

THE BIOLOGICAL TRACE ELEMENTS, OR PERIPATETICS THROUGH THE PERIODIC TA BLE.

J. CHRONIC DI SEASES

$$
\text { 18, } 217-228(1965)
$$

AGRICULTURAL FACTORS AFFECIING THE OAILY INTAKE OF FRESH FALLOUT BY DA IRY COWS.

UCRL-12479 (1965)

EFFECTIVE HALF-LIFE OF FALLOUT RAOIOYUCL IDES JN PLANTS WITH SPECIAL EM PHASIS ON IODINE- 131 .

UCRL -12388 (1965)

TRACE ELEMENTS IN HUMAN TISSUE. PART III. SUBJECTS FROM AFriza, the ne AR AND FAR EAST. AND EURCPE.

ORNL-P-439 (1964)

TRACE ELEMENTS IN hUMAN TISSUE. PART III. SUBJECTS fROM AFRI:A, THE Ne AR AND FAR EAST AND EUROPE.

HEALTH PHYS.

11. $403-451(1965)$

UPTAKE OF RAOIONUCLIDES BY FRESH WATER ALGAE AYG FISH. DPSPU $-63-30-38 \quad(1903)$

UPTAKE OF RADIONUCLIDES EY FRESH WATER ALGAE ANO FISH. HEALTH PHYS.

10. 243- $247(1964)$

1-131, I-133, AND COW MILK.

UCRL -14146 (1965)

TRACE ELEMENTS IN hUMAN TISSUE. PART II. AOULT SUBJeCtS fRom the UNITE D STATES.

HEALTH PHYS.

9. 103‥ 145 (1963) 
1835 CARRITT DE+

1848

BLACK W A P+

1851

1852

1859

$\stackrel{\substack{\omega \\ i}}{i}$

\section{CULKIN F+}

KRUMHOL Z L A

1907

1911

LEWIS G Jt

PRECIPITATION OF FISSION PRODUCT ELEMENTS ON THE CCEAN BOTTOM SY PHYSI CAL, CHEMICAL, AND BIOLOGICAL PROCESSES.

-EFFECTS OF ATOMIC RADIATION ON OCEANOGRAPHY AND FISHERIES, THE." REPGR $T$ DF THE COMMITTEE ON EFFECTS OF ATOMIC RADIATION ON OCEANDGRAPHY AND FI SHERIES OF THE NATIONAL ACADEMY OF SCIENCES STUDY OF THE BIOLOGICAL EFFE SHERIES OF THE NATIONAL ACADEMY OF SCIENCES STUDY OF THE BIOLOGICAL EFFE
CTS OF ATOMIC RADIATIOY. NASHINGTON, D. C. VATIONAL ACADEMY OF SCIENCE CTS OF ATOMIC RADIATIOV. NASH
S-NATIONAL RESEARCH COSNCIL.

GO- 68 (1757)

INAS-NRC/PUB-551 , 60- $68(1957)$

TRACE ELEMENTS IN IHE COMMON BROWN ALGAE AVD IV SEA WATER.

J. MARINE BIDL. ASSOC. J. K.

$30,575-584(1952)$

CANNED aTLANTIC CRAB MEAT. A NEW AMERICAN FOOD.

IND. ENG. CHEM.

$$
\text { 32. } 592-594(1940)
$$

DETERMINATION OF GULD IN SEA WATER BY RADIOACTIVATION ANALYSIS. ANALYST

$$
82,483 \cdots 488(1957)
$$

GERMANIUM IV SEA-WATER. NATURE

$181, \quad 179-180,19581$

GALLIUM IN SEA WATER.

NATURE

181, 180-180 (1958)

AN ECOLOGICAL SURVEY OF THE LIMNOLOGY OF WHITE JAK CREEK ANO LAKE. ORO-587 (YOL III) (1954)

THE BORON CONTENT OF SEA WATFR AND OF MARINE JRGANISMS.

AM. J. SCI.

$$
\text { 35. } 47 \text { - } 63 \text { (1938) }
$$

GEOCHEMISTRY DF THE RADIOACTIVE ELEMENTS IV THE DCEAN. A IRITICAL SUMM $A R Y$.

DEEP SEA RES.

$$
\text { 3. 93- } 123(1956)
$$

IRON IN MARIVE WATERS.

J. MARINE RES. (SEARS FOUND. MARINE RES.)

13, 1B3- $197(1954)$

THE COPPER CONTENT AND COPPER TOLERANCE JF SJME SPELIES OF MOLLUSKS OF THE SOUTHERV CALIFORNIA COAST.

BIOL. BULL.

75. $224-237(1938)$ 
1918 MULLIN J B+

1920 NAKANISHI M

1921 NEUFELD A H

1922

1926

PUTNAM G L

STENBERG A I

SIMONS L H+

SMALES A A+

1929

1930

SMALES A A+

NODOACK I+

1945

NISHITA H+

THE OCCURRENCE OF CADMIUM IN SEAWATER AND IV MARINE ORGANISMS AND SEDI MENTS.

J. MARINE RES. (SEARS FOUND. MARINE RES.)

$$
\text { 15. 103- } 122(1956)
$$

FLUOROMETRIC MICRODETERMINATION OF URANIUM. V. THE URANIUM ZONTENT OF SEA WATER.

BULL. CHEM. SOC. JAPAN

24, $36-38(1951)$

CONTRIBUTIONS TO THE BIOCHEMISTRY OF BROMIVE. PAZT 1. THE ESTIMATION O F BROMINE I Y PLANT AND ANIMAL TISSUES.

CAN. J. RES. SECT. B

14, $160-194(1936)$

THE GOLD CONTENT OF SEA WATER.

J. CHEM. EDUC.

30. $576-579(2953)$

THE NATURAL ARSENIC CONTENT OF THE TISSUES OF FRESHHATER AND MARINE OR GANISMS.

VOPR. PITANIYA

$$
\text { 8, 61- } 74(1940)
$$

ALUMINUM AND IRON IN ATLANTIC ANU GULF OF MEXICO SURFACE WATERS. ANAL. CHEM.

$$
\text { 25. } 989-990(1953)
$$

THE DETERMINATION DF SUB-MICROGRAM QUANTITIES OF ARSENIC BY RADIOACTIV ATION. PART II. THE DETERMINATION OF ARSENIC IN SEA-WATER.

ANALYST

$$
\text { 77. } 188-195(1952)
$$

DETERMINATI ON BY RADIOACTIVATION OF SMALL AMOUNTS OF RUBIDIUM AND CAES IUM IN SEA-WATER AND RELATED MATERIALS OF GEJCHEMICAL INTEREST. ANALYST

$$
80,37-50(1955)
$$

THE FREQUENCY OF HEAVY METALS IN SEA ORGANISMS. ARKIV 2OOL:

$$
\text { 32. } 1 \text { - } 35(1939)
$$

SUMMARY DF CERTAIN TRENDS IN SOIL-PLANT RELATIONSHIP STUDIES DF THE BI OLOGICAL AVAILABILITY OF FALL-OUT DEBRIS.

$$
\text { UCLA-4OL (1957) }
$$

SPECTROSCOPIC DETERMINATION OF BARIUM IN THE ASH OF MAR INE ORGANISMS. TR. BIOGEOKHIM. LAB. AKAD. NAUK SSSR

$$
\text { 5, } 171-173(1939)
$$




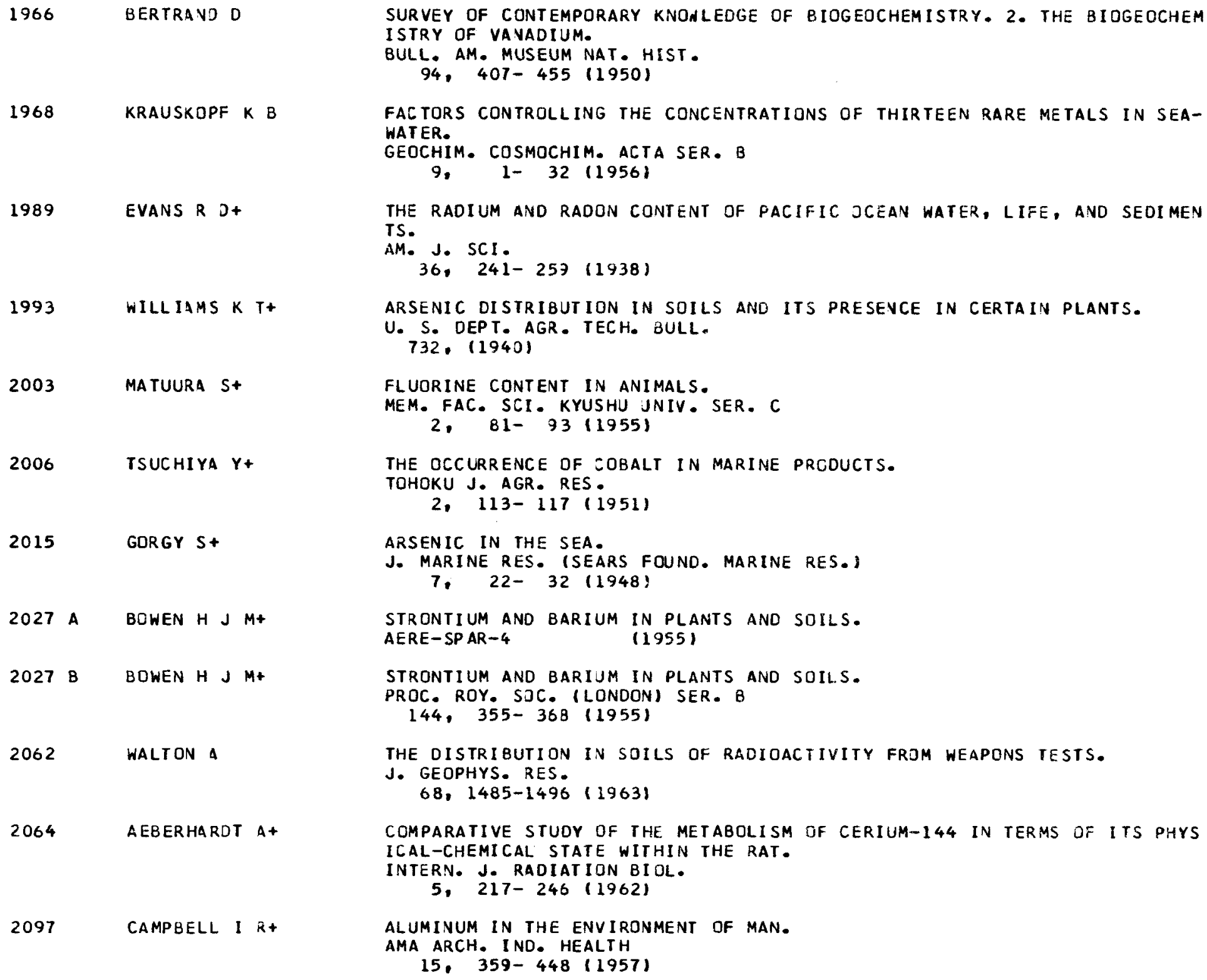

THE RADIUM aND RADON CONTENT OF PACIFIC JCEAN WATER, LIFE, aND SEDIMEN TS. 


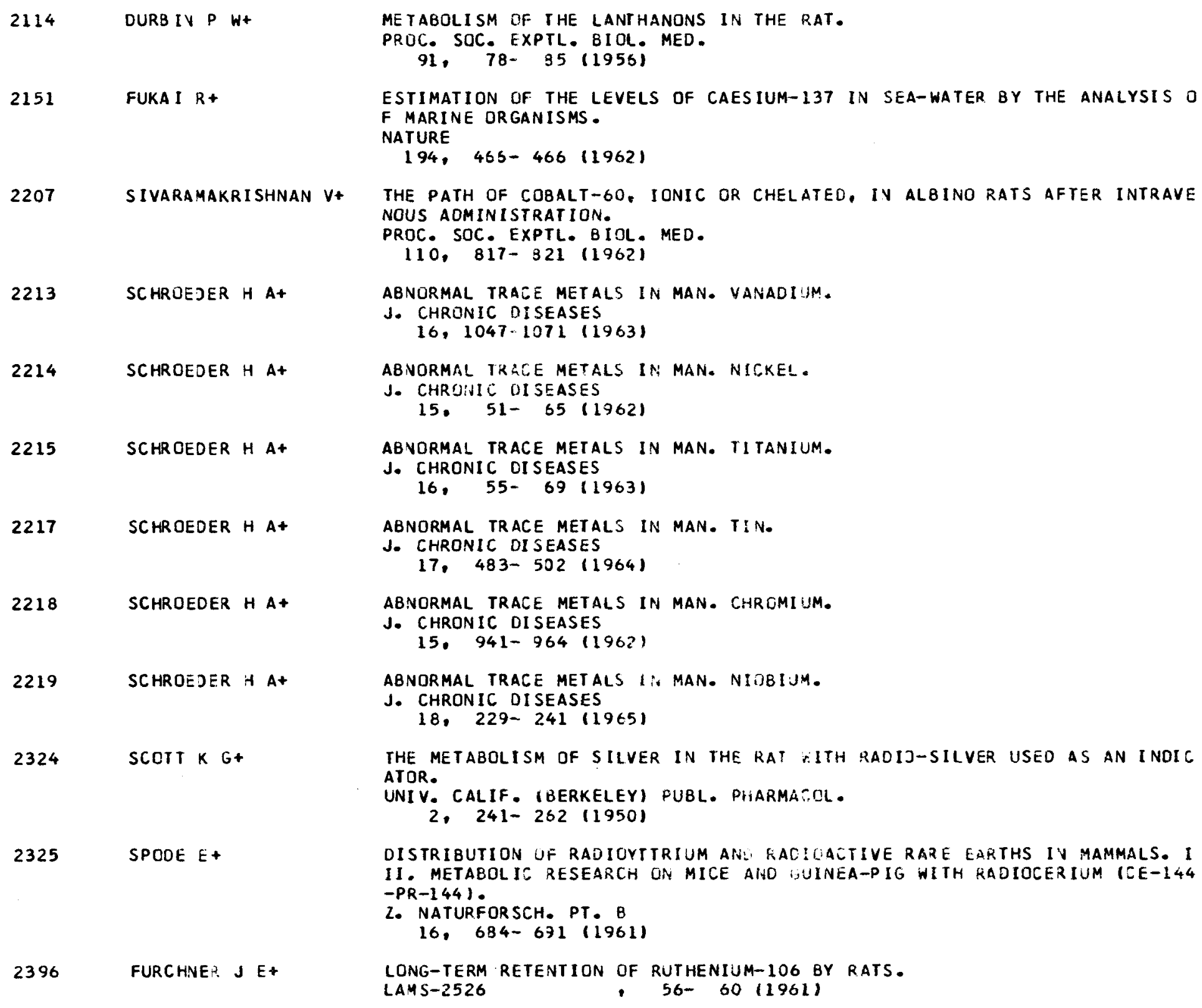

2213 SCHROEJER H A+

2214 SCHROEDER H At

2215 SCHROEDER H A+

2217 SCHROEDER H At

2218 SCHROEDER H A+

2219

SCHROEDER H A+

2324 SCOTT K G+

2325

SPOOE E+

ABNORMAL TRAEE METALS IN MAN. VANADIUM. J. CHRONIC DISEASES

$16,1047-1071$ (1963)

ABNORMAL TRACE METALS IN: MAN. NICKEL.

J. CHROINIC OI SEASES

15. $51-65(1962)$

ABNORMAL TRACE METALS IN MAN. TITANIUM.

J. CHRONIC OISEASES

16. 55-69(1963)

ABNORMAL TRACE METALS IN MAN. TIN.

J. CHRONIC DI SEASES

17. 483- $502(1964)$

ABNORMAL TRACE METALS IN MAN. CHROMIUM.

J. CHRONIC DI SEASES

15. $941-964(1962)$

ABNORMAL TRACE METALS Ii, MAN. NIOBIJM.

J. CHRONIC DISEASES

$18,229-241(1965)$

THE METABOLISM OF SILVER IN THE RAT IITH RADIJ-SILVER USED AS AN INOIC

ATOR. UNIV. CALIF. (BERKELEY) PUBL. PHARMACDL.

2. $241-262(1950)$

DISTRIBUTION UF RADIOVTTRIUM ANL GAC:CIACTIVE RARE EARTHS IV MAMMALS. I II. METABOL IC RESEARCH ON MICE AHD WUINEA-PIG WITH RADIOCERIUM (CE-144 -PR-144).

2. NATURFORSCH. PT. B

16, 684- $671(1961)$

LONG-TERM RETENTION OF RUTHENIUM-106 BY RATS.

LAMS-2526 I56- 60 (1961) 


\begin{tabular}{|c|c|c|}
\hline 2397 & FURCHNER J E+ & $\begin{array}{l}\text { METABOL ISM OF RUTHENIUM-106 CHLORIDE IN MAMMALS. PROGRESS REPORT ON DO } \\
\text { GS. } \\
\text { LAMS-2780 } \\
63-68119621\end{array}$ \\
\hline 2413 & KAWIN 8 & $\begin{array}{l}\text { RETENTION AND LOCALIZATION OF PHOSPHORUS-32 IN RAT OVARY. } \\
\text { HEALTH PHYS- } \\
2,53-56(1959)\end{array}$ \\
\hline 2422 & KLEIBER M+ & $\begin{array}{l}\text { SECRETION IN COW'S MILK OF INTRAVENOUSLY IYJECTED RAOIOACTIVE PHOSPHOR } \\
\text { US P-32. } \\
\text { PROC. SOC. EXPTL. BIOL. MED. } \\
69,354-356(1948)\end{array}$ \\
\hline 2475 & POLIKARPOV $3 G$ & 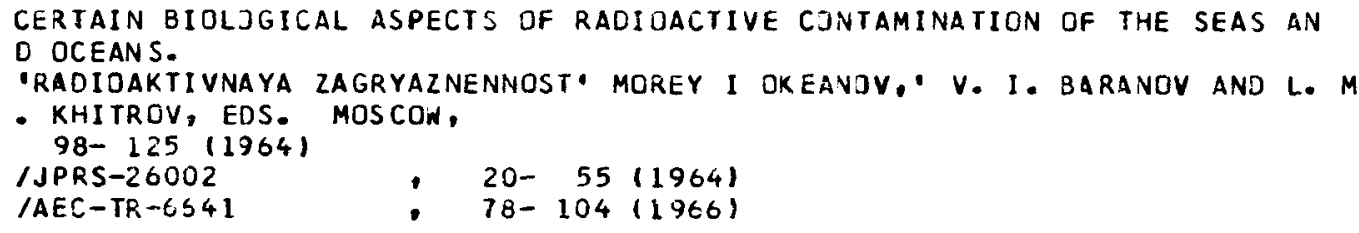 \\
\hline 2520 & MALYUGA D P & $\begin{array}{l}\text { ON THE GEOCHEMISTRY OF NICKEL ANO COEALT DISPERSED IN THE BIOSPHERE. } \\
\text { TR. BIOGEOKHIM. LAB. AKAD. NAUK SSSR } \\
\text { 8. 75- } 141(2946)\end{array}$ \\
\hline 2531 & SWAINE D J & $\begin{array}{l}\text { THE TRACE-ELEMENT CONTENT OF SOILS. } \\
\text { COMMONWEALTH BUR. SOIL SCI. IGT. } 3 R I T .1 \text { TECH. COMMUN. } \\
\quad 48, \quad 1-157(1955)\end{array}$ \\
\hline 2532 & VINOGRADOV A P & $\begin{array}{l}\text { THE GEOCHEMISTRY OF RARE ANO DISPERSED CHEMICAL ELEMENTS IN SOILS. } \\
\text {-GEOCHEMISTRY OF RARE ANO DISPERSED CHEMICAL ELEMENTS IN SOILS, THE, } 2 N \\
\text { DED. TRANSLATEO FROM RUSSIAN. IVEW YGRK, CONSULTANTS GUREAU. } \\
\text { (1959) }\end{array}$ \\
\hline 2536 & FEUILLEN Y $u_{+}$ & $\begin{array}{l}\text { IRON METABOLISM IN INFANTS. I THE INTAKE OF IRJV IN BEEAST FEEDINJ AN } \\
\text { DARTIFICIAL FEEDING IMILK AND MILK FCODSI. } \\
\text { ACTA PAEOIAT. } \\
\quad 41,138-144(1952)\end{array}$ \\
\hline 2538 & MITTELDORF A J+ & $\begin{array}{l}\text { SPECTROCHEMICAL DETERMINATION OF THE MINERAL-ELEMENT CONTENT OF BEEF. } \\
\text { ANAL. CHEM. } \\
24.469-472(1952)\end{array}$ \\
\hline 2539 & OWEN E C & $\begin{array}{l}\text { THE EXCRETION CF BORATE BY THE DAIRY COW. } \\
\text { J. DAIRYRES. } \\
\text { 13. } 243-248(1944)\end{array}$ \\
\hline 2540 & ROBINSDN WO & $\begin{array}{l}\text { THE OCCURRENCE OF RARE EARTHS IN PLANTS AND SOILS. } \\
\text { SOIL SCI. } \\
56,1-6(1943)\end{array}$ \\
\hline
\end{tabular}




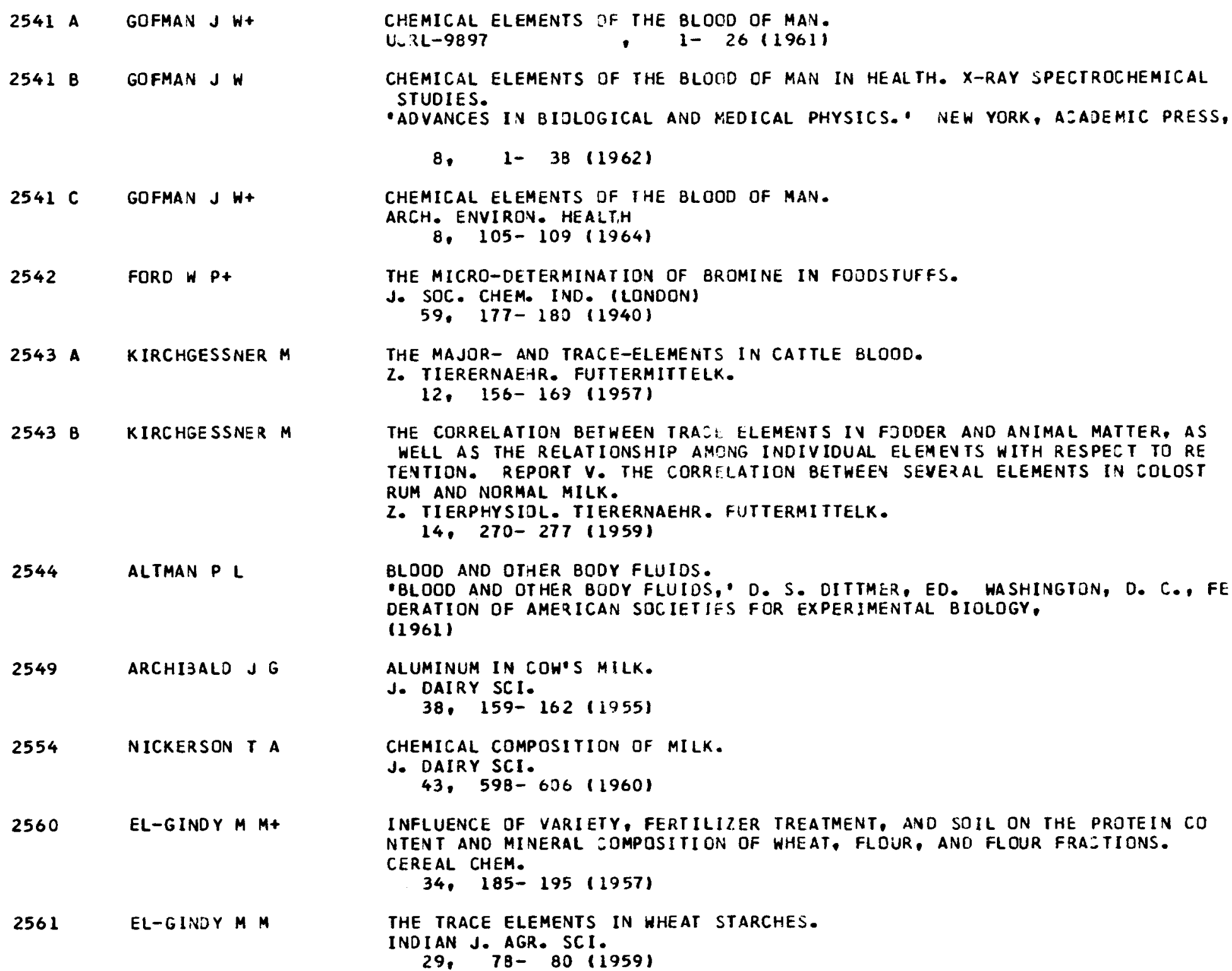

8. 1- 38 (1962)

2541 B GOFMAN J W

CHEMICAL ELEMENTS DF IHE BLOOD OF MAN. ARCH. ENVIRON. HEALTH

THE MICRO-DETERMINATION OF BROMINE IN FOODSTUFFS.

J. SOC. CHEM. IND. (LONDDN)

59. $177-180$ (1940)

THE MAJOR- AND TRACE-ELEMENTS IN CATTLE BLOOD.

2. TIERERNAEHR. FUTTERMITTELK.

12. $156-169(1957)$

THE CORRELATION BETWEEN TRA:: ELEMENTS IV FJODER AND ANIMAL MATTER, AS WELL AS THE RELATIONSHIP AMONG INDIVIDUAL ELEMEVTS WITH RESPECT TO RE TENTION. REPORT V. THE CORRELATION BETWEEN SEVERAL ELEMENTS IN COLOST RUM AND NORMAL MILK.

2. TIERPHYSIOL. TIERERNAEHR. FUTTERMITTELK.

14. $270-277$ (1959)

2544 ALTMAN P L

BLOOD ANO OTHER BODY FLUIOS.

- blood and other budy fluidos,' D. S. oitTMer, ed. hashington, d. C., fe DERATION DF AMERICAN SOCIETIES FOR EXPERIMENTAL BIOLOGY. (1961)

ALUMINUM IN COH'S MILK.

J. DAIRY SCI.

$$
\text { 38. 159- } 162 \text { (1955) }
$$

CHEMICAL COMPOSITION OF MILK.

J. DAIRY SCI.

43. 59B-606 (1960)

INFLUENCE OF VARIETY, FERTILIZER TREATMENT, AND SOIL ON THE PROTEIN CO NTENT AND MINERAL LOMPOSITION OF WHEAT, FLOUR, ANO FLOUR FRA:TIONS. CEREAL CHEM.

$$
\text { 34. } 185-195 \text { (1957) }
$$

THE TRACE ELEMENTS IN HHEAT STARCHES. INDIAN J. AGR. SCI.$$
\text { 29. } 78-80(1959)
$$ 


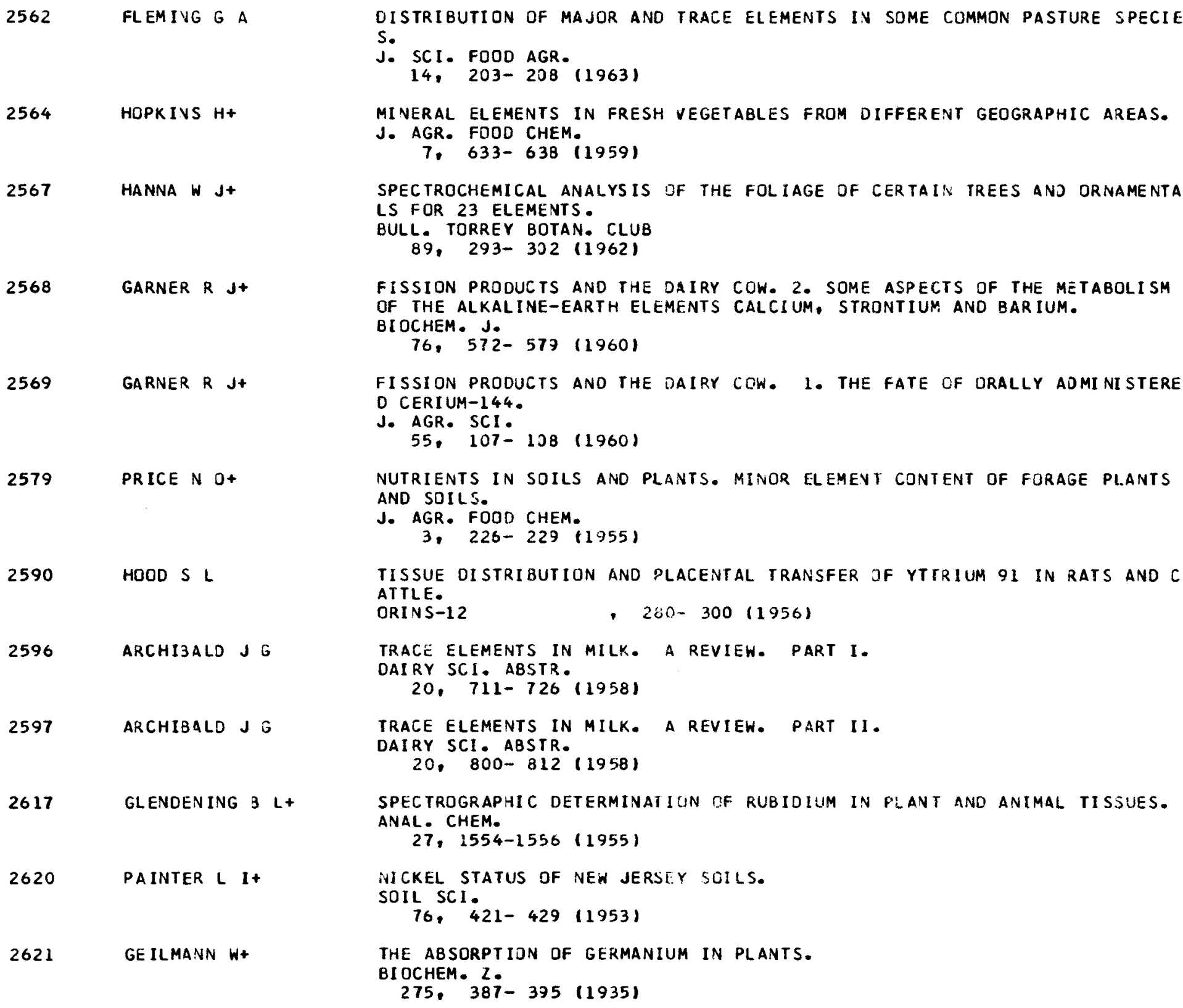


2626 BARSHAD I

2666 MERLINI M+

2669 ROSOFF B

2697 WILSON D O+

2702 A GOLDBERG E

2702 B GOLDBERG E D

2715 POLIKARPOV G G

2716 R ICE I R

2717 RICE T R+

2722

PENDLETON R C

2751 DAVIDOV R+
MOL YBDENUM CONTENT OF PASIJRE PLANTS IN RELATION IO TOXICIYY TO CATILE

SOIL SCI.

$$
66,187-195(1948)
$$

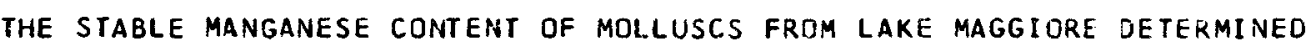
BY ACTIVATION ANALYSIS.

LIMNOL. OCEAYOG.

$$
\text { 10, } 371-378(1965)
$$

METABOLISM OF SCANDIUM-46 IN MAN.

INTERN. J. APPL. RADIATION ISOTOPES

16. $479-485(1965)$

REMOVAL OF PU-239, W-185 AND PB-210 FROM SOILS.

$$
\text { BNWL-SA-128 (1965) }
$$

OPERATION WIGWAM. EFFECTS OF NUCLEAR EXPLOSION ON MARINE BIOLOGY. EL EMENTAL COMPOSITION OF SOME PELAGIC FISHES.

$$
\text { WT-1013 111- } 115 \text { (1957) }
$$

ELEMENTAL COMPOSITION OF SOME PELAGIC FISHES. LIMNOL. OCEAYOG. SUPPL.

$$
\text { 7. } 72-75(1962)
$$

ABILITY OF SOME BLACK SEA ORGANISMS TO ACCUMULATE FISSION PRDDUCTS. SCIENCE

$$
\text { 133. } 1127-1128(1961)
$$

REVIEW OF ZINC IN ECOLOGY.

'RADIOECOLOGY, $V$. SCHJLTZ AND A. W. KLEMENT, EDS., PROCEEDINGS OF THE F IRST NATIONAL SYMPOSIJM HELO AT FORT COLLINS, COLO,. SEPT. 10-15, 1961. IRST NATIONAL SYMPOSIJM HELO AT FORT COLLINS, COLO., SEPT. 10-15, 1961.
NEW YORK, REINHOLD PUBL. CORP. ANO WASHINGTON, D. C. THE AMERICAN INST ITUTE OF BI OLOGICAL SCIENCES,

$619-631(1763)$

UPTAKE, ACCUMULAT ION AND LOSS OF RADIOACTIVE CER IUM-144 BY MARI NE PLAN KTONIC ALGAE.

LIMNOL. OCEANOi;.

$$
\text { 4. } 277-290119591
$$

ABSORPTION DF CESIWHI-137 BY COMPONENTS GF AN AQUATIC COMMUNITY.

-PKOC. INTE ZN. COR. ON THE PEACEFUL USES OF ATOMIC ENERGY, 2NE, GENEVA, 1958." NEW YORK, UNITEO NATIONS,

$18,417-433(1958)$

/A/CONF.15/P/392 (1958)

SULFUR CONTENT OF MILK.

MOLDCHN. PROM.

21. 35- $36(1960)$

(ISSUE 12) 


\begin{tabular}{|c|c|c|}
\hline 2767 & HAMILTJN J Wt & $\begin{array}{l}\text { AMOUNT AND CHEMICAL FORM UF SELENIUM IN VEGETABLE PLANTS. } \\
\text { J. AGR. FOOD CHEM. } \\
12,371-374(1964)\end{array}$ \\
\hline 2793 & MAENO M+ & $\begin{array}{l}\text { COPPER CONTENT OF MILK ANO ITS PRODUCTS AND THEIR VARIETIES. } \\
\text { J. JAPAN. SOC. FOOD NUTR. } \\
4.204-205(1952)\end{array}$ \\
\hline 2805 & HARD ING-BARLOW I & $\begin{array}{l}\text { STUDIES ON THE TRACE ELEMENT CONTENT OF HUMAY IISSUES. } \\
\text { CAPE TOWN U THESIS (19CI) }\end{array}$ \\
\hline 2817 & TAUCINS E+ & $\begin{array}{l}\text { TRACE ELEMENT CONTENT IN TISSUES ANO PRODUCTS OF AGRICULTURAL ANIMALS } \\
\text { IDOMESTIC) OF THE LATVIAN SSR. } \\
\text { TR. SEKTORA FIZIOL. ZHIVOIN. AKAII. NAUK LATV. SSSR INST. } 3 \text { IOL. } \\
3.165-205(1962)\end{array}$ \\
\hline 2819 & BOWEN H J M & $\begin{array}{l}\text { THE DETERMINATION OF TUNGSTEN IN BIJLOGICAL MATERIAL OY ACTIVATICN ANA } \\
\text { LYSIS. } \\
\text { BIOCHEM. J. } \\
77 \text {. } 73-82(1960)\end{array}$ \\
\hline 2824 & COMAR C L+ & 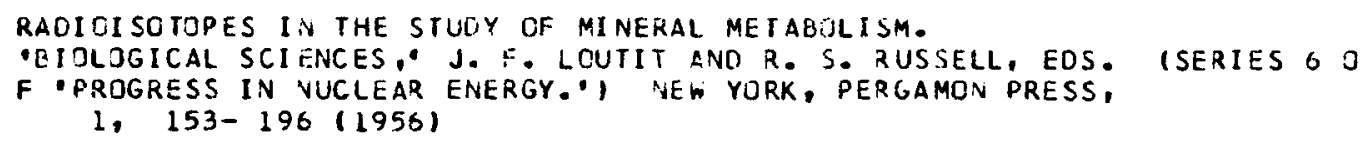 \\
\hline 2826 & LIEB IG G F+ & $\begin{array}{l}\text { EFFECTS GF GALLIUM AND INOIUM ON THE GROWTH ITF CITRUS PLANTS IN SOLUTI } \\
\text { ON CLLTURES. } \\
\text { SOIL SCI } \\
56,173-185(1943)\end{array}$ \\
\hline 2843 & HIYAMA $Y+$ & $\begin{array}{l}\text { HAZARD EVALUATION OF RADIOACTIVE CONTAMINATION OF THE SEA AND DECONTAM } \\
\text { INATION OF RADIOACTIVITY IN FRESH WAIER. } \\
\text { 'PROC. INTERN. CONF. ON THE PEACEFUL USES JF ATJMIC ENERGY. 3RD, GENEVA, } \\
\text { I964. INEW YORK. UNITED NATIONS. } \\
140.97-106(1955) \\
\text { /A/CONF.28/P/849 } \\
\end{array}$ \\
\hline 2855 & NEWTON HP+ & $\begin{array}{l}\text { RESPCNSE OF CROP PLANTS IO I ANO ER. } \\
\text { SOIL SCI. } \\
73.127-134(1952)\end{array}$ \\
\hline 2858 & WARREN H V+ & $\begin{array}{l}\text { GOLO AND SILVER CONTENI IF SOME TREES AND HORSETAILS IN BRITISH CGLUMB } \\
\text { IA. } \\
\text { BULL. GEOL. SOC. AM. } \\
\text { Ól, } 123-128(1950)\end{array}$ \\
\hline 2873 & BATES $\mathrm{r} \mathrm{H}+$ & $\begin{array}{l}\text { METABOLISM OF RADIOACTIVE STRONTIUM IN RAT. PART I. THE WHOLE-BODY R } \\
\text { ETENTION AND TISSUE DISTRIBUTIDN CHARACTERISTICS OF SR-85. PART II A } \\
\text { TIEMPTS TO INFLUENCE ELIMINATION BY PHYSIOLOGICAL AND OTHER PROCEDURES } \\
\text { PG-R-662(CC) } \\
\end{array}$ \\
\hline
\end{tabular}




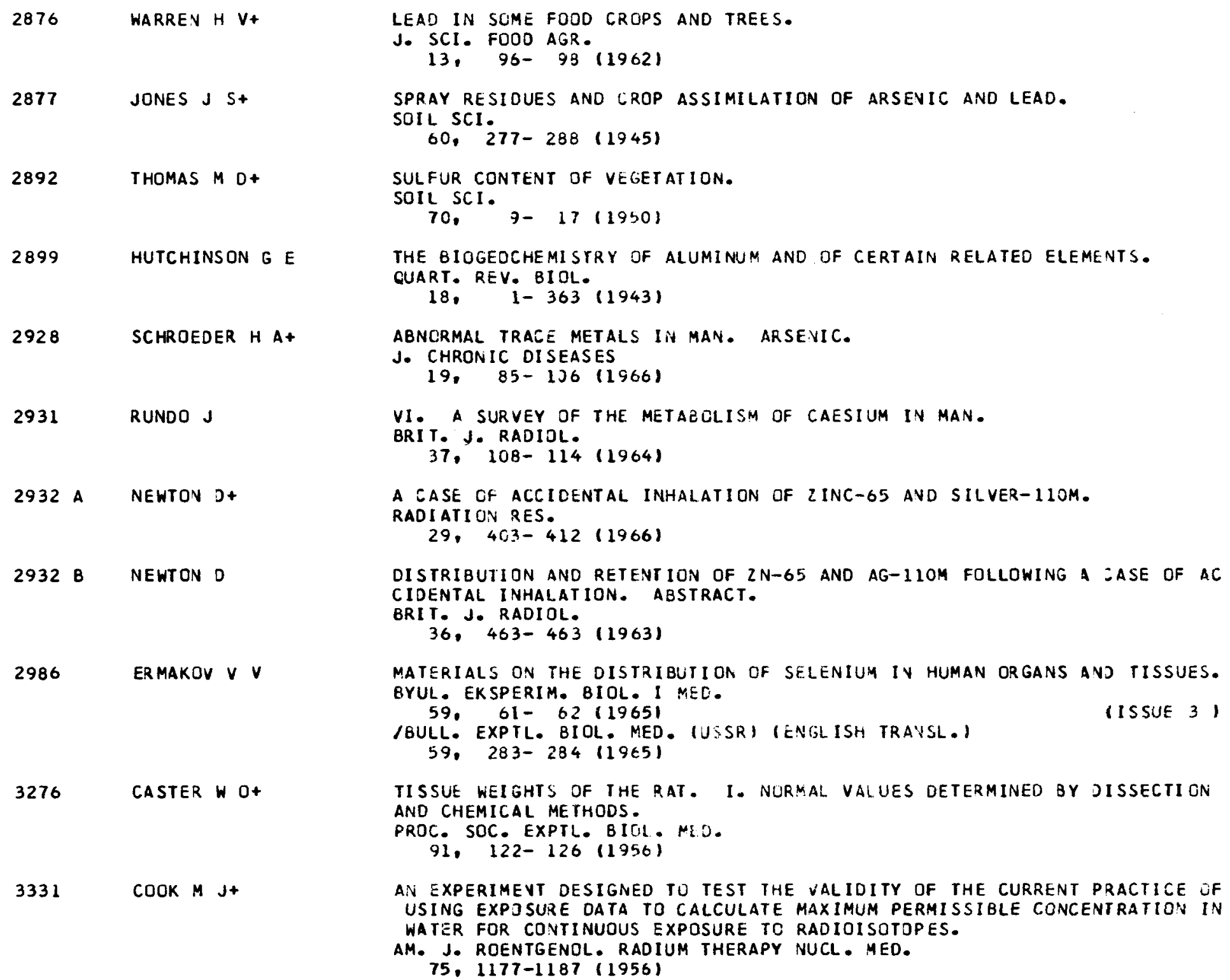

DISTRIBUTION AND RETENTION OF $2 N-65$ AND AG-11OM FOLLOWING A ZASE OF AC CIDENTAL INHALATION. ABSTRACT. CIDENTAL INHALAT 36, J63-463 (1963)

MATERIALS ON THE DISTRIBUTION OF SELENIUM IV HUMAN ORGANS ANJ TISSUES. BYUL. EKSPERIM. BIOL. I MEC.

59. $61-62(1965)$

/BULL. EXPTL. BIOL. MED. (USSR) (ENGLISH TRAYSL. I

(ISSUE 3 )

59. $283-284(1965)$

TISSUE WEIGHTS OF IHE RAT. I. NURMAL VALUES DETERMINED BY DISSECTION AND CHEMICAL METHODS.

PROC. SOC. EXPTL. BIOL. MIO.

91. $122-126(1956)$

AN EXPERIMEVT DESIGNED TO TEST THE VALIDITY OF THE CURRENT PRACTICE OF USING EXPOSURE DATA TO CALCULATE MAXIMUM PERMISSIBLE CONCENTRATION IN WATER FOR CONTINUOUS EXPOSURE TO RADIOISOTDPES.

AM. J. ROENTGENOL. RADIUM THERAPY NUCL. MED.

75. $1177-1187$ (1956) 
3493 ESSINGTON E+

3606 GLASER R

3636

GRUMMITT WE

3734 HIYAMA Y

3868 TIPTON I H+

3869 A TIPTON I H+

3869 B TIPTON I H+

$3885 \quad$ KOCZY F Ft

3970

4081

4105

JACOBSON L+

EFFECT OF CHELATING AGENTS DiN THE UPTAKE DF Y-91, RU-106, ZE-144, ANO PM-147 BY GEANS GROWN ON A CALCAREOUS SOIL.

SOIL SCI.

$$
\text { 95. } 331-337(1963)
$$

THE RADIOACTIVITY OF SEVERAL SUBMERGED WATER PLANTS.

LIMNOLOGICA

$$
\begin{array}{rrr}
1,51- & 63(1962) \\
\text { /AEC-TR-5753 } & (1963)
\end{array}
$$

ENVIRONMENTAL SURVEYS FOR NUCLEAR POWER STATIONS.

AECL-1656 (1962)

ICRER-1120 $(1962)$

FISSION PRGDUCTS IN WATER-AREA AND AGUATIC JRGANISMS. A/AC.82/G/R.4(PT.8) (1950)

STABLE ELEMENT METABOLISM. TRACE ELEMENTS IV HUMAN TISSUE, RIB AND VE RTEBRA.

ORNL -3697

- $179-185(1964)$

TRACE ELEMENTS IN DIETS AND EXCRETA.

HEALTH PHYS.

12, $1383-1689(1966)$

STABLE ELEMENT MeTABOLISM. TRACE ElEMENTS IN DIETS AND EXCRETA. PREL IMINARY STUOY.

ORNL -3697

- $185-188(1964)$

RADIOACTIVITY IN OCEANOGRAPHY.

-NUCl. RADIATION IN GEDPHYSICS, H. ISRAEl. A. KREBS, anO R. D. evans, e OS. VEW YORK. ACADEMIC PRESS INC.:

$18-46(1962)$

THE METABOLISM JF magneSIJM ANO CALCIUM BY THE PAT. ARCH. BIOCHEM. QIOPHYS.

$$
84,278-285(1.959)
$$

PHOSPHORUS-32 PRESENT IN THE PROCESS SEWER MD IV FISH FROM THE OTTAWA RIVER NEAR THE CHALK RIVER PROJECT OH ATOMIC ENEREY UF CAVADA LIMITED E.RI -8

$(1963)$

THE UPTAKE BY PLANTS OF PLUTONIUM ANL SOME PRJOUCTS OH NUGLEAR FISSION ADSURBEU OY SOIL COLLOICS. SOIL SCl.

$$
\text { 65. } 125-134(1948)
$$

4162 MILLER D F

COMPOSITION OF CEREAL GRAINS AND FORAGES. NAS-NRC/PUB-585 (1958) 
4254 YAMAGATA

4278 A OPHEL $1 \mathrm{~L}$

4278 B OPHEL I L

4293 OVERSTREET R+

4351

4400

REVELLE $R+$

4419

RICHMDND C R

4510

4576

4752

4756
THE CONCENTRATION OF COMMCN POTASSIUM, RUBIDIUM AND CESIUM IN JAPANESE DIET.

J. RADIATION RES.

$$
\text { 3. } 158-169(1962)
$$

THE FATE OF RAOIOSTRCNT IUM IN A FRESHWATER COMMUNITY.

AECL -1642
ICRER -1122

THE FATE OF RAOIOSTRONTIUM IN A FRESHWATER COMMUNITY.

-RADIOECGLOGY. $V$. SCHULTZ AND A. W. KLEMENT, EDS., PROCEEDINGS OF THE $F$ IRST YATIDNAL SYMPOSIUM HELO AT FORT COLLINS, COLO., SEPT 10-15, 1961. IRST VATIDNAL SYMPOSIUM HELO AT FORT COLLINS, COLO., SEPT . 10-15. NEW YORK, REINHOLD PUBL. CORP. AND WASHINGTOV. O. C.. THE AMERICAN INST ITUTE OF BIOLOGICAL SCIENCES,

$213-216(1963)$

PROGRESS REPORT ON METABOLISM OF FISSION PRODUCTS FOR PERIOJ ENDING OC TOBER 15, 1943.

MODC-1011 $\quad(19431$

SOME DATA AND COMMENTS ON SOOIUM-22 IN FOOD CHAIVS. HEAL TH PHYS.

$$
\text { 12. } 353-359(1960)
$$

GENERAL CONSIDERATIONS CONCERNING THE OCEAN AS A RECEPTACLE FOR ARTIFI CIALLY RADIOACTIVE MATERIALS.

-EFFECTS OF ATOMIC RADIATION ON OCEANOGRAPHY AND FISHERIES, THE \& REPOR T OF THE COMMITTEE ON EFFECTS OF ATOMIC RAOIATION ON OCEANOGRAPHY AND FI SHERIES OF THE NATIONAL ACADEMY OF SCIENCES STUDY OF THE OIOLOGICAL EFFE CTS DF ATOMIC RADIATION. WASHINGTON. D. C. NATIONAL ACADEMY OF SCIENCE S-NATIONAL RESEARCH COJICIL, S-NATIONAL RES 259571 $1-25 \quad 11957)$
NASTNRC IP UB-551

$$
1-25(1957)
$$

RETENTION ANU EXCRETION OF RADI CNUCLIDES OF THE ALKALI MEITLS BY FIVE MAMMALI AN SPECIES.
LA-2207
$11558\}$

THE METABOLISM OF CARRIER-FREE FISSION PRODUCTS IN THE RAT. $M D D C-1275$ (1947)

VARIATIONS IN ELEMENTAL CONCENTRATIONS IV THE COLUMBIA RIVER. HW-SA-3147 (1963)

THE ABUNDANCE OF RARE EARTHS IN ANIMAL BONES.

Z. ANDRG. ALLGEM. CHEM.
$240,21-30(1938)$

ON THE OCCURRENCE OF BERYLLIUM, BORON, COBALT, ANC MERCURY IN HUMAN II SSUES.

J. BIOL. CHEM.

209, 857-865 (1954) 


\begin{tabular}{|c|c|c|}
\hline 4761 & LENGEMANN $F W^{*}$ & $\begin{array}{l}\text { METABOLISM OF SOME FISSION PRODUCTS BY FARM AYIMIL } \\
\text { FAD-AE-5 } \\
92-115(1964)\end{array}$ \\
\hline 4930 & SOLDAT J K & $\begin{array}{l}\text { SOME RADIOACTIVE MATERIALS MEASUREO IN VARIOUS WATERS I I IHE UNITEO } \\
\text { ATES. A LITERATURE SEARCH. } \\
\text { HW-70706 } \\
\text { (1961) }\end{array}$ \\
\hline 4939 & SCOTT R C+ & $\begin{array}{l}\text { DATA ON URANIUM AND RADIUM IN GROUND WATER IN THE UNITEE STATES, } 1954 \\
\text { TO } 1957 \text {. } \\
\text { U. S. GEOL. SURV. PROFESS. PAPERS } \\
\text { 426, }(1952)\end{array}$ \\
\hline
\end{tabular}

$4940 \quad$ SCOTT R C +

4941 A KOCH R C+

$4941 B \quad K O C H R$

4947 POPOVA O N+

5001 GUSTAFSON P F+

5034 SUGAWARA K+

5037 SUGAWARA $K+$

5125 SCHROEJER HAt
RAOIOCHEMICAL ANALYSES OF GPOUNC ANO SURFACE WATER IN COLORADO, 1954-1

961.: WATER CONSERV. BOARD, BASIC DATA SER., REPT.

$$
\text { 7. }(1961)
$$

APPLICATION OF ACTIVATION ANALY SIS TO THE DETERMINATION OF TRACE-ELEME NT CONCENTRATIONS IN MEAT.

NSEC-56 119621

J. FOOO SCI.

27. $309-323(1962)$

ACTIVATION ANALYSIS OF FOCOS FOF CHARACTERILAT:ON OF THE INDUCED RAOIO ACTIVITIES. FINAL REPORT NUMBEK 3, MAY 10, 1960-FEBRUARY 9, 1961. $A D-267374$ (1961)

DISTRIBUTION IN PLANTS OF RADIUM ABSDHBED FRJM THE SOIL.

FIZIOL. RAST.

11. 435- $441(1964)$

/PLANT PHYSIOL. (USSEI (ENGLISH TRANSL.)

11 . $371-375(1964)$

CAESIUM-13\% IN EDIBLE FRESHWATER FISH.

NATURE

211. $843-844(1966)$

IODINE DISTRIBUTION IN THE WESTERR PACIFIC OCEAN.

J. EARTH SCI. NAGOYA JNIV.

5. $81-102(1957)$

THE DISTRIBUTION OF SOME MINOR BIO-ELEMENTS IY WESTERN PAEIFIC WATERS. - UNESCO SYMPOSIUM DN PHYSICAL OCEANOGRAPHY, PROCEEDINGS OF THE SYMPGSIUM HELO IN TOKYO, 1955." TOKYO, UNESCO AIVO JAPAN SCCIETY FOR THE PROMOTIO $N$ OF SC,IENCE.

$169-174$ i 17551

ESSENTIAL rRACE METALS IN MAN. MANGANES. A STUDY IN MOMEJSTASIS.

J. CHRCNIC DISEASES

19. $545-571(1966)$ 
J. CHRONIC DI SEASES

19. $573-586$ (1966)

5164 ASSAF S A+

INORGANIC ELEMENTS IN BEEF MUSCLE AND THEIR RELATIVE DEGREE OF BINDING IN AQUEOUS BEEF MUSCLE EXTRACTS.

J. AGR. FOOD CHEM.

14. 487-489 (1966)

5166 A HAMILTON JG

THE METABO

ND PLANTS.

$M D O C-1160$

(1947)

5166 B HAMILTON J 3

5168 A DURB IN $P$

5168 B DURB IN P W+

5169 HAMILTON J G

5170 HAMILTON J 3

5171 HAMILTON J

5172 HAMILTON J 3

5173 HAMILTON J 3

5174 HAMILTON J

THE METABOLISM OF THE FISSION PRODUCTS AVD THE HEAVIEST ELEMENTS. RADIOLOGY

$$
49,325-343(1947)
$$

THE OISTRIBUTION OF RAOIOISOTOPES OF SOME HEAVY METALS IN THE RAT. UCRL -3607

THE DISTRIBUTION OF RADIOISOTOPES OF SOME HEAVY METALS IN THE RAT. UNIV. CALIF. (BERKELEY) PJBL. PHARMACOL.

$$
\text { 3. } 1 \text { - } 34 \text { (1957) }
$$

I. THE METABOLIC PROPERTIEs of PLUTONIUM AND ALLIED materials. medica L AND HEALTH DIVISIONS QUARTERLY REPORT, OCTOBER 1947 TO JANUARY 1948. $U C R L-41$ - 4- $23(1948)$

I. THE METABJLIC PROPERTIES OF PLUTONIUM AND ALLIED MATERIALS. MEDICA AND HEALTH DIVISIONS QUARTERLY REPORT, JANUARY, FEBRUARY, MARCH 1948

UCRL-98 4- 40 (1948)

I. THE METABDLIC PROPERTIES OF PLUTONIUM ANO ALLIED MATERIALS. MEdica L AND HEALTH DIVISIONS QUARTERLY REPORT, APRIL, MAY ANO JUNE 1948. UCRL-157

I. THE METABOLIC PROPERTIES OF PLUTONIUM AVD ALLIED MATERIALS. MEDICA L AND HEALTH DIVISIONS QUARTERLY REPORT, JULY, AUGUST, SEPTEMBER, 1948 UCRL-193

- 4- $22(1948)$

1. THE METABOLIC PROPERTIES OF PLUTONIUM AND ALLIED MATERIALS. MEDICA 1 AND HEALTH DIVISIONS QUARTERLY REPORT, OCTOBER, NOVEMBER, DECEMBER, 1948 .

UCRL -270

1. THE METABOLIC PROPERTIES OF PLUTONIUM AVD ALLIED MATERIALS. MEDICA $L$ AND HEALTH DIVISIONS QUARTERLY REPORT, JANUARY, FEBRUARY, MARCH 1949 UCRL-332 
5175 HAMLLTON J

5177 HAMILTON J St

5178 HAMILTON J $3+$

5179

HAMILTON J G+

5180 HAMILTON J G+

5181

5182

5183

HAMILTON J 3+

5184 HAMILTON J $3+$

5185

I. THE METABOLIC PROPERTIEs of PLUtonium aNo allied Materials. medica $L$ ANO HEALTH DIVISIONS QUARTERLY REPORT, APRIL. MAY, JUNE 1949. $\begin{array}{lll}\text { UCRL }-414 & 4-24(1949) \\ \text { IAECU }-592 & 4-24(1949)\end{array}$

I. THE METABOLIC PROPERTIES OF PLUTONIUM aNo allied Materials. medica 1 ANO HEALTH DIVIS IUNS QUARTERLY REPDRT, OCTJBER, NUVEMBER AND DECEMBE R. 1949 . RCRL -587

- 4- $33(1950)$

I. THE METABOLIC PROPERTIES OF PLUTONIUM aYd allied MATERIALS. MEOICA $L$ AND HEALTH PHYSICS QUARTERLY REPORT. JANUARY, FEBRLARY AND MARCH, 19 50.

UCRL-683 4- $64(1950)$

IAECD-2901 $4-64(1950)$

I. THE METABolic properties of plutonium and allied Materials. medica $L$ AND HEALTH PHYSICS QUARTERLY REPORT, APRIL. MAY AND JUNE 1950. UCRL-806 IAECO-2928 $4-45(19501$

1. THE METABDLIC PROPERTIES OF PLUTONIUM ANU ALLIfed MATERIALS. MEDICA $L$ AND HEALTH PHYSICS QUARTERLY REPORT, JULY, AUGUST AND SEPTEMBER 1950 IAECD-3141

$$
\begin{aligned}
& 4-42(1950) \\
& 4-\quad 42(1950)
\end{aligned}
$$

1. THE METABOLIC PROPERTIES OF YARIOUS ELEMENTS. MEOICAL ANS HEALTH P HYSICS QUARTERLY REPORT, OCTOBER, NOVEMBER, AND DECEMBER, 1950. UCRL-1143 IAECD -3200

$$
\text { - 4- } 31 \text { (1951) }
$$

I. THE METABOLIC PROPERTIES OF VARIOUS MATERIALS. MEDICAL and health PHYSICS QUARTERLY REPORT, JANUARY, FEBRUARY AND MARCH, 1951. UCRL -1282

$$
\begin{array}{r}
\text { 4- } 40(1951) \\
4-\quad 40(1951)
\end{array}
$$

- 4- 40119511

I. THE METABOLIC PROPERTIES OF VARIOUS MATERIALS. MEDICAL aVD heALTH PHYSICS QUARTERLY REPORT, APRIL, MAY AND JUVE, 1951.

UCRL -1437

- $4-30(1951)$

IAECD -3282

- 4- 30119511

1. THE METABOLIC PROPERTIES OF VARIOUS MATERIALS. MEOICAL AND HEALTH PHYSICS QUARTERLY REPORT, JULY, AUGUST, AND SEPTEMBER : 1951.

UCRL -1561

$4-21(1951)$
$21(1951)$

I. THE METABOLIC PROPERTIES OF VARIOUS MATERIALS. MEDICAL AND HEALTH PHYSICS QUARTERLY REPORT, OCTOBER, NOVEMBER, AND DECEMBER 1951. UCRL-1694 2- 38 (1952) 
5193 HAMILTON J $\mathrm{j}^{+}$

5194

HAMILTON J $\hat{~}+$

5195

5196

5197
HAMILTON J j+

HAMILTON J $3+$

HAMILTJN J G+

DURE IV $P \mathrm{~W}$

DEKOCK P C +

KOBIASHVILI $V I$

LOUNAMAa j

SARTOR J O+

ASHIKAWA J K+

GOLOSEHMIOT $\vee M$
THE METABOLIC PROPERTIES OF VARIOUS MATERIALS. TRACER STUOIES. MEOI AL AND HEALTH PHYSICS QUARTERLY REPORT, OCTJBER, NOVEMEER, JEZEMEER, I 953.

UCRL-2553

$$
\text { , 24- } 49(1354)
$$

THE METAECLI: PROPERTIES OF VARICUS MATERIALS. TRACER STUDIES. MEOIC AL AND HEALTH PHYSICS OJARTERLY REPORT, JAVUARY, FEBRUARY, MAREH, 1954

UCRL -2605

$$
\text { - 3- } 25(1954)
$$

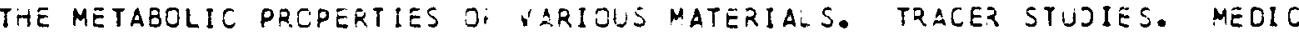
AL AND HEALTH PHYSICS QJARTESLY REPQRT, APRIL, MAY, JUNE 1954.

$U C R L-266$

$$
\text { , } 21-63(1954)
$$

THE METABOLIC PROPERTIES OF VARIOUS MATERIALS. TRACER STUDIES. MEDIO AL AND HEALTH PHYSIES 2ULRTERLY REPORT, JULY, AUGUST, SEPTEMBER, 1954. UCRL-2823

$$
\text { , 3- } 25(1954)
$$

STUDIES CF RADIJACTIVITY ANO IRRADIATION. TRACER STUOIES ANO EFFESTS OF IOVIZING ZAOIATION. ASTATINE STUEIES. YEOICAL AND HEALTH PHYSICS QUARTERLY REPORT, OCTOBEP, NOVEMEER, CECEMBER 1954.

UCRL-288I$$
\text { 4- } 27 \text { (1955! }
$$

the metaejlism of the lanthanons in the rat. II. Time studies th the TISSUE DEPJSITION OF IVTRAVENOUSLY AOMINISTEREO 2ADIOISOTOPES. UCRL -3191

$$
\text { (1955) }
$$

UPTAKE OF CHELATED METALS 3 Y PLANTS.

SOIL SCI.

$$
84, \quad 55-62(1957)
$$

BIJGEOCHEMICAL VALUE OF TJNGSTEN UISPERSEO IY VATURE.

SOOBSHCH. AKAD. YAUK GRUZ. $S \$ R$

$$
\text { 35. } 83-\text { iS (1964) }
$$

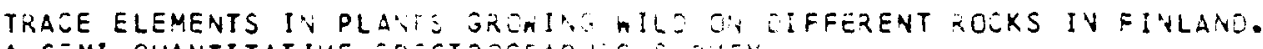
A SEMI-QUANTITATIVE SDECTROGRAPHIC S.IVEY.

ANN. BOTAN. SJC. ZOCL. BOTAN. FELNICAE 'VANAMO

$$
\text { 29. } 119561
$$

LPTAKE OF RADIONUCLILES EY PLANTS.

SRI -PROJ-MU-5J=5 $1: 0001$

ISRI-PROJMUL5893 I:SEC

/AD-649009 (1136)

COPPER METABJLISM IN NORMAL LUNG-EVAYS RATS.

UCRL-3530 (1956)

BOJK

'GEJCHEMISTRY,' A. MUIR, EL. OXFCRD, OXFORD UVIVERSITY PRESS,

(1 754 ) 
5223 JACKSON M L

5225

HASK IN L A+

5227

MENZEL R G

5229

ROMNEY E M+

5230

RICHARDS F A

5231

SHAW D M

5236

5237

5243

5244

BEATH O A+

5261

5273

KITTLE C F+

CHEMICAL COMPOSITION OF SOILS.

-chemistry jf the soll,. f. e. bear, ed. second edition. american chem ICAL SOCIETY MONOGRAPH SERIES NO. 160. NEW YORK, REINHOLD PUBLISHINE CO RPDRATION.

71- $141(1764)$

METEORITIC, SOLAR AND TERRESTRIAL RARE-EARTH DISTRIBUTIONS. -PHYSICS AND CHEMISTRY OF THE EARTH.' NEW YDRK, PFRGAMON PRESS,
7. $167-321$ (1966)

SOIL-PLANT RELATIONSHIPS OF RADIUACTIVE ELEMENTS.

HEALTH PHYS.

11. $1325-1332$ (1905)

NEUTRON ACTIVATION PRODUCTS FROM PROJECT SEDAN IN PLANTS ANJ SOILS. SOIL SCI. SOC. AM. PROC.

$30, \quad 770-773(1966)$

SOME CURRENI ASPECTS OF CHEMICAL OCEANOGRAPHY.

- PHYSICS AND CHEMISTRY OF THE EARTH.' NEW YORK, PERGAMON PRESS,

2. $77-128(1957)$

THE GEOCHEMISTRY OF GALLIJM. INOIUM. THALLIUM. A REVIEW.

-PHYSICS AND CHEMISTRY OF THE EARTH. NEW YORK, PERGAMON PRESS, 2. $164-211(1957)$

55-FE IN THE MARINE ENVIRONMENT AND IN PEGPLE WHO CONSUME OCEAN FISH. BNWL $-235-2$

, $50-54(1966)$

NATURAL AND ARTIFICIAL RADIONUCLIOE CONCENTRATIJN IN FARM PRJDUCE IRRI GATED WITH COLUMBIA RIVER WATER.

BNWL-235-2

ABNORMAL TRACE ELEMENTS IN MAN. TELLURIUM.

J. CHRONIC DISEASES

$20,147-16 i(1967)$

SELENIUM AND OTHER TOXIC MINERALS IN SOILS AND VECETATION. WYOMING AGR. EXPT. STA. BJLL.

$206,(1935)$

DISTRIBUTION AND EXCRETION OF RAOIOACTIVE HAFIVIUM-1UL SOOIUM MANUELGTE IV THE RAT.

PRDC. SOC. EXPTL. BIOL. MED.

$$
\text { 76, } 278-282(1951)
$$

BIOLOGICAL STUDIES ON THE DISTRIBUTION OF RADIOACIIVE METALS WITH SPEC IAL REFERENCE TO DAMAGING LEVELS OF TISSUE COVCEVTRATICN. FOURTH QUA? TERLY REPORT.

IID-22714

(1953) 

5274 ROSOFF B+ FATE OF MOL YBDENUM-99 IN MAN.
NATURE
$202,410-411(1964)$

5275 BORISOV $V P$

COMPARISON OF THE DISTRIBUTION OF MOL YBOENUM-99 AND PHOSPHORUS-32 IN A NIMALS.

-RASPREDELENIE, BIOLOGICHESKOE OEISTVIE, USKDREVIE VYVEDENIYA RADIOAKTIM YKH IZOTOPOV, 1. I. MDSKALEV, ED. MOSCOW, MEDITSINA,

$71-77(1764)$

5280 TIPTON I Ht

5294

5295

5306

5307

5309

5312

FOSTER R F

5314 KOCZYrF
STABLE ELEMENT METABOL ISM. ELEMENTAL COMPOSITION OF STANDARD MAN. CRNL -4007 - 240- $241(1966)$

DZIEWIATKOWSKI D D FATE OF INGESTED SULFIDE SILFUR LABELED WITH RAOIOACTIVE SULFUR IN THE

J. BIOL. CHEM.

161. 723-729(1945)

THE OISTRIBUTION OF RADIOPHOSPHORUS AND RAOIOGOLO IN RATS AFTER ORAL, INTRAPERITOVEAL AND SUBCUTANEOUS ADMINISTRATION.

J. FRANKLIN INST.

230. $125-13 J^{\circ}(1940)$

BOOK

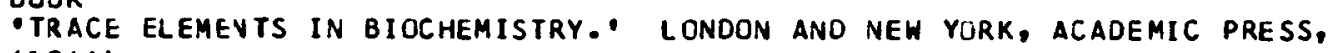
(1966)

INTERNAL DOSE ESTIMATION. REVISION OF STANDARD MAN.

ORNL-4007 213-216 (1966)

BIOLOGICAL AND ENVIRONMENTAL BEHAVIOUR OF RUTHENIUM AND RHODIUM.

'RADIOECOLOGY, V. SCHULT 2 AND A. W. KLEMENT. EDS., PROCEEDINGS OF THE F IRST NATIONAL SYMPOS IUM HELO AT FORT COLLINS, COLO.. SEPT. 10-15, 1961. NEW YORK, REINHOLD P'SBL. CORP. AND WASHINGTON, D. C.. THE AMERICAN INST ITUTE OF BIOLOGICAL SCIENCES.

509- $519(1963)$

ENVIRONMENTAL BEHAVIOR OF CHROMIUM ANO NEPTUNIUM.

-RADIOECOLOGY." $V$. SCHULTZ ANO A. W. KLEMENT, EDS., PROCEEJINGS OF THE $F$ IRST NATIONAL SYMPOSIUM HELD AT FORT COLLINS, COLO., SEPT. I0-15, 1961.

NEW YORK, REINHOLD PUBL. CORP. AND WASHINGION, D. C.. THE AMERICAN INST ITUTE OF BIOLGGICAL SCIENCES,

ITUTE OF BIOLOGICA
$569-576(1963)$

THE NATURAL RAOI UATI IYE SERIES IN ORGANIC MATERIAL.

-RADIDECOLOGY," V. STHJLIZ AND A. H. KLEMENT. EDS.: PROCEEDINGS OF THE F IRST NATIONAL SYMPUSIJM HELD AT FURT COLLINS, COLO., SEPT. 10-15, 1961. NEW YORK, REINHOLD PUBL. CORP. AND WASHINGTON, D. C.. THE AMERICAN INST ITUTE OF BIOLJGICAL SCIENCES,

$611-613(1963)$ 
$5318 \quad B I S H O P M+$

5329

LOWMAN F G+

5335

BRYAN G W+

5340

5373

5387

5395

5416

5417
EXCRETION AND RETENTION CF RADIOACTIVE STRONTIUM IN NORMAL MEN FOLLOWI NG A SINGLE INTRAVENOUS INJECTION.

INTERN. S. RADIATION BIOL. 2. $125-142(1960)$

INTERACTIONS OF THE ENVIRONMENTAL AND BIOLOGICAL FACTORS ON THE OISTRI BUTION OF TRACE ELEMENTS IN THE MARINE EVVIRONMENT.

- DI SPOSAL DF RADIOACTIVE WASTES INTO SEAS. DCEANS AND SURFACE HATERS." PRJCEEDINGS OF THE SYMPOSIJM HELD BY THE INTERNATIONAL ATOMIC ENERGY AGE INCY AT VIENNA, 16-20 MAY 1966. VIENNA, INTERNATIONAL ATOMI: ENERGY AGEN

$249-266(1966)$

$15 M-72 / 15$

ICONF-6605

(1966)

- $249-266(1966)$

ACCUMULATIOV OF RADIONJCLIDES BY AQUATIC ORGANISMS OF ECONOMIC IMPORTA NCE IN THE UNITED KINGDOM.

-DISPJSAL DF RADIOACTIVE NASTES INTO SEAS, JCEANS AND SURFA-E WATERS." PROCEEDINGS OF THE SYMPOSIUM HELD BY THE IVTERNATIONAL ATOMI: ENERGY AGE NCY AT VIENNA, 16-20 MAY 1966. VIENNA. INTERNATIONAL ATOMIL ENERGY AGEN $C Y$,

$623-637(1966)$

$1 \mathrm{SM}-72 / 39$

ICONF-660507

11966

, $623-627(1966)$

COMPARATIVE ELEMENTAL ANALYSES OF A STANDARD PLAVt MATERIAL. ANALYST

92. 124- 131 (1967)

RELATION OF ASH CONSTITUENTS OF PASTURE PLANTS TO THE OXIOATION-REDUCT IOV PJTENTIALS OF NUTRIENTS. SOIL SCI

$$
30,421-430(1930)
$$

THE COMPOSITION OF MILK.

-FUNDAMENTALS OF DAIRY CHEMISTRY, B. H. WEBB AVD A. H. JOHNSON, EDS. ESTPORT, CONN.. THE AVI PJBLISHING, COMPAVY, INC.,

$1-36(1965)$

UPTAKE OF RAOIOACTIVITY BY AQUATIC PLANTS AND LDCATIDN IN THE CELLS. II. UPTAKE OF CERIUM-144 BY THE FRESHWATER PLANT NITELLA OPA:A. J. EXPTL. BOTANY

18. $34-53$ (1967)

THE INTERRELATIONSHIP BETWEEN THALLIUM AND POIASSIUM IN ANIMALS. J. PHARMACOL. EXPTL. THERAP.

155, $187-201(1967)$

FAILURE TO CONFIRM A PROLONGATION OF THE BIOLOGICAL HALF-LIFE OF 22-NA IV HYPERTENSIVE PAT IENTS.

CIRCULATION RES.

19. $750-754(1966)$ 


$\begin{array}{ll}5427 & \text { MENA I+ } \\ 5443 & \text { BEASLEY T M+ } \\ 5464 & \text { HAMPSON M A }\end{array}$

547

SCHRDEDER H A +

5480

5524

BUROVINA I V+

5570 HILSON D $0+$

5571 BURTON 0 A

5572 BURTON $C A+$

5573 TAMPLIN A R
CHRONIC MANGANESE POISONING. CLINICAL PICTURE aNd MANGANESE TURNOVER. NEUROLOGY

$$
\text { 17. } 128-136(1967)
$$

DISTRIBUTION AND EXCRETION OF TECHNETIUM IN HUMANS.

HEALTH PHYS.

$$
\text { 12. 1425-1435 (1966) }
$$

UPTAKE OF RADIOACTIVITY BY AQUATIC PLANTS AVD LOCATION IN THE CELLS I. THE EFFECT OF PH ON THE STRONTIUM-90 AND YTTRIUM-90 UPTAKE BY THE G REEN ALGA ULVA LACTUCA AND THE EFFECT OF STABLE YTTRIUM ON YTTRIUM-9O UPT AKE.

J. EXPTL. BOTANY

$$
\text { 18. } 17-33(1967)
$$

ESSENTIAL TRACE METALS IN MAN. ZINC. RELATION TO ENVIRONMENTAL CADMI UM.

J. CHRONIC DI SEASES

$$
\text { 20. } 179-210 \text { (1967) }
$$

ABNORMAL TRACE METALS IN MAN. gERMANIUM.

J. CHRONIC DI SEASES

$$
\text { 20, } 211-224(1967)
$$

LITHIUM, SODIUM, POTASSIUM, RUBIDIUM AND CESIUM IN THE MUSCLES OF MARI NE ORGANISMS OF THE BARENTS AND BLACK SEAS.

DOKL. ACAD. NAUK SSSR

149, 413- 415 (1963)

IOOKL. EARTH SCI. SECT. (ENGLISH TRANSL.)

$149,170-172(1965)$

ICEA-TR-R-1891 (1966)

REMOVAL OF PU-239, W-185, AND PG-210 FROM SOIL BY PLANTS ANJ ION EXTRA CTING SOLUTIONS.

$\mathrm{HW}-80500$

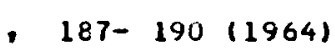

A GRAPHICAL METHOD FOR CALCULATING THE DOSE IV MAN FROM INDIVIDUAL RAD IONUCLIDES IN FALLOUT.

UCRL-14922 (1966)

THE AVAILABLE DISINTEgRATION ENERGY OF ALL RADIOVUCLIDES FOR USE IN DO SIMETRY PROBLEMS. I. RADIONUCLIDES WITH HALF-LIVES OF 12 HOURS OR GRE ATER.

UCRL -50164

(1966)

PREDICTION OF THE MAXIMUM DOSAGE TO MAN FROM THE FALLOUT OF NUCLEAR DE VICES. PART I. ESTIMATION OF THE MAXIMUM CONTAMINATION OF AGRICULTURA $L$ LAND.

UCRL-50163 (PT.I)

(1967) 
$5574 \quad$ NG Y C +

5581 PHELPS D K

$5592 \quad$ ICRP

5593 A ICRP

5593 B ICRP

5594 GEIGY CHEM CO

5595

5596

5597

PREDICTION OF THE MAXIMUM DOSAGE TO MAN FROM THE FALLOUT OF NUCLEAR DE VICES. PART II. ESTIMATION OF THE MAXIMUM DOSE FRDM INTERNAL EMITTERS UCRL-50163(PT.11) (1966)

PARTITIONING OF THE STABLE ELEMENTS, FE, ZN, SC, AND SM WITH A BENTHIC COMMUNITY. ANASCO BAY. PUERTO RICO.

CRADIOECOLOGICAL CONCENTRATION PROCESSES, B. ABERg AND Fo P HUNGATE,

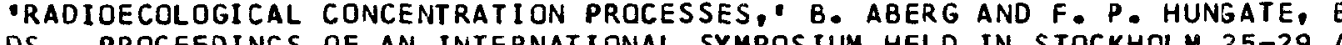
DS. PROCEEDINGS OF AN INTERNATIONAL SYMPOSIUM HELD IN STOCKHOLM 25-29 A PRIL, 1966. LONDON, PERGAMON PRESS,

$721-734(1967)$

ICDNF-660405

- $721-734(1967)$

1962 SUPPLEMENT TO REPORT OF COMMITTEE II ON PERMISSIBLE DOSE FOR INTE RNAL RADIATION (ICRP PUBLICATION 2 I.

-RAOIATION PROTECTION. RECOMMENDATIONS DF THE INTERNATIONAL COMMISSI ON ON RADIOLOGICAL PROTECTION (AS AMENDED 1959 AND ZEVISED 1962).1 OXFORD, PERGAMON PRESS.

$37-68(1964)$

/ICRP-PUBL-B

- $37-68(1964)$

REPORT OF COMMITTEE II ON PERMISSIBLE DOSE FOR INTERNAL RADIATION. - RADIATION PROTECTION. RECOMMENDATIONS OF THE IVTERNATIONAL COMMISSION ON RADIOLOGICAL PROTECTION (1959)." OXFORD, PERGAMON PRESS , $(1960)$

II CRP-PUBL-2

(1960)

REPORT OF COMMITTEE II ON PERMISSIBLE DOSE FOR IVTERNAL RADIATION. HEALTH PHYS.

$$
\text { 3. } 1-383(1960)
$$

TABLE. NORMAL BODY MEASUREMENTS DURING GROWTH, 12 MONTHS-2 YEARS.

- DOCUMENTA GE IGY SEIENTIFIC TABLES IFIFTH ED.1. ' BASLE, SWITZERLAND, $S$. KARGER, KARGER,
$258-258(1759)$

TABLE. CHEMICAL COMPOSITION OF THE BODY AND SOME OF ITS ORGANS.

- DOCUMENTA GEIGY SCIENT IFIC TABLES (FIFTH ED.)." BASLE, SWITZERLAND, S. KARGER,

$282-282(1759)$

TABLE. BODY AND DRGAN WEIGHTS IN MAN.

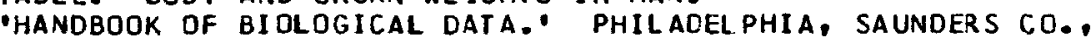
$162-162(1356)$

TABLE. ORGAN WEIGHTS FROM BIRTH TO MATURITY. MAN, NORTH AMERICAN.

-GROWTH INCLUDING REPRODUCTION AND MORPHOLOGICAL DEVELOPMENT,' P. L. ALT MAN AND D. S. DITTMER, EDS. WASHINGTON, D. C., FEDERATION OF AMERICAN S OCIETIES FOR EXPERIMENTAL BIOLOGY,

$347-347$ (1962) 
5599 ALLAWAY W H+

5600

MITCHELL R L+

5601

REDISKE J H+

5604

MITCHELL R L

5605

5608

5708

5757

KATZ J J+

5758

5759

5761
SOREMARK $R$

CANNON H L

HDDGKISS W S+

KATZ J J+

SEABORG G T

JUNGE $こ E$
SYMPOSIUM ON NUTRITION, FORAGE AND PASTURES. SELENIUM IN FORAGES AS R ELATED TO THE GEOGRAPHIC DISTRIBUTION OF MUSCULAR DYSTROPHY IN LIVESTO

ANIMAL SCI.

23, $271-277(1964)$

THE LEAD CONTENT OF PASTURE HERBAGE.

J. SCI. FOOD AGR.

$17,437-440(1966)$

THE ABSORPTION OF FISSION PRODUCTS BY PLANTS. HW-36734 (1955)

THE DISTRIBUTION OF TRACE ELEMENTS IN SOILS AVD GRASSES. PROC. NUTR. SOC. (ENGL. SCOT.)

$$
\text { 1. } 183-189(1944)
$$

BOTANICAL PROSPECTING FOR ORE DEPQSITS.

SCIENCE

$$
\text { 132. } 591-598(1960)
$$

SPECTROGRAPHIC IDENTIFICATION OF MINOR ELEMENTS IN HAY AND GRAIN MIXTU RES.

TRANS. KENTUCKY ACAD. SCI.

$$
\text { 9. } 17-20(1941)
$$

VANADIUM IN SOME BIOLOGICAL SPECIMENS.

J. NUTR.

$$
\text { 92, } 183-190(1967)
$$

CHAPTER VI. NEPTUNIUM.

'CHEMISTRY of the actinide elements, the." london, methuen and co. lto. '204- $238(1957)$

CHAPTER VII. PLUTONIUM.

'CHEMISTRY OF tHe aCtinide elements, the." london, methuen and co. lto.

'239- 330 (1957)

CHAPTER 3. THE ACTINIDE SERIES.

-COMPREHENSIVE INORGANIC CHEMISTRY. PRINCETOV, NEW JERSEY, D. VAN NOST RAND COMPANY, INC.

$$
\text { 1. } 161-223(i 953)
$$

TABLE 1. ATMOSPHERIC GASES OTHER THAN OXYGEN AND NITROGEN.

-AIR CHEMISTRY AND RADIOACTIVITY.' INTERNATIONAL GEOPHYSIOS SERIES, VOL - 4. NEW YORK, ACADEMIC PRESS,

3- 3 (1963) 


$\begin{array}{ll}5763 & \text { KENNA B T+ } \\ 5764 & \text { KURODA P K } \\ 5765 & \text { MORGAN J W } \\ 5768 & \text { KOENIG H+ } \\ 5769 & \text { MAZOR E+ } \\ 5770 \text { A } & \text { OSBURN W S } \\ 5770 \text { B } & \text { OSBORN W S }\end{array}$

5776 KOVALEVSKIY A L

5777

TYUTINA N A+

5778

WR IGHT T L+

5779
ISOLATION OF NATURALLY OCCURRING TECHNETIUM.

J. INDRG. NUCL. CHEM.

23. $142-144(1961)$

RADIOCHEMICAL MEASUREMENT OF THE NATURAL FISSION RATE OF URANIUM AND T HE NATURAL OCCURRENCE OF BA-140.

J. INDRG. NUCL. CHEM.

$$
\text { 3. 345- } 348 \text { (1957) }
$$

THE SIMULTANEOUS DETERMINATION OF RHENIUM AND OSMIUM IN ROCKS BY NEUTR ON ACTIVATION ANALYSIS. ANAL. CHIM. ACTA

$$
\text { 32. } 8-16(1965)
$$

HELIUM, NEON AND ARGON IN THE OCEANS.

DEEP SEA RES.

$$
\text { 11. 243- } 247 \text { (1964) }
$$

RARE GASES IN PACIFIC OCEAN WATER. DEEP SEA RES.

$$
11 \text {, } 929-932 \text { (1964) }
$$

PRIMORDIAL RADIONUCLIDES. THEIR DISTRIBUTION, MOVEMENT, AND BIOLOGICA L EFFECT WITHIN TERRESTRIAL ECOSYSTEMS.

COO-1445-1 (1965)

PRIMORDIAL RADIONUCLIDES. THEIR DISTRIBUTION, MOVEMENT, AND POSSIBLE EFFECT WITHIN TERRESTRIAL ECOSYSTEMS. HEALTH PHYS.

$$
\text { 11. 1275-1295 (1965) }
$$

BIOGEOCHEMI STRY OF TUNGSTEN IN PLANTS.

GEOKHIMIYA

1966, 737- 744 (1966)

IGEOCHEM. INTERN.

3. $555-562(1966)$

AN EXPERIMENT IN BIOGEOCHEMICAL SAMPLING AVD THE METHOD OF DETERMINATI ON OF NIOBIUM IN PLANTS.

GEOKHIMIYA

1959. 550- 554 (1959)

/GEOCHEMISTRY (USSR) (ENGLISH TRANSL.)

1959, 668- 675 (1959)

GEOCHEMISTRY OF THE PLATINUM METALS.

U. S. GEOL. SURV. BULL.

1214. (1965)

ISER A I

DATA FOR GEOCHEMISTRY. CHAPTER G. CHEMICAL COMPOSITION OF RIVERS AND LAKES.

U. S. GEOL. SURV. PROFESS. PAPERS 440. $(1963)$

ISER G $)$ 


\section{CHERDYNTSEV $V$ V+ PLUTONIUM-239 IN NATURE. GEOKHIMIYA \\ 1965, 1257-1259 (1965) \\ IGEOCHEM. INTERN.}

$$
\text { 2. } 918-920 \text { (1965) }
$$

5782 DAS SARMA B+

5786 BAEDECKER P A+

$5788 \quad$ BROOKS R R+

5791 DURUM W H+

5792 FLEISEHER M

5796 MEEHAN W R+

5797 PACHADZHANOV D

$5799 \quad$ RAZIN L V+

5801 VINOGRADOV A P
STUDIES ON GEOCHEMISTRY OF PLATINUM.

ECON. GEOL.

61. $592-597(1966)$

THE DISTRIBUTION OF SOME NDBLE METALS IN METEORITES ANO NATJRAL MATERI ALS.

GEOCHIM. COSMOCHIM. ACTA

$$
\text { 29, } 329-342(1965)
$$

SOME OBSERVATIONS ON THE DISTRIBUTION OF THALLIUM, CADMIUM AND BISMLTH IN SILICATE ROCKS AND THE SIGNIFICANCE OF COVALENCY ON THEIR DEGREE D F ASSOCIATION WITH OTHER ELEMENTS.

GEOCHIM. COSMOCHIM. ACTA

$$
\text { 23, } 100-115(1961)
$$

IMPLICATIONS OF THE MINOR ELEMENT CONTENT OF SOME MAJOR STREAMS OF THE WORLD.

GEOCHIM. COSMOCHIM. ACTA

$$
\text { 27. } 1-11(1963)
$$

SUMMARY OF NEW DATA ON ROCK SAMPLES G-1 AND W-1. 1962-1965. GEOCHIM. COSMOCHIM. ACTA 29. 1263-1283 (1965)

OCCURRENCE OF BERYLLIUM AS A TRACE ELEMENT IN EVVIRONMENTAL MATERIALS. ENVIRON. SCI. TECHNOL.

$$
\text { 1. } 839-844(1967)
$$

GEOCHEMISTRY OF NIOB IUM AND TANTALUM IN CLAYS.

GEOKHIMIYA

1963 , 930- $942(1963)$

/GEOCHEMISTRY (USSR) (ENGLISH TRANSL.

1963. 963-976 (1963)

CONCENTRATION AND DISTRIBUTION PATTERNS OF PLATINUM GROUP METALS IN UL TRABASIC AND ALKALIC ROCKS.

DOKL. ACAD. NAUK SSSR

162, 918- 921 (1965)

CODKL. EARTH SCI. SECT. (ENGLISH TRANSL.)

162. 207- $210(1965)$

AVERAGE CONTENTS OF CHEMICAL ELEMENTS IN THE PRIVCIPAL TYPES OF IGNEOU $S$ ROCKS OF THE EARTH'S CRUST.

GEOKHIMIYA

1962, 555- 571 (1962)

GEOCHEMISTRY (USSR) (ENGLISH TRANSL -

1962. 641- 664 (1962) 


\begin{tabular}{|c|c|c|}
\hline 5803 & NODDAEK I+ & $\begin{array}{l}\text { THE GEQCHEMISTRY OF RHENIJM. } \\
\text { 2. PHYSIK. CHEM. (LEIPZIG) } \\
154,207-244(1931)\end{array}$ \\
\hline 5804 & TRUOG E & $\begin{array}{l}\text { SOIL AS A MEDIUM FOR PLANT GROWTH. } \\
\text { 'MINERAL NUTRIIIION OF PLANTS, E. TRUOG, ED. MADISON, THE UNIVERSITY OF } \\
\text { WISCONSIN PRESS, } \\
28-55(1951)\end{array}$ \\
\hline 5813 & HAMPSON B L & $\begin{array}{l}\text { RESTRICTED DISPERSION OF ZIRCONIUM-95 AND OF NIDBIUM-95 AFTER RELEASE } \\
\text { TO THE SEA IN NUCLEAR FUEL REPROCESSING EFFLUENT. } \\
\text { HEALTH PHYS. } \\
13,1093-1103(1967)\end{array}$ \\
\hline 5840 & GLASSTONE $S$ & $\begin{array}{l}\text { CHAPTER 5. NATURAL RADIOACTIVITY. } \\
\text { "SOURCEBOOK ON ATOMIC ENERGY." THIRD EDITION. PRINCETON, NEW JERSEY, D } \\
\text { VAN NOSTRAND COMPANY, INC.. } \\
135-156(1967)\end{array}$ \\
\hline 5869 & MCCORMACK K R+ & $\begin{array}{l}\text { LOVG-TERM STUDIES OF SOLITARY AUTONOMOUS THYROID NODULES. } \\
\text { J. NUCL. MED. } \\
8.701-708(1967)\end{array}$ \\
\hline 5879 & TUREKIAN K K+ & $\begin{array}{l}\text { ESTIMATES OF THE AVERAGE ABUNDANCE OF CU, MN, PB, TI, NI, AND CR IN SU } \\
\text { RFACE WATERS OF MAINE. } \\
\text { BULL. GEOL. SOC. AM. } \\
\text { 67, } 1129-1131(1956)\end{array}$ \\
\hline 5892 & SILVEY W D & $\begin{array}{l}\text { OCCURRENCE OF SELECTED MINDR ELEMENTS IN THE WATERS OF CALIFORNIA. } \\
\text { U. S. GEOL. SURV. WATER SUPPLY PAPERS } \\
\text { 1535, }(1967)\end{array}$ \\
\hline 5909 & THOMPSDN R C+ & $\begin{array}{l}\text { DISTRIBUTION AND RETENTION OF RUTHENIUM IN RATS FOLLOWING INTRAPERITON } \\
\text { EAL ADMINISTRATION. } \\
\text { HW-35917 } \\
\end{array}$ \\
\hline 5910 & BALLOU J E+ & 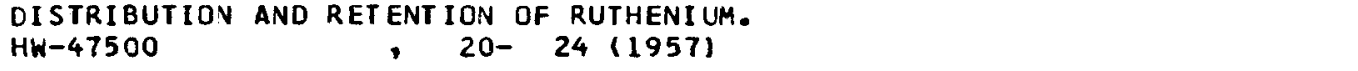 \\
\hline 5911 & WEEKS M H+ & $\begin{array}{l}\text { ABSORPTION, DISTRIBUTION, AND RETENTION DF RUTHENIUM IN RATS FOLLOWING } \\
\text { CHRONIC ORAL ADMINISTRATION. } \\
\text { HW-35917 } \\
\text { M } 74-81(1955)\end{array}$ \\
\hline 5936 & STRAIN W H+ & $\begin{array}{l}\text { RETENTION DF RADIOCOBALT BY HAIR, BONE AND VASCULAR TISSUE. } \\
\text { J. NUCL. MED. } \\
\quad 6,831-837(1965)\end{array}$ \\
\hline 5938 & US PHS & $\begin{array}{l}\text { ACTIVITY MASS RELATIONSHIP- SPECIFIC ACTIVITY. } \\
\text { 'RADIOLOGICAL HEALTH HANDBOOK.' REVISED EOITION. WASHINGTON, D. C.. U. } \\
\text { S. PUBLIC HEALTH SERVICE, } \\
103-103(1960) \\
\text { IPB-121784R } \quad, 103-103(1960)\end{array}$ \\
\hline
\end{tabular}


5943 A HARRISON F L

5943 B HARRISON F L

5948 CUTSHALL N H

5968 A STROMINGER D+

5968 B STROMINGER J+

5968 C STROMINGER J+

5969

KARAMYAN K A

5973

PILLAI $\vee K$

5991

5992

5994
ACCUMULATION AND DISTRIBUTION OF MANGANESE-54 AND ZINC-65 IN FRESHWATE R CLAMS.

(1967)

ACCUMULATION AND DISTRIBUTION OF MANGANESE-54 AND ZINC-65 IN FRESTWATE R CLAMS.

-RADIOECOLOGY, PROCEEDINGS OF THE SECOND NATIONAL SYMPOSIUM HELD IN ANN ARBOR, MICH.: MAY 15-17, 1967. TO BE PUBLISHED.

(196X)

ICONF-670503

(196x)

CHROMIUM-51 IN THE COLUMBIA RIVER AND ADJACENT PACIFIC OCEAN. RLO-1750-22(VOL.4) PAP.- 3 (1967)

TABLE OF ISOTOPES. UCRL-1 $928(R E V .2)$

(1958)

TABLE OF ISOTOPES.

REV. MODERN PHYS.

30, $585-904(1968)$

TABLE OF ISOTOPES.

-RAOIOLOGICAL HEALTH HANOBOOK. - REVISED EOITION. WASHINGTON, D. C., U. S. PUBLIC HEALTH SERVICE,

$195-458(2960)$

IPB-121784R

- $195-458(1960)$

CORRELATION BETHEEN RHENIJM, SELENIUM AND TELLURIUM IN THE MOLYBDENITE

$S$ OF THE KADZHARAN COPPER-MOLYBDENUM DEPOSIT.

GEOKHIMIYA

1962, $171-174(1962$

IGEOCHEMISTRY (USSR) (ENGLISH TRANSL.)

1962, $194-198(1962)$

CHEMICAL STUDIES ON INDIAN SEAWEEDS. 1. MINERAL CONSTITUENTS.

PROC. INDIAN ACAD. SCI. SECT. B

$44, \quad 3-29(1956)$

UPTAKE OF PROMETHIUM-147 BY A FRESH hATER ALGAE, CHLORELLA PYRENOIODSA

AED-CONF-66-2BJ-6 (1966)

CHAPTER 5. MINOR ELEMENTS IN SEA WATER.

- Chemical oceanography, J. P. RILEY and g. SKIRROW, EDS. LONDON AND Ne $W$ YORK, ACADEMIC PRESS,

$$
\text { 1. 163-196 (1965) }
$$

THE CHEMISTRY AND ANALYSIS OF TRACE METALS IN SEA WATER. SECTION 3. NEUTRON ACTIVATION ANALYSIS OF RUTHENIUM IN SEA WATER.

IID-23295 (SECT.3) (1966) 
5995 HAYES D $W+$

5996 HEDGES D H+

5997

VINOGRADOVA $Z$ A

5998

VINOGRADOV A P

5999 BROOKS R R+

6000

FUKA I R+

6001

6002

CULKIN F+

6003 YOUNG E G+

6004
THE CHEMISTRY AND ANALYSIS OF TRACE METALS IV SEA HATER. SE:TION 4. RARE EARTH DISTRIBUTION IN WATERS OF GULF OF MEXICO.

TID-23295(SECT.4) (1966)

THE CHEMISTRY AND ANALYS IS OF TRACE METALS IN SEA hateR. SECTION 5. ZIRCONIUM ANALYSIS OF SEA WATER.

TID-23295(SECT.5) (1966)

ELEMENTAL COMPOSITION OF THE BLACK SEA PLANKTON.

DOKL. ACAD. NAUK SSSR

147, 1453-146) (1962)

IDOKL. EARTH SCI. SECT. (ENGLISH TRANSL.)

147. 217- $219(19641$

BOJK.

-elementary chemical composition of marive jRganisms. The, transl. by J - EFRON AND J. k. SETLOW. NEW haVEN, SEARS fOUNDATION fOR MARINE RESEAR CH, YALE UNIVERSITY. (1953)

THE BIOGEOCHEMISTRY OF TRACE ELEMENT UPTAKE BY SUME NEW ZEALAND BIVALV

LIMNOL. OCEANOG.

$10,521-527(1965)$

ACTIVATION ANALYSES OF VANADIUM, ARSENIC, MOLYBDENUM, TUNGSTEN, RHENIU M. AND GOLD IN MARINE ORGANISMS.

LIMNOL. OCEANOG.

7. $186-200$ (1962)

TRACE ELEMENT ANALYSES OF SOME MARINE ORGANISMS

-INTER-AMERICAN SYMPOSIUM ON THE PEACEFUL APPLICATION OF NUCLEAR ENERGY, 5TH, 1964. WASHINGTON, D. C.. PAN AMERICAV UNION $233-239(1365)$

THE OCCURRENCE OF GALLIUM IN MARINE ORGAVISMS.

J. MARINE BIOL. ASSOC. U. K. 37. $607-615(1958)$

THE DCCURRENCE JF INORGANIC ELEMENTS IN MaRIVE algae OF THE atLANTIC P ROVINCES OF CANADA.

CAN. J. BOTANY

36. 301- 31J (1958)

CHEMICAL STUDIES ON THE OCEAN (PART 78). CHEMICAL STUDIES ON THE SEAW EEDS (VI). THE CONTENT OF CALCIUM, MAGNESIUM AND PHOSPHORUS IN SEAWEE DS.

RECORDS OCEANOG. WORKS JAPAN, SPEC. NO.

4. 73- 78 (1960) 


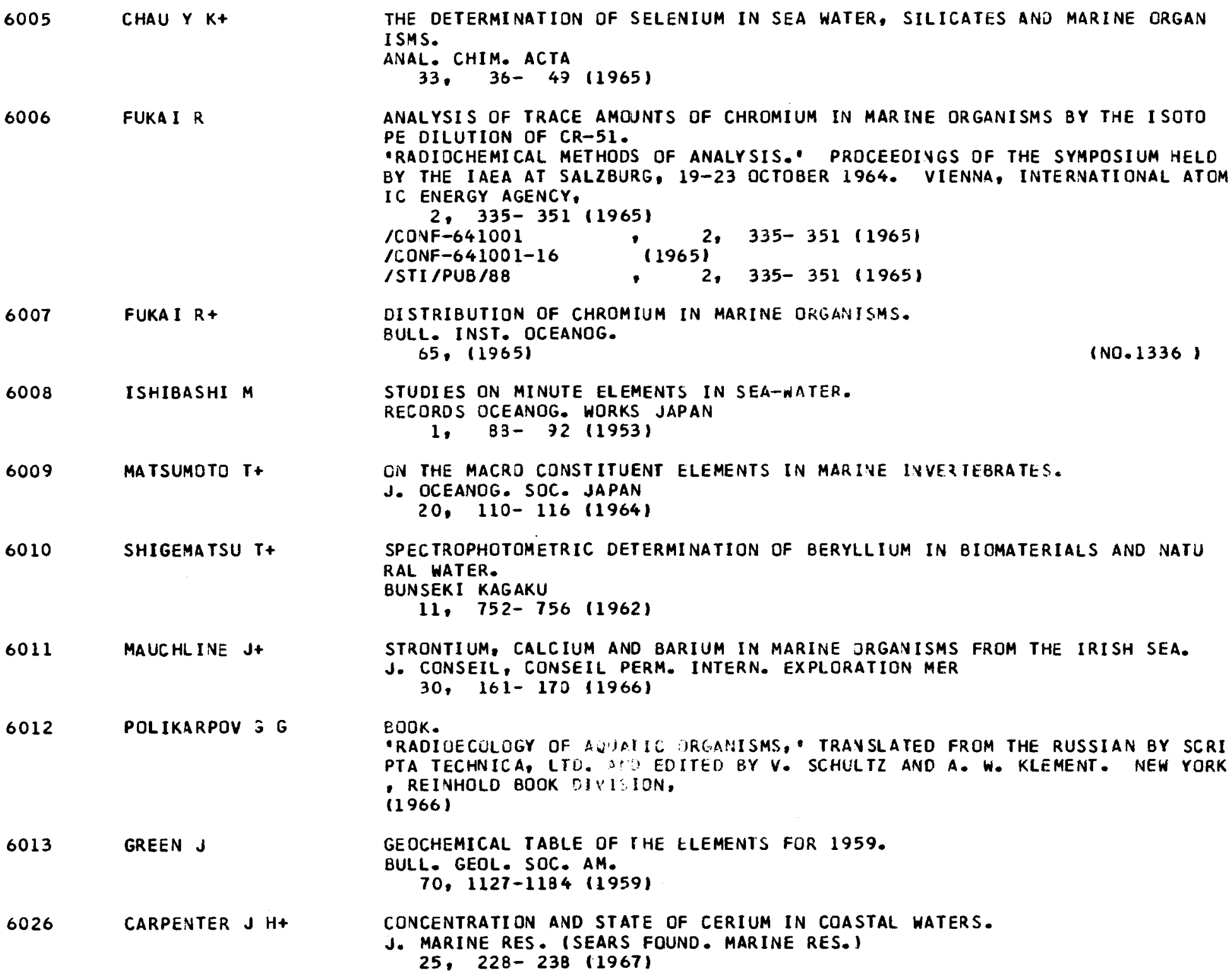

SPECTROPHOTOMETRIC DETERMINATION OF BERYLLIUM IN BIOMATERIALS AND NATU RAL WATER.

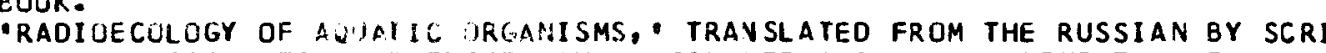
PTA TECHNICA, LTO. A O EDITED BY $V$. SCHULTZ AND A. W. KLEMENT. NEW YORK - REINHOLD BOOK DIYISION, (1966)

GEOCHEMICAL TABLE OF IHE ELEMENTS FOR 1959.

BULL. GEOL. SOC. AM.$$
\text { 70, } 1127-1184(1959)
$$

CONCENTRATION AND STATE OF CERIUM IN COASTAL WATERS.

J. MARINE RES. (SEARS FOUND. MARINE RES.)

$25, \quad 228-238(1967)$ 
$6045 \quad$ SHIBUYA M+

6048 IWASHIMA Y+

6049 WARD E E

6050 ATEN A H

6051 MERLINI M+
STUDY ON THE NEUTRON ACT IVATION ANALYSIS OF INORGANIC MINOR FLEMENTS I N PLANTS AND SOILS (I). THE DETERMINATION OF IVDIVIDUAL RARE EARTH ELE MENTS IN RICE PLANTS ANO SOILS BY ACTIVATION ANALYSIS AND FOEUSINO CHR OMATOGRAPHY

- PROC. JAPAN CONFERENCE ON RADIOISOTOPES, FIFTH.' HELD IN TOKYO, MAY 21 $-23,1963$. TOKYO, JAPAN ATOMIC INDUSTRIAL FORUM, INC., $228-230(1763)$

ICONF-447

$(1963)$

ENVIRONMENTAL CONTAMINATION WITH RADIORUTHENIUM 1961-1965. J. RADIATION RES.

$$
\text { T, } 91-111(1966)
$$

UPTAKE OF PLUTONIUM BY THE LOBSTER HOMARUS VULGARIS. NATURE

209, 625-626(1966)

PERMISSIBLE CONCENTRATIONS OF RADIONUCL IDES IN SEA WATER HEALTH PHYS.

$$
\text { 6, 114- } 125(1961)
$$

ACTIVATION ANALYSIS IN STUDIES OF AN AQUATIC ECOSYSTEM.

- NuCl. aCt IVATION TECHNIQUES IN THE LIFE SCIENCES." proceEdings DF THE SYMPOSIUM HELD BY IHE IAEA IN AMSTERDAM, 8-12 MAY 1967. VIENNA, INTERNA TIONAL ATOMIC ENERGY AGENCY.

615- $629(1767)$

ISTI/PUB/155
ISM $91 / 7$

6052 GOLDBERG E J+

RARE-EARTH DI STRIBUTIONS IN THE MARINE ENVIROYMENT.

J. GEOPHYS. RES.

$68,4209-4217(1963)$

6054 HAMAGULHI H+

$6056 \quad$ KAMEUS K

6058 KRUMHOLZ L L
INORGANIC CONSTITUENTS IN BI OLOGICAL MATERIALS. XIV. THALLIUM, SELENI UM AND ARSENIC CONTENTS OF MINAMATA OISTRICTS.

J. CHEM. SOC. JAPAN. PJRE CHEM. SECT.

81. $920-927(1960)$

STUDY ON ABUNDANCE OR RARE EARTH ELEMENTS IV MARINE. GRGANISMS.

J. RADIATION RES.

3. 89- 103 (1962)

ECOLOGICAL FACTORS INVOLVED IN THE UPTAKE, ACCUMULAIION, ANO LOSS DF R

ADIONUCLIDES BY AQUATIC ORGANISMS. -EFFECTS OF ATOMIC RADIATION ON OCEANOGRAPHY AND FISHERIES, THE.- REPOR $T$ OF THE COMMITTEE ON EFFECTS OF ATOMIC RADIATION ON OCEANOGRAPHY AND FI SHERIES OF THE NATIONAL ACADEMY OF SCIENCES STUDY OF THE BIOLOGICAL EFFE CTS OF ATOMIC RADIATION. WASHINGTON, D. C.. VATIONAL ACADEMY OF SCIENCE S-NATIONAL RESEARCH COUNCIL.

69- $79(1957)$

JNAS-NRC/PUB-551 , 69- $79(1957)$ 


$\begin{array}{ll}6059 & \text { SCHUTZ DF } \\ 6060 & \text { ROBERTSON DE } \\ 6062 & \text { WILLIAMS K T+ } \\ 6064 & \text { WATSON D G+ } \\ 6079 & \text { CLINE J F } \\ 6083 & \text { SAINSBURY C L+ } \\ 6085 & \text { FUKA } 1 \text { R }\end{array}$

6086

VAN DEN WINKEL P+

ADAMEK A+

THE GEOGRAPHICAL ANO VERTILAL DISTRIBUTION OF SEVERAL TRACE ELEMINIS, I N SEA HATER.

TID-21067

$(1964)$

TRACE ELEMENTS IN MARINE DRGANISMS.

BNHL-481-2 $56-59(1967)$

OCCURRENCE OF SELENIUM IN THE COLORADO RIVER AND SOME OF ITS TRIBUTARI

ES.

7, $431-432$ (1935)

RADIONUCLIDES IN COLUMBIA RIVER GIOTA.

BNWL-280 126- $127(1966)$

UPTAKE OF 241-AM AND 23\%-PIS BY PLANTS.

BNHL-714 $8.24-8.25(1968)$

GEOCHEMICAL CYCLE GF SELECTED TRACE ELEMENTS IN THE TIN-TUNGSTEN-BERYL LIUM DISTRICT. WESTERN SEWARD PENINSULA, ALASKA. A RECONNAISSANCE STU or.

U. S. GEOL. SURV. BULL.

1242, (1968)

(SER F I

DISTRIBUTION OF COBALT IN MARINE ORGANISMS.

-I AEA RADIOACTIVITY IN THE SEA SERIES." VIENYA, INTERNATIONAL aTOMIC EN ERGY AGENCY,

23. (1968)

SEPARATION SCHEME FOR THE DETERMINATICN DF NINE ELEMENTS IN BIOLOGICAL MATER I AL.

-NUCl. ACTIVATION TECHNIQUES IN THE LIFE SCIENCES.' PROCEEDINGS OF IHE SYMPOSIUM HELD BY THE IAEA IN AMSTERDAM, 8- 12 MAY 1967. VIENNA, INTERNA TI ONAL ATOMIC ENERGY AGENCY.

$159-172(1967)$

ISTI/PUB/155

$1 \mathrm{SM}-91 / 30$

(156-1721:46:

SUBSTOICHIOMETRIC DETERMINATION OF TRACES OF THALLIUM AND PLATINUM BY ACTIVATION ANALYSIS.

- NuCl. activation techniglis in THE LIFE SCIENCES.- PROCEedings OF the SYMPOSILIM HELI) BY $\cdots$ IAFA IN ANS?EKDAM, 8-12 MAY 1967. VIENNA, INTERNA TIONAL UTOMIC ES

$189-1441136$

/STI/PUB/15S

$/ S M-91 / 51$

$$
\begin{aligned}
& \text { "NCY, } \\
& \text { (189- } 194 \text { (1967) }
\end{aligned}
$$

TRACE ANALYSIS OF BIOLOGICAL MATERIALS BY MASS SPECTROMETRY AND ISOTOP E DILUT ION. SURVEY PAPER.

- NUCL. ACIIVATION TECHNIQUES IN THE LIFE SCIENCES. PROCEEDINGS OF THE SYMPOSIUM HELD BY THE IAEA IN AMSTERDAM, B-12 MAY 1967. VIENNA, INTERNA TIONAL ATOMIC ENERGY AGENCY.

$211-228(1767)$

/STI /PUB /155

$/ S M-91 / 69$
(1967) $228(1967)$ 
6089 GIRARDI F+

6091

6093

6094

6095

6097

6098

6099

6104
VERKHOVSKAYA I N+

SHACKLETTE + T

TURNER R C +

WALDBOTT G L

BELOZEROV E $S$

ROSHOLT J N+

FRANCIS : $w+$

SHIMP N F+
ELEMENTAL ANALYSIS OF A BIOLOGICAL STANDARD REFERENCE MATERIAL BY NONDESTRUCTIVE METHODS. COMPARI SON BETWEEN RADIJACTIVATION A VALYSIS ANO $X$-RAY FLUORESCENCE SPECTROSCOPY

-NUCL. ACTIVATION TECHNIQUES IN THE LIFE SCIENCES.' PROCEEDINGS DF THE SYMPOSIUM HELD BY THE IAEA IN AMSTERDAM, 8-12 MAY 1967. VIENNA, INTERNA TIONAL ATOMIC ENERGY AGENCY,

229- $246(1767)$

ISTI/PUB/155 229- 246 (1967)

$/ S M-91 / 10$

$(1967)$

THE NATURALLY OCCURRING ALPHA-RAY ACTIVITY OF FODDS.

HEALTH PHYS.

$$
\text { 1. } 268-275(1958)
$$

FLUORIDE IN FOOD.

AM. J. CLIN. NUTR.

12. $455-462(1963)$

THE TOPOGRAPHY OF GALLIUM IN THE HEALTHY HUMAN BOOY. BYUL. EKSPERIM. BIOL. I MED.

62. 61- $63(1966)$

62, $1141-1142(1966)$

(ISSUE 10 )

EVOLUTION OF THE ISOTOPIC COMPOSITION OF URANIUM AND THORIUM IN SOIL P ROF ILES.

GEOL. SOC. AM. BULL.

$77,987-1004(1966)$

THE MIGRATION OF NATURAL RADIOACTIVE ELEMENTS UNDER NATURAL SONDITIONS AND THEIR DISTRIBUTION ACCORDING TO BIOTIC AND ABIOTIC ENVIRONMENTAL AND THEIR DISTRIBUTION ACCORDING TO BIOTIC AND ABIOTIC ENVIRONMENTAL COMPONENTS.

- Radioecological concentration processes, B. aberg and f. P. hungate, e DS. PROCEEDINGS OF AN INTERNATIONAL SYMPOSIUM HELD IN STOZKHOLM 25-29 A PRIL, 1966. LONDON, PERGAMON PRESS,

313- $328(1967)$

ICONF-660405

- $313-328(1967)$

ELEMENT CONTENT OF BRYOPHYTES.

U. S. GEOL. SURV. BULL.

$1198 .(1965)$

ISER D ,

RADIOACTIVE INGROWTH OF POLONIUM-210 IM TUEACCJ PLANTS.

J. AGR. FODD CHEM.

15. 704- 706 (1967)

SPECTROCHEMICAL ANALYSIS OF SOILS AND BIOLOGICAL MATERIALS. SOIL SCI .

$$
\text { 83, 51- } 64 \text { (1957) }
$$


6105 BOWEN H J

6106 MOISEENKO U I

6108

MURAKAMI Y+

6110

SPECTOR WS

6111

SCHROEDER HA+

6145

YAMAGATA $N+$

6146

NELSON $0 \mathrm{j+}$

6147

KEVERN N R +

6149

GUSTAFSON P F

THE DETERMINATION

TION ANALYS
AERE-R 4309

(1963)

BIOGEOCHEMI CAL SURVEYS IN PROSPECTING FOR URANIUM IN MARSHY AREAS. GEOKHIMIYA

1959. 96- 98 (1959)

(GEOCHEMISTRY (USSR) (ENGLISH TRANSL.)

1959. 117- 121 (1959)

CHROMIUM AYD MANGANESE IN JAPANESt DIET.

J. RADIATION RES.

6. $105-110(1965)$

TABLE 55. VERTEBRATE TISSUE AND ORGANS. CHEMICAL COMPOSITION. PART IV. MUSCLE. SECTION 1. MAN, CAT, CATILE, DOG, RABEIT, RAT. SECTION 2

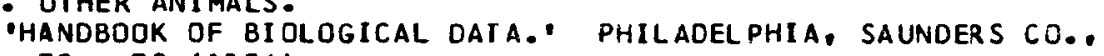
$72-72(1756)$

CHAPTER 4. COMPOSITION OF HUMAN DIET.

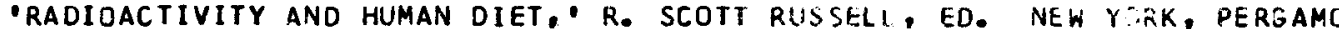
N PRESS.

$65-83(1966)$

essential trace metals in man. cobalt.

J. CHRONIC DI SEASES

20. $869-889(1967)$

THE OIFFEREVT DISTRIBUTION OF RUBIOIUM AND CESIUM IN NATURAL PLANTS.

BULL. CHEM. SOC. JAPAN

(1959)

CESIUM AND POTASSIUM IN WHITE CRAPPIE AND OTHER CLINCH RIVER FISH. ORNL-4007

EFFECT OF TROPHIC LEVEL ON RAOIONUCLIDE ACCUMULATION BY FISH. ORNL-4007 $88-88$ (1966)

COMMENTS ON RACIOYiCLIOFS IH AQUATIC ECOSYSTEMS.

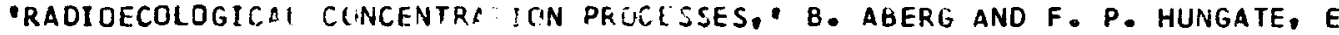
DS. PRCCEED?: " IF AN INI : PAATIONAL SYMPOSIUM HELC IN STOCKHOLM 25-29 A

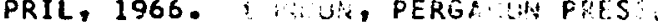

$853-858(196)$

ICONF-660405

- $853-858(i \div 67)$

CALCIUM AND STRONTIUM IN SWEDISH WATERS AND FISH AND ACCUMULATION OF 9 O-SR.

-RADIOECOLOGICAL CONCENTRATION PROCESSES, B. ABERG, AND F. P. HUNGATE, E DS. PROCEEDINGS OF AN INTERNATICNAL SYMPOSIUM HELII IN STOCKHOLM 25-29 A PRIL, 1966 . LONDON, PERGAMON PRESS,

879- 896 (1767)

/CONF-6E 0405

- $879.896(1967)$ 


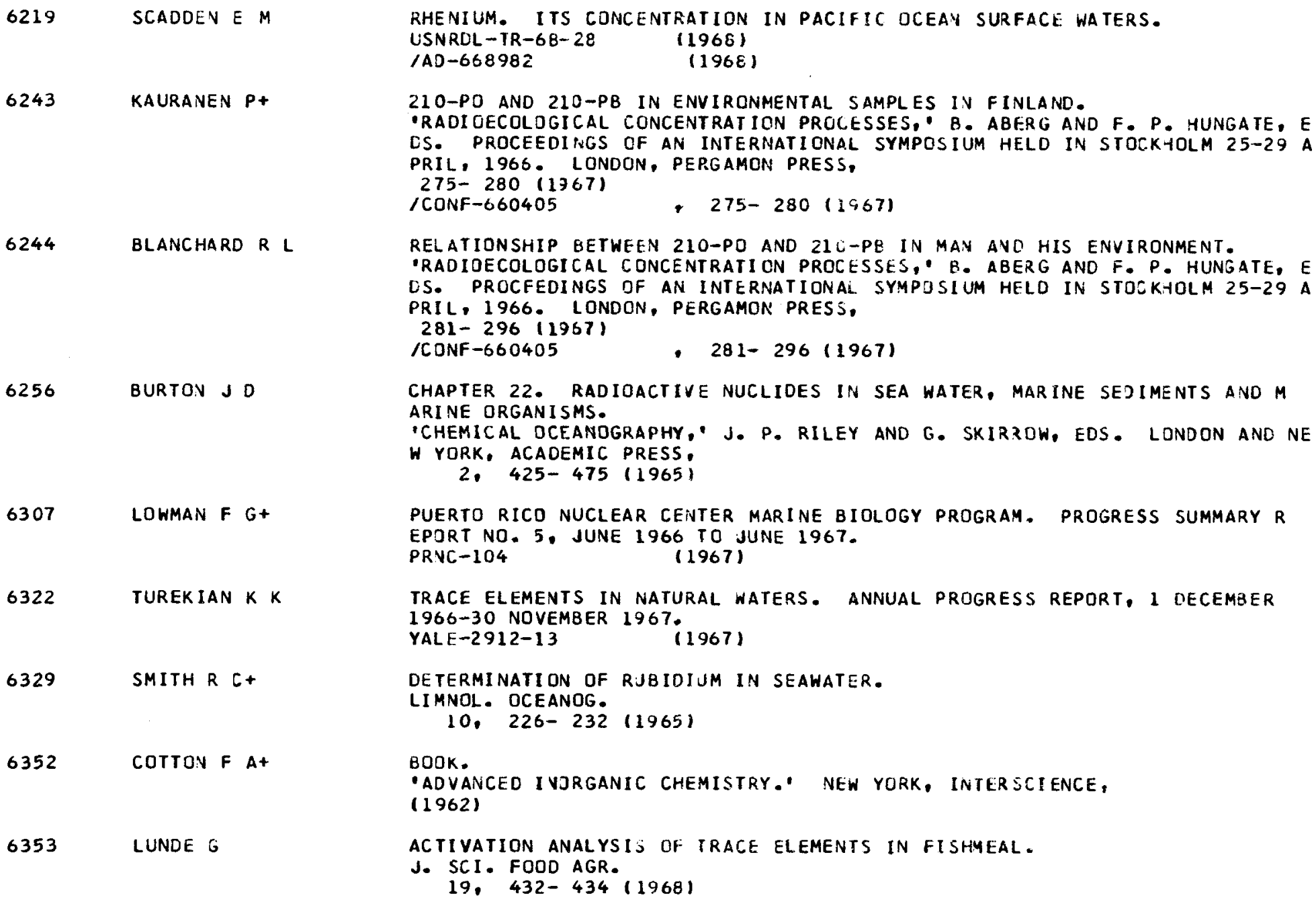

RELATIONSHIP BETWEEN 210-PO AND $21 \mathrm{CL}-\mathrm{PB}$ IN MAN ANO HIS ENVIRONMENT. -RADIOECOLOGICAL CONCENTRATI ON PROCESSES, B. aberg ano F. P. hungate, E CS. PROCFEDINGS DF AN INTERNATIONAL SYMPOSIUM HELD IN STOCKHOLM 25-29 A PRIL, 1966. LONDON, PERGAMON PRESS,

CHAPTER 22. RADIOACTIVE NUCLIDES IN SEA WATER, MARINE SEJIMENTS AND M ARINE ORGANISMS.

'CHEMICAL DCEANDGRAPHY,' J. P. RILEY AND G. SKIRROW, EDS. LONDON AND NE W YORK. ACADEMIC PRESS. 2. 425- 475 (1965)

PUERTO RICD NUCLEAR CENTER MARINE BIOLOGY PROGRAM. PROGRESS SUMMARY R EPORT NO. 5. JUNE 1966 TO JUNE 1967.

PRVC-104 (1967)

trace ELEMENTS IN NATURAL WATERS. ANNUAL PROGRESS REPORT, 1 DeCEMBER 1966-30 NOVEMBER 1967

YALE-2912-13 (1967)

DETERMINATION OF RJBIDIUM IN SEAHATER.

LIMNOL. OCEANOG.$$
\text { 10. 226- } 232(1965)
$$

BOOK.

'ADVANCED I VJRGANIC CHEMISTRY." NEW YORK, INTERSCIENCE, (1962)

ACTIVATION ANALYSIS OF TRACE ELEMENTS IN FISHMEAL.

J. SCI. FOOD AGR.

19. $432-434$ (1968) 